Pacific Northwest

National Laboratory

Operated by Battelle for the

U.S. Department of Energy

\title{
Summary of Uncertainty Estimation Results for Hanford Tank Chemical and Radionuclide Inventories
}

T.A. Ferryman

B. G. Amidan

G. Chen

S. A. Hartley

C. A. LoPresti

J. G. Hill

T. J. DeForest

F. Gao

K. M. Remund

B. C. Simpson (Lockheed Martin Hanford Corporation)

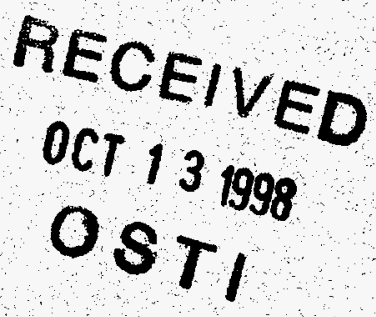

September 1998

Prepared for the U.S. Department of Energy under Contract DE-AC06-76RLO 1830

\section{MASTER JA'}

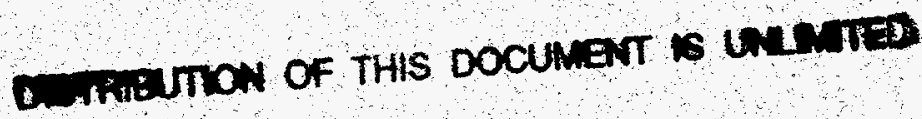




\title{
DISCLAIMER
}

This report was prepared as an account of work sponsored by an agency of the United States Government. Neither the United States Government nor any agency thereof, nor Battelle Memorial Institute, nor any of their employees, makes any warranty, express or implied, or assumes any legal liability or responsibility for the accuracy, completeness, or usefulness of any information, apparatus, product, or process disclosed, or represents that its use would not infringe privately owned rights. Reference herein to any specific commercial product, process, or service by trade name, trademark, manufacturer, or otherwise does not necessarily constitute or imply its endorsement, recommendation, or favoring by the United States Government or any agency thereof; or Battelle Memorial Institute. The views and opinions of authors expressed herein do not necessarily state or reflect those of the United States Government or any agency thereof.

\author{
PACIFIC NORTHWEST NATIONAL LABORATORY \\ operated by \\ BATTELLE \\ for the \\ UNITED STATES DEPARTMENT OF ENERGY \\ under Contract DE-AC06-76RLO 1830
}

This document was printed on recycled paper. 


\section{DISCLAIMER}

Portions of this document may be illegible in electronic image products. Images are produced from the best available original document. 


\title{
Summary of Uncertainty Estimation Results for Hanford Tank Chemical and Radionuclide Inventories
}

\author{
T. A. Ferryman \\ B. G. Amidan \\ G. Chen \\ S. A. Hartley \\ C. A. LoPresti \\ J. G. Hill \\ T. J. DeForest \\ F. Gao \\ K. M. Remund
}

B. C. Simpson (Lockheed Martin Hanford Corporation)

September 1998

Prepared for the U.S. Department of Energy under Contract DE-AC06-76RLO 1830

Pacific Northwest National Laboratory Richland, Washington 99352 


\section{Summary}

The exact physical and chemical nature of 55 million gallons of radioactive waste held in 177 underground waste tanks at the Hanford Site is not known in sufficient detail to support safety, retrieval, and immobilization missions. The Hanford Engineering Analysis "Best-Basis" team has made point estimates of the inventories in each tank. The purpose of this study is to estimate probability distributions for each of the analytes and tanks for which the Hanford Best-Basis team has made point estimates. Uncertainty intervals can then be calculated for the Best-Basis inventories and should facilitate the cleanup missions.

The methodology used to generate the results published in the Tank Characterization Database (TCD) and summarized in this paper is based on scientific principles, sound technical knowledge of the realities associated with the Hanford waste tanks, the chemical analysis of actual samples from the tanks, the Hanford Best-Basis research, and historical data records. The methodology builds on research conducted by Pacific Northwest National Laboratory (PNNL) over the last few years.

Based on the process histories, waste storage practices, and historical data records, some of the waste can be partitioned into more homogeneous subsets that can be identified with tanks and locations within tanks. Using this method, we can maximize the information extracted from the relatively few samples we have for each tank, combining sample data information from similar tanks to generate a sample-based estimate of the chemical and radionuclide concentrations of each of the many waste subsets.

By multiplying the concentrations for a specific constituent (micrograms of the constituent per gram of waste, or microcuries of the constituent per gram of waste) by the density (grams per liter) by the volume in a tank (liters), we can estimate the mass of a particular constituent (or radionuclide activity) in each tank. This methodology provides that estimate, but does so in a probabilistic framework, using and getting probability distributions instead of single numbers. The methodology is described in detail in PNNL-11842, Methodology for Uncertainty Estimation of Hanford Tank Chemical and Radionuclide Inventories and Concentrations (Chen, et al. 1998).

Appendix A of this report summarizes the results of the study. The full set of results (in percentiles, 1 99) is available through the TCD, (http://twins.pnl.gov:8001) Appendix A consists of a very large table with a row for every tank/analyte combination and the following columns:

- Tank name

- Analyte name

- Units (kilograms or Curies)

- The May 1998 Best-Basis inventory estimate (used in implementation of the methodology)

- The September 1998 Best-Basis inventory estimate (at the time of generating this report)

- Best-Basis inventory uncertainty estimate information:

- The median ( 50 percentile) estimated inventories

- Four tail values representing the uncertainty:

- low (1 percentile) estimated inventory

- medium low (10 percentile) estimated inventory

- medium high (90 percentile) estimated inventory

- high (99 percentile) estimated inventory

- The mean estimated inventory

- The standard deviation estimated inventory.

The majority of Best-Basis inventory estimates are "near" the median of the uncertainty distributions calculated using the methodology for each tank/analyte. Specifically, $85 \%$ of the tank/analyte values for 
the May Best-Basis values were inside the $80 \%$ interval between the $10^{\text {th }}$ percentile and the $90^{\text {th }}$ percentile. The September Best-Basis values were inside the same interval $77 \%$ of the time. As one would expect, there are estimates that differ. The 3 primary sources of these differences are:

- minor differences in the details associated with applying the methodologies

- the impact of being able to perform custom analysis for the individual versus developing a basic methodology to be applied to all the tanks

- the difference in convention associated with making point estimates in an environment of uncertainty; that is, "to err on the side of conservatism" as often done when making point estimates versus "to increase the uncertainty interval" as generally done when estimating probability distributions.

The uncertainty estimates are based on consistently applied sound scientific principles and reasonable assumptions based on available information regarding waste behavior in the Hanford tanks. However, the methodology and its results have not been independently validated. Furthermore, there were refinements identified too late in the project timeline to be implemented. These activities are anticipated to be done during fiscal year 1999.

Due to the initial nature of the results, a certain degree of caution is necessary when using or interpreting these values. At this time, when the Best-Basis Inventory Estimate lies in the "fat part of the distribution" (e.g., between the $10^{\text {th }}$ and $90^{\text {th }}$ percentiles) it could be considered as nominal confirmation of the estimate, given the current state of information available. When the Best-Basis Inventory Estimate lies in the "tails of the distribution (e.g.; below the $10^{\text {th }}$ percentile or above the $90^{\text {th }}$ percentile), there remains substantial uncertainty or incompleteness regarding the information associated with the estimate and additional caution should be exercised, with an eye towards potential improvements or refinements. 


\section{Contents}

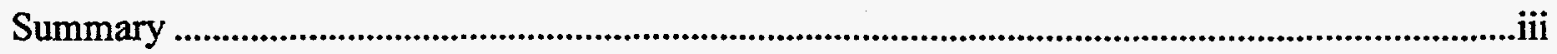

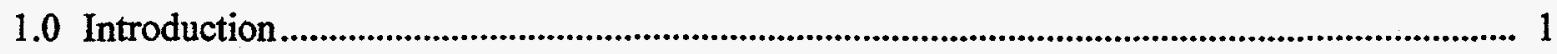

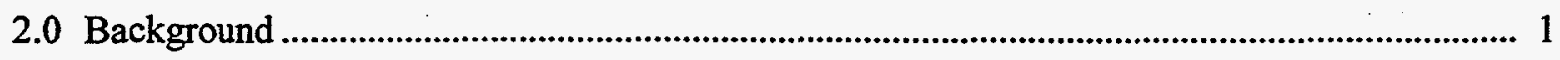

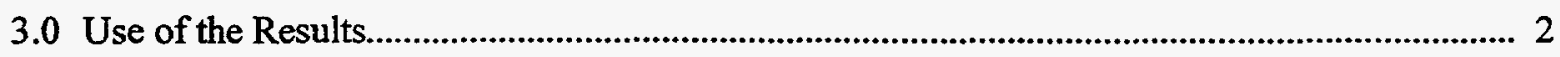

4.0 Comparison of the Best-Basis Inventory Estimates and the Best-Basis Inventory Uncertainty Estimates

4.1 Differences in methodology.

3

4.2 The individual or the class

3

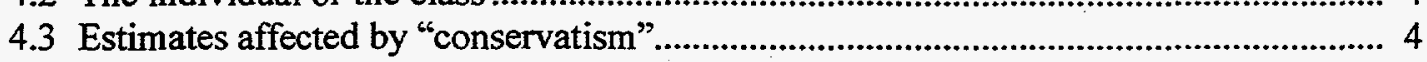

5.0 Use of Best-Basis Inventory Uncertainty Estimates ......................................................... 4

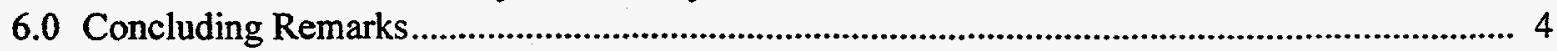

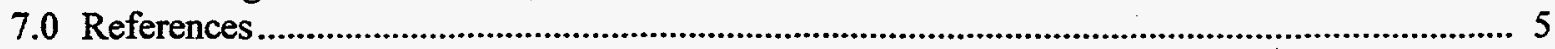

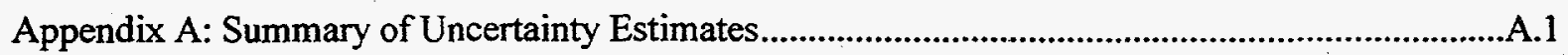




\section{Acronyms}

FY

HEABB

LMHC

PNNL

SOW

TCD

TCR

TWINS fiscal year

Hanford Engineering Analysis Best Basis

Lockheed Martin Hanford Company

Pacific Northwest National Laboratory

statement of work

Tank Characterization Database

tank characterization report

Tank Waste Information System 


\subsection{Introduction}

The purpose of this effort by Pacific Northwest National Laboratory (PNNL) is to quantify uncertainties associated with estimates of concentrations, waste volumes and densities, and total inventories of 72 analytes in the 177 nuclear waste tanks at Hanford. The estimated uncertainties are associated with the estimates of inventories determined by the Hanford Engineering Analysis Best-Basis (HEABB) (Kupfer et al. 1997) task group from the available sample data, process flow sheet data, waste-type characterizations, historical records of tank transfers (Agnew et al. 1997), and other available information. HEABB estimates are contained in the Tank Characterization Reports (TCRs), published by Westinghouse Hanford Company and Lockheed Martin Hanford Company (LMHC).

The uncertainty estimates have been established for all 177 tanks based on the latest data from the Tank Waste Information System/Tank Characterization Database (TWINS/TCD) available in May 1998. The estimates are contained in the TWTNS/TCD and are accessible online at http://twins.pnl.gov:8001.

The activity this report covers was conducted as part of the LMHC 1N4G "Characterization Data Development" work breakdown structure. This work is specifically covered under 1N4GG30C, "Historic Data and Model Confidence Evaluations."

\subsection{Background}

Hanford has approximately 55 million gallons of radioactive waste stored in 177 underground tanks. The inventories of the chemical and radioactive constituents of these wastes need to be known with greater accuracy. Additionally, we need to quantify the variabilities associated with the estimates. Due to the complex nature of the waste and the difficulties of working in such a hazardous environment, it is not practical to "simply" sample the waste many times and average those values. (Currently, there is a considerable amount of sample data, but the available information does not represent wastes from every tank, nor does it include every constituent of interest. Additionally, the waste in any given tank may be so heterogeneous as to bring in to question the representativeness of the 1 or 2 sample cores taken from the tank.) There are models that address every tank and constituent, but they have not been shown to be accurate enough to use in lieu of sampling data.

A major effort was established to estimate the inventories for 72 constituents ( 26 chemicals and 46 radionuclides) in order to satisfy several identified programmatic needs. A team of experts was assembled to review all available sample data and model estimates and come up with point estimates that represented the best possible estimate for each tank and each of the 72 constituents. These constituents represent greater than $99.9 \%$ of the chemical mass and total radioactivity in the tank inventory. The selected chemical and radioactive analytes also represent the greatest tank waste concerns from a safe storage, retrieval, pretreatment, disposal, and regulatory perspective based on requirements in applicable Data Quality Objectives (DQO's). This effort is associated with what has become known as the BestBasis Inventory estimates.

It is intended that the Best-Basis Inventory Estimates represents the best possible estimate of the tank contents so they will be used consistently throughout the Hanford technical community.

\footnotetext{
${ }^{1}$ Pacific Northwest National Laboratory is operated for the U.S. Department of Energy by Battelle under Contract DE-AC06-76RLO 1830.
} 
Some end-users of the inventory data need uncertainty estimates to bound assumptions regarding source terms in support of safety analyses, risk assessments, process designs, and other areas. Consequently, in addition to the single-point estimates of tank inventories, reliable mean concentration estimates and uncertainties are required.

This need resulted in a Statement of Work task for PNNL to create an initial estimate of uncertainty of the inventories.

The data to be used for this effort were documented by LoPresti et al. (1997) in PNNL-11792, Identification of Tanks and Analyte Data from TCD. The report was updated in May 1998 directly from the TWINS/TCD.

The methodology used to generate the results published in the TCD and summarized in this paper is based on scientific principles, sound technical knowledge of the realities associated with the Hanford waste tanks, the chemical analysis of actual samples taken from the tanks, the Hanford Best-Basis research, and historical data records. The methodology builds on research conducted by PNNL over the last few years.

Based on process histories, waste storage practices, and historical records, some of the waste can be partitioned into more homogeneous subsets that can be identified to tanks and locations within tanks. Using this method, we can maximize the information extracted from the relatively few available samples, combining sample data information from tanks with similar wastes to generate a sample based estimate of the chemical and radionuclide concentrations for each of the many waste types.

By multiplying the concentrations for a specific constituent (micrograms of the constituent per gram of waste, or microcuries of the constituent per gram of waste) by the density (grams per liter) by the volume in a tank (liters), we can get an estimate of the mass of a particular constituent (or radionuclide activity) in each tank. This methodology provides this estimate, but does so in a probabilistic framework, using and getting probability distributions instead of single numbers. The methodology is described in detail by Chen et al. (1998) in PNNL-1 1842, Methodology for Uncertainty Estimation of Hanford Tank Chemical and Radionuclide Inventories and Concentrations.

\subsection{Use of the Results}

The full set of results is available through the TCD, http://twins.pnl.gov:8001. This report summarizes those results. Appendix A consists of a very large table with a row for every tank/analyte combination and the following columns:

- Tank name

- Analyte name

- Units (kilograms or Curies)

- The May 1998 Best-Basis inventory estimate (when extracted for use in the methodology)

- The September 1998 Best-Basis inventory estimate (at the time of generating this report)

- Best-Basis inventory uncertainty estimate information:

- The median ( 50 percentile) estimated inventories

- Four tail values representing the extreme uncertainty:

- low (1 percentile) estimated inventory

- medium low (10 percentile) estimated inventory

- medium high (90 percentile) estimated inventory 
- high (99 percentile) estimated inventory

- The mean estimated inventory

- The standard deviation estimated inventory.

Note: the reader is cautioned that most distributions for this data are not normal (Gaussian) and therefore any use of the mean and standard deviation must keep the full distribution in mind.

The numbers provided support the calculation of uncertainty intervals. For example, tank A-101 has the following entry for the analyte $\mathrm{Al}$, aluminum:

$\frac{\text { Tank }}{\mathrm{A} 101} \quad \frac{\text { Anal }}{\mathrm{Al}} \frac{\mathrm{Un}}{\mathrm{kg}} \frac{B B-M a y}{1.47 \mathrm{E}+05} \quad \frac{B B-S e p}{1.47 \mathrm{E}+05} \frac{1 \% \text {-ile }}{1.14 \mathrm{E}+05} \frac{10 \% \text {-ile }}{1.30 \mathrm{E}+05} \frac{50 \% \text {-ile }}{1.49 \mathrm{E}+05} \frac{90 \% \text {-ile }}{1.69 \mathrm{E}+05} \frac{99 \% \text {-ile }}{1.83 \mathrm{E}+05} \frac{\frac{\text { Mean }}{1.49 \mathrm{E}+05}}{\frac{\text { Std Dev. }}{1.50 \mathrm{E}+04}}$

The following expressions serve as an example of the kinds of intervals one might calculate:

- The methodology estimates that the amount of aluminum in tank A-101 is likely to be less than $169,000 \mathrm{~kg}$ (using the entry in the 90 percentile column).

- The methodology estimates that the amount of the aluminum in tank A-101 isvery likely (99\%) to be less than $183,000 \mathrm{~kg}$ (using the entry in the 99 percentile column).

- The methodology estimates the amount of the analyte in the tank is likely bemore than 130,000 $\mathrm{kg}$ (using the entry in the 10 percentile column).

- The methodology estimates the amount of the analyte in the tank that has an equal chance of being above or below $149,000 \mathrm{~kg}$ (using the entry in the 50 percentile column).

- The methodology estimates the amount of the analyte in the tank is very likely to be between 114,000 and $183,000 \mathrm{~kg}$ (using the entry in the 1 and 99 percentile columns).

- The Best-Basis estimates the amount of the analyte in the tank is $147,000 \mathrm{~kg}$.

\subsection{Comparison of the Best-Basis Inventory Estimates and the Best-Basis Inventory Uncertainty Estimates}

A large number of tank/analyte combinations have results with the Best-Basis Inventory Estimate in the middle of the distribution; i.e., between the 10 and 90 percentiles. In fact, $85 \%$ of the tank/analyte values for the May Best-Basis values were inside the $80 \%$ interval between the $10^{\text {th }}$ percentile and the $90^{\text {th }}$ percentile. The September Best-Basis values were inside the same interval $77 \%$ of the time. Naturally, we would like to see the Best-Basis Inventory Estimates very near the 50 percentile for all cases. However, there are a number of very good reasons that this is not going to occur. Several of these reasons are discussed below. Many instances of this apparent disagreement are expected to be mitigated considerably as a result of work anticipated to be conducted in FY 1999.

\footnotetext{
${ }^{2}$ Two columns report Best-Basis values. The "May 98" column reports the value that was used in the statistical analysis, since we needed to capture the values with sufficient time left in the fiscal year to do the analysis. The "Sept 98" column reports the value that was considered "correct" in September 1998, when this report was written. In the majority of tank/analyte cases, these numbers are the same. On those occasions when there is a difference, the "Sept 98 " column represents the better value.
} 


\subsection{Differences in methodology}

Both methods use models that have the same essential character: estimate the concentration, density (if necessary), and volume for each waste type; combine to get an estimate for the inventory of the waste type; and sum over all waste types. The differences include how parts of the products were estimated. For concentrations and densities, the Best-Basis Inventory Estimates often use sample-based concentrations from the tank being estimated, or from one or two other tanks with the same waste type. This methodology uses an average of sample results from many samples of the same waste type from many tanks.

In FY 1999, we plan to refine the estimates of the concentrations and densities by mapping them to more groups and considering more information in the assignment process.

\subsection{The individual or the class}

The Best-Basis Inventory Estimates were created by a team of knowledgeable scientists and engineers by studying data for each individual tank and making the best judgments possible at the time of the review. This methodology created estimates by considering the tanks and analytes in context of a set of rules that drive the calculations.

In FY 1998, we created these rules following a tractable and repeatable set of procedures. In FY 1999, we plan to improve the rules and create a set of tractable and repeatable exceptions to the rules, where appropriate, that will reflect the exceptions for individual tank/analytes as documented by the Best-Basis reports.

\subsection{Estimates affected by "conservatism"}

Individuals make the best estimates they can, but when faced with a situation that has a high chance for error, they will often choose, intentionally or unintentionally, "to err on the side of conservatism." Hanford estimates of waste concentration or inventory are made in a situation with a great amount of uncertainty due to the nature of the limited data that is available. When this uncertainty combines with thoughts of the magnitude of the damage that can occur if a disaster occurs, a person is naturally tempted to set an estimated value to a higher value. In short, higher concentration, volume and/or inventory estimates are generally viewed as more conservative and are often used to reflect lack of knowledge. The manner in which "conservatism" is implemented in this methodology is to increase the values of the uncertainty parameters, not change the values of the centers of the estimates.

This is not likely to change in FY 1999.

\subsection{Use of Best-Basis Inventory Uncertainty Estimates}

The uncertainty estimates are based on consistently applied sound scientific principles and reasonable assumptions based on available information regarding waste behavior in the Hanford tanks. However, the methodology and its results have not been independently validated. Furthermore, there were refinements identified too late in the project timeline to be implemented. These activities are anticipated to be done during fiscal year 1999.

Due to the initial nature of the results, a certain degree of caution is necessary when using or interpreting these values. At this time, when the Best-Basis Inventory Estimate lies in the "fat part of the distribution" (e.g., between the $10^{\text {th }}$ and $90^{\text {th }}$ percentiles) it could be considered as nominal confirmation of the 
estimate, given the current state of information available. When the Best-Basis Inventory Estimate lies in the "tails of the distribution (e.g.; below the $10^{\text {th }}$ percentile or above the $90^{\text {th }}$ percentile), there remains substantial uncertainty or incompleteness regarding the information associated with the estimate and additional caution should be exercised, with an eye towards potential improvements or refinements.

\subsection{Concluding Remarks}

These uncertainty estimates are based on sound scientific principles, consistently applied, and provide reasonable estimates of the uncertainty in the inventories in the Hanford tanks. We have identified a number of improvements and refinements in the estimates that should be made; most important is the updating of the results to reflect the latest sampling and Best-Basis estimates. We have yet to conduct studies that independently assess the quality of the methodology. These improvements are envisioned to be conducted during FY 1999. 


\subsection{References}

Agnew, S. F. 1997. Hanford Tank Chemical and Radionuclide Inventories: HDW Model Rev.4, LA-UR-96-3860, Los Alamos National Laboratory, Los Alamos, New Mexico.

Chen, G.; T. A. Ferryman; K. M. Remund; S. A. Hartley; F. Gao; C. A. LoPresti; T. J. DeForest; J. G. Hill; C. A. Weier; B. G. Amidan, D. K Gemeinhart and B. C. Simpson. 1998. Methodology for Uncertainty Estimation of Hanford Tank Chemical and Radionuclide Inventories and Concentrations, PNNL-11842, Pacific Northwest National Laboratory, Richland Washington.

Kupfer, M. J.; A. L. Boldt; B. A. Higley; K. M. Hodgson; L. W. Shelton; B. C. Simpson; and R. A. Watrous (LMHC); M. D. LeClair (SAIC); G. L. Borsheim (BA); R. T. Winward (MA); R. M. Orme (NHC); N. G. Colton (PNNL); S. L. Lambert and D. E. Place (SESC); and W. W. Schulz (W²S). 1997. Standard Inventories of Chemicals and Radionuclides in Hanford Site Tank Wastes, HNF-SD-WM-TI740, Rev. 0, Lockheed Martin Hanford Company, Richland, Washington.

LoPresti, C. A.; K. M. Remund; F. M. Ryan; T. A. Ferryman; T. J. DeForest; C. A. Weier; S. A. Hartley; G. Chen; J. G. Hill; and F. Gao. 1997. Identification of Tanks and Analyte Data from TCD, PNNL-11792, Pacific Northwest National Laboratory, Richland Washington. 
Appendix A

Summary of Uncertainty Estimates 


\begin{tabular}{|c|c|c|c|c|c|c|c|c|c|c|c|}
\hline ank & nal. & In & $\frac{B B}{\text { ay } 98}$ & $\frac{B B}{D \operatorname{tg8}}$ & $1 \%$-ile & $10 \%$-ile & $50 \%$-ile & $90 \%$-ile & 99\%-ile & Mean & Std Dev \\
\hline 101 & Al & kg & $1 . \overline{47 E+05}$ & $1.47 \mathrm{E}+05$ & $1.14 \mathrm{E}+05$ & $1.30 \mathrm{E}+05$ & $1.49 \mathrm{E}+05$ & $1.69 E+05$ & $1.83 \mathrm{E}+05$ & $1.49 \mathrm{E}+05$ & $1.50 \mathrm{E}+04$ \\
\hline & $\mathbf{B i}$ & kg & $3.78 E+02$ & $3.78 \mathrm{E}+02$ & $2.99 \mathrm{E}-02$ & $E-01$ & $.07 \mathrm{E}+02$ & $:+03$ & $E+03$ & $E+02$ & $E+02$ \\
\hline & Ca & $\mathrm{kg}$ & $7.82 E+02$ & $7.82 \mathrm{E}+02$ & $2.44 \mathrm{E}+02$ & $12 E+03$ & $62 E+03$ & $47 \mathrm{~F}+03$ & $22 F+04$ & $3.07 E+03$ & $20 \mathrm{~F}+03$ \\
\hline & Cl & kg & $2.41 \mathrm{E}+04$ & $41 E+04$ & & & $44 \mathrm{E}+04$ & $60 \mathrm{E}+04$ & $75 \mathrm{E}+04$ & $4 E+04$ & \\
\hline & $\mathrm{CO} 3$ & kg & $1.69 \mathrm{E}+05$ & $1.69 E+05$ & & & $80 \mathrm{E}+05$ & +05 & $E+05$ & $E+05$ & \\
\hline & $\mathrm{Cr}$ & & $43 E+03$ & $E+03$ & +03 & +04 & +04 & -04 & $E+04$ & $E+04$ & +03 \\
\hline & $\mathbf{F}$ & & $E+03$ & $\mathrm{C}+03$ & -02 & -01 & -03 & & +04 & +03 & +03 \\
\hline & e & & $1.39 \mathrm{E}+03$ & $1.39 E+03$ & $E+02$ & +02 & $55 E+03$ & +03 & $E+03$ & $E+03$ & $E+03$ \\
\hline & $\mathbf{t}$ & & $5.90 \mathrm{E}-00$ & $.00 E+01$ & & $E+01$ & & & $3 E+01$ & $\mathrm{E}-00$ & $9 \mathrm{E}-00$ \\
\hline & $Y$ & & $.58 E+04$ & $58 \mathrm{E}+04$ & & & & & $E+04$ & & $E+03$ \\
\hline 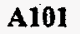 & $\mathbf{a}$ & & $1.00 \mathrm{E}+01$ & $.00 \mathrm{E}+01$ & -02 & & & & +02 & & +01 \\
\hline & $\mathbf{M n}$ & $\mathrm{kg}$ & $1.10 \mathrm{E}+02$ & $1.10 \mathrm{E}+02$ & & & & & $E+03$ & +02 & +02 \\
\hline & $\mathbf{N a}$ & kg & $9.55 E+05$ & $9.55 \mathrm{E}+05$ & & & & & & & \\
\hline & $\mathbf{N i}$ & kg & $2.11 E+02$ & $2.11 E+02$ & & & & & & & \\
\hline & $\mathrm{NO} 2$ & kg & $4.61 E+05$ & $4.61 \mathrm{E}+05$ & & & & & & +05 & +04 \\
\hline & NO3 & & $.21 E+05$ & $E+05$ & & & & & +06 & +06 & \\
\hline & 1 & & $2.44 \mathrm{E}+04$ & $\mathbf{N A}$ & & & & & +04 & & \\
\hline & $\mathbf{P b}$ & & $5.02 E+02$ & $5.02 \mathrm{E}+02$ & & & & & & & \\
\hline & PC & kg & $7.57 \mathrm{E}+04$ & $2.47 E+04$ & & & & & & & \\
\hline & S & k & $3.99 E+04$ & NA & & & & & & & \\
\hline & $\mathbf{S i}$ & k & $2.57 \mathrm{E}+03$ & $2.57 E+03$ & & & & & & & \\
\hline 101 & so & & $1.20 \mathrm{E}+05$ & $3.99 \mathrm{E}+04$ & & .04 & & & & & \\
\hline & $\mathrm{Sr}$ & & $3.78 E+01$ & $3.78 \mathrm{E}+01$ & & .02 & & & +02 & & +02 \\
\hline & 1 & & $1.96 E+04$ & $1.96 E+04$ & & & & & & & \\
\hline & $\mathbf{U}$ & & $1.60 \mathrm{E}+03$ & $1.60 \mathrm{E}+03$ & & & & & & & \\
\hline & $\mathbf{Z r}$ & & $8.84 \mathrm{E}+01$ & $8.84 \mathrm{E}+01$ & & & & & & & \\
\hline & & & $3.20 \mathrm{E}-03$ & $E-03$ & & & & & & & \\
\hline & Am241 & 0 & $1.97 \mathrm{E}+02$ & $7.47 \mathrm{E}+01$ & & & & & & & \\
\hline 01 & 243 & 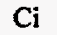 & 7.30E-03 & $2.75 E-03$ & & & & & & & -01 \\
\hline & Ba137 & 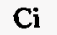 & $1.18 \mathrm{E}+06$ & +06 & & & & & & & \\
\hline & C14 & & 1.1 & & & & & & & & \\
\hline & & $c$ & & 3.0 & & & & & & & \\
\hline & $\mathrm{Cm} 242$ & $c$ & $5.40 \mathrm{E}-01$ & -01 & & & & & & & \\
\hline 01 & $\mathrm{Cm} 243$ & C & $4.90 \mathrm{E}-02$ & $1.85 \mathrm{E}-02$ & -04 & -02 & & & & & -01 \\
\hline 01 & $\mathrm{Cm} 244$ & C & $4.00 \mathrm{E}-01$ & $1.50 \mathrm{E}-01$ & .03 & & & & +01 & & -00 \\
\hline & Co60 & c & $1.27 E+02$ & $1.27 \mathrm{E}+02$ & & & & & & & \\
\hline & Cs134 & & $1.26 \mathrm{E}+01$ & 1.26 & & & & & & & \\
\hline & & & & 06 & & & & & & +06 & \\
\hline & & & +01 & 01 & & & & & & 01 & \\
\hline 01 & Eu154 & C & $4.66 \mathrm{E}+02$ & +02 & & -01 & & & -03 & & +02 \\
\hline 101 & Eul55 & $\mathrm{C}$ & $1.70 E+03$ & $9.61 E+02$ & & & & & & & +03 \\
\hline & I129 & & $1.68 \mathrm{E}-00$ & $2-00$ & & & & & & & \\
\hline & & & & & & & & & & & \\
\hline & & & & & & & & & & & \\
\hline & & 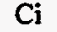 & $7.03 E+02$ & +02 & & & & & & & \\
\hline & Np237 & $c$ & $3.00 \mathrm{E}-00$ & $3.00 \mathrm{E}-00$ & & & & & +02 & & \\
\hline Al01 & Pa231 & C & $1.40 \mathrm{E}-02$ & $1.40 \mathrm{E}-02$ & -01 & & & & -02 & & \\
\hline 1 & & & $5.05 \mathrm{E}-00$ & & & & & & & & +01 \\
\hline & & & & & & & & & & 502 & \\
\hline & & 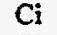 & & & & & & & & & \\
\hline & & & & & & & & & & & \\
\hline 1 & $\mathbf{P u}$ & C & -03 & -04 & & & & & & & -02 \\
\hline & Ra226 & c & $5.20 \mathrm{E}-04$ & $5.20 \mathrm{E}-04$ & & & & & & & \\
\hline$A 101$ & R2228 & & 1.11E-00 & $1.11 \mathrm{E}-00$ & & & & & & 00 & \\
\hline Al & $\mathbf{R}$ & & & & & & & & & & \\
\hline & & & $6.51 \mathrm{E}+02$ & & & & & & $\div 03$ & & \\
\hline Al01 & Se & & & & & & & & & & -00 \\
\hline & & & & & & & & & & +04 & \\
\hline & Sr89/90 & c & & $1.52 \mathrm{E}+05$ & & & & & & & \\
\hline & & C & $8.69 E+02$ & $8.69 E+02$ & & & & & & & \\
\hline & & & $2.60 \mathrm{E}-02$ & $2.60 \mathrm{E}-02$ & & & & & & $E-02$ & $E-02$ \\
\hline , & & c & $1.20 \mathrm{E}-01$ & & & $E+01$ & & & & E-01 & $E-01$ \\
\hline & & C & & & & & & & & & $1.40 \mathrm{E}+01$ \\
\hline 11 & Tritium & $\mathbf{C i}$ & $7.31 E+02$ & $7.31 E+02$ & 6.2 & $E+02$ & $7.31 \mathrm{E}+02$ & $E+02$ & $E+02$ & $E+02$ & $E+01$ \\
\hline A1 & U232 & $\mathrm{Ci}$ & $\mathbf{3 . 3 8}$ & 01 & $E+01$ & $E+01$ & 3.381 & $9.16 \mathrm{E}-00$ & $E+01$ & $3.99 \mathrm{E}-00$ & $3.70 \mathrm{E}-00$ \\
\hline & 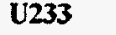 & $C_{1}$ & $1.30 \mathrm{E}+01$ & 3.54E-60 & $0.00 E+01$ & $0.00 \mathrm{E}+01$ & $1.30 E+01$ & $3.52 \mathrm{E}+01$ & $5.50 \mathrm{E}+01$ & $1.53 \mathrm{E}+01$ & $1.42 \mathrm{E}+01$ \\
\hline
\end{tabular}




\begin{tabular}{|c|c|c|c|c|}
\hline A101 & 0234 & $\mathrm{Ci}$ & $\frac{\text { May } \overline{98}}{2.19 E-00}$ & $\frac{\text { Sept98 }}{5.99 \mathrm{E}-01}$ \\
\hline A101 & U235 & $\mathrm{Ci}$ & 8.70巨-02 & 238E-02 \\
\hline A101 & U236 & $\mathrm{Ci}$ & $7.00 \mathrm{E}-02$ & $1.93 \mathrm{E}-02$ \\
\hline A101 & U238 & $\mathrm{Ci}$ & $3.00 \mathrm{E}-00$ & $5.34 \mathrm{E}-01$ \\
\hline A101 & Y90 & $\mathrm{Ci}$ & $1.52 E+05$ & $1.52 \mathrm{E}+05$ \\
\hline A101 & Zr93 & $\mathrm{Ci}$ & $5.81 \mathrm{E}+01$ & $5.81 E+01$ \\
\hline A102 & Al & kg & $7.05 E+03$ & $7.05 \mathrm{E}+03$ \\
\hline A102 & $\mathbf{B i}$ & kg & $2.59 \mathrm{E}+02$ & $2.59 E+02$ \\
\hline A102 & $\mathrm{Ca}$ & kg & $4.10 E+02$ & $4.10 \mathrm{E}+02$ \\
\hline A102 & Cl & kg & $2.10 E+03$ & $2.10 E+03$ \\
\hline A102 & $\mathrm{CO3}$ & $\mathrm{kg}$ & $5.71 \mathrm{E}+03$ & $5.71 \mathrm{E}+03$ \\
\hline A102 & $\mathrm{Cr}$ & kg & $1.88 \mathrm{E}+03$ & $1.88 \mathrm{E}+03$ \\
\hline A102 & $\mathbf{F}$ & kg & $7.30 \mathrm{E}+01$ & $7.30 E+01$ \\
\hline $\mathbf{A 1 0 2}$ & $\mathbf{F e}$ & $\mathrm{kg}$ & $4.31 E+03$ & $4.31 E+03$ \\
\hline A102 & Hg & $\mathrm{kg}$ & 1.15E-01 & $0.00 \mathrm{E}+01$ \\
\hline A102 & $\mathbf{K}$ & kg & $7.54 \mathrm{E}+02$ & $7.54 \mathrm{E}+02$ \\
\hline A102 & La & kg & $2.70 \mathrm{E}+01$ & $2.70 \mathrm{E}+01$ \\
\hline A102 & $\mathbf{M n}$ & kg & $7.10 \mathrm{E}+02$ & $7.10 \mathrm{E}+02$ \\
\hline A102 & $\mathbf{N} \mathbf{2}$ & kg & $4.00 \mathrm{E}+04$ & $4.00 \mathrm{E}+04$ \\
\hline A102 & $\mathbf{N i}$ & kg & $1.20 \mathrm{E}+02$ & $1.20 \mathrm{E}+02$ \\
\hline Al02 & NO2 & $\mathrm{kg}$ & $2.19 E+04$ & $2.19 E+04$ \\
\hline A102 & NO3 & kg & $3.40 \mathrm{E}+04$ & $3.40 \mathrm{E}+04$ \\
\hline 102 & $\mathbf{P}$ & kg & $2.60 \mathrm{E}+03$ & NA \\
\hline A102 & $\mathbf{P b}$ & $\mathrm{kg}$ & $3.32 E+02$ & $3.32 E+02$ \\
\hline A102 & PO4 & kg & $8.06 E+03$ & $2.63 E+03$ \\
\hline A102 & $\mathbf{S}$ & kg & $1.18 E+03$ & NA \\
\hline A102 & Si & kg & $2.56 E+03$ & $2.56 \mathrm{E}+03$ \\
\hline A102 & SO4 & kg & $3.54 \mathrm{E}+03$ & $1.18 \mathrm{E}+03$ \\
\hline A102 & $\mathbf{S r}$ & kg & $1.60 \mathrm{E}+01$ & $1.60 \mathrm{E}+01$ \\
\hline A102 & TOC & kg & $2.89 E+03$ & $2.89 \mathrm{E}+03$ \\
\hline Al02 & $\mathbf{U}$ & kg & $5.83 E+03$ & $5.83 \mathrm{E}+03$ \\
\hline Al02 & $\mathbf{Z r}$ & $\mathbf{k g}$ & $2.41 \mathrm{E}+02$ & $2.41 E+02$ \\
\hline A102 & Ac227 & $\mathbf{C i}$ & 2.52E-03 & $2.52 E-03$ \\
\hline A102 & Am241 & $\mathrm{Ci}$ & $3.15 E+02$ & $3.15 \mathrm{E}+02$ \\
\hline A102 & Am243 & $\mathbf{C i}$ & $8.88 \mathrm{E}-03$ & $1.63 \mathrm{E}-02$ \\
\hline A102 & Ba137 & $\mathrm{Ci}$ & $2.97 E+04$ & $2.97 E+04$ \\
\hline A102 & C14 & $\mathbf{C i}$ & $3.00 \mathrm{E}-01$ & $3.00 \mathrm{E}-01$ \\
\hline A102 & Cd113 & $\mathrm{Ci}$ & $4.91 E+01$ & $4.91 E+01$ \\
\hline A102 & $\mathrm{Cm} 242$ & $\mathrm{Ci}$ & 1.59E-01 & 2.93E-01 \\
\hline Al02 & Cm243 & $\mathrm{Ci}$ & 1.44E-02 & $2.64 \mathrm{E}-02$ \\
\hline A102 & Cm244 & $\mathrm{Ci}$ & 5.19E-01 & $9.53 \mathrm{E}-01$ \\
\hline A102 & Co60 & $\mathrm{Ci}$ & $8.70 E+01$ & $8.70 E+01$ \\
\hline A102 & Cs134 & $\mathrm{Ci}$ & 6.81E-01 & $6.81 \mathrm{E}-01$ \\
\hline A102 & Cs137 & $\mathrm{Ci}$ & $3.14 E+04$ & $3.14 E+04$ \\
\hline A102 & Eu152 & $\mathrm{Ci}$ & 4.28E-00 & $4.28 \mathrm{E}-00$ \\
\hline Al02 & Eu154 & $\mathrm{Ci}$ & $1.85 \mathrm{E}+02$ & $1.85 E+02$ \\
\hline Al02 & Eu155 & $\mathrm{Ci}$ & $2.62 \mathrm{E}+02$ & $2.62 E+02$ \\
\hline Al02 & 1129 & $\mathrm{Ci}$ & $1.00 \mathrm{E}-02$ & $1.00 \mathrm{E}-02$ \\
\hline A102 & Nb93 & $\mathrm{Ci}$ & $1.60 \mathrm{E}+01$ & $1.60 E+01$ \\
\hline A102 & Ni59 & C & $7.17 \mathrm{E}-00$ & $7.17 E-00$ \\
\hline A102 & Ni63 & C & $7.06 \mathrm{E}+02$ & $7.06 \mathrm{E}+02$ \\
\hline A102 & Np237 & C & 1.35E-01 & 1.35E-01 \\
\hline Al02 & Pa231 & $\mathrm{Ci}$ & $4.16 \mathrm{E}-03$ & $4.16 \mathrm{E}-03$ \\
\hline A102 & Pu238 & $\mathrm{Ci}$ & 5.61E-00 & $1.96 \mathrm{E}+01$ \\
\hline A102 & Pu239 & $\mathrm{Ci}$ & $5.30 \mathrm{E}+02$ & $5.30 \mathrm{E}+02$ \\
\hline A102 & Pu240 & $\mathrm{C}$ & $2.81 \mathrm{E}+01$ & $9.82 \mathrm{E}+01$ \\
\hline A102 & Pu241 & C & $3.98 \mathrm{E}+02$ & $1.39 E+03$ \\
\hline Al02 & Pu242 & Ci & 2.34E-03 & $8.15 E-03$ \\
\hline A102 & $\mathbf{R a 2 2 6}$ & C & 4.83E-04 & 4.83E-04 \\
\hline A102 & Ra228 & $\mathrm{Ci}$ & $5.28 \mathrm{E}-02$ & $5.28 \mathrm{E}-02$ \\
\hline A102 & Ru106 & $\mathrm{Ci}$ & $3.09 \mathrm{E}-02$ & $3.09 \mathrm{E}-02$ \\
\hline A102 & Sb125 & $\mathrm{Ci}$ & $3.00 \mathrm{E}+01$ & $3.00 \mathrm{E}+01$ \\
\hline A102 & Se79 & $\mathrm{Ci}$ & $4.35 E-00$ & $4.35 \mathrm{E}-00$ \\
\hline A102 & Sm151 & C & $1.62 \mathrm{E}+04$ & $1.62 E+04$ \\
\hline $\mathbf{A 1 0 2}$ & Sr89/90 & $\mathrm{Ci}$ & $1.35 E+05$ & $1.35 E+05$ \\
\hline Al02 & Tc99 & Ci & $2.64 E+01$ & $2.64 \mathrm{E}+01$ \\
\hline A102 & Th22 & C & $1.22 \mathrm{E}-03$ & $1.22 E-03$ \\
\hline A102 & Th232 & $\mathrm{Ci}$ & $5.72 E-03$ & $5.72 \mathrm{E}-03$ \\
\hline
\end{tabular}

\section{$0.00 \mathrm{E}+01 \quad 0.00 \mathrm{E}+01$}

$0.00 \mathrm{E}+01$

$0.00 \mathrm{E}+01$

$0.00 \mathrm{E}+01$

$0.00 \mathrm{E}+01$

$0.00 \mathrm{E}+01$

$9.03 \mathrm{E}+02$

$2.49 \mathrm{E}-02$

$5.82 \mathrm{E}+01$

$3.69 \mathrm{E}+02$

$3.13 \mathrm{E}+02$

$1.59 \mathrm{E}+02$

4.92E-02

$7.16 \mathrm{E}+02$

$0.00 \mathrm{E}+01$

$3.53 \mathrm{E}+01$

3.61E-01

$6.65 \mathrm{E}+01$

$1.10 \mathrm{E}+04$

9.13E-00

$3.22 \mathrm{E}+03$

$9.73 \mathrm{E}+02$

4.42E-01

$2.94 \mathrm{E}+01$

$2.17 E+01$

$2.40 \mathrm{E}+01$

$3.91 \mathrm{E}+01$

$3.62 \mathrm{E}+01$

$5.25 \mathrm{E}-02$

$5.90 \mathrm{E} \div 02$

$7.81 \mathrm{E} \div 02$

$1.56 \mathrm{E}-02$

$0.00 \mathrm{E} \div 01$

$0.00 \mathrm{E} \div 01$

$1.70 \mathrm{E}-03$

$1.96 \mathrm{E}+01$

$0.00 \mathrm{E}+01$

$0.00 \mathrm{E}+01$

3.05E-02

2.76E-03

$9.96 \mathrm{E}-02$

5.03E-02

4.50E-04

$5.27 \mathrm{E}+03$

$0.00 \mathrm{E}+01$

$0.00 \mathrm{E}+01$

$0.00 \mathrm{E}+01$

$0.00 \mathrm{E}+01$

$0.00 \mathrm{E}+01$

$2.29 \mathrm{E}-01$

$2.26 \mathrm{E}+01$

$2.59 \mathrm{E}-02$

$0.00 \mathrm{E}+01$

$1.08 \mathrm{E}-00$

$1.02 \mathrm{E}+02$

$5.39 \mathrm{E}-00$

$7.64 \mathrm{E}+01$

4.49E-04

$0.00 \mathrm{E}+01$

$0.00 \mathrm{E}+01$

$0.00 \mathrm{E}+01$

$0.00 \mathrm{E}+01$

$0.00 \mathrm{E}+01$

$0.00 \mathrm{E}+01$

$8.91 E+01$

$0.00 E+01$

$0.00 \mathrm{E}+01$ $0.00 E+01$

\section{0\%-ile}

$0.00 \mathrm{E}+01$

$0.00 \mathrm{E}+01$

$0.00 \mathrm{E}+01$

8.03E+03

$0.00 \mathrm{E}+01$

$2.46 \mathrm{E}+03$

9.22E-01

$1.55 \mathrm{E}+02$

7.02E +02

$1.37 \mathrm{E}+03$

$5.16 \mathrm{E}+02$

$4.55 \mathrm{E}-00$

$1.50 \mathrm{E}+03$

$0.00 \mathrm{E}+01$

$2.07 \mathrm{E}+02$

2.23E-00

$2.17 \mathrm{E}+02$

$1.91 \mathrm{E}+04$

4.67E +01

$6.67 \mathrm{E}+03$

$9.54 \mathrm{E}+03$

$2.01 \mathrm{E}+01$

$8.66 \mathrm{E}+01$

$1.38 \mathrm{E}+02$

$8.93 \mathrm{E}+01$

$1.52 \mathrm{E}+02$

$2.80 \mathrm{E}+02$

$1.46 \mathrm{E}-01$

$1.27 \mathrm{E}+03$

$2.52 \mathrm{E}+03$

1.36E-01

$0.00 \mathrm{E}+01$

1.09E-01

4.01E-03

$8.96 \mathrm{E}+03$

8.40E-03

$0.00 \mathrm{E}+01$

$7.18 \mathrm{E}-02$

6.51E-03

2.34E-01

4.82E-01

2.05E-01

$1.51 \mathrm{E}+04$

$0.00 E+01$

$0.00 \mathrm{E}+01$

$0.00 \mathrm{E}+01$

$3.32 \mathrm{E}-03$

$0.00 \mathrm{E}+01$

$1.86 \mathrm{E}-00$

$1.83 \mathrm{E}+02$

6.10E-02

$0.00 \mathrm{E}+01$

$2.53 E-00$

$2.39 \mathrm{E}+02$

$1.27 \mathrm{E}+01$

$1.80 \mathrm{E}+02$

$1.06 \mathrm{E}-03$

$0.00 E+01$

$0.00 \mathrm{E}+01$

7.17E-03

$0.00 \mathrm{E}+01$

$3.76 \mathrm{E}-01$

$0.00 \mathrm{E}+01$

$1.88 \mathrm{E}+04$

4.87E-00

$0.00 \mathrm{E}+01$

$0.00 \mathrm{E}+01$ 50\%-ile

\subsection{E-00}

8.70E-02

7.00E-02

3.00E-00.

1.52E+05

$5.81 \mathrm{E}+01$

$5.17 E+03$

$2.30 \mathrm{E}+01$

$4.21 \mathrm{E}+02$

$1.24 \mathrm{E}+03$

$7.13 E+03$

$1.04 \mathrm{E}+03$

$5.34 \mathrm{E}+02$

$2.92 \mathrm{E}+03$

1.15E-01

$5.75 \mathrm{E}+02$

$1.33 \mathrm{E}+01$

4.47E+02

$3.26 \mathrm{E}+04$

$1.69 \mathrm{E}+02$

$1.29 \mathrm{E}+04$

$2.77 \mathrm{E}+04$

$5.34 \mathrm{E}+02$

$1.68 \mathrm{E}+02$

$2.23 \mathrm{E}+03$

$5.44 \mathrm{E}+02$

$5.62 \mathrm{E}+02$

$1.90 \mathrm{E}+03$

$1.39 \mathrm{E}+01$

$2.77 \mathrm{E}+03$

$4.90 \mathrm{E}+03$

$7.23 \mathrm{E}+01$

2.52E-03

$7.70 \mathrm{E}+01$

8.88E-03

$2.97 \mathrm{E}+04$

$3.00 \mathrm{E}-01$

4.91E+01

1.59E-01

1.44E-02

5.19E-01

4.38E-00

6.81E-01

$3.79 \mathrm{E}+04$

$4.28 \mathrm{E}-00$

$1.85 \mathrm{E}+02$

$2.62 \mathrm{E}+02$

$1.00 \mathrm{E}-02$

$1.60 \mathrm{E}+01$

$7.17 E-00$

$7.06 \mathrm{E}+02$

1.35E-01

4.16E-03

5.61E-00

$5.30 \mathrm{E}+02$

$2.81 \mathrm{E}+01$

$3.98 \mathrm{E}+02$

$2.34 \mathrm{E}-03$

4.83E-04

5.28E-02

3.09E-02

$3.00 \mathrm{E}+01$

4.35E-00

$1.62 \mathrm{E}+04$

$9.08 \mathrm{E}+04$

$2.64 \mathrm{E}+01$

$1.22 \mathrm{E}-03$

5.72E-03 90\%-ile

\subsection{E-00}

$2.36 \mathrm{E}-01$

$1.90 \mathrm{E}-01$

8.13E-00

3.77E+05

$9.11 \mathrm{E}+02$

$8.47 \mathrm{E}+03$

$1.05 \mathrm{E}+02$

$2.21 \mathrm{E}+03$

$1.85 \mathrm{E}+03$

$1.92 \mathrm{E}+04$

$1.74 \mathrm{E}+03$

$1.99 \mathrm{E}+03$

$4.75 \mathrm{E}+03$

2.63E-01

$1.10 \mathrm{E}+03$

$3.17 E+01$

$9.05 \mathrm{E}+02$

$4.89 \mathrm{E}+04$

$7.95 \mathrm{E}+02$

$2.16 \mathrm{E}+04$

$5.41 \mathrm{E}+04$

$1.68 \mathrm{E}+03$

$2.70 \mathrm{E}+02$

$6.43 \mathrm{E}+03$

$1.54 \mathrm{E}+03$

$1.34 \mathrm{E}+03$

$5.23 \mathrm{E}+03$

$5.85 \mathrm{E}+01$

$5.58 \mathrm{E}+03$

$7.84 \mathrm{E}+03$

$5.58 \mathrm{E}+02$

$1.05 \mathrm{E}-02$

$5.99 \mathrm{E}+02$

$3.76 \mathrm{E}-02$

$6.33 \mathrm{E}+04$

$9.09 \mathrm{E}-01$

$1.12 \mathrm{E}+02$

$6.73 \mathrm{E}-01$

$6.09 \mathrm{E}-02$

$2.20 \mathrm{E}-00$

$1.45 \mathrm{E}+01$

$1.45 \mathrm{E}-00$

$7.48 \mathrm{E}+04$

$1.79 \mathrm{E}+01$

$7.72 \mathrm{E}+02$

$1.09 \mathrm{E}+03$

$1.74 \mathrm{E}-02$

1. $30 \mathrm{E}+02$

$3.44 \mathrm{E}+01$

$3.38 \mathrm{E}+03$

5.71E-01

1.06E-02

$2.37 E+01$

$2.24 \mathrm{E}+03$

$1.19 \mathrm{E}+02$

$1.68 \mathrm{E}+03$

$9.90 \mathrm{E}-03$

2.18E-03

2.38E-01

6.16E-02

$1.47 \mathrm{E}+02$

$1.25 \mathrm{E}+0 \mathrm{I}$

$6.76 \mathrm{E}+04$

$7.48 \mathrm{E}+05$

$6.93 \mathrm{E}+01$

3.11E-03

$1.46 \mathrm{E}-02$

9.27E-00

$3.68 \mathrm{E}-01$

$2.96 \mathrm{E}-01$

$1.27 \mathrm{E}+01$

$1.03 E+06$

$1.55 \mathrm{E}+03$ 
Tank Anal. Un

$A 102$ Tin126 Ci $\frac{\text { May98 }}{6.95 \mathrm{E}-00}$

$\begin{array}{llll}\mathrm{A102} & \text { Tritium } & \mathrm{Ci} & 3.29 \mathrm{E}+01 \\ \mathrm{A102} & \mathrm{U} 232 & \mathrm{Ci} & 1.56 \mathrm{E}-01\end{array}$

$\begin{array}{llll}\mathrm{A} 102 & \mathrm{U} 233 & \mathrm{Ci} & 5.97 \mathrm{E}-01 \\ \mathrm{A102} & \mathrm{U} 234 & \mathrm{Ci} & 9.50 \mathrm{E}-02\end{array}$

$\begin{array}{llll}A 102 & \mathrm{U} 235 & \mathrm{Ci} & 3.76 \mathrm{E}-03\end{array}$

$\mathrm{A102} \mathrm{U} 236 \mathrm{Ci}$ 3.08E-03

$A 102$ U238 Ci 1.33E-01

$\begin{array}{llll}\mathrm{A} 102 & \mathrm{Y} 90 & \mathrm{Ci} & 1.35 \mathrm{E}+05\end{array}$

$\begin{array}{llll}\mathrm{A} 102 & \mathrm{Zr} 93 & \mathrm{Ci} & 1.91 \mathrm{E}+01\end{array}$

Al03 Al $\mathrm{kg}$ 3.34E+04

$\begin{array}{llll}\mathrm{A} 103 & \mathrm{Bi} & \mathrm{kg} & 1.85 \mathrm{E}+02\end{array}$

$\mathrm{A103} \mathrm{Ca}$ kg $3.23 \mathrm{E}+03$

$\mathrm{A103} \mathrm{Cl}$

103

A103

A103

$\begin{array}{llll}\mathrm{A103} & \mathrm{Fe} & \mathrm{kg} & 1.54 \mathrm{E}+03 \\ \mathrm{Al03} & \mathrm{Hg} & \mathrm{kg} & 2.45 \mathrm{E}-00\end{array}$

$\begin{array}{llll}\mathrm{A103} & \mathrm{K} & \mathrm{kg} & \mathbf{5 . 1 2 E}+03\end{array}$

$\mathrm{Al03} \mathrm{La}$ kg 3.75E-00

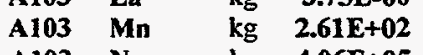

$\mathrm{Al03} \mathrm{Na}$ kg $4.06 \mathrm{E}+05$

$\begin{array}{llll}\mathrm{A} 103 \mathrm{Ni} & \mathrm{kg} & \mathbf{2 . 4 4 E}+02\end{array}$

$\mathrm{A} 103 \mathrm{NO2} \mathrm{kg} \quad 1.40 \mathrm{E}+05$

$\mathrm{AlO3}_{\mathrm{NO3}} \mathrm{kg} \quad 2.20 \mathrm{E}+05$

$A 103 P$ kg $1.25 E+04$

$\mathrm{Al03} \mathrm{Pb} \quad \mathrm{kg} \quad 2.06 \mathrm{E}+02$

$\mathrm{Al03} \mathrm{PO}_{4} \mathrm{~kg} \cdot 3.86 \mathrm{E}+04$

$\begin{array}{llll}\mathrm{A} 103 \mathrm{~S} & \mathrm{~kg} & 3.40 \mathrm{E}+04\end{array}$

Al03 Si

$\mathrm{Al03} \mathrm{SO}_{4}$

$\begin{array}{llll}\mathrm{A} 103 & \mathrm{Sr} & \mathrm{kg} & 2.20 \mathrm{E}+01 \\ \mathrm{~A} 103 & \text { TOC } & \mathrm{kg} & 1.52 \mathrm{E}+04\end{array}$

$\begin{array}{llll}\mathrm{A} 103 \mathrm{U} & \mathrm{kg} & 2.66 \mathrm{E}+03\end{array}$

$\begin{array}{llll}\mathrm{A} 103 & \mathrm{Zr} & \mathrm{kg} & 3.69 \mathrm{E}+02\end{array}$

$\begin{array}{llll}\mathrm{A} 103 & \mathrm{Ac22} & \mathrm{Ci} & 1.51 \mathrm{E}-03\end{array}$

Al03 Am241 Ci 2.32E+02

A103 Am243 Ci 3.36E-03

A103 Ba137 Ci 2.77E+05

$\mathrm{A} 103 \mathrm{C} 14$ Ci 4.92E-00

Al03 Cdl13 Ci 1.29E+02

Al03 Cm242 Ci 2.32E-01

A103 Cm243 Ci 2.09E-02

$\begin{array}{llll}\mathrm{A} 103 \mathrm{Cm} 244 & \mathrm{Ci} & 1.68 \mathrm{E}-01\end{array}$

$\begin{array}{llll}A 103 & \text { Co60 } & \text { Ci } & 2.35 E+01\end{array}$

A103 Csi34 Ci 5.33E-00

$\begin{array}{llll}A 103 & \text { Csi37 Ci } & \text { 2.93E+05 }\end{array}$

A103 Euls2 Ci 7.17E-00

Al03 Eu154 Ci $9.44 E+02$

A103 Eu155 Ci 4.35E+02

$\begin{array}{llll}\mathrm{A} 103 & 1129 & \mathrm{Ci} & 2.64 \mathrm{E}-03\end{array}$

$\mathrm{A} 103$ Nb93 $\mathrm{Ci} \quad 1.86 \mathrm{E}+01$

Al03 Ni59 Ci $3.66 \mathrm{E}-00$

$\mathrm{A} 103 \mathrm{Ni} 63 \mathrm{Ci} 3.60 \mathrm{E}+02$

A103 Np237 Ci 1.25E-00

$\begin{array}{llll}\mathrm{A} 103 & \mathrm{~Pa} 231 & \mathrm{C} j & 5.88 \mathrm{E}-03\end{array}$

$\begin{array}{llll}\text { A } 103 \text { Pu238 Ci 1.92E-00 } & \text { Ci }\end{array}$

A103 Pu239 C Ci 2.81E+02

A103 Pu240 Ci Incl. in 239
A

A103 Pu24l Ci 1.33E+02

A103 Pu242 Ci 7.27E-04

A103 Ra226 Ci 257E-04

$\mathrm{A} 103$ Ra228 Ci 4.93E-01

A103 Ru106 Ci 1.08E-02

$\begin{array}{llll}A 103 & \text { Sbl25 } & \text { Ci } & \text {.73E+02 }\end{array}$

$\begin{array}{llll}A 103 & \text { Se } 79 & \text { Ci } & \text { 5.22E-00 }\end{array}$

Al03 Sml51 Ci 1.85E+04
$B B$ Sept98

6.95E-00

$3.29 \mathrm{E}+01$

$3.59 \mathrm{E}-00$

$1.38 \mathrm{E}+01$

2.19E-00

8.67E-02

$7.10 \mathrm{E}-02$

$1.95 \mathrm{E}-00$

$1.35 \mathrm{E}+05$

$1.91 \mathrm{E}+01$

$3.34 \mathrm{E}+04$

$1.85 \mathrm{E}+02$

3.23E +03

$1.02 \mathrm{E}+04$

$3.81 \mathrm{E}+04$

$2.86 \mathrm{E}+03$

$1.57 \mathrm{E}+03$

$1.54 \mathrm{E}+03$

$0.00 \mathrm{E}+01$

$5.12 \mathrm{E}+03$

$0.00 \mathrm{E}+01$

$2.61 \mathrm{E}+02$

$4.06 \mathrm{E}+05$

$2.44 E+02$

$1.40 \mathrm{E}+05$

$2.20 \mathrm{E}+05$

NA

$2.06 \mathrm{E}+02$

$1.26 \mathrm{E}+04$

NA

$2.06 \mathrm{E}+04$

$3.40 \mathrm{E}+04$

$2.20 \mathrm{E}+01$

$1.52 \mathrm{E}+04$

$2.66 \mathrm{E}+03$

$3.69 \mathrm{E}+02$

1.51E-03

$2.32 E+02$

8.19E-03

2.77E+05

4.92E-00

$1.29 \mathrm{E}+02$

5.65E-01

5.09E-02

4.11E-01

$2.35 \mathrm{E}+01$

5.33E-00

2.93E+05

7.17E-00

$9.44 \mathrm{E}+02$

$4.35 \mathrm{E}+02$

2.64E-03

$1.86 \mathrm{E}+01$

$3.66 \mathrm{E}-00$

$3.60 \mathrm{E}+02$

$1.25 \mathrm{E}-00$

$5.88 \mathrm{E}-03$

7.23E-00

$2.40 \mathrm{E}+02$

$4.15 \mathrm{E}+01$

$5.03 E+02$

2.74E-03

2.57E-04

4.93E-01

1.08E-02

$2.73 \mathrm{E}+02$

5.22E-00

$1.85 \mathrm{E}+04$ 1\%-ile

$0.00 \mathrm{E}+01 \quad 0.00 \mathrm{E}+01$

$8.56 \mathrm{E}-00 \quad 1.82 \mathrm{E}+01$

$0.00 \mathrm{E}+01 \quad 0.00 \mathrm{E}+01$

$0.00 E+01 \quad 0.00 E+01$

$0.00 \mathrm{E}+01 \quad 0.00 \mathrm{E}+01$

$0.00 \mathrm{E}+01 \quad 0.00 \mathrm{E}+01$

$0.00 \mathrm{E}+01 \quad 0.00 \mathrm{E}+01$

$0.00 \mathrm{E}+01$

$0.00 \mathrm{E}+01$

$0.00 \mathrm{E}+01$

$2.46 \mathrm{E}+03$

$3.47 \mathrm{E}-02$

$1.13 \mathrm{E}+02$

$2.62 \mathrm{E}+01$

$4.84 \mathrm{E}+02$

$1.28 \mathrm{E}+01$

8.62E-02

$1.47 \mathrm{E}+02$

$0.00 \mathrm{E}+01$

$2.63 \mathrm{E}+01$

$6.88 \mathrm{E}-02$

$6.86 \mathrm{E}-01$

$1.32 \mathrm{E}+05$

$5.47 \mathrm{E}+01$

$4.53 \mathrm{E}+02$

$2.82 \mathrm{E}+02$

$2.13 \mathrm{E}-00$

2.37E-01

$2.36 \mathrm{E}+01$

$2.71 \mathrm{E}+01$

$3.37 \mathrm{E}+01$

$6.45 \mathrm{E}+01$

$2.08 \mathrm{E}-02$

$3.86 \mathrm{E}+01$

$1.64 \mathrm{E}+01$

9.13E-03

$0.00 \mathrm{E}+01$

8.18E-04

4.36E-06

$0.00 \mathrm{E}+01$

$0.00 \mathrm{E}+01$

$0.00 E+01$

3.01E-04

2.71E-05

2.18E-04

4.42E-02

$0.00 \mathrm{E}+01$

$4.26 \mathrm{E}+03$

$0.00 \mathrm{E}+01$

$0.00 \mathrm{E}+01$

$0.00 \mathrm{E}+01$

$0.00 E+01$

$0.00 \mathrm{E}+01$

$0.00 E+01$

$0.00 \mathrm{E}+01$

1.62E-03

$0.00 E+01$

2.49E-03

3.65E-01

3.65E-01

1.73E-01

9.44E-07

$0.00 \mathrm{E}+01$

$0.00 \mathrm{E}+01$

$0.00 E+01$

$0.00 E+01$

$0.00 \mathrm{E}+01$

$0.00 \mathrm{E}+01$
$0.00 \mathrm{E}+01$

$2.73 E+04$

$0.00 \mathrm{E}+01$

$2.61 \mathrm{E}+04$

$4.88 \mathrm{E}-01$

$5.33 \mathrm{E}+02$

$1.84 \mathrm{E}+02$

$2.09 \mathrm{E}+03$

$9.34 \mathrm{E}+01$

8.47E-00

$5.03 \mathrm{E}+02$

$0.00 \mathrm{E}+01$

$1.65 \mathrm{E}+03$

$2.74 \mathrm{E}-01$

$4.35 \mathrm{E}-00$

$2.92 \mathrm{E}+05$

$2.17 \mathrm{E}+02$

$4.82 \mathrm{E}+03$

$1.98 \mathrm{E}+04$

$3.10 \mathrm{E}+01$

$1.31 \mathrm{E}-00$

$1.10 \mathrm{E}+02$

$1.08 \mathrm{E}+02$

$1.69 \mathrm{E}+02$

$2.51 \mathrm{E}+02$

8.31E-02

$2.43 E+03$

$9.42 \mathrm{E}+01$

6.10E-02

$0.00 \mathrm{E}+01$

$4.77 \mathrm{E}-01$

$1.16 \mathrm{E}-04$

$0.00 \mathrm{E}+01$

$0.00 \mathrm{E}+01$

$0.00 \mathrm{E}+01$

8.01E-03

7.22E-04

$5.80 \mathrm{E}-03$

$3.56 \mathrm{E}-01$

$0.00 \mathrm{E}+01$

$2.76 \mathrm{E}+04$

$0.00 E+01$

$0.00 E+01$

$0.00 E+01$

$0.00 E+01$

$0.00 \mathrm{E}+01$

$0.00 \mathrm{E}+01$

4.32E-02

$0.00 E+0$

$6.63 \mathrm{E}-02$

$9.71 \mathrm{E}-00$

$9.71 \mathrm{E}-00$

4. $59 \mathrm{E}-00$

2.51E-05

$0.00 \mathrm{E}+01$

$0.00 \mathrm{E}+01$

$0.00 \mathrm{E}+01$

$0.00 \mathrm{E}+01$

$0.00 E+01$

$0.00 \mathrm{E}+01$
$0.00 \mathrm{E}+01$

50\%-ile

6.95E-00

$3.29 \mathrm{E}+01$

1.56E-01

5.97E-01

9.50E-02

3.76E-03

3.08E-03

$1.33 \mathrm{E}-01$

$1.35 \mathrm{E}+05$

$1.91 \mathrm{E}+01$

$5.73 \mathrm{E}+04$

$2.20 \mathrm{E}+02$

$2.11 E+03$

$4.84 \mathrm{E}+03$

$1.67 \mathrm{E}+05$

$7.56 \mathrm{E}+03$

$1.83 \mathrm{E}+04$

$6.88 \mathrm{E}+03$

2.45E-00

$5.79 \mathrm{E}+03$

$2.00 \mathrm{E}+02$ 
Tank Anal. Un

\begin{tabular}{|c|c|c|c|}
\hline A103 & Sr89/90 & $\mathbf{C i}$ & $\frac{M a y 98}{1.48 E+05}$ \\
\hline A103 & Te99 & $\mathrm{Ci}$ & $2.36 E+02$ \\
\hline A103 & Th229 & $\mathbf{C i}$ & $1.14 \mathrm{E}-02$ \\
\hline A103 & Th232 & $\mathrm{Ci}$ & 5.34E-02 \\
\hline$A 103$ & Tin126 & $\mathbf{C i}$ & 7.94E-00 \\
\hline A103 & Tritium & $\mathrm{Ci}$ & $3.00 \mathrm{E}+02$ \\
\hline A103 & $\mathbf{U} 232$ & $\mathrm{Ci}$ & 1.45E-00 \\
\hline Al03 & U233 & $\mathbf{C i}$ & $5.57 E-00$ \\
\hline $\mathbf{A 1 0 3}$ & U234 & $\mathbf{C i}$ & $8.87 E-01$ \\
\hline A103 & U235 & $\mathbf{C i}$ & 3.51E-02 \\
\hline A103 & U236 & $\mathrm{Ci}$ & $2.86 \mathrm{E}-02$ \\
\hline A103 & U238 & $\mathrm{Ci}$ & $1.24 \mathrm{E}-00$ \\
\hline A103 & Y90 & $\mathrm{Ci}$ & $1.48 E+05$ \\
\hline A103 & Zr93 & $\mathrm{Ci}$ & $2.53 \mathrm{E}+01$ \\
\hline A104 & Al & $\mathrm{kg}$ & $8.07 E+03$ \\
\hline A104 & $\mathbf{B i}$ & kg & $2.06 \mathrm{E}-01$ \\
\hline Al04 & $\mathrm{Ca}$ & $\mathrm{kg}$ & $1.74 E+03$ \\
\hline A104 & Cl & kg & $5.88 \mathrm{E}+01$ \\
\hline A104 & $\mathrm{CO3}$ & kg & $1.47 E+03$ \\
\hline A104 & $\mathrm{Cr}$ & kg & $7.67 E+01$ \\
\hline A104 & F & kg & $6.28 \mathrm{E}-01$ \\
\hline A104 & $\mathbf{F e}$ & kg & $2.96 E+04$ \\
\hline A104 & $\mathrm{Hg}$ & kg & $6.20 \mathrm{E}-03$ \\
\hline A104 & $\mathbf{K}$ & kg & $2.58 \mathrm{E}+01$ \\
\hline A104 & La & kg & $0.00 E+01$ \\
\hline Al04 & Mn & kg. & $4.14 E+03$ \\
\hline A104 & $\mathbf{N a}$ & kg & $1.34 E+04$ \\
\hline A104 & $\mathrm{Ni}$ & kg & $8.56 \mathrm{E}+02$ \\
\hline A104 & NO2 & kg & $3.07 E+03$ \\
\hline A104 & NO3 & kg & $2.70 \mathrm{E}-05$ \\
\hline A104 & $\mathbf{P}$ & kg & $1.87 E+02$ \\
\hline A104 & $\mathbf{P b}$ & kg & $1.05 \mathrm{E}-00$ \\
\hline A104 & PO4 & kg & $5.79 E+02$ \\
\hline A104 & $\mathbf{S}$ & $\mathrm{kg}$ & $6.80 \mathrm{E}+02$ \\
\hline A104 & $\mathrm{Si}$ & kg & $2.47 E+03$ \\
\hline A104 & SO4 & $\mathrm{kg}$ & $2.04 E+03$ \\
\hline Al04 & $\mathrm{Sr}$ & kg & $0.00 \mathrm{E}+01$ \\
\hline A104 & TOC & kg & $0.00 \mathrm{E}+01$ \\
\hline A104 & U & kg & $4.83 E+01$ \\
\hline A104 & $\mathbf{Z r}$ & kg & 3.55E-04 \\
\hline Al04 & Ac227 & $\mathrm{Ci}$ & $3.99 E-03$ \\
\hline A104 & Am241 & $\mathrm{Ci}$ & $1.75 \mathrm{E} \div 02$ \\
\hline A104 & $\operatorname{Am} 243$ & $\mathrm{Ci}$ & $3.80 \mathrm{E}-03$ \\
\hline A104 & Ba137 & $\mathrm{Ci}$ & $7.35 E \div 04$ \\
\hline A104 & C14 & $\mathrm{Ci}$ & $1.18 \mathrm{E}-00$ \\
\hline A104 & Cd113 & $\mathrm{Ci}$ & $1.53 E+01$ \\
\hline Al04 & $\mathrm{Cm} 242$ & $\mathrm{Ci}$ & $1.87 \mathrm{E}-01$ \\
\hline A104 & $\mathrm{Cm} 243$ & $\mathrm{Ci}$ & 1.03E-02 \\
\hline A104 & $\mathrm{Cm} 244$ & $\mathrm{Ci}$ & $8.06 E-02$ \\
\hline Al04 & Co60 & $\mathrm{Ci}$ & $4.85 E+02$ \\
\hline Al04 & Cs134 & $\mathrm{Ci}$ & $2.00 \mathrm{E}-01$ \\
\hline A104 & Cs137 & $\mathrm{Ci}$ & $7.77 \mathrm{E}+04$ \\
\hline A104 & Eu152 & $\mathrm{Ci}$ & $7.85 \mathrm{E}-00$ \\
\hline A104 & Eu154 & $\mathrm{Ci}$ & $2.86 E+03$ \\
\hline A104 & Eu155 & $\mathrm{Ci}$ & $5.13 E+02$ \\
\hline A104 & 1129 & $\mathrm{Ci}$ & $1.60 \mathrm{E}-02$ \\
\hline A104 & Nb93 & $\mathrm{Ci}$ & $2.03 E+01$ \\
\hline A104 & Ni59 & $\mathrm{Ci}$ & $1.18 \mathrm{E}+01$ \\
\hline Al04 & Ni63 & $\mathrm{Ci}$ & $1.16 \mathrm{E}+03$ \\
\hline A104 & Np237 & $\mathrm{Ci}$ & 2.62E-02 \\
\hline A104 & Pa231 & $\mathrm{Ci}$ & $5.48 E-03$ \\
\hline A104 & Pu238 & $\mathrm{Ci}$ & 2.95E-01 \\
\hline A104 & Pu239 & $\mathrm{Ci}$ & $7.23 E+02$ \\
\hline A104 & Pu240 & $\mathrm{Ci}$ & $2.44 E-00$ \\
\hline A104 & Pu241 & $\mathrm{Ci}$ & $2.15 E+01$ \\
\hline A104 & Pu242 & $\mathrm{Ci}$ & $9.36 \mathrm{E}-05$ \\
\hline Al04 & $\operatorname{Ra226}$ & $\mathrm{Ci}$ & $7.65 \mathrm{E}-04$ \\
\hline A104 & $\operatorname{Ra} 228$ & $\mathrm{Ci}$ & $8.48 E-05$ \\
\hline
\end{tabular}

Sept98

1.48E+05

$2.36 \mathrm{E}+02$

1.14E-02

5.34E-02

$7.94 \mathrm{E}-00$

$3.00 \mathrm{E}+02$

1.64E-00

$6.27 \mathrm{E}-00$

9.99E-01

$3.95 \mathrm{E}-02$

$3.22 \mathrm{E}-02$

8.88E-01

$1.48 \mathrm{E}+05$

$2.53 \mathrm{E}+01$

$8.07 \mathrm{E}+03$

$0.00 \mathrm{E}+01$

$1.74 \mathrm{E}+03$

$5.88 \mathrm{E}+01$

$1.47 \mathrm{E}+03$

$7.67 \mathrm{E}+01$

6.28E-01

$2.96 \mathrm{E}+04$

$0.00 E+01$

2.58E+01

$0.00 E+01$

$4.14 \mathrm{E}+03$

$1.34 E+04$

$8.56 \mathrm{E}+02$

$3.07 E+03$

2.70E-05

NA

1.05E-00

$1.89 \mathrm{E}+02$

NA

$2.47 \mathrm{E}+03$

$6.80 \mathrm{E}+02$

$7.03 \mathrm{E}+01$

$0.00 E+01$

$4.83 \mathrm{E}+01$

3.55E-04

3.99E-03

$1.75 \mathrm{E}+02$

$3.80 \mathrm{E}-03$

$7.35 \mathrm{E}+04$

1.18E-00

$1.53 \mathrm{E}+01$

1.87E-01

1.03E-02

8.06E-02

$4.85 \mathrm{E}+02$

2.00E-01

$7.77 \mathrm{E}+04$

7.85E-00

$2.86 \mathrm{E}+03$

$5.13 E+02$

1.60E-02

$2.03 E+01$

$1.18 \mathrm{E}+01$

$1.16 E+03$

$2.62 \mathrm{E}-02$

5.48E-03

$1.36 \mathrm{E}+01$

$7.23 \mathrm{E}+02$

$1.14 \mathrm{E}+02$

$9.95 \mathrm{E}+02$

4.33E-03

7.65E-04

8.48E-05 1\%-ile

$3.53 \mathrm{E} \div 03$

$0.00 \mathrm{E}+01$

$0.00 \mathrm{E}+01$

$0.00 \mathrm{E}+01$

$0.00 \mathrm{E}+01$

$6.78 \mathrm{E}+01$

$0.00 \mathrm{E}+01$

$0.00 \mathrm{E}+01$

$0.00 \mathrm{E}+01$

$0.00 \mathrm{E}+01$

$0.00 \mathrm{E}+01$

$0.00 \mathrm{E}+01$

$0.00 \mathrm{E}+01$

$0.00 \mathrm{E}+01$

$2.77 \mathrm{E}-06$

8.29E-09

$1.46 \mathrm{E}-05$

1.02E-06

2.78E-05

$6.79 \mathrm{E}-07$

1.12E-08

$2.36 \mathrm{E}-05$

$0.00 \mathrm{E}+01$

$3.16 \mathrm{E}-07$

1.44E-09

4.40E-07

$1.48 \mathrm{E}-04$

9.22E-06

2.25E-05

$1.90 \mathrm{E}-06$

6.44E-07

2.56E-08

1.23E-06

$1.31 \mathrm{E}-06$

6.31E-06

5.91E-06

$1.80 \mathrm{E}-09$

2.91E-07

4.67E-06

$1.89 \mathrm{E}-10$

$0.00 \mathrm{E}+01$

$2.14 \mathrm{E}-10$

$0.00 \mathrm{E}+01$

$0.00 \mathrm{E}+01$

$0.00 \mathrm{E}+01$

$0.00 \mathrm{E}+01$

$0.00 \mathrm{E}+01$

$0.00 \mathrm{E}+01$

$0.00 \mathrm{E}+01$

4.88E-09

$0.00 \mathrm{E}+01$

2.85E-04

$0.00 \mathrm{E}+01$

$0.00 \mathrm{E}+01$

$0.00 \mathrm{E}+01$

$0.00 \mathrm{E}+01$

$0.00 \mathrm{E}+01$

$0.00 \mathrm{E}+01$

$0.00 \mathrm{E}+01$

$0.00 \mathrm{E}+01$

$0.00 \mathrm{E}+01$

$0.00 \mathrm{E}+01$

$0.00 \mathrm{E}+01$

$0.00 \mathrm{E}+01$

$0.00 \mathrm{E}+01$

$0.00 \mathrm{E}+01$

$0.00 E+01$

$0.00 \mathrm{E}+01$
$10 \%$-ile

$7.98 \mathrm{E}+04 \quad 3.45 \mathrm{E}+05$

$0.00 \mathrm{E}+01 \quad 2.36 \mathrm{E}+02$

$0.00 \mathrm{E}+01 \quad 1.14 \mathrm{E}-02$

$0.00 \mathrm{E}+01$

$0.00 \mathrm{E}+01$

$1.69 \mathrm{E}+02$

$0.00 \mathrm{E}+01$

$0.00 \mathrm{E}+01$

$0.00 \mathrm{E}+01$

$0.00 \mathrm{E}+01$

$0.00 \mathrm{E}+01$

$0.00 \mathrm{E}+01$

$3.04 \mathrm{E}+04$

$0.00 \mathrm{E}+01$

$2.74 \mathrm{E}-05$

2.65E-07

1.87E-04

4.47E-06

1.92E-04

5.76E-06

$1.53 \mathrm{E}-06$

$1.56 \mathrm{E}-04$

$0.00 \mathrm{E}+01$

$3.83 \mathrm{E}-06$

4.86E-08

$1.59 \mathrm{E}-05$

$6.81 \mathrm{E}-04$

9.89E-05

2.16E-04

$1.15 \mathrm{E}-04$

1.22E-05

2.94E-07

2.55E-05

2.06E-05

$6.36 \mathrm{E}-05$

5.95E-05

8.64E-08

$1.93 \mathrm{E}-06$

3.82E-05

$5.93 \mathrm{E}-08$

$0.00 \mathrm{E}+01$

2.59E-06

$0.00 \mathrm{E}+01$

$0.00 \mathrm{E}+01$

$0.00 \mathrm{E}+01$

$0.00 E+01$

$0.00 \mathrm{E}+01$

$0.00 E+01$

$0.00 \mathrm{E}+01$

$1.56 \mathrm{E}-07$

$0.00 \mathrm{E}+01$

$1.80 \mathrm{E}-03$

$0.00 E+01$

$0.00 \mathrm{E}+01$

$0.00 \mathrm{E}+01$

$0.00 \mathrm{E}+0 \mathrm{I}$

$0.00 \mathrm{E}+01$

$0.00 \mathrm{E}+01$

$0.00 \mathrm{E}+01$

$0.00 \mathrm{E}+01$

$0.00 \mathrm{E}+01$

$0.00 \mathrm{E}+01$

$0.00 \mathrm{E}+01$

$0.00 \mathrm{E}+01$

$0.00 \mathrm{E}+01$

$0.00 \mathrm{E}+01$

$0.00 \mathrm{E}+01$

$0.00 \mathrm{E}+01$
5.34E-02

7.94E-00

$3.00 \mathrm{E}+02$

1.45E-00

5.57E-00

8.87E-01

3.51E-02

2.86E-02

$1.24 \mathrm{E}-00$

$1.48 \mathrm{E}+05$

$2.53 \mathrm{E}+01$

$3.39 \mathrm{E}+02$

$1.87 \mathrm{E}-00$

$1.16 E+03$

$6.97 \mathrm{E}+01$

$2.71 \mathrm{E}+03$

$8.29 \mathrm{E}+01$

$1.89 \mathrm{E}-00$

$1.94 E+03$

$6.20 \mathrm{E}-03$

$4.56 \mathrm{E}+01$

$1.01 \mathrm{E}+02$

$1.06 \mathrm{E}+04$

$1.18 \mathrm{E}+03$

$2.04 \mathrm{E}+03$

$5.64 \mathrm{E}+02$

$1.11 \mathrm{E}+02$

2.47E-00

$2.21 \mathrm{E}+02$

$1.85 \mathrm{E}+02$

$8.04 E+02$

$5.04 \mathrm{E}+02$

$3.59 \mathrm{E}-01$

$3.35 \mathrm{E}+01$

$5.25 \mathrm{E}+02$

9.91E-02

3.99E-03

9.30E-00

$3.80 \mathrm{E}-03$

$7.35 \mathrm{E}+04$

1.18E-00

$1.53 \mathrm{E}+01$

$1.87 \mathrm{E}-01$

1.03E-02

8.06E-02

1.34E-00

$2.00 \mathrm{E}-01$

$2.36 \mathrm{E}+04$

$7.85 \mathrm{E}-00$

$2.86 \mathrm{E}+03$

$5.13 \mathrm{E}+02$

1.60E-02

$2.03 E+01$

$1.18 \mathrm{E}+01$

$1.16 \mathrm{E}+03$

2.62E-02

5.48E-03

2.95E-01

$7.23 \mathrm{E}+02$

2.44E-00

$2.15 E+01$

9.36E-05

7.65E-04

8.48E-05
3.00E-01

90\%-ile

$9.95 \mathrm{E}+05$

$1.04 \mathrm{E}+03$

4.45E-02

2.09E-01

$3.30 \mathrm{E}+01$

$4.39 \mathrm{E}+02$

$5.66 \mathrm{E}-00$

$2.18 \mathrm{E}+01$

$3.46 \mathrm{E}-00$

$1.37 \mathrm{E}-01$

$1.12 \mathrm{E}-01$

4.84E-00

$4.36 \mathrm{E}+05$ 


\begin{tabular}{|c|c|c|c|c|c|c|c|c|c|c|c|}
\hline ank & Anal. & In & May $\frac{B B}{98}$ & Sept98 & $1 \%$-ile & $10 \%$-ile & $50 \%$-ile & $90 \%-i l e$ & 99\%-ile & Mean & Std Dev \\
\hline 104 & 1106 & $\mathrm{Ci}$ & $\overline{1.33 \mathrm{E}-03}$ & $\overline{1.33 E-03}$ & $0.00 \mathrm{E}+01$ & $0.00 \mathrm{E}+01$ & 1.33E-03 & $6.07 \mathrm{E}-03$ & $1.44 \mathrm{E}-02$ & $2.49 \mathrm{E}-03$ & $3.81 \mathrm{E}-03$ \\
\hline & Sb125 & $\mathrm{Ci}$ & $83 \mathrm{E}+02$ & $4.83 E+02$ & $0.00 \mathrm{E}+01$ & $0.00 \mathrm{E}+01$ & $4.83 E+02$ & $7.61 \mathrm{E}+03$ & $2+04$ & +03 & $37 E+03$ \\
\hline & Se79 & $\mathrm{Ci}$ & $5.34 \mathrm{E}-00$ & $5.34 \mathrm{E}-00$ & $0.00 \mathrm{E}+01$ & $0.00 \mathrm{E}+01$ & & $3.10 \mathrm{E}+01$ & $1.33 \mathrm{E}+02$ & $1.32 E+01$ & $70 \mathrm{E}+01$ \\
\hline A & Sm151 & $\mathrm{Ci}$ & $2.05 E+04$ & $2.05 E+04$ & $0.00 \mathrm{E}+01$ & $0.00 \mathrm{E}+01$ & $2.05 E+04$ & $02 \mathrm{E}+05$ & $4.40 E+05$ & $E+04$ & $.00 \mathrm{E}+05$ \\
\hline nd & Sr89/90 & $\mathrm{Ci}$ & $2.93 \mathrm{E}+06$ & $2.93 E+06$ & $1.01 \mathrm{E}-04$ & $48 \mathrm{E}-02$ & $2.50 \mathrm{E}+05$ & +06 & $1.78 \mathrm{E}+07$ & & +06 \\
\hline 104 & Tc99 & $\mathrm{Ci}$ & $8.29 \mathrm{E}-00$ & $8.29 E-00$ & $E+01$ & & & & & +01 & $E+01$ \\
\hline 104 & Th229 & $\mathrm{Ci}$ & 3.93E-05 & $3 \mathrm{E}-05$ & +01 & & $3.93 E-05$ & -04 & $\mathrm{E}-04$ & -05 & $8 \mathrm{E}-04$ \\
\hline & Th232 & $\mathrm{Ci}$ & 15E-06 & .15E-06 & $0.00 \mathrm{E}+01$ & $\mathrm{JE}+01$ & 9.15E-06 & $4.32 E-05$ & $1.24 \mathrm{E}-04$ & & $E-05$ \\
\hline & n126 & $\mathrm{Ci}$ & 71E-00 & & $0.00 \mathrm{E} \div 01$ & $\mathrm{EE}+01$ & $8.71 E-00$ & $6.50 \mathrm{E}+01$ & $2.46 \mathrm{E}+02$ & $2.59 E+01$ & $26 E+01$ \\
\hline & Tritium & $\mathrm{Ci}$ & $6.06 \mathrm{E}-01$ & $6.06 \mathrm{E}-01$ & $0.00 E+01$ & $0 E+01$ & $6.06 \mathrm{E}-01$ & $1.47 \mathrm{E}-00$ & $2.31 \mathrm{E}-00$ & $6.87 \mathrm{E}-01$ & $5.76 \mathrm{E}-01$ \\
\hline 104 & U232 & $\mathrm{Ci}$ & $57 \mathrm{E}-03$ & $5.37 \mathrm{E}-03$ & $E+01$ & $00 E+01$ & $5.37 \mathrm{E}-03$ & $\mathrm{E}-02$ & & $E-02$ & $\mathrm{IE}-02$ \\
\hline 104 & U233 & C & $2.08 \mathrm{E}-02$ & & $E+01$ & $E+01$ & -02 & -02 & -01 & $E-02$ & E-02 \\
\hline 104 & U234 & $\mathrm{Ci}$ & $1.67 \mathrm{E}-02$ & $1.67 \mathrm{E}-02$ & $0.00 \mathrm{E}+01$ & $E+01$ & $1.67 \mathrm{E}-02$ & $E-02$ & $E-01$ & 5E-02 & E-02 \\
\hline 104 & U235 & $\mathrm{Ci}$ & $6.92 \mathrm{E}-04$ & 6.92E-04 & +01 & & 6.9 & $\varepsilon-03$ & & $\varepsilon-03$ & $E-03$ \\
\hline & U236 & $\mathrm{Ci}$ & 4.58E-04 & 4.58 & +01 & & & & & -04 & -03 \\
\hline & v2 & $\mathrm{Ci}$ & $1.61 \mathrm{E}-02$ & $1.61 \mathrm{E}-02$ & +01 & 01 & -02 & .02 & & -02 & $\varepsilon-02$ \\
\hline 04 & Y90 & $\mathrm{Ci}$ & $2.93 \mathrm{E}+06$ & $2.93 E+06$ & +01 & +01 & +06 & +07 & -08 & $E+07$ & $E+07$ \\
\hline & Zr93 & $\mathrm{Ci}$ & $2.24 \mathrm{E}+01$ & $2.24 \mathrm{E}+01$ & $0.00 \mathrm{E}+01$ & $\div+01$ & $2.24 \mathrm{E}+01$ & +03 & & +02 & +03 \\
\hline IUS & Al & $\mathrm{kg}$ & $1.14 E+04$ & $1.14 E+04$ & -08 & & +01 & & & +02 & +02 \\
\hline & $\mathbf{B i}$ & kg & $0.00 \mathrm{E}+01$ & $0.00 \mathrm{E}+01$ & E-09 & & & & +02 & +01 & +01 \\
\hline & $\mathrm{Ca}$ & $\mathrm{kg}$ & $7.01 E+02$ & $7.01 E+02$ & E-06 & 05 & +02 & & & +03 & +03 \\
\hline 05 & $\mathrm{Cl}$ & kg & $5.38 \mathrm{E}+01$ & $5.38 \mathrm{E}+01$ & 07 & & 5.56 & & & & \\
\hline & $\cos$ & kg & $1.05 E+03$ & +03 & 05 & & & & & +03 & +03 \\
\hline 05 & $\mathrm{Cr}$ & $\mathrm{kg}$ & 2.43 & +01 & 1. & & 2.4 & & -02 & +01 & +02 \\
\hline 105 & $\mathbf{F}$ & kg & $0.00 E+01$ & $0.00 \mathrm{E}+01$ & $E-09$ & E-08 & -01 & +02 & +02 & +01 & $i+02$ \\
\hline 05 & $\mathrm{Fe}$ & $\mathrm{kg}$ & $2.65 E+04$ & $2.65 E+04$ & -06 & -05 & +03 & & & +03 & \\
\hline & $\mathbf{H g}$ & $\mathrm{kg}$ & $0.00 E+01$ & $0.00 E+01$ & & & & & & & +01 \\
\hline 05 & $\mathbf{K}$ & $\mathrm{kg}$ & $1.29 E+01$ & $1.29 \mathrm{E}+01$ & & & & & & & \\
\hline & $\mathbf{L a}$ & kg & 0.00 & 0.0 & 10 & & & & & & \\
\hline 05 & $\mathbf{M n}$ & kg & $8.98 \mathrm{E}+02$ & $8.98 \mathrm{E}+02$ & $8-09$ & -07 & -80 & & 1. & +01 & +01 \\
\hline A105 & $\mathrm{Na}$ & $\mathrm{kg}$ & $7.60 \mathrm{E}+04$ & $7.60 \mathrm{E}+04$ & $=-05$ & -04 & 5.4 & -04 & +04 & 103 & +04 \\
\hline 05 & $\mathrm{Ni}$ & $\mathrm{kg}$ & $2.46 E+02$ & $2.46 \mathrm{E}+02$ & -06 & & & & 104 & & +03 \\
\hline & $\mathrm{NO} 2$ & kg & $1.94 E+03$ & $1.94 E+03$ & & & & & & & \\
\hline & NO & $\mathrm{kg}$ & $2.75 \div>$ & $2.75 E-13$ & & & 9.0 & & & & +03 \\
\hline & $\mathbf{P}$ & kg & $0.00 \mathrm{E}+01$ & NA & -08 & & -00 & & 103 & -02 & $:+02$ \\
\hline A105 & $\mathbf{P b}$ & $\mathrm{kg}$ & $0.00 \mathrm{E}+01$ & $0.00 \mathrm{E}+01$ & $E-09$ & $6-07$ & -00 & -01 & +01 & -00 & $:+01$ \\
\hline Al05 & PO4 & $\mathrm{kg}$ & $0.00 E+01$ & $0.00 \mathrm{E}+01$ & $=-08$ & & -01 & 02 & +03 & -02 & +02 \\
\hline 05 & $\mathbf{S}$ & $\mathrm{kg}$ & $2.97 \mathrm{E}+02$ & NA & & & -02 & & +03 & & +03 \\
\hline & $S$ & kg & $6.70 \mathrm{E}+03$ & $6.70 E+03$ & & & & & +03 & & \\
\hline & 504 & $\mathrm{~kg}$ & 8.9 & 8. & & & & & & -03 & +03 \\
\hline 05 & $\mathrm{Sr}$ & kg & $0.00 E+01$ & +01 & & & -01 & -01 & -01 & -00 & $E-00$ \\
\hline A105 & TOC & $\mathrm{kg}$ & $0.00 \mathrm{E}+01$ & $0.00 \mathrm{E}+01$ & -08 & -07 & -00 & +02 & +03 & +01 & $\div 02$ \\
\hline A105 & $\mathbf{v}$ & kg & $3.55 E-00$ & $3.55 \mathrm{E}-00$ & -07 & & 3.8 & & +03 & 02 & +02 \\
\hline 105 & $\mathbf{Z r}$ & kg & $0.00 \mathrm{E}+01$ & $0.00 \mathrm{E}+01$ & & & & & & & +01 \\
\hline 05 & & $\mathrm{Ci}$ & 13 & & & & & & & & \\
\hline 15 & & $\mathrm{Ci}$ & & & & & & & -03 & +02 & +03 \\
\hline 05 & Am243 & $\mathrm{Ci}$ & -03 & 8.4 & 01 & & & & & .01 & $E-00$ \\
\hline A105 & Ba137 & $\mathrm{Ci}$ & $1.64 E+05$ & $1.64 E+05$ & +01 & 01 . & 1.6 & 106 & -06 & -05 & +06 \\
\hline A105 & C14 & $\mathrm{Ci}$ & $1.71 \mathrm{E}-00$ & $1.71 \mathrm{E}-00$ & +01 & -01 & -00 & +01 & +01 & -00 & +01 \\
\hline A105 & Cd113 & $\mathrm{Ci}$ & $1.58 \mathrm{E}+02$ & $1.58 \mathrm{E}+02$ & +01 & +01 & -02 & 103 & t03 & -02 & +03 \\
\hline 105 & $\operatorname{Cm} 242$ & $\mathrm{Ci}$ & 2E-01 & & & & & & & & +01 \\
\hline 105 & 243 & $\mathrm{Ci}$ & -02 & & & & & & & & \\
\hline & & $\mathrm{Ci}$ & & & & & & & & & +01 \\
\hline 105 & Co60 & $\mathrm{Ci}$ & $2.36 \mathrm{E}-00$ & .00 & & & & & & -01 & $E+01$ \\
\hline 05 & Cs134 & $\mathrm{Ci}$ & $5.62 \mathrm{E}-01$ & 5.62E-01 & +01 & & & & & $-\infty 0$ & -00 \\
\hline 105 & Cs137 & $\mathrm{Ci}$ & $1.73 E+05$ & $1.73 E+05$ & $\varepsilon-04$ & -03 & 2.6 & -05 & +06 & +04 & +05 \\
\hline 5 & Eu152 & $\mathrm{Ci}$ & $9.13 E-00$ & 00 & & & & & & & $8+01$ \\
\hline 105 & Eu154 & $\mathrm{Ci}$ & $7.37 E+03$ & $7.37 E+03$ & $8+01$ & & & +05 & & & +04 \\
\hline 105 & Eu155 & $\mathrm{Ci}$ & $6.80 E+02$ & 6.80 & & & & +04 & & & +03 \\
\hline 105 & & $\mathrm{Ci}$ & & & & & & & & -02 & $E-02$ \\
\hline & & $\mathrm{Ci}$ & $2.77 \mathrm{E}+01$ & $2.77 \mathrm{E}+01$ & & & & & & -03 & $E+03$ \\
\hline & & $\mathrm{Ci}$ & $8.48 E-00$ & 8.481 & & & & & -02 & & $E+02$ \\
\hline & & $\mathrm{Ci}$ & $8.52 E+02$ & $8.52 E+02$ & +01 & $E+01$ & & & & $\varepsilon+03$ & $E+04$ \\
\hline & $N$ & $\mathrm{Ci}$ & $2.59 E-02$ & $2595-0 ?$ & & & & & & & $E-00$ \\
\hline & Paz31 & $\mathrm{Ci}$ & $6.46 E-03$ & $6.46 \mathrm{E}-03$ & $0.00 \mathrm{E}+01$ & & & & & $E-02$ & $E-02$ \\
\hline & Puz & $\mathrm{Ci}$ & & 6.78 & +01 & $2.59 \mathrm{E}-07$ & 6.7 & $E+02$ & +03 & $2.11 E+02$ & $E+02$ \\
\hline Al05 & Puz39 & $\mathrm{Ci}$ & $2.10 \mathrm{E}+02$ & $1.76 E+02$ & $0.00 E+01$ & 7.99E-06 & $2.10 E+02$ & $1.10 E+04$ & +04 & $6.51 E+03$ & $2.81 E+04$ \\
\hline 1105 & Pu240 & $\mathrm{Ci}$ & cl. in 239 & $3.35 \mathrm{E}+01$ & $0.00 \mathrm{E}+01$ & $7.99 \mathrm{E}-06$ & $2.10 E+02$ & $1.10 \mathrm{E}+04$ & $9.99 \mathrm{E}+04$ & $6.51 E \div 03$ & $2.81 E+04$ \\
\hline
\end{tabular}




\begin{tabular}{|c|c|c|c|c|c|c|c|c|c|c|c|}
\hline$n$ & al. & $\ln$ & May $\frac{B B}{98}$ & Sept98 & $1 \%$-ile & ile & $50 \%-i l e$ & 90\%-ile & $9 \%$-ile & Mean & Dev \\
\hline 105 & 241 & $\mathbf{C i}$ & $4.83 \mathrm{E}+02$ & $4.83 E+02$ & $0.00 \mathrm{E}+01$ & $E-05$ & $4.83 E+02$ & $2.54 E+04$ & $E+05$ & $50 \mathrm{E}+04$ & $6.49 E+04$ \\
\hline & 1242 & $\mathrm{Ci}$ & $2.79 \mathrm{E}-03$ & $2.79 \mathrm{E}-03$ & $0.00 \mathrm{E}+01$ & & & E-01 & & -02 & $5 \mathrm{E}-01$ \\
\hline 105 & $\operatorname{Ra226}$ & $\mathrm{Ci}$ & 5.32E-04 & 5.32E-04 & $0.00 \mathrm{E}+01$ & $0.00 \mathrm{E}+01$ & 32E-04 & $3.33 \mathrm{E}-02$ & $E-01$ & $1.03 \mathrm{E}-02$ & $5 E-02$ \\
\hline 105 & $\operatorname{Ra228}$ & C & $4.81 \mathrm{E}-09$ & $4.81 \mathrm{E}-09$ & $0.00 \mathrm{E}+01$ & $.00 \mathrm{E}+01$ & 4.81E-09 & $3.01 \mathrm{E}-07$ & $1.12 \mathrm{E}-06$ & 9.31E-08 & $2.49 \mathrm{E}-07$ \\
\hline 105 & Ru106 & $\mathrm{Ci}$ & $1.00 E-02$ & $1.00 \mathrm{E}-02$ & $0.00 \mathrm{E}+01$ & & $1.00 \mathrm{E}-02$ & $9.17 \mathrm{E}-02$ & & $3.27 \mathrm{E}-02$ & $9 \mathrm{E}-02$ \\
\hline 105 & Sb125 & C & $2.42 \mathrm{E}+02$ & $2.42 \mathrm{E}+02$ & $0.00 \mathrm{E}+01$ & $00 \mathrm{E}+01$ & $2.42 \mathrm{E}+02$ & $E \div 03$ & $4.34 E+04$ & $3.17 \mathrm{E}+03$ & $21 E+03$ \\
\hline 05 & & $\mathrm{Ci}$ & $8.02 \mathrm{E}-00$ & $8.02 \mathrm{E}-00$ & $E+01$ & $E+01$ & $8.02 \mathrm{E}-00$ & $E+01$ & $E+02$ & $E+01$ & $3 E+01$ \\
\hline 05 & n151 & $\mathrm{Ci}$ & $E+04$ & $2.99 \mathrm{E}+04$ & $0 \mathrm{E}+01$ & $E+01$ & $2.99 E+04$ & $E+05$ & $E+06$ & $1.77 \mathrm{E}+05$ & $19 \mathrm{E}+05$ \\
\hline & Sr89/90 & $\mathrm{Ci}$ & $4.71 E+06$ & $4.71 E+06$ & $9 \mathrm{E}-07$ & $E-03$ & $6.10 \mathrm{E}+04$ & $6 \mathrm{E}+06$ & $E+07$ & $1.62 E+06$ & $.01 \mathrm{E}+06$ \\
\hline & & $\mathbf{C} \mathbf{i}$ & $1.21 \mathrm{E}+01$ & $1.21 E+01$ & $0.00 \mathrm{E}+01$ & +01 & $1.21 E+01$ & & & & $1 E+02$ \\
\hline & h229 & 0 & $7.51 \mathrm{E}-07$ & $7.51 \mathrm{E}-07$ & $E+01$ & +01 & $7.51 \mathrm{E}-07$ & & & -06 & DEE-06 \\
\hline & h232 & $\mathrm{Ci}$ & $4.34 \mathrm{E}-10$ & $4.34 \mathrm{E}-10$ & $E+01$ & +01 & $4.34 \mathrm{E}-10$ & & & -09 & $\mathrm{E}-09$ \\
\hline 05 & Tin126 & $\mathbf{C i}$ & $1.25 \mathrm{E}+01$ & $1.25 \mathrm{E}+01$ & $0.00 \mathrm{E}+01$ & $E+01$ & $1.25 E+01$ & $E+02$ & $E+02$ & $E+01$ & $E+02$ \\
\hline & Tritium & $\mathrm{Ci}$ & $9.18 E-00$ & $9.18 \mathrm{E}-00$ & $E+01$ & & $8 E-60$ & & & & $E+01$ \\
\hline & U232 & & 6.0 & $9.88 \mathrm{E}-08$ & & & & & & & \\
\hline & & $\mathrm{C}$ & E-08 & 2.33E-09 & 01 & & & & & & \\
\hline & & $\mathrm{C}$ & 7.44E-03 & $1.22 \mathrm{E}-03$ & 01 & & -03 & & -01 & -02 & \\
\hline & & 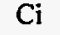 & $3.10 \mathrm{E}-04$ & $5.08 \mathrm{E}-05$ & $E+01$ & & $3.10 \mathrm{E}-04$ & $E-03$ & -02 & -03 & E-03 \\
\hline & & 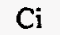 & & $3.32 \mathrm{E}-05$ & +01 & & $03 E-04$ & & -03 & & $E-03$ \\
\hline & 38 & & 25E-03 & $1.19 \mathrm{E}-03$ & +01 & & E-103 & & & & $E-02$ \\
\hline 05 & Y90 & 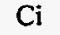 & $4.71 E+06$ & $4.71 \mathrm{E}+06$ & $i+01$ & & $4.71 E+06$ & & & & $E+08$ \\
\hline & $\mathbf{Z r} 9$ & & $3.68 \mathrm{E}+01$ & $3.68 \mathrm{E}+01$ & & & 01 & & & & +03 \\
\hline 06 & Al & $k$ & $1.67 E+04$ & $1.67 E+04$ & 01 & & 02 & & & & +03 \\
\hline & E & & $1.34 \mathrm{E}+02$ & $1.34 \mathrm{E}+02$ & 01 & & -60 & +01 & & +01 & $\div 01$ \\
\hline & c & $\mathrm{h}$ & $3.86 \mathrm{E}+03$ & $3.86 \mathrm{E}+03$ & & & -03 & +04 & & +04 & $E+04$ \\
\hline & & & +03 & 5.8 & & & & & & +02 & $E+02$ \\
\hline & Co3 & & $1.59 E+04$ & $1.59 E+04$ & $: 03$ & & +04 & & & +04 & +04 \\
\hline & $\mathrm{Cr}$ & k & +03 & $3.71 E+03$ & & +01 & -02 & -02 & & & \\
\hline 06 & $\mathbf{F}$ & & +02 & +02 & & & $8-00$ & -01 & & & +01 \\
\hline 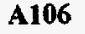 & $\mathbf{F}$ & & $1.93 E+04$ & $1.93 E+04$ & +02 & & $1.26 E+04$ & -04 & & +04 & $E+04$ \\
\hline & $\mathbf{t}$ & & Q-01 & $0.00 \mathrm{E}+01$ & & & & & & & $E-00$ \\
\hline 06 & $\mathbf{K}$ & k & $1.65 E+03$ & $1.65 E+03$ & & & & & & & $E+02$ \\
\hline & La & & $7.55 \mathrm{E}+01$ & $7.55 \mathrm{E}+01$ & & & $E-00$ & & & & \\
\hline & & $\mathrm{k}$ & +03 & 1. & & & & & & & \\
\hline & & & +04 & $i+04$ & & & +04 & 105 & & .04 & +04 \\
\hline & I & & +02 & $4.58 \mathrm{E}+02$ & +02 & & +03 & +04 & & & $E+04$ \\
\hline & & & +04 & $6.10 \mathrm{E}+04$ & & & & & -04 & t04 & $E+04$ \\
\hline & NO3 & $\mathrm{k}$ & & $5.73 E+04$ & & & +03 & & & +03 & $i+03$ \\
\hline & $\mathbf{P}$ & $\mathrm{kg}$ & +04 & NA & 01 & & & & & & \\
\hline & $\mathbf{P b}$ & $\mathrm{kg}$ & 7.6 & $E+02$ & & & & 10I & & -01 & +01 \\
\hline & PO4 & $\mathrm{kg}$ & +04 & $3.95 E+04$ & & & +02 & +03 & +03 & +03 & +03 \\
\hline & $\mathbf{S}$ & $\mathrm{kg}$ & $1.10 E+03$ & NA & +01 & & $E+03$ & +03 & & t03 & +03 \\
\hline & s & & +04 & $2.82 E+04$ & & & -03 & -04 & & +03 & $8+04$ \\
\hline & & kg & & $3.29 E+03$ & & & & & & & $E+04$ \\
\hline & Sr & kg & & & & & & & & & \\
\hline & & $\mathrm{kg}$ & & & & & & & & 04 & \\
\hline & $\mathbf{U}$ & $\mathrm{kg}$ & -02 & +02 & & & -02 & +03 & & -03 & +03 \\
\hline & $\mathbf{Z r}$ & $\mathrm{kg}$ & $\mathrm{E}+02$ & $8.83 E+02$ & $E-02$ & & -01 & -00 & +01 & -00 & -00 \\
\hline & & $\mathrm{Ci}$ & & 2 & & & & & & & \\
\hline & & $\mathrm{Ci}$ & & +03 & & & & & & & +04 \\
\hline & & $\mathrm{Ci}$ & & & & & & & & & \\
\hline & & $\mathrm{Ci}$ & & & & & & & & & \\
\hline & & $\mathrm{C} \mathbf{i}$ & & +01 & & & & & & & +01 \\
\hline & & $\mathrm{Ci}$ & $3.86 \mathrm{E}+02$ & $3.86 E+02$ & +01 & & $3.86 \mathrm{E}+02$ & & & & $E+03$ \\
\hline & $C m 24$ & $\mathrm{C} \mathbf{i}$ & $E-00$ & & & & & & & & $E+01$ \\
\hline & & $\mathrm{Ci}$ & $E-01$ & $1.32 \mathrm{E}-01$ & & & 1.32E-01 & & $E+01$ & $E-01$ & $\mathrm{E}-00$ \\
\hline A106 & $\mathrm{Cm} 24$ & $\mathrm{Ci}$ & $4.96 E-00$ & $4.96 \mathrm{E}-00$ & & & & & $E+02$ & +01 & $E+02$ \\
\hline A106 & $\mathrm{Co60}$ & $\mathrm{Ci}$ & 1.63 & 1.6 & & & & & & & $E+02$ \\
\hline A106 & & $\mathrm{Ci}$ & & & & & & & & & $6 \mathrm{E}-00$ \\
\hline AlO & & $\mathrm{Ci}$ & & & & & & & +06 & +05 & $E+05$ \\
\hline & & $\mathrm{C}$ & & & & & & & & & $E+01$ \\
\hline & & $\mathrm{Ci}$ & & $1.06 \mathrm{E}+03$ & & & & & & & $E+03$ \\
\hline & & $\mathrm{Ci}$ & $2.61 \mathrm{E}+03$ & $2.61 \mathrm{E}+0$ & +01 & & 2.6 & & & & $E+03$ \\
\hline & II & $\mathrm{Ci}$ & $1.88 E-01$ & $1.88 \mathrm{E}-61$ & $E-02$ & $E-02$ & $1.88 \mathrm{E}-01$ & -01 & $=-00$ & $:-01$ & 2E-01 \\
\hline A106 & Nb93 & $\mathrm{Ci}$ & $1.58 E+02$ & $1.58 E+02$ & $E-00$ & $E+01$ & $1.58 E+02$ & $E+03$ & $E+03$ & $E+03$ & $E+03$ \\
\hline $\boldsymbol{A}$ & Nis9 & $\mathrm{Ci}$ & $7.65 E+01$ & $7.65 E+01$ & $E-00$ & $E+01$ & $7.65 \mathrm{E}+01$ & $E+02$ & $E+03$ & $E+02$ & $5.90 \mathrm{E}+02$ \\
\hline 1 & Ni6 & $\mathrm{Ci}$ & $7.53 E$ & $7.53 \mathrm{E}+03$ & & & & & & & $5.81 \mathrm{E}+04$ \\
\hline & & $\mathrm{Ci}$ & & & & & & $E-O D$ & +01 & $F_{0}$ & \\
\hline
\end{tabular}


Tank Anal. Un

\begin{tabular}{|l|l|}
\hline A106 & Pa231 \\
\hline A106 & Pu238 \\
A106 & Pu239 \\
A106 & Pu240 \\
\hline A106 & Pu241 \\
A106 & Pu242 \\
A106 & Ra226 \\
A106 & Ra228 \\
\hline A106 & Ru106 \\
A106 & Sb125 \\
A106 & Se79 \\
\hline A106 & Sm151 \\
\hline A106 & Sr89/90 \\
A106 & Tc99 \\
A106 & Th229 \\
\hline A106 & Th232 \\
\hline A106 & Tin126 \\
A106 & Tritium \\
A106 & U232 \\
A106 & U233 \\
\hline A106 & U234 \\
A106 & U235 \\
\hline A106 & U236 \\
\hline A106 & U238 \\
\hline A106 & Y90 \\
A106 & Zr93 \\
\hline AN101 & Al \\
\hline A1101 & Bi \\
\hline
\end{tabular}

AN101 Bi

AN101 $\mathrm{Ca}$

AN101 Cl

AN101 $\mathrm{CO} 3$

AN101 $\mathrm{Cr}$

AN101 $F$

AN101 Fe

AN101 Hg

AN101 K

AN101 La

AN101 Mn

AN101 Na

AN101 Ni

AN101 $\mathrm{NO2}$

AN101 NO3

AN101 P

AN101 Pb

AN101 PO4

AN101 S

AN101 Si

AN101 S04

ANio1 $\mathrm{Sr}$

AN101 TOC

AN101 $U$

AN101 Zr

AN101 Ac227

AN101 Am241

AN101 Am243

AN101 Bal37

AN101 C14

AN101 CdIl3

AN101 Cm242

AN101 $\mathrm{Cm} 243$

AN101 $\mathrm{Cm} 244$

AN101 C060

AN101 Cs134

AN101 Cs137

AN101 Eu152

AN101 Eu154

AN101 Eu155

AN101 1129

kg

kg

kg

$\mathrm{kg}$

$\mathrm{kg}$

$\mathrm{kg}$

\begin{tabular}{|c|c|}
\hline Un & $B B$ \\
\hline $\mathrm{Ci}$ & $\frac{\text { May } 98}{4.02 \mathrm{E}-02}$ \\
\hline $\mathrm{Ci}$ & $5.29 \mathrm{E}+01$ \\
\hline $\mathrm{Ci}$ & $1.44 E+03$ \\
\hline $\mathrm{Ci}$ & $2.64 \mathrm{E}+02$ \\
\hline $\mathrm{Ci}$ & $3.75 E+03$ \\
\hline $\mathrm{Ci}$ & $2.21 \mathrm{E}-02$ \\
\hline $\mathrm{Ci}$ & $5.11 E-03$ \\
\hline $\mathrm{Ci}$ & $8.39 E-02$ \\
\hline $\mathrm{C}$ & $2.92 \mathrm{E}-8$ \\
\hline C & $7.36 E+0$ \\
\hline & \\
\hline
\end{tabular}

$\frac{\text { Sept98 }}{4.02 \mathrm{E}-02}$

$5.34 E+01$

$1.44 \mathrm{E}+03$

2.67E+02

$3.79 \mathrm{E}+03$

2.23E-02

5.11E-03

8.39E-02

2.92E-01

$7.36 \mathrm{E}+01$

$4.25 \mathrm{E}+01$

$1.59 \mathrm{E}+05$

$8.34 \mathrm{E}+05$

$9.71 \mathrm{E}+01$

1.98E-03

8.27E-03

$6.83 \mathrm{E}+01$

$8.65 \mathrm{E}+01$

2.48E-01

9.53E-01

2.18E-01

8.75E-03

6.95E-03

$1.97 \mathrm{E}-01$

$8.34 \mathrm{E}+05$

$1.84 \mathrm{E}+02$

$9.26 \mathrm{E}+03$

$0.00 \mathrm{E}+01$

3.36E-00

$1.46 \mathrm{E}+03$

$5.42 \mathrm{E}+03$

$9.91 \mathrm{E}+01$

$2.10 E+02$

$1.20 \mathrm{E}-00$

$0.00 \mathrm{E}+01$

kg 8

$\mathrm{kg}$ NA

1.59E+03

$\mathrm{kg}$

kg $3.03 \mathrm{E}+02$

$\mathrm{kg} \quad$ 7.28E-00

kg $\quad 9.39 \mathrm{E}+02$

kg $4.50 \mathrm{E}+02$

$\mathrm{kg} \quad 3.80 \mathrm{E}-00$

$\mathrm{kg} \quad 1.35 \mathrm{E}+03$

$\mathrm{kg}$

kg

1.76E-01

$1.61 \mathrm{E}-04$

9.74E-01

3.57E-04

$4.19 \mathrm{E}+04$

5.76E-00

$1.44 \mathrm{E}+01$

2.05E-02

$1.91 \mathrm{E}-03$

$1.90 \mathrm{E}-02$

$2.28 \mathrm{E}-00$

$7.73 \mathrm{E}-00$

$4.43 E+04$

$5.76 \mathrm{E}-01$

$9.96 E+01$

$3.37 \mathrm{E}+01$

8.10E-02

$1.59 \mathrm{E}+03$

$0.00 \mathrm{E}+01$

$2.60 \mathrm{E}+02$

5.67E+04

1.50E-00

2.13E+04

$4.30 \mathrm{E}+04$

NA

$7.28 \mathrm{E}-00$

$9.39 \mathrm{E}+02$

NA

$3.80 \mathrm{E}-00$

$1.35 \mathrm{E}+03$

$0.00 \mathrm{E}+01$

$1.16 \mathrm{E}+03$

$1.76 \mathrm{E}-01$

1.61E-04

2.28E-02

8.37E-07

$5.37 \mathrm{E}+04$

$5.76 \mathrm{E}-00$

$1.44 E+01$

4.79E-05

4.49E-06

4.44E-05

$2.28 \mathrm{E}-00$

$7.73 \mathrm{E}-00$

$5.68 \mathrm{E}+04$

$5.76 \mathrm{E}-01$

$9.96 \mathrm{E}+01$

$3.37 \mathrm{E}+01$

8.10E-02
$1 \%$-ile

$10 \%$-ile

$4.36 \mathrm{E}-04$
$1.62 \mathrm{E}-02$

$4.40 \mathrm{E}-01$

8.07E-02

$1.15 \mathrm{E}-00$

$6.76 \mathrm{E}-06$

2.50E-04

$4.10 \mathrm{E}-03$

$0.00 \mathrm{E}+01$

$0.00 \mathrm{E}+01$

2.63E-00

$0.00 \mathrm{E}+01$

$1.77 \mathrm{E}+02$

7.03E-01

2.15E-05

8.96E-05

4.54E-00

$3.02 \mathrm{E}+01$

$3.22 \mathrm{E}-03$

$1.24 \mathrm{E}-02$

2.82E-03

1.13E-04

9.00E-05

3.50E-03

$0.00 E+01$

$4.86 \mathrm{E}-00$

$3.35 \mathrm{E}+03$

$7.56 \mathrm{E}-04$

2.50E-00

$1.18 \mathrm{E}+02$

2.70E+03

$4.16 \mathrm{E}+01$

$3.81 \mathrm{E}-03$

2.88E-00

NA

4.33E-02

$1.07 \mathrm{E}-04$

$1.04 E+02$

$3.41 E+04$

$4.25 \mathrm{E}-00$

4.07E+03

$1.09 \mathrm{E}+04$

$2.17 E+02$

$2.06 \mathrm{E}-03$

$3.19 \mathrm{E}+02$

4.17E-01

4.14E+01

$4.07 \mathrm{E}+02$

$1.87 \mathrm{E}+01$

$3.64 \mathrm{E}+02$

6.12E-03

$8.13 \mathrm{E}-00$

$1.15 \mathrm{E}+02$

$6.80 \mathrm{E}-04$

1.35E-03

2.21E-02

7.35E-02

$0.00 \mathrm{E}+01$

$1.00 \mathrm{E}+01$

$0.00 \mathrm{E}+01$

$3.57 \mathrm{E}+05$

6.98E-00

3.73E-04

$1.56 \mathrm{E}-03$

$1.65 \mathrm{E}+01$

$5.33 \mathrm{E}+01$

$5.59 \mathrm{E}-02$

2.15E-01

$4.90 \mathrm{E}-02$

$1.96 \mathrm{E}-03$

$1.56 \mathrm{E}-03$

6.08E-02

1. $28 \mathrm{E}+05$

$3.30 \mathrm{E}+01$

$4.89 \mathrm{E}+03$.

$1.19 \mathrm{E}-02$

$2.28 \mathrm{E}+01$

$6.14 \mathrm{E}+02$

$5.01 \mathrm{E}+03$

$1.49 \mathrm{E}+02$

$8.33 \mathrm{E}+01$

$2.87 \mathrm{E}+01$

$\mathrm{NA}$

8.21E-0I

$2.90 \mathrm{E}-01$

$1.39 \mathrm{E}+02$

$5.09 \mathrm{E}+04$

$2.46 \mathrm{E}+01$

$8.65 \mathrm{E}+03$

$2.70 \mathrm{E}+04$

$1.35 E+03$

4.75E-02

$2.30 \mathrm{E}+03$

$1.14 \mathrm{E}+02$

$9.75 \mathrm{E}+01$

$8.11 \mathrm{E}+02$

$2.40 \mathrm{E}+01$

$7.81 \mathrm{E}+02$

$2.86 \mathrm{E}+0$

4.15E-04

$0.00 \mathrm{E}+01$

$1.79 \mathrm{E}+01$

$0.00 \mathrm{E}+01$

$0.00 \mathrm{E}+01$

$0.00 \mathrm{E}+01$

$0.00 \mathrm{E}+01$

$0.00 \mathrm{E}+01$

$0.00 \mathrm{E}+01$

$0.00 \mathrm{E}+01$

$0.00 \mathrm{E}+01$

$0.00 \mathrm{E}+01$

3.57E-05

$0.00 \mathrm{E}+01$

1.69E-00

$0.00 \mathrm{E}+01$

$0.00 \mathrm{E}+01$

$0.00 \mathrm{E}+01$

$0.00 \mathrm{E}+01$ 50\%-ile

4.02E-02 1.78E-01

$5.29 \mathrm{E}+01$

$1.44 \mathrm{E}+03$

$2.64 \mathrm{E}+02$

$3.75 \mathrm{E}+03$

5.11E-03

8.39E-02

2.92E-01

$7.36 \mathrm{E}+01$

$4.25 \mathrm{E}+01$

$1.59 \mathrm{E}+05$

$2.27 \mathrm{E}+06$

$9.71 \mathrm{E}+01$

$1.98 \mathrm{E}-03$

8.27E-03

$6.83 \mathrm{E}+01$

8.65E +01

2.97E-01

1.14E-00

$2.60 \mathrm{E}-01$

1.04E-02 
Tank Anal. Un

$\begin{array}{ll}\text { AN101 } & \text { Nb93 } \\ \text { AN101 } & \text { Ni59 } \\ \text { AN101 } & \text { Ni63 } \\ \text { AN101 } & \text { Np237 } \\ \text { AN101 } & \text { Pa231 } \\ \text { AN101 } & \text { Pu238 } \\ \text { AN101 } & \text { Pu239 } \\ \text { AN101 } & \text { Pu240 } \\ \text { AN101 } & \text { Pu241 } \\ \text { AN101 } & \text { Pu242 } \\ \text { AN101 } & \text { Ra226 } \\ \text { AN101 } & \text { Ra228 } \\ \text { AN101 } & \text { Ru106 } \\ \text { AN101 } & \text { Sb125 } \\ \text { AN101 } & \text { Se79 }\end{array}$

AN101 Sm151 AN101 Sr89/90 AN101 Tc99 AN101 Th229 AN101 Th232 AN101 Tin126 AN101 Tritium AN101 U232 AN101 U233 AN101 U234 AN101 U235 AN101 U236 AN101 U238 AN101 Y90 AN101 Zr93

AN102 Al

AN102 Bi

AN102 $\mathrm{Ca}$

AN102 Cl

AN102 $\mathrm{CO} 3$

AN102 Cr

AN102 F

AN102 Fe

AN102 $\mathrm{Hg}$

AN102 K

AN102 La

AN102 Mn

AN102 Na

AN102 Ni

AN102 NO2

AN102 NO3

AN102 P

AN102 Pb

AN102 $\mathrm{PO} 4$

AN102 S

AN102 Si

AN102 $\mathrm{SO} 4$

AN102 Sr

AN102 TOC

AN102 U

AN102 Zr

AN102 Ac227

AN102 Am241

AN102 Am243

AN102 Ba137

AN102 $\mathrm{Cl}$

AN102 Cd113

AN102 Cm242

AN102 Cm243

AN102 $\mathrm{Cm} 244$

AN102 C060

AN102 Cs134

AN102 Cs137
May 98

$2.09 \mathrm{E}-00$

$\begin{array}{ll}\mathrm{Ci} & 3.56 \mathrm{E}-01 \\ \mathrm{Ci} & \mathbf{3 . 4 7 E}+01\end{array}$

$3.47 \mathrm{E}+01$
$1.59 \mathrm{E}-01$

7.17E-04

2.48E-01

2.65E-02

Ci Incl. in 239

$1.82 \mathrm{E}+01$

9.12E-05

2.64E-05

3.05E-02

1.09E-03

$2.88 \mathrm{E}+01$

$5.80 \mathrm{E}-01$

$2.05 \mathrm{E}+03$

$1.74 \mathrm{E}+02$

4.21E+01

7.13E-04

3.06E-03

8.78E-01

$4.19 E+01$

1.08E-01

4.14E-01

1.60E-01

$6.51 \mathrm{E}-03$

5.54E-03

1.74E-01

i $\quad 1.74 \mathrm{E}+02$

2.84E-00

$6.29 E+04$

$1.29 E+03$

g $2.68 \mathrm{E}+03$

kg 1.53E+04

kg $2.79 E+05$

kg 1.81E+03

kg $7.43 E+03$

kg $\quad 1.04 \mathrm{E}+03$

kg 9.67E-00

$1.55 \mathrm{E}+04$

$1.49 E+01$

kg $3.89 \mathrm{E}+02$

kg $\quad 1.02 E+06$

$\mathrm{kg} \quad 1.65 \mathrm{E}+03$

kg $3.30 E+05$

kg $9.02 E+05$

$\mathrm{kg} \quad 6.33 \mathrm{E}+03$

kg $1.38 \mathrm{E}+02$

$\begin{array}{ll}\mathrm{kg} & 1.38 \mathrm{E}+02 \\ \mathrm{~kg} & 1.96 \mathrm{E}+04\end{array}$

kg $2.16 E+04$

kg $7.64 \mathrm{E}+02$

g 6.49E+04

$1.01 E+01$

$1.07 E+05$

$1.56 \mathrm{E}+03$

$2.80 \mathrm{E}+02$

$4.90 \mathrm{E}-03$

$8.13 E+02$

$1.38 \mathrm{E}-02$

$1.40 \mathrm{E}+06$

$6.48 \mathrm{E}-01$

$5.65 E+02$

$1.39 \mathrm{E}-03$

5.13E-01

$1.54 \mathrm{E}+01$

$7.59 \mathrm{E}+02$

Ci $1.97 \mathrm{E}+01$

Ci $1.49 \mathrm{E}+06$

\section{Sept98}

2.09E-00

3.56E-01

$3.47 \mathrm{E}+01$

1.59E-01

7.17E-04

2.48E-01

$2.26 \mathrm{E}-02$

$3.87 \mathrm{E}-03$

5.24E-02

9.12E-05

$2.64 \mathrm{E}-05$

3.05E-02

$1.09 \mathrm{E}-03$

$2.88 \mathrm{E}+01$

$5.80 \mathrm{E}-01$

2.05E +03

$1.74 \mathrm{E}+02$

$4.21 \mathrm{E}+01$

7.13E-04

3.06E-03

8.78E-01

$4.19 E+01$

1.08E-01

4.14E-01

$1.60 \mathrm{E}-01$

$6.48 \mathrm{E}-03$

$5.54 \mathrm{E}-03$

1.44E-01

$1.74 \mathrm{E}+02$

2.84E-00

$6.29 \mathrm{E}+04$

$0.00 \mathrm{E}+01$

$2.68 \mathrm{E}+03$

$1.53 \mathrm{E}+04$

$2.79 \mathrm{E}+05$

$1.81 \mathrm{E}+03$

$7.43 E+03$

$1.04 \mathrm{E}+03$

$0.00 \mathrm{E}+01$

$1.55 \mathrm{E}+04$

$0.00 \mathrm{E}+01$

$3.89 \mathrm{E}+02$

$1.02 \mathrm{E}+06$

$1.65 \mathrm{E}+03$

$3.30 \mathrm{E}+05$

$9.02 \mathrm{E}+05$

NA

$1.33 \mathrm{E}+03$

$1.96 \mathrm{E}+04$

NA

$7.64 \mathrm{E}+02$

$6.49 \mathrm{E}+04$

$1.01 \mathrm{E}+01$

$1.07 \mathrm{E}+05$

$1.56 \mathrm{E}+03$

$2.80 \mathrm{E}+02$

4.90E-03

$8.13 E+02$

$3.20 \mathrm{E}-02$

$1.40 \mathrm{E}+06$

$2.20 \mathrm{E}+02$

$5.65 \mathrm{E}+02$

5.13E-01

1.39E-03

$1.54 \mathrm{E}+01$

$7.59 \mathrm{E}+02$

$6.19 \mathrm{E}+01$

$1.49 \mathrm{E}+06$
$1 \%$-ile

$0.00 \mathrm{E}+01$

$0.00 \mathrm{E}+01$

$0.00 \mathrm{E}+01$

$0.00 \mathrm{E}+01$

$0.00 \mathrm{E}+01$

$0.00 \mathrm{E}+01$

$0.00 \mathrm{E}+01$

$0.00 \mathrm{E}+01$

$0.00 \mathrm{E}+01$

$0.00 \mathrm{E}+01$

1.01E-05

$1.17 \mathrm{E}-02$

$0.00 \mathrm{E}+01$

$0.00 \mathrm{E}+01$

7.65E-02

$0.00 \mathrm{E}+01$

$5.03 \mathrm{E}+01$

$7.97 \mathrm{E}-00$

$0.00 \mathrm{E}+01$

$0.00 \mathrm{E}+01$

$0.00 \mathrm{E}+01$

2.97E-01

$0.00 \mathrm{E}+01$

$0.00 \mathrm{E}+01$

$0.00 \mathrm{E}+01$

$0.00 \mathrm{E}+01$

$0.00 \mathrm{E}+01$

$0.00 \mathrm{E}+01$

$0.00 \mathrm{E}+01$

$0.00 \mathrm{E}+01$

$3.39 \mathrm{E}+04$

$6.31 \mathrm{E}-05$

6.97E-00

$6.29 \mathrm{E}+03$

$1.88 \mathrm{E}+05$

$5.70 \mathrm{E}+02$

6.32E-05

3.72E-03

$0.00 \mathrm{E}+01$

3.00E-02

$1.07 \mathrm{E}-00$

$4.90 \mathrm{E}-04$

$7.60 \mathrm{E}+05$

$1.40 \mathrm{E}+03$

$2.44 E+05$

$5.61 \mathrm{E}+05$

$5.67 \mathrm{E}+02$

1.56E-03

1.27E+04

$6.94 \mathrm{E}+02$

5.55E-06

4.45E+04

2.68E-05

$9.13 \mathrm{E}+04$

4.15E-02

$7.85 \mathrm{E}-00$

$0.00 \mathrm{E}+01$

$4.91 E+02$

4.22E-03

$0.00 \mathrm{E}+01$

$0.00 \mathrm{E}+01$

$0.00 \mathrm{E}+01$

4.25E-04

1.57E-01

4.71E-00

$5.16 \mathrm{E}+02$

$0.00 \mathrm{E}+01$

$6.46 \mathrm{E}+05$

\section{$10 \%$ ile}

3.81E-01

$6.79 \mathrm{E}-02$

6.62E-00

8.29E-03

$0.00 \mathrm{E}+01$

$1.29 \mathrm{E}-02$

$1.38 \mathrm{E}-03$

$1.38 \mathrm{E}-03$

9.49E-01

4.76E-06

1.53E-05

$1.77 \mathrm{E}-02$

5.31E-05

$0.00 \mathrm{E}+01$

$1.58 \mathrm{E}-01$

$0.00 E+01$

$1.33 E+02$

1. $60 \mathrm{E}+01$

$0.00 E+01$

$0.00 \mathrm{E}+01$

$0.00 \mathrm{E}+01$

$3.39 \mathrm{E}-00$

$0.00 \mathrm{E}+01$

$0.00 \mathrm{E}+01$

$0.00 \mathrm{E}+01$

$0.00 \mathrm{E}+01$ 
Tank Anal. Un

$\begin{array}{ll}\text { AN102 } & \text { Cu152 } \\ \text { AN102 } & \text { Eu154 } \\ \text { AN102 } & \text { Eu155 } \\ \text { AN102 } & \text { I129 } \\ \text { AN102 } & \text { Nb93 } \\ \text { AN102 } & \text { Ni59 } \\ \text { AN102 } & \text { Ni63 } \\ \text { AN102 } & \text { Np237 } \\ \text { AN102 } & \text { Pa231 } \\ \text { AN102 } & \text { Pu238 } \\ \text { AN102 } & \text { Pu239 } \\ \text { AN102 } & \text { Pu240 } \\ \text { AN102 } & \text { Pu241 } \\ \text { AN102 } & \text { Pu242 } \\ \text { AN102 } & \text { Ra226 } \\ \text { AN102 } & \text { Ra228 } \\ \text { AN102 } & \text { Ru106 } \\ \text { AN102 } & \text { Sb125 } \\ \text { AN102 } & \text { Se79 }\end{array}$

AN102 Se79

AN102 Sm151

AN102 Sr89/90

AN102 Tc99

AN102 Th229

AN102 Th232

AN102 Tin126

AN102 Tritium

AN102 U232

AN102 U233

AN102 U234

AN102 U235

AN102 U236

AN102 U238

AN102 Y90

AN102 Zr93

AN103 Al

AN103 Bi

AN103 Ca

AN103 Cl

AN103 $\mathrm{CO} 3$

AN103 Cr

AN103 F

AN103 Fe

AN103 Hg

AN103 K

AN103 La

AN103 Mn

AN103 Na

AN103 Ni

AN103 NO2

AN103 NO3

AN103 $P$

AN103 Pb

AN103 PO4

AN103 S

AN103 Si

AN103 SO4

AN103 Sr

AN103 TOC

AN103 U

AN103 Zr

AN103 Ac227

AN103 Am241

AN103 Am243

AN103 Ba137

AN103 C14

AN103 Cd113

AN103 Cm242

AN103 Cm243

$\mathrm{kg}$
BB $\frac{\text { May } 98}{4.07 \mathrm{E}+01}$ $3.75 \mathrm{E}+02$ i $3.22 \mathrm{E}+02$ i.17E-00

Ci 7.38E+01

i.15E+01

Ci 1.14E+03

4.77E-01

2.35E-02

Ci 1.17E+01

$5.75 \mathrm{E}+01$

i Incl. in 239

$6.72 \mathrm{E}+02$

3.59E-03

Ci 7.95E-04

1.72E-00

4.85E-02

$1.59 E+02$

1.01E-00

$7.34 \mathrm{E}+04$

$3.68 \mathrm{E}+05$

$4.97 E+01$

3.99E-02

$1.83 \mathrm{E}-01$

$3.15 E+01$

Ci 1.27E-00

5.33E-00

Ci 2.04E+01

4.22E-00

Ci 1.68E-01

Ci 1.50E-01

Ci 5.36E-00

Ci $3.68 \mathrm{E}+05$

Ci 1.03E+02

kg $2.39 \mathrm{E}+05$

kg $1.12 \mathrm{E}+03$

kg $6.10 \mathrm{E}+03$

kg 2.75E+04

$\mathrm{kg} \quad 8.46 \mathrm{E}+04$

kg $2.82 E+03$

$\mathrm{kg} \quad 3.86 \mathrm{E}+03$

kg $\quad 7.74 E+02$

kg $\quad 4.63 \mathrm{E}+04$

kg 8.11E +02

kg $1.12 \mathrm{E}+02$

kg $\quad 1.27 \mathrm{E}+06$

kg 2.25E+02

kg $\quad 4.94 E+05$

kg $7.71 E+05$

kg 3.16E+03

kg $\quad 7.95 \mathrm{E}+02$

kg $\quad 9.80 \mathrm{E}+03$

$\mathrm{kg} \quad 4.40 \mathrm{E}+03$

kg $2.35 \mathrm{E}+03$

$\mathrm{kg} \quad 1.32 \mathrm{E}+04$

kg 1.12E+02

$\mathrm{kg} \quad 1.20 \mathrm{E}+04$

g $5.62 E+03$

kg 1.13E+02

Ci 3.55E-03

Ci $2.17 \mathrm{E}+01$

Ci 2.09E-03

Ci $2.19 \mathrm{E}+06$

Ci $1.12 \mathrm{E}+02$

Ci $3.02 \mathrm{E}+02$

Ci $\quad 6.23 E-01$

Ci 4.85E-00 1\%-ile

\section{$0.00 \mathrm{E}+01$}

$0.00 \mathrm{E}+01$

$0.00 E+01$

$0.00 \mathrm{E}+01$

$3.47 \mathrm{E}-00$

$0.00 \mathrm{E}+01$

$0.00 E+01$

$1.46 \mathrm{E}-01$

$0.00 \mathrm{E}+01$

3.58E-00

$1.76 \mathrm{E}+01$

$1.76 \mathrm{E}+01$

$2.06 \mathrm{E}+02$

$1.10 \mathrm{E}-03$

$0.00 \mathrm{E}+01$

$0.00 \mathrm{E}+01$

$0.00 \mathrm{E}+01$

$0.00 E+01$

1.21E-02

$0.00 E+01$

$2.74 \mathrm{E}+05$

$0.00 E+01$

$0.00 \mathrm{E}+01$

$0.00 E \div 01$

$0.00 E \div 01$

5.06E-01

$0.00 E+01$

$0.00 E+01$

$0.00 \mathrm{E} \div 01$

$0.00 E+01$

$0.00 \mathrm{E}+01$

$0.00 E+01$

$0.00 E+01$

4.84E-00

$1.72 \mathrm{E}+05$

$2.58 \mathrm{E}+01$

$5.65 \mathrm{E}+01$

$2.80 E+04$

$1.63 \mathrm{E}+02$

$8.45 E+02$

$4.96 \mathrm{E}+02$

7.72E-00

NA

4.27E+04

$2.45 \mathrm{E}+01$

$1.18 \mathrm{E}-02$

$1.25 E+06$

$1.26 \mathrm{E}+01$

$6.15 E+05$

$6.98 E+05$

9.52E-01

$4.59 E+02$

$5.86 \mathrm{E}+02$

$7.83 \mathrm{E}+02$

$4.88 \mathrm{E}+02$

$1.51 \mathrm{E}+03$

4.64E-00

$4.83 E+03$

$2.44 \mathrm{E}+02$

9.55E-00

$0.00 \mathrm{E}+01$

$0.00 E+01$

$0.00 E+01$

$5.11 \mathrm{E}+05$

$0.00 E+01$

$0.00 E+01$

$0.00 E+01$

$0.00 \mathrm{E}+01$
$10 \%$-ile

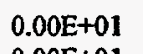

$0.00 \mathrm{E}+01$

$0.00 \mathrm{E}+01$

$9.44 \mathrm{E}-01$

$1.13 \mathrm{E}+01$

$4.89 \mathrm{E}-01$

$4.85 \mathrm{E}+01$

$2.68 \mathrm{E}-01$

$0.00 \mathrm{E}+01$

$6.58 \mathrm{E}-00$

$3.23 \mathrm{E}+01$

$3.23 \mathrm{E}+01$

$3.78 \mathrm{E}+02$

2.02E-03

$3.19 \mathrm{E}-05$

$6.89 \mathrm{E}-02$

$0.00 \mathrm{E}+01$

$0.00 \mathrm{E}+01$

4.49E-01

$0.00 \mathrm{E}+01$

$2.81 \mathrm{E}+05$

$0.00 \mathrm{E}+01$

$0.00 \mathrm{E}+01$

$0.00 \mathrm{E}+01$

$0.00 \mathrm{E}+01$

8.62E-01

$0.00 \mathrm{E}+01$

$0.00 \mathrm{E}+01$

$0.00 \mathrm{E}+01$

$0.00 \mathrm{E}+01$

$0.00 \mathrm{E}+01$

$0.00 \mathrm{E}+01$

$8.50 \mathrm{E}+04$

$1.57 \mathrm{E}+01$

$1.81 \mathrm{E}+05$

$4.45 \mathrm{E}+01$

$1.35 \mathrm{E}+02$

$2.92 \mathrm{E}+04$

$5.71 \mathrm{E}+04$

$1.23 \mathrm{E}+03$

$1.08 \mathrm{E}+03$

$2.34 \mathrm{E} \div 02$

4.64E+04

$3.73 \mathrm{E}+01$

$3.39 \mathrm{E}+01$

$1.28 \mathrm{E}+06$

$1.37 \mathrm{E}+02$

$6.38 \mathrm{E}+05$

$7.60 \mathrm{E}+05$

$1.13 \mathrm{E}+03$

$5.20 \mathrm{E}+02$

$1.36 \mathrm{E}+03$

$8.59 \mathrm{E}+02$

$8.52 \mathrm{E}+02$

$2.34 \mathrm{E}+03$

5.03E-00

$9.85 \mathrm{E} \div 03$

$6.23 \mathrm{E}+02$

$1.14 E+01$

$0.00 \mathrm{E}+01$

$0.00 E \div 01$

3.35E-04

$1.02 \mathrm{E}+06$

$0.00 E+01$

$0.00 \mathrm{E}+01$

9.98E-02

7.77E-01

50\%-ile

4.07E+01

$3.75 \mathrm{E}+02$

3.22E+02

3.17E-00

$7.38 \mathrm{E}+01$

$1.15 E+01$

$1.14 \mathrm{E}+03$

4.77E-01

2.35E-02

1.17E+01

$5.75 \mathrm{E}+01$

$5.75 \mathrm{E}+01$

$6.72 \mathrm{E}+02$

$3.59 \mathrm{E}-03$

7.95E-04

1.72E-00

4.85E-02

$1.59 \mathrm{E}+02$

1.01E-00

$7.34 \mathrm{E}+04$

$2.97 \mathrm{E}+05$

$4.97 \mathrm{E}+01$

3.99E-02

1.83E-01

$3.15 E+01$

1.27E-00

5.33E-00

2.04E+01

4.22E-00 
Tank Anal. Un

$\begin{array}{ll}\text { AN103 } & \text { Cm244 } \\ \text { AN103 } & \text { C060 } \\ \text { AN103 } & \text { Cs134 } \\ \text { AN103 } & \text { Cs137 } \\ \text { AN103 } & \text { Eu152 } \\ \text { AN103 } & \text { Eu154 } \\ \text { AN103 } & \text { Eu155 }\end{array}$

AN103 I129

AN103 Nb93

AN103 Ni59

AN103 Ni63

AN103 Np237

AN103 Pa231

AN103 Pu238

AN103 Pu239

AN103 Pu240

AN103 Pu241

AN103 Pu242

AN103 Ra226

AN103 Ra228

AN103 Ru106

AN103 Sb125

AN103 Se79

AN103 Sm151

AN103 Sr89/90

AN103 Tc99

AN103 Th229

AN103 Th232

AN103 Tin126

AN103 Tritium

AN103 U232

AN103 U233

AN103 U234

AN103 U235

AN103 U236

AN103 U238

AN103 Y90

AN103 Zr93

AN104 Al

AN104 Bi

AN104 Ca

AN104 Cl

AN104 $\mathrm{CO} 3$

AN104 $\mathrm{Cr}$

AN104 F

AN104 Fe

AN104 Hg

AN104 K

AN104 La

AN104 Mn

AN104 Na

AN104 Ni

AN104 NO2

AN104 NO3

AN104 P

AN104 Pb

AN104 PO4

AN104 S

AN104 Si

AN104 $\mathrm{SO} 4$

AN104 Sr

AN104 TOC

AN104 U

AN104 Zr

AN104 Ac227

AN104 Am241

AN104 Am243

AN104 Bal37
$B B$

May98

Ci $1.12 E+02$

Ci $1.89 \mathrm{E}+02$

Ci 2.31E+06

Ci $1.58 \mathrm{E}+01$

Ci $\quad 6.04 \mathrm{E}+02$

i. $30 \mathrm{E}+03$

Ci 8.32E-00

Ci $4.32 \mathrm{E}+01$

Ci $7.58 \mathrm{E}-00$

Ci $\quad 7.47 \mathrm{E}+02$

Ci 3.10E+01

Ci 1.43E-02

Ci $1.26 \mathrm{E}+01$

Ci 1.23E+01

Ci Incl. in 239

Ci $1.06 \mathrm{E}+03$

Ci $\quad 4.97 \mathrm{E}-03$

Ci 5.81E-04

Ci 1.05E-00

Ci 5.33E-02

$8.65 \mathrm{E}+02$

Ci $1.23 \mathrm{E}+01$

$4.36 \mathrm{E}+04$

$8.03 \mathrm{E}+03$

$4.56 \mathrm{E}+02$

Ci 2.43E-02

Ci 1.03E-01

Ci $1.88 \mathrm{E}+01$

Ci $9.31 \mathrm{E}-00$

Ci 3.59E-00

Ci 1.38E+01

Ci 4.87E-00

Ci 1.89E-01

Ci 2.85E-01

Ci 4.84E-00

Ci $8.03 E+03$

Ci $5.96 E+01$

kg $1.43 \mathrm{E}+05$

kg $\quad 6.67 \mathrm{E}+02$

kg $\quad 8.55 E+02$

kg 2.99E+04

kg 2.10E+05

kg $5.19 \mathrm{E}+03$

kg 2.16E+03

kg $3.95 E+02$

kg

kg 2.49E+04

$\mathrm{kg} \quad 3.34 \mathrm{E}+02$

kg 7.46E+01

kg 1.19E+06

kg 2.26E+02

kg $4.48 \mathrm{E}+05$

kg $\quad 6.80 \mathrm{E}+05$

kg 5.52E+03

kg $\quad 3.82 \mathrm{E}+02$

kg $1.71 \mathrm{E}+04$

kg 1.24E+04

kg 1.59E+03

kg $3.71 \mathrm{E}+04$

kg 6.67E+01

kg 1.87E+04

kg $3.34 \mathrm{E}+03$

kg 6.42E+01

Ci 2.86E-03

Ci $5.00 \mathrm{E}+01$

Ci 1.24E-03

Ci $2.20 \mathrm{E}+06$
$B B$

Sept98

6.07E-02

$1.12 \mathrm{E}+02$

$1.89 \mathrm{E}+02$

$2.31 E+06$

$1.58 \mathrm{E}+01$

$6.04 \mathrm{E}+02$

$3.30 \mathrm{E}+03$

8.32E-00

$4.38 \mathrm{E}+01$

7.58E-00

$7.47 \mathrm{E}+02$

$3.10 \mathrm{E}+01$

1.43E-02

5.46E-01

$1.02 \mathrm{E}+01$

2.12E-00

$1.06 \mathrm{E}+03$

$2.16 \mathrm{E}-04$

5.81E-04

1.05E-00

5.33E-02

$8.65 \mathrm{E}+02$

$1.23 E+01$

$4.36 \mathrm{E}+04$

$8.03 \mathrm{E}+03$

$4.56 \mathrm{E}+02$

2.43E-02

1.03E-01

$1.88 \mathrm{E}+01$

9.31E-00

1.30E-01

4.97E-01

1.76E-01

6.82E-03

1.03E-02

1.38E-01

8.03E +03

$5.96 \mathrm{E}+01$

$1.43 E+05$

$1.08 \mathrm{E}+02$

$7.18 \mathrm{E}+02$

$2.99 \mathrm{E}+04$

$2.16 \mathrm{E}+05$

$5.19 \mathrm{E}+03$

$2.45 \mathrm{E}+03$

$3.27 \mathrm{E}+02$

$0.00 \mathrm{E}+01$

$2.49 \mathrm{E}+04$

$1.91 \mathrm{E}+02$

$7.46 \mathrm{E}+01$

$1.19 E+06$

$1.99 \mathrm{E}+02$

$4.47 E+05$

$6.80 \mathrm{E}+05$

NA

$3.82 \mathrm{E}+02$

$1.71 \mathrm{E}+04$

NA

$2.45 E+03$

$3.70 \mathrm{E}+04$

$3.80 \mathrm{E}+01$

$1.98 \mathrm{E}+04$

$4.80 \mathrm{E}+02$

6.42E+01

2.86E-03

$5.00 \mathrm{E}+01$

2.77E-03

$2.20 \mathrm{E}+06$ 1\%-ile

\section{$0.00 \mathrm{E}+01$}

$0.00 \mathrm{E}+01$

$4.41 \mathrm{E}+01$

$5.65 \mathrm{E}+05$

$0.00 \mathrm{E}+01$

$0.00 \mathrm{E}+01$

$0.00 E+01$

$3.84 \mathrm{E}-0 \mathrm{I}$

3.53E- 00

1.34E-01

$1.32 \mathrm{E}+01$

$0.00 \mathrm{E}+01$

$0.00 \mathrm{E}+01$

$0.00 E+01$

$0.00 \mathrm{E}+01$

$0.00 \mathrm{E}+01$

$0.00 \mathrm{E}+01$

$0.00 \mathrm{E}+01$

$8.49 \mathrm{E}-05$

1.53E-01

$0.00 \mathrm{E}+01$

$5.71 \mathrm{E}+01$

2.35E-01

$0.00 \mathrm{E}+01$

$0.00 \mathrm{E}+01$

$0.00 \mathrm{E}+01$

$0.00 \mathrm{E}+01$

$0.00 \mathrm{E}+01$

$0.00 \mathrm{E}+01$

7.89 E-00

$0.00 \mathrm{E}+01$

$0.00 \mathrm{E}+01$

$0.00 \mathrm{E}+01$

$0.00 \mathrm{E}+01$

$0.00 \mathrm{E}+01$

$0.00 \mathrm{E}+01$

$0.00 \mathrm{E}+01$

4.87E-00

$1.27 \mathrm{E}+05$

$1.95 \mathrm{E}+01$

$6.70 \mathrm{E}+01$

$2.79 \mathrm{E}+04$

$1.27 \mathrm{E}+04$

$2.67 \mathrm{E}+03$

$3.03 \mathrm{E}-06$

$0.00 \mathrm{E}+01$

NA

$3.58 \mathrm{E}+01$

3.54E-05

1.17E+06

4.54E-00

$3.85 \mathrm{E}+05$

$5.68 \mathrm{E}+05$

$1.78 \mathrm{E}-02$

$1.20 \mathrm{E}+02$

$4.56 \mathrm{E}+03$

$6.76 \mathrm{E}+03$

$4.76 E+02$

$1.18 \mathrm{E}+04$

$6.23 \mathrm{E}-00$

$3.20 E+03$

8.64E- 02

9.12E-00

$0.00 E+01$

$0.00 \mathrm{E}+0$ ?

$0.00 \mathrm{E}+01$

$1.87 \mathrm{E}+05$

$10 \%$-ile

2.08E-00

2.57E-00

8.84E+01

$7.49 \mathrm{E}+05$

$0.00 \mathrm{E}+01$

$0.00 \mathrm{E}+01$

$0.00 \mathrm{E}+01$

$3.89 \mathrm{E}-00$

4.50E-00

2.42E-00

$2.38 \mathrm{E}+02$

4.97E-00

$0.00 \mathrm{E}+01$

2.02E-00

$1.97 \mathrm{E}-00$

$1.97 \mathrm{E}-00$

$1.70 \mathrm{E}+02$

7.96E-04 
Tank Anal. Un

$\begin{array}{ll}\text { AN104 } & \text { C14 } \\ \text { AN104 } & \text { Cd113 } \\ \text { AN104 } & \text { Cm242 } \\ \text { AN104 } & \text { Cm243 } \\ \text { AN104 } & \text { Cm244 } \\ \text { AN104 } & \text { Co60 } \\ \text { AN104 } & \text { Cs134 } \\ \text { AN104 } & \text { Cs137 } \\ \text { AN104 } & \text { Eu152 } \\ \text { AN104 } & \text { Eu154 } \\ \text { AN104 } & \text { Eu155 } \\ \text { AN104 } & \text { I129 }\end{array}$

AN104 Nb93

AN104 Ni59

AN104 Ni63

AN104 Np237

AN104 Pa231

AN104 Pu238

AN104 Pu239

AN104 Pu240

AN104 Pu241

AN104 Pu242

AN104 Ra226

AN104 Ra228

AN104 Ru106

AN104 Sb125

AN104 Se79

AN104 Sm151

AN104 Sr89/90

AN104 Te99

AN104 Th229

AN104 Th232

AN104 Tin126

AN104 Tritium

AN104 U232

AN104 U233

AN104 U234

AN104 U235

AN104 U236

AN104 U238

AN104 Y90

AN104 Zr93

AN105 Al

AN105 Bi

AN105 Ca

AN105 Cl

AN105 CO3

AN105 Cr

AN105 F

AN105 Fe

AN105 $\mathrm{Hg}$

AN105 K

AN105 La

AN105 Mn

AN105 Na

AN105 Ni

AN105 NO2

AN105 NO3

AN105 P

AN105 Pb

AN105 PO4

AN105 S

AN105 Si

AN105 SO4

AN105 Sr

AN105 TOC

AN105 U

AN105 Zr

\section{Ci $\frac{\text { May }}{1.04 \mathrm{E}+02}$}

Ci $2.90 \mathrm{E}+02$

Ci $4.80 \mathrm{E}-01$

Ci $1.20 \mathrm{E}+01$

Ci $1.49 \mathrm{E}+02$

i. $1.23 \mathrm{E}+02$

2.32E+06

Ci $1.45 \mathrm{E}+01$

i $6.76 \mathrm{E}+02$

i $4.73 E+03$

Ci $\quad 7.53 \mathrm{E}-00$

Ci 4.02E+01

Ci 5.61E-00

Ci $\quad 5.52 \mathrm{E}+02$

Ci $3.07 \mathrm{E}+01$

Ci 1.28E-02

Ci $\quad 7.97 E-00$

9.70E-00

Ci Incl. in 239

Ci $6.64 \mathrm{E}+02$

Ci 3.06E-03

Ci 4.63E-04

1.03E-00

i 3.23E-02

Ci $7.45 \mathrm{E}+02$

i 1.13E+01

Ci $4.00 \mathrm{E}+04$

Ci $9.38 \mathrm{E}+04$

Ci $3.45 \mathrm{E}+02$

Ci 2.39E-02

Ci 1.09E-01

Ci $1.72 \mathrm{E}+01$

Ci 4.33E+01

Ci 3.17E-00

Ci $1.21 \mathrm{E}+01$

Ci 3.59E-00

Ci 1.40E-01

Ci 1.93E-01

Ci 3.86E-00

Ci $\quad 9.38 \mathrm{E}+04$

Ci $5.53 \mathrm{E}+01$

kg $1.56 \mathrm{E}+05$

kg. $\quad 2.81 \mathrm{E}+02$

$\mathrm{kg} \quad 1.10 \mathrm{E}+03$

kg 4.08E+04

kg $\quad 1.97 \mathrm{E}+05$

kg $\quad 6.06 \mathrm{E}+03$

$\mathrm{kg} \quad 4.34 \mathrm{E}+03$

$\mathrm{kg} \quad 3.06 \mathrm{E}+02$

$\begin{array}{lr}\mathrm{kg} & \mathrm{NA} \\ \mathrm{kg} & 2.54 \mathrm{E}+04\end{array}$

kg $5.81 \mathrm{E}+02$

kg $\quad 5.94 \mathrm{E}+01$

kg 1.10E+06

kg $1.29 \mathrm{E}+02$

kg $\quad 5.04 \mathrm{E}+05$

kg $\quad 7.28 \mathrm{E}+05$

kg $\quad 6.56 \mathrm{E}+03$

kg $2.65 E+02$

kg 2.03E+04

kg $\quad 7.63 \mathrm{E}+03$

kg $\quad 9.50 \mathrm{E}+02$

kg 2.29E +04

kg $3.21 \mathrm{E}+01$

kg $3.00 \mathrm{E}+04$

kg 2.42E+02

$\mathrm{kg} \quad 4.35 \mathrm{E}+01$
$B B$ Sept98

$1.04 \mathrm{E}+02$

$2.90 \mathrm{E}+02$

1.17E-01

4.92E-01

$1.18 \mathrm{E}+01$

$1.49 \mathrm{E}+02$

$1.23 \mathrm{E}+02$

$2.32 \mathrm{E}+06$

$1.45 \mathrm{E}+01$

$6.76 \mathrm{E}+02$

$8.99 \mathrm{E}+02$

$1.51 \mathrm{E}-00$

$4.02 \mathrm{E}+01$

5.61E-00

$5.52 \mathrm{E} \div 02$

2.77E-00

1.28E-02

$3.76 \mathrm{E}-01$

8.10E-00

$1.60 \mathrm{E}-00$

$3.14 \mathrm{E}+01$

$1.45 \mathrm{E}-04$

4.63E-04

1.03E-00

3.23E- 102

$7.45 \mathrm{E}+02$

$1.13 \mathrm{E}+01$

$4.00 \mathrm{E}+04$

$9.38 \mathrm{E}+04$

$3.45 \mathrm{E}+02$

2.39E-02

1.09E-01

$1.72 E+01$

$4.33 \mathrm{E}+01$

$1.76 \mathrm{E}-01$

6.74E-01

1.99E-01

7.77E-03

1.07E-02

1.60E-01

$9.38 \mathrm{E}+04$

$5.53 \mathrm{E}+01$

$1.56 \mathrm{E}+05$

$1.96 \mathrm{E}+02$

$1.32 \mathrm{E}+03$

$4.08 E+04$

$1.97 \mathrm{E}+05$

$6.06 \mathrm{E}+03$

$4.01 \mathrm{E}+03$

$3.06 E+02$

2.20E-00

$2.54 \mathrm{E}+04$

$1.31 \mathrm{E}+02$

$5.94 E+01$

$1.10 \mathrm{E}+06$

$1.29 \mathrm{E}+02$

$5.04 E+05$

$7.28 \mathrm{E}+05$

$\mathrm{NA}$
$2.65 \mathrm{E}+02$

$2.03 E+04$

NA

$9.50 \mathrm{E}+02$

$2.29 \mathrm{E}+04$

$2.54 \mathrm{E}+01$

$3.00 \mathrm{E}+04$

$2.42 \mathrm{E}+02$

$4.35 \mathrm{E}+01$ 1\%-ile

$0.00 \mathrm{E}+01$

$0.00 E+01$

$0.00 \mathrm{E}+01$

$0.00 \mathrm{E}+01$

$0.00 \mathrm{E}+01$

4.30E-08

$1.05 \mathrm{E}+01$

$6.99 \mathrm{E}+05$

$0.00 \mathrm{E}+01$

$0.00 \mathrm{E}+01$

$0.00 \mathrm{E}+01$

$6.99 \mathrm{E}-01$

4.28E-00

$0.00 \mathrm{E}+01$

$0.00 \mathrm{E}+01$

$0.00 \mathrm{E}+01$

$0.00 \mathrm{E}+01$

$0.00 \mathrm{E}+01$

$0.00 \mathrm{E}+01$

$0.00 \mathrm{E}+01$

$0.00 \mathrm{E}+01$

$0.00 \mathrm{E} \div 01$

1.82E-05

4.05E-02

$0.00 \mathrm{E}+01$

$3.32 \mathrm{E}+01$

$0.00 \mathrm{E}+01$

$0.00 \mathrm{E}+01$

$0.00 \mathrm{E}+01$

$0.00 \mathrm{E}+01$

$0.00 \mathrm{E}+01$

$0.00 \mathrm{E}+01$

$0.00 \mathrm{E}+01$

$3.64 \mathrm{E}+01$

$0.00 \mathrm{E}+01$

$0.00 \mathrm{E}+01$

$0.00 \mathrm{E}+01$

$0.00 \mathrm{E}+01$

$0.00 \mathrm{E}+01$

$0.00 \mathrm{E}+01$

$0.00 \mathrm{E}+01$

$5.89 \mathrm{E}-00$

$1.45 \mathrm{E}+05$

$2.76 \mathrm{E}+01$

$2.45 E+02$

$3.73 \mathrm{E}+04$

$1.48 \mathrm{E}+05$

$4.28 \mathrm{E}+03$

$5.73 E+01$

$0.00 \mathrm{E}+01$

$\mathrm{NA}$
$.53 \mathrm{E}+04$

$2.88 \mathrm{E}+01$

$0.00 \mathrm{E}+01$

$1.18 \mathrm{E}+06$

$0.00 \mathrm{E}+01$

4. $47 \mathrm{E}+05$

$5.87 \mathrm{E}+05$

$3.30 \mathrm{E}+03$

$1.17 \mathrm{E}+02$

$2.88 \mathrm{E}+03$

$3.81 \mathrm{E}+03$

$4.60 \mathrm{E}+02$

$3.74 \mathrm{E}+03$

$5.58 \mathrm{E}-00$

$6.10 \mathrm{E}+03$

$2.77 \mathrm{E}+02$

7.24E-00

$10 \%$-ile

$0.00 \mathrm{E}+01$

$0.00 \mathrm{E}+01$

$0.00 \mathrm{E}+01$

$0.00 \mathrm{E}+01$

$0.00 \mathrm{E}+01$

$9.70 \mathrm{E}-00$

$4.87 E+01$

$9.69 \mathrm{E}+05$

$0.00 E+01$

$0.00 \mathrm{E}+0 \mathrm{I}$

$0.00 \mathrm{E}+01$

$3.54 \mathrm{E}-00$

$5.24 \mathrm{E}-00$

$0.00 \mathrm{E}+01$

$0.00 \mathrm{E}+01$

$0.00 E+01$

$0.00 \mathrm{E}+01$

$0.00 \mathrm{E}+01$

$0.00 \mathrm{E}+0 \mathrm{I}$

$0.00 \mathrm{E}+01$

$0.00 \mathrm{E}+01$

$0.00 \mathrm{E}+01$

$2.10 \mathrm{E}-05$

4.67E-02

$0.00 \mathrm{E}+01$

$1.49 \mathrm{E}+02$

$3.91 \mathrm{E}-00$ 
Tank Anal. Un

$\begin{array}{ll}\text { AN105 Ac227 } \\ \text { AN105 Am241 } \\ \text { AN105 } & \text { Am243 } \\ \text { AN105 } & \text { Ba137 } \\ \text { AN105 C14 } & \text { C14 } \\ \text { AN105 } & \text { Cd113 } \\ \text { AN105 } & \text { Cm242 } \\ \text { AN105 } & \text { Cm243 } \\ \text { AN105 } & \text { Cm244 } \\ \text { AN105 } & \text { Co60 } \\ \text { AN105 } & \text { Cs134 } \\ \text { AN105 } & \text { Cs137 } \\ \text { AN105 Eu152 } & \text { C15 } \\ \text { AN105 Eu154 } \\ \text { AN105 Eu155 } \\ \text { AN105 } & \text { I129 }\end{array}$

AN105 Nb93

AN105 Ni59

AN105 Ni63

AN105 Np237

AN105 Pa231

AN105 Pu238

AN105 Pu239

AN105 Pu240

AN105 Pu241

AN105 Pu242

AN105 Ra226

AN105 Ra228

AN105 Ru106

AN105 Sb125

AN105 Se79

AN105 Sm151

AN105 Sr89/90

AN105 Tc99

AN105 Th229

AN105 Th232

AN105 Tin126

AN105 Tritium

AN105 U232

AN105 U233

AN105 U234

AN105 U235

AN105 U236

AN105 U238

AN105 Y90

AN105 Zr93

AN106 Al

AN106 Bi

AN106 Ca

AN106 Cl

AN106 CO3

AN106 Cr

AN106 F

AN106 Fe

AN106 $\mathrm{Hg}$

AN106 K

AN106 La

AN106 Mn

AN106 Na

AN106 Ni

AN106 NO2

AN106 NO3

AN106 P

AN106 Pb

AN106 PO4

AN106 S

AN106 Si

AN106 SO4
$B B$

May98

$5.65 \mathrm{E}-03$
$1.01 \mathrm{E}+01$

1.88E-02

$7.94 \mathrm{E}+05$

$1.98 \mathrm{E}+02$

$5.52 \mathrm{E}+02$

9.87E-01

1.70E-01

$4.10 \mathrm{E}-00$

$1.24 \mathrm{E}+02$

$2.08 \mathrm{E}+02$

$8.36 \mathrm{E}+05$

$2.69 E+01$

$5.65 \mathrm{E} \div 02$

$2.26 \mathrm{E}+03$

$1.10 \mathrm{E}+01$

$7.76 \mathrm{E}+01$

Ci 1.11E+01

Ci 1.09E+03

Ci 2.58E+01

Ci $2.49 E-02$

Ci 1.18E+01

6.43E-00

Ci Incl. in 239

Ci $9.20 \mathrm{E}+02$

Ci $\quad 4.57 \mathrm{E}-03$

Ci 9.14E-04

Ci 2.03E-00

Ci 5.60E-02

Ci $1.33 \mathrm{E}+03$

Ci 2.19E+01

C.72E+04

$3.36 \mathrm{E}+04$

$1.17 \mathrm{E}+03$

4.71E-02

2.12E-01

$3.32 \mathrm{E}+01$

$\mathrm{Ci}$

Ci

$\mathrm{Ci}$

C

Ci 2.49E-01

Ci $\quad 6.53 \mathrm{E}-00$

Ci $3.36 \mathrm{E}+04$

Ci $\quad 1.06 \mathrm{E}+02$

kg $9.40 \mathrm{E}+02$

kg $2.60 \mathrm{E}+01$

kg 5.91E-00

kg $\quad 4.96 \mathrm{E}+02$

kg $3.54 \mathrm{E}+03$

kg 4.65E+01

kg $\quad 5.70 \mathrm{E}+02$

kg $\quad 3.21 \mathrm{E}+01$

kg 4.03E-01

$\mathrm{kg} \quad 1.26 \mathrm{E}+03$

$\mathrm{kg} \quad 3.90 \mathrm{E}-01$

$\mathrm{kg} \quad 9.44 \mathrm{E}+01$

kg 1.33E+04

kg 7.76E+01

kg $3.54 E+03$

kg 1.14E+04

$\mathrm{kg} \quad 4.36 \mathrm{E}+02$

kg 2.60E+01

$\mathrm{kg} \quad 1.35 \mathrm{E}+03$

kg 2.39E+02

kg 2.12E+02

kg

\section{Sept98}

5.65E-03

$1.01 \mathrm{E}+01$

4.68E-04

$1.95 \mathrm{E}+06$

$1.98 \mathrm{E}+02$

$5.52 \mathrm{E}+02$

2.46E-02

1.71E-01

4.10E-00

$1.24 \mathrm{E}+02$

$2.08 \mathrm{E}+02$

$2.06 \mathrm{E}+06$

$2.69 \mathrm{E}+01$

$5.65 \mathrm{E}+02$

$1.66 \mathrm{E}+03$

$1.10 \mathrm{E}+01$

$7.76 \mathrm{E}+01$

$1.11 \mathrm{E}+01$

$1.09 \mathrm{E}+03$

$2.58 \mathrm{E}+01$

2.49E-02

2.16E-01

$5.50 \mathrm{E}-00$

1.04E-00

$1.68 \mathrm{E}+01$

8.33E-05

9.14E-04

2.03E-00

$5.60 \mathrm{E}-02$

$1.33 \mathrm{E}+03$

$2.19 E+01$

$7.72 E+04$

$3.36 \mathrm{E}+04$

$1.17 \mathrm{E}+03$

4.71E-02

2.12E-01

$3.32 \mathrm{E}+01$

1.76E-00

1.10E-01

4.25E-01

9.66E-02

3.79E-03

4.38E-03

8.07E-02

$3.36 \mathrm{E}+04$

$1.06 \mathrm{E}+02$

$9.40 \mathrm{E}+02$

$0.00 \mathrm{E}+01$

5.91E-00

$4.96 E+02$

$3.54 \mathrm{E}+03$

4.65E +01

$5.70 \mathrm{E}+02$

$3.21 \mathrm{E}+01$

$0.00 \mathrm{E}+01$

$1.26 \mathrm{E}+03$

2.54E-01

$9.44 E+01$

$1.33 E+04$

$7.76 E+01$

$3.54 \mathrm{E}+03$

$1.14 E+04$

NA

$2.60 E+01$

$1.35 E+03$

$2.12 E+02$

$7.16 E+02$
$1 \%$ ile

\section{$0.00 E+01$}

$2.99 E+02$

$0.00 \mathrm{E}+01$

$9.49 \mathrm{E}+03$

$0.00 \mathrm{E}+01$

$0.00 \mathrm{E}+01$

$0.00 \mathrm{E}+01$

$0.00 \mathrm{E}+01$

$0.00 \mathrm{E}+01$

$3.29 \mathrm{E}+02$

$2.49 \mathrm{E}-00$

$9.24 \mathrm{E}+05$

$0.00 \mathrm{E}+01$

$0.00 \mathrm{E}+01$

$0.00 \mathrm{E}+01$

8.51E-01

$0.00 \mathrm{E}+01$

$0.00 \mathrm{E}+01$

$0.00 \mathrm{E}+01$

$0.00 \mathrm{E}+01$

$6.16 \mathrm{E}-04$

$0.00 \mathrm{E}+01$

$0.00 \mathrm{E}+01$

$0.00 \mathrm{E}+01$

$0.00 \mathrm{E}+01$

$0.00 \mathrm{E}+01$

$3.80 \mathrm{E}-04$

8.45E-01

$0.00 \mathrm{E}+01$

$1.98 \mathrm{E}+02$

$0.00 \mathrm{E}+01$

$0.00 \mathrm{E}+01$

$1.80 \mathrm{E}+05$

$0.00 \mathrm{E}+01$

$1.16 \mathrm{E}-03$

5.24E-03

$0.00 \mathrm{E}+01$

$1.19 \mathrm{E}+03$

$1.56 \mathrm{E}-01$

$5.98 \mathrm{E}-01$

1.36E-01

5.34E-03

$6.16 \mathrm{E}-03$

1.61E-01

$3.34 E+03$

$0.00 \mathrm{E}+01$

$5.31 \mathrm{E}+02$

$0.00 \mathrm{E}+01$

2.95E-01

I.61E+02

$1.15 \mathrm{E}-00$

$0.00 \mathrm{E}+01$

$0.00 \mathrm{E}+01$

$0.00 \mathrm{E}+01$

$0.00 \mathrm{E}+01$

$4.22 \mathrm{E}+01$

5.21E-01

$0.00 \mathrm{E}+01$

$1.46 \mathrm{E}+04$

$0.00 \mathrm{E}+01$

1. $32 \mathrm{E}+03$

$3.27 \mathrm{E}+03$

$0.00 \mathrm{E}+01$

$0.00 \mathrm{E}+0 \mathrm{I}$

$4.99 \mathrm{E}+01$

8.97E-00

$0.00 \mathrm{E}+01$

$0.00 \mathrm{E}+01$

\section{$10 \%$-ile}

$3.33 \mathrm{E}+02$

$1.23 \mathrm{E}-03$

3.63E+05

$2.62 \mathrm{E}+01$

$0.00 \mathrm{E}+01$

6.47E-02

$1.11 \mathrm{E}-02$

2.69E-01

3.67E+02

$9.50 \mathrm{E}+01$

$1.29 \mathrm{E}+06$

$0.00 \mathrm{E}+01$

$0.00 \mathrm{E}+01$

$0.00 \mathrm{E}+01$

$4.94 \mathrm{E}-00$

$0.00 \mathrm{E}+01$

$0.00 \mathrm{E}+01$

$0.00 \mathrm{E}+01$

$1.69 \mathrm{E}-00$

$1.48 \mathrm{E}-03$

7.73E-01

4.21E-01

4.21E-01

$6.03 \mathrm{E}+01$ 


\begin{tabular}{|c|c|c|c|c|c|c|c|c|c|c|c|}
\hline ank & Anal. & Jn & $\frac{B B}{998}$ & $\frac{B B}{9+98}$ & $1 \%$-ile & $10 \%$-ile & $50 \%$-ile & 90\%-ile & 99\%-ile & Mean & Std Dev \\
\hline & $\mathrm{Sr}$ & $\mathrm{kg}$ & $0 . \overline{00 \mathrm{E}+01}$ & $0.00 \mathrm{E}+01$ & $6.18 \mathrm{E}-02$ & $2.30 \mathrm{E}-01$ & $1.09 \mathrm{E}+01$ & $5.16 \mathrm{E}+01$ & $9.55 \mathrm{E}+01$ & $1.87 \mathrm{E}+01$ & $2.24 \mathrm{E}+01$ \\
\hline & TOC & kg & $6.49 \mathrm{E}+02$ & $6.49 E+02$ & $0.00 \mathrm{E}+01$ & $.80 E+02$ & $1.90 \mathrm{E}+03$ & $6.18 E+03$ & $1.27 E+04$ & $2.73 E+03$ & $69 E+03$ \\
\hline N106 & & kg & $5.14 \mathrm{E}+02$ & $4.18 E+02$ & $0.00 \mathrm{E}+01$ & $7.98 \mathrm{E}-01$ & $5.60 \mathrm{E}+02$ & $2.88 \mathrm{E}+03$ & $5.83 E+03$ & $.05 E+03$ & $35 E+03$ \\
\hline N106 & $\mathbf{Z r}_{\mathbf{r}}$ & $\mathbf{k g}$ & $3.12 E+01$ & $3.12 E+01$ & $1.60 \mathrm{E}-02$ & $2.33 \mathrm{E}-01$ & $1.24 \mathrm{E}+02$ & $1.59 \mathrm{E}+03$ & $3.29 E+03$ & $5.33 E+02$ & $.10 E+02$ \\
\hline 106 & Ac227 & $\mathrm{Ci}$ & $1.24 \mathrm{E}-04$ & $1.24 \mathrm{E}-04$ & $0.00 \mathrm{E}+0 \mathrm{I}$ & $0.00 \mathrm{E}+01$ & $1.24 \mathrm{E}-04$ & $6.54 \mathrm{E}-04$ & $1.39 \mathrm{E}-03$ & $2.45 \mathrm{E}-04$ & $3.24 \mathrm{E}-04$ \\
\hline 106 & $\mathbf{m} 241$ & $\mathrm{Ci}$ & $1.14 E+01$ & $1.14 \mathrm{E}+01$ & $0.00 \mathrm{E}+01$ & $0.00 \mathrm{E}+01$ & $2.44 E-01$ & $3.35 \mathrm{E}+02$ & $6.91 E+02$ & $9.93 E+01$ & $1.70 \mathrm{E}+02$ \\
\hline 106 & $n 243$ & $\mathrm{Ci}$ & 61E-04 & $8.61 E-04$ & $0.00 \mathrm{E}+01$ & $0.00 \mathrm{E}+01$ & 8.61E-04 & $6.22 \mathrm{E}-03$ & -02 & E- 03 & 89E-03 \\
\hline 106 & 2137 & $\mathrm{Ci}$ & $1.94 E+04$ & $1.94 \mathrm{E}+04$ & $0.00 \mathrm{E}+01$ & $18 \mathrm{E}+03$ & $1.94 E+04$ & +04 & $E+04$ & $1 E+04$ & $.37 E+04$ \\
\hline 106 & 14 & $\mathrm{Ci}$ & $3.57 \mathrm{E}-00$ & 3.57E-00 & $0.00 E+01$ & $2.33 \mathrm{E}-01$ & $3.57 \mathrm{E}-00$ & $E+01$ & $E+01$ & $4.82 \mathrm{E}-00$ & 4.61E-00 \\
\hline N106 & d113 & $\mathrm{Ci}$ & $1.09 E+01$ & $1.09 E+01$ & $0.00 \mathrm{E}+01$ & $6.35 \mathrm{E}-01$ & $1.09 E+01$ & $4.13 E+01$ & $7.81 \mathrm{E}+01$ & $1.67 E+01$ & $1.78 E+01$ \\
\hline N106 & $\mathrm{Cm} 242$ & $\mathrm{Ci}$ & $2.16 \mathrm{E}-02$ & $2.16 \mathrm{E}-02$ & $0.00 \mathrm{E}+01$ & $0.00 E+01$ & $2.16 \mathrm{E}-02$ & $1.56 \mathrm{E}-01$ & $E-01$ & $0 \mathrm{E}-02$ & $7.25 \mathrm{E}-02$ \\
\hline N106 & $\mathrm{Cm} 243$ & $\mathrm{Ci}$ & $2.17 \mathrm{E}-03$ & 2.17E-03 & $0.00 \mathrm{E}+01$ & $0.00 \mathrm{E}+01$ & 2.17E-03 & $1.57 \mathrm{E}-02$ & $E-02$ & .03 & $7.28 \mathrm{E}-03$ \\
\hline N106 & Cm244 & $\mathrm{Ci}$ & $3.09 E-02$ & 3.09E-02 & $0.00 \mathrm{E}+01$ & $0.00 \mathrm{E}+01$ & $3.09 \mathrm{E}-02$ & $2.23 \mathrm{E}-01$ & E-01 & $7.58 \mathrm{E}-02$ & $1.04 \mathrm{E}-01$ \\
\hline & $\mathrm{Co60}$ & $\mathrm{Ci}$ & $4.87 \mathrm{E}-00$ & 4.87E-00 & $E+01$ & $2.21 \mathrm{E}-01$ & $2.87 \mathrm{E}-00$ & $1.02 \mathrm{E}+01$ & $E+01$ & $\mathrm{E}=00$ & $E-00$ \\
\hline 16 & s134 & $\mathrm{Ci}$ & $5.28 \mathrm{E}-00$ & $5.28 \mathrm{E}-00$ & +01 & -00 & 5.2 & -01 & +01 & & $E-00$ \\
\hline 106 & Cs137 & $\mathrm{Ci}$ & $2.05 \mathrm{E}+04$ & $2.05 E+04$ & +03 & $1.52 \mathrm{E}+04$ & $4.00 \mathrm{E}+04$ & +04 & +05 & $c+v 4$ & $E+04$ \\
\hline & 1152 & $\mathrm{Ci}$ & $5.36 \mathrm{E}-01$ & $5.36 \mathrm{E}-01$ & +01 & +01 & $5.36 \mathrm{E}-01$ & $E-00$ & -00 & $1.06 \mathrm{E}-00$ & $1.40 \mathrm{E}-00$ \\
\hline & Eu154 & $\mathrm{Ci}$ & $7.89 E+01$ & $7.89 \mathrm{E}+01$ & $E+01$ & +01 & $7.89 \mathrm{E}+01$ & $4.16 \mathrm{E}+02$ & +02 & $1.56 \mathrm{E}+02$ & $2.06 E+02$ \\
\hline AN106 & u155 & $\mathrm{Ci}$ & $3.32 \mathrm{E}+01$ & $3.32 E+01$ & $:+01$ & +01 & +01 & $:+02$ & +02 & $E+01$ & $6 E+01$ \\
\hline AN106 & 1129 & $\mathrm{Ci}$ & $5.07 \mathrm{E}-02$ & $5.07 \mathrm{E}-02$ & 0.0 & & & -02 & & $E-02$ & $\mathrm{E}-02$ \\
\hline N106 & Nb93 & $\mathbf{C i}$ & $1.62 \mathrm{E}-00$ & $1.62 \mathrm{E}-00$ & +01 & +01 & 1.6 & +01 & & $=00$ & +01 \\
\hline 106 & 59 & $\mathbf{C i}$ & 2.57E-01 & 2.57E-01 & & & 2.5 & & & & $E-01$ \\
\hline 106 & Ni63 & $\mathrm{Ci}$ & $2.21 E+01$ & $2.21 \mathrm{E}+01$ & 01 & & 2.2 & +01 & +02 & 3. & $E+01$ \\
\hline N106 & Np237 & $\mathrm{Ci}$ & $9.40 \mathrm{E}-02$ & $9.40 \mathrm{E}-02$ & $:+01$ & +01 & $9.40 \mathrm{E}-02$ & $E-01$ & -00 & $E-01$ & $6 \mathrm{E}-01$ \\
\hline 106 & Pa231 & $\mathrm{Ci}$ & 5.10E-04 & 5.10E-04 & $\$ 01$ & +01 & E-04 & $E-03$ & -03 & $E-03$ & $1.33 \mathrm{E}-03$ \\
\hline & $\mathbf{u} 238$ & $\mathrm{Ci}$ & $5.28 \mathrm{E}-01$ & $5.28 E-01$ & & & & $8-00$ & & -00 & $E-00$ \\
\hline 06 & Pu239 & $\mathrm{Ci}$ & 9.11E-00 & $9.11 E-00$ & +01 & +01 & & +01 & +02 & +01 & $E+01$ \\
\hline 106 & Pu240 & $\mathrm{Ci}$ & $1.85 \mathrm{E}-00$ & $1.85 \mathrm{E}-00$ & $E+01$ & +01 & $\mathrm{E}-\mathbf{0 0}$ & $E+01$ & & $E-00$ & $E-00$ \\
\hline 06 & Pu241 & $\mathrm{Ci}$ & $4.78 E+01$ & $4.78 E+01$ & 01 & +01 & +01 & +02 & +02 & $:+02$ & $E+02$ \\
\hline 106 & 4242 & $\mathrm{Ci}$ & $2.06 \mathrm{E}-04$ & $2.06 \mathrm{E}-04$ & +01 & +01 & -04 & $8-03$ & -03 & 5 & E-04 \\
\hline AN106 & a226 & $\mathrm{Ci}$ & $2.00 \mathrm{E}-05$ & $2.00 \mathrm{E}-05$ & $\therefore 01$ & +01 & E-05 & $E-04$ & -04 & $E-05$ & $E-05$ \\
\hline & $\operatorname{a228}$ & $\mathrm{Ci}$ & 2.81E-02 & $2.81 \mathrm{E}-02$ & & & & & & & $E-02$ \\
\hline & Ru106 & $\mathrm{Ci}$ & $1.78 \mathrm{E}-03$ & $1.78 \mathrm{E}-03$ & $\div 01$ & 01 & 1.7 & -03 & & & $E-03$ \\
\hline & Sb125 & $\mathrm{Ci}$ & $3.12 E+01$ & $3.12 \mathrm{E}+01$ & 01 & -01 & 3.1 & +02 & +02 & 5. & +01 \\
\hline & Se79 & $\mathrm{Ci}$ & 4.58E-01 & 4.58E-01 & 01 & & -01 & .00 & -00 & 6 & $E-01$ \\
\hline 106 & Smls1 & $\mathrm{Ci}$ & $1.62 E+03$ & $1.62 \mathrm{E}+03$ & $=01$ & +01 & $i+03$ & +03 & +04 & +03 & $E+03$ \\
\hline 106 & Sr89/90 & $\mathrm{Ci}$ & $1.37 E+02$ & $1.37 E+02$ & +01 & -01 & 003 & +04 & +04 & 9. & $E+04$ \\
\hline & Tc99 & $\mathrm{Ci}$ & $2.63 E+01$ & $2.63 E+01$ & +01 & -01 & -01 & +02 & +02 & 01 & +01 \\
\hline & Th229 & $\mathrm{Ci}$ & $6.53 \mathrm{E}-04$ & $6.53 E-04$ & & & & & & & \\
\hline & Th232 & $\mathrm{Ci}$ & 2.71E-03 & 03 & 01 & +01 & 2.7 & -02 & & & -03 \\
\hline 106 & Tin126 & $\mathrm{Ci}$ & 6.99E-01 & $6.99 \mathrm{E}-01$ & +01 & -02 & 6.9 & -00 & & .00 & $E-00$ \\
\hline N106 & Tritium & $\mathrm{Ci}$ & $2.75 E+01$ & $2.75 E+01$ & $E-02$ & -00 & $i+01$ & $E+01$ & +01 & +01 & $\varepsilon+01$ \\
\hline AN106 & U232 & $\mathrm{Ci}$ & $9.92 \mathrm{E}-02$ & $5.52 \mathrm{E}-02$ & +01 & +01 & -02 & $E-01$ & -00 & -01 & $E-01$ \\
\hline AN106 & U233 & $\mathrm{Ci}$ & $3.80 \mathrm{E}-01$ & $2.12 \mathrm{E}-01$ & & & & & & & $E-01$ \\
\hline & 34 & $\mathrm{Ci}$ & 2.27E-01 & $1.91 \mathrm{E}-01$ & 01 & & & & & & -01 \\
\hline & & $\mathrm{Ci}$ & & 03 & & & & -02 & -02 & 02 & \\
\hline & 36 & $\mathrm{Ci}$ & $1.50 \mathrm{E}-02$ & 1.38 & & 01 & 1. & 02 & & 2 & -02 \\
\hline 106 & U238 & $\mathrm{Ci}$ & 2.01E-01 & $1.39 \mathrm{E}-01$ & $E+01$ & +01 & E-01 & E-00 & $E=00$ & $E-01$ & $5.23 \mathrm{E}-01$ \\
\hline AN106 & Y90 & $\mathrm{Ci}$ & $1.37 \mathrm{E}+02$ & $1.37 \mathrm{E}+02$ & $=01$ & +01 & -02 & $E+02$ & +03 & +02 & $2.50 \mathrm{E}+02$ \\
\hline 106 & Zr93 & $\mathrm{Ci}$ & $2.20 \mathrm{E}-00$ & $2.20 \mathrm{E}-00$ & $E+01$ & $E+01$ & $2.20 \mathrm{E}-00$ & $E+01$ & $E+01$ & $E-00$ & $1.47 \mathrm{E}+01$ \\
\hline AN107 & Al & $\mathrm{kg}$ & $2.61 E+04$ & $2.36 E+04$ & & & +05 & $\div 05$ & +05 & +05 & $E+04$ \\
\hline & $\mathbf{B i}$ & kg & & $0.00 \mathrm{E}+01$ & & & & & & +02 & $E+02$ \\
\hline & $\mathrm{Ca}$ & $\mathrm{kg}$ & -03 & +03 & & & & & & & +02 \\
\hline & Cl & $\mathrm{kg}$ & $1.13 E+04$ & $7.44 \mathrm{E}+03$ & +03 & +04 & $2.36 E+04$ & +04 & t04 & +04 & $8.09 E+03$ \\
\hline AN107 & $\mathrm{CO} 3$ & $\mathrm{~kg}$ & $2.94 \mathrm{E}+05$ & $3.24 E+05$ & $=+03$ & +04 & $1.52 \mathrm{E}+05$ & $E \div 05$ & +05 & $E+05$ & $3 E+04$ \\
\hline AN107 & $\mathrm{Cr}$ & $\mathbf{k g}$ & $1.15 E+03$ & $1.15 E+03$ & +01 & +03 & $E+04$ & +04 & +04 & $:+04$ & $E+03$ \\
\hline AN107 & F & $\mathrm{kg}$ & $5.42 E+03$ & $2.00 \mathrm{E}+03$ & $E+01$ & +03 & +04 & $E+04$ & +04 & +04 & $1.27 E+04$ \\
\hline AN107 & $\mathrm{Fe}$ & kg & $9.99 E+03$ & $1.06 E+04$ & $0.00 \mathrm{E}+01$ & +01 & $4.54 \mathrm{E}+03$ & $E+04$ & $E+04$ & $E+03$ & $5.75 E+03$ \\
\hline AN107 & Hg & kg & $8.41 E-00$ & $1.50 \mathrm{E}-00$ & $E+01$ & & & +01 & & +01 & $E-00$ \\
\hline & $\mathbf{K}$ & kg & $7.97 E+03$ & $7.04 E+03$ & $E+02$ & & $1.80 \mathrm{E}+04$ & $E+04$ & $E+04$ & 1. & $E+04$ \\
\hline & La & $\mathrm{kg}$ & $6.73 \mathrm{E}-00$ & $1.04 E+02$ & & & $1.76 \mathrm{E}+$ & & & & $+E+02$ \\
\hline & $\mathbf{M}$ & $\mathrm{kg}$ & $2.20 \mathrm{E}+03$ & $2.20 \mathrm{E}+03$ & $0.00 E+01$ & & 1.01 & & & & $3 E+03$ \\
\hline & $\mathbf{N a}$ & $\mathrm{kg}$ & $8.00 \mathrm{E}+05$ & $8.00 E+05$ & $5.44 E+05$ & $E+05$ & $6+05$ & +06 & $E+06$ & 05 & $1.90 E+05$ \\
\hline & $\mathbf{N i}$ & kg & $1.82 E+03$ & $2.07 E+03$ & $0.00 E+01$ & & 5.1 & & & $E+02$ & $5.74 E+02$ \\
\hline & $\mathrm{NO} 2$ & $\mathrm{~kg}$ & $2.08 E+05$ & $2.45 \mathrm{E}+05$ & $4.55 E+04$ & $1.52 \mathrm{E}+05$ & $3.45 \mathrm{E}+05$ & $5.33 E+05$ & $6.81 E+05$ & $3.48 \mathrm{E}+05$ & $1.47 E+05$ \\
\hline AN107 & $\mathrm{NO3}$ & kg & $9.24 E+05$ & $9.03 E+05$ & $7.53 E+04$ & $4.19 E+05$ & $9.12 E+05$ & $1.53 E+06$ & $2.12 \mathrm{E}+06$ & $9.61 E+05$ & $4.40 \mathrm{E}+05$ \\
\hline AN107 & $\mathbf{P}$ & $\mathrm{kg}$ & $5.13 E+03$ & NA & $0.00 \mathrm{E}+01$ & $2.21 E+03$ & $1.31 E+04$ & $2.86 E+04$ & $3.92 \mathrm{E}+04$ & $1.42 \mathrm{E}+04$ & $1.01 E+04$ \\
\hline AN107 & $\mathbf{P b}$ & kg & $1.46 \mathrm{E}+03$ & $1.64 E+03$ & $0.00 \mathrm{E}+01$ & $1.16 \mathrm{E}+02$ & $5.00 \mathrm{E}+02$ & $1.12 E+03$ & $1.70 E+03$ & $5.71 E+02$ & $3.95 \mathrm{E}+02$ \\
\hline
\end{tabular}


Tank Anal. Un

AN107 PO4

AN107 S

AN107 Si

AN107 SO4

AN107 Sr

AN107 TOC

AN107 U

AN107 $\mathrm{Zr}$

AN107 Ac227

AN107 Am241

AN107 Am243

AN107 Ba137

AN107 C14

AN107 Cd113

AN107 Cm242

AN107 Cm243

AN107 Cm244

AN107 C060

AN107 Cs134

AN107 Cs137

AN107 Eu152

AN107 Eu154

AN107 Eu155

AN107 I129

AN107 Nb93

AN107 Ni59

AN107 Ni63

AN107 Np237

AN107 Pa231

AN107 Pu238

AN107 Pu239

AN107 Pu240

AN107 Pu241

AN107 Pu242

AN107 Ra226

AN107 Ra228

AN107 Ru106

AN107 Sb125

AN107 Se79

AN107 Sm151

AN107 Sr89/90

AN107 Te99

AN107 Th229

AN107 Th232

AN107 Tin126

AN107 Tritium

AN107 U232

AN107 U233

AN107 U234

AN107 U235

AN107 U236

AN107 U238

AN107 Y90

AN107 Zr93

AP101 Al

AP101 Bi

AP101 C

AP101 Cl

AP101 $\mathrm{CO3}$

AP101 Cr

AP101 F

AP101 Fe

AP101 Hg

AP101 K

AP101 La

AP101 Mn

AP101 Na

AP101 $\mathrm{Ni}$
May98

$1.59 \mathrm{E}+04$

$1.98 \mathrm{E}+04$

$7.95 \mathrm{E}+03$

$5.93 E+04$

$0.00 \mathrm{E}+01$

$2.26 \mathrm{E}+05$

$6.95 \mathrm{E}+03$

4.42E+01

$3.26 \mathrm{E}-03$

$3.65 \mathrm{E}+03$

9.91E-03

$1.42 \mathrm{E}+06$

$1.48 \mathrm{E}+02$

$3.99 \mathrm{E}+02$

6.33E-01

6.33E-02

4.63E-01

$2.00 \mathrm{E}+02$

$5.71 \mathrm{E}+01$

1.50E+06

$2.00 \mathrm{E}+01$

$3.03 \mathrm{E}+03$

$1.22 \mathrm{E}+03$

2.25E-00

$5.10 \mathrm{E}+01$

$6.49 \mathrm{E}-00$

Ci $\quad 6.42 \mathrm{E}+02$

$3.98 \mathrm{E}-00$

1.60E-02

$5.98 \mathrm{E}-00$

$2.61 E+02$

Ci Incl. in 239

$4.29 \mathrm{E}+02$

Ci 2.29E-03

$5.29 \mathrm{E}-04$

2.05E-00

$3.56 \mathrm{E}-02$

$\mathrm{Ci} \quad 9.51 \mathrm{E}+02$

Ci $1.45 \mathrm{E}+01$

Ci $5.08 \mathrm{E}+04$

$5.54 \mathrm{E}+05$

$1.16 \mathrm{E}+03$

4.74E-02

2.32E-01

$2.18 E+01$

$9.29 \mathrm{E}+02$

5.48E-00

$2.10 \mathrm{E}+01$

Ci 2.69E-00

Ci 1.04E-01

Ci 9.51E-02

Ci 4.07E-00

Ci $\quad 5.54 \mathrm{E}+05$

Ci 7.13E+01

kg 4.18E+04

kg $\quad 3.44 \mathrm{E}+01$

g $2.37 \mathrm{E}+02$

kg $5.68 \mathrm{E}+03$

kg $6.03 E+04$

$\mathrm{kg} \quad 6.62 \mathrm{E}+02$

(c.90E+03

$\mathrm{kg} 2.35 \mathrm{E}+01$

kg 8.16E-01

kg $1.09 \mathrm{E}+05$

$\mathrm{kg} \quad 6.83 \mathrm{E}-01$

$\mathrm{kg} \quad 1.97 \mathrm{E}+02$

kg 6.31E+05

kg

\section{Sept98}

\section{$4.10 \mathrm{E}+03$}

NA

$7.95 E+03$

$4.05 E+04$

$0.00 \mathrm{E}+01$

$1.59 \mathrm{E}+05$

$1.86 \mathrm{E}+03$

$4.42 \mathrm{E}+01$

$3.26 \mathrm{E}-03$

$2.65 \mathrm{E}+03$

1.08E-01

$1.22 \mathrm{E}+06$

$1.48 \mathrm{E}+02$

$3.99 \mathrm{E}+02$

6.93E-00

6.59E-01

5.06E-00

$2.00 \mathrm{E}+02$

$5.71 \mathrm{E}+01$

$1.29 \mathrm{E}+06$

$2.00 \mathrm{E}+01$

$3.03 E+03$

$1.22 \mathrm{E}+03$

2.25E-00

$5.10 \mathrm{E}+01$

6.49E-00

$6.42 E+02$

$3.98 \mathrm{E}-00$

$1.60 \mathrm{E}-02$

7.08E-00

$2.22 \mathrm{E}+02$

$3.92 \mathrm{E}+01$

$5.08 \mathrm{E}+02$

2.72E-03

$5.29 \mathrm{E}-04$

2.05E-00

$3.56 \mathrm{E}-02$

$9.51 \mathrm{E}+02$

$1.45 \mathrm{E}+01$

$5.08 \mathrm{E}+04$

$5.54 \mathrm{E}+05$

$1.33 \mathrm{E}+02$

$4.74 \mathrm{E}-02$

2.32E-01

$2.18 \mathrm{E}+01$

$9.29 \mathrm{E}+02$

1.47E-00

5.62E-01

7.19E-01

2.79E-02

2.54E-02

$6.21 \mathrm{E}-01$

$5.54 \mathrm{E}+05$

$7.13 \mathrm{E}+01$

4.18E+04

$0.00 \mathrm{E}+01$

$2.37 \mathrm{E}+02$

$5.68 \mathrm{E}+03$

$6.03 \mathrm{E}+04$

$6.62 E+02$

$9.90 \mathrm{E}+03$

$2.35 \mathrm{E}+01$

$0.00 \mathrm{E}+01$

$1.09 \mathrm{E}+05$

$0.00 E+01$

$0.00 \mathrm{E}+01$

$6.31 E+05$

3.85E+01
$1 \%$-ile

$2.55 \mathrm{E}+03$

$10 \%$-ile

$6.18 \mathrm{E}+02$

$9.49 \mathrm{E}+03$

50\%-ile

$3.45 \mathrm{E}+03$

$3.72 \mathrm{E}+02$

$0.00 \mathrm{E}+01$

$8.93 \mathrm{E}+03$

$8.30 \mathrm{E}-00$

$1.70 \mathrm{E}+05$

$3.54 \mathrm{E}+01$

$8.95 \mathrm{E}-00$

$0.00 \mathrm{E}+01$

$0.00 \mathrm{E}+01$

$6.91 \mathrm{E}-03$

$2.89 \mathrm{E}+05$

$0.00 \mathrm{E}+01$

$0.00 \mathrm{E}+01$

4.41E-01

4.41E-02

3.23E-01

$7.98 \mathrm{E}-00$

$1.16 \mathrm{E}+01$

3.93E+05

$0.00 \mathrm{E}+01$

$0.00 \mathrm{E}+01$

$0.00 \mathrm{E}+01$

6.75E-01

$0.00 \mathrm{E}+01$

$0.00 \mathrm{E}+01$

$0.00 \mathrm{E}+01$

$2.77 \mathrm{E}-00$

$0.00 \mathrm{E}+01$

4.17E-00

$1.82 \mathrm{E}+02$

$1.82 \mathrm{E}+02$

$2.99 \mathrm{E}+02$

$1.60 \mathrm{E}-03$

$0.00 \mathrm{E}+01$

$0.00 \mathrm{E}+01$

$0.00 E+01$

$0.00 \mathrm{E}+01$

$1.55 \mathrm{E}-00$

$0.00 \mathrm{E}+01$

$6.41 \mathrm{E}+02$

$0.00 \mathrm{E}+01$

$0.00 \mathrm{E}+01$

$0.00 \mathrm{E}+01$

$0.00 \mathrm{E}+01$

$5.21 \mathrm{E}+02$

$0.00 \mathrm{E}+01$

$0.00 \mathrm{E}+01$

$0.00 \mathrm{E}+01$

$0.00 \mathrm{E}+01$

$0.00 \mathrm{E}+01$

$0.00 \mathrm{E}+01$

$0.00 \mathrm{E}+01$

$0.00 \mathrm{E}+01$

$7.08 \mathrm{E}+02$

$2.80 \mathrm{E}-03$

$1.92 \mathrm{E}-00$

$1.39 \mathrm{E}-00$

$2.19 E+03$

1.43E-03

$1.92 \mathrm{E}+02$

7.13E-01

$0.00 \mathrm{E}+01$

$4.38 \mathrm{E}-03$

$4.55 \mathrm{E}-03$

$1.08 \mathrm{E}-03$

$1.82 \mathrm{E}+04$

1.01E-03

$3.58 \mathrm{E}+04$

$1.34 \mathrm{E}+04$

$3.86 \mathrm{E}+03$

$3.98 \mathrm{E}+04$

$2.43 \mathrm{E}+02$

$1.79 \mathrm{E}+05$

1.17E+04 
Tank Anal. Un

$\begin{array}{ll}\text { AP101 } & \text { NO2 } \\ \text { AP101 } & \text { NO3 } \\ \text { AP101 } & \text { P } \\ \text { AP101 } & \text { Pb } \\ \text { AP101 } & \text { PO4 } \\ \text { AP101 } & \text { S } \\ \text { AP101 } & \text { Si } \\ \text { AP101 } & \text { SO4 } \\ \text { AP101 } & \text { Sr } \\ \text { AP101 } & \text { TOC } \\ \text { AP101 } & \text { U } \\ \text { AP101 } & \text { Zr }\end{array}$

AP101 Am 241

AP101 Am243

AP101 Ba137

AP101 C14

AP101 Cd113

AP101 Cm242

AP101 Cm243

AP101 Cm244

AP101 Co60

AP101 Cs134

AP101 Cs137

AP101 Eu152

AP101 Eu154

AP101 Eu155

AP101 I129

AP101 Nb93

AP101 Ni59

AP101 Ni63

AP101 Np237

AP101 Pa231

AP101 Pu238

AP101 Pu239

AP101 Pu240

AP101 Pu241

AP101 Pu242

AP101 Ra226

AP101 Ra228

AP101 Rul06

AP101 Sb125

AP101 Se79

AP101 Sm151

AP101 Sr89/90

AP101 Te99

AP101 Th229

AP101 Th232

AP101 Tin126

AP101 Tritium

AP101 U232

AP101 U233

AP101 U234

AP101 U235

AP101 U236

AP101 U238

AP101 Y90

AP101 Zr93

AP102 Al

AP102 Bi

AP102 Ca

AP102 Cl

AP102 $\mathrm{CO} 3$

AP102 Cr

AP102 F

AP102 Fe

AP102 Hg

AP102 K
May $\frac{B 8}{98}$

kg $\quad \overline{1.74 E+05}$

kg 5.68E +05

kg 1.38E+03

kg 1.90E+01

kg $4.28 \mathrm{E}+03$

g. 4.93E+03

kg 5.12E+02

i. $1.48 \mathrm{E}+04$

kg 1.69E-02

$1.36 \mathrm{E}+04$

$\begin{array}{ll}\mathrm{kg} & 1.54 \mathrm{E}+02\end{array}$

$7.50 \mathrm{E}+01$

2.05E-04

8.44E-01

2.02E-03

$7.49 \mathrm{E}+05$

2.22E-01

$1.63 \mathrm{E}+01$

3.59E-02

$3.76 \mathrm{E}-03$

6.30E-02

$7.36 \mathrm{E}-00$

Ci $1.40 \mathrm{E}+01$

Ci $7.49 E+05$

Ci 8.38E-01

Ci 1.49E-02

$5.35 \mathrm{E}+01$

Ci $6.80 \mathrm{E}-02$

2.59E-00

Ci 3.14E-01

Ci.09E+01

Ci 1.13E-00

Ci $7.88 \mathrm{E}-04$

$\mathrm{Ci} \quad$ 1.11E-00

Ci 7.88E-01

Ci Incl. in 239

Ci $1.00 \mathrm{E}+02$

Ci 4.39E-04

Ci 3.36E-05

Ci 4.19E-02

Ci 3.66E-03

Ci $\quad 5.77 \mathrm{E}+01$

Ci 7.31E-01

Ci $2.60 \mathrm{E}+03$

Ci $7.06 \mathrm{E}+02$

Ci $2.21 \mathrm{E}+02$

Ci 9.74E-04

Ci 4.03E-03

Ci 1.13E-00

Ci 4.12E+01

Ci 1.47E-01

Ci 5.64E-01

Ci $4.65 \mathrm{E}-01$

Ci 1.79E-02

Ci 3.35E-02

Ci 5.18E-02

Ci $\quad 7.06 E+02$

Ci 3.46E-00

$\mathrm{kg} \quad 4.85 \mathrm{E}+04$

kg $\quad 6.07 \mathrm{E}+02$

kg $3.34 \mathrm{E}+02$

$\mathrm{kg} \quad 1.21 \mathrm{E}+04$

kg 1.12E+05

kg . $2.58 \mathrm{E}+03$

kg $\quad 7.02 E+02$

kg $1.59 \mathrm{E}+01$

kg 3.98E-00

kg $5.39 E+03$
BB Sept98

$\overline{1.74 \mathrm{E}+05}$

$5.68 \mathrm{E}+05$

NA

$1.90 \mathrm{E}+01$

$4.28 \mathrm{E}+03$

NA

$5.12 \mathrm{E}+02$

$1.48 \mathrm{E}+04$

1.69E-02

$1.36 \mathrm{E}+04$

$1.54 \mathrm{E}+02$

$7.50 \mathrm{E}+01$

6.41E-04

8.44E-01

7.79E-05

$7.11 E+05$

2.20E-01

$6.54 \mathrm{E}+01$

$1.38 \mathrm{E}-03$

7.00E-02

1.53E-00

$1.42 \mathrm{E}+01$

$1.10 \mathrm{E}+01$

$7.49 E+05$

3.34E-00

$3.16 \mathrm{E}+02$

$1.75 \mathrm{E}+02$

2.40E-01

9.08E-00

1.42E-00

$1.42 \mathrm{E}+02$

$7.85 \mathrm{E}-01$

2.87E-03

2.25E-00

$6.24 \mathrm{E}-01$

1.49E-01

4.33E-00

1.80E-05

3.28E-04

$1.91 \mathrm{E}-01$

3.42E-02

$1.10 E+02$

1.67E-00

$8.99 \mathrm{E}+03$

$7.06 \mathrm{E}+02$

$2.21 E+02$

4.51E-03

2.59E-02

$5.41 \mathrm{E}+01$

$5.94 \mathrm{E}+01$

2.23E-02

8.53E-02

$7.04 \mathrm{E}-02$

2.70E-03

5.06E-03

5.13E-02

$7.06 \mathrm{E}+02$

$1.25 \mathrm{E}+01$

$4.85 \mathrm{E}+04$

$0.00 \mathrm{E}+01$

$3.34 \mathrm{E}+02$

$1.21 \mathrm{E}+04$

$1.12 E+05$

$2.58 \mathrm{E}+03$

$7.02 \mathrm{E}+02$

$1.59 \mathrm{E}+01$

$0.00 \mathrm{E}+01$

$5.39 E+03$ 1\%-ile.

$10 \%$-ile

$1.02 \mathrm{E}+02 \quad 2.42 \mathrm{E}+03$

$7.77 \mathrm{E}+03 \quad 1.19 \mathrm{E}+04$

$1.06 \mathrm{E}-03 \quad 4.68 \mathrm{E}-03$

$1.41 \mathrm{E}-04 \quad 9.31 \mathrm{E}-04$

$1.82 \mathrm{E}-03 \quad 2.53 \mathrm{E}-02$

$1.12 \mathrm{E}-03 \quad 5.29 \mathrm{E}-02$

9.74E-04

$1.32 \mathrm{E}+02$

$1.66 \mathrm{E}-05$

7.38E-00

2.31E-03

4.15E-03

$0.00 \mathrm{E}+01$

$5.20 \mathrm{E}-05$

3.37E-07

$0.00 \mathrm{E}+01$

$0.00 \mathrm{E}+01$

$0.00 \mathrm{E}+01$

5.99E-06

6.27E-07

$1.05 \mathrm{E}-05$

1.64E-05

$0.00 \mathrm{E}+01$

7.27E-02

$0.00 E+01$

$0.00 \mathrm{E}+01$

$0.00 \mathrm{E}+01$

$0.00 \mathrm{E}+01$

$0.00 E+01$

$0.00 E+01$

$0.00 \mathrm{E}+01$

$1.88 \mathrm{E}-04$

$0.00 E+01$

1.85E-04

$1.31 \mathrm{E}-04$

1.31E-04

1.67E-02

$7.32 \mathrm{E}-08$

$0.00 E+01$

$0.00 E+01$

$0.00 E+01$

$0.00 E+01$

$0.00 E+01$

$0.00 \mathrm{E}+01$

$5.73 \mathrm{E}-02$

$0.00 \mathrm{E}+0$ !

$0.00 \mathrm{E}+0 \mathrm{I}$

$0.00 \mathrm{E}+0 \mathrm{l}$

$0.00 \mathrm{E}+0 \mathrm{l}$

$1.61 E+01$

$0.00 E+01$

$0.00 E+01$

$0.00 \mathrm{E}+0 \mathrm{I}$

$0.00 \mathrm{E}+01$

$0.00 \mathrm{E}+0 \mathrm{I}$

$0.00 \mathrm{E}+01$

$0.00 \mathrm{E}+01$

$0.00 \mathrm{E}+01$

$3.02 E+04$

$0.00 \mathrm{E}+01$

$5.58 \mathrm{E}-04$

$4.22 \mathrm{E}+02$

$6.49 \mathrm{E}+04$

$6.10 \mathrm{E}+02$

8.93E-06

$0.00 \mathrm{E}+01$

$0.00 \mathrm{E}+01$

3.55E-06

1.12E-01

$2.67 \mathrm{E}+02$

$7.56 \mathrm{E}-04$

$1.48 \mathrm{E}+02$

$1.93 \mathrm{E}-02$

6.14E-02

$0.00 E+01$

9.80E-04

2.16E-06

$5.11 E+04$

$2.78 \mathrm{E}-02$

$0.00 \mathrm{E}+01$

3.84E-05

4.02E-06

6.74E-05

9.38E-05

$9.56 \mathrm{E}-01$

$5.25 E+03$

$0.00 \mathrm{E}+01$

$0.00 E+01$

$0.00 \mathrm{E}+01$

$0.00 E+01$

$0.00 E+01$ 
Tank Anal. Un

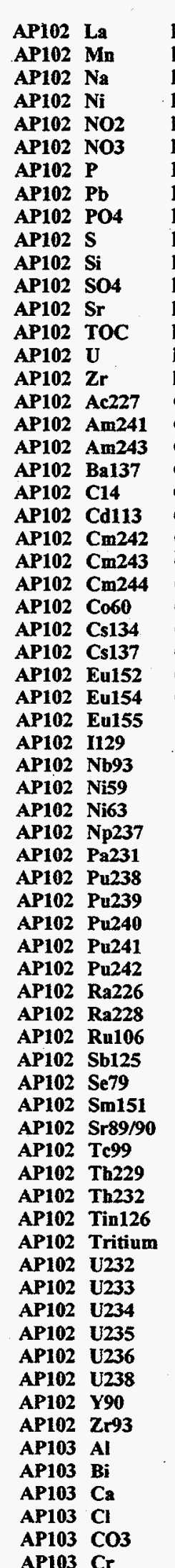

Marg8 $7.42 \mathrm{E}-00$

kg 2.33E+02

kg $4.26 \mathrm{E}+05$

$\mathrm{kg} \quad 1.11 \mathrm{E}+02$

$\begin{array}{ll}\mathrm{kg} & 1.59 \mathrm{E}+05 \\ \mathrm{k} & 3.27 \mathrm{E}+05\end{array}$

kg 1.57E+04

kg 1.38E+01

g $4.85 \mathrm{E}+04$

kg $\quad 6.30 \mathrm{E}+03$

kg 2.01E-00

kg $1.89 \mathrm{E}+04$

g $0.00 \mathrm{E}+01$

g $1.37 \mathrm{E}+04$

$1.16 \mathrm{E}+02$

Ci $1.96 \mathrm{E}-03$

$1.75 \mathrm{E}-00$

5.06E-03

$8.81 \mathrm{E}+05$

2.09E-00

$2.05 \mathrm{E}+02$

4.68E-03

1.73E-01

2.66E-01

$3.19 \mathrm{E}+02$

$1.44 \mathrm{E}+01$

$9.31 \mathrm{E}+05$

9.74E-00

$1.47 \mathrm{E}+03$

$5.82 \mathrm{E}+02$

1.10E-00

$2.76 \mathrm{E}+01$

4.55E-00

$4.47 E+02$

4.18E-00

9.17E-03

6.81E-01

$1.03 E+02$

$1.79 \mathrm{E}+01$

$2.15 E+02$

Ci 1.18E-03

Ci 3.14E-04

$\mathrm{Ci} \quad$ 5.81E-01

Ci 1.69E-02

Ci 4.19E+02

Ci 8.82E-01

Ci $2.74 \mathrm{E}+04$

Ci $5.88 \mathrm{E}+03$

Ci 3.58E+02

Ci 1.35E-02

5.73E-02

Ci 1.18E+01

$1.09 \mathrm{E}+01$

Ci 2.01E-00

Ci 7.71E-00

Ci 1.64E-00

Ci $\quad 6.56 \mathrm{E}-02$

Ci 5.27E-02

Ci 2.08E-00

Ci $\quad 5.88 E+03$

Ci $3.83 \mathrm{E}+01$

$\mathrm{kg} 2.15 \mathrm{E}+01$

kg 1.60E-01

kg 2.18E+01

kg 4.43E-00

kg 2.26E+02

kg
$\frac{B B}{t 98}$

$7.42 \mathrm{E}-00$

$2.33 \mathrm{E}+02$

$4.26 \mathrm{E}+05$

$1.11 E+02$

$1.59 \mathrm{E}+05$

$3.27 \mathrm{E}+05$

NA

$1.38 \mathrm{E}+01$

$4.85 E+04$

NA

2.01E-00

$1.89 \mathrm{E}+04$

$0.00 \mathrm{E}+01$

$1.37 \mathrm{E}+04$

$1.93 \mathrm{E}+01$

$1.16 \mathrm{E}+02$

1.96E-03

1.75E-00

6.61E-05

$8.81 \mathrm{E}+05$

2.09E-00

$2.05 \mathrm{E}+02$

4.68E- 03

1.73E-01

2.66E-01

$3.19 \mathrm{E}+02$

$1.44 \mathrm{E}+01$

$9.31 \mathrm{E}+05$

9.74E-00

$1.47 \mathrm{E}+03$

$5.82 \mathrm{E}+02$

1.10E-00

$2.76 \mathrm{E}+01$

$4.55 \mathrm{E}-00$

$4.47 \mathrm{E}+02$

$4.18 \mathrm{E}-00$

9.17E-03

8.07E-03

2.67E-01

4.61E-02

5.56E-01

3.05E-06

3.14E-04

5.81E-01

$1.69 \mathrm{E}-02$

$4.19 \mathrm{E}+02$

8.82E-01

$2.74 E+04$

$5.88 \mathrm{E}+03$

$3.58 \mathrm{E}+02$

1.35E-02

5.73E-02

1.18E +01

$1.09 E+01$

8.82E-03

3.38E-02

7.17E-03

2.88E-04

2.31E-04

6.44E- 03

$5.88 \mathrm{E}+03$

$3.83 \mathrm{E}+01$

$2.15 \mathrm{E}+01$

$0.00 E+01$

$2.18 E+01$

4.43E-00

$2.26 \mathrm{E}+02$

3.79E-01 1\%-ile

7.69E-08

$1.57 \mathrm{E}+03$

$2.98 \mathrm{E}+05$

$3.95 \mathrm{E}+01$

$4.47 \mathrm{E}+04$

$9.31 \mathrm{E}+04$

2.33E-02

$0.00 \mathrm{E}+01$

$3.84 \mathrm{E}+04$

$8.28 \mathrm{E}-06$

$4.18 \mathrm{E}+02$

$1.18 \mathrm{E}+04$

$3.55 \mathrm{E}+02$

$6.84 \mathrm{E}+03$

$0.00 \mathrm{E}+01$

4.13E-07

$0.00 \mathrm{E}+01$

$0.00 \mathrm{E}+01$

$0.00 \mathrm{E}+01$

$0.00 \mathrm{E}+01$

$0.00 \mathrm{E}+01$

$0.00 \mathrm{E}+01$

$0.00 \mathrm{E}+01$

$0.00 \mathrm{E}+01$

$0.00 \mathrm{E}+0 \mathrm{l}$

$4.59 \mathrm{E}+01$

$0.00 \mathrm{E}+01$

$4.67 \mathrm{E}+04$

$0.00 \mathrm{E}+01$

$0.00 \mathrm{E}+01$

$0.00 \mathrm{E}+01$

$0.00 \mathrm{E}+01$

$0.00 \mathrm{E}+01$

$0.00 E+01$

$0.00 E+01$

$0.00 \mathrm{E}+01$

$0.00 E+01$

$0.00 \mathrm{E}+01$

$0.00 \mathrm{E}+01$

$0.00 \mathrm{E}+01$

$0.00 \mathrm{E}+01$

$0.00 \mathrm{E}+01$

$0.00 E+01$

$0.00 \mathrm{E}+01$

$0.00 \mathrm{E}+01$

$0.00 \mathrm{E}+01$

$0.00 E+01$

$0.00 E+01$

$4.64 E+03$

$0.00 E+01$

$0.00 \mathrm{E}+01$

$0.00 \mathrm{E}+01$

$0.00 E+0$ !

$9.98 \mathrm{E}-00$

$0.00 \mathrm{E}+01$

$0.00 E+01$

$0.00 \mathrm{E}+0 \mathrm{I}$

$0.00 \mathrm{E}+01$

$0.00 \mathrm{E}+01$

$0.00 \mathrm{E}+01$

$4.00 \mathrm{E}+03$

$0.00 \mathrm{E}+01$

$7.89 \mathrm{E}-05$

$0.00 \mathrm{E}+01$

2.54E-06

6.36E-06

2.50E-04

1.67E-07
$10 \%$-ile

$3.75 \mathrm{E}-04$

$1.84 \mathrm{E}+03$

$3.51 \mathrm{E}+05$

$7.35 \mathrm{E}+01$

$8.94 \mathrm{E}+04$

$1.81 \mathrm{E}+05$

9.81E-00

8.18E-05

$4.08 \mathrm{E}+04$

2.02E-01

$9.91 \mathrm{E}+02$

$1.45 \mathrm{E}+04$

$3.65 \mathrm{E}+02$

1. $03 \mathrm{E}+04$

7.18E-07

$1.31 \mathrm{E}+02$

$0.00 \mathrm{E}+01$

7.24E-06

$0.00 \mathrm{E}+01$

$2.51 \mathrm{E}+05$

3.31E-01

$0.00 \mathrm{E}+01$

$0.00 E+01$

$0.00 E+01$

$0.00 \mathrm{E}+01$

$5.86 \mathrm{E}+01$

$4.10 \mathrm{E}-00$

$3.54 \mathrm{E}+05$

$0.00 \mathrm{E}+01$

$0.00 \mathrm{E}+01$

$0.00 \mathrm{E}+01$

2.66E-01

$0.00 \mathrm{E}+01$

$0.00 \mathrm{E}+01$

$0.00 \mathrm{E}+01$

$0.00 \mathrm{E}+01$

$0.00 \mathrm{E}+01$

$0.00 \mathrm{E}+01$

$0.00 \mathrm{E}+01$

$0.00 \mathrm{E}+01$

$0.00 \mathrm{E}+01$

$0.00 \mathrm{E}+01$

$0.00 \mathrm{E}+01$

$0.00 \mathrm{E}+01$

$0.00 \mathrm{E}+01$

$0.00 \mathrm{E}+01$

3.97E-01

$0.00 \mathrm{E}+01$

$5.04 \mathrm{E}+03$

$0.00 \mathrm{E}+01$

$0.00 \mathrm{E}+01$

$0.00 \mathrm{E}+01$

$0.00 \mathrm{E}+01$

$1.03 \mathrm{E}+01$

$0.00 \mathrm{E}+01$

$0.00 E+01$

$0.00 E+01$

$0.00 E+01$

$0.00 \mathrm{E}+01$

$0.00 E+01$

$4.76 \mathrm{E}+03$

$0.00 E+01$

$1.33 \mathrm{E}+01$

7.19E-03

6.70E-00

$7.24 \mathrm{E}-01$

$1.22 \mathrm{E}+02$

$3.03 \mathrm{E}-02$

$50 \%$ ille

\section{$4.17 \mathrm{E}+01$}

$2.17 \mathrm{E}+03$

$4.20 E+05$

$1.14 \mathrm{E}+02$

$1.45 \mathrm{E}+05$

$2.95 \mathrm{E}+05$

$1.35 E+04$

$7.24 \mathrm{E}-00$

$4.37 E+04$ 
Tank Anal. U

\begin{tabular}{|c|c|}
\hline AP103 & $\pi$ \\
\hline AP103 & Fe \\
\hline AP103 & $\mathrm{Hg}$ \\
\hline $\begin{array}{l}\text { AP103 } \\
\text { AP103 }\end{array}$ & $\begin{array}{l}\mathbf{K} \\
\mathbf{L a}\end{array}$ \\
\hline AP103 & $\mathbf{M n}$ \\
\hline AP103 & $\mathbf{N a}$ \\
\hline AP103 & $\mathrm{Ni}$ \\
\hline AP103 & $\mathrm{NO} 2$ \\
\hline AP103 & NO3 \\
\hline AP103 & $\mathbf{P}$ \\
\hline AP103 & $\mathbf{P b}$ \\
\hline AP103 & PO4 \\
\hline AP103 & $\mathbf{S}$ \\
\hline AP103 & Si \\
\hline AP103 & SO4 \\
\hline AP103 & Sr \\
\hline AP103 & TOC \\
\hline AP103 & 0 \\
\hline AP103 & $\begin{array}{l}\mathrm{Zr} \\
\mathrm{Ac} 227\end{array}$ \\
\hline $\begin{array}{l}\text { AP103 } \\
\text { AP103 }\end{array}$ & $\operatorname{Am} 241$ \\
\hline AP103 & Am243 \\
\hline AP103 & Ba137 \\
\hline AP103 & C14 \\
\hline AP103 & Cd113 \\
\hline AP103 & $\mathrm{Cm} 242$ \\
\hline AP103 & $\mathrm{Cm} 243$ \\
\hline AP103 & $\operatorname{Cm} 244$ \\
\hline AP103 & Co60 \\
\hline AP103 & Cs134 \\
\hline AP103 & Cs137 \\
\hline AP103 & Eu152 \\
\hline AP103 & Eu154 \\
\hline AP103 & Eu155 \\
\hline AP103 & 1129 \\
\hline AP103 & Nb93 \\
\hline AP103 & Ni59 \\
\hline AP103 & Ni63 \\
\hline AP103 & Np237 \\
\hline AP103 & Pa231 \\
\hline $\begin{array}{l}\text { AP103 } \\
\text { AP103 }\end{array}$ & $\begin{array}{l}\text { Pu238 } \\
\text { Pu230 }\end{array}$ \\
\hline AP103 & Puz40 \\
\hline
\end{tabular}

AP103 Pu241

AP103 Pu242

AP103 Ra226

AP103 Ra228

AP103 Ru106

AP103 Sb125

AP103 Se79

AP103 Sm151

AP103 Sr89/90

AP103 Tc99

AP103 Th229

AP103 Th232

AP103 Tin126

AP103 Tritium

AP103 U232

AP103 U233

AP103 U234

AP103 U235

AP103 U236

AP103 U238

AP103 Y90

AP103 Zr93

AP104 Al

AP104 Bi

\section{May $\frac{B B}{98}$}

kg 7.18E-00

kg 7.30E-02

kg 1.50E-04

$\begin{array}{ll}\mathrm{kg} & 3.09 \mathrm{E}+01 \\ \mathrm{~kg} & 2.32 \mathrm{E}-03\end{array}$

kg 2.60E-03

kg $\quad 4.59 \mathrm{E}+02$

kg 5.69E-00

kg $\quad 1.13 \mathrm{E}+02$

kg 3.55E+02

$\mathrm{kg} \quad 3.88 \mathrm{E}-00$

kg 3.50E-02

$\mathrm{kg} \cdot 1.20 \mathrm{E}+01$

kg 1.05E+01

kg 2.99E+01

kg 3.15E+01

kg $0.00 \mathrm{E}+01$

$\mathrm{kg} \quad 9.57 \mathrm{E}-00$

$\mathrm{kg} \quad 3.20 \mathrm{E}-01$

$\mathrm{kg} \quad 3.28 \mathrm{E}-01$

Ci 1.13E-05

9.87E-04

Ci 8.18E-05

Ci $\quad 5.47 E+02$

Ci 3.04E-01

6.56E-01

1.01E-02

$1.59 \mathrm{E}-03$

$3.16 \mathrm{E}-03$

Ci 9.60E-01

3.90E+01

Ci $\quad 5.77 \mathrm{E}+02$

Ci 1.27E-01

Ci 1.46E-00

Ci $2.31 \mathrm{E}-00$

Ci 2.90E-03

Ci 1.35E-01

$\mathrm{Ci}$ 9.02E-03

Ci 8.92E-01

$\mathrm{Ci} \quad 4.18 \mathrm{E}-00$

Ci 3.44E-05

Ci 6.41E-01

Ci 6.06E-04

Ci Incl. in 239

Ci 1.61E-00

Ci 8.80E-06

Ci 2.15E+01

Ci 1.96E-04

Ci 1.60E-04

Ci $3.74 \mathrm{E}+01$

Ci $\quad 9.75 \mathrm{E}-04$

Ci 1.35E+02

Ci 1.70E-01

Ci 9.40E-02

$\mathrm{Ci} \quad 4.56 \mathrm{E}-06$

Ci 1.92E-05

Ci 2.02E+01

Ci $\quad 6.76 \mathrm{E}-04$

Ci 2.60E-03

Ci 5.70E-04

Ci 2.95E-05

Ci 2.42E-04

Ci 1.10E-04

Ci 1.70E-01

Ci 1.72E-01

kg $\quad 4.06 E-00$ kg $\frac{\text { Sept98 }}{1.12 \mathrm{E}+01}$

7.30E-02

$0.00 \mathrm{E}+01$

$3.09 E+01$

2.32E-03

$2.60 \mathrm{E}-03$

$4.59 \mathrm{E}+02$

5.69E-00

$1.13 \mathrm{E}+02$

$3.55 \mathrm{E}+02$

NA

3.50E-02

$1.20 \mathrm{E}+01$

NA

$2.99 \mathrm{E}+01$

$3.15 E+01$

$0.00 \mathrm{E}+01$

9.57E-00

3.20E-01

$3.28 \mathrm{E}-01$

1.13E-05

9.87E-04

$7.29 \mathrm{E}-08$

$5.47 E+02$

4.00E-04

6.56E-01

8.99E-06

1.42E-06

2.81E-06

$9.60 \mathrm{E}-01$

$3.90 \mathrm{E}+01$

$5.77 \mathrm{E}+02$

1.27E-01

1.46E-00

2.31E-00

$2.90 \mathrm{E}-03$

$1.35 \mathrm{E}-01$

9.02E-03

8.92E-01

4.18E-00

3.44E-05

2.77E-05

4.88E-04

1.12E-04

2.19E-03

1.19E-08

$2.15 \mathrm{E}+01$

1.96E-04

$1.60 \mathrm{E}-04$

$3.74 \mathrm{E}+01$

$9.75 \mathrm{E}-04$

$1.35 \mathrm{E}+02$

1.70E-01

9.40E-02

4.56E-06

1.92E-05

5.88E-02

4.50E-01

3.08E-05

1.18E-04

$1.48 \mathrm{E}-04$

5.66E-06

1.10E-05

1.07E-04

1.70E-01

1.72E-01

4.06E-00

$0.00 \mathrm{E}+01$ 1\%-ile

$10 \%$-ile

$2.02 \mathrm{E}-04$
$0.00 \mathrm{E}+01$

$0.00 \mathrm{E}+01$

4.99E-06

$0.00 \mathrm{E}+01$

$1.48 \mathrm{E}-06$

$9.26 \mathrm{E}-04$

$0.00 \mathrm{E}+01$

8.09E-06

4.43E-04

$6.60 \mathrm{E}-07$

2.46E-10

4.44E-07

1.17E-07

$0.00 \mathrm{E}+01$

$2.46 \mathrm{E}-06$

$0.00 \mathrm{E}+01$

$1.40 \mathrm{E}-06$

$0.00 E+01$

$0.00 \mathrm{E}+01$

$0.00 \mathrm{E}+01$

$0.00 \mathrm{E}+01$

$0.00 \mathrm{E}+01$

$0.00 \mathrm{E}+01$

$0.00 \mathrm{E}+01$

$0.00 \mathrm{E}+01$

$0.00 \mathrm{E}+01$

$0.00 \mathrm{E}+01$

$0.00 \mathrm{E}+01$

$0.00 \mathrm{E}+01$

$0.00 \mathrm{E}+01$

7.77E-05

$0.00 \mathrm{E}+01$

$0.00 \mathrm{E}+01$

$0.00 \mathrm{E}+01$

$0.00 \mathrm{E}+01$

$0.00 \mathrm{E}+01$

$0.00 \mathrm{E}+01$

$0.00 \mathrm{E}+01$

$0.00 \mathrm{E}+0 \mathrm{l}$

$0.00 \mathrm{E}+01$

$0.00 \mathrm{E}+01$

$0.00 \mathrm{E}+01$

$0.00 \mathrm{E}+01$

$0.00 \mathrm{E}+01$

$0.00 \mathrm{E}+01$

$0.00 \mathrm{E}+01$

$0.00 E+01$

$0.00 E+01$

$0.00 \mathrm{E}+0 \mathrm{I}$

$0.00 \mathrm{E}+0$ !

$0.00 \mathrm{E}+01$

$0.00 \mathrm{E}+0 \mathrm{I}$

$0.00 \mathrm{E}+0 \mathrm{I}$

$0.00 \mathrm{E}+01$

$0.00 \mathrm{E}+01$

$0.00 \mathrm{E}+01$

$0.00 E+01$

$0.00 E+01$

$0.00 \mathrm{E}+01$

$0.00 E+01$

$0.00 E+01$

$0.00 \mathrm{E}+01$

$0.00 \mathrm{E}+01$

$0.00 E+01$

$0.00 E \div 01$

2.81E-06

$0.00 \mathrm{E} \div 01$

$1.10 \mathrm{E}+01$

$5.07 \mathrm{E}-00$

9.14E-07

7.23E- 03

$1.18 \mathrm{E}-02$

4.83E-02

$1.60 \mathrm{E}+02$

9.80E-04

$1.96 \mathrm{E}-01$

$3.96 \mathrm{E}+01$

4.70E-02

$1.25 \mathrm{E}-02$

$1.22 \mathrm{E}-01$

8.53E-01

5.72E-03

1.74E-00

$5.95 \mathrm{E}-04$

$3.06 \mathrm{E}-01$

2.69E-04

$1.77 \mathrm{E}-04$

$7.00 \mathrm{E}-09$

$0.00 \mathrm{E}+01$

4.15E-08

$0.00 \mathrm{E}+01$

$1.23 \mathrm{E}-02$

4.00E-03

5.13E-06

8.07E-07

1.60E-06

$1.07 \mathrm{E}-05$

$0.00 \mathrm{E}+01$

3.53E-01

$7.86 \mathrm{E}-05$

9.04E-04

$1.43 \mathrm{E}-03$

3.64E-05

2.65E-05

$1.99 \mathrm{E}-05$

1.97E-03

2.12E-03

$0.00 \mathrm{E}+01$

$3.25 \mathrm{E}-04$

3.08E-07

3.08E-07

8.17E-04

4.47E-09

$1.09 \mathrm{E}-01$

9.94E-07

3.08E-07

1.36E-01

4.90E-06

8.36E-02

3.07E-08

$0.00 E+01$

$0.00 E+01$

$0.00 \mathrm{E}+01$

50\%-ile 
Tank Anal. Un

$\begin{array}{ll}\text { AP104 } & \mathrm{Ca} \\ \text { AP104 } & \mathrm{Cl} \\ \text { AP104 } & \mathrm{CO} \\ \text { AP104 } & \mathrm{Cr} \\ \text { AP104 } & \text { F } \\ \text { AP104 } & \mathrm{Fe} \\ \text { AP104 } & \mathrm{Hg}\end{array}$

AP104 $\mathrm{K}$

AP104 La

AP104 Mn

AP104 Na

AP104 Ni

AP104 NO2

AP104 NO3

AP104 P

AP104 Pb

AP104 PO4

AP104 S

AP104 Si

AP104 SO4

AP104 Sr

AP104 TOC

AP104U

AP104 Zr

AP104 Ac227

AP104 Am241

AP104 Am243

AP104 Ba137

AP104 C14

AP104 Cd113

AP104 Cm242

AP104 Cm243

AP104 Cm244

AP104 Co60

AP104 Cs134

AP104 Cs137

AP104 Eu152

AP104 Eu154

AP104 Eu155

AP104 I129

AP104 Nb93

AP104 Ni59

AP104 Ni63

AP104 Np237

AP104 Pa231

AP104 Pu238

AP104 Pu239

AP104 Pu240

AP104 Pu241

AP104 Pu242

AP104 Ra226

AP104 Ra228

AP104 Ru106

AP104 Sb125

AP104 Se79

AP104 Sm151

AP104 Sr89/90

AP104 TC99

AP104 Th229

AP104 Th232

AP104 Tin126

AP104 Tritium

AP104 U232

AP104 U233

AP104 U234

AP104 U235

AP104 U236

AP104 U238
$B B$

$\frac{\text { May98 }}{1.12 \mathrm{E}+01}$

351E-00

2.88E+02 2.88E+02

$\mathrm{kg} \quad 1.68 \mathrm{E}-00 \quad 1.68 \mathrm{E}-00$

$\mathrm{kg} \quad 1.11 \mathrm{E}+01$

$\mathrm{kg} \quad 4.90 \mathrm{E}-02$

$\mathrm{kg} \quad 0.00 \mathrm{E}+01$

kg 8.40E-01

$\mathrm{kg} \quad 0.00 \mathrm{E}+01$

kg $\quad 9.80 \mathrm{E}-03$

$\mathrm{kg} \quad 4.46 \mathrm{E}+02$

kg 3.21E-02

$\mathrm{kg} \quad 1.07 \mathrm{E}+02$

$2.75 \mathrm{E}+02$

kg 2.09E+01

kg $0.00 \mathrm{E}+01$

kg $\quad 6.46 \mathrm{E}+01$

kg $\quad 1.84 \mathrm{E}+01$

$\mathrm{kg} \quad 1.98 \mathrm{E}-00$

kg $\quad 5.51 \mathrm{E}+01$

kg $0.00 \mathrm{E}+01$

kg 1.16E+01

kg 3.27E-00

kg $0.00 \mathrm{E}+01$

Ci . $0.00 \mathrm{E}+01$

Ci 1.42E-02

0.00E+01

$3.59 \mathrm{E}+02$

1.57E-02

$0.00 \mathrm{E}+01$

$0.00 E+01$

6.16E-04

1.47E-02

4.03E-02

3.52E-01

Ci $3.72 \mathrm{E}+02$

C.00E+01

Ci 1.11E-01

Ci 8.29E-01

Ci 5.90E-04

Ci $0.00 \mathrm{E}+01$

$0.00 \mathrm{E}+01$

Ci $0.00 \mathrm{E}+01$

Ci 2.74E-02

Ci $0.00 E+01$

Ci 3.90E-03

Ci 3.50E-03

Ci Incl. in 239

Ci $0.00 \mathrm{E}+01$

Ci $0.00 \mathrm{E}+01$

Ci 4.81E-00

Ci 0.00E+01

Ci $1.39 \mathrm{E}+01$

Ci $0.00 \mathrm{E}+01$

$\mathrm{Ci}$ 3.32E-05

$0.00 \mathrm{E}+01$

8.07E-00

$7.12 \mathrm{E}-02$

$0.00 \mathrm{E}+01$

$0.00 \mathrm{E}+01$

$0.00 E+01$

$1.36 \mathrm{E}-00$

$0.00 \mathrm{E}+01$

$0.00 \mathrm{E}+01$

$0.00 \mathrm{E}+01$

Ci $0.00 \mathrm{E}+01$

Ci $0.00 E+01$

Ci $0.00 \mathrm{E}+01$
$\frac{B B}{\text { Sept98 }}$
$\frac{1.12 \mathrm{E}+01}{3.51 \mathrm{E}-00}$
$2.88 \mathrm{E}+02$
$1.68 \mathrm{E}-00$
$1.11 \mathrm{E}+01$

4.90E-02

$0.00 \mathrm{E}+01$

8.40E-01

$0.00 \mathrm{E}+01$

$9.80 \mathrm{E}-03$

$4.46 \mathrm{E}+02$

3.21E-02

$1.07 \mathrm{E}+02$

$2.75 E+02$

NA

$0.00 \mathrm{E}+01$

$6.46 \mathrm{E}+01$

NA

1.98E-00

$5.51 \mathrm{E}+01$

1.93E-04

$1.16 \mathrm{E}+01$

3.27E-00

$0.00 E+01$

1.28E-05

1.42E-02

1.10E-06

$3.59 \mathrm{E}+02$

1.57E-02

$7.38 \mathrm{E}-01$

4.27E-05

6.16E-04

$1.47 \mathrm{E}-02$

4.03E-02

3.52E-01

3.72E +02

1.44E-01

1.11E-01

8.29E-01

5.90E-04

1.53E-01

1.02E-02

1.01E-00

2.74E-02

3.89E-05

3.90E-03

2.87E-03

6.34E-04

1.59E-02

6.98E-08

1.09E-04

1.10E-02

$1.81 \mathrm{E}-04$

4.23E +01

3.32E-05

$1.53 \mathrm{E}+02$

8.07E-00

7.12E-02

5.15E-06

2.16E-05

6.64E-02

$1.36 \mathrm{E}-00$

$5.29 \mathrm{E}-04$

2.03E-03

$1.47 \mathrm{E}-03$

5.65E-05

1.01E-04 1.09E-03 1\%-ile

\section{$3.26 \mathrm{E}-06$}

$4.54 \mathrm{E}-07$

7.38E-05

2.33E-05

2.77E-06

$0.00 \mathrm{E}+01$

$0.00 \mathrm{E}+01$

$1.36 \mathrm{E}-07$

2.23E-09

9.67E-08

$1.81 \mathrm{E}-04$

$0.00 \mathrm{E}+01$

$1.53 \mathrm{E}-06$

3.75E-05

$3.86 \mathrm{E}-06$

$3.07 \mathrm{E}-09$

$5.06 \mathrm{E}-06$

$1.39 \mathrm{E}-05$

$0.00 \mathrm{E}+01$

$5.97 \mathrm{E}-05$

$0.00 \mathrm{E}+01$

$1.39 \mathrm{E}-06$

$0.00 \mathrm{E}+01$

$0.00 \mathrm{E}+01$

$0.00 \mathrm{E}+01$

$0.00 \mathrm{E}+01$

$0.00 \mathrm{E}+01$

$0.00 \mathrm{E}+01$

$0.00 \mathrm{E}+01$

$0.00 \mathrm{E}+01$

$0.00 \mathrm{E}+01$

$0.00 \mathrm{E}+01$

$0.00 \mathrm{E}+01$

$0.00 \mathrm{E}+01$

$0.00 \mathrm{E}+01$

$1.31 \mathrm{E}-05$

$0.00 \mathrm{E}+01$

$0.00 \mathrm{E}+01$

$0.00 \mathrm{E}+01$

$0.00 \mathrm{E}+01$

$0.00 \mathrm{E}+01$

$0.00 \mathrm{E} \div 01$

$0.00 \mathrm{E}+01$

$0.00 \mathrm{E}+01$

$0.00 \mathrm{E}+01$

$0.00 \mathrm{E}+01$

$0.00 E+01$

$0.00 \mathrm{E}+01$

$0.00 \mathrm{E}+01$

$0.00 \mathrm{E}+01$

$0.00 E+01$

$0.00 E+01$

$0.00 \mathrm{E}+01$

$0.00 E+01$

$0.00 E+01$

$0.00 E+01$

$0.00 E+0$

$0.00 E+01$

$0.00 \mathrm{E}+01$

$0.00 E+01$

$0.00 \mathrm{E}+0$ !

$0.00 \mathrm{E}+01$

$0.00 \mathrm{E}+01$

$0.00 \mathrm{E}+01$

$0.00 E+01$

$0.00 \mathrm{E}+01$

$0.00 \mathrm{E}+01$

$0.00 E+01$

10\%-ile

$2.45 \mathrm{E}+01$

$1.70 \mathrm{E}-00$

$2.41 \mathrm{E}+02$

$4.05 E+01$

7.36E-00

$1.17 \mathrm{E}+01$

$0.00 \mathrm{E}+01$

9.49E-02

2.79E-02

4.78E-02

$6.17 \mathrm{E}+02$

$1.02 \mathrm{E}+01$

$3.96 \mathrm{E}-00$

$1.31 \mathrm{E}+02$

$1.27 \mathrm{E}+01$

3.57E-02

$5.02 \mathrm{E}+01$

4. $63 \mathrm{E}+01$

$1.94 \mathrm{E}-02$

$8.28 \mathrm{E}+01$

4.47E-03

$7.68 \mathrm{E}-01$

7.17E-04 
Tank Anal. Un

$\begin{array}{ll}\text { AP104 } & \text { Y90 } \\ \text { AP104 } & \text { Zr93 } \\ \text { AP105 } & \text { Al } \\ \text { AP105 } & \text { Bi } \\ \text { AP105 } & \text { Ca } \\ \text { AP105 } & \text { Cl } \\ \text { AP105 } & \text { CO3 } \\ \text { AP105 } & \text { Cr } \\ \text { AP105 } & \text { F } \\ \text { AP105 } & \text { Fe } \\ \text { AP105 } & \text { Hg } \\ \text { AP105 } & \text { K } \\ \text { AP105 } & \text { La } \\ \text { AP105 } & \text { Mn } \\ \text { AP105 } & \text { Na } \\ \text { AP105 } & \text { Ni } \\ \text { AP105 } & \text { NO2 } \\ \text { AP105 } & \text { NO3 } \\ \text { AP105 } & \text { P } \\ \text { AP105 } & \text { Pb } \\ \text { AP105 } & \text { PO4 } \\ \text { AP105 } & \text { S } \\ \text { AP105 } & \text { Si } \\ \text { AP105 } & \text { SO4 } \\ \text { AP105 } & \text { Sr } \\ \text { AP105 }\end{array}$

AP105 TOC

AP105 U

AP105 $\mathrm{Zr}$

AP105 Ac227

AP105 Am241

AP105 Am243

AP105 Ba137

AP105 C14

AP105 Cd113

AP105 Cm242

AP105 Cm243

AP105 Cm244

AP105 Co60

AP105 Cs134

AP105 Cs137

AP105 Eu152

AP105 Eu154

AP105 Eu155

AP105 1129

AP105 Nb93

AP105 Ni59

AP105 Ni63

AP105 Np237

AP105 Pa231

AP105 Pu238

AP105 Pu239

AP105 Pu240

AP105 Pu241

AP105 Pu242

AP105 Ra226

AP105 Ra228

AP105 Ru106

AP105 Sb125

AP105 Se79

AP105 Sm151

AP105 Sr89/90

AP105 Tc99

AP105 Th229

AP105 Th232

AP105 Tin126

AP105 Tritium

AP105 U232

API05 U233
$B B$

May

Ci 8.07E-00

$\begin{array}{ll}\mathrm{Ci} & 0.00 \mathrm{E}+01 \\ \mathrm{~kg} & 4.99 \mathrm{E}+03\end{array}$

kg $7,30 \mathrm{E}+01$

kg $\quad 8.94 \mathrm{E}+02$

kg $9.48 \mathrm{E}+02$

kg 3.99E+03

kg $\quad 6.20 \mathrm{E}+02$

kg 4.70E+03

kg 9.03E-01

kg 1.14E-00

kg 1.77E+04

kg 7.97E-01

kg 2.61E+02

kg $2.37 \mathrm{E}+05$

kg 1.37E-00

kg $\quad 4.70 \mathrm{E}+03$

$\mathrm{kg} \quad 5.68 \mathrm{E}+05$

$\mathrm{kg} \quad 1.19 \mathrm{E}+02$

$\mathrm{kg} \quad 6.40 \mathrm{E}+01$

$3.70 \mathrm{E}+02$

kg 2.07E+02

kg 2.33E+01

$\mathrm{kg} \quad 6.21 \mathrm{E}+02$

$0.00 E+01$

g $\quad 4.63 \mathrm{E}+02$

$3.42 \mathrm{E}+01$

g.59E+01

3.22E-04

$1.06 \mathrm{E}-01$

2.28E-03

Ci $3.30 \mathrm{E}+04$

Ci $9.40 \mathrm{E}-02$

Ci $2.66 \mathrm{E}+01$

Ci 5.14E-102

4.00E-03

1.04E-01

2.13E-00

Ci 1.09E+01

Ci $3.47 E+04$

Ci 1.26E-00

Ci 7.10E-00

Ci $4.86 \mathrm{E}+01$

Ci 1.25E-01

Ci 4.11E-00

Ci 5.63E-01

Ci $5.52 \mathrm{E}+01$

Ci 4.72E-01

Ci 1.30E-03

Ci 6.61E-02

Ci 5.51E-02

Ci Incl. in 239

Ci 1.35E +02

Ci $\quad 5.67 E-03$

$\mathrm{Ci} 3.96 \mathrm{E}+02$

Ci 6.40E-02

Ci $2.44 \mathrm{E}+02$

Ci 1.04E+01

Ci $\quad 5.79 \mathrm{E}-02$

Ci 4.08E+03

Ci $\quad 6.02 E+01$

Ci $1.77 \mathrm{E}+01$

Ci 1.49E-03

Ci 1.06E-02

Ci 6.42E+01

Ci 2.84E-00

Ci 2.25E-01

Ci $\quad 8.63 \mathrm{E}-01$
$B B$ Sept98

8.07E-00

1.94E-01

$7.13 \mathrm{E}+04$

$0.00 \mathrm{E}+01$

$2.57 \mathrm{E}+02$

$1.85 \mathrm{E}+04$

$8.38 \mathrm{E}+04$

$9.76 \mathrm{E}+02$

$1.91 \mathrm{E}+04$

9.70E+01

$0.00 \mathrm{E}+01$

3.70E+04

$0.00 \mathrm{E}+01$

$2.00 \mathrm{E}+01$

$5.83 \mathrm{E}+05$

$4.80 \mathrm{E}+01$

$3.46 \mathrm{E}+05$

$7.98 \mathrm{E}+05$

NA

$1.61 \mathrm{E}+02$

$1.13 E+04$

NA

$3.94 \mathrm{E}+02$

2.25E+04

$0.00 \mathrm{E}+01$

8.13E+03

$2.89 \mathrm{E}+02$

$1.80 \mathrm{E}+01$

2.94E-03

$6.20 \mathrm{E}-00$

5.91E-04

$5.90 \mathrm{E}+05$

2.20E-00

$2.83 E+02$

9.95E-03

7.16E-02

$1.72 \mathrm{E}-00$

$5.24 \mathrm{E}+01$

$1.41 \mathrm{E}+02$

$6.21 \mathrm{E}+05$

$1.39 \mathrm{E}+01$

$1.92 \mathrm{E}+02$

$8.69 \mathrm{E}+02$

$1.45 \mathrm{E}-00$

$3.99 \mathrm{E}+01$

5.60E-00

$5.51 \mathrm{E}+02$

2.66E-00

1.29E-02

1.09E-01

$1.42 \mathrm{E}-00$

3.44E-01

$1.03 E+01$

4.25E-05

4.73E-04

1.03E-00

3.51E-02

$7.65 E+02$

$1.13 \mathrm{E} \div 01$

$3.98 \mathrm{E}+04$

$1.36 \mathrm{E}+04$

$3.48 \mathrm{E}+02$

2.01E-02

9.97E-02

$1.71 \mathrm{E}+01$

$1.91 \mathrm{E}+01$

3.79E-02

1.45E-01 1\%-ile

$0.00 \mathrm{E}+01$

$0.00 \mathrm{E}+01$

$3.69 \mathrm{E}+03$

$0.00 \mathrm{E}+01$

$2.96 \mathrm{E}-00$

$7.59 \mathrm{E}+02$

$1.47 \mathrm{E}+04$

$1.77 \mathrm{E}+01$

$6.04 \mathrm{E}+03$

$0.00 \mathrm{E}+01$

$0.00 \mathrm{E}+01$

$6.20 \mathrm{E}+03$

$1.80 \mathrm{E}+02$

$0.00 \mathrm{E}+01$

$1.13 \mathrm{E}+05$

$1.65 \mathrm{E}-01$

$1.67 \mathrm{E}+04$

$6.41 \mathrm{E}+04$

$0.00 \mathrm{E}+01$

$1.13 \mathrm{E}+01$

$9.61 \mathrm{E}+01$

$1.22 \mathrm{E}+03$

$5.24 \mathrm{E}+01$

$1.91 \mathrm{E}+03$

$3.14 \mathrm{E}-01$

$1.89 \mathrm{E}+03$

8.23E-00

$0.00 \mathrm{E}+01$

$0.00 \mathrm{E}+01$

$0.00 \mathrm{E}+01$

$0.00 \mathrm{E}+01$

$0.00 \mathrm{E}+01$

$0.00 \mathrm{E}+01$

$0.00 \mathrm{E}+01$

$0.00 \mathrm{E}+01$

$0.00 \mathrm{E} \div 01$

$0.00 \mathrm{E}+01$

9.47E-01

$0.00 \mathrm{E}+01$

$4.34 \mathrm{E}+04$

$0.00 \mathrm{E}+01$

$0.00 E+01$

$0.00 \mathrm{E}+01$

$0.00 \mathrm{E}+01$

$0.00 E+01$

$0.00 E+01$

$0.00 \mathrm{E}+01$

$0.00 \mathrm{E}+01$

$0.00 \mathrm{E}+01$

$0.00 \mathrm{E}+01$

$0.00 E+01$

$0.00 E+01$

$0.00 \mathrm{E}+01$

$0.00 E+01$

$5.34 \mathrm{E}-00$

8.63E-04

$0.00 \mathrm{E}+01$

$0.00 \mathrm{E}+01$

$0.00 E \div 01$

$0.00 \mathrm{E}+01$

9.94E-00

$0.00 \mathrm{E}+01$

$0.00 \mathrm{E}+01$

$0.00 \mathrm{E}+01$

$0.00 \mathrm{E}+01$

2.02E-00

$0.00 \mathrm{E}+01$

$0.00 \mathrm{E}+01$

$10 \%$-ile

1.67E-04

$0.00 \mathrm{E}+01$

$5.65 \mathrm{E}+03$

$5.22 \mathrm{E}-00$

8.56E-00

$1.05 \mathrm{E}+03$

$2.47 \mathrm{E}+04$

$7.49 \mathrm{E}+01$

8.26E +03

$1.92 \mathrm{E}-00$

$0.00 \mathrm{E}+01$

$7.42 \mathrm{E}+03$

$2.08 \mathrm{E}+02$

$3.95 \mathrm{E}-00$

$1.26 \mathrm{E}+05$

$1.41 \mathrm{E}-00$

$2.26 \mathrm{E}+04$

$8.24 \mathrm{E}+04$

$1.88 \mathrm{E}+02$

$2.56 \mathrm{E}+01$

$2.15 \mathrm{E}+02$

$2.18 \mathrm{E}+03$

$7.95 \mathrm{E}+01$

$4.92 \mathrm{E}+03$

4.84E- 01

$2.55 \mathrm{E}+03$

$8.44 \mathrm{E}+01$

$2.99 \mathrm{E}-01$

$0.00 \mathrm{E}+01$

$0.00 \mathrm{E}+01$

4.84E-05

$1.10 \mathrm{E}+04$

$1.20 \mathrm{E}-02$

$0.00 \mathrm{E}+01$

$1.09 \mathrm{E}-03$

8.49E-05 
Tank Anal. Un

$\begin{array}{ll}\text { AP105 } & \text { U234 } \\ \text { AP105 } & \text { U235 } \\ \text { AP105 } & \text { U236 } \\ \text { AP105 } & \text { U238 } \\ \text { AP105 } & \text { Y90 } \\ \text { AP105 } & \mathrm{Zr} 3 \\ \text { AP106 } & \text { Al } \\ \text { AP106 } & \text { Bi } \\ \text { AP106 } & \text { Ca } \\ \text { AP106 } & \text { Cl } \\ \text { AP106 } & \text { CO3 } \\ \text { AP106 } & \text { Cr } \\ \text { AP106 } & \text { F } \\ \text { AP106 } & \text { Fe } \\ \text { AP106 } & \text { Hg } \\ \text { AP106 } & \text { K } \\ \text { AP106 } & \text { La } \\ \text { AP106 } & \text { Mn } \\ \text { AP106 } & \text { Na } \\ \text { AP106 } & \text { Ni } \\ \text { AP106 NO2 } & \text { NO2 } \\ \text { AP106 } & \text { PO3 } \\ \text { AP106 } & \text { P } \\ \text { AP106 } & \text { Pb } \\ \text { AP106 } & \text { PO4 }\end{array}$

AP106 PO4

AP106 S

AP106 Si

AP106 SO4

AP106 Sr

AP106 TOC

AP106 U

AP106 $\mathrm{Zr}$

AP106 Ac227

AP106 Am241

AP106 Am243

AP106 Ba137

A.P106 C14

AP106 Cd113

AP106 Cm242

AP106 $\mathrm{Cm} 243$

AP106 Cm244

AP106 Co60

AP106 Cs134

AP106 Cs137

AP106 Eu152

AP106 Eul54

AP106 Eul55

AP106 1129

AP106 Nb93

AP106 Ni59

AP106 Ni63

AP106 Np237

AP106 Pa231

AP106 Pu238

AP106 Pu239

AP106 Pu240

AP106 Pu241

AP106 Pu242

AP106 Ra226

AP106 Ra228

AP106 Ru106

AP106 Sb125

AP106 Se79

AP106 Sm15!

AP106 Sr89/90

AP106 Tc99

AP106 Th229

AP106 Th232
$B B$

$\frac{\text { May } 98}{6.46 \mathrm{E}-01}$

Ci 2.51E-02

Ci 7.59E-02

Ci 5.51E-01

Ci $6.02 E+01$

5.52E-00

$2.61 E+03$

$\mathrm{kg} \quad$ 9.44E-00

kg 9.44E-00

kg 6.34E+02

kg $7.28 \mathrm{E}+03$

kg 5.23E+01

$\mathrm{kg} \quad 8.24 \mathrm{E}+02$

kg 4.73E-00

kg 6.70E-01

$7.01 \mathrm{E}+03$

$\mathrm{kg} \quad 4.73 \mathrm{E}-00$

kg 9.44E-01

kg 3.59E+04

2.12E-00

kg 1.04E+04

$4.18 E+04$

$\mathrm{kg} \quad 9.09 \mathrm{E}+02$

kg $\quad 9.44 \mathrm{E}-00$

kg 2.82E+03

kg 1.35E+03

kg 2.15E+01

kg $\quad 4.05 \mathrm{E}+03$

kg 9.44E-01

kg $\quad 1.34 \mathrm{E}+03$

g.71E+01

kg 9.44E-01

Ci 2.31E-04

Ci 2.26E-01

Ci 2.10E-03

Ci 4.57E+04

4.29E-00

Ci $1.62 \mathrm{E}+01$

Ci $\quad 4.77 \mathrm{E}-02$

Ci 5.59E-03

Ci $\quad 7.02 E-02$

$\mathrm{Ci} \quad 6.84 \mathrm{E}-00$

Ci $\quad 5.30 \mathrm{E}+01$

$\mathrm{Ci} \quad 4.35 \mathrm{E}+04$

$\mathrm{Ci}$ 9.55E-01

Ci 4.80E-02

Ci $\quad 7.17 \mathrm{E}+01$

Ci 5.90E-02

Ci 2.84E-00

Ci 3.00E-01

Ci 2.93E+01

Ci 1.21E-01

Ci 8.12E-04

Ci 1.06E-00

Ci 3.65E-02

Ci Incl. in 239

Ci $9.70 \mathrm{E}+01$

Ci 4.16E-04

Ci 3.85E-05

Ci 3.20E-02

Ci 3.90E-03

Ci 8.98E+01

Ci 7.90E-01

Ci $2.84 \mathrm{E}+03$

Ci $3.28 \mathrm{E}+01$

Ci $3.04 \mathrm{E}+01$

Ci $\quad 7.46 \mathrm{E}-04$

Ci 3.10E-03
$B B$

Sept98

1.33E-01

5.09E-03

$9.69 \mathrm{E}-03$

9.63E-02

$1.36 \mathrm{E}+04$

$5.46 \mathrm{E}+01$

$3.98 \mathrm{E}+03$

$0.00 \mathrm{E}+01$

$1.39 \mathrm{E}+01$

$8.70 \mathrm{E}+02$

1.01E+04

$7.55 \mathrm{E}+01$

$1.20 \mathrm{E}+03$

$7.00 \mathrm{E}-00$

$0.00 \mathrm{E}+01$

$1.05 \mathrm{E}+04$

$0.00 \mathrm{E}+01$

$1.40 \mathrm{E}-00$

$5.25 E+04$

3.20E-00

$1.51 \mathrm{E}+04$

$6.16 \mathrm{E}+04$

NA

$1.39 \mathrm{E}+01$

$1.01 \mathrm{E}+03$

NA

2.57E+01

$1.90 \mathrm{E}+03$

$1.40 \mathrm{E}-00$

$1.74 \mathrm{E}+03$

$6.94 \mathrm{E}+01$

1.40E-00

2.31E-04

3.70E-01

3.26E-05

$6.23 \mathrm{E}+04$

4.29E-00

1.62E+01

$7.39 \mathrm{E}-04$

8.66E-05

$1.09 \mathrm{E}-03$

$1.09 \mathrm{E}+01$

$5.30 \mathrm{E}+01$

$6.58 \mathrm{E}+04$

9.55E-01

4.80E-02

$7.17 \mathrm{E}+01$

5.90E-02

2.84E-00

3.00E-01

2.93E+01

$1.21 \mathrm{E}-01$

8.12E-04

3.25E-02

4.40E-02

1.05E-02

2.99E-01

$1.28 \mathrm{E}-05$

3.85E-05

$3.20 \mathrm{E}-02$

$3.90 \mathrm{E}-03$

$8.98 \mathrm{E}+01$

$7.90 \mathrm{E}-01$

$2.84 \mathrm{E}+03$

$2.17 \mathrm{E}+02$

$3.04 \mathrm{E}+01$

7.46E-04

3.10E-03
$1 \%$-ile

$0.00 \mathrm{E}+01$

$0.00 \mathrm{E}+01$

$0.00 \mathrm{E}+01$

$0.00 \mathrm{E}+01$

4.72E-01

$0.00 \mathrm{E}+01$

$1.30 \mathrm{E}-01$

$0.00 \mathrm{E}+01$

$0.00 \mathrm{E}+01$

$0.00 \mathrm{E}+01$

$0.00 \mathrm{E}+01$

3.38E-02

$4.90 \mathrm{E}-01$

$0.00 E+01$

$0.00 E+01$

$2.64 \mathrm{E}+02$

$0.00 \mathrm{E}+01$

$0.00 \mathrm{E}+01$

$9.79 \mathrm{E}-01$

$0.00 \mathrm{E}+01$

$0.00 \mathrm{E}+01$

$0.00 \mathrm{E}+01$

$4.77 \mathrm{E}+04$

$0.00 \mathrm{E}+01$

$0.00 \mathrm{E}+01$

$1.12 \mathrm{E}+02$

$0.00 \mathrm{E}+01$

$0.00 \mathrm{E}+01$

$0.00 \mathrm{E}+01$

8.64E-02

$0.00 \mathrm{E}+01$

$2.06 \mathrm{E}-02$

$0.00 \mathrm{E}+01$

$0.00 \mathrm{E}+01$

$0.00 \mathrm{E}+01$

$0.00 \mathrm{E}+01$

$0.00 \mathrm{E}+01$

$0.00 \mathrm{E}+01$

$0.00 \mathrm{E}+01$

$0.00 \mathrm{E}+01$

$0.00 \mathrm{E}+01$

$0.00 \mathrm{E} \div 01$

$0.00 \mathrm{E}+01$

$0.00 \mathrm{E}+01$

$0.00 \mathrm{E}+01$

$0.00 \mathrm{E}+01$

$0.00 E+01$

$0.00 \mathrm{E}+01$

$0.00 \mathrm{E}+01$

$0.00 \mathrm{E}+01$

$0.00 \mathrm{E}+01$

$0.00 \mathrm{E}+01$

$0.00 \mathrm{E}+01$

$0.00 \mathrm{E}+01$

$0.00 \mathrm{E}+01$

$0.00 \mathrm{E}+01$

$0.00 \mathrm{E} \div 01$

$0.00 \mathrm{E}+01$

$0.00 \mathrm{E} \div 01$

$0.00 \mathrm{E}+01$

$0.00 \mathrm{E}+01$

$0.00 \mathrm{E}+01$

$0.00 \mathrm{E}+01$

$0.00 \mathrm{E}+01$

$0.00 E+01$

$0.00 \mathrm{E}+01$

$0.00 \mathrm{E}+01$

$0.00 \mathrm{E}+01$

10\%-ile

$0.00 \mathrm{E}+01$

$0.00 \mathrm{E}+01$

$0.00 \mathrm{E}+01$

$0.00 \mathrm{E}+01$

$1.71 \mathrm{E}-00$

$0.00 \mathrm{E}+01$

7.03E-01

$0.00 \mathrm{E}+01$

$8.26 \mathrm{E}-01$

2.68E-02

$3.47 \mathrm{E}+02$

8.39E-02

1.01E-00

$0.00 \mathrm{E}+01$

$0.00 E+01$

$1.18 \mathrm{E}+03$

7.07E-0I

$0.00 \mathrm{E}+01$

$5.03 \mathrm{E}+03$

$0.00 E+01$

6.47E-01

$2.08 \mathrm{E}+03$

$5.14 \mathrm{E}+04$

4.21E-04

$1.39 \mathrm{E}+02$

$1.98 \mathrm{E}+02$

$1.64 \mathrm{E}-03$

2.00E-01

1.42E-01

$3.32 \mathrm{E}+02$

$0.00 \mathrm{E}+01$

$9.16 \mathrm{E}-02$

$0.00 \mathrm{E}+01$

$0.00 \mathrm{E}+01$

$0.00 \mathrm{E}+01$ 
Tank Anal. Un

AP106 Tin126 AP106 Tritium AP106 U232 AP106 U233

AP106 U234

AP106 U235

AP106 U236

AP106 U238

AP106 Y90

AP106 Zr93

AP107 Al

AP107 Bi

AP107 Ca

AP107 CI

AP107 CO3

AP107 $\mathrm{Cr}$

AP107 F

AP107 Fe

AP107 Hg

AP107 K

AP107 La

AP107 Mn

AP107 Na

AP107 Ni

AP107 NO2

AP107 NO3

AP107 P

AP107 Pb

AP107 PO4

AP107 S

AP107 Si

AP107 S04

AP107 Sr

AP107 TOC

AP107 U

AP107 $\mathrm{Zr}$

AP107 Ac227

AP107 Am241

AP107 Am243

AP107 Ba137

AP107 C14

AP107 Cdl13

AP107 Cm242

AP107 $\mathrm{Cm} 243$

AP107 Cm244

AP107 C060

AP107 Cs134

AP107 Cs137

AP107 Eu152

AP107 Eu154

AP107 Eu155

AP107 1129

AP107 Nb93

AP107 Ni59

AP107 Ni63

AP107 Np237

AP107 Pa231

AP107 Pu238

AP107 Pu239

AP107 Pu240

AP107 Pu241

AP107 Pu242

AP107 Ra226

AP107 Ra228

AP107 Ru106

AP107 Sb125

AP107 Se79

AP107 Sm151
$B B$ May 98

$1.23 \mathrm{E}-00$

5.72E+01

1.13E-01

4.31E-01

$3.98 \mathrm{E}-01$

$1.53 \mathrm{E}-02$

2.90E-02

i 3.21E-01

Ci 3.28E+01

Ci 3.72E-00

g $2.89 \mathrm{E}+01$

g $\quad 0.00 \mathrm{E}+01$

g. 3.01E+01

kg 2.73E-00

kg $1.78 E+02$

$\mathrm{kg} \quad 0.00 \mathrm{E}+01$

kg $3.68 \mathrm{E}+02$

$\mathrm{kg} \quad 9.33 \mathrm{E}-00$

kg $0.00 E+01$

kg 6.50E-01

kg $0.00 \mathrm{E}+01$

kg $\quad 0.00 \mathrm{E}+01$

kg 1.23E+03

kg $0.00 \mathrm{E}+01$

2.63E +02

g $5.51 \mathrm{E}+02$

$0.00 \mathrm{E}+01$

k $0.00 \mathrm{E}+01$

$0.00 \mathrm{E}+01$

kg $0.00 \mathrm{E}+01$

$0.00 \mathrm{E}+01$

g $0.00 \mathrm{E}+01$

$0.00 \mathrm{E}+01$

$2.60 \mathrm{E}+01$

$6.30 \mathrm{E}-01$

$0.00 E+01$

$0.00 \mathrm{E}+01$

$0.00 \mathrm{E}+01$

$0.00 E+01$

$1.48 E+01$

$1.92 \mathrm{E}-03$

$0.00 E+01$

$0.00 \mathrm{E}+01$

$0.00 \mathrm{E}+01$

$0.00 \mathrm{E}+01$

$0.00 E+01$

$0.00 \mathrm{E}+01$

$1.56 \mathrm{E}+01$

$0.00 \mathrm{E}+01$

$0.00 E+01$

$0.00 \mathrm{E}+01$

$0.00 \mathrm{E}+01$

$0.00 \mathrm{E}+01$

$0.00 \mathrm{E}+01$

$0.00 \mathrm{E}+01$

$2.84 \mathrm{E}-03$

$0.00 \mathrm{E}+01$

$0.00 \mathrm{E}+01$

9.37E-03

Ci Incl. in 239

Ci $0.00 \mathrm{E}+01$

Ci $0.00 \mathrm{E}+01$

$0.00 \mathrm{E}+01$

Ci $0.00 E+01$

$0.00 E+01$

$0.00 \mathrm{E}+01$

$0.00 \mathrm{E}+01$

$0.00 E+01$
$\frac{B B}{408}$ Sept98 $1.23 \mathrm{E}-00$ $5.72 \mathrm{E}+01$ 9.01E-03 $3.45 E-02$

3.19E-02

1.22E-03

2.33E-03

2.31E-02

2.17E+02

3.72E-00

$2.89 \mathrm{E}+01$

$0.00 \mathrm{E}+01$

$3.01 \mathrm{E}+01$

2.73E-00

$1.78 \mathrm{E}+02$

$0.00 \mathrm{E}+01$

$3.68 \mathrm{E}+02$

9.33E-00

$0.00 \mathrm{E}+01$

$6.50 \mathrm{E}-01$

$0.00 \mathrm{E}+01$

$0.00 \mathrm{E}+01$

$1.23 \mathrm{E}+03$

$0.00 \mathrm{E}+01$

$2.63 \mathrm{E}+02$

$5.51 \mathrm{E}+02$

NA

$0.00 \mathrm{E}+01$

$0.00 \mathrm{E}+01$

NA

$0.00 \mathrm{E}+01$

$0.00 \mathrm{E}+01$

$0.00 \mathrm{E}+01$

$2.60 \mathrm{E}+01$

$6.30 \mathrm{E}-01$

$0.00 \mathrm{E}+01$

$0.00 \mathrm{E}+01$

$0.00 \mathrm{E}+01$

$0.00 \mathrm{E}+01$

$1.48 \mathrm{E}+01$

1.92E-03

$0.00 \mathrm{E}+01$

$0.00 E+01$

$0.00 \mathrm{E}+01$

$0.00 \mathrm{E}+01$

$0.00 \mathrm{E}+01$

$0.00 \mathrm{E}+01$

$1.56 \mathrm{E}+01$

$0.00 E+01$

$0.00 \mathrm{E}+01$

$0.00 \mathrm{E}+01$

$0.00 \mathrm{E}+01$

$0.00 \mathrm{E}+01$

$0.00 \mathrm{E}+01$

$0.00 \mathrm{E}+01$

2.84E-03

$0.00 \mathrm{E}+01$

4.82E-04

7.75E-03

1.72E-03

4.29E-02

$1.89 \mathrm{E}-07$

$0.00 \mathrm{E}+01$

$0.00 \mathrm{E}+01$

$0.00 E+01$

$0.00 \mathrm{E}+01$

$0.00 \mathrm{E}+01$

$0.00 \mathrm{E}+01$
$1 \%$ ile

$10 \%$-ile

$0.00 \mathrm{E}+01$
$4.66 \mathrm{E}+01$

$0.00 \mathrm{E}+01$

$0.00 \mathrm{E}+01$

$0.00 \mathrm{E}+01$

$0.00 \mathrm{E}+01$

$0.00 \mathrm{E}+01$

$0.00 \mathrm{E}+01$

$0.00 \mathrm{E}+01$

$0.00 \mathrm{E}+01$

$1.98 \mathrm{E}-06$

$1.61 \mathrm{E}-10$

7.06E-07

1.32E-06

2.78E-05

6.52E-09

9.89E-06

$0.00 \mathrm{E}+01$

$0.00 \mathrm{E}+01$

2.64E-05

3.82E-09

5.95E- 10

3.51E-05

6.48E-11

2.35E-05

6.12E-06

9.70E-07

$0.00 \mathrm{E}+01$

1.06E-07

4.87E-07

3.91E-08

$1.59 \mathrm{E}-06$

$0.00 \mathrm{E}+01$

4.56E-07

$0.00 \mathrm{E}+01$

$0.00 \mathrm{E}+01$

$0.00 \mathrm{E}+01$

$0.00 \mathrm{E}+01$

$0.00 \mathrm{E}+01$

$0.00 \mathrm{E}+01$

$0.00 \mathrm{E}+01$

$0.00 \mathrm{E}+01$

$0.00 \mathrm{E}+01$

$0.00 \mathrm{E}+01$

$0.00 \mathrm{E}+01$

$0.00 E+01$

$0.00 \mathrm{E}+01$

$1.87 \mathrm{E}-05$

$0.00 \mathrm{E}+01$

$0.00 \mathrm{E}+01$

$0.00 \mathrm{E}+01$

$0.00 \mathrm{E}+01$

$0.00 \mathrm{E}+01$

$0.00 \mathrm{E}+01$

$0.00 \mathrm{E}+01$

$0.00 \mathrm{E}+01$

$0.00 \mathrm{E}+01$

$0.00 \mathrm{E}+01$

$0.00 \mathrm{E}+01$

$0.00 \mathrm{E}+01$

$0.00 \mathrm{E}+01$

$0.00 \mathrm{E}+01$

$0.00 \mathrm{E}+01$

$0.00 \mathrm{E}+01$

$0.00 \mathrm{E}+01$

$0.00 \mathrm{E}+01$

$0.00 \mathrm{E}+01$

$0.00 \mathrm{E}+01$

$0.00 \mathrm{E}+01$

$4.95 \mathrm{E}+01$

$0.00 \mathrm{E}+01$

$0.00 \mathrm{E}+01$

$0.00 \mathrm{E}+01$

$0.00 \mathrm{E}+01$

$0.00 \mathrm{E}+01$

$0.00 \mathrm{E}+01$

$5.04 \mathrm{E}-00$

3.78E-02 
Tank Anal. Un

$\begin{array}{ll}\text { AP107 } & \text { Sr89/90 } \\ \text { AP107 } & \text { Tc99 } \\ \text { AP107 } & \text { Th229 } \\ \text { AP107 } & \text { Th232 } \\ \text { AP107 } & \text { Tin126 } \\ \text { AP107 } & \text { Tritium } \\ \text { AP107 } & \text { U232 } \\ \text { AP107 } & \text { U233 } \\ \text { AP107 } & \text { U234 } \\ \text { AP107 } & \text { U235 } \\ \text { AP107 } & \text { U236 } \\ \text { AP107 } & \text { U238 } \\ \text { AP107 } & \text { Y90 } \\ \text { AP107 } & \text { Zr93 } \\ \text { AP108 } & \text { Al }\end{array}$

AP108 Al

AP108 Bi

AP108 Ca

AP108 Cl

AP108 $\mathrm{CO} 3$

AP108 Cr

AP108 F

AP108 Fe

AP108 Hg

AP108 K

AP108 La

AP108 Mn

AP108 Na

AP108 Ni

AP108 NO2

AP108 NO3

AP108 P

AP108 Pb

AP108 PO4

AP108 S

AP108 Si

AP108 SO4

AP108 Sr

AP108 TOC

AP108 U

AP108 Zr

APi08 Ac227

AP108 Am241

AP108 Am 243

AP108 Ba137

AP108 C14

AP108 Cd113

AP108 Cm242

AP108 Cm243

AP108 Cm244

AP108 Co60

AP108 Cs134

AP108 Cs 137

AP108 Eu152

AP108 Eu154

AP108 Eu155

AP108 1129

AP108 Nb93

AP108 Ni59

AP108 Ni63

AP108 Np237

AP108 Pa231

AP108 Pu238

AP108 Pu239

AP108 Pu240

AP108 Pu241

AP108 Pu242

AP108 Ra226

AP108 Ra228
$\frac{B B}{\text { May98 }}$

2.49E-01

$0.00 \mathrm{E}+01$

$0.00 \mathrm{E}+01$

$0.00 \mathrm{E}+01$

3.09E-00

$0.00 \mathrm{E}+01$

$0.00 \mathrm{E}+01$

$0.00 \mathrm{E}+01$

$0.00 \mathrm{E}+01$

$0.00 \mathrm{E}+01$

$0.00 \mathrm{E}+01$

$1.63 \mathrm{E}+01$

$0.00 \mathrm{E}+01$

$2.55 \mathrm{E}+03$

$9.90 \mathrm{E}+01$

7.03E-00

$7.47 \mathrm{E}+02$

$1.74 \mathrm{E}+04$

$7.50 \mathrm{E}+01$

$3.93 \mathrm{E}+03$

$1.34 \mathrm{E}-00$

4.00E-03

$8.52 \mathrm{E}+03$

1.60E-00

1.90E-01

$5.90 \mathrm{E}+04$

$4.90 \mathrm{E}+02$

$1.39 \mathrm{E}+04$

$5.20 \mathrm{E}+04$

$1.54 \mathrm{E}+03$

5.30E-00

$4.78 \mathrm{E}+03$

$1.40 \mathrm{E}+03$

$1.50 \mathrm{E}+03$

$4.21 \mathrm{E}+03$

$0.00 \mathrm{E}+01$

$3.36 \mathrm{E}+03$

$1.42 \mathrm{E}+01$

$2.18 E+02$

6.15E-04

9.80E-01

6.00E-03

$5.78 \mathrm{E}+04$

$9.80 \mathrm{E}-02$

$4.56 \mathrm{E}+01$

1.10E-01

5.00E-02

1.30E-00

$1.47 \mathrm{E}+01$

i $2.08 E+02$

Ci $6.11 \mathrm{E}+04$

Ci 2.50E-00

Ci $4.40 \mathrm{E}+01$

Ci 1.09E +02

Ci 2.30E-02

$\mathrm{Ci} \quad$ 7.31E-00

Ci 1.14E-00

Ci 1.12E +02

Ci 3.57E-01

$\mathrm{Ci}$ 2.20E-03

Ci 7.30E-01

Ci 3.90E-01

Ci Incl. in 239

Ci $\quad 3.32 \mathrm{E}+02$

Ci 1.40E-03

Ci 1.03E-04

Ci 1.10E-01

\section{$\frac{B B}{\operatorname{Bap} 98}$ \\ $\frac{163 \mathrm{E}+01}{1.01}$}

$2.49 \mathrm{E}-01$

$0.00 \mathrm{E}+01$

$0.00 \mathrm{E}+01$

$0.00 \mathrm{E}+01$

$3.09 \mathrm{E}-00$

1.02E-04

3.91E-04

2.82E-04

1.09E-05

$1.94 \mathrm{E}-05$

2.10E-04

$1.63 \mathrm{E}+01$

$0.00 \mathrm{E}+01$

2.55E+03

$0.00 \mathrm{E}+01$

7.03E-00

$7.47 \mathrm{E}+02$

$1.74 \mathrm{E}+04$

$7.50 \mathrm{E}+01$

$3.93 \mathrm{E}+03$

$1.34 \mathrm{E}-00$

$0.00 \mathrm{E}+01$

$8.52 \mathrm{E}+03$

$0.00 \mathrm{E}+01$

$0.00 \mathrm{E}+01$

$5.90 \mathrm{E}+04$

$4.90 \mathrm{E}+02$

1.39E+04

$5.20 \mathrm{E}+04$

NA

5.30E-00

$1.56 \mathrm{E}+03$

NA

$1.50 \mathrm{E}+03$

$4.21 \mathrm{E}+03$

$7.77 \mathrm{E}-04$

3.36E+03

$1.42 \mathrm{E}+01$

2.18E +02

6.15E-04

9.80E-01

$7.74 \mathrm{E}-05$

$5.78 \mathrm{E}+04$

$9.80 \mathrm{E}-02$

$4.56 \mathrm{E}+01$

$6.46 \mathrm{E}-03$

$5.00 \mathrm{E}-02$

1.30E-00

$1.47 \mathrm{E}+01$

2.08E+02

$6.11 \mathrm{E}+04$

2.50E-00

$4.40 \mathrm{E}+01$

$1.09 \mathrm{E}+02$

2.30E-02

7.31E-00

1.14E-00

1.12E+02

3.57E-01

2.20E-03

7.30E-01

3.16E-01

7.44E- 02

1.83E-00

9.49E-06

1.03E-04

1.10E-01 1\%-ile

$0.00 \mathrm{E}+01$

$0.00 \mathrm{E}+01$

$0.00 \mathrm{E}+01$

$0.00 \mathrm{E}+01$

$0.00 \mathrm{E}+01$

$0.00 \mathrm{E}+01$

$0.00 \mathrm{E}+01$

$0.00 \mathrm{E}+01$

$0.00 \mathrm{E}+01$

$0.00 \mathrm{E}+01$

$0.00 \mathrm{E}+01$

$0.00 \mathrm{E}+01$

$0.00 \mathrm{E}+01$

$0.00 \mathrm{E}+01$

$1.13 \mathrm{E}-01$

3.86E-04

$1.64 \mathrm{E}-00$

$1.50 \mathrm{E}-02$

$2.38 \mathrm{E}+03$

4.07E-03

$1.06 \mathrm{E}+03$

$4.65 \mathrm{E}-01$

$0.00 \mathrm{E}+01$

$3.30 \mathrm{E}+03$

$1.25 \mathrm{E}-03$

$3.84 \mathrm{E}-03$

$5.27 \mathrm{E}+03$

1.15E-02

2.67E-02

$1.74 \mathrm{E}-00$

3.18E-02

3.83E-04

6.35E-01

1.15E+03

$9.56 \mathrm{E}-05$

$3.32 \mathrm{E}+02$

9.60E-04

7.01E-02

2.29E-03

3.68E-05

$0.00 \mathrm{E}+01$

$0.00 \mathrm{E}+01$

$0.00 \mathrm{E}+01$

$0.00 \mathrm{E}+01$

$0.00 \mathrm{E}+01$

$0.00 E+01$

$0.00 \mathrm{E}+01$

$0.00 \mathrm{E}+01$

$0.00 \mathrm{E}+01$

$2.24 \mathrm{E}-06$

$0.00 \mathrm{E}+01$

3.34E-03

$0.00 \mathrm{E}+01$

$0.00 \mathrm{E}+01$

$0.00 \mathrm{E}+01$

$0.00 \mathrm{E}+01$

$0.00 \mathrm{E}+01$

$0.00 \mathrm{E}+01$

$0.00 \mathrm{E}+01$

$0.00 \mathrm{E}+01$

$0.00 \mathrm{E}+01$

$0.00 \mathrm{E}+01$

$0.00 \mathrm{E}+01$

$0.00 \mathrm{E}+01$

$0.00 \mathrm{E}+01$

$0.00 \mathrm{E}+01$

$0.00 \mathrm{E}+01$

$0.00 \mathrm{E}+01$

$10 \%$-ile

$7.93 \mathrm{E}-03$

50\%-ile

90\%-ile

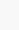

\section{9\%-ile}

Mean

Std Dev

8.66E-00

$0.00 \mathrm{E}+01 \quad 0.00 \mathrm{E}+01$

$0.00 \mathrm{E}+01$

$0.00 \mathrm{E}+01$

$0.00 \mathrm{E}+01$

$2.45 \mathrm{E}-01$

$0.00 \mathrm{E}+01$

3.09E-00

$0.00 \mathrm{E}+01$

$0.00 \mathrm{E}+01$

$0.00 \mathrm{E}+01$

$0.00 \mathrm{E}+01$

$0.00 \mathrm{E}+01$

$0.00 \mathrm{E}+01$

$1.49 \mathrm{E}-02$

$0.00 \mathrm{E}+01$

2.29E +01

9.15E-03 
Tank Anal. Un

AP108 Ru106

AP108 Se79 $\mathrm{Ci}$ 7.40E-02

AP108 Sm151 Ci 7.30E +03

AP108 $5 r 89 / 90 \quad \mathrm{Ci}$

AP108 Te99 Ci

AP108 Th229

AP108 Th232

AP108 Tin126 Ci

AP108 U232

AP108 U233

AP108 U234

AP108 U235

AP108 U236

AP108 U238

AP108 Y90

AP108 Zr93

AW101 Al

AW101 Bi

AW101 Ca

AW101 Cl

AW101 $\mathrm{CO} 3$

AW101 Cr

AW101 F

AW101 Fe

AW101 Hg

AW101 K

AW101 La

AW101 Mn

AW101 Na

AW101 Ni

AW101 NO2

AW101 NO3

AW101 P

AW101 Pb

AW101 PO4

AW101 S

AW101 Si

AW101 SO4

AW101 Sr

AW101 TOC

AW101 U

AW101 Zr

AW101 Ac227

AW101 Am241

AW101 Am243

AW101 Bal37

AW101 C14

AW101 Cdill

AW101 Cm242

AW101 Cm243

AW101 Cm244

AW101 C060

AW101 Cs134

AW101 Cs137

AW101 Eu152

AW101 Eu154

AW101 Eul55

AW101 โ129

AW101 Nb93

AW101 Ni59

AW101 Ni63

AW101 Np237

AW101 Pa231

AW101 Pu238

AW101 Pu239

AW101 Pu240
$B B$

$3.24 \mathrm{E}+01$

$1.73 \mathrm{E}+01$

2.60E-03

1.04E-02

3.16E-00

4.30E-00

4.00E-01

1.52E-00

Ci 1.37E-00

Ci 5.30E-02

Ci 9.90E-02

Ci $4.80 \mathrm{E}-03$

Ci $3.24 \mathrm{E}+01$

Ci $\quad 9.76 \mathrm{E}-00$

kg $1.10 \mathrm{E}+05$

kg $\quad 9.73 \mathrm{E} \div 02$

$\mathrm{kg} \quad 4.61 \mathrm{E}+03$

kg 2.35E+04

kg $\quad 9.77 \mathrm{E}+04$

kg $\quad 8.88 \mathrm{E}+02$

$9.62 E+03$

g $2.83 \mathrm{E}+04$

$\quad 1.60 \mathrm{E}+01$

g $\quad 1.64 E+05$

8.29E-00

kg $\quad 3.64 \mathrm{E}+03$

kg $\quad 9.87 \mathrm{E}+05$

kg $4.08 \mathrm{E}+03$

$\mathrm{kg} \quad 4.01 \mathrm{E}+05$

kg $8.57 \mathrm{E}+05$

kg $\quad 1.97 \mathrm{E}+04$

kg 1.08E+03

$\mathrm{kg} \quad 6.11 \mathrm{E}+04$

kg $3.07 \mathrm{E}+03$

kg 1.04E+03

kg $9.22 \mathrm{E}+03$

kg NA

g $1.03 E+03$

$1.23 E+03$

$4.02 \mathrm{E}-03$

$3.88 \mathrm{E}+02$

3.11E-02

$1.73 E+06$

$1.24 \mathrm{E}+02$

$3.62 \mathrm{E}+02$

$6.98 \mathrm{E}-01$

7.12E-02

$1.00 \mathrm{E}-00$

Ci $1.73 E+02$

Ci $2.65 E+02$

Ci 1.83E+06

$\mathrm{Ci} \quad 1.79 \mathrm{E}+01$

Ci $2.69 \mathrm{E}+03$

Ci $1.14 E+03$

Ci 1.78E-00

Ci $5.24 E+01$

Ci 7.18E-00

Ci $\quad 7.07 E+02$

Ci 3.31E-00

$\mathrm{Ci} \quad$ 1.71E-02

Ci 8.34E+01

Ci 8.17E+02

Ci 2.25E+02
$B B$ Sept98 1.20E-02 $1.58 \mathrm{E}+02$

7.40E-02

$7.30 \mathrm{E}+03$

$3.24 \mathrm{E}+01$

$1.73 \mathrm{E}+01$

2.60E-03

1.04E-02

$3.16 \mathrm{E}-00$

4.30E-00

1.79E-04

6.78E-04

$6.80 \mathrm{E}-03$

2.59E-04

5.50E-04

4.73E-03

$3.24 \mathrm{E}+01$

9.76E-00

1.31E+05

$4.44 \mathrm{E}+02$

$8.40 \mathrm{E}+02$

$2.30 \mathrm{E}+04$

$8.73 \mathrm{E}+04$

$1.03 \mathrm{E}+03$

4.62E +03

$8.50 \mathrm{E}+02$

$0.00 \mathrm{E}+01$

$1.53 \mathrm{E}+05$

$0.00 \mathrm{E}+01$

$4.90 \mathrm{E}+02$

$1.07 \mathrm{E}+06$

$1.40 \mathrm{E}+02$

$4.36 \mathrm{E}+05$

$7.52 \mathrm{E}+05$

NA

4.52E +02

8.37E+03

NA

$1.48 \mathrm{E}+03$

$7.10 \mathrm{E}+03$

$4.59 \mathrm{E}+01$

$1.81 \mathrm{E}+04$

$2.83 E+03$

$1.96 \mathrm{E}+02$

4.02E-03

$5.86 \mathrm{E}+01$

4.71E-03

$1.86 \mathrm{E}+06$

$1.24 \mathrm{E}+02$

$3.62 \mathrm{E}+02$

1.05E-01

2.30E-01

5.53E-00

$1.59 \mathrm{E}+02$

$2.65 \mathrm{E}+02$

$1.97 E+06$

$1.79 \mathrm{E}+01$

$4.86 \mathrm{E}+02$

$2.12 \mathrm{E}+03$

$1.16 \mathrm{E}+01$

$5.24 \mathrm{E}+01$

$7.18 \mathrm{E}-00$

$7.07 E+02$

3.31E-00

1.71E-02

6.05E-00

$5.92 \mathrm{E}+01$

$1.63 \mathrm{E}+01$ 1\%-ile

$10 \%$-ile

$0.00 \mathrm{E}+01$

$0.00 \mathrm{E}+01$

$8.00 \mathrm{E}-03$

$0.00 \mathrm{E}+01$

$5.92 \mathrm{E}-04$

$0.00 \mathrm{E}+01$

$0.00 \mathrm{E} \div 01$

$0.00 \mathrm{E}+01$

$0.00 \mathrm{E}+01$

2.38E-00

$0.00 E+01$

$0.00 \mathrm{E}+01$

$0.00 E+01$

$0.00 \mathrm{E}+01$

$0.00 \mathrm{E}+01$

$0.00 \mathrm{E}+01$

$0.00 \mathrm{E}+01$

$0.00 \mathrm{E}+01$

$1.09 \mathrm{E}+05$

$1.19 \mathrm{E}+02$

$3.70 \mathrm{E}+02$

$5.37 \mathrm{E}+01$

$7.78 E+03$

$1.52 \mathrm{E}+02$

$3.06 \mathrm{E}+02$

$9.20 \mathrm{E}-00$

$0.00 \mathrm{E}+01$

$1.73 \mathrm{E}+05$

$7.87 \mathrm{E}+01$

$2.24 \mathrm{E}+01$

$9.92 \mathrm{E}+05$

$3.75 \mathrm{E}+01$

$7.10 \mathrm{E}+01$

$4.03 E+05$

$1.61 E+02$

$1.69 \mathrm{E}+02$

$1.11 \mathrm{E}+03$

$1.28 \mathrm{E}+03$

$6.76 \mathrm{E}+02$

$2.25 \mathrm{E}+02$

$1.58 \mathrm{E}+01$

8.83E+03

$1.15 \mathrm{E}+03$

$4.10 \mathrm{E}+01$

$0.00 \mathrm{E}+01$

$0.00 \mathrm{E}+01$

$0.00 \mathrm{E}+01$

$0.00 \mathrm{E}+01$

$0.00 \mathrm{E}+01$

$0.00 \mathrm{E}+01$

$0.00 \mathrm{E}+01$

$0.00 \mathrm{E}+01$

$0.00 \mathrm{E}+01$

$1.40 \mathrm{E}-06$

$0.00 E+01$

$7.24 \mathrm{E}+04$

$0.00 \mathrm{E}+01$

$0.00 \mathrm{E}+01$

$0.00 \mathrm{E}+01$

$0.00 \mathrm{E}+01$

$2.23 \mathrm{E}+01$

$1.50 \mathrm{E}-01$

$1.47 \mathrm{E}+01$

$0.00 \mathrm{E}+01$

$3.74 \mathrm{E}-03$

$0.00 \mathrm{E}+01$

$0.00 \mathrm{E}+01$

$0.00 \mathrm{E}+01$

6.91E-04

$0.00 \mathrm{E}+01$

$3.42 \mathrm{E}-02$

$0.00 \mathrm{E}+01$

2.17E+01

$0.00 \mathrm{E}+01$

$0.00 \mathrm{E}+01$

$0.00 \mathrm{E}+01$

$0.00 \mathrm{E}+01$

2.67E-00

$0.00 \mathrm{E}+01$

$0.00 \mathrm{E}+01$

$0.00 \mathrm{E}+01$

$0.00 \mathrm{E}+01$

$0.00 \mathrm{E}+01$

$0.00 \mathrm{E}+01$

4.77E-00

$0.00 \mathrm{E}+01$ 
Tank Anal. Un

AW101 Pu241

AW101 Pu242 $\mathrm{Ci}$.33E+03

AW101 Ra226 Ci 6.38E-04

AW101 Ra228 Ci 1.31E-00

AW101 Ru106 Ci 6.07E-02

AW101 Sb125 Ci 1.18E+03

AW101 Se79 Ci 1.48E+01

AW101 Sm151 Ci $\quad$ 5.24E+04

AW101 Sr89/90 Ci 3.13E+04

AW101 Tc99 Ci $9.22 \mathrm{E}+02$

AW101 Th229 Ci 3.04E-02

AW101 Th232 Ci 1.27E-01

AW101 Tin126 $\mathrm{Ci} \quad 2.26 \mathrm{E}+01$

AW101 Tritium C

AW101 U232

AW101 U233

AW101 U234

AW101 U235

AW101 U236

AW101 U238

AW101 Y90

AW101 Zr93

AW102 Al

AW102 Bi

AW102 Ca

AW102 Cl

AW102 CO3

AW102 Cr

AW102 F

AW102 Fe

AW102 Hg

AW102 K

AW102 La

AW102 Mn

AW102 Na

AW102 Ni

AW102 NO2

AW102 NO3

AW102 P

AW102 Pb

AW102 PO4

AW102 S

AW102 Si

AW102 $\mathrm{SO}_{4}$

AW102 Sr

AW102 TOC

AW102 U

AW102 Zr

AW102 Ac227

AW102 Am241

AW102 Am243

AW102 Ba137

AW102 C14

AW102 Cd113

AW102 Cm242

AW102 Cm243

AW102 Cm244

AW102 C060

AW102 Cs134

AW102 Cs137

AW102 Eul52

AW102 Eu154

AW102 Eu155

AW102 1129

AW102 Nb93

AW102 Nis9

AW102 Ni63

AW102 Np237

Ci 7.75E-05
$5.67 \mathrm{E}+02$

kg $\quad 4.49 E+02$

$\mathrm{kg} \quad 1.15 \mathrm{E}-00$

kg 2.65E +02

kg 1.20E-01

kg $8.41 \mathrm{E}+01$

kg $3.75 E+04$

$\mathrm{kg} \quad 6.87 \mathrm{E}+01$

kg $9.26 E+03$

kg $2.65 E+04$

kg 2.52E+02

kg $5.41 \mathrm{E}+01$

kg $\quad 7.81 \mathrm{E}+02$

$\mathrm{kg} \quad 1.23 \mathrm{E}+03$

kg 3.11E+03

kg $3.69 E+03$

$\mathrm{kg} \quad 0.00 \mathrm{E}+01$

$\mathrm{kg} \quad 1.03 \mathrm{E}+03$

$\mathrm{kg} \quad 3.72 \mathrm{E}+03$

kg 2.71E+01

$\mathrm{Ci} \quad 1.68 \mathrm{E}-04$

Ci $3.34 \mathrm{E}+02$

Ci 3.88E-04

Ci $1.47 \mathrm{E}+04$

Ci 6.50E-00

Ci 1.95E+01

1.55E-06

4.65E-05

$1.28 \mathrm{E}+01$

5.45E-01

$1.55 E+04$

$1.03 \mathrm{E}-00$

$1.47 E+02$

$6.01 E+01$

$6.20 \mathrm{E}-02$

2.54E-00

4.31E-01

$4.30 \mathrm{E}+01$

1.11E-01
$B B$ Sept98

$6.04 \mathrm{E}+02$

2.31E-03

6.38E-04

1.31E-00

6.07E-02

$1.18 \mathrm{E}+03$

$1.48 \mathrm{E}+01$

$5.24 \mathrm{E}+04$

$5.51 \mathrm{E}+03$

$7.93 \mathrm{E}+02$

3.04E-02

1.27E-01

$2.26 \mathrm{E}+01$

$1.49 \mathrm{E}+01$

5.69E-01

2.18E-00

$1.27 \mathrm{E}-00$

4.89E-02

8.79E-02

9.42E-01

$5.51 \mathrm{E}+03$

$7.15 \mathrm{E}+01$

$2.73 \mathrm{E}+03$

$0.00 \mathrm{E}+01$

$4.08 \mathrm{E}+02$

$2.82 \mathrm{E}+03$

$2.12 \mathrm{E}+02$

$2.45 E+02$

$4.49 \mathrm{E}+02$

$0.00 \mathrm{E}+01$

$1 \%$-ile

$0.00 \mathrm{E}+01$

$0.00 \mathrm{E}+01$

2.82E-04

$5.79 \mathrm{E}-01$

$0.00 \mathrm{E}+01$

$8.36 \mathrm{E}+01$

$0.00 \mathrm{E}+01$

$0.00 \mathrm{E}+01$

$0.00 \mathrm{E}+01$

$1.33 \mathrm{E}+01$

6.65E-03

$2.78 \mathrm{E}-02$

$0.00 \mathrm{E}+01$

$7.49 \mathrm{E}+02$

9.91E-01

3.81E-00

2.21E-00

8.53E-02

1.53E-01

$1.92 \mathrm{E}-00$

$0.00 \mathrm{E}+01$

$3.04 \mathrm{E}+01$

$10.00 \mathrm{E}+01$

$1.57 \mathrm{E}-04$

2.81E-00

$1.16 \mathrm{E}-01$

1.12E+03

$4.27 \mathrm{E}+02$

$9.34 \mathrm{E}+03$

$3.66 \mathrm{E}-02$

$0.00 \mathrm{E}+01$

$6.40 \mathrm{E}+01$

8.67E-04

$0.00 \mathrm{E}+01$

$8.41 \mathrm{E}+01$

$3.75 \mathrm{E}+04$

$6.87 \mathrm{E}+01$

$9.26 \mathrm{E}+03$

$2.65 E+04$

NA

$5.41 \mathrm{E}+01$

$7.81 \mathrm{E}+02$

NA

$3.11 E+03$

$3.69 \mathrm{E}+03$

$0.00 \mathrm{E}+01$

$1.03 \mathrm{E}+03$

$3.72 \mathrm{E}+03$

$2.71 E+01$

$1.68 \mathrm{E}-04$

$3.34 \mathrm{E}+02$

3.11E-02

$1.47 \mathrm{E}+04$

6.50E-00

$1.95 \mathrm{E}+01$

3.20E-03

5.77E-02

9.63E-01

$1.28 \mathrm{E}+01$

5.45E-01

$1.55 \mathrm{E}+04$

$1.03 \mathrm{E}-00$

$1.47 E+02$

$6.01 E+01$

$6.20 \mathrm{E}-02$

2.54E-00

4.31E-01

$4.30 \mathrm{E}+01$

1.11E-01

4.59E-05

$6.96 \mathrm{E}+03$

$1.36 \mathrm{E}-00$

4.71E-01

$1.99 \mathrm{E}+01$

$1.30 \mathrm{E}-01$

$1.58 \mathrm{E}-04$

$1.19 \mathrm{E}+01$

$1.80 \mathrm{E}+02$

$2.13 \mathrm{E}+01$

3.17E+02

2.02E-03

$2.50 \mathrm{E}+02$

$1.55 \mathrm{E}-00$

5.34E-01

$0.00 \mathrm{E}+01$

$0.00 \mathrm{E}+01$

$0.00 \mathrm{E}+01$

$0.00 \mathrm{E}+01$

9.79E-01

$0.00 \mathrm{E}+01$

$0.00 \mathrm{E}+01$

$0.00 \mathrm{E}+01$

$0.00 \mathrm{E}+01$

2.36E-04

$0.00 \mathrm{E}+01$

$5.08 \mathrm{E}-03$

$0.00 E+01$

$0.00 \mathrm{E}+01$

$0.00 \mathrm{E}+01$

$0.00 \mathrm{E}+01$

$0.00 \mathrm{E}+01$

$0.00 \mathrm{E}+01$

$0.00 \mathrm{E}+01$
$10 \%$-ile

4.57E+02

$1.75 \mathrm{E}-03$

2.98E-04

$6.11 \mathrm{E}-01$

4.32E-03

4.19E+02

2.21E-00

$0.00 \mathrm{E}+01$

4.85E-01

$2.75 \mathrm{E}+02$

$1.16 \mathrm{E}-02$

4.84E-02

$1.75 \mathrm{E}-00$

8.33E+02

$1.73 \mathrm{E}-00$

6.64E-00

$3.85 \mathrm{E}-00$

1.49E-01

2.67E-01

3.35E-00

$0.00 E+01$

$3.31 \mathrm{E}+01$

$2.60 \mathrm{E}+03$

2.49E-02

$1.17 \mathrm{E}+01$

8.72E +01

$2.23 E+03$

$1.27 E+03$

$1.45 \mathrm{E}+04$

$2.02 \mathrm{E}+01$

$0.00 \mathrm{E}+01$

$5.04 \mathrm{E}+02$

$3.15 \mathrm{E}-01$

$0.00 \mathrm{E}+01$

50\%-ile

$8.33 E+03$

3.19E-02

6.38E-04

1.31E-00

6.07E-02

$1.18 \mathrm{E}+03$

$1.48 \mathrm{E}+01$

$5.24 \mathrm{E}+04$

$2.09 \mathrm{E}+04$ 
Tank Anal. Un

AW102 Pa231

AW102 Pu238

AW102 Pu239

AW102 Pu240

AW102 Pu241

AW102 Pu242

AW102 Ra226

AW102 Ra228

AW102 Ru106

AW102 Sb125

AW102 Se79

AW102 Sm151

AW102 Sr89/90

AW102 Te99

AW102 Th229

AW102 Th232

AW102 Tin126

AW102 Tritium

AW102 U232

AW102 U233

AW102 U234

AW102 U235

AW102 U236

AW102 U238

AW102 Y90

AW102 Zr93

AW103 Al

AW103 Bi

AW103 Ca

AW103 CI

AW103 CO3

AW103 Cr

AW103 F

AW103 Fe

AW103 Hg

AW103 K

AW103 La

AW103 Mn

AW103 Na

AW103 Ni

AW103 NO2

AW103 NO3

AW103 P

AW103 Pb

AW103 PO4

AW103 S

AW103 Si

AW103 504

AW103 Sr

AW103 TOC

AW103 U

AW103 Zr

AW103 Ac227

AW103 Am241

AW103 Am243

AW103 Ba137

AW103 C14

AW103 CdI13

AW103 Cm242

AW103 $\mathrm{Cm} 243$

AW103 Cm244

AW103 Co60

AW103 Cs134

AW103 Cs137

AW103 Eul52

AW103 Eu154

AW103 Eu155

AW103 1129
$B B$ May 98

Ci $\overline{8.01 \mathrm{E}-04}$

Ci $9.64 \mathrm{E}+01$

Ci Incl. in 239

Ci $3.02 \mathrm{E}+01$

Ci 1.89E-04

Ci 2.84E-05

Ci $\quad 6.20 \mathrm{E}-02$

Ci $1.88 \mathrm{E}-03$

Ci $4.42 \mathrm{E}+01$

Ci 7.15E-02

Ci $2.51 E+03$

$1.19 \mathrm{E}+05$

Ci $3.18 \mathrm{E}+01$

Ci $1.47 \mathrm{E}-03$

Ci 7.75E-03

Ci 1.08E-00

Ci $2.46 \mathrm{E}+01$

Ci 1.65E-01

$\mathrm{Ci} \quad 6.38 \mathrm{E}-01$

Ci 2.17E-01

Ci 8.79E-03

Ci $7.49 \mathrm{E}-03$

Ci 2.84E-01

Ci 1.19E+05

Ci 3.53E-00

kg $1.21 \mathrm{E}+04$

kg 2.22E-01

kg $\quad 1.73 E+03$

kg $7.77 \mathrm{E}+01$

$\mathrm{kg} \cdot 1.66 \mathrm{E}+04$

$\mathrm{kg} \quad 3.04 \mathrm{E}+03$

$\mathrm{kg} \quad 1.27 \mathrm{E}+05$

$\mathrm{kg} \quad 1.58 \mathrm{E}+03$

$\mathrm{kg} \quad 5.80 \mathrm{E}+02$

kg $3.35 \mathrm{E}+04$

$\mathrm{kg} \quad 1.65 \mathrm{E}+03$

kg $\quad 5.17 E+02$

kg 2.82E+05

kg $3.43 E+02$

$\mathrm{kg} \quad 7.02 \mathrm{E}+02$

$\mathrm{kg} 2.00 \mathrm{E}+05$

$\mathrm{kg} \quad 1.08 \mathrm{E}+01$

kg $1.57 \mathrm{E} \div 02$

$\begin{array}{ll}\mathrm{kg} & 3.34 \mathrm{E}+01 \\ \mathrm{~kg} & 1.08 \mathrm{E}+01\end{array}$

kg $3.19 \mathrm{E} \div 04$

kg $3.24 \mathrm{E}+01$

kg 3.92E+01

kg 5.38E+02

kg $\quad 1.84 \mathrm{E}+04$

kg $\quad 1.67 \mathrm{E}+05$

Ci 3.88E-06

Ci $2.38 \mathrm{E}+02$

Ci 8.22E-03

Ci 1.25E+05

Ci 1.25E-00

$\mathrm{Ci} \quad 3.37 \mathrm{E}-00$

Ci 1.34E-01

2.25E-02

1.50E-01

Ci 7.84E-00

Ci $8.39 E+01$

Ci $132 \mathrm{E}+05$

Ci 1.53E-00

Ci 5.89E+01

Ci $2.77 \mathrm{E}+02$

Ci 4.62E-03
$B B$ Sept98

8.01E-04

5.89E-00

$7.78 \mathrm{E}+01$

$1.86 \mathrm{E}+01$

$5.54 \mathrm{E}+02$

2.28E-03

2.84E-05

$6.20 \mathrm{E}-02$

$1.88 \mathrm{E}-03$

$4.42 \mathrm{E}+01$

7.15E-02

$2.51 E+03$

$1.19 \mathrm{E}+05$

3.18E+01

$1.47 \mathrm{E}-03$

7.75E-03

$1.08 \mathrm{E}-00$

$2.46 \mathrm{E}+01$

4.71E-01

$1.80 \mathrm{E}-00$

1.70E-00

$6.53 \mathrm{E}-02$

1.22E-01

$1.24 \mathrm{E}-00$

$1.19 \mathrm{E}+05$

3.53E-00

$1.21 \mathrm{E}+04$

2.22E-01

$1.73 \mathrm{E}+03$

$7.77 \mathrm{E}+01$

$1.66 \mathrm{E}+04$

$3.04 \mathrm{E}+03$

$1.27 \mathrm{E}+05$

$1.58 \mathrm{E}+03$

$0.00 \mathrm{E}+01$

$3.35 \mathrm{E}+04$

$1.65 \mathrm{E}+03$

$5.17 \mathrm{E}+02$

$2.82 \mathrm{E}+05$

$3.43 \mathrm{E}+02$

$7.02 \mathrm{E}+02$

$2.00 \mathrm{E}+05$

NA

1.57E+02

3.34E+01

NA

$3.19 E+04$

$3.24 \mathrm{E}+01$

$3.92 \mathrm{E}+01$

$5.38 \mathrm{E}+02$

$1.84 \mathrm{E}+04$

$1.67 \mathrm{E}+05$

3.88E-06

$2.38 \mathrm{E}+02$

8.22E-03

$1.25 E+05$

$1.25 \mathrm{E}-00$

3.37E-00

$1.34 \mathrm{E}-01$

2.25E-02

$1.50 \mathrm{E}-01$

7.84E-00

$8.39 E+01$

$1.32 \mathrm{E}+05$

$1.53 \mathrm{E}-00$

$5.89 \mathrm{E}+01$

$2.77 \mathrm{E}+02$

4.62E- 03 1\%-ile

$0.00 \mathrm{E}+01$
$0.00 \mathrm{E}+01$

$0.00 \mathrm{E}+01$

$0.00 \mathrm{E}+01$

$0.00 \mathrm{E}+01$

$0.00 \mathrm{E}+01$

$7.08 \mathrm{E}-08$

1.55E-04

$0.00 \mathrm{E}+01$

$0.00 \mathrm{E}+01$

$1.74 \mathrm{E}-02$

$0.00 \mathrm{E}+01$

1.17E+01

$0.00 \mathrm{E}+01$

$0.00 \mathrm{E}+01$

$0.00 \mathrm{E}+01$

$0.00 \mathrm{E}+01$

$6.90 \mathrm{E}-01$

$0.00 \mathrm{E}+01$

$0.00 \mathrm{E}+01$

$0.00 \mathrm{E}+01$

$0.00 \mathrm{E}+01$

$0.00 \mathrm{E}+01$

$0.00 \mathrm{E}+01$

$0.00 \mathrm{E}+01$

$0.00 \mathrm{E}+01$

$1.51 \mathrm{E}+02$

$4.18 \mathrm{E}+01$

$5.16 \mathrm{E}+01$

$1.25 \mathrm{E}+02$

$2.71 E+03$

$2.97 \mathrm{E}+01$

$1.27 \mathrm{E}+04$

$9.96 \mathrm{E}-00$

$0.00 \mathrm{E}+01$

$6.06 \mathrm{E}+02$

$8.80 \mathrm{E}-01$

$5.08 \mathrm{E}-02$

$4.64 \mathrm{E}+04$

$3.62 \mathrm{E}-01$

$2.44 \mathrm{E}+03$

$5.86 \mathrm{E}+03$

$1.74 \mathrm{E}+03$

$1.73 \mathrm{E}+02$

$1.42 \mathrm{E}+02$

$8.83 E+02$

$2.70 \mathrm{E}+02$

$8.28 \mathrm{E}+02$

$1.44 \mathrm{E}+01$

$1.02 \mathrm{E}+03$

$3.36 \mathrm{E}+03$

$1.43 \mathrm{E}+04$

8.45E-09

7.07E-01

4.49E-05

$0.00 \mathrm{E}+01$

$0.00 \mathrm{E}+01$

$0.00 \mathrm{E}+01$

7.33E-04

$1.23 \mathrm{E}-04$

8.20E-04

$1.16 \mathrm{E}+01$

$0.00 E+01$

$1.04 \mathrm{E}+04$

3.33E-03

$1.28 \mathrm{E}-01$

6.03E-01

2.81E-05

$10 \%$-ile

$0.00 \mathrm{E}+01$

$1.14 \mathrm{E}-02$

$2.48 \mathrm{E}-00$

$2.48 \mathrm{E}-00$

$7.76 \mathrm{E}-01$

4.86E-06

$2.95 \mathrm{E}-06$

$6.44 \mathrm{E}-03$

$9.67 \mathrm{E}-05$

4.82E-02

$2.85 \mathrm{E}-02$

$9.38 \mathrm{E}+01$ 
Tank Anal. Un

AW103 Nb93 AW103 Ni59

AW103 Ni63

AW103 Np237

AW103 Pa231

AW103 Pu238

AW103 Pu239

AW103 Pu240

AW103 Pu241

AW103 Pu242

AW103 Ra226

AW103 Ra228

AW103 Ru106

AW103 Sb125

AW103 Se79

AW103 Sm151

AW103 Sr89/90

AW103 Te99

AW103 Th229

AW103 Th232

AW103 Tin126

AW103 Tritium

AW103 U232

AW103 U233

AW103 U234

AW103 U235

AW103 U236

AW103 U238

AW103 Y90

AW103 Zr93

AW104 Al

AW104 Bi

AW104 Ca

AW104 Cl

AW104 CO3

AW104 Cr

AW104 F

AW104 Fe

AW104 Hg

AW104 K

AW104 La

AW104 Mn

AW104 Na

AW104 Ni

AW104 NO2

AW104 NO3

AW104 P

AW104 Pb

AW104 PO4

AW104 S

AW104 Si

AW104 SO4

AW104 Sr

AW104 TOC

AW104 U

AW104 Zr

AW104 Ac227

AW104 Am241

AW104 Am243

AW104 Ba137

AW104 C14

AW104 Cd113

AW104 Cm242

AW104 Cm243

AW104 Cm244

AW104 C060

AW104 Cs134 AW104 Cs137
Un

$\frac{B B}{\operatorname{May} 98}$

i.

Ci $\quad$ 1.96E-01

Ci 8.82E-01

Ci 2.07E-05

Ci $4,42 \mathrm{E}+01$

Ci $1.35 \mathrm{E}+03$

Ci Incl in 239 Incl.

cl. in 239

Ci 1.70E+04 1.70E+04

Ci 6.35E-02 6.35E-02

Ci $\quad 6.53 E-07 \quad 6.53 E-07$

Ci 3.32E-04 3.32E-04

Ci $7.10 \mathrm{E}+02 \quad 7.10 \mathrm{E}+02$

Ci $\quad 1.89 \mathrm{E}+02 \quad 1.89 \mathrm{E}+02$

Ci 6.65E-02

Ci $2.30 \mathrm{E}+02$

$1.96 \mathrm{E}+04$

$1.80 \mathrm{E}+02$

Ci 7.73E-06

Ci 2.81E-05

1.05E-01

4.13E+01

Ci $2.82 \mathrm{E}-03$

Ci 5.10E-03

Ci $\quad 6.73 E-00$

Ci 2.56E-01

Ci 5.54E-01

Ci 4.62E-00

Ci $1.96 \mathrm{E}+04$

Ci $3.21 \mathrm{E}-01$

kg 2.65E+04

kg $4.20 \mathrm{E}-0.1$

kg 8.19E+03

$\mathrm{kg} \quad 4.26 \mathrm{E}+03$

kg $3.60 \mathrm{E}+04$

$\mathrm{kg} \quad 1.62 \mathrm{E}+03$

kg 1.92E+03

kg 4.02E+04

$\mathrm{kg} \quad 7.97 \mathrm{E}-00$

kg 1.13E+04

kg 3.97E-03

kg $\quad 1.28 \mathrm{E}+03$

$\mathrm{kg} \quad 1.76 \mathrm{E}+05$

kg $2.81 \mathrm{E}+03$

$\mathrm{kg} \quad 8.18 E+04$

kg 1.37E+05

$\mathrm{kg} \quad 6.39 \mathrm{E}+02$

kg 3.53E+01

$\mathrm{kg} \quad 1.98 \mathrm{E}+03$

kg $\quad 3.61 E+02$

$\mathrm{kg} \quad 3.88 \mathrm{E}-00$

$\mathrm{kg} \quad 1.08 \mathrm{E}+03$

kg $\quad 0.00 E+01$

$\quad 1.94 \mathrm{E}+03$

$8.50 E+03$

$1.60 \mathrm{E}+03$

$1.66 \mathrm{E}-06$

4.50E-01

7.53E-05

$2.57 E+05$

6.07E-02

$1.88 \mathrm{E}-01$

2.04E-13

3.22E-04

1.54E-03

1.38E-01

7.49E-01

$2.72 \mathrm{E}+05$
$6.65 \mathrm{E}-02$

$2.30 \mathrm{E}+02$

1.96E +04

$1.80 \mathrm{E}+02$

7.73E-06

2.81E-05

$1.05 \mathrm{E}-01$

$4.13 \mathrm{E}+01$

2.82E-03

5.10E-03

6.73E-00

2.56E-01

5.54E-01

$4.62 \mathrm{E}-00$

$1.96 \mathrm{E}+04$

3.21E-01

$5.97 \mathrm{E}+04$

$0.00 \mathrm{E}+01$

$3.67 \mathrm{E}+03$

$1.12 \mathrm{E}+04$

$2.05 \mathrm{E}+04$

$2.68 \mathrm{E}+03$

$3.44 \mathrm{E}+04$

$6.43 \mathrm{E}+03$

$0.00 \mathrm{E}+01$

$1.15 \mathrm{E}+04$

$0.00 \mathrm{E}+01$

$2.10 \mathrm{E}+03$

$5.42 \mathrm{E}+05$

$2.81 \mathrm{E}+03$

$2.04 \mathrm{E}+05$

$3.29 \mathrm{E}+05$

NA

$2.80 \mathrm{E}+03$

$4.35 \mathrm{E}+03$

NA

$2.35 \mathrm{E}+03$

$1.75 \mathrm{E}+04$

$5.00 \mathrm{E}-03$

$2.75 \mathrm{E}+03$

$2.37 \mathrm{E}+04$

$1.10 \mathrm{E}+03$

$1.66 \mathrm{E}-06$

4.50E-01

$7.53 \mathrm{E}-05$

$2.60 \mathrm{E}+05$

6.07E-02

$1.88 \mathrm{E}-01$

2.04E-03

3.22E-04

$1.54 \mathrm{E}-03$

$1.38 \mathrm{E}-01$

$7.49 \mathrm{E}-\mathrm{-O1}$

$2.75 E+05$

$1 \%$-ile

$10 \%$-ile

50\%-ile

90\%-ile

$\begin{array}{ll}0.00 \mathrm{E}+01 & 0.00 \mathrm{E}+01 \\ 0.00 \mathrm{E}+01 & 1.96 \mathrm{E}-02\end{array}$

$0.00 \mathrm{E}+01 \quad 2.26 \mathrm{E}-00$

$4.82 \mathrm{E}-03$

$1.79 \mathrm{E}-01$

$0.00 \mathrm{E}+01$

$2.42 \mathrm{E}-01 \quad 8.96 \mathrm{E}-00$

$7.38 \mathrm{E}-00 \quad 2.74 \mathrm{E}+02$

$7.38 \mathrm{E}-00$

$2.74 \mathrm{E}+02$

$9.30 \mathrm{E}+01 \quad 3.45 \mathrm{E}+03$

3.47E-04 1.29E-02

$0.00 \mathrm{E}+01$

$1.54 \mathrm{E}-07$
$1.84 \mathrm{E}-05$

$.00 \mathrm{E}+01$

$2.52 \mathrm{E}-00$

7.84E-05

$00 \mathrm{E}+01$

$1.56 \mathrm{E}+02$

$0.00 \mathrm{E}+01$

$8.30 \mathrm{E}-04 \quad 1.94 \mathrm{E}-02$

5.01E-01

$3.92 \mathrm{E}+01$

$6.72 \mathrm{E}+01$

$0.00 \mathrm{E}+01$

$0.00 \mathrm{E}+01$

$0.00 \mathrm{E}+01$

$0.00 \mathrm{E}+01$

$1.89 \mathrm{E}+01$

$0.00 \mathrm{E}+01$

$0.00 \mathrm{E}+01$

$0.00 \mathrm{E}+01$

$0.00 \mathrm{E}+01$

$0.00 \mathrm{E}+01$

$0.00 \mathrm{E}+01$

$0.00 \mathrm{E}+01$

$0.00 \mathrm{E}+01$

$1.05 \mathrm{E}+04$

$1.12 \mathrm{E}+01$

$1.52 \mathrm{E}+02$

$2.79 \mathrm{E}+03$

$3.99 \mathrm{E}+03$

$1.85 \mathrm{E}+03$

$4.09 \mathrm{E}+04$

$2.50 \mathrm{E}+02$

$0.00 \mathrm{E}+01$

$6.62 \mathrm{E}+01$

$1.73 \mathrm{E}+02$

$7.86 \mathrm{E}+01$

$2.46 \mathrm{E}+05$

$1.70 \mathrm{E}+01$

$4.69 \mathrm{E}+04$

$3.56 \mathrm{E}+04$

$2.16 \mathrm{E}+02$

$4.48 \mathrm{E}-00$

$4.39 \mathrm{E}+01$

$4.88 \mathrm{E}+03$

$7.19 \mathrm{E}+01$

$8.31 E+03$

$4.26 \mathrm{E}-01$

$3.02 \mathrm{E}+03$

$4.36 \mathrm{E}+02$

$3.16 \mathrm{E}+02$

$0.00 \mathrm{E}+01$

$1.08 \mathrm{E}-00$

$1.69 \mathrm{E}-06$

$0.00 \mathrm{E}+01$

$0.00 \mathrm{E}+01$

$0.00 E+01$

4.57E-05

7.21E-06

3.45E-05

5.03E-01

$0.00 \mathrm{E}+01$

$9.72 \mathrm{E}+03$

$1.86 \mathrm{E}+01$

$0.00 \mathrm{E}+01$

$0.00 \mathrm{E}+01$

$3.31 \mathrm{E}-02$

$2.81 \mathrm{E}+01$

$0.00 \mathrm{E}+01$

$0.00 \mathrm{E}+01$

$0.00 \mathrm{E}+01$

$0.00 \mathrm{E}+01$

$0.00 \mathrm{E}+01$

$0.00 \mathrm{E}+01$

3.72E+03

$0.00 \mathrm{E}+01$

$1.81 \mathrm{E}+04$

$3.52 \mathrm{E}+01$

$1.32 \mathrm{E}+03$

$3.37 \mathrm{E}+03$ 
Tank Anal. Un

AW104 Eu152
AW104 Eu154
AW104 Eu155
AW104 1129
AW104 Nb93
AW104 Ni59

AW104 Ni63

AW104 Pa231

AW104 Pu238

AW104 Pu239

AW104 Pu240

AW104 Pu241

AW104 Pu242

AW104 Ra226

AW104 Ra228

AW104 Ru106

AW104 Sb125

AW104 Se79

AW104 Sm151

AW104 Sr89/90

AW104 Te99

AW104 Th229

AW104 Th232

AW104 Tin126

AW104 Tritium

AW104 U232

AW104 U233

AW104 U234

AW104 U235

AW104 U236

AW104 U238

AW104 Y90

AW104 Zr93

AW105 A]

AW105 B

AW105 Ca

AW105 Cl

AW105 CO3

AW105 Cr

AW105 F

AW105 Fe

AW105 Hg

AW105 K

AW105 La

AW105 Mn

AW105 Na

AW105 Ni

AW105 NO2

AW105 NO3

AW105 P

AW105 Pb

AW105 PO4

AW105 S

AW105 Si

AW105 SO4

AW105 Sr

AW105 TOC

AW105 U

AW105 Zr

AW105 Ac227

AW105 Am241

AW105 Am 243

AW105 Ba137.

AW105 C14

AW105 Cd113

AW105 Cm242

AW105 Cm243
BB

May $\frac{B 8}{98}$

$2.79 \mathrm{E}-02$

$1.66 \mathrm{E}-00$

4.10E-00

8.57E-04

2.36E-02

5.82E-03

6.18E-01

$1.76 \mathrm{E}-03$

7.39E-06

$1.09 \mathrm{E}+02$

$8.82 \mathrm{E}+02$

$2.68 \mathrm{E}+02$

$1.11 E+04$

4.15E-02

2.67E-07

5.71E-04

9.78E-00

1.96E-00

6.87E-03

$2.42 E+01$

$1.17 E+03$

4.43E-01

1.33E-05

5.84E-05

$1.05 \mathrm{E}-02$

7.41E-01

2.76E-03

$7.07 \mathrm{E}-03$

4.12E-00

1.57E-01

3.39E-01

2.83E-00

$1.17 \mathrm{E}+03$

Ci 3.34E-02

$3.53 \mathrm{E}+03$

g $1.12 \mathrm{E}+02$

$7.30 \mathrm{E}+03$

kg $\quad 7.43 \mathrm{E}+02$

kg $\quad 1.14 \mathrm{E}+04$

kg 1.17E+03

$\mathrm{kg} \quad 7.76 \mathrm{E}+04$

g $2.77 \mathrm{E}+03$

kg 3.47E+02

kg 1.19E+04

kg 4.43E +02

kg $1.42 \mathrm{E}+03$

kg $1.64 \mathrm{E}+05$

2.00E+02

kg 1.15E+04

kg $5.49 \mathrm{E}+04$

kg $\quad 6.81 \mathrm{E}+02$

kg $1.08 \mathrm{E}+03$

kg $2.11 \mathrm{E}+03$

kg $\quad 5.47 \mathrm{E}+02$

kg $5.31 E+03$

$\mathrm{kg} \quad 1.64 \mathrm{E}+03$

$\mathrm{kg} \quad 1.20 \mathrm{E}+01$

kg $\quad 8.38 \mathrm{E}+03$

kg $\quad 1.28 \mathrm{E}+04$

kg $9.88 \mathrm{E}+04$

1.18E-06

$4.72 \mathrm{E}+02$

3.32E-03

$5.08 \mathrm{E}+04$

2.26E-00

$1.33 \mathrm{E}-00$

8.76E-02

$7.40 E-00$ $\frac{\text { Sept98 }}{2.79 \mathrm{E}-02}$

2.79E-02

$4.10 \mathrm{E}-00$

8.57E-04

2.36E-02

5.82E-03

6.18E-01

$1.76 \mathrm{E}-03$

7.39E-06

$1.09 \mathrm{E}+02$

$8.82 \mathrm{E}+02$

$2.68 \mathrm{E}+02$

$1.11 \mathrm{E}+04$

4.15E-02

2.67E-07

5.71E-04

9.78E-00

$1.96 \mathrm{E}-00$

6.87E-03

2.42E+01

$1.27 \mathrm{E}+03$

$1.79 \mathrm{E}+02$

1.33E-05

5.84E-05

1.05E-02

7.41E-01

$7.69 E-03$

1.97E-02

$1.15 E+01$

4.37E-01

9.46E-01

7.89E-00

$1.27 \mathrm{E}+03$

3.34E-02

$3.53 \mathrm{E}+03$

$1.12 \mathrm{E}+02$

$7.30 \mathrm{E}+03$

$7.43 E+02$

$1.14 \mathrm{E}+04$

$1.17 \mathrm{E}+03$

$7.76 \mathrm{E}+04$

$2.77 \mathrm{E}+03$

$0.00 \mathrm{E}+01$

$1.19 \mathrm{E}+04$

$4.43 E+02$

$1.42 E+03$

$1.64 E+05$

$2.00 E+02$

$5.49 E+04$

NA

$1.08 \mathrm{E}+03$

$2.11 E+03$

NA

$5.31 E+03$

$1.64 \mathrm{E}+03$

$1.20 \mathrm{E}+01$

$8.38 \mathrm{E}+03$

$1.28 E+04$

$9.88 \mathrm{E}+04$

$1.18 \mathrm{E}-06$

$4.72 E+02$

3.32E-03

$5.08 E+04$

$2.26 \mathrm{E}-00$

$1.33 \mathrm{E}-00$

8.76E-02

$7.40 \mathrm{E}-00$
$1.15 E+04$
$1 \%-i l e$

$10 \%$-ile

50\%-ile

$0.00 \mathrm{E}+01$

$0.00 \mathrm{E}+01$

$0.00 \mathrm{E}+01$

2.41E-04

$0.00 \mathrm{E}+01$

$1.16 \mathrm{E}-05$

$1.24 \mathrm{E}-03$

$3.94 \mathrm{E}-05$

3.80E-07

2.44E-00

$1.97 \mathrm{E}+01$

6.00E-00

$2.48 \mathrm{E}+02$

9.29E-04

$0.00 \mathrm{E}+01$

$0.00 \mathrm{E}+01$

$0.00 \mathrm{E}+01$

$0.00 \mathrm{E}+01$

$0.00 \mathrm{E}+01$

$0.00 \mathrm{E}+01$

$6.58 \mathrm{E}+01$

$0.00 \mathrm{E}+01$

6.83E-07

$3.00 \mathrm{E}-06$

$0.00 \mathrm{E}+01$

4.90E-01

1.42E-04

3.63E-04

2.12E-01

8.06E-03

$1.74 \mathrm{E}-02$

$1.45 \mathrm{E}-01$

$0.00 \mathrm{E}+01$

$0.00 \mathrm{E}+01$

$1.45 \mathrm{E}+02$

$3.23 \mathrm{E}+01$

$2.05 \mathrm{E}+02$

$9.57 \mathrm{E}+01$

$3.64 \mathrm{E}+03$

$6.09 \mathrm{E}+01$

$3.79 E \div 03$

$2.21 E \div 02$

$0.00 \mathrm{E}+01$

$1.67 \mathrm{E}+03$

$1.35 \mathrm{E}-00$

$1.44 \mathrm{E}+01$

4.31E+04

$1.27 \mathrm{E}+02$

$1.88 \mathrm{E}+03$

$1.30 \mathrm{E}+04$

$1.19 \mathrm{E}+03$

$1.08 \mathrm{E}+02$

$2.69 \mathrm{E}+02$

$6.62 \mathrm{E}+02$

$1.95 \mathrm{E}+02$

$6.91 \mathrm{E}+02$

$9.82 \mathrm{E}-00$

$9.37 \mathrm{E}+02$

4.17E+03

$8.93 E+03$

$1.72 \mathrm{E}-08$

$4.98 \mathrm{E}-01$

$6.18 \mathrm{E}-05$

$0.00 \mathrm{E}+01$

$0.00 \mathrm{E}+0$ I

$0.00 \mathrm{E}+01$

$1.63 \mathrm{E}-03$

$1.38 \mathrm{E}-01$

$0.00 E+01$

$0.00 \mathrm{E}+01$

$0.00 E+01$

$4.26 \mathrm{E}-04$

$0.00 E+01$

$1.23 \mathrm{E}-03$

$1.30 \mathrm{E}-01$

2.02E-04

$1.80 \mathrm{E}-06$

$1.25 \mathrm{E}+01$

$1.01 \mathrm{E}+02$

$3.07 \mathrm{E}+01$

$1.27 \mathrm{E}+03$

$4.76 \mathrm{E}-03$

2.01E-09

4.30E-06

$1.18 \mathrm{E}-00$ 
Tank Anal. Un

AW105 Cm244
AW105 Co60
AW105 Cs134
AW105 Cs137
AW105 Eu152
AW105 Eu154
AW105 Eu155
AW105 I129
AW105 Nb93
AW105 Ni59
AW105 Ni63
AW105 Np237
AW105 Pa231
AW105 Pu238
AW105 Pu239
AW105 Pu240
AW105 Pu241
AW105 Pu242
AW105 Ra226
AW105 Ra228
AW105 Ru106
AW105 Sb125
AW105 Se79
AW105 Sm151
AW105 Sr89/90
AW105 T

AW105 Tc99

AW105 Th229

AW105 Th232

AW105 Tin126

AW105 Tritium

AW105 U232

AW105 U233

AW105 U234

AW105 U235

AW105 U236

AW105 U238

AW105 Y90

AW105 Zr93

AW106 Al

AW106 Bi

AW106 Ca

AW106 C

AW106 CO3

AW106 Cr

AW106 F

AW106 Fe

AW106 Hg

AW106 K

AW106 La

AW106 Mn

AW106 Na

AW106 Ni

AW106 NO2

AW106 NO3

AW106 P

AW106 Pb

AW106 PO4

AW106 S

AW106 Si

AW106 SO4

AW106 Sr

AW106 TOC

AW106 U

AW106 Zr

AW106 Ac227

AW106 Am241

AW106 Am243

AW106 Bal37
May $\overline{98}$

$1 \overline{77 \mathrm{E}+02}$

Ci 3.15E-00

$3.38 \mathrm{E}+01$

Ci $5.37 \mathrm{E}+04$

Ci 9.95E-01

Ci 2.73E+02

$1.99 \mathrm{E}+02$

Ci 1.72E-03

Ci 5.20E-02

Ci 1.35E-01

$1.56 \mathrm{E}+01$

Ci 1.25E-02

Ci 6.75E-06

Ci 6.91E+01

Ci $\quad 8.21 E+02$

Ci Incl. in 239 I

Ci 1.48E+04

$\begin{array}{ll}\mathrm{Ci} & \text { 5.53E-02 } \\ \mathrm{Ci} & 2.06 \mathrm{E}-07\end{array}$

$\begin{array}{ll}\mathrm{Ci} & 2.06 \mathrm{E}-07 \\ \mathrm{Ci} & 9.01 \mathrm{E}-11\end{array}$

$\begin{array}{ll}\mathrm{Ci} & 9.01 \mathrm{E}-11 \\ \mathrm{Ci} & 4.91 \mathrm{E}+02\end{array}$

Ci $1.86 \mathrm{E}+03$

Ci $2.56 \mathrm{E}-02$

Ci $8.83 \mathrm{E}+01$

$\mathrm{Ci}$

$\mathrm{Ci}$

$\mathrm{Ci}$

$\mathrm{Ci}$

4.00E-02

$1.36 \mathrm{E}+01$

Ci 1.13E-03

Ci 1.16E-05

Ci 5.09E-00

Ci 1.94E-01

Ci 4.19E-01

Ci 3.49E-00

Ci 2.63E+05

Ci 1.23E-01

kg $\quad 5.26 \mathrm{E}+04$

kg - $1.31 \mathrm{E}+03$

kg $1.99 \mathrm{E}+03$

kg 3.58E+03

kg $\quad 1.81 \mathrm{E}+04$

kg $6.35 \mathrm{E}+03$

kg $\quad 1.89 E+03$

kg $\quad 7.57 \mathrm{E}+02$

kg 7.37E-00

$\mathrm{kg} \quad 3.63 \mathrm{E}+03$

$\mathrm{kg} \quad 7.53 \mathrm{E}-01$

kg $2.64 \mathrm{E}+02$

$\mathrm{kg} \quad 3.86 \mathrm{E}+05$

kg $\quad 5.25 E+02$

$\mathrm{kg} \quad 1.25 \mathrm{E}+05$

kg 4.37E+05

$\mathrm{kg} \quad 1.13 \mathrm{E}+04$

kg $1.89 \mathrm{E}+02$

kg 3.50E+04

kg 1.15E+04

kg $\quad 4.26 \mathrm{E}+03$

kg $\quad 3.45 E+04$

kg $\quad 0.00 \mathrm{E}+01$

kg 3.59E+03

kg $2.33 E+03$

$\mathrm{kg} \quad 1.74 \mathrm{E}+02$

Ci 1.07E-03

Ci $2.50 \mathrm{E}+02$

Ci 2.49E-03

Ci $4.95 E+04$
$B B$

Sept98

$1.77 \mathrm{E}+02$

3.15E-00

3.38E +01

5.37E+04

9.95E-01

$2.73 E+02$

$1.99 \mathrm{E}+02$

1.72E-03

5.20E-02

1.35E-01

$1.56 \mathrm{E}+01$

1.25E-02

6.75E-06

$6.91 E+01$

$8.21 \mathrm{E}+02$

ncl. in 239

$1.48 \mathrm{E}+04$

5.53E-02

2.06E-07

9.01E-11

$4.91 \mathrm{E}+02$

$1.86 \mathrm{E}+03$

2.56E-02

$8.83 \mathrm{E}+01$

$2.63 \mathrm{E}+05$

$1.08 \mathrm{E}+02$

1.03E-08

3.45E- 11

4.00E-02

$1.36 \mathrm{E}+01$

1.13E-03

1.16E-05

5.09E-00

1.94E-01

4.19E-01

3.49E-00

$2.63 \mathrm{E}+05$

1.23E-01

$5.12 \mathrm{E}+04$

$0.00 \mathrm{E}+01$

$1.94 \mathrm{E}+03$

$3.49 \mathrm{E}+03$

$1.81 \mathrm{E}+04$

$6.18 \mathrm{E}+03$

$1.84 \mathrm{E}+03$

$7.36 \mathrm{E}+02$

$0.00 \mathrm{E}+01$

$3.53 E+03$

$0.00 \mathrm{E}+01$

$2.56 \mathrm{E}+02$

$3.76 E+05$

$5.10 \mathrm{E}+02$

$1.21 E+05$

$4.25 E+05$

NA

I. $84 \mathrm{E}+02$

$3.40 \mathrm{E}+04$

NA

4.15E +03

$3.36 E+04$

2.14E-00

$3.59 E+03$

$2.33 E+03$

$1.69 E+02$

$1.07 \mathrm{E}-03$

$2.50 \mathrm{E}+02$

2.27E-02

$4.95 E+04$ 1\%-ile

$3.28 \mathrm{E}-00$

$9.08 \mathrm{E}-00$

$0.00 \mathrm{E}+01$

$7.47 \mathrm{E}+03$

$1.45 \mathrm{E}-02$

$3.99 \mathrm{E}-00$

2.91E-00

$0.00 \mathrm{E}+01$

$0.00 \mathrm{E}+01$

$7.44 \mathrm{E}-03$

8.60E-01

2.33E-04

$0.00 \mathrm{E}+01$

$1.29 \mathrm{E}-00$

$1.53 \mathrm{E}+01$

$1.53 \mathrm{E}+01$

$2.75 \mathrm{E}+02$

1.03E-03

$0.00 E+01$

$0.00 \mathrm{E}+01$

8.38E-00

$0.00 \mathrm{E}+01$

$1.40 \mathrm{E}-04$

$1.29 \mathrm{E}-00$

$2.40 \mathrm{E}+02$

9.69E-01

$0.00 \mathrm{E}+01$

$0.00 \mathrm{E}+01$

$0.00 \mathrm{E}+01$

$6.68 \mathrm{E}-00$

$0.00 \mathrm{E}+01$

$0.00 \mathrm{E} \div 01$

$0.00 \mathrm{E}+01$

$0.00 \mathrm{E}+01$

$0.00 \mathrm{E}+01$

$0.00 \mathrm{E}+01$

$0.00 \mathrm{E}+01$

$0.00 \mathrm{E}+01$

$5.79 \mathrm{E}+01$

$0.00 \mathrm{E}+01$

$3.93 \mathrm{E}-00$

2.27E- 00

$3.38 \mathrm{E}+04$

$5.02 \mathrm{E}-02$

$2.00 \mathrm{E}+04$

$0.00 \mathrm{E}+01$

$0.00 \mathrm{E}+01$

$1.46 \mathrm{E}-00$

$2.56 \mathrm{E}-03$

$0.00 \mathrm{E}+01$

$1.16 \mathrm{E}+05$

2.92E- 03

$3.56 \mathrm{E}+01$

$1.63 \mathrm{E}+04$

6.72E-02

$5.76 \mathrm{E}-00$

$1.63 \mathrm{E}+02$

$1.25 \mathrm{E}-01$

1.53E-01

$1.17 \mathrm{E}+04$

2.18E-03

$2.12 E+03$

4.52E-02

$1.83 \mathrm{E}-02$

$0.00 E+01$

$0.00 \mathrm{E}+01$

$0.00 E+01$

$0.00 E+01$

$10 \%$-ile

4.16E+01

$3.65 \mathrm{E}+01$

$1.02 \mathrm{E}+01$

$3.17 \mathrm{E}+04$

$1.86 \mathrm{E}-01$

$5.09 \mathrm{E}+01$

3.71E+01

$5.72 \mathrm{E}-04$

$0.00 \mathrm{E}+01$

3.72E-02

4.30E-00

2.95E-03

$0.00 E+01$

$1.63 \mathrm{E}+01$

$1.94 \mathrm{E}+02$

$1.94 \mathrm{E}+02$

$3.49 \mathrm{E}+03$

$1.30 \mathrm{E}-02$

$7.26 \mathrm{E}-08$

$3.18 \mathrm{E}-11$

$1.05 \mathrm{E}+02$

$0.00 \mathrm{E}+01$

8.60E-03

$1.65 \mathrm{E}+01$

$6.84 \mathrm{E}+03$

$1.79 \mathrm{E}+01$

$0.00 E+01$

$0.00 \mathrm{E}+01$

$1.06 \mathrm{E}-02$

$9.60 \mathrm{E}-00$

$0.00 \mathrm{E}+01$

$0.00 \mathrm{E} \div 01$

$0.00 \mathrm{E}+01$

$0.00 \mathrm{E} \div 01$

$0.00 \mathrm{E}+01$

$0.00 \mathrm{E}+01$ 
Tank Anal. Un

AW106 C14
AW106 Cd113

AW106 Cm242

AW106 Cm243

AW106 Cm244

AW106 Co60

AW106 Cs134

AW106 Cs137

AW106 Eu152

AW106 Eu154

AW106 Eu155

AW106 1129

AW106 Nb93

AW106 Ni59

AW106 Ni63

AW106 Np237

AW106 Pa231

AW106 Pu238

AW106 Pu239

AW106 Pu240

AW106 Pu241

AW106 Pu242

AW106 Ra226

AW106 Ra228

AW106 Ru106

AW106 Sb125

AW106 Se79

AW106 Sm151

AW106 Sr89/90

AW106 Te99

AW106 Th229

AW106 Th232

AW106 Tin126

AW106 Tritium

AW106 U232

AW106 U233

AW106 U234

AW106 U235

AW106 U236

AW106 U238

AW106 Y90

AW106 Zr-93

AX101 Al

AX101 Bi

AX101 $\mathrm{Ca}$

AX101 Cl

AX101 CO3

AX101 $\mathrm{Cr}$

AX101 F

AX101 Fe

AX101 Hg

AX101 $\mathrm{K}$

AX101 La

Ax101 Mn

AX101 Na

AX101 Ni

AX101 NO2

AX101 NO3

AX101 P

AX101 Pb

AX101 PO4

AX101 S

AX101 Si

AX101 $\mathrm{SO}_{4}$

Ax101 Sr

AX101 TOC

AX101 U

AX101 Zr
$B B$

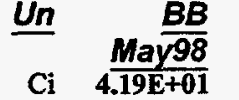

Ci $1.25 E+02$

Ci 4.94E-04

$\mathrm{Ci} \quad 9.96 \mathrm{E}-06$

Ci 2.99E-04

Ci $3.49 E+01$

Ci 3.51E-00

Ci 5.24E+04

Ci $\quad 6.61 \mathrm{E}-00$

Ci $\quad 9.24 \mathrm{E}+02$

Ci $\quad 3.86 \mathrm{E}+02$

Ci 3.99E-01

Ci 1.63E+01

Ci $2.77 \mathrm{E}-00$

Ci $2.77 \mathrm{E}+02$

Ci 7.14E-01

Ci 5.15E-03

Ci 2.84E-00

Ci $7.40 \mathrm{E}+01$

Ci Incl. in 239

Ci $1.94 E+02$

Ci 1.21E-03

Ci $1.83 \mathrm{E}-04$

Ci 3.99E-01

$\mathrm{Ci} \quad 1.21 \mathrm{E}-02$

Ci $\quad 2.84 \mathrm{E}+02$

Ci 4.60E-00

Ci $1.61 \mathrm{E}+04$

Ci $\quad 8.94 \mathrm{E}+04$

Ci $2.04 \mathrm{E}+02$

Ci $9.47 \mathrm{E}-03$

Ci 4.99E-02

Ci 6.95E-00

Ci 1.58E+02

Ci 1.07E-00

Ci 4.10E-00

Ci 1.40E-00

Ci 5.64E-02

$\mathrm{Ci}$ 4.81E-02

Ci 1.83E-00

Ci $\quad 8.94 E+04$

Ci $2.28 \mathrm{E}+01$

kg $9.29 \mathrm{E}+04$

kg 2.56E+03

kg 7.14E+03

kg 2.11E+04

kg $\quad 9.17 \mathrm{E}+04$

kg $1.81 \mathrm{E}+04$

kg 1.17E+03

kg 8.37E+03

kg 5.08E-00

kg 1.16E+04

kg $4.34 \mathrm{E}+02$

kg $\quad 6.09 \mathrm{E}+03$

kg 7.75E+05

$\mathrm{kg} \quad 1.19 \mathrm{E}+03$

kg $3.51 \mathrm{E}+05$

$\mathrm{kg} \quad 5.23 \mathrm{E}+05$

kg 4.37E+04

kg 3.50E +03

kg $1.35 \mathrm{E}+05$

kg $\quad 1.90 \mathrm{E}+04$

kg $\quad 5.80 \mathrm{E}+04$

kg $\quad 5.70 \mathrm{E}+04$

kg 1.61E+02

kg 4.09E+04

kg $\quad 4.91 \mathrm{E}+03$

kg $\quad 3.47 \mathrm{E}+03$
$B B$ Sept98 $\overline{4.19 \mathrm{E}+01}$ $1.25 \mathrm{E}+02$

4.16E-01

4.36E-02

$7.76 \mathrm{E}-01$

$3.49 \mathrm{E}+01$

3.51E-00

$5.24 \mathrm{E}+04$

6.61E-00

$9.24 E+02$

$3.86 \mathrm{E}+02$

3.99E-01

$1.63 E+01$

2.77E-00

$2.77 \mathrm{E}+02$

$7.14 \mathrm{E}-01$

$5.15 \mathrm{E}-03$

4.39E-00

$5.97 \mathrm{E}+01$

$1.42 \mathrm{E}+01$

$4.02 \mathrm{E}+02$

$1.73 \mathrm{E}-03$

$1.83 \mathrm{E}-04$

$3.99 \mathrm{E}-01$

1.21E-02

$2.84 \mathrm{E}+02$

4.60E-00

$1.61 \mathrm{E}+04$

$8.94 E+04$

$2.04 \mathrm{E}+02$

$9.47 \mathrm{E}-03$

4.99E-02

$6.95 \mathrm{E}-00$

$1.58 \mathrm{E}+02$

3.08E-01

$1.18 \mathrm{E}-00$

$1.07 \mathrm{E}-00$

4.09E-02

$7.68 \mathrm{E}-02$

$7.77 \mathrm{E}-01$

$8.94 \mathrm{E}+04$

$2.28 \mathrm{E}+01$

$1.24 \mathrm{E}+05$

$0.00 \mathrm{E}+01$

$6.90 \mathrm{E}+02$

$2.27 E+04$

$1.42 \mathrm{E}+05$

$3.43 E+03$

$1.38 \mathrm{E}+03$

$3.36 \mathrm{E}+03$

$0.00 \mathrm{E}+01$

$1.93 \mathrm{E}+04$

$0.00 E+01$

$0.00 E+01$

$8.39 E+05$

$0.00 \mathrm{E}+01$

$4.34 \mathrm{E}+05$

$6.87 E+05$

NA

$3.99 \mathrm{E}+02$

$1.76 \mathrm{E}+04$

NA

$5.63 E+02$

$3.59 \mathrm{E}+04$

$2.13 E+01$

$1.43 E+04$

$1.29 E+03$

$0.00 E+01$ 1\%-ile

$10 \%$-ile

$0.00 \mathrm{E}+01$

$0.00 E+01$

$0.00 \mathrm{E}+01$

$0.00 \mathrm{E}+01$

$0.00 \mathrm{E}+01$

$0.00 E+01$

$0.00 \mathrm{E}+01$

$3.36 \mathrm{E}-02$

$0.00 \mathrm{E}+01$

$0.00 \mathrm{E}+01$

$0.00 \mathrm{E}+01$

$0.00 \mathrm{E}+01$

$0.00 \mathrm{E}+01$

$0.00 \mathrm{E}+01$

$0.00 \mathrm{E}+01$

$0.00 \mathrm{E}+01$

$0.00 \mathrm{E}+01$

$0.00 \mathrm{E}+01$

$0.00 \mathrm{E}+01$

$0.00 \mathrm{E}+01$

$0.00 \mathrm{E}+01$

$0.00 E+01$

$0.00 \mathrm{E}+01$

$0.00 \mathrm{E}+01$

$0.00 \mathrm{E}+01$

$0.00 \mathrm{E}+01$

$3.93 \mathrm{E}-02$

$0.00 \mathrm{E}+01$

$6.48 \mathrm{E}+02$

$0.00 \mathrm{E}+01$

$0.00 \mathrm{E}+01$

$0.00 \mathrm{E}+01$

$0.00 \mathrm{E}+01$

$5.71 \mathrm{E}+01$

$0.00 \mathrm{E}+01$

$0.00 E+01$

$0.00 \mathrm{E}+01$

$0.00 \mathrm{E}+01$

$0.00 \mathrm{E}+01$

$0.00 \mathrm{E}+01$

2.14E+03

$0.00 \mathrm{E}+01$

$5.59 \mathrm{E}+03$

3.45E-02

$2.48 \mathrm{E}+02$

$2.50 \mathrm{E}+01$

$5.03 \mathrm{E}+02$

5.02E-00

$1.70 \mathrm{E}-01$

$2.33 \mathrm{E}+02$

$0.00 \mathrm{E}+01$

$1.20 \mathrm{E}+01$

$1.20 \mathrm{E}-02$

4.31E-0I

$3.00 \mathrm{E}+05$

$4.81 \mathrm{E}+01$

$4.37 \mathrm{E}+02$

1. $10 \mathrm{E}+02$

1.19E-00

1.24E-01

$5.22 \mathrm{E}-00$

$3.05 \mathrm{E}+01$

$6.65 \mathrm{E}+01$

$7.72 \mathrm{E}+01$

$1.38 \mathrm{E}-02$

1. $23 \mathrm{E}+02$

7.33E-00

4.48E-03

$5.27 \mathrm{E}-00$

$0.00 \mathrm{E}+01$

$3.99 \mathrm{E}-08$

8.04E-10

2.41E-08

9.43E-02

$0.00 \mathrm{E}+01$

$1.56 \mathrm{E}-00$

$0.00 \mathrm{E}+01$

$0.00 \mathrm{E}+01$

$0.00 \mathrm{E}+01$

$0.00 \mathrm{E}+01$ 
Tank Anal. Un

$\begin{array}{ll}\text { AX101 } & \text { Ac227 } \\ \text { AX101 } & \text { Am241 } \\ \text { AX101 } & \text { Am243 } \\ \text { AX101 } & \text { Ba137 } \\ \text { AX101 } & \text { C14 } \\ \text { AX101 } & \text { Cd113 } \\ \text { AX101 } & \text { Cm242 } \\ \text { AX101 } & \text { Cm243 } \\ \text { AX101 } & \text { Cm244 } \\ \text { AX101 } & \text { Co60 } \\ \text { AX101 } & \text { Cs134 } \\ \text { AX101 } & \text { Cs137 } \\ \text { AX101 } & \text { Eu152 } \\ \text { AX101 } & \text { Eu154 } \\ \text { AX101 } & \text { Eu155 } \\ \text { AX101 } & \text { I129 } \\ \text { AX101 } & \text { Nb93 } \\ \text { AX101 } & \text { Ni59 } \\ \text { AX101 } & \text { Ni63 } \\ \text { AX101 } & \text { Np237 } \\ \text { AX101 } & \text { Pa231 } \\ \text { AX101 } & \text { Pu238 } \\ \text { AX101 } & \text { Pu239 } \\ \text { AX101 } & \text { Pu240 }\end{array}$

AX101 Pu240

AX101 Pu241

AX101 Pu242

AX101 Ra226

AX101 Ra228

AX101 Ru106

AX101 Sb125

AX101 Se79

AX101 Sm151

AX101 Sr89/90

AX101 Te99

AX101 Th229

AX101 Th232

AX101 Tin126

AX101 Tritium

AX101 U232

AX101 U233

AX101 U234

AX101 U235

AX101 U236

AX101 U238

AX101 Y90

AX101 Zr93

AX102 Al

$\mathrm{AX102} \mathrm{Bi}$

AX102 Ca

$\mathrm{Ax} 102 \mathrm{Cl}$

$\mathrm{AX102} \mathrm{CO} 3$

AX102 Cr

AX102 F

$\mathrm{AX102} \mathrm{Fe}$

AX102 $\mathrm{Hg}$

AX102 K

AX102 La

AX102 Mn

AX102 Na

AX102 Ni

AX102 NO2

AX102 NO3

AX102 P

AX102 Pb

AX102 PO4

AX102 S

AX102 Si

AX102 504
$\frac{B B}{\text { May } 98}$

$2.94 \mathrm{E}+03$

8.60E-03

$5.71 E+05$

7.91E-00

$3.55 \mathrm{E}+01$

$1.37 \mathrm{E}-01$

1.24E-02

5.02E-01

$7.00 \mathrm{E}+02$

1.11E-01

$6.04 \mathrm{E}+05$

3.61E-00

$8.49 \mathrm{E}+01$

$2.21 \mathrm{E}+02$

1.03E-01

1.43E+01

6.93E-00

$6.81 \mathrm{E}+02$

$1.60 \mathrm{E}-03$

3.60E-03

5.42E-00

$5.02 E+03$

Incl. in 239

$3.84 \mathrm{E}+02$

2.30E-03

4.66E-04

2.11E-09

$3.00 \mathrm{E}-02$

9.19E-01

$3.86 \mathrm{E}-00$

$1.44 E+04$

$1.25 E+06$

$4.58 \mathrm{E}+02$

3.51E-07

2.85E-11

6.20E-00

9.43E-01

9.31E-08

Ci $1.31 \mathrm{E}-09$

Ci 3.90E-04

$\mathrm{Ci} \quad$ 1.52E-05

Ci 2.57E-05

3.12E-04

Ci $1.25 \mathrm{E}+06$

Ci $1.67 \mathrm{E}+01$

$\mathrm{kg} \quad 3.26 \mathrm{E}+03$

kg 3.09E+01

$\mathrm{kg} \quad 3.00 \mathrm{E}+02$

ig 3.30E +02

kg 1.01E+04

kg $\quad 1.60 \mathrm{E}+02$

kg $1.80 \mathrm{E}+02$

kg 4.09E+03

kg 2.27E-01

kg 2.00E +02

kg 4.61E-01

kg $3.80 \mathrm{E}+02$

kg 3.03E+04

kg $1.90 \mathrm{E}+02$

kg $7.30 \mathrm{E}+03$

kg 2.01E+04

kg 6.23E+02

$\mathrm{kg} \quad 3.95 \mathrm{E}+01$

kg $1.93 \mathrm{E}+03$

$\mathrm{kg} \quad 2.76 \mathrm{E}+03$

kg $6.60 \mathrm{E}+02$

kg

\section{Sept98}

$\frac{\text { Sept98 }}{4.66 \mathrm{E}-03}$

$4.38 \mathrm{E}+02$

1.79E-02

$7.11 \mathrm{E}+05$

$6.00 \mathrm{E}-00$

$2.98 \mathrm{E}+02$

$6.60 \mathrm{E}-01$

$5.90 \mathrm{E}-02$

1.08E-00

$1.14 \mathrm{E}+02$

$1.10 \mathrm{E}+01$

$7.52 \mathrm{E}+05$

$1.66 \mathrm{E}+01$

$5.32 \mathrm{E}+02$

$1.58 \mathrm{E}+03$

2.00E-02

$4.83 \mathrm{E}+01$

$1.19 \mathrm{E}+01$

$1.17 \mathrm{E}+03$

2.59E-00

$1.46 \mathrm{E}-02$

$1.40 \mathrm{E}+01$

$3.99 E+02$

$7.28 \mathrm{E}+01$

$9.87 \mathrm{E}+02$

$5.66 \mathrm{E}-03$

8.29E-04

1.02E-00

$5.00 \mathrm{E}-02$

$5.62 \mathrm{E}+02$

$1.35 \mathrm{E}+01$

$4.82 \mathrm{E}+04$

$1.36 \mathrm{E}+05$

$2.67 \mathrm{E}+02$

2.35E-02

1.10E-0I

$2.07 \mathrm{E}+01$

$6.20 \mathrm{E}+02$

$7.89 \mathrm{E}-01$

3.02E-00

4.84E-01

$1.92 \mathrm{E}-02$

$1.56 \mathrm{E}-02$

4.31E-01

$1.36 \mathrm{E}+05$

$6.41 E+01$

$3.49 \mathrm{E}+03$

$3.26 \mathrm{E}+01$

$2.95 \mathrm{E}+02$

$3.29 \mathrm{E}+02$

$1.02 \mathrm{E}+04$

$1.60 \mathrm{E}+02$

$1.77 \mathrm{E}+02$

$4.09 \mathrm{E}+03$

$0.00 \mathrm{E}+01$

$2.00 \mathrm{E}+02$

4.52E-01

$3.77 \mathrm{E}+02$

$3.16 E+04$

$2.17 E+02$

$7.21 \mathrm{E}+03$

$2.26 \mathrm{E}+04$

NA

$3.92 \mathrm{E}+01$

$6.25 \mathrm{E}+02$

NA

$1.21 \mathrm{E}+03$

$2.77 \mathrm{E}+03$ 1\%-ile

\section{$0.00 \mathrm{E}+01$}

$0.00 \mathrm{E}+01$

5.61E-05

$0.00 \mathrm{E}+01$

$0.00 \mathrm{E}+01$

$0.00 \mathrm{E}+01$

8.94E-04

8.09E-05

3.27E-03

7.79E-02

$0.00 \mathrm{E}+01$

$9.90 \mathrm{E}+03$

$0.00 \mathrm{E}+01$

$0.00 \mathrm{E}+01$

$0.00 \mathrm{E}+01$

$0.00 \mathrm{E}+0 \mathrm{I}$

$0.00 \mathrm{E}+0 \mathrm{l}$

$0.00 \mathrm{E}+0 \mathrm{l}$

$0.00 \mathrm{E}+01$

1.04E-05

$0.00 \mathrm{E}+01$

$3.54 \mathrm{E}-02$

$3.27 \mathrm{E}+0 \mathrm{l}$

$3.27 \mathrm{E}+01$

2.50E-00

1.50E-05

$0.00 \mathrm{E}+0 \mathrm{I}$

$0.00 \mathrm{E}+0 \mathrm{l}$

$0.00 \mathrm{E}+0 \mathrm{I}$

$0.00 \mathrm{E}+0 \mathrm{I}$

$0.00 \mathrm{E}+0 \mathrm{I}$

$0.00 \mathrm{E}+0 \mathrm{l}$

$8.74 \mathrm{E}+03$

$0.00 \mathrm{E}+01$

$0.00 \mathrm{E}+01$

$0.00 \mathrm{E}+0 \mathrm{I}$

$0.00 \mathrm{E}+01$

2.32E-0I

$0.00 \mathrm{E}+01$

$0.00 \mathrm{E}+01$

$0.00 \mathrm{E}+01$

$0.00 \mathrm{E}+01$

$0.00 \mathrm{E}+01$

$0.00 \mathrm{E}+01$

$0.00 \mathrm{E}+01$

$0.00 \mathrm{E}+01$

4.93E-05

2.84E-09

3.65E-06

1.36E-05

I.14E-04

7.11E-06

6.78E-09

$5.46 \mathrm{E}-06$

$0.00 \mathrm{E} \div 0 \mathrm{I}$

8.55E-07.

3.63E-09

4.45E-07

8.58E-04

8.00E-07

1.49E-04

9.07E-04

1.08E-05

5.81E-08

3.62E-05

4.24E-06

2.75E-06

$2.58 \mathrm{E}-05$

10\%-ile 50\%-ile

\section{$0.00 \mathrm{E}+01$}

2.13E-01

8.00E-04

$1.23 \mathrm{E}+04$

$0.00 \mathrm{E}+01$

$0.00 \mathrm{E}+01$

$1.28 \mathrm{E}-02$

$1.15 \mathrm{E}-03$

4.67E-02

$1.44 \mathrm{E}-00$

$2.40 \mathrm{E}-03$

$8.78 \mathrm{E}+04$

$0.00 E+01$

$0.00 \mathrm{E}+01$

$0.00 \mathrm{E}+01$

$0.00 \mathrm{E}+01$

$0.00 \mathrm{E}+01$

$0.00 \mathrm{E}+01$

$0.00 \mathrm{E}+01$

$1.49 \mathrm{E}-04$

$0.00 \mathrm{E}+01$

$5.04 \mathrm{E}-01$

4. $67 \mathrm{E}+02$

4.67E+02

$3.57 \mathrm{E}+01$

2.14E-04

$0.00 E+01$

$0.00 \mathrm{E}+01$

$0.00 \mathrm{E}+01$

$0.00 \mathrm{E}+01$

$0.00 \mathrm{E}+01$

$0.00 \mathrm{E}+01$

$2.41 E+05$ 
Tank Anal. Un

$\begin{array}{ll}\text { AX102 } & \text { Sr } \\ \text { AX102 } & \text { TOC } \\ \text { AX102 } & \text { U } \\ \text { AX102 } & \text { Zr } \\ \text { AX102 } & \text { Ac227 } \\ \text { AX102 } & \text { Am241 } \\ \text { AX102 } & \text { Am243 } \\ \text { AX102 } & \text { Ba137 } \\ \text { AX102 } & \text { C14 } \\ \text { AX102 } & \text { Cd113 } \\ \text { AX102 } & \text { Cm242 } \\ \text { AX102 } & \text { Cm243 } \\ \text { AX102 } & \text { Cm244 } \\ \text { AX102 } & \text { Co60 } \\ \text { AX102 } & \text { Cs134 } \\ \text { AX102 } & \text { Cs137 } \\ \text { AX102 } & \text { Eu152 } \\ \text { AX102 } & \text { Eu154 } \\ \text { AX102 } & \text { Eu155 } \\ \text { AX102 } & \mathbf{I 1 2 9}\end{array}$

AX102 1129

AX102 Nb93

AX102 Ni59

AX102 Ni63

AX102 Np237

AX102 Pa231

AX102 Pu238

AX102 Pu239

AX102 Pu240

AX102 Pu241

AX102 Pu242

AX102 Ra226

AX102 Ra228

AX102 Ru106

AX102 Sb125

AX102 Se79

AX102 Sm151

AX102 $\mathbf{5 r 8 9 / 9 0}$

AX102 Te99

AX102 Th229

AX102 Th232

AX102 Tin126

AX102 Tritium AX102 U232

AX102 U233

AX102 U234

AX102 U235

AX102 U236

AX102 U238

AX102 Y90

AX102 Zr93

AX103 Al

AX103 Bi

AX103 Ca

AX103 Cl

$\mathrm{AX}_{103 \mathrm{CO}}$

AX103 Cr

AX103 F

AX103 Fe

AX103 Hg

AX103 K

AX103 La

AX103 Mn

AX103 $\mathrm{Na}$

AX103 Ni

AX103 NO2

AX103 NO3

AX103 P

AX103 Pb
May $\frac{B B}{98}$

9.70E-02

$3.29 \mathrm{E}+03$

$4.20 \mathrm{E}+02$

7.90E-00

$1.14 \mathrm{E}-03$

$1.33 \mathrm{E}+03$

$1.48 \mathrm{E}-01$

$4.92 \mathrm{E}+04$

5.10E-00

$1.17 \mathrm{E}+02$

$1.79 \mathrm{E}-00$

2.18E-01

8.96E-00

$6.61 \mathrm{E}-00$

5.04E-01

$5.20 \mathrm{E}+04$

$2.11 E+01$

$1.66 \mathrm{E}+03$

$1.03 E+03$

$7.28 \mathrm{E}-02$

$1.56 \mathrm{E}+01$

$1.57 \mathrm{E}-00$

$1.61 \mathrm{E}+02$

$1.33 \mathrm{E}-01$

5.91E-04

$6.07 \mathrm{E}+01$

$4.15 \mathrm{E}+02$

$1.48 \mathrm{E}+02$

$4.20 \mathrm{E}+03$

3.03E-02

2.34E-04

3.79E-02

$5.85 E-01$

$3.08 \mathrm{E}+01$

$5.29 \mathrm{E}-00$

$1.55 \mathrm{E}+04$

$2.68 \mathrm{E}+05$

$3.77 \mathrm{E}+01$

8.82E-04

$3.86 \mathrm{E}-03$

8.35E-00

$3.24 \mathrm{E}+01$

1.26E-01

4.82E-01

$9.90 \mathrm{E}-02$

$3.96 \mathrm{E}-03$

3.23E-03

$1.27 \mathrm{E}-01$

$2.68 \mathrm{E}+05$

$2.33 \mathrm{E}+01$

$1.50 \mathrm{E}+04$

$2.81 \mathrm{E}+02$

$2.45 \mathrm{E}+03$

$2.55 \mathrm{E}+03$

$1.00 \mathrm{E}+04$

$2.35 \mathrm{E}+03$

$1.28 \mathrm{E}+02$

$2.16 \mathrm{E}+04$

5.89E-01

$1.37 \mathrm{E}+03$

$4.75 \mathrm{E}+01$

$1.06 \mathrm{E}+03$

$9.83 E+04$

$1.08 \mathrm{E}+03$

$3.86 \mathrm{E}+04$

$6.30 \mathrm{E}+04$

$4.89 \mathrm{E}+03$

$3.89 \mathrm{E}+02$
$\frac{B B}{408}$ Sept98

$\overline{1.32 E+02}$

$3.29 \mathrm{E}+03$

$2.35 \mathrm{E}+02$

2.08E-60

$1.14 \mathrm{E}-03$

$1.33 E+03$

1.48E-01

$5.27 \mathrm{E}+04$

5.10E-00

$1.17 \mathrm{E}+02$

1.79E-00

2.18E-01

8.96E-00

6.61E-00

5.04E-01

$5.57 \mathrm{E}+04$

$2.11 E+01$

$1.66 \mathrm{E}+03$

$1.03 E+03$

$7.28 \mathrm{E}-02$

$1.56 \mathrm{E}+01$

$1.57 \mathrm{E}-00$

$1.61 \mathrm{E}+02$

1.33E-01

5.91E-04

$6.07 \mathrm{E}+01$

$4.15 \mathrm{E}+02$

$1.48 \mathrm{E}+02$

$4.20 \mathrm{E}+03$

3.03E-02

2.34E-04

3.79E-02

5.85E-01

$3.08 \mathrm{E}+01$

$5.29 \mathrm{E}-00$

$1.55 \mathrm{E}+04$

$1.72 \mathrm{E}+05$

$3.77 \mathrm{E}+01$

8.82E-04

$3.86 \mathrm{E}-03$

8.35E-00

$3.24 \mathrm{E}+01$

1.11E-01

4.26E-01

$8.75 \mathrm{E}-02$

$3.50 \mathrm{E}-03$

$2.86 \mathrm{E}-03$

7.84E-02

$1.72 \mathrm{E}+05$

$2.33 \mathrm{E}+01$

$1.22 \mathrm{E}+04$

$8.58 \mathrm{E}+01$

$2.44 \mathrm{E}+03$

$2.55 \mathrm{E}+03$

$1.02 \mathrm{E}+04$

$9.43 E+02$

$3.78 \mathrm{E}+02$

$1.87 \mathrm{E}+04$

$0.00 \mathrm{E}+01$

$1.23 E \div 03$

$9.04 \mathrm{E}-01$

$4.60 E+02$

$1.06 E+05$

$1.00 \mathrm{E}+03$

$3.53 E+04$

$5.52 \mathrm{E}+04$

NA

$1.77 \mathrm{E}+02$

1\%-ile

$10 \%$-ile

$4.25 \mathrm{E}-10$
$7.09 \mathrm{E}-06$

$7.41 \mathrm{E}-07 \quad 9.47 \mathrm{E}-06$

$2.73 \mathrm{E}-10$

$1.92 \mathrm{E}-06$

$0.00 E+01$

$0.00 \mathrm{E}+0 \mathrm{I}$

$0.00 \mathrm{E}+01$

$7.18 \mathrm{E}-05$

$0.00 \mathrm{E}+01 \quad 6.21 \mathrm{E}-08$

$0.00 \mathrm{E}+01 \quad 0.00 \mathrm{E}+01$

$0.00 \mathrm{E}+01 \quad 0.00 \mathrm{E}+01$

$0.00 \mathrm{E}+01 \quad 0.00 \mathrm{E}+01$

$0.00 \mathrm{E}+01 \quad 7.51 \mathrm{E}-07$

$0.00 \mathrm{E}+01$

$0.00 \mathrm{E}+01$

2.39E-08

$9.15 \mathrm{E}-08$

3.76E-06

4.19E-07

$0.00 \mathrm{E}+01$

$3.68 \mathrm{E}-04$

$0.00 \mathrm{E}+01$

$0.00 \mathrm{E}+01$

$0.00 \mathrm{E}+01$

$0.00 \mathrm{E}+01$

$0.00 \mathrm{E}+0 \mathrm{I}$

$0.00 E+01$

$0.00 \mathrm{E}+01$

$0.00 E+01$

$0.00 \mathrm{E}+0 \mathrm{I}$

$0.00 \mathrm{E}+01$

$0.00 \mathrm{E}+01$

$0.00 E+01$

$0.00 \mathrm{E}+01$

$0.00 E+01$

$0.00 \mathrm{E}+01$

$0.00 \mathrm{E}+01$

$0.00 \mathrm{E}+01$

$0.00 \mathrm{E}+01$

$0.00 \mathrm{E}+01$

$0.00 E+01$

5.53E-05

$0.00 \mathrm{E}+01$

$0.00 \mathrm{E}+01$

$0.00 \mathrm{E}+01$

$0.00 E+01$

$0.00 E+01$

$0.00 E+01$

$0.00 \mathrm{E}+01$

$0.00 E+01$

$0.00 E+01$

$0.00 E+01$

$0.00 E+01$

$0.00 E+01$

$0.00 \mathrm{E}+01$

$6.26 \mathrm{E}+03$

2.10E-02

1.39E+02

$2.22 \mathrm{E}+03$

$5.80 \mathrm{E}+03$

$9.03 \mathrm{E}+02$

$5.85 \mathrm{E}-02$

$5.44 \mathrm{E}+01$

$0.00 \mathrm{E}+01$

$8.68 \mathrm{E}-00$

$3.85 \mathrm{E}-03$

4.57E-01

$9.67 \mathrm{E}+04$

$0.00 \mathrm{E}+01$

$1.40 \mathrm{E}-03$

$0.00 \mathrm{E}+01$

$0.00 \mathrm{E}+01$

$0.00 \mathrm{E}+01$

$0.00 \mathrm{E}+01$

$0.00 \mathrm{E}+01$

$0.00 \mathrm{E}+01$

$0.00 \mathrm{E}+01$

$5.58 \mathrm{E}-08$

$0.00 \mathrm{E}+01$

$2.55 \mathrm{E}-05$

1.74E-04

6.21E-05

$1.76 \mathrm{E}-03$

1.27E-08

$0.00 \mathrm{E}+01$

$0.00 \mathrm{E}+01$

$0.00 \mathrm{E}+01$

$0.00 \mathrm{E}+01$

$0.00 \mathrm{E}+01$

$0.00 \mathrm{E}+01$

9.47E-03

$0.00 \mathrm{E}+01$

$0.00 \mathrm{E}+01$

$0.00 \mathrm{E}+01$

$0.00 \mathrm{E}+01$

$0.00 \mathrm{E}+01$

$0.00 E+01$

$0.00 \mathrm{E}+01$ 
Tank Anal. Un

$\begin{array}{ll}\text { AX103 } & \text { PO4 } \\ \text { AX103 } & \text { S } \\ \text { AX103 } & \text { Si } \\ \text { AX103 } & \text { SO4 } \\ \text { AX103 } & \text { Sr } \\ \text { AX103 } & \text { TOC } \\ \text { AX103 } & \text { U } \\ \text { AX103 } & \text { Zr } \\ \text { AX103 } & \text { Ac227 } \\ \text { AX103 } & \text { Am241 } \\ \text { AX103 } & \text { Am243 } \\ \text { AX103 } & \text { Ba137 } \\ \text { AX103 } & \text { C14 } \\ \text { AX103 } & \text { Cd113 } \\ \text { AX103 } & \text { Cm242 } \\ \text { AX103 } & \text { Cm243 } \\ \text { AX103 } & \text { Cm244 } \\ \text { AX103 } & \text { Co60 } \\ \text { AX103 } & \text { Cs134 } \\ \text { AX103 } & \text { Cs137 } \\ \text { AX103 } & \text { Eu152 } \\ \text { AX103 } & \text { Eu154 } \\ \text { AX103 } & \text { Eu155 } \\ \text { AX103 } & \text { i129 } \\ \text { AX103 }\end{array}$

AX103 Nb93

AX103 Ni59

AX103 Ni63

AX103 Np237

AX103 Pa231

AX103 Pu238

AX103 Pu239

AX103 Pu240

AX103 Pu241

AX103 Pu242

AX103 R2226

AX103 Ra228

AX103 Ru106

AX103 Sb125

AX103 Se79

$\begin{array}{ll}\text { AX103 } & \text { Sm151 } \\ \text { AX103 } & \text { Sr89/90 }\end{array}$

AX103 Tc99

AX103 Th229

AX103 Th232

AX103 Tin126

AX103 Tritium

AX103 U232

AX103 U233

AX103 U234

AX103 U235

AX103 U236

AX103 U238

AX103 Y90

AX103 Zr93

AX104 Al

AX104 Bi

AX104 Ca

AX104 Cl

$\mathrm{AX104}_{\mathrm{CO} 3}$

AX104 Cr

AX104 F

AX104 Fe

AX104 Hg

Ax104 K

AX104 La

AX104 Mn

AX104 Na

AX104 Ni

\section{May98}

$\begin{array}{ll}\mathrm{kg} & 1.51 \mathrm{E}+04 \\ \mathrm{~kg} & 2.88 \mathrm{E}+03\end{array}$

kg $9.24 \mathrm{E}+03$

$\mathrm{kg} \quad 8.64 \mathrm{E}+03$

$\mathrm{kg} 1.94 \mathrm{E}+01$

kg $4.73 \mathrm{E}+03$

$\mathrm{kg} \quad 4.58 \mathrm{E}+03$

kg 3.72E+02

Ci 2.40E-03

$2.71 E+02$

7.00E-03

$1.27 \mathrm{E}+05$

Ci $\quad 9.80 \mathrm{E}-01$

Ci $1.47 \mathrm{E}+02$

Ci 2.40E-01

$1.90 \mathrm{E}-02$

$4.80 \mathrm{E}-01$

$8.46 \mathrm{E}+02$

$1.60 \mathrm{E}-00$

$1.34 \mathrm{E}+05$

8.21E-00

$4.40 \mathrm{E}+03$

$4.20 \mathrm{E}+03$

$1.50 \mathrm{E}-02$

$2.44 E+01$

6.83E-00

$6.85 \mathrm{E}+02$

$3.20 \mathrm{E}-01$

$6.00 \mathrm{E}-03$

5.45E-00

$1.32 \mathrm{E}+03$

Ci Incl. in 239

$3.87 \mathrm{E}+02$

$2.20 \mathrm{E}-03$

4.36E-04

1.10E-01

2.80E-02

$6.65 \mathrm{E}+02$

7.04E-00

$2.60 \mathrm{E}+04$

$3.82 \mathrm{E}+06$

$5.58 \mathrm{E}+01$

3.00E-03

$1.20 \mathrm{E}-02$

$1.09 \mathrm{E}+01$

$7.98 \mathrm{E}+01$

$3.40 \mathrm{E}-01$

1.31E-00

2.20E-01

$9.00 \mathrm{E}-03$

$7.00 \mathrm{E}-03$

3.05E-01

$3.82 \mathrm{E}+06$

$3.27 \mathrm{E}+01$

$1.78 \mathrm{E}+03$

$0.00 E+01$

$6.84 \mathrm{E}+02$

$1.09 \mathrm{E}+02$

$3.86 E+02$

$8.40 E+01$

$0.00 \mathrm{E}+01$

$7.89 \mathrm{E}+03$

$0.00 \mathrm{E}+01$

$\mathrm{kg} \quad 3.52 \mathrm{E}+03$

$0.00 \mathrm{E}+01$

kg $\quad 1.23 \mathrm{E}+02$

$2.45 \mathrm{E}+03$

kg $\frac{\text { Sept98 }}{3.89 E+03}$

$\mathrm{NA}$
$4 \mathrm{E}+03$

$9.74 \mathrm{E}+03$
$8.81 \mathrm{E}+03$

5.82E-00

$3.83 \mathrm{E}+03$

$7.16 E+02$

$1.02 \mathrm{E}+02$

2.38E-03

$5.82 \mathrm{E}+01$

$1.83 \mathrm{E}-03$

$1.50 \mathrm{E}+05$

$1.25 \mathrm{E}-00$

$1.47 \mathrm{E}+02$

$6.16 \mathrm{E}-02$

4.95E-03

$1.25 \mathrm{E}-01$

$8.32 \mathrm{E}+02$

$1.60 \mathrm{E}-00$

$1.59 \mathrm{E}+05$

8.21E-00

$4.40 \mathrm{E}+03$

$4.20 \mathrm{E}+03$

$9.24 \mathrm{E}-03$

$2.44 E+01$

$6.83 \mathrm{E}-00$

$6.85 \mathrm{E}+02$

$3.20 \mathrm{E}-01$

$6.05 \mathrm{E}-03$

$2.82 \mathrm{E}+01$

$7.46 \mathrm{E}+02$

$1.41 \mathrm{E}+02$

$2.00 \mathrm{E}+03$

$1.15 \mathrm{E}-02$

$4.36 \mathrm{E}-04$

1.14E-01

2.28E-02

$6.65 E+02$

$7.04 \mathrm{E}-00$

$2.60 \mathrm{E}+04$

$8.92 E+05$

$5.69 \mathrm{E}+01$

2.65E-03

$1.23 \mathrm{E}-02$

$1.09 E+01$

$7.98 \mathrm{E}+01$

4.12E-01

$1.58 \mathrm{E}-00$

2.68E-01

1.06E-02

8.58E-03

2.39E-01

$8.92 \mathrm{E}+05$

$3.27 \mathrm{E}+01$

$1.77 \mathrm{E}+03$

$0.00 \mathrm{E}+01$

$6.80 \mathrm{E}+02$

$7.32 \mathrm{E}+01$

$3.86 \mathrm{E}+02$

$8.40 \mathrm{E}+01$

$0.00 \mathrm{E}+01$

$7.85 \mathrm{E}+03$

$0.00 \mathrm{E}+01$

$2.93 \mathrm{E}+03$

$0.00 \mathrm{E}+01$

$1.23 E+02$

$2.45 \mathrm{E}+03$

$4.06 \mathrm{E}+02$
$1 \%$-ile

$4.87 \mathrm{E}-00$

$3.37 \mathrm{E}+02$

$5.97 \mathrm{E}-00$

$.27 \mathrm{E}+02$

$2.90 \mathrm{E}-03$

$1.06 \mathrm{E}+03$

$1.07 \mathrm{E}+01$

$1.88 \mathrm{E}-03$

$0.00 \mathrm{E}+01$

6.42E-05

8.48E-06

$0.00 \mathrm{E}+0 \mathrm{l}$

$0.00 \mathrm{E}+01$

$0.00 \mathrm{E}+01$

2.91E-04

2.30E-05

5.82E-04

3.19E-03

$0.00 \mathrm{E}+01$

4.37E+03

$0.00 \mathrm{E}+01$

$0.00 \mathrm{E}+01$

$0.00 \mathrm{E}+01$

$1.46 \mathrm{E}-03$

$0.00 \mathrm{E}+01$

4.41E-01

$4.42 \mathrm{E}+0$

$3.88 \mathrm{E}-04$

$0.00 \mathrm{E}+01$

6.61E-03

$1.60 \mathrm{E}-00$

$1.60 \mathrm{E}-00$

4.69E-0]

2.67E-06

$0.00 \mathrm{E}+01$

$0.00 \mathrm{E}+01$

$0.00 \mathrm{E}+01$

$0.00 \mathrm{E}+01$

$0.00 \mathrm{E}+01$

$0.00 \mathrm{E}+01$

$3.20 \mathrm{E}+02$

$0.00 \mathrm{E}+01$

$0.00 \mathrm{E}+01$

$0.00 \mathrm{E}+01$

$0.00 \mathrm{E}+01$

$5.77 \mathrm{E}+01$

$0.00 \mathrm{E}+01$

$0.00 \mathrm{E}+01$

$0.00 \mathrm{E}+01$

$0.00 \mathrm{E}+01$

$0.00 \mathrm{E}+01$

$0.00 E+01$

$0.00 E+01$

$0.00 E+01$

1.33E-07

$1.58 \mathrm{E}-09$

2.27E-06

5.93E-07

$7.86 \mathrm{E}-06$

6.18E-08

2.33E-09

2.16E-06

$0.00 E+01$

$7.91 \mathrm{E}-08$

4.20E-10

3.51E-09

7.70E-05

8.33E-07

$10 \%$-ile

$2.00 \mathrm{E}+01$

$1.66 \mathrm{E}+03$

$2.70 \mathrm{E}+02$

$3.30 \mathrm{E}+03$

3.52E-02

$1.84 \mathrm{E}+03$

$6.66 \mathrm{E}+01$

$1.13 E-02$

$0.00 \mathrm{E}+01$

5.45E-02

4.30E-04

$3.18 \mathrm{E}+04$

4.05E-02

$0.00 \mathrm{E}+01$

1.47E-02

1.17E-03

2.95E-02

9.61E-01

4.01E-01

$3.64 \mathrm{E}+04$

$0.00 \mathrm{E}+01$

$0.00 \mathrm{E}+01$

$0.00 \mathrm{E}+01$

$7.42 \mathrm{E}-03$

$0.00 \mathrm{E}+01$

1.95E-00

$1.96 \mathrm{E}+02$

1.97E-02

$0.00 \mathrm{E}+01$

3.35E-01

8.11E+01

$8.11 E+01$

$2.38 \mathrm{E}+01$

I. $35 \mathrm{E}-04$

$0.00 E+01$

$0.00 \mathrm{E}+01$

4.01E-03

$0.00 \mathrm{E}+01$ 
Tank Anal. Un

$\begin{array}{ll}\text { AX104 } & \text { NO2 } \\ \text { AX104 } & \text { NO3 } \\ \text { AX104 } & \text { P } \\ \text { AX104 } & \text { Pb } \\ \text { AX104 } & \text { PO4 } \\ \text { AX104 } & \text { S } \\ \text { AX104 } & \text { Si } \\ \text { AX104 } & \text { SO4 } \\ \text { AX104 } & \text { Sr } \\ \text { AX104 } & \text { TOC } \\ \text { AX104 } & \text { U } \\ \text { AX104 } & \text { Zr } \\ \text { AX104 } & \text { Ac227 } \\ \text { AX104 } & \text { Am241 } \\ \text { AX104 } & \text { Am243 } \\ \text { AX104 } & \text { Ba137 } \\ \text { AX104 } & \text { C14 } \\ \text { AX104 } & \text { Cd113 } \\ \text { AX104 } & \text { Cm242 } \\ \text { AX104 } & \text { Cm243 } \\ \text { AX104 } & \text { Cm244 } \\ \text { AX104 } & \text { Co60 } \\ \text { AX104 } & \text { Cs134 } \\ \text { AX104 } & \text { Cs137 } \\ \text { AX104 } & \text { Eu152 } \\ \text { AX104 } & \text { Eu154 } \\ \text { AX104 } & \text { Eu155 } \\ \text { AX104 } & \text { I129 } \\ \text { AX104 } & \text { Nb93 } \\ \text { AX104 } & \text { Ni59 } \\ \text { AX104 } & \text { Ni63 } \\ \text { AX104 } & \text { Np237 } \\ \text { AX104 } & \text { Pa231 } \\ \text { AX104 } & \text { Pu238 } \\ \text { AX104 } & \text { Pu239 } \\ \text { AX104 } & \text { Pu240 } \\ \text { AX104 } & \text { Pu241 } \\ \text { AX104 } & \text { Pu242 } \\ \text { AX104 } & \text { Ra226 } \\ \text { AX104 } & \text { Ra228 } \\ \text { AX104 } & \text { Ru106 } \\ \text { AX104 } & \text { Su125 } \\ \text { AX104 } & \text { S125 } \\ \text { AX104 } & \text { Se79 } \\ \text { AX104 } & \text { Sm151 } \\ \text { AX104 } & \text { Sr89/90 } \\ \text { AX104 } & \text { Tc99 } \\ \text { AX104 } & \text { Th229 } \\ \text { AX104 } & \text { Th232 } \\ \text { AX104 } & \text { Tin126 } \\ \text { AX104 } & \text { Tritium } \\ \text { AX104 } & \text { U232 } \\ \text { AX104 } & \text { U233 } \\ \text { AX104 } & \text { U234 } \\ \text { AX104 } & \text { U235 } \\ \text { AX104 } & \text { U236 } \\ \text { AX104 } & \text { U238 } \\ \text { AX104 } & \text { Y90 } \\ \text { AX104 } & \text { Zr93 } \\ \text { AY101 } & \text { Al } \\ \text { AY101 } & \text { Bi } \\ \text { AY101 } & \text { Ca } \\ \text { AY101 } & \text { Cl } \\ \text { AY101 } & \text { CO3 } \\ \text { AY101 } & \text { Cr } \\ \text { AY101 } & \text { F } \\ \text { AY101 } & \text { Fe } \\ \text { AY101 } & \text { Hg } \\ \text { AY10 } & \text { K } \\ \text { a }\end{array}$

AY101 $K$
BB May98 kg $\overline{1.02 \mathrm{E}+02}$ kg $\quad 2.03 \mathrm{E}+03$ kg $2.75 \mathrm{E}+02$

$\mathrm{kg} 1.09 \mathrm{E}+02$

kg $8.52 \mathrm{E}+02$

kg $3.45 \mathrm{E}+02$

kg $\quad 1.86 \mathrm{E}+03$

$\mathrm{kg} \quad 1.04 \mathrm{E}+03$

kg $2.90 \mathrm{E}+01$

$\mathrm{kg} \quad 0.00 \mathrm{E}+01$

$\mathrm{kg}$ 1.10E-01

kg $0.00 \mathrm{E}+01$

Ci $0.00 \mathrm{E}+01$

$1.02 \mathrm{E}+02$

$3.10 \mathrm{E}-03$

$3.08 \mathrm{E}+04$

6.30E-01

$5.82 \mathrm{E}+01$

$9.29 \mathrm{E}-02$

7.10E-03

2.19E-01

Ci $3.47 \mathrm{E}+02$

Ci $2.07 \mathrm{E}-01$

Ci $3.26 \mathrm{E}+04$

Ci 3.36E-00

Ci $1.87 \mathrm{E}+03$

Ci $1.79 \mathrm{E}+03$

Ci $8.60 \mathrm{E}-03$

Ci $1.02 \mathrm{E}+01$

Ci 3.12E-00

Ci $3.13 \mathrm{E}+02$

Ci 9.50E-03

Ci 2.40E-03

Ci 2.50E-00

Ci $4.15 \mathrm{E}+02$

Ci 1.23E+01

Ci $1.78 \mathrm{E}+02$

Ci 1.00E-03

Ci 1.96E-04

Ci 1.77E-09

Ci 1.00E-02

Ci $2.83 E+02$

Ci $2.95 \mathrm{E}-00$

Ci 1.10E+04

C

$\mathrm{Ci}$

$\mathrm{Ci}$

C

$\mathrm{Ci}$

C

$\mathrm{Ci} 270 \mathrm{E}-\mathrm{A3}$

Ci 1.14E-04

$\mathrm{Ci} \quad 7.47 \mathrm{E}-05$

Ci 2.70E-03

Ci $1.58 \mathrm{E}+06$

Ci $1.36 \mathrm{E}+01$

kg $1.88 \mathrm{E}+04$

kg $1.55 \mathrm{E}+02$

kg $3.64 \mathrm{E}+03$

kg 2.04E+03

kg $\quad 1.20 \mathrm{E}+05$

kg 7.25E+03

kg $5.58 \mathrm{E}+02$

kg $4.28 \mathrm{E}+04$

kg 1.15E-00

kg $1.10 E+03$
BB Sept98

$1.02 \mathrm{E}+02$

$2.02 \mathrm{E}+03$

NA

$9.38 \mathrm{E}+01$

$2.76 \mathrm{E}+02$

NA

$1.85 \mathrm{E}+03$

$3.43 E+02$

$2.60 \mathrm{E}+01$

$0.00 \mathrm{E}+01$

$1.10 \mathrm{E}-01$

$0.00 \mathrm{E}+01$

$1.06 \mathrm{E}-03$

$1.02 \mathrm{E}+02$

3.10E-03

$3.07 \mathrm{E}+04$

6.30E-01

$5.82 \mathrm{E}+01$

$9.29 \mathrm{E}-02$

7.10E-03

2.19E-01

$3.47 \mathrm{E}+02$

2.07E-01

$3.24 \mathrm{E}+04$

$3.36 \mathrm{E}-00$

$1.86 \mathrm{E}+03$

$1.78 \mathrm{E}+03$

8.64E-03

$1.02 E+01$

3.12E-00

3.13E +02

9.54E-03

$2.40 E-03$

$1.13 \mathrm{E}+01$

2.93E+02

$5.59 \mathrm{E}+01$

$8.05 E+02$

4.65E-03

1.96E-04

1.77E-09

1.01E-02

$2.81 E+02$

2.95E-00

$1.10 \mathrm{E}+04$

$1.57 \mathrm{E}+06$

4.47E-00

2.77E-07

$1.60 \mathrm{E}-10$

4.61E-00

3.38E-00

$3.06 \mathrm{E}-09$

$7.22 \mathrm{E}-11$

3.77E-05

1.57E-06

1.03E-06

$3.67 \mathrm{E}-05$

$1.57 \mathrm{E}+06$

$1.36 \mathrm{E}+01$

$1.88 \mathrm{E}+04$

$0.00 \mathrm{E}+01$

$3.64 \mathrm{E}+03$

$2.04 \mathrm{E}+03$

$1.20 \mathrm{E}+05$

$7.25 \mathrm{E}+03$

$5.58 \mathrm{E}+02$

$4.28 \mathrm{E}+04$

$0.00 \mathrm{E}+01$

$1.10 \mathrm{E}+03$ 1\%-ile

3.78E-06

1.40E-06

4.30E-08

2.55E-09

$1.69 \mathrm{E}-07$

1.35E-07

1.53E-07

2.07E-06

4.33E-10

2.50E-08

2.92E-07

4.76E-11

$0.00 \mathrm{E}+01$

$0.00 \mathrm{E}+01$

$0.00 \mathrm{E}+01$

$0.00 \mathrm{E}+01$

$0.00 \mathrm{E}+01$

$0.00 \mathrm{E}+01$

$0.00 \mathrm{E}+01$

$0.00 \mathrm{E}+01$

$0.00 \mathrm{E}+01$

$1.34 \mathrm{E}-10$

$0.00 \mathrm{E}+01$

$1.07 \mathrm{E}-04$

$0.00 \mathrm{E}+01$

$0.00 \mathrm{E}+01$

$0.00 \mathrm{E}+01$

$0.00 \mathrm{E}+01$

$0.00 \mathrm{E}+01$

$0.00 \mathrm{E}+01$

$0.00 \mathrm{E}+01$

$0.00 \mathrm{E}+01$

$0.00 \mathrm{E}+01$

$0.00 \mathrm{E}+0 \mathrm{I}$

$0.00 \mathrm{E}+01$

$0.00 \mathrm{E}+01$

$0.00 E+01$

$0.00 \mathrm{E}+01$

$0.00 \mathrm{E}+01$

$0.00 \mathrm{E}+0 \mathrm{l}$

$0.00 \mathrm{E}+01$

$0.00 \mathrm{E}+0 \mathrm{I}$

$0.00 \mathrm{E}+01$

$0.00 E+01$

$2.74 \mathrm{E}-06$

$0.00 \mathrm{E}+01$

$0.00 E+01$

$0.00 \mathrm{E}+01$

$0.00 \mathrm{E}+01$

$0.00 E+01$

$0.00 \mathrm{E}+01$

$0.00 E+01$

$0.00 E+01$

$0.00 E+01$

$0.00 E+01$

$0.00 \mathrm{E}+01$

$0.00 \mathrm{E} \div 01$

$0.00 E+01$

$3.79 \mathrm{E}+04$

4.14E-01

$7.18 \mathrm{E}+02$

I. $01 \mathrm{IE}+02$

$6.42 \mathrm{E}+04$

$4.83 E+02$

$2.71 \mathrm{E}-00$

$1.07 \mathrm{E}+04$

$0.00 E+01$

$1.70 \mathrm{E}+01$ 10\%-ile

5.77E-05

1.50E-05

4.32E-07

6.13E-08

$1.02 \mathrm{E}-06$

5.45E-06

9.49E-06

$1.69 \mathrm{E}-05$

7.64E-09.

$1.65 \mathrm{E}-07$

1.65E-06

2.12E-09

$0.00 \mathrm{E}+01$

$5.77 \mathrm{E}-08$

$6.93 \mathrm{E}-10$

$0.00 \mathrm{E}+01$

$0.00 \mathrm{E}+01$

$0.00 E+01$

2.08E-08

$1.59 \mathrm{E}-09$

4.90E-08

3.93E-08

$0.00 \mathrm{E}+01$

$9.89 \mathrm{E}-04$

$0.00 \mathrm{E}+01$

$0.00 E+01$

$0.00 \mathrm{E}+01$

$0.00 E+01$

$0.00 \mathrm{E}+01$

$0.00 E+01$

$0.00 \mathrm{E}+01$

2.12E-09

$0.00 \mathrm{E}+01$

$5.59 \mathrm{E}-07$

1.45E-05

2.75E-06

3.98E-05

$2.24 \mathrm{E}-10$

$0.00 E+01$

$0.00 \mathrm{E}+01$

$0.00 \mathrm{E}+01$

$0.00 \mathrm{E}+01$

$0.00 \mathrm{E}+01$

$0.00 \mathrm{E}+01$

$8.06 \mathrm{E}-04$

$0.00 \mathrm{E}+01$

$0.00 \mathrm{E} \div 01$

$0.00 \mathrm{E}+01$

$0.00 \mathrm{E}+01$

$0.00 \mathrm{E}+01$

$0.00 \mathrm{E}+01$

$0.00 \mathrm{E}+01$

$0.00 \mathrm{E}+01$

$0.00 \mathrm{E}+01$

$0.00 \mathrm{E}+01$

$0.00 \mathrm{E}+01$

$0.00 \mathrm{E}+01$

$0.00 \mathrm{E}+01$

$4.54 \mathrm{E}+04$

4. $60 \mathrm{E}-00$

$1.45 E+03$

$2.29 \mathrm{E}+02$

$7.94 \mathrm{E}+04$

$1.08 \mathrm{E}+03$

4. $74 \mathrm{E}+02$

$1.24 \mathrm{E}+04$

$0.00 \mathrm{E}+01$

$6.44 \mathrm{E}+01$

50\%-ile

$3.44 \mathrm{E}+02$

$1.36 \mathrm{E}+02$

2.12E-00

$3.29 \mathrm{E}-01$

9.62E-00

$4.31 \mathrm{E}+01$ 
Tank Anal. Un

AY101 La
AY101 $\mathrm{Mn}$
AY101
Na
AY101 Ni
AY101 NO2
AY101 NO3
AY101 P
AY101 Pb
AY101 PO4

AY101 S

AY101 Si

AY101 SO4

AY101 Sr

AY101 TOC

AY101 U

AY101 Zr

AY101 Ac227

AY101 Am241

AY101 Am243

AY101 Ba137

AY101 C14

AY101 Cd113

AY101 Cm242

AY101 Cm243

AY101 Cm244

AY101 Co60

AY101 Cs134

AY101 Cs137

AY101 Eu152

AY101 Eu154

AY101 Eu155

AY101 1129

AY101 Nb93

AY101 Ni59

AY101 Ni63

AY101 Np237

AY101 Pa231

AY101 Pu238

AY101 Pu239

AY101 Pu240

AY101 Pu241

AY101 Pu242

AY101 Ra226

AY101 Ra228

AY101 Ru106

AY101 Sb125

AY101 Se79

AY101 Sm151

AY101 Sr89/90

AY101 Te99

AY101 Th229

AY101 Th232

AY101 Tin126

AY101 Tritium

AY101 U232

AY101 U233

AY101 U234

AY101 U235

AY101 U236

AY101 $\mathbf{U 2 3 8}$

AY101 Y90

AY101 Zr93

AY102 Al

AY102 Bi

AY102 Ca

AY102 Cl

AY102 $\mathrm{CO3}$

AY102 Cr
BB

May 98

$\begin{array}{ll}\mathrm{kg} & 6.91 \mathrm{E}+03 \\ \mathrm{~kg} & 1.05 \mathrm{E}+04\end{array}$

$\begin{array}{ll}\mathrm{kg} & 1.05 \mathrm{E}+04 \\ \mathrm{~kg} & 1.76 \mathrm{E}+05\end{array}$

$\mathrm{kg} \quad 7.85 \mathrm{E}+02$

$\mathrm{kg} \quad 1.24 \mathrm{E}+05$

kg $8.80 \mathrm{E}+04$

kg $1.33 E+03$

kg $2.29 \mathrm{E}+03$

kg $4.13 E+03$

$\mathrm{kg} \quad 6.83 \mathrm{E}+03$

$\mathrm{kg} \quad 6.15 \mathrm{E}+02$

$\mathrm{kg} \quad 2.05 \mathrm{E}+04$

$\mathrm{kg} \quad 0.00 \mathrm{E}+01$

kg $3.11 E+04$

kg $1.09 \mathrm{E}+04$

296E+03

Ci 1.16E-02

$1.02 \mathrm{E}+04$

$4.20 \mathrm{E}-01$

4.18E+05

$5.78 \mathrm{E}+01$

$6.35 \mathrm{E}+02$

$5.74 \mathrm{E}-00$

$6.70 \mathrm{E}-01$

$2.53 \mathrm{E}+01$

$1.41 \mathrm{E}+03$

$1.06 \mathrm{E}+01$

$4.42 \mathrm{E}+05$

Ci $\quad 8.14 \mathrm{E}+01$

Ci 5.70E+03

Ci $\quad 4.27 E+03$

$7.97 \mathrm{E}-01$

$1.09 \mathrm{E}+02$

Ci 3.46E+01

Ci 3.43E+03

Ci 1.40E-00

Ci 2.02E-02

Ci $1.80 \mathrm{E}+02$

Ci 2.41E+03

Ci Incl. in 239

Ci $1.25 E+04$

Ci 8.81E-02

Ci 2.25E-03

Ci 1.45E-01

Ci 1.64E-00

Ci $3.37 \mathrm{E}+02$

Ci $3.26 E+01$

Ci 1.09E+05

Ci 3.75E+06

Ci 4.12E+02

Ci 3.47E-03

Ci 1.44E-02

Ci $5.11 \mathrm{E}+01$

Ci 3.29E+02

Ci 6.11E-01

Ci 2.35E-00

Ci 1.04E-00

Ci 4.23E-02

Ci 3.57E-02

Ci 1.10E-00

Ci 3.75E+06

Ci $1.48 \mathrm{E}+02$

$\mathrm{kg} \quad 6.04 \mathrm{E}+03$

kg 4.04E-01

kg 2.23E+03

kg $1.64 \mathrm{E}+03$

kg $\quad 9.74 \mathrm{E}+03$

$\mathrm{kg} \quad 6.13 \mathrm{E}+02$
Sept98

$6.91 \mathrm{E}+03$

$1.05 \mathrm{E}+04$

$1.76 \mathrm{E}+05$

$7.85 \mathrm{E}+02$

$1.24 \mathrm{E}+05$

$8.80 \mathrm{E}+04$

NA

$2.29 \mathrm{E}+03$

4.13E+03

NA

$6.15 E+02$

2.05E+04

$3.55 \mathrm{E}+01$

$3.11 \mathrm{E}+04$

$1.09 \mathrm{E}+04$

$2.96 \mathrm{E}+03$

1.16E-02

$1.02 E+04$

$1.04 \mathrm{E}-00$

$4.18 E+05$

$5.78 \mathrm{E}+01$

$6.35 \mathrm{E}+02$

$1.42 \mathrm{E}+01$

$1.66 \mathrm{E}-00$

$6.28 \mathrm{E}+01$

$1.41 \mathrm{E}+03$

$1.06 \mathrm{E}+01$

$4.42 \mathrm{E}+05$

$8.14 \mathrm{E}+01$

$5.70 \mathrm{E}+03$

$4.27 \mathrm{E}+03$

7.97E-01

$1.09 \mathrm{E}+02$

$3.46 \mathrm{E}+01$

$3.43 \mathrm{E}+03$

$1.40 \mathrm{E}-00$

2.02E-02

$2.06 E+02$

$1.85 \mathrm{E}+03$

$5.59 \mathrm{E}+02$

$1.43 E+04$

1.01E-01

2.25E-03

1.45E-01

1.64E-00

$3.37 \mathrm{E}+02$

$3.26 \mathrm{E}+01$

$1.09 \mathrm{E}+05$

$1.48 \mathrm{E}+06$

4.12E +02

3.47E-03

1.44E-02

$5.11 \mathrm{E}+01$

$3.29 \mathrm{E}+02$

2.34E-00

8.99E-00

3.99E-00

1.62E-01

1.37E-01

3.64E-00

$1.48 \mathrm{E}+06$

$1.48 \mathrm{E}+02$

$6.06 \mathrm{E}+03$

$0.00 \mathrm{E}+01$

$2.24 \mathrm{E}+03$

$1.64 \mathrm{E}+03$

$9.70 \mathrm{E}+03$

$6.14 \mathrm{E}+02$
$1 \%$ ile

1.80E-02

$1.33 E+03$

$1.38 \mathrm{E}+05$

$6.10 \mathrm{E}+02$

$4.17 \mathrm{E}+04$

$8.20 \mathrm{E}+02$

$8.16 \mathrm{E}-00$

$1.73 E+03$

$4.97 \mathrm{E}+02$

$3.85 \mathrm{E}+03$

$1.56 \mathrm{E}+03$

$1.73 \mathrm{E}+04$

$2.97 \mathrm{E}+02$

8.72E+03

$9.65 \mathrm{E}+02$

$3.95 \mathrm{E}+02$

$0.00 \mathrm{E}+01$

$5.86 \mathrm{E}-00$

4.45E-02

$0.00 \mathrm{E}+01$

$0.00 \mathrm{E}+01$

$0.00 \mathrm{E}+01$

$6.08 \mathrm{E}-01$

$7.10 \mathrm{E}-02$

2.68E-00

$4.74 \mathrm{E}+01$

$0.00 \mathrm{E}+01$

$4.24 \mathrm{E}+03$

$0.00 \mathrm{E}+01$

$0.00 \mathrm{E}+01$

$0.00 \mathrm{E}+01$

$0.00 \mathrm{E}+01$

$0.00 \mathrm{E}+01$

6.84E-00

$6.78 \mathrm{E}+02$

$1.48 \mathrm{E}-01$

9.62E-03

$1.91 \mathrm{E}+01$

$2.55 \mathrm{E}+02$

$2.55 \mathrm{E}+02$

$1.32 \mathrm{E}+03$

9.33E-03

$0.00 \mathrm{E}+01$

$0.00 \mathrm{E}+01$

$5.52 \mathrm{E}-01$

$0.00 \mathrm{E}+01$

6.52E-00

$0.00 \mathrm{E}+01$

$10.00 E+03$

$0.00 E+01$

$1.65 \mathrm{E}-03$

$6.86 \mathrm{E}-03$

$0.00 \mathrm{E}+01$

$1.58 \mathrm{E}+02$

2.91E-01

1.12E-00

4.95E-01

2.01E-02

1.70E-02

$5.24 \mathrm{E}-01$

4.78E +04

$0.00 E+01$

$8.37 \mathrm{E}+01$

$2.84 \mathrm{E}-02$

$9.59 \mathrm{E}+01$

$2.53 \mathrm{E}+01$

4.77E +02

$2.46 \mathrm{E}-00$

10\%-ile

$6.05 \mathrm{E}-01$

$1.55 \mathrm{E}+03$

$1.14 \mathrm{E}+03$

$7.77 \mathrm{E}+04$

$1.49 \mathrm{E}+04$

$1.01 \mathrm{E}+02$

$1.82 \mathrm{E}+03$

$2.09 \mathrm{E}+03$

$4.18 \mathrm{E}+03$

$2.18 \mathrm{E}+03$

$1.92 \mathrm{E}+04$

$3.04 \mathrm{E}+02$

$1.14 \mathrm{E}+04$

$1.13 E+03$

4. $12 \mathrm{E}+02$

$0.00 \mathrm{E}+01$

$1.76 \mathrm{E}+01$

$7.46 \mathrm{E}-02$

$1.67 \mathrm{E}+04$

$1.50 \mathrm{E}+01$

$0.00 \mathrm{E}+01$ 
Tank Anal. Un

$\begin{array}{ll}\text { AY102 } & \text { F } \\ \text { AY102 } & \text { Fe } \\ \text { AY102 } & \text { Hg } \\ \text { AY102 } & \text { K } \\ \text { AY102 } & \text { La } \\ \text { AY102 } & \text { Mn } \\ \text { AY102 } & \text { Na } \\ \text { AY102 } & \text { Ni } \\ \text { AY102 } & \text { NO2 } \\ \text { AY102 } & \text { NO3 } \\ \text { AY102 } & \text { P } \\ \text { AY102 } & \text { Pb } \\ \text { AY102 } & \text { PO4 } \\ \text { AY102 } & \text { S } \\ \text { AY102 } & \text { Si } \\ \text { AY102 } & \text { SO4 } \\ \text { AY102 } & \text { Sr }\end{array}$

AY102 $\mathrm{Sr}$

AY102 TOC

AY102 U

AY102 Zr

AY102 Ac227

AY102 Am241

AY102 Am243

AY102 Ba137

AY102 C14

AY102 Cd113

AY102 Cm242

AY102 Cm243

AY102 Cm244

AY102 C060

AY102 Cs134

AY102 Cs137

AY102 Eu152

AY102 Eu154

AY102 Eu155

AY102 1129

AY102 Nb93

AY102 Ni59

AY102 Ni63

AY102 Np237

AY102 Pa231

AY102 Pu238

AY102 Pu239

AY102 Pu240

AY102 Pu241

AY102 Pu242

AY102 Ra226

AY102 Ra228

AY102 Ru106

AY102 Sb125

AY102 Se79

AY102 Sm151

AY102 Sr89/90

AY102 Te99

AY102 Th229

AY102 Th232

AY102 Tin126

AY102 Tritium

AY102 U232

AY102 U233

AY102 U234

AY102 U235

AY102 U236

AY102 U238

AY102 Y90

AY102 Zr93

AZ101 AI

AZ101 Bi

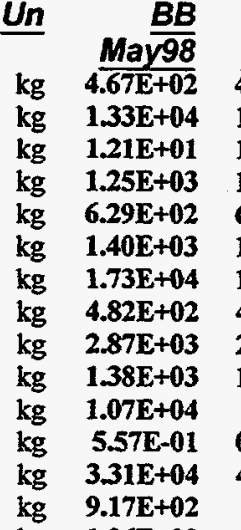

$\frac{B \operatorname{Bg}}{4.68 \mathrm{E}+02}$

$4.68 \mathrm{E}+02$

$1.21 \mathrm{E}+01$

$1.23 \mathrm{E}+03$

$6.32 \mathrm{E}+02$

$1.40 \mathrm{E}+03$

$1.72 \mathrm{E}+04$

$4.84 E+02$

$2.84 \mathrm{E}+03$

$1.36 \mathrm{E}+03$

NA

$0.00 \mathrm{E}+01$

$4.64 \mathrm{E}+03$

NA

$1.96 \mathrm{E}+03$

$9.05 \mathrm{E}+02$

$2.75 \mathrm{E}+03$

$1.14 \mathrm{E}+02$

$9.24 \mathrm{E}+03$

$2.40 \mathrm{E}+03$

$9.14 \mathrm{E}+01$

3.40E-03

$2.87 \mathrm{E}+03$

3.58E-02

$4.40 \mathrm{E}+04$

$1.59 \mathrm{E}-01$

$2.57 \mathrm{E}+01$

1.01E-00

3.20E-00

$7.73 \mathrm{E}+01$

$1.07 \mathrm{E}+02$

$4.27 \mathrm{E}+02$

$4.38 \mathrm{E}+04$

$1.83 \mathrm{E}+01$

$5.14 \mathrm{E}+03$

$1.18 \mathrm{E}+03$

1.91E-01

5.85E-00

$2.04 \mathrm{E}+01$

$2.02 \mathrm{E}+03$

2.11E-01

1.45E-03

$3.15 \mathrm{E}+01$

$5.74 E+02$

Ci Incl. in 239

Ci 2.35E+03

Ci 1.41E-02

Ci 7.50E-04

6.96E-04

1.75E-01

$3.71 \mathrm{E}+02$

8.58E-01

$5.88 \mathrm{E}+03$

$4.09 \mathrm{E}+06$

3.97E-00

$1.75 \mathrm{E}-05$

$6.97 \mathrm{E}-05$

2.56E-00

$2.46 \mathrm{E}+02$

2.36E-03

8.93E-03

1.26E-01

4.79E-03

1.01E-02

8.83E-02

$4.09 \mathrm{E}+06$

7.34E-00

$4.90 \mathrm{E}+04$

$3.50 \mathrm{E}+01$
$1.14 \mathrm{E}+02$

$9.16 \mathrm{E}+03$

$2.42 E+03$

9.17E+01

$3.40 \mathrm{E}-03$

$2.87 \mathrm{E}+03$

2.18E-01

$4.38 \mathrm{E}+04$

1.59E-01

$2.57 \mathrm{E}+01$

6.16E-00

$3.23 \mathrm{E}-00$

$7.75 \mathrm{E}+01$

$1.09 \mathrm{E}+02$

$4.27 \mathrm{E}+02$

$4.63 \mathrm{E}+04$

$1.83 \mathrm{E}+01$

$5.16 \mathrm{E}+03$

$1.18 \mathrm{E}+03$

$1.91 \mathrm{E}-01$

5.85E-00

$2.04 \mathrm{E}+01$

$2.02 \mathrm{E}+03$

2.11E-01

1.45E-03

$1.74 \mathrm{E}+02$

$4.71 \mathrm{E}+02$

$1.05 \mathrm{E}+02$

1.29E-02

7.50E-04

6.96E-04

1.75E-01

$3.72 E+02$

8.61E-01

$5.88 \mathrm{E}+03$

$2.47 \mathrm{E}+06$

3.99E-00

1.75E-05

6.97E-05

2.56E-00

$2.46 \mathrm{E}+02$

2.16E-02

8.19E-02

1.15E-00

4.39E-02

9.31E-02

8.05E-01

$2.47 \mathrm{E}+06$

7.34E-00

$4.90 \mathrm{E}+04$

$3.50 E+01$
$2.16 \mathrm{E}+03$

1\%-ile

$10 \%$-ile

50\%-ile

90\%-ile

3.64E-01 $\quad 1.09 \mathrm{E}-00 \quad 6.96 \mathrm{E}+02$

$5.84 \mathrm{E}+01 \quad 8.14 \mathrm{E}+02$

$0.00 \mathrm{E}+01 \quad 1.07 \mathrm{E}-00$

$6.65 \mathrm{E}-00 \quad 2.87 \mathrm{E}+01$

$1.11 \mathrm{E}-02 \quad 3.24 \mathrm{E}-01$

2.37E-01

$2.86 \mathrm{E}-00$

$2.98 \mathrm{E}+03 \quad 9.26 \mathrm{E}+03$

$4.18 \mathrm{E}+01$

$8.68 \mathrm{E}+02$

$\begin{array}{ll}1.26 \mathrm{E}+02 & 1.93 \mathrm{E}+03 \\ 3.32 \mathrm{E}+01 & 2.56 \mathrm{E}+02\end{array}$

$3.32 \mathrm{E}+01$

$8.96 \mathrm{E}-00$

$4.45 \mathrm{E}+01$

9.02E-01

$5.13 \mathrm{E}+01 \quad 2.82 \mathrm{E}+02$

$1.90 \mathrm{E}+01 \quad 1.55 \mathrm{E}+02$

$9.09 \mathrm{E}-00 \quad 2.57 \mathrm{E}+02$

$1.56 \mathrm{E}+01$

$2.42 \mathrm{E}+02$

$3.86 \mathrm{E}+03$

$1.21 \mathrm{E}+01$

$3.29 \mathrm{E}+03$

$7.55 \mathrm{E}-00$

$3.87 \mathrm{E}+01$

$4.09 \mathrm{E}+04$

$4.35 \mathrm{E}+03$

$2.24 \mathrm{E}+04$

$2.84 \mathrm{E}+04$

$2.21 E+03$

$6.77 \mathrm{E}-00$

$1.75 \mathrm{E}+03$

$5.39 \mathrm{E}+02$

$1.23 \mathrm{E}+03$

$1.85 \mathrm{E}+03$

$9.10 \mathrm{E}-03 \quad 1.69 \mathrm{E}-01 \quad 1.67 \mathrm{E}-00$

$4.59 \mathrm{E}+01 \quad 3.46 \mathrm{E}+02 \quad 2.20 \mathrm{E}+03$

$\begin{array}{lll}6.68 \mathrm{E}-00 & 6.65 \mathrm{E}+01 \quad 3.05 \mathrm{E}+02\end{array}$

3.55E-03 3.81E-02 3.91E-00

$0.00 \mathrm{E}+01 \quad 0.00 \mathrm{E}+01 \quad 3.40 \mathrm{E}-03$

$0.00 \mathrm{E}+01 \quad 2.02 \mathrm{E}-02 \quad 2.87 \mathrm{E}+01$

$0.00 \mathrm{E}+01 \quad 3.70 \mathrm{E}-03$

$0.00 \mathrm{E}+01 \quad 0.00 \mathrm{E}+01$

3.58E-02

$0.00 \mathrm{E}+01$

$0.00 \mathrm{E}+01$

$0.00 \mathrm{E}+01$

$0.00 \mathrm{E}+01$

$2.28 \mathrm{E}-00$

$1.04 \mathrm{E}-0 \mathrm{l}$

$0.00 \mathrm{E}+01$

$0.00 \mathrm{E}+01$

$1.67 \mathrm{E}-05$

$0.00 \mathrm{E}+01$

$4.86 \mathrm{E}-01$

$0.00 \mathrm{E}+01$

$0.00 \mathrm{E}+01$

$0.00 \mathrm{E}+01$

$0.00 \mathrm{E}+01$

$0.00 \mathrm{E}+01$

$1.06 \mathrm{E}-02$

$1.05 \mathrm{E}-00$

$0.00 \mathrm{E}+01$

$0.00 \mathrm{E}+01$

$0.00 \mathrm{E}+01$

$0.00 \mathrm{E}+01$

$0.00 E+01$

$0.00 \mathrm{E}+0 \mathrm{I}$

$0.00 \mathrm{E}+01$

$0.00 \mathrm{E}+01$

$0.00 \mathrm{E}+01$

$0.00 E+01$

$0.00 \mathrm{E}+01$

$0.00 \mathrm{E}+0 \mathrm{I}$

$0.00 \mathrm{E}+0 \mathrm{I}$

$2.28 \mathrm{E}+02$

$0.00 \mathrm{E}+01$

$0.00 \mathrm{E}+01$

$0.00 \mathrm{E}+0 \mathrm{I}$

$0.00 \mathrm{E}+01$

$5.78 \mathrm{E}+01$

$0.00 \mathrm{E}+01$

$0.00 \mathrm{E}+01$

$0.00 \mathrm{E}+0$

$0.00 \mathrm{E}+01$

$0.00 \mathrm{E}+0 \mathrm{I}$

$0.00 \mathrm{E}+01$

$1.38 \mathrm{E}+02$

$0.00 E+01$

$2.21 E+03$

3.31E-01

$7.98 \mathrm{E}-00$

$1.01 \mathrm{E}-01$

$0.00 \mathrm{E}+01$

2.72E-00

$0.00 \mathrm{E}+01$

$0.00 \mathrm{E}+0 \mathrm{I}$

$0.00 \mathrm{E}+01$

$3.81 \mathrm{E}-03$

$0.00 \mathrm{E}+01$

$3.92 \mathrm{E}-00$

$3.88 \mathrm{E}+02$

$2.18 \mathrm{E}-02$

$2.88 \mathrm{E}-04$

$3.25 \mathrm{E}-00$

$5.93 \mathrm{E}+01$

$5.93 \mathrm{E}+01$

$2.43 E+02$

$1.46 \mathrm{E}-03$

$0.00 E+01$ 
Tank Anal. Un

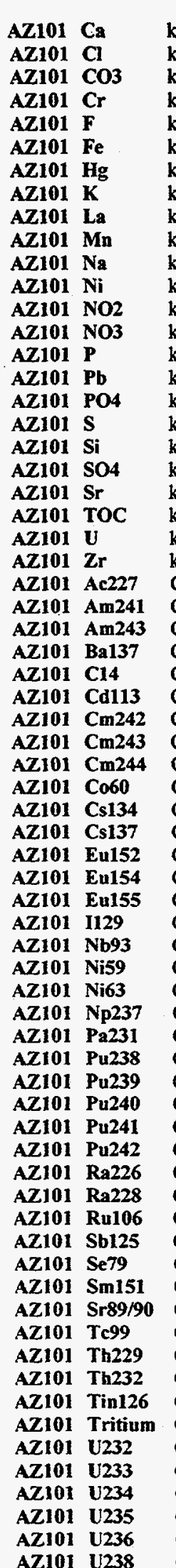

May 98

$\begin{array}{ll}\mathrm{kg} & 5 . \overline{70 \mathrm{E}+02} \\ \mathrm{~kg} & 6.40 \mathrm{E}+02\end{array}$

kg 1.07E+05

kg 2.64E+03

kg $\quad 6.06 \mathrm{E}+03$

kg 2.34E+04

kg 2.60E-01

kg 1.57E+04

kg 8.90E+02

kg $5.22 \mathrm{E}+03$

kg 3.52E+05

kg $\quad 1.36 \mathrm{E}+03$

kg 2.09E+05

kg $2.42 \mathrm{E}+05$

kg 1.52E+03

kg $\quad 4.55 \mathrm{E}+02$

kg 4.72E+03

$\mathrm{kg} \quad 1.92 \mathrm{E}+04$

kg 1.38E+03

kg $5.75 E+04$

kg $\quad 1.17 E+02$

kg $6.06 \mathrm{E}+03$

kg $2.46 \mathrm{E}+03$

kg $8.24 \mathrm{E}+03$

Ci 1.00E-03

$2.26 \mathrm{E}+04$

$9.98 \mathrm{E}-00$

$6.93 \mathrm{E}+06$

4.30E-01

$2.12 \mathrm{E}+03$

$2.54 \mathrm{E}+01$

4.36E-00

$1.02 \mathrm{E}+02$

$4.61 \mathrm{E}+03$

4.34E+04

Ci $7.43 E+06$

$2.73 \mathrm{E}+02$

Ci $\quad 5.76 \mathrm{E}+04$

Ci $7.15 E+04$

Ci 7.10E-00

$8.74 \mathrm{E}+01$

Ci $1.99 \mathrm{E}+01$

Ci 2.30E+03

Ci 1.96E+01

Ci 3.10E-03

Ci $1.65 \mathrm{E}+02$

Ci $9.58 \mathrm{E}+02$

$\mathrm{Ci} \cdot 2.72 \mathrm{E}+02$

Ci $\quad 9.99 \mathrm{E}+03$

$7.40 \mathrm{E}-12$

1.80E-04

1.30E-08

$1.06 \mathrm{E}+05$

$1.31 \mathrm{E}+05$

4.13E+01

$1.42 \mathrm{E}+05$

$6.36 \mathrm{E}+06$

$1.10 \mathrm{E}+03$

$1.20 \mathrm{E}-06$

$1.60 \mathrm{E}-08$

$6.57 \mathrm{E}+01$

4.00E-00

4.60E-04

2.70E-04

1.20E-00

4.40E-02

9.90E-02

8.20E-01
$\frac{\frac{B B}{S e p t 98}}{5.70 E+02}$

$6.40 \mathrm{E}+02$

$1.07 \mathrm{E}+05$

$2.64 \mathrm{E}+03$

$6.06 \mathrm{E}+03$

$2.34 \mathrm{E}+04$

$0.00 \mathrm{E}+01$

$1.57 \mathrm{E}+04$

$8.90 \mathrm{E}+02$

$5.22 \mathrm{E}+03$

$3.52 \mathrm{E}+05$

$1.36 \mathrm{E}+03$

$2.09 \mathrm{E}+05$

$2.42 \mathrm{E}+05$

NA

4.55E+02

4.72E +03

NA

$1.38 \mathrm{E}+03$

$5.75 \mathrm{E}+04$

1.17E +02

$6.06 \mathrm{E}+03$

$2.46 \mathrm{E}+03$

$8.24 \mathrm{E}+03$

$1.00 \mathrm{E}-03$

$2.26 \mathrm{E}+04$

9.98E-00

$7.03 E+06$

4.30E-01

$2.12 \mathrm{E}+03$

$2.54 \mathrm{E}+01$

4.36E-00

$1.09 \mathrm{E}+02$

$4.61 \mathrm{E}+03$

$4.34 \mathrm{E}+04$

$7.43 \mathrm{E}+06$

$2.73 \mathrm{E}+02$

$5.76 \mathrm{E}+04$

$7.15 E+04$

7.10 E-00

8.74E+01

$1.99 \mathrm{E}+01$

$2.30 E+03$

$1.96 \mathrm{E}+01$

3.10E-03

$1.65 \mathrm{E}+02$

$9.58 \mathrm{E}+02$

$2.72 \mathrm{E}+02$

$9.99 \mathrm{E}+03$

$7.40 \mathrm{E}-02$

$1.80 \mathrm{E}-04$

1.10E-08

$4.85 \mathrm{E}+04$

$1.31 \mathrm{E}+05$

4.13E+01

$1.42 \mathrm{E}+05$

$6.36 \mathrm{E}+06$

$1.10 E+03$

$1.20 \mathrm{E}-06$

1.60E-08

$6.57 \mathrm{E}+01$

$4.00 \mathrm{E}-00$

4.46E-02

1.70E-01

1.18E-00

4.51E-02

9.59E-02

8.19E-01 1\%-ile

$7.54 \mathrm{E}+01$

$2.13 \mathrm{E}+01$

$5.83 \mathrm{E}+04$

2.79E-01

$3.89 \mathrm{E}+01$

$1.15 \mathrm{E}+02$

$0.00 \mathrm{E}+01$

7.14E-00

$1.83 \mathrm{E}-02$

$1.68 \mathrm{E}-01$

$1.59 \mathrm{E}+05$

$1.89 \mathrm{E}+01$

$4.96 \mathrm{E}+03$

$4.27 \mathrm{E}+01$

$3.93 \mathrm{E}-00$

$6.89 \mathrm{E}-02$

$1.75 \mathrm{E}+01$

$3.39 \mathrm{E}+01$

$4.44 \mathrm{E}+01$

$4.88 \mathrm{E}+04$

8.86E-03

9.13E-00

7.78E-00

5.10E-03

$0.00 \mathrm{E}+01$

$3.76 \mathrm{E} \div 01$

1.69E-03

$3.20 \mathrm{E}+06$

$0.00 \mathrm{E}+01$

$0.00 \mathrm{E}+01$

$4.30 \mathrm{E}-03$

7.38E-04

$1.73 \mathrm{E}-02$

7.61E-04

$2.00 \mathrm{E}+04$

4.47E+06

$0.00 \mathrm{E}+01$

$0.00 \mathrm{E}+01$

$0.00 \mathrm{E}+01$

$1.79 \mathrm{E}-01$

$0.00 \mathrm{E}+01$

$0.00 \mathrm{E}+01$

$0.00 \mathrm{E}+01$

3.32E-03

$0.00 \mathrm{E}+01$

2.79E-02

$1.62 \mathrm{E}-01$

4.60E-02

$1.69 \mathrm{E}-00$

$1.25 \mathrm{E}-05$

$0.00 \mathrm{E}+01$

$0.00 \mathrm{E}+01$

$0.00 \mathrm{E}+01$

$0.00 \mathrm{E}+01$

$1.28 \mathrm{E}+01$

$0.00 E+01$

$3.86 \mathrm{E}+03$

$0.00 \mathrm{E}+01$

$0.00 \mathrm{E}+01$

$0.00 \mathrm{E}+01$

$0.00 E+01$

8.27E-01

$0.00 \mathrm{E}+01$

$0.00 \mathrm{E}+01$

$0.00 \mathrm{E}+01$

$0.00 \mathrm{E}+01$

$0.00 \mathrm{E}+01$

$0.00 \mathrm{E}+01$ 10\%-ile

$4.98 \mathrm{E}+02$

$9.02 \mathrm{E}+01$

$7.92 \mathrm{E}+04$

$1.66 \mathrm{E}-00$

$1.80 \mathrm{E}+03$

$9.65 \mathrm{E}+02$

$0.00 \mathrm{E}+01$

2.73E+01

$2.85 \mathrm{E}-01$

$1.53 \mathrm{E}-00$

$2.31 \mathrm{E}+05$

$1.09 E+02$

$7.58 \mathrm{E}+04$

4.13E+04

$1.25 \mathrm{E}+01$

1. $05 \mathrm{E}-00$

$4.84 \mathrm{E}+02$

$4.06 \mathrm{E}+02$

$5.75 \mathrm{E}+02$

$5.29 \mathrm{E}+04$

$1.17 \mathrm{E}-01$

$9.05 \mathrm{E}+01$

$1.10 \mathrm{E}+03$

6.05E-02

$0.00 \mathrm{E}+01$

$4.76 \mathrm{E}+01$

$7.96 \mathrm{E}-02$

$4.84 \mathrm{E}+06$

$6.59 \mathrm{E}-02$

$0.00 \mathrm{E}+01$

2.03E-01

$3.48 \mathrm{E}-02$

$8.14 \mathrm{E}-01$

$8.85 \mathrm{E}+01$

$3.03 \mathrm{E}+04$

$5.10 \mathrm{E}+06$

$0.00 \mathrm{E}+01$

$0.00 \mathrm{E}+01$

$0.00 \mathrm{E}+01$

8.71E-01

$0.00 \mathrm{E}+01$

$7.28 \mathrm{E}-01$

$8.41 E+01$

$1.56 \mathrm{E}-0 \mathrm{l}$

4.65E-04

$1.32 \mathrm{E}-00$

7.64E-00

2.17E-00

$7.97 \mathrm{E}+01$

$5.90 \mathrm{E}-04$

$0.00 \mathrm{E}+01$

$0.00 \mathrm{E}+01$

$1.59 \mathrm{E}+04$

$0.00 E+01$

$2.58 \mathrm{E}+01$

$0.00 E+01$

$1.14 \mathrm{E}+04$

$0.00 \mathrm{E}+01$

$1.80 \mathrm{E}-07$

$2.40 \mathrm{E}-09$

$0.00 \mathrm{E}+01$

$1.91 \mathrm{E}-00$

$6.90 \mathrm{E}-05$

4.05E-05

$1.80 \mathrm{E}-01$

6.60E-03

$1.49 \mathrm{E}-02$

$1.23 \mathrm{E}-01$

50\%-ile

\section{$1.81 \mathrm{E}+03$}

$7.11 \mathrm{E}+02$

$1.07 \mathrm{E}+05$

$1.78 \mathrm{E}+03$

$5.01 \mathrm{E}+03$

$4.82 E+03$

2.60E-01

$1.99 \mathrm{E}+04$

$3.70 \mathrm{E}+01$ 
Tank Anal. Un

AZ101 Y90

AZ101 Zr93

AZ102 Al

AZ102 Bi

AZ102 Ca

$A Z 102 \mathrm{Cl}$

AZ102 CO3

AZ102 Cr

AZ102 F

AZ102 Fe

AZ102 Hg

AZ102 K

AZ102 La

AZ102 Mn

AZ102 Na

AZ102 Ni

AZ102 NO2

AZ102 NO3

AZ102 $P$

AZ102 Pb

AZ102 PO4

AZ102 S

AZ102 Si

AZ102 504

AZ102 Sr

AZ102 TOC

AZ102 U

AZ102 $\mathrm{Zr}$

AZ102 Ac227

AZ102 Am241

AZ102 Am243

AZ102 Ba137

AZ102 C14

AZ102 Cd113

AZ102 Cm242

AZ102 Cm243

AZ102 Cm244

AZ102 Co60

AZ102 Cs134

AZ102 Cs137

AZ102 Eul52

AZ102 Eu154

AZ102 Eu155

AZ102 1129

AZ102 Nb93

AZ102 Ni59

AZ102 Ni63

AZ102 Np237

AZ102 Pa231

AZ102 Pu238

AZ102 Pu239

AZ102 Pu240

AZ102 Pu241

AZ102 Pu242

AZ102 Ra226

AZ102 Ra228

AZ102 Ru106

AZ102 Sb125

AZ102 Se79

AZ102 Sm151

AZ102 Sr89/90

AZ102 Tc99

AZ102 Th229

AZ102 Th232

AZ102 Tin126

AZ102 Tritium

AZ102 . U232

AZ102 U233

\section{May $\frac{B B}{98}$ \\ $6 \overline{6.36+06}$}

$1.99 \mathrm{E}+02$

$3.09 \mathrm{E}+04$

$1.56 \mathrm{E}-00$

$1.10 \mathrm{E}+03$

$3.20 \mathrm{E}+01$

$1.28 \mathrm{E}+05$

$3.95 \mathrm{E}+03$

$3.69 \mathrm{E}+03$

$4.84 \mathrm{E}+04$

kg 1.20E-02

kg 1.11E +04

kg $\quad 1.67 \mathrm{E}+03$

kg 1.06E+03

kg 1.99E+05

kg $3.28 \mathrm{E}+03$

kg 1.04E+05

kg $\quad 7.95 \mathrm{E}+04$

kg 1.48E+02

kg $4.00 \mathrm{E}+02$

$4.57 E+02$

2.07E+04

$3.43 E+03$

$6.21 \mathrm{E}+04$

$1.21 \mathrm{E}+02$

$6.22 \mathrm{E}+03$

$9.58 \mathrm{E}+03$

$6.72 \mathrm{E}+03$

4.80E-04

$3.77 \mathrm{E}+04$

4.42E-00

$3.98 \mathrm{E}+06$

8.30E-01

$1.22 \mathrm{E}+03$

$1.12 \mathrm{E}+01$

1.77E-00

$3.93 \mathrm{E}+01$

$3.42 E+03$

$4.12 E+04$

4.32E+06

$1.42 \mathrm{E}+02$

$2.31 \mathrm{E}+04$

i $6.69 \mathrm{E}+04$

$1.52 \mathrm{E}-00$

$4.33 \mathrm{E}+01$

$1.08 \mathrm{E}+01$

i $1.25 \mathrm{E}+03$

$1.17 \mathrm{E}+01$

$1.80 \mathrm{E}-03$

Ci $1.81 \mathrm{E}+02$

$1.25 \mathrm{E}+03$

i $3.72 \mathrm{E}+02$

$1.71 \mathrm{E}+04$

i $3.40 \mathrm{E}-02$

i $\quad 8.50 \mathrm{E}-05$

i.00E-09

$7.62 \mathrm{E}+04$

$9.27 \mathrm{E}+04$

$2.28 \mathrm{E}+01$

8.02E+04

3.70E+06

$5.99 \mathrm{E}+02$

6.80E-07

9.39E-09

$3.54 \mathrm{E}+01$

$1.47 \mathrm{E}+04$

$7.30 \mathrm{E}-04$

$3.60 \mathrm{E}-04$
$\underline{B B}$

$\frac{\operatorname{sep} 38}{636 \mathrm{E}+06}$

$1.99 \mathrm{E}+02$

$3.09 \mathrm{E}+04$

$1.56 \mathrm{E}-00$

$1.10 \mathrm{E}+03$

$3.20 \mathrm{E}+01$

$1.28 \mathrm{E}+05$

$3.95 \mathrm{E}+03$

$3.69 \mathrm{E}+03$

$4.84 \mathrm{E}+04$

$0.00 \mathrm{E}+01$

$1.11 \mathrm{E}+04$

$1.67 \mathrm{E}+03$

$1.06 \mathrm{E}+03$

$1.99 \mathrm{E}+05$

$3.28 \mathrm{E}+03$

$1.04 E+05$

$7.95 \mathrm{E}+04$

NA
$\mathrm{E}+02$

$4.00 \mathrm{E}+02$

$4.57 \mathrm{E}+02$

NA

$3.43 \mathrm{E}+03$

$6.21 \mathrm{E}+04$

$1.21 \mathrm{E}+02$

$6.22 E+03$

$9.58 \mathrm{E}+03$

$6.72 \mathrm{E}+03$

$4.80 \mathrm{E}-04$

$1.48 E+04$

4.42E-00

$4.09 \mathrm{E}+06$

8.30E-01

$1.22 \mathrm{E}+03$

$1.12 \mathrm{E}+01$

$1.77 \mathrm{E}-00$

$4.21 \mathrm{E}+01$

$3.42 \mathrm{E}+03$

4.12E +04

$4.32 \mathrm{E}+06$

$1.42 \mathrm{E}+02$

$2.31 \mathrm{E}+04$

$2.66 \mathrm{E}+04$

1.52E-00

$4.33 \mathrm{E}+01$

$1.08 \mathrm{E}+01$

$1.25 \mathrm{E}+03$

$1.17 \mathrm{E}+01$

$1.80 \mathrm{E}-03$

$1.81 \mathrm{E}+02$

$1.25 \mathrm{E}+03$

3.72E +02

$1.71 \mathrm{E}+04$

3.40E-02

8.50E-05

6.00E-09

$7.62 \mathrm{E}+04$

$9.27 \mathrm{E}+04$

$2.28 \mathrm{E}+01$

8.02E+04

$3.70 \mathrm{E}+06$

$5.99 \mathrm{E}+02$

$6.80 \mathrm{E}-07$

9.39E-09

$3.54 \mathrm{E}+01$

$1.87 \mathrm{E}+03$

$1.85 \mathrm{E}-02$

$6.71 \mathrm{E}-02$ 1\%-ile

$10 \%$-ile

50\%-ile

90\%-ile

$2.57 \mathrm{E}+04 \quad 1.03 \mathrm{E}+05$

$0.00 \mathrm{E}+01$

4.74E+02

1.55E-01

$3.40 \mathrm{E}+02$

$8.25 \mathrm{E}+01$

$5.74 \mathrm{E}+04$

$1.55 \mathrm{E}-00$

$2.35 \mathrm{E}+01$

$5.62 \mathrm{E}+02$

$0.00 \mathrm{E}+01$

2.23E +01

4.07E-02

$6.89 \mathrm{E}-01$

$6.82 \mathrm{E}+04$

$2.43 \mathrm{E}+01$

$2.01 \mathrm{E}+03$

$2.25 \mathrm{E}+02$

$5.78 \mathrm{E}-00$

$4.37 \mathrm{E}-01$

2.55E+01

$1.25 \mathrm{E}+02$

$2.65 \mathrm{E}+02$

$5.16 \mathrm{E}+04$

3.82E-02

$5.01 \mathrm{E}+02$

$1.42 \mathrm{E}+02$

$1.77 \mathrm{E}-02$

$0.00 \mathrm{E}+01$

2.32E-01

$1.43 \mathrm{E}-02$

$1.10 \mathrm{E}+06$

$0.00 \mathrm{E}+01$

$0.00 \mathrm{E}+01$

$3.63 \mathrm{E}-02$

$5.74 \mathrm{E}-03$

1.27E-01

$2.29 \mathrm{E}-01$

$1.14 \mathrm{E}+04$

$2.73 \mathrm{E}+06$

$0.00 \mathrm{E}+01$

$0.00 \mathrm{E}+01$

$0.00 \mathrm{E}+01$

$1.20 \mathrm{E}-01$

$0.00 \mathrm{E}+01$

$0.00 \mathrm{E}+01$

$0.00 \mathrm{E}+01$

$3.79 \mathrm{E}-02$

$0.00 \mathrm{E}+01$

$5.87 \mathrm{E}-01$

4.05E-00

$1.21 \mathrm{E}-00$

$5.54 \mathrm{E}+01$

1.10E-04

$0.00 \mathrm{E}+01$

$0.00 \mathrm{E}+01$

$1.48 \mathrm{E}+02$

$0.00 \mathrm{E}+01$

$7.89 \mathrm{E}-00$

$0.00 \mathrm{E}+01$

$5.85 \mathrm{E}+03$

$0.00 \mathrm{E}+01$

$0.00 \mathrm{E}+01$

$0.00 \mathrm{E}+01$

$0.00 \mathrm{E}+01$

$5.56 \mathrm{E}+03$

$0.00 \mathrm{E}+01$

$0.00 \mathrm{E}+01$

$0.00 \mathrm{E}+01$

$1.80 \mathrm{E}+03$

$1.84 \mathrm{E}-00$

$9.26 \mathrm{E}+02$

$1.88 \mathrm{E}+02$

$7.94 \mathrm{E}+04$

5.67E-00

$1.38 \mathrm{E}+03$

$2.40 \mathrm{E}+03$

$0.00 \mathrm{E}+01$ 
Tank Anal. Un

\begin{tabular}{|c|c|}
\hline AZ102 & U234 \\
\hline AZ102 & U235 \\
\hline AZ102 & U236 \\
\hline AZ102 & U238 \\
\hline AZ102 & Y90 \\
\hline AZ102 & Zr93 \\
\hline B101 & Al \\
\hline B101 & Bi \\
\hline B101 & $\mathrm{Ca}$ \\
\hline B101 & Cl \\
\hline B101 & $\cos$ \\
\hline B101 & $\mathrm{Cr}$ \\
\hline B101 & $\mathbf{F}$ \\
\hline B101 & $\mathrm{Fe}$ \\
\hline B101 & Hg \\
\hline B101 & $\mathbf{K}$ \\
\hline B101 & La \\
\hline B101 & Mn \\
\hline B101 & $\mathrm{Na}$ \\
\hline B101 & $\begin{array}{l}\mathbf{N i} \\
\mathbf{N O P}\end{array}$ \\
\hline B101 & NO2 \\
\hline B101 & $\begin{array}{l}\text { NO3 } \\
\text { P }\end{array}$ \\
\hline $\begin{array}{l}\text { B101 } \\
\text { B101 }\end{array}$ & $\mathrm{Pb}$ \\
\hline B101 & PO4 \\
\hline B101 & $\mathbf{S}$ \\
\hline B101 & $\mathrm{Si}$ \\
\hline B101 & SO4 \\
\hline B101 & $\mathbf{S r}$ \\
\hline B101 & TOC \\
\hline B101 & $\mathbf{U}$ \\
\hline B101 & $\mathbf{Z r}$ \\
\hline B101 & Ac227 \\
\hline B101 & Am241 \\
\hline B101 & Am243 \\
\hline B101 & Ba137 \\
\hline B101 & C14 \\
\hline B101 & Cd113 \\
\hline B101 & $\mathrm{Cm} 242$ \\
\hline B101 & $\mathrm{Cm} 243$ \\
\hline B101 & $\operatorname{Cm} 244$ \\
\hline B101 & Co60 \\
\hline B101 & Cs134 \\
\hline B101 & Cs137 \\
\hline B101 & Eul52 \\
\hline B101 & Eu154 \\
\hline $\begin{array}{l}\text { B101 } \\
\text { B101 }\end{array}$ & $\begin{array}{l}\text { Eu155 } \\
1129\end{array}$ \\
\hline B101 & Nb93 \\
\hline B101 & Ni59 \\
\hline B101 & Ni63 \\
\hline B101 & Np237 \\
\hline B101 & Pa231 \\
\hline B101 & Pu238 \\
\hline B101 & Pu239 \\
\hline B101 & Pu240 \\
\hline B101 & Pu241 \\
\hline B101 & Pu242 \\
\hline B101 & Ra226 \\
\hline B101 & Ra228 \\
\hline B101 & Rul06 \\
\hline B101 & Sb125 \\
\hline B101 & Se79 \\
\hline B101 & Sml51 \\
\hline B101 & Sr89/90 \\
\hline B101 & Tc99 \\
\hline B101 & Th229 \\
\hline
\end{tabular}

May 98 2.24E-00 $\mathrm{Ci} \quad$ 8.83E-02 Ci 1.84E-01

Ci 1.53E-00

Ci 3.70E +06

Ci 1.11E+02

kg $9.40 \mathrm{E}+03$

kg $3.99 \mathrm{E}+03$

c $1.14 E+03$

kg $2.50 \mathrm{E}+02$

g.89E+03

$3.50 \mathrm{E}+02$

$1.56 \mathrm{E}+02$

$6.42 E+03$

g 1.28E-00

g $4.60 \mathrm{E}+02$

kg $4.20 \mathrm{E}+02$

cg $1.37 \mathrm{E}+02$

kg $8.00 E+04$

2.01E+03

kg 3.40E+04

1.15E+05

kg 1.42E+03

$9.30 \mathrm{E}+02$

g $4.40 \mathrm{E}+03$

9.93E+03

g $1.97 \mathrm{E}+03$

2.98E+04

kg 2.07E+02

kg $3.11 E+02$

kg $4.95 E+03$

kg $\quad 9.20 \mathrm{E}+01$

$3.16 \mathrm{E}-03$

$1.12 \mathrm{E}+03$

1.72E-01

Ci $1.51 \mathrm{E}+04$

Ci $\quad 56 \mathrm{E}-01$

$1.06 \mathrm{E}+02$

2.37E-00

2.81E-01

$1.07 \mathrm{E}+01$

8.63E-01

3.15E-02

Ci $1.59 \mathrm{E}+04$

Ci 3.19E+01

Ci $1.57 \mathrm{E}+03$

Ci $1.65 E+03$

Ci 6.75E-03

Ci $1.43 \mathrm{E}+01$

Ci $1.58 \mathrm{E}+01$

Ci 1.57E+03

Ci 1.47E-02

Ci 1.98E-04

5.00E+01

Ci $6.70 \mathrm{E}+02$

Ci Incl, in 239

Ci $5.54 \mathrm{E}+03$

Ci 3.91E-02

Ci 7.04E-04

Ci 1.47E-08

Ci 7.06E-01

Ci 5.16E-00

Ci 4.92E-00

Ci $1.42 \mathrm{E}+04$

Ci $7.24 \mathrm{E}+05$

3.51E-00

1.73E-06

$1.95 \mathrm{E}-10$
Sept98

4.64E-00

1.77E-01

3.82E-01

3.19E-00

$3.70 \mathrm{E}+06$

$1.11 E+02$

1.62E+04

$2.04 \mathrm{E}+03$

$1.22 \mathrm{E}+03$

$2.50 \mathrm{E}+02$

$4.29 \mathrm{E}+03$

$3.60 \mathrm{E}+02$

$1.56 \mathrm{E}+02$

$1.52 \mathrm{E}+03$

$0.00 \mathrm{E}+01$

$7.80 \mathrm{E}+02$

$0.00 \mathrm{E}+01$

$0.00 \mathrm{E}+01$

$1.57 \mathrm{E}+05$

$2.99 \mathrm{E}+03$

$3.40 \mathrm{E}+04$

1.15E+05

NA

$1.24 \mathrm{E}+03$

$4.40 \mathrm{E}+03$

NA

8.70E +02

$2.98 \mathrm{E}+04$

$1.21 \mathrm{E}+02$

$3.11 \mathrm{E}+02$

$3.47 \mathrm{E}+03$

$1.21 \mathrm{E}+02$

3.16E-03

$1.11 \mathrm{E}+03$

$1.16 \mathrm{E}-01$

$1.51 \mathrm{E}+04$

5.46E-01

$1.06 \mathrm{E}+02$

1.61E-00

$1.90 \mathrm{E}-01$

7.25E-00

8.63E-01

3.15E-02

$1.59 \mathrm{E}+04$

$3.19 \mathrm{E}+01$

$1.57 \mathrm{E}+03$

$1.65 \mathrm{E}+03$

$6.75 \mathrm{E}-03$

$1.43 \mathrm{E}+01$

$1.58 \mathrm{E}+01$

$1.57 \mathrm{E}+03$

1.47E-02

$1.98 \mathrm{E}-04$

$5.41 \mathrm{E}+01$

$5.15 E+02$

$1.50 \mathrm{E}+02$

$3.75 \mathrm{E}+03$

2.65E-02

7.04E-04

1.47E-08

7.06E-01

5.16E-00

4.92E-00

$1.42 \mathrm{E}+04$

$2.23 E+03$

3.51E-00

1.73E-06

$1.95 \mathrm{E}-10$
$1 \%$-jle

$0.00 \mathrm{E}+01$

$0.00 \mathrm{E}+01$

$0.00 \mathrm{E}+01$

$0.00 \mathrm{E}+01$

4.51E+03

$0.00 \mathrm{E}+01$

$5.70 \mathrm{E}+02$

$3.91 \mathrm{E}-01$

$2.26 \mathrm{E}+02$

$1.89 \mathrm{E}+02$

$6.67 \mathrm{E}+02$

4.00E-01

$5.23 \mathrm{E}-01$

$3.21 \mathrm{E}+02$

$0.00 \mathrm{E}+01$

$3.06 \mathrm{E}-00$

$1.54 \mathrm{E}-02$

7.02E-02

$1.80 \mathrm{E}+04$

$4.30 \mathrm{E}+01$

$3.36 \mathrm{E}+02$

$5.17 \mathrm{E}+01$

$5.13 E+03$

$3.17 \mathrm{E}+01$

$1.57 \mathrm{E}+02$

$4.59 \mathrm{E}+01$

$2.84 \mathrm{E}+02$

$8.71 \mathrm{E}+01$

2.42E-02

$3.74 \mathrm{E}+01$

$1.92 \mathrm{E}+02$

$6.59 \mathrm{E}-03$

$0.00 \mathrm{E}+01$

$0.00 \mathrm{E}+01$

$0.00 \mathrm{E}+01$

$0.00 \mathrm{E}+01$

$0.00 \mathrm{E}+01$

$0.00 \mathrm{E}+01$

$0.00 \mathrm{E}+01$

$0.00 \mathrm{E}+01$

$0.00 \mathrm{E}+01$

$1.57 \mathrm{E}-02$

$0.00 \mathrm{E}+01$

$1.25 \mathrm{E}+02$

$0.00 \mathrm{E}+01$

$0.00 \mathrm{E}+01$

$0.00 E+01$

$9.49 \mathrm{E}-05$

$0.00 \mathrm{E}+01$

8.83E-02

8.77E-00

$0.00 \mathrm{E}+01$

$0.00 \mathrm{E}+01$

$0.00 \mathrm{E}+01$

$0.00 \mathrm{E}+01$

$0.00 E+01$

$0.00 \mathrm{E}+01$

$0.00 \mathrm{E}+01$

$0.00 \mathrm{E}+01$

$0.00 E+01$

$0.00 \mathrm{E}+01$

$0.00 \mathrm{E}+01$

$0.00 \mathrm{E}+01$

$0.00 \mathrm{E}+01$

$4.97 \mathrm{E}+01$

$0.00 \mathrm{E}+01$

$0.00 \mathrm{E}+01$

$0.00 \mathrm{E}+01$
$10 \%$-ile

3.19E-01

$1.26 \mathrm{E}-02$

2.62E-02

$2.18 \mathrm{E}-01$

$3.76 \mathrm{E}+05$

$0.00 \mathrm{E}+01$

4.64E+03

$2.27 \mathrm{E}+02$

$6.48 \mathrm{E}+02$

$4.00 \mathrm{E}+02$

$2.11 \mathrm{E}+03$

$2.66 \mathrm{E}-00$

$1.72 \mathrm{E}+01$

$2.01 \mathrm{E}+03$

$0.00 \mathrm{E}+01$

$1.25 \mathrm{E}+01$

$1.61 \mathrm{E}-01$

$1.36 \mathrm{E}-00$

$4.90 \mathrm{E}+04$

$7.21 \mathrm{E}+02$

$1.90 \mathrm{E}+03$

$1.81 \mathrm{E}+04$

$8.92 \mathrm{E}+03$

$1.10 \mathrm{E}+02$

$8.54 \mathrm{E}+03$

$6.36 \mathrm{E}+02$

$7.86 \mathrm{E}+02$

$1.07 \mathrm{E}+03$

1.14E-01

$2.22 \mathrm{E}+02$

$7.20 \mathrm{E}+02$

$3.68 \mathrm{E}-02$

$0.00 \mathrm{E}+01$

7.25E-04

8.03E-03

$2.07 \mathrm{E}+03$

$6.98 \mathrm{E}-02$

$0.00 \mathrm{E}+01$

$1.11 \mathrm{E}-01$

$1.31 \mathrm{E}-02$

$5.00 \mathrm{E}-01$

5.08E-01

4.32E-03

$2.47 \mathrm{E}+03$

$0.00 \mathrm{E}+01$

$0.00 \mathrm{E}+01$

$0.00 \mathrm{E}+01$

$2.69 \mathrm{E}-03$

$0.00 \mathrm{E}+01$

3.52E-00

$3.50 \mathrm{E}+02$

6.87E-04

2.92E-05

2.34E-00

$3.13 E+01$

$3.13 \mathrm{E}+01$

$2.59 \mathrm{E}+02$

$1.83 \mathrm{E}-03$

$0.00 \mathrm{E}+01$

$0.00 \mathrm{E}+01$

9.91E-02

$0.00 E+01$

$0.00 \mathrm{E}+01$

$0.00 \mathrm{E}+01$

$4.54 \mathrm{E}+04$

$0.00 \mathrm{E}+01$

2.55E-07

2.88E-11

50\%-ile

$2.24 \mathrm{E}-00$

8.83E-02

$1.84 \mathrm{E}-01$

1.53E-00

3.70E+06

$1.11 \mathrm{E}+02$

2.35E+04

$1.68 \mathrm{E}+03$

$1.83 \mathrm{E}+03$ 


\begin{tabular}{|c|c|c|c|c|c|c|c|c|c|c|c|}
\hline nk & al. & In & May $\frac{B 8}{B 8}$ & Sept98 & $1 \%$-ile & $0 \%$-ile & $50 \%$ & $90 \%$-ile & $9 \%$-ile & Mean & Dev \\
\hline B101 & n126 & $\mathrm{Ci}$ & $\overline{7.79 \mathrm{E}-00}$ & $7.79 \mathrm{E}-00$ & $.00 \mathrm{E}+01$ & $0.00 \mathrm{E}+01$ & $7.79 \mathrm{E}-00$ & $1.85 \mathrm{E}+01$ & $98 \mathrm{E}+01$ & $E-00$ & $99 \mathrm{E}-00$ \\
\hline & ritium & $\mathrm{Ci}$ & $3.27 \mathrm{E}-00$ & $3.27 \mathrm{E}-00$ & $9.76 \mathrm{E}-01$ & $E-00$ & $3.27 \mathrm{E}-00$ & $5.19 \mathrm{E}-00$ & $6 \mathrm{E}-00$ & -00 & $E-00$ \\
\hline 101 & U232 & $\mathrm{Ci}$ & 5.41E-05 & 1.92E-05 & $0.00 E+01$ & $7.98 \mathrm{E}-06$ & 5.41E-05 & $1.77 \mathrm{E}-04$ & $68 \mathrm{E}-04$ & E-05 & E-05 \\
\hline 101 & U233 & $\mathbf{C i}$ & 2.78E-06 & $9.89 \mathrm{E}-07$ & $0.00 \mathrm{E}+01$ & $4.10 \mathrm{E}-07$ & 2.78E-06 & $9.10 \mathrm{E}-06$ & $89 \mathrm{E}-05$ & $.03 E-06$ & $33 \mathrm{E}-06$ \\
\hline B101 & U234 & $\mathrm{Ci}$ & $3.22 \mathrm{E}-00$ & $1.14 \mathrm{E}-00$ & $0.00 \mathrm{E}+01$ & $4.75 \mathrm{E}-01$ & $3.22 \mathrm{E}-00$ & $1.05 \mathrm{E}+01$ & $2.19 \mathrm{E}+01$ & $1.67 \mathrm{E}-00$ & \\
\hline 01 & 235 & $\mathrm{Ci}$ & $1.44 \mathrm{E}-01$ & $5.10 \mathrm{E}-02$ & $0.00 \mathrm{E}+01$ & $2.12 \mathrm{E}-02$ & 01 & & & & \\
\hline & 36 & $\mathrm{Ci}$ & $.56 \mathrm{E}-02$ & 08E-03 & $0.00 \mathrm{E}+01$ & $3.78 \mathrm{E}-03$ & $56 \mathrm{E}-02$ & $8 E-02$ & 74E-01 & -02 & $E-02$ \\
\hline & & $\mathrm{Ci}$ & $3.26 \mathrm{E}-00$ & $16 E-00$ & $0.00 \mathrm{E}+01$ & -01 & -00 & & +01 & $:-00$ & $E-00$ \\
\hline 01 & Y90 & $\mathrm{Ci}$ & $24 \mathrm{E}+05$ & $23 E+03$ & $0.00 \mathrm{E}+01$ & 8.08E+04 & $.24 E+05$ & $E+06$ & $0 \mathrm{E} \div 07$ & $3.43 E+06$ & $1.25 E+07$ \\
\hline 01 & $\mathrm{r} 93$ & $\mathrm{Ci}$ & $.15 E+01$ & $.15 E+01$ & $0.00 \mathrm{E}+01$ & $0.00 \mathrm{E}+01$ & $15 E+01$ & $6.43 E+02$ & $E+03$ & $2.01 E+02$ & $7 E+02$ \\
\hline B102 & Al & $\mathrm{kg}$ & $3.25 E+03$ & $5.59 \mathrm{E}+03$ & $3.50 \mathrm{E}-04$ & $3.89 \mathrm{E}+02$ & $56 \mathrm{E}+03$ & $E+04$ & $E \div 04$ & $5.83 E+03$ & $E+03$ \\
\hline 102 & $\mathbf{B i}$ & k & $1.38 \mathrm{E}+03$ & $7.05 E+02$ & $3.14 \mathrm{E}-05$ & $1.55 \mathrm{E}+01$ & $87 \mathrm{E}+02$ & $E+03$ & $3 E+03$ & $5.36 \mathrm{E}+02$ & $E+02$ \\
\hline 102 & $\mathrm{Ca}$ & kg & $3.95 E+02$ & $4.23 \mathrm{E}+02$ & $1.73 \mathrm{E}-05$ & $1.77 \mathrm{E}+01$ & $.80 \mathrm{E}+02$ & $E+02$ & $E \div 03$ & $E+02$ & $E+02$ \\
\hline B102 & $\mathrm{Cl}$ & $\mathrm{kg}$ & $3.66 \mathrm{E}+02$ & $2.02 E+02$ & $1.06 \mathrm{E}-05$ & $3.41 \mathrm{E}+01$ & $1.85 E+02$ & +02 & $E+02$ & $2.30 \mathrm{E}+02$ & \\
\hline 02 & $\mathrm{CO}$ & kg & $4.28 \mathrm{E}+03$ & $1.48 E+03$ & $1.02 \mathrm{E}-04$ & +02 & $2.57 \mathrm{E}+03$ & & +04 & & \\
\hline 02 & $\mathrm{Cr}$ & $\mathrm{k}$ & 1.2 & $1.24 \mathrm{E}+02$ & $1.12 \mathrm{E}-05$ & $=-00$ & 01 & & +02 & & \\
\hline 02 & $\mathbf{F}$ & $\mathbf{K}$ & $5.45 E+03$ & $1.01 E \div 04$ & $1.77 \mathrm{E}-04$ & +01 & $1 E+03$ & +03 & $E+04$ & +03 & \\
\hline 102 & $\mathbf{F e}$ & $\mathrm{kg}$ & 2.22 & $5.27 \mathrm{E}+02$ & 5.41E-05 & +01 & $33 \mathrm{E}+02$ & & $E+03$ & & \\
\hline B102 & Hg & $\mathrm{kg}$ & $4.90 \mathrm{E}-00$ & $7.30 \mathrm{E}-01$ & 0.00 & +01 & 0E-00 & & $E+01$ & & \\
\hline 02 & $\mathbf{K}$ & kg & $1.58 E+02$ & $2.70 \mathrm{E}+02$ & & $E-00$ & $1.57 \mathrm{E}+02$ & & & & +03 \\
\hline 102 & La & kg & $1.48 E+02$ & $0.00 \mathrm{E}+01$ & $7.09 \mathrm{E}-07$ & $E-02$ & 3.51E-00 & & $E+01$ & & +01 \\
\hline & Mn & $\mathrm{kg}$ & $4.80 \mathrm{E}+01$ & $0.00 \mathrm{E}+01$ & & & & & +02 & & +01 \\
\hline 102 & $\mathbf{N a}$ & $\mathrm{kg}$ & $4.31 E+04$ & $5.43 E+04$ & 1.4 & +03 & -04 & & +04 & & +04 \\
\hline B102 & $\mathrm{Ni}$ & kg & $6.95 E+02$ & $4.60 \mathrm{E}+01$ & 2.68E-06 & $E-01$ & -00 & & $E+02$ & $\$+01$ & +01 \\
\hline B102 & NO2 & $\mathrm{kg}$ & $1.70 E+03$ & $1.93 \mathrm{E}+03$ & $1.64 \mathrm{E}-04$ & +02 & -03 & & +04 & +03 & +03 \\
\hline 102 & NO3 & $\mathbf{k}$ & $4.60 \mathrm{E}+04$ & $2.39 E+04$ & & & & & & & +04 \\
\hline 102 & $\mathbf{P}$ & $\mathrm{kg}$ & $4.81 E+03$ & NA & & +02 & & & & & +03 \\
\hline & $\mathbf{P b}$ & $\mathrm{kg}$ & $3.22 \mathrm{E}+02$ & $E+02$ & & & & & & & \\
\hline 102 & $\mathbf{P O}$ & $\mathrm{kg}$ & $1.49 \mathrm{E}+04$ & $2.12 \mathrm{E}+04$ & & +03 & 03 & & +04 & & \\
\hline B102 & $\mathbf{s}$ & $\mathrm{kg}$ & $6.80 \mathrm{E}+03$ & NA & 2.5 & +02 & 03 & & +04 & +03 & +03 \\
\hline B102 & SI & kg & $6.81 \mathrm{E}+02$ & $3.02 \mathrm{E}+02$ & & & & & & & +02 \\
\hline & SO4 & kg & 2.04 & $3.74 \mathrm{E}+04$ & & +02 & & & +04 & & \\
\hline 12 & $\mathrm{Sr}$ & $\mathrm{kg}$ & $7.20 \mathrm{E}+01$ & $4.20 \mathrm{E}+01$ & -06 & $E-01$ & & & & & \\
\hline & TO & kg & 1.40 & +01 & & & & & & & \\
\hline 102 & $\mathbf{U}$ & $\mathrm{kg}$ & +03 & $1.20 \mathrm{E}+03$ & -04 & 102 & 03 & & +04 & -03 & +03 \\
\hline 102 & $\mathrm{Zr}$ & $\mathrm{k}$ & $3.20 \mathrm{E}+01$ & $4.20 \mathrm{E}+01$ & E-07 & $E-02$ & 4.3 & -02 & $E+02$ & +01 & +01 \\
\hline 102 & Ac227 & $\overline{\mathrm{Ci}}$ & $6.44 \mathrm{E}-04$ & $6.44 \mathrm{E}-04$ & $E+01$ & & & & $E-02$ & -03 & -03 \\
\hline & & $\mathrm{Ci}$ & $1.54 \mathrm{E}-01$ & $1.96 \mathrm{E}-02$ & & & & & & & +03 \\
\hline & & $\mathrm{Ci}$ & $3.21 E-06$ & $4.09 \mathrm{E}-07$ & & & & & & & \\
\hline & & $\mathrm{Ci}$ & & 4.1 & +01 & & & & & & \\
\hline 102 & C & $\mathrm{Ci}$ & $1.34 \mathrm{E}-01$ & -01 & +01 & .02 & & & -00 & & \\
\hline 102 & & $\mathrm{Ci}$ & $2.44 \mathrm{E}-01$ & 2.44E-01 & +01 & & & & & & \\
\hline & & C & $3.97 E-04$ & 5.05E-05 & +01 & & & & & & -04 \\
\hline & & $\mathrm{Ci}$ & & & & & & & & & \\
\hline & & $\mathrm{Ci}$ & & & & & & & & & \\
\hline 02 & C & $\mathrm{Ci}$ & $8.05 E-02$ & 8.0 & & & & & & & \\
\hline 102 & Cs134 & $\mathrm{Ci}$ & $3.44 \mathrm{E}-03$ & $3.44 E-03$ & 0.0 & -04 & & -02 & -02 & .03 & \\
\hline 102 & Cs137 & $\mathrm{Ci}$ & $5.58 E+03$ & $4.41 \mathrm{E}+03$ & -03 & +02 & & & & & +04 \\
\hline & & $\mathrm{Ci}$ & E-02 & $1.57 \mathrm{E}-02$ & & $E+01$ & & & -01 & & -02 \\
\hline & & $\mathrm{Ci}$ & & & & & & & & & \\
\hline & & $\mathrm{Ci}$ & & & & & & & & & \\
\hline & & $\mathrm{Ci}$ & & & & & & & & & \\
\hline 12 & & $\mathrm{Ci}$ & & 02 & & & & & & -01 & \\
\hline 102 & & $\mathrm{Ci}$ & $6.19 \mathrm{E}-02$ & $6.19 \mathrm{E}-02$ & 0 & & & & & & \\
\hline B102 & Ni63 & $\mathrm{Ci}$ & $5.59 \mathrm{E}-00$ & 5.59E-00 & & & 5.59 & & $1.23 E+02$ & $\mp 01$ & +01 \\
\hline B102 & Np237 & $\mathrm{Ci}$ & -03 & 3.54E-03 & & & & & $=-02$ & & -03 \\
\hline B102 & & $\mathrm{Ci}$ & & & & & & & & & \\
\hline B102 & & $\mathrm{Ci}$ & & & & & & & & & \\
\hline 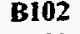 & & $\mathrm{Ci}$ & & & & & & & & & \\
\hline 102 & & $\mathrm{Ci}$ & & & & & & & & & \\
\hline & & $\mathrm{Ci}$ & $1.98 E+01$ & 2.52E-00 & & & & & +02 & & \\
\hline & & $\mathrm{Ci}$ & 5.74E-05 & $7,30 \mathrm{E}-06$ & & & & & -04 & 9 & -04 \\
\hline B102 & 77 & $\mathrm{Ci}$ & 4.65E-06 & 4.65E-06 & 0.0 & $: 01$ & & -0 & EE-05 & -05 & $E-05$ \\
\hline B102 & Ra228 & $\mathrm{Ci}$ & $1.39 \mathrm{E}-03$ & $1.39 E-03$ & $0.00 \mathrm{E}+01$ & $0.00 \mathrm{E}+01$ & 1.3 & $E-03$ & $7 E-02$ & -03 & $E-03$ \\
\hline B102 & Ru106 & $\mathrm{Ci}$ & $1.10 \mathrm{E}-05$ & $1.10 \mathrm{E}-05$ & $0.00 \mathrm{E}+01$ & $1.37 \mathrm{E}-07$ & $1.10 \mathrm{E}-05$ & $4.60 \mathrm{E}-05$ & $1.09 \mathrm{E}-04$ & $3 E-05$ & $E-05$ \\
\hline B102 & Sb125 & $\mathrm{Ci}$ & 3.27E-01 & $3.27 \mathrm{E}-01$ & $0: 00 E+01$ & $0.00 \mathrm{E}+01$ & $3.27 E-01$ & $E-00$ & $2.32 \mathrm{E}-00$ & E-01 & $E-01$ \\
\hline B102 & Se79 & $\mathrm{Ci}$ & $1.53 E-02$ & $1.53 \mathrm{E}-02$ & $E+01$ & & & & $1.19 \mathrm{E}-01$ & -02 & \\
\hline n? & 51 & $\mathrm{Ci}$ & $60 E+01$ & $60 E+01$ & $0.00 \mathrm{E}+01$ & $.00 E+01$ & -01 & $3.87 \mathrm{E}+02$ & $8.84 \mathrm{E}+02$ & $1.37 \mathrm{E}+02$ & $1.96 \mathrm{E}+02$ \\
\hline
\end{tabular}


Tank Anal. Un

\section{B102 Sr89/90 Ci}

$\mathrm{B} 102 \mathrm{Te99} \mathrm{Ci}$ 7.59E-01

B102 Th229 Ci 1.20E-04

B102 Th232 Ci 9.95E-05

B102 Tin126 Ci 2.29E-02

$\begin{array}{llll}\text { B102 Tritium } & \mathrm{Ci} & \text { 6.17E-01 } \\ \mathrm{B102} & \mathrm{C} 232 & \mathrm{Ci} & \mathbf{5 7 E}-02\end{array}$

$\begin{array}{llll}\mathrm{B} 102 & \mathrm{U} 233 & \mathrm{Ci} & 6.05 \mathrm{E}-02\end{array}$

B102 U234 Ci 2.08E-00

$\mathrm{B} 102 \mathrm{U} 235 \mathrm{Ci}$ 9.32E-02

$\mathrm{B} 102 \mathrm{U} 236 \mathrm{Ci}$ 1.60E-02

B102 U238 Ci 2.11E-00

$\begin{array}{llll}B 102 & \text { Y90 Ci } & 1.16 \mathrm{E}+04\end{array}$

$\mathrm{B102} \mathrm{Zr} 93$ Ci $7.33 \mathrm{E}-02$

$\begin{array}{llll}\mathrm{B} 103 \mathrm{Al} & \mathrm{kg} & 4.64 \mathrm{E}+03\end{array}$

$\mathrm{B103} \mathrm{Bi} \quad \mathrm{kg} \quad 1.97 \mathrm{E}+03$

$\begin{array}{llll}\mathrm{B103} \mathrm{Ca} & \mathrm{kg} & 5.64 \mathrm{E}+02\end{array}$

$\begin{array}{llll}\mathrm{B} 103 & \mathrm{Cl} & \mathrm{kg} & \mathbf{5 . 2 2 E}+02\end{array}$

$\begin{array}{llll}\mathrm{B103} & \mathrm{CO} 3 & \mathrm{~kg} & 3.40 \mathrm{E}+03\end{array}$

$\begin{array}{lllll}\text { B103 } & \text { Cr } & \mathrm{kg} & 1.73 \mathrm{E}+02 \\ \text { B103 } & \text { F } & \mathrm{kg} & \mathbf{7 . 7 8 E}+03\end{array}$

$B 103$ Fe $\mathrm{kg} 3.17 \mathrm{E}+03$

$\mathrm{B103} \mathrm{Hg}$. $\mathrm{kg} \quad 8.46 \mathrm{E}-01$

$B 103 \mathrm{~K}$ kg 2.30E+02

$B 103 \mathrm{La}$ kg 2.07E+02

B103 Mn

B103 Na

B103 Ni

B103 NO2

B103 NO3

B103 P

$\begin{array}{llll}\mathrm{B103} & \mathrm{Pb} & \mathrm{kg} & 4.60 \mathrm{E}+02 \\ \mathrm{~B} 103 & \mathrm{PO4} & \mathrm{kg} & 2.13 E+04\end{array}$

$\begin{array}{llll}B 103 & S & k g & 9.67 E+03\end{array}$

$\mathrm{B103} \mathrm{Si} \quad \mathrm{kg} \quad 9.72 \mathrm{E}+02$

$\begin{array}{llll}\mathrm{B} 103 & \mathrm{SO} 4 & \mathrm{~kg} & 2.90 \mathrm{E}+04\end{array}$

B103 Sr kg 1.02E+02

$B 103$ TOC $\mathrm{kg} \quad 2.47 \mathrm{E}+02$

$B 103 U \quad \mathrm{~kg} \quad 2.44 \mathrm{E}+03$

$\begin{array}{llll}B 103 & \mathrm{Zr} & \mathrm{kg} & 4.50 \mathrm{E}+01\end{array}$

$\begin{array}{llll}\mathrm{B} 103 & \mathrm{Ac} 227 & \mathrm{Ci} & 3.79 \mathrm{E}-05\end{array}$

B103 Am241 Ci 1.40E-00

B103 Am243 Ci 1.35E-06

$\begin{array}{llll}\mathrm{B} 103 & \mathrm{Ba} 137 & \mathrm{Ci} & 9.68 \mathrm{E}+03\end{array}$

$\begin{array}{llll}\mathrm{B} 103 & \mathrm{Cl} & \mathrm{Ci} & 1.41 \mathrm{E}-01\end{array}$

B103 Cd113 Ci 2.73E-01

$\begin{array}{llll}\mathrm{B} 103 & \mathrm{Cm} 242 & \mathrm{Ci} & 2.97 \mathrm{E}-04\end{array}$

$\begin{array}{llll}\mathrm{B} 103 & \mathrm{Cm} 243 \mathrm{Ci} & 6.07 \mathrm{E}-06\end{array}$

B103 Cm244 Ci 3.18E-05

$\begin{array}{llll}\mathrm{B} 103 & \mathrm{C} 060 & \mathrm{Ci} & 2.48 \mathrm{E}-02\end{array}$

$\begin{array}{llll}\mathrm{B} 103 & \mathrm{Cs} 134 & \mathrm{C} i & 5.84 \mathrm{E}-04\end{array}$

$\begin{array}{llll}\mathrm{B} 103 & \mathrm{Cs} 137 & \mathrm{Ci} & 1.02 \mathrm{E}+04\end{array}$

$\begin{array}{llll}\mathrm{B} 103 & \mathrm{Cu} 152 & \mathrm{Ci} & 1.70 \mathrm{E}-02\end{array}$

$B 103$ Eul54 Ci 4.37E-01

B103 Eu155 Ci $1.36 \mathrm{E}-00$

$\begin{array}{llll}B 103 & 1129 & C i & 1.49 E-03\end{array}$

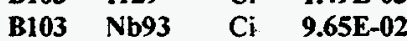

B103 Ni59 Ci $1.34 \mathrm{E}-01$

B103 Ni63 Ci 1.21E+01

B103 Np237 Ci $4.86 \mathrm{E}-03$

B103 Pa231 Ci 7.71E-05

$\mathrm{B} 103$ Pu238 $\mathrm{Ci}$ 4.00E-02

B103 Pu239 Ci 7.70E+01

B103 Pu240 Ci Incl. in 239

B103 Pu241 Ci 1.96E-00

$\begin{array}{llll}\text { B103 Pu242 } & \text { Ci } & 8.66 E-06\end{array}$

B103 Ra226 Ci 8.22E-06

B103 Ra228
$B B$

Sept98

$6.16 \mathrm{E}+02$

$7.59 \mathrm{E}-01$

$1.20 \mathrm{E}-04$

.95E-05

$2.29 \mathrm{E}-02$

6.17E-01

2.98E-03

$1.15 \mathrm{E}-02$

3.96E-01

$1.77 \mathrm{E}-02$

3.05E-03

4.01E-01

$6.16 \mathrm{E}+02$

7.33E-02

$7.96 \mathrm{E}+03$

$1.01 E+03$

$6.00 \mathrm{E}+02$

$.00 \mathrm{E}+02$

$3.40 \mathrm{E}+03$

$1.80 \mathrm{E}+02$

$1.44 \mathrm{E}+04$

$7.50 \mathrm{E}+02$

$0.00 \mathrm{E}+01$

$3.85 \mathrm{E}+02$

$0.00 \mathrm{E}+01$

$0.00 \mathrm{E}+01$

$7.74 \mathrm{E}+04$

$1.05 \mathrm{E}+02$

$2.75 \mathrm{E}+03$

$3.41 \mathrm{E}+04$

$\mathrm{NA}$

$6.10 \mathrm{E}+02$

3.02E+04

NA

$4.30 \mathrm{E}+02$

$5.32 \mathrm{E}+04$

$6.00 \mathrm{E}+01$

$2.47 \mathrm{E}+02$

$1.71 \mathrm{E}+03$

$6.00 \mathrm{E}+01$

3.79E-05

1.35E-00

9.27E-06

$8.80 \mathrm{E}+03$

1.41E-01

2.73E-01

2.04E-03

4.17E-05

2.18E-04

2.48E-02

$5.84 \mathrm{E}-04$

$9.30 \mathrm{E}+03$

1.70E-02

4.37E-01

$1.36 \mathrm{E}-00$

$1.49 \mathrm{E}-03$

9.65E-02

$1.34 \mathrm{E}-01$

$1.21 \mathrm{E}+01$

4.86E- 03

7.71E-05

3.91E-01

$7.06 \mathrm{E}+01$

5.48E-00

$1.35 \mathrm{E}+01$

5.95E-05

8.22E-06

$7.46 \mathrm{E}-11$ 1\%-ile

$0.00 \mathrm{E}+01 \quad 3.58 \mathrm{E}+02$

$0.00 \mathrm{E}+01 \quad 5.64 \mathrm{E}-03$

$0.00 \mathrm{E}+01 \quad 1.08 \mathrm{E}-05$

$0.00 \mathrm{E}+01 \quad 8.98 \mathrm{E}-06$

$0.00 \mathrm{E}+01 \quad 0.00 \mathrm{E}+01$

$0.00 \mathrm{E}+01 \quad 1.17 \mathrm{E}-01$

$0.00 \mathrm{E}+01 \quad 1.42 \mathrm{E}-03$

$0.00 \mathrm{E}+01 \quad 5.46 \mathrm{E}-03$

$0.00 \mathrm{E}+01 \quad 1.88 \mathrm{E}-01$

$0.00 \mathrm{E}+01 \quad 8.41 \mathrm{E}-03$

$0.00 \mathrm{E}+01 \quad 1.44 \mathrm{E}-03$

$0.00 \mathrm{E}+01 \quad 1.90 \mathrm{E}-01$

$0.00 \mathrm{E}+01$

$2.15 \mathrm{E}+02$

$0.00 \mathrm{E}+01 \quad 9.57 \mathrm{E}-04$

$1.07 \mathrm{E}+01$

$1.74 \mathrm{E}+02$

$3.16 \mathrm{E}-01$

9.39E-01

$9.52 \mathrm{E}-00$

$8.76 \mathrm{E}+01$

$3.60 \mathrm{E}+01$

$1.26 \mathrm{E}+02$

$4.18 \mathrm{E}+01$

3.41E-01

$1.60 \mathrm{E}-01$

$1.53 \mathrm{E}-00$

$0.00 \mathrm{E}+01$

4.31E-02

$3.20 \mathrm{E}-03$

$1.26 \mathrm{E}-01$

$1.10 \mathrm{E}+03$

$1.35 \mathrm{E}-0.2$

3.47E-00

1.35E +02

$5.38 \mathrm{E}+02$

2.35E-00

$1.27 \mathrm{E} \div 02$

2.19E-00

$1.13 \mathrm{E}-00$

9.67E-00

7.93E-03

$4.96 \mathrm{E}-01$

$4.29 \mathrm{E}+01$

$1.15 \mathrm{E}-03$

$0.00 \mathrm{E}+01$

$1.59 \mathrm{E}-03$

$4.48 \mathrm{E}-10$

$0.00 \mathrm{E}+01$

$0.00 \mathrm{E}+01$

$0.00 \mathrm{E}+01$

9.86E-08

$2.02 \mathrm{E}-09$

$1.06 \mathrm{E}-08$

9.53E-04

$0.00 \mathrm{E}+01$

$3.22 \mathrm{E} \div 01$

$0.00 \mathrm{E}+01$

$0.00 \mathrm{E}+01$

$0.00 \mathrm{E}+01$

$0.00 \mathrm{E}+01$

$0.00 \mathrm{E}+01$

$0.00 \mathrm{E}+01$

$0.00 \mathrm{E}+01$

$1.61 \mathrm{E}-06$

$0.00 \mathrm{E}+01$

1.33E-05

$2.56 \mathrm{E}-02$

2.56E-02

6.51E-04

$2.88 \mathrm{E}-09$

$0.00 \mathrm{E}+01$

$0.00 \mathrm{E}+01$
$3.58 \mathrm{E}+02$

$3.13 \mathrm{E}-00$

$2.59 \mathrm{E}+01$

$5.11 \mathrm{E}+01$

$0.00 \mathrm{E}+01$

4.58E-01

2.37E- 02

2.14E-00

$1.68 \mathrm{E}+04$

$2.51 \mathrm{E}-01$

$1.31 E+02$

$7.88 \mathrm{E}+03$

$3.70 \mathrm{E}+03$

4.72E +01

$3.30 \mathrm{E} \div 03$

$1.91 \mathrm{E}+02$

$8.20 \mathrm{E}+01$

$3.05 \mathrm{E}+02$

$1.93 \mathrm{E}-01$

$6.76 \mathrm{E}-00$

$4.10 \mathrm{E}+02$

2.22E-02

$0.00 \mathrm{E}+01$

4.13E-02

4.20E-08

$1.09 \mathrm{E}+03$

6.40E-03

$0.00 \mathrm{E}+01$

9.25E-06

$1.89 \mathrm{E}-07$

$9.90 \mathrm{E}-07$

1.23E-02

$6.55 \mathrm{E}-05$

$1.00 \mathrm{E}+03$

$0.00 \mathrm{E}+01$

$0.00 \mathrm{E}+01$

$0.00 \mathrm{E}+01$

3.13E-04

$0.00 \mathrm{E}+01$

$5.94 \mathrm{E}-04$

$5.36 \mathrm{E}-02$

$1.51 \mathrm{E}-04$

$1.08 \mathrm{E}-05$

$1.25 \mathrm{E}-03$

$2.40 \mathrm{E}-00$

$2.40 \mathrm{E}-00$

6.10E-02

2.70E-07

$0.00 E+01$

$0.00 \mathrm{E} \div 01$

50\%-ile

$9.86 \mathrm{E}+03$

90\%-ile

99\%-jle

$2.69 \mathrm{E}+05$

6.17E-00

$1.76 \mathrm{E}-03$

$1.20 \mathrm{E}-04$

$5.97 \mathrm{E}-04$

$1.46 \mathrm{E}-03$

$1.40 \mathrm{E}-01$

2.29E-02

$4.95 \mathrm{E}-04$

$6.17 \mathrm{E}-01$

1.57E-02

75E 00

6.05E-02

2.08E-00

$7.82 \mathrm{E}-02$

2.62E-00

$2.30 \mathrm{E}-01$

$3.01 \mathrm{E}-01$

$8.86 \mathrm{E}-01$

.32E-02

$1.60 \mathrm{E}-02$

2.11E-00

$1.16 \mathrm{E}+04$ 


\begin{tabular}{|c|c|c|c|c|c|c|c|c|c|c|c|}
\hline ank & Anal. & Un & $\frac{B B}{9 y 98}$ & ept98 & $\%$-ile & $10 \%$-ile & 50\%-ile & $90 \%$-ile & 99\%-ile & Mean & Std Dev \\
\hline 03 & 06 & $\mathrm{Ci}$ & SIE-09 & & $00 \mathrm{E}+01$ & $.00 \mathrm{E}+01$ & -09 & E-08 & E-08 & E-08 & 8 \\
\hline & b125 & $\mathrm{Ci}$ & $.22 E-02$ & $.22 \mathrm{E}-02$ & $0.00 \mathrm{E}+01$ & $10 E+01$ & & $81 E-02$ & $.15 E-01$ & $8 \mathrm{E}-02$ & $37 \mathrm{E}-02$ \\
\hline 03 & e 79 & $\mathbf{C i}$ & $2.40 \mathrm{E}-02$ & $40 \mathrm{E}-02$ & $0.00 E+01$ & & & -02 & OE-01 & $3.03 \mathrm{E}-02$ & \\
\hline 103 & m151 & $\mathrm{Ci}$ & $95 E+01$ & $8.95 E+01$ & $0.00 E+01$ & $00 \mathrm{E}+01$ & $8.95 \mathrm{E}+01$ & $98 \mathrm{E}+02$ & $9.59 \mathrm{E}+02$ & $.76 E+02$ & $.22 E+02$ \\
\hline 03 & r89/90 & $\mathrm{Ci}$ & $63 E+03$ & $.29 E+03$ & $.00 \mathrm{E} \div 01$ & $12 \mathrm{E}+02$ & $.61 \mathrm{E}+04$ & $6.15 E+04$ & $.31 E+05$ & $2.66 \mathrm{E}+04$ & $5.51 \mathrm{E}+04$ \\
\hline 103 & c99 & $\mathrm{Ci}$ & $90 \mathrm{E}-01$ & $.90 \mathrm{E}-01$ & $.00 \mathrm{E}+01$ & $00 \mathrm{E}+01$ & $.90 \mathrm{E}-01$ & $83 E-00$ & $.90 \mathrm{E}-00$ & $1.12 \mathrm{E}-00$ & $21 E-00$ \\
\hline 03 & 229 & $\mathrm{Ci}$ & $.45 \mathrm{E}-08$ & 1.45E-08 & $0 E \div 01$ & $03 E-09$ & & $.17 E-08$ & 1.38E-07 & $2.34 \mathrm{E}-08$ & 84E-08 \\
\hline 03 & 232 & $\mathrm{Ci}$ & $3.28 \mathrm{E}-11$ & $3.28 \mathrm{E}-11$ & $E+01$ & $59 \mathrm{E}-12$ & 11 & $7 \mathrm{E}-10$ & F-10 & & \\
\hline B103 & Tin126 & $\mathrm{Ci}$ & 3.61 & $3.61 \mathrm{E}$ & 0 & -01 & & & & & \\
\hline 03 & Tritium & $\mathrm{Ci}$ & 7.6 & 7.6 & & & & & & & -01 \\
\hline 3 & $\mathbf{U}_{2}$ & $\mathrm{Ci}$ & & & & $E=06$ & US & $E-04$ & $=04$ & $6.72 \mathrm{E}-05$ & 8.14E-05 \\
\hline 03 & $\mathbf{U}$ & $\mathrm{Ci}$ & 2.21E-06 & 4.69E-07 & +01 & $3.09 \mathrm{E}-07$ & & $7.88 \mathrm{E}-06$ & 2.11E-05 & $3.57 \mathrm{E}-06$ & $4.33 E-06$ \\
\hline $\boldsymbol{S}$ & U234 & $\mathrm{Ci}$ & $2.65 \mathrm{E}-00$ & $5.63 \mathrm{E}-01$ & $0.00 \mathrm{E}+01$ & $3.71 E-01$ & $2.65 \mathrm{E}-00$ & $9.44 \mathrm{E}-00$ & $2.53 \mathrm{E}+01$ & $4.28 \mathrm{E}-00$ & $5.19 E-00$ \\
\hline $0 \Omega$ & $\mathbf{U} 235$ & $\mathrm{Ci}$ & $1.18 \mathrm{E}-01$ & 2.52E-02 & +01 & $1.65 \mathrm{E}-02$ & -01 & $4.21 \mathrm{E}-01$ & $1.13 \mathrm{E}-00$ & $1.91 \mathrm{E}-01$ & 2.31E-01 \\
\hline 10 & U236 & $\mathrm{Ci}$ & $2.01 \mathrm{E}-02$ & $4.28 \mathrm{E}-03$ & $E+01$ & 2.81E-03 & -02 & E-02 & $:-01$ & $E-02$ & $3.93 \mathrm{E}-02$ \\
\hline B103 & U238 & $\mathrm{Ci}$ & $2.68 \mathrm{E}-00$ & 5.71E-01 & $E+01$ & $3.75 \mathrm{E}-01$ & -00 & $9.55 \mathrm{E}-00$ & +01 & $3 E-00$ & $5.25 \mathrm{E}-00$ \\
\hline 103 & Y90 & $\mathrm{Ci}$ & $2.63 E+03$ & $1.29 E+03$ & +01 & $E+01$ & 03 & +04 & +04 & $E+03$ & $E+03$ \\
\hline 03 & Zr9. & $\mathrm{Ci}$ & -01 & E-01 & 01 & +01 & & & & $E-00$ & $E-00$ \\
\hline & Al & kg & 3.5 & $3.56 E+03$ & +02 & $E+03$ & & +04 & +04 & +04 & +04 \\
\hline B104 & $\mathbf{B i}$ & kg & 2.2 & +04 & +04 & $E+04$ & +04 & $8+04$ & $6.22 \mathrm{E}+04$ & $E+04$ & $9.29 \mathrm{E}+03$ \\
\hline B104 & Ca & kg & $6.30 \mathrm{E}+02$ & $6.30 E+02$ & $E+02$ & $7.14 \mathrm{E}+02$ & +03 & $E+03$ & $6.73 E+03$ & $1.74 E+03$ & $1.14 E+03$ \\
\hline B104 & Cl & kg & $4.08 \mathrm{E}+03$ & $4.08 E+03$ & +02 & $1.35 \mathrm{E}+03$ & & +03 & $\mathrm{DE}+03$ & $E+03$ & $5.76 \mathrm{E}+02$ \\
\hline B104 & $\mathrm{CO} 3$ & kg & $1.12 E+04$ & $1.12 E+04$ & $:+02$ & $E+03$ & +04 & $E+04$ & $E+04$ & $E+04$ & $E+03$ \\
\hline 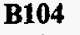 & $\mathrm{Cr}$ & kg & $9.90 \mathrm{E}+02$ & $9.90 \mathrm{E}+02$ & +02 & -02 & 33 & +03 & 03 & -03 & $E+02$ \\
\hline 4 & $\mathbf{F}$ & kg & +03 & +03 & 3 & 03 & & & & & $\varepsilon+04$ \\
\hline 4 & $\mathrm{Fe}$ & kg & 2.0 & 2.0 & +03 & $E+04$ & 04 & $E+04$ & 04 & $2+04$ & $1.12 E+04$ \\
\hline B104 & Hg & kg & $9.56 \mathrm{E}-00$ & $3.86 \mathrm{E}+01$ & $1.20 \mathrm{E}-01$ & 2.91E-00 & -00 & $E+01$ & $E+01$ & $E+01$ & $1.40 E+01$ \\
\hline B104 & $\mathbf{K}$ & kg & $4.16 E+02$ & $4.16 E+02$ & $E+01$ & $E+02$ & -03 & $\div 03$ & +04 & $E+03$ & $E+03$ \\
\hline 104 & La & kg & $0.00 \mathrm{E}+01$ & $0.00 \mathrm{E}+01$ & $=01$ & & & & +02 & -01 & +01 \\
\hline 04 & Mn & kg & $0.00 \mathrm{E}+01$ & $0.00 \mathrm{E}+01$ & -01 & 01 & & 02 & -03 & -02 & -02 \\
\hline 104 & $\mathrm{Na}$ & kg & 2.20 & $\div 05$ & 05 & 55 & & & +02 & 05 & \\
\hline B & $\mathrm{Ni}$ & $k$ & 02 & +02 & 00 & +01 & 8. & +02 & +02 & $E+02$ & $E+02$ \\
\hline B104 & $\mathrm{NO2}$ & kg & $4.73 E+03$ & $4.73 E+03$ & $\therefore+03$ & $E+03$ & 104 & $E+04$ & +04 & $E+04$ & $E+04$ \\
\hline B104 & NO3 & kg & $1.96 E+05$ & $1.96 E+05$ & +04 & $:+05$ & 05 & -05 & +05 & $E+05$ & $E+05$ \\
\hline 04 & $\mathbf{P}$ & kg & $9.99 E+04$ & NA & 04 & & & & & 04 & \\
\hline 4 & $\mathbf{P b}$ & kg & +01 & $0.00 \mathrm{E}+01$ & & & & & & & \\
\hline 4 & $\mathbf{P C}$ & $\mathrm{kg}$ & 3 & 05 & & & & & t05 & -04 & +04 \\
\hline 104 & $\mathbf{S}$ & kg & +04 & NA & +03 & +03 & 04 & \pm 04 & +04 & $E+04$ & $E+03$ \\
\hline B104 & Si & kg & $1.10 \mathrm{E}+04$ & $1.10 E+04$ & +03 & $5.03 E+03$ & -04 & $\div+04$ & +04 & $E+04$ & $5.12 E+03$ \\
\hline B104 & SO4 & kg & $1.26 E+05$ & $4.21 E+04$ & +03 & $1.77 \mathrm{E}+04$ & 4. & -04 & +05 & $E+04$ & $E+04$ \\
\hline B104 & Sr & $\mathrm{kg}$ & $0.00 \mathrm{E}+01$ & $0.00 E+01$ & +01 & -02 & & +02 & & 2 & +02 \\
\hline 104 & TOC & $\mathrm{kg}$ & & & & & & & & & +02 \\
\hline & $\mathbf{U}$ & & & & 02 & 03 & & 04 & -04 & +03 & $E+03$ \\
\hline B104 & $\mathbf{Z r}$ & kg & $1.38 E+01$ & $1.38 E+01$ & -00 & +01 & 02 & $E \div 02$ & +02 & $E+02$ & $1.87 E+02$ \\
\hline B104 & Ac227 & $\mathrm{Ci}$ & $6.00 \mathrm{E}-05$ & $6.00 \mathrm{E}-05$ & \pm 01 & $0.00 \mathrm{E}+01$ & 05 & -04 & -04 & E-04 & $1.26 \mathrm{E}-04$ \\
\hline B104 & Am241 & $\mathrm{Ci}$ & 4.14E-01 & 3.64E-01 & $E-00$ & $3.28 \mathrm{E}+01$ & 02 & 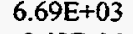 & 04 & 03 & +03 \\
\hline 104 & $\operatorname{Am} 24$ & $\mathrm{Ci}$ & -06 & 2.5 & & & & & & & \\
\hline 104 & & c & & & & & & & & & +04 \\
\hline & & $\mathrm{Ci}$ & & & & & & & & -01 & -01 \\
\hline 104 & 113 & $\mathrm{Ci}$ & 01 & -01 & .03 & 1.56 & 01 & -00 & 00 & -01 & $7.53 \mathrm{E}-01$ \\
\hline B104 & Cm242 & $\mathrm{Ci}$ & 8.32E-04 & $730 \mathrm{E}-04$ & -05 & 2.92 & 04 & 1. & 03 & -04 & $6.26 \mathrm{E}-04$ \\
\hline B104 & Cm243 & $\mathrm{Ci}$ & $1.71 E-05$ & $1.50 \mathrm{E}-05$ & -0 & 06 & 05 & 3. & 05 & -05 & $1.29 \mathrm{E}-05$ \\
\hline B104 & $\operatorname{Cm} 244$ & $\mathrm{Ci}$ & $6.8^{\circ}$ & E-05 & $E-06$ & & & & & & \\
\hline B104 & Co60 & $\mathrm{Ci}$ & 4 & 4.4 & & & & & & & +02 \\
\hline B104 & & $\mathrm{Ci}$ & & & & & & & & & -03 \\
\hline & & $\mathrm{Ci}$ & & +04 & & & & & & 104 & $6.92 E+04$ \\
\hline 104 & 152 & $\mathrm{Ci}$ & -02 & E-02 & $\div 01$ & $E+01$ & 02 & -01 & .01 & $E-02$ & $9.39 E-02$ \\
\hline 104 & 154 & $\mathrm{Ci}$ & $8.79 E-01$ & $8.79 E-01$ & +01 & $0.00 \mathrm{E}+01$ & & 0 & .00 & -00 & -00 \\
\hline B104 & Eul55 & $\mathrm{Ci}$ & $3.28 \mathrm{E}-00$ & $3.28 \mathrm{E}-00$ & +01 & $0.00 \mathrm{E}+01$ & 00 & $E+01$ & 3. & -00 & E-00 \\
\hline B104 & I1 & $\mathrm{Ci}$ & $2.73 \mathrm{E}-03$ & 2.73E-03 & $\therefore+01$ & -04 & & $\mathrm{E}_{-03}$ & & -03 & E-03 \\
\hline B104 & NI & $\mathrm{Ci}$ & 1.76 & $1.76 \mathrm{E}$ & & & & & & & \\
\hline B104 & Ni59 & $\mathrm{C}$ & & & & & & & & & $E-01$ \\
\hline B1 & & $\mathrm{Ci}$ & & $8+01$ & +01 & $1.70 \mathrm{E}-00$ & & +01 & r02 & $E+01$ & $2.93 E+01$ \\
\hline 104 & Np237 & $\mathrm{Ci}$ & $8.95 E-03$ & $8.95 E-03$ & E-04 & $3.14 \mathrm{E}-03$ & & $E-02$ & $E-02$ & EE-02 & $6.73 \mathrm{E}-03$ \\
\hline 104 & 231 & $\mathrm{Ci}$ & & 1.31 E-04 & $0.00 \mathrm{E}+01$ & & & $E-0$ & & $2.20 \mathrm{E}-04$ & $2.84 \mathrm{E}-04$ \\
\hline & & $\mathrm{Ci}$ & $4.43 E-01$ & $3.89 \mathrm{E}-01$ & 4.57E-02 & $1.56 \mathrm{E}-01$ & & $8.68 E-01$ & $1.75 E-00$ & $5.03 \mathrm{E}-01$ & $3.33 \mathrm{E}-01$ \\
\hline & & $\mathrm{Ci}$ & 8.671 & $7.61 E+01$ & & $3.05 E+01$ & & & & $9.85 E+01$ & $6.52 E+01$ \\
\hline & & $\mathrm{Ci}$ & & & & $-\infty 0$ & E-00 & +01 & -01 & $7.31 \mathrm{E}-00$ & \\
\hline
\end{tabular}




\begin{tabular}{|c|c|c|c|c|c|c|c|c|c|c|c|}
\hline ink & Inal. & $n$ & May 98 & Sept98 & $1 \%$-ile & $10 \%$-ile & $50 \%$-ile & $90 \%$-ile & 99\%-ile & Mean & ev \\
\hline 104 & Pu241 & $\mathbf{C i}$ & $1 . \overline{41 E+01}$ & $\overline{1.24 \mathrm{E}+01}$ & $1.45 \mathrm{E}-00$ & $E-00$ & $1.41 E+01$ & $2.76 \mathrm{E}+01$ & $5.56 \mathrm{E}+01$ & $1.60 \mathrm{E}+01$ & $1.06 E+01$ \\
\hline 8104 & Pu242 & $\mathbf{C i}$ & $6.14 E-05$ & 5.39E-05 & & & $6.14 \mathrm{E}-05$ & & $E-04$ & $E-05$ & $62 \mathrm{E}-05$ \\
\hline B104 & $\operatorname{Ra226}$ & $\mathrm{Ci}$ & $1.17 \mathrm{E}-05$ & $1.17 \mathrm{E}-05$ & $0.00 \mathrm{E} \div 01$ & E-06 & 1.17E-05 & $3.03 E-05$ & $7.35 \mathrm{E}-05$ & $1.54 \mathrm{E}-05$ & $1.50 \mathrm{E}-05$ \\
\hline B104 & $\operatorname{Ra} 228$ & $\mathrm{Ci}$ & $1.60 \mathrm{E}-10$ & $1.60 \mathrm{E}-10$ & $0.00 \mathrm{E}+01$ & 3.23E-11 & $1.60 \mathrm{E}-10$ & $4.14 \mathrm{E}-10$ & 1.01E-09 & 2.11 E-10 & 2.05E-10 \\
\hline B104 & Ru106 & $\mathrm{Ci}$ & $2.06 \mathrm{E}-08$ & $2.06 \mathrm{E}-08$ & $0.00 \mathrm{E}+01$ & $5.45 \mathrm{E}-09$ & $2.06 \mathrm{E}-08$ & 4.34E-08 & $7.02 \mathrm{E}-08$ & 2.33E-08 & $1.55 \mathrm{E}-08$ \\
\hline B104 & Sb125 & $\mathrm{Ci}$ & 4.53E-02 & 4.53E-02 & $0.00 \mathrm{E}+01$ & $0.00 \mathrm{E}+01$ & 4.53E-02 & $1.08 \mathrm{E}-01$ & $2.11 \mathrm{E}-01$ & $5.15 \mathrm{E}-02$ & \\
\hline B104 & Se79 & $\mathbf{C i}$ & $4.40 E-02$ & $4.40 \mathrm{E}-02$ & $0.00 \mathrm{E}+01$ & $E-02$ & $4.40 \mathrm{E}-02$ & & $1.43 \mathrm{E}-01$ & $4.74 \mathrm{E}-02$ & $5 \mathrm{E}-02$ \\
\hline B104 & n151 & $\mathrm{Ci}$ & $1.64 E+02$ & $1.64 \mathrm{E}+02$ & +01 & $E+01$ & $1.64 \mathrm{E}+02$ & $7.24 \mathrm{E}+02$ & $1.53 \mathrm{E}+03$ & $2.76 \mathrm{E}+02$ & $E+02$ \\
\hline B104 & $9 / 90$ & $\mathrm{Ci}$ & $1.02 \mathrm{E}+04$ & $1.02 E+04$ & +03 & & & $E+05$ & $5.50 \mathrm{E}+05$ & $1.20 \mathrm{E}+05$ & $E+05$ \\
\hline B104 & Te99 & $\mathrm{Ci}$ & 1.45E-00 & $1.45 \mathrm{E}-00$ & E-01 & $E-01$ & & 58E-00 & $4.94 \mathrm{E}+01$ & $2.44 \mathrm{E}-00$ & $6.68 \mathrm{E}-00$ \\
\hline B104 & Th229 & $\mathrm{Ci}$ & $3.12 \mathrm{E}-08$ & $3.12 \mathrm{E}-08$ & $E+01$ & $E-09$ & E-08 & 21E-07 & $3.41 \mathrm{E}-07$ & $5.24 \mathrm{E}-08$ & $6.77 \mathrm{E}-08$ \\
\hline B104 & 232 & $\mathrm{Ci}$ & $5.93 E-11$ & $5.93 \mathrm{E}-11$ & $0.00 \mathrm{E}+01$ & $E-11$ & & 2.29E-10 & E-10 & & $1.29 E-10$ \\
\hline B104 & in126 & $\mathrm{Ci}$ & 6.63E-02 & $6.63 \mathrm{E}-02$ & & & $6.63 \mathrm{E}-02$ & & $4.52 \mathrm{E}-01$ & $9.94 \mathrm{E}-02$ & $9.71 \mathrm{E}-02$ \\
\hline B104 & Tritium & $\mathrm{Ci}$ & $1.30 \mathrm{E}-00$ & 1.30E-00 & $7.01 \mathrm{E}-01$ & $E-01$ & & & $1 E-00$ & $E-00$ & E-01 \\
\hline B104 & U232 & $\mathrm{Ci}$ & $1.90 \mathrm{E}-04$ & $2.82 E-05$ & +01 & & & & $2.08 \mathrm{E}-03$ & E-04 & $\mathrm{E}-04$ \\
\hline B104 & $\mathbf{U} 233$ & $\mathrm{Ci}$ & $8.78 \mathrm{E}-06$ & $1.31 \mathrm{E}-06$ & & & & & -05 & & \\
\hline 104 & & $\mathrm{Ci}$ & 8.17E-00 & $1.22 \mathrm{E}-00$ & & & & 01 & 01 & & +01 \\
\hline B104 & & $\mathrm{Ci}$ & $3.61 \mathrm{E}-01$ & 5.37E-02 & & & & -00 & $=-00$ & & -01 \\
\hline B104 & & $\mathrm{Ci}$ & $8.15 E-02$ & $1.21 \mathrm{E}-02$ & & & & $=01$ & -01 & & $E-01$ \\
\hline & & $\mathrm{Ci}$ & $E-00$ & $1.24 \mathrm{E}-00$ & & & & & $E+01$ & & $E+01$ \\
\hline 04 & Y90 & $\mathrm{Ci}$ & $1.02 E+04$ & $1.02 E+04$ & +01 & & & & $E+04$ & & +04 \\
\hline 04 & Zr93 & $\mathbf{C i}$ & $2.09 E-01$ & 2.09E-01 & & & & & $E-00$ & & $E-01$ \\
\hline B105 & Al & $\mathrm{kg}$ & $7.41 \mathrm{E}+02$ & $7.41 \mathrm{E}+02$ & & & & & +05 & & +04 \\
\hline 05 & $\mathbf{B}$ & k & +03 & $7.17 E+03$ & & & & & +04 & & +03 \\
\hline B105 & $\mathrm{Cz}$ & 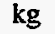 & $1.88 E+03$ & $1.88 E+03$ & +01 & +02 & +02 & +03 & +03 & 103 & +02 \\
\hline B105 & $\mathrm{Cl}$ & & $1.57 E+03$ & $1.57 \mathrm{E}+03$ & & & & & -03 & & +02 \\
\hline B105 & $\mathrm{CO3}$ & $\mathrm{k}$ & $6.28 E+03$ & $6.28 E+03$ & & & & & +04 & & +03 \\
\hline B105 & $\mathrm{Cr}$ & & $1.04 \mathrm{E}+02$ & $1.04 \mathrm{E}+02$ & & & & & +03 & & +02 \\
\hline B105 & $\mathbf{F}$ & & $7.73 E+03$ & $7.73 E+03$ & 02 & & & & 104 & & +04 \\
\hline B105 & $\mathbf{F e}$ & $\mathbf{k}$ & $2.59 \mathrm{E}+03$ & $2.59 \mathrm{E}+03$ & -02 & +03 & 03 & 104 & +04 & 103 & +03 \\
\hline B105 & $\mathbf{H g}$ & $\mathrm{k}$ & $2.82 \mathrm{E}-00$ & $3.82 \mathrm{E}-00$ & +01 & -01 & & -00 & +01 & & $E-00$ \\
\hline B105 & $\mathbf{K}$ & & $3.14 E+02$ & $3.14 E+02$ & & +01 & & & +04 & & +03 \\
\hline B105 & La & $\mathbf{n}$ & $0.00 \mathrm{E}+01$ & $0.00 \mathrm{E}+01$ & $=-02$ & $E-01$ & & & $E+02$ & $:+01$ & $E+01$ \\
\hline B105 & Mn & $k$ & $0.00 \mathrm{E}+01$ & $0.00 \mathrm{E}+01$ & & 01 & & & +02 & -02 & +02 \\
\hline 05 & $\mathbf{N a}$ & 1 & +05 & $1.40 \mathrm{E}+05$ & & .04 & & & -05 & -05 & -04 \\
\hline B105 & $\mathbf{N i}$ & $\mathrm{kg}$ & $2.50 \mathrm{E}+02$ & $2.50 \mathrm{E}+02$ & -01 & -00 & & -02 & +02 & -02 & +02 \\
\hline B105 & NO2 & $\mathrm{gg}$ & $3.63 E+04$ & $3.63 \mathrm{E}+04$ & +02 & $\div 03$ & & +04 & 04 & -03 & +03 \\
\hline B105 & NO3 & $\mathrm{kg}$ & $1.31 E+05$ & $1.31 \mathrm{E}+05$ & & +04 & & +05 & & -05 & +05 \\
\hline B105. & $\mathbf{P}$ & $\mathrm{k}$ & $2.12 \mathrm{E}+04$ & $\mathbf{N A}$ & +04 & +04 & & & +04 & +04 & +03 \\
\hline 5 & $\mathbf{P b}$ & $\mathrm{k}$ & $i+01$ & ++01 & & & & -02 & -02 & -02 & +02 \\
\hline 05 & PO4 & $\mathrm{kg}$ & +04 & $6.58 \mathrm{E}+04$ & & +04 & & -05 & 05 & -04 & +04 \\
\hline B105 & $\mathbf{s}$ & & $6.97 \mathrm{E}+03$ & NA & +02 & +03 & & -04 & +04 & -04 & +04 \\
\hline B105 & Si & $\mathbf{k g}$ & $1.11 \mathrm{E}+03$ & $1.11 \mathrm{E}+03$ & +02 & $\therefore+03$ & & +03 & +03 & $E+03$ & $E+02$ \\
\hline B105 & SO4 & $\mathrm{kg}$ & $2.09 E+04$ & $2.09 E+04$ & +02 & +03 & & +05 & 05 & -04 & +04 \\
\hline & $\mathrm{Sr}$ & kg & & $i+01$ & & & & & & & +02 \\
\hline 5 & & $\mathrm{~kg}$ & & -00 & & & & & & & +02 \\
\hline 05 & $\mathbf{U}$ & $\mathrm{kg}$ & $9.79 E+03$ & $9.79 \mathrm{E}+03$ & & +02 & & 03 & +03 & 03 & +02 \\
\hline B105 & $\mathbf{Z r}$ & kg & $7.74 \mathrm{E}-00$ & $7.74 \mathrm{E}-00$ & -01 & -00 & & +02 & +03 & +02 & +02 \\
\hline B105 & Ac227 & $\mathbf{C i}$ & -05 & 8.07E-05 & & +01 & & -04 & -04 & -04 & $E-04$ \\
\hline B105 & A & $\mathrm{Ci}$ & E-01 & $4.76 \mathrm{E}-01$ & & & & & & $E+03$ & +03 \\
\hline B105 & & $\mathrm{Ci}$ & & & & & & & & -06 & -06 \\
\hline B105 & B & $\mathrm{Ci}$ & $2.32 E+04$ & $2.32 E+04$ & & & & & & -04 & +04 \\
\hline & & $\mathrm{Ci}$ & & & & & & & & & \\
\hline 105 & & $\mathrm{Ci}$ & 01 & 6.33E-01 & & -02 & & & -00 & -01 & -01 \\
\hline B105 & & $\mathrm{Ci}$ & .04 & $7.43 E-04$ & & -04 & & & & & -04 \\
\hline B105 & 243 & $\mathrm{Ci}$ & $1.50 \mathrm{E}-05$ & 1.52E-05 & -07 & E-06 & & & $E-05$ & $=-05$ & E-05 \\
\hline B105 & $\mathrm{Cm} 244$ & $\mathrm{Ci}$ & $7.65 E-05$ & $7.76 \mathrm{E}-05$ & & & & & & E-05 & $\mathrm{E}-05$ \\
\hline B105 & Co60 & $\mathrm{Ci}$ & & & & & & & & & $i+01$ \\
\hline B105 & & $\mathrm{Ci}$ & & & & & & & & & $E-03$ \\
\hline B105 & & $\mathrm{Ci}$ & & $E+04$ & & & & -04 & +04 & & +04 \\
\hline B & & $\mathrm{Ci}$ & & $4.04 \mathrm{E}-02$ & & & & & & -0.2 & $=-02$ \\
\hline 35 & & $\mathrm{Ci}$ & E-00 & $1.03 \mathrm{E}-000$ & & $:+01$ & & -00 & $E-00$ & -00 & $E-00$ \\
\hline B105 & Eu155 & $\mathrm{Ci}$ & $3.08 \mathrm{E}-00$ & $3.08 \mathrm{E}-00$ & $E+01$ & $0.00 \mathrm{E}+01$ & & & $E+01$ & $E-00$ & $E-00$ \\
\hline B105 & 1129 & $\mathrm{Ci}$ & $3.43 E-03$ & $3.43 \mathrm{E}-03$ & $E+01$ & $1.13 \mathrm{E}-03$ & & $E-03$ & 8.72E-03 & $E-03$ & $E-03$ \\
\hline B105 & Nb93 & $\mathrm{Ci}$ & 2.22E-01 & 2.22E-01 & +01 & $E+01$ & E-01 & $2.63 E-00$ & $E-00$ & $E-01$ & $4 \mathrm{E}-00$ \\
\hline B105 & Ni59 & $\mathrm{Ci}$ & $3.12 \mathrm{E}-01$ & $3.12 \mathrm{E}-01$ & +01 & $=-04$ & & $E-00$ & $E-00$ & $9.17 \mathrm{E}-01$ & $3 E-00$ \\
\hline B105 & Ni63 & $\mathrm{Ci}$ & $2.80 \mathrm{E}+01$ & $2.80 \mathrm{E}+01$ & $E+01$ & & & $E+02$ & $4.78 E+02$ & $E+01$ & $\mathrm{E}+02$ \\
\hline & & $C:$ & $1.12 \mathrm{E}-02$ & & $3.96 \mathrm{E}-04$ & $0 \mathrm{E}-03$ & $1.12 \mathrm{E}-02$ & $2.98 \mathrm{E}-02$ & $5.02 \mathrm{E}-02$ & $1.42 E-02$ & $1.13 \mathrm{E}-02$ \\
\hline
\end{tabular}




\begin{tabular}{|c|c|c|c|c|c|c|c|c|c|c|c|}
\hline ank & nal. & In & May $\frac{B B}{98}$ & Sept98 & $1 \%$-ile & $10 \%$-ile & 50\%-ile & $90 \%$-ile & $99 \%$-ile & Mean & Std Dev \\
\hline 3105 & Pa231 & $\mathrm{Ci}$ & $1 \longdiv { 1 . 7 4 \mathrm { E } - 0 4 }$ & $\overline{1.74 \mathrm{E}-04}$ & $0.00 \mathrm{E}+01$ & $0.00 \mathrm{E}+01$ & $1.74 \mathrm{E}-04$ & $4.66 \mathrm{E}-04$ & 8.72E-04 & $2.14 \mathrm{E}-04$ & $1.97 \mathrm{E}-04$ \\
\hline B105 & Pu238 & $\mathrm{Ci}$ & $1.66 \mathrm{E}-01$ & $1.68 \mathrm{E}-01$ & $5.88 \mathrm{E}-03$ & $4.15 \mathrm{E}-02$ & $1.66 \mathrm{E}-01$ & $4.41 \mathrm{E}-01$ & $7.44 \mathrm{E}-01$ & $1 E-01$ & $1.67 \mathrm{E}-01$ \\
\hline B105 & Pu239 & $\mathrm{Ci}$ & $3.06 \mathrm{E}+01$ & $3.10 \mathrm{E}+01$ & $1.08 \mathrm{E}-00$ & $7.65 \mathrm{E}-00$ & $3.06 E+01$ & $8.14 \mathrm{E}+01$ & $1.37 \mathrm{E}+02$ & $3.89 \mathrm{E}+01$ & $3.08 \mathrm{E}+01$ \\
\hline B105 & Pu240 & $\mathrm{Ci}$ & $2.35 \mathrm{E}-00$ & $2.38 \mathrm{E}-00$ & 8.32E-02 & $5.88 \mathrm{E}-01$ & 2.35E-00 & $6.25 \mathrm{E}-00$ & $1.05 \mathrm{E}+01$ & $2.98 \mathrm{E}-00$ & $2.37 \mathrm{E}-00$ \\
\hline B105 & Pu241 & $\mathbf{C i}$ & 5.61E-00 & $5.68 \mathrm{E}-00$ & $1.99 \mathrm{E}-01$ & $1.40 \mathrm{E}-00$ & 5.61E-00 & $1.49 \mathrm{E}+01$ & $2.51 E+01$ & $7.12 \mathrm{E}-00$ & $5.65 \mathrm{E}-00$ \\
\hline B105 & Pu242 & $\mathrm{Ci}$ & $2.47 \mathrm{E}-05$ & $2.50 \mathrm{E}-05$ & $8.74 \mathrm{E}-07$ & $6.18 E-06$ & 2.47E-05 & $6.57 \mathrm{E}-05$ & $1.11 \mathrm{E}-04$ & $3.14 \mathrm{E}-05$ & $2.49 E-05$ \\
\hline 105 & $\operatorname{Ra226}$ & $\mathbf{C i}$ & 1.58E-05 & $1.58 \mathrm{E}-05$ & $0.00 \mathrm{E}+01$ & $0.00 \mathrm{E}+01$ & $1.58 \mathrm{E}-05$ & $5.33 \mathrm{E}-05$ & $9.60 \mathrm{E}-05$ & $2.19 \mathrm{E}-05$ & $2.29 \mathrm{E}-05$ \\
\hline 105 & $\mathbf{R a 2 2 8}$ & $\mathrm{Ci}$ & $1.59 \mathrm{E}-10$ & $1.59 \mathrm{E}-10$ & $0.00 \mathrm{E}+01$ & $0.00 \mathrm{E}+01$ & $1.59 \mathrm{E}-10$ & $5.36 \mathrm{E}-10$ & $9.66 \mathrm{E}-10$ & $2.21 \mathrm{E}-10$ & $2.30 \mathrm{E}-10$ \\
\hline 05 & Ru106 & $\mathrm{Ci}$ & 2.29E-08 & $2.29 \mathrm{E}-08$ & $0.00 \mathrm{E}+01$ & $1.16 \mathrm{E}-09$ & 2.29E-08 & -08 & $1.02 \mathrm{E}-07$ & $E-08$ & -08 \\
\hline 05 & Sb125 & $\mathbf{C i}$ & $5.25 \mathrm{E}-02$ & $5.25 \mathrm{E}-02$ & $0.00 E+01$ & $0.00 \mathrm{E}+01$ & $5.25 \mathrm{E}-02$ & $1.32 \mathrm{E}-01$ & $2.13 \mathrm{E}-01$ & $\mathrm{E}-02$ & $=-02$ \\
\hline 105 & Se79 & $\mathrm{Ci}$ & 5.53E-02 & $5.53 E-02$ & $0.00 \mathrm{E}+01$ & $0.00 \mathrm{E}+01$ & $5.53 E-02$ & $1.33 E-01$ & $2.14 \mathrm{E}-01$ & $6.25 \mathrm{E}-02$ & $5.17 \mathrm{E}-02$ \\
\hline 105 & Sm151 & $\mathrm{Ci}$ & $2.06 \mathrm{E}+02$ & $2.06 \mathrm{E}+02$ & $0.00 \mathrm{E}+01$ & $0.00 \mathrm{E}+01$ & $2.06 \mathrm{E}+02$ & $9.92 E+02$ & $1.85 E+03$ & $3.70 \mathrm{E}+02$ & $4.41 E+02$ \\
\hline B105 & Sr89/90 & $\mathrm{Ci}$ & $6.73 E+03$ & $6.73 E+03$ & $2.47 \mathrm{E}+02$ & $1.52 E+03$ & $4.87 \mathrm{E}+04$ & $1.30 \mathrm{E}+05$ & $2.22 E+05$ & $6 \mathrm{E}+04$ & $5.30 \mathrm{E}+04$ \\
\hline B105 & Tc99 & $\mathrm{Ci}$ & 1.82E-00 & $1.82 \mathrm{E}-00$ & $0.00 \mathrm{E}+01$ & $0.00 E+01$ & 1.82E-00 & $5.09 \mathrm{E}-00$ & $1.11 \mathrm{E}+01$ & $0 \mathrm{E}-00$ & $9 \mathrm{E}-00$ \\
\hline B105 & Th229 & $\mathrm{Ci}$ & $3.09 \mathrm{E}-08$ & $3.09 \mathrm{E}-08$ & $0.00 \mathrm{E}+01$ & $0.00 \mathrm{E}+01$ & 3.09E-08 & $8.27 \mathrm{E}-08$ & E-07 & $3.79 \mathrm{E}-08$ & $\mathrm{E}-08$ \\
\hline B105 & Th232 & $\mathbf{C i}$ & $7.54 \mathrm{E}-11$ & $7.54 \mathrm{E}-11$ & $0.00 \mathrm{E}+01$ & $0.00 \mathrm{E}+01$ & $7.54 \mathrm{E}-11$ & -10 & -10 & E-11 & -11 \\
\hline B105 & Tin126 & $\mathrm{Ci}$ & 8.32E-02 & 8.32E-02 & +01 & E-03 & 8.32E-02 & -01 & -01 & $E-02$ & -02 \\
\hline$B 105$ & Tritium & $\mathrm{Ci}$ & 1.79E-00 & $1.79 \mathrm{E}-00$ & E-01 & $9.66 \mathrm{E}-01$ & $E-00$ & $=-00$ & -00 & $E-00$ & -01 \\
\hline B105 & U232 & $\mathbf{C i}$ & $6.99 \mathrm{E}-05$ & $6.99 \mathrm{E}-05$ & $0.00 \mathrm{E}+01$ & $0.00 E+01$ & 6.99E-05 & $E-04$ & $E-04$ & $E-05$ & $E-05$ \\
\hline B105 & U233 & $\mathbf{C i}$ & 3.30E-06 & $3.30 \mathrm{E}-06$ & $0.00 E+01$ & $0.00 \mathrm{E}+01$ & E-06 & $E-06$ & $E-05$ & $4.05 \mathrm{E}-06$ & $E-06$ \\
\hline B105 & U234 & $\mathbf{C i}$ & $3.22 \mathrm{E}-00$ & $3.22 \mathrm{E}-00$ & $0.00 \mathrm{E}+01$ & $0.00 \mathrm{E}+01$ & $3.22 \mathrm{E}-00$ & $E-00$ & $E+01$ & $E-00$ & $E-00$ \\
\hline B105 & U235 & $\mathrm{Ci}$ & $1.43 E-01$ & $1.43 \mathrm{E}-01$ & $0.00 \mathrm{E}+01$ & $0.00 \mathrm{E}+01$ & $1.43 \mathrm{E}-01$ & $E-01$ & & $E-01$ & 2E-01 \\
\hline B105 & U236 & $\mathbf{C i}$ & $3.06 \mathrm{E}-02$ & $3.06 \mathrm{E}-02$ & $E+01$ & $E+01$ & E-02 & $\varepsilon-02$ & & E-02 & $E-02$ \\
\hline B105 & U238 & $\mathrm{Ci}$ & $3.27 E-00$ & $3.27 \mathrm{E}-00$ & $0.00 \mathrm{E}+01$ & $E+01$ & E-00 & -00 & -01 & $E-00$ & -00 \\
\hline 3105 & Y90 & $\mathrm{Ci}$ & $6.73 E+03$ & $6.73 E+03$ & $0.00 \mathrm{E}+01$ & $0.00 \mathrm{E}+01$ & $6.73 E+03$ & +04 & $: 04$ & $E+03$ & +03 \\
\hline B105 & Zr93 & $\mathbf{C i}$ & $2.63 \mathrm{E}-01$ & $2.63 \mathrm{E}-01$ & $0.00 \mathrm{E}+01$ & $0.00 \mathrm{E}+01$ & 2.63E-01 & $E-00$ & $E-00$ & $4 \mathrm{E}-00$ & $6 E-00$ \\
\hline & Al & kg & $3.45 E+03$ & $3.45 E+03$ & $0.00 \mathrm{E}+01$ & $E+01$ & +03 & & & $E+03$ & $E+03$ \\
\hline B106 & $\mathbf{B i}$ & kg & $4.35 E+03$ & $4.35 E+03$ & $1.37 E+03$ & $E+03$ & $E+03$ & & & $E+03$ & $E \div 02$ \\
\hline B106 & Ca & $\mathrm{kg}$ & $1.04 \mathrm{E}+03$ & $1.04 E+03$ & +02 & $E+02$ & $1.21 \mathrm{E}+03$ & +03 & +03 & $E+03$ & \\
\hline B106 & Cl & $\mathrm{kg}$ & $9.24 \mathrm{E}+02$ & +02 & +02 & +02 & +02 & -03 & r03 & $E+02$ & 02 \\
\hline B106 & $\mathrm{CO3}$ & $\mathrm{kg}$ & $9.27 \mathrm{E}+02$ & $9.27 E+02$ & $0.00 \mathrm{E}+01$ & $E+01$ & $2.00 E+03$ & +04 & +04 & $E+03$ & +03 \\
\hline B106 & $\mathrm{Cr}$ & kg & $2.40 \mathrm{E}+02$ & $2.40 \mathrm{E}+02$ & $0.00 \mathrm{E}+01$ & $0.00 \mathrm{E}+01$ & $2.95 E+02$ & +02 & $E+03$ & $E+02$ & +02 \\
\hline B106 & $\mathbf{F}$ & $\mathrm{kg}$ & $2.72 E+03$ & $2.72 E+03$ & $0.00 E+01$ & $E+02$ & +03 & +03 & +03 & $E+03$ & +03 \\
\hline B106 & $\mathrm{Fe}$ & kg & $9.15 E+03$ & $9.15 E+03$ & $4.60 \mathrm{E}+03$ & $E+03$ & $9.73 E+03$ & $:+04$ & $E+04$ & $E+03$ & \\
\hline B106 & $\mathbf{H g}$ & kg & $1.76 \mathrm{E}-00$ & +01 & +01 & +01 & & & & $E-00$ & -00 \\
\hline B106 & K & kg & $2.30 \mathrm{E}+02$ & $2.30 \mathrm{E}+02$ & :01 & +01 & -02 & +03 & -03 & +02 & +02 \\
\hline B106 & $\mathbf{L a}$ & kg & $2.20 \mathrm{E}+01$ & $0.00 E+01$ & $0.00 \mathrm{E}+01$ & $E+01$ & $7.93 \mathrm{E}-00$ & $E+01$ & $E+01$ & $E+01$ & $E+01$ \\
\hline B106 & $\mathbf{M n}$ & kg & $8.60 \mathrm{E}+01$ & $8.60 \mathrm{E}+01$ & $E+01$ & $E+01$ & $E+01$ & +02 & +02 & $E+01$ & +01 \\
\hline B106 & $\mathbf{N a}$ & kg & $6.82 \mathrm{E}+04$ & $6.82 \mathrm{E}+04$ & $E+04$ & $E+04$ & $8+04$ & +04 & +05 & $E+04$ & +04 \\
\hline B106 & Ni & kg & $3.20 \mathrm{E}+01$ & $3.20 \mathrm{E}+01$ & $E+01$ & $E+01$ & $c+01$ & +02 & +02 & $E+01$ & \\
\hline & NO2 & kg & 93 & $i+03$ & & 02 & -03 & .04 & & $\div 03$ & \\
\hline B106 & NO3 & kg & $4.44 E+04$ & $4.44 \mathrm{E}+04$ & +04 & $\div 04$ & +05 & -05 & -05 & +05 & +05 \\
\hline B106 & $\mathbf{P}$ & kg & $4.36 E+04$ & NA & $7.27 E+03$ & $E+04$ & $E+04$ & $i+04$ & +04 & $E+04$ & +03 \\
\hline B106 & $\mathbf{P b}$ & $\mathrm{kg}$ & $2.05 \mathrm{E}+02$ & $2.05 E+02$ & $E+01$ & $E+02$ & $\varepsilon+02$ & & +02 & $E+02$ & $E+01$ \\
\hline B106 & PO4 & kg & $1.35 E+05$ & $4.41 E+04$ & $6.81 E+03$ & $E+04$ & $E+04$ & +04 & +04 & $E+04$ & $E+03$ \\
\hline B106. & $\mathbf{S}$ & kg & $1.01 \mathrm{E}+04$ & NA & & & +03 & & & $E+03$ & +03 \\
\hline B106 & Si & kg & $\therefore+03$ & $E+03$ & & -02 & & & & +02 & \\
\hline 06 & SO4 & $\mathrm{kg}$ & +04 & +04 & 7.7 & +03 & 9.2 & & -04 & +03 & +03 \\
\hline B106 & $\mathrm{Sr}$ & $\mathrm{kg}$ & $i+02$ & $2.37 E+02$ & $2.58 \mathrm{E}+01$ & $E+0 \mid$ & $Q+02$ & +02 & +02 & $E+02$ & +01 \\
\hline B106 & TOC & kg & $1.01 E+03$ & $1.01 E+03$ & $0.00 \mathrm{E}+01$ & $0 \mathrm{E}+02$ & $8+02$ & +03 & +03 & $E+02$ & +02 \\
\hline B106 & $\mathbf{U}$ & kg & $7.30 \mathrm{E}+03$ & $7.30 E+03$ & $0.00 \mathrm{E}+01$ & $E \div 02$ & $E+02$ & -03 & +03 & $E+03$ & +02 \\
\hline B106 & $\mathbf{Z r}$ & kg & $4.10 E+01$ & $4.10 E+01$ & $E+01$ & $E+01$ & +01 & & +02 & +02 & +02 \\
\hline B106 & & $\mathrm{Ci}$ & & & & & & & & & \\
\hline B106 & & $\mathrm{Ci}$ & & & 01 & 01 & 2.8 & & -04 & +03 & +03 \\
\hline B106 & Am243 & $\mathrm{Ci}$ & -06 & 3.11 & -01 & $E+01$ & E-06 & .05 & & $E-06$ & -06 \\
\hline B106 & Bal37 & $\mathrm{Ci}$ & $1.04 \mathrm{E}+04$ & $1.04 E+04$ & $0.00 \mathrm{E}+01$ & $E+03$ & 1.0 & +04 & +04 & $E+04$ & +03 \\
\hline B106 & C14 & $\mathrm{Ci}$ & $2.48 \mathrm{E}-01$ & $2.48 \mathrm{E}-01$ & $0.00 \mathrm{E}+01$ & $0 \mathrm{E}+01$ & E-01 & -00 & -00 & $E-01$ & -01 \\
\hline B106 & Cd113 & $\mathrm{Ci}$ & $5.93 \mathrm{E}-01$ & 5.93E-01 & $0.00 \mathrm{E}+01$ & $E+01$ & -01 & -00 & -00 & $E-01$ & $E-01$ \\
\hline B106 & $\mathrm{Cm} 242$ & $\mathrm{Ci}$ & $7.09 \mathrm{E}-04$ & $5.84 \mathrm{E}-04$ & $0.00 \mathrm{E}+01$ & $0.00 \mathrm{E}+01$ & 7.09E-04 & $E-03$ & $E-03$ & $1.27 \mathrm{E}-03$ & E-03 \\
\hline B106 & Cm243 & $\mathrm{Ci}$ & $2.25 \mathrm{E}-05$ & 1.85 & & $E+01$ & & & -04 & E-05 & $=05$ \\
\hline B106 & Cm244 & $\mathrm{Ci}$ & & & & $E+01$ & & & & E-04 & $E-04$ \\
\hline B106 & Co60 & $\mathrm{Ci}$ & $2.00 E+\theta]$ & $7.46 \mathrm{E}-02$ & $0.00 \mathrm{E}+01$ & $0 \mathrm{E}+01$ & & +01 & +01 & $E-00$ & $E-00$ \\
\hline B106 & Cs134 & $\mathrm{Ci}$ & $2.80 \mathrm{E}+01$ & 2.32E-03 & $0.00 \mathrm{E}+01$ & $3.81 E-00$ & $2.80 \mathrm{E}+01$ & $E+01$ & +02 & $E+01$ & $E+01$ \\
\hline & Cs137 & $\mathrm{Ci}$ & $1.10 E+04$ & $1.10 E+04$ & $0.00 \mathrm{E}$ & $E+03$ & $1.34 E$ & $2.90 E+04$ & +04 & $1.49 E+04$ & $E+03$ \\
\hline & Eu152 & $\mathrm{Ci}$ & $3.59 \mathrm{E}-02$ & 3.59E-02 & $0.00 \mathrm{E}+01$ & $00 E+01$ & $3.59 \mathrm{E}-02$ & & $3.25 \mathrm{E}-01$ & $2 \mathrm{E}-02$ & \\
\hline B106 & Euls & $\mathrm{Ci}$ & $5.10 E+01$ & $1.23 \mathrm{E}-00$ & 0.00 & $0.00 \mathrm{E}+01$ & 5.1 & $E+02$ & $4.61 E+02$ & & $E+02$ \\
\hline B106 & Eu155 & $\mathrm{Ci}$ & $1.14 \mathrm{E}+02$ & 2.72E-00 & $0.00 \mathrm{E}+01$ & $0.00 \mathrm{E}+01$ & $1.14 E+02$ & $5.85 E+02$ & $1.03 E+03$ & $2.07 \mathrm{E}+02$ & $2.54 \mathrm{E}+02$ \\
\hline B106 & 1129 & $\mathrm{Ci}$ & $3.25 E-03$ & $3.25 \mathrm{E}-03$ & $0.00 E+01$ & $1.12 E-03$ & 3.25E-03 & $5.77 \mathrm{E}-03$ & $7.66 E-03$ & $3.34 \mathrm{E}-03$ & $1.76 \mathrm{E}-03$ \\
\hline
\end{tabular}


Tank Anal. Un

\begin{tabular}{|c|c|c|c|}
\hline & & & \\
\hline B106 & Nb93 & $\mathrm{Ci}$ & $1.99 \mathrm{E}-01$ \\
\hline B106 & Ni59 & $\mathrm{Ci}$ & $2.78 E-01$ \\
\hline B106 & Ni63 & $\mathrm{Ci}$ & $2.50 \mathrm{E}+01$ \\
\hline B106 & Np237 & $\mathrm{Ci}$ & $1.02 \mathrm{E}-02$ \\
\hline B106 & Pa231 & $\mathrm{Ci}$ & $1.56 \mathrm{E}-04$ \\
\hline B106 & Pu238 & $\mathrm{Ci}$ & $1.19 \mathrm{E}-01$ \\
\hline B106 & Pu239 & $\mathrm{Ci}$ & $2.13 E+01$ \\
\hline B106 & Pu240 & $\mathrm{Ci}$ & $1.65 E-00$ \\
\hline B106 & Pu241 & $\mathrm{Ci}$ & $4.12 E-00$ \\
\hline B106 & Pu242 & $\mathrm{Ci}$ & 1.83E-05 \\
\hline B106 & Ra226 & $\mathrm{Ci}$ & $7.52 \mathrm{E}+02$ \\
\hline B106 & Ra228 & $\mathrm{Ci}$ & 4.34E-04 \\
\hline B106 & Ru106 & $\mathrm{Ci}$ & 3.79E-06 \\
\hline B106 & Sb125 & $\mathrm{Ci}$ & $1.57 \mathrm{E}-01$ \\
\hline B106 & Se79 & $\mathrm{Ci}$ & 4.98E-02 \\
\hline B106 & Sm151 & $\mathrm{Ci}$ & $1.85 \mathrm{E}+02$ \\
\hline B106 & Sr89/90 & $\mathrm{Ci}$ & $5.46 E+03$ \\
\hline B106 & Tc99 & $\mathrm{Ci}$ & $1.72 E-00$ \\
\hline B106 & Th229 & $\mathrm{Ci}$ & 1.02E-05 \\
\hline B106 & Th232 & $\mathrm{Ci}$ & $2.85 \mathrm{E}-05$ \\
\hline B106 & Tin126 & $\mathrm{Ci}$ & $7.48 \mathrm{E}-02$ \\
\hline B106 & Tritium & $C \mathbf{i}$ & $1.67 \mathrm{E}-00$ \\
\hline B106 & $\mathbf{U} 232$ & $\mathbf{C i}$ & $2.12 \mathrm{E}-03$ \\
\hline B106 & $\mathbf{U} 233$ & $\mathrm{Ci}$ & 7.93E-03 \\
\hline B106 & U234 & $\mathrm{Ci}$ & $2.26 \mathrm{E}-00$ \\
\hline B106 & U235 & $\mathrm{Ci}$ & $1.00 \mathrm{E}-01$ \\
\hline B106 & U236 & $\mathrm{Ci}$ & $2.11 E-02$ \\
\hline B106 & U238 & $\mathbf{C i}$ & $2.29 \mathrm{E}-00$ \\
\hline B106 & Y90 & $\mathrm{Ci}$ & $5.47 \mathrm{E}+03$ \\
\hline B106 & Zr93 & $\mathrm{Ci}$ & $2.37 \mathrm{E}-01$ \\
\hline B107 & Al & $\mathrm{kg}$ & $1.30 E+04$ \\
\hline B107 & $\mathbf{B i}$ & kg & $1.45 E+04$ \\
\hline B107 & $\mathrm{Ca}$ & kg & $1.08 \mathrm{E}+03$ \\
\hline B107 & Cl & kg & $7.82 \mathrm{E}+02$ \\
\hline B107 & $\mathrm{CO3}$ & kg & $7,30 E+03$ \\
\hline B107 & $\mathrm{Cr}$ & kg & $6.90 \mathrm{E}+02$ \\
\hline B107 & $\mathbf{F}$ & $\mathrm{kg}$ & $8.26 E+03$ \\
\hline B107 & $\mathrm{Fe}$ & kg & $1.25 \mathrm{E}+04$ \\
\hline B107 & Hg & $\mathrm{kg}$ & $2.80 \mathrm{E}-01$ \\
\hline B107 & $\mathbf{K}$ & $\mathrm{kg}$ & $2.34 \mathrm{E}+02$ \\
\hline B107 & La & $\mathrm{kg}$ & $3.10 \mathrm{E}+01$ \\
\hline B107 & Mn & kg & $1.23 \mathrm{E}+02$ \\
\hline B107 & $\mathbf{N a}$ & kg & $8.02 E+04$ \\
\hline B107 & $\mathbf{N i}$ & kg & $6.00 \mathrm{E}+01$ \\
\hline B107 & NO2 & $\mathrm{kg}$ & $1.10 \mathrm{E}+04$ \\
\hline B107 & NO3 & $\mathrm{kg}$ & $7.92 E+04$ \\
\hline B107 & $\mathbf{P}$ & kg & $6.59 E+04$ \\
\hline B107 & $\mathbf{P b}$ & $\mathrm{kg}$ & $2.60 \mathrm{E} \div 02$ \\
\hline B107 & PO4 & $\mathrm{kg}$ & $2.04 E+05$ \\
\hline B107 & $\mathbf{S}$ & kg & $8.08 \mathrm{E}+03$ \\
\hline B107 & $\mathbf{S i}$ & kg & $6.10 \mathrm{E}+03$ \\
\hline B107 & SO4 & kg & $2.42 E+04$ \\
\hline B107 & $\mathbf{S r}$ & $\mathrm{kg}$ & $2.59 E+02$ \\
\hline B107 & TOC & $\mathrm{kg}$ & $8.17 E+02$ \\
\hline B107 & $\mathbf{U}$ & $\mathrm{kg}$ & $5.48 \mathrm{E}+03$ \\
\hline B107 & $\mathbf{Z r}$ & $\mathrm{kg}$ & $9.80 \mathrm{E}+0 \mathrm{I}$ \\
\hline B107 & Ac227 & $\mathrm{Ci}$ & $2.46 \mathrm{E}-05$ \\
\hline B107 & Am241 & $\mathrm{Ci}$ & 2.09E-02 \\
\hline B107 & Am243 & $\mathrm{Ci}$ & $7.89 \mathrm{E}-08$ \\
\hline B107 & Ba137 & $\mathrm{Ci}$ & $1.65 E+04$ \\
\hline B107 & C14 & $\mathrm{Ci}$ & $1.80 \mathrm{E}-01$ \\
\hline B107 & Cd113 & $\mathrm{Ci}$ & 1.13E-01 \\
\hline B107 & $\mathrm{Cm} 242$ & Ci & $2.38 E-05$ \\
\hline B107 & $\mathrm{Cm} 243$ & $\mathrm{Ci}$ & $4.29 \mathrm{E}-07$ \\
\hline B107 & $\operatorname{Cm} 244^{\prime}$ & $\mathrm{Ci}$ & $2.97 \mathrm{E}-06$ \\
\hline B107 & $\mathrm{Co60}$ & $\mathrm{Ci}$ & $1.06 E+01$ \\
\hline B107 & Cs134 & $\mathrm{Ci}$ & 2.02E-04 \\
\hline B107 & Cs137 & $\mathrm{Ci}$ & $1.74 \mathrm{E} \div 0 \mathrm{~d}$ \\
\hline
\end{tabular}

\section{Sept98 \\ $\overline{1.99 \mathrm{E}-01}$}

2.78E-01

$2.50 \mathrm{E}+01$

1.02E-02

1.56E-04

9.78E-02

$1.75 \mathrm{E}+01$

$1.36 \mathrm{E}-00$

$3.40 \mathrm{E}-00$

$1.50 \mathrm{E}-05$

$1.39 \mathrm{E}-05$

4.34E-04

3.79E-06

1.57E-01

4.98E-02

$1.85 \mathrm{E}+02$

$5.46 \mathrm{E}+03$

$1.72 \mathrm{E}-00$

1.02E-05

2.85E-05

$7.48 \mathrm{E}-02$

$1.67 \mathrm{E}-00$

2.25E-03

8.43E-03

2.40E-00

1.06E-01

2.24E-02

2.44E-00

$5.47 \mathrm{E}+03$

2.37E-01

$2.81 \mathrm{E}+04$

$1.06 \mathrm{E}+04$

$5.47 \mathrm{E}+02$

$9.97 \mathrm{E}+02$

$4.97 \mathrm{E}+03$

$2.86 \mathrm{E}+02$

$2.50 \mathrm{E}+04$

$1.59 \mathrm{E}+04$

$5.23 E+01$

$5.10 \mathrm{E}+02$

$0.00 E+01$

$1.06 \mathrm{E}+02$

$1.64 \mathrm{E}+05$

$4.00 \mathrm{E}+01$

$3.41 E+03$

$1.51 \mathrm{E}+05$

NA

$5.15 E+02$

$7.66 \mathrm{E}+04$

NA

$5.85 E+03$

8.67E+04

$1.21 \mathrm{E}+02$

4.08E +02

$2.23 E+03$

$1.33 \mathrm{E}+02$

9.82E-04

1.05E-01

3.95E-07

$2.05 \mathrm{E}+04$

6.03E-01

1.29E-00

1.19E-04

2.15E-06

1.49E-05

8.17E-00

$3.26 E-00$ 2.17E+04
$1 \%$-ile

\section{$0.00 \mathrm{E}+01$}

$0.00 \mathrm{E}+01$

$0.00 \mathrm{E}+01$

$0.00 \mathrm{E}+01$

$0.00 \mathrm{E}+01$

$0.00 \mathrm{E}+01$

$0.00 \mathrm{E}+01$

$0.00 \mathrm{E}+01$

$0.00 \mathrm{E}+01$

$0.00 \mathrm{E}+01$

$0.00 \mathrm{E}+01$

$0.00 \mathrm{E}+01$

$0.00 \mathrm{E}+01$

$0.00 \mathrm{E}+01$

$0.00 \mathrm{E}+01$

$0.00 \mathrm{E}+01$

$0.00 \mathrm{E}+01$

$0.00 \mathrm{E}+01$

$0.00 \mathrm{E}+01$

$0.00 \mathrm{E}+01$

$0.00 \mathrm{E}+01$

7.57E-01

$0.00 \mathrm{E}+01$

$0.00 \mathrm{E}+01$

$0.00 \mathrm{E}+01$

$0.00 \mathrm{E} \div 01$

$0.00 \mathrm{E}+01$

$0.00 \mathrm{E}+01$

$0.00 \mathrm{E}+01$

$0.00 \mathrm{E}+01$

$2.91 \mathrm{E}+02$

$5.47 \mathrm{E}+03$

$3.68 \mathrm{E}+01$

$1.78 \mathrm{E}+02$

$2.29 \mathrm{E}+02$

$1.22 \mathrm{E}+01$

$2.37 \mathrm{E}+02$

$3.05 \mathrm{E}+03$

$0.00 \mathrm{E}+01$

$3.77 \mathrm{E}+01$

$6.88 \mathrm{E}-02$

4.39E-00

$3.23 \mathrm{E}+04$

9.41E-01

$4.25 \mathrm{E}+02$

$9.72 \mathrm{E}+03$

$2.37 \mathrm{E}+03$

4.01E-00

$1.26 \mathrm{E}+03$

$2.04 \mathrm{E}+02$

$3.65 \mathrm{E}+02$

$1.29 \mathrm{E}+03$

6.90E-00

$5.72 \mathrm{E}-00$

$4.54 \mathrm{E}+01$

$1.03 \mathrm{E}-00$

$0.00 \mathrm{E}+01$

$7.22 \mathrm{E}-01$

3.49E-09

$1.19 \mathrm{E}+03$

$0.00 \mathrm{E}+01$

$0.00 \mathrm{E}+01$

$1.05 \mathrm{E}-06$

$1.90 \mathrm{E}-08$

1.32E-07

$7.08 \mathrm{E}-03$

$1.46 \mathrm{E}-05$

$1.28 \mathrm{E}+03$

\section{0\%-ile}

$0.00 \mathrm{E}+01$

$50 \%$-ile

1.99E-01

2.00E+01 2.78E-01

$0.00 \mathrm{E}+01$

$0.00 \mathrm{E}+01$

$2.50 \mathrm{E}+01$

$1.02 \mathrm{E}-02$

$0.00 \mathrm{E}+01$

1.56E-04

1.19E-01

$0.00 \mathrm{E}+01$

$0.00 \mathrm{E}+01$

2.13E +01

$0.00 \mathrm{E}+01 \quad 4.12 \mathrm{E}-00$

$0.00 \mathrm{E}+01$

$0.00 \mathrm{E}+01$

$1.83 \mathrm{E}-05$

$7.52 \mathrm{E}+02$
$4.34 \mathrm{E}-04$

$0.00 \mathrm{E}+01 \quad 4.34 \mathrm{E}-04$

$5.09 \mathrm{E}-07$

$0.00 \mathrm{E}+01$

$1.46 \mathrm{E}-02$

$0.00 \mathrm{E}+01$

$2.99 \mathrm{E}+03$

$0.00 \mathrm{E}+01$

$0.00 \mathrm{E}+01$

$0.00 \mathrm{E}+01$

$0.00 \mathrm{E}+01$

$1.12 \mathrm{E}-00$

$0.00 \mathrm{E}+01$

$0.00 \mathrm{E}+01$

$0.00 \mathrm{E}+01$

$0.00 \mathrm{E}+01$

$0.00 \mathrm{E}+01$

$0.00 \mathrm{E}+01$

$0.00 \mathrm{E}+01$

$0.00 \mathrm{E}+01$

$1.23 \mathrm{E}+03$

$8.29 \mathrm{E}+03$

$2.03 \mathrm{E}+02$

$4.83 \mathrm{E}+02$

$3.17 \mathrm{E}+03$

$2.85 \mathrm{E}+02$

$1.06 \mathrm{E}+03$

$5.82 \mathrm{E}+03$

$2.85 \mathrm{E}-02$

$1.24 \mathrm{E}+02$

8.76E-01

$2.66 \mathrm{E}+01$

$4.97 \mathrm{E}+04$

$5.98 \mathrm{E}-00$

$2.61 \mathrm{E}+03$

$3.55 \mathrm{E}+04$

$7.65 \mathrm{E}+03$

$4.90 \mathrm{E}+01$

$5.85 \mathrm{E}+03$

$1.25 \mathrm{E}+03$

$2.31 \mathrm{E}+03$

$4.25 \mathrm{E}+03$

4.64E+01

$1.94 \mathrm{E}+02$

$3.54 \mathrm{E}+02$

$5.00 \mathrm{E}-00$

3.42E-06

$1.16 \mathrm{E}+01$

2.39E-08

$5.85 \mathrm{E}+03$

2.25E-03

$1.15 \mathrm{E}-02$

7.20E-06

$1.30 \mathrm{E}-07$

8.99E-07

$1.38 \mathrm{E}-01$

7.16E-05

$5.56 \mathrm{E}+03$
3.79E-06

1.57E-01

4.98E-02

$1.85 \mathrm{E}+02$

3.52E+04

1.72E-00

1.02E-05

2.85E-05

7.48E-02

1.67E-00

2.12E-03

$7.93 \mathrm{E}-03$

2.26E- 00

1.00E-01

2.11E-02

2.29E-00

$5.47 \mathrm{E}+03$

2.37E-01

$1.03 E+04$

$1.37 \mathrm{E}+04$

$5.45 \mathrm{E}+02$

$8.56 \mathrm{E}+02$

$8.54 \mathrm{E}+03$

$6.68 \mathrm{E}+02$

$5.43 E+03$

$1.13 \mathrm{E}+04$

2.80E-01

$2.86 \mathrm{E}+02$

$3.90 \mathrm{E}-00$

$6.28 \mathrm{E}+01$

$7.57 \mathrm{E}+04$

$1.95 \mathrm{E}+01$

$8.72 \mathrm{E}+03$

$1.01 \mathrm{E}+05$

$1.53 \mathrm{E}+04$

$2.50 \mathrm{E}+02$

$1.54 \mathrm{E}+04$

$3.65 \mathrm{E}+03$

$5.53 E+03$

$9.56 \mathrm{E}+03$

$1.48 \mathrm{E}+02$

$5.52 \mathrm{E}+02$

$1.37 \mathrm{E}+03$

$3.64 \mathrm{E}+01$

2.46E-05

$5.61 \mathrm{E}+01$

$7.89 \mathrm{E}-08$

$1.65 \mathrm{E}+04$

$1.80 \mathrm{E}-01$

$1.13 E-01$

2.38E-05

4.29E- -07

2.97E-06

3.17E-00

2.02E-04

$1.53 E+04$

90\%-ile

$99 \%$-ile

$5.16 \mathrm{E}-00 \quad 1.03 \mathrm{E}+01$

$1.57 \mathrm{E}-00 \quad 3.18 \mathrm{E}-00$

$1.41 \mathrm{E}+02 \quad 2.86 \mathrm{E}+02$

4.82E-02 8.62E-02

$4.07 \mathrm{E}-04 \quad 6.96 \mathrm{E}-04$

$5.63 \mathrm{E}-01$

$1.01 \mathrm{E}+02$

$7.80 \mathrm{E}-00$

$1.95 \mathrm{E}+01$ 


\begin{tabular}{|c|c|c|c|c|c|c|c|c|c|c|c|}
\hline ank & nal. & $n$ & May $\frac{B B}{98}$ & Sept98 & $1 \%$-ile & $10 \%$-ile & $50 \%$-ile & $90 \%$-ile & 99\%-ile & Mean & Std Dev \\
\hline 3107 & 152 & $\mathbf{C i}$ & $\overline{5.38 \mathrm{E}-03}$ & $5.66 \mathrm{E}-02$ & $0.00 \mathrm{E}+01$ & $7.48 \mathrm{E}-04$ & $5.38 E-03$ & $3.80 \mathrm{E}-02$ & $1.07 \mathrm{E}-01$ & 1.37E-02 & 2.22E-02 \\
\hline B107 & Eu154 & $\mathbf{C i}$ & $3.20 \mathrm{E}+01$ & $2.99 E+01$ & $0.00 \mathrm{E}+01$ & $4.45 \mathrm{E}-00$ & $3.20 \mathrm{E}+01$ & $2.26 \mathrm{E}+02$ & $6.36 E+02$ & $13 E+01$ & $1.32 E+02$ \\
\hline B107 & Eu155 & $\mathbf{C i}$ & $6.10 E+01$ & $4.62 \mathrm{E}+01$ & $0.00 \mathrm{E}+01$ & 8.48E-00 & $6.10 E+01$ & $4.31 E+02$ & $1.21 E+03$ & $1.55 \mathrm{E}+02$ & $2.52 \mathrm{E}+02$ \\
\hline B107 & 1129 & $\mathbf{C i}$ & $7.07 \mathrm{E}-04$ & $7.93 E-00$ & $0.00 \mathrm{E}+01$ & $2.40 \mathrm{E}-04$ & $7.07 E-04$ & $1.21 \mathrm{E}-03$ & $1.81 E-03$ & $7.20 \mathrm{E}-04$ & $3.88 \mathrm{E}-04$ \\
\hline B107 & $\mathrm{Nb93}$ & $\mathbf{C i}$ & $4.70 \mathrm{E}-02$ & $2.21 \mathrm{E}-01$ & $0.00 \mathrm{E}+01$ & $0.00 \mathrm{E}+01$ & $4.70 \mathrm{E}-02$ & $2.70 \mathrm{E}-01$ & $4.42 \mathrm{E}-01$ & $1.03 \mathrm{E}-01$ & $1.15 \mathrm{E}-01$ \\
\hline 107 & Ni59 & $\mathbf{C i}$ & 1.55E-02 & 1.15E-01 & $46 \mathrm{E}-05$ & $E-03$ & $1.55 \mathrm{E}-02$ & & & & \\
\hline 107 & Ni63 & $\mathrm{Ci}$ & $1.36 \mathrm{E}-00$ & $1.04 \mathrm{E}+01$ & $2.16 \mathrm{E}-03$ & $3.70 \mathrm{E}-01$ & $1.36 \mathrm{E}-00$ & $4.93 \mathrm{E}-00$ & $2.13 \mathrm{E}+01$ & $2.57 \mathrm{E}-00$ & $3.97 \mathrm{E}-00$ \\
\hline 107 & Np237 & $\mathbf{C i}$ & $2.26 \mathrm{E}-03$ & $1.84 \mathrm{E}-02$ & $1.00 \mathrm{E}-04$ & -04 & -03 & $4.59 \mathrm{E}-03$ & -03 & & $E-03$ \\
\hline B107 & 2231 & $\mathbf{C i}$ & $5.20 \mathrm{E}-05$ & $1.51 \mathrm{E}-03$ & $0.00 \mathrm{E}+01$ & $E-06$ & 5.20E-05 & 2.37E-04 & E-04 & -05 & $E-04$ \\
\hline B107 & Pu238 & $\mathbf{C i}$ & $3.34 \mathrm{E}-02$ & $1.67 \mathrm{E}-01$ & $1.48 \mathrm{E}-03$ & $1.01 \mathrm{E}-02$ & 3.34E-02 & $6.78 \mathrm{E}-02$ & $1.38 \mathrm{E}-01$ & $3.82 \mathrm{E}-02$ & $2.75 \mathrm{E}-02$ \\
\hline B107 & Pu239 & $\mathbf{C i}$ & $9.30 \mathrm{E}+01$ & $5.21 \mathrm{E}+01$ & $4.12 \mathrm{E}-00$ & $2.81 \mathrm{E}+01$ & $9.30 \mathrm{E}+01$ & $1.89 E+02$ & $3.83 E+02$ & $1.06 \mathrm{E}+02$ & 7.67E+01 \\
\hline B107 & Pu240 & $\mathrm{Ci}$ & Incl. in 239 & $3.08 \mathrm{E}-00$ & $4.12 \mathrm{E}-00$ & +01 & $9.30 \mathrm{E}+01$ & $1.89 \mathrm{E}+02$ & $3.83 E+02$ & $1.06 \mathrm{E}+02$ & $7.67 \mathrm{E}+01$ \\
\hline B107 & Pu241 & $\mathbf{C i}$ & 4.05E-01 & $2.03 E-00$ & $1.79 \mathrm{E}-02$ & $1.23 E-01$ & $4.05 E-01$ & $8.22 \mathrm{E}-01$ & $1.67 \mathrm{E}-00$ & 3E-01 & 4E-01 \\
\hline B107 & Pu242 & $\mathrm{Ci}$ & $1.24 \mathrm{E}-06$ & $6.20 \mathrm{E}-06$ & $5.49 \mathrm{E}-08$ & $E-07$ & $1.24 \mathrm{E}-06$ & 2.52E-06 & & E-06 & E-06 \\
\hline B107 & Ra226 & $\mathbf{C i}$ & 4.83E-06 & $7.76 \mathrm{E}-06$ & $0.00 \mathrm{E}+01$ & & & & -05 & & \\
\hline B107 & $\operatorname{Ra228}$ & $\mathbf{C i}$ & $9.22 \mathrm{E}-07$ & $1.06 \mathrm{E}-02$ & +01 & -07 & 9.221 & & & & -06 \\
\hline B107 & Ru106 & $\mathrm{Ci}$ & $1.98 \mathrm{E}-07$ & $8.43 \mathrm{E}-05$ & +01 & -08 & 1.98 & $E-07$ & -07 & & $E-07$ \\
\hline B107 & Sb125 & $\mathbf{C i}$ & $7.07 \mathrm{E}-03$ & $3.03 E-00$ & $0.00 \mathrm{E}+01$ & $0.00 \mathrm{E}+01$ & 7.07E-03 & $1.68 \mathrm{E}-02$ & -02 & $E-03$ & -03 \\
\hline B107 & Se79 & $\mathrm{Ci}$ & $1.15 E-02$ & $1.43 E-01$ & 3E-04 & & 1.15E-02 & $2.36 \mathrm{E}-02$ & $6.46 \mathrm{E}-02$ & $9 \mathrm{E}-02$ & $E-02$ \\
\hline B107 & $\operatorname{Sm} 151$ & $\mathrm{Ci}$ & $4.33 E+01$ & $2.14 E+02$ & $0.00 \mathrm{E}+01$ & & $4.33 E+01$ & $3.06 \mathrm{E}+02$ & $E+02$ & $1.10 \mathrm{E}+02$ & $E+02$ \\
\hline B107 & Sr89/90 & $\mathrm{Ci}$ & $2.24 E+04$ & $3.81 \mathrm{E}+04$ & $2.12 \mathrm{E}+02$ & +03 & $2.78 E+04$ & +05 & & $E+04$ & +04 \\
\hline B107 & Te99 & $\mathrm{Ci}$ & $2.60 \mathrm{E}+01$ & $1.75 E+01$ & $8-00$ & & 2.6 & & & & +02 \\
\hline B107 & Th229 & $\mathrm{Ci}$ & 4.31E-07 & $3.78 \mathrm{E}-04$ & +01 & 08 & & & & & -06 \\
\hline B107 & Th232 & $\mathbf{C i}$ & 1.97E-06 & $1.28 E-03$ & +01 & -07 & 1.9 & & & -06 & -06 \\
\hline B107 & Tin126 & $\mathrm{Ci}$ & $1.70 \mathrm{E}-02$ & 8.89E-02 & $0.00 \mathrm{E}+01$ & -03 & 1.70 & $E-02$ & $E=01$. & $\mathrm{E}-02$ & $E-02$ \\
\hline B107 & Tritium & $\mathrm{Ci}$ & $2.14 \mathrm{E}-01$ & $3.58 \mathrm{E}-00$ & & & 2.14E-01 & & -01 & & \\
\hline B107 & U232 & $\mathrm{Ci}$ & $1.56 \mathrm{E}-04$ & $1.16 \mathrm{E}-05$ & +01 & & & & & & \\
\hline B107 & U233 & $\mathrm{Ci}$ & 1.57E-04 & $1.17 \mathrm{E}-05$ & \pm 01 & & & & & & \\
\hline 107 & U234 & $\mathbf{C i}$ & 9.90E-00 & $7.35 \mathrm{E}-01$ & 01 & & 00 & 4.5 & -02 & 01 & +01 \\
\hline B107 & U235 & $\mathbf{C i}$ & 4.45E-01 & $3.31 \mathrm{E}-02$ & +01 & -02 & 4.4 & $-\infty 0$ & -00 & -01 & -00 \\
\hline B107 & U236 & $\mathbf{C i}$ & $6.32 \mathrm{E}-02$ & $4.69 \mathrm{E}-03$ & +01 & -02 & $6.32 \mathrm{E}-02$ & $2.89 \mathrm{E}-01$ & $E-01$ & $E-01$ & -01 \\
\hline B107 & U238 & $\mathrm{Ci}$ & $1.00 \mathrm{E}+01$ & $7.45 \mathrm{E}-01$ & +01 & -00 & $8+01$ & +01 & +02 & $E+01$ & $E+01$ \\
\hline B107 & Y90 & $\mathrm{Ci}$ & $2.24 \mathrm{E}+04$ & $3.81 E+04$ & +01 & & & & & +04 & +04 \\
\hline & Zr93 & $\mathrm{Ci}$ & $5.46 \mathrm{E}-02$ & $2.89 \mathrm{E}-01$ & & & & & & & \\
\hline 108 & Al & $\mathrm{kg}$ & $3.03 E+04$ & +04 & & & 04 & & & & +04 \\
\hline B108 & $\mathbf{B i}$ & $\mathrm{kg}$ & $3.66 \mathrm{E}+03$ & $3.66 \mathrm{E}+03$ & +02 & +03 & -03 & +03 & -03 & +03 & +03 \\
\hline B108 & $\mathrm{Ca}$ & $\mathrm{kg}$ & $9.67 E+02$ & $9.67 E+02$ & +01 & +02 & $E+02$ & +02 & +03 & +02 & +02 \\
\hline B108 & $\mathrm{Cl}$ & $\mathrm{kg}$ & $5.00 E+02$ & $5.00 \mathrm{E}+02$ & +02 & & -02 & & & +02 & +02 \\
\hline B108 & $\cos$ & $\mathrm{kg}$ & $2.11 E+03$ & $2.11 E+03$ & & & & & & +03 & +03 \\
\hline & $\mathrm{Cr}$ & $\mathrm{kg}$ & $2.18 E+02$ & $2.18 \mathrm{E}+02$ & & & & & & 102 & \\
\hline 08 & $\mathbf{F}$ & $\mathrm{kg}$ & $1.36 E+04$ & $1.36 \mathrm{E}+04$ & -03 & -03 & 1.3 & & & 04 & +03 \\
\hline B108 & $\mathbf{F e}$ & kg & $4.14 E+03$ & $4.14 E+03$ & $2.67 \mathrm{E}+02$ & +02 & 2.1 & -03 & +03 & +03 & $E+03$ \\
\hline B108 & Hg & $\mathrm{kg}$ & $3.60 \mathrm{E}-00$ & $1.08 \mathrm{E}+01$ & $E+01$ & $8-01$ & 3.6 & & +01 & $E-00$ & $E-00$ \\
\hline B108 & $\mathbf{K}$ & $\mathrm{kg}$ & $6.08 E+02$ & $6.08 \mathrm{E}+02$ & & & -02 & & +04 & +03 & +03 \\
\hline B108 & $\mathbf{L a}$ & $\mathrm{kg}$ & $0.00 E+01$ & $0.00 E+01$ & & & & & & -01 & \\
\hline & $\mathbf{M n}$ & $\mathbf{k g}$ & 02 & $E+02$ & & & & & & & \\
\hline B108 & $\mathbf{N a}$ & $\mathrm{kg}$ & & -04 & 1.8 & 04 & & & & .04 & +04 \\
\hline B108 & $\mathrm{Ni}$ & $\mathrm{kg}$ & $1.21 \mathrm{E}+02$ & $1.21 \mathrm{E}+02$ & $E-01$ & $E-00$ & -01 & +02 & -02 & +01 & $:+02$ \\
\hline B108 & NO2 & $\mathrm{kg}$ & $6.33 E+03$ & $6.33 E+03$ & +02 & +03 & +03 & 04 & 04 & +03 & +03 \\
\hline B108 & NO3 & $\mathrm{kg}$ & $4.01 E+04$ & $4.01 E+04$ & +03 & -04 & & & & +04 & $E+04$ \\
\hline B108 & $\mathbf{P}$ & $\mathrm{kg}$ & $1.61 \mathrm{E}+04$ & NA & & & & & & & $:+03$ \\
\hline B108 & $\mathbf{P b}$ & kg & $i+02$ & $8.86 E+02$ & & & & & & & +02 \\
\hline B108 & $F$ & $\mathrm{~kg}$ & & $E+04$ & & & & & & & +04 \\
\hline B108 & $\mathbf{S}$ & kg & +04 & $\mathbf{N A}$ & & & & & & -04 & +03 \\
\hline B108 & $\mathbf{S}$ & $\mathrm{kg}$ & $1.68 E+03$ & $1.68 E+03$ & -02 & & & & & & $E+02$ \\
\hline B108 & so & $\mathrm{kg}$ & $3.78 E+04$ & $3.78 E+04$ & & .04 & & & 04 & $\div 04$ & $E+04$ \\
\hline B108 & Sr & kg & $0.00 E+01$ & $1.65 E+02$ & $E-00$ & $E+01$ & $5.59 E+01$ & $E+02$ & & +01 & $E+01$ \\
\hline B108 & TOC & kg & $2.34 E+02$ & $2.34 E+02$ & & & & & & +02 & $E+02$ \\
\hline & $\mathbf{U}$ & kg & & $4.09 E+03$ & & & & & & & $E+02$ \\
\hline B108 & $\mathbf{Z r}$ & $\mathrm{kg}$ & $5.20 E+01$ & $1.45 \mathrm{E}+01$ & & & +01 & & & +02 & $E+02$ \\
\hline B108 & Ac227 & $\mathrm{Ci}$ & $4.15 \mathrm{E}-05$ & $4.15 E-05$ & +01 & & & & & $E-05$ & $E-05$ \\
\hline B108 & Am241 & $\mathrm{Ci}$ & 2.13E-01 & $3.26 E-02$ & $3.70 \mathrm{E}-01$ & & $8.86 \mathrm{E}$ & 6.4 & +04 & $E+03$ & $3.29 E+03$ \\
\hline B10 & Am243 & $\mathrm{Ci}$ & $1.45 \mathrm{E}-06$ & 2.23E-07 & $787 F_{-0} 08$ & $4.14 E-07$ & $1.45 \%$ & & & & $8 \mathrm{E}-06$ \\
\hline B108 & Bal & $\mathrm{Ci}$ & $8.10 E+03$ & $8.10 E+03$ & $2.39 E+02$ & $\div 03$ & 8.10 & 2.0 & 3.5 & +03 & +03 \\
\hline B108 & C14 & $\mathrm{Ci}$ & 1.2 & $9 E-01$ & $0.00 \mathrm{E}+01$ & 2.61E-02 & 1.25 & & & & $E-01$ \\
\hline B108 & Cd113 & $\mathrm{Ci}$ & 3.07E-01 & 3.07E-01 & $0.00 \mathrm{E}+01$ & $E-03$ & $3.07 E-01$ & $7.26 \mathrm{E}-01$ & $1.27 \mathrm{E}-00$ & DE-01 & $6 E-01$ \\
\hline B108 & Cm242 & $\mathrm{Ci}$ & $3.20 \mathrm{E}-04$ & 4.91E-05 & $1.74 \mathrm{E}-0.5$ & $9.14 E-05$ & $3.20 \mathrm{E}-04$ & $8.52 \mathrm{E}-04$ & E-03 & 4.05E-04 & $3.26 \mathrm{E}-04$ \\
\hline 108 & Cm243 & $\mathrm{Ci}$ & $6.52 \mathrm{E}-06$ & $1.00 \mathrm{E}-06$ & 3.54E-07 & $1.86 \mathrm{E}-06$ & 6.52E-06 & $1.74 \mathrm{E}-05$ & 2.89E-05 & $8.26 E-06$ & $6.64 \mathrm{E}-0.6$ \\
\hline
\end{tabular}




\begin{tabular}{|c|c|c|c|c|c|c|c|c|c|c|c|}
\hline ank & Inal. & In & May $\frac{B B}{98}$ & Sept98 & $1 \%$-ile & $10 \%$-ile & $50 \%$-ile & $90 \%$-ile & 99\%-ile & Mean & Std Dev \\
\hline 108 & & $\mathbf{C i}$ & $41 \mathrm{E}-05$ & & $E-06$ & $E-06$ & E-05 & -05 & E-04 & $20-00$ & $(2-6)$ \\
\hline 108 & Co60 & $\mathrm{Ci}$ & 2.75E-02 & $2.75 \mathrm{E}-02$ & & & & & & & $\vdots+01$ \\
\hline 108 & s134 & $\mathbf{C i}$ & 6.38E-04 & $38 E-04$ & E-05 & $3 \mathrm{E}-04$ & 8E-04 & $1.62 \mathrm{E}-03$ & 77E-03 & & $9 \mathrm{E}-04$ \\
\hline 3108 & Cs137 & $\mathbf{C i}$ & $8.60 \mathrm{E}+03$ & $.60 \mathrm{E}+03$ & $1.24 E+03$ & $3.58 E+03$ & $.17 E+04$ & $83 E+04$ & $4.77 \mathrm{E}+04$ & $1.41 \mathrm{E}+04$ & $1.05 E+04$ \\
\hline 108 & 152 & $\mathbf{C i}$ & $1.85 \mathrm{E}-02$ & $1.85 \mathrm{E}-02$ & $.00 \mathrm{E}+01$ & $0.00 \mathrm{E}+01$ & $1.85 \mathrm{E}-02$ & $7.20 \mathrm{E}-02$ & $1.23 \mathrm{E}-01$ & $2.73 \mathrm{E}-02$ & $3.03 E-02$ \\
\hline 108 & u154 & $\mathrm{Ci}$ & $4.81 \mathrm{E}-01$ & $4.81 \mathrm{E}-01$ & $.00 \mathrm{E}+01$ & $0.00 \mathrm{E}+01$ & $4.81 \mathrm{E}-01$ & $1.87 \mathrm{E}-00$ & $3.20 \mathrm{E}-00$ & $7.09 \mathrm{E}-01$ & $87 \mathrm{E}-01$ \\
\hline 108 & 155 & $\mathbf{C i}$ & 1.50E-00 & $1.50 \mathrm{E}-00$ & $00 \mathrm{E}+01$ & $0.00 \mathrm{E}+01$ & $1.50 \mathrm{E}-00$ & $5.84 \mathrm{E}-00$ & $8 \mathrm{~F}-$ & $2.21 \mathrm{E}-00$ & $5 E-00$ \\
\hline 108 & 29 & $\mathrm{Ci}$ & -03 & 1.68 & 01 & 44 & & $3.22 \mathrm{E}-03$ & & -03 & -03 \\
\hline B108 & & $\mathrm{Ci}$ & N-01 & 01 & 01 & +01 & & $1.02 \mathrm{E}-00$ & & & \\
\hline 108 & 59 & $\mathrm{Ci}$ & & $1.46 \mathrm{E}-01$ & 01 & & & $1.26 \mathrm{E}-00$ & & $E-01$ & \\
\hline B108 & Ni63 & $\mathrm{Ci}$ & $1.31 \mathrm{E}+01$ & $1.31 \mathrm{E}+01$ & $E+01$ & $3.73 \mathrm{E}-01$ & $1.31 E+01$ & $1.13 E+02$ & $\mathrm{E}+02$ & $3.78 \mathrm{E}+01$ & $6 \mathrm{E}+01$ \\
\hline B108 & p237 & $\mathrm{Ci}$ & 5.50E-03 & $5.50 \mathrm{E}-03$ & $\mathrm{E}-04$ & $E-03$ & $5.50 \mathrm{E}-03$ & $1.46 \mathrm{E}-02$ & IE-02 & $6.97 \mathrm{E}-03$ & $.60 \mathrm{E}-03$ \\
\hline B108 & Pa231 & $\mathrm{Ci}$ & 8.92E-05 & 8.92E-05 & $0.00 \mathrm{E}+01$ & $3.43 \mathrm{E}-06$ & $8.92 \mathrm{E}-05$ & 2.37E-04 & $6.30 \mathrm{E}-04$ & $1.15 \mathrm{E}-04$ & $1.19 \mathrm{E}-04$ \\
\hline 108 & u238 & $\mathrm{Ci}$ & $6.78 \mathrm{E}-02$ & $1.04 E-02$ & $\varepsilon-03$ & $E-02$ & -02 & $1.81 E-0.1$ & $E-01$ & -02 & $6.91 \mathrm{E}-02$ \\
\hline 108 & Pu239 & $\mathrm{Ci}$ & $22 \mathrm{E}+02$ & 2.02E-00 & $E+01$ & $E+01$ & +02 & $5.91 \mathrm{E}+02$ & $E+02$ & +02 & $E+02$ \\
\hline 108 & $\mathbf{u} 240$ & $\mathrm{Ci}$ & cl. in 239 & $1.50 \mathrm{E}-01$ & +01 & 01 & +02 & $5.91 \mathrm{E}+02$ & -02 & 02 & -02 \\
\hline 108 & 1 & $\mathrm{Ci}$ & 10 & 01 & & & & & & & \\
\hline & 2 & $\mathrm{Ci}$ & E-06 & 1 & & & & .05 & & & \\
\hline B108 & $\operatorname{Ra226}$ & $\mathrm{Ci}$ & 8.14E-06 & 8.14E-06 & -01 & 07 & 06 & $77 \mathrm{E}-05$ & -05 & -05 & -05 \\
\hline 08 & $\operatorname{Ra} 228$ & $\mathrm{Ci}$ & 8.44E-11 & 8.44E-11 & :+01 & $E-12$ & -11 & 2.87E-10 & $=-10$ & $E-10$ & $1.23 \mathrm{E}-10$ \\
\hline 108 & Ru106 & $\mathrm{Ci}$ & 1.02E-08 & $1.02 \mathrm{E}-08$ & $0.00 \mathrm{E}+01$ & $1.80 \mathrm{E}-09$ & -08 & $2.45 \mathrm{E}-08$ & $3.96 \mathrm{E}-08$ & E-08 & E-09 \\
\hline 108 & b125 & $\mathrm{Ci}$ & $2.43 \mathrm{E}-02$ & $\varepsilon-02$ & +01 & & & $6.43 \mathrm{E}-02$ & & & $E-02$ \\
\hline 108 & 79 & $\mathbf{C i}$ & $\mathrm{E}-02$ & -02 & +01 & $E-03$ & 20 & $A$ & & & \\
\hline 108 & 11 & $\mathrm{Ci}$ & 2 & 1. & & & & +02 & & & \\
\hline 108 & 39/90 & $\mathrm{Ci}$ & $1.05 E+04$ & 04 & -02 & 03 & 04 & +04 & & & +04 \\
\hline 08 & Tc99 & $\mathrm{Ci}$ & $8.95 \mathrm{E}-01$ & 8.95E-01 & +01 & 02 & -01 & .00 & 00 & -00 & $=-00$ \\
\hline 08 & Th229 & $\mathbf{C i}$ & $1.64 \mathrm{E}-08$ & $1.64 \mathrm{E}-08$ & +01 & 10 & -08 & $4.35 \mathrm{E}-08$ & -07 & & $=-088$ \\
\hline 108 & Th232 & $\mathbf{C i}$ & $3.74 \mathrm{E}-11$ & -11 & +01 & 12 & & & 10 & & -11 \\
\hline 108 & 26 & $\mathrm{Ci}$ & E-02 & 02 & 01 & 03 & & 0 & & & \\
\hline 108 & Tritium & $\mathrm{Ci}$ & 8.54E-01 & 1 & & & & & & & \\
\hline B108 & & $\mathrm{Ci}$ & 05 & 05 & 01 & & & .04 & & & \\
\hline B108 & U233 & $\mathrm{Ci}$ & 2.63E-06 & 1.10E-06 & $\varepsilon+01$ & $E-07$ & -06 & $6.98 \mathrm{E}-06$ & -05 & -06 & E-06 \\
\hline B108 & $\mathbf{U 2 3 4}$ & $\mathrm{Ci}$ & $3.22 \mathrm{E}-00$ & $1.35 E-00$ & $\varepsilon+01$ & $E-01$ & -00 & $8.55 \mathrm{E}-00$ & +01 & -00 & $E-00$ \\
\hline 18 & 5 & $\mathrm{Ci}$ & -01 & -02 & +01 & & & -01 & -00 & 1 & $E-01$ \\
\hline 08 & 36 & $\mathrm{Ci}$ & E-02 & 02 & & & & & & & \\
\hline 108 & & $\mathrm{Ci}$ & 00 & & & & & & & & \\
\hline B108 & Y90 & $\mathrm{Ci}$ & 1.05E +04 & $1.05 E+04$ & +01 & +01 & +04 & $E+04$ & +04 & +04 & $=04$ \\
\hline B108 & Zr93 & $\mathrm{Ci}$ & $1.29 E-01$ & $1.29 E-01$ & $E+01$ & +01 & E-01 & $9 \mathrm{E}-00$ & $E-00$ & -01 & $E-01$ \\
\hline B109 & $\mathbf{A} \mid$ & kg & $5.70 E+04$ & $5.27 E+04$ & $E+04$ & +04 & +04 & $E+04$ & +04 & +04 & +03 \\
\hline 09 & B & kg & +03 & +03 & & -03 & 03 & 3 & 03 & & +03 \\
\hline 109 & $\mathrm{Cz}$ & $\mathrm{kg}$ & +03 & & & & & & & & 02 \\
\hline 109 & $\mathrm{Cl}$ & $\mathbf{k g}$ & & & & & & +03 & & & \\
\hline B109 & $\mathrm{cos}$ & kg & $6.87 E+03$ & $7.82 \mathrm{E}+03$ & +01 & +02 & +03 & $1.74 E+04$ & +04 & +03 & $E+03$ \\
\hline B109 & $\mathrm{Cr}$ & kg & $1.77 E+03$ & $1.93 E+03$ & $E+01$ & +02 & +03 & $1.92 \mathrm{E}+03$ & +03 & +03 & $E+02$ \\
\hline B109 & $\mathbf{F}$ & kg & $2.39 \mathrm{E}+04$ & $2.15 E+04$ & +04 & +04 & $8+04$ & $E+04$ & $E+04$ & $E+04$ & $E+03$ \\
\hline B109 & Fe & kg & 3.7 & $i+03$ & & & & +03 & 03 & 13 & +03 \\
\hline 109 & Hg & kg & 01 & & & & & & 02 & I & -01 \\
\hline & $\mathbf{K}$ & $\mathbf{k}$ & 12 & 4 & & & & & & & \\
\hline B109 & $\mathbf{L}$ & kg & & & & & -01 & 01 & +01 & & +01 \\
\hline B109 & Mn & kg & $3.28 \mathrm{E}+02$ & $0.00 \mathrm{E}+01$ & $=00$ & +01 & +02 & $4.74 \mathrm{E}+02$ & +02 & +02 & $E+02$ \\
\hline B109 & Na & $\mathbf{k g}$ & $1.79 E+05$ & $1.78 E+05$ & $E+04$ & $i+05$ & $i+05$ & $E+05$ & +05 & 05 & $E+04$ \\
\hline B109 & $\mathrm{Ni}$ & kg & +02 & +02 & & & & -02 & -02 & 2 & +02 \\
\hline B109 & $\mathbf{N}$ & kg & 13 & 03 & & & & & & & +03 \\
\hline B & & $\mathrm{kg}$ & & $E+04$ & & & & & & & \\
\hline B & $\mathbf{P}$ & kg & & & & & & -04 & & & +03 \\
\hline B109 & $\mathbf{P b}$ & kg & $1.63 \mathrm{E}+03$ & $1.56 \mathrm{E}+03$ & $E+02$ & $E+02$ & +02 & $6.42 \mathrm{E}+02$ & $E+02$ & $E+02$ & $E+02$ \\
\hline B109 & PO4 & kg & $7.32 E+04$ & $8.90 E+04$ & +04 & $5.75 \mathrm{E}+04$ & 8.0 & $1.06 \mathrm{E}+05$ & $E+05$ & $E+04$ & $E+04$ \\
\hline B109 & $\mathbf{S}$ & kg & $8.43 E+04$ & NA & $1.34 E+04$ & $E+04$ & +04 & $3.41 E+04$ & +04 & $E+04$ & +03 \\
\hline B109 & $\mathbf{S i}$ & kg & $2.88 E+03$ & +03 & $E+01$ & +02 & -03 & $2.39 E+03$ & +03 & $\varepsilon+03$ & $E+02$ \\
\hline B109 & SO4 & kg & $2.53 E+05$ & $6.92 \mathrm{E}+04$ & $4.63 E+04$ & -04 & 04 & 105 & +05 & $E+04$ & $E+04$ \\
\hline B10 & $\mathbf{S r}$ & kg & 0 & 1. & & & & & & +02 & +02 \\
\hline B109 & $\mathbf{I}$ & $\mathrm{kg}$ & & 3 & & & & +03 & & & \\
\hline B & $\mathbf{U}$ & kg & & & & & & $3.18 \mathrm{E}+03$ & 03 & +03 & +02 \\
\hline B109 & $\mathbf{Z r}$ & kg & $4.90 E-00$ & $1.51 \mathrm{E}+02$ & +01 & & & $5.69 \mathrm{E}+02$ & $:+03$ & $E+02$ & $E+02$ \\
\hline B10 & Ac227 & $\mathrm{Ci}$ & $8.21 E-03$ & $8.21 E-03$ & $0.00 E+01$ & $0.00 \mathrm{E}+01$ & $\mathbf{E}-\mathbf{0 3}$ & $3.60 \mathrm{E}-02$ & $E-02$ & $1.35 \mathrm{E}-02$ & $1.57 \mathrm{E}-02$ \\
\hline 10 & & $\mathrm{Ci}$ & 4.32E-00 & $1.14 E-00$ & $1.62 E-00$ & $3.12 \mathrm{E}-00$ & $1.30 E+02$ & $1.33 E+04$ & $2.67 \mathrm{E}+04$ & $4.18 E+03$ & $6.27 E+03$ \\
\hline B109 & Am243 & $\mathrm{Ci}$ & $1.41 E-04$ & $3.72 E-05$ & $0.00 \mathrm{E}+01$ & $2.81 E-05$ & $1.41 E-04$ & 3.39E-04 & 5.61E-04 & $1.70 \mathrm{E}-04$ & $1.30 \mathrm{E}-04$ \\
\hline & & $\mathrm{Ci}$ & & $10 \mathrm{E}+04$ & $.00 E+01$ & $42 \mathrm{E}+03$ & $1.60 E+04$ & $3.14 \mathrm{E}+04$ & $1.68 \mathrm{E}+04$ & $.74 E+04$ & +04 \\
\hline
\end{tabular}


Tank Anal. Un

\begin{tabular}{|c|c|c|c|}
\hline & & & $\overline{98}$ \\
\hline B109 & C14 & $\mathrm{Ci}$ & $\frac{20}{20}$ \\
\hline B109 & Cd113 & $\mathrm{Ci}$ & $8.41 \mathrm{E}-00$ \\
\hline B109 & Cm242 & $\mathrm{Ci}$ & $37 \mathrm{E}-04$ \\
\hline B109 & Cm243 & $\mathrm{Ci}$ & .25E-05 \\
\hline B109 & Cm244 & $\mathrm{Ci}$ & $9.22 \mathrm{E}-05$ \\
\hline B109 & Co60 & $\mathrm{Ci}$ & $5.52 E-00$ \\
\hline B109 & Cs134 & $\mathrm{Ci}$ & $1.71 \mathrm{E}-01$ \\
\hline B109 & Cs137 & $\mathrm{Ci}$ & $1.70 \mathrm{E}+04$ \\
\hline 109 & Eu152 & C & 2.87 \\
\hline 109 & Eu154 & $\mathrm{Ci}$ & $6.74 \mathrm{E}+01$ \\
\hline B109 & Eu155 & C & $1.81 \mathrm{E}+01$ \\
\hline B109 & 1129 & $\mathrm{Ci}$ & \\
\hline B109 & 693 & $\mathrm{Ci}$ & \\
\hline B109 & Ni59 & $\mathrm{Ci}$ & \\
\hline B109 & $\mathrm{Ni63}$ & $\mathbf{C i}$ & $2.45 E+01$ \\
\hline B109 & Np237 & $\mathrm{Ci}$ & 1.17E-01 \\
\hline B109 & Pa231 & $\mathrm{Ci}$ & $1.24 \mathrm{E}-02$ \\
\hline B109 & Pu238 & C & 3.18E-00 \\
\hline B109 & Pu239 & $\mathrm{Ci}$ & $1.44 \mathrm{E}+02$ \\
\hline B109 & Pu240 & $\mathrm{Ci}$ & 2.41 \\
\hline B109 & Pu241 & $\mathrm{Ci}$ & $2.51 E+02$ \\
\hline B109 & Pu242 & $\mathrm{Ci}$ & 7.16E-04 \\
\hline B109 & $\mathbf{R a 2 2 6}$ & $\mathrm{Ci}$ & $1.83 \mathrm{E}-05$ \\
\hline B109 & $\operatorname{Ra228}$ & $\mathrm{Ci}$ & $9.01 E-02$ \\
\hline B109 & Ru106 & $\mathrm{Ci}$ & $6.76 \mathrm{E}-04$ \\
\hline B109 & Sb125 & $\mathrm{Ci}$ & $2.46 E+01$ \\
\hline B109 & Se79 & $\mathrm{Ci}$ & 01 \\
\hline B109 & Sm151 & $\mathrm{Ci}$ & 1.0 \\
\hline B109 & Sr89/90 & $\mathrm{Ci}$ & $8.53 E+03$ \\
\hline B109 & Te99 & $\mathrm{Ci}$ & $3.38 \mathrm{E}+01$ \\
\hline B109 & Th229 & $\mathrm{Ci}$ & $3.22 E-03$ \\
\hline B109 & Th232 & $\mathrm{Ci}$ & 1.09E-02 \\
\hline B109 & $\operatorname{Tin} 126$ & $\mathrm{Ci}$ & 01 \\
\hline B109 & Tritium & $\mathrm{Ci}$ & $2.36 \mathrm{E}+01$ \\
\hline B109 & U232 & $\mathrm{Ci}$ & $3.24 E-01$ \\
\hline B109 & U233 & $\mathbf{C i}$ & 1.25E-00 \\
\hline B109 & U234 & $\mathrm{Ci}$ & $2.40 \mathrm{E}-00$ \\
\hline B109 & U235 & $\mathrm{Ci}$ & $1.04 E-01$ \\
\hline B109 & U236 & C & -02 \\
\hline B109 & U238 & $\mathrm{Ci}$ & $2.44 \mathrm{E}-00$ \\
\hline B109 & Y90 & $\mathrm{Ci}$ & $8.53 E+03$ \\
\hline B109 & Zr93 & $\mathrm{Ci}$ & $1.50 \mathrm{E}-00$ \\
\hline $\mathbf{B 1 1 0}$ & Al & kg & $1.42 \mathrm{E}+03$ \\
\hline B110 & $\mathbf{B i}$ & kg & $2.32 E+04$ \\
\hline B110 & $\mathrm{Ca}$ & k & 1.01 \\
\hline B110 & Cl & kg & $1.54 E+03$ \\
\hline B110 & $\mathrm{CO3}$ & kg & $5.63 E+03$ \\
\hline B110 & $\mathrm{Cr}$ & kg & $1.01 E+03$ \\
\hline B110 & $\mathbf{F}$ & kg & $2.37 E+03$ \\
\hline B110 & $\mathbf{F e}$ & kg & $2.26 \mathrm{E}+04$ \\
\hline B110 & Hg & kg & 0.00 \\
\hline B110 & $\mathbf{K}$ & kg & $3.90 \mathrm{E}+02$ \\
\hline B110 & $\mathbf{L a}$ & kg & $3.98 E+01$ \\
\hline B110 & $\mathbf{M n}$ & kg & $8.36 E+01$ \\
\hline B110 & $\mathbf{N a}$ & kg & $1.22 E+05$ \\
\hline B110 & $\mathrm{Ni}$ & kg & $2.33 E+01$ \\
\hline B110 & NO2 & kg & $1.29 E+04$ \\
\hline B110 & NO3 & kg & 2.34 \\
\hline B110 & $\mathbf{P}$ & kg & $6.10 \mathrm{E}+04$ \\
\hline B110 & $\mathbf{P b}$ & kg & $6.61 \mathrm{E}+02$ \\
\hline B110 & PO4 & kg & $1.89 E+05$ \\
\hline B110 & $\mathbf{S}$ & kg & $1.44 E+04$ \\
\hline B110 & $\mathbf{S i}$ & kg & $1.17 E+04$ \\
\hline B110 & SO4 & kg & $4.32 E+04$ \\
\hline B110 & $\mathrm{Sr}$ & kg & $2.64 E+02$ \\
\hline 8110 & TOC & $\mathrm{kg}$ & 4.77E+02 \\
\hline B110 & $\mathbf{U}$ & kg & $2.60 \mathrm{E}+02$ \\
\hline B110 & $\mathbf{Z r}$ & kg . & $7.82 \mathrm{E}-00$ \\
\hline
\end{tabular}

BB Sept98

3.88E-00

8.41E-00

2.48E-04

$5.94 \mathrm{E}-06$

2.44E-05

5.52E-00

1.71E-01

$3.17 \mathrm{E}+04$

2.87E-01

$6.74 \mathrm{E}+01$

$1.81 \mathrm{E}+01$

$6.53 \mathrm{E}-02$

$1.08 \mathrm{E}-00$

2.65E-01

2.45E+01

1.17E-01

$1.24 \mathrm{E}-02$

8.41E-01

$3.81 \mathrm{E}+01$

$6.37 \mathrm{E}-00$

$6.63 \mathrm{E}+01$

$1.89 \mathrm{E}-04$

1.83E-05

9.01E-02

6.76E-04

$2.46 \mathrm{E}+01$

3.02E-01

$1.07 \mathrm{E}+03$

$8.53 \mathrm{E}+03$

$3.38 \mathrm{E}+01$

3.22E-03

1.09E-02

4.53E-01

$2.36 \mathrm{E}+01$

5.06E-01

1.95E-00

3.74E-00

$1.62 \mathrm{E}-01$

5.99E-02

3.71E-00

$8.53 E+03$

$1.50 \mathrm{E}-00$

$1.42 E+03$

$2.32 \mathrm{E}+04$

$1.01 \mathrm{E}+03$

$1.54 \mathrm{E}+03$

$5.63 \mathrm{E}+03$

$1.01 \mathrm{E}+03$

$2.37 \mathrm{E}+03$

$2.26 \mathrm{E}+04$

$0.00 \mathrm{E}+01$

$3.90 \mathrm{E}+02$

$3.98 \mathrm{E}+01$

$8.36 \mathrm{E}+01$

$1.22 \mathrm{E}+05$

$2.33 E+01$

$1.29 \mathrm{E}+04$

$2.34 E+05$

NA

$6.61 E+02$

$6.16 \mathrm{E}+04$

NA

$1.17 E+04$

$1.44 \mathrm{E}+04$

$2.64 \mathrm{E}+02$

$4.77 E+02$

$2.60 \mathrm{E}+02$

$7.82 \mathrm{E}-00$ 1\%-ile

\section{$0.00 \mathrm{E}+01 \quad 4.43 \mathrm{E}-01$} $0.00 \mathrm{E}+01 \quad 0.00 \mathrm{E}+01$

$0.00 \mathrm{E}+01 \quad 1.87 \mathrm{E}-04$

$0.00 \mathrm{E}+01 \quad 4.49 \mathrm{E}-06$

$0.00 \mathrm{E}+01 \quad 1.84 \mathrm{E}-05$

$2.86 \mathrm{E}-01 \quad 5.71 \mathrm{E}-01$

$0.00 \mathrm{E}+01 \quad 4.72 \mathrm{E}-02$

$2.80 \mathrm{E}+03$

$0.00 E+01$

$0.00 \mathrm{E}+01$

$0.00 \mathrm{E}+01$

$0.00 \mathrm{E}+01$

$0.00 \mathrm{E}+01$

$0.00 \mathrm{E}+01$

$0.00 \mathrm{E}+01$

$0.00 \mathrm{E}+01$

$0.00 \mathrm{E}+01 \quad 2.87 \mathrm{E}+01$

$0.00 \mathrm{E}+01 \quad 4.81 \mathrm{E}-00$

$0.00 \mathrm{E}+01 \quad 5.01 \mathrm{E}+01$

$0.00 \mathrm{E}+01 \quad 1.43 \mathrm{E}-04$

$0.00 \mathrm{E}+01 \quad 9.85 \mathrm{E}-07$

$0.00 \mathrm{E}+01$

$0.00 \mathrm{E}+01$

$0.00 \mathrm{E}+01$

$0.00 \mathrm{E}+01$

$0.00 \mathrm{E}+01$

$0.00 \mathrm{E}+01$

$0.00 \mathrm{E}+01$

$0.00 \mathrm{E}+01$

$0.00 \mathrm{E}+01$

$1.12 \mathrm{E}+01$

$0.00 \mathrm{E}+01$

$0.00 \mathrm{E}+01$

$0.00 \mathrm{E}+01$

$0.00 \mathrm{E}+01$

$0.00 \mathrm{E}+01$

$0.00 \mathrm{E}+01$

$0.00 \mathrm{E}+01$

$0.00 \mathrm{E}+01$

$3.21 \mathrm{E}+02$

$8.92 \mathrm{E}+03$

$2.33 \mathrm{E}+02$

$3.37 \mathrm{E}+02$

$3.37 \mathrm{E}+02$

$8.92 \mathrm{E}+0 \mathrm{I}$

$1.60 \mathrm{E}+02$

$5.57 \mathrm{E}+03$

$0.00 \mathrm{E}+01$

$4.76 \mathrm{E}+01$

$1.64 \mathrm{E}-01$

$6.58 \mathrm{E}-00$

$6.05 \mathrm{E}+04$

$2.31 \mathrm{E}+0 \mathrm{I}$

$1.31 \mathrm{E}+03$

1. $56 \mathrm{E}+04$

$4.03 E+03$

8.72E-00

$2.01 \mathrm{E}+03$

$3.08 \mathrm{E}+02$

$9.40 \mathrm{E}+02$

$1.63 \mathrm{E}+03$

$1.20 \mathrm{E}+01$

$1.46 \mathrm{E}-00$

$3.81 \mathrm{E}+01$

$1.45 \mathrm{E}-00$
$10 \%$-ile

8.37E+03

$0.00 E+01$

$0.00 E+01$

$0.00 \mathrm{E}+01$

2.22E-02

4.05E-04

4.19E-03

$3.88 \mathrm{E}-01$

2.33E-02

$0.00 \mathrm{E}+01$

6.34E-01

$4.85 \mathrm{E}-03$

1.61E-04

50\%-ile

3.88E-00

8.41E-00

9.37E-04

2.25E-05

9.22E-05

4.29E-00

1.71E-01

$2.09 E+04$

2.87E-01

$6.74 E+01$

$1.81 \mathrm{E}+01$

6.53E-02

1.08E-00

2.65E-01

$2.45 E+01$

1.17E-01

1.24E-02

$3.18 \mathrm{E}-00$

$1.44 \mathrm{E}+02$

$2.41 \mathrm{E}+01$

$2.51 \mathrm{E}+02$

7.16E-04

1.83E-05

9.01E-02

6.76E-04

$2.46 \mathrm{E}+01$

3.02E-01

1.31E-01

$0.00 \mathrm{E}+01$

$3.16 \mathrm{E}+03$

$1.07 \mathrm{E}+03$

$7.77 \mathrm{E}+04$

$0.00 \mathrm{E}+01$

$0.00 \mathrm{E}+01$

$0.00 \mathrm{E}+01$

$0.00 \mathrm{E}+01$

$1.69 \mathrm{E}+01$

$0.00 \mathrm{E}+01$

$0.00 \mathrm{E}+01$

$0.00 \mathrm{E}+01$

$0.00 \mathrm{E}+01$

$0.00 \mathrm{E}+01$

$0.00 \mathrm{E}+01$

$3.26 \mathrm{E}+02$

$5.63 \mathrm{E}-04$

$1.81 E+03$

$1.36 \mathrm{E}+04$

$5.53 \mathrm{E}+02$

$7.59 \mathrm{E}+02$

$4.99 \mathrm{E}+03$

$5.02 E+02$

$1.24 \mathrm{E}+03$

$9.20 \mathrm{E}+03$

$0.00 \mathrm{E}+01$

$1.88 \mathrm{E}+02$

$1.21 \mathrm{E}-00$

$3.92 \mathrm{E}+01$

$7.93 \mathrm{E}+04$

$5.26 \mathrm{E}+01$

$4.40 \mathrm{E}+03$

$5.88 \mathrm{E}+04$

$1.24 \mathrm{E}+04$

$7.86 \mathrm{E}+01$

$1.01 \mathrm{E}+04$

$1.58 \mathrm{E}+03$

$3.50 \mathrm{E}+03$

5.57E+03

$7.87 \mathrm{E}+01$

$2.54 \mathrm{E}+02$

$5.09 \mathrm{E}+02$

$7.90 \mathrm{E}-00$

3.22E-03

1.09E-02

4.53E-01

2.36E+01

3.24E-01

1.25E-00

2.40E-00

1.04E-01

3.84E-02

2.44E-00

$8.53 \mathrm{E}+03$

1.50E-00

$1.62 \mathrm{E}+04$

$2.11 E+04$

$1.26 \mathrm{E}+03$

$1.31 \mathrm{E}+03$

$1.25 \mathrm{E}+04$

$1.10 \mathrm{E}+03$

$7.95 \mathrm{E}+03$

$1.74 \mathrm{E}+04$

$0.00 E+01$

$4.28 \mathrm{E}+02$

4.96E-00

$1.01 \mathrm{E}+02$

$1.12 E+05$

$1.46 \mathrm{E}+02$

$1.40 \mathrm{E}+04$

$1.59 \mathrm{E}+05$

2.35E +04

$3.70 \mathrm{E}+02$

$2.34 \mathrm{E}+04$

$4.68 \mathrm{E}+03$

90\%-ile

$$
\begin{array}{rr}
2.08 \mathrm{E}+01 & 3.60 \mathrm{E}+01 \\
1.77 \mathrm{E}+01 & 2.70 \mathrm{E}+01 \\
2.25 \mathrm{E}-03 & 3.73 \mathrm{E}-03 \\
5.41 \mathrm{E}-05 & 8.96 \mathrm{E}-05 \\
2.22 \mathrm{E}-04 & 3.67 \mathrm{E}-04 \\
1.60 \mathrm{E}+01 & 2.68 \mathrm{E}+01
\end{array}
$$

3.36E-01

$3.77 \mathrm{E}+04$

$1.26 \mathrm{E}-00$

$2.96 \mathrm{E}+02$

$7.94 \mathrm{E}+01$

1.15E-01

$1.51 \mathrm{E}+01$

$2.36 \mathrm{E}-00$

$2.18 \mathrm{E}+02$

2.82E-01

$3.28 \mathrm{E}-02$

7.65E-00

$3.46 \mathrm{E}+02$

$5.80 \mathrm{E}+01$

$6.04 \mathrm{E}+02$

1.72E-03

6.03E-05

$2.97 \mathrm{E}-01$

1.29E-03

$5.77 \mathrm{E}+01$

4.88E-01

$4.70 \mathrm{E}+03$

4.01E+05

$8.52 \mathrm{E}+01$

8.52E-03 
Tank Anal. Un

\begin{tabular}{|c|c|c|c|c|c|c|c|c|c|c|c|}
\hline & & & & & & & & & & & \\
\hline & & Ci & $456 \mathrm{E}-04$ & $4.56 \mathrm{E}-04$ & $0.00 \mathrm{E}+01$ & -05 & 4 & 3 & 2 & 10 & \\
\hline B110 & n241 & $\mathrm{Ci}$ & $9.07 \mathrm{E}+01$ & $9.08 \mathrm{E}+01$ & 4.82 & $2.70 \mathrm{E}+01$ & $1.04 \mathrm{E}+02$ & $7.15 E+02$ & $2.98 \mathrm{E}+03$ & $3.01 \mathrm{E}+02$ & $21 \mathrm{E}+02$ \\
\hline B110 & 243 & $\mathrm{Ci}$ & $1.34 \mathrm{E}-03$ & 2.78E-03 & $1.37 \mathrm{E}-04$ & $E-04$ & -03 & 7E-03 & & 3 & $:-02$ \\
\hline B110 & Ba137 & $\mathrm{Ci}$ & $1.76 \mathrm{E}+04$ & $1.76 \mathrm{E}+04$ & & 3 & & & & & \\
\hline B110 & 14 & $\mathrm{Ci}$ & .01 & -01 & $0.00 \mathrm{E}+01$ & $7.87 \mathrm{E}-03$ & $2.81 \mathrm{E}-01$ & 37E-01 & $9.01 \mathrm{E}$ & & \\
\hline B110 & $\$ 113$ & & $49 E+01$ & $2.49 \mathrm{E}+01$ & \pm 01 & -00 & $2.49 \mathrm{E}+01$ & $E+01$ & & $4 \mathrm{E}+01$ & +01 \\
\hline B110 & $\mathbf{m} 242$ & $\mathbf{C i}$ & -02 & $E-02$ & -03 & & E-02 & $\mathrm{E}-01$ & $E-00$ & -01 & . \\
\hline B110 & n243 & & E-03 & $6.40 \mathrm{E}-02$ & -04 & $E-03$ & $3.06 \mathrm{E}-03$ & $E-02$ & 7.69E-02 & E-03 & $3 E-02$ \\
\hline B110 & Cm244 & c & E-02 & $1.54 \mathrm{E}-00$ & 03 & & $9.40 \mathrm{E}-02$ & -01 & -00 & -01 & FE-01 \\
\hline B110 & Co60 & 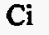 & $3.74 E-01$ & $3.74 \mathrm{E}-01$ & $1.09 \mathrm{E}-01$ & & $5.91 \mathrm{E}-00$ & $E+01$ & $6.45 E+02$ & $3.70 \mathrm{E}+01$ & $1.19 \mathrm{E}+02$ \\
\hline 110 & 134 & c & -02 & $8.87 \mathrm{E}-0$ & -02 & -02 & E-02 & E-01 & & -01 & $9 \mathrm{E}-01$ \\
\hline $\mathbf{0}$ & 137 & & +04 & $1.86 E+04$ & 03 & +04 & $E+04$ & $E+05$ & & +04 & $E+04$ \\
\hline 110 & 152 & C & $\varepsilon-00$ & 1.45 & 01 & & & +01 & & $E-00$ & $E-00$ \\
\hline 10 & 154 & $\mathrm{Ci}$ & 8 & 8.301 & & & & & & & +02 \\
\hline 110 & 5 & $\mathrm{Ci}$ & & 1.08 & & -01 & +02 & +02 & & & $E+02$ \\
\hline B110 & & 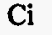 & $6-02$ & $4.50 \mathrm{E}-02$ & & -02 & 02 & .02 & 01 & $8-02$ & $:-02$ \\
\hline 10 & 93 & C & -00 & $4.37 \mathrm{E}-00$ & +01 & +01 & 00 & +01 & 01 & $E-00$ & $1.08 \mathrm{E}+01$ \\
\hline 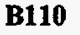 & & 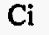 & -00 & $1.34 \mathrm{E}-00$ & -01 & -01 & 00 & & & $\mathrm{E}-00$ & $4.27 \mathrm{E}-00$ \\
\hline 10 & 33 & 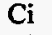 & +02 & $1.35 E+02$ & & +01 & & & & +02 & +02 \\
\hline 110 & 237 & c & E-01 & $1.40 \mathrm{E}-01$ & & -02 & & & & & -00 \\
\hline 110 & 231 & c & -03 & 1.03 & & -04 & & & & & -03 \\
\hline 10 & 238 & C & & 4.02 & & & & & & & -01 \\
\hline B110 & 39 & $\mathrm{Ci}$ & +01 & 8.9 & & 01 & 9. & & & +02 & +02 \\
\hline B11 & 240 & $\mathrm{Ci}$ & +01 & $9.76 \mathrm{E}-00$ & 00 & .00 & 1. & 01 & 02 & +01 & $E+01$ \\
\hline B110 & Pu241 & $\mathrm{Ci}$ & $8.82 \mathrm{E}+01$ & $8.20 \mathrm{E}+01$ & & -01 & & +02 & 03 & +02 & +02 \\
\hline B11 & 242 & $\mathrm{Ci}$ & 4 & $;-04$ & & & & & & 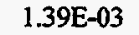 & -013 \\
\hline 1110 & 226 & $\mathrm{Ci}$ & -05 & -05 & & & & & & & \\
\hline & F & $\mathrm{Ci}$ & & 79 & & & & & & & 09 \\
\hline B110 & 106 & $\mathrm{Ci}$ & -03 & 4.3 & 01 & & & & & & -03 \\
\hline B11 & 125 & $\mathrm{Ci}$ & $1.67 \mathrm{E}-00$ & $1.67 E-00$ & +01 & 0.0 & 1. & -00 & -00 & $E-00$ & $E-00$ \\
\hline B110 & $\mathbf{s}$ & $\mathrm{Ci}$ & $1.27 \mathrm{E}-00$ & $1.27 E-00$ & 02 & & & .00 & 00 & $=-00$ & $E-00$ \\
\hline B & 151 & c & +03 & 4.73 & & & & & & 04 & +04 \\
\hline & 9/90 & $\mathrm{Ci}$ & +05 & 1.3 & & & & & & & +05 \\
\hline & & $\mathrm{Ci}$ & 1 & 2. & & & & & & & \\
\hline B11 & 229 & $\mathrm{Ci}$ & 1.25 & 1.2 & & & & & & & \\
\hline B110 & Th232 & $\mathrm{Ci}$ & $7.18 \mathrm{E}-11$ & $7.18 \mathrm{E}-11$ & -01 & 11 & & 10 & 10 & 10 & $E-10$ \\
\hline B1 & n126 & $\mathrm{Ci}$ & $1.98 \mathrm{E}-00$ & $1.98 \mathrm{E}-00$ & -01 & 01 & & 00 & 01 & 00 & $E-00$ \\
\hline B110 & itium & $\mathrm{C}$ & $1.48 \mathrm{E}-00$ & -00 & & & & & & 10 & $=-01$ \\
\hline & 32 & $\mathrm{Ci}$ & & & & & & & & & us \\
\hline & & c & & & & & & & & & \\
\hline 110 & 34 & $\mathrm{Ci}$ & -01 & 8.57E-02 & & 02 & & & & & -01 \\
\hline B110 & U235 & $\mathrm{Ci}$ & 1.35E-02 & $3.84 \mathrm{E}-03$ & 01 & .03 & 02 & 02 & 01 & $E-02$ & $E-02$ \\
\hline B & 36 & $c$ & 2.15 & 6.2 & & & & & & 03 & $E-03$ \\
\hline B & 38 & $c$ & & & & & & & & & -01 \\
\hline & 0 & $\mathrm{Ci}$ & & & & & & & & & 3 \\
\hline & & c & & & & -01 & & & & & \\
\hline B1 & $\mathbf{A}$ & kg & $9.58 \mathrm{E}+02$ & $9.58 \mathrm{E}+02$ & -02 & +03 & -04 & -04 & 04 & +04 & +03 \\
\hline B11 & $\mathbf{B i}$ & $\mathrm{kg}$ & $2.15 E+04$ & $2.15 E+04$ & +03 & +04 & +04 & -04 & 04 & +04 & +03 \\
\hline B] & C & $\mathrm{kg}$ & $7.34 E+02$ & $7.34 \mathrm{E}+02$ & 02 & +02 & & 94 & 14 & +03 & $E+03$ \\
\hline B111 & C & $\mathrm{kg}$ & & & & & & & & & +02 \\
\hline 111 & C & kg & 2 & & & & & & & & \\
\hline & & k & & & & & & & & & \\
\hline & $\mathbf{F}$ & $\mathrm{kg}$ & +03 & 03 & & +03 & & 04 & & 03 & +03 \\
\hline B1 & Fe & kg & $1.88 \mathrm{E}+04$ & $1.88 E+04$ & & $\div 04$ & 1.8 & & .04 & +04 & $E+03$ \\
\hline B1 & $\mathbf{H g}$ & kg & 9.93E-00 & 9.93E-00 & 0. & $E-\infty 0$ & 0 & & 01 & +01 & $E \div 01$ \\
\hline B1 & $\mathbf{K}$ & $\mathrm{kg}$ & $7.18 \mathrm{E}+02$ & 7.18 & 7. & 1 & +02 & & $E+03$ & 02 & $E+02$ \\
\hline B1 & $\mathbf{L}$ & kg & 01 & 1.2 & & & & & & & +01 \\
\hline B1 & $\mathbf{M}$ & kg & & & & & & & & & +02 \\
\hline & 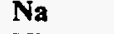 & $k_{\ell}$ & & & & & & & & & \\
\hline B1 & I & $k_{\varepsilon}$ & & & & & & & & 03 & $E+03$ \\
\hline & & kg & & & & & & & & 104 & $E+04$ \\
\hline B1 & No & $\mathrm{kg}$ & $8.72 E+04$ & $8.72 E+04$ & & & & & & $:+05$ & $E+04$ \\
\hline B1 & $\mathbf{P}$ & kg & $5.13 E+04$ & NA & & +04 & +04 & & & $E+04$ & $E+03$ \\
\hline B1 & $\mathbf{P}$ & kg & $1.67 E+03$ & 1.6 & & & & & & & +02 \\
\hline B1 & PO & kg & $1.59 E+05$ & $5.18 E+04$ & & & & & & -04 & \\
\hline & $\mathbf{S}$ & $\mathrm{kg}$ & & & & & & $9.92 E+03$ & E+04 & $E+03$ & $4.58 \mathrm{E}+03$ \\
\hline B11 & Si & $\mathrm{kg}$ & & & & & & $E+04$ & +04 & $E+03$ & $6.97 E+03$ \\
\hline 28 & SO4 & kg & $3.72 E+04$ & & $2.12 E+03$ & $6.10 \mathrm{E}+03$ & $1.28 E+04$ & $2.32 \mathrm{E}+04$ & $6.98 \mathrm{E}+04$ & $1.50 \mathrm{E}+04$ & I.12E+04 \\
\hline
\end{tabular}


Tank Anal. Un

\begin{tabular}{|c|c|c|c|c|c|c|c|c|c|c|c|}
\hline & & & 198 & t98 & 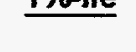 & 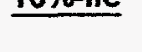 & $\overline{1}$ & 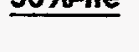 & 6 & & \\
\hline 111 & Sr & kg & $2 \overline{32 E+02}$ & +02 & $9.85 \mathrm{E}-00$ & $6.10 \mathrm{E}+01$ & $1.90 \mathrm{E}+02$ & $4.76 \mathrm{E}+02$ & $1.14 \mathrm{E}+03$ & $2.45 \mathrm{E}+02$ & $.23 E+02$ \\
\hline 3111 & TOC & kg & $9.32 E+02$ & $9.32 E+02$ & $1.28 \mathrm{E}+01$ & $2.82 \mathrm{E}+02$ & $7.22 \mathrm{E}+02$ & -03 & $8 E+03$ & $69 E+02$ & $4.01 E+02$ \\
\hline B111 & $\mathbf{U}$ & kg & $2.10 \mathrm{E}+02$ & $2.10 \mathrm{E}+02$ & $1.07 \mathrm{E}+02$ & $7.16 \mathrm{E}+02$ & $.13 E+03$ & $7.79 E+03$ & $2.20 \mathrm{E}+04$ & $.53 E+03$ & $29 E+03$ \\
\hline B111 & $\mathbf{Z r}$ & kg & $1.53 \mathrm{E}+01$ & $1.53 E+01$ & $9.45 \mathrm{E}-01$ & $6.94 \mathrm{E}-00$ & $8.51 E+01$ & $2.85 E+02$ & $5.90 \mathrm{E}+02$ & $.21 E+02$ & $1.33 E+02$ \\
\hline 111 & Ac227 & $\overline{\mathrm{Ci}}$ & $3.93 E-03$ & $3.93 \mathrm{E}-03$ & $0.00 \mathrm{E}+01$ & $7.04 \mathrm{E}-04$ & $3.93 \mathrm{E}-03$ & $2.72 \mathrm{E}-02$ & $7.79 \mathrm{E}-02$ & $9.97 \mathrm{E}-03$ & $E-02$ \\
\hline 111 & Am241 & & $9.01 \mathrm{E}+01$ & $7.41 \mathrm{E}+01$ & $4.10 \mathrm{E}-00$ & $2.34 \mathrm{E}+01$ & $69 \mathrm{E}+01$ & $5.11 E+02$ & $13 E+03$ & $4.73 E+02$ & $35 E+03$ \\
\hline 11 & 0243 & & -02 & 27E-03 & 09E-04 & 6E-03 & 16E-02 & -01 & -01 & $\mathrm{E}-02$ & $3 \mathrm{E}-01$ \\
\hline 111 & Ba137 & c & $1.59 E+05$ & $1.59 E+05$ & $1.75 \mathrm{E}+04$ & $4.82 \mathrm{E}+0.4$ & $59 \mathrm{E}+05$ & $6.10 \mathrm{E}+05$ & $9 E+06$ & $2.81 E+05$ & $67 E+05$ \\
\hline 3111 & $\mathrm{Cl4}$ & $\mathbf{C i}$ & $1.70 \mathrm{E}-00$ & $1.70 \mathrm{E}-00$ & $0.00 \mathrm{E}+01$ & $2.50 \mathrm{E}-01$ & $1.70 \mathrm{E}-00$ & $.72 \mathrm{E}-00$ & $3 E-00$ & $1.89 \mathrm{E}-00$ & $1.38 \mathrm{E}-00$ \\
\hline B111 & Cd113 & $\mathbf{C i}$ & $2.16 E+02$ & $2.16 \mathrm{E}+02$ & $0.00 \mathrm{E}+01$ & $2.84 \mathrm{E}+01$ & $2.16 E+02$ & & & $3.53 E+02$ & $4.01 \mathrm{E}+02$ \\
\hline B111 & $\mathrm{Cm} 242$ & $\mathbf{C i}$ & $3.45 E-01$ & $6.77 \mathrm{E}-02$ & $1.22 \mathrm{E}-02$ & $E-02$ & $3.45 \mathrm{E}-01$ & & $5 \mathbf{E}+01$ & $2.18 \mathrm{E}-00$ & $39 E-00$ \\
\hline B111 & $\mathrm{Cm} 243$ & $\mathrm{Ci}$ & $2.65 \mathrm{E}-02$ & $2.00 \mathrm{E}-02$ & & & $2.65 \mathrm{E}-02$ & & $E-\infty$ & $1.67 \mathrm{E}-01$ & $E-01$ \\
\hline B111 & $\mathrm{Cm} 244$ & $\mathrm{Ci}$ & $8.16 \mathrm{E}-01$ & $4.80 \mathrm{E}-01$ & -02 & & $8.16 \mathrm{E}-01$ & $8.01 \mathrm{E}-00$ & $7.01 E+01$ & $5.15 \mathrm{E}-00$ & $4 \mathrm{E}+01$ \\
\hline B111 & Co60 & $\mathrm{Ci}$ & 4.12E-00 & $4.12 \mathrm{E}-00$ & & & & & +02 & $E+01$ & $E+02$ \\
\hline 1 & 134 & Ci & $7.74 \mathrm{E}-01$ & $7.74 \mathrm{E}-01$ & & & & & & $=-00$ & \\
\hline & 37 & C & $1.68 E+05$ & $1.68 E+05$ & 04 & 2.7 & 04 & & & 05 & +05 \\
\hline & & $\mathrm{C}$ & 1.25 & $1.25 E+01$ & 01 & -00 & +01 & & 02 & +01 & \\
\hline B111 & Eu154 & $\mathrm{Ci}$ & $1.81 E+02$ & $1.81 \mathrm{E}+02$ & +01 & & +02 & & & +02 & \\
\hline B111 & Eu155 & $\mathbf{C i}$ & 9.32 & $9.32 E+02$ & & & & & & +03 & \\
\hline B111 & I129 & Ci & $3.41 \mathrm{E}-02$ & $3.41 E-02$ & 0.0 & & & & & & $E-02$ \\
\hline B111 & Nb93 & $\mathbf{C} \mathbf{i}$ & $3.79 E+01$ & $3.79 E+01$ & 0.0 & & & & & & \\
\hline B111 & Ni59 & Ci & +01 & $1.16 E+01$ & & & & & & & \\
\hline B111 & Ni63 & & 1.17 & $1.17 E+03$ & & & & & & & \\
\hline & Np237 & C & 7.61 & $7.61 \mathrm{E}-02$ & & & & & & & \\
\hline B111 & Pa231 & $\mathbf{C i}$ & 8.85 & 8.85E-03 & & & & & & & \\
\hline B111 & Pu238 & $\mathbf{C i}$ & 9.79 & $3.26 \mathrm{E}-00$ & & & & & & & \\
\hline B111 & Pu239 & $\mathbf{C i}$ & $1.04 E+02$ & $9.27 \mathrm{E}+01$ & & & & & -03 & & \\
\hline 1 & Pu240 & $\mathrm{Ci}$ & Incl. in 239 & $1.59 \mathrm{E}+01$ & & & 02 & & & & +03 \\
\hline B111 & Pu241 & $\mathrm{Ci}$ & +02 & $2.09 \mathrm{E}+02$ & 2.4 & 02 & -02 & & & +03 & +04 \\
\hline & Pu242 & $\mathrm{Ci}$ & -03 & $1.20 \mathrm{E}-03$ & & & & & & $E-02$ & \\
\hline B111 & Ra226 & C & $7.29 \mathrm{E}$ & $7.29 \mathrm{E}-04$ & & & & & & & \\
\hline B111 & $\mathbf{R a 2 2 8}$ & & $2.52 \mathrm{E}-03$ & $2.52 \mathrm{E}-03$ & & & & & & & \\
\hline B111 & Ru106 & & $3.76 \mathrm{E}-02$ & $3.76 \mathrm{E}-02$ & & & & & & & \\
\hline 11 & Sb125 & C & +01 & $1.52 E+01$ & & & & & & 01 & \\
\hline B111 & Se79 & C & +01 & $1.10 \mathrm{E}+01$ & & & & & & & \\
\hline B111 & Sm151 & $C$ & 4.10 & $4.10 \mathrm{E}+04$ & 0.0 & & & & & & \\
\hline B111 & Sr89/90 & $\mathrm{Ci}$ & $2.64 \mathrm{E}+05$ & $2.64 \mathrm{E}+05$ & & & & & +07 & +06 & +06 \\
\hline B111 & Tc99 & $\mathrm{Ci}$ & $1.21 E+02$ & $1.21 \mathrm{E}+02$ & & 01 & 02 & & & +02 & +02 \\
\hline B111 & & $\mathrm{Ci}$ & & -05 & & & & & & & \\
\hline B111 & Th232 & $\mathrm{Ci}$ & 3.11 & Q-04 & & & & & & -04 & \\
\hline B111 & Tin126 & C & 1.71 & $1.71 E+01$ & & & & & & -01 & \\
\hline B111 & Tritium & $\mathrm{Ci}$ & $1.32 E+01$ & $1.32 E+01$ & & & & & & $=01$ & -00 \\
\hline B111 & $\mathbf{U} 232$ & $\mathrm{Ci}$ & $5.40 \mathrm{E}-03$ & $2.68 \mathrm{E}-03$ & & & & & & $E-03$ & -02 \\
\hline B111 & & $\mathrm{Ci}$ & & $1.03 E-02$ & & & & & & .02 & \\
\hline & & $\mathrm{Ci}$ & & 12 & & & & & & & \\
\hline 11 & & $\mathrm{Ci}$ & 6.13 & -03 & & & & & & & \\
\hline B111 & U236 & $\mathrm{Ci}$ & 1.64 & 8.13E-04 & & 04 & & & & & \\
\hline B111 & U238 & $\mathrm{Ci}$ & $1.43 \mathrm{E}-01$ & $7,01 \mathrm{E}-02$ & & & 01 & -01 & & $\mathrm{E}-01$ & -01 \\
\hline B111 & Y90 & $\mathrm{Ci}$ & $2.64 E+05$ & $2.64 E+05$ & & & & +06 & +07 & \pm+06 & +06 \\
\hline B111 & Zr93 & $\mathrm{Ci}$ & & $5.05 E+01$ & & & & & & $E+01$ & $E+02$ \\
\hline & Al & kg & & $1.61 \mathrm{E}+02$ & & & & & & & +03 \\
\hline 2 & $\mathrm{Bi}$ & k & & & & & & & & & \\
\hline B112 & $\mathrm{Ca}$ & $\mathrm{kg}$ & 1.19 & $1.19 E+02$ & & & & & & & +02 \\
\hline B112 & Cl & kg & $1.79 E+02$ & $1.79 E+02$ & & & $\mathbf{E}+02$ & & & \pm 02 & $E+02$ \\
\hline B112 & $\mathrm{CO3}$ & $\mathrm{kg}$ & $2.13 E+03$ & $2.13 E+03$ & & +02 & $\mathbf{E}+03$ & & +04 & $E+03$ & $E+03$ \\
\hline B112 & $\mathrm{Cr}$ & kg & & $1.53 E+02$ & & & & & & $E+02$ & $E+02$ \\
\hline B112 & $\mathbf{F}$ & $\mathrm{kg}$ & $2.75 E+02$ & $2.75 E+02$ & & & & & & & $E+03$ \\
\hline B112 & $\mathrm{Fe}$ & $\mathrm{kg}$ & $2.85 E+03$ & $2.85 E+03$ & & & & & & & +03 \\
\hline & Hg & kg & & & & & & & & & $E-00$ \\
\hline B112 & $\mathbf{K}$ & kg & $4.96 \mathrm{E}$ & $4.96 \mathrm{E}+01$ & & & & & & -02 & +02 \\
\hline B112 & $\mathbf{L a}$ & kg & $3.08 \mathrm{E}-00$ & $3.08 E-00$ & & & & & & & $E-00$ \\
\hline B112 & Mn & kg & $1.16 E+01$ & $1.16 E+01$ & & $1.03 \mathrm{E}-00$ & 1.0 & +01 & $4.99 \mathrm{E}+02$ & $E+01$ & +02 \\
\hline B112 & $\mathbf{N a}$ & kg & $1.54 E+04$ & $1.54 E+04$ & 7.57E-04 & $3.29 \mathrm{E}+03$ & $1.83 E+04$ & $4.00 \mathrm{E}+04$ & $6.19 E+04$ & $2.05 E+04$ & $E+04$ \\
\hline B112 & $\mathbf{N i}$ & $\mathrm{kg}$ & $2.99 \mathrm{E}-00$ & $2.99 \mathrm{E}-00$ & & $1.10 \mathrm{E}-00$ & & $6.07 \mathrm{E}+01$ & $1.98 \mathrm{E}+02$ & $2.29 \mathrm{E}+01$ & $4.11 E+01$ \\
\hline B112 & $\mathrm{NO2}$ & kg & $4.40 E+03$ & $4.40 \mathrm{E}+03$ & & $4.80 \mathrm{E}+02$ & $3.16 E+03$ & $1.05 \mathrm{E}+04$ & $1.91 E+04$ & $4.51 E+03$ & $4.35 E+03$ \\
\hline B112 & NO3 & kg & $2.14 E+04$ & $2.14 E+04$ & -03 & $E+03$ & $2.01 E+04$ & $E+04$ & $E+04$ & $E+04$ & $2.11 E+04$ \\
\hline-1. & $\mathbf{P}$ & $\mathrm{kg}$ & & NA & & & & & & $E+03$ & \\
\hline & & kg & $2.50 \mathrm{E}+02$ & $2.50 E+02$ & $3.46 \mathrm{E}-06$ & $3.85 E-00$ & $3.31 \mathrm{E}+01$ & $1.42 \mathrm{E}+02$ & $3.95 E+02$ & $6.03 \mathrm{E}+01$ & $8.04 E+01$ \\
\hline
\end{tabular}




\begin{tabular}{|c|c|c|c|c|c|c|c|c|c|c|c|}
\hline ank & Anal. & $U_{n}$ & May $\frac{B B}{98}$ & Sept98 & $1 \%$-ile & $10 \%$-ile & $50 \%$-ile & $90 \%$-jle & 99\%-ile & Mean & Std Dev \\
\hline 12 & P04 & kg & $5.80 \mathrm{E}+03$ & $.80 \mathrm{E}+03$ & 04 & $E+02$ & 3 & $E+03$ & 4 & +03 & 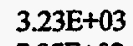 \\
\hline 112 & $\mathbf{S}$ & $\mathbf{k}$ & $6.13 E+02$ & NA & & $E+01$ & & & & & +02 \\
\hline 112 & Si & $\mathbf{k}$ & $1.57 \mathrm{E}+03$ & $1.57 E+03$ & $2 E-05$ & $E+01$ & $4 E+02$ & $2 E+03$ & & $7 E+02$ & $7.67 \mathrm{E}+02$ \\
\hline 12 & & kg & $1.84 \mathrm{E}+03$ & $1.84 E+03$ & $7.30 \mathrm{E}-05$ & $E+02$ & $9 E+03$ & $.87 \mathrm{E}+03$ & $1.09 E+04$ & $91 \mathrm{E}+03$ & $2.15 E+03$ \\
\hline 12 & $\mathbf{s}+$ & kg & $3.42 \mathrm{E}+01$ & $3.42 E+01$ & $1.32 \mathrm{E}-06$ & $2.25 \mathrm{E}-00$ & $2.06 \mathrm{E}+01$ & $5.82 E+01$ & $3 E+02$ & $3.10 \mathrm{E}+01$ & $3.76 \mathrm{E}+01$ \\
\hline 112 & & $\mathrm{~kg}$ & $9.99 \mathrm{E}+01$ & $99 \mathrm{E}+01$ & $2.91 \mathrm{E}-05$ & $2.02 \mathrm{E}+01$ & $2.46 \mathrm{E}+02$ & $1.91 E+03$ & $3 E+03$ & $E+02$ & +03 \\
\hline 112 & $\mathbf{U}$ & kg & $3.23 \mathrm{E}+01$ & $3.23 E+01$ & 2.58E-05 & $1.90 \mathrm{E}+01$ & $2.41 \mathrm{E}+02$ & $1.50 \mathrm{E}+03$ & -03 & $E+02$ & +02 \\
\hline 112 & $\mathbf{Z r}$ & $\mathrm{kg}$ & $1.64 \mathrm{E}-00$ & 1.64 & $E-06$ & & 9.36 & & & $E+02$ & \\
\hline 112 & 27 & $\mathrm{Ci}$ & 1.21 & 1.21 & & & 4 & & & & \\
\hline 12 & & $\mathbf{C i}$ & 1.2 & $1.07 E-01$ & 62E-07 & -01 & $6.95 \mathrm{E}-00$ & $8.53 E+01$ & & $3.21 \mathrm{E}+01$ & $7.52 E+01$ \\
\hline 12 & & $\mathrm{Ci}$ & $1.02 \mathrm{E}-04$ & 3.71E-06 & $00 \mathrm{E}+01$ & $1.26 \mathrm{E}-05$ & $1.02 \mathrm{E}-04$ & $4.41 \mathrm{E}-04$ & $1.29 \mathrm{E}-03$ & $7 \mathrm{E}-04$ & $4.04 \mathrm{E}-04$ \\
\hline 112 & Ba137 & $\mathrm{Ci}$ & $1.20 \mathrm{E}+04$ & $1.20 \mathrm{E}+04$ & $0.00 \mathrm{E}+01$ & $1.14 E+03$ & $1.20 E+04$ & $4.56 \mathrm{E}+04$ & $E+04$ & $E+04$ & $2.14 \mathrm{E}+04$ \\
\hline 112 & & $\mathrm{Ci}$ & 2.54E-01 & 2.54E-01 & $E+01$ & $E-02$ & 54E-01 & $.87 \mathrm{E}-01$ & $\mathrm{E}-00$ & $E-01$ & $5.79 \mathrm{E}-01$ \\
\hline$?$ & & $\mathrm{Ci}$ & $4.94 \mathrm{E}-00$ & 4.94E-00 & $E+01$ & $E-01$ & 94E-00 & $20 \mathrm{E}+01$ & $E+01$ & $E-00$ & $.26 \mathrm{E}+01$ \\
\hline & 242 & c & $5.27 \mathrm{E}-05$ & $1.91 E-06$ & $0.00 E+01$ & $E-06$ & 5.27E-05 & $E-04$ & $E-04$ & E-04 & E-04 \\
\hline 12 & & $\mathrm{Ci}$ & 1.08E-06 & $3.92 \mathrm{E}-08$ & $E+01$ & 77 & 06 & $4 . \quad>>$ & -05 & E-06 & -06 \\
\hline & & $\mathrm{Ci}$ & 1.49E-05 & 5.42E-07 & $2+01$ & & & & 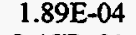 & -05 & $8-05$ \\
\hline 12 & & $\mathrm{Ci}$ & 2.28 & 2.28 & 97 & -02 & & 00 & & -00 & +01 \\
\hline 12 & & $\mathrm{Ci}$ & 1.05E-01 & $1.05 E-01$ & $E+01$ & -02 & -01 & 01 & -01 & $E-01$ & -01 \\
\hline 12 & avr & $\mathrm{Ci}$ & $1.27 \mathrm{E}+04$ & $1.27 E+04$ & $0 E-04$ & $:+03$ & $8.73 E+03$ & $3.26 E+04$ & $E+04$ & $E+04$ & \\
\hline & & L 1 & 2.87E-01 & $2.87 E-01$ & $E+01$ & & -01 & -00 & $E-00$ & & \\
\hline & 54 & c & $2.11 E+01$ & $2.11 E+01$ & $0.00 \mathrm{E}+01$ & -00 & $11 \mathrm{E}+01$ & +02 & +02 & $E+01$ & $E+02$ \\
\hline & & $\mathbf{C i}$ & $1.74 E+01$ & $1.74 E+01$ & +01 & 01 & 01 & 02 & +02 & -01 & +02 \\
\hline & & $\mathrm{Ci}$ & 5.72 & 5. 7 & 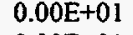 & & & & & & $:-03$ \\
\hline 112 & 3 & C & $6.64 \mathrm{E}-01$ & 6.641 & 01 & -02 & 6.6 & & +02 & & +01 \\
\hline 2 & 39 & $\mathrm{Ci}$ & 2.24E-01 & 2.24E-01 & +01 & -02 & -01 & $8-00$ & $E-00$ & E-01 & $E-00$ \\
\hline & & $\mathrm{Cl}_{1}$ & $2.22 \mathrm{E}+01$ & $2.22 \mathrm{E}+01$ & +01 & -00 & 01 & +02 & $E+02$ & $E+01$ & +02 \\
\hline & & $\mathrm{CI}_{1}$ & 1.4 & 1.4 & & & & & & & \\
\hline & & & 6.2 & 6.2 & 1 & & & & & & \\
\hline & & C & & & +01 & -02 & & & & & 8-01 \\
\hline 112 & 39 & $\mathbf{C i}$ & $1.54 E+01$ & $3.56 \mathrm{E}-01$ & +01 & $=00$ & -01 & 01 & -02 & -01 & +01 \\
\hline 12 & 240 & $\mathbf{C i}$ & Incl. in 239 & 4.97E-02 & $E+01$ & -00 & -01 & +01 & $E+02$ & t01 & $E+01$ \\
\hline & Pu241 & $\mathrm{Ci}$ & $1.27 \mathrm{E}+01$ & $4.62 \mathrm{E}-01$ & +01 & -00 & -01 & Hol & +02 & +01 & +01 \\
\hline & 242 & $\mathbf{C i}$ & E-05 & 2.2 & +01 & & & & & & \\
\hline & 226 & 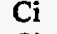 & .06 & 8.8 & & & & & & & \\
\hline 12 & 228 & $\mathbf{C i}$ & 1.09E-01 & 1.09E-01 & -01 & 03 & 01 & & & & -01 \\
\hline B112 & Ru106 & $\mathrm{Ci}$ & $4.28 \mathrm{E}-04$ & $4.28 E-04$ & :01 & -05 & $4.28 \mathrm{E}-04$ & -03 & -03 & $E-04$ & $E-04$ \\
\hline 12 & Sb125 & $\mathbf{C i}$ & $1.02 E+01$ & $1.02 \mathrm{E}+01$ & +01 & -01 & $\mathbf{E}+01$ & +01 & +01 & +01 & +01 \\
\hline & Se79 & c & E-01 & $1.90 \mathrm{E}-01$ & +01 & 02 & & & .00 & -01 & $E-01$ \\
\hline & & & +02 & 6.58 & & & & & & +03 & +03 \\
\hline & & $\mathbf{C i}$ & 04 & & & & & & & 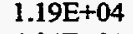 & \\
\hline 2 & & $\mathrm{Ci}$ & & & & & & & +02 & +01 & +02 \\
\hline 12 & Th229 & $\mathrm{Ci}$ & 2.51E-03 & 2.5 & $=01$ & $8-04$ & -03 & $E-02$ & -02 & -03 & $E-03$ \\
\hline 12 & 2332 & $\mathbf{C i}$ & $4.51 \mathrm{E}-03$ & 4.51 & $E+01$ & .04 & 03 & .02 & -02 & -02 & $E-02$ \\
\hline & 1126 & c & $2.84 \mathrm{E}-01$ & 2.84 & -01 & -02 & & & 00 & -01 & -01 \\
\hline & ium & $c$ & & & & & & & & & -00 \\
\hline & & c & & & & & & & & & \\
\hline & & $\mathrm{Ci}$ & -00 & & & & & & & & \\
\hline 2 & & $\mathrm{Ci}$ & $1.68 \mathrm{E}-01$ & $1.28 \mathrm{E}-02$ & $E+01$ & -03 & & & -00 & 3. & $=-01$ \\
\hline 112 & $\mathbf{U}$ & $\mathbf{C i}$ & $6.26 \mathrm{E}-03$ & $4.79 \mathrm{E}-04$ & $E+01$ & -04 & $6.26 \mathrm{E}-03$ & 4. & $E-01$ & -02 & $\mathrm{E}-02$ \\
\hline & & C & $5.38 \mathrm{E}-03$ & 4.11E-04 & $E+01$ & -04 & -03 & & -02 & 1 & $E-02$ \\
\hline 12 & $\mathbf{U}$ & $\mathrm{Ci}$ & -01 & 1.0 & 101 & & & & & & $=-00$ \\
\hline & $\mathbf{Y}$ & 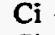 & & & & & & & & & \\
\hline & & & & & & & & & & & +01 \\
\hline 11 & $A$ & k & 4.7 & & +01 & & 501 & & & 02 & +02 \\
\hline 1 & B & kg & +04 & 1.3 & $E+02$ & 03 & $8+03$ & & & $E+03$ & +03 \\
\hline B201 & $\mathrm{Ca}$ & kg & $1.68 E+03$ & $1.68 E+03$ & BE-02 & +01 & $5.65 E+01$ & +02 & +03 & $E+02$ & $E+02$ \\
\hline B201 & C & kg & $2.27 E+02$ & $2.27 E+02$ & $4.66 \mathrm{E}+01$ & 6 & $8.66 \mathrm{E}+01$ & 1.2 & $E+02$ & $E+01$ & $E+01$ \\
\hline B & $\mathrm{CO3}$ & kg & $0.00 \mathrm{E}+01$ & 1.55 & $E+01$ & 4 & .03 & 3. & 0 & +03 & $E+03$ \\
\hline B & $\mathrm{Cr}$ & kg & 02 & 4.59 & 02 & 2. & 12 & $E+0$ & & +02 & +02 \\
\hline B & $\mathbf{F}$ & $k$ & 8 & 8.0 & & & & & & +02 & +02 \\
\hline & $\mathbf{F}$ & & & & & & & & & & +02 \\
\hline B201 & Hg & $\mathbf{k g}$ & $8.24 \mathrm{E}-02$ & & & & & -0 & & -01 & E-00 \\
\hline $\mathbf{B 2 0}$ & $\mathbf{K}$ & kg & $7.99 \mathrm{E}+02$ & $7.99 \mathrm{E}-$ & & & $6.73 E+02$ & $8.89 \mathrm{E}+0$ & & $6.52 \mathrm{E}+02$ & $E+02$ \\
\hline & $\mathbf{L}$ & $\mathbf{k g}$ & $2.08 E+03$ & $2.08 E+03$ & $6 F+0 ?$ & $7.36 \mathrm{E}+02$ & $1.27 E+03$ & & & $1.29 E+03$ & $4.66 \mathrm{E}+02$ \\
\hline & Mr & kg & $2.64 E+03$ & $2.64 \mathrm{E}+03$ & $2.15 E+02$ & $8.14 \mathrm{E}+02$ & $1.60 \mathrm{E}+03$ & $2.60 \mathrm{E}+0$ & $4.00 \mathrm{E}+03$ & $1.68 E+03$ & $7.60 \mathrm{E}+02$ \\
\hline & $\mathbf{N a}$ & kg & $.25 E+03$ & $5.25 \mathrm{E}+03$ & $2.39 E+03$ & $E+03$ & $4.31 E+03$ & $6.43 E+03$ & $E+03$ & $4.59 E+03$ & $1.49 E+03$ \\
\hline & Tiv & $\mathrm{kg}$ & $.59 E+01$ & $6.59 E+01$ & $3.04 \mathrm{E}-00$ & $1.12 \mathrm{E}+01$ & $2.42 \mathrm{E}+01$ & $4.57 \mathrm{E}+01$ & $8.06 E+01$ & $2.71 \mathrm{E}+01$ & $1.55 E+01$ \\
\hline
\end{tabular}




\begin{tabular}{|c|c|c|c|c|c|c|c|c|c|c|c|}
\hline ank & Anal. & $n$ & May98 & Sept98 & $1 \%$-ile & $10 \%$-ile & $50 \%-j l e$ & $90 \%$-ile & $99 \%$-ile & Mean & Std Dev \\
\hline 201 & $\mathrm{NO} 2$ & $\mathrm{~kg}$ & $1 . \overline{21 E+02}$ & $1.21 \mathrm{E}+02$ & $1.73 \mathrm{E}-01$ & $3.88 \mathrm{E}+0 \mathrm{I}$ & $1.70 \mathrm{E}+02$ & $6.31 E+02$ & $4.80 E+03$ & $4.09 \mathrm{E}+02$ & $8.52 E+02$ \\
\hline 201 & NO3 & $\mathrm{kg}$ & $6.78 E+03$ & $6.78 \mathrm{E}+03$ & $2.13 E+03$ & $4.89 \mathrm{E}+03$ & $7.17 \mathrm{E}+03$ & $43 E+04$ & $2.91 \mathrm{E}+04$ & 8.77E +03 & $46 \mathrm{E}+03$ \\
\hline 3201 & $\mathbf{P}$ & $\mathrm{kg}$ & $2.28 E+03$ & NA & $1.85 \mathrm{E}+02$ & $2.35 E+02$ & $3.21 \mathrm{E}+02$ & $6.64 E+02$ & $2.00 \mathrm{E}+03$ & $4.25 \mathrm{E}+02$ & $11 E+02$ \\
\hline B201 & $\mathbf{P b}$ & kg & $1.87 E+02$ & $1.87 E+02$ & $0.00 \mathrm{E}+01$ & $1.30 \mathrm{E}-00$ & $1.12 \mathrm{E}+01$ & $1.05 \mathrm{E}+02$ & $8.80 \mathrm{E}+02$ & $5.22 \mathrm{E}+01$ & $1.37 \mathrm{E}+02$ \\
\hline 3201 & PO4 & $\mathbf{k g}$ & $7.05 \mathrm{E}+03$ & $2.30 \mathrm{E}+03$ & $6.02 \mathrm{E}+01$ & $2.03 E+02$ & $4.09 E+02$ & $1.36 \mathrm{E}+03$ & $3.72 E+03$ & $6.43 E+02$ & $6.97 \mathrm{E}+02$ \\
\hline B201 & $\mathbf{s}$ & $\mathrm{kg}$ & $4.79 E+01$ & NA & $5.76 \mathrm{E}-01$ & $6.27 \mathrm{E}-00$ & $2.21 E+01$ & & $27 \mathrm{E}+02$ & $5.57 \mathrm{E}+01$ & $E+01$ \\
\hline 201 & $\mathbf{S i}$ & $\mathrm{kg}$ & $2.78 E+03$ & $2.78 \mathrm{E}+03$ & 2.45E-00 & $4.56 \mathrm{E}+01$ & $1.36 E+02$ & $3.38 \mathrm{E}+02$ & $1.87 E+03$ & $2.42 \mathrm{E}+02$ & $E+02$ \\
\hline 01 & SO4 & $\mathrm{kg}$ & $1.44 \mathrm{E}+02$ & $4.79 \mathrm{E}+01$ & $1.64 \mathrm{E}+01$ & $4.19 E+01$ & $9.80 \mathrm{E}+01$ & $3.94 \mathrm{E}+02$ & $17 \mathrm{E}+03$ & $2 \mathrm{E}+02$ & $E+02$ \\
\hline 201 & $\mathbf{S}$ & & $1.27 \mathrm{E}+02$ & $1.27 \mathrm{E}+02$ & $7.86 \mathrm{E}-00$ & $E+01$ & $35 E+01$ & $9.72 \mathrm{E}+01$ & $1.28 \mathrm{E}+02$ & $37 \mathrm{E}+01$ & $61 \mathrm{E}+01$ \\
\hline 201 & TOC & & $3.25 E+02$ & $3.25 \mathrm{E}+02$ & $7.36 \mathrm{E}-00$ & $6.80 \mathrm{E}+01$ & $3.27 E+02$ & $8.44 E+02$ & $2.15 E+03$ & $4.55 \mathrm{E}+02$ & $4.77 \mathrm{E}+02$ \\
\hline 201 & $\mathbf{U}$ & & $2.15 E+01$ & $2.15 E+01$ & $0.00 \mathrm{E}+01$ & $7.64 \mathrm{E}-01$ & 32E-00 & $8.35 \mathrm{E}+01$ & $1 \mathrm{E}+02$ & $2.66 \mathrm{E}+01$ & $5.30 \mathrm{E}+01$ \\
\hline 201 & $\mathbf{Z r}$ & kg & $1.47 \mathrm{E}-00$ & $1.47 \mathrm{E}-00$ & $2.71 E-02$ & $6.03 E-02$ & E-01 & $E+01$ & $E+01$ & $3.73 E-00$ & BE-00 \\
\hline 8201 & Ac227 & $\mathrm{Ci}$ & $4.34 \mathrm{E}-08$ & 4.34E-08 & $0.00 \mathrm{E}+01$ & $0.00 \mathrm{E}+01$ & E-08 & $1.47 \mathrm{E}-07$ & E-07 & $5.87 \mathrm{E}-08$ & E-08 \\
\hline 8201 & Am241 & $\mathrm{Ci}$ & $4.26 \mathrm{E}-00$ & $4.26 \mathrm{E}-00$ & $3.37 \mathrm{E}-01$ & $1.44 \mathrm{E}-00$ & $E-00$ & $1.51 \mathrm{E}+02$ & $5.88 \mathrm{E}+02$ & $5.02 \mathrm{E}+01$ & $E+02$ \\
\hline 3201 & Am243 & $\mathrm{Ci}$ & 3.08E-09 & $3.46 \mathrm{E}-05$ & 5.6 & -09 & 09 & & E-08 & E-09 & $\mathrm{E}-09$ \\
\hline B201 & Ba137 & $\mathrm{Ci}$ & $1.04 E+02$ & $1.04 E+02$ & +01 & -00 & +02 & +03 & 103 & $3.32 \mathrm{E}+02$ & +02 \\
\hline B201 & C14 & $\mathrm{Ci}$ & $4.35 E-02$ & 4.35E-02 & +01 & -03 & -02 & -01 & -01 & $5.86 \mathrm{E}-02$ & -02 \\
\hline B201 & Cd113 & $\mathrm{Ci}$ & $4.89 \mathrm{E}-04$ & $4.89 \mathrm{E}-04$ & $0.00 \mathrm{E}+01$ & $E-05$ & Q-04 & $4.56 \mathrm{E}-03$ & -02 & $2.28 \mathrm{E}-03$ & -03 \\
\hline B201 & Cm242 & $\mathrm{Ci}$ & $3.70 \mathrm{E}-06$ & $4.16 \mathrm{E}-02$ & -07 & $=-06$ & & -06 & & $E-06$ & $E-06$ \\
\hline B201 & Cm243 & $\mathrm{Ci}$ & $7.98 \mathrm{E}-08$ & $9.04 \mathrm{E}-03$ & $E-08$ & -08 & & -07 & -07 & E-08 & -08 \\
\hline B201 & $\mathrm{Cm} 244$ & $\mathrm{Ci}$ & $7.84 \mathrm{E}-08$ & 2.17E-01 & -08 & -08 & & -07 & -07 & E-08 & $E-08$ \\
\hline B201 & Co60 & $\mathrm{Ci}$ & $5.60 \mathrm{E}-05$ & $5.60 \mathrm{E}-05$ & +01 & -02 & & & & E-01 & -00 \\
\hline B201 & Cs134 & C & 2.78E-06 & $2.78 \mathrm{E}-06$ & 1 & -07 & & 05 & & -06 & -05 \\
\hline B201 & Cs137 & $\mathrm{Ci}$ & $1.10 E+02$ & $1.10 \mathrm{E}+02$ & 02 & +01 & -02 & -03 & -03 & +03 & 103 \\
\hline B201 & Eu152 & $\mathrm{Ci}$ & 1.82E-04 & $1.82 \mathrm{E}-04$ & +01 & +01 & -04 & $8-04$ & $E-03$ & E-04 & $E-04$ \\
\hline B201 & Eu154 & $\mathrm{Ci}$ & $6.02 \mathrm{E}-01$ & $6.02 E-01$ & & & & & & & \\
\hline B201 & Eu155 & & $1.64 \mathrm{E}-02$ & $1.64 \mathrm{E}-02$ & +01 & +01 & & & -01 & & -02 \\
\hline B201 & 1129 & C & $2.29 \mathrm{E}-06$ & $2.29 \mathrm{E}-06$ & & -06 & & 06 & -06 & & \\
\hline B201 & Nb93 & C & $1.45 E-04$ & $1.45 \mathrm{E}-04$ & & & & & & & \\
\hline B201 & Ni59 & C & 4.96E-05 & $4.96 \mathrm{E}-05$ & 01 & -06 & 05 & -04 & 04 & -05 & -05 \\
\hline B201 & Ni63 & Ci & $4.57 \mathrm{E}-03$ & $4.57 \mathrm{E}-03$ & +01 & -04 & -03 & $=-02$ & -02 & $E-03$ & -03 \\
\hline B201 & Np237 & & 7.51E-06 & $7.51 \mathrm{E}-06$ & & -06 & & & 05 & 06 & -06 \\
\hline B201 & Pa231 & $\mathrm{Ci}$ & $1.00 \mathrm{E}-07$ & $1.00 \mathrm{E}-07$ & -01 & -08 & & -06 & -06 & & \\
\hline & & & $3.21 \mathrm{E}-04$ & $9.49 \mathrm{E}-01$ & & & & & & & \\
\hline B201 & Pu239 & $\mathbf{C i}$ & $1.55 E+02$ & $1.42 \mathrm{E}+02$ & 01 & 01 & 02 & & & 02 & +02 \\
\hline B201 & Puz40 & $\mathbf{C i}$ & Icl. in 239 & $1.25 E+01$ & -01 & +01 & 02 & +02 & +02 & +02 & +02 \\
\hline B201 & Pu241 & $\mathbf{C i}$ & 1.35E-02 & $4.13 E+01$ & -03 & -03 & .02 & -02 & -02 & $E-02$ & -02 \\
\hline & Pu242 & $\mathbf{C i}$ & $6.23 \mathrm{E}-08$ & 1.91E-04 & & -08 & & & -07 & & -08 \\
\hline B201 & 226 & & 8.22E-09 & $8.22 \mathrm{E}-09$ & & -01 & & & -08 & & \\
\hline & & c & 5.29 & 5.29 & & & & & & 13 & -13 \\
\hline 01 & Ru106 & $\mathbf{C i}$ & $4.20 \mathrm{E}-11$ & $4.20 \mathrm{E}-11$ & & & & & & 11 & -11 \\
\hline B201 & Sb125 & $\mathrm{Ci}$ & $6.46 \mathrm{E}-05$ & $6.46 \mathrm{E}-05$ & +01 & -07 & 05 & -04 & -04 & -05 & -05 \\
\hline B201 & Se79 & $\mathbf{C i}$ & $3.68 \mathrm{E}-05$ & $3.68 \mathrm{E}-05$ & -06 & -05 & & & .04 & -05 & -05 \\
\hline B201 & Sm151 & $\mathrm{Ci}$ & $1.39 \mathrm{E}-01$ & $1.39 \mathrm{E}-01$ & 01 & 01 & & & -01 & -01 & -01 \\
\hline B201 & Sr89/90 & $\mathbf{C i}$ & $2.87 E+02$ & $2.87 E+02$ & & +01 & & & -04 & -03 & \\
\hline & & $\mathbf{C i}$ & & & & & & & & & \\
\hline 11 & 29 & $\mathrm{Ci}$ & $1.02 \mathrm{E}-10$ & 0 & & 11 & & & 09 & & -09 \\
\hline B201 & Th232 & $\mathbf{C i}$ & $4.62 \mathrm{E}-14$ & 4.62 & 01 & -15 & 14 & 13 & -12 & $E-13$ & E-13 \\
\hline B201 & Tin126 & $\mathbf{C i}$ & $5.55 \mathrm{E}-05$ & $5.55 \mathrm{E}-05$ & +01 & -06 & 05 & 04 & -03 & 94 & -04 \\
\hline B201 & Tritium & $\mathrm{Ci}$ & 5.63E-04 & $5.63 \mathrm{E}-04$ & & & & & & $r$ & -05 \\
\hline B201 & U232 & $\mathrm{Ci}$ & $5.36 \mathrm{E}-08$ & -07 & & & & & & & -07 \\
\hline B201 & U233 & $\mathrm{Ci}$ & & & & & & & & & \\
\hline & & & & & & & & & & & \\
\hline B201 & $\mathrm{U} 2$ & $\mathrm{Ci}$ & 1.19E-04 & 3.15E-04 & & & & & -03 & -04 & .03 \\
\hline B201 & U236 & C & 2.33E-05 & $6.17 E-05$ & 01 & .06 & -05 & -04 & $E-03$ & $E-04$ & $E-04$ \\
\hline B201 & U238 & $\mathrm{Ci}$ & $2.71 E-03$ & $7.18 \mathrm{E}-03$ & $0 \mathbf{1}$ & $E-04$ & -03 & 02 & -01 & $E-02$ & -02 \\
\hline B201 & Y90 & $\mathrm{Ci}$ & $2.87 E+02$ & $2.87 \mathrm{E}+02$ & & +01 & +02 & & +04 & $E+03$ & $=03$ \\
\hline B201 & Zr93 & $\mathrm{Ci}$ & & & & & & & & & -02 \\
\hline & Al & 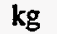 & & & & & & & & & +02 \\
\hline B202 & Bi & kg & & & & & & & & +03 & +03 \\
\hline 02 & $\mathrm{Ca}$ & $\mathrm{kg}$ & $1.91 \mathrm{E}+02$ & $1.91 \mathrm{E}+02$ & & & & & +03 & $E+02$ & +02 \\
\hline 02 & CI & kg & $1.02 E+02$ & $1.02 E+02$ & & & -01 & +02 & +02 & $E+01$ & $E+01$ \\
\hline B202 & $\operatorname{co3}$ & kg & $2.21 E+02$ & $2.21 \mathrm{E}+02$ & & & & & $E+03$ & $E+03$ & $E+03$ \\
\hline B202 & $\mathrm{Cr}$ & kg & $2.96 \mathrm{E}+02$ & $2.96 \mathrm{E}$ & $1.22 \mathrm{E}+02$ & +02 & & +02 & +02 & $3.84 \mathrm{E}+02$ & +02 \\
\hline B202 & $\mathbf{F}$ & kg & $7.63 \mathrm{E}+02$ & $7.63 E+02$ & $5.84 \mathrm{E}+01$ & $5.05 E+02$ & $7.12 E+02$ & $8.80 \mathrm{E}+02$ & $2.64 E+03$ & $7.53 \mathrm{E}+02$ & $E+02$ \\
\hline B202 & $\mathrm{Fe}$ & kg & $8.00 \mathrm{E}+02$ & $8.00 E+02$ & $1.35 E+02$ & $2.61 \mathrm{E}+02$ & $4.67 E+02$ & $1.79 E+03$ & $E+03$ & $7.50 \mathrm{E}+02$ & $E+02$ \\
\hline B202 & Hg & kg & 4.00E-02 & $4.00 \mathrm{E}-02$ & $0.00 \mathrm{E}+01$ & $5.27 \mathrm{E}-03$ & $4.00 \mathrm{E}-02$ & $3.88 \mathrm{E}-01$ & 3.13E-00 & $1.92 \mathrm{E}-01$ & $5.00 \mathrm{E}-01$ \\
\hline B202 & $\mathbf{K}$ & kg & $8.11 \mathrm{E}+02$ & $8.11 E+02$ & $5.39 \mathrm{E}+01$ & $3.73 E+02$ & $6.25 E+02$ & $8.29 E+02$ & $1.00 \mathrm{E}+03$ & $6.08 \mathrm{E}+02$ & $1.95 \mathrm{E}+02$ \\
\hline
\end{tabular}


Tank Anal. Un

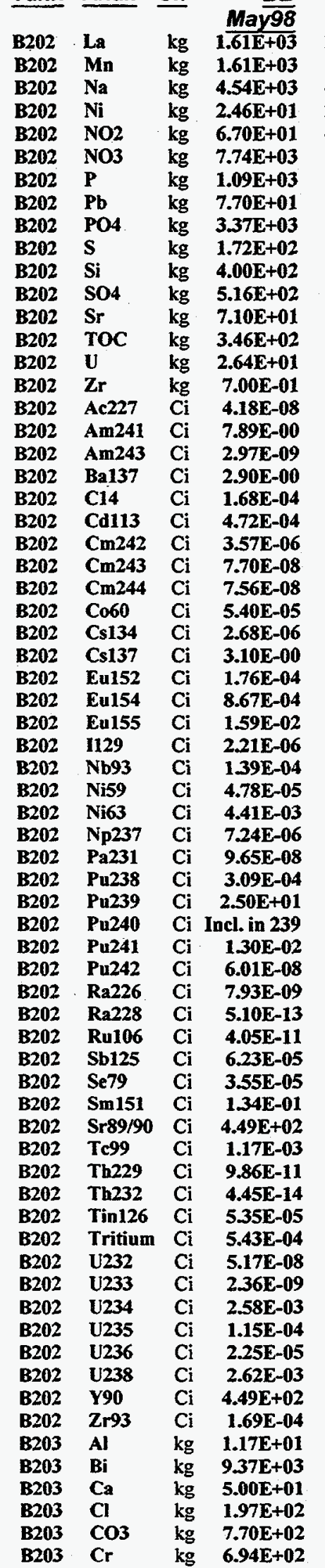
Sept98

$1.61 \mathrm{E}+03$
$1.61 \mathrm{E}+03$

$4.54 \mathrm{E}+03$

$2.46 \mathrm{E}+01$

$6.70 \mathrm{E}+01$

$7.74 \mathrm{E}+03$

NA

$7.70 E+01$
$1.10 E+03$

NA

$4.00 \mathrm{E}+02$

$1.72 \mathrm{E}+02$

$7.10 \mathrm{E}+01$

$3.46 \mathrm{E}+02$

2.64E+01

7.00E-01

$4.18 \mathrm{E}-08$

7.89E-00

6.41E-05

2.90E-00

1.68E-04

4.72E-04

$7.70 \mathrm{E}-02$

1.66E-03

$1.63 \mathrm{E}-03$

5.40E-05

2.68E-06

3.10E-00

$1.76 \mathrm{E}-04$

8.67E-04

1.59E-02

2.21E-06

$1.39 \mathrm{E}-04$

4.78E-05

4.41E-03

7.24E-06

9.65E-08

3.19E-01

$4.61 \mathrm{E}+01$

4.05E-00

$1.34 \mathrm{E}+01$

6.19E-05

7.93E-09

5.10E-13

4.05E-11

6.23E-05

3.55E-05

1.34E-01

$4.49 \mathrm{E}+02$

1.17E-03

9.86E-11

4.45E-14

5.35E-05

5.43E-04

1.74E-07

7.94E-09

8.68E-03

3.86E-04

7.57E-05

8.81E-03

$4.49 \mathrm{E}+02$

1.69E-04

1.17E+01

$9.37 \mathrm{E}+03$

$5.00 \mathrm{E}+01$

$1.97 \mathrm{E}+02$

$7.70 \mathrm{E}+02$

$6.94 E+02$
$1 \%$-ile

\section{$2.32 \mathrm{E}+02$}

$2.08 \mathrm{E}+02$

$2.19 \mathrm{E}+03$

$3.08 \mathrm{E}-00$

$1.48 \mathrm{E}-00$

$1.88 \mathrm{E}+03$

$1.61 \mathrm{E}+02$

2.82E-04

4.47E+01

$6.02 \mathrm{E}-01$

3.08E-00

$1.57 \mathrm{E}+01$

6.31E-00

7.22E-00

$1.45 \mathrm{E}-02$

$2.26 \mathrm{E}-02$

$0.00 \mathrm{E}+01$

3.36E-01

5.36E-10

$0.00 \mathrm{E}+01$

$0.00 \mathrm{E}+01$

$0.00 \mathrm{E}+01$

6.44E-07

1.39E-08

$1.36 \mathrm{E}-08$

$7.98 \mathrm{E}-04$

$0.00 \mathrm{E}+01$

$1.22 \mathrm{E}-00$

$0.00 \mathrm{E}+01$

$0.00 \mathrm{E}+01$

$0.00 \mathrm{E}+01$

$9.97 \mathrm{E}-07$
$2.60 \mathrm{E}-05$

$0.00 \mathrm{E}+01$

$0.00 \mathrm{E}+01$

$1.31 \mathrm{E}-06$

$1.78 \mathrm{E}-11$

5.57E-05

4.51E-00

4.51E-00

2.35E-03

$1.08 \mathrm{E}-08$

$0.00 E+01$

$0.00 \mathrm{E}+01$

9.44E-12

$0.00 \mathrm{E}+01$

$5.60 \mathrm{E}-06$

$0.00 \mathrm{E}+01$

$0.00 \mathrm{E}+01$

$0.00 \mathrm{E}+01$

$1.82 \mathrm{E}-14$

8.22E-18

$0.00 \mathrm{E}+01$

$3.49 \mathrm{E}-04$

9.55E-12

4.36E-13

4.76E-07

2.12E-08

4.16E-09

4.84E- 07

$0.00 \mathrm{E}+01$

$3.16 \mathrm{E}-05$

$0.00 E+01$

$1.43 \mathrm{E}+03$

$0.00 E+01$

8.42E+01

$1.03 E+02$

$2.48 \mathrm{E}+02$
$10 \%-i l e$

$6.74 E+02$

$7.64 \mathrm{E}+02$

$2.90 \mathrm{E}+03$

$1.11 \mathrm{E}+01$

$3.89 \mathrm{E}+01$

$4.66 \mathrm{E}+03$

$2.15 E+02$

$1.35 \mathrm{E}-00$

$1.81 \mathrm{E}+02$

$5.55 \mathrm{E}-00$

4.22E+01

$3.88 \mathrm{E}+01$

$2.87 \mathrm{E}+01$

$6.91 \mathrm{E}+01$

$7.36 \mathrm{E}-01$

$5.75 \mathrm{E}-02$

$0.00 \mathrm{E}+01$

$1.71 E-00$

1.47E-09

1.11E-0।

3.28E-05

$6.22 \mathrm{E}-05$

$1.76 \mathrm{E}-06$

$3.80 \mathrm{E}-08$

3.73E-08

2.68E-02

$1.03 \mathrm{E}-07$

$1.48 \mathrm{E}+01$

$0.00 \mathrm{E}+01$

$0.00 \mathrm{E}+01$

$0.00 \mathrm{E}+01$

1.55E-06

6.62E-05

5.79E-06

5.34E-04

3.57E-06

1.77E-08

1.53E-04

1. $23 \mathrm{E}+01$

$1.23 \mathrm{E}+01$

6.42E-03

2.97E-08

$0.00 \mathrm{E}+01$

$0.00 \mathrm{E}+01$

2.12E-11

$0.00 \mathrm{E}+01$

$1.46 \mathrm{E}-05$

$0.00 \mathrm{E}+01$

$1.37 \mathrm{E}+02$

9.78E-06

$1.81 \mathrm{E}-11$

8.15E-15

7.05E-06

4.41E-04

9.47E-09

4.32E-10

4.73E-04

2.11E-05

4.12E-06

4.80E-04

$6.36 \mathrm{E}+01$

$8.05 \mathrm{E}-05$

3.32E-00

$4.93 E+03$

$2.01 E+01$

$1.17 \mathrm{E}+02$

$7.64 E+02$

$4.28 \mathrm{E}+02$ 50\%-ile

\section{$1.18 \mathrm{E}+03$}

$1.51 \mathrm{E}+03$

4.01E+03

$2.28 \mathrm{E}+01$

$1.64 \mathrm{E}+02$

$6.76 \mathrm{E}+03$

$1.01 \mathrm{E}+01$

$3.85 \mathrm{E}+02$

$2.00 \mathrm{E}+01$

$1.28 \mathrm{E}+02$

$9.23 \mathrm{E}+01$

$5.85 \mathrm{E}+01$

$3.04 \mathrm{E}+02$

$3.96 \mathrm{E}-00$

$1.21 \mathrm{E}-01$

4.18E-08

$4.80 \mathrm{E}-00$

2.97E-09

$2.90 \mathrm{E}-00$

1.68E-04

4.72E-04

3.57E-06

7.70E-08

$7.56 \mathrm{E}-08$

1.73E-01

2.68E-06

$3.51 E+02$

1.76E-04

8.67E-04

1.59E-02

2.21E-06

1.39E-04

4.78E-05

4.41E-03

7.24E-06

9.65E-08

3.09E-04

$2.50 \mathrm{E}+01$

$2.50 \mathrm{E}+01$

$1.30 \mathrm{E}-02$

6.01E-08

7.93E-09

5.10E-13

4.05E-11

6.23E-05

3.55E-05

1.34E-01

$9.42 \mathrm{E}+02$

$1.17 \mathrm{E}-03$

9.86E-11

4.45E-14

5.35E-05

5.43E-04

5.17E-08

2.36E-09

2.58E-03

1.15E-04

2.25E-05

2.62E-03

$4.49 E+02$

1.69E-04

$9.57 \mathrm{E}+01$

$9.58 \mathrm{E}+03$

$9.67 \mathrm{E}+01$

$1.50 \mathrm{E}+02$

$2.44 \mathrm{E}+03$

$7.08 \mathrm{E}+02$

90\%-ile

\section{$1.72 \mathrm{E}+03$}

$2.39 \mathrm{E}+03$

$5.99 \mathrm{E}+03$

$4.43 \mathrm{E}+01$

$6.35 \mathrm{E}+02$

$1.33 \mathrm{E}+04$

$6.82 \mathrm{E}+02$

$9.81 \mathrm{E}+01$ 
Tank Anal. Un

\begin{tabular}{|c|c|}
\hline B203 & $\mathbf{F}$ \\
\hline B203 & $\mathrm{Fe}$ \\
\hline B203 & Hg \\
\hline B203 & $\mathbf{K}$ \\
\hline B203 & $\mathbf{L a}$ \\
\hline B203 & Mn \\
\hline B203 & $\mathbf{N a}$ \\
\hline B203 & $\mathrm{Ni}$ \\
\hline B203 & NO2 \\
\hline B203 & NO3 \\
\hline B203 & $\mathbf{P}$ \\
\hline B203 & $\mathbf{P b}$ \\
\hline B203 & PO4 \\
\hline B203 & $\mathbf{S}$ \\
\hline B203 & $\mathbf{S i}$ \\
\hline B203 & SO4 \\
\hline B203 & Sr \\
\hline B203 & TOC \\
\hline B203 & $\mathbf{U}$ \\
\hline B203 & $\mathbf{Z r}$ \\
\hline B203 & Ac227 \\
\hline B203 & Am241 \\
\hline B203 & Am243 \\
\hline B203 & Ba137 \\
\hline B203 & $\mathrm{C14}$ \\
\hline B203 & Cd113 \\
\hline B203 & $\mathrm{Cm} 242$ \\
\hline B203 & $\mathrm{Cm} 243$ \\
\hline B203 & Cm244 \\
\hline B203 & Co60 \\
\hline B203 & Cs134 \\
\hline B203 & Cs137 \\
\hline B203 & Eu152 \\
\hline B203 & Eu154 \\
\hline B203 & Eu155 \\
\hline B203 & 1129 \\
\hline B203 & $\mathrm{Nb93}$ \\
\hline B203 & Ni59 \\
\hline B203 & Ni63 \\
\hline B203 & Np237 \\
\hline B203 & Pa231 \\
\hline B203 & Pu238 \\
\hline B203 & Pu239 \\
\hline B203 & Pu240 \\
\hline B203 & Pu241 \\
\hline B203 & Pu242 \\
\hline B203 & $\mathbf{R a 2 2 6}$ \\
\hline B203 & $\mathbf{R a 2 2 8}$ \\
\hline B203 & Ru106 \\
\hline B203 & Sb125 \\
\hline B203 & Se79 \\
\hline B203 & Sm151 \\
\hline B203 & Sr89/90 \\
\hline B203 & Tc99 \\
\hline B203 & Th229 \\
\hline B203 & Th232 \\
\hline B203 & Tin126 \\
\hline B203 & Tritium \\
\hline B203 & U232 \\
\hline B203 & U233 \\
\hline B203 & U234 \\
\hline B203 & U235 \\
\hline B203 & U236 \\
\hline B203 & U238 \\
\hline B203 & Y90 \\
\hline B203 & Zr93 \\
\hline B204 & Al \\
\hline
\end{tabular}

B204 Bi

\section{May $\frac{B 8}{98}$} kg $1 . \overline{77 E+03}$ $9.93 E+02$
$0.00 E+01$ $1.17 \mathrm{E}+03$ kg $2.35 \mathrm{E}+03$ 3.18E+03 $6.66 \mathrm{E}+03$ kg 4.11E+01

$\mathrm{kg} \quad 1.70 \mathrm{E}+02$

kg $1.46 \mathrm{E}+04$

kg 1.49E+03

kg $0.00 \mathrm{E}+01$

$\mathrm{kg} \quad 1.60 \mathrm{E}+02$

$\mathrm{kg} \quad 2.08 \mathrm{E}+02$

$\mathrm{kg} \quad 4.80 \mathrm{E}+02$

kg 1.11E+02

kg $4.83 \mathrm{E}+03$

$\mathrm{kg} \quad 1.45 \mathrm{E}+01$ $0.00 \mathrm{E}+01$

7.75E-08

6.78E-04

5.51E-09

$3.49 E+01$

3.12E-04

8.74E-04

$6.62 \mathrm{E}-06$

1.43E-07

1.40E-07

1.00E-04

4.97E-06

$3.69 \mathrm{E}+01$

3.25E-04

1.61E-03

2.94E-02

4.08E-06

2.58E-04

$8.86 \mathrm{E}-05$

8.17E-03

1.34E-05

1.79E-07

5.73E-04

8.29E-02

$7.27 \mathrm{E}-03$

2.40E-02

1.11E-07

1.47E-08

9.44E-13

7.50E-11

1.15E-04

6.57E-05

2.49E-01

$3.25 \mathrm{E}+01$

2.16E-03

1.83E-10

8.25E-14

9.91E-05

1.01E-03

9.57E-08

4.37E-09

4.77E-03

2.12E-04

4.16E-05

4.84E-63

$3.26 \mathrm{E}+01$

3.12E-04

$1.44 \mathrm{E}+01$

$1.07 \mathrm{E}+04$
$B B$ Sept98 $\overline{1.77 \mathrm{E}+03}$ $9.93 E+02$ $0.00 \mathrm{E}+01$ $1.17 \mathrm{E}+03$

$2.35 \mathrm{E}+03$

$3.18 \mathrm{E}+03$

$6.66 \mathrm{E}+03$

4.11E+01

$1.70 \mathrm{E}+02$

$1.46 \mathrm{E}+04$

NA

$0.00 \mathrm{E}+01$

$1.51 \mathrm{E}+03$ NA

$2.08 \mathrm{E}+02$

$1.60 \mathrm{E}+02$

$1.11 \mathrm{E}+02$

$4.83 E+03$

$1.45 \mathrm{E}+01$

$0.00 \mathrm{E}+01$

$7.75 \mathrm{E}-08$

3.57E-01

2.90E-06

$3.49 \mathrm{E}+01$

3.12E-04

8.74E-04

$3.49 \mathrm{E}-03$

7.51E-05

$7.38 \mathrm{E}-05$

1.00E-04

$4.97 \mathrm{E}-06$

$3.69 \mathrm{E}+01$

3.25E-04

1.61E-03

2.94E-02

4.08E-06

2.58E-04

8.86E-05

8.17E-03

1.34E-05

1.79E-07

3.02E-01

$4.37 \mathrm{E}+01$

3.83E-00

1.27E+01

$5.86 \mathrm{E}-05$

$1.47 \mathrm{E}-08$

$9.44 \mathrm{E}-13$

$7.50 \mathrm{E}-11$

1.15E-04

6.57E-05

2.49E-01

$3.25 \mathrm{E}+01$

2.16E-03

1.83E-10

8.25E-14

9.91E-05

1.01E-03

9.57E-08

4.37E-09

4.77E-03

2.12E-04

4.16E-05

4.84E-03

$3.26 \mathrm{E}+01$

3.12E-04

$1.44 \mathrm{E}+01$

$1.07 \mathrm{E}+04$
$1 \%$-ile $10 \%$-ile

$6.40 \mathrm{E}+01 \quad 9.94 \mathrm{E}+02$

$2.59 \mathrm{E}+02$

$0.00 \mathrm{E}+01$

$9.88 \mathrm{E}+01$

$4.24 \mathrm{E}+02$

$4.09 \mathrm{E}+02$

$4.25 \mathrm{E}+03$

6.57E-00

9.55E-02

$3.47 \mathrm{E}+03$

$3.33 \mathrm{E}+02$

$0.00 \mathrm{E}+01$

8.07E+01

7.75E-01

2.21E-00

$2.94 \mathrm{E}+01$

$1.32 \mathrm{E}+01$

$1.10 \mathrm{E}+01$

$0.00 \mathrm{E}+01$

3.31E-02

$0.00 \mathrm{E}+01$

$6.08 \mathrm{E}-01$

8.62E-10

$0.00 \mathrm{E}+01$

$0.00 \mathrm{E}+01$

$0.00 \mathrm{E}+01$

$1.04 \mathrm{E}-06$

2.24E-08

$2.19 \mathrm{E}-08$

$0.00 \mathrm{E}+01$

$0.00 \mathrm{E}+01$

$1.92 \mathrm{E}-01$

$0.00 \mathrm{E}+01$

$0.00 \mathrm{E}+01$

$0.00 \mathrm{E}+01$

$2.06 \mathrm{E}-06$

3.97E-05

$0.00 \mathrm{E}+01$

$0.00 \mathrm{E}+01$

$2.10 \mathrm{E}-06$

$0.00 \mathrm{E}+01$

$8.96 \mathrm{E}-05$

1.30E-02

$1.14 \mathrm{E}-03$

3.75E-03

$1.74 \mathrm{E}-08$

$0.00 \mathrm{E}+01$

$0.00 \mathrm{E}+01$

$1.86 \mathrm{E}-11$

$0.00 \mathrm{E}+01$

$1.14 \mathrm{E}-05$

$0.00 \mathrm{E}+01$

$0.00 \mathrm{E}+01$

$0.00 \mathrm{E}+01$

$0.00 \mathrm{E}+01$

$0.00 \mathrm{E}+01$

$0.00 \mathrm{E}+01$

6.63E-04

$0.00 \mathrm{E}+01$

$0.00 E+01$

$0.00 \mathrm{E}+01$

$0.00 \mathrm{E}+01$

$0.00 \mathrm{E}+01$

$0.00 \mathrm{E}+01$

$0.00 \mathrm{E}+01$

4.80E-05

$1.02 \mathrm{E}-01$

$1.37 \mathrm{E}+03$
$4.86 \mathrm{E}+02 \quad 8.67 \mathrm{E}+02$

$0.00 \mathrm{E}+01 \quad 0.00 \mathrm{E}+01$

$7.38 \mathrm{E}+02$

$1.34 \mathrm{E}+03$

$1.53 \mathrm{E}+03$

$5.46 \mathrm{E}+03$

$2.00 \mathrm{E}+01$

$6.05 \mathrm{E}+01$

$8.84 \mathrm{E}+03$

$4.17 \mathrm{E}+02$

$3.45 \mathrm{E}+02$

$1.02 \mathrm{E}+01$

$7.75 \mathrm{E}+01$

7.17E+01

$5.38 \mathrm{E}+01$

$1.19 \mathrm{E}+02$

$1.01 \mathrm{E}-00$

$1.04 \mathrm{E}-01$

$00 \mathrm{E}+01$

$2.58 \mathrm{E}-00$

2.75E-09

$2.18 \mathrm{E}-00$

$5.52 \mathrm{E}-05$

8.35E-05

3.31E-06

$7.15 \mathrm{E}-08$

$7.00 \mathrm{E}-08$

4.23E-02

$3.10 \mathrm{E}-07$

$2.26 \mathrm{E}+01$

$0.00 \mathrm{E}+01$

$0.00 \mathrm{E}+01$

$0.00 E+01$

$3.05 \mathrm{E}-06$

$1.25 \mathrm{E}-04$

9.85E-06

9.08E-0.

6.70E-06

2.33E-08

$2.86 \mathrm{E}-04$

4.14E- 02

3.63E-03

1.20E-02

$5.55 \mathrm{E}-08$

$0.00 \mathrm{E}+01$

$0.00 \mathrm{E}+01$

$3.97 \mathrm{E}-\mathrm{L} 1$

$0.00 \mathrm{E}+01$

2.91E-05

$0.00 \mathrm{E}+01$

$2.20 \mathrm{E}+01$

$1.06 \mathrm{E}-04$

2.38E-11

$1.07 \mathrm{E}-14$

9.46E-06

8.33E-04

$1.24 \mathrm{E}-08$

$5.68 \mathrm{E}-10$

$6.20 \mathrm{E}-04$

$2.76 \mathrm{E}-05$

5.41E-06

$6.29 \mathrm{E}-04$

6.18E-01

1.51E-04

$1.99 \mathrm{E}-00$

$4.73 E+03$

$1.21 \mathrm{E}+03$

$2.30 \mathrm{E}+03$

$2.92 \mathrm{E}+03$

$7.40 \mathrm{E}+03$

$4.21 \mathrm{E}+01$

2.38E+02

$1.26 \mathrm{E}+04$

$5.55 \mathrm{E}+02$

$1.85 \mathrm{E}+01$

$7.23 \mathrm{E}+02$ 
Tank Anal. Un

\begin{tabular}{|c|c|c|c|c|c|c|}
\hline & Ca & & & Sept98 & & \\
\hline 3204 & $\mathrm{CI}$ & & $55 E+02$ & $\begin{array}{l}6.72 E+01 \\
1.55 E+02\end{array}$ & $\begin{array}{r}9.35 \mathrm{E}-00 \\
8.34 \mathrm{E}+01\end{array}$ & $\begin{array}{l}4.24 E+01 \\
1.13 E+02\end{array}$ \\
\hline 204 & 03 & & $2 E+03$ & $2.72 E+03$ & $8 E+02$ & $3 \mathrm{E}+02$ \\
\hline 204 & $\mathrm{Cr}$ & & $7.15 E+02$ & $7.15 E+02$ & $50 \mathrm{E}+02$ & $65 \mathrm{E}+02$ \\
\hline 204 & & & $58 E+03$ & $1.58 E+03$ & $1.46 \mathrm{E}+02$ & $.07 E+03$ \\
\hline 204 & we & & $E+02$ & $9 \mathrm{E}+02$ & $61 E+02$ & $16 E+02$ \\
\hline 204 & & & $0 E+01$ & $00 \mathrm{E}+01$ & $0.00 E+01$ & $00 \mathrm{E}+01$ \\
\hline 204 & & & $9 E+03$ & $.29 E+03$ & $E+02$ & $15 E+02$ \\
\hline 204 & & & +03 & $E+03$ & $E+02$ & $30 E+0$ \\
\hline 204 & & & 03 & 03 & 02 & $+c$ \\
\hline 204 & & & & U. & & +0 \\
\hline $20>>>$ & & & & & & \\
\hline 204 & & & & & & +01 \\
\hline 204 & [03 & & $10 \mathrm{~L}+\mathrm{U}_{4}$ & LTUt & & $1 E+03$ \\
\hline 20 & $\mathbf{F}$ & & $1.56 \mathrm{E}+03$ & NA & $E+02$ & $1 E+02$ \\
\hline 204 & $\mathbf{F}$ & & +01 & $0.00 \mathrm{E}+01$ & -03 & $1.84 \mathrm{E}-0 \mathrm{C}$ \\
\hline 204 & $\mathbf{F}$ & & +03 & $1.58 \mathrm{E}+03$ & & $E+0$ \\
\hline 204 & & & H01 & $\mathbf{N}$ & & +0 \\
\hline 20 & & & & $e+n<-1$ & & +01 \\
\hline 204 & & & & 1 & & \\
\hline 204 & & & 01 & 8.61 & & $i+01$ \\
\hline 204 & TOC & & 103 & 4.7 & Fol & $E+02$ \\
\hline 20 & L & & 01 & $1.42 \mathrm{E}+01$ & -04 & $8 E-00$ \\
\hline 20 & $\mathbf{Z r}$ & & & 0.0 & & $E-0$ \\
\hline 204 & Ac22 & & & 7.59 & & $E+0$ \\
\hline 20 & $A$ & & & & & $E-0$ \\
\hline 204 & & & 5.4 & 3.5 & & E-09 \\
\hline B204 & Ba157 & & +01 & $3.42 \mathrm{E}+01$ & -01 & $4 \mathrm{E}-00$ \\
\hline 20 & C1 & & -04 & $3.05 E-04$ & -01 & E-05 \\
\hline D 200 & & & & 8.5 & & - $-0 \Omega$ \\
\hline B204 & 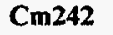 & & & 4.2 & & \\
\hline 20 & $c$ & & & & & \\
\hline 20 & $\mathrm{Cm} 2$ & & 07. & 8. & & $E-08$ \\
\hline B204 & $\mathrm{Co60}$ & & -05 & 9.81E-05 & & $\mathrm{E}-02$ \\
\hline B204 & Cs134 & & -06 & $4.8^{\circ}$ & & E-07 \\
\hline $\mathbf{B 2} \mathbf{2}$ & Cs1 & & & & & $E+01$ \\
\hline $\mathbf{B} \mathbf{2} \mathbf{0}$ & E & & & 04 & & to $-2-1$ \\
\hline B20 & & & & & & \\
\hline B204 & $\mathbf{E v}$ & & 2.8 & $2.88 \mathrm{E}-02$ & & $E+01$ \\
\hline B204 & 1129 & c & .06 & $4.00 \mathrm{E}-06$ & -06 & $0 \mathrm{E}-06$ \\
\hline B204 & Nb9 & & & 2.5 & & E-04 \\
\hline $20>-3$ & vit & & & & & -05 \\
\hline B & ii & & & & & \\
\hline & & & & & & \\
\hline B204 & Pa231 & & 1.7 & $1.75 E-07$ & 01 & $E-08$ \\
\hline B204 & Pu238 & & 5.6 & $3.64 E-01$ & & E-04 \\
\hline 2 & Pu2: & & & 5. & & $E-02$ \\
\hline B & Puz & & & & & \\
\hline & $\mathbf{P u}$ & & & & & \\
\hline & $\mathbf{P u} 2$ & & & & & -08 \\
\hline & & & 08 & E-08 & 101 & $E+01$ \\
\hline 4 & Ra22 & & & 9.2 & -01 & $E+01$ \\
\hline 4 & Ru1 & & 7.3 & $7.35 \mathrm{E}-11$ & & E-11 \\
\hline B & Sb1 & & & 11 & & $E+01$ \\
\hline B & $\mathrm{Se}$ & & & & & \\
\hline & & & & & & \\
\hline & Sr & & & & & -0 \\
\hline & & $c$ & & & & $\mathrm{E}-0$ \\
\hline B2 & Th22 & c & 10 & $1.79 \mathrm{E}-10$ & $E+01$ & $9 E-11$ \\
\hline B20 & Th2 & $c$ & 8 & 8.0 & & $E-1$ \\
\hline B204 & Tin1 & C & 9. & 9.7 & 01 & $E-05$ \\
\hline 204 & Trit & $\mathrm{c}$ & & $9.8>>$ & & $F=0$ \\
\hline 204 & U232 & & & & & 08 \\
\hline & $\mathbf{U}$ & & & & & \\
\hline & & & 4.68 & & & $1 \mathrm{E}-0$ \\
\hline & $\mathbf{U} 2$ & C & 2.08E-04 & $2.08 \mathrm{~F}$ & & $7 \mathrm{E}-0$ \\
\hline & & & 4.08 & 4.081 & & $6.81 \mathrm{E}-06$ \\
\hline & & & & & $0.00 \mathrm{E}+01$ & 703 \\
\hline
\end{tabular}

50\%-ile

\section{$1.46 \mathrm{E}+02 \quad 1.15 \mathrm{E}+03$}

$1.50 \mathrm{E}+02 \quad 2.30 \mathrm{E}+02$

$2.70 \mathrm{E}+03 \quad 7.35 \mathrm{E}+03$

$7.58 \mathrm{E}+02 \quad 1.11 \mathrm{E}+03$

$1.46 \mathrm{E}+03 \quad 2.10 \mathrm{E}+03$

$9.88 \mathrm{E}+02 \quad 3.48 \mathrm{E}+03$

$0.00 \mathrm{E}+01 \quad 0.00 \mathrm{E}+01$

$1.19 \mathrm{E}+03 \quad 1.56 \mathrm{E}+03$

$2.25 \mathrm{E}+03 \quad 3.19 \mathrm{E}+03$

$2.82 \mathrm{E}+03 \quad 4.44 \mathrm{E}+03$

$7.60 \mathrm{E}+03$

$7.68 \mathrm{E}+01$

$2.18 \mathrm{E}+02$

$1.18 \mathrm{E}+04 \quad 1.51 \mathrm{E}+04$

$5.09 \mathrm{E}+02$

$1.66 \mathrm{E}+01$

$6.31 \mathrm{E}+02$

$1.16 \mathrm{E}+02$

$2.38 \mathrm{E}+02$

$3.41 \mathrm{E}+02$

$1.07 \mathrm{E}+02$

$6.04 \mathrm{E}+02$

$7.91 \mathrm{E}-00$

2.37E-01

7.59E-08

$7.38 \mathrm{E}-00$

5.40E-09

$3.42 \mathrm{E}+01$

3.05E-04

8.56E-04

6.48E-06

1.40E-07

1.37E-07

2.68E-01

4.87E-06

$1.70 \mathrm{E}+02$

3.19E-04

1.57E-03

2.88E-02

4.00E-06

2.53E-04

8.68E-05

8.01E-03

1.31 E-65

1.75E-07

5.61E-04

8.10E-02

7.10E-03

2.36E-02

1.09E-07

1.44E-08

9.25E-13

$7.35 \mathrm{E}-11$

1.13E-04

6.44E-05

2.44E-01

$7.45 \mathrm{E}+02$

$2.12 \mathrm{E}-03$

1.79E-10

8.08E-14

9.72E-05

9.86E-04

9.38E-08

4.28E-09

4.68E-03

2.08E-04

4.08E-05

$4.75 E-03$
$1.11 \mathrm{E}+04$

$5.14 \mathrm{E}+02$

$99 \%$-ile

$3.82 \mathrm{E}+03$

$4.39 \mathrm{E}+02$

$1.05 \mathrm{E}+04$

$1.81 \mathrm{E}+03$

$4.82 \mathrm{E}+03$

$6.70 \mathrm{E}+03$

$0.00 \mathrm{E}+01$

$1.98 \mathrm{E}+03$

$4.59 \mathrm{E}+03$

$6.93 \mathrm{E}+03$

$1.72 \mathrm{E}+04$

$2.48 \mathrm{E}+03$

$7.71 \mathrm{E}+03$

$3.44 \mathrm{E}+04$

$3.40 \mathrm{E}+03$

$1.84 \mathrm{E}+02 \quad 1.42 \mathrm{E}+03$

$9.68 \mathrm{E}+02$

$8.98 \mathrm{E}+02$

$5.65 \mathrm{E}+02$

$2.53 \mathrm{E}+03$

$1.66 \mathrm{E}+02$

$1.53 \mathrm{E}+03$

$7.78 \mathrm{E}+01$

$1.17 \mathrm{E}+01$

2.41E-07

$2.21 \mathrm{E}+02$

$1.06 \mathrm{E}-08$

$9.44 \mathrm{E}+02$

9.52E-04

9.46E-03

$1.27 \mathrm{E}-05$

2.74E-07

$2.68 \mathrm{E}-07$

3.21E-00

$1.34 \mathrm{E}-04$

4.68E +03

$1.01 \mathrm{E}-03$

4.99E-03

9.15E-02

6.42E-06

$1.25 \mathrm{E}-02$

$5.85 \mathrm{E}-04$

$5.40 \mathrm{E}-02$

2.56E-05

1.74E-06

$1.10 \mathrm{E}-03$

1. $58 \mathrm{E}-01$

1.39E-02

4.62E-02

2.13E-07

3.27E-08

$2.10 \mathrm{E}-12$

$2.76 \mathrm{E}-10$

2.59E-04

4.80E-04

$7.75 \mathrm{E}-01$

$8.51 \mathrm{E}+03$

$4.52 \mathrm{E}-03$

$1.78 \mathrm{E}-09$

8.01E-13

1.07E-03

1.13E-03

9.30E-07

$4.25 \mathrm{E}-08$

4.64E-02

$2.06 \mathrm{E}-03$

4.05E-04

$4.71 \mathrm{E}-02$
$6.27 \mathrm{E}+03$

$2.35 \mathrm{E}+03$

$3.24 E+03$

$6.74 \mathrm{E}+03$

$2.25 \mathrm{E}+02$

$3.59 \mathrm{E}+03$

$3.18 \mathrm{E}+02$

$3.78 \mathrm{E}+01$

$4.86 \mathrm{E}-07$

$1.07 \mathrm{E}+03$

2.98E-08

$1.73 \mathrm{E}+03$

$1.40 \mathrm{E}-03$

7.31E-02

3.58E-05

$7.74 \mathrm{E}-07$

7.57E-07

9.73E-00

$2.46 \mathrm{E}-04$

$8.57 \mathrm{E}+03$

2.04E-03

$1.01 \mathrm{E}-02$

$1.84 \mathrm{E}-01$

$1.27 \mathrm{E}-05$

4.03E-02

$2.83 \mathrm{E}-03$

2.61E-01

7.24E-05

7.11E-06

3.10E-03

4.48E-01

3.92E-02

$1.30 \mathrm{E}-01$

6.02E-07

5.12E-08

3.29E-12

5.37E-10

5.29E-04

$1.28 \mathrm{E}-03$

$1.56 \mathrm{E}-00$

$3.35 \mathrm{E}+04$

8.23E-03

$7.27 \mathrm{E}-09$

$3.28 \mathrm{E}-12$

8.30E-03

$1.23 \mathrm{E}-03$

3.81E-06

$1.74 \mathrm{E}-07$

$1.90 \mathrm{E}-01$

8.45E-03

$1.66 \mathrm{E}-03$

$1.93 \mathrm{E}-01$
Mean StdDev

4.35E+02 7.95E+02

$1.73 \mathrm{E}+02 \quad 1.23 \mathrm{E}+02$

$3.36 \mathrm{E}+03 \quad 2.40 \mathrm{E}+03$

$7.94 \mathrm{E}+02 \quad 3.41 \mathrm{E}+02$

$1.60 \mathrm{E}+03 \quad 1.21 \mathrm{E}+03$

$1.51 \mathrm{E}+03 \quad 1.36 \mathrm{E}+03$

$0.00 \mathrm{E}+01 \quad 0.00 \mathrm{E}+01$

$1.16 \mathrm{E}+03 \quad 3.71 \mathrm{E}+02$

$2.27 \mathrm{E}+03 \quad 7.89 \mathrm{E}+02$

$2.95 \mathrm{E}+03 \quad 1.31 \mathrm{E}+03$

$8.04 \mathrm{E}+03 \quad 2.45 \mathrm{E}+03$

$2.30 \mathrm{E}+02 \quad 5.04 \mathrm{E}+02$

$6.99 \mathrm{E}+02 \quad 1.54 \mathrm{E}+03$

$1.26 \mathrm{E}+04 \quad 9.63 \mathrm{E}+03$

$6.42 \mathrm{E}+02 \quad 4.98 \mathrm{E}+02$

$8.87 \mathrm{E}+01 \quad 2.34 \mathrm{E}+02$

$7.71 \mathrm{E}+02 \quad 8.60 \mathrm{E}+02$

$3.21 \mathrm{E}+02 \quad 4.86 \mathrm{E}+02$

$4.17 \mathrm{E}+02 \quad 8.64 \mathrm{E}+02$

$9.11 \mathrm{E}+02 \quad 1.33 \mathrm{E}+03$

$1.08 \mathrm{E}+02 \quad 4.51 \mathrm{E}+01$

$8.19 \mathrm{E}+02 \quad 8.25 \mathrm{E}+02$

$3.01 \mathrm{E} \div 01 \quad 5.81 \mathrm{E}+01$

3.40E-00 7.71E-00

$1.01 \mathrm{E}-07 \quad 1.09 \mathrm{E}-07$

$7.54 \mathrm{E}+01 \quad 2.11 \mathrm{E}+02$

$6.56 \mathrm{E}-09 \quad 5.08 \mathrm{E}-09$

$2.40 \mathrm{E}+02 \quad 4.19 \mathrm{E}+02$

3.97E-04 3.33E-04

4.57E-03 1.21E-02

$7.88 \mathrm{E}-06 \quad 6.10 \mathrm{E}-06$

$1.70 \mathrm{E}-07 \quad 1.32 \mathrm{E}-07$

$1.67 \mathrm{E}-07 \quad 1.29 \mathrm{E}-07$

1.18E-00 2.20E-00

3.41E-05 5.97E-05

$1.19 E+03 \quad 2.08 E+03$

4.24E-04 4.57E-04

$2.09 \mathrm{E}-03 \quad 2.25 \mathrm{E}-03$

3.83E-02 4.12E-02

4.70E-06 3.68E-06

3.63E-03 8.23E-03

2.61E-04 5.74E-04

2.41E-02 5.30E-02

1.59E-05 1.23E-05

6.72E-07 1.30E-06

6.82E-04 5.28E-04

$9.84 \mathrm{E}-02 \quad 7.62 \mathrm{E}-02$

8.63E-03 6.68E-03

$2.87 \mathrm{E}-02 \quad 2.22 \mathrm{E}-02$

$1.32 \mathrm{E}-07 \quad 1.03 \mathrm{E}-07$

$1.57 \mathrm{E}-08 \quad 1.25 \mathrm{E}-08$

$1.01 \mathrm{E}-12 \quad 8.03 \mathrm{E}-13$

$1.16 \mathrm{E}-10 \quad 1.10 \mathrm{E}-10$

1.32E-04 1.15E-04

1.73E-04 2.53E-04

$3.24 \mathrm{E}-01 \quad 3.49 \mathrm{E}-01$

$2.84 \mathrm{E}+03 \quad 6.42 \mathrm{E}+03$

2.37E-03 1.84E-03

6.87E-10 1.33E-09

3.10E-13 5.99E-13

5.19E-04 1.37E-03

9.77E-04 1.22E-04

3.60E-07 6.96E-07

$1.64 \mathrm{E}-08 \quad 3.18 \mathrm{E}-08$

$1.80 \mathrm{E}-02 \quad 3.47 \mathrm{E}-02$

$7.98 \mathrm{E}-04 \quad 1.54 \mathrm{E}-03$

1.57E-04 3.03E-04

$1.82 \mathrm{E}-02 \quad 3.52 \mathrm{E}-02$ 
Tank Anal. Un

$\begin{array}{ll}\text { B204 } & \text { Y90 } \\ \text { B204 } & \text { Zr93 } \\ \text { BX101 } & \text { Al } \\ \text { BX101 } & \text { Bi } \\ \text { BX101 } & \text { Ca } \\ \text { BX101 } & \text { Cl } \\ \text { BX101 } & \text { CO3 } \\ \text { BX101 } & \text { Cr } \\ \text { BX101 } & \text { F } \\ \text { BX101 } & \text { Fe } \\ \text { BX101 } & \text { Hg } \\ \text { BX101 } & \text { K } \\ \text { BX101 } & \text { La } \\ \text { BX101 } & \text { Mn } \\ \text { BX101 } & \text { Na } \\ \text { BX101 } & \mathrm{Ni} \\ \text { BX101 } & \text { Na }\end{array}$

BX101 NO2

BX101 NO3

BX101 P

BX101 Pb

BX101 PO4

BX101 S

BX101 Si

BX101 SO4

BX101 Sr

BX101 TOC

BX101 U

BX101 Zr

BX101 Ac227

BX101 Am241

BX101 Am243

BX101 Ba137

BX101 C14

BX101 Cd113

BX101 Cm242

BX101 Cm243

BX101 $\mathrm{Cm} 244$

BX101 Co60

BX101 Cs134

BX101 Cs137

BX101 Eu152

BX101 Eu154

BX101 Eu155

BX101 1129

BX101 Nb93

BX101 Ni59

BX101 Ni63

BX101 Np237

BX101 Pa231

BX101 Pu238

BX101 Pu239

BX101 Pu240

BX101 Pu241

BX101 Pu242

BX101 Ra226

BX101 Ra228

BX101 Ru106

BX101 Sb125

BX101 Se79

BX101 Sm151

BX101 Sr89/90

BX101 Te99

BX101 Th229

BX101 Th232

BX101 Tin126

BX101 Tritium

BX10I U232

BX101 U233

\section{May $\frac{B B}{98}$}

$3 . \overline{19 E+01}$

3.06E-04

$1.96 \mathrm{E}+04$

$1.75 \mathrm{E}+02$

$1.07 \mathrm{E}+03$

$2.86 \mathrm{E}+02$

$6.59 \mathrm{E}+03$

$1.98 \mathrm{E}+03$

$2.34 \mathrm{E}+02$

$1.63 \mathrm{E}+03$

$5.70 \mathrm{E}-03$

$3.94 \mathrm{E}+02$

1.04E-03

$4.00 \mathrm{E}+02$

$2.46 \mathrm{E}+04$

$3.90 \mathrm{E}+01$

$6.60 \mathrm{E}+03$

$1.12 \mathrm{E}+04$

$2.97 \mathrm{E}+03$

$1.99 \mathrm{E}+02$

$9.19 \mathrm{E}+03$

$1.59 \mathrm{E}+02$

$5.72 \mathrm{E}+03$

$4.76 \mathrm{E}+02$

$1.40 \mathrm{E}+01$

$1.07 E+03$

$5.65 \mathrm{E}+03$

$8.80 \mathrm{E}+01$

$1.39 \mathrm{E}-03$

$2.01 \mathrm{E}+02$

$1.52 \mathrm{E}-02$

$1.58 \mathrm{E}+04$

4.33E-01

8.16E-01

3.94E-01

4.19E-02

1.12E-00

2.45E-01

3.59E-03

$1.67 \mathrm{E}+04$

7.42E-00

5.22E-00

$4.26 \mathrm{E}+02$

2.54E-03

$1.86 \mathrm{E}-01$

9.31E-00

Ci $9.22 \mathrm{E}+02$

Ci 5.04E-03

7.36E-05

$1.24 \mathrm{E}+01$

$7.40 \mathrm{E}+01$

Ci Incl. in 239

$8.70 \mathrm{E}+02$

$5.66 \mathrm{E}-03$

3.25E-04

$1.87 \mathrm{E}-03$

$7.85 \mathrm{E}-02$

$1.28 \mathrm{E}-00$

$5.06 \mathrm{E}-02$

$1.84 \mathrm{E}+02$

$2.97 \mathrm{E}+04$

1.32E-00

4.37E-05

2.31E-04

$7.87 \mathrm{E}-02$

$1.40 \mathrm{E}-00$

4.18E-03

$1.54 \mathrm{E}-02$

\section{Sept98}

$3.19 \mathrm{E}+01$

3.06E-04

$3.16 \mathrm{E}+04$

$1.50 \mathrm{E}+02$

$5.20 \mathrm{E}+02$

$2.97 \mathrm{E}+02$

$6.86 \mathrm{E}+03$

$1.74 \mathrm{E}+03$

$2.40 \mathrm{E}+02$

$1.76 \mathrm{E}+03$

$0.00 \mathrm{E}+01$

3.00E+02

$0.00 \mathrm{E}+01$

$4.23 \mathrm{E}+02$

2.05E+04

$4.30 \mathrm{E}+01$

$6.90 \mathrm{E}+03$

$1.36 \mathrm{E}+04$

NA

$2.60 \mathrm{E}+02$

2.35E+03

NA

$5.20 \mathrm{E}+02$

$4.95 E+02$

$1.40 \mathrm{E}+01$

$1.45 \mathrm{E}+03$

$1.06 \mathrm{E}+04$

$3.46 \mathrm{E}+02$

1.39E-03

$1.73 \mathrm{E}+02$

1.31E-02

$1.66 \mathrm{E}+04$

1.35E-01

8.16E-01

$3.39 \mathrm{E}-01$

3.61E-02

9.68E-01

7.18E-00

3.59E-03

$1.75 \mathrm{E}+04$

$7.42 \mathrm{E}-00$

5.22E-00

$4.26 \mathrm{E}+02$

1.20E-02

$1.86 \mathrm{E}-01$

9.31E-00

$9.22 \mathrm{E}+02$

5.04E-03

$736 \mathrm{E}-05$

$4.68 \mathrm{E}-00$

$8.22 \mathrm{E}+01$

$1.78 \mathrm{E}+01$

$3.28 \mathrm{E}+02$

2.14E-03

3.25E-04

$1.87 \mathrm{E}-03$

$7.85 \mathrm{E}-02$

$1.28 \mathrm{E}-00$

5.06E-02

$1.84 \mathrm{E}+02$

$6.12 \mathrm{E}+04$

9.05E-00

4.37E-05

2.31E-04

7.87E-02

$1.40 \mathrm{E}-00$

$9.65 \mathrm{E}-04$

3.54E-03
$1 \%$-ile

$0.00 \mathrm{E}+01 \quad 2.73 \mathrm{E}-02$

5.05E-05 $\quad 1.49 \mathrm{E}-04$

$1.30 \mathrm{E}+01 \quad 4.00 \mathrm{E}+02$

2.05E-02 4.73E-01

$1.28 \mathrm{E}+01 \quad 3.58 \mathrm{E}+02$

5.71E-01 3.14E+01

$6.27 \mathrm{E}+01 \quad 2.96 \mathrm{E}+03$

$8.95 \mathrm{E}-02 \quad 7.98 \mathrm{E}-00$

3.46E-02 1.07E-00

$1.35 \mathrm{E}-00 \quad 3.03 \mathrm{E}+02$

$0.00 \mathrm{E}+01 \quad 1.05 \mathrm{E}-03$

$5.01 \mathrm{E}-01 \quad 1.09 \mathrm{E}+01$

$1.11 \mathrm{E}-03 \quad 5.31 \mathrm{E}-02$

$1.25 \mathrm{E}-02 \quad 6.25 \mathrm{E}-01$

$1.90 \mathrm{E}+02 \quad 6.78 \mathrm{E}+03$

$3.93 \mathrm{E}-00 \quad 3.26 \mathrm{E}+02$

$2.16 \mathrm{E}+01$

$1.66 \mathrm{E}+01$

$3.83 \mathrm{E}+02$

$2.98 \mathrm{E}-00$

$1.61 \mathrm{E}+02$

9.19E-01

$6.86 \mathrm{E}-00 \quad 3.32 \mathrm{E}+02$

$2.96 \mathrm{E}-00 \quad 7.20 \mathrm{E}+01$

$2.27 \mathrm{E}-00 \quad 1.02 \mathrm{E}+02$

$4.59 \mathrm{E}-00 \quad 2.14 \mathrm{E}+02$

$6.69 \mathrm{E}-04 \quad 6.51 \mathrm{E}-02$

$1.66 \mathrm{E}-00$

$1.10 \mathrm{E}-00$

$6.51 \mathrm{E}-02$
$5.02 \mathrm{E}+01$

$8.70 \mathrm{E}+02$

2.77E-03

4.34E-02

$0.00 \mathrm{E}+01$

$0.00 \mathrm{E}+01$

$1.67 \mathrm{E}-03$

0.00E+01 1.74E-04

$0.00 \mathrm{E}+01 \quad 2.00 \mathrm{E}+03$

$0.00 \mathrm{E}+01 \quad 8.02 \mathrm{E}-02$

$0.00 \mathrm{E}+01 \quad 1.51 \mathrm{E}-01$

$0.00 \mathrm{E}+01 \quad 4.51 \mathrm{E}-03$

$0.00 \mathrm{E}+01 \quad 4.79 \mathrm{E}-04$

$0.00 \mathrm{E}+01 \quad 1.28 \mathrm{E}-02$

$2.32 \mathrm{E}-04 \quad 7.79 \mathrm{E}-02$

$0.00 \mathrm{E}+01 \quad 4.54 \mathrm{E}-04$

5.53E-00 2.58E+02

$0.00 \mathrm{E}+01$

$0.00 \mathrm{E}+01$

$0.00 \mathrm{E}+01$

$0.00 \mathrm{E}+01 \quad 0.00 \mathrm{E}+01$

$0.00 \mathrm{E}+01 \quad 4.85 \mathrm{E}-04$

7.07E-04 1.22E-02

$0.00 \mathrm{E}+01 \quad 1.32 \mathrm{E}-00$

$0.00 \mathrm{E}+01 \quad 1.30 \mathrm{E}+02$

$0.00 \mathrm{E}+01 \quad 5.77 \mathrm{E}-05$

$0.00 \mathrm{E}+01 \quad 0.00 \mathrm{E}+01$

$0.00 \mathrm{E}+01 \quad 1.42 \mathrm{E}-01$

$0.00 \mathrm{E}+01 \quad 8.47 \mathrm{E}-01$

$0.00 \mathrm{E}+01$

8.47E-01

$0.00 \mathrm{E}+01 \quad 9.95 \mathrm{E}-00$

$0.00 \mathrm{E}+01 \quad 6.48 \mathrm{E}-05$

$0.00 \mathrm{E}+01 \quad 5.13 \mathrm{E}-05$

$0.00 \mathrm{E}+01 \quad 2.95 \mathrm{E}-04$

$0.00 \mathrm{E}+01 \quad 8.09 \mathrm{E}-03$

$0.00 \mathrm{E}+01 \quad 0.00 \mathrm{E}+01$

$0.00 \mathrm{E}+01 \quad 8.95 \mathrm{E}-03$

$0.00 \mathrm{E}+01 \quad 0.00 \mathrm{E}+01$

$6.30 \mathrm{E}-03 \quad 4.70 \mathrm{E}+02$

$0.00 \mathrm{E}+01 \quad 9.42 \mathrm{E}-02$

$0.00 \mathrm{E}+01 \quad 0.00 \mathrm{E}+01$

$0.00 \mathrm{E}+01 \quad 0.00 \mathrm{E}+01$

$0.00 \mathrm{E}+01 \quad 1.45 \mathrm{E}-02$

$5.47 \mathrm{E}-03 \quad 4.24 \mathrm{E}-01$

$0.00 \mathrm{E}+01 \quad 0.00 \mathrm{E}+01$

$0.00 \mathrm{E}+01 \quad 0.00 \mathrm{E}+01$

50\%-ile

90\%-ile

99\%-ile

$3.19 E+01$

$3.06 \mathrm{E}-04$

$2.94 \mathrm{E}+03$

5.11E-00

$1.87 \mathrm{E}+03$

$1.32 \mathrm{E}+02$

$1.44 E+04$

$4.30 \mathrm{E}+01$

9.56E-00

$2.56 \mathrm{E}+03$

$5.70 \mathrm{E}-03$

$5.98 \mathrm{E}+01$

7.11E-01

$7.33 \mathrm{E}-00$ 
Tank Anal. Un

BX101 U234

BX101 U235

BX101 U236

BX101 U238

BX101 Y90

BX101 Zr93

BX102 Al

BX102 Bi

BX102 Ca

BX102 Cl

BX102 $\mathrm{CO} 3$

BX102 Cr

BX102 F

BX102 Fe

BX102 Hg

BX102 K

BX102 La

BX102 Mn

BX102 Na

BX102 Ni

BX102 NO2

BX102 NO3

BX102 P

BX102 Pb

BX102 PO4

BX102 S

BX102 Si

BX102 SO4

BX102 Sr

BX102 TOC

BX102 U

BX102 Zr

BX102 Ac227

BX102 Am241

BX102 Am243

BX102 Ba137

BX102 $\mathrm{Cl} 14$

BX102 Cd1 13

BX102 Cm242

BX102 Cm243

BX102 Cm244

BX102 C060

BX102 Cs134

BX102 Cs137

BX102 Eu152

BX102 Eu154

BX102 Eul55

BX102 1129

BX102 Nb93

BX102 Ni59

BX102 Ni63

BX102 Np237

BX102 Pa231

BX102 Pu238

BX102 Pu239

BX102 Pu240

BX102 Pu241

BX102 Pu242

BX102 Ra226

BX102 Ra228

BX102 Ru106

BX102 Sbl25

BX102 Se79

BX102 Sm151

BX102 Sr89/90 BX102 Tc99

BX102 Th229

BX102 Tb232
May $\frac{B B}{98}$

Ci $\overline{151 \mathrm{E}+01}$

Ci 6.79E-01

Ci $\quad 9.64 \mathrm{E}-02$

Ci $1.53 \mathrm{E}+01$

Ci $2.97 \mathrm{E}+04$

Ci 2.33E-01

kg 1.37E+04

kg $1.16 \mathrm{E}+02$

kg $1.32 \mathrm{E}+03$

kg $\quad 1.83 \mathrm{E}+02$

kg $4.26 \mathrm{E}+03$

kg 1.32E+03

kg 1.55E+02

kg 2.41E+03

$\mathrm{kg} 2.24 \mathrm{E}+01$

kg 2.57E+02

kg 2.50E+01

kg 2.67E+02

kg 1.61E+04

kg 2.60E+01

kg $4.26 \mathrm{E}+03$

kg 7.13E+03

kg $\quad 1.97 \mathrm{E}+03$

kg 1.33E+02

kg $\quad 6.11 \mathrm{E}+03$

kg 1.03E+02

$\mathrm{kg} \quad 4.48 \mathrm{E}+04$

$\mathrm{kg} \quad 3.08 \mathrm{E}+02$

kg $1.00 \mathrm{E}+01$

kg $7.03 E+02$

$\mathrm{kg} \quad 3.76 \mathrm{E}+03$

kg $5.90 \mathrm{E}+01$

Ci 3.15E-03

Ci 1.94E-02

Ci 1.25E-07

Ci 1.02E+04

Ci 2.39E-01

$\mathrm{Ci} \quad 6.74 \mathrm{E}-02$

$2.01 E-04$

$5.26 \mathrm{E}-06$

4.12E-06

6.24E-03

7.75E-04

$1.08 \mathrm{E}+04$

$1.79 \mathrm{E}-02$

$9.74 \mathrm{E}-02$

$1.71 \mathrm{E}-00$

$3.99 \mathrm{E}-04$

2.65E-02

8.79E-03

$7.77 \mathrm{E}-01$

1.30E-03

4.65E-03

1.18E-00

Ci $4.90 \mathrm{E}+01$

Ci Inel. in 239

$9.41 \mathrm{E}+01$

2.65E-04

1.20E-05

$1.02 \mathrm{E}-03$

3.54E-06

1.39E-02

$6.51 \mathrm{E}-03$

$2.46 \mathrm{E}+01$

$2.02 E+04$

$2.14 \mathrm{E}-01$

4.61E-04

4.75E-05
Sept98

3.48E-00

$1.57 \mathrm{E}-01$

2.22E-02

$3.53 \mathrm{E}-00$

$6.12 \mathrm{E}+04$

2.33E-01

$6.57 \mathrm{E}+03$

$1.38 \mathrm{E}+02$

$1.72 \mathrm{E}+03$

$1.02 \mathrm{E}+01$

$1.02 \mathrm{E}+04$

$1.54 \mathrm{E}+03$

$0.00 \mathrm{E}+01$

$2.39 \mathrm{E}+03$

3.63E-00

$2.62 \mathrm{E}+02$

$0.00 \mathrm{E}+01$

$2.67 \mathrm{E}+02$

$1.97 \mathrm{E}+04$

$2.64 \mathrm{E}+01$

2.15E +02

$5.96 \mathrm{E}+03$

NA

$1.01 E+02$

1.10E+04

NA

4.84E+04

$7.72 \mathrm{E}+02$

$1.01 \mathrm{E}+01$

$4.98 \mathrm{E}+02$

$7.89 \mathrm{E}+02$

6.00E+01

3.15E-03

$1.76 \mathrm{E}+02$

1.14E-03

$7.66 \mathrm{E}+03$

2.30E-01

6.74E-02

1.83E-00

4.78E-02

3.74E-02

$1.02 \mathrm{E}+01$

$7.75 \mathrm{E}-04$

$8.10 \mathrm{E}+03$

1.79E-02

9.74E-02

1.71E-00

5.37E-03

2.65E-02

8.79E-03

7.77E-01

1.30E-03

4.65E-03

1.01E-00

$4.25 \mathrm{E}+01$

7.52E-00

8.12E+01

2.28E-04

$1.20 \mathrm{E}-05$

1.02E-03

3.54E-06

1.39E-02

6.51E-03

$2.46 \mathrm{E}+01$

2.04E+04

5.99E-00

4.61E-04

4.75E-05 1\%-ile

$0.00 \mathrm{E}+01$

$0.00 \mathrm{E}+01$

$0.00 \mathrm{E}+01$

$0.00 \mathrm{E}+01$

$0.00 \mathrm{E}+01$

8.86E-04

$1.82 \mathrm{E}+03$

4.41E-01

$1.42 \mathrm{E}+02$

$1.22 \mathrm{E}+01$

$9.28 \mathrm{E}+02$

7.73E- 00

$1.14 \mathrm{E}-00$

$1.90 \mathrm{E}+02$

8.06E-01

9.07E-00

$1.96 \mathrm{E}-01$

$2.16 \mathrm{E}-00$

$1.68 \mathrm{E}+04$

4.72E-00

$1.11 \mathrm{E}+02$

$2.38 \mathrm{E}+02$

8.11E+01

$1.32 \mathrm{E}+02$

$1.70 \mathrm{E}+01$

$5.21 \mathrm{E}+01$

$5.13 \mathrm{E}+01$

3.68E-01

$1.61 \mathrm{E}+01$

$9.66 \mathrm{E}+01$

$3.68 \mathrm{E}-02$

$0.00 \mathrm{E}+01$

9.60E- 01

1.92E-09

$3.27 \mathrm{E}+02$

1.92E-02

2.43E-03

$3.08 \mathrm{E}-06$

8.07E-08

6.32E-08

$1.47 \mathrm{E}-01$

$2.48 \mathrm{E}-05$

$5.61 \mathrm{E}+02$

$0.00 \mathrm{E}+01$

$0.00 \mathrm{E}+01$

$0.00 \mathrm{E}+01$

$0.00 \mathrm{E}+01$

$1.94 \mathrm{E}-04$

2.34E-04

2.06E-02

$1.99 \mathrm{E}-05$

$0.00 \mathrm{E}+01$

$1.81 \mathrm{E}-02$

$7.52 \mathrm{E}-01$

7.52E-01

1.44E-00

4.07E-06

1.10E-07

9.32E-06

1.63E-07

$5.97 \mathrm{E}-05$

3.36E-04

$0.00 \mathrm{E}+01$

$121 \mathrm{E}+01$

$0.00 \mathrm{E}+01$

$0.00 \mathrm{E}+01$

$0.00 \mathrm{E}+01$

10\%-ile

$0.00 \mathrm{E}+01$

$0.00 \mathrm{E}+01$

$0.00 \mathrm{E}+01$

$0.00 \mathrm{E}+01$

$1.10 \mathrm{E}+02$

$1.53 \mathrm{E}-02$

$2.88 \mathrm{E}+03$

$7.69 \mathrm{E}-00$

$4.46 \mathrm{E}+02$

$3.06 \mathrm{E}+01$

2.31E+03

$2.76 \mathrm{E}+01$

$7.00 \mathrm{E}-00$ 
Tank Anal. Un

BX102 Tin126 BX102 Tritium Ci BX102 U232 BX102 U233 BX102 U234 BX102 U235 BX102 U236 BX102 U238 BX102 Y90 BX102 Zr93 BX103 Al BX103 Bi BX103 Ca BX103 Cl BX103 $\mathrm{CO3}$ BX103 Cr BX103 F BX103 Fe BX103 $\mathrm{Hg}$ BX103 K BX103 La BX103 Mn BX103 Na BX103 Ni BX103 NO2 BX103 NO3 BX103 P BX103 Pb BX103 PO4 BX103 S BX103 Si BX103 SO4 BX103 $\mathrm{Sr}$ BX103 TOC BX103 U BX103 Zr BX103 Ac227 BX103 Am241 BX103 Am243 BX103 Ba137 BX103 $\mathrm{Cl} 4$ BX103 Cd113 BX103 Cm242 BX103 Cm243 BX103 Cm244 BX103 Co60 BX103 Cs134 BX103 Cs137 BX103 Eu152 BX103 Eul54 BX103 Eut55 BX103 1129 BX103 Nb93 BX103 Ni59 BX103 Ni63 BX103 Np237 BX103 Pa231 BX103 Pu238 BX103 Pu239 BX103 Pu240 BX103 Pu241 BX103 Pu242 BX103 Ra226 BX103 Ra228 BX103 Ru106 BX103 Sb125 BX103 Se79 BX103 Sm15!

\section{May $\frac{B B}{98}$ \\ 9.68E-03}

9.31E-02

5.26E-02

2.04E-01

Ci $1.22 \mathrm{E}+01$

Ci 5.49E-01

Ci $8.46 \mathrm{E}-02$

$\begin{array}{ll}\text { Ci } & 8.46 \mathrm{E}-02 \\ \mathrm{Ci}+01\end{array}$

Ci 2.02E+04

Ci 3.10E-02

kg 2.91E+04

$\begin{array}{ll}\mathrm{kg} & 2.91 \mathrm{E}+04 \\ \mathrm{~kg} & 2.58 \mathrm{E}+02\end{array}$

kg $1.58 \mathrm{E}+03$

$\mathrm{kg} \quad 4.76 \mathrm{E}+02$

$\mathrm{kg} \quad 1.06 \mathrm{E}+04$

kg $2.95 E+03$

$\mathrm{kg} \quad 3.50 \mathrm{E}+02$

kg $2.40 E+03$

kg $3.59 \mathrm{E}+01$

$\mathrm{kg} \quad 6.21 \mathrm{E}+02$

kg $\quad 5.50 \mathrm{E}+01$

kg $\quad 5.90 \mathrm{E}+02$

kg $3.88 \mathrm{E}+04$

kg $\quad 5.80 \mathrm{E}+01$

kg 1.09E+04

$\mathrm{kg} \quad 1.86 \mathrm{E}+04$

kg $4.39 \mathrm{E}+03$

kg

kg

$\mathrm{kg}$

$\mathrm{kg}$

$\mathrm{kg}$

$\mathrm{kg}$
$\mathrm{kg}$
$\mathrm{kg}$

8.34E+03

Ci 5.07E-03

Ci 4.15E-01

Ci 1.36E-05

Ci $2.59 \mathrm{E}+04$

$\mathrm{Ci} \quad 8.80 \mathrm{E}-01$

Ci 8.74E-01

Ci 1.03E-03

$\mathrm{Ci}$ 7.33E-05

Ci 6.31E-04

$\mathrm{Ci}$ 4.42E-01

Ci 1.83E-02

Ci 2.74E+04

Ci 6.07E-02

Ci 5.97E-00

Ci 5.05E-00

Ci $\quad$ 5.87E-03

$\mathrm{Ci} \quad 1.47 \mathrm{E}-01$

$\mathrm{Ci}$ 3.16E-02

$\mathrm{Ci}$ 2.91E-00

$\mathrm{Ci}$ 1.16E-02

Ci 7.48E-03

Ci 1.89E-00

Ci $1.09 \mathrm{E}+02$

Ci Incl. in 239

Ci $1.51 \mathrm{E}+02$

Ci 4.27E-04

Ci 2.89E-05

Ci 6.12E-03

Ci 6.63E-05

Ci 1.93E-00

Ci 3.95E-02

Ci $1.43 E+02$
$\frac{B B}{\operatorname{seg}}$ 9.68E-03 9.31E-02 1.12E-03 4.34E-03 2.61E-01

1.17E-02

$1.80 \mathrm{E}-03$

2.63E-01

$2.04 E+04$

$3.10 \mathrm{E}-02$

$1.36 \mathrm{E}+04$

$3.06 \mathrm{E}+02$

$2.47 \mathrm{E}+03$

$9.02 E+01$

$2.40 \mathrm{E}+04$

$3.46 E+03$

$9.24 \mathrm{E}-00$

$2.36 \mathrm{E}+03$

5.81E-00

$6.48 \mathrm{E}+02$

$0.00 \mathrm{E}+01$

$5.92 \mathrm{E}+02$

$4.77 \mathrm{E}+04$

$6.00 \mathrm{E}+01$

$1.46 \mathrm{E}+03$

$1.67 \mathrm{E}+04$

$$
\text { NA }
$$

$2.24 \mathrm{E}+02$

$2.44 E+04$

NA

$1.65 E+04$

$2.02 \mathrm{E}+03$

$2.23 \mathrm{E}+01$

$1.25 \mathrm{E}+03$

$1.75 \mathrm{E}+03$

$1.33 \mathrm{E}+02$

5.07E-03

$3.89 \mathrm{E}+02$

1.28E-02

$1.92 \mathrm{E}+04$

$5.26 \mathrm{E}-01$

8.74E-01

$9.66 \mathrm{E}-01$

6.87E-02

5.92E-01

$2.36 \mathrm{E}+01$

$1.83 \mathrm{E}-02$

$2.03 \mathrm{E}+04$

6.07E-02

5.97E-00

5.05E-00

1.37E-02

$1.47 \mathrm{E}-01$

$3.16 \mathrm{E}-02$

2.91E-00

$1.16 \mathrm{E}-02$

7.48E-03

2.25E-00

$9.43 \mathrm{E}+01$

$1.67 \mathrm{E}+01$

$1.80 \mathrm{E}+02$

5.08E-04

2.89E-05

6.12E-03

$6.63 \mathrm{E}-05$

$1.93 \mathrm{E}-00$

3.95E-02

$1.43 \mathrm{E}+02$
$1 \%$-ile

$10 \%$-ile

50\%-ile

3.48E-04 1.98E-03

$3.01 \mathrm{E}-02 \quad 4.80 \mathrm{E}-02$

$0.00 \mathrm{E}+01$

$0.00 \mathrm{E}+01$

$0.00 \mathrm{E}+01$

$0.00 \mathrm{E}+01$

$0.00 \mathrm{E}+01$

$0.00 \mathrm{E}+01$

$0.00 \mathrm{E}+01$

$2.26 \mathrm{E}-04$

$9.50 \mathrm{E}+02$

2.73E-01

4.63E+01

$1.16 \mathrm{E}+01$

$1.03 \mathrm{E}+03$

7.94E-00

$1.46 \mathrm{E}-00$

8.81E+01

1.23E-00

4.41E-00

1.37E-01

4.03E-00

4.13E+03

$3.86 \mathrm{E}-00$

$1.40 \mathrm{E}+02$

$3.31 \mathrm{E}+02$

1.32E+02

$6.48 \mathrm{E}-01$

$1.92 \mathrm{E}+02$

2.05E+01

$1.30 \mathrm{E}+01$

$6.75 \mathrm{E}+01$

4.95E-01

$1.17 \mathrm{E}+01$

$1.39 \mathrm{E}+02$

$5.88 \mathrm{E}-02$

$0.00 \mathrm{E}+01$

$1.07 \mathrm{E}-00$

$3.03 \mathrm{E}-07$

$2.58 \mathrm{E}+03$

2.91E-02

$3.00 \mathrm{E}-02$

2.29E-05

$1.63 \mathrm{E}-06$

$1.40 \mathrm{E}-05$

$1.87 \mathrm{E}-01$

$1.82 \mathrm{E}-03$

$1.19 E+03$

$0.00 \mathrm{E}+01$

$0.00 \mathrm{E}+01$

$0.00 \mathrm{E}+01$

$0.00 E+01$

$1.13 \mathrm{E}-03$

$2.29 \mathrm{E}-03$

2.11E-01

2.58E-04

$0.00 \mathrm{E} \div 01$

4.20E-02

2.42E-00

2.42E-00

3.36E-00

$9.50 \mathrm{E}-06$

6.76E-07

1.43E-04

3.08E-06

$5.06 \mathrm{E}-03$

9.97E-04

$0.00 \mathrm{E}+01$

$0.00 \mathrm{E}+01$

$0.00 \mathrm{E}+01$

$0.00 \mathrm{E}+01$

$0.00 \mathrm{E}+01$

$0.00 \mathrm{E}+01$

$0.00 \mathrm{E}+01$

$1.72 \mathrm{E}+03$

$5.68 \mathrm{E}-03$

$2.35 \mathrm{E}+03$ 
Tank Anal. Un BX103 Sr89/90 BX103 Te99 BX103 Th229 BX103 Th232 BX103 Tin126 BX103 Tritium BX103 U232 BX103 U233 BX103 U234 BX103 U235 BX103 U236 BX103 U238 BX103 Y90 BX103 Zr93 BX104 Al BX104 Bi BX104 Ca BX104 Cl BX104 $\mathrm{CO} 3$ BX104 Cr BX104 F BX104 Fe BX104 Hg BX104 K BX104 La BX104 Mn BX104 Na BX104 Ni BX104 NO2 BX104 NO3 BX104 P BX104 Pb BX104 PO4 BX104 S BX104 Si BX104 $\mathrm{SO4}$ BX104 Sr BX104 TOC BX104 U

BX104 Zr BX104 Ac227

BX104 Am241 BX104 Am243 BX104 Ba137 BX104 C14 BX104 Cd113 BX104 Cm242 BX104 Cm243 BX104 Cm244 BX104 Co60 BX104 Cs134 BX104 Cs137 BX104 Eu152 BX104 Eu154 BX104 Eu155 BX104 1129

BX104 Nb93 BX104 Ni59

BX104 Ni63 BX104 Np237 BX104 Pa231 BX104 Pu238 BX104 Pu239 BX104 Pu240 BX104 Pu241 BX104 Pu242 BX104 Ra226 BX104 Ra228

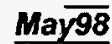
$4 \overline{39 \mathrm{E}+04}$ Ci 3.05E-00 6.06E-04 5.92E-02 1.95E-00 9.53E-02 3.69E-01 i $2.85 \mathrm{E}+01$ $1.28 \mathrm{E}-00$ $\mathrm{Ci} 1.93 \mathrm{E}-01$ Ci $2.89 \mathrm{E}+01$

\section{Ci $4.39 \mathrm{E}+04$}

Ci 1.94E-01

kg $6.78 \mathrm{E}+04$

kg $3.25 \mathrm{E}+02$

$\mathrm{kg} \quad 1.12 \mathrm{E}+03$

kg $7.10 \mathrm{E}+02$

kg $\quad 1.57 \mathrm{E}+04$

kg $3.83 \mathrm{E}+03$

kg 5.29E+02

kg 3.77E+03

kg 3.09E-01

kg $\quad 9.39 \mathrm{E}+02$

kg 8.13E+01

kg $\quad 9.07 E+02$

kg $4.76 \mathrm{E}+04$

kg $\quad 8.80 \mathrm{E}+01$

kg 1.65E +04

kg $\quad 3.29 \mathrm{E}+04$

kg $\quad 1.67 \mathrm{E}+03$

kg $5.57 \mathrm{E}+02$

$\mathrm{kg} \quad 5.16 \mathrm{E}+03$

kg 3.90E+02

kg $1.11 \mathrm{E}+03$

kg 1.17E+03

3.01E+01

g $3.18 \mathrm{E}+03$

g $2.26 \mathrm{E}+04$

g $1.97 \mathrm{E}+02$

Ci $233 \mathrm{E}-04$

Ci $1.17 \mathrm{E}+01$

Ci 4.25E-04

Ci $4.27 E+04$

Ci 7.05E-00

Ci $\quad 1.81 \mathrm{E}+01$

Ci 3.38E-02

i $3.11 \mathrm{E}-03$

Ci 2.89E-02

$9.90 \mathrm{E}+01$

Ci 6.77E-01

Ci $4.51 \mathrm{E}+04$

Ci 9.01E-01

Ci $1.26 \mathrm{E}+02$

Ci $5.46 \mathrm{E}+01$

Ci $9.16 \mathrm{E}-02$

Ci 2.50E-00

Ci 4.43E-01

$\mathrm{Ci} \quad 4.33 E+01$

Ci 1.73E-01

Ci 8.83E-04

Ci 3.06E-01

Ci $\quad 1.65 E+02$

Ci Incl. in 239

Ci 2.08E+01

Ci 1.14E-04

Ci 4.99E-05

Ci 2.55E-02
Sept98

$4.51 \mathrm{E}+04$

$1.59 \mathrm{E}+01$

8.42E-04

$6.06 \mathrm{E}-04$

5.92E-02

$1.95 \mathrm{E}-00$

$1.93 \mathrm{E}-03$

$7.46 \mathrm{E}-03$

$5.78 \mathrm{E}-01$

2.59E-02

$3.90 \mathrm{E}-03$

$5.84 \mathrm{E}-01$

4.51E+04

$1.94 \mathrm{E}-01$

$7.24 \mathrm{E}+04$

$3.47 \mathrm{E}+02$

$1.20 \mathrm{E}+03$

$7.49 \mathrm{E}+02$

$1.67 \mathrm{E}+04$

$4.08 \mathrm{E}+03$

$2.08 \mathrm{E}+02$

$4.02 \mathrm{E}+03$

$0.00 \mathrm{E}+01$

$7.35 \mathrm{E}+02$

$0.00 \mathrm{E}+01$

$9.68 \mathrm{E}+02$

$5.05 E+04$

$9.77 \mathrm{E}+01$

$1.74 \mathrm{E}+04$

$3.46 E+04$

NA

$5.95 E+02$

$5.50 \mathrm{E}+03$

NA

$1.18 \mathrm{E}+03$

$1.23 \mathrm{E}+03$

$3.21 \mathrm{E}+01$

$3.38 \mathrm{E}+03$

2.41E+04

$7.92 \mathrm{E}+02$

2.33E-04

$3.90 \mathrm{E}+02$

1.42E-02

$4.95 E+04$

3.37E-01

$1.81 \mathrm{E}+01$

1.13E-00

$1.04 \mathrm{E}-01$

9.65E-01

$1.64 \mathrm{E}+01$

$6.77 \mathrm{E}-01$

$5.23 \mathrm{E}+04$

9.01E-01

$1.26 \mathrm{E}+02$

$5.46 \mathrm{E}+01$

3.11E-02

2.50E-00

4.43E-01

$4.33 E+01$

$1.73 \mathrm{E}-01$

8.83E-04

$5.59 \mathrm{E}-00$

$1.98 \mathrm{E}+02$

$3.28 \mathrm{E}+01$

$3.81 \mathrm{E}+02$

2.09E-03

4.99 -05

2.55E-02
$1 \%$-ile

$2.46 \mathrm{E}+02$

$10 \%$-ile

$7.25 \mathrm{E}+03$

$1.47 \mathrm{E}-01$

$0.00 \mathrm{E}+01$

$00 \mathrm{E}+01$

$0.00 \mathrm{E}+01$

$1.20 \mathrm{E}-02$

$7.55 \mathrm{E}-01$

$0.00 \mathrm{E}+01$

$0.00 \mathrm{E}+01$

$0.00 \mathrm{E}+01$

$0.00 \mathrm{E}+01$

$0.00 \mathrm{E}+01$

$0.00 E+01$

$5.78 \mathrm{E}+03$

2.82E-02

$1.81 \mathrm{E}+02$

1.57E-00

$4.79 \mathrm{E}+02$

$2.92 \mathrm{E}+01$

$9.27 \mathrm{E}+03$

$2.49 \mathrm{E}+01$

3.32E-00

$1.71 \mathrm{E}+02$

$5.32 \mathrm{E}-02$

9.32E-00

$1.09 \mathrm{E}-01$

2.81E-00

$1.45 \mathrm{E}+04$

$2.80 \mathrm{E}+01$

$2.26 \mathrm{E}+02$

$1.58 \mathrm{E}+03$

$7.77 \mathrm{E}+02$

$1.91 \mathrm{E}-00$

$2.16 \mathrm{E}+03$

$1.67 \mathrm{E}+02$

$2.53 \mathrm{E}+01$

$5.61 \mathrm{E}+02$

$3.85 \mathrm{E}-01$

$2.58 \mathrm{E}+01$

$6.30 \mathrm{E}+03$

1.19E-01

1.14E-05

5.31E-02

1.39E-05

$5.97 \mathrm{E}+03$

1.71E-00

$3.11 \mathrm{E}-00$

1.10E-03

1.01E-04

9.42E-04

6.22E-02

9.47E-02

$8.42 E+02$

4.40E-02

$6.15 \mathrm{E}-00$

2.66E-00

$1.91 \mathrm{E}-02$

$3.19 \mathrm{E}-01$

5.29E-02

5.17E-00

5. $64 \mathrm{E}-03$

3.19E-06

9.97E-03

$5.38 \mathrm{E}-00$

$5.38 \mathrm{E}-00$

$6.78 \mathrm{E}-01$

3.72E-06

9.98E-06

$5.10 \mathrm{E}-03$

50\%-ile

$5.47 \mathrm{E}+04$

8.42E-04

6.06E-04

5.92E-02

1.95E-00

9.53E-02

3.69E-01

$2.85 \mathrm{E}+01$

$1.28 \mathrm{E}-00$ 


\begin{tabular}{|c|c|c|c|c|c|c|c|c|c|c|c|}
\hline ank & Anal. & In & $\frac{B B}{y 98}$ & Sept98 & $1 \%$-ile & $10 \%$-ile & $50 \%$-ile & $90 \%$-ile & 99\%-ile & Mean & Std Dev \\
\hline 2107 & 106 & $\mathrm{Ci}$ & $1.44 \mathrm{E}-03$ & $\overline{1.44 \mathrm{E}-03}$ & $0.00 \mathrm{E}+01$ & $1.02 \mathrm{E}-04$ & $1.44 \mathrm{E}-03$ & 1.13E-02 & $36 \mathrm{E}-02$ & $4.47 \mathrm{E}-03$ & $1.17 \mathrm{E}-02$ \\
\hline X104 & Sb125 & $\mathrm{Ci}$ & $3.27 \mathrm{E}+01$ & $3.27 \mathrm{E}+01$ & $0.00 \mathrm{E}+01$ & $0.00 \mathrm{E} \div 01$ & $3.27 E+01$ & $1.46 E+02$ & $53 \mathrm{E}+02$ & $5.70 E+01$ & $28 E+01$ \\
\hline 104 & Se79 & $\mathrm{Ci}$ & $7.01 E-01$ & $7.01 \mathrm{E}-01$ & $6.23 E-03$ & 1.18E-01 & $7.01 \mathrm{E}-01$ & $2.73 \mathrm{E}-00$ & $.23 E+01$ & $1.40 \mathrm{E}-00$ & $2.56 \mathrm{E}-00$ \\
\hline X104 & Sm151 & $\mathrm{Ci}$ & $2.47 E+03$ & $2.47 E+03$ & $0.00 \mathrm{E}+01$ & $1.21 \mathrm{E}+02$ & $2.47 E+03$ & $2.68 E+04$ & $5.79 \mathrm{E}+04$ & $1.01 E+04$ & $.35 E+04$ \\
\hline K104 & Sr89/90 & $\mathrm{Ci}$ & $6.64 E+04$ & $1.38 E+05$ & $2.15 \mathrm{E}-00$ & $6.83 E+02$ & $6.20 E+03$ & $3.99 \mathrm{E}+04$ & $1.39 \mathrm{E}+05$ & $1.79 E+04$ & $.73 E+04$ \\
\hline 104 & Tc99 & $\mathrm{Ci}$ & $4.75 E+01$ & $2.47 \mathrm{E}+01$ & $0.00 \mathrm{E}+01$ & $4.74 \mathrm{E}-01$ & $4.75 E+01$ & $.82 \mathrm{E}+02$ & $5.57 \mathrm{E}+02$ & $1.02 \mathrm{E}+02$ & $1.28 \mathrm{E}+02$ \\
\hline & Th229 & $\mathbf{C i}$ & & $5.98 \mathrm{E}-04$ & $0.00 \mathrm{E}+01$ & $E-06$ & & 20E-03 & $85 E-03$ & $9.47 \mathrm{E}-04$ & $1.10 \mathrm{E}-03$ \\
\hline & $\operatorname{Th} 232$ & $\mathrm{Ci}$ & $1.73 E-03$ & $1.73 E-03$ & $0.00 \mathrm{E}+01$ & $6.25 \mathrm{E}-06$ & $1.73 E-03$ & $6.37 \mathrm{E}-03$ & $1.40 \mathrm{E}-02$ & $2.74 \mathrm{E}-03$ & $.19 \mathrm{E}-03$ \\
\hline & n126 & $\mathbf{C i}$ & $1.06 \mathrm{E}-00$ & $1.06 \mathrm{E}-00$ & & & $1.06 \mathrm{E}-00$ & $5.58 \mathrm{E}-00$ & $2.26 E+01$ & E-00 & $4.67 \mathrm{E}-00$ \\
\hline & itium & $\mathrm{Ci}$ & $4.52 \mathrm{E}+01$ & $4.52 \mathrm{E}+01$ & $6.54 \mathrm{E}-00$ & $1.88 \mathrm{E}+01$ & $4.52 E+01$ & $8.76 E+01$ & $1.28 \mathrm{E}+02$ & $5.00 \mathrm{E}+01$ & $2.78 \mathrm{E}+01$ \\
\hline & U232 & $\mathrm{Ci}$ & $1.31 \mathrm{E}-01$ & $4.86 \mathrm{E}-02$ & $0.00 \mathrm{E}+01$ & $E-04$ & $1.31 \mathrm{E}-01$ & $.82 \mathrm{E}-01$ & $1.06 \mathrm{E}-00$ & $2.07 \mathrm{E}-01$ & $.41 \mathrm{E}-01$ \\
\hline 04 & U233 & $\mathrm{Ci}$ & 5.03E-01 & $1.86 \mathrm{E}-01$ & $0.00 \mathrm{E}+01$ & $E-03$ & & $.85 \mathrm{E}-00$ & $18 \mathrm{E}-00$ & E-01 & 27E-01 \\
\hline 104 & U234 & $\mathrm{Ci}$ & $2.15 E+01$ & $7.95 \mathrm{E}-00$ & $0.00 \mathrm{E}+01$ & $E-02$ & $2.15 \mathrm{E}+01$ & $7.92 \mathrm{E}+01$ & $1.74 \mathrm{E}+02$ & $3.40 \mathrm{E}+01$ & $3.96 \mathrm{E}+01$ \\
\hline $\mathbf{K 1 0 4}$ & U235 & $\mathrm{Ci}$ & 9.65E-01 & 3.57E-01 & $0.00 \mathrm{E}+01$ & $E-03$ & 9.65E-01 & $3.55 \mathrm{E}-00$ & 7.83E-00 & E-00 & $1.78 \mathrm{E}-00$ \\
\hline 104 & U236 & $\mathrm{Ci}$ & $1.41 \mathrm{E}-01$ & $5.22 \mathrm{E}-02$ & $0.00 \mathrm{E}+01$ & -04 & 1.41E-01 & $5.19 \mathrm{E}-01$ & $1.14 \mathrm{E}-00$ & $E-01$ & \\
\hline & U238 & $\mathrm{Ci}$ & $2.18 E+01$ & $8.05 \mathrm{E}-00$ & +01 & .02 & +01 & $8.03 E+01$ & $1.77 \mathrm{E}+02$ & $E+01$ & $\div 01$ \\
\hline 104 & Y90 & $\mathrm{Ci}$ & $6.64 \mathrm{E}+04$ & $1.38 E+05$ & $0.00 \mathrm{E}+01$ & -03 & +04 & $4.31 E+05$ & $1.50 \mathrm{E}+06$ & $E+05$ & $8+05$ \\
\hline & Zr93 & $\mathrm{Ci}$ & $3.44 \mathrm{E}-00$ & $3.44 \mathrm{E}-00$ & $1.22 \mathrm{E}-01$ & $E-01$ & $E-00$ & $93 E+02$ & $2.76 E+03$ & $E+02$ & +02 \\
\hline & Al & $\mathrm{kg}$ & $8.01 E+03$ & $1.01 E+04$ & $E-00$ & +01 & +02 & $93 E+02$ & $5 E+03$ & $E+02$ & $E+02$ \\
\hline & 1 & kg & $1.75 E+02$ & $2.27 \mathrm{E}+02$ & $E-02$ & & & $2.74 \mathrm{E}+01$ & $6.18 E+01$ & $E+01$ & $5 E+01$ \\
\hline 105 & $\mathrm{Ca}$ & $\mathrm{kg}$ & $1.41 \mathrm{E}+03$ & $1.83 E+03$ & $1.30 \mathrm{E}+01$ & +02 & $8.31 E+02$ & $8.21 E+03$ & $3.07 E+04$ & $E+03$ & $E+03$ \\
\hline & $\mathrm{Cl}$ & $\mathrm{kg}$ & $4.19 \mathrm{E}+02$ & $3.65 E+02$ & & & -01 & $E+02$ & $E+02$ & +01 & $E+01$ \\
\hline & 0 & $\mathrm{~kg}$ & +03 & +03 & 02 & .03 & $1 . ?$ & +05 & +05 & +04 & +04 \\
\hline 05 & $r$ & $\mathrm{~kg}$ & $1.96 \mathrm{E}+03$ & +03 & -01 & -00 & 3.2 & -02 & $E+02$ & +01 & $E+01$ \\
\hline 105 & $\mathbf{F}$ & $\mathrm{kg}$ & $2.19 E+02$ & $2.65 E+02$ & $1.67 \mathrm{E}-01$ & $E-00$ & $1.01 E+01$ & $6.54 \mathrm{E}+01$ & $1.58 \mathrm{E}+02$ & $E+01$ & $4 E+01$ \\
\hline & $\mathrm{Fe}$ & $\mathrm{kg}$ & $1.34 E+03$ & $1.75 E+03$ & $E-01$ & & 9.2 & $1.02 E+04$ & +04 & +03 & $E+03$ \\
\hline & $\mathbf{H g}$ & $\mathrm{kg}$ & 2.76E-02 & $0.00 \mathrm{E}+01$ & $E-05$ & -03 & $2.76 \mathrm{E}-02$ & $1.88 \mathrm{E}-01$ & $\mathrm{E}-00$ & $E-02$ & $1 \mathrm{E}-01$ \\
\hline 105 & $\mathbf{K}$ & $\mathrm{kg}$ & $4.84 E+02$ & $4.80 \mathrm{E}+02$ & 4.2 & -00 & 1.6 & $9.37 E+01$ & $E+02$ & -01 & $E+01$ \\
\hline & $\mathbf{L a}$ & kg & $6.65 \mathrm{E}-102$ & $0.00 E+01$ & 2. & & & $4 E-00$ & $E+01$ & -00 & -00 \\
\hline 05 & $\mathbf{M n}$ & $\mathrm{kg}$ & $3.38 \mathrm{E}+02$ & $4.39 E+02$ & $E-02$ & -01 & $\varepsilon-00$ & $2.78 \mathrm{E}+01$ & $E+01$ & +01 & +01 \\
\hline 105 & $\mathbf{N a}$ & $\mathrm{kg}$ & $3.22 E+04$ & $3.47 E+04$ & $5.93 \mathrm{E}+02$ & $4.81 E+03$ & $1.89 \mathrm{E}+04$ & $5.55 \mathrm{E}+04$ & $1.05 E+05$ & +04 & $E+04$ \\
\hline 105 & Ni & $\mathrm{kg}$ & $3.90 \mathrm{E}+01$ & $4.51 \mathrm{E}+01$ & & & +01 & $9.13 E+02$ & +03 & & $E+03$ \\
\hline 105 & $\mathrm{NO} 2$ & $\mathrm{~kg}$ & $9.26 \mathrm{E}+03$ & $8.33 E+03$ & -00 & +01 & $E \div 02$ & $2 E+03$ & $E+03$ & +02 & $E+03$ \\
\hline & NO3 & $\mathrm{kg}$ & +04 & $1.20 \mathrm{E}+04$ & & & & -03 & & & \\
\hline & 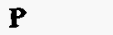 & kg & $4.55 E+03$ & NA & +01 & +02 & +03 & $1.76 \mathrm{E}+04$ & $E+04$ & -03 & +03 \\
\hline 105 & $\mathbf{P b}$ & $\mathrm{kg}$ & $1.30 \mathrm{E}+02$ & $1.67 \mathrm{E}+02$ & $2.71 \mathrm{E}-02$ & $E-01$ & $3.65 E-00$ & $2.47 E+01$ & $1.44 \mathrm{E}+02$ & $E+01$ & $E+01$ \\
\hline 105 & PO4 & $\mathrm{kg}$ & $1.41 E+04$ & $1.81 \mathrm{E}+04$ & +01 & +02 & +03 & $4.14 E+04$ & $E+05$ & +04 & $E+04$ \\
\hline & 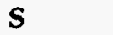 & $\mathrm{kg}$ & $2.09 E+02$ & NA & $E-00$ & 01 & & F03 & r04 & -03 & $E+03$ \\
\hline & $\mathrm{Si}$ & $\mathrm{kg}$ & $9.39 E+03$ & $1.22 E+04$ & & & & & $E+02$ & & +02 \\
\hline & SO4 & $\mathrm{kg}$ & 6.2 & 5.8 & & -02 & & -03 & & +03 & +03 \\
\hline & $\mathrm{Sr}$ & $\mathrm{kg}$ & $1.30 E+01$ & $1.66 \mathrm{E}+01$ & .02 & -01 & -01 & -00 & +01 & $E-00$ & $E-00$ \\
\hline 05 & TOC & $\mathrm{kg}$ & $1.18 E+03$ & $9.97 \mathrm{E}+02$ & $E-01$ & $E-00$ & $4.34 E+01$ & $4.46 E+02$ & $1.56 \mathrm{E}+03$ & $E+02$ & $E+02$ \\
\hline 15 & $U$ & $\mathrm{~kg}$ & $9.97 \mathrm{E}+02$ & $1.30 \mathrm{E}+03$ & $E-00$ & +03 & -04 & $6.85 E+04$ & $E+05$ & +04 & $E+04$ \\
\hline & $\mathbf{Z r}$ & $\mathrm{k}$ & $7.60 \mathrm{E}+01$ & $9.87 \mathrm{E}+01$ & -03 & & & $2.28 E+01$ & -02 & & +01 \\
\hline 05 & Ac227 & $\mathrm{Ci}$ & E-05 & & & & & & & & \\
\hline & Am & $\mathrm{Ci}$ & 2.2 & 2.8 & & & & -01 & -02 & 01 & +02 \\
\hline & Am243 & $\mathrm{Ci}$ & -05 & E-02 & $E-08$ & .06 & & -04 & -03 & -04 & $E-03$ \\
\hline 05 & Bal37 & $\mathrm{Ci}$ & $1.77 \mathrm{E}+04$ & $1.52 E+04$ & $\varepsilon+02$ & +03 & 104 & 8.8 & +05 & -04 & +04 \\
\hline 105 & C14 & $\mathrm{Ci}$ & $1.04 \mathrm{E}-00$ & $3.90 \mathrm{E}-01$ & +01 & -01 & 00 & & & & -00 \\
\hline & Cd113 & $\mathrm{Ci}$ & $\varepsilon-00$ & E-00 & 3. & & & 101 & -01 & & $E+01$ \\
\hline & $\mathrm{Cm} 242$ & $\mathrm{Ci}$ & & & & & & & & & -01 \\
\hline & Cm243 & $\mathrm{Ci}$ & & & & & & & & & -02 \\
\hline & $\mathrm{Cm} 244$ & $\mathrm{Ci}$ & & & & & & & & & -01 \\
\hline & $\mathrm{Co60}$ & $\mathrm{Ci}$ & 1.6 & 1.7 & 03 & & & -00 & $=01$ & -01 & $E-00$ \\
\hline & Cs134 & $\mathrm{Ci}$ & $5.76 \mathrm{E}-02$ & 5.76E-02 & $E-04$ & -03 & -02 & $2.87 \mathrm{E}-01$ & $7 \mathrm{E}-01$ & -01 & E-01 \\
\hline & Cs137 & $\mathrm{Ci}$ & $1.87 E+04$ & $1.61 \mathrm{E}+04$ & $E+01$ & +02 & +03 & $7.14 \mathrm{E}+03$ & $E+04$ & $\div 03$ & $E+03$ \\
\hline$=$ & Eu152 & $\mathrm{Ci}$ & $E_{-}-02$ & $8.48 E-02$ & & & & & & & -01 \\
\hline & Eu154 & $\mathrm{C} \mathbf{i}$ & +01 & $1.09 E+01$ & & & $i+01$ & 1.7 & +02 & +01 & +01 \\
\hline & Eu15 & $\mathrm{Ci}$ & & & & & & & & & $E+01$ \\
\hline 105 & 1129 & $\mathrm{Ci}$ & -03 & $9.79 \mathrm{E}-03$ & 01 & & & & & -02 & $1 \mathrm{E}-02$ \\
\hline & Nb93 & $\mathrm{Ci}$ & 2.53E-01 & $2.53 \mathrm{E}-01$ & & & -01 & $1.36 \mathrm{E}+01$ & $\div 01$ & $=00$ & $E+01$ \\
\hline & Ni59 & $\mathrm{Ci}$ & $5.00 \mathrm{E}-02$ & $5.00 \mathrm{E}-02$ & $\mathrm{E}-04$ & & E-02 & $5.56 \mathrm{E}-01$ & & $6 \mathrm{E}-01$ & $E-01$ \\
\hline & Ni63 & $\mathrm{Ci}$ & 4.73E-00 & 4.73E-00 & $3.83 \mathrm{E}-02$ & 3.7 & & $5.26 \mathrm{E}+01$ & $3.59 \mathrm{E}+02$ & $E+01$ & $E+01$ \\
\hline & Np237 & $\mathrm{Ci}$ & 1.71E-02 & $1.71 E-02$ & $4.61 E-05$ & -03 & $1.71 \mathrm{E}-0$ & 2.32E- & $1.44 \mathrm{E}-00$ & & $8 E-00$ \\
\hline & 31 & $\mathrm{Ci}$ & $1.23 E-04$ & $1.23 E-04$ & $0.00 \mathrm{E}+01$ & $0.00 \mathrm{E}+01$ & $1.23 \mathrm{E}-04$ & $5.18 \mathrm{E}-04$ & $1.37 \mathrm{E}-03$ & $2.11 \mathrm{E}-04$ & $2.70 \mathrm{E}-04$ \\
\hline BX105 & Pu238 & $\mathrm{Ci}$ & $2.60 \mathrm{E}-02$ & $1.37 \mathrm{E}-00$ & 7.01E-05 & -03 & $2.60 \mathrm{E}-02$ & $3.53 \mathrm{E}-0 \mathrm{l}$ & $2.19 \mathrm{E}-00$ & $2.44 \mathrm{E}-01$ & $1.80 \mathrm{E}-00$ \\
\hline & Pu239 & $\mathrm{Ci}$ & $6.40 \mathrm{E}+01$ & $7.34 E+01$ & $1.73 \mathrm{E}-01$ & $E-00$ & $6.40 \mathrm{E}+01$ & $8.68 \mathrm{E}+02$ & $5.38 E+03$ & $6.01 E+02$ & $4.43 E+03$ \\
\hline & Pu240 & $\mathrm{Ci}$ & el. in 239 & $9.15 E-00$ & $1.73 \mathrm{E}-01$ & $4.11 \mathrm{E}-00$ & $6.40 \mathrm{E}+01$ & $8.68 \mathrm{E}+02$ & $5.38 \mathrm{E}+03$ & $6.01 \mathrm{E}+02$ & $4.43 E+03$ \\
\hline
\end{tabular}




\begin{tabular}{|c|c|c|c|c|c|c|c|c|c|c|c|}
\hline$a k$ & al. & Un & $\frac{B B}{y 98}$ & $\frac{B B}{p t 98}$ & $1 \%$ ile & 0\%-ile & $50 \%$-ile & $0 \%$-ile & 9\%-ile & Yean & 200 \\
\hline & 241 & $\mathrm{Ci}$ & & $65+01$ & $E-03$ & $E-01$ & $5-00$ & $E+01$ & $E+02$ & $E+01$ & $1.16 E+$ \\
\hline 55 & 42 & $\mathrm{Ci}$ & $9.15 \mathrm{E}-06$ & $4.83 \mathrm{E}-04$ & 08 & & & & 4 & & \\
\hline & 226 & $\mathrm{Ci}$ & $\mathbf{L}=\mathbf{v s}$ & 2.62E-05 & & & & & & & \\
\hline & & $\mathrm{Ci}$ & $\mathrm{E}-03$ & $3.62 \mathrm{E}-03$ & & & & & & & \\
\hline & Ru106 & $\mathrm{Ci}$ & & $1.22 E-04$ & $00 \mathrm{E}+01$ & $E-07$ & & $1.35 \mathrm{E}-03$ & & & \\
\hline & & $\mathrm{Ci}$ & & 2.95E-00 & $00 \mathrm{E}+01$ & & & & $7 E+01$ & & \\
\hline & Se79 & $\mathrm{Ci}$ & & $6.93 \mathrm{E}-02$ & $.99 \mathrm{E}-04$ & & E- 02 & & -00 & & \\
\hline & & & rog & $47 \mathrm{E}+02$ & & & $47 E+02$ & & & & \\
\hline & 90 & & $F+0$ & $36 E+04$ & & +02 & $E+03$ & $8 F+04$ & $F+m$ & & . \\
\hline & c99 & $\mathrm{Ci}$ & $1+01$ & $1.20 \mathrm{E}+01$ & & & & & $E+02$ & $E+01$ & \\
\hline & h229 & $\mathrm{Ci}$ & 8. & 8.44E-05 & & & & & & & \\
\hline & & Ci & & & & & & & & & \\
\hline 5 & & $\mathrm{Ci}$ & 1.0 & - & $E-04$ & & & & & & \\
\hline & Tritiun & . & 3.9 & 3.91E-00 & $E-01$ & & & 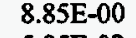 & & -00 & \\
\hline & U232 & $\mathrm{Ci}$ & 1.39E-02 & $2.48 E-04$ & +01 & & -02 & -02 & & 9 & \\
\hline & 233 & & -02 & & & & & & & & \\
\hline & 234 & & $39 \mathrm{E}+01$ & $4.29 \mathrm{E}-01$ & & & & & +02 & & \\
\hline & 3235 & & & $1.93 \mathrm{E}-02$ & 01 & & & & & & \\
\hline & U236 & $\mathrm{Ci}$ & & 2.7 & & & & & & & \\
\hline & U238 & $\mathrm{Ci}$ & 2.4 & & & & & & & & \\
\hline & Y90 & C & $2.60 \mathrm{E}+04$ & $3.36 E+04$ & 01 & & 04 & & 06 & 05 & +05 \\
\hline & Zr93 & C & {$[-01$} & -01 & 2. & 02 & & 01 & +01 & -00 & +01 \\
\hline & Al & & $1.75 E+04$ & $7.97 \mathrm{E}+03$ & & & & & +04 & & +03 \\
\hline & 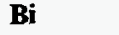 & & $1.56 \mathrm{E}+02$ & $1.82 E+02$ & & & & & & & \\
\hline & a & & +02 & $1.46 \mathrm{E}+03$ & & & & & & & \\
\hline & Cl & & & 2.4 & & & & & & & \\
\hline & 03 & k & +03 & +03 & & & & & -05 & +04 & \\
\hline & $-r$ & & $1.76 E+03$ & +03 & 1.4 & 01 & -02 & 02 & +03 & +02 & +02 \\
\hline & $\mathbf{F}$ & & $2.08 \mathrm{E}+02$ & 1.5 & & & & & U3 & 02 & +03 \\
\hline & Fe & & $1.46 E+03$ & +03 & & & & & & & \\
\hline & $\mathbf{H g}$ & k & -01 & 0.0 & & & & & & & \\
\hline & $\mathbf{K}$ & k & & & & & & & & & \\
\hline 06 & La & & +01 & 0.0 & & & & -01 & -01 & -00 & 101 \\
\hline & Mn & $\mathbf{k}$ & +02 & +02 & & & +01 & -02 & & +01 & +02 \\
\hline & a & & 04 & & & & & & & -04 & +04 \\
\hline & & & +01 & & & & & & & & .0. \\
\hline & 02 & $n$ & & & & & & & & & \\
\hline & & & & 7. & & & & & & 04 & \\
\hline & & & +03 & NA & -04 & 02 & 1.3 & -03 & -04 & 03 & +03 \\
\hline & $\mathbf{P b}$ & $k_{q}$ & +02 & +02 & -06 & & & -01 & 02 & & +01 \\
\hline & 04 & & & +04 & & & & & & & 104 \\
\hline & $\mathbf{S}$ & & & NA & & & & & & & \\
\hline & Si & & & & & & & & & & \\
\hline & & g & +02 & 4.1 & & & -03 & 03 & & 03 & \\
\hline & $\mathrm{Sr}$ & $\mathrm{g}$ & +01 & +01 & 07 & & -00 & 01 & -02 & +01 & 501 \\
\hline & TOC & kg & +02 & 6.5 & & & & & & & \\
\hline & U & & & & & & & & & & \\
\hline & & & & & & & & & & & \\
\hline & $=227$ & & & & & & & & & & \\
\hline & & & & & & & & & & & \\
\hline & Am243 & $\mathrm{Ci}$ & & & & & & & & & \\
\hline & Ba137 & $\mathrm{Ci}$ & +04 & 1.01 & & & & & & & \\
\hline & C14 & $\mathrm{Ci}$ & $=-00$ & 4.5 & & & & & & & +01 \\
\hline & di & $\mathrm{Ci}$ & & & & & & & & & +0 \\
\hline & $\operatorname{m} 24$ & C & & & & & & & & & \\
\hline & & & & & & & & & & & \\
\hline & & $c$ & & & & & & & & & \\
\hline & Co60 & C & 1.3 & 1.3 & & & & & & & \\
\hline & Cs134 & $\mathrm{Ci}$ & $2.28 \mathrm{E}-01$ & $2.28 \mathrm{E}-01$ & & & & & & & \\
\hline & Cs13 & C & $43 E+04$ & $1.07 E+04$ & & & & & & & \\
\hline & Eu15 & $\mathrm{Ci}$ & & & & & & & & & \\
\hline & Euls & $\mathrm{C}$ & & & & & & & +03 & & +0 \\
\hline & Eul & $\mathrm{Ci}$ & +01 & & +01 & & & $E+02$ & $E+03$ & $E+02$ & $E+02$ \\
\hline & 1129 & $\mathrm{Ci}$ & & & & & & & & & -01 \\
\hline & Nb93 & $\mathrm{Ci}$ & & & & & & & & & \\
\hline & & C & & & & & & & & & \\
\hline & & $c$ & & & & & & & & & \\
\hline & & C & & $9.29 \mathrm{E}-02$ & $E+01$ & $E-03$ & $9.29 E-02$ & $1.22 \mathrm{E}-00$ & $E-00$ & $E-01$ & -0 \\
\hline
\end{tabular}


Tank Anal. Un

\begin{tabular}{|c|c|}
\hline BX106 & a23 \\
\hline BX106 & Pu238 \\
\hline BX106 & Pu239 \\
\hline BX106 & $P u 240$ \\
\hline BX106 & Pu241 \\
\hline BX106 & Pu242 \\
\hline BX106 & $\mathbf{R a 2 2 6}$ \\
\hline BX106 & Ra228 \\
\hline BX106 & Ru106 \\
\hline BX106 & Sb125 \\
\hline BX106 & Se79 \\
\hline $\begin{array}{l}\text { BX106 } \\
\text { BX106 }\end{array}$ & Sm151 \\
\hline
\end{tabular}

BX106 $\$ r 89 / 90$

BX106 Tc99

BX106 Th229

BX106 Th232

BX106 Tin126

BX106 Tritium

BX106 U232

BX106 U233

BX106 U234

BX106 U235

BX106 U236

BX106 U238

BX106 Y90

BX106 Zr93

BX107 Al

BX107 Bi

BX107 Ca

BX107 Cl

$\mathrm{BX} 107 \mathrm{CO}$

BX107 $\mathrm{Cr}$

BX107 F

BX107 Fe

BX107 $\mathrm{Hg}$

BX107 K

BX107 La

BX107 Mn

BX107 Na

BX107 Ni

BX107 NO2

BX107 NO3

BX107 P

BX107 Pb

BX107 $P 04$

BX107 S

BX107 Si

BX107 $\mathrm{SO}_{4}$

BX107 Sr

BX107 TOC

BX107 U

BX107 $\mathrm{Zr}$

BX107 Ac227

BX107 Am241

BX107 Am243

BX107 Ba137

BX107 C14

BX107 Cd113

BX107 Cm242

BX107 Cm243

BX107 Cm244

BX107 C060

BX107 Cs134

BX107 C\$137

BX107 Eu152

BX107 Eu154

BX107 Eu155

BX107 1129

\section{Un $\quad \frac{B B}{M a y 98}$}

2.90E-01

Ci $\quad 6.60 \mathrm{E}+01$

Ci Incl. in 239

$\begin{array}{lr}\text { Ci } & 2.09 \mathrm{E}+01 \\ \mathrm{Ci} & 1.01 \mathrm{E}-04\end{array}$

$1.84 \mathrm{E}-05$

2.00E-01

8.57E-04

$2.09 \mathrm{E}+01$

3.76E-01

$1.30 \mathrm{E}+03$

$2.65 \mathrm{E}+04$

$2.77 \mathrm{E}+01$

4.62E-03

8.97E-03

5.63E-01

$1.90 \mathrm{E}+01$

$1.05 \mathrm{E}-00$

4.04E-00

$1.85 \mathrm{E}-00$

8.08E-02

$1.95 \mathrm{E}-02$

2.18E-00

$2.65 \mathrm{E}+04$

$1.83 \mathrm{E}-00$

$2.69 \mathrm{E}+04$

$4.19 \mathrm{E}+04$

$7.45 \mathrm{E}+02$

$2.14 E+03$

$2.18 E+03$

$1.82 E+03$

$1.73 E+04$

$2.09 E+04$

kg 1.00E-00

$\quad 4.95 E+02$
$1.50 E+01$

$\begin{array}{ll}\mathrm{kg} & 1.50 \mathrm{E}+01 \\ \mathrm{~kg} & 1.21 \mathrm{E}+02\end{array}$

$\begin{array}{ll}\mathrm{kg} & 1.21 \mathrm{E}+02 \\ \mathrm{~kg} & 1.92 \mathrm{E}+05\end{array}$

$230 \mathrm{E}+0$

$\begin{array}{ll}\mathrm{kg} & 2.30 \mathrm{E}+01 \\ \mathrm{~kg} & 1.53 \mathrm{E}+04\end{array}$

$\begin{array}{ll}\mathrm{kg} & 2.58 \mathrm{E}+05 \\ \mathrm{~kg} & 1.34 \mathrm{E}+05\end{array}$

$\begin{array}{ll}\mathrm{kg} & 1.34 \mathrm{E}+05 \\ \mathrm{~kg} & 1.18 \mathrm{E}+02\end{array}$

kg 4.14E+05

kg $2.50 \mathrm{E}+04$

$\mathrm{kg} \quad 1.28 \mathrm{E}+04$

kg $7.50 \mathrm{E}+04$

kg $3.16 E+02$

$\mathrm{kg} \quad 1.50 \mathrm{E}+03$

$\mathrm{kg} \quad 9.10 \mathrm{E}+03$

kg $2.56 \mathrm{E}+02$

Ci

Ci

Ci

$\mathrm{Ci}$

$\mathrm{Ci}$

$\mathrm{Ci}$

$\mathrm{Ci}$
$\mathrm{Ci}$

$\mathrm{Ci}$

$\mathrm{Ci}$

$$
\mathrm{Ci}
$$

$327 \mathrm{E}+04$

Ci $\quad 1.85 \mathrm{E}-00$

Ci 3.22E-00

Ci $2.97 E-03$
BB

Sept98

1.14E-03

2.18E-00

$7.81 \mathrm{E}+01$

$1.34 \mathrm{E}+01$

$1.57 \mathrm{E}+02$

7.58E-04

1.84E-05

2.00E-01

8.57E-04

$2.09 \mathrm{E}+01$

$3.76 \mathrm{E}-01$

$1.30 \mathrm{E}+03$

$2.68 \mathrm{E}+04$

$2.77 \mathrm{E}+01$

4.62E-03

8.97E-03

5.63E-01

$1.90 \mathrm{E}+01$

2.01E-01

$7.71 \mathrm{E}-01$

3.53E-01

$1.54 \mathrm{E}-02$

3.72E-03

3.47E-01

$2.68 \mathrm{E}+04$

$1.83 \mathrm{E}-00$

$2.69 \mathrm{E}+04$

4.19E+04

$7.45 \mathrm{E}+02$

$2.14 \mathrm{E}+03$

$2.18 \mathrm{E}+03$

$1.82 \mathrm{E}+03$

$1.73 \mathrm{E}+04$

$2.09 \mathrm{E}+04$

$1.06 \mathrm{E}-00$

$4.95 \mathrm{E}+02$

$1.50 \mathrm{E}+01$

$1.21 \mathrm{E}+02$

$1.92 \mathrm{E}+05$

$2.29 \mathrm{E}+01$

$1.53 \mathrm{E}+04$

2.58E+05

NA

1.18E +02

$1.35 \mathrm{E}+05$

NA

$1.28 \mathrm{E}+04$

$2.52 \mathrm{E}+04$

$3.16 \mathrm{E}+02$

$1.50 \mathrm{E}+03$

$9.10 \mathrm{E}+03$

$2.56 \mathrm{E}+02$

4.80E-05

$2.44 \mathrm{E}+01$

3.29E-04

$2.99 \mathrm{E}+04$

$4.89 \mathrm{E}-01$

$4.88 \mathrm{E}-01$

7.01E-02

3.17E-03

2.56E-02

$1.26 \mathrm{E}-01$

1.72E-03

$3.16 E+04$

4.02E-02

1.85E-00

3.22E-00

2.97E-03

$1 \%$-ile

$10 \%$-ile

50\%-ile

$0.00 \mathrm{E}+01 \quad 0.00 \mathrm{E}+01$

$0.00 \mathrm{E}+01 \quad 1.34 \mathrm{E}-02$

$0.00 \mathrm{E}+01$

$0.00 \mathrm{E}+01$

$0.00 \mathrm{E}+01$

$0.00 \mathrm{E}+01$

$0.00 \mathrm{E}+01$

$0.00 \mathrm{E}+01$

$0.00 \mathrm{E}+01$

$0.00 \mathrm{E}+01$

$0.00 \mathrm{E}+01$

$0.00 \mathrm{E}+01$

$2.08 \mathrm{E}-05$

$0.00 \mathrm{E}+01$

$0.00 \mathrm{E}+01$

$0.00 \mathrm{E}+01$

$0.00 \mathrm{E}+01$

$0.00 \mathrm{E}+01$

$0.00 \mathrm{E}+01$

$0.00 \mathrm{E}+01$

$0.00 \mathrm{E}+01$

$0.00 \mathrm{E}+01$

$0.00 \mathrm{E}+01$

$0.00 \mathrm{E}+01$

$0.00 \mathrm{E}+01$

$0.00 \mathrm{E}+01$

$1.82 \mathrm{E}+02$

$1.33 \mathrm{E}+04$

$7.46 \mathrm{E}+01$

$3.37 \mathrm{E}+02$

$6.39 \mathrm{E}+01$

$2.24 E+01$

$1.90 \mathrm{E}+02$

$6.58 \mathrm{E}+03$

$0.00 E+01$

$5.66 \mathrm{E}+01$

$6.79 \mathrm{E}-02$

$7.41 \mathrm{E}-00$

8.72E $\div 04$

$1.49 \mathrm{E}-00$

$8.80 \mathrm{E}+02$

$2.00 \mathrm{E}+04$

$5.63 \mathrm{E}+03$

8.48E-00

3.04E-00

3.04E-00

9.62E-01

4.65E-06

$1.15 \mathrm{E}-06$

$1.25 \mathrm{E}-02$

$5.29 \mathrm{E}-05$

$9.16 \mathrm{E}-01$

2.62E-02

$6.54 \mathrm{E}+01$

$6.23 \mathrm{E}+01$

$1.43 \mathrm{E}-00$

$0.00 \mathrm{E}+01$

$0.00 \mathrm{E}+01$

$3.30 \mathrm{E}-02$

$3.57 \mathrm{E}-00$

$0.00 \mathrm{E}+01$

$0.00 \mathrm{E}+01$

$0.00 \mathrm{E}+01$

$0.00 \mathrm{E}+01$

$0.00 \mathrm{E}+01$

$0.00 \mathrm{E}+01$

$2.12 \mathrm{E}+02$

9.75E-02

$1.85 \mathrm{E}+03$

$1.93 \mathrm{E}+04$

$4.53 \mathrm{E}+02$

$1.03 \mathrm{E}+03$

$6.23 \mathrm{E}+03$

$5.85 \mathrm{E}+02$

$1.92 \mathrm{E}+03$

$1.28 \mathrm{E} \div 04$

$1.16 \mathrm{E}-01$

$2.48 \mathrm{E}+02$

1.39E-00

4.77E +01

$1.14 \mathrm{E}+05$

$1.26 \mathrm{E}+01$

$5.29 \mathrm{E}+03$

$7.95 \mathrm{E}+04$

$1.78 \mathrm{E}+04$

$1.07 \mathrm{E}+02$

$3.05 \mathrm{E}+03 \quad 1.41 \mathrm{E}+04$

$2.62 \mathrm{E}+02 \quad 2.09 \mathrm{E}+03$

8.71E+02 $\quad 4.81 \mathrm{E}+03$

$1.64 \mathrm{E}+03 \quad 7.56 \mathrm{E}+03$

$1.55 \mathrm{E}+01 \quad 1.00 \mathrm{E}+02$

$1.52 \mathrm{E}-00 \quad 3.44 \mathrm{E}+02$

$4.36 \mathrm{E}+01 \quad 6.83 \mathrm{E}+02$

$1.71 \mathrm{E}-00 \quad 1.06 \mathrm{E}+01$ 


\begin{tabular}{|c|c|c|c|c|c|c|c|c|c|c|c|}
\hline nk & al. & In & $\frac{B B}{998}$ & $\frac{B B}{\operatorname{sept98}}$ & $1 \%$-ile & $10 \%$-ile & $50 \%$-ile & 90\%-ile & 99\%-ile & Mean & Std Dev \\
\hline 07 & Nb93 & $\mathrm{Ci}$ & 37E-01 & $1.37 \mathrm{E}-01$ & $0.00 \mathrm{E}+01$ & $00 \mathrm{E}+01$ & 01 & $38 \mathrm{E}-01$ & 00 & 01 & 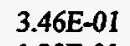 \\
\hline 07 & Ni59 & $\mathrm{Ci}$ & 36E-02 & $1.36 E-02$ & $E+01$ & & $\mathrm{E}-02$ & & & & -01 \\
\hline 107 & Ni63 & $\mathrm{Ci}$ & $=00$ & $3.94 \mathrm{E}-00$ & $0.00 E+01$ & & & $33 E+01$ & & $25 \mathrm{E}-00$ & $5 E+01$ \\
\hline 107 & Np237 & $\mathbf{C i}$ & $7.95 E-03$ & $7.95 \mathrm{E}-03$ & 48E-04 & $.51 E-03$ & E-03 & & $2 \mathrm{E}-02$ & $8.95 \mathrm{E}-03$ & $6.30 \mathrm{E}-03$ \\
\hline X107 & Pa231 & $\mathrm{Ci}$ & $1.06 E-04$ & $1.06 E-04$ & $0.00 E+01$ & $1.89 \mathrm{E}-05$ & 6E-04 & $4.96 \mathrm{E}-04$ & $40 \mathrm{E}-03$ & E-04 & $2.82 \mathrm{E}-04$ \\
\hline 6107 & Pu238 & $\mathrm{Ci}$ & $2.68 \mathrm{E}-01$ & $6.48 \mathrm{E}-01$ & $17 \mathrm{E}-02$ & $8.46 \mathrm{E}-02$ & & $5.34 \mathrm{E}-01$ & $1.09 \mathrm{E}-00$ & $3.02 \mathrm{E}-01$ & $8-01$ \\
\hline & 10239 & $\mathrm{Ci}$ & $.08 E+02$ & $95 \mathrm{E}+01$ & E-00 & $3.41 \mathrm{E}+01$ & $E+02$ & $15 \mathrm{E}+02$ & $38 \mathrm{E}+02$ & $1.22 E+02$ & +01 \\
\hline 107 & 1240 & $\mathrm{Ci}$ & Incl. in 239 & 47E-00 & $4.73 E-00$ & $11 E+01$ & $3 E+02$ & $15 E+02$ & $4.38 E+02$ & $1.22 \mathrm{E}+02$ & $8.56 \mathrm{E}+01$ \\
\hline 107 & Pu241 & $\mathrm{Ci}$ & $1.04 \mathrm{E}+01$ & $2.52 \mathrm{E}+01$ & $4.56 \mathrm{E}-01$ & & +01 & $2.07 \mathrm{E}+01$ & 01 & & \\
\hline BX107 & Pu242 & $\mathrm{Ci}$ & E-05 & $1.14 E-04$ & & & & & & & \\
\hline 107 & $\mathbf{R a 2 2 6}$ & $\mathrm{Ci}$ & $E-06$ & 9.42E-06 & & 06 & & & .05 & & \\
\hline 7 & $\mathbf{R a} 228$ & $\mathrm{Ci}$ & 3. & 3.85E-06 & & -07 & E-06 & 05 & 5 & & \\
\hline 07 & Ru106 & $\mathrm{Ci}$ & $1.26 \mathrm{E}-05$ & $1.26 \mathrm{E}-05$ & $E+01$ & -06 & $1.26 \mathrm{E}-05$ & -05 & 4.62E-05 & $E-05$ & $E-06$ \\
\hline & Sb125 & $\mathrm{Ci}$ & $4.46 \mathrm{E}-01$ & $4.46 \mathrm{E}-01$ & $0.00 \mathrm{E}+01$ & $0.00 \mathrm{E}+01$ & $4.46 \mathrm{E}-01$ & 00 & -00 & -01 & $4.26 \mathrm{E}-01$ \\
\hline & Se79 & $\mathrm{Ci}$ & 3.48E-02 & 3.48E-02 & E-05 & $E-02$ & C-02 & 02 & -01 & -02 & $E-02$ \\
\hline & Sm151 & $\mathrm{Ci}$ & $1.29 E+02$ & $1.29 \mathrm{E}+02$ & $0.00 \mathrm{E}+01$ & +01 & $\mathrm{E}+02$ & $: 03$ & +03 & +02 & +02 \\
\hline 7 & $89 / 90$ & $\mathrm{Ci}$ & $1.86 E+04$ & $1.79 E+04$ & \pm 01 & & $E+04$ & & +05 & +05 & +05 \\
\hline & Te99 & $\mathrm{Ci}$ & $6.94 E+01$ & $6.94 E+01$ & & & & & & & +02 \\
\hline & Th22 & $\mathrm{Ci}$ & 07 & 7 & & & -07 & & .06 & & \\
\hline 7 & Th232 & $\mathrm{Ci}$ & 06 & $-1-06$ & 01 & 1 & -06 & 06 & -05 & -06 & -06 \\
\hline 07 & Tin126 & $\mathrm{Ci}$ & $5.21 \mathrm{E}-02$ & $5.21 \mathrm{E}-02$ & $0.00 \mathrm{E}+01$ & $=-03$ & $8-02$ & $E-01$ & $4.22 \mathrm{E}-01$ & $E-02$ & $9.54 \mathrm{E}-02$ \\
\hline & Tritium & $\mathrm{Ci}$ & $1.09 \mathrm{E}-00$ & $1.00 \mathrm{E}-00$ & E-01 & -01 & -00 & & -00 & -60 & $E-01$ \\
\hline 7 & $\mathrm{~J} 232$ & $\mathrm{Ci}$ & $5.04 \mathrm{E}-04$ & $7.52 \mathrm{E}-05$ & $E+01$ & & & & & & -03 \\
\hline 107 & 233 & $\mathrm{Ci}$ & $7.06 E-04$ & 1.05E-04 & $E+01$ & 04 & -04 & & -03 & & \\
\hline & U234 & $\mathrm{Ci}$ & $2.01 \mathrm{E}+01$ & $3.00 \mathrm{E}-00$ & & & & & & & \\
\hline 107 & U235 & $\mathrm{Ci}$ & 8.98E-01 & 01 & 01 & & & & -01 & & -00 \\
\hline 07 & U236 & $\mathrm{Ci}$ & $1.56 \mathrm{E}-01$ & 2.33E-02 & $E+01$ & $=02$ & $E-01$ & 01 & $2.06 \mathrm{E}-00$ & $E-01$ & $E-01$ \\
\hline 7 & U238 & $\mathrm{Ci}$ & $1.40 \mathrm{E}-00$ & 3.04E-00 & $E+01$ & 01 & -00 & 00 & +01 & .00 & $E-00$ \\
\hline & 90 & $\mathbf{C i}$ & $1.86 \mathrm{E}+04$ & $1.79 E+04$ & $\div+01$ & & & & -05 & & -04 \\
\hline & Zr93 & $\mathrm{Ci}$ & $1.67 \mathrm{E}-01$ & $1.67 \mathrm{E}-01$ & $: 01$ & & & & & & \\
\hline & I & kg & 1.0 & 1.0 & +01 & -02 & -03 & & +03 & & +03 \\
\hline 08 & $\mathbf{B i}$ & kg & $1.26 E+03$ & $1.26 \mathrm{E}+03$ & +02 & +02 & +03 & 03 & +03 & -03 & +03 \\
\hline 08 & $\mathbf{C a}$ & kg & $2.93 E+02$ & $2.93 \mathrm{E}+02$ & $=-00$ & -01 & 01 & 02 & +02 & 02 & +02 \\
\hline & Cl & kg & $1.80 \mathrm{E} \div 02$ & $1.80 \mathrm{E}+02$ & 01 & & 02 & & +02 & & +01 \\
\hline & 03 & kg & $4.42 E+02$ & +02 & & & & & 03 & & \\
\hline & $\mathbf{r}$ & kg & +01 & 01 & & & & & & & \\
\hline 08 & $\mathbf{F}$ & kg & & +02 & -01 & 02 & 02 & & +03 & & -02 \\
\hline 08 & $\mathbf{F e}$ & kg & $2.57 \mathrm{E}+03$ & $2.57 E+03$ & +02 & +02 & +03 & 03 & +03 & 03 & -03 \\
\hline 08 & Hg & kg & $1.97 \mathrm{E}-00$ & $8.28 \mathrm{E}-00$ & $\varepsilon+01$ & -01 & -00 & -00 & +01 & .00 & $2-00$ \\
\hline 08 & $\mathbf{K}$ & kg & $2.63 E+01$ & $2.63 E+01$ & $E-00$ & -01 & 01 & 2 & +02 & 01 & +01 \\
\hline 08 & $a$ & kg & $2.22 \mathrm{E}+01$ & $0.00 \mathrm{E}+01$ & & & & & 01 & & 10 \\
\hline & In & kg & & 01 & & & & & & & \\
\hline & $\mathbf{N a}$ & kg & & & 03 & -03 & -04 & & -04 & 04 & \\
\hline 98 & $\mathbf{N i}$ & $\mathbf{k g}$ & $8.20 \mathrm{E}-00$ & $8.20 E-00$ & 01 & -01 & -00 & 01 & +01 & -00 & +01 \\
\hline 08 & $\mathrm{NO} 2$ & kg & $2.29 E+03$ & $2.29 E+03$ & +01 & +02 & +03 & -03 & +03 & 03 & +03 \\
\hline 108 & 103 & kg & $2.48 E+04$ & $2.48 E+04$ & +02 & & -04 & 3 & +04 & 04 & +04 \\
\hline 8 & 1 & kg & $\mathbf{0 3}$ & NA & & & & & & & -03 \\
\hline & Pb & kg & & +01 & & & & & & & \\
\hline & & kg & $1.89 \mathrm{E}+04$ & $6.17 E+03$ & & 02 & +03 & & -04 & & 103 \\
\hline & $\mathbf{S}$ & $\mathrm{kg}$ & $2.30 E+03$ & NA & +01 & +02 & +02 & 103 & +03 & +02 & +02 \\
\hline 108 & $\mathbf{S i}$ & $\mathrm{kg}$ & $4.36 E+02$ & $4.36 E+02$ & +01 & 02 & +02 & 103 & +03 & +02 & $E+02$ \\
\hline 8 & SO4 & $\mathrm{kg}$ & $6.90 \mathrm{E}+03$ & $2.30 \mathrm{E}+03$ & $E+01$ & +02 & +03 & 103 & +03 & 3 & +03 \\
\hline & $\mathrm{Sr}$ & kg & $6.22 E+01$ & $6.22 \mathrm{E}+01$ & & & & & +02 & 01 & +01 \\
\hline & C & kg & 8. & 8. & & & & & & & +02 \\
\hline BX & & kg & & 1. & & & & & & 02 & \\
\hline & $\mathbf{Z r}$ & $\mathrm{kg}$ & & & & & & & & & $\div 01$ \\
\hline 108 & Ac227 & $\mathrm{Ci}$ & -06 & $3.44 \mathrm{E}-06$ & $E+01$ & .07 & -06 & -05 & $E-05$ & -06 & $E-05$ \\
\hline BX108 & Am241 & $\mathrm{Ci}$ & $2.62 \mathrm{E}+01$ & $5.05 \mathrm{E}-00$ & BE-01 & 1. & 8.22E-00 & +02 & $E+02$ & $E+01$ & $E+02$ \\
\hline BX10 & Am243 & $\mathrm{Ci}$ & $1.34 E-07$ & $3.48 \mathrm{E}-05$ & -09 & -08 & -07 & -07 & $E-07$ & 1 & $=07$ \\
\hline BX10 & Ba13 & $\mathrm{Ci}$ & $1.94 \mathrm{E}+03$ & $1.94 E+03$ & 01 & +02 & -03 & -03 & .04 & +03 & +03 \\
\hline BX108 & C14 & $\mathrm{Ci}$ & 8.42E-02 & $1.11 \mathrm{E}-02$ & +01 & & & & & & -02 \\
\hline & Cd1 & $\mathrm{Ci}$ & 2.67 & $E-1$ & 0 & & & & & & -02 \\
\hline X108 & $\mathrm{m} 2$ & $\mathrm{Ci}$ & 5.41 & & & & & -04 & & & $E-05$ \\
\hline & & $\mathrm{Ci}$ & & E-04 & & & & & $E-0$ & $E-06$ & 4E-06 \\
\hline & Cm244 & $\mathrm{Ci}$ & 3.17E-06 & 8.21E-04 & $9.49 E-08$ & E-07 & $3.17 \mathrm{E}-06$ & 8.47E-06 & & $E-06$ & $3.57 \mathrm{E}-06$ \\
\hline & Co60 & $\mathrm{Ci}$ & & $2.43 E-03$ & $0.00 \mathrm{E}+01$ & $4.48 \mathrm{E}-02$ & 5.65E-01 & & $7.99 \mathrm{E}+01$ & $3.58 \mathrm{E}-00$ & $1.27 \mathrm{E}+01$ \\
\hline & & $\mathrm{Ci}$ & $8.88 \mathrm{E}-05$ & $8.88 E-05$ & & $1.89 \mathrm{E}-05$ & $8.88 E-05$ & $4.23 E-04$ & $9.97 \mathrm{E}-04$ & $1.66 \mathrm{E}-04$ & $1.99 \mathrm{E}-04$ \\
\hline & & 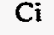 & & & & $.65 \mathrm{E}+02$ & 3.4 & -04 & & +03 & \\
\hline
\end{tabular}


Tank Anal. Un

\begin{tabular}{ll|} 
BX108 & Eu152 \\
BX108 & Eu154 \\
BX108 & Eu155 \\
BX108 & I129 \\
BX108 & Nb93 \\
BX108 & Ni59 \\
\hline
\end{tabular}

BX108 Ni63

BX108 Np237

BX108 Pa231

BX108 Pu238

BX108 Pu239

BX108 Pu240

BX108 Pu241

BX108 Pu242

BX108 Ra226

BX108 Ra228

BX108 Ru106

BX108 Sb125

BX108 Se79

BX108 Sm151

BX108 Sr89/90

BX108 Tc99

BX108 Th229

BX108 Th232

BX108 Tin126

BX108 Tritium

BX108 U232

BX108 U233

BX108 U234

BX108 U235

BX108 U236

BX108 U238

BX108 Y90

BX108 Zr93

BX109 Al

BX109 Bi

BX109 Ca

BX109 Cl

BX109 CO3

BX109 Cr

BX109 F

BX109 Fe

BX109 Hg

BX109 K

BX109 La

BX109 Mn

BX109 Na

BX109 Ni

BX109 NO2

BX109 NO3

BX109 P

BX109 Pb

BX109 PO4

BX109 S

BX109 Si

BX109 SO4

BX109 Sr

BX109 TOC

BX109U

BX109 Zr

BX109 Ac227

BX109 Am241

BX109 Am243

BX109 Ba137

BX109 C14

BX109 Cd113

BX109 Cm242

BX109 Cm243

\section{May $\frac{B B}{98}$ \\ $3.06 \mathrm{E}-03$}

$5.75 \mathrm{E}-00$

$1.37 \mathrm{E}+01$

5.94E-01

9.40E-03

2.84E-01

4.74E-04

7.43E-06

2.47E-02

5.22E- -00

Ci Incl. in 239

9.98E-01

6.75E-07

$1.30 \mathrm{E}-11$

9.50E-10

2.19E-03

Ci 2.34E-03

8.72E-00

$1.43 \mathrm{E}+04$

$4.96 \mathrm{E}-00$

2.51E-09

3.19E-12

3.51E-03

5.21E-02

2.66E-05

1.35E-06

$1.50 \mathrm{E}-00$

6.70E-02

1.24E-02

1.52E-00

1.43E+04

$1.11 \mathrm{E}-02$

$2.72 \mathrm{E}+03$

$1.61 \mathrm{E}+03$

$3.32 E+03$

$1.45 E+03$

$1.60 \mathrm{E}+04$

$1.50 \mathrm{E}+02$

$3.48 \mathrm{E}+02$

$2.40 \mathrm{E}+04$

2.41E-00

$2.05 \mathrm{E}+02$

$0.00 \mathrm{E}+01$

$1.63 E+02$

$1.15 E+05$

$3.53 \mathrm{E}+01$

$1.98 \mathrm{E}+04$

$2.11 \mathrm{E}+05$

$8.91 \mathrm{E}+03$

$7.32 \mathrm{E}+02$

$2.76 \mathrm{E}+04$

$6.43 \mathrm{E}+03$

$6.03 \mathrm{E}+02$

$1.93 \mathrm{E}+04$

$6.49 \mathrm{E}+02$

$4.49 \mathrm{E}+02$

$1.80 \mathrm{E}+04$

2.88E-00

6.01E-05

$4.15 \mathrm{E}-01$

2.90E-06

$1.33 E+04$

2.09E-01

5.15E-01

8.14E-03

1.67E-04
$\frac{B B}{\operatorname{sen}}$

$\frac{\mathrm{ept}}{3.06 \mathrm{E}-03}$

4.31E-02

2.40E-01

$1.45 \mathrm{E}-04$

9.40E-03

3.15E-03

2.84E-01

4.74E-04

7.43E-06

3.32E-02

4.79E-00

4.25E-01

$1.34 \mathrm{E}-00$

6.08E-06

6.75E-07

$1.30 \mathrm{E}-11$

9.50E-10

$2.19 \mathrm{E}-03$

2.34E-03

8.72E-00

$1.43 \mathrm{E}+04$

4.96E-00

2.51 E-09

3.19E-12

3.51 E-03

5.21 E- 02

1.01 E-05

5.11 E-07

5.69E-01

2.54E-02

4.68E-03

5.78E-01

$1.43 E+04$

$1.11 \mathrm{E}-02$

$2.72 \mathrm{E}+03$

$1.61 \mathrm{E}+03$

$3.32 E+03$

$1.45 \mathrm{E}+03$

$1.60 \mathrm{E}+04$

$1.50 \mathrm{E}+02$

$3.48 \mathrm{E}+02$

$2.40 \mathrm{E}+04$

$1.08 \mathrm{E}+01$

$2.05 \mathrm{E}+02$

$0.00 \mathrm{E}+01$

$1.63 E+02$

$1.15 \mathrm{E}+05$

$3.53 \mathrm{E}+01$

$1.98 \mathrm{E}+04$

$2.11 E+05$

NA

$7.32 \mathrm{E}+02$

2.76E+04

NA

$6.03 E+02$

$1.93 E+04$

$6.49 \mathrm{E}+02$

$4.49 \mathrm{E}+02$

$1.80 \mathrm{E}+04$

2.88E-00

6.01E-05

8.08E-01

5.66E-06

$1.33 \mathrm{E}+04$

2.09E-01

5.15E- $\theta 1$

1.59E-02

3.25E-04
$1 \%$-ile

(1)

$0.00 \mathrm{E} \div 01$

$0.00 \mathrm{E}+01$

$0.00 \mathrm{E}+01$

$0.00 \mathrm{E}+01$

$0.00 \mathrm{E}+01$

$0.00 \mathrm{E}+01$

$0.00 \mathrm{E}+01$

1.42E-05

$0.00 \mathrm{E}+01$

7.39E-04

1.56E-01

$1.56 \mathrm{E}-01$

2.99E-02

$1.35 \mathrm{E}-07$

$0.00 \mathrm{E}+01$

$0.00 \mathrm{E}+01$

$0.00 \mathrm{E}+01$

$0.00 \mathrm{E}+01$

$0.00 \mathrm{E}+01$

$0.00 \mathrm{E}+01$

$3.08 \mathrm{E}+01$

1.17E-01

$0.00 \mathrm{E}+01$

$0.00 \mathrm{E}+01$

$0.00 \mathrm{E}+01$

7.04E-03

$0.00 \mathrm{E}+01$

$0.00 \mathrm{E}+01$

$0.00 \mathrm{E}+01$

$0.00 \mathrm{E}+01$

$0.00 \mathrm{E}+01$

$0.00 \mathrm{E}+01$

$0.00 \mathrm{E}+01$

$0.00 \mathrm{E}+01$

$1.76 \mathrm{E}+02$

$7.14 \mathrm{E}+03$

$7.11 \mathrm{E}+01$

$2.09 \mathrm{E}+02$

8.77E +01

8.37E-00

$1.16 \mathrm{E}+02$

$3.59 \mathrm{E}+03$

$0.00 \mathrm{E}+01$

$3.81 \mathrm{E}+01$

$6.31 \mathrm{E}-02$

$6.38 \mathrm{E}-00$

4.63E+04

3.52E-00

$6.77 \mathrm{E} \div 02$

$9.88 \mathrm{E}+03$

$3.41 \mathrm{E}+03$

5.13E-00

$1.72 \mathrm{E}+03$

$1.32 \mathrm{E}+02$

4. $72 \mathrm{E}+02$

$1.17 \mathrm{E}+03$

9.10E-00

$7.85 \mathrm{E}-01$

$4.52 E+01$

$4.19 \mathrm{E}-01$

$0.00 E+01$

1.71E-00

$1.76 \mathrm{E}-07$

$7.32 \mathrm{E}+02$

$0.00 E+01$

$0.00 E+01$

4.95E-04

1.02E-05

$10 \%$-ile

$2.50 \mathrm{E}-04 \quad 3.06 \mathrm{E}-03$

$4.69 \mathrm{E}-01$

$1.12 \mathrm{E}-00$

$1.65 \mathrm{E}-01$

$0.00 \mathrm{E}+01$

$5.88 \mathrm{E}-0.4$

$5.30 \mathrm{E}-02$

$1.18 \mathrm{E}-04$

$8.56 \mathrm{E}-07$

$6.14 \mathrm{E}-03$

$1.30 \mathrm{E}-00$

$1.30 \mathrm{E}-00$

2.48E-01

1.12E-06

$1.13 \mathrm{E}-07$

2.18E-12

$1.97 \mathrm{E}-10$

$0.00 \mathrm{E}+01$ 
Tank Anal. Un

\begin{tabular}{|c|c|}
\hline BX109 & $\mathrm{Cm} 244$ \\
\hline BX109 & Co60 \\
\hline BX109 & Cs134 \\
\hline BX109 & Cs137 \\
\hline BX109 & Eu152 \\
\hline BX109 & Eu154 \\
\hline BX109 & Eu155 \\
\hline BX109 & I129 \\
\hline BX109 & Nb93 \\
\hline BX109 & Ni59 \\
\hline BX109 & Ni63 \\
\hline BX109 & Np237 \\
\hline BX109 & Pa231 \\
\hline BX109 & Pu238 \\
\hline BX109 & Pu239 \\
\hline BX109 & Pu240 \\
\hline BX109 & Pu241 \\
\hline BX109 & Pu242 \\
\hline BX109 & $\operatorname{Ra226}$ \\
\hline BX109 & Ra228 \\
\hline BX109 & Ru106 \\
\hline BX109 & Sb125 \\
\hline & 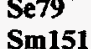 \\
\hline
\end{tabular}

BX109 Sm151

BX109 Sr89/90

BX109 Tc99

BX109 Th229

BX109 Th232

BX109 Tin126

BX109 Tritium

BX109 U232

BX109 U233

BX109 U234

BX109 U235

BX109 U236

BX109 U238

BX109 Y90

BX109 Zr93

BX110 Al

BX110 Bi

BX110 Ca

BX110 Cl

BX110 CO3

BX110 Cr

BX110 F

BX110 Fe

BX110 Hg

BX110 K

BX110 La

BX110 Mn

BX110 Na

BX110 Ni

BX110 NO2

BX110 NO3

BX110 P

BX110 Pb

BX110 PO4

BX110 S

BX110 Si

BX110 SO4

BX110 Sr

BX110 TOC

BX110 U

BX110 $\mathrm{Zr}$

BX110 Ac227

BX110 Am241

BX110 Am243

BX110 Ba137
May $\frac{B 8}{688 E-05}$

$4.90 \mathrm{E}-02$

$7.27 \mathrm{E}-04$

$1.41 \mathrm{E}+04$

4.44E-01

8.79E-01

$3.34 E+01$

2.73E-03

$1.76 \mathrm{E}-01$

5.94E-02

5.36E-00

8.96E-03

1.31E-04

6.84E-02

8.80E-00

8.40E-01

2.95E-00

1.35E-05

1.18E-05

1.69E-09

2.06E-08

4.54E- 02

4.41E-02

$1.64 \mathrm{E}+02$

$1.99 \mathrm{E}+05$

$1.45 \mathrm{E}-00$

Ci 3.28E-07

$\mathrm{Ci} \quad 5.94 \mathrm{E}-11$

Ci 6.63E-02

$1.47 \mathrm{E}-00$

4.58E-05

Ci 2.10E-06

$\mathrm{Ci}$ 1.91E-00

Ci 8.44E-02

Ci 1.95E-02

Ci 1.94E-00

Ci 1.99E+05

Ci 2.09E-01

kg 1.58E+04

kg $\quad 1.38 \mathrm{E}+04$

kg 2.07E+03

kg 1.35E+03

kg 3.64E+04

kg $\quad 1.41 \mathrm{E}+03$

kg $\quad 9.83 E+03$

kg $7.62 \mathrm{E}+03$

$\mathrm{kg} \quad 1.01 \mathrm{E}+01$

kg $8.69 \mathrm{E}+02$

$\mathrm{kg} \quad 7.13 E-02$

kg $2.66 \mathrm{E}+02$

kg $\quad 1.26 \mathrm{E}+05$

kg $\quad 3.20 \mathrm{E}+01$

kg 2.73E+04

kg $\quad 1.56 \mathrm{E}+05$

$\mathrm{kg} \quad 4.94 \mathrm{E}+03$

kg $\quad 1.87 \mathrm{E}+02$

kg $1.53 \mathrm{E}+04$

kg $2.98 \mathrm{E}+03$

kg $6.70 E+03$

kg $\quad 8.95 \mathrm{E}+03$

kg 1.22E+02

kg 2.07E+03

$\mathrm{kg} \quad 9.33 \mathrm{E}+02$

kg 6.40E+01

$\mathrm{Ci}$ 3.28E-04

Ci 7.22E-00

Ci 2.46E-04

Ci $6.23 \mathrm{E}+04$

\section{Sept98}

\subsection{E-04}

4.90E-02

7.27E-04

$1.41 \mathrm{E}+04$

4.44E-01

8.79E-01

$3.34 \mathrm{E}+01$

2.73E-03

$1.76 \mathrm{E}-01$

5.94E-02

5.36E-00

8.96E-03

$1.31 \mathrm{E} 04$

1.33E-01

$1.72 \mathrm{E}+01$

1.64E-00

$5.76 \mathrm{E}-00$

2.63E-05

1.18E-05

1.69E-09

2.06E-08

4.54E-02

4.41E- 02

$1.64 \mathrm{E}+02$

$1.99 \mathrm{E}+05$

$1.45 \mathrm{E}-00$

$3.28 \mathrm{E}-07$

5.94E-11

6.63E-02

$1.47 \mathrm{E}-00$

$1.42 \mathrm{E}-04$

6.50E-06

5.91E-00

2.61E-01

6.04E-02

6.01E-00

$1.99 \mathrm{E}+05$

2.09E-01

$1.58 \mathrm{E}+04$

$1.38 E+04$

$2.07 \mathrm{E}+03$

$1.35 \mathrm{E}+03$

$3.64 E+04$

$1.41 \mathrm{E}+03$

$9.83 \mathrm{E}+03$

$7.62 \mathrm{E}+03$

$4.97 E+01$

$8.69 E+02$

7.13E-02

$2.66 \mathrm{E}+02$

$1.26 \mathrm{E}+05$

$3.20 \mathrm{E}+01$

$2.73 E+04$

$1.56 \mathrm{E}+05$

NA

$1.87 \mathrm{E}+02$

$1.53 \mathrm{E}+04$

NA

$6.70 E+03$

$8.95 E+03$

$1.22 \mathrm{E}+02$

$2.07 \mathrm{E}+03$

$9.33 \mathrm{E}+02$

$6.40 \mathrm{E}+01$

3.28E-04

$7.22 \mathrm{E}-00$

2.46E-04

$6.23 \mathrm{E}+04$

\section{$1 \%$-ile}

4.19E-06

7.54E-04

$4.00 \mathrm{E}-05$

$1.16 \mathrm{E}+03$

$0.00 \mathrm{E}+01$

$0.00 \mathrm{E}+01$

$0.00 \mathrm{E}+01$

$0.00 \mathrm{E}+01$

$0.00 \mathrm{E}+01$

4.42E-03

$3.99 \mathrm{E}-01$

$5.45 \mathrm{E}-04$

$0.00 \mathrm{E}+01$

4.16E-03

$5.36 \mathrm{E}-01$

$5.11 \mathrm{E}-02$

$1.80 \mathrm{E}-01$

8.22E-07

$0.00 \mathrm{E}+01$

$0.00 \mathrm{E}+01$

$0.00 \mathrm{E}+01$

$0.00 \mathrm{E}+01$

1. $62 \mathrm{E}-03$

$0.00 \mathrm{E}+01$

$3.70 \mathrm{E}+02$

$1.13 \mathrm{E}-01$

$0.00 \mathrm{E}+01$

$0.00 \mathrm{E}+01$

$0.00 \mathrm{E}+01$

$7.29 \mathrm{E}-01$

$0.00 \mathrm{E}+01$

$0.00 \mathrm{E}+01$

$0.00 \mathrm{E}+01$

$0.00 \mathrm{E}+01$

$0.00 \mathrm{E}+01$

$0.00 \mathrm{E}+01$

$0.00 \mathrm{E}+01$

$0.00 \mathrm{E}+01$

$3.90 \mathrm{E}+03$

$6.53 \mathrm{E}+03$

$1.44 \mathrm{E}+02$

$4.58 \mathrm{E}+02$

$6.38 \mathrm{E}+02$

$5.04 \mathrm{E}+02$

$8.32 \mathrm{E}+02$

$3.46 \mathrm{E}+03$

$0.00 \mathrm{E}+01$

$2.04 \mathrm{E}+02$

3.53E-01

$1.32 \mathrm{E}+01$

1. $11 \mathrm{E}+05$

$5.26 \mathrm{E}-00$

$3.02 \mathrm{E}+03$

1.25E+05

$6.79 \mathrm{E}+03$

$1.91 \mathrm{E}+01$

$8.43 \mathrm{E}+03$

$6.51 E+02$

$1.06 \mathrm{E}+03$

$1.92 \mathrm{E}+03$

2. $12 \mathrm{E}+01$

$2.40 \mathrm{E}+02$

$1.27 \mathrm{E}+02$

$1.25 \mathrm{E}-00$

$0.00 \mathrm{E}+01$

1.94E-00

$1.88 \mathrm{E}-05$

$0.00 \mathrm{E}+01$

\section{$10 \%$-ile}

2.41E-05

$2.26 \mathrm{E}-01$

$2.60 \mathrm{E}-04$

$6.40 \mathrm{E}+03$

7.91E-02

1.57E-01

$5.95 \mathrm{E}-00$

8.48E-04

$4.89 \mathrm{E}-03$

2.13E-02

1.92E-00

3.13E-03

2.41E-05

2.39E-02

$3.08 \mathrm{E}-00$

2.94E-01

1.03E-00

4.72E-06

2.10E-06

3.01E-10

5.66E-09

$0.00 \mathrm{E}+01$

$1.72 \mathrm{E}-02$

$2.92 \mathrm{E}+01$

$4.25 \mathrm{E} \div 03$

$5.59 \mathrm{E}-01$

6.02E-08

1.09E-11

8.51E-03

$9.86 \mathrm{E}-01$

8.41E-06

3.86E-07 


\begin{tabular}{|c|c|c|c|c|c|c|c|c|c|c|c|}
\hline ank & Anal. & Un & May98 & $\operatorname{Sept98}$ & $1 \%$-ile & $10 \%$-ile & $50 \%$-ile & $0 \%$-ile & $99 \%$-ile & Mean & itd Dev \\
\hline & 14 & $\mathbf{C i}$ & $5.47 \mathrm{E}-00$ & $5.47 \mathrm{E}-00$ & $0.00 \mathrm{E}+01$ & $7.48 \mathrm{E}-01$ & $5.47 \mathrm{E}-00$ & $1.30 \mathrm{E}+01$ & $1.94 \mathrm{E}+01$ & $6.29 \mathrm{E}-00$ & $4.66 \mathrm{E}-00$ \\
\hline$x 110$ & d113 & $\mathbf{C i}$ & $1.18 E+01$ & $1.18 \mathrm{E}+01$ & $0.00 E+01$ & $E=00$ & $1.18 \mathrm{E}+01$ & $34 \mathrm{E}+01$ & $96 \mathrm{E}+01$ & $1.83 E+01$ & $1.98 \mathrm{E}+01$ \\
\hline $\mathbf{X 1 1 0}$ & $\mathrm{Cm} 242$ & $\mathbf{C i}$ & 4.94E-04 & $4.94 \mathrm{E}-04$ & $3.78 \mathrm{E}-05$ & $1.84 \mathrm{E}-04$ & $4.94 E-04$ & $9.33 E-04$ & $1.79 \mathrm{E}-03$ & 5.47E-04 & $3.49 \mathrm{E}-04$ \\
\hline 2110 & $\mathrm{Cm} 243$ & $\mathbf{C i}$ & $01 \mathrm{E}-05$ & $1.01 E-05$ & $7.72 \mathrm{E}-07$ & $3.75 \mathrm{E}-06$ & $1.01 \mathrm{E}-05$ & $1.91 \mathrm{E}-05$ & & & $.14 \mathrm{E}-06$ \\
\hline & $\mathrm{Cm} 244$ & $\mathrm{Ci}$ & 5.61E-05 & $5.61 \mathrm{E}-05$ & $4.29 \mathrm{E}-06$ & $2.09 \mathrm{E}-05$ & $5.61 \mathrm{E}-05$ & $1.06 \mathrm{E}-04$ & 3E-04 & $6.21 \mathrm{E}-05$ & $3.97 \mathrm{E}-05$ \\
\hline & 060 & $\mathbf{C i}$ & $07 \mathrm{E}-00$ & $5.07 \mathrm{E}-00$ & $1.75 E-01$ & $1.69 \mathrm{E}-00$ & $1.29 \mathrm{E}+01$ & $5.21 \mathrm{E}+01$ & $4.41 E+02$ & $3.20 \mathrm{E}+01$ & $7.89 \mathrm{E}+01$ \\
\hline & s134 & $\mathbf{C i}$ & $2.47 \mathrm{E}-01$ & $2.47 \mathrm{E}-01$ & $0.00 \mathrm{E}+01$ & $5.61 \mathrm{E}-02$ & $47 \mathrm{E}-01$ & $53 \mathrm{E}-01$ & 2E-01 & -01 & E-01 \\
\hline & s137 & $\mathrm{Ci}$ & $E+04$ & $6.59 \mathrm{E}+04$ & $7.19 \mathrm{E}+03$ & $2.10 E+04$ & $E+04$ & $36 \mathrm{E}+05$ & $\mathrm{E}+05$ & $E+04$ & $E+04$ \\
\hline & Eu152 & Ci & 33E-01 & 33E-01 & $0.00 \mathrm{E}+01$ & $0.00 \mathrm{E}+01$ & 33E-01 & $13 \mathrm{E}-00$ & $1 \mathrm{E}-00$ & $1.22 \mathrm{E}-00$ & 1.57E-00 \\
\hline 110 & Eu154 & $\mathbf{C i}$ & $8.57 E+01$ & $8.57 E+01$ & $0.00 E+01$ & $0.00 E+01$ & $8.57 E+01$ & $3.65 E+02$ & $20 \mathrm{E} \div 02$ & $1.42 \mathrm{E}+02$ & $1.84 E+02$ \\
\hline 110 & Eu155 & $\mathbf{C i}$ & $4.47 E+01$ & $4.47 E+01$ & $0.00 E+01$ & $0.00 \mathrm{E}+01$ & $.47 E+01$ & $1.91 E+02$ & $4.28 \mathrm{E}+02$ & \pm+01 & $9.58 \mathrm{E}+01$ \\
\hline 110 & 1129 & $\mathbf{C i}$ & $5.92 \mathrm{E}-02$ & $5.92 \mathrm{E}-02$ & $0.00 \mathrm{E}+01$ & $2.08 \mathrm{E}-02$ & $5.92 \mathrm{E}-02$ & $3 \mathrm{E}-02$ & $1.23 E-01$ & $5.94 \mathrm{E}-02$ & $5 \mathrm{E}-02$ \\
\hline BX110 & $\mathrm{Nb93}$ & $\mathbf{C i}$ & $1.64 \mathrm{E}-00$ & $1.64 \mathrm{E}-00$ & $0.00 \mathrm{E}+01$ & $0.00 \mathrm{E}+01$ & $1.64 \mathrm{E}-00$ & 8.73E-00 & $1.56 \mathrm{E}+01$ & $E-00$ & $3.79 E-00$ \\
\hline BX110 & i59 & $\mathbf{C i}$ & $5.96 \mathrm{E}-01$ & $5.96 \mathrm{E}-01$ & $0.00 \mathrm{E}+01$ & +01 & 5067 & E-00 & $E-00$ & & E-01 \\
\hline 110 & i63 & $\mathrm{Ci}$ & $5.90 \mathrm{E}+01$ & $5.90 \mathrm{E}+01$ & $0.00 \mathrm{E}+01$ & +01 & $90 \mathrm{E}+01$ & +02 & +02 & 7.4 & +01 \\
\hline & p237 & $\mathbf{C i}$ & $1.04 \mathrm{E}-01$ & $1.04 \mathrm{E}-01$ & $E-03$ & -02 & E-01 & & -01 & & $E-02$ \\
\hline & 2331 & $\mathbf{C i}$ & $1.63 E-03$ & $1.63 E-03$ & $0.00 \mathrm{E}+01$ & -04 & -03 & .03 & E-02 & .03 & $E-03$ \\
\hline & 4238 & $\mathrm{Ci}$ & $5.81 \mathrm{E}-01$ & $5.81 \mathrm{E}-01$ & $E-02$ & $E-01$ & 5.81E-01 & & $E-00$ & & $E-01$ \\
\hline 10 & Pu239 & $\mathrm{Ci}$ & $3.87 E+01$ & $3.87 \mathrm{E}+01$ & & $E+01$ & & & $E+02$ & & $E+01$ \\
\hline 110 & Pu240 & $\mathbf{C i}$ & 4.70E-00 & $4.70 \mathrm{E}-00$ & E-01 & $E-00$ & $4.70 \mathrm{E}-00$ & $E-00$ & $E+01$ & & $E-00$ \\
\hline 110 & Pu241 & $\mathbf{C i}$ & $3.65 E+01$ & $3.65 E+01$ & $2.79 \mathrm{E}-00$ & +01 & $3.65 \mathrm{E}+01$ & +01 & $E+02$ & & +01 \\
\hline & 1242 & $\mathbf{C i}$ & $1.73 \mathrm{E}-04$ & $1.73 E-04$ & 1.2 & & 04 & & -04 & & \\
\hline 10. & 2226 & $\mathbf{C i}$ & 2.57E-05 & 2.57E-05 & $\div 01$ & 06 & 2.5 & & -04 & & -05 \\
\hline 10 & La228 & $\mathbf{C i}$ & $2.70 \mathrm{E}-01$ & 2.70E-01 & +01 & $E-02$ & $2.70 \mathrm{E}-01$ & -01 & E-00 & -01 & $\varepsilon-01$ \\
\hline 110 & Ru106 & $\mathrm{Ci}$ & $1.01 \mathrm{E}-03$ & $1.01 \mathrm{E}-03$ & $0.00 E+01$ & $2.67 \mathrm{E}-04$ & 1.01E-03 & $2.10 \mathrm{E}-03$ & $3.71 \mathrm{E}-03$ & -03 & $E-04$ \\
\hline & b125 & $\mathbf{C i}$ & $2.27 E+01$ & $2.27 \mathrm{E}+01$ & & & & & +02 & & +01 \\
\hline 110 & Se79 & $\mathbf{C i}$ & $4.68 \mathrm{E}-01$ & 4.68E-01 & $E-02$ & -01 & $4.68 \mathrm{E}-01$ & -01 & $\mathrm{E}-00$ & -01 & $E-01$ \\
\hline & Sm151 & $\mathrm{Ci}$ & $1.62 E+03$ & $1.62 \mathrm{E}+03$ & +01 & & & +03 & +04 & & +03 \\
\hline & 190 & $\mathbf{C i}$ & $1.13 E+04$ & +04 & +03 & 104 & +04 & -05 & +05 & -04 & +05 \\
\hline 110 & Tc99 & $\mathrm{Ci}$ & $3.06 \mathrm{E}+01$ & $3.06 \mathrm{E}+01$ & $=-00$ & +01 & $3.06 E+01$ & +01 & +02 & +01 & +02 \\
\hline 110 & h229 & $\mathrm{Ci}$ & $6.24 E-03$ & $6.24 E-03$ & $0.00 E+01$ & -03 & & & -02 & & $=-02$ \\
\hline & h232 & $\mathbf{C i}$ & $9.98 \mathrm{E}-03$ & $9.98 \mathrm{E}-03$ & & -03 & & -02 & $:-01$ & & \\
\hline 110 & Tin126 & $\mathbf{C i}$ & $7.00 \mathrm{E}-01$ & $7.00 \mathrm{E}-01$ & +01 & -01 & -01 & & -00 & & -00 \\
\hline & Tritium & $\mathrm{Ci}$ & $2.11 \mathrm{E}+01$ & $i+01$ & +01 & & & & & & \\
\hline 10 & U232 & $\mathrm{Ci}$ & $8-00$ & $1.51 \mathrm{E}-00$ & +01 & & -00 & -00 & 01 & -00 & -00 \\
\hline 110 & U233 & $\mathrm{Ci}$ & $5.77 E-00$ & $5.77 \mathrm{E}-00$ & +01 & -00 & $5.77 \mathrm{E}-00$ & $E+01$ & $E+01$ & -00 & $E+01$ \\
\hline 110 & U234 & $\mathrm{Ci}$ & 9.32E-00 & $9.32 \mathrm{E}-00$ & +01 & & & +01 & +02 & 01 & $E+01$ \\
\hline 110 & U235 & $\mathrm{Ci}$ & 4.12E-01 & 4.12E-01 & +01 & & & -00 & -00 & -01 & $E-01$ \\
\hline 110 & 236 & $\mathrm{Ci}$ & $9.14 E-02$ & E-02 & +01 & & & & -00 & & \\
\hline & & $\mathrm{Ci}$ & & & & & & & +02 & -01 & +01 \\
\hline 10 & Y90 & $\mathrm{Ci}$ & $1.13 E+04$ & +04 & 01 & 03 & 1. & +04 & +04 & -04 & +04 \\
\hline 110 & Zr93 & $\mathrm{Ci}$ & $2.26 \mathrm{E}-00$ & $2.26 \mathrm{E}-00$ & +01 & +01 & $E-00$ & $E+01$ & +01 & & $=-00$ \\
\hline 111 & Al & $\mathrm{kg}$ & $2.26 \mathrm{E}+04$ & $i+04$ & +03 & +04 & $8+04$ & +04 & $\div 04$ & -04 & $E+03$ \\
\hline 111 & $\mathbf{B i}$ & kg & $2.82 \mathrm{E}+03$ & $E+03$ & & & & +03 & +03 & & +03 \\
\hline & $\mathrm{Ca}$ & kg & 7. & +02 & & & & 102 & & & +02 \\
\hline & $\mathrm{Cl}$ & kg & & & & & & .03 & -03 & & +02 \\
\hline & $\mathrm{CO3}$ & $\mathrm{kg}$ & +05 & +03 & -02 & +03 & +04 & +04 & +05 & -04 & +04 \\
\hline 111 & $\mathrm{Cr}$ & $\mathrm{kg}$ & $1.78 \mathrm{E}+03$ & $1.93 E+03$ & +02 & +03 & $2.47 E+03$ & $E+03$ & +03 & .03 & $\therefore+02$ \\
\hline BX111 & $\mathbf{F}$ & $\mathrm{kg}$ & $7.33 E+03$ & $4.13 E+03$ & +02 & +03 & +03 & +03 & +04 & & $E+03$ \\
\hline BX111 & $\mathrm{Fe}$ & $\mathrm{kg}$ & $2.11 E+03$ & $2.64 E+03$ & $E+02$ & +03 & $E+03$ & +03 & +03 & & +03 \\
\hline BX111 & $\mathbf{H g}$ & kg & $E-00$ & $1.02 \mathrm{E}+01$ & +01 & & & +01 & +01 & & -00 \\
\hline 111 & $\mathbf{K}$ & kg & & & & & & & & & +03 \\
\hline & La & kg & & & & & & & & & +01 \\
\hline 11 & $\mathbf{M n}$ & kg & $9.63 \mathrm{E}+01$ & 5.30 & & 00 & & +02 & +02 & & +02 \\
\hline & $\mathrm{Na}$ & $\mathbf{k g}$ & $2.60 \mathrm{E}+05$ & $2.14 E+05$ & 105 & +05 & -05 & 3 & +05 & & +04 \\
\hline 111 & $\mathbf{N i}$ & $\mathrm{kg}$ & $1.23 E+02$ & $1.03 E+02$ & -01 & -00 & $7.06 \mathrm{E}+01$ & $E+02$ & $E+02$ & $\mathrm{E}+02$ & $E+02$ \\
\hline BX111 & $\mathrm{NO} 2$ & kg & $3.20 E+04$ & $1.06 E+04$ & $E+02$ & +03 & $1.65 E+04$ & +04 & $E+04$ & $E+04$ & $E+03$ \\
\hline BX111 & NO3 & kg & $4.00 \mathrm{E}+05$ & $3.63 E+05$ & $=+05$ & & $4.44 E+05$ & +05 & +05 & & $E+05$ \\
\hline & $\mathbf{P}$ & kg & & & & & & & & -04 & $E+03$ \\
\hline BX111 & $\mathbf{P b}$ & kg & $7 \mathrm{E}+02$ & $1.08 \mathrm{E}+02$ & +01 & & 1.3 & +02 & +02 & +02 & +01 \\
\hline & PO & kg & $1.87 \mathrm{E}+04$ & $3.21 E+04$ & & & 2.891 & & & & $E+03$ \\
\hline & $\mathbf{S}$ & $\mathrm{kg}$ & $5.63 E+03$ & & & & & & & & $E+03$ \\
\hline & Si & $\mathrm{kg}$ & $1.64 \mathrm{E}+03$ & $i+03$ & $3.44 \mathrm{E}+02$ & $1.02 E+03$ & 2.5 & +03 & +03 & +03 & $E+03$ \\
\hline & SO4 & $\mathrm{kg}$ & $1.69 E+04$ & $3.62 E \div 03$ & +02 & +03 & 5.5 & +04 & +04 & +03 & $E+03$ \\
\hline BX111 & $\mathbf{S r}$ & kg & $9.50 \mathrm{E}+01$ & $9.60 E+01$ & $3.81 \mathrm{E}-00$ & $1.61 \mathrm{E}+01$ & $8.70 E+01$ & $2.56 \mathrm{E}+02$ & $4.28 \mathrm{E}+02$ & $1.14 \mathrm{E}+02$ & $9.86 \mathrm{E}+01$ \\
\hline BX111 & TOC & kg & $4.66 E+03$ & $1.00 E+03$ & $4.14 E+01$ & $3.29 \mathrm{E}+02$ & $2.33 E+03$ & $4.71 \mathrm{E}+03$ & $6.92 E+03$ & $7 E+03$ & $1.65 E+03$ \\
\hline BX111 & $\mathbf{U}$ & kg & $5.98 \mathrm{E}+02$ & $1.15 E+03$ & $1.58 \mathrm{E}+0 \mathrm{l}$ & $1.60 \mathrm{E}+02$ & $7.36 E+03$ & $2.42 E+04$ & $4.01 E+04$ & $E+03$ & $1.02 E+04$ \\
\hline 1 & $\mathbf{Z r}$ & kg & 1.10E+01 & $2.30 E+01$ & $1.47 \mathrm{E}-01$ & $1.21 \mathrm{E}-00$ & $5.83 \mathrm{E}+01$ & $1.18 E+03$ & $2.27 E+03$ & $3.79 E+02$ & $5.41 \mathrm{E}+02$ \\
\hline
\end{tabular}


Tank Anal. Un

\begin{tabular}{|c|c|c|c|c|}
\hline & & & May98 & Sept98 \\
\hline BX111 & Ac & $\mathrm{Ci}$ & $\overline{1.32 \mathrm{E}-03}$ & $\overline{1.32 \mathrm{E}-03}$ \\
\hline BX111 & Am241 & $\mathrm{Ci}$ & 3.02E+01 & $1.36 \mathrm{E}+01$ \\
\hline BX111 & Am243 & $\mathrm{Ci}$ & $1.04 \mathrm{E}-03$ & $4.69 \mathrm{E}-04$ \\
\hline BX111 & Ba137 & $\mathrm{Ci}$ & $1.02 \mathrm{E}+05$ & $723 E+04$ \\
\hline BX111 & $\mathrm{Cl}$ & $\mathrm{Ci}$ & $2.30 \mathrm{E}+01$ & $1.77 \mathrm{E}+01$ \\
\hline BX111 & Cd113 & $\mathrm{Ci}$ & $4.94 E+01$ & $4.94 E+01$ \\
\hline BX111 & Cm242 & $\mathrm{Ci}$ & 5.17E-04 & 2.33E-04 \\
\hline BX111 & Cm243 & $\mathrm{Ci}$ & $1.06 \mathrm{E}-05$ & 4.78E-06 \\
\hline BX111 & Cm244 & $\mathrm{Ci}$ & $1.46 \mathrm{E}-04$ & 6.58E-05 \\
\hline BX111 & Co60 & $\mathrm{Ci}$ & $2.16 \mathrm{E}+01$ & $2.16 \mathrm{E}+01$ \\
\hline BX111 & Cs134 & $\mathrm{Ci}$ & $1.05 E-00$ & $1.05 \mathrm{E}-00$ \\
\hline BX111 & Cs137 & $\mathrm{Ci}$ & $1.08 E+05$ & $7.64 \mathrm{E}+04$ \\
\hline BX111 & Eu152 & $\mathrm{Ci}$ & E-00 & -00 \\
\hline BX111 & Eu154 & $\mathrm{Ci}$ & $3.64 E+02$ & $3.64 \mathrm{E}+02$ \\
\hline BX111 & Eu155 & $\mathrm{Ci}$ & $1.84 \mathrm{E}+02$ & $1.84 \mathrm{E}+02$ \\
\hline BX111 & I129 & $\mathrm{Ci}$ & $2.49 \mathrm{E}-01$ & $1.90 \mathrm{E}-01$ \\
\hline BX111 & Nb93 & $\mathrm{Ci}$ & $6.75 \mathrm{E}-00$ & $6.75 E-00$ \\
\hline BX111 & Ni59 & $\mathrm{Ci}$ & $2.46 \mathrm{E}-00$ & $2.46 \mathrm{E}-00$ \\
\hline BX111 & Ni63 & $\mathrm{Ci}$ & $2.44 \mathrm{E}+02$ & $2.44 \mathrm{E}+02$ \\
\hline BX111 & Np237 & $\mathrm{Ci}$ & 4.31E-01 & 1.9 \\
\hline 111 & Pa231 & $\mathrm{Ci}$ & 6.7 & 2.9 \\
\hline BX111 & Pu238 & $\mathrm{Ci}$ & 1.77E-00 & 7.98E-01 \\
\hline BX111 & Pu239 & $\mathrm{Ci}$ & $6.82 \mathrm{E}+01$ & $3.07 E+01$ \\
\hline BX111 & Pu240 & $\mathbf{C i}$ & $1.12 E+01$ & $5.04 \mathrm{E}-00$ \\
\hline BX111 & Pu241 & $\mathrm{Ci}$ & $1.26 \mathrm{E}+02$ & $5.68 E+01$ \\
\hline BX111 & Pu242 & $\mathrm{Ci}$ & $6.06 E-04$ & 2.73E-04 \\
\hline 11 & $\operatorname{Ra226}$ & Ci & -05 & 4.0 \\
\hline BX111 & $\operatorname{Ra228}$ & $\mathrm{Ci}$ & 1.15E-00 & $1.15 E-00$ \\
\hline BX111 & Ru106 & $\mathrm{Ci}$ & $4.30 \mathrm{E}-03$ & 4.30E-03 \\
\hline BX111 & Sb125 & $\mathbf{C i}$ & $9.66 \mathrm{E}+01$ & $9.66 \mathrm{E}+01$ \\
\hline BX111 & Se79 & $\mathrm{Ci}$ & $1.94 E-00$ & $1.94 E-00$ \\
\hline BX111 & Sm151 & $C \mathbf{i}$ & $6.69 E+03$ & $6.69 E+03$ \\
\hline B) & Sr89/90 & $\mathrm{Ci}$ & -04 & $2.29 \mathrm{E}+04$ \\
\hline BX111 & Te99 & $\mathrm{Ci}$ & $1.28 \mathrm{E}+02$ & $9.83 E+01$ \\
\hline BX111 & Th229 & $C \mathrm{i}$ & $2.66 \mathrm{E}-02$ & $1.15 E-02$ \\
\hline BX111 & Th232 & $\mathbf{C i}$ & 4.25E-02 & $1.84 \mathrm{E}-02$ \\
\hline BX111 & Tin126 & $\mathrm{Ci}$ & -00 & 2.89E-00 \\
\hline BX111 & Tritium & $\mathrm{Ci}$ & 8.85 & 6.801 \\
\hline BX111 & U232 & $\mathrm{Ci}$ & 6.42E-00 & 7.60E-01 \\
\hline BX111 & U233 & $\mathrm{Ci}$ & $2.46 \mathrm{E}+01$ & 2.91E-00 \\
\hline BX111 & U234 & $\mathrm{Ci}$ & $3.51 \mathrm{E}-00$ & $4.20 \mathrm{E}-01$ \\
\hline BX111 & U235 & $\mathrm{Ci}$ & $1.43 \mathrm{E}-01$ & $1.70 \mathrm{E}-02$ \\
\hline BX111 & U236 & $\mathrm{Ci}$ & 02 & $9.00 \mathrm{E}-03$ \\
\hline BX111 & U238 & $\mathrm{Ci}$ & -00 & 3.8 \\
\hline BX111 & Y90 & $\mathrm{Ci}$ & $2.74 E+04$ & $2.29 E+04$ \\
\hline BX111 & Zr93 & $\mathrm{Ci}$ & $9.34 E-00$ & 9.34E-00 \\
\hline BX112 & Al & kg & $1.11 E+04$ & 1.11E+04 \\
\hline BX112 & $\mathbf{B i}$ & $\mathrm{kg}$ & +04 & $1.42 \mathrm{E}+04$ \\
\hline BX112 & $\mathrm{Ca}$ & $\mathrm{kg}$ & $2.04 \mathrm{E}+03$ & $2.04 E+03$ \\
\hline 112 & CI & $\mathrm{kg}$ & 8.6 & 8.5 \\
\hline BX112 & $\mathrm{CO3}$ & $\mathrm{kg}$ & 03 & $\mathbf{8 . 8 4}$ \\
\hline BX112 & $\mathrm{Cr}$ & kg & $1.05 E+03$ & $1.05 E+03$ \\
\hline BX112 & $\mathbf{F}$ & $\mathrm{kg}$ & $8.70 \mathrm{E}+03$ & $8.71 E+03$ \\
\hline BX112 & $\mathbf{F e}$ & $\mathrm{kg}$ & $7.70 \mathrm{E}+03$ & $7.69 \mathrm{E}+03$ \\
\hline BX112 & $\mathbf{H g}$ & kg & 7.05E-00 & $4.21 \mathrm{E}+01$ \\
\hline BX112 & $\mathbf{K}$ & kg & 3.82 & $3.33 E+02$ \\
\hline BX112 & La & kg & 03 & $0.00 \mathrm{I}$ \\
\hline BX112 & Mn & $\mathrm{kg}$ & 2.6 & 2.631 \\
\hline BX112 & $\mathbf{N a}$ & kg & $6.68 E+04$ & $6.68 E+04$ \\
\hline BX112 & $\mathbf{N i}$ & kg & $2.26 \mathrm{E}-00$ & $9.60 \mathrm{E}+01$ \\
\hline BX112 & $\mathrm{NO} 2$ & kg & $2.09 E+04$ & $2.10 \mathrm{E}+04$ \\
\hline BX112 & NO3 & kg & $6.15 E+04$ & $6.14 \mathrm{E}+04$ \\
\hline BX112 & p & kg & $4.76 E+04$ & NA \\
\hline BX112 & $\mathbf{P b}$ & kg & $1.01 E-00$ & $1.02 \mathrm{E}-00$ \\
\hline BX112 & PO4 & $\mathbf{k g}$ & $1.47 \mathrm{E}+05$ & $E+0$ \\
\hline BX112 & & kg & $1.77 \mathrm{E}+03$ & NA \\
\hline BX112 & $\mathbf{S i}$ & kg & $6.83 E+03$ & $6.83 E+03$ \\
\hline BX112 & SO4 & $\mathrm{kg}$ & $5.30 E+03$ & $5.30 \mathrm{E}+03$ \\
\hline
\end{tabular}

$1 \%$-ile

\section{$0.00 \mathrm{E}+01$}

$6.10 \mathrm{E}-01$

4.26E-05

$0.00 \mathrm{E}+01$

$0.00 \mathrm{E}+01$

$0.00 \mathrm{E}+01$

2.12E-05

4.34E-07

$5.98 \mathrm{E}-06$

$0.00 \mathrm{E}+01$

$0.00 \mathrm{E}+01$

$1.31 \mathrm{E}+03$

$0.00 \mathrm{E}+01$

$0.00 \mathrm{E}+01$

$0.00 \mathrm{E}+01$

$0.00 \mathrm{E}+01$

$0.00 \mathrm{E}+01$

$0.00 \mathrm{E}+01$

$0.00 \mathrm{E}+01$

$1.77 \mathrm{E}-02$

$0.00 \mathrm{E}+01$

7.25E-02

2.79E-00

$4.59 \mathrm{E}-01$

5.16E-00

2.48E-05

$0.00 \mathrm{E}+01$

$0.00 \mathrm{E}+01$

$0.00 \mathrm{E}+01$

$0.00 E+01$

$0.00 E+01$

$0.00 \mathrm{E}+01$

$2.58 \mathrm{E}+02$

$0.00 \mathrm{E}+01$

$0.00 \mathrm{E}+01$

$0.00 \mathrm{E}+01$

$0.00 E+01$

$4.67 \mathrm{E} \div 01$

$0.00 \mathrm{E}+0 \mathrm{I}$

$0.00 \mathrm{E}+0 \mathrm{I}$

$0.00 \mathrm{E}+0 \mathrm{l}$

$0.00 \mathrm{E}+01$

$0.00 \mathrm{E}+0$ !

$0.00 \mathrm{E}+01$

$0.00 \mathrm{E}+0$ !

$0.00 \mathrm{E}+01$

$1.76 \mathrm{E}+02$

$5.95 \mathrm{E}+03$

$7.73 \mathrm{E}+0 \mathrm{I}$

$1.97 \mathrm{E}+02$

$1.79 \mathrm{E}+02$

$1.85 \mathrm{E}+01$

$1.83 \mathrm{E}+02$

$3.04 \mathrm{E}+03$

$0.00 \mathrm{E}+01$

$3.75 \mathrm{E}+01$

3.02E-02

5.07E-00

$3.82 \mathrm{E}+04$

$2.96 \mathrm{E}-00$

$5.66 \mathrm{E}+02$

$9.79 \mathrm{E}+03$

$4.10 \mathrm{E}+03$

$7.08 \mathrm{E}-00$

$1.78 E+03$

$1.95 \mathrm{E}+02$

$5.17 E+02$

$1.26 \mathrm{E}+03$
$10 \%$-ile

$0.00 \mathrm{E}+01$

$3.36 \mathrm{E}-00$

2.63E-04

$0.00 \mathrm{E}+01$

$0.00 \mathrm{E}+01$

6.10E-00

$1.31 \mathrm{E}-04$

2.68E-06

3.70E-05

2.88E-01

$0.00 \mathrm{E}+01$

$1.32 \mathrm{E}+04$

$0.00 \mathrm{E}+01$

$0.00 \mathrm{E}+01$

$0.00 \mathrm{E}+01$

$6.33 \mathrm{E}-02$

$6.60 \mathrm{E}-02$

$0.00 \mathrm{E}+01$

$0.00 \mathrm{E}+01$

$1.09 \mathrm{E}-01$

$0.00 \mathrm{E}+01$

4.48E-01

$1.73 \mathrm{E}+01$

2.84E-00

$3.19 E+01$

$1.53 \mathrm{E}-04$

$0.00 \mathrm{E}+01$

$0.00 \mathrm{E}+01$

$1.07 \mathrm{E}-03$

$0.00 \mathrm{E}+01$

$2.68 \mathrm{E}-01$

$0.00 \mathrm{E}+01$

$7.74 \mathrm{E}+03$

$1.43 \mathrm{E}+01$

$0.00 \mathrm{E}+01$

$0.00 \mathrm{E}+01$

$3.57 \mathrm{E}-01$

$6.55 \mathrm{E}+01$

$0.00 \mathrm{E}+01$

$0.00 \mathrm{E}+01$

$0.00 \mathrm{E}+01$

$0.00 \mathrm{E}+01$

$0.00 \mathrm{E}+01$

$0.00 \mathrm{E}+01$

$0.00 \mathrm{E}+01$

9.13E-02

1.30E+03

$8.58 \mathrm{E}+03$

$2.46 \mathrm{E}+02$

$5.01 E+02$

$3.13 \mathrm{E}+03$

$2.89 \mathrm{E}+02$

$1.16 \mathrm{E}+03$

$5.59 \mathrm{E}+03$

$1.30 \mathrm{E}-00$

$1.20 \mathrm{E}+02$

$7.94 \mathrm{E}-01$

$2.48 E+01$

$5.21 \mathrm{E}+04$

$1.02 \mathrm{E}+01$

$2.92 E+03$

$3.88 \mathrm{E}+04$

$8.68 \mathrm{E}+03$

$6.35 E+01$

$7.20 \mathrm{E}+03$

$1.20 \mathrm{E}+03$

$2.18 \mathrm{E}+03$

$4.44 \mathrm{E}+03$
$50 \%$-ille

1.32E-03

$8.76 \mathrm{E}+01$

1.04E-03

$1.02 \mathrm{E}+05$

$2.30 \mathrm{E}+01$

$4.94 E+01$

5.17E-04

1.06E-05

1.46E-04

$2.45 \mathrm{E}+01$

1.05E-00

$1.25 E+05$

3.04E-00

$3.64 \mathrm{E}+02$

$1.84 \mathrm{E}+02$

2.49E-01

6.75E-00

2.46E-00

$2.44 \mathrm{E}+02$

4.31E-01

6.79E-03

1.77E-00

$6.82 \mathrm{E}+01$

$1.12 \mathrm{E}+01$

$1.26 \mathrm{E}+02$

6.06E-04

9.35E-05

1.15E-00

4.30E-03

$9.66 \mathrm{E}+01$

$1.94 \mathrm{E}-00$

$6.69 \mathrm{E}+03$

8.88E+04

$1.28 \mathrm{E}+02$

2.66E-02

4.25E-02

2.89E-00

$8.85 E+01$

6.42E-00

2.46E+01

3.51E-00

1.43E-01

7.61E-02

5.44E-00

$2.74 E+04$

9.34E-00

$1.06 \mathrm{E}+04$

$1.37 \mathrm{E}+04$

$6.09 \mathrm{E}+02$

$8.57 \mathrm{E}+02$

$8.14 \mathrm{E}+03$

$6.73 \mathrm{E}+02$

$5.69 \mathrm{E}+03$

$1.10 \mathrm{E}+04$

7.05E-00

$2.90 \mathrm{E}+02$

4.14E-00

6.11E +01

$7.70 \mathrm{E}+04$

$3.10 \mathrm{E}+01$

$8.60 \mathrm{E}+03$

$1.03 \mathrm{E}+05$

$1.59 \mathrm{E}+04$

$2.41 \mathrm{E}+02$

$1.69 \mathrm{E}+04$

$3.71 E+03$

$5.47 \mathrm{E}+03$

$9.64 \mathrm{E}+03$ 90\%-ile

6.49E-03 1.12E-02

$2.98 \mathrm{E}+03$

$3.95 \mathrm{E}-03$

$2.19 \mathrm{E}+05$

$6.41 E+01$

$1.23 \mathrm{E}+02$

$1.96 \mathrm{E}-03$

4.03E-05

5.55E-04

$8.32 \mathrm{E}+01$

2.25E-00

$2.53 \mathrm{E}+05$

$1.49 \mathrm{E}+01$

$1.79 \mathrm{E}+03$

$9.05 \mathrm{E}+02$

4.67E-01

$1.38 \mathrm{E}+02$

$1.14 \mathrm{E}+01$

$1.13 E+03$

$1.64 \mathrm{E}-00$

2.61E-02

$6.73 E-00$

$2.59 \mathrm{E}+02$

$4.26 \mathrm{E}+01$

4.79E+02

2.30E-03

3.23E-04

$3.98 \mathrm{E}-00$

9.31E-03

$2.28 \mathrm{E}+02$

$5.27 \mathrm{E}-00$

$3.29 \mathrm{E}+04$

$1.95 \mathrm{E}+05$

$1.05 \mathrm{E}+03$

$1.02 \mathrm{E}-01$

$1.64 \mathrm{E}-01$

$7.19 \mathrm{E}-00$

$1.16 \mathrm{E}+02$

$2.47 \mathrm{E}+01$

$9.47 \mathrm{E}+01$

$1.35 \mathrm{E}+01$

5.50E-01

2.93E-01

$2.09 \mathrm{E}+01$

$8.15 \mathrm{E}+04$

$1.90 \mathrm{E}+02$

$1.94 \mathrm{E}+04$

$2.00 \mathrm{E}+04$

$1.32 E+03$

$1.24 \mathrm{E}+03$

1.37E+04

$1.11 \mathrm{E}+03$

1.21E+04

$1.90 \mathrm{E}+04$

$2.83 \mathrm{E}+01$

$8.82 \mathrm{E}+02$

$2.47 \mathrm{E}+01$

$1.24 \mathrm{E}+02$

$1.12 \mathrm{E}+05$

$2.02 \mathrm{E}+02$

$2.50 \mathrm{E}+04$

$1.81 \mathrm{E}+05$

$2.56 \mathrm{E}+04$

$9.00 \mathrm{E}+02$

$3.05 \mathrm{E}+04$

$7.96 \mathrm{E}+03$

9.17E+03

$2.01 \mathrm{E}+04$

$5.80 \mathrm{E}+03$

$6.99 \mathrm{E}-03$

$3.39 \mathrm{E}+05$

$1.07 \mathrm{E}+02$

$2.58 \mathrm{E}+02$

$3.48 \mathrm{E}-03$

7.13E-05

9.81E-04 


\begin{tabular}{|c|c|c|c|c|c|c|c|c|c|c|c|}
\hline ank & Anal. & Un & $B B$ & $\frac{B B}{t 98}$ & $1 \%$-ile & $10 \%$-ile & $50 \%$-ile & $90 \%-i l e$ & $99 \%$-ile & Mean & Std Dev \\
\hline 2 & Sr & kg & $1.07 \mathrm{E}+02$ & $1.07 \mathrm{E}+02$ & $9.47 \mathrm{E}-00$ & $5.22 \mathrm{E}+01$ & $1.45 E+02$ & $3.55 \mathrm{E}+02$ & $9.28 \mathrm{E}+02$ & $1.90 E+02$ & $1.70 \mathrm{E}+02$ \\
\hline 1112 & TOC & $\mathrm{kg}$ & $7.80 \mathrm{E}+02$ & $7.80 \mathrm{E}+02$ & $8.78 \mathrm{E}-00$ & $2.07 \mathrm{E}+02$ & $.66 \mathrm{E}+02$ & $1.05 E+03$ & $1.86 \mathrm{E}+03$ & $.11 E+02$ & $3.56 \mathrm{E}+02$ \\
\hline $\mathbf{X 1 1 2}$ & $\mathrm{U}$ & kg & $8.48 \mathrm{E}+02$ & $8.48 \mathrm{E}+02$ & $5.86 \mathrm{E}+01$ & $3.94 \mathrm{E}+02$ & $.37 E+03$ & $75 E+03$ & $1.60 \mathrm{E}+04$ & $42 E+03$ & $13 E+03$ \\
\hline 6112 & $\mathbf{Z r}$ & $\mathrm{kg}$ & $3.63 E+02$ & $6.35 \mathrm{E}+01$ & $28 \mathrm{E}-01$ & $4.59 \mathrm{E}-00$ & $57 E+01$ & $79 \mathrm{E}+02$ & +02 & $7.07 \mathrm{Y}$ & \\
\hline 12 & Ac227 & $\overline{\mathrm{Ci}}$ & $3.65 E-05$ & $3.65 \mathrm{E}-05$ & $0.00 \mathrm{E}+01$ & 3.24E-06 & $3.65 \mathrm{E}-05$ & $2.41 \mathrm{E}-04$ & 43E-04 & $9.12 \mathrm{E}-05$ & $1.42 \mathrm{E}-04$ \\
\hline 12 & Am241 & $\mathrm{Ci}$ & -01 & $1.65 \mathrm{E}-00$ & $1.04 \mathrm{E}-00$ & $1.16 \mathrm{E}+01$ & $E+01$ & $40 \mathrm{E}+02$ & $.58 E+03$ & $.45 \mathrm{E}+02$ & $5.54 \mathrm{E}+02$ \\
\hline 112 & Am243 & $\mathrm{Ci}$ & $7.01 E-06$ & 2.71E-05 & 4.37E-07 & $2.29 \mathrm{E}-06$ & 01E-06 & $1.48 \mathrm{E}-05$ & $02 \mathrm{E}-05$ & $8.18 E-06$ & $.95 \mathrm{E}-06$ \\
\hline 112 & Ba137 & $\mathbf{C i}$ & $3.99 E+04$ & $3.97 E+04$ & $3.25 \mathrm{E}+03$ & $1.44 \mathrm{E}+04$ & $99 E+04$ & $2.08 \mathrm{E}+05$ & $66 \mathrm{E}+05$ & $7.22 E+04$ & $47 \mathrm{E}+04$ \\
\hline $\mathbf{X 1 1 2}$ & C14 & $\mathbf{C i}$ & $.31 E-01$ & 2.31E-01 & $0.00 \mathrm{E}+01$ & $2.58 \mathrm{E}-03$ & -31E-01 & $4.86 \mathrm{E}-01$ & $7.29 \mathrm{E}-01$ & $2.43 \mathrm{E}-01$ & $80 \mathrm{E}-01$ \\
\hline 112 & Cd113 & $\mathbf{C i}$ & $5.84 \mathrm{E}-01$ & $5.84 \mathrm{E}-01$ & $0.00 \mathrm{E}+01$ & $1.07 \mathrm{E}-01$ & $5.84 \mathrm{E}-01$ & $2.35 \mathrm{E}-00$ & $5.05 E-00$ & $9.79 E-01$ & $.10 E-00$ \\
\hline 112 & Cm242 & $\mathrm{Ci}$ & $9.59 E-04$ & $3.71 E-03$ & $5.98 \mathrm{E}-05$ & $E-04$ & $9.59 \mathrm{E}-04$ & $.02 E-03$ & E-03 & $1.12 E-03$ & $4 E-04$ \\
\hline 112 & $\mathrm{Cm} 243$ & $\mathbf{C i}$ & 3.79E-05 & $1.47 \mathrm{E}-04$ & E-06 & $1.24 \mathrm{E}-05$ & 3.79E-05 & $7.98 \mathrm{E}-05$ & E-04 & $4.42 E-05$ & E-05 \\
\hline 112 & $\operatorname{Cm} 244$ & $\mathbf{C i}$ & $2.29 \mathrm{E}-04$ & $8.86 \mathrm{E}-04$ & $E-05$ & $E-05$ & $2.29 \mathrm{E}-04$ & $4.82 \mathrm{E}-04$ & $E-04$ & $2.67 \mathrm{E}-04$ & $1.94 \mathrm{E}-04$ \\
\hline 112 & Co60 & $\mathbf{C i}$ & 1.73E-01 & 1.73E-01 & & -01 & 3.33E-00 & $\mathrm{E}+01$ & $E+02$ & +01 & $E+01$ \\
\hline 12 & Cs134 & $\mathrm{Ci}$ & 1.1 & 1.11E-02 & & & E-02 & -02 & & & \\
\hline 12 & Cs137 & $\mathrm{Ci}$ & $4.22 E+04$ & $4.20 E+04$ & +03 & +03 & $1.72 E+04$ & +04 & -05 & +04 & $E+04$ \\
\hline & Eu152 & $\mathbf{C i}$ & -02 & $4.72 \mathrm{E}-02$ & +01 & -03 & $4.72 \mathrm{E}-02$ & E-01 & $E-01$ & -01 & $E-01$ \\
\hline 112 & Eu154 & $\mathbf{C i}$ & 2.56 & $2.56 \mathrm{E}-00$ & $0.00 \mathrm{E}+01$ & $2.27 \mathrm{E}-01$ & $2.56 \mathrm{E}-00$ & $1.69 \mathrm{E}+01$ & +01 & $6.40 \mathrm{E}-00$ & $9.95 \mathrm{E}-00$ \\
\hline 112 & Eu155 & $\mathbf{C i}$ & 3.19E-00 & 3.19E-00 & $8+01$ & -01 & 3.19E-00 & +01 & & -00 & $E+01$ \\
\hline 112 & I129 & $\mathrm{Ci}$ & $3.32 \mathrm{E}-03$ & 3.32E-03 & +01 & -03 & 3.32E-03 & $E-03$ & -03 & -03 & $E-03$ \\
\hline & Nb93 & $\mathrm{Ci}$ & $1.41 \mathrm{E}-01$ & 1.41E-01 & +01 & 0.0 & & & & & \\
\hline & Ni59 & $\mathrm{Ci}$ & 9.9 & 9.9 & & -02 & & 01 & .00 & 01 & \\
\hline 112 & Ni63 & Ci & $8.96 \mathrm{E}-00$ & $8.96 \mathrm{E}-00$ & & -00 & .00 & +01 & +02 & 01 & +01 \\
\hline 112 & Np237 & $\mathbf{C i}$ & -03 & $8.43 E-03$ & 04 & $8-03$ & -03 & $\varepsilon-02$ & $E-02$ & -03 & $E-03$ \\
\hline 112 & Pa231 & $\mathbf{C i}$ & $8.59 \mathrm{E}-05$ & 8.59E-05 & +01 & $E-05$ & $8.59 E-05$ & $E-04$ & $E-03$ & -04 & $2.14 \mathrm{E}-04$ \\
\hline 112 & Pu238 & $\mathrm{Ci}$ & 2.59E-01 & $1.00 E-00$ & & 8.02 & 2.59E-01 & -01 & & -01 & $E-01$ \\
\hline 112 & Pu239 & $\mathbf{C i}$ & $3.37 E+01$ & $1.30 \mathrm{E}+02$ & & +01 & 01 & +01 & +02 & +01 & $E+01$ \\
\hline & Pu240 & $\mathrm{Ci}$ & 3.18E-00 & $1.23 E+01$ & & & & & & & \\
\hline 12 & Pu241 & $\mathrm{Ci}$ & 1.12 & 4.3 & & 00 & 1.1 & -01 & & 01 & -00 \\
\hline 3X112 & Pu242 & $\mathrm{Ci}$ & $5.09 \mathrm{E}-05$ & $1.97 \mathrm{E}-04$ & -06 & -05 & $\varepsilon-05$ & -04 & -04 & -05 & $E-05$ \\
\hline 112 & Ra226 & $\mathrm{Ci}$ & $7.06 \mathrm{E}-06$ & $7.06 \mathrm{E}-06$ & 01 & $E-06$ & $7.06 \mathrm{E}-06$ & $E-05$ & -05 & -06 & E-05 \\
\hline 12 & Ra228 & C & $1.89 \mathrm{E}-03$ & $1.89 \mathrm{E}-03$ & 01 & & 03 & & & & -03 \\
\hline & Ru106 & $\mathrm{Ci}$ & $2.14 \mathrm{E}-05$ & E-05 & & & & & & & \\
\hline & Sb125 & $\mathrm{Ci}$ & 6.6 & 6.6 & 01 & +01 & 01 & 00 & & & -01 \\
\hline 112 & Se79 & $\mathrm{Ci}$ & $3.64 \mathrm{E}-02$ & 3.64E-02 & -03 & $E-02$ & $3.64 \mathrm{E}-02$ & -02 & -01 & $:-02$ & $E-02$ \\
\hline 12 & Sm15l & $\mathrm{Ci}$ & $1.33 \mathrm{E}+02$ & $1.33 E+02$ & +01 & +01 & $1.33 E+02$ & +02 & -03 & 02 & $E+02$ \\
\hline 12 & Sr89/90 & $\mathrm{Ci}$ & $4.92 \mathrm{E}+03$ & $4.91 E+03$ & -02 & +03 & +04 & +05 & 105 & 04 & $E+04$ \\
\hline 12 & Te99 & $\mathrm{Ci}$ & 1.7 & -00 & & & & & & & -01 \\
\hline & Th229 & $\mathrm{Ci}$ & 4.3 & 4.3 & & & & & & & \\
\hline & Th & $\mathrm{Ci}$ & & 04 & 01 & 05 & 04 & -04 & & 04 & -04 \\
\hline 12 & Tin126 & $\mathrm{Ci}$ & $5.49 \mathrm{E}-02$ & $5.49 \mathrm{E}-02$ & 01 & -02 & -02 & $E-01$ & -01 & -02 & -01 \\
\hline 12 & Tritium & $\mathrm{Ci}$ & $1.36 \mathrm{E}-00$ & $1.36 \mathrm{E}-00$ & 01 & -01 & -00 & -00 & $E-00$ & -00 & $E-01$ \\
\hline 12 & U232 & $\mathrm{Ci}$ & $4.87 \mathrm{E}-03$ & $1.72 \mathrm{E}-04$ & 01 & -03 & -63 & 02 & & 03 & $E-02$ \\
\hline 12 & U233 & c & 1.8 & 6.3 & & & & & & & -02 \\
\hline & - $>$ & C & & 2. & & & & 01 & 02 & & \\
\hline & & $\mathrm{Ci}$ & & 02 & & -02 & & -00 & -00 & 01 & -01 \\
\hline 12 & U236 & $\mathrm{Ci}$ & 8.06 & $2.83 \mathrm{E}-03$ & +01 & $=-02$ & .02 & $=-01$ & $=00$ & $E-01$ & $E-01$ \\
\hline 112 & U238 & $\mathrm{Ci}$ & 8.05E-00 & 2.83E-01 & $\div 01$ & $E-\infty 0$ & E-00 & +01 & 102 & +01 & +01 \\
\hline BX112 & Y90 & $\mathrm{Ci}$ & $4.92 \mathrm{E}+03$ & $4.91 \mathrm{E}+03$ & +01 & +02 & +03 & -04 & & 04 & +04 \\
\hline 112 & Zr93 & C & & & & & & & & & -01 \\
\hline & Al & kg & 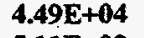 & 4. & & & & & & & 04 \\
\hline & $\mathbf{B i}$ & $\mathbf{k g}$ & & 03 & & & & & 03 & 02 & +02 \\
\hline & $\mathrm{Ca}$ & kg & $4.86 E+03$ & $4.86 E+03$ & +01 & +02 & 2.0 & +03 & +04 & $\varepsilon+03$ & $E+03$ \\
\hline BY101 & $\mathrm{Cl}$ & kg & $4.55 E+03$ & $4.55 E+03$ & $E-00$ & +01 & $1.04 E+03$ & +03 & +03 & +03 & $E+03$ \\
\hline BY101 & $\mathrm{CO} 3$ & $\mathrm{~kg}$ & $1.88 \mathrm{E}+05$ & $1.88 \mathrm{E}+05$ & $E+03$ & +04 & $1.36 E+05$ & +05 & +05 & -05 & $\varepsilon+05$ \\
\hline BY101 & $\mathrm{Cr}$ & kg & $3.32 \mathrm{E}+03$ & $3.32 E+03$ & 6. & 9 & 1.4 & -04 & & & +03 \\
\hline 01 & $\mathbf{F}$ & kg & & 1.3 & & & & 04 & 04 & & +04 \\
\hline & Fe & kg & & & & & & & & & +04 \\
\hline & $\mathbf{H g}$ & kg & & $E+01$ & 01 & +01 & & -01 & -01 & -01 & +01 \\
\hline 101 & $\mathbf{K}$ & kg & $4.12 E+03$ & $4.12 \mathrm{E}+03$ & & +03 & 5.9 & +04 & & -03 & $E+03$ \\
\hline & $\mathbf{L a}$ & $\mathrm{kg}$ & $5.94 \mathrm{E}-01$ & $0.00 \mathrm{E}+01$ & & & 4.7 & & & +01 & $E+01$ \\
\hline & Mn & kg & $2.43 E+02$ & $2.43 E+02$ & 0.0 & $E-01$ & 2.0 & +03 & +03 & -02 & $E+02$ \\
\hline BY101 & $\mathbf{N a}$ & $\mathbf{k g}$ & $4.50 \mathrm{E}+05$ & $4.50 \mathrm{E}+05$ & $3.38 \mathrm{E}+05$ & $F+05$ & $6.07 E+05$ & +05 & +05 & $E+05$ & $E+05$ \\
\hline BY101 & $\mathbf{N}$ & kg & $2,41 E+03$ & $2.41 E+03$ & $6.20 \mathrm{E}-02$ & $4.88 \mathrm{E}-00$ & $7.84 \mathrm{E}+02$ & $E+03$ & $3.54 \mathrm{E}+03$ & $E+02$ & $E+02$ \\
\hline BY10 & $\mathrm{NO} 2$ & kg & $6.43 E+04$ & $6.43 E+04$ & 1781 & $9.14 \mathrm{E}+01$ & $2.77 E+04$ & $E+04$ & +05 & E+04 & $3.14 E+04$ \\
\hline & & kg & & $\mathbf{E}+05$ & +05 & $: 05$ & $7.81 E+05$ & $1.12 \mathrm{E}+06$ & $2.45 E+06$ & $:+05$ & $3.41 E+05$ \\
\hline & $\mathbf{P}$ & kg & $4.19 \mathrm{E}+04$ & NA & $2.57 E+04$ & +04 & $5.75 \mathrm{E}+04$ & +04 & & -04 & $1.69 E+04$ \\
\hline & $\mathbf{P b}$ & $\mathrm{kg}$ & & $i+02$ & $0.00 \mathrm{E}+01$ & $3.01 E-00$ & $1.72 E+02$ & $4.49 \mathrm{E}+02$ & $7.09 \mathrm{E}+02$ & $2.03 E+02$ & $1.82 \mathrm{E}+02$ \\
\hline
\end{tabular}




\begin{tabular}{|c|c|c|c|c|c|c|c|c|c|c|c|}
\hline ank & Anal. & Un & $\operatorname{May} 98$ & $\operatorname{Sept98}$ & $1 \%$-ile & $10 \%$-ile & $50 \%$-ile & $90 \%$-ile & 99\%-ile & Mean & Std Dev \\
\hline & PO4 & $\mathrm{kg}$ & $1.30 \mathrm{E}+05$ & $4.23 \mathrm{E}+04$ & $4.08 \mathrm{E}+04$ & $7.43 E+04$ & $1.20 \mathrm{E}+05$ & $1.79 \mathrm{E}+05$ & $2.64 \mathrm{E}+05$ & $1.25 E+05$ & $4.59 \mathrm{E}+04$ \\
\hline & S & $\mathrm{kg}$ & $3.18 \mathrm{E}+04$ & NA & 2.32E-00 & $E+02$ & $6.61 \mathrm{E}+03$ & $8 E+04$ & & $E+03$ & $1 E+03$ \\
\hline Y101 & Si & kg & $3.30 \mathrm{E}+03$ & $3.30 E+03$ & $1.78 \mathrm{E}-00$ & $.75 \mathrm{E}+03$ & $8.31 E+03$ & $1.49 \mathrm{E}+04$ & $.26 \mathrm{E}+04$ & $8.65 E+03$ & $4.74 E+03$ \\
\hline & so & $\mathrm{kg}$ & $9.54 E+04$ & $3.18 E+04$ & 3.19E-00 & $6.89 \mathrm{E}+02$ & $1.88 \mathrm{E}+04$ & $5.48 E+04$ & $9.05 \mathrm{E}+04$ & $2.38 E+04$ & $2.25 \mathrm{E}+04$ \\
\hline 101 & $\mathrm{Sr}$ & $\mathrm{kg}$ & $2.35 E+03$ & $2.35 E+03$ & $0.00 \mathrm{E}+01$ & $1.64 \mathrm{E}-01$ & $2.21 \mathrm{E}+02$ & $7.74 E+02$ & $1.26 \mathrm{E}+03$ & $3.08 E+02$ & $3.28 \mathrm{E}+02$ \\
\hline Y101 & TOC & kg & $1.23 E+04$ & $1.23 E+04$ & $1.32 \mathrm{E}-00$ & $21 E+03$ & $8.72 \mathrm{E}+03$ & $1.65 \mathrm{E}+04$ & $2.31 E+04$ & $9.13 \mathrm{E}+03$ & $5.35 \mathrm{E}+03$ \\
\hline 101 & $\mathbf{U}$ & kg & $7.69 \mathrm{E}+03$ & $7.69 \mathrm{E}+03$ & $0.00 \mathrm{E}+01$ & $1 E \div 03$ & $2.18 E+04$ & $6.63 E+04$ & $1.44 E+05$ & $3.05 \mathrm{E}+04$ & \\
\hline Y101 & $\mathbf{Z r}$ & kg & $1.70 \mathrm{E}+02$ & $1.70 \mathrm{E}+02$ & $0.00 \mathrm{E}+01$ & 19E-02 & $2.54 E+02$ & $5.42 \mathrm{E}+03$ & $1.08 \mathrm{E}+04$ & $1.77 \mathrm{E}+03$ & +03 \\
\hline & Ac227 & $\overline{\mathrm{Ci}}$ & $2.62 \mathrm{E}-03$ & $2.62 \mathrm{E}-03$ & $0 E+01$ & & $2.62 \mathrm{E}-03$ & $4 \mathrm{E}-02$ & -02 & & \\
\hline & Am241 & & $5.90 \mathrm{E}+01$ & $5.90 \mathrm{E}+01$ & $E+01$ & & $1.08 \mathrm{E}+02$ & $7.49 \mathrm{E}+03$ & +04 & +03 & +03 \\
\hline & Am 243 & & $2.04 \mathrm{E}-03$ & $2.04 \mathrm{E}-03$ & $0 \mathrm{E}+01$ & -06 & $2.04 E-03$ & $2.91 \mathrm{E}-02$ & $E-02$ & $4 E-03$ & $31 E-02$ \\
\hline & Ba137 & $\mathbf{C i}$ & $2.46 E+05$ & $2.52 \mathrm{E}+0.5$ & $0.00 \mathrm{E}+01$ & +01 & $46 \mathrm{E}+05$ & & $E+05$ & +05 & $E+05$ \\
\hline & $\mathrm{Cl4}$ & $\mathrm{Ci}$ & $4.54 \mathrm{E}+01$ & $4.54 E+01$ & $0.00 \mathrm{E}+01$ & $E+01$ & $4.54 E+01$ & $1.15 \mathrm{E}+02$ & & & $E+01$ \\
\hline YY101 & Cd113 & $\mathrm{Ci}$ & $9.67 E+01$ & $9.67 \mathrm{E}+01$ & $0.00 E+01$ & $\therefore+01$ & $9.67 E+01$ & & $E+02$ & $E+02$ & $E+02$ \\
\hline 101 & $\mathrm{Cm} 242$ & $\mathrm{Ci}$ & $7.94 \mathrm{E}-04$ & $7.94 \mathrm{E}-04$ & $0.00 \mathrm{E}+01$ & $5 \mathrm{E}-06$ & $7.94 \mathrm{E}-04$ & $1.13 E-02$ & $2.06 \mathrm{E}-02$ & $3.67 \mathrm{E}-03$ & $\mathrm{E}-03$ \\
\hline & $\mathrm{Cm} 243$ & $\mathrm{Ci}$ & $1.60 \mathrm{E}-05$ & $1.60 \mathrm{E}-05$ & $E+01$ & -08 & $1.60 \mathrm{E}-05$ & -04 & & & \\
\hline I & $\mathrm{Cm} 244$ & $\mathrm{Ci}$ & $2.71 \mathrm{E}-04$ & $2.71 \mathrm{E}-04$ & $E+01$ & -07 & $2.71 \mathrm{E}-04$ & -03 & -03 & -03 & \\
\hline l & Co60 & $\mathrm{Ci}$ & $4.22 \mathrm{E}+01$ & $4.22 \mathrm{E}+01$ & $E+01$ & -03 & $5.67 \mathrm{E}+01$ & +02 & +02 & +01 & \\
\hline & Cs134 & $\mathrm{Ci}$ & $2.05 \mathrm{E}-60$ & $2.05 \mathrm{E}-00$ & $E+01$ & +01 & $2.05 \mathrm{E}-00$ & & -00 & $-\infty 0$ & $=00$ \\
\hline & Cs137 & $\mathbf{C i}$ & $2.60 E+05$ & $2.67 \mathrm{E}+05$ & $E+01$ & & $.02 E+05$ & & & & \\
\hline & Eu152 & $\mathbf{C i}$ & $5.93 \mathrm{E}-00$ & 5.93E-00 & $E+01$ & & & & & & +01 \\
\hline 101 & Eu154 & $\mathrm{Ci}$ & $7.11 E+02$ & $7.11 \mathrm{E}+02$ & $E+01$ & +01 & $7.11 \mathrm{E}+02$ & $E+03$ & +03 & $E+03$ & $E+03$ \\
\hline 101 & Eu155 & $\mathrm{Ci}$ & $3.60 \mathrm{E}+02$ & $3.60 \mathrm{E}+02$ & $E+01$ & & $3.60 \mathrm{E}+02$ & +03 & +03 & $\varepsilon+02$ & +02 \\
\hline 01 & 129 & $\mathrm{Ci}$ & $4.86 \mathrm{E}-01$ & $4.86 \mathrm{E}-01$ & -01 & & -01 & $-\infty$ & & -01 & \\
\hline 101 & $\mathrm{Nb93}$ & $\mathrm{Ci}$ & $1.32 \mathrm{E}+01$ & $1.32 \mathrm{E}+01$ & $\mathrm{E}+01$ & +01 & +01 & +02 & +02 & $E+01$ & $E+02$ \\
\hline & Ni59 & $\mathrm{Ci}$ & $4.82 \mathrm{E}-00$ & $4.82 \mathrm{E}-00$ & $E+01$ & & -00 & & & & \\
\hline & $\mathrm{Ni} 63$ & $\mathrm{Ci}$ & $4.78 \mathrm{E}+02$ & $4.78 E+02$ & $0.00 \mathrm{E}+01$ & 01 & & & +03 & & +02 \\
\hline 101 & Np237? & $\mathrm{Ci}$ & $8.43 E-01$ & 8.43E-01 & $E+01$ & & & & +01 & & \\
\hline & Pa231 & $\mathrm{Ci}$ & $1.33 \mathrm{E}-02$ & 1.33E-02 & $E+01$ & & & & & & \\
\hline 101 & Pu238 & $\mathrm{Ci}$ & $3.36 \mathrm{E}-00$ & $1.55 \mathrm{E}-00$ & $E+01$ & -03 & 3.3 & -01 & 01 & +01 & +01 \\
\hline 101 & Pu239 & $\mathrm{Ci}$ & $6.50 E+01$ & $5.55 E+01$ & $E+01$ & & -01 & +02 & & & +02 \\
\hline 101 & $\mathrm{Pu} 240$ & $\mathrm{Ci}$ & Incl. in 239 & $9.50 \mathrm{E}-00$ & $E+01$ & & -01 & & +03 & & +02 \\
\hline 101 & u241 & $\mathrm{Ci}$ & $2.42 \mathrm{E}+02$ & $1.11 E+02$ & $E \div 01$ & & $42 E+02$ & & & +03 & $\$ 03$ \\
\hline & u242 & Ci & $1.16 E-03$ & $5.36 \mathrm{E}-04$ & $:+01$ & & & & & & \\
\hline$\{101$ & 9226 & $\mathrm{Ci}$ & 2.01E-04 & $2.01 \mathrm{E}-04$ & +01 & & -04 & & & -04 & -04 \\
\hline 101 & $\operatorname{Ra228}$ & C & $2.25 \mathrm{E}-00$ & $2.25 \mathrm{E}-00$ & +01 & -01 & -00 & -00 & -01 & $8-00$ & -00 \\
\hline 101 & Ru106 & $\mathbf{C i}$ & $8.41 E-03$ & $8.41 E-03$ & $E+01$ & -04 & -03 & -02 & -02 & $E-02$ & -02 \\
\hline 101 & Sb125 & $\mathrm{Ci}$ & $1.89 \mathrm{E}+02$ & $1.89 \mathrm{E}+02$ & & & -02 & & & 102 & +02 \\
\hline 101 & Se79 & C & 3.79E-00 & 3.79E-00 & $E+01$ & & & & & & $=00$ \\
\hline & Sm151 & $\mathrm{c}$ & 4 & 1.3 & 01 & & .04 & & & & +04 \\
\hline 101 & Sr89/90 & $\mathbf{C i}$ & $2.28 \mathrm{E}+05$ & 2.20 & & -02 & +05 & -05 & & 05 & +05 \\
\hline 101 & Te99 & $\mathbf{C i}$ & $2.51 \mathrm{E}+02$ & $2.51 E+02$ & $E+01$ & -01 & +02 & +03 & +03 & 02 & +02 \\
\hline BY101 & Th229 & $\mathrm{Ci}$ & $5.20 \mathrm{E}-02$ & $5.20 \mathrm{E}-02$ & $E+01$ & -01 & -02 & & & & -02 \\
\hline BY101 & Th232 & $\mathbf{C i}$ & 8.32E-02 & 8.32E-02 & $E+01$ & +01 & & & & & $8-01$ \\
\hline & Tin126 & $\mathrm{Ci}$ & & $5.66 \mathrm{E}-00$ & & & & & & & $=-00$ \\
\hline & Tritium & $\mathbf{C i}$ & 1.7 & 1.73 & & & & & & & \\
\hline 01 & U232 & C & +01 & -00 & & & 1.2 & & -02 & +01 & +01 \\
\hline$Y 101$ & U233 & $\mathbf{C i}$ & $4.81 \mathrm{E}+01$ & 5.53E-00 & $E+01$ & +01 & 4.81 & +02 & +02 & +01 & +01 \\
\hline BY101 & U234 & $\mathrm{Ci}$ & $2.27 \mathrm{E}+01$ & $2.61 \mathrm{E}-00$ & $E+01$ & +01 & $2.27 \mathrm{E}+01$ & & +02 & $\div 01$ & $E+01$ \\
\hline BY101 & U235 & $\mathbf{C i}$ & 9.92E-01 & 1.14E-01 & $E+01$ & +01 & & & & .00 & E-00 \\
\hline BY101 & U236 & $\mathbf{C i}$ & $2.36 \mathrm{E}-01$ & $2.72 \mathrm{E}-02$ & & & & & & & -01 \\
\hline Y101 & U238 & $\mathrm{Ci}$ & $2.66 \mathrm{E}+01$ & & & & & & & & +01 \\
\hline & Y90 & $c$ & 2.2 & 2.2 & & & & & & & +05 \\
\hline 101 & Zr93 & C & $1.83 \mathrm{E}+01$ & $1.83 E+01$ & & & & & & +02 & +02 \\
\hline & Al & k & $6.75 E+04$ & $6.75 E+04$ & +03 & & 104 & & -04 & +04 & +04 \\
\hline BY102 & $\mathbf{B i}$ & kg & $1.00 E+02$ & $1.00 \mathrm{E}+02$ & +01 & & -01 & & +03 & $E+02$ & $E+02$ \\
\hline BY102 & $\mathrm{Ca}$ & kg & $5.52 \mathrm{E}+02$ & $5.52 \mathrm{E}+02$ & $E+02$ & +02 & $1.12 E+03$ & +03 & +04 & $E+03$ & $E+03$ \\
\hline BY102 & $\mathrm{Cl}$ & $\mathbf{k g}$ & $1.92 \mathrm{E}+03$ & $1.92 \mathrm{E}+03$ & $E+02$ & & $2.34 E+03$ & & $E+03$ & $E+03$ & $E+02$ \\
\hline & $\cos$ & kg & & & & & & & & & $i+04$ \\
\hline BY102 & $\mathrm{Cr}$ & kg & 2.9 & 2.94 & & +03 & $2.60 \mathrm{E}+03$ & +03 & +03 & +03 & +03 \\
\hline 102 & $\mathbf{F}$ & kg & $2.83 E+04$ & $2.83 \mathrm{E}+04$ & & & 1.391 & & & 1. & +03 \\
\hline 102 & $\mathrm{Fe}$ & kg & $9.65 \mathrm{E}+02$ & $9.65 E+02$ & & & $1.73 E+03$ & & & $E+03$ & +03 \\
\hline & Hg & $\mathbf{k g}$ & $8.60 \mathrm{E}-00$ & $0.00 \mathrm{E}+01$ & & & & & & $E+01$ & \\
\hline & $\mathbf{K}$ & $\mathrm{kg}$ & $3.05 E+03$ & $3.05 E+03$ & $5.43 \mathrm{E}-01$ & $E+02$ & $\mathbf{3 . 8 7}$ & & & $E+03$ & +03 \\
\hline & La & kg & $3.53 E-01$ & $0.00 \mathrm{E}+01$ & $2.05 \mathrm{E}+02$ & $3.90 \mathrm{E}+02$ & $6.02 E+02$ & $8.28 E+02$ & $1.03 E+03$ & $6.07 E+02$ & +02 \\
\hline & $\mathbf{M n}$ & $\mathrm{kg}$ & $7.00 \mathrm{E}+01$ & $7.00 \mathrm{E}+01$ & $3.84 \mathrm{E}-02$ & $1.93 \mathrm{E}+01$ & $3.96 \mathrm{E}+02$ & $9.14 \mathrm{E}+02$ & $1.33 E+03$ & $4.49 \mathrm{E}+02$ & $3.33 E+02$ \\
\hline & $\mathrm{N}_{2}$ & $\mathrm{~kg}$ & $4.20 \mathrm{E}+05$ & $4.20 \mathrm{E}+05$ & $1.55 \mathrm{E}+05$ & $E+05$ & $439 E+05$ & & & $E+05$ & \\
\hline & $\mathbf{N}$ & $\mathrm{kg}$ & & $1.90 \mathrm{E}+02$ & $00 \mathrm{E}+01$ & $1 \mathrm{E}-00$ & $4.59 \mathrm{E}+02$ & 1. & $2.33 E+0$. & $5.90 \mathrm{E}+02$ & $5.77 \mathrm{E}+$ \\
\hline
\end{tabular}




\begin{tabular}{|c|c|c|c|c|c|c|c|c|c|c|c|}
\hline$a n k$ & nal. & $n$ & Mar $\frac{B B}{98}$ & Sept98 & $1 \%$-ile & $10 \%$-ile & $50 \%$-ile & $90 \%$-ile & $99 \%$-ile & Mean & Std Dev \\
\hline 102 & $\mathbf{0 2}$ & kg & $2 . \overline{19 E+04}$ & $2.19 \bar{E}+04$ & $2.70 E+03$ & $1.26 \mathrm{E}+04$ & $3.03 E+04$ & $5.14 \mathrm{E}+04$ & $7.36 \mathrm{E}+04$ & $3.16 \mathrm{E} \div 04$ & $.53 E+04$ \\
\hline 102 & & kg & $1.49 \mathrm{E}+05$ & $1.49 \mathrm{E}+05$ & $5.06 \mathrm{E}+04$ & $9.73 E+04$ & $1.92 \mathrm{E}+05$ & $E+05$ & $8 \mathrm{E}+06$ & $E+05$ & $38 \mathrm{E}+05$ \\
\hline 102 & & kg & $4.20 \mathrm{E}+04$ & NA & $E+03$ & -03 & 04 & $E+04$ & $0 \mathrm{E}+04$ & $9 E+04$ & $5.65 E+03$ \\
\hline 102 & $\mathbf{P b}$ & kg & $1.47 \mathrm{E}+02$ & $1.47 \mathrm{E}+02$ & 52E-01 & $97 E+01$ & $.22 \mathrm{E}+02$ & $2.33 \mathrm{E}+02$ & $33 \mathrm{E}+02$ & $1.32 \mathrm{E}+02$ & $7.72 \mathrm{E}+01$ \\
\hline 102 & PO4 & kg & $1.30 E+05$ & $4.24 E+04$ & $73 E+03$ & $59 E+04$ & $.24 E+04$ & $5.81 \mathrm{E}+04$ & $.22 \mathrm{E}+04$ & $3.52 E+04$ & $1.75 E+04$ \\
\hline 10? & 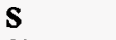 & kg & $9.08 \mathrm{E}+04$ & NA & $85 \mathrm{E}+03$ & $16 \mathrm{E}+04$ & $32 \mathrm{E}+04$ & +04 & $13 E+04$ & $E \div 04$ & $E+03$ \\
\hline & $\mathbf{S i}$ & kg & $6.84 E+03$ & $\mathbf{E}+\mathbf{0 3}$ & $7 E+01$ & +03 & -03 & $E+03$ & $95 \mathrm{E}+03$ & $E+03$ & $5 E+03$ \\
\hline & & $\mathrm{kg}$ & $72 \mathrm{E}+05$ & $9.08 E+04$ & $2.52 \mathrm{E}+04$ & $20 \mathrm{E}+04$ & $.70 E+04$ & $E+05$ & $6 E+05$ & & $E+04$ \\
\hline & & & $\mathrm{E}+02$ & $E+02$ & $E+01$ & $6 \mathrm{E}-02$ & +01 & +02 & $6 E+02$ & +02 & $\mathrm{E}+02$ \\
\hline & & $\mathrm{k}$ & $6.86 E+03$ & $6.86 \mathrm{E}+03$ & $2.18 E+03$ & $9 E+03$ & $E+03$ & $E+04$ & $42 \mathrm{E}+04$ & $3 E+03$ & $9 E+03$ \\
\hline & 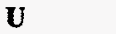 & $\mathrm{kg}$ & $6.71 E+02$ & $6.71 E+02$ & $0.00 \mathrm{E}+01$ & $E+03$ & $66 \mathrm{E}+03$ & +04 & $2 E+04$ & $E+04$ & $E+04$ \\
\hline & $\mathbf{Z r}$ & kg & $1.56 E+01$ & $56 \mathrm{E}+01$ & $0.00 \mathrm{E}+01$ & $4 \mathrm{E}-02$ & $84 \mathrm{E}+01$ & $E+03$ & $\mathrm{E}+03$ & +02 & $E+02$ \\
\hline & Ac227 & $\mathrm{Ci}$ & 2.33E-03 & 2.33E-03 & $0.00 \mathrm{E}+01$ & $0 E+01$ & & $E-03$ & $.26 \mathrm{E}-02$ & $E-03$ & E-03 \\
\hline & n241 & C & $5.38 E+01$ & $4.06 E+01$ & $0.00 \mathrm{E}+01$ & $\mathrm{DE}+01$ & $8.08 \mathrm{E}+01$ & +03 & 47E+03 & $E+03$ & +03 \\
\hline & $\mathbf{m} 243$ & $\mathrm{Ci}$ & $1.86 E-03$ & $1.41 \mathrm{E}-03$ & +01 & & & & $1 \mathrm{E}-02$ & & \\
\hline & a137 & $\mathbf{C i}$ & $1.33 E+$ & $1.33 E+05$ & +01 & & 1.3 & 05 & $E+05$ & +05 & +05 \\
\hline & 14 & $\mathrm{Ci}$ & $4.12 \mathrm{E}+01$ & $\mathbf{E}+\mathbf{0 1}$ & +01 & -00 & 01 & -01 & +02 & 01 & +01 \\
\hline & 113 & C & $8.84 \mathrm{E}+01$ & $\mathrm{E}+01$ & $\varepsilon+01$ & +01 & 01 & +02 & $6 E+02$ & +02 & +01 \\
\hline & 1242 & $\mathrm{Ci}$ & $2.88 \mathrm{E}-03$ & $2.17 E-03$ & $E+01$ & $E-06$ & & -03 & -02 & -03 & \\
\hline & $\mathrm{m} 243$ & $C$ & 2.12E-04 & $1.60 \mathrm{E}-04$ & +01 & & & & -03 & & \\
\hline & $\mathrm{Cm} 244$ & $\mathbf{C i}$ & $1.68 \mathrm{E}-03$ & $1.27 \mathrm{E}-03$ & $E+01$ & & & $\varepsilon-03$ & & $2.14 \mathrm{E}-03$ & E-03 \\
\hline & Co60 & C & $3.89 \mathrm{E}+01$ & $3.89 \mathrm{E}+01$ & $: 01$ & & & & & & \\
\hline & s134 & C & 1.92 & $1.92 \mathrm{E}-00$ & +01 & & & -00 & & -00 & .00 \\
\hline 2 & s137 & C & $1.40 \mathrm{E}+05$ & $1.40 \mathrm{E}+05$ & +01 & & 1.8 & -05 & & 05 & \\
\hline & u152 & $\mathrm{Ci}$ & $5.39 \mathrm{E}-00$ & 5.39E-00 & $0.00 \mathrm{E}+01$ & $E+01$ & -00 & +01 & +01 & $E-00$ & -00 \\
\hline & Eu154 & C & $6.52 \mathrm{E}+02$ & $6.52 \mathrm{E}+02$ & +01 & & & & & & \\
\hline & Eu155 & C & $3.27 \mathrm{E}+02$ & $3.27 \mathrm{E}+02$ & $i+01$ & & & & & & +02 \\
\hline & 1129 & C & 4.48E-01 & $4.48 \mathrm{E}-01$ & +01 & & & & & & \\
\hline & Nb93 & C & $1.20 \mathrm{E}+01$ & $1.20 \mathrm{E}+01$ & & & & 02 & 02 & -02 & +02 \\
\hline 02 & Ni59 & C & 4.31E-00 & 4.31E-00 & 01 & & & 01 & 01 & -00 & -00 \\
\hline 02 & Ni63 & C & $4.28 E+02$ & $4.28 \mathrm{E}+02$ & +01 & 01 & 02 & -03 & +03 & $E+02$ & +02 \\
\hline & Np237 & 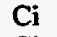 & $7.76 \mathrm{E}-01$ & $7.76 \mathrm{E}-01$ & +01 & & & & & & \\
\hline & Pa231 & C & $1.19 \mathrm{E}-02$ & $1.19 \mathrm{E}-02$ & +01 & & & & & & \\
\hline & Pu238 & C & $3.02 E-00$ & .00 & 01 & & & & & & \\
\hline & Pu239 & C & $1.40 \mathrm{E}+02$ & 8.17E+01 & +01 & & 1.4 & 02 & -02 & $i+02$ & +02 \\
\hline 02 & u240 & C & $1.89 \mathrm{E}+01$ & $1.40 \mathrm{E}+01$ & +01 & -02 & +01 & -01 & +02 & $8+01$ & +01 \\
\hline & u241 & C & $2.17 \mathrm{E}+02$ & $1.64 \mathrm{E}+02$ & $\div 01$ & & & & & +02 & +02 \\
\hline & 1242 & C & $1.05 \mathrm{E}-03$ & & & & & & & & \\
\hline & Ra226 & c & $1.74 \mathrm{E}-04$ & 04 & 01 & & & & & & \\
\hline & $\operatorname{Ra228}$ & c & 2.0 & & & & & & & .00 & \\
\hline & Ru106 & C & $7.69 \mathrm{E}-03$ & $7.69 \mathrm{E}-03$ & 01 & & & -02 & .02 & -02 & -02 \\
\hline & Sb125 & C & $1.74 E+02$ & $1.74 E+02$ & +01 & 01 & -02 & +03 & & +02 & +02 \\
\hline & Se79 & C & $3,45 \mathrm{E}-00$ & $3.45 \mathrm{E}-00$ & $E+01$ & & & -00 & .00 & -00 & $=-00$ \\
\hline & Sm151 & $\mathrm{Ci}$ & $1.19 \mathrm{E}+04$ & $1.19 E+04$ & & & & & & & +04 \\
\hline & $89 / 90$ & $\mathrm{Ci}$ & $3.80 \mathrm{E}+04$ & $3.80 \mathrm{E}+04$ & & & & & & -05 & +05 \\
\hline & c99 & C & & 02 & & & & & & & -02 \\
\hline & h229 & C & -02 & & 01 & & & 01 & & & \\
\hline & h232 & $\mathrm{Ci}$ & $7.63 \mathrm{E}-02$ & $7.63 \mathrm{E}-02$ & +01 & 03 & & $=-01$ & .01 & -01 & $\begin{array}{:}-01 \\
-1\end{array}$ \\
\hline 02 & in126 & $\mathrm{Ci}$ & $5.16 E-00$ & 5.16E-00 & +01 & & & & & -00 & -00 \\
\hline & ritiun & $\mathrm{Ci}$ & $1.59 E+02$ & $1.59 E+02$ & -01 & & & +02 & & +02 & $=01$ \\
\hline & U232 & $\mathrm{Ci}$ & +01 & & & & & & & -01 & $\div 01$ \\
\hline & II233 & $\mathrm{Ci}$ & & & & & & & & & +01 \\
\hline & U234 & C & 1.24 & & & & & & & -01 & 101 \\
\hline & U235 & $\mathrm{Ci}$ & $5.34 \mathrm{E}-01$ & 9.95E-03 & +01 & & & $E-00$ & 00 & E-01 & -01 \\
\hline & U236 & $\mathrm{Ci}$ & $1.61 \mathrm{E}-01$ & $3.01 E-03$ & +01 & & & -01 & 00 & -01 & -01 \\
\hline & U238 & $\mathrm{Ci}$ & $1.59 E+01$ & $2.24 E-01$ & +01 & & & $E+01$ & & +01 & +01 \\
\hline & Y90 & $\mathrm{Ci}$ & $3.80 \mathrm{E}+04$ & $3.80 \mathrm{E}+04$ & +01 & & & +05 & & $E+04$ & +04 \\
\hline & Zr93 & $\mathrm{Ci}$ & $1.67 E+01$ & $1.67 \mathrm{E}+01$ & +01 & & & & & $E+02$ & $E+02$ \\
\hline & Al & kg & & & & & & & & & +04 \\
\hline & $\mathbf{B i}$ & kg & & & & & & & & +02 & +02 \\
\hline & $\mathrm{Ca}$ & k & $1.05 E+03$ & $1.05 \mathrm{E}+03$ & & & & 3. & & 1.5 & +03 \\
\hline & Cl & k & $4.40 \mathrm{E}$ & $4.43 E$ & +01 & & & & & $E+03$ & +03 \\
\hline & $\mathrm{CO}$ & $\mathbf{K}$ & $2.50 \mathrm{E}+05$ & $2.50 \mathrm{E}+05$ & & & & & & $E+05$ & +05 \\
\hline & $\mathrm{Cr}$ & kg & $3.50 \mathrm{E}+03$ & $3.50 \mathrm{E}+03$ & $E+01$ & & & $E+04$ & & $E+03$ & +03 \\
\hline & $\mathbf{F}$ & kg & $1.24 E+04$ & $1.24 E+04$ & 7.57E-0! & $E+01$ & 1.9 & $E+04$ & $7.89 E+04$ & $2,24 \mathrm{E}+04$ & $7 E+04$ \\
\hline & Fe & kg & $1.73 E+03$ & $1.73 E+03$ & $1.74 \mathrm{E}+01$ & 1.0 & $6.88 E+03$ & $E+04$ & $1 E+04$ & $9.06 \mathrm{E}+03$ & +03 \\
\hline & $\mathbf{H g}$ & kg & 5.12E+01 & $6.54 \mathrm{E}-00$ & $0.00 \mathrm{E}+01$ & $0.00 \mathrm{E}+01$ & $5.12 E+01$ & $E+02$ & $E+02$ & $7.73 \mathrm{E}+01$ & $8.74 E+01$ \\
\hline & $\mathbf{K}$ & $\mathrm{kg}$ & $4.39 E+03$ & $4.39 \mathrm{E}+03$ & $2.60 \mathrm{E}+01$ & $1.75 E+03$ & $6.30 \mathrm{E}+03$ & $1.09 \mathrm{E}+04$ & $1.53 E+04$ & $6.35 E+03$ & $3.56 \mathrm{E}+03$ \\
\hline
\end{tabular}


Tank Anal. Un

\begin{tabular}{ll} 
BY103 & La \\
BY103 & Mn \\
BY103 & Na \\
BY103 & Ni \\
BY103 & NO2 \\
BY103 & NO3 \\
BY103 & P \\
BY103 & Pb \\
BY103 & PO4 \\
BY103 & S \\
BY103 & Si \\
BY103 & SO4 \\
BY103 & Sr \\
BY103 & TOC \\
BY103 & U \\
BY103 & Zr \\
BY103 & Ac227 \\
BY103 & Am241 \\
BY103 & Am243 \\
BY103 & Ba137 \\
BY103 & C14 \\
BY103 & Cd113 \\
BY103 & Cm242 \\
BY103 & Cm243 \\
BY103 & Cm244 \\
BY103 & Co60 \\
BY103 & Cs134 \\
BY103 & Cs137 \\
BY103 & Eu152 \\
BY103 & Eu154 \\
BY103 & Eu155 \\
BY103 & I129 \\
BY103 & Nb93 \\
BY103 & Ni59 \\
BY103 & Ni63 \\
\hline BY103 & Np237 \\
\hline Cing
\end{tabular}

BY103 Np237

BY103 Pa231

BY103 Pu238

BY103 Pu239

BY103 Pu240

BY103 Pu241

BY103 Pu242

BY103 Ra226

BY103 Ra228

BY103 Ru106

BY103 Sb125

BY103 Se79

BY103 Sm151

BY103 Sr89/90

BY103 Te99

BY103 Th229

BY103 Th232

BY103 Tin126

BY103 Tritium

BY103 U232

BY103 U233

BY103 U234

BY103 U235

BY103 U236

BY103 U238

BY103 Y90

BY103 Zr93

BY104 Al

BY104 Bi

BY104 Ca

BY104 Cl

BY104 CO3

BY104 Cr
$B B$

May 98

$\begin{array}{lr}\mathrm{kg} & \overline{6.63 \mathrm{E}-01} \\ \mathrm{~kg} & 1.83 \mathrm{E}+02\end{array}$

kg $\quad 5.44 \mathrm{E}+05$

kg $3.41 E+02$

kg 6.11E+04

kg 8.51E+05

kg 2.08E+04

kg 2.39E+02

kg $6.44 \mathrm{E}+04$

kg $3.49 \mathrm{E}+04$

kg 2.04E+03

kg 1.05E+05

kg 1.68E+02

kg $\quad 9.96 \mathrm{E}+03$

kg 1.30E+03

kg 5.10E+01

Ci 8.48E-03

$6.59 \mathrm{E}+01$

2.28E-03

$2.14 E+05$

Ci $5.03 E+01$

$1.08 \mathrm{E}+02$

1.17E-03

2.62E-05

Ci 3.08E-04

4.71E+01

Ci 2.29E-00

Ci $\quad 2.26 \mathrm{E}+05$

Ci 6.64E-00

7.95E+02

$4.03 E+02$

5.42E-01

$1.47 \mathrm{E}+01$

5.37E-00

$5.33 \mathrm{E}+02$

$9.40 \mathrm{E}-01$

2.31E-02

$5.86 \mathrm{E}-00$

$7.94 \mathrm{E}+01$

Ci Incl. in 239

$4.40 \mathrm{E}+02$

1.78E-03

2.03E-04

2.52E-00

9.40E-03

$2.11 \mathrm{E}+02$

4.22E-00

$1.46 \mathrm{E}+04$

$7.96 \mathrm{E}+04$

$2.80 \mathrm{E}+02$

$5.89 \mathrm{E}-02$

9.30E-02

6.31E-00

$1.93 E+02$

$1.41 \mathrm{E}+01$

$5.41 \mathrm{E}+01$

$4.30 \mathrm{E}-00$

1.61E-01

1.41E-01

8.43E-00

$7.96 \mathrm{E}+04$

$2.04 \mathrm{E}+01$

$6.50 \mathrm{E}+04$

$6.15 \mathrm{E}+02$

$2.68 \mathrm{E}+03$

$5.01 \mathrm{E}+03$

$1.60 \mathrm{E}+05$

$9.88 \mathrm{E}+03$
$B B$

Sept98

$0.00 \mathrm{E}+01$

$1.83 \mathrm{E}+02$

$5.44 \mathrm{E}+05$

$3.41 \mathrm{E}+02$

$6.22 \mathrm{E}+04$

$8.51 \mathrm{E}+05$

NA

$2.39 \mathrm{E}+02$

$2.10 \mathrm{E}+04$

NA

$2.04 \mathrm{E}+03$

$3.42 \mathrm{E}+04$

$1.68 \mathrm{E}+02$

$9.96 \mathrm{E}+03$

$1.30 \mathrm{E}+03$

$5.10 \mathrm{E}+01$

8.48E-03

$6.59 \mathrm{E}+01$

2.28E-03

$2.14 \mathrm{E}+05$

$5.03 \mathrm{E}+01$

$1.08 \mathrm{E}+02$

1.17E-03

2.62E-05

3.08E-04

4.71E+01

2.29E-00

$2.26 \mathrm{E}+05$

6.64E-00

$7.95 \mathrm{E}+02$

$4.03 \mathrm{E}+02$

5.42E-01

$1.47 \mathrm{E}+01$

5.37E-00

$5.33 \mathrm{E}+02$

9.40E-01

2.31E-02

$1.78 \mathrm{E}-00$

$6.76 \mathrm{E}+01$

$1.18 \mathrm{E}+01$

$1.34 \mathrm{E}+02$

5.41E-04

2.03E-04

2.52E-00

$9.40 \mathrm{E}-03$

2.11E+02

4.22E-00

$1.46 \mathrm{E}+04$

$7.96 \mathrm{E}+04$

$2.80 \mathrm{E}+02$

5.89E-02

9.30E-02

6.31E-00

$1.93 \mathrm{E}+02$

$1.69 \mathrm{E}-00$

$6.47 \mathrm{E}-00$

5.14E-01

1.93E-02

$1.69 \mathrm{E}-02$

4.34E-01

$7.96 \mathrm{E}+04$

$2.04 \mathrm{E}+01$

$6.50 \mathrm{E}+04$

$6.33 \mathrm{E}+01$

$2.68 \mathrm{E}+03$

$5.01 E+03$

$1.60 \mathrm{E}+05$

$.9 .88 \mathrm{E}+03$ 1\%-ile

\subsection{E-01}

$1.76 \mathrm{E}-00$

$1.42 \mathrm{E}+05$

1.57E-01

$3.42 \mathrm{E}+02$

$1.39 E+03$

$2.07 \mathrm{E}+01$

2.62E-01

$1.89 \mathrm{E}+01$

7.10E-00

$1.11 \mathrm{E}+01$

$1.15 \mathrm{E}+01$

7.54E-01

$4.93 \mathrm{E}+01$

$5.67 \mathrm{E}+01$

$0.00 \mathrm{E}+01$

$0.00 \mathrm{E}+01$

1.47E-00

$0.00 \mathrm{E}+01$

$0.00 \mathrm{E}+01$

$0.00 \mathrm{E}+01$

$0.00 \mathrm{E}+01$

$0.00 \mathrm{E}+01$

$0.00 \mathrm{E}+01$

$0.00 \mathrm{E}+01$

$2.26 \mathrm{E}-01$

$0.00 \mathrm{E}+01$

$1.71 \mathrm{E}+03$

$0.00 \mathrm{E}+01$

$0.00 \mathrm{E}+01$

$0.00 \mathrm{E}+01$

$0.00 \mathrm{E}+01$

$0.00 \mathrm{E}+01$

$0.00 \mathrm{E}+01$

$0.00 \mathrm{E}+01$

$0.00 \mathrm{E}+01$

$0.00 \mathrm{E}+01$

$0.00 E+01$

$0.00 \mathrm{E}+01$

$0.00 \mathrm{E}+01$

$0.00 \mathrm{E}+01$

$0.00 \mathrm{E}+01$

$0.00 \mathrm{E}+01$

$0.00 \mathrm{E}+01$

$0.00 \mathrm{E}+01$

$0.00 \mathrm{E}+01$

$0.00 E+01$

$0.00 \mathrm{E}+01$

$0.00 \mathrm{E}+01$

$0.00 \mathrm{E}+01$

$0.00 \mathrm{E}+01$

$0.00 \mathrm{E}+01$

$0.00 \mathrm{E}+01$

4.51E+01

$0.00 \mathrm{E}+01$

$0.00 \mathrm{E}+01$

$0.00 \mathrm{E}+01$

$0.00 \mathrm{E}+01$

$0.00 \mathrm{E}+01$

$0.00 \mathrm{E}+01$

$0.00 \mathrm{E}+01$

$0.00 \mathrm{E}+01$

$2.58 \mathrm{E}+04$

1.50E-00

$4.93 \mathrm{E}+02$

$2.22 \mathrm{E}+03$

8.27E+04

$4.47 \mathrm{E}+03$

$10 \%$-ije

5.42E-01

$4.31 \mathrm{E}+01$

$3.13 \mathrm{E}+05$

$3.31 \mathrm{E}-00$

$3.40 \mathrm{E}+03$

$2.51 \mathrm{E}+04$

$1.54 \mathrm{E}+02$

$1.74 \mathrm{E}-00$

$4.16 \mathrm{E}+02$

$3.65 \mathrm{E}+01$

$7.19 \mathrm{E}+01$

$9.77 \mathrm{E}+01$

1.22E+01

$2.40 \mathrm{E}+03$

$3.08 \mathrm{E}+02$

8.07E-01

$0.00 E+01$

$2.53 \mathrm{E}-00$

$1.48 \mathrm{E}-05$

$0.00 \mathrm{E}+01$

$0.00 \mathrm{E}+01$

$0.00 \mathrm{E}+01$

7.60E-06

1.70E-07

2.00E-06

$5.00 \mathrm{E}-01$

$0.00 \mathrm{E}+01$

$1.09 \mathrm{E}+04$

$0.00 \mathrm{E}+01$

$0.00 \mathrm{E}+01$

$0.00 \mathrm{E}+01$

$0.00 \mathrm{E}+01$

$0.00 E+01$

$0.00 \mathrm{E}+01$

$0.00 \mathrm{E}+01$

6.11E-03

$0.00 \mathrm{E} \div 01$

$3.81 \mathrm{E}-02$

5.16E-01

$5.16 \mathrm{E}-01$

2.86E-00

$1.16 \mathrm{E}-05$

$0.00 \mathrm{E}+01$

$0.00 \mathrm{E}+01$

$0.00 \mathrm{E}+01$ 
Tank Anal. Un

BY104 F BY104 Fe BY104 Hg BY104 K BY104 La BY104 Mn BY104 Na BY104 Ni BY104 NO2 BY104 NO3 BY104 P BY104 Pb BY104 PO4 BY104 S BY104 Si BY104 SO4 BY104 Sr BY104 TOC BY104 U BY104 Zr BY104 Ac227 BY104 Am241 BY104 Am243 BY104 Ba137 BY104 Cl4 BY104 Cd113 BY104 Cm242 BY104 Cm243 BY104 Cm244 BY104 Co60 BY104 Cs134 BY104 Cs137 BY104 Eu152 BY104 Eu154 BY104 Eu155 BY104 I129 BY104 Nb93 BY104 Ni59 BY104 Ni63 BY104 Np237 BY104 Pa231 BY104 Pu238 BY104 Pu239 BY104 Pu240 BY104 Pu241 BY104 Pu242 BY104 Ra226 BY104 Ra228 BY104 Ru106 BY104 Sb125 BY104 Se79 BY104 Sm151 BY104 Sr89/90 BY104 Tc99 BY104 Th229 BY104 Th232 BY104 Tin126 BY104 Tritium BY104 U232

BY104 U233 BY104 U234 BY104 U235 BY104 U236 BY104 U238 BY104 Y90 BY104 Zr93 BY105 A! BY105 Bi
BB May 98 kg $\overline{1.00 \mathrm{E}+04}$ kg $\quad 8.83 \mathrm{E}+03$ kg 4.84E-00 kg 7.32E+03 kg $\mathrm{kg}$ kg kg kg kg kg kg kg kg

$\mathrm{kg} \quad 1.12 \mathrm{E}+05$

kg $\quad 5.03 E+03$

kg 1.47E+04

$\mathrm{kg} \quad 7.06 \mathrm{E}+03$

kg 2.85E+01

Ci 1.35E-03

$$
3.00 \mathrm{E}+01
$$

1.03E-03

$$
2.08 \mathrm{E}+05
$$$$
2.28 \mathrm{E}+01
$$$$
4.91 \mathrm{E}+01
$$

7.01E-03$$
2.02 \mathrm{E}-04
$$

$4.19 \mathrm{E}+01$

1.05E-00

$2.20 \mathrm{E}+05$

$3.34 \mathrm{E}-00$

$3.59 \mathrm{E}+02$

$2.08 \mathrm{E}+02$

2.47E-01

$6.80 \mathrm{E}-00$

4.68E-00

$4.44 \mathrm{E}+02$

4.32E-01

$6.80 \mathrm{E}-03$

1.70E-00

Ci $6.22 \mathrm{E}+01$

Ci 1.05E+01

Ci 1.22E+02

Ci 5.88E-04

Ci 1.03E-04

Ci 1.13E-00

Ci 4.23E-03

Ci $\quad 9.50 \mathrm{E}+01$

Ci 1.94E-00

Ci $6.73 E+03$

$8.84 \mathrm{E}+05$

Ci $1.28 \mathrm{E}+02$

Ci 2.61E-02

Ci 4.18E-02

$\mathrm{Ci}$ 2.91E-00

Ci $\quad 8.84 \mathrm{E}+01$

Ci 6.31E-00

Ci 2.42E+01

Ci 1.75E-00

Ci 6.50E-02

Ci 5.73E-02

Ci 3.62E-00

Ci $\quad 8.84 \mathrm{E}+05$

Ci 9.37E-00

kg $\quad 6.98 \mathrm{E}+04$

kg $1.23 E+02$
$\underline{B B}$ Sept98 $1.00 \mathrm{E}+04$ $8.83 \mathrm{E}+03$ $0.00 \mathrm{E}+01$

$7.32 \mathrm{E}+03$

$0.00 \mathrm{E}+01$

$1.66 \mathrm{E}+02$

4.75E +05

$2.51 \mathrm{E}+03$

$7.54 \mathrm{E}+04$

$5.64 \mathrm{E}+05$

NA

$4.10 \mathrm{E}+02$

2.42E+04

NA

$9.37 \mathrm{E}+02$

$3.74 \mathrm{E}+04$

$5.03 \mathrm{E}+03$

$1.47 \mathrm{E}+04$

$7.06 \mathrm{E}+03$

$2.85 \mathrm{E}+01$

1.35E-03

$3.00 \mathrm{E}+01$

1.03E-03

$2.08 \mathrm{E}+05$

$2.28 \mathrm{E}+01$

$4.91 \mathrm{E}+01$

7.01E-03

1.44E-04

2,02E-04

$4.19 \mathrm{E}+01$

$1.05 \mathrm{E}-00$

$2.20 \mathrm{E}+05$

3.34E-00

$3.59 \mathrm{E}+02$

$2.08 \mathrm{E}+02$

2.47E-01

$6.80 \mathrm{E}-00$

4.68E-00

$4.44 \mathrm{E}+02$

4.32E-01

$6.80 \mathrm{E}-03$

2.36E-03

8.64E-02

1.46E-02

1.70E-01

8.16E-07

1.03E-04

1.13E-00

4.23E-03

$9.50 \mathrm{E}+01$

1.94E-00

$6.73 E+03$

$8.84 \mathrm{E}+05$

$1.28 \mathrm{E}+02$

2.61E-02

4.18E-02

2.91E-00

8.84E+01

$1.02 \mathrm{E}+01$

$3.90 \mathrm{E}+01$

2.82E-00

1.05E-01

$9.24 \mathrm{E}-02$

2.36E-00

$8.84 \mathrm{E}+05$

9.37E-00

$6.98 \mathrm{E}+04$

$1.23 \mathrm{E}+02$ 1\%-ile

$10 \%$-ile

\section{0\%-ile}

90

$2.27 E+03 \quad 4.81 E+03$

$2.41 \mathrm{E}+03 \quad 7.31 \mathrm{E}+03$

$0.00 \mathrm{E}+01 \quad 4.01 \mathrm{E}-01$

$1.22 \mathrm{E}+03$

$3.50 \mathrm{E}-00$

$3.06 \mathrm{E}+01$

$2.58 \mathrm{E}+05$

$2.97 \mathrm{E}+02$

$5.11 \mathrm{E}+04$

$2.47 \mathrm{E}+05$

$1.73 \mathrm{E}+03$

$1.44 \mathrm{E}+02$

$5.44 \mathrm{E}+03$

$5.33 \mathrm{E}+03$

$1.90 \mathrm{E}+02$

$1.82 \mathrm{E}+04$

$4.28 \mathrm{E}+02$

$6.81 \mathrm{E}+03$

$1.73 \mathrm{E}+03$

$0.00 \mathrm{E}+01$

$0.00 \mathrm{E}+01$

$1.17 \mathrm{E}+01$

7.18E-05

$8.23 \mathrm{E}+04$

$0.00 \mathrm{E}+01$

$0.00 \mathrm{E}+01$

$4.89 \mathrm{E}-04$

$1.00 \mathrm{E}-05$

$1.41 \mathrm{E}-05$

2.82E-00

4.15E-01

$1.22 \mathrm{E}+05$

$0.00 \mathrm{E}+01$

$0.00 \mathrm{E}+01$

$0.00 \mathrm{E}+01$

$1.03 \mathrm{E}-01$

$0.00 \mathrm{E}+01$

$0.00 \mathrm{E}+01$

$0.00 \mathrm{E}+01$

3.01E-02

$0.00 \mathrm{E}+01$

$1.19 \mathrm{E}-01$

4.34E-00

7.32E-01

8.51E-00

4.10E-05

$0.00 \mathrm{E}+01$

$0.00 \mathrm{E} \div 01$

$0.00 \mathrm{E}+01$

$0.00 \mathrm{E}+01$

3.31E-01

$0.00 \mathrm{E}+01$

$1.15 \mathrm{E}+05$

4.87E-00

$0.00 \mathrm{E}+01$

$0.00 \mathrm{E}+01$

$0.00 \mathrm{E}+01$

$6.02 \mathrm{E}+01$

$0.00 \mathrm{E}+01$

$0.00 \mathrm{E}+01$

$0.00 \mathrm{E}+01$

$0.00 \mathrm{E}+01$

$0.00 \mathrm{E}+01$

$0.00 \mathrm{E}+01$

$0.00 \mathrm{E}+01$

$0.00 \mathrm{E}+01$

$2.67 \mathrm{E}+04$

$5.30 \mathrm{E}-00$

$2.93 \mathrm{E}+03$

$1.59 \mathrm{E} \div 01$

$1.40 \mathrm{E}+02$

$3.03 \mathrm{E}+05$

$2.82 \mathrm{E}+03$ 
Tank Anal. Un

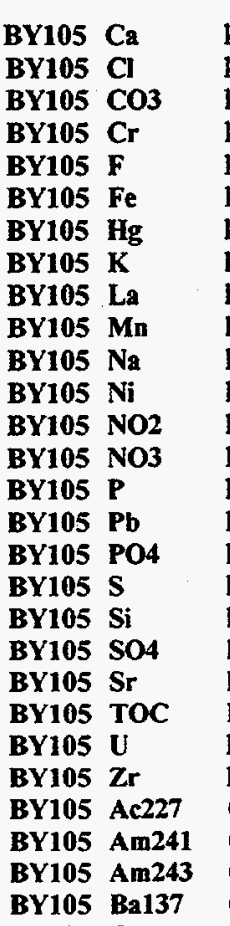

BY105 Cd113

BY105 Cm242

BY105 Cm243

BY105 Cm244

BY105 C060

BY105 Cs134

BY105 Cs137

BY105 Eul52

BY105 Eul54

BY105 Eu155

BY105 1129

BY105 Nb93

BY105 Ni59

BY105. Ni63

BY105 Np237

BY105 Pa231

BY105 Pu238

BY105 Pu239

BY105 Pu240

BY105 Pu241

BY105 Pu242

BY105 Ra226

BY105 Ra228

BY105 Ru106

BY105 Sb125

BY105 Se79

BY105 Sm151

BY105 Sr89/90

BY105 Te99

BY105 Th229

BY105 Th232

BY105 Tin126

BY105 Tritium

BY105 U232

BY105 U233

BY105 U234

BY105 U235

BY105 U236

BY105 U238
$B B$

May98

$\begin{array}{ll}\mathrm{kg} & 3 . \overline{80 \mathrm{E}+04} \\ \mathrm{~kg} & 7.37 \mathrm{E}+03\end{array}$

$2.47 \mathrm{E}+05$

$4.66 \mathrm{E}+03$

$1.50 \mathrm{E}+04$

2.82E+04

kg 9.27E-00

g $\quad 6.67 \mathrm{E}+03$

5.72E-01

3.10E +02

$6.15 \mathrm{E}+05$

g $\quad 6.96 \mathrm{E}+03$

k $9.59 \mathrm{E}+04$

$\begin{array}{ll}\mathrm{kg} & 8.51 \mathrm{E}+05 \\ \mathrm{~kg} & 4.92 \mathrm{E}+04\end{array}$

kg $2.04 \mathrm{E}+03$

$\mathrm{kg} 1.52 \mathrm{E}+05$

gg $4.99 \mathrm{E}+04$

k 7.35E+03

1.50E+05

$6.89 \mathrm{E}+03$

$2.42 \mathrm{E}+04$

$2.14 E+04$

$3.20 \mathrm{E}+02$

2.55E-03

$5.71 \mathrm{E}+01$

$1.97 \mathrm{E}-03$

$4.94 \mathrm{E}+05$

$4.37 \mathrm{E}+01$

$9.35 E+01$

7.02E-03

$1.44 \mathrm{E}-04$

3.23E-04

$4.06 \mathrm{E}+01$

$1.99 \mathrm{E}-00$

$5.22 \mathrm{E}+05$

6.05E-00

$6.86 \mathrm{E}+02$

$3.72 \mathrm{E}+02$

4.70E-01

$1.29 \mathrm{E}+01$

$6.78 \mathrm{E}-00$

$6.53 \mathrm{E}+02$

8.19E-01

1.29E-02

$\mathrm{Ci} \quad 3.24 \mathrm{E}-00$

Ci $1.22 \mathrm{E}+02$

Ci Incl. in 239

Ci 2.33E+02

Ci 1.12E-03

$\mathrm{Ci}$ 1.94E-04

Ci 2.17E-00

Ci 8.10E-03

Ci $1.82 \mathrm{E}+02$

Ci 3.68E-00

Ci $1.27 \mathrm{E}+04$

Ci $6.20 \mathrm{E}+05$

Ci $2.43 E+02$

Ci 5.01E-02

Ci 8.01E-02

Ci 5.51E-00

Ci $1.68 \mathrm{E}+02$

Ci $1.21 \mathrm{E}+01$

Ci 4.63E+01

Ci $1.16 \mathrm{E}+01$

Ci 4.97E-01

Ci 1.63E-01

Ci 1.53E+01
$B B$

Sept98

$3.80 \mathrm{E}+04$

$7.37 \mathrm{E}+03$

$2.47 \mathrm{E}+05$

$4.66 \mathrm{E}+03$

$1.50 \mathrm{E}+04$

$2.82 \mathrm{E}+04$

$0.00 \mathrm{E}+01$

$6.67 \mathrm{E}+03$

$0.00 \mathrm{E}+01$

3.10E+02

$6.15 \mathrm{E}+05$

$6.96 \mathrm{E}+03$

$9.59 \mathrm{E}+04$

8.51E+05

NA

2.04E+03

$4.97 \mathrm{E}+04$

NA

$7.35 \mathrm{E}+03$

$4.99 \mathrm{E}+04$

$6.89 \mathrm{E}+03$

$2.42 \mathrm{E}+04$

$2.14 \mathrm{E}+04$

$3.20 \mathrm{E}+02$

2.55E-03

$1.43 \mathrm{E}+02$

4.92E-03

$4.94 E+05$

4.37E+01

$9.35 \mathrm{E}+01$

$1.75 \mathrm{E}-02$

$3.59 \mathrm{E}-04$

8.06E-04

$4.06 \mathrm{E}+01$

1.99E-00

$5.22 \mathrm{E}+05$

6.05E-00

$6.86 \mathrm{E}+02$

$3.72 E+02$

4.70E-01

$1.29 \mathrm{E}+01$

6.78E-00

$6.53 E+02$

8.19E-01

$1.29 \mathrm{E}-02$

2.88E-00

$1.04 \mathrm{E}+02$

$1.77 \mathrm{E}+01$

$2.07 \mathrm{E}+02$

9.96E-04

1.94E-04

2.17E-00

8.10E-03

$1.82 \mathrm{E}+02$

3.68E-00

$1.27 \mathrm{E}+04$

$6.20 \mathrm{E}+05$

$2.43 \mathrm{E}+02$

5.01E-02

8.01E-02

5.51E-00

$1.68 \mathrm{E}+02$

7.72E-00

$2.96 \mathrm{E}+01$

7.43E- 00

3.17E-01

1.04E-01

7.14E-00
$1 \%-i l e$

$3.03 E+03$

$8.47 E \div 02$

$1.27 \mathrm{E}+04$

$6.60 \mathrm{E}+01$

$1.70 \mathrm{E}+02$

$2.42 \mathrm{E}+03$

$0.00 \mathrm{E}+01$

$9.66 \mathrm{E}+02$

2.03E-00

$1.68 \mathrm{E}+01$

$3.83 \mathrm{E}+05$

$2.49 \mathrm{E}+02$

$1.86 \mathrm{E}+04$

$6.90 \mathrm{E}+05$

$1.12 \mathrm{E}+03$

$1.16 \mathrm{E}+02$

$3.29 \mathrm{E}+03$

$1.39 \mathrm{E}+03$

$7.10 \mathrm{E}+02$

$5.22 \mathrm{E}+03$

$3.22 \mathrm{E}+01$

$3.87 \mathrm{E}+03$

$4.67 \mathrm{E}+03$

4.24E-02

$0.00 \mathrm{E}+01$

$1.16 \mathrm{E}+01$

$4.55 \mathrm{E}-05$

$0.00 \mathrm{E}+01$

$0.00 \mathrm{E}+01$

$0.00 \mathrm{E}+01$

1.62E-04

3.32E-06

$7.46 \mathrm{E}-06$

2.32E-00

$0.00 \mathrm{E}+01$

$3.95 \mathrm{E}+04$

$0.00 \mathrm{E}+01$

$0.00 \mathrm{E}+01$

$0.00 \mathrm{E}+01$

3.67E-02

$0.00 \mathrm{E}+01$

$0.00 \mathrm{E}+01$

$0.00 \mathrm{E}+01$

$1.89 \mathrm{E}-02$

$0.00 \mathrm{E}+01$

$7.48 \mathrm{E}-02$

$2.82 \mathrm{E}-00$

2.82E-00

5.38E-00

2.59E-05

$0.00 \mathrm{E}+01$

$0.00 \mathrm{E}+01$

$0.00 \mathrm{E}+01$

$0.00 \mathrm{E}+01$

$0.00 \mathrm{E}+01$

$0.00 \mathrm{E}+01$

$7.65 \mathrm{E}+04$

$0.00 \mathrm{E}+01$

$0.00 \mathrm{E}+01$

$0.00 \mathrm{E}+01$

$0.00 \mathrm{E}+01$

$9.65 \mathrm{E}+01$

$0.00 \mathrm{E}+01$

$0.00 \mathrm{E}+01$

$0.00 \mathrm{E}+01$

$0.00 \mathrm{E}+01$

$0.00 \mathrm{E}+01$

$0.00 \mathrm{E}+01$

$10 \%$-ile

50\%-ile

\section{$2.25 \mathrm{E}+04$}

$1.88 \mathrm{E}+03$

$4.09 \mathrm{E}+04$

$2.64 \mathrm{E}+02$

$1.86 \mathrm{E}+03$

$9.26 \mathrm{E}+03$

9.62E-01

$2.96 \mathrm{E}+03$

$1.35 \mathrm{E}+01$

$1.29 \mathrm{E}+02$

$4.48 \mathrm{E}+05$

$2.80 \mathrm{E}+03$

$3.40 \mathrm{E}+04$

$8.29 \mathrm{E}+05$

$7.67 \mathrm{E}+03$

$4.60 \mathrm{E}+02$

$1.08 \mathrm{E}+04$

$5.48 \mathrm{E}+03$

$1.81 \mathrm{E}+03$

$1.63 \mathrm{E}+04$

$3.80 \mathrm{E}+02$

$7.93 \mathrm{E}+03$

$1.35 \mathrm{E}+04$

$2.66 \mathrm{E}+01$

$0.00 \mathrm{E}+01$

$1.09 \mathrm{E}+02$

$4.59 \mathrm{E}-04$

$1.29 \mathrm{E}+05$

$0.00 \mathrm{E}+01$

9.70E- 00

1.63E-03

3.35E-05

$7.52 \mathrm{E}-05$

$5.38 \mathrm{E}-00$

$5.21 \mathrm{E}-01$

$1.81 \mathrm{E}+05$

$0.00 E+01$

$0.00 \mathrm{E}+01$ 


\begin{tabular}{|c|c|}
\hline BY105 & Y90 \\
\hline BY105 & Zr93 \\
\hline BY106 & Al \\
\hline BY106 & $\mathbf{B i}$ \\
\hline BY106 & Ca \\
\hline BY106 & $\mathrm{Cl}$ \\
\hline BY106 & $\mathrm{CO} 3$ \\
\hline BY106 & $\mathrm{Cr}$ \\
\hline BY106 & $\mathbf{F}$ \\
\hline BY106 & $\mathrm{Fe}$ \\
\hline BY106 & $\mathbf{H g}$ \\
\hline BY106 & $\mathbf{K}$ \\
\hline BY106 & $\mathbf{L a}$ \\
\hline BY106 & Mn \\
\hline BY106 & Na \\
\hline BY106 & $\mathrm{Ni}$ \\
\hline BY106 & NO2 \\
\hline BY106 & NO3 \\
\hline BY106 & $P$ \\
\hline BY106 & $\mathbf{P b}$ \\
\hline BY106 & PO4 \\
\hline BY106 & $\mathbf{S}$ \\
\hline BY106 & Si \\
\hline BY106 & SO4 \\
\hline BY106 & Sr \\
\hline BY106 & roc \\
\hline BY106 & $\mathbf{U}$ \\
\hline BY106 & $\mathbf{Z r}$ \\
\hline BY106. & Ac227 \\
\hline BY106 & Am 241 \\
\hline BY106 & $\operatorname{Am} 243$ \\
\hline BY106 & Bal37 \\
\hline BY106 & C14 \\
\hline BY106 & Cd113 \\
\hline BY106 & $\mathrm{Cm} 242$ \\
\hline BY106 & $\mathrm{Cm} 243$ \\
\hline BY106 & $\mathrm{Cm} 244$ \\
\hline BY106 & Co60 \\
\hline BY106 & Cs134 \\
\hline BY106 & Cs137 \\
\hline BY106 & Eul52 \\
\hline BY106 & Eul54 \\
\hline BY106 & Eut55 \\
\hline BY106 & 1129 \\
\hline BY106 & $\mathrm{Nb93}$ \\
\hline BY106 & Ni59 \\
\hline BY106 & Ni63 \\
\hline BY106 & Np237 \\
\hline BY106 & Pa231 \\
\hline BY106 & Pu238 \\
\hline BY106 & Pu239 \\
\hline BY106 & Pu240 \\
\hline BY106 & Pu241 \\
\hline BY106 & Pu242 \\
\hline BY106 & $\operatorname{Ra226}$ \\
\hline BY106 & Ra228 \\
\hline BY106 & Rul06 \\
\hline BY106 & Sbl25 \\
\hline BY106 & Se79 \\
\hline BY106 & Sm 151 \\
\hline BY106 & Sr89/90 \\
\hline BY106 & Tc99 \\
\hline BY106 & Th229 \\
\hline BY106 & Th232 \\
\hline BY106 & Tin126 \\
\hline BY106 & Tritium \\
\hline $\begin{array}{l}\text { BY106 } \\
\text { BY106 }\end{array}$ & $\begin{array}{l}\text { U232 } \\
\text { U233 }\end{array}$ \\
\hline
\end{tabular}

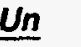

$\frac{\mathrm{MB}}{6.20 \mathrm{E}+05}$ Ci $\quad 6.20 \mathrm{E}+05$ kg 1.21E+05 $\mathrm{kg} \quad 1.95 \mathrm{E}+02$

kg $\quad 6.11 \mathrm{E}+03$

kg 7.29E+03

$\mathrm{kg} \quad 1.47 \mathrm{E}+05$

kg 5.19E+03

$\mathrm{kg} \quad 1.82 \mathrm{E}+04$

kg 2.11E+04

kg 1.50E+01

kg $\quad 8.74 \mathrm{E}+03$

kg 9.23E-01

kg 3.39E+01

kg $\quad 8.27 \mathrm{E}+05$

kg 1.84E+04

kg 1.14E+05

kg 1.16E+06

kg 4.93E+04

kg $2.28 \mathrm{E}+02$

kg 1.53E+05

kg $\quad 4.00 \mathrm{E}+04$

kg $\quad 6.51 \mathrm{E}+02$

$\mathrm{kg} \quad 1.20 \mathrm{E}+05$

kg $\quad 1.57 \mathrm{E}+02$

kg 2.15E+04

kg $5.80 \mathrm{E}+02$

kg $3.00 \mathrm{E}+01$

Ci 4.04E-03

Ci $9.19 \mathrm{E}+01$

Ci 3.17E-03

Ci $\quad 6.66 \mathrm{E}+05$

Ci $7.01 \mathrm{E}+01$

Ci 1.50E+02

Ci 5.20E-03

Ci 1.06E-04

Ci $4.65 \mathrm{E}-04$

Ci $\quad 6.55 \mathrm{E}+01$

Ci 3.20E-00

Ci $\quad 7.04 E+05$

Ci 9.43E-00

Ci 1.11E+03

Ci $\quad 5.74 E+02$

Ci $\quad 7.56 \mathrm{E}-01$

Ci $2.06 \mathrm{E}+01$

Ci 8.84E-00

Ci 8.65E +02

Ci 1.31E-00

Ci 2.07E-02

Ci $\quad 5.23 E-00$

Ci 6.14E+01

Ci $3.21 \mathrm{E}+01$

Ci $3.76 \mathrm{E}+02$

Ci 1.81E-03

Ci 2.90E-04

Ci 3.50E-00

Ci 1.31E-02

Ci $2.94 \mathrm{E}+02$

Ci 5.90E-00

Ci $2.04 E+04$

Ci $4.96 \mathrm{E}+05$

Ci $3.91 \mathrm{E}+02$

Ci 8.08E-02

$1.29 \mathrm{E}-01$

8.82E-00

$2.70 \mathrm{E}+02$

Ci $1.95 \mathrm{E}+01$

Ci $7.48 \mathrm{E}+01$
$B B$ Sept98 $\widehat{6.20 \mathrm{E}+05}$ $1.78 \mathrm{E}+01$ $1.21 \mathrm{E}+05$ $1.95 E+02$

$6.11 \mathrm{E}+03$

$7.29 \mathrm{E}+03$

$1.47 \mathrm{E}+05$

$5.19 E+03$

$1.82 \mathrm{E}+04$

$2.11 \mathrm{E}+04$

$0.00 \mathrm{E}+01$

$8.74 E+03$

$0.00 \mathrm{E}+01$

$3.39 \mathrm{E}+01$

8.27E +05

$1.84 \mathrm{E}+04$

$1.14 \mathrm{E}+05$

$1.16 \mathrm{E}+06$

NA

$2.28 \mathrm{E}+02$

$4.98 \mathrm{E}+04$ NA

$6.51 \mathrm{E}+02$

$4.00 \mathrm{E}+04$

$1.57 \mathrm{E}+02$

2.15E+04

$5.80 \mathrm{E}+02$

$3.00 \mathrm{E}+01$

4.04E-03

$1.23 \mathrm{E}+02$

$4.23 \mathrm{E}-03$

$6.66 \mathrm{E}+05$

$7.01 E+01$

$1.50 \mathrm{E}+02$

6.93E-03

1.42E-04

$6.21 \mathrm{E}-04$

$6.55 \mathrm{E}+01$

$3.20 \mathrm{E}-00$

$7.04 \mathrm{E}+05$

9.43E-00

$1.11 E+03$

$5.74 E+02$

$7.56 \mathrm{E}-01$

$2.06 \mathrm{E}+01$

8.84E-00

$8.65 \mathrm{E}+02$

1.31E-00

2.07E-02

1.46E-00

$5.24 \mathrm{E}+01$

8.97E-00

$1.05 E+02$

5.05E-04

$2.90 \mathrm{E}-04$

3.50E-00

1.31E-02

$2.94 \mathrm{E}+02$

$5.90 \mathrm{E}-00$

2.04E+04

$4.96 \mathrm{E}+05$

$3.91 \mathrm{E}+02$

8.08E-02

$1.29 \mathrm{E}-01$

8.82E-00

$2.70 \mathrm{E}+02$

8.53E-01

3.27E-00 1\%-ile

$10 \%$-ile

$0.00 E+01 \cdot 1.03 E+05$

$0.00 E+01$

$6.79 \mathrm{E}+04$

2.37E-00

$1.72 \mathrm{E}+03$

$4.49 \mathrm{E}+03$

$1.21 \mathrm{E}+05$

$6.33 \mathrm{E}+02$

$2.19 \mathrm{E}+03$

$5.76 \mathrm{E}+03$

$0.00 \mathrm{E}+01$

$9.60 \mathrm{E}+02$

$1.75 \mathrm{E}-00$

$4.85 \mathrm{E}-00$

$6.50 \mathrm{E}+05$

$5.00 \mathrm{E}+01$

$5.55 \mathrm{E}+04$

$8.52 \mathrm{E}+05$

$3.05 \mathrm{E}+03$

$1.21 \mathrm{E}+02$

$1.23 \mathrm{E}+03$

$3.24 \mathrm{E}+03$

$1.05 \mathrm{E}+02$

$1.08 \mathrm{E}+04$

$3.32 \mathrm{E}+01$

$1.25 \mathrm{E}+04$

$6.25 \mathrm{E}+02$

$1.15 \mathrm{E}+04$

$0.00 \mathrm{E}+01$

$1.01 \mathrm{E}+01$

5.83E-05

$3.16 \mathrm{E}+05$

$0.00 \mathrm{E}+01$

$0.00 \mathrm{E}+01$

9.57E-05

$1.95 \mathrm{E}-06$

8.56E-06

$1.59 \mathrm{E}-00$

$1.52 \mathrm{E}-00$

$2.93 \mathrm{E}+05$

$0.00 \mathrm{E}+01$

$0.00 \mathrm{E}+01$

$0.00 \mathrm{E}+01$

2.15E-01

$0.00 \mathrm{E}+01$

$0.00 \mathrm{E}+01$

$0.00 \mathrm{E}+01$

2.41E-02

$0.00 \mathrm{E}+01$

9.62E-02

$1.13 \mathrm{E}-00$

5.91E-01

6.92E-00

3.33E-05

$0.00 \mathrm{E}+01$

$0.00 \mathrm{E}+01$

$0.00 \mathrm{E}+01$

$0.00 \mathrm{E}+01$

$0.00 \mathrm{E}+01$

$0.00 \mathrm{E}+01$

$8.63 E+04$

$0.00 \mathrm{E}+01$

$0.00 \mathrm{E}+01$

$0.00 \mathrm{E}+01$

$0.00 \mathrm{E}+01$

$1.99 E+02$

$0.00 \mathrm{E}+0$

$0.00 \mathrm{E}+01$

$5.34 \mathrm{E}-01$

$8.65 \mathrm{E}+04$

$4.13 \mathrm{E}+02$

$4.87 \mathrm{E}+03$

$5.64 \mathrm{E}+03$

$1.73 \mathrm{E}+05$

$2.58 \mathrm{E}+03$

$1.01 \mathrm{E}+04$

$1.02 \mathrm{E}+04$

$2.00 \mathrm{E}-00$

$4.23 \mathrm{E}+03$

$1.07 \mathrm{E}+01$

$8.45 \mathrm{E}+01$

$7.15 \mathrm{E}+05$

$1.93 \mathrm{E}+03$

$8.68 \mathrm{E}+04$

$1.00 \mathrm{E}+06$

$7.48 \mathrm{E}+03$

$4.01 \mathrm{E}+02$

$7.70 \mathrm{E}+03$

8.27E +03

$7.75 \mathrm{E}+02$

$2.96 \mathrm{E}+04$

$3.12 \mathrm{E}+02$

$1.56 \mathrm{E}+04$

$4.72 \mathrm{E}+03$

$1.38 \mathrm{E}+04$

$0.00 \mathrm{E}+01$

$7.64 \mathrm{E} \div 01$

$7.45 \mathrm{E}-04$

$4.62 \mathrm{E}+0.5$

9.57E-00

$2.00 \mathrm{E}+01$

$1.22 \mathrm{E}-03$

2.49E-05

$1.09 \mathrm{E}-04$

3.74E-00

2.22E-00

3.70E+05

$0.00 \mathrm{E}+01$

$0.00 \mathrm{E}+01$

$0.00 \mathrm{E}+01$

4.31E-01

$0.00 \mathrm{E}+01$

$0.00 \mathrm{E}+01$

$0.00 \mathrm{E}+01$

3.08E-01

$1.37 \mathrm{E}-03$

$1.23 E-00$

$1.44 \mathrm{E}+01$

$7.54 \mathrm{E}-00$

8.84E +01

$4.25 \mathrm{E}-0.04$

$0.00 \mathrm{E}+01$

$0.00 \mathrm{E}+01$

$3.24 \mathrm{E}-03$

$0.00 \mathrm{E}+01$

$2.08 \mathrm{E}-00$

$0.00 \mathrm{E}+01$

$2.67 \mathrm{E}+05$

$7.30 \mathrm{E}+01$

5.34E-03

8.52E-03

$1.18 \mathrm{E}-00$

$2.26 \mathrm{E}+02$

$1.29 \mathrm{E}-00$ 50\%-ile

4. $94 \mathrm{E}-00$

$6.20 \mathrm{E}+05$

$1.78 \mathrm{E}+01$

$1.09 \mathrm{E}+05$

$1.11 E+03$

$1.03 E+04$

$7.36 \mathrm{E}+03$

$2.43 E+05$

$4.95 \mathrm{E}+03$

$2.14 \mathrm{E}+04$

$2.15 E+04$ 
Tank Anal. Un

\begin{tabular}{|c|c|c|c|c|}
\hline BY106 & U234 & $\mathrm{Ci}$ & $\frac{\text { May98 }}{5.32 \mathrm{E}-00}$ & $\frac{\text { Sept98 }}{2.32 \mathrm{E}-01}$ \\
\hline BY106 & U235 & $\mathrm{Ci}$ & $1.97 \mathrm{E}-01$ & $8.61 \mathrm{E}-03$ \\
\hline BY106 & U236 & $\mathrm{Ci}$ & $1.76 \mathrm{E}-01$ & 7.71E-03 \\
\hline BY106 & U238 & $\mathrm{Ci}$ & $1.11 \mathrm{E}+01$ & $1.93 \mathrm{E}-01$ \\
\hline BY106 & Y90 & $\mathrm{Ci}$ & $4.96 \mathrm{E}+05$ & $4.96 E+05$ \\
\hline BY106 & $\mathrm{Zr} 93$ & $\mathrm{Ci}$ & $2.85 \mathrm{E}+01$ & $2.85 E+01$ \\
\hline BY107 & Al & kg & $6.58 \mathrm{E}+04$ & $7.22 \mathrm{E}+04$ \\
\hline BY107 & $\mathbf{B i}$ & kg & $1.70 \mathrm{E}+02$ & $8.10 \mathrm{E}+01$ \\
\hline BY107 & $\mathrm{Ca}$ & kg & $3.42 \mathrm{E}+03$ & $3.47 E+03$ \\
\hline BY107 & $\mathrm{Cl}$ & $\mathrm{kg}$ & $4.56 E+03$ & $4.97 E+03$ \\
\hline BY107 & $\mathrm{CO3}$ & $\mathrm{kg}$ & $7.44 E+04$ & $8.20 E+04$ \\
\hline BY107 & $\mathrm{Cr}$ & $\mathrm{kg}$ & $5.94 \mathrm{E}+03$ & $6.54 E+03$ \\
\hline BY107 & $\mathbf{F}$ & $\mathrm{kg}$ & $6.69 \mathrm{E}+03$ & $7.38 E+03$ \\
\hline BY107 & Fe & $\mathrm{kg}$ & $8.51 \mathrm{E}+03$ & $9.40 \mathrm{E}+03$ \\
\hline BY107 & Hg & kg & 5.16E-00 & $7.65 \mathrm{E}-00$ \\
\hline BY107 & $\mathbf{K}$ & kg & $6.04 \mathrm{E}+03$ & $6.68 E+03$ \\
\hline BY107 & La & kg & $9.30 \mathrm{E}+01$ & $0.00 \mathrm{E}+01$ \\
\hline BY107 & $\mathbf{M n}$ & kg & $2.15 E+02$ & $1.19 E+02$ \\
\hline BY107 & $\mathbf{N a}$ & kg & $4.30 \mathrm{E}+05$ & $4.73 E+05$ \\
\hline BY107 & $\mathbf{N i}$ & $\mathrm{kg}$ & $6.80 \mathrm{E}+03$ & $7.50 E+03$ \\
\hline BY107 & NO2 & kg & $7.38 \mathrm{E}+04$ & $8.05 E+04$ \\
\hline BY107 & NO3 & kg & $3.78 \mathrm{E}+05$ & $4.16 E+05$ \\
\hline BY107 & $\mathbf{P}$ & kg & $4.26 E+04$ & NA \\
\hline BY107 & $\mathbf{P b}$ & kg & $6.63 E+02$ & $6.28 \mathrm{E}+02$ \\
\hline BY107 & PO4 & kg & $1.32 E+05$ & $4.75 E+04$ \\
\hline BY107 & $\mathbf{S}$ & $\mathrm{kg}$ & $2.85 E+04$ & NA \\
\hline BY107 & $\mathbf{S i}$ & kg & $2.14 E+03$ & $2.36 E+03$ \\
\hline BY107 & SO4 & kg & $8.55 E+04$ & $3.13 E+04$ \\
\hline BY107 & $\mathrm{Sr}$ & kg & $2.44 \mathrm{E}+03$ & $2.69 E+03$ \\
\hline BY107 & TOC & kg & $7.63 E+03$ & $8.40 E+03$ \\
\hline BY107 & $\mathbf{U}$ & kg & $7.73 E+03$ & $8.54 E+03$ \\
\hline BY107 & $\mathbf{Z r}$ & kg & $2.44 E+02$ & $9.49 \mathrm{E}+01$ \\
\hline BY107 & Ac227 & $\mathrm{Ci}$ & $1.00 \mathrm{E}-03$ & $1.14 E-03$ \\
\hline BY107 & Am241 & $\mathrm{Ci}$ & $4.58 \mathrm{E}+02$ & $1.59 E+01$ \\
\hline BY107 & Am243 & $\mathrm{Ci}$ & $1.00 \mathrm{E}-03$ & 5.44E-04 \\
\hline BY107 & Ba137 & $\mathrm{Ci}$ & $2.04 E+05$ & $2.28 E+05$ \\
\hline BY107 & C14 & $\mathrm{Ci}$ & $1.93 \mathrm{E}+01$ & $1.93 E+01$ \\
\hline BY107 & Cd113 & $\mathrm{Ci}$ & $4.15 E+01$ & $4.15 \mathrm{E}+01$ \\
\hline BY107 & $\mathrm{Cm} 242$ & $\mathrm{Ci}$ & $4.00 \mathrm{E}-03$ & $2.75 E-03$ \\
\hline BY107 & Cm243 & $\mathrm{Ci}$ & NA & $5.64 E-05$ \\
\hline BY107 & $\mathrm{Cm} 244$ & $\mathrm{Ci}$ & NA & $1.02 E-04$ \\
\hline BY107 & Co60 & $\mathrm{Ci}$ & $1.80 \mathrm{E}+01$ & $1.80 \mathrm{E}+01$ \\
\hline BY107 & Css134 & $\mathrm{Ci}$ & $8.82 \mathrm{E}-01$ & $8.82 \mathrm{E}-01$ \\
\hline BY107 & Cs137 & $\mathrm{Ci}$ & $2.15 E+05$ & $2.41 E+05$ \\
\hline BY107 & Eul52 & $\mathrm{Ci}$ & $2.74 E-00$ & 2.74E-00 \\
\hline BY107 & Eu154 & $\mathrm{Ci}$ & $3.03 E+02$ & $3.03 E+02$ \\
\hline BY107 & Eul55 & $\mathrm{Ci}$ & $1.70 \mathrm{E}+02$ & $1.70 E+02$ \\
\hline BY107 & 1129 & $\mathrm{Ci}$ & $2.09 \mathrm{E}-01$ & $2.09 E-01$ \\
\hline BY107 & Nb93 & $\mathrm{Ci}$ & $5.73 \mathrm{E}-00$ & $5.73 E-00$ \\
\hline BY107 & Ni59 & $\mathrm{Ci}$ & 3.07E-00 & $3.07 E-00$ \\
\hline BY107 & Ni63 & $\mathrm{Ci}$ & $2.96 E+02$ & $2.96 \mathrm{E}+02$ \\
\hline BY107 & Np237 & $\mathrm{Ci}$ & $3.64 \mathrm{E}-01$ & $3.64 \mathrm{E}-01$ \\
\hline BY107 & Pa231 & $\mathrm{Ci}$ & $6.00 \mathrm{E}-03$ & 5.73E-03 \\
\hline BY107 & Pu238 & $\mathrm{Ci}$ & $1.48 E-00$ & 9.24E-01 \\
\hline BY107. & Pu239 & $\mathrm{Ci}$ & $5.73 E+01$ & $3.58 E+01$ \\
\hline BY107 & Pu240 & $\mathrm{Ci}$ & $9.36 \mathrm{E}-00$ & $5.85 \mathrm{E}-00$ \\
\hline BY107 & Pu24I & $\mathrm{Ci}$ & $1.05 E+02$ & $6.57 \mathrm{E}+01$ \\
\hline BY107 & Pu242 & $\mathrm{Ci}$ & NA & $3.16 E-04$ \\
\hline BY107 & $\operatorname{Ra226}$ & $\mathrm{Ci}$ & NA. & $8.54 \mathrm{E}-05$ \\
\hline BY107 & Ra228 & $\mathrm{Ci}$ & 9.59E-01 & $9.59 \mathrm{E}-01$ \\
\hline BY107 & Rul06 & $\mathrm{Ci}$ & $4.00 \mathrm{E}-03$ & $3.58 \mathrm{E}-03$ \\
\hline BY107 & Sbl25 & $\mathrm{Ci}$ & $8.04 E+01$ & $8.04 E+01$ \\
\hline BY107 & Se79 & $\mathrm{Ci}$ & $1.64 E-00$ & $1.64 \mathrm{E}-00$ \\
\hline BY107 & Sm151 & $\mathrm{Ci}$ & $3.42 \mathrm{E}-00$ & $5.68 \mathrm{E}+03$ \\
\hline BY10 & Sr89/90 & $\mathrm{Ci}$ & $3.01 E+04$ & $3.36 E+04$ \\
\hline BY10 & Tc99 & $\mathrm{Ci}$ & $1.08 E+02$ & $1.08 \mathrm{E}+02$ \\
\hline 1107 & Th229 & $\mathrm{Ci}$ & $2.20 \mathrm{E}-02$ & $2.21 \mathrm{E}-02$ \\
\hline BY107 & Th232 & $\mathrm{Ci}$ & 3.50E-02 & $3.54 \mathrm{E}-02$ \\
\hline
\end{tabular}

$1 \%-j l e$

$0.00 \mathrm{E}+01$

3.52E-01

$0.00 \mathrm{E}+01 \quad 1.30 \mathrm{E}-02$

$0.00 \mathrm{E}+01 \quad 7.34 \mathrm{E}-01$

$0.00 \mathrm{E}+01 \quad 1.05 \mathrm{E}+05$

$0.00 \mathrm{E}+01 \quad 0.00 \mathrm{E}+01$

$2.96 \mathrm{E}+04 \quad 3.81 \mathrm{E}+04$

$1.52 \mathrm{E}+03 \quad 2.23 \mathrm{E}+03$

$1.25 \mathrm{E}+03 \quad 2.81 \mathrm{E}+03$

$2.16 \mathrm{E}+03 \quad 2.66 \mathrm{E}+03$

$2.30 \mathrm{E}+04 \quad 3.80 \mathrm{E}+04$

$2.87 \mathrm{E}+03 \quad 3.84 \mathrm{E}+03$

$1.42 \mathrm{E}+03 \quad 3.52 \mathrm{E}+03$

$4.65 \mathrm{E}+03 \quad 7.22 \mathrm{E}+03$

$0.00 \mathrm{E}+01 \quad 7.90 \mathrm{E}-01$

$3.38 \mathrm{E}+02 \quad 1.63 \mathrm{E}+03$

$2.39 \mathrm{E}+02 \quad 2.86 \mathrm{E}+02$

$2.55 \mathrm{E}+01 \quad 8.24 \mathrm{E}+01$

$2.40 \mathrm{E}+05 \quad 2.81 \mathrm{E}+05$

$3.26 \mathrm{E}+01 \quad 1.27 \mathrm{E}+03$

$3.45 \mathrm{E}+04 \quad 4.34 \mathrm{E}+04$

$1.84 \mathrm{E}+05 \quad 2.35 \mathrm{E}+05$

$5.70 \mathrm{E}+03$

$1.14 \mathrm{E}+02$

$6.07 \mathrm{E}+03$

$3.97 \mathrm{E}+03$

$9.98 \mathrm{E}+02$

$1.19 \mathrm{E}+04$

$6.96 \mathrm{E}+02$

$4.22 \mathrm{E}+03$

$2.54 \mathrm{E}+04$

$1.86 \mathrm{E}-00$

$0.00 \mathrm{E}+01$

$1.05 \mathrm{E}+01$

9.45E-05

$5.32 \mathrm{E}+04$

$0.00 \mathrm{E}+01$

$0.00 \mathrm{E}+01$

3.78E-04

NA

NA

1.31E-00

$2.30 \mathrm{E}-01$

$9.20 \mathrm{E}+04$

$0.00 \mathrm{E}+01$

$0.00 \mathrm{E}+01$

$0.00 \mathrm{E}+01$

$7.20 \mathrm{E}-02$

$0.00 \mathrm{E}+01$

$0.00 \mathrm{E}+01$

$0.00 \mathrm{E}+01$

3.44E-02

$0.00 \mathrm{E}+01$

1.40E-01

$5.41 \mathrm{E}-00$

8.84E-01

9.92E-00

NA

NA

$1.06 \mathrm{E}-01$

$0.00 \mathrm{E}+01$

$0.00 \mathrm{E}+01$

4.80E-02

$0.00 E+01$

$2.84 E+04$

$0.00 \mathrm{E}+01$

$0.00 \mathrm{E}+01$

$0.00 \mathrm{E}+01$

$9.26 \mathrm{E}+03$

$2.68 \mathrm{E}+02$

$1.23 \mathrm{E}+04$

$5.71 \mathrm{E}+03$

$1.65 \mathrm{E}+03$

$1.76 \mathrm{E}+04$

$9.18 \mathrm{E}+02$

$5.51 \mathrm{E}+03$

$3.25 \mathrm{E}+04$

$2.28 \mathrm{E}+01$

$0.00 \mathrm{E}+01$

$4.77 \mathrm{E}+01$

3.01E-04

$1.11 \mathrm{E}+05$

$3.24 \mathrm{E}-00$

$6.36 \mathrm{E}-00$

$1.20 \mathrm{E}-03$

NA

2.64E-00

4.81E-01

$1.43 \mathrm{E}+05$

$0.00 \mathrm{E}+01$

$0.00 \mathrm{E}+01$

$0.00 \mathrm{E}+01$

$1.23 \mathrm{E}-01$

6.10E-01

$0.00 \mathrm{E}+01$

$0.00 \mathrm{E}+01$

$1.09 \mathrm{E}-01$

$7.40 \mathrm{E}-04$

4.45E-01

$1.72 \mathrm{E}+01$

$2.82 \mathrm{E}-00$

$3.16 \mathrm{E}+01$

NA

2.12E-01

$1.01 E-03$

$0.00 \mathrm{E}+01$

$6.69 \mathrm{E}-01$

$0.00 \mathrm{E}+01$

$1.12 \mathrm{E}+05$

$1.95 \mathrm{E}+01$

2.71E-03

4.32E-03
$1.42 \mathrm{E}+04$

$3.48 \mathrm{E}+04$ 50\%-ile

5.32E-00

$1.60 \mathrm{E}+01$

$1.97 \mathrm{E}-01$

$1.11 \mathrm{E}+01$

$4.96 \mathrm{E}+05$

$2.85 E+01$

$5.04 \mathrm{E}+04$

$3.50 \mathrm{E}+03$

$5.89 \mathrm{E}+03$

$3.50 \mathrm{E}+03$

$6.10 \mathrm{E}+04$

$5.06 \mathrm{E}+03$

$7.30 \mathrm{E}+03$

$1.41 \mathrm{E}+04$

5.16E-00

$3.86 \mathrm{E}+03$

$3.49 \mathrm{E}+02$

$2.86 \mathrm{E}+02$

$3.46 \mathrm{E}+05$

$3.23 E+03$

$5.81 \mathrm{E}+04$

$3.05 \mathrm{E}+05$

$7.08 \mathrm{E}+02$

$2.17 E+04$

$8.61 \mathrm{E}+03$

$3.23 \mathrm{E}+03$

$2.65 \mathrm{E}+04$

$2.48 \mathrm{E}+03$

$7.70 \mathrm{E}+03$

$4.29 \mathrm{E}+04$

$1.80 \mathrm{E}+02$

$1.00 \mathrm{E}-03$

$2.68 E+02$

1.00E-03

$2.04 \mathrm{E}+05$

$1.93 \mathrm{E}+01$

$4.15 E+01$

4.00E-03

NA

2.14E+01

8.82E-01

$2.26 \mathrm{E}+05$

2.74E-00

$3.03 \mathrm{E}+02$

$1.70 \mathrm{E}+02$

2.09E-01

5.73E- 00

3.07E-00

$2.96 \mathrm{E}+02$

$3.64 \mathrm{E}-01$

6.00E-03

1.48E-00

$5.73 \mathrm{E}+01$

9.36E-00

$1.05 \mathrm{E}+02$

NA.

90\%-ile

99\%-ile

$5.94 \mathrm{E}-01$

5.30E-01

$3.34 \mathrm{E}+01$

1.70E+06

$8.61 \mathrm{E}+01$

$7.12 \mathrm{E}+04$

$5.21 \mathrm{E}+03$

$1.01 \mathrm{E}+04$

$4.71 \mathrm{E}+03$

$8.85 \mathrm{E}+04$

$6.53 \mathrm{E}+03$

$1.17 \mathrm{E}+04$

$2.46 \mathrm{E}+04$

$1.49 \mathrm{E}+01$

$6.90 \mathrm{E}+03$

$4.26 \mathrm{E}+02$

$6.56 \mathrm{E}+02$

$4.08 E+05$

$5.59 \mathrm{E}+03$

$7.38 \mathrm{E}+04$

$3.89 E+05$

$1.99 \mathrm{E}+04$

$1.69 \mathrm{E}+03$

$1.18 \mathrm{E}+04$

$5.50 \mathrm{E}+03$

$3.64 \mathrm{E}+04$

$7.51 \mathrm{E}+03$

$1.02 \mathrm{E}+04$

$6.05 \mathrm{E}+04$

$1.53 \mathrm{E}+03$

$3.50 \mathrm{E}-03$

$3.78 \mathrm{E}+03$

$5.84 \mathrm{E}-03$

$4.20 \mathrm{E}+05$

$3.86 \mathrm{E}+0 \mathrm{I}$

$1.20 \mathrm{E}+02$

$233 \mathrm{E}-02$

NA

NA

7.04E+01

$1.82 \mathrm{E}-00$

4.17E +05

9.58E-00

$1.06 \mathrm{E}+03$

$5.94 \mathrm{E}+02$

$3.32 \mathrm{E}-01$

$4.96 \mathrm{E}+01$

7.33E-00

$7.07 \mathrm{E}+02$

2.12E-00

$1.49 \mathrm{E}-02$

8.64E-00

$3.34 \mathrm{E}+02$

$5.46 \mathrm{E}+01$

$6.13 E+02$

NA

9.59E-01

4.00E-03

8.04E+01

1.64E-00

3.42E- 00

$3.11 \mathrm{E}+05$

$1.08 \mathrm{E}+02$

$2.20 \mathrm{E}-02$

$3.50 \mathrm{E}-02$

$4.37 \mathrm{E}+01$

$1.62 \mathrm{E}-00$

$1.44 \mathrm{E}-00$

$9.11 \mathrm{E}+01$

$3.44 \mathrm{E}+06$

$1.48 \mathrm{E}+02$

$1.08 \mathrm{E}+05$

$7.84 \mathrm{E}+03$

$1.43 \mathrm{E}+04$

$8.20 \mathrm{E}+03$

$1.18 \mathrm{E}+05$

$8.05 E+03$

$1.57 \mathrm{E}+04$

$4.02 \mathrm{E}+04$

$2.89 \mathrm{E}+01$

$3.12 \mathrm{E}+04$

$4.96 \mathrm{E}+02$

$2.27 \mathrm{E}+03$

4.62E+05

$8.29 \mathrm{E}+03$

8.81E+04

$1.20 \mathrm{E}+06$

$2.55 E+04$

$3.10 \mathrm{E}+03$

$4.81 \mathrm{E}+04$

1.49E+04

$1.01 \mathrm{E}+04$

4.74E+04

$1.42 \mathrm{E}+04$

$1.25 \mathrm{E}+04$

$8.98 \mathrm{E}+04$

$5.61 \mathrm{E}+03$

$5.78 \mathrm{E}-03$

$6.63 \mathrm{E}+03$

$1.11 \mathrm{E}-02$

$6.41 \mathrm{E}+05$

$5.95 \mathrm{E}+01$

$2.32 \mathrm{E}+02$

4.42E-02

$1.51 \mathrm{E}+02$

2.77E-00

$6.13 E+05$

$1.58 \mathrm{E}+01$

$1.75 \mathrm{E}+03$

$9.83 \mathrm{E}+02$

$6.87 \mathrm{E}-01$

$1.82 \mathrm{E}+02$

1.22E+01

NA 
Tank Anal. Un

BY107 Tin126 BY107 Tritium $\mathrm{Ci} \quad 2.45 \mathrm{E}-00$ BY107 U232 Ci 5.35E-00 BY107 U233 Ci 2.05E+01 BY107 U234 Ci 2.80E-00 BY107 U235 Ci 1.13E-01 BY107 U236 Ci $6.20 \mathrm{E}-02$ BY107 U238

BY107 Y90 BY107 Zr93

BY108 Al BY108 Bi BY108 Ca BY108 Cl BY108 $\mathrm{CO3}$ BY108 $\mathrm{Cr}$ BY108 F BY108 Fe BY108 $\mathrm{Hg}$ BY108 K

BY108 La

BY108 Mn

BY108 Na

BY108 Ni

BY108 NO2

BY108 NO3

BY108 P

BY108 Pb

BY108 PO4

BY108 S

BY108 Si

BY108 504

BY108 $\mathrm{Sr}$

BY108 TOC

BY108 U

BY108 Zr

BY108 Ac227

BY108 Am241

BY108 Am243

BY108 Ba137

BY108 C14

BY108 Cd113

BY108 Cm242

BY108 Cm243

BY108 Cm244

BY108 C060

BY108 Cs134

BY108 Cs137

BY108 Eu152

BY108 Eu154

BY108 Eu155

BY108 1129

BY108 Nb93

BY108 Nis9

BY108 Ni63

BY108 Np237

BY108 Pa231

BY108 Pu238

BY108 Pu239

BY108 Pu240

BY108 Pu241

BY108 Pu242

BY108 Ra226

BY108 Ra228

BY108 Ru106

BY108 Sb125

BY108 Se79

BY108 Sm151
BB

Sept98

$2.45 \mathrm{E}-00$

$7.46 \mathrm{E}+01$

5.91E-00

$2.27 \mathrm{E}+01$

3.10E-00

1.25E-01

$6.86 \mathrm{E}-02$

2.85E-00

$3.36 \mathrm{E}+04$

$7.90 \mathrm{E}-00$

$5.17 E+04$

$6.44 E+02$

$4.38 \mathrm{E}+03$

$2.00 \mathrm{E}+03$

$3.47 \mathrm{E}+04$

$3.32 \mathrm{E}+02$

8.59E+03

$9.35 \mathrm{E}+03$

$0.00 \mathrm{E}+01$

$3.45 \mathrm{E}+03$

$0.00 \mathrm{E}+01$

$2.72 \mathrm{E}+02$

$2.12 \mathrm{E}+05$

$3.26 \mathrm{E}+03$

$3.55 E+04$

2.61E+05

NA
$\mathbf{E}+02$

$5.71 \mathrm{E}+02$

$3.38 \mathrm{E}+04$

NA

$1.99 \mathrm{E}+03$

$3.04 \mathrm{E}+04$

$4.15 E+03$

$5.82 \mathrm{E}+03$

$1.23 \mathrm{E}+04$

$4.51 \mathrm{E}+01$

5.36E-04

$2.47 \mathrm{E}+01$

3.71E-04

$3.32 \mathrm{E}+05$

8.35E-00

$1.80 \mathrm{E}+01$

6.66E-03

$1.36 \mathrm{E}-04$

1.28E-04

7.64E-00

3.87E-01

$3.51 \mathrm{E}+05$

1.42E-00

$6.96 \mathrm{E}+01$

$9.14 \mathrm{E}+01$

9.07E-02

2.58E-00

$3.09 \mathrm{E}-00$

$2.87 \mathrm{E}+02$

1.62E-01

2.54E-03

8.57E- 00

$5.13 \mathrm{E}+01$

$8.40 \mathrm{E}-00$

$9.51 \mathrm{E}+01$

4.58E-04

4.71E-05

4.05E-01

1.51E-03

$3.40 \mathrm{E}+01$

$7.33 \mathrm{E}-01$

2.55E+03 1\%-ile

50\%-ile

$0.00 \mathrm{E}+01$

$4.84 \mathrm{E}+01$

$0.00 \mathrm{E}+01$

$0.00 \mathrm{E}+01$

$0.00 \mathrm{E}+01$

$0.00 \mathrm{E}+01$

$0.00 \mathrm{E}+01$

$0.00 \mathrm{E}+01$

$0.00 \mathrm{E}+01$

$0.00 \mathrm{E}+01$

$1.16 \mathrm{E}+04$

$1.99 \mathrm{E}+01$

$3.39 \mathrm{E}+02$

$5.14 \mathrm{E}+02$

$6.05 \mathrm{E}+03$

$9.94 \mathrm{E}-00$

$1.98 \mathrm{E}+03$

$1.10 \mathrm{E}+03$

$0.00 \mathrm{E}+01$

$4.02 \mathrm{E}+02$

4.05E-00

$1.83 \mathrm{E}+01$

$8.55 \mathrm{E}+04$

$1.99 \mathrm{E}+02$

$2.23 \mathrm{E}+04$

7.34E+04

$1.59 \mathrm{E}+03$

$7.43 \mathrm{E}+01$

$4.83 \mathrm{E}+03$

$2.57 \mathrm{E}+03$

$1.36 \mathrm{E}+02$

$1.07 \mathrm{E}+04$

$5.39 \mathrm{E}+01$

$1.17 \mathrm{E}+03$

$6.18 \mathrm{E}+02$

$0.00 \mathrm{E}+01$

$0.00 \mathrm{E}+01$

$1.47 \mathrm{E}+01$

$1.73 E-06$

$3.70 \mathrm{E}+04$

$0.00 \mathrm{E}+01$

$0.00 \mathrm{E}+01$

3.10E-05

6.33E-07

5.96E-07

2.88E-00

4.32E-02

$3.11 \mathrm{E}+04$

$0.00 \mathrm{E}+01$

$0.00 \mathrm{E}+01$

$0.00 E+01$

$1.73 \mathrm{E}-02$

$0.00 \mathrm{E}+01$

$0.00 E+01$

$0.00 \mathrm{E}+01$

7.54E-04

$0.00 E+01$

4.05E-02

2.78E-01

$1.81 \mathrm{E}-02$

$2.05 \mathrm{E}-01$

9.87E-07

$0.00 E+01$

$0.00 E+01$

$0.00 \mathrm{E}+01$

$0.00 \mathrm{E}+01$

$1.48 \mathrm{E}-01$

$0.00 E+01$

\subsection{E-01}

$6.06 \mathrm{E}+01$

$6.60 E-01$

2.53E-00

3.45E-01

$1.39 \mathrm{E}-02$

$7.64 \mathrm{E}-03$

$5.44 \mathrm{E}-01$

$5.43 \mathrm{E}+03$

8.41E-01

$1.90 \mathrm{E}+04$

$4.63 \mathrm{E}+02$

$4.66 \mathrm{E}+03$

$9.70 \mathrm{E}+02$

$2.13 E+04$

$1.57 \mathrm{E}+02$

$3.13 \mathrm{E}+03$

$6.81 E+03$

9.71E-02

$1.24 \mathrm{E}+03$

$1.49 \mathrm{E}+01$

$9.85 \mathrm{E}+01$

$1.23 \mathrm{E}+05$

$2.83 \mathrm{E}+03$

$3.13 \mathrm{E}+04$

$1.06 \mathrm{E}+05$

$5.01 E+03$

$3.79 E+02$

$9.41 \mathrm{E}+03$

$3.93 \mathrm{E}+03$

$7.97 \mathrm{E}+02$

$1.48 \mathrm{E}+04$

$1.98 \mathrm{E}+02$

$3.06 \mathrm{E}+03$

$5.44 \mathrm{E}+03$

$2.85 \mathrm{E}+01$

$0.00 \mathrm{E}+01$

$1.21 \mathrm{E}+02$

7.04E-05

$1.40 \mathrm{E}+05$

$1.01 \mathrm{E}-00$

$1.01 \mathrm{E}-00$

$1.26 \mathrm{E}-03$

2.58E-05

2.43E-05

$5.49 \mathrm{E}-00$

1.64E-01

$9.62 \mathrm{E}+04$

$0.00 \mathrm{E}+01$

$0.00 \mathrm{E}+01$

$0.00 \mathrm{E}+01$

3.73E-02

$3.27 \mathrm{E}-01$

$0.00 \mathrm{E}+01$

$0.00 \mathrm{E}+01$

3.08E-02

$0.00 \mathrm{E}+01$

1.65E-00

$1.13 \mathrm{E}+01$

7.38E-01

8.37E-00

4.02E-05

$0.00 \mathrm{E}+01$

$0.00 \mathrm{E}+01$

$1.31 \mathrm{E}-04$

$0.00 \mathrm{E}+01$

$3.25 \mathrm{E}-01$

$0.00 \mathrm{E}+01$

\subsection{E-00}

$7.46 \mathrm{E}+01$

5.35E-00

$2.05 \mathrm{E}+01$

2.80E-00

$1.13 \mathrm{E}-01$

$6.20 \mathrm{E}-02$

$4.41 \mathrm{E}-00$

$3.01 \mathrm{E}+04$

7.90E-00 
Tank Anal. Un

BY108 Sr89/90 Ci

BX108 Te99

BY108 Th229

BY108 Th232

BY108 Tin126

BY108 Tritium

BY108 U232

BY108 U233

BY108 U234

BY108 U235

BY108 U236

BY108 U238

BY108 Y90

BY108 Zr93

BY109 Al

BY109 Bi

BY109 Ca

BY109 Cl

BY109 CO3

BY109 $\mathrm{Cr}$

BY109 F

BY109 Fe

BY109 $\mathrm{Hg}$

BY109 K

BY109 La

BY109 Mn

BY109 $\mathrm{Na}$

BY109 Ni

BY109 NO2

BY109 NO3

BY109 P

BY109 Pb

BY109 PO4

BY109 S

BY109 Si

BY109 SO4

BY109 $\mathrm{Sr}$

BY109 TOC

BY109 U

BY109 Zr

BY109 Ac227

BY109 Am241

BY109 Am243

BY109 Ba137

BY109 C14

BY109 Cd113

BY109 Cm242

BY109 Cm243

BY109 Cm244

BY109 C060

BY109 Cs134

BY109 Cs137

BY109 Eu152

BY109 Eu154

BY109 Eu155

BY109 I129

BY109 Nb93

BY109 Ni59

BY109 Ni63

BY109 Np237

BY109 Pa231

BY109 Pu238

BY109 Pu239

BY109 Pu240

BY109 Pu241

BY109 Pu242

BY109 Ra226

BY109 Ra228 $\frac{\text { May98 }}{1.95 \mathrm{E}+05}$

9.36E-03

1.50E-02

1.10E-00

$3.29 \mathrm{E}+01$

2.26E-00

Ci $\quad 8.66 \mathrm{E}-00$

Ci 6.56E-01

Ci 2.46E-02

Ci 2.08E-02

Ci 1.33E-00

Ci $1.95 \mathrm{E}+05$

Ci 3.53E-00

$\mathrm{kg} \quad 4.82 \mathrm{E}+04$

kg $3.45 E+02$

kg $\quad 1.77 \mathrm{E}+03$

kg $\quad 4.26 \mathrm{E}+03$

$\mathrm{kg} \quad 2.18 \mathrm{E}+05$

kg $\quad 3.23 E+03$

kg 1.10E +04

kg $\quad 1.00 \mathrm{E}+04$

kg $\quad 1.06 \mathrm{E}+01$

kg $\quad 4.00 \mathrm{E}+03$

kg 6.57E-01

kg $\quad 4.79 \mathrm{E}+02$

kg $5.16 \mathrm{E}+05$

kg $5.51 \mathrm{E}+02$

kg $5.93 E+04$

kg $7.90 \mathrm{E}+05$

kg 4.34E +04

kg $3.96 \mathrm{E}+02$

kg $\quad 1.35 \mathrm{E}+05$

kg $3.34 \mathrm{E}+04$

$\mathrm{kg} \quad 6.26 \mathrm{E}+03$

kg $\quad 1.00 \mathrm{E}+05$

kg $\quad 1.66 \mathrm{E}+02$

kg $\quad 9.27 \mathrm{E}+03$

kg 4.17E+03

kg 2.36E+02

$\mathrm{Ci} \quad 2.89 \mathrm{E}-03$

Ci $6.52 \mathrm{E}+01$

Ci 2.26E-03

Ci $2.11 \mathrm{E}+05$

Ci $\quad 5.01 \mathrm{E}+01$

$\mathrm{Ci} \quad 1.07 \mathrm{E}+02$

Ci $8.73 \mathrm{E}-04$

Ci $\quad 1.77 E-05$

Ci 2.99E-04

4.66E+01

Ci 2.27E-00

Ci 2.23E+05

Ci 6.56E-00

Ci 7.87E+02

Ci $3.98 \mathrm{E}+02$

Ci 5.37E-01

Ci $1.46 \mathrm{E}+01$

Ci 5.32E-00

Ci $\quad 5.28 \mathrm{E}+02$

Ci 9.32E-01

$\mathrm{Ci}$ 1.47E-02

Ci 3.71E-00

Ci $1.77 \mathrm{E}+02$

Ci $2.28 \mathrm{E}+01$

Ci $2.67 \mathrm{E}+02$

Ci 1.29E-03

2.19E-04

Ci 2.49E-00
Sept98

$1.95 \mathrm{E}+05$
$4.69 \mathrm{E}+01$

9.36E-03

$1.50 \mathrm{E}-02$

1.10E-00

$3.29 \mathrm{E}+01$

$1.68 \mathrm{E}+01$

$6.42 \mathrm{E}+01$

4.86E-00

1.83E-01

1.54E-01

4.10E-00

$1.95 \mathrm{E}+05$

3.53E-00

$5.98 \mathrm{E}+04$

$2.53 E+02$

$5.72 E+02$

$2.35 E+03$

$2.94 \mathrm{E}+04$

$2.89 \mathrm{E}+03$

$7.41 \mathrm{E}+04$

$2.20 \mathrm{E}+03$

$0.00 \mathrm{E}+01$

$3.72 \mathrm{E}+03$

$0.00 \mathrm{E}+01$

$5.83 \mathrm{E}+02$

$3.70 \mathrm{E}+05$

$3.00 \mathrm{E}+02$

$4.52 \mathrm{E}+04$

$1.16 \mathrm{E}+05$

NA

$2.75 E+02$

$1.30 \mathrm{E}+05$

NA

$9.29 \mathrm{E}+03$

$1.22 \mathrm{E}+05$

$1.75 \mathrm{E}+02$

1.19E+04

$1.99 \mathrm{E}+03$

$9.25 \mathrm{E}+01$

2.89E-03

4.02E +01

$1.39 \mathrm{E}-03$

$1.41 \mathrm{E}+05$

$3.43 E+01$

$1.07 \mathrm{E}+02$

$5.39 \mathrm{E}-04$

1.09E-05

1.85E-04

$4.66 \mathrm{E}+01$

$1.56 \mathrm{E}-00$

$1.49 \mathrm{E}+05$

$6.56 \mathrm{E}-00$

$7.87 \mathrm{E}+02$

$3.98 \mathrm{E}+02$

$3.68 \mathrm{E}-01$

$1.46 \mathrm{E}+01$

5.32E-00

$5.28 \mathrm{E}+02$

9.32E-01

1.47E-02

2.29E-00

$8.22 E+01$

$1.41 \mathrm{E}+01$

$1.65 E+02$

$7.95 \mathrm{E}-04$

2.19E-04

$2.49 \mathrm{E}-0 \mathrm{O}$
$1 \%$-ile

$3.32 \mathrm{E}+04$

$10 \%$-ile

$1.38 \mathrm{E}-00$

$0.00 \mathrm{E}+01$

$0.00 \mathrm{E}+01$

$0.00 \mathrm{E}+01$

$1.78 \mathrm{E}+01$

$0.00 \mathrm{E}+01$

$0.00 \mathrm{E}+01$

$0.00 \mathrm{E}+01$

$0.00 \mathrm{E}+01$

$0.00 \mathrm{E}+01$

$0.00 \mathrm{E}+01$

$0.00 \mathrm{E}+01$

$0.00 \mathrm{E}+01$

$2.74 \mathrm{E}+04$

$0.00 \mathrm{E}+01$

$1.11 \mathrm{E}+02$

$1.79 E+03$

$2.60 \mathrm{E}+03$

$1.04 \mathrm{E}+03$

$8.43 \mathrm{E}+04$

4.59E-02

$0.00 \mathrm{E}+01$

7.44E-00

2.98E-02

2.80E-02

4.49E+05

$3.08 \mathrm{E}-01$

$2.89 \mathrm{E}+04$

$1.97 \mathrm{E}+04$

$2.51 \mathrm{E}+04$

9.59E-00

$6.91 \mathrm{E}+04$

$5.19 \mathrm{E}+04$

$8.89 E+03$

$1.53 \mathrm{E}+05$

5.23E-04

$1.14 \mathrm{E}+04$

$0.00 \mathrm{E}+01$

$0.00 \mathrm{E}+01$

$0.00 \mathrm{E}+01$

$0.00 \mathrm{E}+01$

$0.00 \mathrm{E}+01$

$0.00 \mathrm{E}+01$

$0.00 \mathrm{E}+01$

$0.00 \mathrm{E}+01$

$0.00 \mathrm{E}+01$

$0.00 \mathrm{E}+01$

$0.00 \mathrm{E}+01$

$0.00 \mathrm{E}+01$

$0.00 \mathrm{E}+01$

$1.02 \mathrm{E}+02$

$0.00 \mathrm{E}+01$

$0.00 \mathrm{E}+01$

$0.00 \mathrm{E}+01$

$0.00 \mathrm{E}+01$

$0.00 \mathrm{E}+01$

$0.00 \mathrm{E}+01$

$0.00 \mathrm{E}+01$

$0.00 \mathrm{E}+01$

$0.00 \mathrm{E}+01$

$0.00 \mathrm{E}+01$

$0.00 \mathrm{E}+01$

$0.00 \mathrm{E}+01$

$0.00 \mathrm{E}+01$

$0.00 \mathrm{E}+01$

$0.00 E+01$

$0.00 \mathrm{E}+01$

$1.50 \mathrm{E}+05$

$1.35 \mathrm{E}+01$

$0.00 \mathrm{E}+01$

$0.00 \mathrm{E}+01$

6.18E-02

$2.46 \mathrm{E}+01$

$0.00 \mathrm{E}+01$

$0.00 \mathrm{E}+01$

$0.00 \mathrm{E}+01$

$0.00 \mathrm{E}+01$

$0.00 \mathrm{E}+01$

$0.00 \mathrm{E}+01$

2.10E+04

4.47E-01 


\begin{tabular}{|c|c|c|c|c|c|c|c|c|c|c|c|}
\hline Tank & Inal. & Un & May $\frac{B B}{98}$ & Sept98 & $1 \%$ ile & $10 \%$-ile & $50 \%$-ile & $90 \%$-ile & 99\%-ile & Mean & Std Dev \\
\hline 100 & 06 & $\mathrm{Ci}$ & $9.30 \mathrm{E}-03$ & $6.37 \mathrm{E}-03$ & $0.00 \mathrm{E}+01$ & $O E-03$ & - & $10-v 2$ & 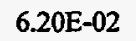 & $0 E-02$ & 20-V \\
\hline BY109 & Sb125 & $\mathrm{Ci}$ & $2.09 \mathrm{E}+02$ & $2.09 E+02$ & $0.00 \mathrm{E}+01$ & $0.00 \mathrm{E}+01$ & +02 & +03 & & $E+02$ & +02 \\
\hline Y109 & Se79 & $\mathrm{Ci}$ & 4.19E-00 & $2.87 \mathrm{E}-00$ & $5.93 \mathrm{E}-01$ & $2.23 \mathrm{E}-00$ & $.19 E-00$ & $.58 \mathrm{E}-00$ & $.85 \mathrm{E}-00$ & $4.33 \mathrm{E}-00$ & $1.76 \mathrm{E}-00$ \\
\hline 109 & 1151 & $\mathrm{Ci}$ & $1.45 E+04$ & $1.45 E+04$ & $0.00 \mathrm{E}+01$ & $0.00 \mathrm{E}+01$ & $1.45 E+04$ & $22 E+04$ & $34 \mathrm{E}+04$ & $3 E+04$ & $21 E+04$ \\
\hline Y109 & $89 / 90$ & $\mathrm{Ci}$ & $2.45 E+05$ & $1.05 E+05$ & $0.00 \mathrm{E}+01$ & $7.03 E+02$ & $2.22 \mathrm{E}+05$ & $92 \mathrm{E}+05$ & $.17 \mathrm{E}+05$ & $E+05$ & $1.83 E+05$ \\
\hline 109 & Te99 & $\mathrm{Ci}$ & $2.78 \mathrm{E}+02$ & $1.91 E+02$ & $0.00 \mathrm{E}+01$ & $00 \mathrm{E}+01$ & $2.78 E+02$ & $78 \mathrm{E}+02$ & $.85 E+02$ & $3.15 E+02$ & $2.59 \mathrm{E}+02$ \\
\hline 109 & Th229 & $\mathrm{Ci}$ & $5.75 E-02$ & $5.75 E-02$ & $0.00 \mathrm{E}+01$ & $0 \mathrm{E}+01$ & & $97 \mathrm{E}-01$ & $4.56 \mathrm{E}-01$ & & \\
\hline & Th232 & $\mathrm{Ci}$ & $9.20 \mathrm{E}-02$ & $9.20 \mathrm{E}-02$ & $0.00 \mathrm{E}+01$ & $0.00 \mathrm{E}+01$ & $9.20 \mathrm{E}-02$ & $3.15 \mathrm{E}-01$ & 7.30E-01 & & $E-01$ \\
\hline & n126 & $\mathrm{Ci}$ & $6.26 \mathrm{E}-00$ & $6.26 E-00$ & 501 & $E+01$ & $26 \mathrm{E}-00$ & $E+01$ & $08 \mathrm{E}+01$ & $=00$ & $E-00$ \\
\hline & Tritium & $\mathrm{Ci}$ & $1.91 \mathrm{E}+02$ & $1.31 \mathrm{E}+02$ & $1.47 \mathrm{E}+02$ & $E+02$ & $1.91 E+02$ & $8 E+02$ & $2.37 \mathrm{E}+02$ & +02 & +01 \\
\hline 109 & U232 & $\mathrm{Ci}$ & $1.39 E+01$ & 4.17E-01 & $0.00 \mathrm{E}+01$ & $E+01$ & $1.39 \mathrm{E}+01$ & $5 E+01$ & $10 E+02$ & $: 01$ & +01 \\
\hline & U233 & $\mathrm{Ci}$ & $5.32 E+01$ & $1.60 \mathrm{E}-00$ & +01 & $0 \mathrm{E}+01$ & 5.32E+01 & $2 E+02$ & $4.22 \mathrm{E}+02$ & $E+01$ & $E+01$ \\
\hline 109 & 1234 & $\mathrm{Ci}$ & $2.25 \mathrm{E}+01$ & $6.76 \mathrm{E}-01$ & +01 & $0 \mathrm{E}+01$ & $2.25 E+01$ & $69 \mathrm{E}+01$ & $1.79 \mathrm{E}+02$ & $E+01$ & $E+01$ \\
\hline BY109 & U235 & $\mathrm{Ci}$ & $9.82 \mathrm{E}-01$ & $2.95 \mathrm{E}-02$ & +01 & $0 \mathrm{E}+01$ & $9.82 \mathrm{E}-01$ & $3.36 E-00$ & $7.79 \mathrm{E}-00$ & E-00 & $1.62 \mathrm{E}-00$ \\
\hline 109 & U236 & $\mathrm{Ci}$ & $2.45 \mathrm{E}-01$ & $7.36 \mathrm{E}-03$ & +01 & $0 \mathrm{E}+01$ & $.45 \mathrm{E}-01$ & BE-01 & $1.94 \mathrm{E}-00$ & 3E-01 & 5E-01 \\
\hline & U238 & $\mathrm{Ci}$ & $2.68 \mathrm{E}+01$ & $6.64 \mathrm{E}-01$ & & $E+01$ & $2.68 \mathrm{E}+01$ & 01 & $2.13 E+02$ & +01 & $E+01$ \\
\hline 9 & Y90 & $\mathrm{Ci}$ & $2.45 E+05$ & $1.05 E+05$ & 01 & 01 & $2.45 E+05$ & $E+05$ & $E+06$ & +05 & +05 \\
\hline & and & $\mathrm{Ci}$ & $2.02 E+01$ & $2.02 E+01$ & -01 & +01 & $2.02 E+01$ & $E+02$ & $E+02$ & +02 & +02 \\
\hline & Al & kg & $4.09 \mathrm{E}+04$ & $4.09 E+04$ & 2.6 & $E+04$ & $4.75 E+04$ & $7 \mathrm{E}+04$ & $71 E+05$ & +04 & +04 \\
\hline & $\mathbf{B i}$ & kg & $1.05 E+02$ & $1.05 \mathrm{E}+02$ & & & $3.76 \mathrm{E}+03$ & $E+03$ & +03 & +03 & +03 \\
\hline 110 & Ca & kg & $9.36 \mathrm{E}+03$ & $9.36 E+03$ & +03 & $E+03$ & $1.19 E+04$ & $E+04$ & $2.69 E+04$ & $E+04$ & +03 \\
\hline 110 & Cl & kg & $5.94 E+03$ & $5.94 E+03$ & +03 & $: 03$ & $4.49 E+03$ & +03 & $E+04$ & +03 & +03 \\
\hline & $\cos$ & kg & $2.85 E+05$ & $2.85 \mathrm{E}+05$ & & & 2.10 & -05 & 05 & +05 & .04 \\
\hline 10 & $\mathrm{Cr}$ & $\mathrm{kg}$ & $6.20 \mathrm{E}+03$ & $6.20 \mathrm{E}+03$ & & & & -03 & 03 & -03 & \\
\hline 10 & $x_{2}$ & kg & $1.16 E+04$ & $1.16 E+04$ & 03 & 03 & 04 & +04 & $1.86 E+04$ & +04 & +03 \\
\hline & $\mathrm{Fe}$ & kg & $1.37 \mathbf{E}+04$ & $1.37 E+04$ & & 03 & $2.36 \mathrm{E}+04$ & $4.47 E+04$ & $44 E+04$ & 04 & +04 \\
\hline & $\mathbf{H g}$ & kg & 7.35E-00 & $1.18 E+01$ & +01 & $27 \mathrm{E}-01$ & & $29 \mathrm{E}+01$ & $61 E+01$ & $E-00$ & E-00 \\
\hline 110 & $\mathbf{K}$ & kg & $5.01 \mathrm{E}+03$ & $5.01 E+03$ & +03 & $E+03$ & $4.96 E+03$ & $7.67 \mathrm{E}+03$ & $5.74 E+04$ & +03 & $i+03$ \\
\hline 10 & $\mathbf{L a}$ & kg & $3.53 \mathrm{E}-01$ & e-01 & & & 11 & 01 & $E+02$ & -01 & 01 \\
\hline 10 & In & kg & $2.28 \mathrm{E}+02$ & $2.28 \mathrm{E}+02$ & 01 & +02 & 3.35 & +02 & $4.66 \mathrm{E}+03$ & +02 & +02 \\
\hline BY110 & la & kg & $4.95 E+05$ & $4.95 E+05$ & -05 & $E+05$ & 4.301 & $E \div 05$ & $5.48 E+05$ & $:+05$ & $E+04$ \\
\hline 110 & INI & kg & $4.40 E+03$ & $4.40 \mathrm{E}+03$ & -02 & -03 & $6.80 \mathrm{E}+03$ & $E+04$ & $1.61 E+04$ & & +03 \\
\hline 110 & NO2 & kg & $7.75 E+04$ & $7.75 E+04$ & +04 & $E+04$ & $7.29 E+04$ & $E+04$ & $E+04$ & +04 & +04 \\
\hline & NO3 & kg & $3.75 E+05$ & $3.75 E+05$ & -05 & $E+05$ & $3.12 E+05$ & $E+05$ & $E+06$ & $E+05$ & +05 \\
\hline 10 & & kg & $4.28 E+04$ & NA & 03 & 04 & 04 & +04 & .04 & 04 & -03 \\
\hline 110 & b & $\mathrm{kg}$ & $1.37 E+03$ & $1.37 E+03$ & 102 & +02 & -03 & +03 & +03 & +03 & +03 \\
\hline BY110 & PO4 & kg & $1.33 E+05$ & $4.33 E+04$ & +04 & $E+04$ & $2.56 \mathrm{E}+04$ & $4.34 \mathrm{E}+04$ & $6.43 E+04$ & +04 & +04 \\
\hline 10 & 3 & kg & $4.20 \mathrm{E}+04$ & NA & 103 & $E+03$ & 1.221 & $E+04$ & $E+04$ & $: 04$ & $E+03$ \\
\hline 10 & Si & kg & $1.48 E+03$ & $1.48 \mathrm{E}+03$ & -03 & & 3.49 & 03 & -04 & -03 & $E+03$ \\
\hline & SO4 & kg & $1.26 E+05$ & +04 & & & & & & 04 & -03 \\
\hline & r & kg & $4.29 E+03$ & 4.29 & & & & & & 03 & -03 \\
\hline 10 & roc & $\mathrm{kg}$ & $8+04$ & +04 & 6.0 & & & 04 & & -04 & +03 \\
\hline 110 & $\mathbf{U}$ & kg & $1.40 E+04$ & $1.40 E+04$ & +03 & $E+04$ & $2.57 E+04$ & $5 E+04$ & +05 & +04 & +04 \\
\hline 110 & $\mathbf{Z r}$ & kg & $3.60 \mathrm{E}+01$ & $3.60 \mathrm{E}+01$ & -00 & $\varepsilon+0.1$ & $2.63 E+02$ & $E+02$ & 04 & 02 & +03 \\
\hline 10 & Ac227 & $\mathrm{Ci}$ & $1.60 \mathrm{E}-03$ & $1.60 \mathrm{E}-03$ & & & & & -02 & & -03 \\
\hline BY110 & Am241 & $\mathrm{Ci}$ & $3.55 \mathrm{E}+01$ & $3.55 E+01$ & & +02 & 02 & 03 & 03 & 03 & -03 \\
\hline & $\mathrm{m} 243$ & $C i$ & 3 & 3 & & & & & & 03 & -03 \\
\hline 10 & Ba137 & $\mathrm{Ci}$ & +05 & 1.84 & 04 & 05 & 05 & 05 & 05 & +05 & $E+05$ \\
\hline & C14 & $\mathrm{Ci}$ & $2.70 E+01$ & $2.70 \mathrm{E}+01$ & 01 & 00 & 2.7 & 01 & $+0 !$ & $:+01$ & +01 \\
\hline B & Cd113 & $\mathrm{Ci}$ & $5.80 \mathrm{E}+01$ & $5.80 \mathrm{E}+01$ & -01 & & 5.8 & $\varepsilon+02$ & 02 & & $E+01$ \\
\hline & $\mathrm{Cm} 242$ & $\mathrm{Ci}$ & $6.72 \mathrm{E}-03$ & $6.72 \mathrm{E}-03$ & & & & & & & E-02 \\
\hline & $\mathrm{Cm} 243$ & $\mathrm{Ci}$ & & & & & & & & & \\
\hline & Cm244 & $\mathrm{Ci}$ & & 04 & & & & & & & -04 \\
\hline & Co60 & $\mathrm{Ci}$ & 2.51 & -01 & & & 2.28 & & & 01 & +01 \\
\hline & Cs134 & $\mathrm{Ci}$ & $1.24 \mathrm{E}-00$ & $1.24 \mathrm{E}-00$ & 01 & 01 & 00 & 00 & 00 & $E-00$ & $E-00$ \\
\hline & Cs137 & $C \mathrm{i}$ & $1.95 \mathrm{E}+05$ & $1.95 E+05$ & 05 & 05 & 05 & +05 & $1.04 E+06$ & +05 & +05 \\
\hline & Eu152 & $\mathrm{Ci}$ & $3.86 \mathrm{E}-00$ & $3.86 \mathrm{E}-00$ & 0. & 01 & 3.86 & -01 & 01 & .00 & -00 \\
\hline BY & Eu154 & $\mathrm{Ci}$ & $i+02$ & $4.24 \mathrm{E}+02$ & & 01 & & -03 & & -02 & 102 \\
\hline BY110 & Eu155 & $C i$ & $2.39 E+02$ & $2.39 E+02$ & & +01 & $2.39 \mathrm{E}+02$ & $E+02$ & $E+03$ & \pm 02 & $E+02$ \\
\hline BY110 & I129 & $\mathrm{Ci}$ & 2.92E-01 & 2.92E-01 & & & & $E-01$ & & & $E-01$ \\
\hline & Nb93 & $\mathrm{Ci}$ & $8.04 E-00$ & & & & & $=01$ & $E+02$ & $E+01$ & $E+01$ \\
\hline & Ni59 & $\mathrm{Ci}$ & 4.95E-00 & 4.95E-00 & & & DO & $1.22 \mathrm{E}+01$ & $1.82 \mathrm{E}+01$ & E-00 & 4.65E-00 \\
\hline & Ni63 & $\mathrm{Ci}$ & $4.72 E+02$ & $4.72 E+02$ & & $0 \mathrm{E}+01$ & $4.72 E+02$ & $1.17 \mathrm{E}+03$ & $1.74 E+03$ & $5 E+02$ & $E+02$ \\
\hline & Np237 & $\mathrm{Ci}$ & $5.10 \mathrm{E}-$ & $5.10 \mathrm{E}$ & & & & & & $6 \mathrm{E}-00$ & $E-00$ \\
\hline & $\operatorname{Pa} 231$ & $\mathrm{Ci}$ & $8.03 E-03$ & $8.03 \mathrm{E}-03$ & $0.00 \mathrm{E}+01$ & & 8.031 & $E-02$ & $5.80 \mathrm{E}-02$ & $1.07 \mathrm{E}-02$ & $0 \mathrm{E}-02$ \\
\hline BY110 & Pu238 & $C \mathrm{i}$ & 2.07E-00 & $1.52 \mathrm{E}-00$ & $E-01$ & $6.62 \mathrm{E}-01$ & 2.07E-00 & $1.69 E+01$ & $2.53 E+01$ & 4.31E-00 & $6.14 \mathrm{E}-00$ \\
\hline BY110 & Pu239 & $\mathrm{Ci}$ & $8.11 E+01$ & $5.97 E+01$ & $7.02 E-00$ & $2.60 \mathrm{E}+01$ & $8.11 \mathrm{E}+01$ & $6.64 \mathrm{E}+02$ & $9.92 \mathrm{E}+02$ & $1.69 E+02$ & $2.41 \mathrm{E}+02$ \\
\hline BY110 & Pu240 & $\mathrm{Ci}$ & $32 \mathrm{E}+01$ & $9.70 \mathrm{E}-00$ & $1.14 \mathrm{E}-00$ & $4.22 \mathrm{E}-00$ & $1.32 \mathrm{E}+01$ & $1.08 \mathrm{E}+02$ & $1.62 \mathrm{E}+02$ & $2.75 \mathrm{E}+01$ & $3.92 \mathrm{E}+01$ \\
\hline
\end{tabular}




\begin{tabular}{|c|c|c|c|c|c|c|c|c|c|c|c|}
\hline ank & Inal. & In & May $\frac{B B}{98}$ & Sept98 & $\%$-ile & $10 \%$-ile & $50 \%$-ile & $90 \%$-ile & $99 \%$-ile & Mean & Std Dev \\
\hline 1110 & & $\mathbf{C i}$ & $1.47 E+02$ & $1.08 \mathrm{E}+02$ & $1.27 \mathrm{E}+01$ & $4.70 \mathrm{E}+01$ & $1.47 \mathrm{E}+02$ & $20 \mathrm{E}+03$ & $1.80 \mathrm{E}+03$ & $3.06 \mathrm{E}+02$ & $4.36 \mathrm{E}+02$ \\
\hline 110 & 11242 & $\mathbf{C i}$ & 7.07E-04 & $7.07 E-04$ & $E-05$ & & $.07 E-04$ & 8E-03 & & $1.47 \mathrm{E}-03$ & $10 \mathrm{E}-03$ \\
\hline 110 & 2226 & $\mathbf{C i}$ & $1.22 \mathrm{E}-04$ & $1.22 \mathrm{E}-04$ & $0.00 E+01$ & $0.00 \mathrm{E}+01$ & $1.22 \mathrm{E}-04$ & $4.92 \mathrm{E}-04$ & 9.37E-04 & $1.92 E-04$ & 2.27E-04 \\
\hline 110 & 2228 & $\mathbf{C i}$ & $1.34 \mathrm{E}-00$ & $1.34 \mathrm{E}-00$ & $0.00 E+01$ & $0.00 \mathrm{E}+01$ & $1.34 \mathrm{E}-00$ & $5.40 \mathrm{E}-00$ & $1.03 \mathrm{E}+01$ & $2.10 \mathrm{E}-00$ & $2.49 \mathrm{E}-00$ \\
\hline$Y 110$ & $\mathbf{u 1 0 6}$ & $\mathrm{Ci}$ & $5.00 E-03$ & $5.00 \mathrm{E}-03$ & $0.00 \mathrm{E}+01$ & 8.63E-04 & $5.00 \mathrm{E}-03$ & $1.11 \mathrm{E}-02$ & $2.25 \mathrm{E}-02$ & $5.77 \mathrm{E}-03$ & 4.48E-03 \\
\hline$Y 110$ & b125 & $\mathrm{Ci}$ & $1.12 E+02$ & $1.12 \mathrm{E}+02$ & $0.00 \mathrm{E}+01$ & $1.70 \mathrm{E}-00$ & $1.12 E+02$ & $2.69 E+02$ & $4.84 \mathrm{E}+02$ & $1.30 \mathrm{E}+02$ & $1.08 \mathrm{E}+02$ \\
\hline 311 & Se79 & $\mathbf{C i}$ & $2.30 \mathrm{E}-00$ & $2.30 \mathrm{E}-00$ & $7.27 \mathrm{E}-01$ & $1.32 \mathrm{E}-00$ & & $4.00 \mathrm{E}-00$ & & -00 & \\
\hline & n151 & $\mathrm{Ci}$ & $7.96 \mathrm{E}+03$ & $7.96 E+03$ & $0.00 \mathrm{E}+01$ & $0.00 \mathrm{E}+01$ & $7.96 E+03$ & $3.16 \mathrm{E}+04$ & & $0 E+04$ & +04 \\
\hline & & $\mathbf{C i}$ & $2.63 E+05$ & $2.63 E+05$ & $5.96 \mathrm{E}+04$ & $E+05$ & $5.51 \mathrm{E}+05$ & $E+06$ & $5 \mathrm{E}+06$ & $E+05$ & +05 \\
\hline 110 & c99 & $\mathrm{Ci}$ & $E+02$ & $1.51 E+02$ & 7.75E-00 & $E+01$ & 02 & $10 E+02$ & $9 \mathrm{E}+03$ & $45 E+02$ & $4.00 E+02$ \\
\hline & & $\mathbf{C i}$ & $3.09 E-02$ & $3.09 \mathrm{E}-02$ & $0.00 \mathrm{E}+01$ & 67E-03 & & $23 E-02$ & & $4.11 \mathrm{E}-02$ & $4.23 \mathrm{E}-02$ \\
\hline & 232 & $\mathbf{C i}$ & $4.94 E-02$ & $4.94 \mathrm{E}-02$ & $0.00 E+01$ & 7E-03 & $4.94 \mathrm{E}-02$ & 2E-01 & 7E-01 & $8 \mathrm{E}-02$ & $6.77 \mathrm{E}-02$ \\
\hline 110 & in126 & $\mathbf{C i}$ & $3.44 \mathrm{E}-00$ & $3.44 \mathrm{E}-00$ & $0.00 E+01$ & $3.87 \mathrm{E}-01$ & $3.44 \mathrm{E}-00$ & $7 E+01$ & $E+01$ & E- 00 & E-00 \\
\hline 110 & Tritium & $\mathrm{Ci}$ & $1.04 \mathrm{E}+02$ & $1.04 E+02$ & $7.84 \mathrm{E}+01$ & $8.92 \mathrm{E}+01$ & $1.04 \mathrm{E}+02$ & $1.22 \mathrm{E}+02$ & $1.72 E+02$ & $1.06 \mathrm{E}+02$ & $E+01$ \\
\hline & 1232 & $\mathrm{Ci}$ & $7.46 \mathrm{E}-00$ & 9.17E-00 & $E+01$ & $E-01$ & $7.46 \mathrm{E}-00$ & $E+01$ & $E+01$ & $E-00$ & $E+01$ \\
\hline & 1233 & $\mathrm{Ci}$ & $2.86 E+01$ & $3.51 E+01$ & 01 & -00 & 2.8 & +01 & +02 & $E+01$ & +01 \\
\hline & 234 & $\mathrm{Ci}$ & E-00 & $5.05 \mathrm{E}-00$ & +01 & & $\varepsilon-00$ & +01 & & $E-00$ & -00 \\
\hline & 235 & c & 1.67E-01 & 2.05E-01 & $\begin{array}{l}i+01 \\
\end{array}$ & & E-01 & $E-01$ & $E-00$ & $E-01$ & $E-01$ \\
\hline & U236 & C & $8.86 \mathrm{E}-02$ & $1.09 \mathrm{E}-01$ & & & & $E-01$ & & & $E-01$ \\
\hline & $\mathrm{J} 238$ & C & $6.36 \mathrm{E}-00$ & 4.67E-00 & +01 & E-01 & E-00 & $E+01$ & & & $2-00$ \\
\hline 110 & Y90 & $\mathrm{Ci}$ & $2.63 E+05$ & $2.63 E+05$ & $E+01$ & $E+04$ & $2.63 E+05$ & $E+06$ & $E+06$ & $E+05$ & $E+05$ \\
\hline & Zr93 & $\mathrm{Ci}$ & $1.11 \mathrm{E}+01$ & $1.11 \mathrm{E}+01$ & & & +01 & & & & $E+01$ \\
\hline & Al & kg & $i+04$ & +04 & $3 .($ & 04 & +04 & 04 & & & +04 \\
\hline 11 & B1 & $\mathbf{k}$ & $E+02$ & $0.00 E+01$ & -01 & -00 & $E+02$ & +02 & +03 & & +02 \\
\hline & $\mathrm{Ca}$ & kg & $8.40 E+02$ & $8.40 \mathrm{E}+02$ & & & $3.56 \mathrm{E}+03$ & $E+03$ & +04 & $E+03$ & $8 E+03$ \\
\hline & Cl & $\pi$ & +03 & $2.98 \mathrm{E}+03$ & & & & & +03 & & $8+03$ \\
\hline & $\mathrm{CO3}$ & & $5.33 E+04$ & $2.66 \mathrm{E}+05$ & +05 & $:+05$ & $E+05$ & +05 & & +05 & +04 \\
\hline & $\mathrm{Cr}$ & kg & +03 & +03 & & +03 & 5.4 & & & +03 & +03 \\
\hline 111 & $\mathbf{F}$ & k & +04 & +04 & +03 & +04 & +04 & 104 & +04 & +04 & +03 \\
\hline 111 & $\mathrm{Fe}$ & kg & $1.63 E+04$ & $1.63 E+04$ & :+03 & :03 & +03 & +04 & +04 & $E+03$ & $E+03$ \\
\hline & $\mathbf{H g}$ & $\mathrm{kg}$ & $i+01$ & $0.00 \mathrm{E}+01$ & & +01 & & & & t01 & $E+01$ \\
\hline & $\mathbf{K}$ & kg & $4.65 E+03$ & $4.65 E+03$ & & & & & & & +03 \\
\hline & La & $\mathrm{kg}$ & E-01 & $0.00 \mathrm{E}+01$ & & & & & & & +02 \\
\hline & Mn & $\mathrm{kg}$ & 02 & $2.92 \mathrm{E}+02$ & & & & -02 & & -02 & +02 \\
\hline 111 & $\mathbf{N a}$ & $\mathbf{k}$ & $6.60 \mathrm{E}+05$ & $6.60 \mathrm{E}+05$ & +05 & -05 & 6.6 & t05 & & +05 & +05 \\
\hline 1 & $\mathrm{Ni}$ & $\mathbf{k g}$ & $1.30 E+03$ & $2.34 E+04$ & $E-01$ & $\varepsilon-00$ & 8.0 & $E+03$ & t03 & $E+02$ & +02 \\
\hline & NO2 & kg & $3.88 E+04$ & $3.88 \mathrm{E}+04$ & +03 & +04 & & +04 & & +04 & +04 \\
\hline & NO3 & $\mathrm{kg}$ & $4.18 E+05$ & $4.18 E+05$ & +05 & & & +05 & & & +05 \\
\hline 111 & $\mathbf{P}$ & $\mathrm{k}$ & $1.76 E+04$ & NA & & & & +04 & & & +03 \\
\hline & $\mathbf{P b}$ & & +02 & $2.23 E+02$ & $=00$ & +01 & +02 & $\div 02$ & & $E+02$ & +02 \\
\hline & PO4 & k & +04 & $5.45 E+04$ & +02 & +04 & +04 & +04 & & +04 & +04 \\
\hline & $\mathbf{S}$ & $\mathbf{k g}$ & $3.13 E+04$ & NA & +04 & -04 & +04 & $E+04$ & & +04 & +03 \\
\hline & $\mathbf{S i}$ & kg & $9.42 E+04$ & $9.42 \mathrm{E}+04$ & +04 & +04 & $t+04$ & $E+04$ & 04 & +04 & $E+03$ \\
\hline & SO4 & kg & $9.40 \mathrm{E}+04$ & $9.40 E+04$ & & & & & & & $E+04$ \\
\hline & $\mathrm{Sr}$ & $\mathbf{k}$ & +02 & & & & & & & & +02 \\
\hline & TOC & kg & +04 & 1.67 & .03 & & 104 & -04 & & +04 & +03 \\
\hline & 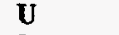 & $\mathrm{k}$ & $2.64 E+04$ & $2.64 E+04$ & $E-00$ & +03 & +04 & $E+04$ & -04 & +04 & +04 \\
\hline 111 & $\mathbf{Z r}$ & $\mathbf{k}$ & $2.40 E+01$ & $2.40 E+01$ & $E-03$ & -02 & +01 & $E+03$ & & +02 & $i+03$ \\
\hline & Ac227 & $\mathbf{C i}$ & -03 & $3.21 \mathrm{E}-03$ & +01 & +01 & & $\mathrm{E}-02$ & & .03 & $=-03$ \\
\hline & & $\mathrm{Ci}$ & +01 & 6.4 & & & & & & $\div 03$ & +03 \\
\hline & & $\mathrm{Ci}$ & & & & & & & & & $E-03$ \\
\hline & Ba137 & $\mathrm{Ci}$ & & 05 & & & & & & 35 & +05 \\
\hline & C14 & $\mathrm{Ci}$ & +01 & $5.59 \mathrm{E}+01$ & & & & & & +01 & +01 \\
\hline & Cd113 & $\mathrm{Ci}$ & $1.20 \mathrm{E}+02$ & $1.20 \mathrm{E}+02$ & $E+01$ & +01 & 1. & +02 & & $E+02$ & +02 \\
\hline & $\mathrm{Cm} 242$ & $\mathrm{Ci}$ & $9.64 E-04$ & 8.47E-04 & +01 & -06 & & -03 & & -03 & $E-04$ \\
\hline & $\mathrm{Cm} 243$ & $\mathrm{Ci}$ & $\varepsilon-05$ & $1.72 \mathrm{E}-05$ & +01 & & & E-05 & & E-05 & E-05 \\
\hline 111 & Cm244 & $\mathrm{Ci}$ & 3.34E-04 & 2.94E-04 & r01 & & & & & $E-04$ & E-04 \\
\hline & Co60 & $\mathrm{Ci}$ & $5.21 E+01$ & & & & & & & $E+01$ & $E+01$ \\
\hline & Cs134 & $\mathrm{Ci}$ & & & & & & & & -00 & $E-00$ \\
\hline & Cs137 & $\mathrm{Ci}$ & $2.26 \mathrm{E}+05$ & $2.26 E+05$ & & & & & & $E+05$ & +05 \\
\hline & Eu152 & $\mathrm{Ci}$ & 7.34 & & & & & & & $E-00$ & \\
\hline & Eu1 & $\mathrm{Ci}$ & $8.80 \mathrm{E}+02$ & $8.80 \mathrm{E}+02$ & $0.00 \mathrm{E}+01$ & +01 & & +03 & & $E+03$ & \\
\hline & Eu15 & $\mathrm{Ci}$ & $4.45 E+02$ & $4.45 E+02$ & $0.00 \mathrm{E}+01$ & $0.00 \mathrm{E}+01$ & 4.4 & $1.45 E+03$ & $E+03$ & $E+02$ & $E+02$ \\
\hline BY111 & I129 & $\mathbf{C} \mathbf{i}$ & $6.01 \mathrm{E}-01$ & $6.01 E-01$ & $\mathrm{E}-02$ & $E-01$ & E-01 & $4 E-00$ & $E-00$ & E-0l & $E-01$ \\
\hline & $\mathrm{Nb93}$ & $\mathrm{Ci}$ & $1.63 E+01$ & $1.63 E+01$ & $E+01$ & +01 & $1.63 E+01$ & $\mathrm{E}+02$ & $E+02$ & $1.60 \mathrm{E}+02$ & $3 E+02$ \\
\hline & Ni59 & $\mathbf{C i}$ & $5.95 E-00$ & & $E+01$ & +01 & $E-00$ & $E+01$ & $E+01$ & $1 E-00$ & $E-00$ \\
\hline & Ni63 & $\mathrm{Ci}$ & $5.91 E+02$ & $5.91 E+02$ & $0.00 E+01$ & & & $2.02 \mathrm{E}+03$ & $3.29 E+03$ & $7.96 \mathrm{E}+02$ & $8.41 E+02$ \\
\hline & & $\mathbf{C i}$ & & & $0.00 E+01$ & $6.10 \mathrm{E}-03$ & $1.04 \mathrm{E}-0$ & $2.57 \mathrm{E}-00$ & 4.12E-00 & $1.19 \mathrm{E}-00$ & $1.02 \mathrm{E}-00$ \\
\hline
\end{tabular}


Tank Anal. Un

\begin{tabular}{|c|c|}
\hline BY111 & Pa231 \\
\hline BY111 & Pu238 \\
\hline BY111 & Pu239 \\
\hline BY111 & Pu240 \\
\hline BY111 & Pu241 \\
\hline BY111 & Pu242 \\
\hline BY111 & $\operatorname{Ra226}$ \\
\hline BY111 & $\mathbf{R a 2 2 8}$ \\
\hline BY111 & Ru106 \\
\hline BY111 & Sb125 \\
\hline BY111 & Se79 \\
\hline BY111 & Sm151 \\
\hline BY111 & Sr89/90 \\
\hline BY111 & Tc99 \\
\hline BY111 & Th229 \\
\hline BY111 & Th232 \\
\hline $\begin{array}{l}\text { BY111 } \\
\text { RY111 }\end{array}$ & Tin126 \\
\hline $\begin{array}{l}\text { BY111 } \\
\text { BY111 }\end{array}$ & $\begin{array}{l}\text { Inthum } \\
\text { U232 }\end{array}$ \\
\hline
\end{tabular}

BY111 1232

BY111 U233

BY111 U234

BY111 U235

BY111 U236

BY111 U238

BY111 Y90

BY111 Zr93

BY112 Al

BY112 Bi

BY112 Ca

BY112 Cl

BY112 $\mathrm{CO}$

BY112 Cr

BY112 F

BY112 Fe

BY112 Hg

BY112 K

BY112 La

BY112 Mn

BY112 Na

BY112 Ni

BY112 NO2

BY112 NO3

BY112 $P$

BY112 Pb

BY112 PO4

BY112 S

BY112 Si

BY112 SO4

BY112 Sr

BY112 TOC

BY112 U

BY112 Zr

BY112 Ac227

BY112 Am241

BY112 Am243

BY112 Ba137

BY112 C14

BY112 Cd113

BY112 Cm242

BY112 Cm243

BY112 $\mathrm{Cm} 244$

BY112 Co60

BY112 Cs134

BY112 Cs137

BY112 Eu152

BY112 Eu154

BY112 Eu155

BY112 1129

\section{$\frac{B B}{98}$ \\ 1.64E-02}

4.15E-00

Ci $1.49 \mathrm{E}+02$

$2.55 \mathrm{E}+01$

$2.99 \mathrm{E}+02$

Ci 1.44E-03

Ci 2.38E-04

2.79E-00

$1.04 \mathrm{E}-02$

$2.34 \mathrm{E}+02$

Ci 4.68E-00

$1.62 \mathrm{E}+04$

$6.15 E+04$

$3.10 \mathrm{E}+02$

6.43E-02

1.03E-01

$7.00 \mathrm{E}-00$

$2.14 \mathrm{E}+02$

Ci 1.55E+01

Ci $5.95 \mathrm{E}+01$

Ci 1.77E+01

$\begin{array}{ll}\mathrm{Ci} & 7.64 \mathrm{E}-01 \\ \mathrm{Ci} & 2.27 \mathrm{E}-01\end{array}$

Ci $2.25 \mathrm{E}+01$

Ci $6.15 E+04$

$\begin{array}{ll}\mathrm{Ci} & 2.26 \mathrm{E}+01 \\ \mathrm{~kg} & 3.10 \mathrm{E}+04\end{array}$

$\begin{array}{ll}\mathrm{kg} & 3.10 \mathrm{E}+04 \\ \mathrm{~kg} & 3.27 \mathrm{E}+03\end{array}$

kg 3.27E+03

$\mathrm{kg} \quad 2.11 \mathrm{E}+03$

kg 3.26E+05

kg $2.84 \mathrm{E}+04$

kg 1.51E+04

$\mathrm{kg} \quad 4.76 \mathrm{E}+03$

$\begin{array}{lr}\mathrm{kg} & 4.76 \mathrm{E}+03 \\ \mathrm{~kg} & \mathbf{7 . 7 9 \mathrm { E } - 0 0}\end{array}$

kg $\quad 2.74 \mathrm{E}+03$

$\mathrm{kg} \quad 3.04 \mathrm{E}-01$

kg $\quad 4.69 \mathrm{E}+02$

$\mathrm{kg} \quad 5.43 \mathrm{E}+05$

kg $7.75 \mathrm{E}+03$

$\mathrm{kg} \quad 3.70 \mathrm{E}+04$

$\mathrm{kg} \quad 1.24 \mathrm{E}+05$

$\mathrm{kg} \quad 8.62 \mathrm{E}+03$

$\mathrm{kg} \quad 3.27 \mathrm{E}+03$

$\mathrm{kg} \quad 2.67 \mathrm{E}+04$

$\mathrm{kg} \quad 1.35 \mathrm{E}+04$

kg $\quad 3.91 \mathrm{E}+03$

$\mathrm{kg} \quad 4.04 \mathrm{E}+04$

$\mathrm{kg} \quad 3.27 \mathrm{E}+02$

kg $1.36 \mathrm{E}+04$

$\mathrm{kg} \quad 1.65 \mathrm{E}+04$

$\mathrm{kg} \quad 3.27 \mathrm{E}+02$

2.08E-03

$4.77 E+01$

$1.65 \mathrm{E}-03$

$1.79 \mathrm{E}+05$

$3.64 \mathrm{E}+01$

$7.81 \mathrm{E}+01$

8.81E-04

$1.80 \mathrm{E}-05$

2.21E-04

i $3.41 \mathrm{E}+01$

$1.66 \mathrm{E}-00$

$1.89 E+05$

$4.81 \mathrm{E}-00$

Ci $5.75 E+02$

$2.91 \mathrm{E}+02$

Ci 3.93E-01
$\frac{B B}{\operatorname{sep}}$

$\frac{\mathrm{ep} 2 \mathrm{8}}{1.64 \mathrm{E}-02}$

$3.65 \mathrm{E}-00$

$1.31 \mathrm{E}+02$

$2.24 \mathrm{E}+01$

$2.63 \mathrm{E}+02$

$1.27 E-03$

2.38E-04

2.79E-00

1.04E-02

$2.34 \mathrm{E}+02$

4.68E-00

$1.62 \mathrm{E}+04$

$6.15 E+04$

$3.10 \mathrm{E}+02$

6.43E-02

1.03E-01

7.00E-00

$2.14 \mathrm{E}+02$

$7.96 \mathrm{E}-00$

$3.05 \mathrm{E}+01$

9.09E-00

3.92E-01

1.16E-01

8.81 E-00

$6.15 \mathrm{E}+04$

$2.26 \mathrm{E}+01$

$3.10 \mathrm{E}+04$

$0.00 \mathrm{E}+01$

$3.27 \mathrm{E}+03$

$2.11 \mathrm{E}+03$

$3.26 \mathrm{E}+05$

$2.84 \mathrm{E}+04$

$1.51 \mathrm{E}+04$

$4.76 E+03$

$0.00 \mathrm{E}+01$

$2.74 E+03$

$0.00 \mathrm{E}+01$

$1.69 \mathrm{E}+02$

$5.43 E+05$

$1.07 \mathrm{E}+04$

$3.70 \mathrm{E}+04$

$1.24 \mathrm{E}+05$

$\mathrm{NA}$

$1.26 \mathrm{E}+03$

$2.67 \mathrm{E}+04$

NA

$3.91 \mathrm{E}+03$

$4.04 \mathrm{E}+04$

$0.00 \mathrm{E}+01$

$1.36 \mathrm{E}+04$

$1.00 E+04$

$1.40 \mathrm{E}+01$

2.08E-03

$1.85 \mathrm{E}-00$

6.40E-04

$1.79 \mathrm{E}+05$

$3.64 \mathrm{E}+01$

$7.81 \mathrm{E}+01$

3.41E-04

$6.98 \mathrm{E}-06$

8.56E-05

$3.41 \mathrm{E}+01$

$1.66 \mathrm{E}-00$

$1.89 \mathrm{E}+05$

$4.81 \mathrm{E}-00$

$5.75 E+02$

2.91E+02

3.93E-01

1\%-ile

$10 \%$ ile

$00 \mathrm{E}+01$

$0.00 \mathrm{E}+01$

$0.00 \mathrm{E}+01$

$0.00 \mathrm{E}+01$

$0.00 \mathrm{E}+01$

$0.00 \mathrm{E}+01$

$0.00 \mathrm{E}+01$

$2.23 \mathrm{E}-03$

$0.00 \mathrm{E}+01$

2.95E-01

$0.00 \mathrm{E}+01$

8.07E-00

$0.00 \mathrm{E}+01$

$0.00 \mathrm{E}+01$

$0.00 \mathrm{E}+01$

$0.00 \mathrm{E}+01$

$1.13 \mathrm{E}+02$

$0.00 \mathrm{E}+01$

$0.00 \mathrm{E}+01$

$0.00 \mathrm{E}+01$

$0.00 \mathrm{E}+01$

$0.00 \mathrm{E}+01$

$0.00 \mathrm{E}+01$

$0.00 E+01$

$0.00 \mathrm{E}+01$

$1.43 E+04$

1.21E-00

$1.24 \mathrm{E}+02$

3.77E+02

$2.34 \mathrm{E}+05$

$1.16 \mathrm{E}+04$

$7.03 \mathrm{E}+02$

$6.07 \mathrm{E}+02$

$0.00 \mathrm{E}+01$

$4.07 \mathrm{E}+01$

$2.64 E+02$

$5.10 \mathrm{E}-01$

$3.88 \mathrm{E}+05$

1. $49 \mathrm{E}+01$

$2.31 E+03$

$2.62 E+03$

$5.07 \mathrm{E}+03$

$2.44 \mathrm{E}-02$

8.74E-01

1.50E-01

$1.75 \mathrm{E}-00$

8.45E-06

$0.00 \mathrm{E}+01$

$0.00 E+01$

$5.54 \mathrm{E}-03$

$0.00 \mathrm{E}+01$

$1.98 \mathrm{E}-00$

$0.00 \mathrm{E}+01$

$8.74 \mathrm{E}+02$

$0.00 \mathrm{E}+01$

$0.00 \mathrm{E}+01$

$0.00 \mathrm{E}+01$

$0.00 \mathrm{E}+01$

$1.56 \mathrm{E}+02$

$0.00 \mathrm{E}+01$

$0.00 \mathrm{E}+01$

$0.00 \mathrm{E}+01$

$0.00 \mathrm{E}+01$

$0.00 \mathrm{E}+01$

$0.00 \mathrm{E}+01$

$0.00 \mathrm{E}+01$

$0.00 \mathrm{E}+01$

$2.08 \mathrm{E}+04$

$2.47 \mathrm{E}+01$

$5.51 \mathrm{E}+02$

9.24E+02

$2.77 \mathrm{E}+05$

1.33E+04

$4.92 \mathrm{E}+03$

$1.75 \mathrm{E}+03$

$0.00 \mathrm{E}+01$

$1.15 \mathrm{E}+03$

$3.05 E+02$

5.44E-00

$4.32 \mathrm{E}+05$

2.09E+02

$1.38 \mathrm{E}+04$

$4.32 \mathrm{E}+04$

$8.32 \mathrm{E}+03$

$1.82 \mathrm{E}+01$

7.53E+01

$3.66 \mathrm{E}+03$

$1.50 \mathrm{E}+04$

$6.45 \mathrm{E}+03$

$2.48 \mathrm{E}+01$

$7.58 \mathrm{E}+03$

$8.52 \mathrm{E}+02$

2.03E-01

$2.16 \mathrm{E}+03$ 


\begin{tabular}{|c|c|c|c|c|c|c|c|c|c|c|c|}
\hline & al. & Un & May $\frac{B B}{98}$ & $\operatorname{Sept98} \frac{B B}{}$ & $1 \%$-jle & $10 \%$-ile & 50\%-ile & $90 \%$-ile & 99\%-ile & Mean & Std Der \\
\hline & & $\mathrm{Ci}$ & $.07 \mathrm{E}+01$ & $07 \mathrm{E}+01$ & $E+01$ & $0.00 \mathrm{E}+01$ & $.07 E+01$ & $11 E+02$ & $8 \mathrm{E}+02$ & 1 & +01 \\
\hline & Nis9 & $\mathrm{Ci}$ & $3.97 \mathrm{E}-00$ & $3.97 \mathrm{E}-00$ & $0.00 \mathrm{E}+01$ & $E+01$ & $3.97 \mathrm{E}-00$ & $E+01$ & +01 & $E-00$ & $4.45 \mathrm{E}-00$ \\
\hline Y112 & Ni63 & $\mathrm{Ci}$ & $3.94 \mathrm{E}+02$ & $3.94 E+02$ & $0.00 \mathrm{E}+01$ & $.00 \mathrm{E}+01$ & $3.94 \mathrm{E}+02$ & $13 E+03$ & $74 \mathrm{E}+03$ & $4.87 \mathrm{E}+02$ & $4.41 E+02$ \\
\hline & Np237 & $\mathrm{Ci}$ & $6.80 \mathrm{E}-01$ & $6.80 \mathrm{E}-01$ & $5.19 \mathrm{E}-03$ & $5.11 \mathrm{E}-02$ & $6.80 \mathrm{E}-01$ & $1.90 \mathrm{E}-00$ & 44E-00 & $6 \mathrm{E}-01$ & $7.74 \mathrm{E}-01$ \\
\hline 112 & Pa231 & $\mathrm{Ci}$ & 1.07E-02 & 1.07E-02 & $0.00 \mathrm{E}+01$ & $98 E-05$ & $1.07 \mathrm{E}-02$ & -02 & $28 \mathrm{E}-02$ & 10E-02 & $1.39 \mathrm{E}-02$ \\
\hline & Pu238 & $\mathbf{C i}$ & $2.71 \mathrm{E}-00$ & $1.05 \mathrm{E}-00$ & $2.07 \mathrm{E}-02$ & 04E-01 & 2.71E-00 & $7.57 \mathrm{E}-00$ & $1.37 \mathrm{E}+01$ & $3.41 \mathrm{E}-00$ & 3.09E-00 \\
\hline & Pu239 & $\mathrm{Ci}$ & $9.72 E+01$ & $3.77 E+01$ & $7.42 \mathrm{E}-01$ & $31 \mathrm{E}-00$ & $9.72 E+01$ & $2.72 \mathrm{E}+02$ & $4.92 E+02$ & $1.22 \mathrm{E}+02$ & $E+02$ \\
\hline & Pu240 & $\mathbf{C i}$ & $1.67 E+01$ & $6.46 \mathrm{E}-00$ & Fn1 & & $1.67 E+01$ & $4.67 \mathrm{E}+01$ & $E+01$ & +01 & +01 \\
\hline 2 & Pu241 & $\mathbf{C i}_{\mathbf{i}}$ & $1.96 \mathrm{E}+02$ & $7.58 \mathrm{E}+01$ & 0 & 01 & $1.96 \mathrm{E}+02$ & & $E+02$ & $E+02$ & $E+02$ \\
\hline & & $\mathbf{C i}$ & & $3.64 \mathrm{E}$ & E-06 & $E-05$ & & $=-03$ & 77E-03 & $E-03$ & $E-03$ \\
\hline 12 & Ra226 & $\mathrm{Ci}$ & $1.48 E-04$ & $1.48 E-04$ & $0.00 \mathrm{E}+01$ & $0.00 \mathrm{E}+01$ & $1.48 \mathrm{E}-04$ & $r$ & J3E-04 & SE-04 & $E-04$ \\
\hline & $\operatorname{Ra} 228$ & $\mathbf{C i}$ & $1.82 \mathrm{E}-00$ & $1.82 \mathrm{E}-00$ & $E+01$ & $0.00 \mathrm{E}+01$ & E-00 & $5.68 \mathrm{E}-00$ & $E+01$ & $E-00$ & 2.57E-00 \\
\hline Y112 & Ru106 & $\mathbf{C i}$ & $6.80 \mathrm{E}-03$ & $6.80 \mathrm{E}-03$ & $E \div 01$ & $1.29 \mathrm{E}-03$ & $6.80 \mathrm{E}-03$ & $1.32 \mathrm{E}-02$ & $19 \mathrm{E}-02$ & $7.22 \mathrm{E}-03$ & $\mathrm{E}-03$ \\
\hline 112 & Sb125 & $\mathrm{Ci}$ & $1.53 E+02$ & $1.53 \mathrm{E}+02$ & $E+01$ & +01 & $1.53 E+02$ & +02 & $E+03$ & $E+02$ & $E+02$ \\
\hline & Se79 & $\mathrm{Ci}$ & $3.06 \mathrm{E}-00$ & $3.06 \mathrm{E}-00$ & $E+01$ & $E-00$ & $3.06 \mathrm{E}-00$ & $\varepsilon-00$ & -00 & $E-00$ & $\mathrm{E}-00$ \\
\hline & $\mathrm{Sm}$ & $\mathbf{C i}$ & $1.06 E+04$ & $1.06 E+04$ & $E+01$ & +01 & $E+04$ & +04 & 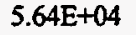 & $E+04$ & $E+04$ \\
\hline 2 & Sr89/9 & $\mathrm{Ci}$ & 1.3 & $1.33 E+05$ & & & & & & & +05 \\
\hline & Te99 & $\mathrm{Ci}$ & 2.0 & 2.0 & +01 & +01 & & & -03 & 02 & $E+02$ \\
\hline 12 & Th & $\mathbf{C i}$ & $4.20 \mathrm{E}-02$ & $4.20 \mathrm{E}-02$ & +01 & $2-04$ & E-02 & -01 & $E-01$ & $\varepsilon-02$ & $E-02$ \\
\hline 12 & Th232 & $\mathrm{Ci}$ & $6.73 \mathrm{E}-02$ & $6.73 \mathrm{E}-02$ & $E+01$ & $E-04$ & $6.73 \mathrm{E}-02$ & $E-01$ & E-01 & $E-02$ & E-02 \\
\hline 112 & 126 & $\mathrm{Ci}$ & 4.57 & 4.57E-00 & +01 & & E-00 & +01 & & -00 & $E-00$ \\
\hline & Tritium & $\mathrm{Ci}$ & $1.40 \mathrm{E}+02$ & $1.40 \mathrm{E}+02$ & $E+01$ & +02 & $\mathrm{E}+02$ & +02 & -02 & +02 & $E+01$ \\
\hline & $\mathbf{U} 232$ & $\mathbf{C i}$ & $1.02 E+01$ & $1.02 E+01$ & +01 & 02 & $\mathbf{E}+01$ & -01 & 01 & +01 & +01 \\
\hline & 33 & $\mathbf{C i}$ & $3.89 E+01$ & $3.89 \mathrm{E}+01$ & 0 & & 11 & & & $: 01$ & +01 \\
\hline & U234 & $\mathrm{Ci}$ & 3.79E-00 & $3.79 \mathrm{E}-00$ & +01 & -02 & -00 & +01 & 01 & $=00$ & $E-00$ \\
\hline 12 & U235 & $\mathbf{C i}$ & $1.49 \mathrm{E}-01$ & $1.49 \mathrm{E}-01$ & +01 & 04 & E-01 & $E-01$ & -01 & $E-01$ & $E-01$ \\
\hline 12 & U236 & $\mathbf{C i}$ & $9.83 \mathrm{E}-02$ & $9.83 \mathrm{E}-02$ & +01 & 04 & 9.83E-02 & -01 & -01 & $=-01$ & $E-01$ \\
\hline & U238 & $\mathbf{C i}$ & $6.82 \mathrm{E}-00$ & $3.34 \mathrm{E}-00$ & +01 & & & +01 & 1 & $=-00$ & $E-00$ \\
\hline & Y90 & $\mathbf{C i}$ & $1.33 E+05$ & $1.33 E+05$ & +01 & & -05 & 05 & & 05 & 105 \\
\hline & Zr93 & $\mathrm{Ci}$ & +01 & $i+01$ & 01 & & 1 & & -02 & & \\
\hline & $\mathbf{A}$ & kg & 04 & $2.03 E+04$ & $\div+03$ & +04 & -04 & 104 & -04 & -04 & +04 \\
\hline C101 & $\mathbf{B i}$ & kg & $2.76 \mathrm{E}+02$ & $2.76 \mathrm{E}+02$ & +02 & +03 & $E+03$ & +03 & +04 & $E+03$ & $E+03$ \\
\hline C101 & $\mathrm{Ca}$ & kg & $2.51 \mathrm{E}+03$ & $2.51 \mathrm{E}+03$ & +01 & +02 & $E+02$ & +03 & +03 & $E+02$ & $E+02$ \\
\hline 01 & Cl & kg & $2.30 \mathrm{E}+02$ & +02 & & & & 03 & & 2 & +02 \\
\hline & $\mathrm{CO} 3$ & kg & $6.84 E+03$ & $E+03$ & & & & & & 3 & \\
\hline & c & kg & 32 & 02 & 01 & 02 & & & & -02 & +02 \\
\hline C101 & $\mathbf{F}$ & kg & $0.00 \mathrm{E}+01$ & $0.00 \mathrm{E}+01$ & :+01 & +02 & +03 & +03 & +03 & $E+03$ & $E+03$ \\
\hline C101 & Fe & $\mathrm{kg}$ & $7.42 \mathrm{E}+03$ & $7.42 E+03$ & $5.56 \mathrm{E}+02$ & +03 & $E+03$ & +03 & -04 & $E+03$ & $E+03$ \\
\hline C101 & Hg & $\mathrm{kg}$ & $1.70 \mathrm{E}+02$ & $4.00 \mathrm{E}+01$ & -00 & +01 & $\mathbf{E}+02$ & +02 & 3 & +02 & $E+02$ \\
\hline C101 & $\mathbf{K}$ & $\mathrm{kg}$ & $4.50 \mathrm{E}+01$ & $\varepsilon+01$ & -01 & & +02 & & & 3. & $E+02$ \\
\hline 101 & 10 & $\mathrm{~kg}$ & & & & & & & & & \\
\hline & & & & +02 & & & & & & -02 & +02 \\
\hline C101 & $\mathbf{N a}$ & $\mathrm{kg}$ & $4.52 E+04$ & $4.52 E+04$ & $E+04$ & +04 & $E+04$ & +04 & -05 & $=\div 04$ & $E+04$ \\
\hline C101 & Ni & $\mathrm{kg}$ & $7.08 \mathrm{E}+02$ & $7.08 \mathrm{E}+02$ & -00 & +01 & $E+01$ & -02 & 03 & $E+01$ & $E+02$ \\
\hline C101 & $\mathrm{NO} 2$ & $\mathrm{~kg}$ & $5.32 E+03$ & $5.32 \mathrm{E}+03$ & -03 & 03 & $E+03$ & -04 & $E+04$ & \pm 04 & $E+03$ \\
\hline C & NO3 & $\mathrm{kg}$ & 3.74 & $3.74 \mathrm{E}+04$ & 03 & & & & & 04 & +04 \\
\hline C101 & $\mathbf{P}$ & kg & 1 & NA & & & & & & & +03 \\
\hline & & $\mathrm{kg}$ & & $3.63 \mathrm{E}+0$ & & 01 & 01 & & & 02 & $E+02$ \\
\hline C101 & PO4 & kg & $3.31 E+03$ & $1.08 E+03$ & +02 & +03 & -03 & & & $E+04$ & $E+04$ \\
\hline C101 & $\mathbf{S}$ & kg & $1.01 E+03$ & NA & $E+02$ & +02 & $E+02$ & -03 & .03 & $E+03$ & $E+03$ \\
\hline C101 & $\mathbf{S}$ & $\mathrm{kg}$ & $1.30 \mathrm{E}+04$ & $1.30 \mathrm{E}+04$ & $E+02$ & +02 & $E+03$ & .03 & $E+03$ & $\div 03$ & $E+03$ \\
\hline C & $\mathbf{S}$ & kg & +03 & 1. & 02 & .02 & & +03 & & 03 & $\div 03$ \\
\hline C101 & & kg & 0. & o. & & & & & & & +02 \\
\hline & & kg & & & & & & & & & +03 \\
\hline & & kg & & & +02 & -03 & & & & +03 & $E+03$ \\
\hline C101 & $\mathbf{Z r}$ & kg & $2.31 \mathrm{E}+02$ & $2.31 E+02$ & E-01 & -00 & $E+01$ & +02 & +03 & $E+01$ & $E+02$ \\
\hline C101 & Ac227 & $\overrightarrow{C i}$ & $1.56 \mathrm{E}-02$ & $1.56 \mathrm{E}-02$ & $E+01$ & $E_{-}-04$ & $1.56 \mathrm{E}-02$ & $E-02$ & BE-01 & $E-02$ & $\mathrm{E}-02$ \\
\hline C10 & Am 2 & $\mathrm{Ci}$ & $5.49 \mathrm{E}+02$ & $5.49 \mathrm{E}+02$ & $F=00$ & $E+01$ & $E+01$ & $E+02$ & 9 & $E+01$ & $E+02$ \\
\hline C101 & Am243 & $\mathrm{Ci}$ & $1.45 E-06$ & $4.81 \mathrm{E}-$ & .08 & .07 & 06 & -06 & -06 & -06 & $E-06$ \\
\hline C101 & Ba137 & $\mathrm{Ci}$ & $1.64 E+03$ & 1.6 & 01 & 4 & 1. & 03 & & 03 & +03 \\
\hline C10 & C1 & C & & & & & & & & & $=-02$ \\
\hline & & c & & & & & & & & -01 & $2 \mathrm{E}-01$ \\
\hline C. & $\mathrm{Cm} 242$ & $\mathrm{Ci}$ & $2.88 \mathrm{E}-03$ & 9.5 & & $E-04$ & $2.88 \mathrm{E}-03$ & -03 & -02 & E-03 & $2.38 \mathrm{E}-03$ \\
\hline C101 & $\mathrm{Cm} 243$ & $\mathrm{Ci}$ & $6.75 E-05$ & $2.24 \mathrm{E}-01$ & $E-06$ & $E-05$ & $6.75 \mathrm{E}-05$ & $E-04$ & 2.35E-04 & $\mathrm{E}-05$ & $5.58 \mathrm{E}-05$ \\
\hline C101 & Cm244 & $\mathrm{Ci}$ & 5.42E-05 & $1.80 \mathrm{E}-01$ & $53 \mathrm{E}-06$ & 1.37E-05 & $5.42 E-05$ & $1.28 \mathrm{E}-04$ & $1.89 \mathrm{E}-04$ & 6. & $4.48 \mathrm{E}-05$ \\
\hline C101 & Co60 & $\mathrm{Ci}$ & 2.84E-02 & $2.84 \mathrm{E}-02$ & $7 \mathrm{E}-01$ & $2.15 \mathrm{E}-00$ & $4.03 E-00$ & $1.24 E+01$ & $8.32 \mathrm{E}+01$ & $E-00$ & $1.48 \mathrm{E}+01$ \\
\hline $\mathrm{C} 101$ & Cs134 & $\mathrm{Ci}$ & $4.46 \mathrm{E}-03$ & $4.46 \mathrm{E}-03$ & $E+01$ & $1.27 \mathrm{E}-03$ & $4.46 \mathrm{E}-03$ & E-03 & -02 & -03 & $3.10 \mathrm{E}-03$ \\
\hline & & $\mathrm{Ci}$ & $73 E+03$ & $1.73 E+03$ & $2.83 E+03$ & $1.28 E+04$ & $3.20 \mathrm{E}+04$ & $6.05 E+04$ & $8.74 E+04$ & $3.45 \mathrm{E}+04$ & $1.88 E+04$ \\
\hline
\end{tabular}


Tank Anal. Un

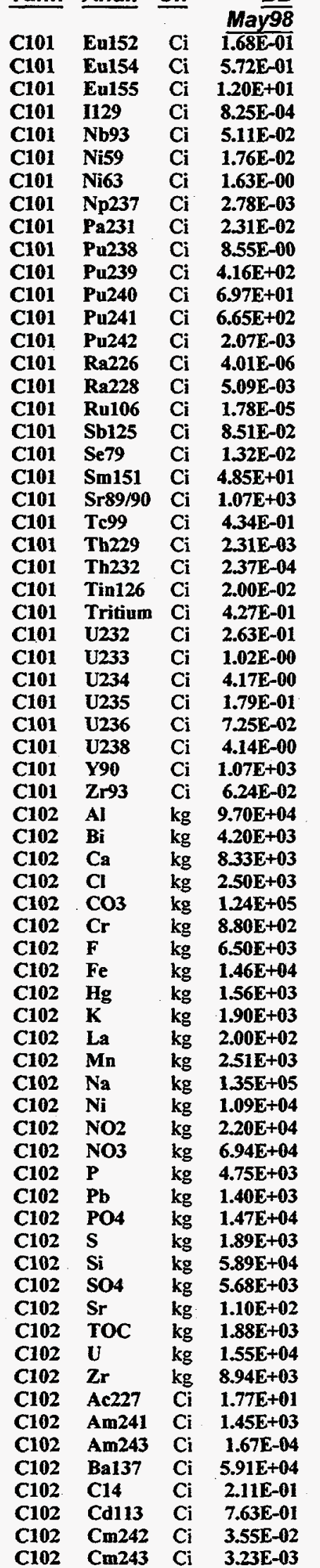

\section{$B B$} Sept98

1.68E-01

$5.72 \mathrm{E}-01$

$1.20 \mathrm{E}+01$

8.25E-04

5.11E-02

$1.76 \mathrm{E}-02$

$1.63 \mathrm{E}-00$

2.78E-03

2.31E-02

8.55E-00

$4.16 \mathrm{E}+02$

$6.97 \mathrm{E}+01$

$6.65 \mathrm{E}+02$

2.07E-03

4.01E-06

5.09E-03

1.78E-05

8.51E-02

1.32E-02

$4.85 E+01$

$1.07 \mathrm{E}+03$

4.34E-01

2.31E-03

2.37E-04

2.00E-02

4.27E-01

8.89E-02

3.45E-01

1.41E-00

6.07E-02

2.46E-02

$1.40 \mathrm{E}-00$

$1.07 \mathrm{E}+03$

$6.24 \mathrm{E}-02$

$9.70 \mathrm{E}+04$

$4.20 \mathrm{E}+03$

$8.33 \mathrm{E}+03$

$2.50 \mathrm{E}+03$

$1.24 \mathrm{E}+05$

$8.80 \mathrm{E}+02$

$6.50 \mathrm{E}+03$

$1.46 \mathrm{E}+04$

$2.73 E+02$

$1.90 \mathrm{E}+03$

$2.00 \mathrm{E}+02$

$2.51 E+03$

$1.35 \mathrm{E}+05$

$1.09 \mathrm{E}+04$

$2.20 \mathrm{E}+04$

$6.94 \mathrm{E}+04$

NA

$1.40 \mathrm{E}+03$

$1.47 \mathrm{E}+04$

NA

$5.89 \mathrm{E}+04$

$5.68 \mathrm{E}+03$

$1.10 \mathrm{E}+02$

$1.88 \mathrm{E}+03$

$1.55 \mathrm{E}+04$

$8.94 \mathrm{E}+03$

$1.77 \mathrm{E}+01$

$1.45 \mathrm{E}+03$

1.37E-01

$5.91 \mathrm{E}+04$

2.11E-01

7.63E-01

$2.90 \mathrm{E}+01$

2.64E- 00 1\%-ile

10\%-ile

50\%-ile

90\%-ile

99\%-ile

$0.00 \mathrm{E}+01$

$9.59 \mathrm{E}-03$

$3.26 \mathrm{E}-02$

$6.85 \mathrm{E}-01$

$1.80 \mathrm{E}-04$

$9.40 \mathrm{E}-03$

$6.01 \mathrm{E}-03$

$5.57 \mathrm{E}-01$

7.01E-04

$1.93 \mathrm{E}-03$

$2.16 \mathrm{E}-00$

$1.05 \mathrm{E}+02$

$1.76 \mathrm{E}+01$

$1.68 \mathrm{E}+02$

5.22E-04

$1.06 \mathrm{E}-06$

$1.35 \mathrm{E}-03$

4.84E-06

$2.46 \mathrm{E}-02$

5.51E-03

2.77E-00

$0.00 \mathrm{E}+01$

$1.05 \mathrm{E}+02$

$2.49 \mathrm{E}+03$

$0.00 \mathrm{E}+01$

$0.00 \mathrm{E}+01$

$0.00 \mathrm{E}+01$

1.65E-04

1. $10 E-01$

$0.00 \mathrm{E}+01$

$0.00 \mathrm{E}+01$

$0.00 \mathrm{E}+01$

$0.00 E+01$

$0.00 \mathrm{E}+01$

$0.00 \mathrm{E}+01$

$1.03 \mathrm{E}-00$

$1.40 \mathrm{E}-03$

$9.32 \mathrm{E}+04$

$5.80 E+02$

$6.16 \mathrm{E}+02$

$4.49 \mathrm{E}+02$

$4.45 \mathrm{E}+03$

$2.28 \mathrm{E}+02$

$1.09 \mathrm{E}+03$

$2.05 E+03$

$2.89 \mathrm{E}+02$

$2.76 \mathrm{E}+02$

$5.91 \mathrm{E}-00$

$6.84 E+01$

$6.57 \mathrm{E}+04$

$9.50 \mathrm{E}+01$

$4.02 \mathrm{E}+03$

1.19E+04

$1.53 \mathrm{E}+03$

$1.10 \mathrm{E}-02$

$1.93 \mathrm{E}-04$

$1.98 \mathrm{E}-05$

3.83E-03

$2.33 \mathrm{E}-01$

$2.20 \mathrm{E}-02$

8.52E-02

3.48E-01

$1.50 \mathrm{E}-02$

$6.06 \mathrm{E}-03$

$3.46 \mathrm{E}-01$

$2.77 \mathrm{E}+01$

$1.15 \mathrm{E}-02$

$1.62 \mathrm{E}+05$

$1.27 E \div 03$

$1.05 \mathrm{E}+03$

$1.11 E+03$

$1.51 \mathrm{E}+04$

$1.02 \mathrm{E}+03$

$3.02 \mathrm{E}+03$

$4.81 \mathrm{E}+03$

$6.92 \mathrm{E}+02$

$7.49 \mathrm{E}+02$

$1.97 \mathrm{E}+01$

$6.28 \mathrm{E}+02$

$1.51 \mathrm{E}+05$

$2.57 \mathrm{E}+02$

$1.36 \mathrm{E}+04$

4.45E+04

$3.51 \mathrm{E}+03$

$2.92 \mathrm{E}+01$

$2.77 \mathrm{E}+03$

$5.06 \mathrm{E}+02$

$6.33 E+02$

$1.08 \mathrm{E}+03$

$1.09 \mathrm{E}+01$

$4.53 \mathrm{E}+02$

$5.03 E+03$

$5.47 \mathrm{E}+02$

$0.00 \mathrm{E}+01$

$1.12 \mathrm{E}+02$

5.81E-06

$0.00 \mathrm{E}+01$

$0.00 \mathrm{E}+01$

$6.62 \mathrm{E}+01$

$5.73 E+03$

$9.20 \mathrm{E}+02$

$1.54 \mathrm{E}+03$

$2.47 \mathrm{E}+03$

$1.41 \mathrm{E}+02$

$1.94 \mathrm{E}+03$

$1.03 \mathrm{E}+04$

$2.64 \mathrm{E}+03$

$1.02 \mathrm{E}+01$

$1.40 \mathrm{E}+02$

2.83E-05

$1.52 \mathrm{E}+04$

1.24E-02

$3.38 \mathrm{E}-01$

$\begin{array}{ll}1.24 \mathrm{E}-03 & 6.02 \mathrm{E}-03 \\ 1.12 \mathrm{E}-04 & 5.48 \mathrm{E}-04\end{array}$

$1.24 \mathrm{E}-03$

5.48E-04

\subsection{E-01}

5.72E-01

8.25E-04

5.11E-02

$1.76 \mathrm{E}-02$

1.63E-00

2.78E-03

2.31E-02

$8.55 \mathrm{E}-00$

$4.16 \mathrm{E}+02$

$6.97 \mathrm{E}+01$

$6.65 \mathrm{E}+02$

2.07E-03

4.01E-06

5.09E-03

1.78E-05

8.51E-02

1.32E-02

$4.85 \mathrm{E}+01$

$9.57 \mathrm{E}+04$

4.34E-01

2.31E-03

2.37E-04

2.00E-02

4.27E-01

2.63E-01

1.02E-00

4.17E-00

1.79E-01

7.25E-02

4.14E-00

$1.07 \mathrm{E}+03$

$6.24 \mathrm{E}-02$

$2.51 \mathrm{E}+05$

$3.06 \mathrm{E}+03$

$2.60 \mathrm{E}+03$

$4.48 \mathrm{E}+03$

$3.35 \mathrm{E}+04$

$4.10 \mathrm{E}+03$

$8.03 E+03$

$1.12 \mathrm{E}+04$

$1.56 \mathrm{E}+03$

1.71E +03

2.82E+01

$3.90 \mathrm{E}+03$

$2.72 \mathrm{E}+05$

$7.24 \mathrm{E}+02$

$5.23 \mathrm{E}+04$

$2.52 \mathrm{E}+05$

$9.30 \mathrm{E}+03$

$1.46 \mathrm{E}+02$

$1.81 \mathrm{E}+04$

$1.82 \mathrm{E}+03$

$4.14 \mathrm{E}+03$

$4.94 \mathrm{E}+03$

$6.72 \mathrm{E}+02$

$6.54 \mathrm{E}+03$

$2.27 \mathrm{E}+04$

$6.27 \mathrm{E}+03$

$1.77 \mathrm{E}+01$

$2.09 E+02$

1.67E-04

$5.91 E+04$

2.11E-01

7.63E-01

3.55E-02

3.23E-03
$1.20 \mathrm{E}+01$

\section{$6.21 \mathrm{E}-01$}

2.11E-00

$4.43 \mathrm{E}+01$

1.94E-03

2.19E-01

4.96E-02

4.60E-00

$6.56 \mathrm{E}-03$

7.34E-02

$2.02 \mathrm{E}+01$

$9.82 \mathrm{E}+02$

$1.64 \mathrm{E}+02$

$1.57 \mathrm{E}+03$

$4.88 \mathrm{E}-03$

9.59E-06

$1.22 \mathrm{E}-02$

4.69E-05

$2.55 \mathrm{E}-01$

$3.28 \mathrm{E}-02$

$1.79 \mathrm{E}+02$

$1.44 \mathrm{E}+06$

1.28E-00

7.34E-03

7.53E-04

$7.95 \mathrm{E}-02$ 


\begin{tabular}{|c|c|c|c|c|c|c|c|c|c|c|c|}
\hline ank & Inal. & $n$ & $\frac{B B}{\operatorname{lay} 98}$ & $\frac{B B}{B \text { Sep }}$ & $1 \%$-ile & $10 \%$-ile & $50 \%-i l e$ & $90 \%-i l e$ & $99 \%-i l e$ & Mean & Std Dev \\
\hline 102 & n244 & $\mathrm{Ci}$ & $7.19 \mathrm{E}-02$ & $5.88 \mathrm{E}+01$ & $2.50 \mathrm{E}-03$ & $1.22 \mathrm{E}-02$ & $7.19 \mathrm{E}-02$ & $1.37 \mathrm{E} \sim 01$ & $2.01 \mathrm{E}-01$ & $7.47 \mathrm{E}-02$ & E- -02 \\
\hline & 2060 & $\mathrm{Ci}$ & $5.00 \mathrm{E}+02$ & $5.00 \mathrm{E}+02$ & $E+01$ & $2.00 \mathrm{E}+01$ & $2.73 \mathrm{E}+01$ & $4.79 E+01$ & $63 E+02$ & $3.39 E+01$ & $.55 E+01$ \\
\hline 102 & Cs134 & $\mathrm{Ci}$ & $1.20 \mathrm{E}-01$ & $1.20 \mathrm{E}-01$ & $0.00 E+01$ & $3.09 \mathrm{E}-02$ & $1.20 \mathrm{E}-01$ & $2.11 E-01$ & $2.86 \mathrm{E}-01$ & $1.21 \mathrm{E}-01$ & $6.70 \mathrm{E}-02$ \\
\hline 102 & Cs137 & $\mathrm{Ci}$ & $6.25 E+04$ & $6.25 E+04$ & $27 \mathrm{E}+04$ & $8.46 E+04$ & $1.91 E+05$ & $3.00 \mathrm{E}+05$ & $3.89 \mathrm{E}+05$ & $1 E+05$ & $19 E+04$ \\
\hline 102 & Eu152 & Ci & $1.03 \mathrm{E}-00$ & $1.03 E-00$ & $0.00 \mathrm{E}+01$ & $5.94 \mathrm{E}-01$ & $1.03 \mathrm{E}-00$ & $96 E-00$ & $19 \mathrm{E}+01$ & $2.13 \mathrm{E}-00$ & $2.63 E-00$ \\
\hline 102 & 154 & $\mathrm{Ci}$ & $9.05 \mathrm{E}+01$ & $9.05 \mathrm{E}+01$ & $0.00 \mathrm{E}+01$ & $5.22 \mathrm{E}+01$ & $9.05 E+01$ & $24 E+02$ & $1.05 \mathrm{E}+03$ & $1.87 \mathrm{E}+02$ & $31 E+02$ \\
\hline $\mathrm{ClO2}$ & 155 & $\mathrm{Ci}$ & $9.15 E+01$ & $9.15 \mathrm{E}+01$ & $0.00 \mathrm{E}+01$ & $5.28 \mathrm{E}+01$ & $15 E+01$ & $.30 E+02$ & $1.06 \mathrm{E}+03$ & $1.90 E+02$ & $34 E+02$ \\
\hline $\mathrm{ClO2}$ & 29 & $\mathrm{Ci}$ & $2.57 \mathrm{E}-03$ & 2.57E-03 & $0.00 \mathrm{E}+01$ & $2.43 \mathrm{E}-04$ & 57E-03 & $36 \mathrm{E}-03$ & E-03 & $2.70 \mathrm{E}-03$ & $.93 \mathrm{E}-03$ \\
\hline $\mathrm{C} 102$ & & $\mathrm{Ci}$ & $1.40 \mathrm{E}-01$ & 1.40E-01 & $0.00 \mathrm{E}+01$ & $0.00 E+01$ & $40 E-01$ & $4 E-01$ & $8.18 \mathrm{E}-01$ & $E-01$ & $E=01$ \\
\hline 102 & 159 & $\mathrm{Ci}$ & 4.09E-02 & $4.09 \mathrm{E}-02$ & $.70 \mathrm{E}-03$ & $1.40 \mathrm{E}-02$ & $4.09 \mathrm{E}-02$ & $3.67 \mathrm{E}-01$ & $E-01$ & $1.20 \mathrm{E}-01$ & $1.89 \mathrm{E}-01$ \\
\hline 102 & & $\mathrm{Ci}$ & $4.02 \mathrm{E}-00$ & $4.02 \mathrm{E}-00$ & $4.62 \mathrm{E}-01$ & $1.38 \mathrm{E}-00$ & $4.02 \mathrm{E}-00$ & $3.61 E+01$ & $8.75 E+01$ & $1.18 \mathrm{E}+01$ & $1.86 \mathrm{E}+01$ \\
\hline 102 & Np237 & $\mathrm{Ci}$ & 8.93E-03 & $8.93 E-03$ & $3.11 E-04$ & $1.51 \mathrm{E}-03$ & $8.93 \mathrm{E}-03$ & $1.70 \mathrm{E}-02$ & $E-02$ & $8 E-03$ & $E-03$ \\
\hline 102 & Pa231 & $\mathrm{Ci}$ & $2.97 \mathrm{E}+01$ & $2.97 E+01$ & $E+01$ & $3.60 \mathrm{E}-00$ & $E+01$ & $2 \mathrm{E}+02$ & +02 & $E+01$ & \\
\hline 102 & Pu238 & $\mathbf{C i}$ & 1.02 & $1.70 \mathrm{E}+02$ & $E-00$ & 11.7 & $E+02$ & $E+02$ & & $E+02$ & +01 \\
\hline 7 & $\mathbf{P}$ & $\mathrm{Ci}$ & $6.96 \mathrm{E}+03$ & $5.88 \mathrm{E}+03$ & +02 & +03 & -03 & $E+04$ & +04 & +03 & +03 \\
\hline 2 & 240 & $\mathrm{Ci}$ & 1. in 239 & $1.08 \mathrm{E}+03$ & +02 & +03 & -03 & $E \div 04$ & +04 & $E+03$ & +03 \\
\hline 02 & Pu241 & $\mathrm{Ci}$ & $90 \mathrm{E}+03$ & $1.31 E+04$ & $E+02$ & $1.34 E+03$ & -03 & $E+04$ & +04 & $E+03$ & +03 \\
\hline C102 & Pu242 & $\mathbf{C i}$ & $3.00 \mathrm{E}-02$ & 4.99E-02 & $E-03$ & $5.09 \mathrm{E}-03$ & -02 & E-02 & -02 & & \\
\hline & 226 & & $7.53 \mathrm{E}-$ & $7.53 \mathrm{E}-04$ & +01 & & & $E-03$ & & & $=03$ \\
\hline 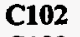 & Ra228 & $\mathrm{Ci}$ & 5.39E-00 & 5.39E-00 & +01 & & 00 & $E+01$ & & & \\
\hline$?$ & Ru106 & $\mathrm{Ci}$ & 8.9 & $8.96 \mathrm{E}-04$ & & & & $=-03$ & & & -03 \\
\hline $\mathrm{Cl02}$ & 25 & $\mathrm{Ci}$ & $0.00 \mathrm{E}+01$ & $0.00 E+01$ & +01 & 0.0 & 01 & +01 & 01 & & \\
\hline C102 & & $\mathrm{C}_{\mathbf{i}}$ & 4.42E-02 & 4.42E-02 & -03 & -02 & -02 & $E-01$ & $E-00$ & E-01 & -01 \\
\hline $\mathrm{ClO2}$ & 151 & $\mathrm{Ci}$ & $1.29 E+02$ & $1.29 \mathrm{E}+02$ & $t=1$ & 01 & +02 & $E+02$ & +03 & & \\
\hline C102 & $39 / 90$ & $\mathbf{C} \mathbf{i}$ & 1.821 & $1.82 E+05$ & +02 & & +05 & +06 & +07 & & +07 \\
\hline 102 & 99 & r & $1.23 \mathrm{E}-00$ & $1.23 E-00$ & +01 & & & & & & -00 \\
\hline & 229 & 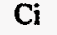 & 1.59E-01 & $1.59 \mathrm{E}-01$ & & & & & & & \\
\hline & & c & $3.21 \mathrm{E}-02$ & 3.2 & +01 & 03 & 3. & -01 & & -02 & \\
\hline $\mathrm{ClO2}$ & 126 & C & -02 & -02 & 02 & .02 & 6. & -01 & .00 & -011 & -01 \\
\hline C102 & itium & $\mathrm{C}$ & $1.46 \mathrm{E}-00$ & $1.46 \mathrm{E}-00$ & -01 & 1.0 & -00 & $E-00$ & $E-00$ & -00 & -01 \\
\hline C102 & & $\mathrm{C}$ & $3.59 \mathrm{E}-00$ & $8.68 E-01$ & +01 & 01 & & +01 & & -00 & -00 \\
\hline 02 & & $\mathbf{C i}$ & $1.40 \mathrm{E}+01$ & 3.37E-00 & & & & & & & \\
\hline & & $c$ & $2.25 E+01$ & 5.44E-00 & & & & & & & \\
\hline 02 & & c & 9.35E-01 & & -01 & & & -00 & -00 & -00 & -00 \\
\hline C102 & & C & $6.29 \mathrm{E}-01$ & $1.52 \mathrm{E}-01$ & +01 & -02 & & $8-00$ & $=-00$ & -01 & -01 \\
\hline $\mathrm{C102}$ & $\mathbf{U}$ & C & $2.14 E+01$ & $5.17 \mathrm{E}-00$ & +01 & -00 & & +01 & +02 & +01 & +01 \\
\hline C102 & $\mathbf{Y}$ & $\mathrm{C}$ & $1.82 E+05$ & $1.82 E+05$ & +02 & & & 6 & & +06 & +06 \\
\hline & & & & & & & & & & & \\
\hline & $A$ & 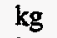 & & & & & & & & & +04 \\
\hline C103 & $\mathbf{B i}$ & $\mathrm{k}$ & $4.30 \mathrm{E}+02$ & 4.30 & -01 & +01 & 02 & 03 & -04 & +03 & +03 \\
\hline $\mathrm{Cl03}$ & $\mathrm{C}_{2}$ & $\mathbf{k}$ & $6.69 \mathrm{E}+03$ & $6.69 \mathrm{E}+03$ & +01 & +02 & 102 & +03 & +04 & +03 & +03 \\
\hline $\mathrm{C103}$ & $\mathrm{Cl}$ & k & $1.20 \mathrm{E}+02$ & $1.20 \mathrm{E}+02$ & +02 & +02 & +03 & $E+03$ & +03 & +03 & +02 \\
\hline & C & k & $2.37 \mathrm{E}+04$ & $2.36 E+04$ & & & -04 & $E+04$ & -04 & 4 & +03 \\
\hline & C & & & & & & & & & & \\
\hline & $\mathbf{F}$ & kg & 3 & & & & & & & & +03 \\
\hline 03 & $\mathrm{Fe}$ & $\mathrm{k}$ & +04 & 5.23 & & & & -04 & -04 & +03 & +03 \\
\hline 3 & Hg & $\mathrm{k}$ & +01 & $2.69 \mathrm{E}+01$ & +01 & 01 & 101 & +02 & -02 & +01 & +01 \\
\hline C & $\mathbf{K}$ & $\mathrm{kg}$ & $9.40 \mathrm{E}+02$ & $9.40 E+02$ & $E+01$ & 2.2 & +02 & $E+$ & & 02 & +02 \\
\hline 03 & $\mathbf{L}$ & $\mathrm{kg}$ & $5.10 \mathrm{E}-02$ & $5.10 \mathrm{E}-02$ & & & & +01 & +01 & 1 & -00 \\
\hline & $\mathbf{N}$ & & & & & & & & & +02 & \\
\hline & $\mathbf{N}$ & $\mathrm{kg}$ & & & & & & & & & +04 \\
\hline 103 & $\mathbf{N}$ & $\mathrm{kg}$ & & -03 & & & & +04 & +04 & 5. & +04 \\
\hline & & kg & +04 & 1.78 & -03 & & & & $E+04$ & :+04 & +03 \\
\hline 3 & $\mathbf{N}$ & $\mathrm{kg}$ & $2.99 \mathrm{E}+03$ & $2.99 E+03$ & 00 & 9. & 04 & +04 & $E+05$ & +04 & +04 \\
\hline C103 & $\mathbf{P}$ & $\mathrm{kg}$ & $9.04 \mathrm{E}+03$ & $\mathbf{N A}$ & $=+02$ & 8. & 03 & $E+04$ & +04 & $\div 03$ & +03 \\
\hline $\mathrm{ClO}$ & $\mathbf{P}$ & $\mathrm{kg}$ & $215 E+03$ & $i+03$ & $F-00$ & & & +02 & $E+02$ & & +02 \\
\hline $\mathrm{C} 103$ & $\mathbf{P}$ & $\mathbf{k g}$ & & $9.14 E+03$ & & & & & & & \\
\hline C103 & $\mathbf{S}$ & kg & $9.60 \mathrm{E}+02$ & NA & & & & & & & +03 \\
\hline $\mathbf{C l}$ & $\mathbf{S}$ & $\mathrm{kg}$ & & +03 & & & & & & & \\
\hline 103 & & kg & $2.88 \mathrm{E}+03$ & $9.60 \mathrm{E}+02$ & & & & & & +03 & +03 \\
\hline$c$ & $\mathbf{S}$ & $\mathrm{kg}$ & $7.00 \mathrm{E}+01$ & $7.00 E+01$ & +01 & 5.4 & -02 & $E+02$ & +03 & $E+02$ & $E+02$ \\
\hline & & kg & $4.10 \mathrm{E}$ & $4.10 E+03$ & & & & & $E+03$ & $E+03$ & \\
\hline & $\mathbf{U}$ & kg & $3.42 \mathrm{E}+03$ & $3.42 E+03$ & 2. & 1.0 & & $\mathrm{~F}+03$ & F+04 & $E+03$ & +03 \\
\hline 10 & $\mathbf{Z}$ & $\mathbf{k g}$ & $7.48 \mathrm{E}+03$ & $7.48 \mathrm{E}+03$ & $E-00$ & 2.54 & & & $E+03$ & & +02 \\
\hline$C 1$ & Ac2 & $\mathrm{Ci}$ & & 3.66E-03 & +01 & $E+01$ & & $E-02$ & $2.92 \mathrm{E}-02$ & & $\varepsilon-03$ \\
\hline C10. & Am241 & $\mathrm{Ci}$ & $9.10 E+02$ & $9.10 \mathrm{E}+02$ & $1: 19 E+01$ & 1.6 & 4.5 & $E+02$ & +03 & +02 & \\
\hline & & $\mathbf{C i}$ & & & & & & & & & \\
\hline & 127 & $\mathrm{Ci}$ & $7.40 \mathrm{E}$ & $7.40 \mathrm{E}+04$ & $9.39 E+03$ & $3.08 \mathrm{E} \div 04$ & 7.40 & $1.64 E+05$ & $E+05$ & $9.43 E+04$ & 8.0 \\
\hline
\end{tabular}




\begin{tabular}{|c|c|c|c|c|c|c|c|c|c|c|c|}
\hline ank & nal. & In & May $\frac{B B}{98}$ & Sept98 & $1 \%$-ile & $10 \%$-ile & $50 \%$-ile & $90 \%$-ile & $99 \%$-ile & Mean & to Dev \\
\hline 103 & C14 & $\mathrm{Ci}$ & $4.40 \mathrm{E}-01$ & $4.40 \mathrm{E}-01$ & $1.41 \mathrm{E}-02$ & $99 \mathrm{E}-01$ & $\mathrm{E}-01$ & $4 \mathrm{E}-01$ & $1 \mathrm{E}-00$ & 1 & $35 \mathrm{E}-01$ \\
\hline & & $\mathrm{Ci}$ & $3.03 \mathrm{E}+01$ & $.03 \mathrm{E}+01$ & $0.00 \mathrm{E}+01$ & & $3.03 \mathrm{E}$ & & & & +01 \\
\hline $\mathrm{ClO3}$ & Cm242 & $\mathbf{C i}$ & $2.18 \mathrm{E}-01$ & $1.19 E-00$ & $3.31 \mathrm{E}-03$ & 6.37E-02 & $2.18 \mathrm{E}-01$ & $.35 \mathrm{E}-00$ & $.06 \mathrm{E}+01$ & -01 & $3.53 \mathrm{E}-00$ \\
\hline 103 & $\operatorname{Cm} 243$ & $\mathrm{Ci}$ & $1.39 \mathrm{E}-02$ & $7.58 \mathrm{E}-02$ & & $4.06 \mathrm{E}-03$ & $1.39 \mathrm{E}-02$ & & & $5.49 \mathrm{E}-02$ & $2.25 \mathrm{E}-01$ \\
\hline 103 & Cm244 & $\mathrm{Ci}$ & $9.61 \mathrm{E}-02$ & $5.23 E-01$ & $6 \mathrm{E}-03$ & $2.81 \mathrm{E}-02$ & $9.61 \mathrm{E}-02$ & $7 \mathrm{E}-01$ & $9 \mathrm{E}-00$ & $3.79 E-01$ & $1.56 \mathrm{E}-00$ \\
\hline & & $\mathrm{Ci}$ & $10 \mathrm{E}+03$ & $10 E+03$ & $2.46 \mathrm{E}-00$ & -00 & $1.19 \mathrm{E}+01$ & $96 E+01$ & $.00 \mathrm{E}+02$ & $E+01$ & \\
\hline & 134 & $\mathrm{Ci}$ & E-00 & $93 \mathrm{E}-00$ & $2.45 \mathrm{E}-01$ & & $1.93 \mathrm{E}-00$ & $E-00$ & +01 & $6 \mathrm{E}-00$ & E-00 \\
\hline & 137 & $\mathrm{Ci}$ & -04 & $80 \mathrm{E}$ & $E+04$ & $E+04$ & $1.22 \mathrm{E}+05$ & $62 E+05$ & $E+05$ & $1.54 \mathrm{E}+05$ & +05 \\
\hline 03 & Eu152 & $\mathrm{Ci}$ & $46 \mathrm{E}-00$ & $3.46 \mathrm{E}-00$ & $.00 E+01$ & $E+01$ & $8.46 E-00$ & $.20 \mathrm{E}+01$ & $75 \mathrm{E}+01$ & $E+01$ & $E+01$ \\
\hline 103 & Eu154 & $\mathrm{Ci}$ & $6.40 E+03$ & $6.40 \mathrm{E}+03$ & $0.00 \mathrm{E}+01$ & $00 E+01$ & $6.40 \mathrm{E}+03$ & $42 E+04$ & $\mathrm{DE}+04$ & $E+03$ & $4 E+04$ \\
\hline & & $\mathrm{Ci}$ & $40 \mathrm{E}+03$ & $5.40 \mathrm{E}+03$ & $0.00 \mathrm{E}+01$ & & $5.40 \mathrm{E}+03$ & $.04 E+04$ & & $E+03$ & $E+03$ \\
\hline & I129 & $\mathrm{Ci}$ & $.00 \mathrm{E}-02$ & $4.00 \mathrm{E}-02$ & $0.00 \mathrm{E}+01$ & EE-03 & $4.00 \mathrm{E}-02$ & & & $E-02$ & $E-02$ \\
\hline 103 & $\mathrm{Nb} 93$ & $\mathrm{Ci}$ & $2.01 \mathrm{E}+01$ & $2.01 E+01$ & $0.00 \mathrm{E}+01$ & E-00 & $2.01 E+01$ & $E+01$ & & $E+01$ & +01 \\
\hline & & $\mathrm{Ci}$ & $1.07 \mathrm{E}+01$ & $1.07 E+01$ & & & $1.07 E+01$ & $2.09 E+02$ & & & +02 \\
\hline & & $\mathrm{Ci}$ & $1.05 E+03$ & $1.05 E+03$ & & & & & & & \\
\hline & & $\mathrm{Ci}$ & 01 & 1.3 & & & & & & & \\
\hline & & $\mathbf{C i}_{\mathbf{i}}$ & 03 & 5.4 & 01 & & & & & & \\
\hline & & $\mathrm{Ci}$ & $3.73 E-00$ & $7.41 \mathrm{E}+01$ & $E-02$ & -00 & 3.7 & +01 & & +01 & +01 \\
\hline & Pu239 & $\mathrm{Ci}$ & $41 E+03$ & $4.48 \mathrm{E}$ & $E+01$ & & & & & & +04 \\
\hline 103 & Pu240 & $\mathrm{Ci}$ & i. in 239 & $6.91 \mathrm{E}+$ & $i+01$ & & & & & & +04 \\
\hline 103 & Pu241 & $\mathrm{Ci}$ & $2.71 E+02$ & $5.38 E+03$ & & & 2.71 & & & & +03 \\
\hline & & $\mathrm{Ci}$ & & & & & & & & & \\
\hline 2 & 226 & $\mathrm{Ci}$ & 6.9 & 6.9 & & & & & & & \\
\hline 03 & Ra228 & $\mathrm{Ci}$ & 5.10E-02 & $5.10 \mathrm{E}-02$ & $=-02$ & & 5.1 & & & & -01 \\
\hline & 106 & $\mathrm{Ci}$ & 2.63E-03 & $2.63 \mathrm{E}-03$ & $3.44 E-05$ & & $2.63 E-03$ & $E-03$ & $E-02$ & -03 & $E-03$ \\
\hline & Sb125 & $\mathrm{Ci}$ & 3.711 & 3.71 & & & & & & & \\
\hline & $\mathbf{s}$ & $\mathrm{Ci}$ & $5.34 \mathrm{E}-00$ & 534 & & & & & & & +01 \\
\hline & & $\mathrm{Ci}$ & +04 & 2.03 & & & & & & & \\
\hline & $9 / 90$ & $\mathrm{Ci}$ & $E+06$ & 1.9 & & & & & & & \\
\hline & 99 & $\mathrm{Ci}$ & $13 E+02$ & 2.13 & & & & +02 & & TuL & +02 \\
\hline & & $\mathrm{Ci}$ & $1.22 \mathrm{E}-03$ & $1.22 \mathrm{E}-03$ & & & & & & & $=-03$ \\
\hline & 232 & $\mathrm{Ci}$ & $5.98 \mathrm{E}-03$ & 5.98 & +01 & & & & & & \\
\hline & 6 & $\mathrm{Ci}$ & & 8.6 & & & & & & & \\
\hline & & $\mathbf{C i}$ & & & & & 3.1 & & & & \\
\hline 03 & 32 & $\mathrm{Ci}$ & -01 & 1.0 & & & & & & & \\
\hline 03 & 33 & $\mathrm{Ci}$ & $5.56 \mathrm{E}-01$ & $3.91 E-01$ & +01 & & -01 & -00 & & & \\
\hline & & $\mathrm{Ci}$ & $1.60 \mathrm{E}-00$ & $1.12 \mathrm{E}-00$ & & & 1.6 & & & & \\
\hline & 5 & $\mathrm{Ci}$ & $6.78 \mathrm{E}-02$ & $4.77 \mathrm{E}-02$ & +01 & & & & & & \\
\hline & & $\mathrm{Ci}$ & & & & & & & & & \\
\hline & & $\mathrm{Ci}$ & & & & & & & & & \\
\hline 03 & $\mathbf{Y}$ & $\mathrm{Ci}$ & $1.98 \mathrm{E}+06$ & $1.98 E+06$ & 01 & & 1.9 & & & & \\
\hline & r93 & $\mathbf{C i}$ & $2.28 \mathrm{E}+01$ & $2.28 E+01$ & +01 & & +01 & & & & \\
\hline & A & $\mathrm{kg}$ & $6.75 E+04$ & $8.07 E+04$ & +04 & +04 & 1.3 & & & & +04 \\
\hline & E & $\mathrm{kg}$ & & & & & & & & & \\
\hline & $\mathrm{Ca}$ & kg & & & & & & & & & \\
\hline 04 & $c$ & kg & & & & & & & & & \\
\hline 04 & $\mathrm{CO} 3$ & kg & $5.50 E+04$ & 6.39 & .04 & & -04 & & & & \\
\hline C104 & $\mathrm{Cr}$ & $\mathrm{kg}$ & $1.66 \mathrm{E}+03$ & $1.80 \mathrm{E}+03$ & +02 & & +03 & +03 & -03 & 2. & +03 \\
\hline & $\mathbf{F}$ & kg & $3.94 \mathrm{E}+04$ & $3.66 \mathrm{E}+04$ & & & & & & & \\
\hline C104 & Fe & kg & +04 & $4.20 \mathrm{E}$ & & & & & & 2 & +04 \\
\hline & & kg & & & & & & & & & +03 \\
\hline & $\mathbf{K}$ & $\mathrm{kg}$ & & & & & & & & & \\
\hline & I & kg & & & & & & & & & \\
\hline & $\mathbf{M}$ & kg & 03 & 7. & & & & & & & \\
\hline & $\mathbf{N}$ & $\mathrm{kg}$ & 05 & 1.9 & & & & & & & \\
\hline & $\mathbf{N}$ & kg & $3.00 \mathrm{E}+03$ & $3.23 E+03$ & +02 & & 03 & & & & +04 \\
\hline C104 & NO2 & kg & $4.16 E+04$ & $4.46 E+04$ & & & & & & & $=04$ \\
\hline C104 & NO3 & kg & $2.23 E+04$ & $E+04$ & & & & & & & $E+04$ \\
\hline & $\mathbf{P}$ & kg & & & & & & & & & $E+04$ \\
\hline & $\mathbf{P}$ & kg & & & & & & & & & \pm+02 \\
\hline & $\mathbf{P}$ & $\mathbf{k g}$ & $2.52 \mathrm{E}+04$ & $5.97 E+03$ & & & & & & & \\
\hline & $\mathbf{S}$ & $\mathbf{k g}$ & & & & & & & & & \\
\hline & $\mathbf{S i}$ & kg & $1.16 E+04$ & & & & & & & & \\
\hline C104 & SO4 & kg & 3.711 & $5.40 \mathrm{E}+$ & $E+02$ & & 3681 & & & & \\
\hline C104 & Sr & kg & $4.12 \mathrm{E}+02$ & $1.18 \mathrm{E}+02$ & $2.97 \mathrm{E}-00$ & $E+01$ & $3.39 E+02$ & $E+02$ & $1.08 \mathrm{E}+04$ & +02 & $E+03$ \\
\hline C104 & TOC & kg & $1.61 \mathrm{E}+04$ & $1.40 \mathrm{E}+04$ & $1.63 E+03$ & $E \div 03$ & $6.71 E+03$ & $E \div 04$ & $2.56 \mathrm{E}+04$ & $E+03$ & $E+03$ \\
\hline C104 & $\mathbf{U}$ & kg & $6.11 \mathrm{E}+04$ & $3.45 E+04$ & $7.97 \mathrm{E}+03$ & $E+04$ & $2.56 E+04$ & $5.93 E+04$ & $1.21 E+05$ & $E+04$ & $2.32 \mathrm{E}+04$ \\
\hline 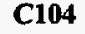 & $\mathbf{Z}_{1}$ & kg & $7.39 E+04$ & & $1.48 E+03$ & $5.85 E \div 03$ & $1.27 \mathrm{E}+04$ & $2.42 \mathrm{E}+04$ & $3.65 E+04$ & $1.40 \mathrm{E}+04$ & $7.40 E+03$ \\
\hline
\end{tabular}




\begin{tabular}{|c|c|c|c|c|c|c|c|c|c|c|c|}
\hline$n k$ & al. & Un & $\frac{B B}{y 98}$ & $\frac{B B}{o t 98}$ & $1 \%$-ile & $10 \%$-ile & $50 \%-i l e$ & $90 \%$-ile & 99\%-ile & Mean & Std Dev \\
\hline & & Ci & 2 & & $E+01$ & +01 & +01 & +02 & $0 \mathrm{E}+03$ & $E+02$ & $36 E+02$ \\
\hline & 41 & $\mathrm{Ci}$ & $15 E+03$ & $78 E+03$ & $E+01$ & 02 & $\mathrm{E}+02$ & +03 & $E+04$ & -02 & +03 \\
\hline & & $\mathrm{Ci}$ & 27E-02 & $3.50 E-01$ & $E-03$ & 70E-03 & $7 \mathrm{E}-02$ & $8 \mathrm{E}-01$ & $2 E-00$ & & \\
\hline 104 & Ba137 & $\mathrm{Ci}$ & $16 E+05$ & $74 \mathrm{E}+04$ & $00 \mathrm{E}+01$ & $9 E+04$ & $16 E+05$ & $07 E+05$ & $09 E+05$ & & $63 E+04$ \\
\hline 104 & C14 & $\mathrm{Ci}$ & $8.40 \mathrm{E}-01$ & $1.18 \mathrm{E}-00$ & $6 \mathrm{E}-01$ & E-01 & $8.40 \mathrm{E}-01$ & .00 & LE-00 & $E-00$ & $.00 \mathrm{E}-00$ \\
\hline 104 & d113 & $\mathrm{Ci}$ & $1.49 \mathrm{E}+02$ & $1.49 E+02$ & $E+01$ & $5 E+01$ & $1.49 E+02$ & $01 E+03$ & $E+03$ & & $4 E+02$ \\
\hline 104 & Cm242 & $\mathrm{Ci}$ & $32 E-01$ & $6.25 \mathrm{E}-00$ & $.89 \mathrm{E}-02$ & $E E-01$ & $5.82 \mathrm{E}-01$ & .00 & $E+01$ & & $3 E+01$ \\
\hline & 1243 & & $\mathrm{E}-02$ & E-01 & $9 E-03$ & & $35 \mathrm{E}-02$ & $\mathrm{E}-01$ & $E-00$ & -01 & $E-01$ \\
\hline 04 & & & $\mathbf{E}-\mathbf{0 0}$ & 01 & 1 & & $2.05 \mathrm{~F}_{-00}$ & $1.30 \mathrm{E}+01$ & $E+02$ & $E-00$ & +01 \\
\hline A4 & C & $\mathrm{Ci}$ & 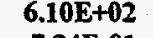 & & & & & & & & \\
\hline 104 & & $\mathrm{Ci}$ & 7 & & & & & & & & \\
\hline 4 & & $\mathbf{C i}$ & CTos & $\mathbf{E}+05$ & $E+04$ & 04 & $1.28 \mathrm{E}+05$ & +05 & +05 & +05 & +04 \\
\hline 4 & Eu152 & $\mathbf{C i}$ & $1.49 E+01$ & $\mathbf{E}+01$ & $7 E-00$ & -00 & $1.49 \mathrm{E}+01$ & $E+02$ & $E+02$ & $E+01$ & $E+01$ \\
\hline & & c & $18+2$ & 1.4 & $E+02$ & & $1.88 \mathrm{E}+03$ & $E+04$ & $E+04$ & +03 & $E+03$ \\
\hline & & $\mathrm{Ci}$ & 1.8 & +02 & $E+02$ & & $1.85 \mathrm{~F}$ & +04 & +04 & +03 & \\
\hline 14 & 29 & c & $1.57 \mathrm{E}-02$ & $1.57 \mathrm{E}-02$ & $E+01$ & & 1.57E-02 & $E-02$ & -02 & -02 & $E-02$ \\
\hline & 1 & & $E+01$ & & 1 & & & 02 & & 11 & +01 \\
\hline & & & & & 0 & & & 02 & & & +02 \\
\hline & $N$ & C & 2.59 & 2.5 & 2.1 & & 2.5 & & & & \\
\hline 04 & 77 & C & 2.79E-02 & 2.79 & 3 & 03 & 02 & -01 & $E-00$ & -01 & -01 \\
\hline 4 & 231 & $\mathrm{Ci}$ & $1.25 \mathrm{E}+02$ & $1.25 E+02$ & $E+01$ & 01 & $1.25 E+02$ & $3.72 \mathrm{E}+02$ & +02 & +02 & +02 \\
\hline 44 & Pu238 & $\mathrm{Ci}$ & $1.02 E+02$ & $2.07 E+02$ & $6 \mathrm{E}-00$ & 01 & $1.02 E+02$ & $6.47 \mathrm{E}+02$ & \pm+03 & +02 & +03 \\
\hline & 1239 & & $5.83 E+03$ & 4.70 & +02 & & & & +05 & +04 & +05 \\
\hline & 40 & & 239 & $9.26 E+02$ & $E+02$ & & 03 & +04 & +05 & 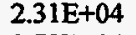 & +05 \\
\hline & 1 & $\mathrm{Ci}$ & & 1.4 & 2 & & & & +05 & & -05 \\
\hline 104 & 11242 & C & -02 & 8.16E-02 & $E-03$ & & 4.0 & 01 & -00 & -01 & \\
\hline 04 & 2226 & $\mathrm{Ci}$ & 4.88E-03 & 4.88E-03 & $E+01$ & & 4.88 & -02 & $e-01$ & E-03 & $=-02$ \\
\hline 04 & $\mathbf{R a 2 2 8}$ & $\mathrm{Ci}$ & $2.22 \mathrm{E}+01$ & $2.22 \mathrm{E}+01$ & $E+01$ & & 2.2 & & -02 & +01 & +02 \\
\hline & 106 & c & & & & & & & & & \\
\hline & & & 0 & & $E-01$ & & & & & & 02 \\
\hline & $\mathbf{s}$ & & 1 & 1.5 & & & & & & & \\
\hline 04 & Sm151 & $\mathrm{Ci}$ & $5.63 \mathrm{E}+04$ & +04 & $E+04$ & -04 & +04 & +05 & +06 & -05 & +05 \\
\hline 94 & 69/90 & $\mathrm{Ci}$ & $6.24 \mathrm{E}+05$ & $6.30 \mathrm{E}+05$ & $E+03$ & -05 & 9.1 & +06 & +07 & $E+06$ & $8+07$ \\
\hline 4 & 10 & $\mathrm{Ci}$ & +03 & $3.10 \mathrm{E}+01$ & +01 & & 3.7 & -03 & +04 & +03 & +03 \\
\hline & & $\mathrm{Ci}$ & & & & & & & & & \\
\hline & & & & & & & & & & & \\
\hline & & & & & & & & & & & \\
\hline 4 & um & $\mathrm{Ci}$ & +01 & +01 & .00 & & 01 & 01 & -01 & 01 & -00 \\
\hline 04 & U232 & $\mathrm{Ci}$ & $1.89 E+01$ & $1.47 \mathrm{E}+01$ & +01 & & -01 & +01 & +02 & +01 & $+0 I$ \\
\hline 04 & & c & & 5.63 & $+0 !$ & & & & 02 & +02 & +01 \\
\hline & & c & & & & & & & & & \\
\hline & & & & & & & & & & & \\
\hline 04 & & C & 01 & -01 & & & & & & & -00 \\
\hline 44 & 8 & C & $1.48 \mathrm{E}+01$ & +01 & $E+01$ & -00 & +01 & +01 & -01 & +01 & \pm 01 \\
\hline 4 & Y9 & c & $6.24 \mathrm{E}+05$ & $6.30 \mathrm{E}+05$ & $E+03$ & 104 & $6.24 E+05$ & +06 & -07 & +06 & $E \div 07$ \\
\hline & $\mathbf{Z r}$ & c & $6.55 \mathrm{E}+01$ & $6.55 E+01$ & $+0 !$ & -01 & +01 & +02 & -02 & +01 & $E+01$ \\
\hline & & & & & & & & & & & +04 \\
\hline & & & & & & & & & & & \\
\hline & C & & & & & & & & & & +02 \\
\hline & Cl & kg & +02 & 1.9 & & & 1.8 & +03 & -03 & -03 & +03 \\
\hline 5 & $\mathrm{CO} 3$ & kg & +03 & $5.15 E+03$ & $E+02$ & +03 & 1.0 & $E+04$ & $E+04$ & +04 & +03 \\
\hline C105 & $\mathrm{Cr}$ & kg & $6.80 E+02$ & $6.80 \mathrm{E}+02$ & +02 & +02 & 1.3) & $E+03$ & -03 & 03 & \pm+03 \\
\hline & $\mathbf{F}$ & kg & & 0.00 & & & & & & & 103 \\
\hline & $\mathbf{F}$ & & & & & & & & & & \\
\hline & $\mathbf{H}$ & & & & & & & & & & \\
\hline & $\mathbf{K}$ & & & & & & & & & & \\
\hline & La & kg & +01 & -01 & & & 8. & 1 & -01 & -01 & $=-00$ \\
\hline$C$ & $\mathbf{M}$ & $\mathbf{k g}$ & $1.26 \mathrm{E}+03$ & $1.26 \mathrm{E}+03$ & 01 & +02 & 1.11 & $E+03$ & & +03 & $E+03$ \\
\hline$C$ & $\mathrm{~N}_{2}$ & kg & $5.65 E+04$ & $5.65 E+04$ & $E+04$ & $5.52 \mathrm{E}+04$ & $9.46 \mathrm{E}+04$ & $E+05$ & $E+05$ & $E+04$ & $E+04$ \\
\hline C & $\mathrm{Ni}$ & $\mathrm{kg}$ & $1.09 E+03$ & $1.09 E+03$ & 1 & & 1.03 & $6 .>-1$ & $E+03$ & +02 & +02 \\
\hline C105 & No & $\mathrm{kg}$ & $1.68 \mathrm{E}+04$ & $1.63 E+04$ & $\mathrm{E}+03$ & 33 & -04 & 04 & & -04 & +04 \\
\hline 105 & N03 & kg & $1.13 E+04$ & $1.13 E+04$ & +0 & & & & & & \\
\hline & $\mathbf{P}$ & $\mathbf{k g}$ & & & & & & & & & $E+03$ \\
\hline & 1 & & & +02 & & & & & -03 & -02 & $E+02$ \\
\hline & $\mathbf{P}$ & $\mathbf{k}$ & $5.30 \mathrm{E}+03$ & $5.30 E+03$ & & & 5.41 & 5.1 & +04 & & $E+04$ \\
\hline & $\mathbf{S}$ & kg & $4.10 E+02$ & $\mathbf{N}$ & & & $7.25 E+02$ & $2.44 E+03$ & $\mathrm{E}+04$ & $E+03$ & $E+03$ \\
\hline & $\mathbf{S}$ & kg & $2.03 E+04$ & +04 & $3.01 E+02$ & $6.38 \mathrm{E}+02$ & $1.47 E+03$ & $5.83 E+03$ & $1.62 E+04$ & $E+03$ & $E+03$ \\
\hline & & ng & & & 102 & +03 & -03 & $.54 E+03$ & $5.32 E+04$ & $E+03$ & r \\
\hline
\end{tabular}


Tank Anal. Un

\begin{tabular}{|c|c|}
\hline C105 & $\mathbf{S r}$ \\
\hline $\begin{array}{l}\text { C105 } \\
\text { C105 }\end{array}$ & TOC \\
\hline 105 & U \\
\hline C105 & $\begin{array}{l}\mathrm{Zr} \\
\text { Ac227 }\end{array}$ \\
\hline C105 & Am241 \\
\hline C105 & Am243 \\
\hline C105 & Ba137 \\
\hline C105 & C14 \\
\hline C105 & Cd113 \\
\hline C105 & Cm242 \\
\hline C105 & Cm243 \\
\hline C105 & Cm244 \\
\hline C105 & Co60 \\
\hline C105 & Cs134 \\
\hline C105 & Cs137 \\
\hline C105 & Eu152 \\
\hline C105 & Eu154 \\
\hline C105 & Eu155 \\
\hline C105 & 1129 \\
\hline C105 & Nb93 \\
\hline C105 & Ni59 \\
\hline C105 & Ni63 \\
\hline C105 & Np237 \\
\hline C105 & P2231 \\
\hline C105 & Pu238 \\
\hline C105 & Pu239 \\
\hline C105 & Pu240 \\
\hline C105 & Pu241 \\
\hline C105 & Pu242 \\
\hline C105 & Ra226 \\
\hline C105 & Ra228 \\
\hline C105 & Ru106 \\
\hline C105 & Sb125 \\
\hline C105 & Se79 \\
\hline C105 & Sm151 \\
\hline C105 & Sr89/90 \\
\hline C105 & Tc99 \\
\hline C105 & Th229 \\
\hline C105 & Th232 \\
\hline C105 & Tin126 \\
\hline C105 & Tritium \\
\hline C105 & U232 \\
\hline C105 & U233 \\
\hline C105 & U234 \\
\hline C105 & U235 \\
\hline C105 & U236 \\
\hline C105 & U238 \\
\hline C105 & Y90 \\
\hline C10 & Zr93 \\
\hline C106 & Al \\
\hline C106. & $\mathrm{Bi}$ \\
\hline C106 & $\mathrm{Ca}$ \\
\hline C106 & CI \\
\hline C106 & $\mathrm{CO3}$ \\
\hline C106 & $\mathrm{Cr}$ \\
\hline $\begin{array}{l}\text { C106 } \\
\text { C106 }\end{array}$ & $\mathbf{F}$ \\
\hline $\begin{array}{l}\text { c106 } \\
\text { C106 }\end{array}$ & $\begin{array}{l}\mathrm{Fe} \\
\mathrm{Hg}\end{array}$ \\
\hline C106 & $\mathbf{K}$ \\
\hline C10 & La \\
\hline C106 & Mn \\
\hline C10 & $\mathbf{N a}$ \\
\hline C106 & $\mathrm{Ni}$ \\
\hline $\begin{array}{l}\text { C106 } \\
\text { C106 }\end{array}$ & $\mathrm{NO2}$ \\
\hline C10 & $\mathbf{P}$ \\
\hline & \\
\hline
\end{tabular}

BB May 98 $9 . \overline{00 \mathrm{E}+01}$ $1.01 \mathrm{E}+03$ $5.18 E+03$ $4.30 \mathrm{E}+02$ 1.18E-05 $7.69 \mathrm{E}+02$ 2.40E-06 $1.18 E+05$ 9.30E-01 2.86E-01 2.90E-03 6.86E-05 $1.15 \mathrm{E}-04$ $1.32 \mathrm{E}+02$ Ci 2.27E-03 Ci $1.25 E+05$

Ci 1.45E-01 Ci 9.95E-01 Ci 8.58E-00 Ci 1.07E-01 Ci 6.81E-02 Ci 2.40E-02 2.27E-00 $\mathrm{Ci}$ 3.80E-03 Ci 2.77E-05 Ci $1.20 \mathrm{E}+01$ Ci $4.59 \mathrm{E}+02$ Ci Incl. in 239

Ci $8.76 \mathrm{E}+02$

Ci 3.34E-03

Ci 2.28E-06

Ci 2.45E-10

Ci 5.48E-07

Ci 8.88E-02

Ci 1.79E-02

$6.48 \mathrm{E}+01$

3.65E+05

$\quad 9.81 \mathrm{E}+01$

Ci 4.78E-08

$$
\text { 2.03E-11 }
$$

$$
\text { 2.76E-02 }
$$

$$
\text { 5.92E-01 }
$$

Ci 2.91E-04

Ci 9.75E-06

Ci 5.53E-00

Ci 2.36E-01

Ci 9.59E-02

Ci 5.67E-00

Ci 3.65E+05

Ci 8.46E-02

kg 3.60E+04

kg 7.30E-00

kg 2.02E+03

$\mathrm{kg} \quad 3.25 \mathrm{E}+02$

kg 1.48E+05

kg $4.75 E+02$

kg $2.71 E+02$

$\mathrm{kg} \quad 4.68 \mathrm{E}+04$

$\mathrm{kg} \quad 2.47 \mathrm{E}+01$

$\mathrm{kg} \quad 7.74 \mathrm{E}+02$

kg 5.67E+01

kg 1.61E+03

kg 1.45E+05

kg $4.60 \mathrm{E}+02$

kg 2.17E+04

kg 1.92E+03

kg 1.43E+04

kg 1.74E+03
$B B$ Sept $\overline{98}$ $\overline{9.00 \mathrm{E}+01}$ $1.01 E+03$ $5.18 \mathrm{E}+03$ $4.30 E+02$

1.18E-05

$7.69 \mathrm{E}+02$

$7.21 \mathrm{E}-03$

$1.18 \mathrm{E}+05$

9.30E-01

2.86E-01

8.73E-00

2.06E-01

3.46E-01

$1.32 \mathrm{E}+02$

2.27E-03

$1.25 \mathrm{E}+05$

1.45E-01

9.95E-01

8.58E-00

1.07E-01

6.81E-02

2.40E-02

2.27E-00

$3.80 \mathrm{E}-03$

2.77E-05

6.24E-00

$3.98 \mathrm{E}+02$

$6.09 \mathrm{E}+01$

$4.54 \mathrm{E}+02$

1.73E-03

2.28E-06

2.45E-10

5.48E-07

8.88E-02

1.79E-02

$6.48 \mathrm{E}+01$

$3.65 \mathrm{E}+05$

$9.81 \mathrm{E}+01$

4.78E-08

2.03E-11

2.76E-02

5.92E-01

8.87E-05

2.98E-06

$1.69 \mathrm{E}-00$

7.21E-02

2.93E-02

$1.73 \mathrm{E}-00$

$3.65 \mathrm{E}+05$

8.46E-02

$3.60 \mathrm{E}+04$

7.30E-00

$2.02 \mathrm{E}+03$

$3.25 \mathrm{E}+02$

$1.48 \mathrm{E}+05$

$4.75 E+02$

$2.71 \mathrm{E}+02$

$4.68 \mathrm{E}+04$

$2.47 \mathrm{E}+01$

$7.74 \mathrm{E}+02$

$5.67 E+01$

$1.61 \mathrm{E}+03$

$1.45 \mathrm{E}+05$

$4.60 \mathrm{E}+02$

$2.17 \mathrm{E}+04$

$1.92 \mathrm{E}+03$

$$
\text { NA }
$$

$1.74 \mathrm{E}+03$ 1\%-ile

$10 \%$-ile

$1.13 \mathrm{E}+01$

$9.57 \mathrm{E}+01$

$3.84 \mathrm{E}+02$

8.44E-01

$0.00 \mathrm{E}+01$

$2.72 \mathrm{E}+01$

7.11E-08

$4.15 \mathrm{E}+03$

$0.00 \mathrm{E}+01$

$1.88 \mathrm{E}-02$

8.60E-05

2.03E-06

$3.41 \mathrm{E}-06$

$3.88 \mathrm{E}-00$

$7.99 \mathrm{E}-05$

$1.49 \mathrm{E}+04$

$0.00 \mathrm{E}+01$

$0.00 \mathrm{E}+01$

$0.00 \mathrm{E}+01$

$0.00 \mathrm{E}+01$

$1.15 \mathrm{E}-03$

3.37E-03

3.19E-01

1.13E-04

$0.00 \mathrm{E}+01$

$3.56 \mathrm{E}-01$

$1.36 \mathrm{E}+01$

$1.36 \mathrm{E}+01$

$2.60 \mathrm{E}+01$

$9.90 \mathrm{E}-05$

8.07E-08

8.67E-12

4.29E-08

$1.62 \mathrm{E}-02$

3.02E-03

$0.00 \mathrm{E}+01$

$1.78 \mathrm{E}+02$

$0.00 \mathrm{E}+01$

$0.00 \mathrm{E}+01$

$0.00 \mathrm{E}+01$

$1.82 \mathrm{E}-03$

$2.08 \mathrm{E}-01$

$0.00 \mathrm{E}+01$

$0.00 \mathrm{E}+01$

$0.00 \mathrm{E}+01$

$0.00 \mathrm{E}+01$

$0.00 \mathrm{E}+0 \mathrm{I}$

$0.00 \mathrm{E}+01$

$1.67 \mathrm{E}+02$

$1.43 \mathrm{E}-03$

$1.43 \mathrm{E}+04$

$3.17 \mathrm{E}+02$

$4.59 \mathrm{E}+02$

$3.39 \mathrm{E}+02$

1.27E+04

$1.32 \mathrm{E}+02$

$1.10 \mathrm{E}+02$

$1.78 \mathrm{E}+03$

$2.89 \mathrm{E}-01$

$1.51 \mathrm{E}+02$

2.30E-00

$2.90 E+01$

$5.14 E+04$

$4.00 \mathrm{E}+02$

$5.22 \mathrm{E}+03$

$3.21 E+03$

9.17E +02

$1.06 \mathrm{E}+01$
$4.81 \mathrm{E}+01$

$6.80 \mathrm{E}+02$

$1.79 \mathrm{E}+03$

$1.11 \mathrm{E}+01$

2.38E-06

3.61E+01

5.03E-07

$3.76 \mathrm{E}+04$

$5.48 \mathrm{E}-02$

$8.88 \mathrm{E}-02$

$6.08 \mathrm{E}-04$

1.44E-05

2.41E-05

$5.89 \mathrm{E}-00$

$7.23 \mathrm{E}-04$

$3.99 \mathrm{E}+04$

$2.92 \mathrm{E}-02$

$2.00 \mathrm{E}-01$

$1.73 \mathrm{E}-00$

$1.79 \mathrm{E}-02$

$1.98 \mathrm{E}-02$

9.09E-03

$8.60 \mathrm{E}-01$

$7.96 \mathrm{E}-04$

2.35E-06

$2.51 \mathrm{E}-00$

$9.62 \mathrm{E}+01$

$9.62 \mathrm{E}+01$

$1.84 \mathrm{E}+02$

$7.00 \mathrm{E}-04$

$4.89 \mathrm{E}-07$

$5.25 \mathrm{E}-11$

$1.91 \mathrm{E}-07$

$3.66 \mathrm{E}-02$

7.97E-03

$1.31 E+01$

$1.08 \mathrm{E}+04$

$0.00 \mathrm{E}+01$

4.05E-09

$1.72 \mathrm{E}-12$

8.57E-03

3.64E-01

$2.46 \mathrm{E}-05$

8.26E-07

4.68E-01

$2.00 \mathrm{E}-02$

8.12E-03

4.80E-01

$1.75 \mathrm{E} \div 04$

2.45E-02

$2.34 \mathrm{E}+04$

$7.33 E \div 02$

$1.48 \mathrm{E}+03$

$6.11 \mathrm{E}+02$

$1.82 \mathrm{E}+04$

$3.25 \mathrm{E}+02$

$3.33 E+02$

$6.89 \mathrm{E}+03$

$4.97 \mathrm{E}-00$

$2.73 E+02$

3.32E-00

$1.29 \mathrm{E}+02$

$7.41 \mathrm{E}+0.4$

$4.08 \mathrm{E}+03$

$1.23 E+04$

$1.11 \mathrm{E}+04$

$1.55 \mathrm{E}+03$

$2.13 \mathrm{E}+01$

50\%-ile

2.10E+02

$2.49 \mathrm{E}+03$

$5.23 \mathrm{E}+03$ 
Tank Anal. Un

\begin{tabular}{|c|c|}
\hline 106 & PO4 \\
\hline & 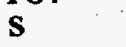 \\
\hline $\begin{array}{l}\text { C106 } \\
\text { C106 }\end{array}$ & $\begin{array}{l}\text { Si } \\
\text { S04 }\end{array}$ \\
\hline C106 & $\begin{array}{l}\text { S04 } \\
\text { Sr }\end{array}$ \\
\hline C106 & TOC \\
\hline C106 & u \\
\hline C106 & $\mathbf{Z}_{\mathbf{r}}$ \\
\hline C106 & Ac227 \\
\hline C106 & Am241 \\
\hline C106 & Am243 \\
\hline C106 & Ba137 \\
\hline C106 & C14 \\
\hline C106 & Cd113 \\
\hline C106 & $\mathrm{Cm} 242$ \\
\hline C106 & $\mathrm{Cm} 243$ \\
\hline C106 & $\mathrm{Cm} 244$ \\
\hline C106 & Co60 \\
\hline C106 & Cs134 \\
\hline C106 & Cs137 \\
\hline C106 & Eu152 \\
\hline C106 & Eu154 \\
\hline C106 & Eu155 \\
\hline C106 & 1129 \\
\hline C106 & Nb93 \\
\hline C106 & Ni59. \\
\hline C106 & Ni63 \\
\hline C106 & Np237 \\
\hline C106 & Pa231 \\
\hline C106 & Pu238 \\
\hline C106 & Pu239 \\
\hline C106 & Pu240 \\
\hline C106 & Pu241 \\
\hline C106 & Pu242 \\
\hline C106 & Ra226 \\
\hline C106 & Ra228 \\
\hline C106 & Ru106 \\
\hline C106 & $\mathrm{Sb} 125$ \\
\hline C106 & Se79 \\
\hline C106 & Sm151 \\
\hline C106 & Sr89/90 \\
\hline C106 & Tc99 \\
\hline C106 & Th229 \\
\hline C106 & Th232 \\
\hline C106 & Tin126 \\
\hline C106 & Tritium \\
\hline C106 & U232 \\
\hline C106 & U233 \\
\hline C106 & U234 \\
\hline C106 & U235 \\
\hline C106 & U236 \\
\hline C106 & U238 \\
\hline C106 & Y90 \\
\hline C106 & $\mathrm{Zr} 93$ \\
\hline C107 & Al \\
\hline C107 & $\mathbf{B i}$ \\
\hline C107 & $\mathrm{Ca}$ \\
\hline C107 & Cl \\
\hline C107 & $\mathrm{CO} 3$ \\
\hline C107 & $\mathrm{Cr}$ \\
\hline C107 & $\mathbf{F}$ \\
\hline C107 & $\mathrm{Fe}$ \\
\hline C107 & Hg \\
\hline C107 & $\mathbf{K}$ \\
\hline C107 & $\mathbf{L a}$ \\
\hline C107 & Mn \\
\hline C107 & $\mathrm{Na}$ \\
\hline C107 & $\mathbf{N i}$ \\
\hline
\end{tabular}

May $\frac{B 8}{8 B}$ 4.41E+04 $6.08 \mathrm{E}+03$ $1.84 \mathrm{E}+04$ $1.82 \mathrm{E}+04$ $3.07 \mathrm{E}+01$ $1.52 \mathrm{E}+04$ $1.40 \mathrm{E}+03$ $5.87 \mathrm{E}+02$ $1.90 \mathrm{E}-02$

$1.12 \mathrm{E}+03$

$7.42 \mathrm{E}-02$

$2.53 E+05$

2.30E-01

$5.65 \mathrm{E}+01$

2.22E-00

$1.62 \mathrm{E}+01$

4.78E-00

$3.42 E+02$

$7.02 \mathrm{E}-01$

$2.67 \mathrm{E}+05$

$5.67 \mathrm{E}+01$

$2.30 \mathrm{E}+03$

$1.36 \mathrm{E}+03$

1.00E-02

$7.03 \mathrm{E}+01$

$7.79 \mathrm{E}+01$

$7.68 \mathrm{E}+03$

9.57E-02

$1.89 \mathrm{E}-02$

$5.32 \mathrm{E}+01$

$2.86 \mathrm{E}+03$

Ci Incl. in 239

$3.74 \mathrm{E}+03$

2.37E-02

3.85E-03

3.02E-04

3.19E-01

$1.73 \mathrm{E}+03$

$1.85 \mathrm{E}+01$

$7.11 \mathrm{E}+04$

$4.77 \mathrm{E}+06$

$2.21 \mathrm{E}+02$

$1.42 \mathrm{E}-04$

3.25E-05

$3.02 \mathrm{E}+01$

$5.75 \mathrm{E}-00$

1.92E-02

7.39E-02

1.46E-00

6.21E-02

2.59E-02

1.49E-00

$4.77 \mathrm{E}+06$

$7.77 \mathrm{E}+01$

$3.95 E+04$

$1.73 E+04$

$1.58 \mathrm{E}+03$

$8.09 \mathrm{E}+02$

$8.03 E+03$

$9.31 \mathrm{E}+02$

$7.76 E+03$

$4.58 \mathrm{E}+04$

$2.00 \mathrm{E}+01$

$2.76 \mathrm{E}+02$

$1.13 \mathrm{E}+02$

$1.82 E+03$

$8.00 E+04$ $1.52 \mathrm{E}+03$ $\frac{\text { Sept98 }}{1.44 \mathrm{E}+04}$

NA

$1.84 \mathrm{E}+04$

$6.08 \mathrm{E}+03$

$3.07 \mathrm{E}+01$

$1.52 \mathrm{E}+04$

$1.40 \mathrm{E}+03$

$5.87 \mathrm{E}+02$

1.90E-02

$1.12 E+03$

5.89E-02

$2.53 \mathrm{E}+05$

2.30E-01

$5.65 \mathrm{E}+01$

1.76E-00

7.49E-01

$1.86 \mathrm{E}+01$

$3.42 \mathrm{E}+02$

7.02E-01

$2.67 \mathrm{E}+05$

$5.67 \mathrm{E}+01$

$230 \mathrm{E}+03$

$2.12 \mathrm{E}+03$

1.00E-02

$7.03 E+01$

$7.79 \mathrm{E}+01$

$7.68 \mathrm{E}+03$

9.57E-02

1.89E-02

$1.16 \mathrm{E}+02$

$2.37 E+03$

$4.85 \mathrm{E}+02$

8.17E +03

5.17E-02

3.85E-03

3.02E-04

3.19E-01

$1.73 \mathrm{E}+03$

$1.85 \mathrm{E}+01$

7.11E+04

4.77E+06

$2.21 \mathrm{E}+02$

1.42E-04

$3.25 \mathrm{E}-05$

$3.02 E+01$

$5.75 E-00$

6.02E-03

2.32E-02

$4.58 \mathrm{E}-01$

$1.95 \mathrm{E}-02$

8.14E-03

4.68E-01

$4.77 \mathrm{E}+06$

$7.77 \mathrm{E}+01$

$3.95 \mathrm{E}+04$

$1.73 \mathrm{E}+04$

$1.58 \mathrm{E}+03$

$8.09 E+02$

8.03E+03

$9.31 \mathrm{E}+02$

$7.76 \mathrm{E}+03$

$4.58 \mathrm{E}+04$

$9.63 \mathrm{E}+01$

$2.76 \mathrm{E}+02$

1.13E+02

$1.82 \mathrm{E}+03$

8.00E+04

$1.52 \mathrm{E}+03$
$1 \%$-ile

$1.05 \mathrm{E}+03$

$5.85 \mathrm{E}+02$

$1.01 \mathrm{E}+03$

$1.55 \mathrm{E}+03$

7.10E-00

$3.26 \mathrm{E}+02$

$5.39 \mathrm{E}+02$

$2.76 \mathrm{E}+01$

$0.00 \mathrm{E}+01$

$1.23 \mathrm{E}+01$

$2.80 \mathrm{E}-03$

4.03E+04

$5.39 \mathrm{E}-02$

6.62E-01

8.39E-02

6.12E-01

$1.81 \mathrm{E}-01$

$1.71 \mathrm{E}-00$

1.12E-01

$1.94 E+04$

$0.00 \mathrm{E}+01$

$0.00 \mathrm{E}+01$

$0.00 \mathrm{E}+01$

$1.49 \mathrm{E}-03$

$2.59 \mathrm{E}+01$

$1.66 \mathrm{E}-00$

$1.63 \mathrm{E}+02$

$3.62 \mathrm{E}-03$

$0.00 \mathrm{E}+01$

2.01E-00

$1.08 \mathrm{E}+02$

$1.08 \mathrm{E}+02$

$1.4 \mathrm{IE}+02$

8.96E-04

2.35E-04

$1.84 \mathrm{E}-05$

2.35E-03

$0.00 \mathrm{E}+01$

3.97E-00

$0.00 \mathrm{E}+01$

$8.76 E+03$

$0.00 \mathrm{E}+01$

$0.00 \mathrm{E}+01$

$0.00 \mathrm{E}+01$

$3.54 \mathrm{E}-01$

2.47E-00

$0.00 \mathrm{E}+01$

$0.00 \mathrm{E}+01$

$0.00 \mathrm{E}+01$

$0.00 \mathrm{E}+01$

$0.00 \mathrm{E}+01$

$0.00 \mathrm{E}+01$

$0.00 \mathrm{E}+01$

$2.87 \mathrm{E}+0 \mathrm{I}$

$1.16 \mathrm{E}+04$

$7.54 \mathrm{E}+03$

2.77E+02

$5.85 \mathrm{E}+02$

$2.28 \mathrm{E}+03$

$2.61 \mathrm{E}+02$

$2.84 \mathrm{E}+02$

$5.88 \mathrm{E}+03$

$0.00 \mathrm{E}+01$

$1.70 \mathrm{E}+02$

$1.51 \mathrm{E}-00$

4.83E+01

$6.76 E+04$

$1.46 \mathrm{E}+01$
$10 \%$-ile

$2.03 E+03$

$1.02 \mathrm{E}+03$

$3.29 \mathrm{E}+03$

$2.80 \mathrm{E}+03$

$2.58 \mathrm{E}+01$

$7.63 E+02$

$1.16 \mathrm{E}+03$

$4.06 \mathrm{E}+01$

$0.00 \mathrm{E}+01$

$1.72 \mathrm{E}+01$

6.84E-03

$8.89 \mathrm{E}+04$

$1.10 \mathrm{E}-01$

$1.14 \mathrm{E}+01$

$2.05 \mathrm{E}-01$
$1.49 \mathrm{E}-00$

4.41E-01

$3.15 \mathrm{E}-00$

2.47E-01

4.14E+04

$0.00 \mathrm{E}+01$

$0.00 \mathrm{E}+01$

$0.00 \mathrm{E}+0 \mathrm{l}$

4.53E-03

$4.30 \mathrm{E}+01$

$2.57 \mathrm{E}+01$

$2.53 E+03$

8.82E-03

$1.63 \mathrm{E}-03$

4. $90 \mathrm{E}-00$

$2.64 \mathrm{E}+02$

$2.64 \mathrm{E}+02$

$3.45 \mathrm{E}+02$

2.19E-03

1.12E-03

8.77E-05

$9.65 \mathrm{E}-02$

$4.87 \mathrm{E}+02$

$7.68 \mathrm{E}-00$

$0.00 E+01$

$5.48 \mathrm{E}+05$

$1.28 \mathrm{E}+01$

1.23E-05

2.81E-06

6.08E-00

3.84E-00

$1.66 \mathrm{E}-03$

6.38E-03

1.26E-01

5.36E-03

2.24E-03

$1.29 \mathrm{E}-01$

$8.23 E+05$

$4.76 \mathrm{E}+01$

$2.07 E+04$

$1.17 \mathrm{E}+04$

$6.54 \mathrm{E}+02$

$1.03 E+03$

$7.36 \mathrm{E}+03$

$6.69 \mathrm{E}+02$

1.52E+03

$9.54 \mathrm{E}+03$

$3.23 \mathrm{E}-00$

$3.10 E+02$

3.23E-00

1. $28 \mathrm{E}+02$

$9.20 \mathrm{E}+04$

$2.67 \mathrm{E}+01$

50\%-ile

$5.35 \mathrm{E}+03$

$2.43 E+03$

$7.64 \mathrm{E}+03$

$6.44 \mathrm{E}+03$

$8.38 \mathrm{E}+01$

$1.72 E+03$

$2.69 \mathrm{E}+03$

$6.14 \mathrm{E}+01$

$1.90 \mathrm{E}-02$

$1.95 \mathrm{E}+02$ 


\begin{tabular}{|c|c|c|c|c|c|c|c|c|c|c|c|}
\hline nk & ral. & In & May98 & $\frac{B B}{\text { ept98 }}$ & $\%$-ile & ile & jle & ile & le & an & $e v$ \\
\hline 107 & - & kg & $2.07 \mathrm{E}+04$ & $2.07 \mathrm{E}+04$ & $4.39 E+03$ & $8.62 E+03$ & $t+04$ & $8 E+04$ & $6.41 E+04$ & 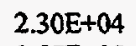 & $31 E+04$ \\
\hline & & $\mathbf{k g}$ & $.17 E+04$ & $5.17 E+04$ & $2.33 E+04$ & & & $5 E+05$ & & & +04 \\
\hline $\mathrm{ClO}$ & $\mathbf{P}$ & kg & $3.89 E+04$ & NA & $4.39 \mathrm{E}+03$ & $1.12 E+04$ & $17 E+04$ & $58 E+04$ & +04 & $E+04$ & $1 E+03$ \\
\hline 107 & $\mathbf{P b}$ & kg & $2.27 E+03$ & $2.27 E+03$ & $3.17 \mathrm{E}+01$ & $1.00 \mathrm{E}+02$ & $3.76 E+02$ & $1.16 \mathrm{E}+03$ & $2.52 \mathrm{E}+03$ & $5.48 E+02$ & $45 \mathrm{E}+02$ \\
\hline 107 & PO & $\mathbf{k g}$ & $2.13 E+05$ & $6.96 \mathrm{E}+04$ & $3.24 \mathrm{E}+03$ & $1.13 E+04$ & $34 E+04$ & $30 E+04$ & $1.33 E+05$ & $2.75 E+04$ & $E+04$ \\
\hline 107 & $\mathbf{S}$ & $\mathrm{kg}$ & $5.67 \mathrm{E}+03$ & $\mathbf{N A}$ & $4.74 E+02$ & +03 & $E+03$ & $40 E+03$ & & $5.51 E+03$ & $E+03$ \\
\hline 107 & $\mathbf{S}$ & $\mathrm{kg}$ & $1.08 E+04$ & $1.08 E+04$ & $1.40 \mathrm{E}+03$ & & & $38 E+04$ & & & \\
\hline 107 & & kg & $1.70 E+04$ & $5.67 E+03$ & $2.01 E+03$ & & +04. & $2.20 \mathrm{E}+04$ & +04 & & \\
\hline 107 & & & $1.40 \mathrm{E}+02$ & $1.40 \mathrm{E}+02$ & & & & $1 E+02$ & $:+03$ & $\mathrm{E}+02$ & +02 \\
\hline 107 & T & & & $E+03$ & $E+02$ & & & $5 E+03$ & & +03 & +03 \\
\hline 07 & $x$ & & & $3.86 \mathrm{E}+03$ & $7.40 \mathrm{E}+02$ & & & & & $E+03$ & +03 \\
\hline & 2 & & 70F? & $1.70 \mathrm{E}+03$ & $3.90 \mathrm{E}-00$ & & & $\mathrm{E}+02$ & & $E+02$ & $E+02$ \\
\hline & Ac227 & $\mathrm{Ci}$ & $3.92 \mathrm{E}-02$ & $3.92 \mathrm{E}-02$ & & & & $0 E-01$ & & & $\mathrm{E}-01$ \\
\hline 107 & & $\mathrm{Ci}$ & $1.61 E+03$ & $1.61 E+03$ & $1.71 \mathrm{E}+01$ & & & $8 E+02$ & & +03 & $E+03$ \\
\hline 07 & 0243 & $\mathrm{Ci}$ & 5.71E-02 & $8.43 \mathrm{E}-02$ & 1.8 & & & 29E-01 & & & $E-00$ \\
\hline & & $\mathrm{Ci}$ & $2.91 E+04$ & $2.91 \mathrm{E}+04$ & 03 & & & $6 E+04$ & & & \\
\hline & & $\mathrm{Ci}$ & 5.95 & $5.95 \mathrm{E}-01$ & 0.0 & & & $E-00$ & & & \\
\hline & & $\mathrm{Ci}$ & ot & $\mathbf{E}+02$ & +01 & & & +02 & & & +02 \\
\hline & & $\mathbf{C i}$ & 9.15 & $E-00$ & -02 & -02 & & +01 & & & +01 \\
\hline & & $\mathrm{Ci}$ & & $E-01$ & & & & & & & +03 \\
\hline & $\mathrm{Cm}$ & $\mathrm{Ci}$ & $3.35 \mathrm{E}-00$ & $4.94 \mathrm{E}-00$ & & & & & & & +02 \\
\hline 07 & $\mathrm{Co60}$ & $\mathrm{Ci}$ & $7.87 \mathrm{E}+02$ & $7.87 E+02$ & & & & & & & +02 \\
\hline & & $\mathrm{Ci}$ & 7.4 & $7.45 \mathrm{E}-01$ & & & & & & & \\
\hline 07 & & $\mathbf{C i}$ & $i+04$ & $3.08 \mathrm{E}+04$ & 1.6 & & & & & & \\
\hline & & $C$ & +01 & $E+01$ & & & & & & & \\
\hline & & c & & +03 & & & & & & & \\
\hline & 5 & c & & & & & & & & & \\
\hline & & $\mathrm{Ci}$ & $7.80 \mathrm{E}-03$ & E-03 & & & & & & & \\
\hline & & $\mathrm{Ci}$ & & 9.5 & & & & & & & \\
\hline & & $\mathrm{Ci}$ & +01 & $4.62 \mathrm{E}+01$ & & & & & & & \\
\hline & & c & +03 & $4.54 \mathrm{E}+03$ & -03 & & & & & +03 & \\
\hline & & $\mathrm{Ci}$ & -02 & $c-02$ & & & & & & & \\
\hline & $P 731$ & $c$ & 507 & $F_{m} M$ & & & & & & & \\
\hline & Pu738 & $\mathrm{Ci}$ & $4.48 \mathrm{E}+01$ & $3.83 \mathrm{E}+01$ & & & & & & & \\
\hline & & $\mathbf{C i}$ & 2.7 & +03 & 4.2 & & & & & & \\
\hline & $\mathbf{u 2 4 0}$ & $\mathrm{Ci}$ & 2.45 & +02 & $E-00$ & & & +03 & & +03 & +03 \\
\hline 07 & Pu241 & C & $3.26 E+03$ & $2.78 E+03$ & & & & & & & \\
\hline & Pu242 & & 1.70E-02 & $1.46 \mathrm{E}-02$ & & & & & & & \\
\hline & $\mathbf{R a 2 2 6}$ & c & 3.11 & & & & & & & & \\
\hline & Pa?28 & $c$ & & & & & & & & & \\
\hline & 06 & c & -01 & 1.9 & & & & & & & \\
\hline & Sb125 & c & $6.22 \mathrm{E}-00$ & $6.22 \mathrm{E}-00$ & & & & & & & \\
\hline & & C & +01 & 01 & & & & & & & \\
\hline & & $c$ & $9.63 \mathrm{E}+04$ & +04 & & & & & & & +05 \\
\hline & & c & & & & & & & & & \\
\hline & & C & & & & & & & & & \\
\hline & & & & & & & & & & & \\
\hline & Th232 & & -04 & E-04 & & & & & & & -04 \\
\hline 107 & Tin126 & & $4.14 E+01$ & +01 & & & & & & & \\
\hline & tiur & s. & $6.64 \mathrm{E}-00$ & $6.64 \mathrm{E}-00$ & & & & & & & -00 \\
\hline & & & & & & & & & & & \\
\hline & & C & & & & & & & & & \\
\hline & & 0 & & & & & & & & & \\
\hline & & & & & & & & & & & $=-00$ \\
\hline & & & & & & & & & & & \\
\hline & & $\mathbf{C i}$ & $E+01$ & $1.29 \mathrm{E}-00$ & $E+01$ & & & & & & +01 \\
\hline & Y90 & $\mathrm{Ci}$ & & & & & & & & & +07 \\
\hline & $\mathrm{ZrS}$ & $\mathrm{Ci}$ & & & & & & & & & \\
\hline & A & k & & & & & & & & & \\
\hline & B & k & & & & & & & & & +03 \\
\hline & C & & & & & & & & & & +02 \\
\hline & $c$ & 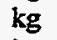 & DT, & & & & & & & & \\
\hline & $\operatorname{co3}$ & $\mathbf{k g}$ & $5.10 \mathrm{E}$ & $5.10 \mathrm{~K}$ & & & & & & & \\
\hline C108 & $\mathrm{Cr}$ & kg & $9.40 \mathrm{E}+01$ & $9.40 E+01$ & $2.48 \mathrm{E}+01$ & +01 & & $5.82 \mathrm{E}+02$ & +03 & +02 & +02 \\
\hline C108 & $\mathbf{F}$ & kg & $1.32 E+03$ & $1.32 E+03$ & $7.46 E+01$ & +02 & +03 & $E+03$ & $E+03$ & $E+03$ & +03 \\
\hline C108 & $\mathrm{Fe}$ & kg & $2.51 \mathrm{E}+03$ & $2.51 E+03$ & $E+02$ & +03 & +03 & $1.00 \mathrm{E}+04$ & $2 \mathrm{E}+04$ & $E+03$ & $3.42 E+03$ \\
\hline C108 & $\mathbf{H g}$ & kg & 2.33E-00 & $9.24 \mathrm{E}-00$ & $E+01$ & & & $-\infty$ & & -00 & $7 \mathrm{E}-00$ \\
\hline 108 & $\mathbf{K}$ & & & & $.95 E+01$ & +01 & +02 & $6.47 \mathrm{E}+02$ & $511 F+03$ & $4.47 \mathrm{E}+02$ & $9.83 E+02$ \\
\hline
\end{tabular}


Tank Anal. Un

\begin{tabular}{|c|c|}
\hline C108 & $\mathbf{L a}$ \\
\hline C108 & Mn \\
\hline 108 & $\mathrm{Na}$ \\
\hline 108 & $\mathbf{N i}$ \\
\hline C108 & NO2 \\
\hline & $\mathrm{NO3}$ \\
\hline $\mathrm{Cl08}$ & $\mathbf{P}$ \\
\hline $\begin{array}{l}\mathrm{C} 108 \\
\mathrm{C} 108\end{array}$ & $\mathbf{P b}$ \\
\hline 108 & PO4 \\
\hline $\begin{array}{l}\mathrm{C} 108 \\
\mathrm{C} 108\end{array}$ & $S$ \\
\hline $\begin{array}{l}\mathrm{C} 108 \\
\mathrm{C} 108\end{array}$ & $\begin{array}{l}\mathrm{Si} \\
\mathrm{SOS}\end{array}$ \\
\hline $\begin{array}{l}\text { C108 } \\
\text { C108 }\end{array}$ & $\begin{array}{l}\mathrm{SOH} \\
\mathrm{Sr}\end{array}$ \\
\hline $\mathrm{Cl} 108$ & TOC \\
\hline C108 & $\mathbf{U}$ \\
\hline C108 & \\
\hline $\mathrm{Cl08}$ & Ac227 \\
\hline $\mathrm{Cl08}$ & Am241 \\
\hline C108 & Am243 \\
\hline $\mathrm{Cl08}$ & Ba137 \\
\hline $\mathrm{Cl08}$ & C14 \\
\hline $\mathrm{Cl} 108$ & Cd113 \\
\hline C108 & Cm242 \\
\hline C108 & $\mathrm{Cm} 243$ \\
\hline $\mathrm{Cl08}$ & Cm244 \\
\hline $\mathrm{Cl08}$ & Co60 \\
\hline C108 & Cs134 \\
\hline $\mathrm{C} 108$ & Cs137 \\
\hline $\mathrm{C} 108$ & Eu152 \\
\hline $\mathrm{C} 108$ & Eu154 \\
\hline C108 & Eu155 \\
\hline $\mathrm{Cl08}$ & 1129 \\
\hline C108 & Nb93 \\
\hline $\mathrm{C} 108$ & Ni59 \\
\hline C108 & Ni63 \\
\hline $\mathrm{C} 108$ & Np237 \\
\hline $\mathrm{Cl08}$ & $P_{2231}$ \\
\hline $\mathrm{C} 108$ & Pu238 \\
\hline C108 & Pu239 \\
\hline C108 & Pu240 \\
\hline C108 & Pu241 \\
\hline $\mathrm{Cl08}$ & Pu242 \\
\hline C108 & Ra226 \\
\hline C108 & $\mathbf{R a} 228$ \\
\hline C108 & Ru106 \\
\hline C108 & Sb125 \\
\hline $\mathrm{ClOS}$ & Se79 \\
\hline C108 & Sm151 \\
\hline C108 & Sr89/90 \\
\hline $\mathrm{Cl08}$ & Te99 \\
\hline C108 & Th229 \\
\hline C108 & Th232 \\
\hline C108 & Tin126 \\
\hline C108 & Tritium \\
\hline C108 & U232 \\
\hline Cl08 & U233 \\
\hline C108 & U234 \\
\hline C108 & U235 \\
\hline C108 & U236 \\
\hline C108 & U238 \\
\hline C108 & Y90 \\
\hline C108 & Zr93 \\
\hline $\mathrm{ClO}$ & \\
\hline C109 & \\
\hline C109 & $\mathrm{C}_{2}$ \\
\hline $\mathrm{C}$ & \\
\hline C109 & $\begin{array}{l}\mathrm{CC} \\
\mathrm{Cr}\end{array}$ \\
\hline
\end{tabular}

\section{May98} $\begin{array}{ll}\mathrm{kg} & 0 . \overline{00 \mathrm{E}+01} \\ \mathrm{~kg} & 3.80 \mathrm{E}+01\end{array}$ $3.29 \mathrm{E}+04$ 2.94E+03 8.65E+03 kg $1.56 \mathrm{E}+04$ 9.11E+03 g $0.00 \mathrm{E}+01$ kg 2.82E+04 kg $\quad 9.73 E+02$ g. $1.35 E+03$ g $2.92 \mathrm{E}+03$ o.00E+01 $1.25 \mathrm{E}+03$ $1.47 \mathrm{E}+02$ 2.39E-00 2.14E-05 Ci 1.15E-01 Ci 7.92E-07

Ci $\quad .58 \mathrm{E}+04$ Ci 6.78E-02 1.62E-01

\section{$2.47 \mathrm{E}-03$} 5.05E-05

\section{$1.86 \mathrm{E}-05$}

1.47E-02

3.36E-03

$9.07 \mathrm{E}+04$

Ci 1.36E-01

258E-01

Ci 1.03E+01

Ci 8.85E-04

Ci 5.75E-02

5.35E-01

$4.82 \mathrm{E}+01$

\subsection{E-03}

4.62E-05

9.19E-03

$3.28 \mathrm{E}-00$

1.52E-01

1.99E-01

$7.98 \mathrm{E}-07$

4.21E-06

$5.27 \mathrm{E}-10$

5.58E-09

1.31E-02

1.43E-02

5.33E+01

$9.45 \mathrm{E}+03$

4.70E-01

1.02E-07

$1.96 \mathrm{E}-11$

2.15E-02

4.12E-01

2.09E-05

$1.25 \mathrm{E}-06$

$1.76 \mathrm{E}-00$

7.92E-02

1.13E-02

3.08E-00

Ci $9.45 \mathrm{E}+03$

Ci $\quad 6.80 \mathrm{E}-02$

kg $2.43 \mathrm{E}+04$

kg 4.93E+02

kg 5.51E+03

kg 2.12E+02

kg 1.58E+03

$\mathrm{kg} \quad 7.22 \mathrm{E}+01$
Sept98

1\%-ile $0.00 \mathrm{E}+01$
$3.80 \mathrm{E}+01$

$3.29 \mathrm{E}+04$

$2.94 \mathrm{E}+03$

$8.65 \mathrm{E}+03$

$1.56 \mathrm{E}+04$

NA

$0.00 \mathrm{E}+01$

2.82E+04

NA

1.35E +03

2.92E +03

$0.00 \mathrm{E}+01$

$1.25 \mathrm{E}+03$

$1.47 \mathrm{E}+02$

2.39E-00

2.14E-05

1.15E-01

7.92E-07

$8.58 \mathrm{E}+04$

6.78E-02

1.62E-01

$2.47 \mathrm{E}-03$

$5.05 \mathrm{E}-05$

$1.86 \mathrm{E}-05$

1.47E-02

3.36E-03

$9.07 \mathrm{E}+04$

$1.36 \mathrm{E}-01$

2.58E-01

$1.03 E+01$

8.85E-04

5.75E-02

5.35E-01

$4.82 \mathrm{E}+01$

2.89E-03

4.62E-05

1.21E-02

$3.08 \mathrm{E}-00$

1.99E-01

2.62E-01

1.05E-06

4.21E-06

5.27E-10

5.58E-09

$1.31 \mathrm{E}-02$

$1.43 \mathrm{E}-02$

$533 \mathrm{E}+01$

$9.45 E+03$

$4.70 \mathrm{E}-01$

1.02E-07

$1.96 \mathrm{E}-11$

2.15E-02

4.12E-01

5.76E-07

3.44E-08

4.85E-02

2.18E-03

3.10E-04

4.91E-02

$9.45 \mathrm{E}+03$

$6.80 \mathrm{E}-02$

$2.43 E+04$

$4.93 E+02$

$5.51 E+03$

$2.12 \mathrm{E}+02$

$1.58 \mathrm{E}+03$

$7.22 E+01$

$3.74 \mathrm{E}+03$

$1.66 \mathrm{E}+01$

$5.23 \mathrm{E}+02$

$3.21 \mathrm{E}+03$

$6.33 \mathrm{E}+02$

$2.15 \mathrm{E}+01$

$5.72 \mathrm{E}+02$

$7.21 \mathrm{E}+01$

$1.82 \mathrm{E}+02$

$3.39 \mathrm{E}+02$

$1.03 E+01$

$4.91 \mathrm{E}+01$

$1.26 \mathrm{E}+02$

$9.21 \mathrm{E}-01$

$0.00 \mathrm{E}+01$

2.69E-00

4.73E-08

$2.95 \mathrm{E}+03$

$0.00 \mathrm{E}+01$

$0.00 \mathrm{E}+01$

1.47E-04

3.02E-06

$1.11 \mathrm{E}-06$

1.07E-01

1.15E-04

$1.56 \mathrm{E}+03$

$0.00 \mathrm{E} \div 01$

$0.00 \mathrm{E}+01$

$0.00 \mathrm{E} \div 01$

2.58E-06

1.30E-03

$0.00 \mathrm{E}+01$

$0.00 \mathrm{E}+01$

$1.73 \mathrm{E}-04$

$0.00 \mathrm{E}+01$

$5.49 \mathrm{E}-04$

$1.96 \mathrm{E}-01$

9.08E-03

$1.19 \mathrm{E}-02$

4.77E-08

$0.00 \mathrm{E}+01$

$0.00 \mathrm{E}+01$

$0.00 \mathrm{E}+01$

$0.00 \mathrm{E}+01$

3.19E-04

$0.00 \mathrm{E}+01$

4.37E+03

$1.76 \mathrm{E}-02$

$0.00 \mathrm{E}+01$

$0.00 \mathrm{E}+01$

$0.00 \mathrm{E}+01$

$4.66 \mathrm{E}-02$

$0.00 \mathrm{E}+01$

$0.00 \mathrm{E}+01$

$0.00 \mathrm{E}+01$

$0.00 \mathrm{E}+01$

$0.00 \mathrm{E}+01$

$0.00 \mathrm{E}+01$

$1.98 \mathrm{E}+02$

$1.54 \mathrm{E}-03$

$3.22 \mathrm{E}+02$

$9.26 \mathrm{E}+01$

$2.21 \mathrm{E}+01$

$3.02 \mathrm{E}+01$

$5.33 \mathrm{E}+02$

$1.21 \mathrm{E}+01$

\section{$2.58 \mathrm{E}-01 \quad 1.06 \mathrm{E}-00$}

$5.26 \mathrm{E}-00 \quad 2.42 \mathrm{E}+01$
$1.44 \mathrm{E}+04$

$1.30 \mathrm{E}+02$

$2.50 \mathrm{E}+03$

$1.27 \mathrm{E}+04$

$2.28 \mathrm{E}+03$

$8.59 \mathrm{E}+01$

$2.02 \mathrm{E}+03$

$4.07 \mathrm{E}+02$

$6.17 \mathrm{E}+02$

$1.24 \mathrm{E}+03$

$4.53 \mathrm{E}+01$

$2.33 \mathrm{E}+02$

$6.16 \mathrm{E}+02$

5.12E-00

$7.76 \mathrm{E}-08$

$1.15 \mathrm{E}+01$

2.38E-07

$2.26 \mathrm{E}+04$

$1.01 \mathrm{E}-02$

$3.60 \mathrm{E}-02$

7.41E-04

$1.52 \mathrm{E}-05$

$5.58 \mathrm{E}-06$

$5.52 \mathrm{E}-0 \mathrm{I}$

8.85E-04

$6.57 \mathrm{E}+03$

4.93E-04

9.36E-04

$3.74 \mathrm{E}-02$

$3.25 \mathrm{E}-04$

$1.02 \mathrm{E}-02$

$7.21 \mathrm{E}-02$

$6.50 \mathrm{E}-00$

8.67E-04

$5.60 \mathrm{E}-06$

2.76E-03

9.84E-01

4.56E- 02

5.97E-02

2.39E-07

5.41E-07

6.78E- 11

1.25E-09

$0.00 \mathrm{E}+01$

4.75E-03

$1.93 \mathrm{E}-01$

$2.36 \mathrm{E} \div 04$

1.65E-01

1.24E-08

2.37E-12

4.78E- 03

$1.98 \mathrm{E}-01$

2.53E-06

1.51E-07

2.13E-0I

9.59E-03

$1.37 \mathrm{E}-03$

3.73E-01

$2.26 \mathrm{E}+03$

1.21E-02

$2.19 \mathrm{E}+03$

$4.23 \mathrm{E}+02$

$7.37 \mathrm{E}+02$

$1.74 \mathrm{E}+02$

$1.81 \mathrm{E}+03$

$6.09 \mathrm{E}+01$

$50 \%$-ile

3.22E-60

$6.33 \mathrm{E}+01$

$3.09 \mathrm{E}+04$

$5.64 \mathrm{E}+02$

$5.83 \mathrm{E}+03$

$3.45 \mathrm{E}+04$

$5.42 \mathrm{E}+03$

2.85E+02

$5.65 E+03$ 
Tank Anal. Un

\begin{tabular}{ll} 
C109 & F \\
C109 & Fe \\
C109 & Hg \\
C109 & K \\
C109 & La \\
C109 & Mn \\
C109 & Na \\
C109 & Ni \\
C109 & NO2 \\
C109 & NO3 \\
C109 & P \\
C109 & Pb \\
C109 & PO4 \\
C109 & S \\
C109 & Si \\
C109 & SO4 \\
C109 & Sr \\
C109 & ToC \\
C109 & U \\
C109 & Zr \\
C109 & Ac227 \\
C109 & Am241 \\
C109 & Am243 \\
C109 & Ba137 \\
C109 & C14 \\
C109 & Cd113 \\
C109 & Cm242 \\
C109 & Cm243 \\
C109 & Cm244 \\
C109 & Co60 \\
C109 & Cs134 \\
C109 & Cs137 \\
C109 & Eu152 \\
C109 & Eu154 \\
C109 & Eu155 \\
C109 & I129 \\
C109 & Nb93 \\
C109 & Ni59 \\
C109 & Ni63 \\
C109 & Np237 \\
C109 & Pa231 \\
C109 & Pu238 \\
C109 & Pu339 \\
C109 & Pu240 \\
C109 & Pu241 \\
C109 & Pu242 \\
C109 & Ra226 \\
C109 & Ra228 \\
C109 & Ru106 \\
C109 & Sb125 \\
C109 & Se79 \\
C109 & Sm151 \\
C109 & Sr89990 \\
C109 & Tc99 \\
C109 & Th229 \\
C109 & Th232 \\
C109 & Tin126 \\
C109 & Tritium \\
C109 & U232 \\
C109 & U233 \\
C109 & U234 \\
C109 & U235 \\
C109 & U236 \\
C109 & U238 \\
C109 & Y90 \\
C109 & Zr93 \\
C110 & Al \\
C110 & Bi \\
& \\
\hline
\end{tabular}

$\underline{B B}$ May98 $\begin{array}{ll}\mathrm{kg} & \mathbf{2 . 0 2 E}+02 \\ \mathrm{~kg} & \mathrm{5.41E}+03\end{array}$ kg 8.02E-01 kg 1.57E+02 kg $\quad 1.27 \mathrm{E}+01$ kg 3.69E +01 kg 2.54E+04 kg $\quad 4.06 E+03$ kg 1.18E+04 kg 1.17E+04 kg $1.59 \mathrm{E}+04$ kg $\quad 9.71 \mathrm{E}+02$ kg 4.93E+04 kg 7.43E+02 kg $1.95 \mathrm{E}+03$ kg 2.23E+03 kg 1.09E+02 kg $\quad 8.24 \mathrm{E}+02$ g.73E+03 $\mathrm{kg} \quad 1.37 \mathrm{E}-00$ Ci 1.29E-03 Ci $\quad 4.46 \mathrm{E}+01$ Ci 8.05E-04 Ci $2.24 \mathrm{E}+05$ Ci 5.70E-03 Ci 6.59E-01 Ci 5.85E-02 $\mathrm{Ci} \quad 3.05 \mathrm{E}-03$ Ci 1.47E-03 Ci 3.30E-02

$\mathrm{Ci} \quad 1.23 \mathrm{E}-02$

Ci 2.37E+05

Ci 2.52E-00

Ci 1.76E-00

Ci $\quad 1.67 \mathrm{E}+02$

Ci 1.54E-03

Ci 2.21E-01

Ci 4.93E-00

Ci $4.68 \mathrm{E}+02$

$\mathrm{Ci} \quad$ 4.85E-03

Ci 1.05E-04

Ci 2.36E-00

Ci $9.23 \mathrm{E}+01$

Ci Incl. in 239

Ci 1.70E+02

Ci 8.35E-04

Ci 2.64E-04

Ci 2.39E-09

Ci 6.60E-05

Ci $\quad 5.47 \mathrm{E}-02$

Ci $5.76 \mathrm{E}-02$

Ci $2.16 \mathrm{E}+02$

Ci 2.21E+05

Ci 8.14E-01

Ci 4.45E-07

Ci 3.31E-11

Ci $\quad 8.98 \mathrm{E}-02$

$\mathrm{C}$

Ci 7.50E-06

Ci 4.45E-07

Ci $\quad 6.22 \mathrm{E}-01$

Ci 2.80E-02

Ci 4.01E-03

Ci 5.48E-00

Ci $2.21 \mathrm{E}+05$

Ci 2.62E-01

kg 1.43E+04

kg $1.66 \mathrm{E}+04$
$B B$ Sept98 $2.02 \mathrm{E}+02$ $5.41 \mathrm{E}+03$ 3.19E-00 $1.57 \mathrm{E}+02$ $1.27 \mathrm{E}+01$ 3.69E +01 $2.54 \mathrm{E}+04$ $4.06 \mathrm{E}+03$ $1.18 \mathrm{E}+04$ 1.17E+04 NA $9.71 \mathrm{E}+02$ $1.61 \mathrm{E}+04$ NA

$1.95 \mathrm{E}+03$ $2.23 E+03$ $1.09 E+02$ 8.24E +02 $3.73 \mathrm{E}+03$ 1.37E-00 $1.29 \mathrm{E}-03$ $4.46 \mathrm{E}+01$ $1.07 \mathrm{E}-03$ $2.24 E+05$ 5.70E-03 6.59E-01 7.73E-02 4.03E-03 1.94E-03 3.30E-02 1.23E-02 $2.37 \mathrm{E}+05$ 2.52E-00 1.76E-00 $1.67 \mathrm{E}+02$ 1.54E-03 2.21E-01 4.93E-00 $4.68 \mathrm{E}+02$ $4.85 \mathrm{E}-03$ $1.05 \mathrm{E}-04$ $1.86 \mathrm{E}-00$ $7.94 \mathrm{E}+01$ $1.29 \mathrm{E}+01$ $1.34 \mathrm{E}+02$ 9.15E-04 2.64E-04 2.39E-09 6.60E-05 $5.47 \mathrm{E}-02$ 5.76E-02 $2.16 \mathrm{E}+02$ $2.21 \mathrm{E}+05$ 8.14E-01 4.45E-07 3.31E-11 8.98E-02 6.08E-01 $1.48 \mathrm{E}-05$ $8.81 \mathrm{E}-07$ $1.23 \mathrm{E}-00$ 5.53E-02 7.93E-03 $1.25 \mathrm{E}-00$ $2.21 \mathrm{E}+05$ $2.62 \mathrm{E}-01$ $1.43 E+04$ $1.66 \mathrm{E}+04$
$1 \%$-ile

\section{$4.09 \mathrm{E}+01 \quad 1.95 \mathrm{E}+02$}

$4.77 \mathrm{E}+02 \quad 2.08 \mathrm{E}+03$

$0.00 \mathrm{E}+01 \quad 1.80 \mathrm{E}-01$

$3.80 \mathrm{E}+01 \quad 2.08 \mathrm{E}+02$

$2.49 \mathrm{E}-01$

$1.80 \mathrm{E}-00$

4.25E-00

$3.68 \mathrm{E}+03$

$2.15 \mathrm{E}+01$

$1.36 \mathrm{E}+04$

$3.78 \mathrm{E}+01$

$1.02 \mathrm{E}+03$

$1.86 \mathrm{E}+03$

$2.58 \mathrm{E}+02$

$6.84 \mathrm{E}+02$

$5.40 \mathrm{E}+03$

$9.50 \mathrm{E}+03$

$1.23 \mathrm{E}+03$

$25 \mathrm{E}+02$

$4.73 \mathrm{E}+01$

2.39E+02

$1.21 \mathrm{E}+03$

$2.85 \mathrm{E}+02$

$2.88 \mathrm{E}+02$

$2.16 \mathrm{E}+02$

3.55E-00

$1.29 \mathrm{E}+02$

$1.10 \mathrm{E}+02$

4.50E-01

$0.00 \mathrm{E}+01$

$2.46 \mathrm{E}-00$

$1.26 \mathrm{E}-05$

$4.15 E+03$

$0.00 \mathrm{E}+01$

$0.00 \mathrm{E}+01$

$9.19 \mathrm{E}-04$

4.79E-05

2.31E-05

$2.26 \mathrm{E}-0$

2.28E-04

$2.08 \mathrm{E}+03$

$0.00 \mathrm{E}+01$

$0.00 \mathrm{E}+01$

$0.00 \mathrm{E}+01$

$2.08 \mathrm{E}-05$

$2.28 \mathrm{E}-03$

$0.00 \mathrm{E}+01$

$0.00 \mathrm{E}+01$

7.62E-05

$0.00 \mathrm{E}+01$

3.71E-02

$1.45 \mathrm{E}-00$

$1.45 \mathrm{E}-00$

2.67E-00

1.31E-05

$0.00 \mathrm{E}+01$

$0.00 \mathrm{E}+01$

$0.00 \mathrm{E}+01$

$0.00 \mathrm{E}+01$

$0.00 \mathrm{E} \div 01$

$0.00 \mathrm{E}+01$

$6.09 \mathrm{E}+03$

2.45E-02

$0.00 \mathrm{E}+01$

$0.00 \mathrm{E}+01$

$0.00 \mathrm{E}+01$

4.88E-02

$0.00 \mathrm{E}+01$

$0.00 \mathrm{E}+01$

$0.00 \mathrm{E}+01$

$0.00 \mathrm{E}+01$

$0.00 \mathrm{E}+01$

$0.00 \mathrm{E}+01$

$2.50 \mathrm{E}+03$

2.71E-03

$1.24 \mathrm{E}+02$

$6.66 \mathrm{E}+03$
$1.09 \mathrm{E}+03$

$4.56 \mathrm{E}+01$

$6.49 \mathrm{E}+02$

$1.18 \mathrm{E}+03$

$5.82 \mathrm{E}-00$

$0.00 \mathrm{E}+01$

$1.88 \mathrm{E}+01$

$1.56 \mathrm{E}-04$

$5.65 \mathrm{E}+04$

8.05E-04

$1.48 \mathrm{E}-01$

$1.13 \mathrm{E}-02$

$5.91 \mathrm{E}-04$

$2.85 \mathrm{E}-04$

$1.02 \mathrm{E}-00$

3.10E-03

$1.42 \mathrm{E}+04$

$0.00 \mathrm{E}+01$

$0.00 \mathrm{E}+01$

$0.00 \mathrm{E}+01$

4.21E-04

$3.41 \mathrm{E}-02$

4.68E-01

$4.44 \mathrm{E}+01$

$9.40 \mathrm{E}-04$

$1.46 \mathrm{E}-06$

4.57E-01

$1.79 \mathrm{E}+01$

$1.79 \mathrm{E}+01$

$3.29 \mathrm{E}+01$

$1.62 \mathrm{E}-04$

3.72E-07

3.36E-12

8.29E-06

$0.00 \mathrm{E}+01$

$1.35 \mathrm{E}-02$

$0.00 \mathrm{E}+01$

$6.35 \mathrm{E}+04$

$1.96 \mathrm{E}-01$

6.18E-09

4.59E-13

2.01E-02

2.81E-01

$1.04 \mathrm{E}-07$

$6.18 \mathrm{E}-09$

8.63E-03

$3.89 \mathrm{E}-04$

$5.56 \mathrm{E}-05$

7.60E-02

$5.29 \mathrm{E}+0.4$

4.04E-02

$1.27 \mathrm{E}+03$

$9.93 \mathrm{E}+03$

50\%-ile

$7.78 \mathrm{E}+03$

8.02E-01

$6.96 \mathrm{E}+02$

$7.05 \mathrm{E}-00$

8.35E+01

$3.46 \mathrm{E}+04$

$2.48 \mathrm{E}+03$

$1.18 \mathrm{E}+04$

$2.69 E+04$

$4.16 \mathrm{E}+03$

8.24E +02

$3.77 \mathrm{E}+03$ 
Tank Anal. Un

\begin{tabular}{ll} 
C110 & Ca \\
C110 & C \\
C110 & CO3 \\
C110 & Cr \\
C110 & F \\
C110 & Fe \\
C110 & Hg \\
C110 & K \\
C110 & La \\
C110 & Mn \\
C110 & Na \\
C110 & Ni \\
C110 & NO2 \\
C110 & N03 \\
C110 & P \\
C110 & Pb \\
C110 & PO4 \\
C110 & S \\
C110 & Si \\
C110 & SO4 \\
C110 & Sr \\
C110 & TOC \\
C110 & U \\
C110 & Zr \\
C110 & Ac227 \\
C110 & Am241 \\
C110 & Am243 \\
C110 & Ba137 \\
C110 & C14 \\
C110 & Cd113 \\
C110 & Cm242 \\
C110 & Cm243 \\
C110 & Cm244 \\
C110 & Co60 \\
C110 & Cs134 \\
C110 & Cs137 \\
C110 & Eu152 \\
C110 & Eu154 \\
C110 & E1155 \\
C110 & 1129 \\
C110 & Nb93 \\
C110 & Ni59 \\
C110 & Ni63 \\
C110 & Np237 \\
C110 & Pa231 \\
C110 & Pu238 \\
C110 & Pu239 \\
C110 & Pu240 \\
C110 & Pu221 \\
C110 & Pu242 \\
C110 & Ra226 \\
C110 & Ra228 \\
C110 & Ru106 \\
C110 & Sb125 \\
C110 & Se79 \\
C110 & Sm151 \\
C110 & Sr89990 \\
C110 & Tc99 \\
C110 & Th229 \\
C110 & Th232 \\
C110 & Tin126 \\
C110 & Tritium \\
C110 & U232 \\
C110 & U233 \\
C110 & U234 \\
C110 & U235 \\
C110 & U236 \\
C110 & U238 \\
& \\
\hline
\end{tabular}

$\frac{B B}{98}$

kg $\frac{\text { May98 }}{1.15 E+03}$

kg $1.08 \mathrm{E}+03$

kg $1.04 \mathrm{E}+04$

kg $4.64 \mathrm{E}+02$

kg $7.49 \mathrm{E}+03$

kg $1.09 \mathrm{E}+04$

$\mathrm{kg}$ 4.00E-01

kg $5.51 \mathrm{E}+02$

kg $0.00 \mathrm{E}+01$

kg $5.20 \mathrm{E}+01$

kg $8.17 \mathrm{E}+04$

kg $2.40 \mathrm{E}+01$

kg 9.17E+03

kg $\quad 1.09 \mathrm{E}+05$

$\mathrm{kg} \quad 6.04 \mathrm{E}+04$

kg 2,55E+02

kg $1.87 \mathrm{E}+05$

$\mathrm{kg} \quad 1.47 \mathrm{E}+04$

$7.07 \mathrm{E}+03$

$4.41 \mathrm{E}+04$

$1.28 \mathrm{E}+02$

$5.00 \mathrm{E}+02$

$2.11 E+03$

$1.70 \mathrm{E}+02$

2.77E-05

$2.90 \mathrm{E}+02$

$5.96 \mathrm{E}-08$

$1.75 \mathrm{E}+04$

4.75E-01

1.25E-01

2.40E-05

3.56E-07

9.12E-07

Ci $3.44 \mathrm{E}+01$

7.32E-05

Ci $1.85 \mathrm{E}+04$

Ci 5.99E-03

Ci . 1.15E-01

Ci $9.05 \mathrm{E}-01$

$2.60 \mathrm{E}+01$

Ci 5.30E-02

$1.75 \mathrm{E}-02$

Ci 1.53E-00

2.55E-03

Ci 5.78E-05

Ci $5.10 \mathrm{E}+01$

Ci $8.24 \mathrm{E}+01$

Ci Incl. in 239

Ci 4.48E-01

Ci 1.37E-06

Ci 5.50E-06

Ci 8.15E-11

2.01E-10

4.61E- 03

$1.34 \mathrm{E}-00$

$4.88 \mathrm{E}+01$

$4.86 \mathrm{E}+03$

$3.41 \mathrm{E}+01$

1.57E-08

$1.91 \mathrm{E}-11$

1.92E-02

1.09E-00

1.34E-04

8.01E-06

$1.13 \mathrm{E}+01$

Ci $5.08 \mathrm{E}-01$

$\mathrm{Ci} \quad 7.21 \mathrm{E}-02$

Ci 5.10E-01
Sept98

$\frac{5 e p 198}{1.15 \mathrm{E}+03}$

$1.08 \mathrm{E}+03$

$1.04 \mathrm{E}+04$

$4.64 \mathrm{E}+02$

$7.49 \mathrm{E}+03$

$1.09 \mathrm{E}+04$

4.00E-01

$5.51 \mathrm{E}+02$

$0.00 \mathrm{E}+01$

$5.20 \mathrm{E}+01$

8.17E+04

$2.40 \mathrm{E}+01$

$9.17 E+03$

$1.09 \mathrm{E}+05$

NA

2.55E+02

$6.10 \mathrm{E}+04$

NA

$7.07 \mathrm{E}+03$

$1.47 \mathrm{E}+04$

$1.28 \mathrm{E}+02$

$6.68 \mathrm{E}+02$

2.11E+03

$1.70 \mathrm{E}+02$

2.77E-05

2.13E-02

$5.96 \mathrm{E}-08$

$1.76 \mathrm{E}+04$

3.16E-01

$1.25 \mathrm{E}-01$

2.40E-05

3.56E-07

9.12E-07

8.55E-03

7.32E-05

$1.86 \mathrm{E}+04$

5.99E-03

1.15E-01

9.05E-01

$7.90 \mathrm{E}-04$

5.30E-02

$1.75 \mathrm{E}-02$

1.53E-00

2.55E-03

$5.78 \mathrm{E}-05$

2.38E-01

$7.46 \mathrm{E}+01$

$4.40 \mathrm{E}-00$

2.81E-00

8.60E-06

5.50E-06

8.15E-11

2.01E-10

4.61E- 03

$1.34 E-00$

$4.88 \mathrm{E}+01$

$4.69 \mathrm{E}+03$

$3.41 \mathrm{E}+01$

1.57E-08

1.91E-11

1.92E-02

1.07E-00

8.25E-06

4.94E-07

6.96E-01

3.13E-02

4.44E-03

7.04E-01 1\%-ile

$10 \%-i l e$

$8.48 \mathrm{E}+01$

$2.43 \mathrm{E}+02$

$1.07 \mathrm{E}+02$

4.05E-00

$1.14 \mathrm{E}+02$

$3.46 \mathrm{E}+03$

$0.00 \mathrm{E}+01$

$3.20 \mathrm{E}+01$

6.55E-02

4.01E-00

$4.31 \mathrm{E}+04$

$4.08 \mathrm{E}-00$

$6.46 \mathrm{E}+02$

$1.15 \mathrm{E}+04$

$2.90 \mathrm{E}+03$

4.53E-00

$1.22 \mathrm{E}+03$

$1.21 E+02$

$4.58 \mathrm{E}+02$

$7.88 \mathrm{E}+02$

9.21E-00

6.11E-01

4.10E+01

7.73E-01

$0.00 \mathrm{E}+01$

7.35E-01

$2.10 \mathrm{E}-09$

$1.30 \mathrm{E}+03$

$0.00 \mathrm{E}+01$

$0.00 \mathrm{E}+01$

$8.46 \mathrm{E}-07$

$1.25 \mathrm{E}-08$

3.21E-08

2.03E-05

5.44E-06

$1.60 \mathrm{E}+03$

$0.00 \mathrm{E}+01$

$0.00 \mathrm{E}+01$

$0.00 \mathrm{E}+01$

$0.00 \mathrm{E}+01$

$0.00 \mathrm{E}+01$

$1.55 \mathrm{E}-03$

$1.36 \mathrm{E}-01$

8.99E-05

$0.00 \mathrm{E}+01$

$1.80 \mathrm{E}-00$

$2.90 \mathrm{E}-00$

$2.90 \mathrm{E}-00$

1.58E-02

4.83E-08

$0.00 \mathrm{E}+01$

$0.00 \mathrm{E}+01$

$0.00 \mathrm{E}+01$

$0.00 \mathrm{E}+01$

$0.00 \mathrm{E}+01$

$0.00 E+01$

1.13E+02

$1.63 \mathrm{E}-00$

$0.00 \mathrm{E}+01$

$0.00 \mathrm{E}+01$

$0.00 \mathrm{E}+01$

$5.54 \mathrm{E}-01$

$0.00 \mathrm{E}+01$

$0.00 E+01$

$0.00 \mathrm{E}+01$

$0.00 \mathrm{E}+01$

$0.00 \mathrm{E}+01$

$0.00 \mathrm{E}+0 \mathrm{I}$

\section{$2.76 \mathrm{E}+02$}

$5.46 \mathrm{E}+02$

$3.47 \mathrm{E}+03$

$3.44 \mathrm{E}+02$

$9.37 E+02$

$5.60 \mathrm{E}+03$

5.44E-02

$1.40 \mathrm{E}+02$

8.06E-01

2.73E+01

$5.83 \mathrm{E}+04$

$1.21 \mathrm{E}+01$

$3.16 \mathrm{E}+03$

$4.05 \mathrm{E}+04$

$9.26 \mathrm{E}+03$

$6.15 \mathrm{E}+01$

7.33E+03

$1.07 \mathrm{E}+03$

$2.60 \mathrm{E}+03$

$3.89 \mathrm{E}+03$

$5.54 \mathrm{E}+01$

$1.86 \mathrm{E}+02$

4.17E+02

5.34E- 00

5.41E-06

$1.34 \mathrm{E}+01$

$1.99 \mathrm{E}-08$

$6.18 \mathrm{E}+03$

1.15E-02

1.70E-02

8.02E-06

1.19E-07

3.05E-07

2.46E-01

$2.59 \mathrm{E}-05$

$6.68 \mathrm{E}+03$

$1.17 \mathrm{E}-03$

2.24E-02

$1.77 \mathrm{E}-01$

7.83E-00

$1.78 \mathrm{E}-03$

$6.15 \mathrm{E}-03$

5.38E-01

8.52E-04

$1.14 \mathrm{E}-05$

$1.70 \mathrm{E}+01$

$2.75 \mathrm{E}+01$

$2.75 \mathrm{E}+01$

$1.50 \mathrm{E}-01$

4.58E-07

$8.30 \mathrm{E}-07$

1.23E-11

$6.02 \mathrm{E}-11$

$0.00 \mathrm{E}+01$

$4.81 \mathrm{E}-01$

9.53E-00

$3.98 \mathrm{E}+03$

$1.26 \mathrm{E}+01$

$3.10 \mathrm{E}-09$

3.77E-12

2.61E-03

7.31E-01

2.64E-05

$1.58 \mathrm{E}-06$

2.23E-00

$1.00 \mathrm{E}-01$

1.42E-02

$1.01 \mathrm{E}-01$

\section{$50 \%$-jle}

$6.99 \mathrm{E}+02$

$9.93 \mathrm{E}+02$

$9.09 \mathrm{E}+03$

$7.91 \mathrm{E}+02$

$5.91 E+03$

$1.25 E+04$

4.00E-01 


\begin{tabular}{|c|c|c|c|c|c|c|c|c|c|c|c|}
\hline$a n k$ & Anal. & In & $\frac{B B}{v 98}$ & $\frac{B B}{\operatorname{tag}}$ & $1 \%$-ile & $10 \%$-ile & $50 \%$-ile & $90 \%$-jle & $99 \%$-ile & Mean & Std Dev \\
\hline 110 & Y90 & $\mathrm{Ci}$ & $4 \overline{86 E+03}$ & $4.69 \mathrm{E}+03$ & $0.00 E+01$ & $5.54 \mathrm{E}+02$ & $4.86 E+03$ & $3.40 E+04$ & $6.79 E+04$ & $1.27 \mathrm{E}+04$ & $1.61 \mathrm{E}+04$ \\
\hline 110 & Zr93 & $\mathrm{Ci}$ & $6.15 \mathrm{E}-02$ & $6.15 \mathrm{E}-02$ & $0.00 \mathrm{E}+01$ & $2.06 \mathrm{E}-03$ & $6.15 E-02$ & $4.10 \mathrm{E}-01$ & $6.44 \mathrm{E}-01$ & $1.54 \mathrm{E}-01$ & $1.74 \mathrm{E}-01$ \\
\hline 111 & Al & kg & $3.58 E+04$ & $3.58 \mathrm{E}+04$ & $7.90 \mathrm{E}+02$ & $84 E+03$ & $1.34 E+04$ & $2.42 \mathrm{E}+04$ & $68 \mathrm{E}+04$ & $1.42 E+04$ & 7.75E+03 \\
\hline 111 & $\mathbf{B i}$ & $\mathrm{kg}$ & $4.62 \mathrm{E}+02$ & $4.62 E+02$ & $1.82 \mathrm{E}+02$ & $E+03$ & $3.11 \mathrm{E}+03$ & $5.92 \mathrm{E}+03$ & +04 & $3.42 E+03$ & $07 \mathrm{E}+03$ \\
\hline 111 & Ca & kg & $3.28 \mathrm{E}+03$ & $3.28 E+03$ & $3.46 \mathrm{E}+01$ & $1.58 \mathrm{E}+02$ & $5.46 E+02$ & $E+03$ & $8 E+03$ & $6.37 E+02$ & $1 E+02$ \\
\hline 111 & Cl & $\mathrm{kg}$ & $.94 \mathrm{E}+02$ & $.94 \mathrm{E}+02$ & $2.66 \mathrm{E}+01$ & $1.42 \mathrm{E}+02$ & $4.05 \mathrm{E}+02$ & $8.38 E+02$ & $.29 E+03$ & $4.56 \mathrm{E}+02$ & $87 E+02$ \\
\hline 11 & $\mathrm{CO3}$ & $\mathrm{kg}$ & $1.96 E+03$ & $1.96 E+03$ & $2.64 \mathrm{E}+02$ & $1.12 E+03$ & $3.43 E+03$ & $6.59 E+03$ & $1 E+03$ & $3.66 \mathrm{E}+03$ & $1 E+03$ \\
\hline 11 & $\mathrm{Cr}$ & kg & $6.22 \mathrm{E}+02$ & $6.22 \mathrm{E}+02$ & $1.76 \mathrm{E}+01$ & $1.15 E+02$ & $3.35 E+02$ & $7.56 \mathrm{E}+02$ & $6 \mathrm{E}+03$ & $4.01 E+02$ & $0 E+02$ \\
\hline 111 & $\mathbf{F}$ & & $3.60 \mathrm{E}+02$ & $3.60 E+02$ & $4.49 \mathrm{E}+01$ & $2.41 \mathrm{E}+02$ & $1.33 E+03$ & $3.44 E+03$ & $7 \mathrm{E}+03$ & $1.65 \mathrm{E}+03$ & $4 E+03$ \\
\hline 111 & $\mathbf{F e}$ & k & $1.53 E+04$ & $1.53 E+04$ & $2.20 \mathrm{E}+02$ & $1.25 E+03$ & $3.48 \mathrm{E}+03$ & $6.85 E+03$ & $1.08 E+04$ & $3.84 E+03$ & $2.29 \mathrm{E}+03$ \\
\hline 111 & Hg & $k$ & $3.36 \mathrm{E}+01$ & $2.31 E+01$ & $0.00 E+01$ & $5.27 \mathrm{E}-00$ & $3.36 E+01$ & $9.88 \mathrm{E}+01$ & $6 E+02$ & $4.65 E+01$ & $82 \mathrm{E}+01$ \\
\hline 111 & $\mathbf{K}$ & $\mathrm{kg}$ & $4.66 \mathrm{E}+01$ & $4.66 \mathrm{E}+01$ & $1.11 \mathrm{E}+01$ & $6.89 \mathrm{E}+01$ & $.09 E+02$ & $4.91 E+02$ & $2.97 \mathrm{E}+03$ & $3.12 E+02$ & $5.32 E+02$ \\
\hline 111 & $\mathbf{L a}$ & kg & $1.21 \mathrm{E}+02$ & $1.21 E+02$ & $2.06 \mathrm{E}-01$ & $9.67 \mathrm{E}-01$ & 2.91E-00 & $E-00$ & $2.10 \mathrm{E}+01$ & 4.19E-00 & $8 \mathrm{E}-00$ \\
\hline 111 & $\mathbf{M n}$ & kg & $\varepsilon+01$ & $8.60 \mathrm{E}+01$ & & +01 & & & +03 & $E+02$ & $\Xi+02$ \\
\hline 111 & $\mathbf{N a}$ & $\mathrm{kg}$ & +03 & $7.56 \mathrm{E}+03$ & 2.4 & +04 & $E+04$ & -04 & +04 & +04 & +04 \\
\hline 111 & $\mathrm{Ni}$ & kg & $5.80 \mathrm{E}+03$ & $5.80 \mathrm{E}+03$ & $8.23 E-00$ & $\div 01$ & $2.32 \mathrm{E}+02$ & $=02$ & $E+03$ & $3.04 E+02$ & $E+02$ \\
\hline 11 & NO2 & Kg & $5.33 E+03$ & $5.33 E+03$ & $3.77 \mathrm{E}+02$ & $2.03 E+03$ & $5.48 E+03$ & +04 & $2.01 E+04$ & $6.32 E+03$ & $4.06 \mathrm{E}+03$ \\
\hline C111 & NO3 & kg & $1.09 E+04$ & $1.09 E+04$ & $1.70 E+03$ & $E+04$ & $3.30 \mathrm{E}+04$ & +04 & +04 & $E+04$ & $E+04$ \\
\hline 111 & $\mathbf{P}$ & $\mathrm{kg}$ & $3.07 E+03$ & NA & 2.78 & +03 & $4.23 E+03$ & +03 & & $E+03$ & $E+03$ \\
\hline 111 & $\mathbf{P b}$ & $\mathrm{kg}$ & $1.60 \mathrm{E}+03$ & $1.60 E+03$ & & +01 & $\mathrm{E}+02$ & & -02 & $E+02$ & $E+02$ \\
\hline 111 & PO4 & kg & $9.52 E+03$ & $9.52 \mathrm{E}+03$ & & & -03 & & & & \\
\hline C111 & $\mathbf{S}$ & $\mathrm{kg}$ & -02 & NA & & & & & & & \\
\hline 111 & Si & kg & $2.50 \mathrm{E}+03$ & $2.50 \mathrm{E}+03$ & 8.3 & +02 & -03 & 03 & -03 & $E+03$ & $E+03$ \\
\hline 111 & SO4 & kg & $8.22 \mathrm{E}+02$ & $8.22 \mathrm{E}+02$ & $1.59 \mathrm{E}+02$ & +02 & $2.22 \mathrm{E}+03$ & +03 & $E+04$ & $E+03$ & $E+03$ \\
\hline $\mathrm{C} 111$ & $\mathbf{S r}$ & $\mathrm{kg}$ & $5.50 \mathrm{E}+01$ & $5.50 \mathrm{E}+01$ & $6.03 \mathrm{E}-00$ & +01 & $1.82 \mathrm{E}+02$ & +02 & $E+03$ & $3.06 E+02$ & $3 E+02$ \\
\hline 11 & TOC & $\mathrm{kg}$ & $3.50 \mathrm{E}+02$ & $3.50 \mathrm{E}+02$ & 3.0 & +02 & $E+02$ & +03 & +03 & +02 & $E+02$ \\
\hline 111 & $\mathbf{U}$ & $\mathrm{kg}$ & $4.23 E+03$ & $4.23 E+03$ & 9.2 & & & & & & +03 \\
\hline 11 & $\mathbf{Z r}$ & $\mathrm{kg}$ & $2.88 E+01$ & $2.88 \mathrm{E}+01$ & & & & & & & +02 \\
\hline 111 & Ac227 & $\mathrm{Ci}$ & 9.57E-06 & 9.57E-06 & 0.0 & $0 . C$ & 9.5 & & -04 & 05 & -05 \\
\hline C111 & $\operatorname{Am} 241$ & $\mathrm{Ci}$ & $5.29 \mathrm{E}-02$ & $5.29 E-02$ & 1.4 & $E-00$ & $2.55 E+01$ & +02 & $E+02$ & $E+01$ & $E+02$ \\
\hline C111. & Am243 & $\mathrm{Ci}$ & 4.25E-07 & $4.25 \mathrm{E}-07$ & 1.3 & $E-07$ & 4.25E-07 & -06 & E-06 & -07 & $E-07$ \\
\hline 1 & Ba137 & $\mathrm{Ci}$ & +04 & +04 & 0.0 & .03 & & & & & \\
\hline 11 & c & $\mathrm{Ci}$ & & 3.1 & & & & & & & \\
\hline & & $\mathrm{Ci}$ & -02 & 8 & & & & & & & \\
\hline C111 & $\mathrm{Cm} 242$ & $\mathrm{Ci}$ & $7.61 \mathrm{E}-04$ & E-04 & 2. & -04 & 7.6 & -03 & 03 & 03 & -03 \\
\hline C111 & $\mathrm{Cm} 243$ & $\mathrm{Ci}$ & $1.67 \mathrm{E}-05$ & $1.67 E-05$ & 5.4 & $E-06$ & -05 & -05 & -04 & -05 & -05 \\
\hline C111 & $\mathrm{Cm} 244$ & $\mathrm{Ci}$ & $1.69 \mathrm{E}-05$ & $1.69 \mathrm{E}-05$ & 5.5 & -06 & 1.6 & .05 & $E-04$ & -05 & $E-05$ \\
\hline 11 & $\mathrm{C} 060$ & $\mathrm{Ci}$ & -03 & -03 & & 01 & & & & & 01 \\
\hline & & $\mathrm{Ci}$ & & 1.6 & & & & & & & \\
\hline & & $\mathrm{Ci}$ & & & & & & -04 & -04 & 104 & \\
\hline C111 & Eu152 & $\mathrm{Ci}$ & 4.09E-02 & $4.09 \mathrm{E}-02$ & 0.0 & t01 & 4.0 & -01 & -01 & -02 & -02 \\
\hline C111 & Eu154 & $\mathrm{Ci}$ & $1.80 \mathrm{E}-01$ & $1.80 \mathrm{E}-01$ & 0.0 & +01 & $1.80 \mathrm{E}-01$ & $E-01$ & $E-00$ & $E-01$ & E-01 \\
\hline C111 & Eut55 & $\mathrm{Ci}$ & $2.89 \mathrm{E}-00$ & $2.89 E-00$ & +01 & +01 & -00 & +01 & +01 & 5. & -00 \\
\hline 2111 & 1129 & $\mathrm{Ci}$ & & $4.13 E-04$ & & & & & & & -04 \\
\hline & & $\mathrm{Ci}$ & & 2 & & & & & & & \\
\hline C111 & 9 & $\mathrm{Ci}$ & -01 & $2.24 \mathrm{E}-01$ & & 03 & & & & .01 & -01 \\
\hline C111 & Ni63 & $\mathrm{Ci}$ & $2.02 E+01$ & $2.02 \mathrm{E}+01$ & 0.0 & 01 & 2.0 & -01 & +02 & +01 & +01 \\
\hline C111 & Np237 & $\mathrm{Ci}$ & $1.35 E-03$ & $1.35 \mathrm{E}-03$ & -05 & $E-04$ & 1.3 & -03 & $E-03$ & -03 & -03 \\
\hline C111 & Pa231 & $\mathrm{Ci}$ & 2.06E-05 & $2.06 \mathrm{E}-05$ & 0.0 & $=-07$ & 2.0 & -05 & 4 & -05 & -05 \\
\hline C1II & Pu238 & $\mathrm{Ci}$ & $1.43 \mathrm{E}-00$ & $3.31 \mathrm{E}-00$ & 4. & -01 & & 00 & $E-00$ & 0 & -00 \\
\hline C111 & Pu239 & $\mathrm{Ci}$ & 02 & & & & & & & & r02 \\
\hline C111 & $\mathbf{P}$ & $\mathrm{Ci}$ & Incl & 3. & & & & & & +02 & +02 \\
\hline C111 & $\mathbf{P u}$ & $\mathrm{Ci}$ & $E+02$ & $2.40 \mathrm{E}+02$ & & 01 & & & & -02 & +02 \\
\hline C111 & Pu242 & $\mathrm{Ci}$ & $3.97 \mathrm{E}-04$ & $9.15 \mathrm{E}-04$ & 1.2 & $=-04$ & 04 & -03 & -03 & -04 & E-04 \\
\hline C111 & Ra226 & $\mathrm{Ci}$ & $1.88 E-06$ & $1.88 \mathrm{E}-06$ & 0.00 & $E-07$ & 1.8 & -06 & $E-05$ & -06 & -06 \\
\hline C111 & $\operatorname{Ra228}$ & $\mathrm{Ci}$ & $1.31 \mathrm{E}-10$ & 1.31E-10 & 0.0 & $E-11$ & 10 & 10 & -09 & -10 & -10 \\
\hline C111 & Ru106 & $\mathrm{Ci}$ & $6.59 \mathrm{E}-08$ & $6.59 \mathrm{E}-08$ & 0. & -09 & $65+2>$ & 07 & 07 & 7. & -08 \\
\hline C11 & Sb125 & $\mathrm{Ci}$ & 1. & 13 & 0.0 & 01 & & & & & \\
\hline & & C & & & & & & & & & -03 \\
\hline Clll & & $\mathrm{Ci}$ & +01 & $2.46 E+01$ & & +01 & & & +02 & -01 & $E+01$ \\
\hline Citl & Sr89/90 & $\mathrm{Ci}$ & $1.14 E+06$ & $1.14 E+06$ & & $E+04$ & 7.7 & -05 & $E+06$ & +05 & $E+05$ \\
\hline C111 & Tc99 & $\mathrm{Ci}$ & $2.19 E-01$ & 2.19E-01 & & & & & $\mathrm{E}-00$ & $E-01$ & $7 E-01$ \\
\hline Cl11 & Th229 & $\mathrm{Ci}$ & $2.54 E-08$ & $2.54 \mathrm{E}-08$ & 0.0 & E-09 & 2.54 & $=-08$ & E-07 & $\varepsilon-08$ & $E-08$ \\
\hline C111 & Th232 & $\mathrm{Ci}$ & 8.83E-12 & $8.83 \mathrm{E}-12$ & $0.00 E+01$ & $F_{-13}$ & $8.83 \mathrm{E}-12$ & & & 1. & $8 E-11$ \\
\hline C1II & Tin126 & $\mathrm{Ci}$ & $1.00 \mathrm{E}-02$ & $1.00 \mathrm{E}-02$ & $0.00 E+01$ & $157 E_{-03}$ & $1.00 \mathrm{E}-02$ & $2.94 E-02$ & $6.74 \mathrm{E}-02$ & $1.38 \mathrm{E}-02$ & $1.44 \mathrm{E}-02$ \\
\hline C111 & Tritium & $\mathrm{Ci}$ & -01 & 1.66 & & & & & $E-01$ & 1. & \\
\hline 11 & U232 & $\mathrm{Ci}$ & $6.02 E-05$ & $2.96 \mathrm{E}-05$ & $0.00 E+01$ & $2.86 E-06$ & $6.02 E-05$ & E-04 & $E-04$ & E-05 & 8.70E-05 \\
\hline C111 & U233 & $\mathbf{C i}$ & 2.70E-06 & $1.33 \mathrm{E}-06$ & $0.00 \mathrm{E}+01$ & $1.28 E-07$ & $2.70 \mathrm{E}-06$ & $8.20 \mathrm{E}-06$ & $1.90 \mathrm{E}-05$ & $3.65 \mathrm{E}-06$ & $3.90 \mathrm{E}-06$ \\
\hline
\end{tabular}


Tank Anal. Un

\begin{tabular}{|c|c|}
\hline C111 & U234 \\
\hline C111 & U235 \\
\hline C111 & U236 \\
\hline C11I & U238 \\
\hline C111 & Y90 \\
\hline C111 & Zr93 \\
\hline C112 & Al \\
\hline C112 & $\mathbf{B i}$ \\
\hline C112 & $\mathrm{Ca}$ \\
\hline C112 & CI \\
\hline C112 & $\mathrm{CO3}$ \\
\hline C112 & $\mathrm{Cr}$ \\
\hline C112 & F \\
\hline C112 & Fe \\
\hline C112 & Hg \\
\hline C112 & $\mathbf{K}$ \\
\hline C112 & $\mathbf{L a}$ \\
\hline C112 & Mn \\
\hline C112 & $\mathrm{Na}$ \\
\hline C112 & $\mathrm{Ni}$ \\
\hline C112 & NO2 \\
\hline C112 & NO3 \\
\hline C112 & $\mathbf{P}$ \\
\hline C112 & $\mathbf{P b}$ \\
\hline C112 & PO4 \\
\hline C112 & $\mathbf{S}$ \\
\hline $\mathrm{Cl12}$ & $\mathrm{Si}$ \\
\hline C112 & SO4 \\
\hline C112 & Sr \\
\hline C112 & TOC \\
\hline C112 & $\mathbf{U}$ \\
\hline C112 & $\mathbf{Z r}$ \\
\hline $\mathrm{Cl12}$ & Ac227 \\
\hline C112 & Am241 \\
\hline C112 & Am243 \\
\hline C112 & Ba137 \\
\hline C112 & C14 \\
\hline C112 & Cd113 \\
\hline C112 & Cm242 \\
\hline C112 & Cm243 \\
\hline C112 & $\mathrm{Cm} 244$ \\
\hline C112 & Co60 \\
\hline C112 & Cs134 \\
\hline C112 & Cs137 \\
\hline C112 & Eu152 \\
\hline C112 & Eu154 \\
\hline C112 & Eul55 \\
\hline C112 & 1129 \\
\hline C112 & Nb93 \\
\hline C112 & Ni59 \\
\hline C112 & Ni63 \\
\hline $\mathrm{C} 112$ & Np237 \\
\hline C112 & Pa231 \\
\hline C112 & Pu238 \\
\hline C112 & Pu239 \\
\hline C112 & Pu240 \\
\hline C112 & Pu241 \\
\hline $\mathrm{Cl12}$ & Pu242 \\
\hline C112 & Ra226 \\
\hline C112 & $\operatorname{Ra228}$ \\
\hline C112 & Ru106 \\
\hline C112 & Sb125 \\
\hline C112 & Se79 \\
\hline C112 & Sm151 \\
\hline $\mathrm{Cl12}$ & Sr89/90 \\
\hline $\mathrm{Cl12}$ & Te99 \\
\hline C112 & Th229 \\
\hline
\end{tabular}

$B B$ May98 $1.26 \mathrm{E}-01$ 2.53E-02 $3.41 \mathrm{E}-00$ $1.14 \mathrm{E}+06$ 3.15E-02 $1.18 \mathrm{E}+04$

$7.40 \mathrm{E}+02$ $1.60 \mathrm{E}+04$

kg $\quad 7.36 \mathrm{E}+02$

kg $\quad 2.26 \mathrm{E}+04$

kg $\quad 1.87 \mathrm{E}+02$

$\mathrm{kg} \quad 4.69 \mathrm{E}+02$

kg $\quad 1.62 \mathrm{E}+04$

kg $3.96 \mathrm{E}+01$

kg $\quad 4.06 \mathrm{E}+02$

kg $3.90 \mathrm{E}+01$

kg 1.92E+02

$\begin{array}{ll}\mathrm{kg} & 1.92 \mathrm{E}+02 \\ \mathrm{~kg} & \mathbf{7 . 6 7 \mathrm { E } + 0 4}\end{array}$

kg $\quad 9.19 \mathrm{E}+03$

kg $3.62 \mathrm{E}+04$

kg $\quad 4.83 \mathrm{E}+04$

kg $\quad 5.78 \mathrm{E}+04$

kg $1.79 \mathrm{E}+05$

$3.10 \mathrm{E}+03$

$1.65 \mathrm{E}+03$

$9.31 E+03$

$2.07 \mathrm{E}+02$

$1.45 E+03$

$3.64 \mathrm{E}+04$

$1.89 \mathrm{E}+01$

2.10E-03

$2.19 \mathrm{E}+02$

1.17E-04

$2.33 \mathrm{E}+05$

2.73E-00

5.09E-01

$9.70 \mathrm{E}-02$

8.77E-01

2.72E-04

4.58E-02

2.03E-02

$2.46 \mathrm{E}+05$

$7.38 E-01$

$6.30 \mathrm{E}+02$

$8.25 \mathrm{E}+02$

2.40E-03

$1.72 \mathrm{E}-01$

9.40E-00

$\mathrm{Ci} \quad 9.21 \mathrm{E} \div 02$

3.47E-01

2.89E- 03

$5.54 \mathrm{E}-00$

$6.95 E+01$

i Incl. in 239

Ci $1.65 \mathrm{E}+02$

$6.02 \mathrm{E}-04$

Ci 4.71E-05

Ci $6.11 \mathrm{E}-04$

$\mathrm{Ci}$ 1.16E-05

Ci $5.60 \mathrm{E}-02$

Ci 2.39E-01

Ci $1.61 \mathrm{E}+02$

$1.25 \mathrm{E}+06$

$7.97 \mathrm{E}+01$

2.77E-04

2.85E-05
BB

Sept98

1.39E-00

6.18E-02

1.24E-02

1.41E-00

$1.14 \mathrm{E}+06$

3.15E-02

$1.18 \mathrm{E}+04$

$7.40 \mathrm{E}+02$

$1.60 \mathrm{E}+04$

$7.36 \mathrm{E}+02$

$2.26 \mathrm{E}+04$

$1.87 \mathrm{E}+02$

$4.69 \mathrm{E}+02$

$1.62 \mathrm{E}+04$

$1.64 \mathrm{E}+01$

$4.06 \mathrm{E}+02$

$3.90 \mathrm{E}+01$

$1.92 \mathrm{E}+02$

$7.67 \mathrm{E}+04$

$9.19 \mathrm{E}+03$

$3.62 \mathrm{E}+04$

$4.83 E+04$

$$
\text { NA }
$$

$1.42 \mathrm{E}+03$

$5.84 \mathrm{E}+04$

NA

$1.65 \mathrm{E}+03$

$9.31 \mathrm{E}+03$

$2.07 \mathrm{E}+02$

$1.45 \mathrm{E}+03$

$3.64 \mathrm{E}+04$

$1.89 \mathrm{E}+01$

2.10E-03

$2.19 \mathrm{E}+02$

$5.00 \mathrm{E}-03$

$2.33 E+05$

2.73E-00

5.09E-01

9.70E-02

3.51E-02

8.42E-01

4.58E-02

2.03E-02

$2.46 \mathrm{E}+05$

7.38E-01

$6.30 \mathrm{E}+02$

$8.25 \mathrm{E}+02$

2.40E-03

$1.72 \mathrm{E}-01$

$9.40 \mathrm{E}-00$

$9.21 \mathrm{E}+02$

3.47E-01

2.89E- 03

5.54E-00

$6.00 \mathrm{E}+01$

9.53E-00

8.27E+01

3.01E-04

4.71E-05

6.11E-04

1.16E-05

5.60E-02

2.39E-01

$1.61 \mathrm{E}+02$

$1.25 \mathrm{E}+06$

$7.97 \mathrm{E}+01$

2.77E-04

2.85E-05
$1 \%$ ille

$0.00 E+01$

$0.00 \mathrm{E}+01$

$0.00 \mathrm{E}+01$

$0.00 \mathrm{E}+01$

$3.41 \mathrm{E}+04$

$0.00 \mathrm{E}+01$

$7.42 \mathrm{E}+03$

$5.59 \mathrm{E}+02$

$8.89 \mathrm{E}+01$

$2.08 \mathrm{E}+02$

$2.13 E+03$

$9.60 \mathrm{E}+01$

$1.81 \mathrm{E}+02$

$1.62 \mathrm{E}+03$

$0.00 \mathrm{E}+01$

$7.02 \mathrm{E}+01$

$1.69 \mathrm{E}-00$

$3.59 \mathrm{E}+01$

$2.04 \mathrm{E}+04$

$2.56 \mathrm{E}+01$

$6.69 \mathrm{E}+03$

$1.29 \mathrm{E}+04$

$1.13 \mathrm{E}+03$

$8.68 \mathrm{E}+01$

$1.15 \mathrm{E}+03$

$1.55 \mathrm{E}+02$

$3.96 \mathrm{E}+02$

$8.75 \mathrm{E}+02$

$2.85 \mathrm{E}+01$

$2.44 \mathrm{E}+02$

$9.29 \mathrm{E}+02$

$1.50 \mathrm{E}-00$

$0.00 \mathrm{E}+01$

$1.24 \mathrm{E}+01$

$1.38 \mathrm{E}-05$

$2.48 \mathrm{E}+04$

$0.00 \mathrm{E}+01$

$0.00 \mathrm{E}+01$

1.15E-02

1.04E-01

$3.21 \mathrm{E}-05$

$1.41 \mathrm{E}-00$

$2.16 \mathrm{E}-03$

1.10E+04

$0.00 E+01$

$0.00 \mathrm{E}+01$

$0.00 \mathrm{E}+01$

4.13E-04

3.16E-03

$0.00 \mathrm{E}+01$

$0.00 \mathrm{E}+01$

4.10E-02

$0.00 \mathrm{E}+01$

$6.55 \mathrm{E}-01$

8.21E-00

8.21E-00

$1.95 \mathrm{E}+01$

7.11E-05

$0.00 \mathrm{E}+01$

$0.00 \mathrm{E}+01$

$0.00 \mathrm{E}+01$

$0.00 \mathrm{E}+01$

6.43E-03

$0.00 \mathrm{E}+01$

$2.96 \mathrm{E}+04$

6.77E-01

$0.00 \mathrm{E}+01$

$0.00 E+01$
$10 \%$-ile

1.35E-01

5.99E-03

$1.20 \mathrm{E}-03$

$1.62 \mathrm{E}-01$

$2.06 \mathrm{E}+05$

4.73E-03

$1.26 \mathrm{E}+04$

$1.11 \mathrm{E}+03$

$1.85 \mathrm{E}+03$

$3.96 \mathrm{E}+02$

$4.56 \mathrm{E}+03$

$2.14 \mathrm{E}+02$

$5.25 \mathrm{E}+02$

$4.04 \mathrm{E}+03$

6.97E-00

$2.98 \mathrm{E}+02$

4.31E-00

$1.18 \mathrm{E}+02$

$3.61 \mathrm{E}+04$

$1.17 \mathrm{E}+03$

$1.16 \mathrm{E}+04$

2.72E+04

$2.97 \mathrm{E}+03$

$2.62 \mathrm{E}+02$

$3.14 \mathrm{E}+03$

$5.40 \mathrm{E}+02$

$7.72 \mathrm{E}+02$

$2.27 E+03$

$1.24 \mathrm{E}+02$

$1.16 \mathrm{E}+03$

$3.33 \mathrm{E}+03$

$1.44 \mathrm{E}+01$

$0.00 \mathrm{E}+01$

4.52E+01

3.62E-05

$8.38 \mathrm{E}+04$

$5.54 \mathrm{E}-01$

8.96E-02

$3.00 \mathrm{E}-02$

2.71E-01

8.41E-05

2.57E-00

7.30E-03

3.17E+04

$0.00 \mathrm{E}+01$

$0.00 \mathrm{E}+01$

$0.00 E+01$

9.02E-04

3.45E- 02

$0.00 \mathrm{E}+01$

$0.00 \mathrm{E}+01$

$1.07 \mathrm{E}-01$

1.73E-04

1.71E-00

$2.15 \mathrm{E} \div 01$

$2.15 E+01$

$5.10 \mathrm{E}+01$

$1.86 \mathrm{E}-04$

$0.00 \mathrm{E}+01$

$0.00 \mathrm{E}+01$

2.00E-06

$7.80 \mathrm{E}-03$

$6.95 \mathrm{E}-02$

$0.00 E+01$

$1.10 \mathrm{E}+05$

$2.35 \mathrm{E}+01$

$1.66 \mathrm{E}-05$

1.71E-06

\section{$50 \%-i l e$}

2.83E-00

1.26E-01

2.53E-02

$3.41 E-00$

1.14E+06

3.15E-02 


\begin{tabular}{|c|c|c|c|c|c|c|c|c|c|c|c|}
\hline Tank & Anal. & n & $\frac{B B}{\sqrt{98}}$ & BB & $1 \%$-ile & $10 \%$-ile & $50 \%$-ile & $90 \%$-ile & $99 \%-i l e$ & Mean & Std Dev \\
\hline 112 & 126 & $\mathrm{Ci}$ & -02 & $6.56 \mathrm{E}-02$ & $0.00 \mathrm{E}+01$ & $1.15 \mathrm{E}-02$ & $6.56 \mathrm{E}-02$ & $2.12 \mathrm{E}-0 \mathrm{i}$ & $6 \mathrm{E}-01$ & $E-02$ & 2 \\
\hline 112 & Tritium & $\mathrm{Ci}$ & $2.81 E+01$ & $2.81 \mathrm{E}+01$ & $1.20 \mathrm{E}+01$ & $1.81 \mathrm{E}+01$ & $2.81 \mathrm{E}+01$ & 4.12E+01 & $E+01$ & 01 & $1.02 \mathrm{E}+01$ \\
\hline 112 & U232 & $\mathrm{Ci}$ & $3.16 \mathrm{E}-02$ & $2.33 \mathrm{E}-01$ & $0.00 \mathrm{E}+01$ & $1.89 \mathrm{E}-03$ & $3.16 \mathrm{E}-02$ & $9.26 \mathrm{E}-02$ & $56 \mathrm{E}-01$ & $E-02$ & $4.96 \mathrm{E}-02$ \\
\hline C112 & U233 & $\mathrm{Ci}$ & $1.22 \mathrm{E}-01$ & $9.03 \mathrm{E}-01$ & $0.00 \mathrm{E}+01$ & & $1.22 \mathrm{E}-01$ & $7 \mathrm{E}-01$ & $89 \mathrm{E}-01$ & $E-01$ & $1.92 \mathrm{E}-01$ \\
\hline 12 & U234 & $\mathrm{Ci}$ & $1.63 \mathrm{E}-00$ & $1.20 \mathrm{E}+01$ & $0.00 \mathrm{E}+01$ & $9.76 \mathrm{E}-02$ & $1.63 \mathrm{E}-00$ & $4.78 \mathrm{E}-00$ & $1.32 \mathrm{E}+01$ & $2.25 \mathrm{E}-00$ & $2.56 \mathrm{E}-00$ \\
\hline 112 & U235 & $\mathrm{Ci}$ & $7.15 \mathrm{E}-02$ & $5.28 \mathrm{E}-01$ & $0.00 \mathrm{E}+01$ & $4.28 \mathrm{E}-03$ & $7.15 \mathrm{E}-02$ & $2.09 \mathrm{E}-01$ & $79 \mathrm{E}-01$ & $9.89 \mathrm{E}-02$ & $1.12 E-01$ \\
\hline $\mathrm{C112}$ & U236 & $\mathrm{Ci}$ & $2.02 \mathrm{E}-02$ & $1.49 \mathrm{E}-01$ & $0.00 \mathrm{E}+01$ & $E-03$ & $2.02 \mathrm{E}-02$ & $5.92 \mathrm{E}-02$ & $4 \mathrm{E}-0 \mathrm{I}$ & $E-02$ & $3.17 \mathrm{E}-02$ \\
\hline $\mathrm{C} 112$ & U238 & $\mathrm{Ci}$ & 9.41E-00 & $1.22 \mathrm{E}+01$ & $0.00 \mathrm{E}+01$ & $5.63 E-01$ & $9.41 \mathrm{E}-00$ & $2.76 E+01$ & $7.63 E+01$ & $1.30 \mathrm{E}+01$ & $1.48 E+01$ \\
\hline 112 & Y90 & $\mathrm{Ci}$ & $1.25 E+06$ & $1.25 E+06$ & $2.80 E+04$ & $2.74 E+05$ & $1.25 E+06$ & $5.01 E+06$ & $1.21 E+07$ & $2.27 \mathrm{E}+06$ & $4.31 E+06$ \\
\hline 12 & $x-93$ & $\mathrm{Ci}$ & $2.04 \mathrm{E}-01$ & $2.04 \mathrm{E}-01$ & $3.75 E-03$ & $4.09 \mathrm{E}-02$ & 2.04E-01 & $7.81 E-01$ & $1.97 \mathrm{E}+01$ & $1.22 \mathrm{E}-00$ & $3.75 \mathrm{E}-00$ \\
\hline 201 & Al & $\mathrm{kg}$ & $1.41 \mathrm{E}+01$ & $5.10 \mathrm{E}+01$ & $2.02 \mathrm{E}-07$ & E-04 & 9.13E-60 & $E+02$ & +03 & $E+02$ & +02 \\
\hline C201 & $\mathbf{B i}$ & $\mathrm{kg}$ & $0.00 \mathrm{E}+01$ & $0.00 \mathrm{E}+01$ & -08 & & $E-01$ & & & $=00$ & \\
\hline C201 & Ca & kg & $6.40 \mathrm{E}+01$ & $2.31 E+02$ & $E-06$ & -03 & 9.55E-00 & & +02 & $E+01$ & \\
\hline C201 & Cl & $\mathrm{kg}$ & $1.76 \mathrm{E}+01$ & $6.37 \mathrm{E}+01$ & $E-06$ & -04 & E-00 & -01 & +01 & +01 & +01 \\
\hline C201 & $\mathrm{CO} 3$ & $\mathrm{~kg}$ & $1.76 \mathrm{E}+02$ & $9.18 E+02$ & $4.46 \mathrm{E}-05$ & -02 & $1.74 E+02$ & +03 & +03 & $6.49 E+02$ & $E+03$ \\
\hline C201 & $\mathrm{Cr}$ & $\mathrm{kg}$ & $1.18 \mathrm{E}-00$ & $4.28 \mathrm{E}-00$ & $2.95 \mathrm{E}-07$ & $3.71 \mathrm{E}-04$ & $2.22 \mathrm{E}-00$ & $3.78 E+01$ & $1.34 \mathrm{E}+02$ & $1.27 \mathrm{E}+01$ & $2.83 E+01$ \\
\hline C201 & $\mathbf{F}$ & $\mathrm{kg}$ & $4.39 \mathrm{E}-00$ & $1.59 \mathrm{E}+01$ & $1.60 \mathrm{E}-08$ & $4.04 E-05$ & $1.71 \mathrm{E}-01$ & +01 & +02 & $E-00$ & $E+01$ \\
\hline C201 & $\mathrm{Fe}$ & $\mathrm{kg}$ & $5.45 E+02$ & $1.98 E+03$ & $1.84 \mathrm{E}-05$ & -04 & +01 & +02 & +03 & +02 & +02 \\
\hline 201 & Hg & $\mathrm{kg}$ & $0.00 E+01$ & $0.00 E+01$ & $0.00 \mathrm{E}+01$ & +01 & +01 & +01 & +01 & +01 & +01 \\
\hline 01 & $\mathbf{K}$ & $\mathrm{kg}$ & $1.09 E+01$ & $1.27 E+01$ & -06 & & & & & +01 & \\
\hline C & $\mathbf{L a}$ & $\mathrm{kg}$ & $0.00 \mathrm{E}+01$ & $2.23 \mathrm{E}+01$ & -09 & 06 & -02 & & & -01 & -01 \\
\hline C201 & $\mathbf{M n}$ & $\mathrm{kg}$ & $0.00 \mathrm{E}+01$ & $0.00 E+01$ & $E-09$ & -05 & $E-01$ & 101 & $E+01$ & $E-00$ & $E+01$ \\
\hline C201 & $\mathbf{N a}$ & $\mathrm{kg}$ & $2.38 E+02$ & $8.60 \mathrm{E}+02$ & $1.48 \mathrm{E}-04$ & $E-02$ & $4.83 E+02$ & +03 & $E+03$ & $7.60 \mathrm{E}+02$ & +02 \\
\hline C201 & $1 \mathrm{NI}$ & $\mathrm{kg}$ & $7.05 E+01$ & $2.55 \mathrm{E}+02$ & -06 & & +01 & & $E+03$ & +02 & +02 \\
\hline C201 & NO2 & $\mathrm{kg}$ & $8.82 \mathrm{E}-00$ & $3.18 E+01$ & $E-05$ & & +02 & & +03 & +02 & +02 \\
\hline 01 & NO3 & $\mathrm{kg}$ & $2.91 \mathrm{E}+02$ & $1.05 E+03$ & -06 & 03 & 01 & & -04 & +02 & \\
\hline 1 & $\mathbf{P}$ & $\mathrm{kg}$ & 4.17 & NA & & & & & & +02 & +02 \\
\hline C201 & $\mathbf{P b}$ & $\mathrm{kg}$ & $2.29 \mathrm{E}+03$ & $1.80 \mathrm{E}+03$ & -06 & -04 & 01 & 02 & 03 & +02 & +02 \\
\hline C201 & PO4 & $\mathrm{kg}$ & $1.29 E+03$ & $3.13 E+02$ & 1.2 & -02 & +01 & +02 & $\div+03$ & 102 & +02 \\
\hline C201 & $\mathbf{S}$ & $\mathrm{kg}$ & $1.77 E+01$ & NA & -06 & -04 & +01 & 01 & $E+02$ & +01 & +01 \\
\hline C201 & $\mathbf{S i}$ & $\mathrm{kg}$ & 3.29 & $1.19 E+02$ & & & & & & & \\
\hline 11 & 04 & $\mathrm{~kg}$ & $5.30 \mathrm{E}+01$ & $5.30 \mathrm{E}+01$ & & & 01 & & & & \\
\hline & $\mathbf{S r}$ & $\mathrm{kg}$ & & 0.0 & .09 & & & & -01 & .00 & $-\infty 0$ \\
\hline C201 & TOC & $\mathrm{kg}$ & $1.82 \mathrm{E}+01$ & $4.75 E+02$ & -06 & -03 & +01 & +02 & $\varepsilon+02$ & $\vdots+01$ & +02 \\
\hline C201 & $\mathbf{U}$ & $\mathrm{kg}$ & $5.80 \mathrm{E}-01$ & $2.12 E-00$ & -05 & -04 & +02 & +03 & +03 & +02 & +02 \\
\hline $\mathrm{C201}$ & $\mathbf{Z r}$ & $\mathrm{kg}$ & $0.00 E+01$ & $0.00 \mathrm{E}+01$ & & & & & & & -00 \\
\hline 1 & 27 & $\mathrm{Ci}$ & & $1.81 E-04$ & & & & & & & \\
\hline & Am & $\mathrm{Ci}$ & & 4.18 & & & & & +02 & +01 & t01 \\
\hline C201 & Am243 & $\mathrm{Ci}$ & 1.15E-04 & $1.00 \mathrm{E}-03$ & +01 & $8-09$ & -04 & -03 & $E-02$ & $E-03$ & $\mathrm{E}-02$ \\
\hline C201 & Ba137 & $\mathrm{Ci}$ & $4,04 E+01$ & $1.63 E+02$ & +01 & +01 & $4.04 E+01$ & +03 & $E+03$ & $E+02$ & +02 \\
\hline C201 & C14 & $\mathrm{Ci}$ & $1.14 \mathrm{E}-02$ & $1.14 \mathrm{E}-02$ & +01 & +01 & -02 & -01 & $E-01$ & 4. & -02 \\
\hline C201 & Cd113 & $\mathrm{Ci}$ & -02 & $5.87 \mathrm{E}-02$ & & & & & & & -00 \\
\hline 01 & $\mathrm{Cm} 242$ & $\mathrm{Ci}$ & 7. & 02 & & & & & & & \\
\hline & & $\mathrm{Ci}$ & & 3.6 & 01 & & & & & & -02 \\
\hline C201 & Cm244 & $\mathrm{Ci}$ & $2.05 E-04$ & $1.79 \mathrm{E}-03$ & +01 & -09 & -04 & -03 & -02 & -03 & $E-02$ \\
\hline C201 & Co60 & $\mathrm{Ci}$ & $1.36 E-03$ & $1.36 \mathrm{E}-03$ & $E-11$ & $\$-07$ & -03 & -01 & -01 & $E-02$ & -01 \\
\hline C201 & Cs134 & $\mathrm{Ci}$ & 4.52E-06 & 4.52E-06 & $E+01$ & +01 & -06 & -04 & $E-04$ & -05 & -05 \\
\hline $\mathrm{C201}$ & Cs13 & $\mathrm{Ci}$ & $4.27 E+01$ & $1.72 E+02$ & & & 01 & & 3 & 32 & -03 \\
\hline C & & $\mathrm{Ci}$ & & & & & & & & & \\
\hline C & & $\mathrm{Ci}$ & & & & & & & & & -00 \\
\hline C201 & Eul55 & $\mathrm{Ci}$ & $2.12 E+01$ & $2.12 E+01$ & +01 & -01 & 2.1 & +02 & +02 & 7.7 & $E+02$ \\
\hline C201 & 1129 & $\mathrm{Ci}$ & $3.18 E-05$ & 3.18E-05 & 0.0 & 0.0 & -05 & -04 & $\varepsilon-04$ & & -04 \\
\hline C201 & Nb93 & $\mathrm{Ci}$ & $1.95 \mathrm{E}-02$ & $1.95 E-02$ & $\therefore+01$ & $=-06$ & -02 & .00 & -01 & 00 & $E-00$ \\
\hline C201 & Ni59 & $\mathrm{Ci}$ & $3 E-01$ & 4.23E-01 & 0. & -01 & 01 & -00 & - & & +01 \\
\hline C201 & $\mathrm{Ni}$ & $\mathrm{Ci}$ & - & 4.1 & & & & & & & \\
\hline C & & $c$ & & & & & & & & & -03 \\
\hline $\mathrm{C} 2$ & & $\mathrm{Ci}$ & & $6.06 \mathrm{E}$ & -01 & & .06 & & & & -05 \\
\hline C201 & Pu238 & $\mathrm{Ci}$ & $335 E-01$ & $2.93 \mathrm{E}-00$ & 0. & & -01 & -00 & +02 & -00 & $E+01$ \\
\hline C201 & Pu239 & $\mathrm{Ci}$ & $1.46 \mathrm{E}+02$ & $1.24 E+02$ & $0.00 \mathrm{E}+01$ & $1.64 \mathrm{E}-03$ & $1.46 E+02$ & +03 & $E+04$ & $E+03$ & $E+04$ \\
\hline C201 & Pu240 & $\mathrm{Ci}$ & Incl. in 239 & $2.04 E+01$ & $0.00 \mathrm{E}+01$ & $1.64 E-03$ & $1.46 \mathrm{E}+02$ & $E \div 03$ & +04 & +03 & $8+04$ \\
\hline C201 & Pu241 & $\mathrm{Ci}$ & $2.42 E+01$ & $2.12 \mathrm{E}+02$ & 0.0 & 2. & 2.4 & 6. & & 4. & +03 \\
\hline C201 & Pu242 & $\mathrm{Ci}$ & aF_os & $1.04 \mathrm{E}-0$ & +01 & & in & -03 & 3 & & \\
\hline C201 & $\operatorname{Ra226}$ & $\mathrm{Ci}$ & & & & & & & & & -03 \\
\hline C20 & $\operatorname{Ra228}$ & $\mathrm{Ci}$ & -10 & 2.09E-10 & 0.0 & $E-14$ & -10 & & -08 & -09 & -08 \\
\hline C201 & Ru106 & $\mathrm{Ci}$ & $9.41 \mathrm{E}-06$ & $9.41 \mathrm{E}-06$ & $E+01$ & +01 & $9.41 E-06$ & -05 & -04 & -05 & $4.46 \mathrm{E}-05$ \\
\hline$n$ & Sb125 & $\mathrm{Ci}$ & $4.66 \mathrm{E}-03$ & $4.66 E-03$ & $0.00 \mathrm{E}+01$ & $0.00 E+01$ & $4.66 \mathrm{E}-03$ & $1.87 \mathrm{E}-01$ & $7.89 \mathrm{E}-01$ & $6.00 \mathrm{E}-02$ & $1.58 \mathrm{E}-01$ \\
\hline C201 & Se79 & $\mathrm{Ci}$ & $5.21 E-03$ & $5.21 E-03$ & $0.00 \mathrm{E}+01$ & $0.00 \mathrm{E}+01$ & & $2.90 \mathrm{E}-02$ & $9.24 \mathrm{E}-02$ & $1.18 \mathrm{E}-02$ & $2.08 \mathrm{E}-02$ \\
\hline $\mathrm{C} 20$ & & $\mathrm{Ci}$ & $.95 E+01$ & $1.95 E+01$ & $0.00 \mathrm{E}+01$ & $0.00 \mathrm{E}+01$ & $1.95 E+01$ & $1.97 \mathrm{E}+02$ & $6.89 \mathrm{E}+02$ & $7.13 \mathrm{E}+01$ & 1.421 \\
\hline
\end{tabular}


Tank Anal. Un

\begin{tabular}{|c|c|}
\hline $\mathrm{C201}$ & Sr89/90 \\
\hline 201 & Te99 \\
\hline C201 & Th229 \\
\hline C201 & Th232 \\
\hline C201 & Tin126 \\
\hline C201 & Tritium \\
\hline C201 & U232 \\
\hline C201 & $\mathbf{U} 233$ \\
\hline C201 & U234 \\
\hline C201 & U235 \\
\hline C201 & U236 \\
\hline C201 & U238 \\
\hline C201 & Y90 \\
\hline C201 & Zr93 \\
\hline $\mathrm{C} 202$ & Al \\
\hline C202 & $\mathbf{B i}$ \\
\hline C202 & Ca \\
\hline C202 & Cl \\
\hline $\mathrm{C2O2}_{2}$ & $\mathrm{CO3}$ \\
\hline C202 & $\mathrm{Cr}$ \\
\hline $\mathrm{C} 202$ & $\mathbf{F}$ \\
\hline C202 & Fe \\
\hline C202 & Hg \\
\hline C202 & $\mathbf{K}$ \\
\hline $\mathrm{C} 202$ & $\mathbf{L a}$ \\
\hline C202 & Mn \\
\hline C202 & $\mathbf{N a}$ \\
\hline C202 & $\mathbf{N i}$ \\
\hline C202 & NO2 \\
\hline C202 & NO3 \\
\hline C202 & $\mathbf{P}$ \\
\hline C202 & $\mathbf{P b}$ \\
\hline C202 & PO4 \\
\hline C202 & $\mathbf{S}$ \\
\hline C202 & $\mathrm{Si}$ \\
\hline C202 & S04 \\
\hline C202 & $\mathrm{Sr}$ \\
\hline C202 & TOC \\
\hline C202 & $\mathbf{U}$ \\
\hline C202 & $\mathbf{Z r}$ \\
\hline C202 & Ac227 \\
\hline C202 & Am241 \\
\hline C202 & $\operatorname{Am} 243$ \\
\hline C202 & Ba137 \\
\hline C202 & C14 \\
\hline C202 & Cd113 \\
\hline C202 & Cm242 \\
\hline C202 & $\mathrm{Cm} 243$ \\
\hline C202 & Cm244 \\
\hline C202 & Co60 \\
\hline C202 & Cs134 \\
\hline C202 & Cs137 \\
\hline C202 & Eu152 \\
\hline $\mathrm{C202}$ & Eu154 \\
\hline $\mathrm{C}_{202}$ & Eu155 \\
\hline C202 & I129 \\
\hline C202 & $\mathrm{Nb93}$ \\
\hline C202 & Ni59 \\
\hline C202 & Ni63 \\
\hline C202 & Np237 \\
\hline $\mathrm{C}_{202}$ & Pa231 \\
\hline $\mathrm{C202}$ & Pu238 \\
\hline C202 & Pu239 \\
\hline $\mathrm{C202}$ & Pu240 \\
\hline$C_{202}$ & Pu241 \\
\hline C202 & Pu242 \\
\hline C20 & $\operatorname{Ra226}$ \\
\hline $\mathrm{mon}$ & $\operatorname{Ra} 228$ \\
\hline
\end{tabular}

May $\frac{B B}{98}$

$1.71 \mathrm{E}+04$

$1.68 \mathrm{E}-02$

$3.79 E-08$

6.65E-13

8.28E-03

4.41E-03

6.16E-06

Ci 3.69E-07

Ci 5.20E-01

Ci 2.34E-02

Ci 3.32E-03

Ci 5.30E-01

Ci 1.71E+04

Ci 2.31E-02

kg 7.10E-00

kg $0.00 \mathrm{E}+01$

$\mathrm{kg} \quad 3.20 \mathrm{E}+01$

kg $8.80 \mathrm{E}-00$

$\mathrm{kg} \quad 8.80 \mathrm{E}+01$

5.90E-01

2.20E-00

$2.73 E+02$

$0.00 E+01$

$1.08 \mathrm{E}+01$

$0.00 E+01$

$0.00 \mathrm{E}+01$

$4.68 \mathrm{E}+02$

$3.53 E+01$

$4.41 \mathrm{E}-00$

$1.46 \mathrm{E}+02$

$2.10 E+02$

$1.15 \mathrm{E}+03$

$6.50 \mathrm{E}+02$

$$
6.73 \mathrm{E}-00
$$

$1.65 \mathrm{E}+01$

$2.02 \mathrm{E}+01$

$0.00 \mathrm{E}+01$

9.10E-00

2.90E-01

$0.00 \mathrm{E}+01$

$1.80 \mathrm{E}-04$

4.79E-00

$1.15 \mathrm{E}-04$

$2.23 E+01$

$1.13 E-03$

5.62E-02

$7.71 \mathrm{E}-03$

4.22E-04

2.05E-04

1.19E-03

4.42E-06

$2.36 \mathrm{E}+01$

3.25E-01

$1.89 \mathrm{E}-01$

$2.12 E+01$

$1.54 \mathrm{E}-05$

$1.84 \mathrm{E}-02$

4.23E-01

4. $15 \mathrm{E}+01$

2.41E-05

4.86E-06

3.35E-01

$1.42 \mathrm{E}+01$

2.33E-00

2.42E+01

1.19E-04

3.68E-05

2.06E-10
$B B$

Sept98

$1.68 \mathrm{E}-02$

3.79E-08

$6.65 \mathrm{E}-13$

8.28E-03

$4.41 \mathrm{E}-03$

8.29E-09

4.96E-10

6.99E-04

3.14E-05

4.46E-06

7.08E-04

$1.71 \mathrm{E}+04$

2.31E-02

$2.68 \mathrm{E}+01$

$0.00 \mathrm{E}+01$

1.22E+02

$3.35 \mathrm{E}+01$

$8.21 \mathrm{E}+02$

2.25E-00

8.38E-00

$1.04 \mathrm{E}+03$

$0.00 \mathrm{E}+01$

6.70E-00

$1.17 \mathrm{E}+01$

$0.00 \mathrm{E}+01$

4.52E +02

$1.34 \mathrm{E}+02$

$1.68 \mathrm{E}+01$

$5.53 \mathrm{E}+02$

NA
$\mathbf{E}+02$

$9.50 \mathrm{E}+02$
$1.10 \mathrm{E}+02$

NA

6.27E+01

$2.02 E+01$

$0.00 \mathrm{E}+01$

$1.68 \mathrm{E}+02$

1.11E-00

$0.00 \mathrm{E}+01$

$1.80 \mathrm{E}-04$

$1.09 \mathrm{E}+01$

2.61E-04

$8.56 \mathrm{E}+01$

1.13E-03

5.62E-02

$1.75 \mathrm{E}-02$

9.61E-04

4.67E-04

1.19E-03

4.42E-06

$9.05 \mathrm{E}+01$

$3.25 \mathrm{E}-01$

1.89E-01

$2.12 E+01$

1.54E-05

1.84E-02

4.23E-01

$4.15 \mathrm{E}+01$

2.41E-05

4.86E- 06

7.63E-01

$3.22 \mathrm{E}+01$

5.30E-00

$5.51 \mathrm{E}+01$

2.71E-04

$3.68 \mathrm{E}-05$

2.06E-10 1\%-ile

$0.00 \mathrm{E}+01$

$0.00 \mathrm{E}+01$

$0.00 \mathrm{E}+01$

$0.00 \mathrm{E}+01$

$0.00 \mathrm{E}+01$

$0.00 \mathrm{E}+01$

$0.00 \mathrm{E}+01$

$0.00 \mathrm{E}+01$

$0.00 \mathrm{E}+01$

$0.00 \mathrm{E}+01$

$0.00 \mathrm{E}+01$

$0.00 \mathrm{E}+01$

$0.00 \mathrm{E}+01$

$0.00 \mathrm{E}+01$

$1.26 \mathrm{E}-07$

3.43E-09

4.58E-07

8.80E-07

5.00E-06

1.19E-07

6.64E-09

7.82E-06

$0.00 \mathrm{E}+01$

6.25E-07

$1.05 \mathrm{E}-09$

$1.60 \mathrm{E}-09$

$7.44 \mathrm{E}-05$

$1.21 \mathrm{E}-06$

$1.28 \mathrm{E}-05$

$1.26 \mathrm{E}-06$

$1.25 \mathrm{E}-06$

2.68E-07

2.07E-06

1.79E-06

6.12E-09

5.24E-06

1.57E-09

2.75E-06

$1.14 \mathrm{E}-07$

$2.09 \mathrm{E}-10$

$0.00 \mathrm{E}+01$

$0.00 \mathrm{E}+01$

$0.00 \mathrm{E}+01$

$0.00 \mathrm{E}+01$

$0.00 \mathrm{E}+01$

$0.00 \mathrm{E}+01$

$0.00 \mathrm{E}+01$

$0.00 \mathrm{E}+01$

$0.00 \mathrm{E}+01$

$1.94 \mathrm{E}-11$

$0.00 E+01$

$4.80 \mathrm{E}-07$

$0.00 \mathrm{E}+01$

$0.00 E+01$

$0.00 E+01$

$0.00 \mathrm{E}+01$

$0.00 \mathrm{E}+01$

$0.00 \mathrm{E}+01$

$0.00 \mathrm{E}+01$

$0.00 \mathrm{E}+01$

$0.00 E+01$

$0.00 \mathrm{E}+01$

$0.00 \mathrm{E}+01$

$0.00 \mathrm{E}+01$

$0.00 \mathrm{E}+01$

$0.00 \mathrm{E}+01$

$0.00 E+01$

$0.00 \mathrm{E}+01$ 10\%-ile

2.33E-02

$0.00 \mathrm{E}+01$

$0.00 \mathrm{E}+01$

$0.00 \mathrm{E}+01$

$0.00 \mathrm{E}+01$

$0.00 \mathrm{E}+01$

$0.00 \mathrm{E}+01$

$0.00 \mathrm{E}+01$

$0.00 \mathrm{E}+01$

$0.00 \mathrm{E}+01$

$0.00 \mathrm{E}+01$

$0.00 \mathrm{E}+01$

$0.00 \mathrm{E}+01$

3.11E-06

3.90E-05

$2.96 \mathrm{E}-07$

7.02E-06

6.74E-06

1.21E-04

$3.86 \mathrm{E}-06$

$3.15 \mathrm{E}-07$

$9.86 \mathrm{E}-05$

$0.00 \mathrm{E}+01$

1.23E-05

4.93E-08

3.65E-07

4.27E-04

1.92E-05

$1.23 \mathrm{E}-04$

$2.54 \mathrm{E}-04$

3.55E-05

2.43E-05

4.82E-05

1.12E-05

2.66E-07

3.54E-05

8.59E-08

3.73E-05

3.43E-05

5.72E-08

$0.00 \mathrm{E}+01$

$0.00 \mathrm{E}+01$

4.61E-10

$1.15 \mathrm{E}-04$

$0.00 \mathrm{E}+01$

$0.00 \mathrm{E}+01$

$3.09 \mathrm{E}-08$

$1.69 \mathrm{E}-09$

8.21E-10

$6.80 \mathrm{E}-09$

2.27E-11

$1.79 \mathrm{E}-04$

$0.00 E+01$

$0.00 \mathrm{E}+01$

$0.00 \mathrm{E}+01$

$0.00 \mathrm{E}+01$

$3.38 \mathrm{E}-08$

$0.00 \mathrm{E}+01$

$0.00 \mathrm{E}+01$

9.65E-11

$0.00 \mathrm{E}+01$

1.34E-06

$5.69 \mathrm{E}-05$

9.33E-06

9.69E-05

4.77E-10

$0.00 E+01$

$0.00 \mathrm{E}+01$

$50 \%$-ile

$3.96 \mathrm{E}+03$

$1.68 \mathrm{E}-02$

3.79E-08

6.65E-13

8.28E-03

4.41E-03

6.16E-06

3.69E-07

$5.20 \mathrm{E}-01$ 


\begin{tabular}{|c|c|c|c|c|c|c|c|c|c|c|c|}
\hline ank & Anal. & Un & $B B$ & Sept98 & $1 \%$-ile & $10 \%$-ile & 50\%-ile & $90 \%$-ile & $99 \%$-ile & Mean & Std Dev \\
\hline 202 & Ru106 & $\mathrm{Ci}$ & $9.42 \mathrm{E}-06$ & $9.42 \mathrm{E}-06$ & $0.00 \mathrm{E}+01$ & $0.00 \mathrm{E}+01$ & $9.42 \mathrm{E}-06$ & $1.06 \mathrm{E}-04$ & $2.97 \mathrm{E}-04$ & $3.65 \mathrm{E}-05$ & $7.14 \mathrm{E}-05$ \\
\hline $\mathrm{C2O2}$ & Sb125 & $\mathrm{Ci}$ & $4.57 \mathrm{E}-03$ & $4.57 \mathrm{E}-03$ & $0.00 \mathrm{E}+01$ & $0.00 \mathrm{E}+01$ & $4.57 \mathrm{E}-03$ & $3.28 \mathrm{E}-01$ & $2.08 \mathrm{E}-00$ & $27 \mathrm{E}-01$ & $97 \mathrm{E}-01$ \\
\hline 202 & Se79 & $\mathrm{Ci}$ & $4.94 \mathrm{E}-03$ & $4.94 E-03$ & $0.00 \mathrm{E}+01$ & $0.00 \mathrm{E} \div 01$ & $4.94 \mathrm{E}-03$ & $4.76 \mathrm{E}-02$ & $1.60 \mathrm{E}-01$ & $79 \mathrm{E}-02$ & $3.43 \mathrm{E}-02$ \\
\hline $\mathrm{C2O2}$ & Sm151 & $\mathrm{Ci}$ & $1.85 E+01$ & $1.85 E+01$ & $0.00 \mathrm{E}+01$ & $0.00 \mathrm{E} \div 01$ & $1.85 \mathrm{E}+01$ & $4.41 E+02$ & $1.84 \mathrm{E}+03$ & $1.59 \mathrm{E}+02$ & $3.69 \mathrm{E}+02$ \\
\hline $\mathrm{C} 202$ & Sr89/90 & $\mathrm{Ci}$ & $1.47 \mathrm{E}+04$ & $1.47 \mathrm{E}+04$ & $0.00 \mathrm{E}+01$ & $3.43 E-03$ & $1.89 \mathrm{E}+03$ & $4.57 \mathrm{E}+04$ & $4.91 E+05$ & $2.78 \mathrm{E}+04$ & $1.37 \mathrm{E}+05$ \\
\hline 202 & Te99 & $\mathrm{Ci}$ & $7.95 \mathrm{E}-03$ & $7.95 \mathrm{E}-03$ & $0.00 \mathrm{E}+01$ & $0.00 \mathrm{E}+01$ & $7.95 E-03$ & $8.05 \mathrm{E}-01$ & $4.51 E-00$ & $2.94 \mathrm{E}-01$ & $z-01$ \\
\hline 202 & Th229 & $\mathrm{Ci}$ & 3.73E-08 & 3.73E-08 & $0.00 \mathrm{E}+01$ & $0 \mathrm{E}+01$ & $3.73 E-08$ & $5.73 \mathrm{E}-07$ & 3E-06 & 2.11E-07 & $E-07$ \\
\hline 202 & Th232 & $\mathrm{Ci}$ & $2.69 \mathrm{E}-13$ & $2.69 \mathrm{E}-13$ & $0.00 \mathrm{E}+01$ & $0.00 \mathrm{E}+01$ & $2.69 \mathrm{E}-13$ & $4.13 E-12$ & -11 & $1.52 \mathrm{E}-12$ & E-12 \\
\hline 202 & Tin126 & $\mathrm{Ci}$ & $7.88 \mathrm{E}-03$ & $7.88 \mathrm{E}-03$ & +01 & $0 E+01$ & $7.88 \mathrm{E}-03$ & -01 & -01 & $6.37 E-02$ & $E-01$ \\
\hline 202 & Tritium & $\mathrm{Ci}$ & $8.19 \mathrm{E}-04$ & $8.19 E-04$ & $0.00 \mathrm{E}+01$ & $E+01$ & $8.19 E-04$ & $4.70 \mathrm{E}-03$ & -02 & $1.74 \mathrm{E}-03$ & $E-03$ \\
\hline 202 & $\mathbf{U} 232$ & $\mathrm{Ci}$ & $2.92 \mathrm{E}-10$ & $2.61 E-08$ & $0.00 \mathrm{E}+01$ & $00 \mathrm{E}+01$ & $2.92 \mathrm{E}-10$ & 4.49E-09 & E-08 & $1.65 \mathrm{E}-09$ & $3.85 \mathrm{E}-09$ \\
\hline 202 & $\mathbf{U} 233$ & $\mathrm{Ci}$ & $7.68 \mathrm{E}-12$ & $6.85 \mathrm{E}-10$ & $0.00 \mathrm{E}+01$ & $00 E+01$ & $7.68 \mathrm{E}-12$ & & -10 & $4.35 \mathrm{E}-11$ & $1 E-10$ \\
\hline 202 & U234 & $\mathrm{Ci}$ & 4.19E-06 & $3.73 \mathrm{E}-04$ & $0.00 \mathrm{E}+01$ & $0 \mathrm{E}+01$ & $4.19 E-06$ & & & E-05 & E-05 \\
\hline 202 & U235 & $\mathrm{Ci}$ & $1.76 \mathrm{E}-07$ & 1.57E-05 & $0.00 \mathrm{E}+01$ & $0.00 \mathrm{E}+01$ & $1.76 \mathrm{E}-07$ & $2.70 \mathrm{E}-06$ & & E-07 & 2E-06 \\
\hline 202 & U236 & $\mathrm{Ci}$ & $9.74 \mathrm{E}-08$ & $8.68 \mathrm{E}-06$ & $0.00 \mathrm{E}+01$ & $\mathrm{E}+01$ & $9.74 \mathrm{E}-08$ & E-06 & & & $\varepsilon-06$ \\
\hline $\mathrm{C202}$ & U238 & $\mathrm{Ci}$ & 4.16E-06 & $3.71 \mathrm{E}-04$ & $E+01$ & $E+01$ & $4.16 \mathrm{E}-06$ & -05 & & -05 & \\
\hline$C 202$ & Y90 & $\mathrm{Ci}$ & $1.47 E+04$ & $1.47 E+04$ & $E+01$ & $E+01$ & $1.47 E+04$ & $E+05$ & 06 & +05 & \\
\hline 202 & Zr93 & C & 2.18E-02 & $2.18 \mathrm{E}-02$ & $0.00 E+01$ & $E-08$ & 2.18E-02 & E-00 & +01 & $E-00$ & $E-00$ \\
\hline $\mathrm{C203}$ & Al & $\mathrm{kg}$ & $4.90 \mathrm{E}+01$ & $9.41 E+01$ & $2.60 \mathrm{E}-07$ & $1.59 \mathrm{E}-01$ & $1.42 E+01$ & $E+02$ & +02 & +02 & +02 \\
\hline $\mathrm{C203}$ & $\mathbf{B i}$ & $\mathrm{kg}$ & $0.00 \mathrm{E}+01$ & $0.00 \mathrm{E}+01$ & 2.41E-08 & 7.37E-03 & $4.07 \mathrm{E}-01$ & $E-00$ & +01 & $E-00$ & $E-00$ \\
\hline 203 & $\mathrm{Ca}$ & $\mathrm{kg}$ & $1.60 E+02$ & $4.27 \mathrm{E}+02$ & $6.19 \mathrm{E}-06$ & 1.17E-00 & $2.54 \mathrm{E}+01$ & $E+02$ & +03 & $E+01$ & +02 \\
\hline 203 & Cl & $\mathrm{kg}$ & $6.14 E+01$ & $1.18 \mathrm{E}+02$ & $1.66 \mathrm{E}-06$ & $4.81 \mathrm{E}-01$ & 5.02E-00 & $E+01$ & +01 & $E-00$ & $E+01$ \\
\hline $\mathrm{C203}$ & $\mathrm{cos}$ & $\mathrm{kg}$ & $6.14 E+02$ & $1.57 E+03$ & 1.7 & 01 & $5.64 E+02$ & -03 & & & +03 \\
\hline 203 & $\mathrm{Cr}$ & $\mathrm{kg}$ & $4.12 E-00$ & $7.91 \mathrm{E}-00$ & 7.0 & & $2.54 \mathrm{E}-00$ & & 02 & +01 & +01 \\
\hline$C 203$ & $\mathbf{F}$ & $\mathrm{kg}$ & $1.54 E+01$ & $2.94 E+01$ & 3.6 & .02 & 3.12E-01 & $E+01$ & +01 & $E-00$ & $E+01$ \\
\hline C203 & $\mathbf{F e}$ & $\mathrm{kg}$ & $1.90 \mathrm{E}+03$ & $3.65 \mathrm{E}+03$ & $4.75 E-05$ & $8.38 \mathrm{E}-00$ & $1.39 \mathrm{E}+02$ & +02 & & & +02 \\
\hline $\mathbf{2 0 3}$ & $\mathbf{H g}$ & $\mathrm{kg}$ & $0.00 \mathrm{E}+01$ & $0.00 \mathrm{E}+01$ & $E+01$ & & $0.00 \mathrm{E}+01$ & $E+01$ & +01 & & $i+01$ \\
\hline $\mathrm{C203}$ & $\mathbf{K}$ & $\mathrm{kg}$ & $1.10 \mathrm{E}+01$ & $2.35 E+01$ & $E-06$ & $E-01$ & 7.43E-00 & $i+01$ & +02 & +01 & $i+01$ \\
\hline $\mathrm{C203}$ & La & kg & $0.00 \mathrm{E}+01$ & $4.12 E+01$ & & & & & & -01 & -01 \\
\hline 203 & $\mathbf{M n}$ & kg & 0.00 & $0.00 \mathrm{E}+01$ & 5. & & -01 & -01 & & -00 & -00 \\
\hline $\mathrm{C203}$ & $\mathrm{Na}$ & kg & $1.92 E+03$ & $1.59 \mathrm{E}+03$ & $2.68 \mathrm{E}-04$ & $i+01$ & $8.16 \mathrm{E}+02$ & +03 & +03 & +03 & $8+03$ \\
\hline $\mathrm{C203}$ & $\mathbf{N i}$ & $\mathrm{kg}$ & $2.46 \mathrm{E}+02$ & $4.71 E+02$ & $3.36 \mathrm{E}-06$ & $E-01$ & $1.33 E+01$ & +02 & & +02 & +02 \\
\hline 03 & NO2 & $\mathrm{kg}$ & $3.09 \mathrm{E}+01$ & $5.88 \mathrm{E}+01$ & 3.8 & & $8.66 \mathrm{E}+01$ & +02 & +03 & & $\varepsilon+02$ \\
\hline 203 & NO3 & kg & $1.02 E+03$ & $1.94 \mathrm{E}+03$ & & $E-00$ & $1.38 \mathrm{E}+02$ & & & & \\
\hline $\mathrm{C203}$ & $\mathbf{P}$ & kg & $1.45 E+03$ & NA & -05 & & 9.39 & +02 & -03 & +02 & +02 \\
\hline $\mathrm{C} 203$ & $\mathbf{P b}$ & kg & $5.72 E+03$ & $3.33 E+03$ & -06 & -01 & +01 & +02 & -02 & +01 & +02 \\
\hline $\mathrm{C203}$ & PO4 & $\mathrm{kg}$ & $4.50 \mathrm{E}+03$ & $1.53 \mathrm{E}+03$ & $6.67 \mathrm{E}-05$ & +01 & $2.60 \mathrm{E}+02$ & -03 & -03 & +02 & +03 \\
\hline $\mathrm{C203}$ & $\mathbf{S}$ & kg & $5.03 E+01$ & $\mathbf{N A}$ & & & 01 & 02 & -02 & +01 & +01 \\
\hline $\mathrm{C} 203$ & Si & kg & $1.15 E+02$ & $2.20 \mathrm{E}+02$ & & & & & & & \\
\hline & SO4 & kg & 02 & +02 & & & 6.6 & -02 & & -02 & -02 \\
\hline C203 & $\mathrm{Sr}$ & kg & $0.00 \mathrm{E}+01$ & $0.00 \mathrm{E}+01$ & -09 & .03 & .02 & -00 & & -00 & $E-00$ \\
\hline C203 & TOC & kg & $6.35 E+01$ & $2.33 E+03$ & -06 & $8-00$ & $2.44 \mathrm{E}+01$ & -02 & & -01 & +02 \\
\hline $\mathrm{C} 203$ & $\mathbf{U}$ & kg & $2.04 E-00$ & $3.91 \mathrm{E}-00$ & -05 & -00 & $4.88 E+02$ & 03 & -03 & +03 & +03 \\
\hline C203 & $\mathbf{Z r}$ & kg & $0.00 E+01$ & $0.00 \mathrm{E}+01$ & .09 & & & & & & \\
\hline C203 & Ac227 & $\mathrm{Ci}$ & & $1.85 \mathrm{E}-04$ & & & & & & & \\
\hline C203 & & $\mathrm{Ci}$ & & 00 & +01 & -01 & -01 & -01 & & -01 & +01 \\
\hline C203 & Ar & $\mathrm{Ci}$ & & 2.39 & -01 & 07 & 04 & -03 & -02 & $E-03$ & E-03 \\
\hline $\mathrm{C203}$ & Ba137 & $\mathrm{Ci}$ & $1.41 \mathrm{E}+02$ & $3.01 \mathrm{E}+02$ & +01. & $=-00$ & $1.41 E+02$ & +03 & 03 & +02 & +03 \\
\hline C203 & C14 & $\mathrm{Ci}$ & $4.24 \mathrm{E}-02$ & 4.24E-02 & $0.00 \mathrm{E}+01$ & $2.00 E-03$ & $4.24 \mathrm{E}-02$ & -01 & & 01 & $=-01$ \\
\hline $\mathrm{C203}$ & Cd113 & $\mathrm{Ci}$ & -02 & 6.65E-02 & ;+01 & & 6.6 & & & -01 & E-01 \\
\hline $\mathrm{C} 203$ & $\mathrm{Cm} 242$ & $\mathrm{Ci}$ & $7.69 \mathrm{E}-03$ & $1.60 \mathrm{E}-02$ & & & & & & & $E-01$ \\
\hline C203 & $\mathrm{Cm} 243$ & $\mathrm{Ci}$ & E-04 & 8.79E-04 & & & & & & & -02 \\
\hline & $\mathrm{Cm} 2$ & $\mathrm{Ci}$ & & & & & & & & & -02 \\
\hline C203 & Co60 & $\mathrm{Ci}$ & 1.9 & $1.90 \mathrm{E}-03$ & 11 & 04 & 7.8 & -01 & -01 & -02 & $E-01$ \\
\hline C203 & Cs134 & $\mathrm{Ci}$ & $4.82 \mathrm{E}-06$ & 4.82E-06 & $E+01$ & E-0? & $4.82 \mathrm{E}-06$ & .05 & -04 & $=-05$ & E-05 \\
\hline C203 & Cs137 & $\mathrm{Ci}$ & $1.49 E+02$ & $3.18 E+02$ & 06 & 00 & $1.16 E+02$ & +03 & 103 & $E+02$ & $E+02$ \\
\hline C203 & Eu152 & $\mathrm{Ci}$ & $3.26 \mathrm{E}-01$ & $3.26 \mathrm{E}-01$ & $E+01$ & & -01 & & & -00 & $E-00$ \\
\hline C203 & Eu154 & $\mathrm{Ci}$ & & 1.98E-01 & & & & & & & $E-00$ \\
\hline $\mathrm{C203}$ & Eu155 & $\mathrm{Cj}$ & & & & & & & +02 & & $E+02$ \\
\hline C203 & 1129 & $\mathrm{Ci}$ & & & & & & & & & $E-04$ \\
\hline C203 & Nb93 & $\mathrm{Ci}$ & $2.28 \mathrm{E}-02$ & $2.28 \mathrm{E}-02$ & +01 & & $2.28 \mathrm{E}-02$ & -00 & -00 & -01 & $6 \mathrm{E}-00$ \\
\hline C203 & $\mathrm{Ni}$ & $\mathrm{C} \mathbf{j}$ & $4.24 E-01$ & $4.24 E-01$ & $0.00 \mathrm{E}+01$ & $\mathrm{E}-02$ & 4.24E-01 & $E-00$ & +01 & $E-00$ & $9 \mathrm{E}-00$ \\
\hline$C 203$ & Ni63 & $\mathrm{Ci}$ & $4.15 E+01$ & $4.15 E+01$ & $0.00 \mathrm{E}+01$ & $1.85 \mathrm{E}-00$ & $4.15 E+01$ & +02 & +03 & +02 & $E+02$ \\
\hline C203 & Np237 & $\mathrm{Ci}$ & & $2.36 \mathrm{E}-04$ & $0.00 \mathrm{E}+01$ & & $2.36 \mathrm{E}-0$ & -03 & & -03 & $E-02$ \\
\hline C203 & Pa231 & $\mathrm{Ci}$ & 9.67E-06 & $9.67 \mathrm{E}-06$ & $0.00 \mathrm{E}+01$ & $0.00 E+01$ & 9.67E-06 & $5.46 \mathrm{E}-05$ & $1.44 E-04$ & E-05 & $2.92 \mathrm{E}-05$ \\
\hline C203 & Pu238 & $\mathrm{Ci}$ & 3.35E-01 & $6.98 \mathrm{E}-01$ & $0.00 \mathrm{E}+01$ & $6.52 \mathrm{E}-04$ & $3.35 E-01$ & $5.02 \mathrm{E}-00$ & $5.32 \mathrm{E}+01$ & $3.83 \mathrm{E}-00$ & $2.86 \mathrm{E}+01$ \\
\hline C203 & Pu239 & $\mathrm{Ci}$ & $4.63 E \div 01$ & $2.96 \mathrm{E}+01$ & $0.00 \mathrm{E}+01$ & 02 & $4.63 E+01$ & -02 & +03 & $5.29 \mathrm{E}+02$ & $6 \mathrm{E}+03$ \\
\hline C203 & Pu240 & $\mathbf{C i}$ & d. in 239 & 4.85E-00 & $0.00 \mathrm{E}+01$ & 9.01E-02 & $4.63 E+01$ & $6.93 E+02$ & $7.35 \mathrm{E} \div 03$ & $5.29 \mathrm{E}+02$ & $3.96 E+03$ \\
\hline
\end{tabular}


Tank Anal. Un

\begin{tabular}{|c|c|}
\hline C203 & Pu241 \\
\hline C203 & Pu242 \\
\hline C203 & Ra226 \\
\hline C203 & Ra228 \\
\hline C203 & Ru106 \\
\hline C203 & Sb125 \\
\hline C203 & Se79 \\
\hline $\mathrm{C}_{203}$ & Sm151 \\
\hline $\mathrm{C203}$ & Sr89/90 \\
\hline C203 & Te99 \\
\hline C203 & Th229 \\
\hline C203 & Th232 \\
\hline C203 & Tin126 \\
\hline C203 & Tritium \\
\hline C203 & U232 \\
\hline C203 & $\mathbf{U} 233$ \\
\hline C203 & U234 \\
\hline C203 & U235 \\
\hline $\mathrm{C203}$ & U236 \\
\hline C203 & U238 \\
\hline C203 & Y90 \\
\hline C203 & Zr93 \\
\hline C204 & Al \\
\hline C204 & $\mathrm{Bi}$ \\
\hline C204 & $\mathrm{Ca}$ \\
\hline C204 & Cl \\
\hline C204 & $\mathrm{CO3}$ \\
\hline C204 & $\mathrm{Cr}$ \\
\hline C204 & $\mathbf{F}$ \\
\hline C204 & $\mathbf{F e}$ \\
\hline C204 & $\mathbf{H g}$ \\
\hline C204 & $\mathbf{K}$ \\
\hline C204 & La \\
\hline C204 & Mn \\
\hline C204 & Na \\
\hline C204 & $\mathrm{Ni}$ \\
\hline C204 & No2 \\
\hline C204 & NO3 \\
\hline C204 & $\mathbf{P}$ \\
\hline C204 & $\mathbf{P b}$ \\
\hline C204 & P04 \\
\hline C204 & $\mathbf{S}$ \\
\hline C204 & Si \\
\hline C204 & SO4 \\
\hline C204 & $\mathrm{Sr}$ \\
\hline $\begin{array}{l}\text { C204 } \\
\text { C204 }\end{array}$ & TOC \\
\hline $\begin{array}{l}\text { C204 } \\
\text { C204 }\end{array}$ & $\mathrm{Zr}$ \\
\hline C204 & Ac227 \\
\hline C204 & Am241 \\
\hline C204 & Am243 \\
\hline C204 & Ba137 \\
\hline C204 & C14 \\
\hline C204 & Cd113 \\
\hline C204 & $\mathrm{Cm} 242$ \\
\hline C204 & $\mathrm{Cm} 243$ \\
\hline C204 & Cm244 \\
\hline C204 & Co60 \\
\hline C204 & Cs134 \\
\hline C204 & Cs137 \\
\hline C204 & Eu152 \\
\hline C204 & Eu154 \\
\hline C204 & Eu155 \\
\hline C204 & 1129 \\
\hline C204 & Nb93 \\
\hline C204 & Ni59 \\
\hline C204 & Ni63 \\
\hline C204 & Np237 \\
\hline
\end{tabular}

May $2 \longdiv { 4 2 \mathrm { E } + 0 1 }$ 1.19E-04 $3.88 \mathrm{E}-05$ 2.19E-10 $9.40 \mathrm{E}-06$ 4.95E-03 6.01E-03 $2.26 \mathrm{E}+01$ $9.32 \mathrm{E}+03$ $4.33 \mathrm{E}-02$ 3.97E-08 1.86E-12 9.47E- 03 1.52E-02 2.47E-05 $1.48 \mathrm{E}-06$ 2.08E-00 9.36E-02 $1.33 \mathrm{E}-02$ 2.11E-00 $9.32 \mathrm{E}+03$ 2.69E-02 2.12E +01 $0.00 \mathrm{E}+01$ $9.60 \mathrm{E}+01$ $2.64 \mathrm{E}+01$ $2.64 \mathrm{E}+02$

1.77E-00

6.59E-00

8.17E+02 $0.00 \mathrm{E}+01$ $1.09 \mathrm{E}+01$ $0.00 E+01$ $0.00 \mathrm{E}+01$ $1.40 \mathrm{E}+03$ $1.06 \mathrm{E}+02$

$1.32 \mathrm{E}+01$

$4.37 \mathrm{E}+02$ $6.30 \mathrm{E}+02$

$3.44 E+03$

$1.95 \mathrm{E}+03$

$2.86 \mathrm{E}+01$

$4.94 \mathrm{E}+01$

$8.58 \mathrm{E}+01$

$0.00 E+01$

$2.73 E+01$

8.75E-01

$0.00 \mathrm{E}+01$

1.82E-04

4.78E-00

1.15E-04

$6.70 \mathrm{E}+01$

2.18E-02

6.13E-02

$7.70 \mathrm{E}-03$

4.22E-04

2.05E-04

1.54E-03

4.62E-06

$7.10 \mathrm{E}+01$

3.25E-01

$1.93 \mathrm{E}-01$

$2.13 E+01$

4.83E-05

2.06E-02

4.23E-01

$4.15 E+01$

1.34E-04
Sept98

$5.04 \mathrm{E}+01$

2.48E-04

3.88E-05

2.19E-10

9.40E-06

4.95E-03

6.01E-03

$2.26 \mathrm{E}+01$

$9.32 \mathrm{E}+03$

4.33E-02

3.97E-08

$1.86 \mathrm{E}-12$

9.47E-03

1.52E-02

1.53E-08

9.15E-10

$1.29 \mathrm{E}-03$

5.80E-05

8.23E-06

$1.31 \mathrm{E}-03$

$9.32 E+03$

2.69E-02

$3.96 \mathrm{E}+01$

$0.00 \mathrm{E}+01$

$1.80 \mathrm{E}+02$

$4.95 \mathrm{E}+01$

$9.66 \mathrm{E}+02$

3.33E-00

$1.24 \mathrm{E}+01$

$1.54 \mathrm{E}+03$

$0.00 \mathrm{E}+01$

9.90E-00

$1.73 \mathrm{E}+01$

$0.00 \mathrm{E}+01$

$6.68 \mathrm{E}+02$

$1.98 \mathrm{E}+02$

$2.47 \mathrm{E}+01$

8.17E+02

NA

$1.40 \mathrm{E}+03$

$1.53 \mathrm{E}+03$

NA

$9.26 \mathrm{E}+01$

$8.58 \mathrm{E}+01$

$0.00 \mathrm{E}+01$

$2.32 \mathrm{E}+03$

$1.64 \mathrm{E}-00$

$0.00 \mathrm{E}+01$

$1.82 \mathrm{E}-04$

1.31 E-01

3.13E-06

$1.27 \mathrm{E}+02$

2.18E-02

6.13E-02

2.10E-04

1.15E-05

5.60E-06

$1.54 \mathrm{E}-03$

4.62E-06

$1.34 \mathrm{E}+02$

3.25E-01

1.93E-01

$2.13 E+01$

4.83E-05

2.06E-02

4.23E-01

$4.15 E+01$

1.30E-04 1\%-ile

$0.00 \mathrm{E}+01$

$0.00 \mathrm{E}+01$

$0.00 \mathrm{E}+01$

$0.00 \mathrm{E}+01$

$0.00 \mathrm{E}+01$

$0.00 \mathrm{E}+01$

$0.00 \mathrm{E}+01$

$0.00 \mathrm{E}+01$

$0.00 \mathrm{E}+01$

$0.00 \mathrm{E}+01$

$0.00 \mathrm{E}+01$

$0.00 \mathrm{E}+01$

$0.00 \mathrm{E}+01$

$0.00 \mathrm{E}+01$

$0.00 \mathrm{E}+01$

$0.00 \mathrm{E}+01$

$0.00 \mathrm{E}+01$

$0.00 \mathrm{E}+01$

$0.00 \mathrm{E}+01$

$0.00 \mathrm{E}+01$

$0.00 \mathrm{E}+01$

$0.00 \mathrm{E}+01$

3.82E-07

$7.39 \mathrm{E}-08$

6.44E-06

$2.48 \mathrm{E}-06$

$1.84 \mathrm{E}-04$

4.61E-07

$5.56 \mathrm{E}-08$

5.03E-05

$0.00 \mathrm{E}+01$

2.41E-06

6.93E-09

1.47E-07

2.12E-04

3.36E-06

2.15E-05

2.81E-05

2.92E-05

3.07E-06

$6.80 \mathrm{E}-05$

$5.74 \mathrm{E}-06$

$5.68 \mathrm{E}-08$

2.46E-05

$1.05 \mathrm{E}-08$

$7.57 \mathrm{E}-06$

$7.55 \mathrm{E}-05$

2.05E-09

$0.00 \mathrm{E}+01$

$0.00 \mathrm{E}+01$

$0.00 \mathrm{E}+01$

$0.00 \mathrm{E}+01$

$0.00 \mathrm{E}+01$

$0.00 \mathrm{E}+01$

$0.00 \mathrm{E}+01$

$0.00 E+01$

$0.00 E+01$

$0.00 \mathrm{E}+01$

$0.00 E+01$

4.38E-05

$0.00 \mathrm{E}+01$

$0.00 \mathrm{E}+01$

$0.00 \mathrm{E}+01$

$0.00 \mathrm{E}+01$

$0.00 \mathrm{E}+01$

$0.00 \mathrm{E}+01$

$0.00 \mathrm{E}+01$

$0.00 \mathrm{E}+01$ 10\%-ile

4.71E-02

2.31E-07

1.50E-06

8.45E-12

4.28E-07

8.32E-05

4.13E-04

$0.00 \mathrm{E}+01$

$6.05 \mathrm{E}-00$

7.31E-04

$0.00 \mathrm{E}+01$

$0.00 \mathrm{E}+01$

$1.36 \mathrm{E}-04$

$1.54 \mathrm{E}-03$

$0.00 E+01$

$0.00 \mathrm{E}+01$

$0.00 \mathrm{E}+01$

$0.00 \mathrm{E}+01$

$0.00 \mathrm{E}+01$

$0.00 \mathrm{E}+01$

$7.86 \mathrm{E}-00$

5.21E-04

$1.41 \mathrm{E}-01$

7.63E-03

$8.86 \mathrm{E}-01$

4.67E-01

$2.88 \mathrm{E}+01$

$1.87 \mathrm{E}-01$

$1.36 \mathrm{E}-02$

$7.90 \mathrm{E}-00$

$0.00 \mathrm{E}+01$

5.41E-01

$1.31 \mathrm{E}-03$

7.86E-03

$8.13 E+01$

$7.82 \mathrm{E}-01$

$7.38 \mathrm{E}-00$

4.65E-00

3.20E-00

2.66E-01

8.07E-00

$1.42 \mathrm{E}-00$

8.18E-03

$4.98 \mathrm{E}-00$

2.27E-03

$1.25 \mathrm{E}-00$

1.33E-00

$5.98 \mathrm{E}-04$

$0.00 \mathrm{E}+01$

1.08E-07

1.13E-07

8.11E-01

1.38E-03

2.44E-04

$7.58 \mathrm{E}-06$

4.16E-07

2.02E-07

9.71E-05

$5.59 \mathrm{E}-08$

2.01E-00

$0.00 \mathrm{E}+01$

$0.00 \mathrm{E}+01$

$0.00 \mathrm{E}+01$

$2.65 \mathrm{E}-06$

2.50E-04

1.67E-02

$1.64 \mathrm{E}-00$

1.32E-07

\section{0\%-ile}

\subsection{E+01}

1.19E-04

3.88E-05

2.19E-10

$9.40 \mathrm{E}-06$

4.95E-03

6.01E-03 


\begin{tabular}{|c|c|c|c|c|c|c|c|c|c|c|c|}
\hline ank & Anal. & Un & May98 & Sept98 & $1 \%$-ile & $10 \%$-ile & $50 \%$-ile & $90 \%$-ile & $99 \%$-ile & Mean & Std Dev \\
\hline 204 & 231 & $\mathrm{Ci}$ & $7.27 \mathrm{E}-06$ & $7.27 \mathrm{E}-06$ & $0.00 \mathrm{E}+01$ & $E+01$ & 7.27E-06 & $4.43 \mathrm{E}-05$ & $1.25 \mathrm{E}-04$ & E-05 & (2) \\
\hline & 1238 & $\mathrm{Ci}$ & $3.35 E-01$ & $9.16 \mathrm{E}-03$ & $0.00 \mathrm{E}+01$ & E-04 & & $5.99 \mathrm{E}-00$ & $6.13 \mathrm{E}+01$ & & $1.36 \mathrm{E}+01$ \\
\hline 204 & Pu239 & $\mathbf{C i}$ & $5.90 \mathrm{E}-01$ & 3.87E-01 & $0.00 \mathrm{E}+01$ & $31 E-04$ & $5.90 \mathrm{E}-01$ & $1.05 \mathrm{E}+01$ & $1.08 \mathrm{E}+02$ & $5.96 \mathrm{E}-00$ & $2.40 \mathrm{E}+01$ \\
\hline 204 & Pu240 & $\mathrm{Ci} \mathrm{I}$ & Incl. in 239 & $6.36 \mathrm{E}-02$ & $0.00 \mathrm{E}+01$ & $5.81 \mathrm{E}-04$ & $5.90 \mathrm{E}-01$ & $1.05 \mathrm{E}+01$ & $1.08 \mathrm{E}+02$ & $5.96 \mathrm{E}-00$ & $2.40 \mathrm{E}+01$ \\
\hline 204 & Pu241 & $\mathrm{Ci}$ & $2.42 \mathrm{E}+01$ & $6.61 \mathrm{E}-01$ & $0.00 \mathrm{E}+01$ & $2.38 \mathrm{E}-02$ & $2.42 \mathrm{E}+01$ & $4.33 \mathrm{E}+02$ & $4.43 E+03$ & $2.44 \mathrm{E}+02$ & $9.83 \mathrm{E}+02$ \\
\hline 204 & Pu242 & $\mathrm{Ci}$ & .19E-04 & $3.25 E-06$ & $0.00 \mathrm{E}+01$ & $1.17 \mathrm{E}-07$ & $119 \mathrm{~F}-\mathrm{n} 4$ & 2.13E-03 & $2.18 \mathrm{E}-02$ & $1.20 \mathrm{E}-03$ & $4.84 E-03$ \\
\hline 204 & $\mathbf{R a 2 2 6}$ & $\mathrm{Ci}$ & $3.78 \mathrm{E}-05$ & $3.78 \mathrm{E}-05$ & $0.00 \mathrm{E}+01$ & & 3.78 & & $5.87 \mathrm{E}-03$ & & $4 E-03$ \\
\hline & Ra228 & $\mathbf{C i}$ & 2.12E-10 & $2.12 \mathrm{E}-10$ & $0.00 \mathrm{E}+01$ & $5.83 \mathrm{E}-12$ & $2.12 \mathrm{E}-10$ & $1.08 \mathrm{E}-08$ & $3.29 \mathrm{E}-08$ & E-09 & $7.54 \mathrm{E}-09$ \\
\hline & Ru106 & $\mathbf{C i}$ & & $9.41 E-06$ & to1 & & & $4.60 \mathrm{E}-05$ & .04 & & $5 \mathrm{E}-05$ \\
\hline 04 & 125 & $\mathrm{Ci}$ & $4.76 \mathrm{E}-03$ & $4.76 \mathrm{E}-03$ & $0.00 \mathrm{E}+01$ & $8.89 \mathrm{E}-05$ & & $1.47 \mathrm{E}-01$ & $6.13 \mathrm{E}-01$ & $5.14 \mathrm{E}-02$ & $1.37 \mathrm{E}-01$ \\
\hline 204 & Se79 & $\mathbf{C i}$ & $5.48 \mathrm{E}-03$ & $5.48 \mathrm{E}-03$ & $0.00 \mathrm{E}+01$ & $3.67 \mathrm{E}-04$ & -03 & $2.63 \mathrm{E}-02$ & $1.17 \mathrm{E}-01$ & $1.15 \mathrm{E}-02$ & $1.98 \mathrm{E}-02$ \\
\hline 204 & m151 & $\mathbf{C i}$ & $2.05 \mathrm{E}+01$ & $2.05 \mathrm{E}+01$ & $0.00 E+01$ & $0.00 \mathrm{E}+01$ & $2.05 E+01$ & $1.63 E+02$ & $5.62 \mathrm{E}+02$ & $6.43 \mathrm{E}+01$ & $1.18 E+02$ \\
\hline 204 & r89/90 & $\mathrm{Ci}$ & $3.76 E+02$ & $3.76 \mathrm{E}+02$ & $0.00 \mathrm{E}+01$ & $7.62 \mathrm{E}-0.0$ & +03 & $7.41 E+04$ & $6.62 E+05$ & $4.75 \mathrm{E}+04$ & $2.02 \mathrm{E}+05$ \\
\hline 204 & Te99 & $\mathbf{C i}$ & $2.56 \mathrm{E}-03$ & $2.56 \mathrm{E}-02$ & $0.00 \mathrm{E}+01$ & $5.19 \mathrm{E}-05$ & $2.56 \mathrm{E}-03$ & $9.60 \mathrm{E}-02$ & 3.35E-01 & $E-02$ & $8.42 \mathrm{E}-02$ \\
\hline 204 & Th229 & $\mathrm{Ci}$ & $3.85 \mathrm{E}-08$ & 3.85E-08 & $0.00 \mathrm{E}+01$ & $0.00 E+01$ & 3.85E-08 & $2.35 \mathrm{E}-07$ & $6.61 \mathrm{E}-07$ & E-08 & $1.65 \mathrm{E}-07$ \\
\hline $\mathrm{C204}$ & Th232 & $\mathbf{C i}$ & $1.06 \mathrm{E}-12$ & $1.06 \mathrm{E}-12$ & 0. & -01 & & $6.46 \mathrm{E}-12$ & $1.82 \mathrm{E}-11$ & $E-12$ & $35 \mathrm{E}-12$ \\
\hline 204 & D126 & Ci & $8.68 \mathrm{E}-03$ & $8.68 \mathrm{E}-03$ & $0.00 \mathrm{E}+01$ & -05 & 8.0 & $5.92 \mathrm{E}-02$ & $\mathrm{E}-01$ & -02 & $7.53 \mathrm{E}-02$ \\
\hline 204 & $\mathrm{um}$ & $\mathbf{C i}$ & $8.00 \mathrm{E}-03$ & $8.00 \mathrm{E}-03$ & $0.00 \mathrm{E}+01$ & $8-04$ & -03 & $2.27 \mathrm{E}-02$ & $4.00 \mathrm{E}-02$ & -02 & $9.02 \mathrm{E}-03$ \\
\hline C204 & U232 & $\mathbf{C i}$ & $1.23 \mathrm{E}-05$ & $6.41 \mathrm{E}-09$ & $0.00 \mathrm{E}+01$ & +01 & -05 & $7.50 \mathrm{E}-05$ & $2.11 \mathrm{E}-04$ & $2.95 \mathrm{E}-05$ & $5.28 \mathrm{E}-05$ \\
\hline C20.4 & 33 & $\mathrm{Ci}$ & $7.38 \mathrm{E}-07$ & $3.84 \mathrm{E}-10$ & $0.00 \mathrm{E}+01$ & & & E-06 & $1.27 \mathrm{E}-05$ & & $3.17 \mathrm{E}-06$ \\
\hline 204 & U234 & $\mathrm{Ci}$ & $1.04 \mathrm{E}-00$ & 5.41E-04 & $0.00 \mathrm{E}+01$ & +01 & $8-00$ & & $1.79 \mathrm{E}+01$ & & E-00 \\
\hline C204 & U235 & $\mathrm{Ci}$ & $4.68 \mathrm{E}-02$ & 2.43E-05 & $0.00 \mathrm{E}+01$ & +01 & & & 8.04E-01 & & 2.01E-01 \\
\hline C204 & U236 & $\mathrm{Ci}$ & $6.64 \mathrm{E}-03$ & $3.45 \mathrm{E}-06$ & & 01 & & & 1.14E-01 & -02 & \\
\hline C204 & U238 & $\mathrm{Ci}$ & $1.05 E-00$ & $5.48 \mathrm{E}-04$ & 01 & +01 & -00 & -00 & 1.8 & -00 & $=-00$ \\
\hline C204 & Y90 & $\mathrm{Ci}$ & $3.76 \mathrm{E}+02$ & $3.76 \mathrm{E}+02$ & $0.00 \mathrm{E}+01$ & -01 & $3.76 \mathrm{E}+02$ & $4.29 E+03$ & $3.84 \mathrm{E}+04$ & +03 & $1.17 \mathrm{E}+04$ \\
\hline C204 & Zr93 & $\mathbf{C i}$ & $2.43 \mathrm{E}-02$ & 2.43E-02 & $0.00 \mathrm{E}+01$ & -04 & -02 & $1.89 \mathrm{E}-00$ & $1.11 E+01$ & $E-01$ & $2.09 \mathrm{E}-00$ \\
\hline & Al & kg & $2.10 \mathrm{E}+05$ & $2.10 \mathrm{E}+05$ & 1.0 & & & & +05 & +05 & $8.30 E+04$ \\
\hline 01 & 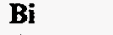 & kg & $1.25 E+02$ & $1.23 E+02$ & & & & $E \div 04$ & $E+04$ & & $E+04$ \\
\hline & $\mathrm{Ca}$ & kg & $8.55 E+02$ & $8.55 E+02$ & & & & & +04 & & $E+03$ \\
\hline 101 & 1 & kg & $9.31 E+03$ & +03 & 02 & 03 & 03 & 04 & +04 & 03 & $E+03$ \\
\hline$\$ 101$ & $\mathrm{CO} 3$ & kg & $4.88 E+04$ & $4.88 E+04$ & +03 & r04 & +04 & $5.39 \mathrm{E}+04$ & $7.47 \mathrm{E}+04$ & +04 & $E+04$ \\
\hline$\$ 101$ & $\mathbf{C r}$ & kg & $1.88 E+04$ & $1.88 \mathrm{E}+04$ & $:+02$ & -03 & 03 & $1.25 \mathrm{E}+04$ & $1.94 \mathrm{E}+04$ & -03 & 4.29E+03 \\
\hline & $\mathbf{F}$ & kg & $6.01 \mathrm{E}+02$ & $6.01 E+02$ & & & & $E+04$ & $8+04$ & & $E+04$ \\
\hline & $\mathbf{F e}$ & kg & $3.53 \mathrm{E}+03$ & $3.53 E+03$ & & & & & +04 & & $E+04$ \\
\hline 01 & $\mathbf{H g}$ & kg & 1 & 01 & 00 & & & 02 & +03 & -02 & +02 \\
\hline S101 & $\mathbf{K}$ & $\mathrm{kg}$ & $2.51 E+03$ & +03 & +02 & -02 & 2.0 & +03 & $E+03$ & +03 & $E+03$ \\
\hline S101 & La & $\mathrm{kg}$ & $3.20 \mathrm{E}-00$ & $3.75 E-00$ & $E-00$ & 1.9 & 2.71 & $4.90 \mathrm{E}+01$ & $1.72 \mathrm{E}+02$ & +01 & $E+01$ \\
\hline 1 & Mn & $\mathrm{kg}$ & $3.99 \mathrm{E}+03$ & $3.99 E+03$ & +01 & +02 & & 03 & $E+04$ & 03 & $E+03$ \\
\hline & $\mathbf{N a}$ & $\mathrm{kg}$ & $3.96 E+05$ & $3.96 E+05$ & & & & & 105 & & +04 \\
\hline & $\mathbf{N i}$ & $\mathrm{kg}$ & 22 & 02 & & & & & & & $E+03$ \\
\hline 1 & NO2 & $\mathrm{kg}$ & $1.32 E+05$ & +05 & & & & 05 & 05 & -04 & +04 \\
\hline 5101 & NO3 & $\mathrm{kg}$ & $4.28 \mathrm{E}+05$ & $4.28 E+05$ & +04 & 04 & 2.8 & $E+05$ & $7.53 E+05$ & -05 & $E+05$ \\
\hline S101 & $\mathbf{P}$ & kg & $9.60 \mathrm{E}+03$ & NA & $3.15 \mathrm{E}+02$ & -03 & 03 & $E+04$ & $7.97 \mathrm{E}+04$ & +04 & $E+04$ \\
\hline $\mathbf{S 1 0 1}$ & $\mathbf{P b}$ & kg & $1.35 \mathrm{E}+02$ & $1.33 E+02$ & $E-00$ & t01 & & & +03 & -02 & $E+02$ \\
\hline & PO & $\mathrm{kg}$ & $2.97 \mathrm{E}+04$ & $9.70 E+03$ & & & & & +05 & -04 & $E+04$ \\
\hline & $\mathbf{S}$ & $\mathrm{kg}$ & & NA & & & & & & & \\
\hline 1 & & kg & $3.50 \mathrm{E}+03$ & $3.50 \mathrm{E}+03$ & -01 & & & -04 & 04 & -03 & 104 \\
\hline 1 & SO4 & kg & $5.73 E+04$ & $1.91 \mathrm{E}+04$ & +02 & +03 & 4.8 & $1.79 E+04$ & $2.13 E+05$ & +04 & $E+04$ \\
\hline S101 & $\mathrm{Sr}$ & $\mathrm{kg}$ & $7.57 \mathrm{E}+02$ & $7.57 \mathrm{E}+02$ & E-01 & 1.3 & 72 & $1.71 E+03$ & $2.04 \mathrm{E}+04$ & +03 & $3.40 \mathrm{E}+03$ \\
\hline S101 & 0 & $\mathrm{~kg}$ & $5.16 E+03$ & $5.16 E+03$ & $: 02$ & +03 & 7.6 & $E+04$ & $E+04$ & -03 & $E+03$ \\
\hline S1 & - & $\mathrm{kg}$ & $1.21 E+04$ & $1.21 E+04$ & +02 & & 04 & & & & +04 \\
\hline S1 & $\mathbf{Z r}$ & $k$ & +01 & +01 & & & & & & -02 & +03 \\
\hline & & $\mathrm{Ci}$ & & & & & & & & -03 & \\
\hline S101 & Am241 & $\mathrm{Ci}$ & +01 & 1.46 & & & & 102 & 03 & +02 & $E+02$ \\
\hline S1 & Am243 & $\mathbf{C i}$ & $2.85 \mathrm{E}-03$ & $4.78 \mathrm{E}-03$ & & 04 & & $5.66 \mathrm{E}-03$ & 03 & -03 & $E-03$ \\
\hline S101 & Ba13 & $\mathrm{Ci}$ & $2.75 E+05$ & $2.75 E+05$ & $E+01$ & +04 & & 4.8 & $E+05$ & -05 & $E+05$ \\
\hline $\mathbf{S 1 0 1}$ & C14 & $\mathbf{C} \mathbf{i}$ & $3.18 E+01$ & $3.18 E+01$ & $0.00 \mathrm{E}+01$ & 01 & 3.1 & $E+01$ & $9.59 \mathrm{E}+01$ & $E+01$ & $2.35 \mathrm{E}+01$ \\
\hline $\mathbf{S 1 0 1}$ & Cd11 & $\mathbf{C i}$ & $8.66 \mathrm{E}+0 \mathrm{I}$ & $8.66 E+01$ & 00 & -01 & 8.6 & 8. & -03 & $\div 02$ & $E+02$ \\
\hline \$101 & & Ci & & & & & & & & & \\
\hline & & $\mathrm{Ci}$ & & & & & & & -02 & -02 & $E-03$ \\
\hline \$101 & Cm244 & $\mathbf{C i}$ & $1.07 E-01$ & $1.80 \mathrm{E}-01$ & & & & 2.13E-01 & 01 & -01 & $E-02$ \\
\hline & Co60 & $\mathrm{Ci}$ & $4.93 E+02$ & $3.38 E+01$ & $E+01$ & +01 & 2.85 & $4.14 \mathrm{E}+01$ & $1.61 E+02$ & $E+01$ & $2.53 E+01$ \\
\hline S101 & Cs134 & $\mathrm{Ci}$ & $2.69 E-00$ & 2.69E-00 & $0.00 \mathrm{E}+01$ & & $269 E_{-00}$ & & & $E-00$ & $1.53 \mathrm{E}-00$ \\
\hline S101 & Cs137 & $\mathrm{Ci}$ & $2.91 E+05$ & $2.91 \mathrm{E}+05$ & $3.70 \mathrm{E}+04$ & $1.29 E+05$ & $2.70 \mathrm{E}+05$ & $4.29 E+05$ & $E+05$ & $E+05$ & $18 E+05$ \\
\hline \$101 & Eu152 & $\mathrm{Ci}$ & $8.50 \mathrm{E}-00$ & $8.50 \mathrm{E}-00$ & $0.00 \mathrm{E}+01$ & $4.07 \mathrm{E}-00$ & $8.50 \mathrm{E}-00$ & $2.13 E+01$ & $9.34 \mathrm{E}+01$ & $1.33 \mathrm{E}+01$ & $1.79 \mathrm{E}+01$ \\
\hline S101 & Eul & $\mathrm{Ci}$ & $5.75 \mathrm{E}+02$ & 5.751 & itol & +02 & & $1.44 \mathrm{E}+03$ & $6.32 E+03$ & $E+02$ & $E+03$ \\
\hline S101 & Eu155 & $\mathrm{Ci}$ & $4.48 E+02$ & $4.48 E+02$ & $0.00 \mathrm{E}+01$ & $2.15 E+02$ & $4.48 E+02$ & $1.12 E+03$ & $4.92 E+03$ & $7.00 E+02$ & $9.46 \mathrm{E}+02$ \\
\hline W & 1129 & $\mathrm{Ci}$ & $4.40 \mathrm{E}-01$ & $4.40 \mathrm{E}-01$ & $0.00 \mathrm{E}+01$ & $9.35 \mathrm{E}-02$ & $4.40 \mathrm{E}-01$ & $8.86 \mathrm{E}-01$ & $1.17 \mathrm{E}-00$ & $4.76 \mathrm{E}-01$ & $3.07 \mathrm{E}-01$ \\
\hline
\end{tabular}


Tank Anal. Un

\begin{tabular}{|c|c|}
\hline S101 & Nb93 \\
\hline S101 & Ni59 \\
\hline S101 & Ni63 \\
\hline S101 & Np237 \\
\hline S101 & Pa231 \\
\hline S101 & Pu238 \\
\hline S101 & Pu239 \\
\hline S101 & Pu240 \\
\hline S101 & Pu241 \\
\hline S101 & Pu242 \\
\hline S101 & $\operatorname{Ra226}$ \\
\hline S101 & Ra228 \\
\hline S101 & Ru106 \\
\hline S101 & Sb125 \\
\hline S101 & Se79 \\
\hline S101 & Sm151 \\
\hline S101 & Sr89/90 \\
\hline S101 & Tc99 \\
\hline S101 & Th229 \\
\hline S101 & Th232 \\
\hline S101 & Tin126 \\
\hline S101 & Tritium \\
\hline S101 & U232 \\
\hline S101 & U233 \\
\hline S101 & U234 \\
\hline S101 & $\mathbf{U} 235$ \\
\hline S101 & U236 \\
\hline S101 & U238 \\
\hline S101 & Y90 \\
\hline S101 & $\mathrm{Zr93}$ \\
\hline S102 & Al \\
\hline S102 & $\mathbf{B i}$ \\
\hline S102 & $\mathrm{Ca}$ \\
\hline S102 & Cl \\
\hline S102 & $\mathrm{CO3}$ \\
\hline S102 & $\mathrm{Cr}$ \\
\hline S102 & $\mathbf{F}$ \\
\hline S102 & $\mathrm{Fe}$ \\
\hline S102 & Hg \\
\hline $\mathbf{S 1 0 2}$ & $\mathbf{K}$ \\
\hline $\mathrm{S} 102$ & La \\
\hline $\mathbf{S 1 0 2}$ & Mn \\
\hline S102 & $\mathbf{N a}$ \\
\hline S102 & $\mathbf{N i}$ \\
\hline S102 & NO2 \\
\hline S102 & $\mathrm{NO3}$ \\
\hline S102 & $\mathbf{P}$ \\
\hline S102 & $\mathbf{P b}$ \\
\hline S102 & $\mathrm{PO} 4$ \\
\hline S102 & $\mathbf{s}$ \\
\hline S102 & Si \\
\hline S102 & SO4 \\
\hline S102 & \\
\hline $\mathbf{S 1 0 2}$ & TOC \\
\hline S102 & $\mathbf{U}$ \\
\hline S102 & $\mathbf{Z r}$ \\
\hline S102 & Ac227 \\
\hline S102 & Am241 \\
\hline S102 & Am243 \\
\hline S102 & Ba137 \\
\hline $\mathbf{S 1 0 2}$ & C14 \\
\hline $\mathbf{S 1 0 2}$ & Cd113 \\
\hline S102 & Cm242 \\
\hline $\mathbf{S 1 0 2}$ & $\mathrm{Cm} 243$ \\
\hline S102 & Cm244 \\
\hline \$102 & Co60 \\
\hline $\mathbf{S 1 0 2}$ & Cs134 \\
\hline$\$ 102$ & Cs137 \\
\hline
\end{tabular}

May 98

\section{$1.92 \mathrm{E}+01$}

- 9.22E-00

$8.77 \mathrm{E}+02$

8.73E-01

7.03E-03

6.94E-00

$4.04 \mathrm{E}+02$

$5.90 \mathrm{E}+01$

$4.05 \mathrm{E}+02$

$1.90 \mathrm{E}-03$

$6.48 \mathrm{E}-04$

1.08E-01

$6.08 \mathrm{E}-03$

$1.42 \mathrm{E}+02$

5.18E-00

$1.84 \mathrm{E}+04$

$5.25 \mathrm{E}+05$

$2.29 \mathrm{E}+02$

2.55E-03

6.97E-03

7.89E-00

$2.73 E+02$

$5.59 \mathrm{E}-01$

2.14E-00

2.72E-00

1.14E-01

7.38E-02

2.73E-00

$5.25 E+05$

$2.50 \mathrm{E}+01$

4.64E+04

2.37E+02

$1.74 E+03$

$1.20 \mathrm{E}+04$

$8.90 \mathrm{E}+04$

$9.10 \mathrm{E}+03$

9.03E +03

$3.13 E+03$

2.71E-00

$2.90 \mathrm{E}+03$

$8.60 \mathrm{E}-00$

$1.04 \mathrm{E}+03$

$6.48 \mathrm{E}+05$

$9.34 \mathrm{E}+01$

$1.14 E+05$

$1.03 \mathrm{E}+06$

$1.48 \mathrm{E}+05$

$2.82 \mathrm{E}+02$

$4.60 \mathrm{E}+05$

$3.05 \mathrm{E}+04$

$1.82 \mathrm{E}+03$

$9.15 \mathrm{E}+04$

$2.44 \mathrm{E}+01$

$1.18 \mathrm{E}+04$

$3.29 \mathrm{E}+03$

$6.21 \mathrm{E}+01$

9.85E-04

$5.74 \mathrm{E}+01$

$1.97 \mathrm{E}-03$

$4.18 \mathrm{E}+05$

$3.42 \mathrm{E}+01$

$8.74 \mathrm{E}+01$

$1.47 \mathrm{E}-01$

1.34E-02

1.33E-01

$3.76 \mathrm{E}+01$

2.48E-00

$4.43 E+05$ $\frac{\text { Sept98 }}{1.92 \mathrm{E}+01}$

9.22E-00

$8.77 \mathrm{E}+02$

8.73E-01

7.03E-03

$1.17 \mathrm{E}+01$

$6.78 \mathrm{E}+02$

$.91 \mathrm{E}+01$

$6.80 \mathrm{E}+02$

3.19E-03

6.48E-04

1.08E-01

6.08E-03

$1.42 \mathrm{E}+02$

$5.18 \mathrm{E}-00$

$1.84 \mathrm{E}+04$

$5.25 \mathrm{E}+05$

$2.29 \mathrm{E}+02$

2.55E-03

6.97E-03

7.89E-00

$2.73 \mathrm{E}+02$

8.81E-01

3.38E-00

4.29E-00

1.79E-01

1.16E-01

4.04E-00

$5.25 \mathrm{E}+05$

2.50E+01

$4.64 \mathrm{E}+04$

$2.37 \mathrm{E}+02$

$1.74 \mathrm{E}+03$

$1.20 \mathrm{E}+04$

$8.90 \mathrm{E}+04$

$9.10 \mathrm{E}+03$

$9.03 \mathrm{E}+03$

$3.13 \mathrm{E}+03$

$0.00 \mathrm{E}+01$

$2.90 \mathrm{E}+03$

4.41E-00

$1.04 \mathrm{E}+03$

$6.48 \mathrm{E}+05$

$9.34 \mathrm{E}+01$

$1.14 \mathrm{E}+05$

$1.03 E+06$

$$
\text { NA }
$$

$2.82 \mathrm{E}+02$

$1.50 \mathrm{E}+05$

NA

$1.82 \mathrm{E}+03$

$3.05 \mathrm{E}+04$

7.43E-00

$1.18 \mathrm{E}+04$

$3.29 \mathrm{E}+03$

$6.21 \mathrm{E}+01$

9.85E-04

$5.74 \mathrm{E}+01$

$1.97 \mathrm{E}-03$

4.18E+05

$3.42 \mathrm{E}+01$

$8.74 E+01$

$1.47 \mathrm{E}-01$

1.34E-02

1.33E-01

$3.76 \mathrm{E}+01$

2.48E-00

$4.43 \mathrm{E}+05$
$1 \%$-ile

$0.00 \mathrm{E}+01$

$0.00 \mathrm{E}+01$

$0.00 \mathrm{E}+01$

$0.00 \mathrm{E}+01$

$0.00 \mathrm{E}+01$

$0.00 \mathrm{E}+01$

$0.00 \mathrm{E}+01$

$0.00 \mathrm{E}+01$

$0.00 \mathrm{E}+01$

$0.00 \mathrm{E} \div 01$

$0.00 \mathrm{E}+01$

$0.00 \mathrm{E}+01$

$0.00 \mathrm{E}+01$

$0.00 \mathrm{E}+01$

7.44E-01

$0.00 \mathrm{E}+01$

$0.00 \mathrm{E}+01$

$0.00 \mathrm{E}+01$

$0.00 \mathrm{E}+01$

$0.00 \mathrm{E}+01$

2.82E-01

$8.97 \mathrm{E}+01$

$0.00 \mathrm{E}+01$

$0.00 \mathrm{E}+01$

$0.00 \mathrm{E}+01$

$0.00 \mathrm{E}+01$

$0.00 \mathrm{E}+01$

$0.00 \mathrm{E}+01$

$0.00 \mathrm{E}+01$

$0.00 \mathrm{E}+01$

$2.54 \mathrm{E}+04$

$0.00 \mathrm{E}+01$

$2.25 \mathrm{E}+01$

$1.06 \mathrm{E}+04$

$1.90 \mathrm{E}+04$

$7.66 \mathrm{E}+03$

$2.58 \mathrm{E}+03$

$1.03 \mathrm{E}+02$

$0.00 \mathrm{E}+01$

3.84E-00

$1.99 \mathrm{E}-01$

$6.90 \mathrm{E}+02$

$5.91 \mathrm{E}+05$

1.42E-01

8. $10 \mathrm{E}+04$

8.57E+05

$3.87 \mathrm{E}+04$

$6.22 \mathrm{E}+01$

$1.44 \mathrm{E}+05$

$1.26 \mathrm{E}+01$

4.52E-00

$6.45 \mathrm{E}+03$

$3.51 \mathrm{E}-02$

$9.54 \mathrm{E}+03$

$2.37 \mathrm{E}+03$

$0.00 \mathrm{E}+01$

$0.00 \mathrm{E}+01$

$1.11 \mathrm{E}-00$

$7.95 \mathrm{E}-04$

$2.99 \mathrm{E}+04$

$0.00 \mathrm{E}+01$

$0.00 \mathrm{E}+0 \mathrm{I}$

$5.93 \mathrm{E}-02$

5.41E- 03

$5.37 \mathrm{E}-02$

$1.63 \mathrm{E}-01$

$1.77 \mathrm{E}-01$

$2.69 \mathrm{E}+05$
$10 \%$-ile

$2.38 \mathrm{E}-00$

2.69E-00

$2.55 \mathrm{E}+02$

$5.26 \mathrm{E}-02$

$5.72 \mathrm{E}-04$

$4.18 \mathrm{E}-01$.

$2.43 \mathrm{E}+01$

$3.56 \mathrm{E}-00$

$2.44 \mathrm{E}+01$

$1.15 \mathrm{E}-04$

1.12E-04

$1.87 \mathrm{E}-02$

$1.09 \mathrm{E}-03$

$9.30 \mathrm{E}-00$

$2.26 \mathrm{E}-00$

$8.82 \mathrm{E}+03$

$8.69 \mathrm{E}+02$

$0.00 \mathrm{E}+01$

2.08E-04

5.67E-04

2.38E-00

$1.69 \mathrm{E}+02$

4.55E-02

$1.74 \mathrm{E}-01$

2.21E-01

9.28E-03

6.01E-03

2.22E-01

$5.53 \mathrm{E}+02$

$3.09 \mathrm{E}-00$

$3.46 \mathrm{E}+04$

6.67E-00

$6.11 \mathrm{E}+02$

$1.13 \mathrm{E}+04$

$5.28 \mathrm{E}+04$

$9.04 \mathrm{E}+03$

$8.03 \mathrm{E}+03$

$1.39 \mathrm{E}+03$

$0.00 \mathrm{E}+01$

$1.59 \mathrm{E}+01$

3.63E-01

$1.08 \mathrm{E}+03$

$6.26 \mathrm{E}+05$

$1.27 \mathrm{E}-00$

$9.66 \mathrm{E}+04$

$9.38 \mathrm{E}+05$

4.27E+04

$1.47 \mathrm{E}+02$

$1.59 \mathrm{E}+05$

$2.09 E+03$

$3.77 \mathrm{E}+01$

$1.78 \mathrm{E}+04$

3.45E-00

$1.15 \mathrm{E}+04$

$3.96 \mathrm{E}+03$

4.27E-01

$0.00 \mathrm{E}+01$

$1.46 \mathrm{E}-00$

$1.32 \mathrm{E}-03$

$1.99 \mathrm{E}+05$

2.01E-00

$0.00 \mathrm{E}+01$

$9.88 \mathrm{E}-02$

9.01E-03

8.94E-02

2.44E-01

$1.18 \mathrm{E}-00$

$3.34 \mathrm{E}+05$

50\%-ile

$1.92 \mathrm{E}+01$

9.22E-00

$8.77 \mathrm{E}+02$

8.73E-01

7.03E-03

$6.94 \mathrm{E}-00$

$4.04 \mathrm{E}+02$ 


\begin{tabular}{|c|c|c|c|c|c|c|c|c|c|c|c|}
\hline nk & Anal. & $n$ & May $\frac{B B}{98}$ & $\frac{B B}{\operatorname{cept} 98}$ & $1 \%$-ile & $10 \%$-ile & $50 \%$-ile & $90 \%$-ile & $99 \%$-ile & Mean & Std Dev \\
\hline 102 & Eu152 & $\mathrm{Ci}$ & $3.95 \mathrm{E}-00$ & $3.95 \mathrm{E}-00$ & $0.00 \mathrm{E}+01$ & $0.00 \mathrm{E}+01$ & $3.95 \mathrm{E}-00$ & $1.53 \mathrm{E}+01$ & $2.40 \mathrm{E}+01$ & $E-00$ & $6.40 \mathrm{E}-00$ \\
\hline 02 & Eu154 & $\mathrm{Ci}$ & $11 E+02$ & $6.11 E+02$ & $0.00 \mathrm{E}+01$ & $00 E+01$ & $6.11 E+02$ & $36 E+03$ & $3.71 E+03$ & +02 & $E+02$ \\
\hline S102 & Eu155 & $\mathrm{Ci}$ & $32 E+02$ & $2.32 \mathrm{E}+02$ & $0.00 \mathrm{E}+01$ & $0.00 \mathrm{E}+01$ & $2.32 \mathrm{E}+02$ & $.96 \mathrm{E}+02$ & $1.41 E+03$ & $43 \mathrm{E}+02$ & $.76 E+02$ \\
\hline S102 & 1129 & $\mathrm{Ci}$ & $70 E-01$ & 4.70E-01 & $5.30 \mathrm{E}-02$ & $2.26 \mathrm{E}-01$ & $4.70 E-01$ & $7.34 \mathrm{E}-01$ & $9.70 \mathrm{E}-01$ & $4.75 E-01$ & $E-01$ \\
\hline 02 & Nb93 & $\mathrm{Ci}$ & $21 E+01$ & $1.21 \mathrm{E}+01$ & $0.00 \mathrm{E}+01$ & $00 E+01$ & $1.21 \mathrm{E}+01$ & $2.03 E+02$ & +02 & 01 & +01 \\
\hline 02 & Ni59 & $\mathrm{Ci}$ & $2.43 \mathrm{E}-00$ & $2.43 E-00$ & $0.00 \mathrm{E}+01$ & $0 \mathrm{E}+01$ & $2.43 E-00$ & $15 E+01$ & $8 E+01$ & $17 \mathrm{E}-00$ & $E-00$ \\
\hline 02 & Ni63 & $\mathrm{Ci}$ & $7 \mathrm{E}+02$ & $2.37 E+02$ & $E+01$ & $00+01$ & $2.37 \mathrm{E}+02$ & $12 E+03$ & $E+03$ & $07 E+02$ & $E+02$ \\
\hline 02 & Np237 & $\mathrm{Ci}$ & $97 \mathrm{E}-01$ & 8.97E-01 & $62 \mathrm{E}-01$ & $6.03 \mathrm{E}-01$ & $8.97 \mathrm{E}-01$ & $.21 \mathrm{E}-00$ & $1.47 \mathrm{E}-00$ & -01 & $6 \mathrm{E}-01$ \\
\hline 02 & Pa231 & $\mathrm{Ci}$ & $4.20 \mathrm{E}-03$ & $4.20 \mathrm{E}-03$ & $0 E+01$ & & $4.20 \mathrm{E}-03$ & & $1.46 \mathrm{E}-02$ & OE-03 & 83E-03 \\
\hline 02 & Pu238 & $\mathrm{Ci}$ & $1.47 \mathrm{E}-00$ & $1.47 \mathrm{E}-00$ & $E-01$ & $\varepsilon-01$ & $1.47 \mathrm{E}-00$ & $1.98 E-00$ & $E-00$ & $E-00$ & $.87 \mathrm{E}-01$ \\
\hline & Pu239 & $\mathbf{C i}$ & $5.42 E+01$ & $5.42 \mathrm{E}+01$ & $2.19 E+01$ & $3.64 E+01$ & $5.42 \mathrm{E}+01$ & $7.29 \mathrm{E}+01$ & 8.87E+01 & $E+01$ & $43 E+01$ \\
\hline 02 & Pu240 & $\mathrm{Ci}$ & 8.98E-00 & $8.98 \mathrm{E}-00$ & $3.62 \mathrm{E}-00$ & $E-00$ & $8.98 \mathrm{E}-00$ & $1.21 \mathrm{E}+01$ & $E+01$ & $E-00$ & $6 \mathrm{E}-00$ \\
\hline 02 & Pu241 & $\mathbf{C i}$ & $9.84 E+01$ & $9.84 \mathrm{E}+01$ & $E+01$ & & $9.84 E+01$ & $1.32 \mathrm{E}+02$ & +02 & +01 & $E+01$ \\
\hline 02 & Pu242 & $\mathrm{Ci}$ & $5.34 \mathrm{E}-04$ & 5.34E-04 & -04 & 04 & 94 & -04 & & & $E-04$ \\
\hline 02 & $\operatorname{Ra226}$ & $\mathrm{Ci}$ & $1.61 \mathrm{E}-04$ & $1.61 \mathrm{E}-04$ & & & 04 & -03 & -03 & -04 & -04 \\
\hline 02 & Ra228 & $\mathrm{Ci}$ & $1.31 \mathrm{E}-01$ & $1.31 \mathrm{E}-01$ & $E+01$ & -02 & -01 & $1.52 \mathrm{E}-00$ & E-00 & -01 & -01 \\
\hline 02 & Ru106 & $\mathrm{Ci}$ & $6.64 \mathrm{E}-03$ & $6.64 \mathrm{E}-03$ & $E+01$ & -04 & $6.64 \mathrm{E}-03$ & -02 & -02 & -03 & $E-03$ \\
\hline $\mathbf{S 1 0 2}$ & Sb125 & $\mathbf{C i}$ & $1.61 \mathrm{E}+02$ & $1.61 E+02$ & $0.00 E+01$ & +01 & $1.61 E+02$ & $7.30 \mathrm{E}+02$ & +03 & +02 & +02 \\
\hline & Se79 & $\mathrm{Ci}$ & $3.41 \mathrm{E}-00$ & 3.41E-00 & $:+01$ & & -00 & -00 & $E-00$ & -00 & $E-00$ \\
\hline 02 & Sm151 & $\mathrm{Ci}$ & $1.20 \mathrm{E}+04$ & $1.20 \mathrm{E}+04$ & $E \div 01$ & & +04 & +04 & +04 & & +04 \\
\hline & Sr89/90 & $\mathrm{Ci}$ & $3.10 \mathrm{E}+05$ & $3.10 \mathrm{E}+05$ & +01 & & & & & -05 & +05 \\
\hline 2 & Tc99 & $\mathrm{Ci}$ & $2.44 E+02$ & $2.44 \mathrm{E}+02$ & -01 & & 02 & -02 & -02 & 02 & +02 \\
\hline & Th229 & $\mathrm{Ci}$ & $3.07 \mathrm{E}-03$ & $3.07 E-03$ & +01 & +01 & -03 & -03 & -02 & -03 & $E-03$ \\
\hline & Th232 & $\mathrm{Ci}$ & $8.69 \mathrm{E}-03$ & $8.69 E-03$ & +01 & +01 & -03 & -02 & -02 & -03 & -03 \\
\hline & Tin126 & $\mathrm{Ci}$ & 5.15E-00 & 5.15E-00 & +01 & & & +01 & & & -00 \\
\hline & Tritium & $\mathrm{Ci}$ & $2.41 \mathrm{E}+02$ & $2.41 \mathrm{E}+02$ & +02 & +02 & +02 & $\div 02$ & +02 & & +01 \\
\hline & $\mathbf{U} 232$ & $\mathrm{Ci}$ & $6.80 \mathrm{E}-01$ & $1.01 \mathrm{E}-00$ & & & & & & & \\
\hline 2 & U233 & $\mathrm{Ci}$ & 2.61E-00 & $3.88 \mathrm{E}-00$ & & & & & & & -00 \\
\hline 12 & U234 & $\mathrm{Ci}$ & $8.09 \mathrm{E}-01$ & $1.20 \mathrm{E}-00$ & +01 & & 01 & -00 & -00 & 01 & -01 \\
\hline 2 & U235 & $\mathrm{Ci}$ & $3.29 \mathrm{E}-02$ & $4.89 \mathrm{E}-02$ & +01 & -01 & -02 & -02 & -01 & -02 & -02 \\
\hline & & $\mathrm{Ci}$ & $2.52 \mathrm{E}-02$ & $3.75 \mathrm{E}-02$ & +01 & & & & & & -02 \\
\hline & U238 & $\mathbf{C i}$ & $9.25 \mathrm{E}-01$ & $1.10 \mathrm{E}-00$ & & & & & & & -01 \\
\hline & $\mathbf{Y}$ & $\mathbf{C i}$ & $3.10 \mathrm{E}+05$ & $3.10 \mathrm{E}+05$ & & & & & & & \\
\hline 2 & Zr93 & $\mathbf{C i}$ & $1.67 \mathrm{E}+01$ & $1.67 \mathrm{E}+01$ & & & & & & & +02 \\
\hline 13 & Al & kg & $2.54 E+04$ & $2.54 \mathrm{E}+04$ & +03 & -04 & 04 & -04 & .04 & -04 & +04 \\
\hline & $\mathbf{B i}$ & kg & $1.57 \mathrm{E}+02$ & $1.57 \mathrm{E}+02$ & & & 02 & -02 & & -02 & +02 \\
\hline & $\mathbf{C a}$ & kg & $2.43 E+02$ & $2.43 E+02$ & & & & 02 & -03 & & +02 \\
\hline & Cl & kg & $5.66 \mathrm{E}+03$ & 5.6 & & & & & & & +03 \\
\hline & $\mathrm{CO} 3$ & $\mathbf{k g}$ & $2.64 E+04$ & $2.66 \mathrm{E}+04$ & & & 04 & 04 & .05 & -04 & +04 \\
\hline 03 & $\mathrm{Cr}$ & kg & $4.51 \mathrm{E}+03$ & $7.86 \mathrm{E}+03$ & -02 & -03 & 03 & 03 & +03 & +03 & +03 \\
\hline 3 & $\mathbf{F}$ & $\mathbf{k g}$ & $4.84 E+03$ & $4.84 E+03$ & & & & & & +03 & +03 \\
\hline & $\mathbf{F e}$ & $\mathrm{kg}$ & $1.72 \mathrm{E}+03$ & $1.72 E+03$ & & -02 & 03 & & 03 & -03 & +03 \\
\hline & Hg & kg & $1.79 E-00$ & $0.00 \mathrm{E}+01$ & & & & & & & -00 \\
\hline & $\mathbf{K}$ & $\mathbf{k g}$ & 1.6 & 1.6 & & & & & & & \\
\hline & $\mathbf{L a}$ & kg & & 00 & & & & & 02 & -01 & +01 \\
\hline $\mathbf{3}$ & Mn & kg & $i+02$ & $7.41 \mathrm{E}+02$ & & 01 & 02 & +03 & -03 & +02 & +02 \\
\hline 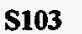 & $\mathbf{N a}$ & $\mathrm{kg}$ & $2.72 \mathrm{E}+05$ & $2.72 E+05$ & & & & & & tos & +04 \\
\hline S103 & $\mathbf{N i}$ & kg & $1.62 E+02$ & $1.62 E+02$ & & & -02 & & +02 & -02 & +02 \\
\hline & NO2 & kg & +04 & & & & & & & & +04 \\
\hline & & $\mathrm{kg}$ & & $3.22 E+05$ & & & & & -06 & & \\
\hline & $=$ & $\mathrm{kg}$ & & NA & & & & & .04 & & \\
\hline S103 & $\mathbf{P b}$ & kg & $1.97 E+02$ & $1.97 E+02$ & & & & & -02 & & \\
\hline & PO4 & kg & $2.65 E+04$ & $1.32 E+04$ & & & & & -04 & & \\
\hline S103 & $\mathbf{s}$ & $\mathrm{kg}$ & $1.36 E+04$ & NA & -01 & -03 & & & +04 & & +03 \\
\hline S103 & $\mathbf{S i}$ & kg & 1.82 & $1.82 \mathrm{E}+03$ & & & & & -03 & & $E+02$ \\
\hline S10 & SO4 & kg & & $1.36 E+04$ & & & & & & & $E+03$ \\
\hline & Sr & kg & & & & & & & -02 & & $=01$ \\
\hline & & kg & & 8.6 & & & & & & & +03 \\
\hline & $\mathbf{U}$ & kg & $1.74 E+03$ & $1.74 E+03$ & & -02 & & & -03 & +02 & +02 \\
\hline & $\mathbf{Z}_{\mathbf{r}}$ & kg & $4.60 E+01$ & $4.60 \mathrm{E}+01$ & & & 3. & & +02 & +02 & $E+02$ \\
\hline S1 & Ac? & $\mathrm{Ci}$ & $1.14 \mathrm{E}-03$ & $1.14 \mathrm{E}-03$ & & 0.0 & & -03 & E-03 & -03 & $\varepsilon-03$ \\
\hline S103 & Am241 & $\mathrm{Ci}$ & $6.18 E+01$ & $6.18 E+01$ & $E-00$ & E-00 & $6.29 E+03$ & $E+04$ & $3.60 \mathrm{E}+04$ & $E+03$ & $9.34 E+03$ \\
\hline $\mathbf{S 1 0 3}$ & Am243 & $\mathbf{C i}$ & 2.14E-03 & $2.14 E-03$ & +01 & $1.44 \mathrm{E}-04$ & $E-03$ & & E-03 & -03 & E-03 \\
\hline S103 & Ba137 & $\mathrm{Ci}$ & $1.84 E+05$ & $1.84 E+05$ & & $4.99 E+04$ & & & $E+05$ & $E+05$ & $E+05$ \\
\hline & C14 & $\mathrm{Ci}$ & & $3.65 \mathrm{E}+01$ & & & & & $E+02$ & +01 & $E+01$ \\
\hline & & $\mathrm{Ci}$ & $9.34 \mathrm{E}+01$ & $9.34 E+01$ & & & & & $E+02$ & +02 & $E+01$ \\
\hline & & $\mathrm{Ci}$ & & & & & & & & & \\
\hline S10 & $\mathrm{Cm}$ & $\mathrm{Ci}$ & $1.48 \mathrm{E}-02$ & $1.48 \mathrm{E}-02$ & $0.00 \mathrm{E}+01$ & $9.95 \mathrm{E}-04$ & $1.48 \mathrm{E}-02$ & $3.48 \mathrm{E}-02$ & $5.26 \mathrm{E}-02$ & $1.66 \mathrm{E}-02$ & 1.33 \\
\hline
\end{tabular}


Tank Anal. Un

\begin{tabular}{|c|c|c|c|c|}
\hline & & & May98 & Sept98 \\
\hline S103 & Cm244 & $\mathrm{Ci}$ & $1.45 \mathrm{E}-01$ & $\overline{1.45 \mathrm{E}-01}$ \\
\hline $\mathbf{S 1 0 3}$ & Co60 & $\mathrm{Ci}$ & $4.04 \mathrm{E}+01$ & $4.04 \mathrm{E}+01$ \\
\hline $\mathbf{S 1 0 3}$ & Cs134 & $\mathrm{Ci}$ & $2.74 \mathrm{E}-00$ & $2.74 E-00$ \\
\hline S103 & Cs137 & $\mathrm{Ci}$ & $1.95 E+05$ & $1.95 E+05$ \\
\hline $\mathbf{S 1 0 3}$ & Eu152 & $\mathrm{Ci}$ & $4.41 \mathrm{E}-00$ & $4.41 \mathrm{E}-00$ \\
\hline S103 & Eu154 & $\mathrm{Ci}$ & $6.55 \mathrm{E}+02$ & $6.55 E+02$ \\
\hline S103 & Eu155 & $\mathrm{Ci}$ & $2.58 \mathrm{E}+02$ & $2.58 E+02$ \\
\hline S103 & 1129 & $\mathrm{Ci}$ & 5.01E-01 & $5.01 \mathrm{E}-01$ \\
\hline \$103 & Nb93 & $\mathrm{Ci}$ & $1.30 \mathrm{E}+01$ & $1.30 E+01$ \\
\hline $\mathbf{S 1 0 3}$ & Ni59 & $\mathrm{Ci}$ & $2.86 \mathrm{E}-00$ & $2.86 \mathrm{E}-00$ \\
\hline S103 & Ni63 & $\mathrm{Ci}$ & $2.78 E+02$ & $2.78 E+02$ \\
\hline $\mathbf{S 1 0 3}$ & Np237 & $\mathrm{Ci}$ & 9.52E-01 & 9.52 \\
\hline S103 & Pa231 & $\mathrm{Ci}$ & 4.52E-03 & \\
\hline $\mathbf{S 1 0 3}$ & Pu238 & $\mathrm{Ci}$ & 1.70E-00 & $1.70 \mathrm{E}-00$ \\
\hline $\mathbf{S 1 0 3}$ & Pu239 & $\mathrm{Ci}$ & $6.68 \mathrm{E}+01$ & $6.68 \mathrm{E}+01$ \\
\hline S103 & Pu240 & $\mathrm{Ci}$ & $1.09 \mathrm{E}+01$ & $1.09 \mathrm{E}+01$ \\
\hline S103 & Pu241 & $\mathrm{Ci}$ & $1.13 E+02$ & $1.13 E+02$ \\
\hline S103 & Pu242 & $\mathrm{Ci}$ & $6.10 \mathrm{E}-04$ & $6.10 \mathrm{E}-04$ \\
\hline $\mathbf{S 1 0 3}$ & Ra226 & $\mathrm{Ci}$ & $1.92 \mathrm{E}-04$ & $1.92 \mathrm{E}-04$ \\
\hline S103 & R2228 & $\mathrm{Ci}$ & 1.51E-01 & -01 \\
\hline S103 & Ru106 & $\mathrm{Ci}$ & 7.17E-03 & 7.17E-03 \\
\hline S103 & Sb125 & $\mathrm{Ci}$ & $1.73 E+02$ & $1.73 E+02$ \\
\hline S103 & Se79 & $\mathrm{Ci}$ & $3.64 \mathrm{E}-00$ & $3.64 E-00$ \\
\hline S103 & Sm151 & $\mathrm{Ci}$ & $1.28 E+04$ & $1.28 \mathrm{E}+04$ \\
\hline S103 & Sr89/90 & $\mathrm{Ci}$ & $1.43 E+05$ & $1.43 E+05$ \\
\hline$\$ 103$ & Te99 & $\mathrm{Ci}$ & $2.60 \mathrm{E}+02$ & $2.60 E+02$ \\
\hline S103 & Th229 & $\mathrm{Ci}$ & $3.54 \mathrm{E}-03$ & -03 \\
\hline S103 & Th232 & $\mathrm{Ci}$ & $1.00 \mathrm{E}-02$ & $1.00 \mathrm{E}-02$ \\
\hline $\mathbf{S 1 0 3}$ & Tin126 & $\mathrm{Ci}$ & $5.49 \mathrm{E}-00$ & $5.49 \mathrm{E}-00$ \\
\hline$\$ 103$ & Tritium & $\mathrm{Ci}$ & $2.54 \mathrm{E}+02$ & $2.54 E+02$ \\
\hline S103 & U232 & $\mathrm{Ci}$ & $7.76 \mathrm{E}-01$ & 01 \\
\hline S103 & U233 & $\mathrm{Ci}$ & 2.97E-00 & -00 \\
\hline S103 & U234 & $\mathrm{Ci}$ & $8.76 \mathrm{E}-01$ & 1 \\
\hline S103 & U235 & $\mathrm{Ci}$ & 3.56E-02 & 2.59E-02 \\
\hline S103 & U236 & $\mathrm{Ci}$ & $2.74 E-02$ & $1.99 \mathrm{E}-02$ \\
\hline $\mathbf{S 1 0 3}$ & U238 & $\mathrm{Ci}$ & $1.01 \mathrm{E}-00$ & 5.81 \\
\hline $\mathbf{S 1 0 3}$ & Y90 & $\mathrm{Ci}$ & $1.43 E+05$ & +05 \\
\hline S103 & Zr93 & $\mathrm{Ci}$ & $1.78 \mathrm{E}+01$ & 01 \\
\hline S104 & Al & kg & $2.14 E+05$ & $2.14 \mathrm{E}+05$ \\
\hline S104 & Bi & $\mathrm{kg}$ & $7.08 E+01$ & $0.00 \mathrm{E}+01$ \\
\hline S104 & $\mathbf{C a}$ & $\mathrm{kg}$ & $7.70 \mathrm{E}+03$ & $7.70 E+03$ \\
\hline S104 & Cl & $\mathrm{kg}$ & $5.84 \mathrm{E}+03$ & $5.84 \mathrm{E}+03$ \\
\hline $\mathbf{S 1 0 4}$ & $\mathrm{CO3}$ & kg & $7.56 \mathrm{E}+03$ & $7.56 \mathrm{E}+03$ \\
\hline S104 & $\mathrm{Cr}$ & kg & $4.29 \mathrm{E}+03$ & \\
\hline S104 & $\mathbf{F}$ & kg & $2.37 \mathrm{E}+02$ & $2.29 \mathrm{E}+02$ \\
\hline S104 & Fe & kg & $3.14 E+03$ & $3.14 E+03$ \\
\hline S104 & $\mathbf{H g}$ & kg & $7.45 \mathrm{E}+01$ & $1.74 E+01$ \\
\hline S104 & $\mathbf{K}$ & kg & $5.48 E+02$ & $5.48 E+02$ \\
\hline S104 & $\mathbf{L a}$ & kg & $2.00 \mathrm{E}-06$ & $0.00 \mathrm{E}+01$ \\
\hline S104 & $\mathbf{M n}$ & $\mathrm{kg}$ & +03 & 03 \\
\hline$\$ 104$ & Na & $\mathrm{kg}$ & $2.21 \mathrm{E}+05$ & $2.21 \mathrm{E}+05$ \\
\hline S104 & $\mathbf{N i}$ & kg & $1.02 E+02$ & $1.02 E+02$ \\
\hline S104 & NO2 & kg & $4.73 E+04$ & $4.73 E+04$ \\
\hline S104 & NO3 & $\mathrm{kg}$ & $3.49 \mathrm{E}+05$ & $3.49 E+05$ \\
\hline S104 & $\mathbf{P}$ & $\mathrm{kg}$ & $2.82 \mathrm{E}+02$ & NA \\
\hline S104 & $\mathbf{P b}$ & $\mathrm{kg}$ & $5.40 \mathrm{E}+01$ & $5.40 E+01$ \\
\hline S104 & PO4 & kg & 8.73E+02 & $2.85 E+02$ \\
\hline S104 & $\mathbf{S}$ & $\mathrm{kg}$ & $4.14 \mathrm{E}+03$ & NA \\
\hline S104 & $\mathbf{S i}$ & $\mathrm{kg}$ & $2.43 E+03$ & $2.43 E+03$ \\
\hline S104 & SO4 & kg & $1.24 E+04$ & $4.14 E+03$ \\
\hline S104 & $\mathbf{S r}$ & $\mathrm{kg}$ & $7.74 \mathrm{E}+02$ & $7.74 E+02$ \\
\hline S104 & TOC & kg & $3.16 E+03$ & $3.16 E+\theta 3$ \\
\hline S104 & $\mathbf{U}$ & $\mathrm{kg}$ & $1.22 E+04$ & $1.22 E+04$ \\
\hline S104 & $\mathbf{Z r}$ & kg & $6.13 E+01$ & $6.13 E+01$ \\
\hline 104 & Ac227 & Ci & $3.62 \mathrm{E}-03$ & 3.62E-03 \\
\hline S104 & Am241 & $\mathrm{Ci}$ & $7.45 \mathrm{E}+01$ & $7.45 E+01$ \\
\hline S104 & Am243 & $\mathrm{Ci}$ & $2.30 E-03$ & $2.30 \mathrm{E}-03$ \\
\hline S104 & Ba137 & $\mathrm{Ci}$ & $1.04 E+05$ & $1.04 E+05$ \\
\hline
\end{tabular}

1\%-ile $10 \%$-ile $50 \%$-ile

\section{$0.00 \mathrm{E}+01$}

$5.68 \mathrm{E}-01$

$0.00 \mathrm{E}+01$

$2.90 \mathrm{E}+04$

$0.00 E+01$

$0.00 \mathrm{E}+01$

$0.00 \mathrm{E}+01$

$1.64 \mathrm{E}-02$

$0.00 \mathrm{E}+01$

$0.00 \mathrm{E}+01$

$0.00 \mathrm{E}+01$

$0.00 \mathrm{E}+01$

$0.00 \mathrm{E}+01$

$0.00 \mathrm{E} \div 01$

$0.00 \mathrm{E}+01$

$0.00 \mathrm{E}+01$

$0.00 \mathrm{E}+01$

$0.00 \mathrm{E}+01$

$0.00 \mathrm{E}+01$

$0.00 \mathrm{E}+01$

$0.00 \mathrm{E}+01$

$0.00 \mathrm{E}+01$

$0.00 \mathrm{E}+0 \mathrm{I}$

$0.00 \mathrm{E}+01$

$0.00 \mathrm{E}+01$

$0.00 \mathrm{E}+0 \mathrm{l}$

$0.00 \mathrm{E}+01$

$0.00 \mathrm{E}+0 \mathrm{I}$

$0.00 \mathrm{E}+0 \mathrm{I}$

$1.20 \mathrm{E}+02$

$0.00 \mathrm{E}+01$

$0.00 \mathrm{E}+01$

$0.00 \mathrm{E}+01$

$0.00 \mathrm{E}+01$

$0.00 \mathrm{E}+01$

$0.00 \mathrm{E}+01$

$0.00 \mathrm{E}+01$

$0.00 \mathrm{E}+01$

$7.54 \mathrm{E}+04$

$0.00 E+01$

$1.53 E+02$

$1.89 E+02$

$2.33 \mathrm{E}+02$

$1.53 \mathrm{E}+02$

$0.00 \mathrm{E}+01$

$7.71 E+01$

1.13E-00

$9.55 \mathrm{E}+01$

$1.62 \mathrm{E}-00$

$5.23 E+01$

$3.67 \mathrm{E}+04$

$3.56 \mathrm{E}-01$

$1.13 \mathrm{E}+03$

2.24E-00

$5.12 \mathrm{E}+01$

$1.88 \mathrm{E}-00$

$4.93 \mathrm{E}-00$

4.04E+01

$8.58 \mathrm{E}+01$

4.62E+01

$3.46 \mathrm{E}-01$

$1.27 \mathrm{E}+02$

$1.08 \mathrm{E}+03$

$0.00 \mathrm{E}+0$ !

$0.00 \mathrm{E}+01$

$7.29 \mathrm{E}+01$

$0.00 E+01$

$0.00 E+01$

\section{$9.74 \mathrm{E}-03$ :}

$7.09 \mathrm{E}+01$
$7.43 \mathrm{E}-01$

$1.02 \mathrm{E}+05 \quad 2.28 \mathrm{E}+05$

$0.00 \mathrm{E}+01$

$0.00 \mathrm{E}+01$

$0.00 \mathrm{E}+01$

$2.24 \mathrm{E}-01$

$0.00 \mathrm{E}+01$

$0.00 \mathrm{E}+01$

$0.00 \mathrm{E}+01$

$6.40 \mathrm{E}-02$

$0.00 \mathrm{E}+01$

$1.14 \mathrm{E}-01$

4.49E-00

7.32E-01

$7.59 \mathrm{E}-00$

4.10E-05

3.22E-05

2.53E-02

$0.00 \mathrm{E}+01$

$1.34 \mathrm{E}+01$

7.18E-01

$0.00 \mathrm{E}+01$

$1.19 \mathrm{E}+03$

$0.00 \mathrm{E}+01$

$0.00 \mathrm{E}+01$

$0.00 \mathrm{E}+01$

$0.00 \mathrm{E}+01$

$1.78 \mathrm{E}+02$

$0.00 \mathrm{E}+01$

$0.00 \mathrm{E}+01$

$0.00 \mathrm{E}+01$

$0.00 \mathrm{E}+01$

$0.00 \mathrm{E}+01$

$0.00 \mathrm{E}+01$

$3.72 \mathrm{E}+03$

$1.33 \mathrm{E}+05$

$8.37 \mathrm{E}+01$

$3.75 \mathrm{E}+02$

$7.65 \mathrm{E}+02$

$8.39 \mathrm{E}+03$

$8.50 \mathrm{E}+02$

1.37E+02

$1.18 \mathrm{E}+03$

$2.32 \mathrm{E}+01$

4.67E+02

$1.32 \mathrm{E}+01$

$4.64 \mathrm{E}+02$

$1.08 \mathrm{E}+05$

$4.06 \mathrm{E}+01$

$2.29 \mathrm{E}+04$

$3.78 \mathrm{E}+02$

$2.55 E+01$

$1.51 E+03$

$3.22 \mathrm{E}+02$

$7.94 \mathrm{E}+02$

1.12E+02

$1.15 E+03$

$5.06 \mathrm{E}+03$

9.22E-00

$1.60 \mathrm{E}-03$

$3.92 \mathrm{E}+01$

2.30E-04

$2.82 \mathrm{E}+04$

7.17E-03
$0.00 \mathrm{E}+01$

$1.01 \mathrm{E}+04$

$7.43 \mathrm{E}+02$
$2.28 \mathrm{E}+05$
$4.41 \mathrm{E}-00$

$6.55 \mathrm{E}+02$

2.58E+02

5.01E-01

$1.30 \mathrm{E}+01$

2.86E-00

$2.78 \mathrm{E}+02$

9.52E-01

4.52E-03

1.70E-00

$6.68 \mathrm{E}+01$

$1.09 \mathrm{E}+01$

1.13E+02

6.10E-04

1.92E-04

1.51E-01

$1.73 \mathrm{E}+02$

$1.28 \mathrm{E}+04$

$4.37 \mathrm{E}+04$

$2.60 \mathrm{E}+02$

$3.54 \mathrm{E}-03$

1.00E-02

5.49E-06

$2.54 \mathrm{E}+02$

7.76E-01

2.97E-00

8.76E-01

$3.56 \mathrm{E}-02$

2.74E-02

1.01E-00

$1.43 \mathrm{E}+05$

$1.78 \mathrm{E}+01$

$2.03 \mathrm{E}+05$

$1.21 \mathrm{E}+03$

$7.31 E+02$

$3.49 \mathrm{E}+03$

2.30E+04

$3.29 \mathrm{E}+03$

$1.62 \mathrm{E}+03$

$5.42 \mathrm{E}+03$

$7.45 \mathrm{E}+01$

$1.30 \mathrm{E}+03$

$1.90 \mathrm{E}+01$

$3.11 \mathrm{E}+03$

$1.43 \mathrm{E}+02$

4.14E+04

$1.94 \mathrm{E}+05$

$4.91 \mathrm{E}+03$

8.05E+01

$9.32 \mathrm{E}+03$

$8.69 \mathrm{E}+02$

$2.78 \mathrm{E}+03$

$2.52 \mathrm{E}+03$

$5.56 \mathrm{E}+02$

$4.88 \mathrm{E}+03$

$1.40 \mathrm{E}+04$

$7.41 \mathrm{E}+01$

3.62E- 03

$1.21 \mathrm{E}+02$

2.30E-03

$1.04 E+05$
3.64E-00

$2.04 \mathrm{E}+05$ 90\%-ile

3.41E-01

$5.06 \mathrm{E}+01$

$4.88 \mathrm{E}-00$

$3.64 \mathrm{E}+05$

$1.71 \mathrm{E}+01$

$2.54 \mathrm{E}+03$

$1.00 \mathrm{E}+03$

$8.26 \mathrm{E}-01$

$1.72 \mathrm{E}+02$

8.37E-00

$8.14 \mathrm{E}+02$

$2.24 \mathrm{E}-00$

$1.36 \mathrm{E}-02$

$4.00 \mathrm{E}-00$

$1.57 \mathrm{E}+02$

$2.56 \mathrm{E}+01$

$2.66 \mathrm{E}+02$

$1.43 \mathrm{E}-03$

6.07E-04

4.77E-01

1.70E-02

$1.14 \mathrm{E}+03$

6.77E-00

$4.97 \mathrm{E}+04$

$2.54 \mathrm{E}+05$

$7.58 \mathrm{E}+02$

1.07E-02

3.01E-02

$1.38 \mathrm{E}+01$

$3.38 \mathrm{E}+02$

$2.34 \mathrm{E}-00$

8.94E-00

2.64E-00

1.07E-01

8.25E-02

3.04E-00

$8.33 \mathrm{E} \div 05$

$2.35 \mathrm{E}+02$

$2.90 \mathrm{E}+05$

$2.73 \mathrm{E}+04$

$5.22 \mathrm{E}+03$

$6.84 \mathrm{E}+03$

$4.04 \mathrm{E}+04$

$8.60 \mathrm{E}+03$

$2.00 \mathrm{E}+04$

$2.41 \mathrm{E}+04$

$7.51 \mathrm{E}+02$

$2.34 \mathrm{E}+03$

$3.54 \mathrm{E}+01$

$6.83 \mathrm{E}+03$

$2.92 \mathrm{E}+05$

$3.25 \mathrm{E}+02$

$9.28 \mathrm{E}+04$

3.97E+05

$4.43 \mathrm{E}+04$

$8.05 \mathrm{E}+02$

$1.31 \mathrm{E}+05$

$5.79 \mathrm{E}+03$

$1.60 \mathrm{E}+04$

$1.29 \mathrm{E}+04$

$1.27 \mathrm{E}+03$

1. $42 \mathrm{E}+04$

4.27E+04

$4.83 \mathrm{E}+02$

9.25E-03

$1.78 \mathrm{E}+03$

$4.59 \mathrm{E}-03$

$1.90 \mathrm{E}+05$

99\%-ile

5.15E-01

8.31E+01

6.93E-00

$4.93 \mathrm{E}+05$

$2.96 \mathrm{E}+01$

$4.39 \mathrm{E}+03$

$1.73 \mathrm{E}+03$

1.12E-00

$2.87 \mathrm{E}+02$

1.33E +01

$1.29 \mathrm{E}+03$

$3.38 \mathrm{E}-00$

$2.35 \mathrm{E}-02$ 
Tank Anal. Un

\begin{tabular}{|c|c|c|c|c|}
\hline & & & Мау 98 & Sept98 \\
\hline S104 & C14 & $\mathrm{Ci}$ & $1.64 \mathrm{E}-00$ & $1.64 \mathrm{E}-00$ \\
\hline S104 & Cd113 & $\mathrm{Ci}$ & 3.13E+01 & $3.13 E+01$ \\
\hline S104 & Cm242 & $\mathrm{Ci}$ & $6.71 \mathrm{E}-02$ & 6.71E-02 \\
\hline S104 & Cm243 & $\mathrm{Ci}$ & $1.54 \mathrm{E}-03$ & $1.54 \mathrm{E}-03$ \\
\hline S104 & Cm244 & $\mathrm{Ci}$ & $1.23 \mathrm{E}-03$ & $1.23 E-03$ \\
\hline S104 & Co60 & $\mathrm{Ci}$ & $6.67 \mathrm{E}-00$ & $6.67 \mathrm{E}-00$ \\
\hline S104 & Cs134 & Ci & 1.42E-00 & $1.42 \mathrm{E}-00$ \\
\hline S104 & Cs137 & $\mathrm{Ci}$ & $1.10 E+05$ & $1.10 E+05$ \\
\hline S104 & Eu152 & $\mathrm{Ci}$ & $8.29 \mathrm{E}-00$ & 8.291 \\
\hline $\mathbf{S 1 0 4}$ & Eu154 & $\mathrm{Ci}$ & $1.60 \mathrm{E}+02$ & 1.60 \\
\hline S104 & Eu155 & $\mathrm{Ci}$ & $4.10 \mathrm{E}+02$ & $4.10 \mathrm{E}+02$ \\
\hline S104 & 1129 & $\mathrm{Ci}$ & $1.16 \mathrm{E}-01$ & $1.16 \mathrm{E}-01$ \\
\hline S104 & Nb93 & $\mathrm{Ci}$ & $1.70 \mathrm{E}+01$ & $1.70 \mathrm{E}+01$ \\
\hline S104 & Ni59 & $\mathrm{Ci}$ & $1.02 \mathrm{E}+01$ & $1.02 E+01$ \\
\hline S104 & Ni63 & $\mathrm{Ci}$ & $9.71 \mathrm{E}+02$ & $9.71 E+02$ \\
\hline S104 & Np237 & $\mathrm{Ci}$ & $2.80 \mathrm{E}-01$ & 2.80E-01 \\
\hline S104 & $\mathbf{P a 2 3 1}$ & $\mathrm{Ci}$ & $6.60 \mathrm{E}$ & $6.60 \mathrm{E}-03$ \\
\hline S104 & Pu238 & $\mathrm{Ci}$ & 6.42 & 7.49 \\
\hline S104 & Pu239 & $\mathbf{C i}$ & $5.15 E+02$ & $4.50 E+02$ \\
\hline S104 & Pu240 & $\mathrm{Ci}$ & Incl. in 239 & $6.52 \mathrm{E}+01$ \\
\hline S104 & Pu241 & $\mathbf{C i}$ & $3.62 E+02$ & $4.22 \mathrm{E}+02$ \\
\hline$\$ 104$ & Pu242 & $\mathbf{C i}$ & $1.67 \mathrm{E}-03$ & $1.95 \mathrm{E}-03$ \\
\hline S104 & $\mathbf{R a 2 2 6}$ & $\mathrm{Ci}$ & $7.28 \mathrm{E}-04$ & $7.28 \mathrm{E}-04$ \\
\hline S104 & Ra228 & $\mathrm{Ci}$ & $9.60 \mathrm{E}-03$ & $9.60 \mathrm{E}-03$ \\
\hline S104 & Ru106 & $\mathrm{Ci}$ & 1.461 & $1.46 \mathrm{E}-03$ \\
\hline \$104 & Sb125 & $\mathrm{Ci}$ & $2.31 E+01$ & $2.31 E+01$ \\
\hline S104 & Se79 & $\mathbf{C i}$ & $4.44 E-00$ & $4.44 \mathrm{E}-00$ \\
\hline S104 & Sm151 & $\mathrm{Ci}$ & $1.58 \mathrm{E}+04$ & $1.58 E+04$ \\
\hline S104 & Sr89/90 & $\mathbf{C i}$ & $5.49 \mathrm{E}+05$ & $5.49 E+05$ \\
\hline S104 & Tc99 & $\mathbf{C i}$ & $4.42 E+01$ & $4.42 \mathrm{E}+01$ \\
\hline S104 & Th229 & $\mathrm{Ci}$ & 2.31E-04 & 2.31E-04 \\
\hline S104 & Th232 & $\mathrm{Ci}$ & $1.29 \mathrm{E}-04$ & 1.29E-04 \\
\hline S104 & Tin126 & $\mathbf{C i}$ & $6.81 \mathrm{E}-00$ & $6.81 \mathrm{E}-00$ \\
\hline S104 & Tritium & $\mathbf{C i}$ & $6.15 \mathrm{E}-00$ & $6.15 E-00$ \\
\hline S104 & U232 & $\mathbf{C i}$ & 4.30E-02 & $8.64 E-02$ \\
\hline S104 & U233 & $\mathbf{C i}$ & $1.64 \mathrm{E}-01$ & $3.30 \mathrm{E}-01$ \\
\hline S104 & U234 & $\mathbf{C i}$ & 2.17E-00 & $4.36 \mathrm{E}-00$ \\
\hline S104 & U235 & $\mathrm{Ci}$ & $9.06 \mathrm{E}-02$ & $1.82 \mathrm{E}-01$ \\
\hline S104 & U236 & $\mathrm{Ci}$ & $6.25 \mathrm{E}-02$ & $1.26 \mathrm{E}-01$ \\
\hline S104 & U238 & $\mathrm{Ci}$ & 2.04E-00 & $4.07 E-00$ \\
\hline S104 & Y90 & $\mathrm{Ci}$ & $5.49 E+05$ & $5.49 \mathrm{E}+05$ \\
\hline S104 & Zr93 & $\mathrm{Ci}$ & $2.10 \mathrm{E}+01$ & $2.10 \mathrm{E}+01$ \\
\hline $\mathbf{S 1 0 5}$ & Al & kg & $4.36 \mathrm{E}+04$ & $4.36 E+04$ \\
\hline S105 & $\mathbf{B i}$ & kg & $9.50 \mathrm{E}+01$ & $9.50 \mathrm{E}+01$ \\
\hline S105 & $\mathrm{Ca}$ & kg & $7.18 \mathrm{E}+02$ & $7.18 E+02$ \\
\hline S105 & $\mathrm{Cl}$ & $\mathrm{kg}$ & $1.07 \mathrm{E}+04$ & $1.07 E+04$ \\
\hline S105 & $\mathrm{CO} 3$ & kg & $2.10 \mathrm{E}+04$ & $2.16 E+04$ \\
\hline S105 & $\mathbf{C r}$ & kg & $1.53 E+04$ & $1.53 E+04$ \\
\hline $\mathbf{S 1 0 5}$ & $\mathbf{F}$ & kg & $1.72 E+04$ & $1.72 E+04$ \\
\hline S105 & $\mathbf{F e}$ & kg & $4.60 \mathrm{E}+03$ & $4.60 \mathrm{E}+03$ \\
\hline S105 & Hg & kg & $1.75 \mathrm{E}-00$ & $0.00 E+01$ \\
\hline S105 & $\mathbf{K}$ & $\mathrm{kg}$ & $3.10 \mathrm{E}+03$ & $3.10 \mathrm{E}+03$ \\
\hline S105 & La & kg & $1.12 \mathrm{E}+02$ & $1.12 E+02$ \\
\hline S105 & Mn & kg & $1.94 E+03$ & $1.94 E+03$ \\
\hline $\mathbf{S 1 0 5}$ & $\mathbf{N a}$ & kg & $5.11 E+05$ & 5.11 \\
\hline S105 & $\mathbf{N i}$ & kg & $4.37 \mathrm{E}+02$ & $4.37 E+02$ \\
\hline S105 & NO2 & kg & $1.61 \mathrm{E} \div 05$ & $1.61 \mathrm{E}+05$ \\
\hline S105 & NO3 & $\mathrm{kg}$ & $4.59 \mathrm{E}+05$ & $4.59 \mathrm{E}+05$ \\
\hline S105 & $\mathbf{P}$ & $\mathrm{kg}$ & $3.07 \mathrm{E}+04$ & NA \\
\hline S105 & $\mathbf{P b}$ & $\mathrm{kg}$ & $5.37 \mathrm{E}+02$ & $5.37 E+02$ \\
\hline S105 & PO4 & kg & $9.52 E+04$ & $9.52 \mathrm{E}+04$ \\
\hline S105 & $\mathbf{S}$ & kg & $1.29 \mathrm{E}+04$ & NA \\
\hline S105 & $\mathbf{S i}$ & kg & $5.58 \mathrm{E}+03$ & $5.58 E+03$ \\
\hline S105 & SO4 & $\mathrm{kg}$ & $3.86 E+04$ & $3.86 E+04$ \\
\hline S105 & Sr & $\mathrm{kg}$ & $2.40 \mathrm{E}+01$ & $2.40 E+01$ \\
\hline S105 & TOC & kg & $2.51 \mathrm{E}+04$ & $2.51 E+04$ \\
\hline S105 & $\mathbf{U}$ & $\mathrm{kg}$ & $2.58 E+03$ & $2.58 E+03$ \\
\hline 05 & $\mathbf{Z r}$ & kg & $1.32 E+02$ & $1.32 \mathrm{E}+02$ \\
\hline
\end{tabular}

1\%-ile $0.00 \mathrm{E}+01$ $0.00 \mathrm{E}+01$ $0.00 \mathrm{E}+01$ $0.00 \mathrm{E}+01$ 9.63E-00 $0.00 \mathrm{E}+01$ $1.02 \mathrm{E}+04$ $0.00 \mathrm{E}+01$ $0.00 \mathrm{E}+01$ $0.00 \mathrm{E}+01$ $0.00 \mathrm{E}+01$ $0.00 \mathrm{E}+01$ $0.00 \mathrm{E}+01$ $0.00 \mathrm{E}+01$ $0.00 \mathrm{E}+01$ $0.00 \mathrm{E}+01$ $0.00 \mathrm{E}+01$ $0.00 \mathrm{E}+01$ $0.00 \mathrm{E}+01$ $0.00 \mathrm{E}+01$ $0.00 \mathrm{E}+01$ $0.00 \mathrm{E}+01$ $0.00 \mathrm{E}+01$ $0.00 \mathrm{E}+01$ $0.00 \mathrm{E}+01$ $7.69 \mathrm{E}-02$ $0.00 \mathrm{E}+01$ $2.40 \mathrm{E}-00$ $0.00 \mathrm{E}+01$ $0.00 \mathrm{E} \div 01$ $0.00 \mathrm{E}+01$ $1.03 E-01$

$1.96 \mathrm{E}-00$ $0.00 \mathrm{E}+0 \mathrm{I}$ $0.00 \mathrm{E}+0 \mathrm{I}$ $0.00 \mathrm{E}+01$ $0.00 \mathrm{E}+01$ $0.00 \mathrm{E}+01$ $0.00 \mathrm{E}+01$ $0.00 E+01$ $0.00 \mathrm{E}+01$

$4.31 E+02$ $0.00 \mathrm{E}+01$

8.04E-01

4.63E-00

$1.86 \mathrm{E}+02$

3.97E-00

$0.00 \mathrm{E}+01$

$8.29 \mathrm{E}-00$

$0.00 \mathrm{E}+01$

$1.20 \mathrm{E}-00$

3.95E-02

7.77E-01

$2.52 \mathrm{E}+05$

4.89E-02

$7.23 \mathrm{E}+01$

$2.15 E+02$

$2.51 E+01$

2.87E-0I

$1.42 \mathrm{E}+0 \mid$

2.08E-00

3.51E-00

8.55E-00

6.5IE-03

4.72E-00

8.60E-00

$0.00 \mathrm{E}+01$
$10 \%$-ile

50\%-ile

90\%-ile

99\%-ile

\subsection{E-02}

$9.73 \mathrm{E}-00$

6.72E-03

$1.54 \mathrm{E}-04$

1.23E-04

$1.30 \mathrm{E}+01$

3.85E-01

$6.84 \mathrm{E}+04$

3.66E-00

$7.06 \mathrm{E}+01$

$1.81 \mathrm{E}+02$

8.54E-03

2.07E-00

$2.86 \mathrm{E}-00$

$2.72 \mathrm{E}+02$

2.81E-02

7.47E-04

6.43E-01

$5.16 \mathrm{E}+01$

$5.16 \mathrm{E}+01$

$3.63 \mathrm{E}+01$

1.67E-04

$1.37 \mathrm{E}-04$

$1.80 \mathrm{E}-03$

2.60E-04

$1.54 \mathrm{E}-00$

$1.40 \mathrm{E}-00$

$6.97 \mathrm{E}+03$

$4.31 E+01$

$0.00 \mathrm{E}+01$

2.61E-05

$1.46 \mathrm{E}-05$

2.12E-00

3.72E-00

4.87E-03

$1.86 \mathrm{E}-02$

2.46E-01

$1.03 \mathrm{E}-02$

7.07E-03

$2.31 \mathrm{E}-01$

$0.00 \mathrm{E}+01$

2.55E-00

$1.71 \mathrm{E}+03$

$3.20 \mathrm{E}-00$

4.17E-00

$6.68 \mathrm{E}+01$

$2.80 \mathrm{E}+04$

$3.08 \mathrm{E}+01$

$4.29 \mathrm{E}-00$

$3.58 \mathrm{E}+02$

$0.00 \mathrm{E}+01$

6.08E-00

$1.50 \mathrm{E}-01$

1.19E+01

$3.53 \mathrm{E}+05$

$5.83 \mathrm{E}-01$

$5.40 \mathrm{E}+02$

$4.04 \mathrm{E}+04$

$3.69 \mathrm{E}+03$

$3.18 \mathrm{E}+01$

$6.20 \mathrm{E}+03$

$7.20 \mathrm{E}+02$

$1.4 \mathrm{IE}+01$

4.42E+03

$1.32 E-00$

4.95E+01

$5.14 \mathrm{E}+01$

$1.21 \mathrm{E}-01$
1.64E-00

$3.13 E+01$

6.71E-02

$1.54 \mathrm{E}-03$

1.23E-03

$1.83 \mathrm{E}+01$

1.42E-00

$1.54 \mathrm{E}+05$

8.29E-00

$1.60 \mathrm{E}+02$

$4.10 \mathrm{E}+02$

1.16E-01

$1.70 \mathrm{E}+01$

$1.02 \mathrm{E}+01$

$9.71 \mathrm{E}+02$

2.80E-01

$6.60 \mathrm{E}-03$

6.42E-00

$5.15 E+02$

$5.15 \mathrm{E}+02$

$3.62 \mathrm{E}+02$

$1.67 \mathrm{E}-03$

7.28E-04

$9.60 \mathrm{E}-03$

1.46E-03

$2.31 \mathrm{E}+01$

4.44E-00

$1.58 \mathrm{E}+04$

$5.00 \mathrm{E}+05$

4.42E+01

2.31E-04

$1.29 \mathrm{E}-04$

$6.81 \mathrm{E}-00$

6.15E-00

4.30E-02

1.64E-01

2.17E-00

9.06E-02

$6.25 \mathrm{E}-02$

2.04E-00

$5.49 \mathrm{E}+05$

$2.10 \mathrm{E}+01$

$4.15 E+04$

$2.33 E+02$

$4.52 \mathrm{E}+02$

$6.34 \mathrm{E}+03$

$7.47 \mathrm{E}+04$

$1.71 \mathrm{E}+04$

$2.64 \mathrm{E}+03$

$2.45 \mathrm{E}+03$

1.75E-00

$1.65 \mathrm{E}+03$

$2.42 \mathrm{E}+01$

$4.24 \mathrm{E}+02$

$5.19 \mathrm{E}+05$

$1.23 \mathrm{E}+02$

$7.44 \mathrm{E}+04$

$8.97 \mathrm{E}+05$

1.17E+04

$1.30 \mathrm{E}+02$

$3.66 \mathrm{E}+04$

$9.93 \mathrm{E}+03$

$1.64 \mathrm{E}+03$

$2.95 E+04$

$4.34 E+01$

$3.94 \mathrm{E}+03$

$6.65 \mathrm{E}+02$ $4.03 E+01$
3.51E-00

$3.15 \mathrm{E}+02$

1.34E-01

3.07E-03

$2.45 \mathrm{E}-03$

$2.41 \mathrm{E}+01$

2.59E-00

$2.52 \mathrm{E}+05$

2.12E+01

4.09E+02

$1.05 \mathrm{E}+03$

2.48E-01

$1.11 \mathrm{E}+02$

2.32E+01

$2.21 \mathrm{E}+03$

$5.58 \mathrm{E}-01$

2.54E-02

$1.28 \mathrm{E}+01$

$1.03 \mathrm{E}+03$

$1.03 \mathrm{E}+03$

$7.22 \mathrm{E}+02$

$3.33 \mathrm{E}-03$

$1.68 \mathrm{E}-03$

2.21E-02

6.75E-03

$5.22 \mathrm{E}+02$

$2.28 \mathrm{E}+01$

$4.04 \mathrm{E}+04$

$7.25 \mathrm{E}+06$

$1.12 \mathrm{E}+02$

$8.90 \mathrm{E}-04$

4.97E-04

$6.86 \mathrm{E}+01$

$1.01 \mathrm{E}+01$

$1.66 \mathrm{E}-01$

$6.32 \mathrm{E}-01$

8.36E-00

$3.49 \mathrm{E}-01$

2.41E-01

$7.86 \mathrm{E}-00$

7.97E+06

$1.37 \mathrm{E}+02$

$1.01 \mathrm{E}+05$

$8.96 \mathrm{E}+02$

$1.33 \mathrm{E}+03$

$1.47 \mathrm{E}+04$

$1.38 \mathrm{E}+05$

4.15E+04

$1.04 \mathrm{E}+04$

$4.73 \mathrm{E}+03$

$4.41 \mathrm{E}-00$

$6.44 \mathrm{E}+03$

$7.01 \mathrm{E}+01$

$1.30 \mathrm{E}+03$

$7.42 \mathrm{E}+05$

$5.29 \mathrm{E}+02$

$1.94 \mathrm{E}+05$

2.01E+06

$2.20 \mathrm{E}+04$

$2.56 \mathrm{E}+02$

$7.20 \mathrm{E}+04$

$2.05 \mathrm{E}+04$

4.64E+03

$5.87 \mathrm{E}+04$

$3.08 E+02$

$9.46 \mathrm{E}+03$

$2.57 \mathrm{E}+03$

$1.48 \mathrm{E}+03$
5.81E-00

$9.78 \mathrm{E}+02$

2.11E-01

4.84E-03

$3.86 \mathrm{E}-03$

$1.14 \mathrm{E}+02$

$3.40 \mathrm{E}-00$

$3.19 \mathrm{E}+05$

$9.49 \mathrm{E}+01$ 
Tank Anal. Un

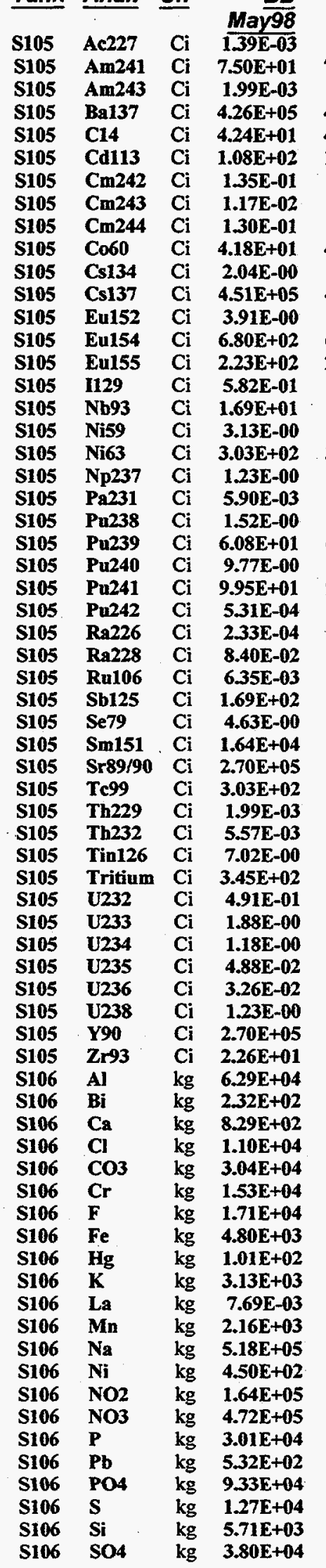

\section{Sept98}

$1.39 \mathrm{E}-03$
$7.50 \mathrm{E}+01$

$1.99 \mathrm{E}-03$

$4.26 \mathrm{E}+05$

$4.24 \mathrm{E}+01$

$1.08 \mathrm{E}+02$

1.35E-01

$1.17 \mathrm{E}-02$

1.30E-01

$4.18 \mathrm{E}+01$

2.04E-00

$4.51 E+05$

3.91E-00

$6.80 \mathrm{E}+02$

$2.23 \mathrm{E}+02$

5.82E-01

$1.69 \mathrm{E}+01$

3.13E-00

$3.03 \mathrm{E}+02$

1.23E-00

$5.90 \mathrm{E}-03$

1.52E-00

$6.08 \mathrm{E}+01$

9.77E-00

$9.95 \mathrm{E}+01$

5.31E-04

2.33E-04

8.40E-02

$6.35 \mathrm{E}-03$

$1.69 \mathrm{E}+02$

4.63E-00

$1.64 \mathrm{E}+04$

$2.70 \mathrm{E}+05$

$3.03 \mathrm{E}+02$

$1.99 \mathrm{E}-03$

5.57E-03

7.02E-00

$3.45 \mathrm{E}+02$

$3.82 \mathrm{E}-01$

1.46E-00

$9.15 \mathrm{E}-01$

3.79E-02

2.53E-02

8.61E-01

2.70E+05

$2.26 \mathrm{E}+01$

$5.40 \mathrm{E}+04$

$3.34 \mathrm{E}+02$

$3.39 E+02$

$9.61 \mathrm{E}+03$

$9.56 \mathrm{E}+04$

$1.68 \mathrm{E}+04$

$6.45 \mathrm{E}+03$

$4.63 E+03$

$2.33 \mathrm{E}+01$

$2.21 \mathrm{E}+03$

$0.00 \mathrm{E}+01$

$1.73 E+02$

$6.24 \mathrm{E}+05$

$8.13 E+01$

$8.81 E+04$

1.05E+06

$$
\text { NA }
$$

$1.94 \mathrm{E}+02$

$6.33 E+04$

$$
\text { NA }
$$

$1.33 \mathrm{E}+03$

$2.30 \mathrm{E}+04$
$1 \%$ ile

$0.00 E+01$

$1.89 \mathrm{E}-01$

$0.00 \mathrm{E}+01$

$0.00 \mathrm{E}+01$

$0.00 \mathrm{E}+01$

$0.00 \mathrm{E}+01$

$0.00 \mathrm{E}+01$

$0.00 \mathrm{E}+01$

$0.00 E+01$

4.31E-02

$0.00 \mathrm{E}+01$

$2.75 E+02$

$0.00 \mathrm{E}+01$

$0.00 \mathrm{E}+01$

$0.00 \mathrm{E}+01$

$0.00 \mathrm{E}+01$

$0.00 E+01$

$0.00 \mathrm{E}+01$

$0.00 E+01$

$0.00 E+01$

$0.00 \mathrm{E}+01$

$0.00 \mathrm{E}+01$

$0.00 E+01$

$0.00 \mathrm{E}+01$

$0.00 \mathrm{E}+01$

$0.00 \mathrm{E}+01$

$0.00 \mathrm{E}+01$

$0.00 \mathrm{E}+01$

$0.00 \mathrm{E}+01$

$0.00 \mathrm{E}+01$

$0.00 \mathrm{E}+01$

$0.00 \mathrm{E}+01$

$0.00 \mathrm{E}+01$

$0.00 \mathrm{E}+01$

$0.00 \mathrm{E}+01$

$0.00 \mathrm{E}+01$

$0.00 \mathrm{E}+01$

$0.00 \mathrm{E}+0 \mathrm{I}$

$0.00 \mathrm{E}+01$

$0.00 \mathrm{E}+0 \mathrm{I}$

$0.00 \mathrm{E}+01$

$0.00 \mathrm{E}+0 \mathrm{I}$

$0.00 \mathrm{E}+01$

$0.00 \mathrm{E}+01$

$0.00 \mathrm{E}+01$

$0.00 \mathrm{E}+01$

$3.41 \mathrm{E}+04$

$0.00 \mathrm{E}+01$

$2.65 \mathrm{E}+01$

$4.86 \mathrm{E}+03$

$7.26 \mathrm{E}+03$

$1.03 \mathrm{E}+04$

$5.34 \mathrm{E}-00$

$4.32 \mathrm{E}+02$

$0.00 \mathrm{E}+01$

$3.05 \mathrm{E}+01$

$5.54 \mathrm{E}-01$

$2.00 \mathrm{E}+01$

$3.82 \mathrm{E}+05$

$3.50 \mathrm{E}-01$

$2.68 \mathrm{E}+04$

$5.77 \mathrm{E}+0.5$

$5.18 \mathrm{E}+03$

6.19E-00

$1.50 \mathrm{E}+04$

$1.29 \mathrm{E}+02$

$2.42 \mathrm{E}+0 \mathrm{l}$

$7.71 \mathrm{E}+02$
$10 \%$ ile

$0.00 \mathrm{E}+01$

$5.38 \mathrm{E}-01$

$1.80 \mathrm{E}-05$

$0.00 \mathrm{E}+01$

$2.78 \mathrm{E}-00$

$0.00 \mathrm{E}+01$

$1.22 \mathrm{E}-03$

$1.06 \mathrm{E}-04$

$1.18 \mathrm{E}-03$

$1.38 \mathrm{E}-01$

$0.00 \mathrm{E}+01$

$1.63 \mathrm{E}+03$

$0.00 \mathrm{E}+01$

$0.00 \mathrm{E}+01$

$0.00 \mathrm{E}+01$

$0.00 \mathrm{E}+01$

$0.00 \mathrm{E}+01$

$0.00 \mathrm{E}+01$

$0.00 E+01$

$1.11 \mathrm{E}-02$

$0.00 \mathrm{E}+01$

$1.37 \mathrm{E}-02$

$5.50 \mathrm{E}-01$

$8.83 \mathrm{E}-02$

$8.99 \mathrm{E}-01$

$4.80 \mathrm{E}-06$

$0.00 \mathrm{E}+01$

$0.00 \mathrm{E}+01$

$0.00 \mathrm{E}+01$

$0.00 \mathrm{E}+01$

$1.09 \mathrm{E}-01$

$0.00 \mathrm{E}+01$

4. $10 \mathrm{E}-00$

$0.00 \mathrm{E}+01$

$0.00 \mathrm{E}+0$

$0.00 \mathrm{E}+01$

$0.00 \mathrm{E}+01$

$2.61 \mathrm{E}+01$

$0.00 \mathrm{E}+01$

$0.00 E+01$

$0.00 \mathrm{E}+0 \mathrm{I}$

$0.00 \mathrm{E}+0$

$0.00 \mathrm{E}+01$

$0.00 E+01$

$0.00 \mathrm{E}+01$

$0.00 \mathrm{E}+0$

$4.93 \mathrm{E}+04$

$5.46 \mathrm{E}+01$

$6.56 \mathrm{E}+01$

$6.29 \mathrm{E}+03$

$4.21 E+04$

$1.25 \mathrm{E}+04$

$9.90 \mathrm{E}+0 \mathrm{I}$

$1.95 \mathrm{E}+03$

$0.00 E+01$

$1.14 \mathrm{E}+02$

$1.98 \mathrm{E}-00$

$1.81 \mathrm{E}+02$

$4.69 \mathrm{E}+05$

$9.50 \mathrm{E}-00$

$4.66 \mathrm{E}+04$

$7.45 \mathrm{E}+05$

$9.95 \mathrm{E}+03$

$6.37 E+01$

$3.36 \mathrm{E}+04$

$3.50 \mathrm{E}+03$

$2.57 \mathrm{E}+02$

$1.20 \mathrm{E}+04$
$50 \%$-ile

1.39E-03

$3.97 \mathrm{E}+01$

1.99E-03

$4.26 E+05$

$4.24 \mathrm{E}+01$

$1.08 \mathrm{E}+02$

1.35E-01

1.17E-02

$1.30 \mathrm{E}-01$

$1.78 \mathrm{E}+01$

2.04E-00

$2.29 \mathrm{E}+05$

3.91E-00

$6.80 \mathrm{E}+02$

$2.23 \mathrm{E}+02$

$5.82 \mathrm{E}-01$

$1.69 \mathrm{E}+01$

3.13E-00

$3.03 E+02$

$1.23 \mathrm{E}-00$

$5.90 \mathrm{E}-03$

1.52E-00

$6.08 \mathrm{E}+01$

9.77E-00

$9.95 \mathrm{E}+01$

5.31E-04

2.33E-04

8.40E-02

6.35E-03

$1.69 \mathrm{E}+02$

4.63E-00

$1.64 \mathrm{E}+04$

$5.97 \mathrm{E}+04$

$3.03 E+02$

$1.99 \mathrm{E}-03$

5.57E-03

7.02E-00

$3.45 \mathrm{E}+02$

4.91E-01

$1.88 \mathrm{E}-00$

1.18E-00

4.88E-02

3.26E-02

$1.23 \mathrm{E}-00$

$2.70 \mathrm{E}+05$

$2.26 \mathrm{E}+01$

$7.08 \mathrm{E}+04$

$5.29 \mathrm{E}+02$

$6.60 \mathrm{E}+02$

$8.32 \mathrm{E}+03$

$8.87 \mathrm{E}+04$

$1.67 \mathrm{E}+04$

$4.33 \mathrm{E}+03$

$3.97 \mathrm{E}+03$

$1.01 \mathrm{E}+02$

2.05E +03

$2.96 \mathrm{E}+01$

$7.03 \mathrm{E}+02$

$5.99 \mathrm{E}+05$

$1.54 \mathrm{E}+02$

$7.73 \mathrm{E}+04$

$9.89 \mathrm{E}+05$

$1.71 \mathrm{E}+04$

$1.70 \mathrm{E}+02$

$5.59 \mathrm{E}+04$

$9.01 \mathrm{E}+03$

$2.34 E+03$

$2.90 \mathrm{E}+04$

90\%-ile

6.23E-03

$3.84 \mathrm{E}+04$

$1.22 \mathrm{E}-02$

$1.84 \mathrm{E}+06$

$1.40 \mathrm{E}+02$

$4.09 \mathrm{E}+02$ 
Tank Anal. Un

S106 $\mathrm{Sr} \quad \mathrm{kg} \quad \frac{\mathrm{May98}}{1.10 \mathrm{E}+02}$

$\begin{array}{llll}\text { S106 } & \text { TOC } & \mathrm{kg} & 2.49 \mathrm{E}+04 \\ \text { S106 } & \mathrm{U} & \mathrm{kg} & \mathbf{4 . 1 6 \mathrm { E } + 0 3}\end{array}$

$\begin{array}{llll}S 106 & \mathrm{Zr} & \mathrm{kg} & 1.42 \mathrm{E}+02\end{array}$

S106 Ac227 Ci 1.61E-03

S106 Am241 Ci 9.80E+02

S106 Am243 Ci 2.96E-03

$\begin{array}{llll}\mathrm{S} 106 & \mathrm{Ba} 137 & \mathrm{Ci} & 4.33 \mathrm{E}+05\end{array}$

$\begin{array}{llll}\mathrm{S} 106 & \mathrm{Cl} & \mathrm{Ci} & \mathbf{5 . 8 4 E}+01\end{array}$

$\begin{array}{llll}\$ 106 & \mathrm{Cd} 113 & \mathrm{Ci} & 1.49 \mathrm{E}+02\end{array}$

S106 Cm242 Ci 1.85E-01

$\begin{array}{llll}\mathrm{S} 106 & \mathrm{Cm} 243 & \mathrm{Ci} & 1.66 \mathrm{E}-02 \\ \mathrm{~S} 106 & \mathrm{Cm} 244 & \mathrm{Ci} & 1.86 \mathrm{E}-01\end{array}$

$\begin{array}{llll}S 106 & \mathrm{C} 060 & \mathrm{Ci} & 5.99 E+01\end{array}$

$\begin{array}{lllll}\mathrm{S} 106 & \mathrm{Cs} 134 & \mathrm{Ci} & \text { 2.92E-00 }\end{array}$

$\begin{array}{llll}\text { S106 Cs137 Ci 4.58E+05 } & \text { C }\end{array}$

$\begin{array}{llll}\text { S106 Eu152 Ci 5.34E-00 } & \end{array}$

$\begin{array}{llll}\$ 106 & \text { Eu154 } & \text { Ci } & 9.78 \mathrm{E}+02\end{array}$

S106 Eu155

S106 I129

S106 Nb93

S106 Nis9

S106 Ni63

S106 Np237

S106 Pa231

S106 Pu238

S106 Pu239

S106 Pu240

S106 Pu241

S106 Pu242

S106 Ra226

S106 Ra228
R

S106 Ru106

S106 Sb125

S106 Se79

S106 Sm151

S106 Sr89/90

S106 Tc99

S106 Th229 C

S106 Th232 Ci

S106 Tin126 Ci

S106. Tritium $\mathrm{C}$

$\begin{array}{lll}\text { S106 U232 Ci } & \end{array}$

$\begin{array}{lll}S 106 & \text { U233 } & \text { C } \\ \text { S106 } & \text { U234 }\end{array}$

S106 U234
S106
U235

S106 U236

S106 U238

$\$ 106 \quad Y 90$

S106 Zr93

$\$ 107$ A

S107

kg $7.80 \mathrm{E}+01$

$\begin{array}{llll}\mathrm{S} 107 & \mathrm{Cl} & \mathrm{kg} & \mathbf{1 . 1 9 E}+03 \\ \mathrm{Sg} & \mathrm{5.08E}+03\end{array}$

$\begin{array}{llll}\mathrm{S} 107 & \mathrm{CO} 3 & \mathrm{~kg} & 2.60 \mathrm{E}+04\end{array}$

$\begin{array}{llll}\mathrm{S} 107 & \mathrm{Cr} & \mathrm{kg} & \mathbf{8 . 6 1 E}+03\end{array}$

$\$ 107 \quad \mathrm{~F} \quad \mathrm{~kg} \cdot 3.48 \mathrm{E}+04$

$\begin{array}{llll}\$ 107 & F e & k g & 5.61 E+03\end{array}$

S107 Hg kg 6.55E+02

$\begin{array}{llll}\mathrm{S107} & \mathrm{K} & \mathrm{kg} & 1.31 \mathrm{E}+03\end{array}$

$\$ 107 \quad \mathrm{La} \quad \mathrm{kg} \quad 1.75 \mathrm{E}-00$

$\begin{array}{llll}S 107 & \mathrm{Mn} & \mathrm{kg} & 1.38 \mathrm{E}+03\end{array}$

$5107 \mathrm{Na} \quad \mathrm{kg} \quad 2.11 \mathrm{E}+05$

$\begin{array}{llll}\mathrm{S} 107 \mathrm{Ni} & \mathrm{kg} & 3.12 \mathrm{E}+03\end{array}$

$\mathrm{S} 107 \mathrm{NO2} \mathrm{kg} \quad 753 \mathrm{E}+04$

$\$ 107$ NO3 kg 1.41E+05

$\begin{array}{llll}S 107 & P & \mathrm{~kg} & 5.22 \mathrm{E}+04\end{array}$

S107 Pb
$B B$ Sept98

$\overline{1.71 \mathrm{E}+01}$

$6.11 \mathrm{E}+03$

$9.61 \mathrm{E}+02$

$1.99 \mathrm{E}+01$

1.61E-03

$1.94 \mathrm{E}+01$

$5.86 \mathrm{E}-04$

$2.66 \mathrm{E}+05$

$5.84 \mathrm{E}+01$

$1.49 \mathrm{E}+02$

3.65E-02

3.29E-03

$3.68 \mathrm{E}-02$

$6.03 E+01$

2.92E-00

$2.81 \mathrm{E}+05$

5.34E-00

$2.03 E+02$

$7.52 \mathrm{E}+02$

8.05E-01

$2.18 \mathrm{E}+01$

$3.82 \mathrm{E}-00$

$3.72 \mathrm{E}+02$

$1.64 \mathrm{E}-00$

7.24E-03

9.96E-01

$5.28 \mathrm{E}+01$

7.80E-00

$5.93 \mathrm{E}+01$

2.83E-04

2.67E-04

8.74E-02

$9.81 \mathrm{E}-03$

$2.46 \mathrm{E}+02$

6.05E-00

2.13E+04

$4.18 \mathrm{E}+04$

$4.18 \mathrm{E}+02$

2.09E-03

6.03E-03

9.15E-00

$4.78 \mathrm{E}+02$

5.82E-02

2.23E-01

3.36E-01

1.40E-02

8.49E-03

3.21E-01

$4.18 \mathrm{E}+04$

$2.96 \mathrm{E}+01$

$1.21 \mathrm{E}+05$

$7.80 \mathrm{E}+01$

$1.19 \mathrm{E}+03$

$5.08 \mathrm{E}+03$

$2.60 \mathrm{E}+04$

$8.61 \mathrm{E}+03$

$3.48 \mathrm{E}+04$

$5.61 E+03$

$1.08 E+02$

$1.31 E+03$

1.75E-00

$1.38 \mathrm{E}+03$

$2.11 E+05$

$3.12 E+03$

$7.53 \mathrm{E}+04$

$1.41 \mathrm{E}+05$

NA

$2.90 \mathrm{E}+02$
$1 \%$-ile

\section{$2.85 \mathrm{E}-02$}

$1.18 \mathrm{E}+03$

$2.03 \mathrm{E}+02$

$0.00 \mathrm{E}+01$

$0.00 \mathrm{E}+01$

6.62E-00

$0.00 \mathrm{E}+01$

$5.02 \mathrm{E}+04$

$0.00 \mathrm{E}+01$

$0.00 \mathrm{E}+01$

$0.00 \mathrm{E}+01$

$0.00 \mathrm{E}+01$

$0.00 \mathrm{E}+01$

$1.24 \mathrm{E}-00$

$3.39 \mathrm{E}-01$

$1.85 \mathrm{E}+05$

$0.00 \mathrm{E}+01$

$0.00 \mathrm{E}+01$

$0.00 \mathrm{E}+01$

$1.83 \mathrm{E}-02$

$0.00 \mathrm{E}+01$

$0.00 \mathrm{E}+01$

$0.00 \mathrm{E}+01$

$0.00 \mathrm{E}+01$

$0.00 \mathrm{E}+01$

$0.00 \mathrm{E}+01$

$0.00 \mathrm{E}+01$

$0.00 \mathrm{E}+01$

$0.00 \mathrm{E}+01$

$0.00 \mathrm{E}+01$

$0.00 \mathrm{E}+01$

$0.00 \mathrm{E}+01$

$0.00 \mathrm{E}+01$

$0.00 \mathrm{E}+01$

$0.00 \mathrm{E}+01$

$0.00 \mathrm{E}+01$

$0.00 \mathrm{E}+01$

$0.00 \mathrm{E}+01$

$0.00 \mathrm{E}+01$

$0.00 \mathrm{E}+01$

$0.00 \mathrm{E}+01$

$2.88 \mathrm{E}+02$

$0.00 E+01$

$0.00 \mathrm{E}+01$

$0.00 \mathrm{E}+01$

$0.00 \mathrm{E}+01$

$0.00 \mathrm{E}+01$

$0.00 \mathrm{E}+01$

$0.00 \mathrm{E}+01$

$0.00 \mathrm{E}+01$

$9.31 \mathrm{E}+04$

$6.77 \mathrm{E}+01$

$3.52 E+02$

$1.31 \mathrm{E}+03$

$3.47 \mathrm{E}+03$

$1.73 \mathrm{E}+03$

8.12E+03

$1.42 \mathrm{E}+03$

$3.03 \mathrm{E}+02$

$6.29 \mathrm{E}+02$

$1.31 \mathrm{E}+01$

$3.44 \mathrm{E}+02$

$1.02 \mathrm{E}+05$

$6.97 \mathrm{E}+02$

$2.18 E+04$

$2.47 \mathrm{E}+04$

$1.49 \mathrm{E}+03$

$7.60 \mathrm{E}+01$ 10\%-ile

$$
\begin{array}{rr}
2.63 \mathrm{E}+01 & 1.35 \mathrm{E}+02 \\
3.36 \mathrm{E}+03 & 6.45 \mathrm{E}+03 \\
8.16 \mathrm{E}+02 & 2.38 \mathrm{E}+03 \\
2.41 \mathrm{E}-00 & 8.82 \mathrm{E}+01 \\
0.00 \mathrm{E}+01 & 1.61 \mathrm{E}-03 \\
1.01 \mathrm{E}+01 & 2.21 \mathrm{E}+02 \\
5.52 \mathrm{E}-04 & 2.96 \mathrm{E}-03 \\
2.10 \mathrm{E}+05 & 4.33 \mathrm{E}+05
\end{array}
$$

5.14E-00

$0.00 \mathrm{E}+01$

3. $45 \mathrm{E}-02$

$3.10 \mathrm{E}-03$

3.47E-02

2.15E-00

1.42E-00

$2.41 \mathrm{E}+05$

$0.00 \mathrm{E}+01$

$0.00 \mathrm{E}+01$

$0.00 \mathrm{E}+01$

3.44E-01

3.42E-01

$0.00 \mathrm{E}+01$

$0.00 \mathrm{E}+01$

$3.06 \mathrm{E}-01$

$0.00 \mathrm{E}+01$

9.41E-01

$4.98 \mathrm{E}+0 \mathrm{I}$

7.35E-00

$5.60 \mathrm{E}+01$

2.67E-04

3.87E-05

1.27E-02

$1.91 \mathrm{E}-03$

$1.82 \mathrm{E}+01$

$1.45 \mathrm{E}-00$

$0.00 \mathrm{E}+01$

$4.52 \mathrm{E}+01$

$0.00 \mathrm{E}+01$

$0.00 \mathrm{E}+01$

$0.00 \mathrm{E}+01$

$0.00 \mathrm{E}+01$

$3.66 \mathrm{E}+02$

$0.00 \mathrm{E}+01$

$0.00 \mathrm{E}+0 \mathrm{I}$

$0.00 \mathrm{E}+0 \mathrm{I}$

$0.00 \mathrm{E}+01$

$0.00 \mathrm{E}+0 \mathrm{I}$

$0.00 E+01$

$0.00 \mathrm{E}+0$

$4.65 \mathrm{E}-0 \mathrm{I}$

$1.48 \mathrm{E}+05$

$2.29 \mathrm{E}+02$

$5.98 \mathrm{E}+02$

$1.99 \mathrm{E}+03$

$2.30 \mathrm{E}+04$

$2.43 E+03$

$1.10 \mathrm{E}+04$

$2.71 E+03$

$4.24 E+02$

$1.23 \mathrm{E}+03$

$1.97 \mathrm{E}+01$

$7.77 \mathrm{E}+02$

$1.63 E+05$

$8.39 \mathrm{E}+02$

$3.06 \mathrm{E}+04$

$5.25 \mathrm{E}+04$

$2.92 \mathrm{E}+03$

$1.04 \mathrm{E}+02$

50\%-ile

$5.84 \mathrm{E}+01$

$1.49 \mathrm{E}+02$

1.85E-01

$1.66 \mathrm{E}-02$

$1.86 \mathrm{E}-01$ 


\begin{tabular}{|c|c|c|c|c|c|c|c|c|c|c|c|}
\hline ank & I. & Un & May98 & Sept98 & $\%$-ile & p\%-ile & 50\%-ile & $90 \%$-ile & 99\%-ile & Mean & $\mathbf{v}$ \\
\hline 07 & PO & $\mathrm{kg}$ & $1 . \overline{62 \mathrm{E}+05}$ & $\overline{5.27 E+04}$ & +03 & & 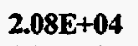 & 100 & 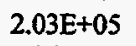 & & \\
\hline & $a$ & & $5.93 E+03$ & NA & & & & & & & \\
\hline & & & $21 E+03$ & $.21 \mathrm{E}+03$ & & & & & & & \\
\hline & & $\mathbf{k}$ & $.78 E+04$ & $93 E+03$ & $E+02$ & $E+03$ & & & $E+05$ & $E+03$ & $6 \mathrm{E}+04$ \\
\hline & & & $5.88 \mathrm{E}+02$ & $.88 \mathrm{E}+02$ & $.42 \mathrm{E}+02$ & $6 \mathrm{E}+02$ & $.62 E+02$ & $E+03$ & $2 E+04$ & & $E+03$ \\
\hline & & $\mathbf{k}$ & $.15 E+03$ & $.15 E+03$ & $2.50 \mathrm{E}+03$ & $73 E+03$ & $54 E+03$ & $E+04$ & $65 E+04$ & & \\
\hline 07 & $\mathbf{T}$ & $\mathrm{kg}$ & $1.67 E+04$ & $1.67 E+04$ & $7.46 \mathrm{E}+03$ & $20 \mathrm{E}+04$ & $12 E+04$ & $57 E+04$ & $96 E+04$ & +04 & $8+04$ \\
\hline 07 & $\mathbf{z}$ & kg & $2.37 E+04$ & $2.37 E+04$ & $E+03$ & $E+03$ & $1.32 E+04$ & $E+04$ & $7 \mathrm{E}+04$ & +04 & +03 \\
\hline & 227 & $\mathrm{Ci}$ & 2.121 & 2.12E-03 & 4 & & & & & & \\
\hline 7 & 1241 & U & $3.09 E+01$ & $3.19 \mathrm{E}+01$ & $E+01$ & $E+02$ & $4.69 \mathrm{E}+02$ & $7 E+03$ & $3.90 \mathrm{E}+03$ & 02 & \\
\hline & & & 1.1 & $1.23 \mathrm{E}-03$ & 4.73E-04 & $5 E-04$ & & $E-03$ & $2 \mathrm{E}-03$ & E-03 & $E-04$ \\
\hline & 37 & & $2.03 E+05$ & $2.03 E+05$ & $E+03$ & $3 E+04$ & & & $E+05$ & +05 & $E+04$ \\
\hline & & & 1.31 & $1.31 E+01$ & $E+01$ & $5 E-01$ & -01 & & $6 E+01$ & & $6 \mathrm{E}+01$ \\
\hline & & & $3.69 \mathrm{E}+01$ & $.69 \mathrm{E}+01$ & $E+01$ & $E+01$ & & +02 & $\mathrm{E}+02$ & $E+01$ & $E+02$ \\
\hline & 1242 & & 1.51E-01 & E-01 & $E-02$ & E-02 & & E-01 & 6E-01 & $E-01$ & $\mathrm{E}-02$ \\
\hline & 1243 & & 1.10E-02 & E-02 & $E-03$ & & & & & & -03 \\
\hline & & & 1.521 & E-01 & & & & & & & \\
\hline & & C & $3.17 \mathrm{E}$ & $\mathbf{E}+02$ & +01 & & 8.5 & 02 & & & \\
\hline & & & E-00 & E-00 & $E-02$ & E-01 & -00 & $E-00$ & $E-00$ & $E-00$ & -01 \\
\hline & & & $2.14 E+05$ & $2.14 E+05$ & $E+04$ & $E+05$ & $2.07 \mathrm{E}+05$ & $E+05$ & $E+05$ & $E+05$ & +04 \\
\hline & & & -00 & $\varepsilon-00$ & $=-00$ & & & & +01 & +01 & +01 \\
\hline & & & $2.47 \mathrm{E}+02$ & +02 & +01 & & & & +03 & & \\
\hline & N & $H_{1}$ & $2.52 \mathrm{E}+02$ & $2.52 \mathrm{E}+02$ & +01 & -02 & +02 & & +03 & +02 & +02 \\
\hline & & & 1.7 & & & & & & & & \\
\hline & & & $6.35 \mathrm{E}-00$ & -00 & +01 & & 00 & -01 & & & \\
\hline & & & $6.28 \mathrm{E}-00$ & $6.28 \mathrm{E}-00$ & $E-00$ & $E-00$ & -00 & 01 & f01 & -00 & \\
\hline & & & 5.911 & 02 & +02 & +02 & & & & & fos \\
\hline & & & 3.58E-01 & $3.58 \mathrm{E}-01$ & & & & & & & \\
\hline & & & -03 & 2.2 & & & & & & & \\
\hline & & 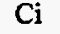 & 7.16 & & & & & & +02 & & \\
\hline & & & $2.20 \mathrm{E}+03$ & +03 & & +03 & & $\div 03$ & +03 & & Fo. \\
\hline & & C & 239 & 3.1 & +02 & & & & & & +0 \\
\hline & & & & & & & & & & & Hus. \\
\hline & & & E-02 & $8-02$ & & & & & & & \\
\hline & & & -04 & & & & & & & & \\
\hline & & & & & & & & & & & \\
\hline & & C & $3.60 \mathrm{E}-03$ & $3.60 \mathrm{E}-03$ & $E-04$ & -03 & -03 & -02 & $E-02$ & -0 & \\
\hline & 125 & C & $6.06 E+01$ & $6.06 E+01$ & $=00$ & & 01 & & & 2 & \\
\hline & & & & & & & & & & & \\
\hline & & & & & & & & & & & \\
\hline & & 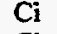 & & & & & & & & & $+0^{2}$ \\
\hline & & 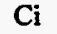 & +01 & 9.30 & +01 & & & & & & \\
\hline & 229 & C & $1.06 \mathrm{E}-03$ & $1.06 \mathrm{E}-03$ & +01 & -04 & & & 03 & & \\
\hline & & & -03 & & & & & & & & \\
\hline & & C & -00 & & & & & & & & \\
\hline & & $\mathrm{Ci}$ & & & & & & & & & \\
\hline & & & & & & & & & & & \\
\hline & & & & & & & & & & & \\
\hline & & $c$ & +01 & & & & & & & & \\
\hline & & & -01 & 01 & & & & & & & \\
\hline & & & 01 & & & & & & & & \\
\hline & & & & & & & & & & & \\
\hline & & & & & & & & & & & \\
\hline & & & & & & & & & & & \\
\hline & A & & & & & & & & & & \\
\hline & $\mathbf{B i}$ & & +02 & & & & & & & & \\
\hline & C & & $8.65 E+02$ & 8.6 & & & & & & & +02 \\
\hline & Cl & & & & & & & & & 10 & +0 \\
\hline & $\cos$ & kg & $t+04$ & $3.23 E+04$ & $:+02$ & & & $E+05$ & 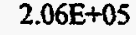 & +04 & $E+04$ \\
\hline & C & 3 & & & & & & & & +04 & \\
\hline & $\mathbf{F}$ & kg & & & & & & & & & \\
\hline & $\mathrm{Fe}$ & $\mathbf{k}$ & & & & & & & & & +02 \\
\hline & H & & & & & & & & & & -0 \\
\hline & K & & & & & & & & & & \\
\hline & L & & & & & & & & $1.22 \mathrm{E}+\mathrm{C}$ & +01 & \\
\hline & $\mathbf{M}$ & kg & & & & & 4.9 & $E+03$ & $E+03$ & $E+02$ & $E+02$ \\
\hline & Na & $\mathrm{kg}$ & $5.56 \mathrm{E}+05$ & $5.56 E \div 05$ & $3.30 \mathrm{E}+05$ & $4.27 E+05$ & $5.77 E+05$ & $7.67 \mathrm{E}+05$ & $9.25 E+05$ & $5.89 \mathrm{E}+05$ & $1.32 \mathrm{E}+05$ \\
\hline & 171 & $\mathbf{k}$ & $74 E+02$ & $.74 E+02$ & $9 E-02$ & $1.17 \mathrm{E}-00$ & $1.42 E+02$ & $5.86 \mathrm{E}+02$ & $1.01 E+03$ & $.22 \mathrm{E}+02$ & $2.51 \mathrm{E}+0$ \\
\hline
\end{tabular}


Tank Anal. Un

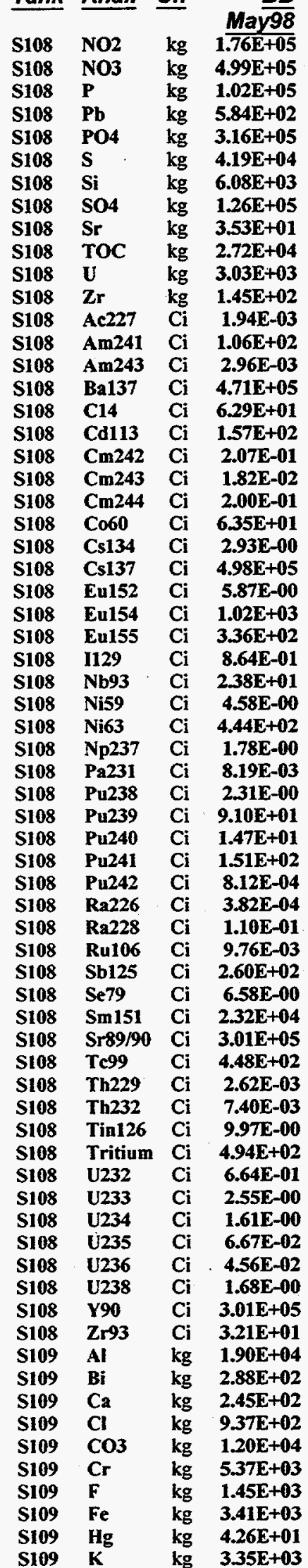

$B B$ Sept98

$\overline{1.76 \mathrm{E}+05}$ $4.99 \mathrm{E}+05$

NA

$5.84 \mathrm{E}+02$

1.03E+05

NA

$6.08 \mathrm{E}+03$

$4.19 \mathrm{E}+04$

$3.53 \mathrm{E}+01$

$2.72 \mathrm{E}+04$

$3.03 \mathrm{E}+03$

$1.45 \mathrm{E}+02$

$1.94 \mathrm{E}-03$

$1.06 \mathrm{E}+02$

$2.96 \mathrm{E}-03$

$4.71 E+05$

$6.29 \mathrm{E}+01$

$1.57 \mathrm{E}+02$

2.07E-01

1.82E-02

2.00E-01

$6.35 \mathrm{E}+01$

$2.93 \mathrm{E}-00$

$4.98 \mathrm{E}+05$

5.87E-00

$1.02 \mathrm{E}+03$

$3.36 \mathrm{E}+02$

8.64E-01

$2.38 \mathrm{E}+01$

$4.58 \mathrm{E}-00$

$4.44 \mathrm{E}+02$

1.78E-00

8.19E-03

2.31E-00

$9.10 E+01$

$1.47 \mathrm{E}+01$

$1.51 \mathrm{E}+02$

8.12E-04

3.28E-04

1.10E-01

$9.76 \mathrm{E}-03$

$2.60 \mathrm{E}+02$

6.58E-00

$2.32 \mathrm{E}+04$

$3.01 \mathrm{E}+05$

$4.48 \mathrm{E}+02$

2.62E-03

$7.40 \mathrm{E}-03$

$9.97 \mathrm{E}-00$

$4.94 \mathrm{E}+02$

4.44E-01

$1.70 \mathrm{E}-00$

$1.08 \mathrm{E}-00$

4.46E-02

3.05E-02

1.01E-00

3.01E+05

$3.21 \mathrm{E}+01$

$1.90 \mathrm{E}+04$

$3.04 \mathrm{E}+02$

$2.45 \mathrm{E}+02$

$9.37 \mathrm{E}+02$

$1.20 \mathrm{E}+04$

$5.33 \mathrm{E}+03$

$1.32 \mathrm{E}+03$

$3.41 \mathrm{E}+03$

9.44E-00

$4.66 \mathrm{E}+03$

\section{1\%-ile \\ 10\%-ile}

$8.60 \mathrm{E}+01$

$8.49 \mathrm{E}+02$

$6.71 \mathrm{E}+01$

$8.39 \mathrm{E}+02$

$5.27 \mathrm{E}+04$

$4.19 \mathrm{E}+03$

4.12E+01

$5.55 \mathrm{E}+01$

$5.76 \mathrm{E}-00$

8.49E+03

$7.20 \mathrm{E}+02$

4.94E-00

$1.93 \mathrm{E}+01$

$4.08 \mathrm{E}+01$

$5.10 \mathrm{E}+03$

3.77E-00

$1.10 \mathrm{E}+02$

$1.15 \mathrm{E}+01$

$1.76 \mathrm{E}+01$

$0.00 \mathrm{E}+01$

$0.00 \mathrm{E}+01$

$4.22 \mathrm{E}-01$

$0.00 \mathrm{E}+01$

$0.00 \mathrm{E}+01$

$0.00 \mathrm{E}+01$

$0.00 \mathrm{E}+01$

$0.00 \mathrm{E}+01$

$0.00 \mathrm{E}+01$

$0.00 \mathrm{E}+0$

$8.96 \mathrm{E}-02$

$0.00 \mathrm{E}+01$

$7.04 \mathrm{E}+02$

$0.00 \mathrm{E}+01$

$0.00 \mathrm{E}+01$

$0.00 \mathrm{E}+01$

$0.00 \mathrm{E}+01$

$0.00 \mathrm{E}+01$

$0.00 \mathrm{E}+01$

$0.00 \mathrm{E}+01$

$0.00 \mathrm{E} \div 01$

$0.00 \mathrm{E}+01$

$0.00 \mathrm{E}+01$

$0.00 \mathrm{E}+01$

$0.00 \mathrm{E}+01$

$0.00 \mathrm{E}+01$

$0.00 \mathrm{E}+01$

$0.00 \mathrm{E}+01$

$0.00 \mathrm{E}+01$

$0.00 \mathrm{E}+01$

$0.00 \mathrm{E}+01$

$0.00 \mathrm{E}+01$

$0.00 \mathrm{E}+01$

$0.00 \mathrm{E}+01$

$0.00 \mathrm{E}+01$

$0.00 \mathrm{E}+01$

$0.00 \mathrm{E}+01$

$0.00 \mathrm{E}+01$

$2.41 \mathrm{E}-00$

$0.00 \mathrm{E}+01$

$0.00 \mathrm{E}+01$

$0.00 \mathrm{E}+01$

$0.00 \mathrm{E}+01$

$0.00 \mathrm{E}+01$

$0.00 \mathrm{E}+01$

$0.00 E+01$

$0.00 \mathrm{E}+01$

$4.43 E+03$

$0.00 \mathrm{E}+01$

$9.20 \mathrm{E}-00$

$5.88 \mathrm{E}+01$

$1.59 \mathrm{E}+03$

$3.07 \mathrm{E}+02$

$0.00 \mathrm{E}+01$

$9.94 \mathrm{E}+01$

$0.00 \mathrm{E}+01$

7.91E-00
$1.31 \mathrm{E}+02$

3.04E-01

$0.00 \mathrm{E}+01$

1.24E-00

$5.18 \mathrm{E}-05$

$0.00 \mathrm{E}+01$

$3.96 \mathrm{E}-02$

$0.00 \mathrm{E}+01$

$3.62 \mathrm{E}-03$

$3.19 \mathrm{E}-04$

$3.50 \mathrm{E}-03$

$3.24 \mathrm{E}-01$

$0.00 \mathrm{E}+0$

$3.83 \mathrm{E}+03$

$0.00 \mathrm{E}+01$

$0.00 \mathrm{E}+01$

$0.00 \mathrm{E}+01$

$0.00 \mathrm{E}+01$

$4.77 \mathrm{E}-02$

$0.00 \mathrm{E}+01$

$0.00 \mathrm{E}+01$

3.12E-02

$0.00 \mathrm{E}+01$

4.04E-02

$1.59 \mathrm{E}-00$

2.57E-01

2.64E-00

1.42E-05

7.64E-06

2.20E-03

$0.00 E+01$

$0.00 \mathrm{E}+01$

6.88E-02

$0.00 \mathrm{E}+01$

$1.93 \mathrm{E}+01$

$0.00 \mathrm{E}+01$

$0.00 \mathrm{E}+0 \mathrm{I}$

$0.00 \mathrm{E}+01$

$0.00 E+01$

$4.24 \mathrm{E}+01$

$0.00 E+01$

$0.00 \mathrm{E}+01$

$0.00 \mathrm{E}+01$

$0.00 \mathrm{E}+01$

$0.00 E+01$

$0.00 E+01$

$0.00 \mathrm{E}+01$

$6.44 \mathrm{E}-02$

$7.94 \mathrm{E}+03$

$2.09 \mathrm{E}+01$

$2.67 \mathrm{E}+01$

$2.52 \mathrm{E}+02$

$3.17 \mathrm{E}+04$

$1.90 \mathrm{E}+03$

$2.86 E+0$ I

$9.63 \mathrm{E}+02$

$0.00 \mathrm{E}+01$

$4.45 \mathrm{E}+01$

\section{$50 \%$-jle}

$8.34 \mathrm{E}+04 \quad 2.04 \mathrm{E}+05$

$1.02 \mathrm{E}+06 \quad 2.19 \mathrm{E}+06$

$1.33 \mathrm{E}+04 \quad 2.33 \mathrm{E}+04$

$1.48 \mathrm{E}+02$

4.17E+04

$1.11 \mathrm{E}+04$

$1.92 \mathrm{E}+03$

$3.34 \mathrm{E}+04$

$5.65 \mathrm{E}+01$

$4.36 \mathrm{E}+03$

$1.01 \mathrm{E}+03$

$5.14 \mathrm{E}+01$

$1.94 \mathrm{E}-03$

$5.86 \mathrm{E}+01$

$2.96 \mathrm{E}-03$

$4.71 \mathrm{E}+05$

$6.29 \mathrm{E}+01$

$1.57 \mathrm{E}+02$

2.07E-01

$1.82 \mathrm{E}-02$

2.00E-01

$2.07 \mathrm{E}+01$

2.93E-00

$2.61 E+05$

5.87E-00

$1.02 \mathrm{E}+03$

$3.36 \mathrm{E}+02$

8.64E-01

$2.38 \mathrm{E}+01$

4.58E-00

$4.44 \mathrm{E}+02$

1.78E-00

8.19E-03

2.31E-00

$9.10 \mathrm{E}+01$

$1.47 \mathrm{E}+01$

$1.51 \mathrm{E}+02$

8.12E-04

3.82E-04

1.10E-01

$9.76 \mathrm{E}-03$

$2.60 \mathrm{E}+02$

6.58E-00

$2.32 E+04$

$8.98 \mathrm{E}+04$

$4.48 \mathrm{E}+02$

2.62E-03

7.40 E- 03

9.97E-00

$4.94 \mathrm{E}+02$

6.64E-01

2.55E-00

$1.61 \mathrm{E}-00$

6.67E-02

4.56E-02

1.68E-00

$3.01 \mathrm{E}+05$

$3.21 \mathrm{E}+01$

$1.74 \mathrm{E}+04$

$3.57 E+02$

$4.95 \mathrm{E}+02$

$1.22 \mathrm{E}+03$

$7.36 \mathrm{E}+04$

$4.22 \mathrm{E}+03$

$2.67 \mathrm{E}+03$

$2.57 \mathrm{E}+03$

$4.26 E+01$

$1.77 \mathrm{E}+03$

$2.63 \mathrm{E}+02$

$7.73 \mathrm{E}+04$

$2.27 \mathrm{E}+04$

$4.99 \mathrm{E}+03$

$6.48 \mathrm{E}+04$

$3.51 \mathrm{E}+02$

$1.01 E+04$

$2.91 \mathrm{E}+03$ 
Tank Anal. Un

\begin{tabular}{|c|c|}
\hline S109 & $\mathbf{L a}$ \\
\hline$\$ 109$ & $\mathbf{M n}$ \\
\hline$\$ 109$ & $\mathbf{N a}$ \\
\hline S109 & $\mathbf{N i}$ \\
\hline \$109 & NO2 \\
\hline S109 & NO3 \\
\hline S109 & $\mathbf{P}$ \\
\hline S109 & $\mathbf{P b}$ \\
\hline S109 & PO4 \\
\hline S109 & $\mathbf{S}$ \\
\hline S109 & Si \\
\hline S109 & SO4 \\
\hline S109 & $\mathbf{S r}$ \\
\hline$\$ 109$ & TOC \\
\hline S109 & $\mathbf{U}$ \\
\hline S109 & $\mathbf{Z r}$ \\
\hline S109 & Ac227 \\
\hline$\$ 109$ & Am241 \\
\hline S109 & Am243 \\
\hline S109 & Ba137 \\
\hline S109 & C14 \\
\hline S109 & Cd113 \\
\hline S109 & Cm242 \\
\hline S109 & Cm243 \\
\hline S109 & Cm244 \\
\hline S109 & $\mathrm{Co60}$ \\
\hline S109 & Cs134 \\
\hline S109 & Cs137 \\
\hline S109 & Eu152 \\
\hline S109 & Eu154 \\
\hline S109 & Eu155 \\
\hline S109 & 1129 \\
\hline S109 & $\mathbf{N b 9 3}$ \\
\hline S109 & Ni59 \\
\hline S109 & Ni63 \\
\hline S109 & Np237 \\
\hline \$109 & Pa231 \\
\hline S109 & Pu238 \\
\hline S109 & Pu239 \\
\hline S109 & Pu240 \\
\hline S109 & Pu241 \\
\hline S109 & Pu242 \\
\hline S109 & Ra226 \\
\hline \$109 & Ra228 \\
\hline S109 & Rul06 \\
\hline S109 & Sb125 \\
\hline S109 & Se79 \\
\hline S109 & Sm151 \\
\hline$\$ 109$ & Sr89/90 \\
\hline S109 & Te99 \\
\hline$\$ 109$ & Th229 \\
\hline S109 & Th232 \\
\hline S109 & Tin126 \\
\hline$\$ 109$ & Tritium \\
\hline S109 & U232 \\
\hline S109 & U233 \\
\hline$\$ 109$ & U234 \\
\hline S109 & U235 \\
\hline S109 & U236 \\
\hline S109 & U238 \\
\hline S109 & Y90 \\
\hline$\$ 109$ & Zr93 \\
\hline S110 & Al \\
\hline$\$ 110$ & Bi \\
\hline S110 & Ca \\
\hline S110 & $\mathrm{Cl}$ \\
\hline & \\
\hline
\end{tabular}

May $\frac{B B}{98}$ $4 . \overline{4.00 \mathrm{E}-03}$ $5.44 \mathrm{E}+01$ $6.25 \mathrm{E}+05$ $6.67 \mathrm{E}+02$ $1.14 E+04$ $1.47 \mathrm{E}+06$ $3.06 \mathrm{E}+04$ $1.48 \mathrm{E}+03$ $9.47 \mathrm{E}+04$ $2.00 \mathrm{E}+04$ $9.77 \mathrm{E}+02$ $6.01 \mathrm{E}+04$ 8.41E-04 $1.51 \mathrm{E}+03$ $1.42 \mathrm{E}+02$ $8.72 \mathrm{E}+01$ 1.82E-03 $1.06 \mathrm{E}+02$ $3.05 \mathrm{E}-03$ $1.00 \mathrm{E}+05$ $6.38 \mathrm{E}+01$ $1.57 \mathrm{E}+02$ 2.10E-01 1.89E-02 2.08E-01 $6.52 \mathrm{E}+01$ 2.86E-00 $1.06 \mathrm{E}+05$ 5.84E-00 $1.04 \mathrm{E}+03$ $3.36 \mathrm{E}+02$ 8.75E-01 $2.36 \mathrm{E}+01$ 4.28E-00 $4.16 \mathrm{E}+02$ 1.78E-00 8.07E-03 3.46E-00 $1.61 \mathrm{E}+02$ $2.47 \mathrm{E}+01$ 2.16E-00 Ci 1.09E-03 3.01E-04 Ci 1.11E-01 1.02E-02 $2.69 \mathrm{E}+02$ Ci 6.54E-00 $2.31 \mathrm{E}+04$ $2.75 \mathrm{E}+05$ $4.54 \mathrm{E}+02$ 2.65E-03 7.51E-03 9.90E-00 $4.90 \mathrm{E}+02$ $6.76 \mathrm{E}-01$ 2.59E-00

Ci 2.30E-00

$\mathrm{Ci}$ 9.60E-02

Ci 6.11E-02

Ci 2.35E-00

Ci 2.75E+05

Ci 3.19E+01

kg 1.12E+05

$\mathrm{kg} \quad 1.72 \mathrm{E}+02$

kg $\quad 6.86 \mathrm{E}+02$

$\mathrm{kg} \quad 8.22 \mathrm{E}+03$

gg $2.77 \mathrm{E}+04$

$\mathrm{kg} \quad 1.04 \mathrm{E}+04$
BB

Sept98

$5.44 \mathrm{E}+01$

$6.24 \mathrm{E}+05$

$6.84 \mathrm{E}+02$

$1.14 \mathrm{E}+04$

$1.45 \mathrm{E}+06$

NA

$1.50 \mathrm{E}+03$

3.09E+04

NA

$9.77 E+02$

$2.00 \mathrm{E}+04$

$0.00 E+01$

$1.51 \mathrm{E}+03$

$1.42 \mathrm{E}+02$

$2.73 \mathrm{E}+01$

1.82E-03

6.63E-00

$1.91 \mathrm{E}-04$

$1.00 \mathrm{E}+05$

$6.38 \mathrm{E}+01$

$1.57 \mathrm{E}+02$

1.32E-02

1.18E-03

1.30E-02

$6.52 \mathrm{E}+01$

2.86E-00

$1.06 \mathrm{E}+05$

5.84E-00

$1.04 \mathrm{E}+03$

$3.36 \mathrm{E}+02$

8.75E-01

$2.36 \mathrm{E}+01$

4.28E-00

$4.16 \mathrm{E}+02$

1.78E-00

8.07E-03

2.17E-01

$1.01 \mathrm{E}+01$

$1.55 \mathrm{E}-00$

$1.35 \mathrm{E}+01$

6.82E-05

3.01E-04

1.11E-01

1.02E-02

$2.69 \mathrm{E}+02$

6.54E-00

$2.31 \mathrm{E}+04$

$2.75 E+05$

$4.54 \mathrm{E}+02$

2.65E-03

7.51E-03

9.90E-00

$4.90 \mathrm{E}+02$

$1.47 \mathrm{E}-02$

5.63E-02

5.00E-02

2.08E-03

1.33E-03

4.74E-02

$2.75 E+05$

$3.19 E+01$

$1.12 \mathrm{E}+05$

$1.78 \mathrm{E}+02$

$6.86 \mathrm{E}+02$

$8.22 \mathrm{E}+03$

$2.81 \mathrm{E}+04$

$1.04 \mathrm{E}+04$
$1 \%$-ile

4.08E-01

2.95E-00

3.37E +05

$7.26 \mathrm{E}-01$

$2.41 E+02$

$8.32 \mathrm{E}+05$

$1.48 \mathrm{E}+03$

2.42E-00

$1.88 \mathrm{E}+03$

$4.93 \mathrm{E}+01$

$1.77 \mathrm{E}+02$

$7.75 \mathrm{E}-02$

$5.73 E+01$

$5.18 \mathrm{E}+01$

$0.00 \mathrm{E}+01$

$0.00 \mathrm{E}+01$

$2.86 \mathrm{E}-00$

$0.00 \mathrm{E}+01$

$0.00 \mathrm{E}+01$

$0.00 \mathrm{E}+01$

$0.00 \mathrm{E}+01$

$0.00 \mathrm{E}+01$

$0.00 \mathrm{E}+01$

$0.00 \mathrm{E}+01$

$3.94 \mathrm{E}-01$

$0.00 \mathrm{E}+01$

$2.45 \mathrm{E}+03$

$0.00 \mathrm{E}+01$

$0.00 \mathrm{E}+01$

$0.00 \mathrm{E}+01$

$0.00 \mathrm{E}+01$

$0.00 \mathrm{E}+01$

$0.00 E+01$

$0.00 \mathrm{E}+01$

$0.00 \mathrm{E}+01$

$0.00 \mathrm{E}+01$

$0.00 \mathrm{E}+01$

$0.00 \mathrm{E}+01$

$0.00 \mathrm{E}+01$

$0.00 \mathrm{E}+01$

$0.00 \mathrm{E}+01$

$0.00 E \div 01$

$0.00 \mathrm{E}+01$

$0.00 \mathrm{E}+01$

$0.00 E+01$

$0.00 \mathrm{E}+01$

$0.00 \mathrm{E}+01$

$0.00 \mathrm{E}+01$

$0.00 \mathrm{E}+01$

$0.00 \mathrm{E}+01$

$0.00 \mathrm{E}+01$

$0.00 \mathrm{E}+01$

$1.71 \mathrm{E}+02$

$0.00 \mathrm{E}+01$

$0.00 \mathrm{E}+01$

$0.00 \mathrm{E}+01$

$0.00 \mathrm{E}+01$

$0.00 \mathrm{E}+01$

$0.00 \mathrm{E}+01$

$0.00 \mathrm{E}+01$

$0.00 \mathrm{E}+01$

$4.70 \mathrm{E}+04$

$4.60 \mathrm{E}-02$

$9.30 \mathrm{E}+01$

$1.64 E+02$

$8.73 \mathrm{E}+03$

$2.14 \mathrm{E}+02$
$10 \%$-ile

8.74E-01

$8.45 \mathrm{E}+01$

$4.46 \mathrm{E}+05$

4.34E-00

$2.32 E+03$

$1.12 \mathrm{E}+06$

$5.56 \mathrm{E}+03$

$4.59 \mathrm{E}+01$

$1.55 \mathrm{E}+04$

$2.96 \mathrm{E}+03$

$1.22 \mathrm{E}+02$

$9.52 \mathrm{E}+03$

9.90E-00

$4.04 \mathrm{E}+02$

$3.49 \mathrm{E}+02$

$1.01 \mathrm{E}-00$

$0.00 \mathrm{E}+01$

4.27E-00

4.19E-04

$1.08 \mathrm{E}+04$

3.69E-00

$0.00 \mathrm{E}+01$

2.88E-02

2.60E-03

2.86E- 02

8.91E-01

3.08E-01

$6.94 \mathrm{E}+03$

$0.00 \mathrm{E}+01$

$0.00 \mathrm{E}+01$

$0.00 \mathrm{E}+01$

7.33E-02

2.44E-01

$0.00 \mathrm{E}+01$

$0.00 \mathrm{E}+01$

2.44E-01

$0.00 \mathrm{E}+01$

4.75E-01

$2.21 E+01$

$3.39 \mathrm{E}-00$

2.97E-01

1.50E-04

2.66E-05

9.82E-03

$7.89 \mathrm{E}-04$

$0.00 E+01$

$1.47 \mathrm{E}-00$

$0.00 \mathrm{E}+01$

$0.00 \mathrm{E}+01$

$1.83 \mathrm{E}-00$

$0.00 E+01$

$0.00 \mathrm{E}+01$

$0.00 \mathrm{E}+01$

$2.93 \mathrm{E}+02$

$0.00 \mathrm{E}+01$

$0.00 \mathrm{E}+01$

$0.00 \mathrm{E}+01$

$0.00 E+01$

$0.00 \mathrm{E}+01$

$0.00 \mathrm{E} \div 01$

$0.00 \mathrm{E}+01$

$3.30 \mathrm{E}-01$

$6.89 \mathrm{E}+04$

$1.44 \mathrm{E}+02$

$2.20 \mathrm{E}+02$

$1.33 \mathrm{E}+03$

$2.54 \mathrm{E}+04$

$1.68 \mathrm{E}+03$

$50 \%$-ile

$2.55 \mathrm{E}+01$

$3.77 \mathrm{E}+02$

$5.91 E+05$

$1.17 \mathrm{E}+02$

$1.92 \mathrm{E}+04$

$1.50 \mathrm{E}+06$

$1.14 \mathrm{E}+04$

1.34E+02

$3.54 \mathrm{E}+04$

8.03E+03 


\begin{tabular}{|c|c|c|c|c|c|c|c|c|c|c|c|}
\hline$n$ & nal. & $\underline{U n}$ & May $\frac{B 8}{B B}$ & Sept98 & $1 \%$-ile & $10 \%$-ile & 50\%-ile & $90 \%$-ile & 99\%-ile & Mean & Std Dev \\
\hline 10 & F & $\mathrm{kg}$ & $7 \overline{73 E+03}$ & $\overline{7.73 E+03}$ & $1.29 \mathrm{E}-00$ & $1.97 \mathrm{E}+02$ & $4.75 E+03$ & ULive & $2.02 E+04$ & $E+03$ & $4.70 E+03$ \\
\hline 10 & $\mathrm{Fe}$ & kg & $4.02 \mathrm{E}+03$ & $4.02 E+03$ & $3.55 \mathrm{E}+02$ & $E+03$ & $E+03$ & $E+04$ & $\mathrm{E}+04$ & & $E+03$ \\
\hline S110 & $\mathbf{H g}$ & kg & $7.58 \mathrm{E}+01$ & $1.74 E+01$ & $4.70 \mathrm{E}-01$ & & $58 E+01$ & $2.75 \mathrm{E}+02$ & $.81 E+02$ & $.26 \mathrm{E}+02$ & $1.47 \mathrm{E}+02$ \\
\hline 10 & $\mathbf{K}$ & $\mathrm{kg}$ & $2.15 \mathrm{E}+03$ & $2.15 E+03$ & 91E+01 & $3.31 E+02$ & $1.53 E+03$ & $4.41 \mathrm{E}+03$ & $.48 \mathrm{E}+03$ & $2.05 E+03$ & $1.73 E+03$ \\
\hline 10 & La & $\mathrm{kg}$ & $6.40 \mathrm{E}+01$ & $6.40 \mathrm{E}+01$ & $4.46 \mathrm{E}-00$ & $.21 E-00$ & $45 E+01$ & $5.40 \mathrm{E}+01$ & 8.71E+01 & $2.82 \mathrm{E}+01$ & $1.96 \mathrm{E}+01$ \\
\hline S110 & Mn & $\mathrm{kg}$ & $2.26 \mathrm{E}+03$ & $2.26 E+03$ & $4.88 \mathrm{E}+01$ & $4.39 \mathrm{E}+02$ & $.58 E+03$ & $3.20 \mathrm{E}+03$ & $7.47 \mathrm{E}+03$ & $1.79 \mathrm{E}+03$ & $3 E+03$ \\
\hline 10 & $\mathrm{Na}$ & $\mathrm{kg}$ & $3.77 E+05$ & $3.77 E+05$ & $2.38 \mathrm{E}+05$ & $3.04 E+05$ & $95 E+05$ & $5.02 \mathrm{E}+05$ & $6.02 E+05$ & +05 & $E+04$ \\
\hline & $\mathbf{N i}$ & $\mathbf{k g}$ & 3.52E +02 & $3.52 \mathrm{E}+02$ & $2.91 \mathrm{E}-00$ & $4 E+01$ & $11 E+02$ & & $57 E+03$ & $E+02$ & $E+02$ \\
\hline & NO2 & kg & $19 E+05$ & $1.19 \mathrm{E}+05$ & $2.90 \mathrm{E}+03$ & $1.54 \mathrm{E}+04$ & $1 E+04$ & 05 & $1.90 \mathrm{E}+05$ & +04 & $E+04$ \\
\hline 10 & 3 & & $3.68 \mathrm{E}+05$ & $3.68 \mathrm{E}+05$ & $1.33 E+03$ & $1.34 \mathrm{E}+05$ & $3 E+05$ & $1.26 \mathrm{E}+06$ & $6 E+06$ & $\div 05$ & $E+05$ \\
\hline & $\mathbf{P}$ & & $5.52 \mathrm{E}+04$ & NA & $1.13 E+03$ & $4.59 \mathrm{E}+03$ & & $E+04$ & $2 E+04$ & & $7 E+03$ \\
\hline & $\mathbf{P b}$ & & $3.36 \mathrm{E}+02$ & $3.36 \mathrm{E}+02$ & $1.20 \mathrm{E}+01$ & $4.64 \mathrm{E}+01$ & $5 \mathrm{E}+02$ & $E+02$ & $19 E \div 03$ & $E+02$ & $2 E+02$ \\
\hline $\mathbf{S 1 1 0}$ & PO4 & $\mathrm{kg}$ & $1.71 \mathrm{E}+05$ & $5.58 \mathrm{E}+04$ & $1.11 E+03$ & & $3.47 \mathrm{E}+04$ & & $1.24 \mathrm{E} \div 05$ & $E+04$ & $9 E+04$ \\
\hline S110 & $\mathbf{S}$ & kg & 2.33E+04 & NA & $E+02$ & $E+03$ & $0 E+03$ & $E+04$ & $6 E+04$ & +03 & $E+03$ \\
\hline & $\mathrm{Si}$ & kg & $4.28 \mathrm{E}+03$ & $4.28 \mathrm{E}+03$ & $1.34 E+02$ & $7.42 E+02$ & $51 \mathrm{E}+03$ & $7.47 E+03$ & $1.73 E+04$ & $E+03$ & $\mathrm{E}+03$ \\
\hline 10 & so & $\mathrm{kg}$ & $6.99 \mathrm{E}+04$ & $2.33 E+04$ & $E+02$ & $E+03$ & $2.01 \mathrm{E}+04$ & & $7 \mathrm{E}+04$ & & \\
\hline & Sr & $\mathrm{kg}$ & $3.80 \mathrm{E}+02$ & $3.80 \mathrm{E}+02$ & -00 & +01 & $3 E+02$ & +02 & $E+03$ & +02 & +03 \\
\hline & 0 & & $1.58 \mathrm{E}+04$ & $1.58 \mathrm{E}+04$ & +02 & $E+03$ & $2 E+03$ & +03 & $E+04$ & -03 & \\
\hline & $\mathbf{U}$ & & $8.21 E+03$ & $8.21 E+03$ & +02 & $E+03$ & $E+03$ & +04 & $E+04$ & & +03 \\
\hline 10 & $\mathbf{Z r}$ & & $1.34 \mathrm{E}+02$ & $1.34 \mathrm{E}+02$ & & $1 E-00$ & $\mathrm{DE}+02$ & +03 & & +02 & \\
\hline 10 & Ac227 & $\mathrm{Ci}$ & 2.58E-03 & 2.58E-03 & $E+01$ & $E+01$ & $2.58 \mathrm{E}-03$ & $E-02$ & -02 & & \\
\hline & Am241 & $\mathrm{Ci}$ & $6.39 \mathrm{E}+01$ & $6.39 \mathrm{E}+01$ & +01 & $3 E+01$ & & +04 & $1 E+04$ & +03 & +03 \\
\hline & $\mathrm{Am} 243$ & $\mathrm{Ci}$ & $2.10 \mathrm{E}-03$ & $2.10 \mathrm{E}-03$ & & & & & $1 E-03$ & & -03 \\
\hline & Ba1: & $\mathrm{Ci}$ & $3.08 \mathrm{E}+05$ & 3.08 & 01 & .04 & & & -05 & & \\
\hline 10 & C14 & $\mathrm{Ci}$ & $3.93 E+01$ & $3.93 E+01$ & +01 & $E-00$ & & +01 & $E+02$ & & +01 \\
\hline S110 & Cd113 & $\mathrm{Ci}$ & 9.791 & $9.79 E+01$ & $E-01$ & $E+01$ & $9.79 E+01$ & +02 & +03 & & \\
\hline & & $\mathrm{Ci}$ & 1.98E-01 & $1.98 \mathrm{E}-01$ & & $\mathrm{E}-02$ & & $E-01$ & E-01 & & -01 \\
\hline $\mathbf{s} 1$ & Cm243 & $\mathrm{Ci}$ & $1.46 \mathrm{E}-02$ & $1.46 \mathrm{E}-02$ & +01 & $E-03$ & & $E-02$ & & & \\
\hline & $\mathrm{Cm} 2$ & $\mathbf{C i}$ & $1.40 \mathrm{E}-01$ & $1.40 \mathrm{E}-01$ & & & & & -01 & & \\
\hline 10 & Co60 & $\mathrm{Ci}$ & $4.20 \mathrm{E}+01$ & $4.20 \mathrm{E}+01$ & .00 & .00 & -01 & +01 & $E+01$ & 01 & +01 \\
\hline S110 & Cs134 & $\mathrm{Ci}$ & 2.13E-00 & $2.13 \mathrm{E}-00$ & +01 & & & -00 & -00 & & -00 \\
\hline S110 & C & $\mathbf{C i}$ & $3.26 \mathrm{E}+05$ & $3.26 \mathrm{E}+05$ & +04 & 04 & 05 & & -05 & & \\
\hline S110 & Eu152 & $\mathrm{Ci}$ & $6.24 \mathrm{E}-00$ & $6.24 \mathrm{E}-00$ & $\$+01$ & +01 & & -01 & +01 & & +01 \\
\hline 0 & Eu154 & $\mathbf{C i}$ & $6.74 E+02$ & $6.74 E+02$ & & $E+01$ & & & -03 & & +03 \\
\hline & & $\mathrm{Ci}$ & $3.38 \mathrm{E}+02$ & +02 & & 01 & & & -03 & & +02 \\
\hline 10 & I129 & $\mathbf{C i}$ & 5.39E-01 & 5.39 & & & & & & & \\
\hline S110 & Nb93 & $\mathbf{C i}$ & $1.39 E+01$ & $1.39 \mathrm{E}+01$ & +01 & $8-01$ & 1.3 & -01 & $E+02$ & & +01 \\
\hline & & $\mathbf{C i}$ & $7.40 \mathrm{E}-00$ & $7.40 \mathrm{E}-00$ & +0.1 & $E-00$ & & & $E+02$ & & +01 \\
\hline S110 & Ni63 & $\mathrm{Ci}$ & $7.01 E+02$ & $7.01 E+02$ & +01 & $\varepsilon+02$ & & -03 & 04 & & +03 \\
\hline & Np23 & $\mathrm{Ci}$ & $1.05 \mathrm{E}-00$ & $1.05 \mathrm{E}-00$ & & & & .00 & & & \\
\hline & & $\mathbf{C i}$ & 4.7 & & & & & & & & \\
\hline & & & 5.93E-00 & 5.93 & & & & & -01 & & \\
\hline$\$ 110$ & Pu239 & $\mathbf{C i}$ & $3.54 \mathrm{E}+02$ & $3.54 \mathrm{E}+02$ & +01 & $E+01$ & & & +03 & +02 & +02 \\
\hline & Pu240 & $\mathrm{Ci}$ & $5.16 \mathrm{E}+01$ & $5.16 E+01$ & +01 & & & -02 & $E+02$ & 101 & +01 \\
\hline & Pu241 & $\mathrm{Ci}$ & $3.56 \mathrm{E}+02$ & $3.56 \mathrm{E}+02$ & & & & +02 & & +02 & +02 \\
\hline & Pu242 & $\mathrm{Ci}$ & $1.67 \mathrm{E}-03$ & & & & & & & & \\
\hline & & $c$ & 04 & 5. & & & & & & & \\
\hline 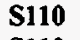 & $\mathbf{R}$ & $\mathrm{Ci}$ & $1.23 \mathrm{E}-01$ & 1.23 & & & & & & 01 & -01 \\
\hline & Rul06 & $\mathrm{Ci}$ & $7.10 \mathrm{E}-03$ & $7.10 \mathrm{E}-03$ & +01 & -03 & 03 & $E-02$ & -02 & -03 & -03 \\
\hline & Sbl25 & $\mathrm{Ci}$ & $1.77 \mathrm{E}+02$ & $1.77 \mathrm{E}+02$ & +01 & & & & & & +03 \\
\hline $\mathbf{S 1 1 0}$ & Se79 & $\mathrm{Ci}$ & $3.89 \mathrm{E}-00$ & $3.89 \mathrm{E}-00$ & $E+01$ & $E-01$ & $39 E-00$ & -00 & $E+01$ & -00 & $E-00$ \\
\hline S110 & Sm151 & $\mathrm{Ci}$ & $1.37 E+04$ & 1.37 & & & & & & & +04 \\
\hline & & $\mathrm{Ci}$ & & & & & & & & & \\
\hline & & $\mathrm{Ci}$ & & & & & & & & & +02 \\
\hline & & C & & & & & & & & & \\
\hline & $\mathbf{T}$ & $\mathrm{Ci}$ & 8.25 & 8.25 & & 9 & & -02 & .02 & & -02 \\
\hline $\mathbf{S 1 1 0}$ & Tin126 & $\mathbf{C i}$ & $5.88 \mathrm{E}-00$ & $5.88 \mathrm{E}-00$ & $=-02$ & $1.81 E-00$ & $5.88 \mathrm{E}-00$ & $E+01$ & $6 \mathrm{E}+01$ & -00 & $E+01$ \\
\hline$\$ 110$ & Tritium & $\mathrm{Ci}$ & $2.88 \mathrm{E}+02$ & $2.88 E+02$ & & $E+02$ & $2.88 E+02$ & $E+02$ & $E+02$ & +02 & +02 \\
\hline$\$ 110$ & U232 & $\mathrm{Ci}$ & & & & $E-02$ & & & $8 E-00$ & & \\
\hline & & $\mathrm{Ci}$ & & & & & & & & & \\
\hline & & $\mathrm{Ci}$ & $2.23 \mathrm{E}-60$ & 2.88 & & & & & $E+01$ & & \\
\hline & & C & $9.33 \mathrm{E}-02$ & $1.20 \mathrm{E}-01$ & & & & $E-01$ & & -01 & E-01 \\
\hline & & C & 5.63E-02 & $7.26 \mathrm{E}-02$ & & & $5.63 \mathrm{E}-02$ & & E-01 & -02 & $E-02$ \\
\hline & & $\mathrm{Ci}$ & $2.29 \mathrm{E}-00$ & $2.74 \mathrm{E}$ & & & 2.291 & & 01 & -00 & $E-00$ \\
\hline \$110 & Y90 & $\mathrm{Ci}$ & $4.05 E+05$ & $4.05 E+05$ & $0.00 \mathrm{E}+01$ & $0.00 \mathrm{E}+01$ & $4.05 E+05$ & $4.47 E+06$ & $3.03 E+07$ & +06 & $E+07$ \\
\hline 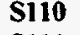 & Zr93 & $\mathrm{Ci}$ & $1.90 \mathrm{E}+01$ & $1.90 \mathrm{E}+01$ & $0.00 \mathrm{E}+01$ & EE-01 & $1.90 \mathrm{E}+01$ & $E+02$ & $4.56 \mathrm{E}+02$ & $E+01$ & $E+01$ \\
\hline S111 & Al & kg & $2.54 \mathrm{E}+05$ & $2.54 \mathrm{E}+05$ & & $8.09 E+04$ & $1.09 E+05$ & $1.37 E+05$ & $1.63 E+05$ & $E+05$ & $2.27 \mathrm{E}+04$ \\
\hline Sin & $\mathbf{B i}$ & kg & $74 F+02$ & $1.74 \mathrm{E}+02$ & $.00 \mathrm{E}+01$ & $1.08 \mathrm{E}+02$ & $5.41 \mathrm{E}+02$ & $8.85 \mathrm{E}+03$ & $2.26 \mathrm{E}+04$ & $2.54 \mathrm{E}+03$ & $5.01 E+03$ \\
\hline
\end{tabular}


Tank Anal. Un

\begin{tabular}{|c|c|}
\hline S111 & $\mathrm{Ca}$ \\
\hline S111 & Cl \\
\hline S111 & $\mathrm{CO3}$ \\
\hline S111 & $\mathrm{Cr}$ \\
\hline S111 & $\mathbf{F}$ \\
\hline S111 & $\mathrm{Fe}$ \\
\hline $\begin{array}{l}\text { S111 } \\
\text { S111 }\end{array}$ & Hg \\
\hline $\begin{array}{l}\text { S111 } \\
\text { S111 }\end{array}$ & $\mathbf{K}$ \\
\hline S111 & $\mathbf{L a}$ \\
\hline $\begin{array}{l}\text { S111 } \\
\text { S111 }\end{array}$ & Mn \\
\hline $\begin{array}{l}\text { S111 } \\
\text { S111 }\end{array}$ & $\mathrm{Na}$ \\
\hline S111 & $\mathbf{N i}$ \\
\hline $\begin{array}{l}\text { S111 } \\
\text { S111 }\end{array}$ & NO2 \\
\hline $\begin{array}{l}\text { S111 } \\
\text { S111 }\end{array}$ & NO3 \\
\hline S111 & $\mathbf{P}$ \\
\hline s111 & $\mathbf{P b}$ \\
\hline S111 & PO4 \\
\hline S111 & $\mathrm{s}$ \\
\hline $\begin{array}{l}\text { S111 } \\
\text { S111 }\end{array}$ & $\mathbf{S i}$ \\
\hline $\begin{array}{l}\text { S111 } \\
\text { S111 }\end{array}$ & SO4 \\
\hline S111 & $\mathrm{Sr}$ \\
\hline 5111 & TOC \\
\hline $\begin{array}{l}\text { S111 } \\
\text { S111 }\end{array}$ & $\mathbf{U}$ \\
\hline $\begin{array}{l}\text { S111 } \\
\text { S111 }\end{array}$ & $\mathbf{Z r}$ \\
\hline S111 & Ac227 \\
\hline S111 & Am241 \\
\hline S111 & Am243 \\
\hline S111 & Bal37 \\
\hline S111 & $\mathrm{C14}$ \\
\hline S111 & Cd113 \\
\hline S111 & $\mathrm{Cm} 242$ \\
\hline S111 & $\mathrm{Cm} 243$ \\
\hline S111 & Cm244 \\
\hline S111 & Co60 \\
\hline S111 & Cs134 \\
\hline S111 & Cs137 \\
\hline S111 & Eu152 \\
\hline S111 & Eu154 \\
\hline S111 & Eu155 \\
\hline S111 & 1129 \\
\hline S111 & Nb93 \\
\hline sil1 & Ni59 \\
\hline S111 & Ni63 \\
\hline S111 & Np237 \\
\hline S111 & Pa231 \\
\hline S111 & Pu238 \\
\hline S111 & Pu239 \\
\hline S111 & Pu240 \\
\hline S111 & Pu241 \\
\hline S111 & Pu242 \\
\hline S111 & Ra226 \\
\hline S111 & Ra228 \\
\hline S111 & Rul06 \\
\hline S111 & Sb125 \\
\hline S111 & Se79 \\
\hline S111 & Sm151 \\
\hline s111 & Sr89/90 \\
\hline S111 & Tc99 \\
\hline S111 & Th229 \\
\hline S111 & Th232 \\
\hline s111 & $\operatorname{Tin} 126$ \\
\hline S111 & Tritium \\
\hline S111 & U232 \\
\hline S111 & U233 \\
\hline S111 & U234 \\
\hline S111 & U235 \\
\hline S111 & U2 \\
\hline & \\
\hline
\end{tabular}

May 98 $4 . \overline{97 \mathrm{E}+02}$ kg $9.00 \mathrm{E}+03$ kg $4.31 E+04$ 1.51E+04 kg 2.39E+03 kg $5.75 E+02$ kg $3.97 \mathrm{E}+01$ kg 2.33E+03 kg $9.80 \mathrm{E}+01$ kg $1.51 \mathrm{E}+02$ 5.81E+05 kg 1.01E+02 gg $9.19 \mathrm{E}+04$ kg $7.07 \mathrm{E}+05$ kg $2.50 \mathrm{E}+04$ kg $1.41 \mathrm{E}+02$ kg $7.75 \mathrm{E}+04$ kg $5.20 \mathrm{E}+04$ kg $7.45 \mathrm{E}+02$ kg $1.56 \mathrm{E}+05$ kg 2.32E+02 $6.60 \mathrm{E}+03$ kg $\quad 6.39 \mathrm{E}+02$ kg $1.50 \mathrm{E}+01$ 3.08E-03 $2.53 E+03$ $3.75 \mathrm{E}-03$ $3.96 \mathrm{E}+05$ $7.15 E+01$ $1.82 \mathrm{E}+02$ 2.87E-01 2.34E-02 2.43E-01

$7.49 \mathrm{E}+01$ $3.91 \mathrm{E}-00$ 4.18E+05 $8.64 \mathrm{E}-00$ $1.22 \mathrm{E}+03$ $4.82 \mathrm{E}+02$ 9.84E- 01 $2.62 \mathrm{E}+01$ $8.27 \mathrm{E}-00$ $7.91 \mathrm{E}+02$ $1.96 \mathrm{E}-00$ 8.81E-03

5.34E-00 $2.81 \mathrm{E}+02$ $4.22 \mathrm{E}+01$ $3.32 \mathrm{E}+02$

Ci 1.65E-03

Ci 5.80E-04

$1.54 \mathrm{E}-01$

$1.25 \mathrm{E}-02$ $3.13 \mathrm{E}+02$

$7.29 \mathrm{E}-00$

$7.29 \mathrm{E}-00$

$5.12 \mathrm{E}+04$

$5.11 \mathrm{E}+02$

$3.66 \mathrm{E}-03$

$1.08 \mathrm{E}-02$

$1.10 \mathrm{E}+01$

$5.56 \mathrm{E}+02$

8.69E-01

3.33E-00

2.32E-00

$9.64 \mathrm{E}-02$

6.40E-02

2.42E-00 $\frac{\text { Sept } \frac{B B}{4978+02}}{497}$

$9.00 \mathrm{E}+03$

$4.31 E+04$

$1.51 \mathrm{E}+04$

$2.39 \mathrm{E}+03$

$5.75 E+02$

8.72E-00

$2.33 E+03$

$9.80 \mathrm{E}+01$

$1.51 \mathrm{E}+02$

$5.81 E+05$

$1.01 \mathrm{E}+02$

$9.19 E+04$

7.07E+05 NA

$1.41 \mathrm{E}+02$

$2.53 \mathrm{E}+04$

NA

$7.45 \mathrm{E}+02$

$5.20 \mathrm{E}+04$

$2.32 \mathrm{E}+02$

$6.60 \mathrm{E}+03$

$6.39 \mathrm{E}+02$

$1.50 \mathrm{E}+01$

3.08E-03

2.53E+03

7.95E-02

$3.96 \mathrm{E}+05$

$7.15 \mathrm{E}+01$

$1.82 \mathrm{E}+02$

$6.09 \mathrm{E}-00$

4.98E-01

5.15E-00

$7.49 \mathrm{E}+01$

$3.91 \mathrm{E}-00$

4.18E+05

8.64E-00

$1.22 \mathrm{E}+03$

4.82E+02

9.84E-01

2.62E+01

8.27E-00

$7.91 \mathrm{E}+02$

$1.96 \mathrm{E}-00$

8.81E-03

5.34E-00

$2.81 \mathrm{E}+02$

$4.22 \mathrm{E}+01$

$3.32 \mathrm{E}+02$

$1.65 \mathrm{E}-03$

5.80E-04

1.54E-01

1.25E-02

$3.13 E+02$

7.29E-00

2.57E+04

$5.12 E+04$

$5.11 E+02$

3.66E-03

1.08E-02

$1.10 \mathrm{E}+01$

$5.56 \mathrm{E}+02$

8.48E-02

$3.25 \mathrm{E}-01$

2.27E-01

9.41E-03

$6.25 \mathrm{E}-03$

2.13E-01
$1 \%-i l e$

$1.36 \mathrm{E}+02$

$7.41 \mathrm{E}+03$

$7.10 \mathrm{E}+04$

$1.25 \mathrm{E}+04$

5.47E-00

$2.24 \mathrm{E}+02$

$0.00 \mathrm{E}+01$

$1.72 \mathrm{E}+02$

$4.07 \mathrm{E}-00$

$4.33 \mathrm{E}+01$

$5.35 \mathrm{E}+05$

7.78E-00

$6.66 \mathrm{E}+04$

$5.64 \mathrm{E}+05$

$6.02 \mathrm{E}+03$

$8.31 \mathrm{E}-00$

$1.80 \mathrm{E}+04$

$9.21 \mathrm{E}+03$

$1.63 \mathrm{E}+02$

$2.56 \mathrm{E}+04$

8.21E-03

$3.05 \mathrm{E}+03$

$7.04 \mathrm{E} \div 02$

$0.00 \mathrm{E} \div 01$

$0.00 E+01$

2.52E+01

$0.00 \mathrm{E}+01$

$5.56 \mathrm{E}+04$

$0.00 \mathrm{E}+01$

$0.00 \mathrm{E}+01$

$0.00 \mathrm{E}+01$

$0.00 \mathrm{E}+01$

$0.00 \mathrm{E}+01$

$3.19 \mathrm{E}-00$

$5.49 \mathrm{E}-01$

$2.79 E+05$

$0.00 \mathrm{E}+01$

$0.00 \mathrm{E}+01$

$0.00 \mathrm{E}+01$

$5.60 \mathrm{E}-02$

$0.00 \mathrm{E}+01$

$0.00 \mathrm{E}+01$

$0.00 \mathrm{E}+01$

$0.00 \mathrm{E}+01$

$0.00 \mathrm{E}+01$

$0.00 \mathrm{E}+01$

$0.00 \mathrm{E}+01$

$0.00 \mathrm{E}+01$

$0.00 \mathrm{E}+01$

$0.00 \mathrm{E}+01$

$0.00 \mathrm{E}+01$

$0.00 \mathrm{E}+01$

$0.00 \mathrm{E}+01$

$0.00 \mathrm{E}+01$

3.31E-01

$0.00 \mathrm{E}+01$

$0.00 \mathrm{E}+01$

$0.00 \mathrm{E}+01$

$0.00 \mathrm{E}+01$

$0.00 \mathrm{E}+01$

$0.00 \mathrm{E}+01$

$4.26 \mathrm{E}+02$

$0.00 \mathrm{E}+01$

$0.00 \mathrm{E}+01$

$0.00 \mathrm{E}+01$

$0.00 \mathrm{E}+01$

$0.00 \mathrm{E}+01$

$0.00 \mathrm{E}+01$ 10\%-ile

$5.03 \mathrm{E}+02$

$8.45 \mathrm{E}+03$

$1.07 \mathrm{E}+05$

$1.43 \mathrm{E}+04$

$3.86 \mathrm{E}+02$

$1.18 \mathrm{E}+03$

$2.80 \mathrm{E}-01$

$8.41 E+02$

$7.09 \mathrm{E}-00$

$3.81 \mathrm{E}+02$

$5.89 \mathrm{E}+05$

4.71E+01

$8.93 \mathrm{E}+04$

$6.59 \mathrm{E}+05$

$8.98 \mathrm{E}+03$

$4.71 \mathrm{E}+01$

$2.86 \mathrm{E}+04$

$1.37 \mathrm{E}+04$

$9.02 \mathrm{E}+02$

$3.78 \mathrm{E}+04$

$5.48 \mathrm{E}+01$

$6.09 \mathrm{E}+03$

$2.06 \mathrm{E}+03$

6.40E-00

$0.00 \mathrm{E}+01$

$3.21 \mathrm{E}+01$

9.38E-04

$1.98 \mathrm{E}+05$

$9.15 \mathrm{E}-00$

$1.29 \mathrm{E}-00$

$7.18 \mathrm{E}-02$

$5.85 \mathrm{E}-03$

$6.08 \mathrm{E}-02$

$5.36 \mathrm{E}-00$

$1.96 \mathrm{E}-00$

$3.22 \mathrm{E}+05$

$0.00 \mathrm{E}+01$

$0.00 \mathrm{E}+01$

$0.00 \mathrm{E}+01$

4.46E-01

$0.00 \mathrm{E}+01$

$5.00 \mathrm{E}-01$

$4.78 \mathrm{E}+01$

4.90E-01

9.50E-04

1.34E-00

$7.03 \mathrm{E}+01$

$1.06 \mathrm{E}+01$

8.30E+01

4.13E-04

1.33E-04

3.54E-02

2.54E-03

$6.10 \mathrm{E}+01$

3.40E-00

$0.00 \mathrm{E}+01$

$1.41 \mathrm{E}+03$

1.28E-00

$3.95 \mathrm{E}-04$

$1.16 \mathrm{E}-03$

7.77E-02

$4.79 \mathrm{E}+02$

9.37E-02

3.59E-01

$2.50 \mathrm{E}-01$

1.04E-02

$6.90 \mathrm{E}-03$

2.61E-01

\section{0\%-ile}

$1.14 \mathrm{E}+03$

$9.89 \mathrm{E}+03$

$1.50 \mathrm{E}+05$

$1.67 \mathrm{E}+04$

$4.16 \mathrm{E}+03$

$3.57 \mathrm{E}+03$

$3.97 \mathrm{E}+01$

$3.85 \mathrm{E}+03$ 


\begin{tabular}{|c|c|c|c|c|c|c|c|c|c|c|c|}
\hline$a n k$ & Anal. & $U_{n}$ & May $\frac{B B}{98}$ & Sept98 & $1 \%$-ile & $10 \%$-ile & 50\%-ile & $90 \%$-ile & 99\%-ile & Mean & Std Dev \\
\hline 11 & Y90 & $\mathbf{C i}$ & $5 . \overline{12 E+04}$ & $\overline{5.12 \mathrm{E}+04}$ & $0 \mathrm{E}+01$ & $E+02$ & $2 \mathrm{E}+04$ & $E+05$ & 4.13E+06 & $4 E+05$ & 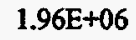 \\
\hline 11 & 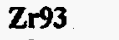 & $\mathrm{Ci}$ & $.57 E+01$ & $3.57 \mathrm{E}+01$ & $00 \mathrm{E}+01$ & $E+01$ & $7 \mathrm{E}+01$ & $50 \mathrm{E}+02$ & $5 \mathrm{E}+02$ & $E+01$ & $38 \mathrm{E}+02$ \\
\hline & Al & kg & $5.22 E+04$ & $322 E+04$ & $17 E+03$ & $E+03$ & $3 E+04$ & $2 E+05$ & $1 E+05$ & $4 E+04$ & 17E+04 \\
\hline 12 & B & kg & $2.36 \mathrm{E}+02$ & $2.36 \mathrm{E}+02$ & $0.00 \mathrm{E}+01$ & $12 E+01$ & $3.58 \mathrm{E}+02$ & $28 E+03$ & $28 \mathrm{E}+03$ & $7 \mathrm{E}+02$ & $.40 \mathrm{E}+02$ \\
\hline & & $\mathrm{k}$ & $10 \mathrm{E}+02$ & $.10 E+02$ & $13 E-00$ & $4 E+01$ & $6 \mathrm{E}+02$ & $4 E+03$ & $35 E \div 03$ & 02 & $5.02 E+02$ \\
\hline & & $\mathrm{kg}$ & $3 E+04$ & $1.23 E+04$ & $+3 \mathrm{E}-00$ & $1 E+02$ & $6 E+03$ & $3 E+04$ & $58 \mathrm{E}+04$ & $E+03$ & $\mathrm{E}+03$ \\
\hline & & kg & $E+04$ & $3.44 E+04$ & $E+02$ & 04 & & $1.50 \mathrm{E}+05$ & $2.17 E+05$ & $F+04$ & $i+04$ \\
\hline & $\mathrm{Cr}$ & $\mathrm{kg}$ & $E+04$ & $1.75 E+04$ & $8 E-00$ & 9 & & & -04 & & \\
\hline & $\mathbf{F}$ & kg & $2.00 \mathrm{E}+$ & 2.001 & 01 & & 3 & & $7 \mathrm{E}+04$ & & \\
\hline & $\mathbf{F}$ & $\mathbf{k}$ & & & 1 & & & 03 & $8.19 E+03$ & +03 & $9 E+03$ \\
\hline & $\mathbf{H g}$ & $k$ & $1.18 \mathrm{E}+01$ & $2.10 \mathrm{E}-0 \mathrm{U}$ & $E+01$ & $E+01$ & - & $8 \mathrm{E} \div 01$ & $4.37 \mathrm{E}+01$ & $E \div 01$ & $1.09 \mathrm{E}+01$ \\
\hline & $\mathbf{K}$ & kg & $3.55 E+03$ & $3.55 E+03$ & 3E-00 & $95 \mathrm{E}+01$ & $0 \mathrm{E}+\mathbf{0 3}$ & $37 E+03$ & $1.24 \mathrm{E}+04$ & $2.92 E+03$ & $3.23 E+03$ \\
\hline & & k & $1.28 E+02$ & $1.28 \mathrm{E}+02$ & $\mathrm{E}-01$ & $.02 \mathrm{E}-01$ & $2.85 \mathrm{E}+01$ & $5 E+01$ & $1.23 E+02$ & $3.47 \mathrm{E}+01$ & $E+01$ \\
\hline & & k & $2.23 E+03$ & $2.23 \mathrm{E}+$ & $2.90 \mathrm{E}-00$ & +01 & & & $2.52 \mathrm{E}+03$ & $E+02$ & $1 E+02$ \\
\hline & & k & $5.84 E+05$ & $5.84 \mathrm{E}+$ & $3.47 E+05$ & $E+05$ & & -05 & $9.43 E+05$ & $E+05$ & $E+05$ \\
\hline & & & $4.99 E+02$ & 4.991 & $\mathrm{E}-02$ & $2 \mathrm{E}-00$ & +02 & -02 & $1.06 \mathrm{E}+03$ & $E+02$ & $E+02$ \\
\hline & & $\mathrm{kg}$ & 1.8 & 1.8 & +02 & -03 & & -05 & $3.09 \mathrm{E}+05$ & +04 & $E+04$ \\
\hline & & kg & $5.25 E+05$ & $5.25 E+05$ & +02 & & & -06 & $3.24 E+06$ & +06 & $E \div 05$ \\
\hline & $\mathbf{F}$ & $\mathbf{k g}$ & 1.0 & N & +01 & 03 & 34 & -04 & $3.59 \mathrm{E}+04$ & +04 & $E+03$ \\
\hline & $\mathbf{P b}$ & $\mathrm{kg}$ & $6.14 E+02$ & $6.14 E+02$ & $7.82 \mathrm{E}-01$ & $E+01$ & $1.57 \mathrm{E}+02$ & $3 E+02$ & $4.08 \mathrm{E}+02$ & $1.61 E+02$ & $9.34 \mathrm{E}+01$ \\
\hline & I & $n_{5}$ & & $1.08 E$ & $E+01$ & & & & $.17 E+05$ & +04 & $E+04$ \\
\hline & $s$ & kg & $4.40 \mathrm{I}$ & $\mathbf{N}$ & $E-00$ & & & & +04 & & $E+03$ \\
\hline & & kg & 6.40 & $6.40 \mathrm{E}+0$ & $E-00$ & -01 & & & & 03 & +03 \\
\hline & & k & & 4.4 & 2 & & & & 04 & & $E+04$ \\
\hline & $s$ & k & 3.92 & $3.92 E+01$ & E-01 & 00 & 01 & .02 & +02 & 102 & +02 \\
\hline & $O C$ & k & $2.86 \mathrm{E}$ & $2.86 E+04$ & $E+01$ & -02 & 4.6 & -04 & $69 E+04$ & +03 & $E+03$ \\
\hline 2 & 1 & k & $3.22 E+03$ & $3.22 \mathrm{E}+03$ & $3.58 \mathrm{E}+01$ & $1.69 \mathrm{E}+02$ & 1.0 & +03 & $.08 \mathrm{E}+03$ & 103 & $E+03$ \\
\hline & $\mathbf{Z r}$ & $\mathrm{kg}$ & 1.5 & $1.53 \mathrm{E}+02$ & $E+01$ & & & & 03 & & +02 \\
\hline & & $\mathrm{Ci}$ & ? & 2.02 & +01 & & & & $1.46 \mathrm{E}-02$ & & -03 \\
\hline & & $\mathrm{Ci}$ & 2 & 1.1 & 10 & & & & 3.7 & 103 & +03 \\
\hline & A & $\mathrm{Ci}$ & 3.1 & 3.1 & +01 & & & & -02 & .03 & -03 \\
\hline 12 & Ba137 & $\mathrm{Ci}$ & $5.51 E+05$ & 5.511 & +01 & $0.00 \mathrm{E}+01$ & 5. & -06 & 06 & $E+05$ & $E+05$ \\
\hline & C & C & $6.68 \mathrm{E}+01$ & $6.68 \mathrm{E}$ & $E+01$ & -01 & & -02 & $E+02$ & 7. & $E+01$ \\
\hline & & $C_{2}$ & $1.66 E+02$ & 1.66 & +01 & & & & & & +02 \\
\hline & & c & 01 & & & & & & & & \\
\hline & & C & & & & & & & & & -02 \\
\hline 12 & $\mathrm{Cn}$ & $\mathrm{Ci}$ & 2.13E-01 & 2.13 & +01 & & & -01 & $1.12 \mathrm{E}-00$ & E-01 & E-01 \\
\hline 12 & Co60 & $\mathrm{Ci}$ & $6.75 E+01$ & $6.75 E+01$ & E-01 & $3.98 \mathrm{E}-01$ & 2.10 & 1 & 01 & +01 & $E+01$ \\
\hline & C & $\mathrm{Ci}$ & 3.11E-00 & 3.11 & $E+01$ & 01 & & & 01 & & $=00$ \\
\hline & & 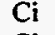 & 05 & 55 & +02 & & & & -05 & & 105 \\
\hline & & & & & & & & & & & r01 \\
\hline & & $\mathrm{Ci}$ & $\mathrm{E}+03$ & & +01 & -01 & & +03 & $7.80 \mathrm{E}+03$ & $i+03$ & +03 \\
\hline 12 & 155 & $\mathrm{Ci}$ & $3.54 \mathrm{E}+02$ & $3.54 \mathrm{E}+02$ & +01 & +01 & 02 & $E+03$ & $2.56 E+03$ & $E+02$ & $8 E+02$ \\
\hline 12 & 1129 & $\mathrm{Ci}$ & 9.17E-01 & 9.17E-01 & $E+01$ & :+01 & 01 & $E-00$ & $3.43 \mathrm{E}-00$ & $E-00$ & $4 \mathrm{E}-01$ \\
\hline & $N$ & $\mathrm{Ci}$ & $2.52 \mathrm{E}+01$ & $2.52 \mathrm{E}$ & $E+01$ & +01 & 01 & $E+02$ & 03 & 02 & $E+02$ \\
\hline & & & & & & & & & 11 & & \\
\hline & & & & & & & & & 3 & +02 & +02 \\
\hline & & & & & 01 & .02 & & & -00 & -00 & $=-00$ \\
\hline 12 & Pa231 & $\mathrm{Ci}$ & 8.67E-03 & 8.67E-03 & $i+01$ & $E+01$ & 8.6 & -02 & $5.17 \mathrm{E}-02$ & $E-02$ & $1.28 \mathrm{E}-02$ \\
\hline \$112 & Pu238 & $\mathrm{Ci}$ & 2.68E-00 & $2.68 \mathrm{E}-60$ & $E+01$ & $E-02$ & 00 & -00 & +01 & -00 & $3 E-00$ \\
\hline 11 & Pu239 & $\mathrm{Ci}$ & $1.11 E+02$ & $1.11 E+02$ & $E+01$ & -00 & 02 & $3.47 \mathrm{E}+02$ & +02 & +02 & $E+02$ \\
\hline $112+2$ & $\mathbf{P}$ & $\mathrm{Ci}$ & 1. & 1.76 & & & & & & & $\$+01$ \\
\hline & & C & & & & & & & & & \\
\hline & & & & & & & & & & & -03 \\
\hline & $\mathbf{R}$ & C & 3.3 & & & & & & & & \\
\hline & $\mathbf{R}$ & $\mathrm{Ci}$ & 1.18E-01 & $1.18 \mathrm{E}-01$ & $:+01$ & 03 & 01 & 01 & 1.2 & -01 & $4 \mathrm{E}-01$ \\
\hline 112 & 106 & $\mathrm{Ci}$ & $1.04 \mathrm{E}-02$ & $1.04 \mathrm{E}-02$ & $E+01$ & $E \div 01$ & 02 & -02 & $3.59 \mathrm{E}-02$ & $4 \mathrm{E}-02$ & 8.74E-03 \\
\hline 112 & 125 & $\mathrm{Ci}$ & $2.77 E+02$ & $2.77 \mathrm{E}+02$ & $E+01$ & +01 & 02 & +03 & +03 & $E+02$ & $4.87 \mathrm{E}+02$ \\
\hline 112 & $\mathbf{S}$ & $\mathrm{Ci}$ & 6.97E-00 & 6.97. & 01 & 1 & & $E+01$ & 1 & 0 & $E-00$ \\
\hline 112 & $\mathbf{S}$ & $\mathrm{C}$ & 6.4 & 2.46 & +01 & 17 & & & & & +04 \\
\hline 112 & $r 89 / 9$ & $C$ & $3.40 \mathrm{E}+05$ & 3. & & & & & & & +05 \\
\hline & & & & & & & & & & & $E+02$ \\
\hline & Th229 & C & $2.80 \mathrm{E}-03$ & $2.80 \mathrm{E}$ & $E+01$ & & & $9.86 \mathrm{E}-03$ & $1.67 \mathrm{E}-02$ & $3.90 \mathrm{E}-03$ & $4.13 E-03$ \\
\hline & Th23 & $\mathrm{Ci}$ & $7.93 E-03$ & $7.93 \mathrm{E}$ & $0 E+01$ & & & & $4.72 \mathrm{E}-02$ & $.11 \mathrm{E}-02$ & $1.17 \mathrm{E}-02$ \\
\hline & $\operatorname{Tin} 126$ & $\mathrm{Ci}$ & $1.06 \mathrm{E}+01$ & $1.06 \mathrm{E}+01$ & $0.00 \mathrm{E}+01$ & & & $2.50 \mathrm{E}+01$ & $3.92 E+01$ & $E+01$ & $9.76 \mathrm{E}-00$ \\
\hline $\mathbf{S 1 1 2}$ & Tritium & $\mathrm{Ci}$ & $5.23 E+02$ & $5.23 E+02$ & $3,45 \mathrm{E}-00$ & & $5.23 \mathrm{E}+02$ & $1.04 E+03$ & I. $.50 \mathrm{E}+03$ & $5.49 \mathrm{E}+02$ & $3.68 \mathrm{E}+02$ \\
\hline & U232 & $\mathrm{Ci}$ & 7.10 & 4.33. & $0.00 \mathrm{E}+01$ & 政 & & 2.50E-00 & 4.23E-00 & OE-01 & 5E-00 \\
\hline & ה & 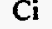 & $2.72 \mathrm{E}-00$ & $1.66 \mathrm{E}-00$ & $0.00 \mathrm{E}+01$ & $0.00 \mathrm{E}+01$ & 2.72E-00 & $9.58 \mathrm{E}-00$ & $1.62 E+01$ & $3.79 \mathrm{E}-00$ & $-\infty 0$ \\
\hline
\end{tabular}


Tank Anal. Un

$\begin{array}{ll}\text { S112 } & \text { U234 } \\ \text { S112 } & \text { U235 } \\ \text { S112 } & \text { U236 } \\ \text { S112 } & \text { U238 } \\ \text { S112 } & \text { Y90 } \\ \text { S112 } & \text { Zr93 } \\ \text { SX101 } & \text { Al }\end{array}$

SX101 Bi

SX101 Ca

SX101 C

SX101 $\mathrm{CO} 3$

SX101 Cr

SX101 F

SX101 Fe

SX101. Hg

SX101 K

SX101 La

SX101 Mn

SX101 Na

SX101 Ni

SX101 NO2

SX101 NO3

SX101 P

SX101 Pb

SX101 PO4

SX101 $s$

SX101 Si

SX101 SO4

SX101 Sr

SX101 TOC

SX101 U

SX101 Zr

SX101 Ac227

SX101 Am241

SX101 Am243

SX101 Ba137

SX101 C14

SX101 Cd113

Sx101 Cm242

SX101 Cm243

Sx101 Cm244

SX101 Co60

SX101 Cs134

SX101 Cs137

SX101 Eu152

SX101 Eu154

SX101 Eu155

SX101 I129

SX101 Nb93

SX101 Ni59

Sx101 Ni63

SX101 Np237

SX101 Pa231

SX101 Pu238

Sx101 Pu239

SX101 Pu240

SX101 Pu241

SX101 Pu242

SX101 Ra226

SX101 R2228

SX101 Ru106

SX101 Sb125

SX101 Se79

SX101 Sm151

SX101 Sr89/90

SX101 Te99

SX101 Th229

SX101 Th232
$B B$

May98

1.87E-00

Ci $\quad 7.76 \mathrm{E}-02$

Ci 5.20E-02

Ci 1.94E-00

Ci $3.40 \mathrm{E}+05$

Ci $3.40 \mathrm{E}+01$

kg 1.04E+05

kg $\quad 2.49 \mathrm{E}+02$

kg $\quad 7.87 \mathrm{E}+02$

kg $\quad 8.87 \mathrm{E}+03$

kg $\quad 1.66 \mathrm{E}+04$

kg 1.13E+04

kg $\quad 6.94 \mathrm{E}+03$

kg $\quad 3.91 \mathrm{E}+03$

$\mathrm{kg} \quad 5.60 \mathrm{E}-01$

kg $2.61 \mathrm{E}+03$

kg $\quad 6.21 \mathrm{E}+01$

kg $2.11 \mathrm{E}+03$

$\mathrm{kg} \quad 4.62 \mathrm{E}+05$

$\mathrm{kg} \quad 3.49 \mathrm{E}+02$

kg $\quad 1.29 \mathrm{E}+05$

kg $\quad 5.87 \mathrm{E}+05$

kg 5.62E+04

kg $3.49 \mathrm{E}+02$

$\mathrm{kg} \quad 1.74 \mathrm{E}+05$

2.82E+04

kg 3.96E+03

kg $\quad 8.46 \mathrm{E}+04$

kg $\quad 3.45 \mathrm{E}+02$

kg $\quad 1.58 \mathrm{E}+04$

g $\quad 7.71 \mathrm{E}+03$

g $\quad 1.23 \mathrm{E}+02$

Ci $4.66 \mathrm{E}-03$

Ci $\quad 8.99 \mathrm{E}+01$

2.80E-03

Ci $3.43 \mathrm{E}+05$

i $1.77 \mathrm{E}+01$

Ci $5.62 \mathrm{E}+01$

Ci $1.36 \mathrm{E}-01$

Ci 5.98E-03

Ci 3.95E-02

Ci $1.72 \mathrm{E}+01$

Ci 2.09E-00

Ci $3.62 \mathrm{E}+05$

$\mathrm{Ci} \quad 1.04 \mathrm{E}+01$

Ci $3.31 \mathrm{E}+02$

Ci $5.23 E+02$

Ci 2.50E-01

Ci $2.03 E+01$

Ci $1.33 \mathrm{E}+01$

Ci $1.26 \mathrm{E}+03$

Ci 5.42E-01

Ci 7.72E-03

Ci 5.64E-00

Ci $3.33 E+02$

Ci $4.91 \mathrm{E}+01$

Ci $3.29 \mathrm{E} \div 02$

Ci 1.58E-03

Ci 9.48E-04

Ci $\quad 4.61 E-02$

Ci 3.29E-03

Ci $\quad 6.81 \mathrm{E}+01$

Ci 5.35E-00

Ci $1.90 \mathrm{E}+04$

Ci 4.11E+05

$1.31 \mathrm{E}+02$

Ci 1.09E-03

Ci 2.57E-03
$\frac{B B}{t 98}$

Sept98

4.74E-02

3.17E-02

1.07E-00

$3.40 \mathrm{E}+05$

$3.40 \mathrm{E}+01$

$1.04 \mathrm{E}+05$

$2.33 \mathrm{E}+02$

$7.87 \mathrm{E}+02$

$8.87 \mathrm{E}+03$

$1.73 \mathrm{E}+04$

$1.13 \mathrm{E}+04$

$6.94 \mathrm{E}+03$

$3.91 \mathrm{E}+03$

$0.00 \mathrm{E}+01$

$2.61 \mathrm{E}+03$

$5.80 \mathrm{E}+01$

$2.11 \mathrm{E}+03$

$4.63 \mathrm{E}+05$

$3.49 \mathrm{E}+02$

$1.29 \mathrm{E}+05$

$5.88 \mathrm{E}+05$

NA

$3.49 \mathrm{E}+02$

$5.68 \mathrm{E}+04$

NA

$3.96 \mathrm{E}+03$

$2.82 \mathrm{E}+04$

$3.45 \mathrm{E}+02$

$1.58 \mathrm{E}+04$

$7.71 \mathrm{E}+03$

$1.23 \mathrm{E}+02$

4.66E-03

$8.99 E+01$

$2.80 \mathrm{E}-03$

$3.28 \mathrm{E}+05$

$1.77 \mathrm{E}+01$

$5.62 \mathrm{E}+01$

1.36E-01

5.98E-03

3.95E-02

$1.72 \mathrm{E}+01$

2.09E-00

$3.47 \mathrm{E}+05$

$1.04 \mathrm{E}+01$

$3.31 \mathrm{E}+02$

$5.23 \mathrm{E}+02$

2.50E-01

$2.03 \mathrm{E}+01$

$1.33 \mathrm{E}+01$

$1.26 \mathrm{E}+03$

5.42E-01

7.72E-03

5.64E-00

$3.33 E+02$

$4.91 E+01$

$3.29 \mathrm{E}+02$

1.58E-03

9.48E-04

4.61E-02

3.29E-03

$6.81 \mathrm{E}+01$

5.35E-00

$1.90 \mathrm{E}+04$

4.01E +05

$1.31 \mathrm{E}+02$

1.09E-03

2.57E-03
$1 \%$-ile

$0.00 \mathrm{E}+01$

$0.00 \mathrm{E}+01$

$0.00 \mathrm{E}+01$

$0.00 \mathrm{E}+01$

$0.00 \mathrm{E}+01$

$0.00 \mathrm{E}+01$

$5.68 \mathrm{E}+04$

$0.00 \mathrm{E}+01$

$1.41 \mathrm{E}+02$

$2.78 \mathrm{E}+02$

$1.02 \mathrm{E}+04$

$2.03 \mathrm{E}+02$

$0.00 \mathrm{E}+01$

$4.36 \mathrm{E}+02$

$5.59 \mathrm{E}-04$

$7.05 \mathrm{E}+01$

2.04E-00

$9.84 \mathrm{E}+01$

$2.79 E+05$

$1.68 \mathrm{E}-00$

$2.76 \mathrm{E}+03$

$6.66 \mathrm{E}+03$

$1.05 \mathrm{E}+03$

1. $30 \mathrm{E}+01$

$1.99 \mathrm{E}+03$

$1.81 \mathrm{E}+02$

$2.23 \mathrm{E}+02$

$3.87 \mathrm{E}+02$

$2.96 \mathrm{E}-00$

$2.93 \mathrm{E}+02$

$7.16 \mathrm{E}+02$

$0.00 \mathrm{E}+01$

$0.00 \mathrm{E}+01$

$3.95 \mathrm{E}+01$

$0.00 \mathrm{E}+01$

$0.00 \mathrm{E}+01$

$0.00 \mathrm{E}+01$

5.61E-02

$0.00 \mathrm{E}+01$

$0.00 \mathrm{E}+01$

$0.00 \mathrm{E}+01$

5.53E-00

$0.00 \mathrm{E}+01$

$2.53 \mathrm{E}+04$

$0.00 \mathrm{E}+01$

$0.00 \mathrm{E}+01$

$0.00 \mathrm{E}+01$

$0.00 \mathrm{E}+01$

$0.00 \mathrm{E}+01$

$0.00 \mathrm{E}+01$

$0.00 \mathrm{E}+01$

$0.00 \mathrm{E}+01$

$0.00 \mathrm{E}+01$

$0.00 \mathrm{E}+01$

$0.00 \mathrm{E}+01$

$0.00 \mathrm{E}+01$

$0.00 \mathrm{E}+0$

$0.00 \mathrm{E}+01$

$0.00 \mathrm{E}+01$

$0.00 \mathrm{E}+01$

$0.00 \mathrm{E}+0$

$0.00 \mathrm{E}+01$

$0.00 \mathrm{E}+01$

$0.00 \mathrm{E}+01$

$0.00 \mathrm{E}+01$

$0.00 \mathrm{E}+01$

$0.00 \mathrm{E}+01$

$0.00 \mathrm{E}+01$

$10 \%$-ile

$0.00 \mathrm{E}+01$

$0.00 \mathrm{E}+01$

$0.00 \mathrm{E}+01$

$0.00 \mathrm{E}+01$

$0.00 \mathrm{E}+01$

$0.00 \mathrm{E}+01$

$8.66 \mathrm{E}+04$

$1.71 E+02$

$2.75 \mathrm{E}+02$

$1.53 \mathrm{E}+03$

$3.10 \mathrm{E}+04$

$2.03 \mathrm{E}+03$

$2.86 \mathrm{E}+02$

$1.87 \mathrm{E}+03$

$1.74 \mathrm{E}-01$

$3.97 \mathrm{E}+02$

$8.94 \mathrm{E}-00$

$5.32 \mathrm{E}+02$ 
Tank Anal. Un

$\begin{array}{ll}\text { SX101 } & \text { Tin126 } \\ \text { SX101 } & \text { Tritium } \\ \text { SX101 } & \text { U232 } \\ \text { SX101 } & \text { U233 } \\ \text { SX101 } & \text { U234 } \\ \text { SX101 } & \text { U235 } \\ \text { SX101 } & \text { U236 } \\ \text { SX101 } & \text { U238 } \\ \text { SX101 } & \text { Y90 } \\ \text { SX101 } & \text { Zr93 } \\ \text { SX102 } & \text { Al } \\ \text { SX102 } & \text { Bi } \\ \text { SX102 } & \text { Ca } \\ \text { SX102 } & \text { Cl } \\ \text { SX102 } & \text { CO3 } \\ \text { SX102 } & \text { Cr } \\ \text { SX102 } & \text { F } \\ \text { SX102 } & \text { Fe } \\ \text { SX102 } & \text { Hg } \\ \text { SX102 } & \text { K } \\ \text { SX102 } & \text { La } \\ \text { SX102 } & \text { Mn } \\ \text { SX102 } & \text { Na } \\ \text { SX102 } & \text { Ni } \\ \text { SX102 } & \text { NO2 } \\ \text { SX102 } & \text { NO3 } \\ \text { SX102 } & \text { P } \\ \text { SX102 } & \text { Pb } \\ \text { SX102 } & \text { PO4 } \\ \text { SX102 } & \text { S } \\ \text { SX102 } & \text { Si } \\ \text { SX102 } & \text { SO4 } \\ \text { Sa }\end{array}$

SX102 SO4

SX102 Sr

SX102 TOC

SX102 U

SX102 Zr

SX102 Ac227

Sx102 Am241

SX102 Am243

SX102 Ba137

SX102 C14

SX102 Cd113

Sx102 Cm242

$\mathrm{Sx} 102 \mathrm{Cm} 243$

SX102 Cm244

SX102 Co60

SX102 Cs134

SX102 Cs137

SX102 Eu152

SX102 Eu154

SX102 Eu155

SX102 1129

SX102 Nb93

SX102 Ni59

SX102 Ni63

SX102 Np237

SX102 Pa231

SX102 Pu238

SX102 Pu239

SX102 Pu240

SX102 Pu241

SX102 Pu242

SX102 Ra226

SX102 Ra228

SX102 Ru106

SX102 Sb125

SX102 Se79

SX102 Sm151
May $\frac{B B}{98}$

$8.19 \mathrm{E}-00$

$2.17 \mathrm{E}+02$

2.33E-01

8.94E-01

1.05E-00

4.27E-02

3.93E-02

9.92E-01

4.11E +05

$2.55 E+01$

$1.17 \mathrm{E}+05$

$2.46 \mathrm{E}+02$

$9.48 \mathrm{E}+02$

$1.18 \mathrm{E}+04$

$6.63 E+04$

$1.83 E+04$

$1.50 \mathrm{E}+04$

$5.30 \mathrm{E}+03$

4.16E-00

$3.23 \mathrm{E}+03$

$1.51 \mathrm{E}+01$

$2.73 E+03$

$5.57 \mathrm{E}+05$

4.78E +02

$1.65 \mathrm{E}+05$

$5.73 E+05$

$8.45 E+04$

$5.04 \mathrm{E}+02$

$2.62 \mathrm{E}+05$

$3.68 \mathrm{E}+04$

$5.83 E+03$

$1.10 \mathrm{E}+05$

$3.53 \mathrm{E}+02$

$2.38 E+04$

$8.44 E+03$

$1.67 \mathrm{E}+02$

$3.31 \mathrm{E}-03$

$1.44 \mathrm{E}+02$

$5.10 \mathrm{E}-03$

$4.46 \mathrm{E}+05$

$8.81 \mathrm{E}+01$

$2.22 E+02$

4.17E-01

3.63E-02

3.50E-01

$9.87 \mathrm{E}+01$

$6.31 \mathrm{E}-00$

$4.72 \mathrm{E}+05$

$1.15 \mathrm{E}+01$

$1.58 \mathrm{E}+03$

$6.65 E+02$

$1.21 \mathrm{E}-00$

$3.04 \mathrm{E}+01$

8.80E-00

$8.48 E+02$

2.28E-00

$1.06 \mathrm{E}-02$

5.01E-00

$2.25 \mathrm{E}+02$

$3.54 \mathrm{E}+01$

$3.29 E+02$

$1.73 \mathrm{E}-03$

5.94E-04

3.83E-01

1.74E-02

$4.26 E+02$

8.54E-00

$3.01 \mathrm{E}+04$
$B B$ Sept98 8.19E-00 $2.17 \mathrm{E}+02$ 6.48E-01 2.48E-00

2.92E-00

1.18E-01

$1.09 \mathrm{E}-01$

2.57E-00

4.01E+05

$2.55 \mathrm{E}+01$

1.17E+05

$2.30 \mathrm{E}+02$

$9.48 \mathrm{E}+02$

$1.18 \mathrm{E}+04$

$6.58 \mathrm{E}+04$

$1.83 \mathrm{E}+04$

$1.50 \mathrm{E}+04$

$5.30 \mathrm{E}+03$

$0.00 \mathrm{E}+01$

$3.23 \mathrm{E}+03$

$1.77 \mathrm{E}+01$

$2.73 E+03$

$5.57 \mathrm{E}+05$

$4.78 \mathrm{E}+02$

$1.65 \mathrm{E}+05$

$5.73 E+05$

NA

$5.04 \mathrm{E}+02$

8.54E+04

NA

$5.83 \mathrm{E}+03$

$3.68 \mathrm{E}+04$

$2.38 \mathrm{E}+04$

8.44E+03

$1.67 \mathrm{E}+02$

$3.31 \mathrm{E}-03$

$1.44 \mathrm{E}+02$

5.10E-03

4.27E+05

$8.81 \mathrm{E}+01$

$2.22 \mathrm{E}+02$

4.17E-01

3.63E-02

$3.50 \mathrm{E}-01$

$9.87 \mathrm{E}+01$

6.31E-00

4.51E+05

$1.15 \mathrm{E}+01$

$1.58 \mathrm{E}+03$

$6.65 \mathrm{E}+02$

1.21E-00

$3.04 \mathrm{E}+01$

$8.80 \mathrm{E}-00$

$8.48 E+02$

2.28E-00

1.06E-02

5.01E-00

$2.25 E+02$

$3.54 \mathrm{E}+01$

$3.29 E+02$

1.73E-03

$5.94 \mathrm{E}-04$

3.83E-01

1.74E-02

$4.26 \mathrm{E}+02$

8.54E-00

$3.01 E+04$ 1\%-ile

10\%-ile

50\%-ile

3.

8.17E-03

$4.78 \mathrm{E}+01$

2.54E-00

$9.44 \mathrm{E}+01$

3.41E-02

$1.31 \mathrm{E}-01$

$0.00 \mathrm{E}+01$

$1.54 \mathrm{E}-01$

6.25E-03

$5.75 \mathrm{E}-03$

$1,45 \mathrm{E}-01$

$0.00 \mathrm{E}+01$

$0.00 \mathrm{E}+01$

$6.45 \mathrm{E}+04$

$8.80 \mathrm{E}+01$

$5.17 \mathrm{E}+02$

$1.68 \mathrm{E}+03$

$9.85 \mathrm{E}+04$

$4.23 \mathrm{E}+03$

$3.37 \mathrm{E}+02$

$9.16 \mathrm{E}+02$

$0.00 \mathrm{E}+01$

6.17E+02

4.33E-00

4. $13 E+02$

$5.82 \mathrm{E}+05$

$3.11 \mathrm{E}+01$

$9.45 \mathrm{E}+04$

$1.94 \mathrm{E}+05$

$7.97 \mathrm{E}+03$

$2.83 \mathrm{E}+01$

$1.41 \mathrm{E}+04$

$4.40 \mathrm{E}+03$

$4.92 \mathrm{E}+02$

$8.71 \mathrm{E}+03$

4.32E +01

$2.44 \mathrm{E}+03$

$1.64 \mathrm{E}+03$

$5.52 \mathrm{E}-00$

$0.00 \mathrm{E}+01$

$2.33 \mathrm{E}+01$

4.48E-04

$6.02 \mathrm{E} \div 04$

$1.07 \mathrm{E}+01$

$0.00 \mathrm{E}+01$

$3.66 \mathrm{E}-02$

$3.19 \mathrm{E}-03$

3.07E-02

$4.49 \mathrm{E}-00$

8.52E-01

$1.98 \mathrm{E}+05$

$0.00 \mathrm{E}+01$

$0.00 \mathrm{E}+01$

$0.00 \mathrm{E}+01$

$0.00 \mathrm{E}+01$

$0.00 \mathrm{E}+01$

$0.00 \mathrm{E}+01$

$0.00 \mathrm{E}+01$

2.00E-01

1.01E-04

4.40E-01

$1.98 \mathrm{E}+01$

3.11E-00

$2.89 \mathrm{E}+01$

$1.52 \mathrm{E}-04$

$1.05 \mathrm{E}-04$

6.75E-02

$0.00 \mathrm{E}+01$

$7.18 \mathrm{E}+01$

$0.00 \mathrm{E}+0$

$1.55 \mathrm{E}-00$

$0.00 \mathrm{E}+01$

8.19E-00

$2.17 \mathrm{E}+02$

2.33E-01

8.94E-01

1.05E-00

4.27E-02

3.93E-02

9.92E-01

4.11E+05

2.55E+01

$1.09 \mathrm{E}+05$

$5.03 \mathrm{E}+02$

$1.33 \mathrm{E}+03$

$1.76 \mathrm{E}+04$

$1.41 \mathrm{E}+05$

$1.18 \mathrm{E}+04$

$6.01 \mathrm{E}+03$

$5.14 \mathrm{E}+03$

4.16E-00

$7.13 \mathrm{E}+03$

7.23E+01

$1.93 \mathrm{E}+03$

$6.52 \mathrm{E}+05$

$5.44 \mathrm{E}+02$

$2.10 \mathrm{E}+05$

$5.89 \mathrm{E}+05$

$1.43 \mathrm{E}+04$

$5.56 \mathrm{E}+02$

3.13E+04

$8.55 E+03$

$1.90 \mathrm{E}+03$

$2.44 \mathrm{E}+04$

$2.07 \mathrm{E}+02$

$3.46 \mathrm{E}+04$

$4.44 \mathrm{E}+03$

$2.70 \mathrm{E}+02$

3.31E-03 
Tank Anal. Un

Sx102 Sr89/90 Ci

SX102 Te99

SX102 Th229 Ci

SX102 Th232 C

SX102 Tritium Ci

SX102 U232

SX102 U233

SX102 U234

SX102 U235

SX102 U236

SX102 U238

SX102 Y90

SX102 Zr93

SX103 Al

SX103 Bi

SX103 Ca

Sx103 Cl

SX103 CO3

SX103 Cr

SX103 F

SX103 Fe

SX103 Hg

SX103 K

SX103 La

SX103 Mn

SX103 Na

SX103 Ni

SX103 NO2

SX103 NO3

SX103 P

SX103 Pb

SX103 PO4

Sx103 S

SX103 Si

SX103 SO4

SX103 Sr

SX103 TOC

SX103 U

SX103 Zr

SX103 Ac227

SX103 Am241

SX103 Am243

Sx103 Ba137

SX103 C14

SX103 Cd113

sX103 Cm242

Sx103 Cm243

sX103 Cm244

SX103 Co60

SX103 Cs134

SX103 Cs137

SX103 Eu152

SX103 Eu154

SX103 Eu155

SX103 I129

SX103 Nb93

SX103 Ni59

SX103 Ni63

SX103 Np237

SX103 Pa231

SX103 Pu238

SX103 Pu239

SX103 Pu240

SX103 Pu241

SX103 Pu242

Sx103 Ra226

SX103 Ra228
May $\overline{98}$

$4 \overline{74 E+05}$

$6.29 E+02$

8.99E-03

2.69E-02

$1.29 \mathrm{E}+01$

$5.92 \mathrm{E}+02$

$1.90 \mathrm{E}-00$

$7.30 \mathrm{E}-00$

2.20E-00

8.18E-02

6.32E-02

2.38E-60

$4.74 \mathrm{E}+05$

$4.19 E+01$

$1.27 \mathrm{E}+05$

$2.76 \mathrm{E}+02$

g 1.45E+04

$1.43 E+04$

$6.71 E+04$

$1.95 \mathrm{E}+04$

$1.59 E+04$

$6.64 \mathrm{E}+03$

4.42E-00

$4.00 \mathrm{E}+03$

kg $1.32 \mathrm{E}+02$

kg $\quad 3.28 \mathrm{E}+03$

kg $\quad 6.77 \mathrm{E}+05$

kg $\quad 6.04 \mathrm{E}+02$

kg 2.14E+05

kg $\quad 6.33 E+05$

kg 1.12E+05

$\mathrm{kg} \quad 6.60 \mathrm{E}+02$

kg $3.46 \mathrm{E}+05$

kg $\quad 4.68 \mathrm{E}+04$

g $\quad 7.54 \mathrm{E}+03$

kg 1.40E+05

3.47E+02

kg 3.09E+04

g.95E +03

kg 2.07E+02

3.55E-03

$1.92 \mathrm{E}+02$

$6.69 \mathrm{E}-03$

$6.01 \mathrm{E}+05$

$9.06 \mathrm{E}+01$

$2.40 \mathrm{E}+02$

$4.00 \mathrm{E}-01$

3.67E-02

$3.56 \mathrm{E}-01$

$1.01 \mathrm{E}+02$

$7.85 E-00$

$6.35 E+05$

$1.40 \mathrm{E}+01$

$1.67 \mathrm{E}+03$

Ci $\quad 7.97 \mathrm{E}+02$

Ci 1.25E-00

Ci $4.08 \mathrm{E}+01$

Ci $\quad 8.76 \mathrm{E}-00$

Ci $\quad 8.54 \mathrm{E}+02$

Ci 2.37E-00

$\mathrm{Ci} \quad 1.46 \mathrm{E}-02$

$\mathrm{Ci} \quad$ 4.76E-00

Ci $1.69 \mathrm{E}+02$

Ci $2.82 \mathrm{E}+01$

Ci $3.06 \mathrm{E}+02$

Ci $1.66 \mathrm{E}-03$

Ci 5.86E-04

Ci $3.95 \mathrm{E}-01$
$B B$

Sept98

$4.60 \mathrm{E}+05$

$6.29 \mathrm{E}+02$

8.99E-03

2.69E-02

$1.29 \mathrm{E}+01$

$5.92 \mathrm{E}+02$

2.92E-00

$1.12 \mathrm{E}+01$

3.10E-00

$1.25 \mathrm{E}-01$

9.69E- 02

2.82E-00

$4.60 \mathrm{E}+05$

$4.19 \mathrm{E}+01$

$1.27 \mathrm{E}+05$

$2.59 \mathrm{E}+02$

$1.45 \mathrm{E}+04$

$1.43 E+04$

$6.76 \mathrm{E}+04$

$1.95 \mathrm{E}+04$

$1.59 \mathrm{E}+04$

$6.64 \mathrm{E}+03$

$0.00 \mathrm{E}+01$

$4.00 \mathrm{E}+03$

$1.32 \mathrm{E}+02$

$3.28 \mathrm{E}+03$

$6.77 \mathrm{E}+05$

$6.04 \mathrm{E}+02$

$2.14 E+05$

$6.33 \mathrm{E}+05$

NA
$\mathrm{E}+02$

$6.60 \mathrm{E}+02$

1.13E+05 NA

$7.54 \mathrm{E}+03$

$4.68 \mathrm{E}+04$

$3.47 \mathrm{E}+02$

$3.09 \mathrm{E}+04$

$8.95 E+03$

$2.07 E+02$

3.55E-03

$1.92 \mathrm{E}+02$

6.69E- 03

$5.35 \mathrm{E}+05$

$9.06 \mathrm{E}+01$

$2.40 \mathrm{E}+02$

4.00E-01

3.67E-02

3.56E-01

$1.01 \mathrm{E}+02$

$7.85 \mathrm{E}-00$

$5.66 \mathrm{E}+05$

$1.40 \mathrm{E}+01$

$1.67 \mathrm{E}+03$

$7.97 \mathrm{E}+02$

$1.25 \mathrm{E}-00$

$4.08 \mathrm{E}+01$

8.76E-00

$8.54 E+02$

$2.37 E-00$

1.46E-02

4.76E-00

$1.69 \mathrm{E}+02$

$2.82 \mathrm{E}+01$

$3.06 \mathrm{E}+02$

$1.66 \mathrm{E}-03$

$5.86 \mathrm{E}-04$

3.95E-01 1\%-ile

\section{$0.00 \mathrm{E}+01$}

$\begin{array}{ll}0.00 \mathrm{E}+01 & 0.00 \mathrm{E}+01 \\ 0.00 \mathrm{E}+01 & 8.56 \mathrm{E}-05\end{array}$

$0.00 \mathrm{E}+01 \quad 2.56 \mathrm{E}-04$

$0.00 \mathrm{E}+01 \quad 0.00 \mathrm{E}+01$

$2.83 \mathrm{E}+02 \quad 4.18 \mathrm{E}+02$

$0.00 \mathrm{E}+01 \quad 1.81 \mathrm{E}-02$

$0.00 \mathrm{E}+01 \quad 6.95 \mathrm{E}-02$

$0.00 \mathrm{E}+01 \quad 2.10 \mathrm{E}-02$

$0.00 \mathrm{E}+01 \quad 7.79 \mathrm{E}-04$

$0.00 \mathrm{E}+01 \quad 6.02 \mathrm{E}-04$

$0.00 \mathrm{E}+01 \quad 2.27 \mathrm{E}-02$

$0.00 \mathrm{E}+01 \quad 3.15 \mathrm{E}+02$

$0.00 \mathrm{E}+01 \quad 0.00 \mathrm{E}+01$

$1.07 \mathrm{E}+04 \quad 3.76 \mathrm{E}+04$

$0.00 \mathrm{E}+01 \quad 1.83 \mathrm{E}+01$

$2.11 \mathrm{E}+01 \quad 4.30 \mathrm{E}+02$

8.31E+03 1.13E+04

$1.56 \mathrm{E}+04 \quad 8.41 \mathrm{E}+04$

$1.21 \mathrm{E}+03$

1.67E-00

$4.13 \mathrm{E}+01$

$0.00 \mathrm{E}+01$

$2.26 \mathrm{E}+01$

5.23E-01

$6.14 \mathrm{E}-00$

$5.05 \mathrm{E}+05$

1.34E-00

$7.76 \mathrm{E}+04$

$4.42 \mathrm{E}+05$

$6.41 \mathrm{E}+03$

$7.65 \mathrm{E}-01$

$2.28 \mathrm{E}+04$

$1.82 \mathrm{E}+01$

$3.04 \mathrm{E}+01$

$1.25 \mathrm{E}+02$

5.32E-03

$7.91 \mathrm{E}+01$

$1.79 \mathrm{E}+02$

$0.00 \mathrm{E}+01$

$0.00 \mathrm{E}+01$

3.22E-00

$0.00 \mathrm{E}+01$

$0.00 \mathrm{E}+01$

$0.00 \mathrm{E}+01$

$0.00 \mathrm{E}+01$

$0.00 \mathrm{E}+01$

$0.00 \mathrm{E}+01$

$0.00 \mathrm{E}+01$

4.68E-01

$0.00 E+01$

$6.40 \mathrm{E}+03$

$0.00 \mathrm{E}+01$

$0.00 \mathrm{E}+01$

$0.00 \mathrm{E} \div 01$

$0.00 \mathrm{E}+01$

$0.00 \mathrm{E}+01$

$0.00 \mathrm{E}+01$

$0.00 \mathrm{E}+01$

$0.00 \mathrm{E}+01$

$0.00 \mathrm{E}+01$

$0.00 \mathrm{E}+01$

$0.00 E+01$

$0.00 E+01$

$0.00 \mathrm{E}+01$

$0.00 \mathrm{E}+01$

$0.00 \mathrm{E}+01$

$0.00 \mathrm{E}+01$

$7.12 \mathrm{E}+03$

$4.82 \mathrm{E}+01$

$4.10 \mathrm{E}+02$

$0.00 \mathrm{E}+01$

$4.74 \mathrm{E}+02$

$1.00 \mathrm{E}-00$

$1.06 \mathrm{E}+02$

$6.55 \mathrm{E}+05$

6.70E-00

$1.23 \mathrm{E}+05$

$7.84 \mathrm{E}+05$

$1.13 \mathrm{E}+04$

$3.37 \mathrm{E}+01$

$3.86 \mathrm{E}+04$ 
Tank Anal. Un

\begin{tabular}{ll} 
SX103 & Ru106 \\
SX103 & Sb125 \\
SX103 & Se79 \\
SX103 & Sm151 \\
SX103 & Sr89/90 \\
SX103 & Te99 \\
SX103 & Th229 \\
SX103 & Th232 \\
SX103 & Tin126 \\
SX103 & Tritium \\
SX103 & U232 \\
SX103 & U233 \\
SX103 & U234 \\
SX103 & U235 \\
SX103 & U236 \\
SX103 & U238 \\
SX103 & Y90 \\
SX103 & Zr93 \\
SX104 & Al \\
SX104 & Bi \\
\hline SX104 & Ca
\end{tabular}

SX104 Ca

SX104 Cl

SX104 CO3

SX104 Cr

SX104 F

SX104 Fe

SX104 Hg

SX104 K

SX104 La

SX104 Mn

SX104 Na

SX104 Ni

SX104 NO2

SX104 NO3

SX104 P

SX104 Pb

SX104 PO4

SX104 S

SX104 Si

SX104 SO4

SX104 Sr

SX104 TOC

SX104 U

SX104 Zr

SX104 Ac227

SX104 Am241

SX104 Am243

SX104 Bai37

SX104 C14

SX104 Cd113

SX104 Cm242

Sx104 Cm243

SX104 Cm244

SX104 Co60

SX104 Cs134

SX104 Cs137

SX104 Eu152

SX104 Eu154

SX104 Eu155

SX104 I129

SX104 Nb93

SX104 Ni59

SX104 Ni63

SX104 Np237

SX104 Pa231

SX104 Pu238

SX104 Pu239

SX104 Pu240
May

1.85E-02

$1.12 \mathrm{E}+01$

$3.97 \mathrm{E}+04$

$5.46 \mathrm{E}+05$

$6.48 \mathrm{E}+02$

$9.26 \mathrm{E}-03$

2.59E-02

$1.71 \mathrm{E}+01$

$6.72 \mathrm{E}+02$

2.01E-00

$7.70 \mathrm{E}-00$

2.55E-00

1.03E-01

8.56E-02

$2.86 \mathrm{E}-00$

$5.46 \mathrm{E}+05$

$5.47 \mathrm{E}+01$

$1.36 \mathrm{E}+05$

$2.55 \mathrm{E}+02$

$1.08 E+03$

$1.35 \mathrm{E}+04$

$5.74 \mathrm{E}+04$

$1.78 \mathrm{E}+04$

$1.86 \mathrm{E}+04$

$6.28 \mathrm{E}+03$

3.29E-00

$3.66 \mathrm{E}+03$

$1.18 \mathrm{E}+02$

$3.23 \mathrm{E}+03$

$6.26 \mathrm{E}+05$

$5.65 \mathrm{E}+02$

$1.97 \mathrm{E}+05$

$5.93 \mathrm{E}+05$

$1.01 \mathrm{E}+05$

$5.96 \mathrm{E}+02$

$3.13 \mathrm{E}+05$

$4.21 \mathrm{E}+04$

$7.00 E+03$

$1.26 \mathrm{E}+05$

$4.03 E+02$

$2.80 \mathrm{E}+04$

$9.70 \mathrm{E}+03$

$2.00 \mathrm{E}+02$

4.41E-03

$1.54 \mathrm{E}+02$

$5.27 \mathrm{E}-03$

$4.94 \mathrm{E}+05$

$7.67 \mathrm{E}+01$

$1.99 \mathrm{E}+02$

$3.76 \mathrm{E}-01$

3.07E-02

2.91E-01

$8.40 \mathrm{E}+01$

$5.84 \mathrm{E}-00$

$5.22 \mathrm{E}+05$

$132 \mathrm{E}+01$

$1.38 \mathrm{E}+03$

$7.32 \mathrm{E}+02$

$1.03 \mathrm{E}-00$

$3.31 \mathrm{E}+01$

$1.20 \mathrm{E}+01$

$1.15 \mathrm{E}+03$

2.03E-00

$1.18 \mathrm{E}-02$

6.03E-00

$2.96 \mathrm{E}+02$

$4.56 E+01$
Sept98

\subsection{E-02}

$4.32 E+02$

1.12E+01

$3.97 \mathrm{E}+04$

$5.19 \mathrm{E}+05$

$6.48 \mathrm{E}+02$

9.26E-03

2.59E-02

$1.71 \mathrm{E}+01$

$6.72 \mathrm{E}+02$

2.62E-00

$1.00 \mathrm{E}+01$

3.33E-00

1.35E-01

1.11E-01

2.99E-00

$5.19 \mathrm{E}+05$

5.47E+01

$1.36 \mathrm{E}+05$

$2.36 \mathrm{E}+02$

$1.08 \mathrm{E}+03$

$1.35 \mathrm{E}+04$

$5.74 \mathrm{E}+04$

$1.78 \mathrm{E}+04$

$1.86 \mathrm{E}+04$

$6.28 \mathrm{E}+03$

$0.00 \mathrm{E}+01$

$3.66 E+03$

$1.18 \mathrm{E}+02$

$3.23 \mathrm{E}+03$

$6.26 \mathrm{E}+05$

$5.65 \mathrm{E}+02$

$1.97 \mathrm{E}+05$

$5.93 E+05$

NA

$5.96 \mathrm{E}+02$

$1.02 \mathrm{E}+05$

NA

$7.00 \mathrm{E}+03$

$4.21 \mathrm{E}+04$

$4.03 \mathrm{E}+02$

$2.80 \mathrm{E}+04$

$9.70 \mathrm{E}+03$

$2.00 \mathrm{E}+02$

4.41E-03

$1.54 \mathrm{E}+02$

5.27E-03

$4.94 \mathrm{E}+05$

$7.67 \mathrm{E}+01$

$1.99 \mathrm{E}+02$

$3.76 \mathrm{E}-01$

3.07E-02

$2.91 \mathrm{E}-01$

$8.40 \mathrm{E}+01$

$5.84 \mathrm{E}-00$

$5.22 \mathrm{E}+05$

$1.32 E+01$

$1.38 \mathrm{E}+03$

$7.32 E+02$

$1.06 \mathrm{E}-00$

$3.31 \mathrm{E}+01$

$1.20 \mathrm{E}+01$

$1.15 E+03$

2.03E-00

1.18E-02

6.03E-00

$2.96 \mathrm{E}+02$

$4.56 \mathrm{E}+01$ 1\%-ile

$0.00 E+01$

$10 \%$-ile

50\%-ile

.

$0.00 \mathrm{E}+01$

$0.00 \mathrm{E}+01$

$0.00 \mathrm{E}+01$

$0.00 \mathrm{E}+01$

$0.00 \mathrm{E}+01$

$0.00 \mathrm{E}+01$

$0.00 \mathrm{E}+01$

$3.30 \mathrm{E}+02$

$0.00 \mathrm{E}+01$

$0.00 \mathrm{E}+01$

$0.00 \mathrm{E}+01$

$0.00 \mathrm{E}+01$

$0.00 \mathrm{E}+01$

$0.00 \mathrm{E}+01$

$0.00 \mathrm{E}+01$

$0.00 \mathrm{E}+01$

$5.72 \mathrm{E}+04$

$0.00 \mathrm{E}+01$

$1.74 \mathrm{E}+02$

$7.67 \mathrm{E}+03$

$1.76 \mathrm{E}+04$

$1.89 \mathrm{E}+03$

$3.06 \mathrm{E}-00$

$3.39 \mathrm{E}+02$

$0.00 \mathrm{E}+01$

$1.97 \mathrm{E}+02$

$4.82 \mathrm{E}-00$

$6.81 \mathrm{E}+01$

4.69E+05

$1.68 \mathrm{E}+01$

$7.87 \mathrm{E}+04$

$4.02 \mathrm{E}+05$

$6.32 \mathrm{E}+03$

$1.03 \mathrm{E}+01$

$2.19 \mathrm{E}+04$

$1.66 \mathrm{E}+02$

$2.48 \mathrm{E}+02$

$1.03 \mathrm{E}+03$

3.14E-00

$6.34 \mathrm{E}+02$

$7.49 \mathrm{E}+02$

$0.00 \mathrm{E}+01$

$0.00 \mathrm{E}+01$

$3.03 E+01$

$0.00 \mathrm{E}+01$

$0.00 \mathrm{E}+01$

$0.00 \mathrm{E}+01$

$0.00 \mathrm{E}+01$

$0.00 \mathrm{E}+01$

$0.00 \mathrm{E}+01$

$0.00 \mathrm{E}+01$

4.15E-00

$0.00 \mathrm{E}+01$

$6.01 \mathrm{E}+04$

$0.00 \mathrm{E}+01$

$0.00 E+01$

$0.00 \mathrm{E}+01$

$5.47 \mathrm{E}-02$

$0.00 \mathrm{E}+01$

$0.00 \mathrm{E}+01$

$0.00 \mathrm{E}+01$

$0.00 \mathrm{E}+01$

$0.00 \mathrm{E}+01$

$0.00 \mathrm{E}+01$

$0.00 \mathrm{E}+01$

$0.00 \mathrm{E}+01$

$0.00 \mathrm{E}+01$

$2.06 \mathrm{E}-00$

$0.00 \mathrm{E}+01$

$1.39 \mathrm{E}+03$

$0.00 \mathrm{E}+01$

$0.00 \mathrm{E}+01$

$0.00 \mathrm{E}+01$

$0.00 \mathrm{E}+01$

$4.82 \mathrm{E}+02$

$0.00 \mathrm{E}+01$

$0.00 \mathrm{E}+01$

$0.00 \mathrm{E}+01$

$0.00 \mathrm{E}+01$

$0.00 \mathrm{E}+01$

$0.00 \mathrm{E}+01$

$5.25 \mathrm{E}+03$

$0.00 \mathrm{E}+01$

$8.80 \mathrm{E}+04$

$1.20 \mathrm{E}+02$

$6.09 \mathrm{E}+02$

$9.82 \mathrm{E}+03$

$7.44 \mathrm{E}+04$

$6.88 \mathrm{E}+03$

$3.93 \mathrm{E}+02$

$1.82 \mathrm{E}+03$

$0.00 \mathrm{E}+01$

$8.68 \mathrm{E}+02$

8.19E-00

$6.15 \mathrm{E}+02$

$5.87 \mathrm{E}+05$ 
Tank Anal. Un

\section{SX104 Pu241 SX104 Pu242 SX104 Ra226 SX104 Ra228 SX104 Ru106 Sx104 Sb125 SX104 Se79} $\begin{array}{ll}\text { SX104 } & \text { Sm151 } \\ \text { SX104 } & \text { Sr89/90 }\end{array}$ $\begin{array}{ll}\text { SX104 } & \text { Sr89/90 } \\ \text { SX104 } & \text { Tc99 }\end{array}$ $\begin{array}{ll}\text { Sx104 } & \text { Tc99 } \\ \text { SX104 } & \text { Th229 }\end{array}$ SX104 Th232 SX104 Tin126 SX104 Tritium SX104 U232

SX104 U233

SX104 U234

SX104 U235

SX104 U236

SX104 U238

SX104 Y90

SX104 Zr93

Sx105 Al

SX105 Bi

SX105 Ca

SX105 Cl

SX105 CO3

SX105 Cr

SX105 F

SX105 Fe

SX105 Hg

SX105 K

SX105 La

SX105 Mn

SX105 Na

SX105 Ni

SX105 NO2

SX105 NO3

SX105 P

SX105 Pb

SX105 PO4

SX105 S

SX105 Si

SX105 SO4

SX105 Sr.

SX105 TOC

SX105 U

SX105 Zr

SX105 Ac227

SX105 Am241

SX105 Am243

SX105 Ba137

SX105 C14

Sx105 Cd113

Sx105 Cm242

Sx105 Cm243

SX105 Cm244

SX105 Co60

SX105 Cs134

SX105 Cs137

SX105 Eu152

SX105 Eu154

SX105 Eu155

SX105 I129

SX105 Nb93

SX105 Ni59

SX105 Ni63

SX105 Np237
$\frac{B B}{98}$

$\frac{\text { May98 }}{3.80 \mathrm{E}+02}$

$1.95 \mathrm{E}-03$

8.29E-104

2.89E-01

1.50E-02

$3.59 \mathrm{E}+02$

9.14E-00

$3.23 \mathrm{E}+04$

$5.43 E+05$

$5.48 \mathrm{E}+02$

6.79E-03

1.93E-02

$1.39 \mathrm{E}+01$

$5.68 \mathrm{E}+02$

1.49E-00

5.70E-00

Ci 2.05E-00

Ci 8.32E-02

Ci 6.68E-02

i $2.28 \mathrm{E}-00$

Ci $5.43 E+05$

4.45E+01

$1.07 \mathrm{E}+05$

$2.99 \mathrm{E}+02$

kg 1.19E+03

1.53E+04

kg $7.58 \mathrm{E}+04$

kg 2.14E+04

kg $\quad 1.80 \mathrm{E}+04$

kg $\quad 6.93 \mathrm{E}+03$

kg 4.69E- 00

kg $\quad 4.82 \mathrm{E}+03$

kg 1.51E+02

kg 3.21E+03

kg $7.34 \mathrm{E}+05$

kg $\quad 6.41 \mathrm{E}+02$

kg 2.31E+05

kg $6.59 \mathrm{E}+05$

kg $1.28 \mathrm{E}+05$

kg 7,37E+02

kg 3.95E+05

kg 5.25E+04

kg 8.08E+03

kg 1.58E+05

$\mathrm{kg} \quad 2.27 \mathrm{E}+02$

3.42E+04

$7.09 E+03$

$2.09 E+02$

4.46E-03

$3.86 \mathrm{E}+03$

$1.00 \mathrm{E}-02$

$6.11 E+05$

$1.09 \mathrm{E}+02$

$2.76 \mathrm{E}+02$

6.52E-01

6.15E-02

$9.81 \mathrm{E}-01$

Ci $1.21 \mathrm{E}+02$

Ci 7.85E-00

Ci $\quad 6.46 \mathrm{E}+05$

Ci $1.74 \mathrm{E}+01$

Ci $1.96 \mathrm{E}+03$

Ci $9.82 \mathrm{E}+02$

Ci 1.49E-00

Ci 4.49E+01

Ci $1.11 \mathrm{E}+01$

Ci 1.09E+03

Ci 2.82E-00
Sept98

$3.80 \mathrm{E}+02$

$1.95 \mathrm{E}-03$

8.29E-04

$2.89 \mathrm{E}-01$

1.50E-02

$3.59 \mathrm{E}+02$

9.14E-00

$3.23 \mathrm{E}+04$

$5.28 \mathrm{E}+05$

$5.48 \mathrm{E}+02$

$6.79 \mathrm{E}-03$

$1.93 \mathrm{E}-02$

$1.39 \mathrm{E}+01$

$5.68 \mathrm{E}+02$

2.60E-00

9.95E-00

3.58E-00

1.45E-01

1.17E-01

$3.24 \mathrm{E}-00$

$5.28 \mathrm{E}+05$

$4.45 E+01$

$1.07 \mathrm{E}+05$

$2.99 E+02$

$1.19 \mathrm{E}+03$

$1.53 \mathrm{E}+04$

$7.66 \mathrm{E}+04$

$2.14 \mathrm{E}+04$

$1.80 \mathrm{E}+04$

$6.93 \mathrm{E}+03$

$0.00 E+01$

$4.82 \mathrm{E}+03$

$1.51 \mathrm{E}+02$

$3.21 \mathrm{E}+03$

$7.34 \mathrm{E}+05$

$6.41 \mathrm{E}+02$

$2.31 \mathrm{E}+05$

$6.59 \mathrm{E}+05$

NA

$7.37 \mathrm{E}+02$

1.29E+05

NA

8.08E+03

$5.25 \mathrm{E}+04$

$2.27 \mathrm{E}+02$

$3.42 E+04$

$7.09 \mathrm{E}+03$

$2.09 E+02$

4.46E-03

$2.64 \mathrm{E}+02$

1.00E-02

$5.80 \mathrm{E}+05$

$1.09 \mathrm{E}+02$

$2.76 \mathrm{E}+02$

6.52E-01

6.15E-02

9.81E-01

$1.21 \mathrm{E}+02$

$7.85 \mathrm{E}-00$

$6.14 E+05$

$1.74 \mathrm{E}+01$

$1.96 \mathrm{E}+03$

$9.82 \mathrm{E}+02$

$1.49 \mathrm{E}-00$

$4.49 \mathrm{E}+01$

$1.11 \mathrm{E}+01$

$1.09 \mathrm{E}+03$

2.82E-00
$1 \%$-ile

\section{$0.00 E+01$}

$10 \%$-ile

$0.00 \mathrm{E}+01$

$8.47 \mathrm{E}+01$

4.35E-04

$1.89 \mathrm{E}-04$

$0.00 \mathrm{E}+01$

$6.59 \mathrm{E}-02$

$1.52 \mathrm{E}-03$

.00E+01

$6.63 \mathrm{E}+01$

$\begin{array}{ll}0.00 \mathrm{E}+01 & 2.26 \mathrm{E}-00 \\ 0.00 \mathrm{E}+01 & 0.00 \mathrm{E}+01\end{array}$

$0.00 \mathrm{E}+01 \quad 2.29 \mathrm{E}+03$

$0.00 \mathrm{E}+01 \quad 0.00 \mathrm{E}+01$

$0.00 \mathrm{E}+01 \quad 6.78 \mathrm{E}-04$

$0.00 \mathrm{E}+01 \quad 1.93 \mathrm{E}-03$

$0.00 E+01 \quad 0.00 E+01$

$3.10 \mathrm{E}+02 \quad 4.18 \mathrm{E}+02$

$0.00 \mathrm{E}+01 \quad 1.49 \mathrm{E}-01$

$0.00 \mathrm{E}+01 \quad 5.69 \mathrm{E}-01$

$0.00 \mathrm{E}+01 \quad 2.05 \mathrm{E}-01$

$0.00 \mathrm{E}+01 \quad 8.31 \mathrm{E}-03$

$0.00 \mathrm{E}+01 \quad 6.67 \mathrm{E}-03$

$0.00 \mathrm{E}+01 \quad 2.28 \mathrm{E}-01$

$0.00 \mathrm{E}+01 \quad 5.02 \mathrm{E}+03$

$0.00 \mathrm{E}+01 \quad 0.00 \mathrm{E}+01$

$2.19 \mathrm{E}+04 \quad 4.65 \mathrm{E}+04$

$0.00 \mathrm{E}+01 \quad 4.02 \mathrm{E}+01$

$3.89 \mathrm{E}+01 \quad 4.95 \mathrm{E}+02$

$8.58 \mathrm{E}+03 \quad 1.17 \mathrm{E}+04$

$1.55 \mathrm{E}+04 \quad 8.66 \mathrm{E}+04$

$1.45 \mathrm{E}+03 \quad 7.49 \mathrm{E}+03$

$0.00 \mathrm{E}+01 \quad 1.09 \mathrm{E}+02$

$1.12 \mathrm{E}+02 \quad 6.83 \mathrm{E}+02$

$0.00 \mathrm{E}+01 \quad 0.00 \mathrm{E}+01$

$3.68 \mathrm{E}+01 \quad 5.36 \mathrm{E}+02$

$1.11 \mathrm{E}-00 \quad 1.93 \mathrm{E}-00$

$1.32 \mathrm{E}+01 \quad 2.49 \mathrm{E}+02$

$5.18 \mathrm{E}+05 \quad 6.84 \mathrm{E}+05$

$1.39 \mathrm{E}-00 \quad 1.41 \mathrm{E}+01$

8.31E+04 1.27E+05

$4.66 \mathrm{E}+05 \quad 8.11 \mathrm{E}+05$

$7.50 \mathrm{E}+03$

2.13E-00

$2.51 E+04$

$3.82 \mathrm{E}+01$

$5.85 \mathrm{E}+01$

$2.58 \mathrm{E}+02$

$3.03 \mathrm{E}-03$

$1.33 \mathrm{E}+02$

$3.16 \mathrm{E}+02$

$0.00 \mathrm{E}+01$

$0.00 \mathrm{E}+01$

7.14E-00

$0.00 \mathrm{E}+01$

$0.00 \mathrm{E}+01$

$0.00 \mathrm{E}+01$

$0.00 \mathrm{E}+01$

$0.00 \mathrm{E}+01$

$0.00 \mathrm{E}+01$

$0.00 \mathrm{E}+01$

1.04E-00

$0.00 \mathrm{E}+01$

$1.62 \mathrm{E}+04$

$0.00 \mathrm{E}+01$

$0.00 \mathrm{E}+01$

$0.00 \mathrm{E}+01$

$0.00 \mathrm{E}+01$

$0.00 \mathrm{E}+01$

$0.00 \mathrm{E}+01$

$0.00 \mathrm{E}+01$

$0.00 \mathrm{E}+01$

$50 \%$-ile

$3.80 \mathrm{E}+02$

$1.95 \mathrm{E}-03$

8.29E-04

2.89E-01

1.50E-02

$3.59 \mathrm{E}+02$

9.14E-00 
Tank Anal. Un

$\begin{array}{ll}\text { SX105 } & \text { Pa231 } \\ \text { SX105 } & \text { Pu238 } \\ \text { SX105 } & \text { Pu239 } \\ \text { SX105 } & \text { Pu240 } \\ \text { SX105 } & \text { Pu241 } \\ \text { SX105 } & \text { Pu242 } \\ \text { SX105 } & \text { Ra226 } \\ \text { SX105 } & \text { Ra228 } \\ \text { SX105 } & \text { Ru106 } \\ \text { SX105 } & \text { Sb125 } \\ \text { SX105 } & \text { Se79 } \\ \text { SX105 } & \text { Sm151 }\end{array}$

SX105 Sr89/90

SX105 Tc99

SX105 Th229

SX105 Th232

Sx105 Tin126

SX105 Tritium.

SX105 U232

SX105 U233

SX105 U234

SX105 U235

SX105 U236

SX105 U238

SX105 Y90

SX105 Zr93

SX106 Al

SX106 Bi

SX106 Ca

SX106 Cl

Sx106 CO3

SX106 Cr

SX106 F

SX106 Fe

SX106 Hg

SX106 K

SX106 La

SX106 Mn

SX106 Na

SX106 Ni

SX106 NO2

SX106 NO3

SX106 $P$

SX106 Pb

SX106 PO4

SX106 S

SX106 Si

SX106 SO4

SX106 Sr

SX106 TOC

SX106 U

SX106 Zr

SX106 Ac227

SX106 Am241

SX106 Am243

SX106 Ba137

SX106 C14

SX106 Cd113

SX106 Cm242

Sx106 Cm243

SX106 Cm244

SX106 Co60

SX106 Cs134

Sx106 Cs137

SX106 Eu152

SX106 Eu154

SX106 Eu155

SX106 I129

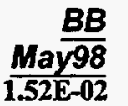

$1.52 \mathrm{E}-02$

$1.36 \mathrm{E}+02$

$3.96 \mathrm{E}+01$

$4.52 \mathrm{E}+02$

$2.49 \mathrm{E}-03$

8.02E-04

4.04E-01

2.15E-02

$5.20 \mathrm{E}+02$

$1.24 \mathrm{E}+01$

$4.38 E+04$

$4.99 E+05$

$7.75 \mathrm{E}+02$

9.05E- 03

2.71E-02

$1.85 \mathrm{E}+01$

$7.59 \mathrm{E}+02$

2.09E-00

8.03E-00

2.56E-00

1.04E-01

8.16E-02

2.93E-00

$4.99 E+05$

$5.99 \mathrm{E}+01$

$4.45 \mathrm{E}+04$

$4.62 \mathrm{E}+02$

$8.96 \mathrm{E}+02$

$1.17 E+04$

$5.21 \mathrm{E}+04$

$1.20 \mathrm{E}+04$

$4.61 \mathrm{E}+03$

$2.79 \mathrm{E}+03$

3.64E-00

$3.37 \mathrm{E}+03$

$2.16 \mathrm{E}+01$

$1.14 \mathrm{E}+03$

$6.13 \mathrm{E}+05$

$2.63 \mathrm{E}+02$

$1.33 \mathrm{E}+05$

$1.08 \mathrm{E}+06$

$1.66 \mathrm{E}+04$

$3.30 \mathrm{E}+02$

$5.13 \mathrm{E}+04$

$1.24 \mathrm{E}+04$

$2.29 \mathrm{E}+03$

$3.73 E+04$

$1.11 E+02$

$1.33 E+04$

$3.60 E+03$

$6.32 \mathrm{E}+01$

$1.99 \mathrm{E}-03$

$1.25 \mathrm{E}+02$

4.34E-03

$3.76 \mathrm{E}+05$

$7.37 \mathrm{E}+01$

$1.89 \mathrm{E}+02$

3.20E-01

2.97E-02

2.93E-01

Ci $8.16 E+01$

Ci 5.57E-00

Ci $\quad 3.98 \mathrm{E}+05$

Ci 8.49E-00

Ci 1.33E+03

Ci $5.01 \mathrm{E}+02$

Ci 1.01E-00

\section{$B B$} Sept 98

\subsection{E-02}

7.51E-00

$2.30 \mathrm{E}+02$

$3.96 \mathrm{E}+01$

$4.52 \mathrm{E}+02$

2.49E-03

8.02E-04

4.04E-01

2.15E-02

$5.20 \mathrm{E}+02$

$1.24 \mathrm{E}+01$

$4.38 \mathrm{E}+04$

$4.80 \mathrm{E}+05$

$7.75 \mathrm{E}+02$

9.50E-03

2.71E-02

$1.85 \mathrm{E}+01$

$7.59 \mathrm{E}+02$

2.13E-00

8.18E-00

2.60E-00

1.06E-01

8.30E-02

2.37E-00

$4.80 \mathrm{E}+05$

$5.99 \mathrm{E}+01$

$4.45 \mathrm{E}+04$

4.62E +02

$8.96 \mathrm{E}+02$

1.17E+04

$5.31 \mathrm{E}+04$

$1.20 \mathrm{E}+04$

$4.61 \mathrm{E}+03$

$2.79 \mathrm{E}+03$

$0.00 \mathrm{E}+01$

$3.37 \mathrm{E}+03$

$2.16 \mathrm{E}+01$

$1.14 E+03$

6.13E +05

$2.63 E+02$

$1.33 \mathrm{E}+05$

$1.08 \mathrm{E}+06$

.NA

$3.30 \mathrm{E}+02$

5.13E+04

NA

$2.29 \mathrm{E}+03$

$3.73 E+04$

$1.11 \mathrm{E}+02$

$1.33 \mathrm{E}+04$

$3.60 \mathrm{E}+03$

$6.32 \mathrm{E}+01$

$1.99 \mathrm{E}-03$

$1.25 E+02$

4.34E-03

$3.57 \mathrm{E}+05$

$7.37 \mathrm{E}+01$

$1.89 \mathrm{E}+02$

3.20E-01

2.97E-02

2.93E-01

$8.16 \mathrm{E}+01$

$5.57 \mathrm{E}-00$

$3.78 \mathrm{E}+05$

8.49E-00

$1.33 \mathrm{E}+03$

$5.01 E+02$

1.01E-00
$1 \%$-ile

$10 \%$-ile

50\%-ile

90\%-ile

$0.00 \mathrm{E}+01 \quad 0.00 \mathrm{E}+01$

$0.00 \mathrm{E}+01 \quad 4.63 \mathrm{E}-01$

$0.00 \mathrm{E}+01$

$8.39 \mathrm{E}-00$

2.44E-00

$0.00 \mathrm{E}+0$

$0.00 \mathrm{E}+01$

$0.00 \mathrm{E}+01 \quad 1.54 \mathrm{E}-04$

$0.00 \mathrm{E}+01 \quad 1.07 \mathrm{E}-04$

$0.00 \mathrm{E}+01$

$0.00 \mathrm{E}+01$

$0.00 \mathrm{E}+01$

$0.00 \mathrm{E}+01$

$0.00 \mathrm{E}+01$

$0.00 \mathrm{E}+01$

$0.00 \mathrm{E}+01$

$0.00 \mathrm{E}+01$

$0.00 \mathrm{E}+01$

$0.00 \mathrm{E}+01$

$3.90 \mathrm{E}+02$

$0.00 \mathrm{E}+01$

$0.00 E+01$

$0.00 \mathrm{E}+01$

$0.00 \mathrm{E}+01$

$0.00 \mathrm{E}+01$

$0.00 E+01$

$0.00 \mathrm{E}+01$

$0.00 E+01$

$9.43 \mathrm{E}+03$

$0.00 \mathrm{E}+01$

$3.07 \mathrm{E}-00$

$7.24 \mathrm{E}+03$

$1.49 \mathrm{E}+04$

$9.38 \mathrm{E}+02$

$0.00 \mathrm{E}+01$

$0.00 \mathrm{E}+01$

$0.00 \mathrm{E}+01$

$2.97 \mathrm{E}+02$

$1.39 \mathrm{E}-01$

$0.00 \mathrm{E}+01$

4.10E+05

$0.00 \mathrm{E}+01$

$8.06 \mathrm{E}+04$

$3.95 \mathrm{E}+05$

$5.09 \mathrm{E}+03$

$1.53 \mathrm{E}-00$

$1.72 \mathrm{E}+04$

$6.33 \mathrm{E}+01$

$0.00 \mathrm{E}+01$

$1.05 \mathrm{E}+03$

$0.00 \mathrm{E}+01$

$6.16 \mathrm{E}+02$

4.88E-00

$0.00 \mathrm{E}+01$

$0.00 \mathrm{E}+01$

$0.00 \mathrm{E}+01$

$0.00 \mathrm{E}+01$

$0.00 \mathrm{E}+01$

$0.00 \mathrm{E}+01$

$0.00 \mathrm{E}+01$

$0.00 \mathrm{E}+01$

$0.00 \mathrm{E}+01$

$0.00 \mathrm{E}+01$

$3.87 \mathrm{E}-01$

$0.00 \mathrm{E}+01$

$8.34 E+04$

$0.00 \mathrm{E}+01$

$0.00 \mathrm{E}+01$

$0.00 \mathrm{E}+01$

$0.00 \mathrm{E}+01$

\subsection{E-02}

7.51E-00

$1.36 \mathrm{E}+02$

$3.96 \mathrm{E}+01$

$4.52 \mathrm{E}+02$

2.49E-03

8.02E-04

4.04E-01

2.15E-02

$5.20 \mathrm{E}+02$

$4.26 \mathrm{E}+01$

$2.24 \mathrm{E}-00$

$0.00 \mathrm{E}+01$

$6.78 \mathrm{E}+03$

$0.00 \mathrm{E}+01$

$0.00 \mathrm{E}+01$

$0.00 \mathrm{E}+01$ 
Tank Anal. Un

$\begin{array}{ll}\text { SX106 } & \text { Nb93 } \\ \text { SX106 } & \text { Ni59 } \\ \text { SX106 } & \text { Ni63 } \\ \text { SX106 } & \text { Np237 } \\ \text { SX106 } & \text { Pa231 } \\ \text { SX106 } & \text { Pu238 } \\ \text { SX106 } & \text { Pu239 } \\ \text { SX106 } & \text { Pu240 } \\ \text { SX106 } & \text { Pu241 } \\ \text { SX106 } & \text { Pu242 } \\ \text { SX106 } & \text { Ra226 } \\ \text { SX106 } & \text { Ra228 } \\ \text { SX106 } & \text { Ru106 } \\ \text { SX106 } & \text { Sb125 }\end{array}$

SX106 Se79

$\begin{array}{ll}\text { SX106 } & \text { Sm151 } \\ \text { SX106 } & \text { Sr89/90 }\end{array}$

SX106 Te99

SX106 Th229

SX106 Th232

SX106 Tin126

SX106 Tritium

SX106 U232

SX106 U233

SX106 U234

SX106 U235

SX106 U236

SX106 U238

SX106 Y90

SX106 Zr93

SX107 Al

SX107 Bi

SX107 Ca

SX107 Cl

SX107 CO3

SX107 Cr

SX107 F

Sx107 Fe

SX107 Hg

SX107 K

SX107 La

SX107 Mn

SX107 Na

SX107 Ni

SX107 NO2

SX107 NO3

SX107 P

SX107 Pb

SX107 PO4

SX107 S

SX107 Si

SX107 SO4

SX107 Sr

SX107 TOC

SX107 U

SX107 Zr

SX107 Ac227

Sx107 Am241

SX107 Am243

SX107 Ba137

SX107 C14

SX107 Cd113

$\begin{array}{lll}\mathrm{Sx} 107 & \mathrm{Cm} 242\end{array}$

Sx107 Cm243

SX107 Cm244

SX107 Co60

SX107 Cs134

$\begin{array}{lll}\text { SX107 } & \text { Cs137 }\end{array}$

\section{May $\overline{98}$}

$2 . \overline{62 \mathrm{E}+01}$

4.80E-00

$4.70 \mathrm{E}+02$

1.92E-00

9.13E-03

$\mathrm{Ci} \cdot 3.00 \mathrm{E}-00$

Ci $1.03 \mathrm{E}+02$

Ci $1.75 \mathrm{E}+01$

Ci $2.03 \mathrm{E}+02$

Ci 1.11E-03

Ci 3.15E-04

Ci 3.07E-01

Ci 1.45E-02

Ci 3.51E+02

Ci 7.35E-00

Ci 2.59E+04

Ci 3.09E+05

Ci 7.20E-03

Ci 2.04E-02

Ci 1.11E+01

5.13E+02

Ci 1.58E-00

Ci 6.05E-00

Ci 1.77E-00

Ci 7.19E-02

Ci 5.55E-02

Ci 2.05E-00

Ci $3.09 \mathrm{E}+05$

Ci 3.61E+01

kg $\quad 6.98 \mathrm{E}+04$

kg $3.14 \mathrm{E}+01$

kg $\quad 1.84 \mathrm{E}+02$

$\mathrm{kg} \quad 1.63 \mathrm{E}+03$

kg 2.84E+03

kg 1.32E+03

kg 1.02E+02

kg $1.11 \mathrm{E}+03$

kg 1.39E-02

kg 2.97E+02

$\mathrm{kg} \quad 0.00 \mathrm{E}+01$

kg $\quad 9.15 \mathrm{E}+02$

kg $\quad 6.95 \mathrm{E}+04$

kg 8.12E+01

kg 2.09E+04

kg $\quad 6.39 \mathrm{E}+04$

kg 3.84E+02

kg $228 \mathrm{E}+01$

$\mathrm{kg} \quad 1.19 \mathrm{E}+03$

kg 3.40E+02

kg $8.60 \mathrm{E}+02$

$\mathrm{kg} \quad 1.02 \mathrm{E}+03$

kg 2.89E+02

kg $6.95 \mathrm{E}+02$

kg $\quad 5.40 E+03$

kg 4.60E+01

4.32E-03

$2.09 E+02$

9.27E-03

$5.04 \mathrm{E}+04$

1.85E-00

$7.29 E-00$

$5.20 \mathrm{E}-01$

4.97E-02

1.48E-00

$1.52 \mathrm{E}-00$

3.75E-01

$5.33 E+04$
Sept98

$2.62 \mathrm{E}+01$

$4.80 \mathrm{E}-00$

$4.70 \mathrm{E}+02$

1.92E-00

9.13E-03

$3.00 \mathrm{E}-00$

$1.03 \mathrm{E}+02$

$1.75 \mathrm{E}+01$

$2.03 \mathrm{E}+02$

1.11E-03

3.15E-04

3.07E-01

$1.45 \mathrm{E}-02$

$3.51 \mathrm{E}+02$

7.35E-00

$2.59 \mathrm{E}+04$

$2.94 \mathrm{E}+05$

$5.25 \mathrm{E}+02$

$7.20 \mathrm{E}-03$

2.04E-02

$1.11 \mathrm{E}+01$

$5.13 E+02$

1.18E-00

4.51E-00

1.32E-00

5.36E-02

4.14E-02

$1.20 \mathrm{E}-00$

$2.94 \mathrm{E}+05$

$3.61 \mathrm{E}+01$

$6.98 \mathrm{E}+04$

$3.14 \mathrm{E}+01$

$1.84 \mathrm{E}+02$

$1.63 \mathrm{E}+03$

$2.84 \mathrm{E}+03$

$1.32 \mathrm{E}+03$

$8.27 \mathrm{E}+01$

$1.11 \mathrm{E}+03$

$0.00 \mathrm{E}+01$

$2.97 \mathrm{E}+02$

$0.00 \mathrm{E}+01$

$9.15 \mathrm{E}+02$

$6.73 \mathrm{E}+04$

$8.12 \mathrm{E}+01$

2.09E +04

$8.43 E+04$

NA

$2.28 \mathrm{E}+01$

1.19E +03

NA

$8.60 \mathrm{E}+02$

$1.02 \mathrm{E}+03$

$2.89 \mathrm{E}+02$

$6.95 \mathrm{E}+02$

$5.29 \mathrm{E}+03$

$4.60 \mathrm{E}+01$

4.32E-03

$2.09 \mathrm{E}+02$

9.27E-03

$5.04 \mathrm{E}+04$

$1.85 \mathrm{E}-00$

$7.29 \mathrm{E}-00$

5.20E-01

4.97E-02

$1.48 \mathrm{E}-00$

1.52E-00

$3.75 \mathrm{E}-01$

$5.33 E+04$
$1 \%$-ile

$0.00 \mathrm{E}+01$

$0.00 \mathrm{E}+01$

$0.00 \mathrm{E}+01$

$0.00 \mathrm{E}+01$

$0.00 \mathrm{E}+01$

$0.00 \mathrm{E}+01$

$0.00 \mathrm{E}+01$

$0.00 \mathrm{E}+01$

$0.00 \mathrm{E}+01$

$0.00 \mathrm{E}+01$

$0.00 \mathrm{E}+01$

$0.00 \mathrm{E}+01$

$0.00 E+01$

$0.00 \mathrm{E}+01$

$0.00 E+01$

$0.00 \mathrm{E}+01$

$0.00 \mathrm{E}+01$

$0.00 \mathrm{E}+01$

$0.00 \mathrm{E}+01$

$0.00 \mathrm{E}+01$

$0.00 \mathrm{E}+01$

$2.42 \mathrm{E}+02$

$0.00 \mathrm{E}+01$

$0.00 \mathrm{E}+01$

$0.00 \mathrm{E}+01$

$0.00 \mathrm{E}+01$

$0.00 \mathrm{E}+01$

$0.00 \mathrm{E}+01$

$0.00 \mathrm{E}+01$

$0.00 \mathrm{E}+01$

$3.12 \mathrm{E}+04$

$0.00 \mathrm{E}+01$

$5.59 \mathrm{E}+01$

4.20E+01

$6.99 \mathrm{E}-01$

4.12E+01

$0.00 \mathrm{E}+01$

$2.89 \mathrm{E}+01$

$5.89 \mathrm{E}-06$

$2.87 \mathrm{E}+01$

4.18E-01

2.11E+01

$1.50 \mathrm{E}+04$

2.22E-03

$5.32 \mathrm{E}+02$

$0.00 \mathrm{E}+01$

$2.43 \mathrm{E}+01$

$2.78 \mathrm{E}-01$

8.21E-02

$2.32 E+01$

$2.52 \mathrm{E}+01$

$2.35 \mathrm{E}+01$

2.60E-01

$5.76 \mathrm{E}+01$

$3.02 \mathrm{E}+02$

$0.00 E+01$

$0.00 \mathrm{E}+01$

$2.66 \mathrm{E}+01$

$0.00 E+01$

$0.00 E+01$

$0.00 \mathrm{E}+01$

3.09E-03

$0.00 \mathrm{E}+01$

$0.00 \mathrm{E}+01$

$0.00 \mathrm{E}+01$

3.55E-00

$0.00 \mathrm{E}+01$

$1.41 \mathrm{E}+03$

$10 \%$-ile

$0.00 \mathrm{E}+01$

$0.00 \mathrm{E}+01$

$0.00 \mathrm{E}+01$

$5.65 \mathrm{E}-03$

$0.00 \mathrm{E}+01$

8.82E-03

$3.03 \mathrm{E}-01$

5.15E-02

5.97E-01

3.26E-06

5.01E-06

4.88E-03

$0.00 \mathrm{E}+01$

$0.00 \mathrm{E}+01$

$1.55 \mathrm{E}-00$

$0.00 \mathrm{E}+01$

$2.44 \mathrm{E}+02$

$0.00 \mathrm{E}+01$

$0.00 \mathrm{E}+01$

$0.00 \mathrm{E}+01$

$0.00 \mathrm{E}+01$

$3.59 \mathrm{E}+02$

$0.00 \mathrm{E}+01$

$0.00 \mathrm{E}+01$

$0.00 \mathrm{E}+01$

$0.00 \mathrm{E}+01$ 


\begin{tabular}{|c|c|c|c|c|c|c|c|c|c|c|c|}
\hline ank & Anal. & Un & May $\frac{B B}{98}$ & Sept98 & $1 \%$-ile & $10 \%$-ile & $50 \%$-ile & 90\%-ile & 99\%-ile & Mean & Std Dev \\
\hline $\mathrm{X} 107$ & $\mathbf{u} 152$ & $\mathrm{Ci}$ & $1 \overline{24 \mathrm{E}+01}$ & $\overline{1.24 \mathrm{E}+01}$ & $0.00 \mathrm{E}+01$ & 4.43E-00 & $1.24 \mathrm{E}+01$ & $4.77 \mathrm{E}+01$ & $1.36 \mathrm{E}+02$ & $2.13 E+01$ & $2.68 \mathrm{E}+01$ \\
\hline X107 & Eu154 & $\mathrm{Ci}$ & $3.66 E+01$ & $3.66 \mathrm{E}+01$ & $0.00 E+01$ & $1.31 \mathrm{E}+01$ & $3.66 \mathrm{E}+01$ & $1.41 E+02$ & $4.02 \mathrm{E}+02$ & $6.29 \mathrm{E}+01$ & $7.92 \mathrm{E}+01$ \\
\hline $\mathbf{X 1 0 7}$ & Eu155 & $\mathrm{Ci}$ & $6.28 \mathrm{E}+02$ & $6.28 \mathrm{E}+02$ & $0.00 \mathrm{E}+01$ & $2.24 \mathrm{E}+02$ & $6.28 \mathrm{E}+02$ & $2.42 E+03$ & $6.89 \mathrm{E}+03$ & $1.08 \mathrm{E}+03$ & $1.36 \mathrm{E}+03$ \\
\hline SX107 & 1129 & $\mathrm{Ci}$ & 2.70E-02 & $2.70 \mathrm{E}-02$ & $0.00 \mathrm{E} \div 01$ & $2.50 \mathrm{E}-03$ & $2.70 \mathrm{E}-02$ & $5.91 \mathrm{E}-02$ & $7.89 \mathrm{E}-02$ & $2.88 \mathrm{E}-02$ & $2.14 \mathrm{E}-02$ \\
\hline SX107 & $\mathrm{Nb93}$ & $\mathrm{Ci}$ & $1.47 \mathrm{E}+01$ & $1.47 \mathrm{E}+01$ & $0.00 \mathrm{E}+01$ & $1.77 \mathrm{E}-00$ & $1.47 E+01$ & $1.41 E+02$ & $1.62 E+03$ & $7.66 \mathrm{E}+01$ & $2.41 E+02$ \\
\hline SX107 & Ni59 & $\mathbf{C i}$ & $1.13 E+01$ & $1.13 E+01$ & $0.00 \mathrm{E}+01$ & $3.22 \mathrm{E}-00$ & +01 & $6 E+01$ & +02 & & \\
\hline $\mathbf{1 0 7}$ & Ni63 & $\mathbf{C i}$ & $1.10 E+03$ & $1.10 \mathrm{E}+03$ & $0.00 \mathrm{E}+01$ & $3.13 E+02$ & $1.10 E+03$ & $2.78 E+03$ & +04 & +03 & \\
\hline $\mathbf{1 0 7}$ & Np237 & $\mathrm{Ci}$ & $6.62 \mathrm{E}-02$ & $6.62 \mathrm{E}-02$ & $0.00 \mathrm{E}+01$ & $\mathrm{E}-03$ & $6.62 \mathrm{E}-02$ & $1.33 \mathrm{E}-01$ & $2.02 \mathrm{E}-01$ & EE-02 & $E-02$ \\
\hline X107 & Pa231 & $\mathrm{Ci}$ & $4.15 E-03$ & $4.15 \mathrm{E}-03$ & $0.00 \mathrm{E}+01$ & $3.68 \mathrm{E}-04$ & $4.15 E-03$ & $1.60 \mathrm{E}-02$ & $2.50 \mathrm{E}-02$ & -03 & -03 \\
\hline X107 & Pu238 & $\mathbf{C i}$ & $8.56 \mathrm{E}-00$ & $8.56 \mathrm{E}-00$ & $0.00 \mathrm{E}+01$ & $5.83 \mathrm{E}-01$ & $8.56 \mathrm{E}-00$ & $1.72 E+01$ & $2.61 E+01$ & $9.04 \mathrm{E}-00$ & $6.34 \mathrm{E}-00$ \\
\hline X107 & Pu239 & $\mathbf{C i}$ & $2.50 \mathrm{E}+02$ & $2.50 \mathrm{E}+02$ & $0.00 \mathrm{E}+01$ & $1.70 \mathrm{E}+01$ & $2.50 \mathrm{E}+02$ & $5.01 \mathrm{E}+02$ & $7.61 \mathrm{E}+02$ & $2.64 \mathrm{E}+02$ & $1.85 \mathrm{E}+02$ \\
\hline $\mathbf{X 1 0 7}$ & Pu240 & $\mathrm{Ci}$ & $4.25 E+01$ & $4.25 E+01$ & $0.00 \mathrm{E}+01$ & $2.90 \mathrm{E}-00$ & $4.25 E+01$ & $8.52 E+01$ & $1.29 E+02$ & $4.49 \mathrm{E}+01$ & $3.15 \mathrm{E}+01$ \\
\hline X107 & Pu241 & $\mathbf{C i}$ & $4.31 E+02$ & $4.31 E+02$ & $0.00 \mathrm{E}+01$ & $2.94 \mathrm{E}+01$ & $4.31 E+02$ & $8.64 \mathrm{E}+02$ & $1.31 \mathrm{E}+03$ & $4.55 \mathrm{E}+02$ & $19 \mathrm{E}+02$ \\
\hline SX107 & Pu242 & $\mathbf{C i}$ & $236 \mathrm{E}-03$ & $2.36 \mathrm{E}-03$ & $0.00 \mathrm{E}+01$ & $1.61 E-04$ & 2.36E-03 & 4.73E-03 & $7.18 \mathrm{E}-03$ & E-03 & $75 \mathrm{E}-03$ \\
\hline SX107 & Ra226 & $\mathrm{Ci}$ & $9.61 \mathrm{E}-04$ & $9.61 \mathrm{E}-04$ & $E+01$ & .04 & & -03 & $E-02$ & E-03 & \\
\hline SX107 & $\mathbf{R a 2 2 8}$ & $\mathrm{Ci}$ & 1.01E-03 & $1.01 \mathrm{E}-03$ & +01 & .04 & -03 & -03 & $3.07 \mathrm{E}-02$ & $E-03$ & $E-03$ \\
\hline 8107 & Ru106 & $\mathrm{Ci}$ & $3.28 \mathrm{E}-04$ & $3.28 \mathrm{E}-04$ & \pm+01 & -05 & $9-04$ & $1.50 \mathrm{E}-03$ & $E-03$ & E-04 & -04 \\
\hline 107 & Sb125 & $\mathrm{Ci}$ & 5.16E-00 & $5.16 \mathrm{E}-00$ & $0.00 \mathrm{E}+01$ & $E-01$ & $5.16 \mathrm{E}-00$ & $1.04 E+02$ & $3.23 E+02$ & $3.31 \mathrm{E}+01$ & $6.90 \mathrm{E}+01$ \\
\hline SX107 & Se79 & $\mathrm{Ci}$ & 3.73E-00 & $3.73 E-00$ & $E-02$ & $E-00$ & 3.73E-00 & $2.10 \mathrm{E}+01$ & +02 & $E+01$ & $E+01$ \\
\hline $\mathbf{X 1 0 7}$ & Sm151 & $\mathrm{Ci}$ & $1.34 \mathrm{E}+04$ & $1.34 \mathrm{E}+04$ & $0.00 \mathrm{E}+01$ & $E+03$ & $Q+04$ & $E+04$ & $E+05$ & $E+04$ & $E+04$ \\
\hline SX107 & Sr89/90 & $\mathbf{C i}$ & $1.98 E+05$ & $1.98 E+05$ & $0.00 \mathrm{E}+01$ & $4.45 \mathrm{E}-00$ & $2.03 E+05$ & 2.77E+06 & $E+07$ & $E+06$ & $\mathrm{E}+06$ \\
\hline SX107 & Te99 & $\mathrm{Ci}$ & $1.42 \mathrm{E}+01$ & $1.42 \mathrm{E}+01$ & $E+01$ & +01 & +01 & $E+01$ & +01 & $E+01$ & +01 \\
\hline SX107 & Th229 & $\mathrm{Ci}$ & 2.58E-05 & $2.58 \mathrm{E}-05$ & +01 & -06 & -05 & -05 & -04 & -05 & -05 \\
\hline SX107 & Th232 & $\mathrm{Ci}$ & $1.36 \mathrm{E}-05$ & $1.36 E-05$ & \pm 01 & -06 & -05 & -05 & $E-05$ & -05 & $E-05$ \\
\hline SX107 & Tin126 & $\mathrm{Ci}$ & 4.85E-00 & $4.85 \mathrm{E}-00$ & $2.05 \mathrm{E}-03$ & $E-00$ & $4.85 \mathrm{E}-00$ & $4.79 E+01$ & $1.59 \mathrm{E}+02$ & $E+01$ & $E+01$ \\
\hline$\times 107$ & Tritium & $\mathrm{Ci}$ & $3.47 \mathrm{E}+01$ & $3.47 \mathrm{E}+01$ & +01 & +01 & & +01 & +01 & & $\vdots+01$ \\
\hline $\mathbf{X 1 0 7}$ & U232 & $\mathrm{Ci}$ & 4.53E-03 & $7.38 \mathrm{E}-02$ & $0.00 \mathrm{E}+01$ & $E-04$ & E3 & $E-02$ & $\varepsilon-02$ & & -03 \\
\hline 107 & U233 & $\mathrm{Ci}$ & 1.73E-02 & 2.82E-01 & $E+01$ & & -02 & $E-02$ & $E-01$ & -02 & $E-02$ \\
\hline SX107 & U234 & $\mathrm{Ci}$ & $1.23 E-01$ & 2.01E-00 & +01 & 02 & -01 & -01 & -01 & -01 & -01 \\
\hline 107 & U235 & $\mathbf{C i}$ & 5.01E-03 & $8.17 E-02$ & $E+01$ & -04 & -03 & $E-02$ & $E-02$ & $E-03$ & $E-03$ \\
\hline $3 \times 107$ & U236 & $\mathrm{Ci}$ & $4.71 E-03$ & $7.67 \mathrm{E}-02$ & $0.00 \mathrm{E}+01$ & -04 & $4.71 \mathrm{E}-03$ & IE-02 & E-02 & $E-03$ & $E-03$ \\
\hline 107 & $\mathbf{U} 238$ & $\mathbf{C i}$ & 1.10E-01 & $1.77 \mathrm{E}-00$ & -01 & & & & -01 & -01 & $E-01$ \\
\hline X107 & Y90 & $\mathrm{Ci}$ & $1.98 \mathrm{E}+05$ & $1.98 \mathrm{E}+05$ & $E+01$ & +01 & +05 & +06 & +07 & +06 & $E+06$ \\
\hline $\mathbf{x 1 0 7}$ & Zr93 & $\mathbf{C i}$ & $1.59 E+01$ & $1.59 \mathrm{E}+01$ & 01 & & 01 & & +03 & & +02 \\
\hline X108 & Al & kg & $3.69 E+04$ & $3.69 \mathrm{E}+04$ & +04 & -04 & 6.2 & -05 & -05 & -04 & +04 \\
\hline X108 & $\mathbf{B i}$ & kg & $4.30 \mathrm{E}+01$ & $4.30 E+01$ & $0.00 \mathrm{E}+01$ & +01 & +02 & +03 & $E+04$ & +03 & $E+03$ \\
\hline $\mathbf{X 1 0 8}$ & $\mathrm{Ca}$ & $\mathbf{k g}$ & $3.69 \mathrm{E}+03$ & $3.69 \mathrm{E}+03$ & +01 & +01 & +02 & +03 & $\div 03$ & +02 & +02 \\
\hline SX108 & Cl & kg & $1.24 E+03$ & $1.24 \mathrm{E}+03$ & $8+01$ & +02 & 9.8 & $E+03$ & $8+03$ & +03 & $E+02$ \\
\hline X108 & $\mathrm{CO} 3$ & kg & $5.52 E+03$ & $5.52 E+03$ & $E-01$ & & & $E+04$ & +04 & +03 & +03 \\
\hline X108 & $\mathrm{Cr}$ & kg & 4.9 & 4.9 & -01 & -02 & & +03 & +03 & +03 & +03 \\
\hline $\mathbf{X 1 0 8}$ & $\mathbf{F}$ & kg & $2.49 \mathrm{E}+02$ & $2.49 E+02$ & +01 & +01 & 4. & +03 & +04 & +03 & +03 \\
\hline SX108 & $\mathrm{Fe}$ & $\mathrm{kg}$ & $9.90 \mathrm{E}+03$ & $9.90 \mathrm{E}+03$ & $E+01$ & +02 & +03 & +03 & +04 & +03 & $E+03$ \\
\hline X108 & $\mathrm{Hg}$ & kg & $0.00 \mathrm{E}+01$ & $0.00 \mathrm{E}+01$ & $E+01$ & +01 & +01 & $E+01$ & +01 & +01 & $: 01$ \\
\hline X108 & $\mathbf{K}$ & kg & $3.64 E+02$ & $3.64 E+02$ & $E+01$ & +02 & +02 & +02 & +03 & -02 & +02 \\
\hline 108 & $\mathbf{L a}$ & kg & $9.15 E+01$ & 9.1 & & .00 & & +01 & +01 & -00 & -00 \\
\hline & Mn & $\mathrm{kg}$ & -03 & & +01 & -02 & & -03 & -03 & +03 & +03 \\
\hline X108 & $\mathbf{N a}$ & kg & $5.53 E+04$ & $5.53 E+04$ & $E+04$ & +04 & -04 & +05 & 105 & +04 & +04 \\
\hline X108 & $\mathbf{N i}$ & kg & $8.87 E+02$ & $8.87 E+02$ & $5 E-02$ & $:+01$ & 4.3 & $E+02$ & +03 & $E+02$ & $E+02$ \\
\hline X108 & $\mathrm{NO} 2$ & $\mathbf{k g}$ & $9.04 E+03$ & $9.04 E+03$ & $3.12 \mathrm{E}+02$ & +03 & 1.2 & +04 & $E+04$ & :+04 & $E+04$ \\
\hline 108 & NO3 & $\mathrm{kg}$ & $8.56 E+04$ & $8.56 E+04$ & E-00 & $E+03$ & +04 & +05 & +05 & +04 & $E+04$ \\
\hline SX108 & $\mathbf{P}$ & kg & $2.68 E+02$ & $\mathbf{N A}$ & $E-00$ & +02 & +03 & $E+04$ & $E+04$ & +03 & $E+03$ \\
\hline X108 & $\mathbf{P b}$ & $\mathrm{kg}$ & $2.45 \mathrm{E}+02$ & $2,45 E+02$ & & & & 02 & 02 & 01 & $=02$ \\
\hline & P04 & kg & 8.29 & $1.43 E+03$ & & & & & 104 & & +04 \\
\hline & $\mathbf{S}$ & $\mathrm{kg}$ & $1.32 E+03$ & $\mathbf{N A}$ & $1.12 \mathrm{E}+01$ & +01 & 2.5 & +03 & $\varepsilon+04$ & +02 & $E+03$ \\
\hline X108 & $\mathbf{S i}$ & kg & $4.36 E+02$ & $4.36 \mathrm{E}+02$ & $E+01$ & +02 & 8.8 & $E+03$ & $E+04$ & $E+03$ & $E+03$ \\
\hline X108 & SO4 & kg & $3.97 E+03$ & $3.97 E+03$ & $E-00$ & +02 & 7.4 & $E+03$ & $E+04$ & $E+03$ & $E+03$ \\
\hline SX108 & $\mathbf{S r}$ & kg & $3.21 E+02$ & $3.21 E+02$ & $4.44 \mathrm{E}-02$ & $E+01$ & $2+02$ & $4.40 E+02$ & $E+03$ & +02 & $E+02$ \\
\hline SX108 & TOC & kg & $0.00 E+01$ & $0.00 \mathrm{E}+01$ & +01 & & 1.5 & $E+03$ & $E+03$ & +03 & $E+03$ \\
\hline & $\mathbf{U}$ & kg & & & & & & & +04 & & +03 \\
\hline XX108 & $\mathbf{Z r}$ & kg & $1.47 E+02$ & $1.47 E+02$ & $E+01$ & -00 & +01 & $1.43 E+02$ & $E+03$ & +02 & $E+02$ \\
\hline & Ac227 & $\mathrm{Ci}$ & $3.50 \mathrm{E}-03$ & $3.50 \mathrm{E}-03$ & $E+01$ & $4.09 \mathrm{E}-04$ & & -03 & $7 E-02$ & -03 & E-03 \\
\hline & Am241 & $\mathrm{Ci}$ & $1.52 E+02$ & $1.52 E+02$ & $1.03 E+01$ & +01 & 3.94 & $1.29 E+02$ & +02 & 7.7 & +02 \\
\hline $5 \mathbf{Y} 108$ & Am243 & $\mathrm{Ci}$ & $6.78 E-03$ & $6.78 E-03$ & $0.00 E+01$ & $3.65 E-04$ & $6.78 \mathrm{E}-03$ & $9 \mathrm{E}-02$ & $0 \mathrm{E}-02$ & $7.63 E-03$ & 7E-03 \\
\hline & Ba13 & $\mathrm{Ci}$ & $3.07 E+04$ & $6.54 E+04$ & $0.00 E+01$ & $7.21 E+03$ & $3.07 E+0$ & $6.10 E+04$ & $8.90 E+04$ & $E+04$ & $2.06 \mathrm{E}+04$ \\
\hline & C14 & $\mathrm{Ci}$ & 1.02E-00 & 1.02 & $0.00 E+01$ & $4.00 E-02$ & $1.02 \mathrm{E}-00$ & $E-00$ & $4.01 E-00$ & $E-00$ & $7 \mathrm{E}-01$ \\
\hline SX108 & Cd113 & $\mathrm{Ci}$ & $3.80 \mathrm{E}-00$ & $3.80 \mathrm{E}-00$ & $7.72 \mathrm{E}-02$ & $1.09 \mathrm{E}-00$ & $3.80 \mathrm{E}-00$ & $4.07 \mathrm{E}+01$ & $1.42 E+02$ & $1.49 E+01$ & $2.89 E+01$ \\
\hline SX108 & $\mathrm{Cm} 242$ & $\mathrm{Ci}$ & 4.03E-01 & $4.03 E-01$ & $0.00 \mathrm{E}+01$ & $2.17 E-02$ & 4.03E-01 & $9.44 \mathrm{E}-01$ & $1.49 \mathrm{E}-00$ & $4.53 \mathrm{E}-01$ & $5 \mathrm{E}-01$ \\
\hline 108 & $\mathrm{Cm} 243$ & $\mathrm{C} \mathbf{j}$ & $3.76 \mathrm{E}-02$ & $3.76 E-02$ & $0.00 \mathrm{E}+01$ & 2.03E-03 & $3.76 \mathrm{E}-02$ & $8.81 E-02$ & $1.39 \mathrm{E}-01$ & $4.23 \mathrm{E}-02$ & $3.31 \mathrm{E}-02$ \\
\hline
\end{tabular}


Tank Anal. Un

\begin{tabular}{|c|c|}
\hline & \\
\hline 108 & Co60 \\
\hline 108 & Cs134 \\
\hline 108 & Cs137 \\
\hline 108 & Eu152 \\
\hline K108 & Eu154 \\
\hline $\mathrm{K108}$ & Eu155 \\
\hline 108 & 1129 \\
\hline 18 & $\mathrm{Nb93}$ \\
\hline K108 & Ni59 \\
\hline SX108 & Ni63 \\
\hline SX108 & Np237 \\
\hline$\times 108$ & Pa231 \\
\hline$\times 108$ & Pu238 \\
\hline $\mathbf{K 1 0 8}$ & Pu239 \\
\hline 6108 & Pu240 \\
\hline 108 & Pu241 \\
\hline 108 & Pu242 \\
\hline SX108 & $\operatorname{Ra} 226$ \\
\hline SX108 & $\operatorname{Ra} 228$ \\
\hline SX108 & Ru106 \\
\hline SX108 & Sb125 \\
\hline SX108 & Se79 \\
\hline 1 & Sm151 \\
\hline SX108 & Sr89/90 \\
\hline SX108 & Te99 \\
\hline SX108 & Th229 \\
\hline SX108 & Th232 \\
\hline SX108 & Tin126 \\
\hline SX10 & Tritium \\
\hline SX108 & $\mathrm{U} 232$ \\
\hline SX108 & U233 \\
\hline SX108 & U234 \\
\hline SX108 & U235 \\
\hline SX1 & U236 \\
\hline & U23 \\
\hline & \\
\hline
\end{tabular}

SX108 Zr93

SX109 A]

SX109 Bi

SX109 Ca

$5 \times 109 \mathrm{C}$

SX109 CO3

Sx109 $\mathrm{Cr}$

SX109 F

SX109 Fe

SX109 Hg

SX109 K

Sx109 La

SX109 Mn

SX109 $\mathrm{Na}$

Sx109 Ni

SX109 NO2

SX109 NO3

SX109 P

Sx109 Pb

SX109 PO4

SX109 S

SX109 Si

SX109 SO4

Sx109 Sr

SX109 TOC

SX109 U

SX109 Zr

SX109 Ac227

Sx109 Am241

SX109 Am243

SX109 Ba137
$\frac{B 8}{\text { May98 }}$

7.15E-01

1.73E-01

3.25E+04

9.46E-00

$1.74 \mathrm{E}+01$

$4.76 \mathrm{E}+02$

$1.46 \mathrm{E}-02$

$9.90 \mathrm{E}-00$

9.40E-00

$9.08 \mathrm{E}+02$

$3.94 \mathrm{E}-02$

2.67E-03

$6.85 \mathrm{E}-00$

$2.22 \mathrm{E}+02$

$3.68 \mathrm{E}+01$

$3.50 \mathrm{E}+02$

$1.89 \mathrm{E}-03$

$7.87 \mathrm{E}-04$

1.58E-08

1.32E-04

2.21E-00

2.50E-00

$8.96 \mathrm{E}+03$

$1.09 \mathrm{E}+06$

$7.65 \mathrm{E}-00$

1.29E-06

5.77E-10

3.19E-00

$1.77 \mathrm{E}+01$

2.32E-06

7.42E-08

4.08E-02

$1.70 \mathrm{E}-03$

$1.14 \mathrm{E}-03$

3.85E-02

$1.09 \mathrm{E}+06$

$1.05 \mathrm{E}+01$

1.19E+05

$6.53 E+01$

$4.86 \mathrm{E}+03$

$3.93 E+03$

$1.61 \mathrm{E}+04$

$2.12 E+04$

$3.23 E+02$

$1.11 \mathrm{E}+04$

$1.56 \mathrm{E}-01$

$9.84 \mathrm{E}+02$

$1.69 \mathrm{E}-06$

$3.89 \mathrm{E}+03$

$2.50 \mathrm{E}+05$

$1.24 \mathrm{E}+03$

$4.45 \mathrm{E}+04$

$4.09 \mathrm{E}+05$

$5.62 \mathrm{E}+02$

$2.59 \mathrm{E}+02$

$1.74 E+03$

$2.11 E+03$

$2.49 \mathrm{E}+03$

$6.34 \mathrm{E}+03$

$1.99 \mathrm{E}+02$

8.18E+02

$1.67 \mathrm{E}+04$

$3.16 \mathrm{E}+01$

4.77E-03

$2.19 E+02$

8.70E-03

$2.44 E+05$

\section{$\frac{B B}{\operatorname{Bg}}$ \\ $\frac{\text { Sept98 }}{1.11 \mathrm{E}-00}$}

7.15E-01

1.73E-01

$6.92 \mathrm{E}+04$

9.46E-00

$1.74 \mathrm{E}+01$

$4.76 E+02$

1.46E-02

9.90E-00

9.40E-00

$9.08 \mathrm{E}+02$

3.94E-02

2.67E-03

6.85E-00

$2.22 \mathrm{E}+02$

$3.68 \mathrm{E}+01$

$3.50 \mathrm{E}+02$

$1.89 \mathrm{E}-03$

7.87E-04

$1.58 \mathrm{E}-08$

1.32E-04

2.21E-00

2.50E-00

$8.96 \mathrm{E}+03$

$1.09 \mathrm{E}+06$

$7.65 \mathrm{E}-00$

$1.29 \mathrm{E}-06$

5.77E-10

3.19E-00

$1.77 \mathrm{E}+01$

1.31E-04

4.19E-06

2.30E-00

9.61E-02

6.46E-02

2.17E-00

$1.09 \mathrm{E}+06$

$1.05 \mathrm{E}+01$

$1.21 \mathrm{E}+05$

$5.41 \mathrm{E}+01$

$4.86 \mathrm{E}+03$

$3.98 \mathrm{E}+03$

$1.81 \mathrm{E}+04$

$2.12 \mathrm{E}+04$

$3.12 \mathrm{E}+02$

1.11E+04

$0.00 \mathrm{E}+01$

$9.93 \mathrm{E}+02$

$1.69 \mathrm{E}-06$

$3.92 \mathrm{E}+03$

2.52E+05

$1.24 \mathrm{E}+03$

$4.51 \mathrm{E}+04$

4.12E+05

NA

$2.59 \mathrm{E}+02$

$1.74 \mathrm{E}+03$

NA

$2.52 \mathrm{E}+03$

$6.87 \mathrm{E}+03$

$2.07 \mathrm{E}+02$

$8.53 \mathrm{E}+02$

$1.69 \mathrm{E}+04$

$3.30 \mathrm{E}+01$

4.77E-03

$2.19 E+02$

8.70E-03 $2.44 E+05$
$1 \%$ ile

\section{$0.00 \mathrm{E}+01$}

$1.59 \mathrm{E}-00$

$0.00 \mathrm{E}+01$

$1.12 \mathrm{E}+03$

$0.00 \mathrm{E}+01$

$0.00 \mathrm{E}+01$

$0.00 \mathrm{E}+01$

$0.00 \mathrm{E}+01$

$0.00 \mathrm{E}+01$

$0.00 \mathrm{E}+01$

$0.00 \mathrm{E}+01$

$0.00 \mathrm{E}+01$

$0.00 \mathrm{E}+01$

$0.00 \mathrm{E}+01$

$0.00 \mathrm{E}+01$

$0.00 \mathrm{E}+01$

$0.00 \mathrm{E}+01$

$0.00 \mathrm{E}+01$

$0.00 \mathrm{E}+01$

$0.00 \mathrm{E}+01$

$0.00 \mathrm{E}+01$

$0.00 \mathrm{E}+01$

$1.48 \mathrm{E}-02$

$0.00 \mathrm{E}+01$

$5.63 \mathrm{E}-00$

$0.00 \mathrm{E}+01$

$0.00 \mathrm{E}+01$

$0.00 \mathrm{E}+01$

$6.48 \mathrm{E}-02$

$3.45 \mathrm{E}-00$

$0.00 \mathrm{E}+01$

$0.00 \mathrm{E}+01$

$0.00 \mathrm{E}+0 \mathrm{I}$

$0.00 \mathrm{E}+0 \mathrm{I}$

$0.00 \mathrm{E}+01$

$0.00 \mathrm{E}+01$

$0.00 \mathrm{E}+01$

$0.00 \mathrm{E}+01$

$5.97 \mathrm{E}+04$

$0.00 \mathrm{E}+01$

$5.30 \mathrm{E}+01$

$1.16 \mathrm{E}+02$

$2.88 \mathrm{E}-00$

$4.38 \mathrm{E}+01$

$0.00 \mathrm{E}+01$

$5.79 \mathrm{E}+01$

$0.00 E+01$

$2.85 \mathrm{E}+01$

4.64E-01

$3.49 \mathrm{E}+01$

$3.04 \mathrm{E}+04$

$1.57 \mathrm{E}-00$

$7.17 \mathrm{E}+02$

$0.00 E+01$

$4.34 \mathrm{E}+01$

$6.32 \mathrm{E}-01$

4.72E-00

$1.28 \mathrm{E}+01$

$2.58 \mathrm{E}+01$

$5.03 \mathrm{E}+01$

$1.77 \mathrm{E}+01$

8.11E+01

$6.18 \mathrm{E}+02$

$0.00 \mathrm{E}+01$

$0.00 \mathrm{E}+01$

$1.67 \mathrm{E}+01$

$0.00 \mathrm{E}+01$

$5.50 \mathrm{E}+03$

$10 \%$-ile

\section{$5.98 \mathrm{E}-02$}

3.19E-00

4.06E-02

$1.74 \mathrm{E}+04$

$1.11 \mathrm{E}-00$

2.03E-00

$5.57 \mathrm{E}+01$

$1.69 \mathrm{E}-03$

$1.19 \mathrm{E}-00$

2.32E-00

$2.24 \mathrm{E}+02$

2.12E-03

2.59E-04

$3.69 \mathrm{E}-01$

$1.20 \mathrm{E}+01$

$1.98 \mathrm{E}-00$

$1.89 \mathrm{E}+01$

1.02E-04

$1.23 \mathrm{E}-04$

2.47E-09

$1.94 \mathrm{E}-05$

$1.25 \mathrm{E}-01$

$6.61 \mathrm{E}-01$

$1.05 \mathrm{E}+03$

1. $24 \mathrm{E}+01$

$0.00 E+01$

1.25E-07

5.60E-11

$9.17 \mathrm{E}-01$

$8.60 \mathrm{E}-00$

2.25E-07

7.21E-09

$3.96 \mathrm{E}-03$

1.65E-04

1.11E-04

3.74E-03

$0.00 \mathrm{E}+01$

$1.26 \mathrm{E}-00$

$9.34 \mathrm{E}+04$

$6.95 \mathrm{E}+01$

$1.15 \mathrm{E}+02$

$9.72 E+02$

$3.35 \mathrm{E}+03$

$4.21 \mathrm{E}+02$

$7.09 \mathrm{E}+01$

$5.28 \mathrm{E}+02$

$0.00 \mathrm{E}+0 \mathrm{I}$ 
Tank Anal. Un

$\begin{array}{ll}\text { SX109 } & \text { C14 } \\ \text { SX109 } & \text { Cd113 } \\ \text { SX109 } & \text { Cm242 } \\ \text { SX109 } & \text { Cm243 } \\ \text { SX109 } & \text { Cm244 } \\ \text { SX109 } & \text { Co60 } \\ \text { SX109 } & \text { Cs134 } \\ \text { SX109 } & \text { Cs137 } \\ \text { SX109 } & \text { Eu152 } \\ \text { SX109 } & \text { Eu154 } \\ \text { SX109 } & \text { Eu155 } \\ \text { SX109 } & \mathbf{1 1 2 9}\end{array}$

SX109 Nb93

SX109 Ni59

SX109 Ni63

SX109 Np237

SX109 Pa231

SX109 Pu238

SX109 Pu239

SX109 Pu240

Sx109 Pu241

SX109 Pu242

SX109 Ra226

SX109 Ra228

SX109 Ru106

SX109 Sb125

SX109 Se79

SX109 Sm151

SX109 Sr89/90.

SX109 Tc99

SX109 Th229

SX109 Th232

Sx109 Tin126

$\begin{array}{ll}\text { SX109 } & \text { Tritium } \\ \text { SX109 } & \text { U232 }\end{array}$

SX109 U233

SX109 U234

SX109 U235

SX109 U236

SX109 U238

SX109 Y90

SX109 Zr93

SX110 Al

SX110 Bi

SX110 Ca

SX110 Cl

SX110 CO3

SX110 Cr

SX110 F

SX110 Fe

SX110 Hg

Sx110 K

SX110 La

Sx110 Mn

SX110 $\mathrm{Na}$

SX110 Ni

SX110 NO2

SX110 NO3

SX110 P

SX110 Pb

SX110 PO4

SX110 S

SX110 Si

SX110 SO4

SX110 Sr

SX110 TOC

SX110 U

SX110 Zr

\section{May $\frac{B B}{88}$ \\ $8.99 \mathrm{E}-00$}

$3.62 \mathrm{E}+01$

3.49E-01

3.32E-02

9.85E-01

$7.98 \mathrm{E}-00$

1.81E-00

$2.58 E+05$

$1.43 \mathrm{E}+01$

$1.91 \mathrm{E}+02$

$7.25 \mathrm{E}+02$

1.32E-01

$2.79 \mathrm{E}+01$

$1.23 \mathrm{E}+01$

$1.19 \mathrm{E}+03$

3.07E-01

9.77E-03

$7.35 \mathrm{E}-00$

$2.22 \mathrm{E}+02$

$3.74 \mathrm{E}+01$

$3.72 \mathrm{E}+02$

2.02E-03

9.67E-04

$1.13 \mathrm{E}-02$

1.83E-03

$2.83 \mathrm{E}+01$

$7.19 \mathrm{E}-00$

$2.57 \mathrm{E}+04$

$1.10 \mathrm{E}+06$

$6.97 \mathrm{E}+01$

2.73E-04

1.52E-04

$1.05 E+01$

$1.74 \mathrm{E}+02$

5.06E-02

$1.94 \mathrm{E}-01$

9.43E-01

3.81E- 02

$3.88 \mathrm{E}-02$

8.28E-01

$1.10 \mathrm{E}+06$

$3.29 \mathrm{E}+01$

$4.06 \mathrm{E}+04$

$1.72 \mathrm{E}+01$

g $\quad 1.09 \mathrm{E}+02$

$9.62 \mathrm{E}+02$

kg $\quad 4.25 \mathrm{E}+03$

kg $7.80 \mathrm{E}+02$

$\mathrm{kg} \quad 4.87 \mathrm{E}+01$

$\mathrm{kg} \quad 6.54 \mathrm{E}+02$

kg 2.46E-02

kg 1.75E+02

g 2.67E-07

g $5.40 \mathrm{E}+02$

3.97E+04

g $4.79 \mathrm{E}+01$

$1.23 E+04$

$4.99 \mathrm{E}+04$

$2.27 \mathrm{E}+02$

g 1.35E+01

$7.02 \mathrm{E}+02$

g $2.02 \mathrm{E}+02$

g $\quad 5.08 \mathrm{E}+02$

g $6.05 \mathrm{E}+02$

$\quad 1.71 \mathrm{E}+02$

kg 7.02E+02

kg 3.13E+03

kg 2.72E+01
$\frac{B B}{98}$

Sept98

8.99E-00

$3.62 \mathrm{E}+01$

3.49E-01

3.32E-02

$9.85 \mathrm{E}-01$

$7.98 \mathrm{E}-00$

$1.81 \mathrm{E}-00$

$2.58 \mathrm{E}+05$

$1.43 \mathrm{E}+01$

$1.91 \mathrm{E}+02$

$7.25 \mathrm{E}+02$

1.32E-01

$2.79 \mathrm{E}+01$

$1.23 \mathrm{E}+01$

$1.19 \mathrm{E}+03$

3.07E-01

9.77E-03

7.35E-00

$2.22 \mathrm{E}+02$

$3.74 \mathrm{E}+01$

$3.72 \mathrm{E}+02$

2.02E-03

9.67E-04

1.13E-02

1.83E-03

$2.83 E+01$

7.19E-00

$2.57 E+04$

$1.10 \mathrm{E}+06$

$6.97 \mathrm{E}+01$

2.73E-04

1.52E-04

$1.05 \mathrm{E}+01$

$1.74 \mathrm{E}+02$

3.51E-01

1.34E-00

6.54E-00

2.64E-01

2.69E-01

5.64E-00

$1.10 \mathrm{E}+06$

$3.29 \mathrm{E}+01$

$4.06 \mathrm{E}+04$

$1.72 \mathrm{E}+01$

$1.09 \mathrm{E}+02$

$9.62 \mathrm{E}+02$

$4.25 \mathrm{E}+03$

$7.80 \mathrm{E}+02$

$4.87 \mathrm{E}+01$

$6.54 \mathrm{E}+02$

$0.00 \mathrm{E}+01$

$1.75 \mathrm{E}+02$

2.67E-07

$5.40 \mathrm{E}+02$

$3.97 \mathrm{E}+04$

$4.79 \mathrm{E}+01$

$1.24 \mathrm{E}+04$

4.97E+04

NA

$1.35 \mathrm{E}+01$

$7.02 E+02$

NA

$5.08 \mathrm{E}+02$

$6.05 \mathrm{E}+02$

$1.71 \mathrm{E}+02$

$7.02 \mathrm{E}+02$

$3.12 E+03$

$2.72 E+01$ 1\%-ile

10\%-ile

50\%-ile

90\%-ile

$0.00 \mathrm{E}+01$

$7.30 \mathrm{E}-02$

$0.00 \mathrm{E}+01$

$0.00 \mathrm{E}+01$

$0.00 \mathrm{E}+01$

$3.84 \mathrm{E}-02$

$3.65 \mathrm{E}-03$

$1.08 \mathrm{E}-01$

$3.46 \mathrm{E}-00$

2.24E-00

$4.08 \mathrm{E}-02$

4.14E-01

$2.76 \mathrm{E}+04$

$0.00 \mathrm{E}+01 \quad 1.89 \mathrm{E}-00$

$0.00 \mathrm{E}+01 \quad 2.52 \mathrm{E}+01$

$0.00 \mathrm{E}+01 \quad 9.58 \mathrm{E}+01$

$0.00 \mathrm{E}+01 \quad 8.89 \mathrm{E}-03$

$0.00 \mathrm{E}+01$

$0.00 \mathrm{E}+01$

4.36E-01

9.93E-01

$0.00 \mathrm{E}+01 \quad 9.61 \mathrm{E}+01$

$0.00 \mathrm{E}+01$

3.38E-02

$0.00 \mathrm{E}+01 \quad 0.00 \mathrm{E}+01$

$0.00 \mathrm{E}+01 \quad 8.09 \mathrm{E}-01$

$0.00 \mathrm{E}+01$

$0.00 \mathrm{E}+01$

$0.00 \mathrm{E}+01$

$2.44 E+01$

4.12E-00

$4.10 \mathrm{E}+01$

$0.00 \mathrm{E}+01$

2.22E-04

$1.14 \mathrm{E}-04$

$0.00 \mathrm{E}+01 \quad 1.34 \mathrm{E}-03$

$0.00 \mathrm{E}+01 \quad 9.18 \mathrm{E}-05$

$0.00 \mathrm{E}+01$

$5.25 \mathrm{E}-02$

$1.26 \mathrm{E}-00$

$2.96 \mathrm{E}-01$

$0.00 \mathrm{E}+01$

$0.00 \mathrm{E}+01$

$0.00 \mathrm{E}+01$

$0.00 \mathrm{E}+01$

$0.00 \mathrm{E}+01$

$0.00 \mathrm{E}+01$

$3.32 \mathrm{E}+01$

$0.00 \mathrm{E}+01$

$0.00 \mathrm{E}+0$ I

$0.00 \mathrm{E}+01$

$0.00 E+01$

$0.00 E+01$

$0.00 \mathrm{E}+01$

$0.00 \mathrm{E}+01$

$0.00 \mathrm{E}+01$

6.63E-04

$0.00 \mathrm{E}+01$

2.21E-06

1.41E-05

7.97E-05

$6.13 \mathrm{E}-06$

$0.00 \mathrm{E}+01$

$1.65 \mathrm{E}-05$

$0.00 \mathrm{E}+01$

5.91E-06

7.20E-08

$1.16 \mathrm{E}-05$

7.27E-04

2.59E-07

$1.93 \mathrm{E}-04$

$7.96 \mathrm{E}-08$

2.41E-06

3.42E-07

$1.75 \mathrm{E}-05$

2.74E-06

7.16E-06

7.70E-06

$1.59 \mathrm{E}-06$

1.50E-05

4.34E-05

$0.00 \mathrm{E}+01$

$8.99 \mathrm{E}-00$

$3.62 \mathrm{E}+01$

3.49E-01

3.32E-02 


\begin{tabular}{|c|c|}
\hline$\$ 110$ & 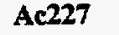 \\
\hline $\mathbf{X 1 1 0}$ & Am241 \\
\hline $\mathbf{X 1 1 0}$ & Am243 \\
\hline Sx110 & Ba137 \\
\hline X110 & $\mathbf{C l}$ \\
\hline SX110 & Cd113 \\
\hline SX110 & Cm242 \\
\hline SX110 & $\mathrm{Cm} 243$ \\
\hline SX110 & $\operatorname{Cm} 244$ \\
\hline SX110 & Co60 \\
\hline SX110 & Cs134 \\
\hline SX110 & Cs137 \\
\hline SX110 & Eu152 \\
\hline SX110 & Eu154 \\
\hline SX110 & Eu155 \\
\hline SX110 & 1129 \\
\hline SX110 & Nb93 \\
\hline SX110 & Ni59 \\
\hline SX110 & Ni63 \\
\hline SX110 & Np237 \\
\hline SX110 & Pa231 \\
\hline SX110 & Pu238 \\
\hline SX110 & Pu239 \\
\hline SX110 & Pu240 \\
\hline SX110 & Pu241 \\
\hline SX110 & Pu242 \\
\hline SX110 & Ra226 \\
\hline SX110 & $\mathrm{Ra} 228$ \\
\hline SX110 & Ru106 \\
\hline SX110 & Sb125 \\
\hline SX110 & Se79 \\
\hline SX110 & Sm151 \\
\hline SX110 & Sr89/90 \\
\hline SX110 & Te99 \\
\hline SX110 & Th229 \\
\hline SX110 & Th232 \\
\hline SX110 & Tin126 \\
\hline SX110 & Tritium \\
\hline SX110 & U232 \\
\hline SX110 & U233 \\
\hline SX110 & U234 \\
\hline SX110 & U235 \\
\hline SX110 & U236 \\
\hline SX110 & U238 \\
\hline Sx110 & Y90 \\
\hline SX110 & Zr93 \\
\hline SX111 & Al \\
\hline SX111 & $\mathbf{B i}$ \\
\hline SX111 & $\mathrm{Ca}$ \\
\hline SX111 & Cl \\
\hline SX111 & $\mathrm{CO3}$ \\
\hline SX111 & $\mathrm{Cr}$ \\
\hline SX111 & $\mathbf{F}$ \\
\hline SX111 & $\mathbf{F e}$ \\
\hline SX111 & Hg \\
\hline SX111 & $\mathbf{K}$ \\
\hline SX111 & La \\
\hline SX111 & Mn \\
\hline SX111 & $\mathbf{N a}$ \\
\hline SX111 & $\mathrm{Ni}$ \\
\hline SX111 & $\mathrm{NO} 2$ \\
\hline SX111 & NO3 \\
\hline SX111 & $\mathbf{P}$ \\
\hline $\begin{array}{l}\text { SX111 } \\
\text { SX111 }\end{array}$ & $\begin{array}{l}\mathrm{Pb} \\
\mathbf{P O 4}\end{array}$ \\
\hline $\begin{array}{l}\text { SX111 } \\
\text { SX111 }\end{array}$ & S \\
\hline 111 & Si \\
\hline & \\
\hline
\end{tabular}

$\frac{B B}{\text { May } 98}$ $4.32 \mathrm{E}-03$
$2.80 \mathrm{E}+02$ 1.24E- 02 $2.98 \mathrm{E}+04$ 2.06E-00 8.78E-00 6.42E-01 6.51E-02 1.97E-00 2.03E-00 5.56E-01 $3.15 E+04$ $1.46 \mathrm{E}+01$ $4.88 \mathrm{E}+01$ $7.45 \mathrm{E}+02$ $3.10 \mathrm{E}-02$ $1.98 \mathrm{E}+01$ $1.06 \mathrm{E}+01$ $1.05 \mathrm{E}+03$ 6.63E- 02 5.59E-03 $9.38 \mathrm{E}-00$ $1.85 \mathrm{E}+02$ $3.56 \mathrm{E}+01$ $4.51 \mathrm{E}+02$ 2.58E-03 9.51E-04 $1.79 \mathrm{E}-03$ 5.03E-04 $7.49 \mathrm{E}-00$ 5.01E-00 $1.80 \mathrm{E}+04$ $1.17 \mathrm{E}+05$ $1.63 \mathrm{E}+01$ 4.43E-05 2.40E-05 6.53E-00 $4.31 E+01$ $7.99 \mathrm{E}-03$ 3.06E-02 1.62E-01 6.48E-03 7.14E-03 $1.06 \mathrm{E}-00$ $1.17 \mathrm{E}+05$ $2.14 \mathrm{E}+01$ $8.22 \mathrm{E}+04$ $3.46 \mathrm{E}+01$ $2.20 \mathrm{E}+02$ $1.95 \mathrm{E}+03$ $3.40 \mathrm{E}+03$ $1.58 \mathrm{E}+03$ $9.87 \mathrm{E}+01$ $1.32 \mathrm{E}+03$ 1.04E-01 $3.55 E+02$ 8.88E-00 $1.09 E+03$ $8.04 E+04$ $9.70 \mathrm{E}+01$ kg $2.50 \mathrm{E}+04$ kg 1.01E +05 kg $\quad 4.59 E+02$ kg $2.73 E+01$ i.42E+03 kg $\quad 4.07 \mathrm{E}+02$ 1.03E+03 $\mathrm{kg} \quad 1.22 \mathrm{E}+03$

\section{Sept98}

$4.32 \mathrm{E}-03$

$2.80 \mathrm{E}+02$

1.24E-02

$2.98 \mathrm{E}+04$

2.06E-00

8.78E-00

6.42E-01

6.51E-02

$1.97 \mathrm{E}-00$

2.03E-00

$5.56 \mathrm{E}-01$

$3.15 \mathrm{E}+04$

$1.46 \mathrm{E}+01$

$4.88 \mathrm{E}+01$

$7.45 \mathrm{E}+02$

3.10E-02

$1.98 \mathrm{E}+01$

$1.06 \mathrm{E}+01$

$1.05 \mathrm{E}+03$

6.63E-02

5.59E-103

$9.38 \mathrm{E}-00$

$1.85 \mathrm{E}+02$

$3.56 \mathrm{E}+01$

$4.51 \mathrm{E}+02$

2.58E-03

9.51E-04

1.79E-03

5.03E-04

7.49E-00

5.01E-00

$1.80 \mathrm{E}+04$

1.17E+05

$1.63 \mathrm{E}+01$

4.43E-05

2.40E-05

6.53E-00

$4.31 \mathrm{E}+01$

6.12E-02

2.34E-01

$1.23 \mathrm{E}-00$

$4.96 \mathrm{E}-02$

5.46E-02

1.04E-00

$1.17 \mathrm{E}+05$

$2.14 \mathrm{E}+01$

8.22E+04

$3.46 \mathrm{E}+01$

$2.20 \mathrm{E}+02$

$1.95 \mathrm{E}+03$

$3.40 \mathrm{E}+03$

$1.58 \mathrm{E}+03$

$9.87 \mathrm{E}+01$

$1.33 \mathrm{E}+03$

$0.00 \mathrm{E}+01$

$3.55 \mathrm{E}+02$

$0.00 \mathrm{E}+01$

$1.09 \mathrm{E}+03$

$8.04 \mathrm{E}+04$

$9.70 \mathrm{E}+01$

$2.50 \mathrm{E}+04$

$1.01 \mathrm{E}+05$

NA

$2.73 \mathrm{E}+01$
$1.42 \mathrm{E}+03$

NA

$1.03 E+03$

$1.22 \mathrm{E}+03$
$1 \%$-ile

$0.00 \mathrm{E}+01$

$10 \%$-ile

$50 \%$-ile

$90 \%-i l e$

$\begin{array}{llll}4.54 \mathrm{E}-07 & 8.05 \mathrm{E}-00 & 3.35 \mathrm{E}+01 & 9.80 \mathrm{E}+02\end{array}$

$0.00 \mathrm{E}+01$

$0.00 \mathrm{E}+01$

4.27E-04

$2.92 \mathrm{E}+03$

$0.00 \mathrm{E}+01$

$5.32 \mathrm{E}-02$

$0.00 \mathrm{E}+01$

1.14E-00

2.24E-03

$0.00 \mathrm{E}+01$

$2.24 \mathrm{E}-03$
$6.79 \mathrm{E}-02$

$1.24 \mathrm{E}-00$

$7.52 \mathrm{E}-08$

$0.00 \mathrm{E}+01$

$5.45 \mathrm{E}-02$

$6.68 \mathrm{E}-04 \quad 6.66 \mathrm{E}+03$

$0.00 \mathrm{E}+01$

$0.00 \mathrm{E}+01$

$0.00 \mathrm{E}+01$

$0.00 \mathrm{E}+01$

$0.00 \mathrm{E}+0$

$0.00 \mathrm{E}+01$

$0.00 \mathrm{E}+01 \quad 2.44 \mathrm{E}-03$

$0.00 \mathrm{E}+01$

$8.46 \mathrm{E}-01$

$1.18 \mathrm{E}-00$

$0.00 \mathrm{E}+01 \quad 1.17 \mathrm{E}+02$

$0.00 \mathrm{E}+01$

$2.28 \mathrm{E}-03$

$0.00 \mathrm{E}+01$

$3.23 \mathrm{E}-01$

$0.00 \mathrm{E}+01$

$0.00 \mathrm{E}+01$

$0.00 \mathrm{E}+01$

$0.00 \mathrm{E}+01$

$0.00 \mathrm{E}+01$

$6.38 \mathrm{E}-00$

$1.23 \mathrm{E}-00$

$1.55 \mathrm{E}+01$

8.89E-05

$0.00 \mathrm{E}+01$

$0.00 \mathrm{E}+01$

$1.01 \mathrm{E}-04$

$1.90 \mathrm{E}-04$

$0.00 \mathrm{E}+01.3 .91 \mathrm{E}-05$

$0.00 \mathrm{E}+01$

2.55E-01

$0.00 \mathrm{E}+01 \quad 6.08 \mathrm{E}-01$

$0.00 \mathrm{E}+01 \quad 0.00 \mathrm{E}+01$

$0.00 \mathrm{E}+01$

2.14E-00

$0.00 \mathrm{E}+01 \quad 0.00 \mathrm{E}+01$

$0.00 \mathrm{E}+01 \quad 0.00 \mathrm{E}+01$

$0.00 \mathrm{E}+01 \quad 0.00 \mathrm{E}+01$

$0.00 \mathrm{E}+01 \quad 8.45 \mathrm{E}-01$

$0.00 \mathrm{E}+01$

$0.00 \mathrm{E}+0 \mathrm{I}$

$0.00 \mathrm{E}+01$

$0.00 \mathrm{E}+01$.

$0.00 E+01$

$0.00 \mathrm{E}+01$

$0.00 E+01$

$0.00 \mathrm{E}+01$

$0.00 \mathrm{E}+01$

$3.59 \mathrm{E}+04$

$0.00 \mathrm{E}+01$

$6.80 \mathrm{E}+01$

$7.21 \mathrm{E}+01$

$1.89 \mathrm{E}-00$

$4.19 E+01$

$0.00 \mathrm{E}+01$

$4.01 E+01$

$1.24 \mathrm{E}-03$

$2.65 E+01$

5.13E-01

$2.83 E+01$

$1.77 \mathrm{E}+04$

2.48E-03

$6.15 E+02$

$1.11 \mathrm{E}-00$

$3.16 \mathrm{E}-00$

$9.83 \mathrm{E}-00$

$0.00 \mathrm{E}+01$

$0.00 \mathrm{E}+01$

$0.00 \mathrm{E}+01$

$0.00 \mathrm{E}+01$

$0.00 E+01$

$0.00 \mathrm{E}+01$

$0.00 E+01$

9.14E-01

$5.85 \mathrm{E}+04$

$5.28 \mathrm{E}+01$

$1.52 \mathrm{E}+02$

$3.56 \mathrm{E}+02$

$3.68 \mathrm{E}+03$

$3.46 \mathrm{E}+02$

$5.77 \mathrm{E}+01$

$5.70 \mathrm{E}+02$

$3.16 \mathrm{E}-02$

$2.09 \mathrm{E}+02$

$5.74 \mathrm{E}-00$

$2.13 \mathrm{E}+02$

4.75E+04

$1.73 \mathrm{E}+01$

$4.84 E+03$

$7.37 \mathrm{E}+03$

$3.97 \mathrm{E}+02$

7.46E-01 1.13E+01

$3.54 \mathrm{E}-01 \quad 7.22 \mathrm{E}+02$

$1.32 E+01$

$1.43 \mathrm{E}+02$

$3.26 \mathrm{E}+02$

$7.65 \mathrm{E}-00$

$3.69 \mathrm{E}+02$

$1.24 \mathrm{E}-02$

$2.98 \mathrm{E}+04$

2.06E-00

$8.78 \mathrm{E}-00$

6.42E-01

$6.51 \mathrm{E}-02$

$1.97 \mathrm{E}-00$

4.93E-00

$5.56 \mathrm{E}-01$

$3.87 \mathrm{E}+04$

$1.46 \mathrm{E}+01$

$4.88 \mathrm{E}+01$

$7.45 \mathrm{E}+02$

3.10E-02

$1.98 \mathrm{E}+01$

$1.06 \mathrm{E}+01$

$1.05 \mathrm{E}+03$

$6.63 \mathrm{E}-02$

5.59E-03

9.38E-00

$1.85 \mathrm{E}+02$

$3.56 \mathrm{E}+01$

$4.51 \mathrm{E}+02$

2.58E-03

9.51E-04

1.79E-03

5.03E-04

7.49E-00

5.01E-00

$1.80 \mathrm{E}+04$ 
Tank Anal. Un

\begin{tabular}{lll} 
& & \\
SX111 & Sr & \\
SX111 & TOC & \\
SX111 & U & \\
SX111 & Zr & r \\
SX111 & Ac227 & \\
SX111 & Am241 \\
SX111 & Am243 \\
SX111 & Ba137 \\
SX111 & C14 \\
SX111 & Cd113 \\
SX111 & Cm242 \\
SX111 & Cm243 \\
SX111 & Cm244 \\
SX111 & Co60 \\
SX111 & Cs134 \\
SX111 & Cs137 \\
SX111 & Eu152 \\
SX111 & Eu154 \\
SX111 & Eu155 \\
SX111 & I129 & C \\
\hline SX111 & Nb93 & C
\end{tabular}

SX111

SX111 Ni59

SX111 Ni63

SX111 Np237

SX111 Pa231

SX111 Pu238

SX111 Pu239

SX111 Pu240

SX111 Pu241

SX111 Pu242

SX111 Ra226

SX111 Ra228

SX111 Ru106

SX111 Sb125

SX111 Se79

SX111 Sm151

SX111 Sr89/90

SX111 Te99

SX111 Th229

SX111 Th232

SX111 Tin126

SX111 Tritium

SX111 U232

SX111 U233

SX111 U234

SX111 U235

SX111 U236

SX111 U238

SX111 Y90

SX111 Zr93

SX112 A!

SX112 Bi

SX112 Ca

SX112 CI

$\mathrm{SX112 \textrm {CO }}$

$\mathrm{SX112} \mathrm{Cr}$

SX112 F

SX112 Fe

SX112 Hg

SX112 K

SX112 La

SX112 Mn

SX112 Na

SX112 Ni

SX112 NO2

SX112 NO3

SX112 P

SX112 Pb
May $\frac{B B}{98}$

3.45E+02

$1.42 \mathrm{E}+03$

$6.32 \mathrm{E}+03$

$5.50 \mathrm{E}+01$

6.33E-03

$3.61 \mathrm{E}+02$

1.60E-02

$6.04 E+04$

$3.16 \mathrm{E}-00$

$1.30 \mathrm{E}+01$

8.49E-01

8.39E-02

2.52E-00

$2.88 \mathrm{E}-00$

$7.47 \mathrm{E}-01$

$6.38 \mathrm{E}+04$

$1.99 \mathrm{E}+01$

$6.93 \mathrm{E}+01$

$1.01 E+03$

4.68E-02

$2.60 \mathrm{E}+01$

$1.60 \mathrm{E}+01$

$1.57 \mathrm{E}+03$

1.07E-01

7.42E-03

$1.31 \mathrm{E}+01$

3.13E+02

$5.65 E+01$

$6.43 E+02$

$3.61 \mathrm{E}-03$

$1.40 \mathrm{E}-03$

2.50E-03

6.77E-04

$1.03 \mathrm{E}+01$

6.59E-00

$2.36 \mathrm{E}+04$

$2.40 \mathrm{E}+05$

$2.47 E+01$

6.22E-05

3.36E-05

8.63E-00

$6.29 \mathrm{E}+01$

1.12E-02

$4.28 \mathrm{E}-02$

2.43E-01

9.81E-03

1.02E-02

2.12E-00

$2.40 \mathrm{E}+05$

$2.82 E+01$

$6.07 E+04$

$2.56 \mathrm{E}+01$

$1.63 \mathrm{E}+02$

$\mathrm{kg} \quad 1.44 \mathrm{E}+03$

$2.51 \mathrm{E}+03$

I.17E+03

$7.29 E+01$

g $\quad 9.77 \mathrm{E}+02$

$7.60 \mathrm{E}-02$

$2.62 \mathrm{E}+02$

6.56E-00

8.07E+02

$5.94 \mathrm{E}+04$

$7.16 \mathrm{E}+01$

$1.85 \mathrm{E}+04$

$7.34 \mathrm{E}+04$

$3.39 \mathrm{E}+02$

$2.02 \mathrm{E}+01$
$\frac{B B}{\sin }$

$\frac{\text { Septas }}{3.45 \mathrm{E}+02}$

$1.42 \mathrm{E}+03$

$6.32 \mathrm{E}+03$

$5.50 \mathrm{E}+01$

6.33E-03

$3.61 \mathrm{E}+02$

$1.60 \mathrm{E}-02$

$6.03 E+04$

$3.16 \mathrm{E}-00$

$1.30 \mathrm{E}+01$

8.49E-01

$8.39 \mathrm{E}-02$

2.52E-00

2.88E-00

7.47E-01

$6.37 \mathrm{E}+04$

$1.99 \mathrm{E}+01$

$6.93 \mathrm{E}+01$

$1.01 \mathrm{E}+03$

$4.68 \mathrm{E}-02$

$2.60 \mathrm{E}+01$

$1.60 \mathrm{E}+01$

$1.57 \mathrm{E}+03$

1.07E-01

7.42E-03

$1.31 \mathrm{E}+01$

$3.13 \mathrm{E}+02$

$5.65 \mathrm{E}+01$

$6.43 \mathrm{E}+02$

3.61E-03

$1.40 \mathrm{E}-03$

2.50E-03

6.77E-04

$1.03 \mathrm{E}+01$

$6.59 \mathrm{E}-00$

$2.36 \mathrm{E}+04$

$2.37 \mathrm{E}+05$

$2.47 \mathrm{E}+01$

6.22E-05

3.36E-05

8.63E-00

$6.29 \mathrm{E}+01$

1.13E-01

4.33E-01

$2.46 \mathrm{E}-00$

9.92E- 02

1.02E-01

2.11E-00

$2.37 \mathrm{E}+05$

$2.82 \mathrm{E}+01$

$6.07 \mathrm{E}+04$

$2.56 \mathrm{E}+01$

$1.63 \mathrm{E}+02$

$1.44 \mathrm{E}+03$

$2.51 \mathrm{E}+03$

$1.17 E+03$

$7.29 \mathrm{E}+01$

$9.77 E+02$

$0.00 \mathrm{E}+01$

$2.62 \mathrm{E}+02$

$0.00 \mathrm{E}+01$

8.07E +02

$5.94 \mathrm{E}+04$

$7.16 \mathrm{E}+01$

$1.85 \mathrm{E}+04$

$7.34 \mathrm{E}+04$

NA

2.02E+01 1\%-ile 10\%-ile

8.30E-03 5.15E+01

$5.70 \mathrm{E}+01 \quad 5.61 \mathrm{E}+02$

$3.42 \mathrm{E}+02 \quad 2.19 \mathrm{E}+03$

$0.00 \mathrm{E}+01$

$5.01 \mathrm{E}-00$

$2.59 \mathrm{E}-03$

$3.90 \mathrm{E}+01$

$3.04 \mathrm{E}+01$

$0.00 \mathrm{E}+01$

$0.00 \mathrm{E}+01$

$1.73 \mathrm{E}-03$

$1.67 \mathrm{E}+04$

2.23E-01

$3.95 \mathrm{E}-00$

$1.55 \mathrm{E}-01$

$0.00 \mathrm{E}+01$

$0.00 \mathrm{E}+01$

$9.20 \mathrm{E}-02$

$9.09 \mathrm{E}-03$

2.73E-01

$5.60 \mathrm{E}-00$

3.94E-00

$0.00 \mathrm{E}+01$

$1.28 \mathrm{E}+03$

2.07E-01

$2.95 \mathrm{E}+04$

$0.00 \mathrm{E}+01 \quad 8.15 \mathrm{E}-00$

$0.00 \mathrm{E}+01 \quad 2.84 \mathrm{E}+01$

$0.00 \mathrm{E}+01 \quad 4.14 \mathrm{E}+02$

$0.00 \mathrm{E}+01 \quad 4.46 \mathrm{E}-03$

$0.00 \mathrm{E}+01 \quad 3.46 \mathrm{E}-00$

$0.00 \mathrm{E}+01$

$3.46 \mathrm{E}-00$
$4.38 \mathrm{E}-00$

$0.00 \mathrm{E}+01 \quad 4.30 \mathrm{E}+02$

$0.00 \mathrm{E}+01$

$1.16 \mathrm{E}-02$

$0.00 \mathrm{E}+01 \quad 7.36 \mathrm{E}-04$

$0.00 \mathrm{E}+01 \quad 1.42 \mathrm{E}-00$

$0.00 \mathrm{E}+01$

$3.39 \mathrm{E}+01$

$0.00 \mathrm{E}+01 \quad 6.12 \mathrm{E}-00$

$0.00 \mathrm{E}+01 \quad 6.97 \mathrm{E}+01$

$0.00 \mathrm{E}+01 \quad 3.91 \mathrm{E}-04$

$0.00 \mathrm{E}+01 \quad 2.78 \mathrm{E}-04$

$0.00 \mathrm{E}+01 \quad 4.97 \mathrm{E}-04$

$0.00 \mathrm{E}+01$

$1.19 \mathrm{E}-04$

$0.00 \mathrm{E}+01$

9.72E-01

$3.22 \mathrm{E}-02 \quad 1.94 \mathrm{E}-00$

$0.00 \mathrm{E}+01 \quad 9.66 \mathrm{E}+03$

$0.00 \mathrm{E}+01$

$3.60 \mathrm{E}-00$

$0.00 \mathrm{E}+01 \quad 0.00 \mathrm{E}+01$

$0.00 \mathrm{E}+01$

6.17E-06

$0.00 \mathrm{E}+01 \quad 3.33 \mathrm{E}-06$

$1.03 \mathrm{E}-01 \quad 2.62 \mathrm{E}-00$

$2.16 \mathrm{E}+01 \quad 3.96 \mathrm{E}+01$

$0.00 \mathrm{E}+01 \quad 1.11 \mathrm{E}-03$

$0.00 \mathrm{E}+01 \quad 4.25 \mathrm{E}-03$

$0.00 \mathrm{E}+01 \quad 2.41 \mathrm{E}-02$

$0.00 \mathrm{E}+01 \quad 9.73 \mathrm{E}-04$

$0.00 \mathrm{E}+01 \quad 1.01 \mathrm{E}-03$

$0.00 \mathrm{E}+01$

$1.01 \mathrm{E}-03$
$2.10 \mathrm{E}-01$

$0.00 \mathrm{E}+01 \quad 0.00 \mathrm{E}+0$

$0.00 \mathrm{E}+01 \quad 3.75 \mathrm{E}-00$

$2.19 \mathrm{E}+04 \quad 4.51 \mathrm{E}+04$

$0.00 \mathrm{E}+01$

$4.51 E+04$

$1.31 \mathrm{E}+02$

$4.66 \mathrm{E}+01$

$4.34 \mathrm{E}+01$

$7.86 \mathrm{E}-00$

$3.15 E+02$

$3.07 \mathrm{E}+03$

$2.98 \mathrm{E}+01 \quad 3.44 \mathrm{E}+02$

$0.00 \mathrm{E}+01 \quad 5.05 \mathrm{E}+01$

$2.49 \mathrm{E}+01 \quad 4.52 \mathrm{E}+02$

5.32E-04 2.09E-02

$2.96 \mathrm{E}+01$

$5.80 \mathrm{E}-01$

$9.96 \mathrm{E}-00$

$1.55 \mathrm{E}+04$

$3.45 \mathrm{E}-03$

$4.28 \mathrm{E}+02$

$1.71 \mathrm{E}-01$

3. $18 \mathrm{E}+01$

$1.84 \mathrm{E}+02$

$4.31 \mathrm{E}-00$

$1.81 E+02$

$3.94 \mathrm{E}+04$

$1.57 \mathrm{E}+01$

$3.80 \mathrm{E}+03$

$7.53 E+03$

$3.97 \mathrm{E}+02$

$9.10 \mathrm{E}-00$

90\%-ile

$50 \%$-ile 
Tank Anal. Un

$\begin{array}{ll}\text { SX112 } & \text { PO4 } \\ \text { SX112 } & \text { S } \\ \text { SX112 } & \text { Si } \\ \text { SX112 } & \text { SO4 } \\ \text { SX112 } & \text { Sr } \\ \text { SX112 } & \text { TOC } \\ \text { SX112 } & \text { U } \\ \text { SX112 } & \text { Zr } \\ \text { SX112 } & \text { Ac227 } \\ \text { SX112 } & \text { Am241 } \\ \text { SX112 } & \text { Am243 } \\ \text { SX112 } & \text { Ba137 } \\ \text { SX112 } & \text { C14 } \\ \text { SX112 } & \text { Cd113 } \\ \text { SX112 } & \text { Cm242 } \\ \text { SX112 } & \text { Cm243 } \\ \text { SX112 } & \text { Cm244 } \\ \text { SX112 } & \text { Co60 } \\ \text { SX112 } & \text { Cs134 } \\ \text { SX112 } & \text { Cs137 } \\ \text { SX112 } & \text { Eu152 } \\ \text { SX112 } & \text { Eu154 } \\ \text { SX112 } & \text { Eu155 } \\ \text { SX112 } & \text { I129 } \\ \text { SX112 } & \text { C }\end{array}$

SX112 Nb93

SX112 Ni59

SX112 Ni63

SX112 Np237

SX112 Pa231

SX112 Pu238

SX112 Pu239

SX112 Pu240

SX112 Pu241

SX112 Pu242

SX112 Ra226

SX112 Ra228

SX112 Ru106

SX112 Sb125

SX112 Se79

SX112 Sm151

SX112 Sr89/90

SX112 Te99

SX112 Th229

SX112 Th232

SX112 Tin126

SX112 Tritium

SX112 U232

SX112 U233

SXI12 U234

SX112 U235

SX112 U236

SX112 U238

SX112 Y90

SX112 Zr93

SX113 Al

SX113 Bi

SX113 Ca

SX113 CI

$5 \times 113 \mathrm{CO}$

SX113 Cr

SXI13 F

Sx113 Fe

SX113 Hg

$S \times 113 k$

SX113 La

SX113 Mn

SX113 $\mathrm{Na}$

SX113 Ni
BB

May98

$1.05 \mathrm{E}+03$

$3.01 \mathrm{E}+02$

$7.59 \mathrm{E}+02$

$9.04 \mathrm{E}+02$

$2.55 \mathrm{E}+02$

$1.05 \mathrm{E}+03$

$4.67 \mathrm{E}+03$

$4.05 \mathrm{E}+01$

4.16E- 03

$2.20 \mathrm{E}+02$

9.72E-03

$4.46 \mathrm{E}+04$

1.97E-00

$7.95 \mathrm{E}-00$

5.29E-01

5.14E-02

$1.54 \mathrm{E}-00$

1.71E-00

4.35E-01

$4.71 \mathrm{E}+04$

1.25E+01

4.13E+01

$6.36 \mathrm{E}+02$

2.90E-02

$1.57 \mathrm{E}+01$

$1.07 E+01$

i $1.05 \mathrm{E}+03$

Ci $\quad 6.83 \mathrm{E}-02$

Ci $\quad 4.47 E-03$

Ci 8.42E-00

Ci $2.23 E+02$

$\mathrm{Ci} \quad 3.90 \mathrm{E}+01$

i $4.18 \mathrm{E}+02$

Ci 2.32E-03

$\mathrm{Ci}$ 9.22E-04

Ci 1.37E-03

Ci 3.89E-04

Ci 6.01E-00

Ci 3.98E-00

Ci 1.43E+04

Ci 1.75E+05

Ci 1.53E+01

Ci 3.43E-05

$1.84 \mathrm{E}-05$

5.21E-00

$3.82 \mathrm{E}+01$

6.13E- 03

2.34E-02

Ci 1.44E-01

Ci 5.83E-03

Ci 5.80E-03

Ci 1.57E-00

Ci $1.75 E+05$

Ci 1.70E+01

$\mathrm{kg} \quad$ 1.58E+03

$\mathrm{kg} \quad 5.70 \mathrm{E}-01$

kg 2.65E+02

kg $3.20 \mathrm{E}+01$

kg 5.55E+01

kg 2.57E+01

kg 1.61E-00

kg $1.36 \mathrm{E}+03$

$\mathrm{kg} \quad 1.70 \mathrm{E}-03$

$\mathrm{kg} \quad 7.69 \mathrm{E}+01$

$\mathrm{kg} \quad 1.45 \mathrm{E}-01$

$\mathrm{kg} \quad 3.66 \mathrm{E}+01$

kg $1.31 \mathrm{E}+03$

$\mathrm{kg}$
$\frac{B B}{\text { Sept98 }}$

$1 \%$-ile

\section{$0.00 E+01$}

$10 \%-j l e$

NA

$7.59 \mathrm{E}+02$

$9.04 \mathrm{E}+02$

$2.55 \mathrm{E}+02$

$1.05 E+03$

$4.67 \mathrm{E}+03$

$4.05 E+01$

4.16E-03

$2.20 \mathrm{E}+02$

9.72E-03

$4.45 E+04$

1.97E-00

$7.95 \mathrm{E}-00$

$5.29 \mathrm{E}-01$

5.14E-02

1.54E-00

1.71E-00

4.35E-01

$4.71 E+04$

$1.25 \mathrm{E}+01$

$4.13 E+01$

$6.36 \mathrm{E}+02$

2.90E-02

$1.57 \mathrm{E}+01$

$1.07 \mathrm{E}+01$

$1.05 \mathrm{E}+03$

$6.83 \mathrm{E}-02$

4.47E-03

8.42E-00

$2.23 \mathrm{E}+02$

$3.90 \mathrm{E}+01$

$4.18 \mathrm{E}+02$

2.32E-03

9.22E-04

1.37E-03

3.89E-04

6.01E-00

3.98E-00

$1.43 E+04$

$1.75 \mathrm{E}+05$

$1.53 E+01$

3.43E-05

$1.84 \mathrm{E}-05$

$5.21 \mathrm{E}-00$

$3.82 \mathrm{E}+01$

$7.65 \mathrm{E}-02$

2.93E-01

$1.80 \mathrm{E}-00$

$7.28 \mathrm{E}-02$

7.25E-02

$1.56 \mathrm{E}-00$

$1.75 E+05$

$1.70 \mathrm{E}+01$

$1.58 \mathrm{E}+03$

4.60E-01

$2.65 \mathrm{E}+02$

$3.20 \mathrm{E}+01$

$5.55 \mathrm{E}+01$

$2.57 \mathrm{E}+01$

$1.61 \mathrm{E}-00$

$1.36 \mathrm{E}+03$

$0.00 \mathrm{E}+01$

$7.69 E+01$

$0.00 \mathrm{E}+01$

$3.66 \mathrm{E}+01$

$1.31 \mathrm{E}+03$

$1.58 E-00$
$2.86 \mathrm{E}+01 \quad 2.73 \mathrm{E}+02$

$1.26 \mathrm{E}+01 \quad 2.97 \mathrm{E}+02$

$1.66 \mathrm{E}-01 \quad 4.76 \mathrm{E}+01$

$8.13 \mathrm{E}+01 \quad 4.94 \mathrm{E}+02$

$3.26 \mathrm{E}+02 \quad 1.73 \mathrm{E}+03$

$0.00 \mathrm{E}+01 \quad 3.96 \mathrm{E}-00$

$0.00 \mathrm{E}+01 \quad 9.40 \mathrm{E}-04$

$1.90 \mathrm{E}+01 \quad 2.97 \mathrm{E}+01$

$0.00 \mathrm{E}+01 \quad 9.90 \mathrm{E}-04$

$0.00 \mathrm{E}+01 \quad 1.24 \mathrm{E}+04$

$0.00 \mathrm{E}+01 \quad 2.06 \mathrm{E}-01$

$5.56 \mathrm{E}-02 \quad 2.19 \mathrm{E}-00$

$0.00 \mathrm{E}+01 \quad 5.39 \mathrm{E}-02$

$0.00 \mathrm{E}+01 \quad 5.24 \mathrm{E}-03$

$0.00 \mathrm{E}+01$

2.54E-00

$0.00 \mathrm{E}+01$

$1.56 \mathrm{E}+03$

$0.00 \mathrm{E}+01$

$0.00 \mathrm{E}+01$

$0.00 \mathrm{E}+01$

$0.00 \mathrm{E}+01$

$0.00 \mathrm{E}+01$

$0.00 \mathrm{E}+01$

$0.00 \mathrm{E}+01$

$0.00 E+01$

$0.00 \mathrm{E}+01$

$0.00 \mathrm{E}+01$

$0.00 \mathrm{E}+01$

$0.00 \mathrm{E}+01$

$0.00 \mathrm{E}+01$

$0.00 E+01$

$0.00 \mathrm{E}+01$

$0.00 \mathrm{E}+01$

$0.00 E+01$

$0.00 \mathrm{E}+01$

4.00E-02

$0.00 E+01$

$0.00 \mathrm{E}+01$

$0.00 \mathrm{E}+01$

$0.00 \mathrm{E}+01$

$0.00 \mathrm{E}+01$

3.65E-02

$1.10 \mathrm{E}+01$

$0.00 \mathrm{E}+01$

$0.00 \mathrm{E}+01$

$0.00 \mathrm{E}+01$

$0.00 \mathrm{E}+01$

$0.00 \mathrm{E}+01$

$0.00 \mathrm{E}+01$

$0.00 \mathrm{E}+01$

$0.00 \mathrm{E}+01$

$4.78 \mathrm{E}-05$

1.59E-07

$9.40 \mathrm{E}-06$

8.87E-07

$7.96 \mathrm{E}-06$

$6.86 \mathrm{E}-07$

$1.76 \mathrm{E}-07$

7.59E-06

$0.00 \mathrm{E}+01$

2.93E-07

7.22E-09

4.38E-07

3.99E-04

$6.00 \mathrm{E}-08$

$5.24 \mathrm{E}-03$
$1.57 \mathrm{E}-01$

4.24E-00

1.21E-01

$2.44 \mathrm{E}+04$

2.82E-00

9.33E-00

$1.44 \mathrm{E}+02$

$3.46 \mathrm{E}-03$

$1.79 \mathrm{E}-00$

$3.04 \mathrm{E}-00$

$2.99 \mathrm{E}+02$

$6.96 \mathrm{E}-03$

$4.90 \mathrm{E}-04$

$8.58 \mathrm{E}-01$

$2.27 \mathrm{E}+01$

$3.97 \mathrm{E}-00$

$4.26 \mathrm{E}+01$

2.36E-04

2.05E-04

$3.04 \mathrm{E}-04$

$6.40 \mathrm{E}-05$

5.31E-01

$1.05 \mathrm{E}-00$

$3.23 E+03$

$5.43 \mathrm{E}-00$

$0.00 \mathrm{E}+01$

$3.76 \mathrm{E}-06$

2.02E-06

$1.43 \mathrm{E}-00$

$2.12 \mathrm{E}+01$

6.72E-04

$2.57 \mathrm{E}-03$

1.58E-02

6.39E-04 
Tank Anal. Un

$\begin{array}{ll}\text { SX113 } & \text { NO2 } \\ \text { SX113 } & \text { NO3 } \\ \text { SX113 } & \text { P } \\ \text { SX113 } & \text { Pb } \\ \text { SX113 } & \text { PO4 } \\ \text { SX113 } & \text { S } \\ \text { SX113 } & \text { Si } \\ \text { SX113 } & \text { SO4 } \\ \text { SX113 } & \text { Sr } \\ \text { SX113 } & \text { TOC } \\ \text { SX113 } & \text { U } \\ \text { SX113 } & \text { Zr }\end{array}$

SX113 Ac227

SX113 Am241

SX113 Am243

SX113 Ba137

SX113 C14

SX113 Cd113

SX113 Cm242

SX113 Cm243

SX113 Cm244

SX113 Co60

SX113 Cs134

SX113 Cs137

SX113 Eu152

SX113 Eu154

SXI13 Eu155

SX113 I129

SX113 Nb93

SX113 Ni59

SX113 Ni63

SX113 Np237

SX113 Pa231

SX113 Pu238

SX113 Pu239

SX113 Pu240

SX113 Pu241

SX113 Pu242

SX113 Ra226

SX113 Ra228

SX113 Ru106

SX113 Sb125

SX113 Se79

SX113 Sm151

SX113 Sr89/90

SX113 Te99

SX113 Th229

SX113 Th232

SX113 Tin126

SX113 Tritium

SX113 U232

SX113 U233

SX113 U234

SX113 U235

SX113 U236

SX113 U238

SX113 Y90

SX113 Zr93

SX114 AI

SX114 Bi

SX114 Ca

SX114 Cl

SX114 CO3

SX114 Cr

SX114 F

SX114 Fe

SX114 Hg

SX114 $K$

\section{$\begin{array}{lr}\text { Un } & \frac{B B}{M a y 98} \\ & \frac{M .07 \mathrm{E}+02}{\mathrm{~kg}} \\ \mathrm{~kg} & 1.64 \mathrm{E}+03 \\ \mathrm{~kg} & 7.49 \mathrm{E}-00 \\ \mathrm{~kg} & 6.80 \mathrm{E}-01 \\ \mathrm{~kg} & 2.32 \mathrm{E}+01 \\ \mathrm{~kg} & 6.67 \mathrm{E}-00\end{array}$}

kg $\quad 1.77 \mathrm{E}+04$

kg $\quad 2.00 \mathrm{E}+01$

kg $5.60 \mathrm{E}-00$

kg 2.32E+01

k $\quad 1.03 \mathrm{E}+02$

1.36E-00

$3.60 \mathrm{E}-05$

6.12E-02

5.72E-07

$9.84 \mathrm{E}+02$

1.71E-02

5.82E-02

$1.22 \mathrm{E}-03$

2.79E-05

2.04E-05

6.75E-03

2.05E-04

$1.04 \mathrm{E}+03$

5.92E-02

1.63E-01

2.50E-00

2.30E-04

1.40E-02

1.12E-01

$1.05 \mathrm{E}+01$

7.77E-04

5.43E-06

5.00E-02

3.57E-00

5.11E-01

3.00E-00

1.38E-05

8.17E-06

7.00E-11

1.89E-08

9.92E-03

3.63E-03

$1.30 \mathrm{E}+01$

$3.86 \mathrm{E}+03$

$1.20 \mathrm{E}-01$

1.34E-08

4.47E-12

5.58E-03

2.21E-01

3.96E-08

1.51E-09

9.01E-04

3.88E-05

1.42E-05

9.19E-04

$1.04 \mathrm{E}+03$

1.71E-02

$1.07 \mathrm{E}+05$

$3.92 \mathrm{E}+01$

$1.17 E+03$

$2.75 E+03$

$6.99 \mathrm{E}+03$

$5.49 \mathrm{E}+03$

$1.60 \mathrm{E}+02$

$3.49 \mathrm{E} \div 03$

$1.17 \mathrm{E}-01$

kg $\quad 5.53 E+02$

\section{$\frac{B B}{\operatorname{sep} 98}$ \\ $4.07 \mathrm{E}+02$ \\ $1.64 \mathrm{E}+03$ \\ NA \\ $6.80 \mathrm{E}-01$ \\ $2.32 \mathrm{E}+01$ \\ NA}

1.77E+04

$2.00 \mathrm{E}+01$

$5.60 \mathrm{E}-00$

$2.32 \mathrm{E}+01$

$1.03 \mathrm{E}+02$

$1.36 \mathrm{E}-00$

3.60E-05

6.12E-02

5.72E-07

$9.84 \mathrm{E}+02$

1.71E-02

5.28E- 02

$1.22 \mathrm{E}-03$

$2.79 \mathrm{E}-05$

2.04E-05

$6.75 \mathrm{E}-03$

2.05E-04

$1.04 \mathrm{E}+03$

5.29E-02

1.63E-01

2.50E-00

2.30E-04

1.40E-02

1.12E-01

$1.05 E+01$

$7.77 \mathrm{E}-04$

5.43E-06

5.00E-02

3.57E-00

5.11E-01

3.00E-00

1.38E-05

8.17E-06

7.00E-11

1.89E-08

9.92E-03

3.63E-03

$1.30 \mathrm{E}+01$

$3.87 \mathrm{E}+03$

$1.20 \mathrm{E}-01$

1.34E-08

4.47E-12

5.58E-03

2.21E-01

$1.48 \mathrm{E}-06$

5.64E-08

3.37E-02

$1.45 E-03$

5.34E-04

3.44E-02

$3.87 \mathrm{E}+03$

$1.71 E-02$

$1.07 \mathrm{E}+05$

$3.92 \mathrm{E}+01$

$1.17 \mathrm{E}+03$

$2.75 \mathrm{E}+03$

$6.99 \mathrm{E}+03$

$5.49 \mathrm{E}+03$

$1.60 \mathrm{E}+02$

$3.49 \mathrm{E}+03$

$0.00 \mathrm{E}+01$

$5.53 E+02$ 1\%-ile

$10 \%$-ile

1.08E-05

$4.43 \mathrm{E}-05 \quad 8.87 \mathrm{E}+01$

$1.04 \mathrm{E}-06$

$5.06 \mathrm{E}-08$

2.47E-06

2.61E-07

$1.99 \mathrm{E}-05$

9.49E-07

1.05E-07

$1.12 \mathrm{E}-06$

$1.91 \mathrm{E}-06$

$1.52 \mathrm{E}-08$

$0.00 \mathrm{E}+01$

2.72E-08

$0.00 \mathrm{E}+01$

$0.00 \mathrm{E}+01$

$0.00 \mathrm{E}+01$

$0.00 \mathrm{E}+01$

$0.00 \mathrm{E}+01$

$0.00 \mathrm{E}+01$

$0.00 \mathrm{E}+01$

5.64E-09

$0.00 \mathrm{E}+01$

2.40E-05

$0.00 \mathrm{E}+01$

$0.00 \mathrm{E}+01$

$0.00 \mathrm{E}+01$

$0.00 \mathrm{E}+01$

$0.00 \mathrm{E}+01$

$0.00 \mathrm{E}+01$

$0.00 \mathrm{E}+01$

$0.00 \mathrm{E}+01$

$0.00 \mathrm{E}+01$

$0.00 \mathrm{E}+01$

$0.00 \mathrm{E}+01$

$0.00 E+01$

$0.00 E+01$

$0.00 \mathrm{E}+01$

$0.00 E+01$

$0.00 \mathrm{E}+01$

$0.00 E+01$

$0.00 E+01$

$0.00 \mathrm{E}+01$

$0.00 \mathrm{E}+01$

$0.00 \mathrm{E}+01$

$0.00 \mathrm{E}+01$

$0.00 \mathrm{E}+01$

$0.00 E+01$

$0.00 \mathrm{E}+01$

$0.00 \mathrm{E}+01$

$0.00 \mathrm{E}+01$

$0.00 \mathrm{E}+01$

$0.00 \mathrm{E}+01$

$0.00 \mathrm{E}+01$

$0.00 \mathrm{E}+01$

$0.00 \mathrm{E}+01$

$0.00 \mathrm{E}+01$

$0.00 \mathrm{E}+01$

$5.41 \mathrm{E}+04$

$0.00 \mathrm{E}+01$

$7.66 \mathrm{E}+01$

$2.23 \mathrm{E}+02$

$3.55 \mathrm{E}+02$

$7.79 \mathrm{E}+01$

$0.00 \mathrm{E}+01$

$8.81 \mathrm{E}+01$

$0.00 \mathrm{E}+01$

$4.75 E+01$

$4.85 \mathrm{E}-00$

2.64E-01

$1.01 \mathrm{E}+01$

$1.53 \mathrm{E}-00$

$2.91 \mathrm{E}+01$

5.43E-00

$3.46 \mathrm{E}-01$

3.34E-00

9.15E-00

5.81E-02

$0.00 \mathrm{E}+01$

$1.70 \mathrm{E}-01$

$1.87 \mathrm{E}-08$

8.15E+01

$5.78 \mathrm{E}-04$

2.41E-03

3.99E-05

9.12E-07

6.67E-07

2.34E-02

1.70E-05

$1.74 \mathrm{E}+02$

$0.00 \mathrm{E}+01$

$0.00 \mathrm{E}+01$

$0.00 E+01$

$1.80 \mathrm{E}-05$

7.33E-04 
Tank Anal. Un

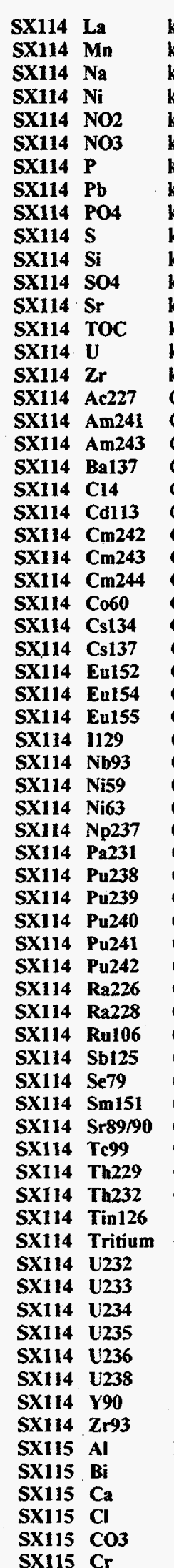

May 9 kg $\frac{1.00 \mathrm{E}+01}{0.00 \mathrm{E}+01}$ kg 250E+03 $250 \mathrm{E}+03$ $\mathrm{kg} \quad 1.30 \mathrm{E}+05 \quad 1.30 \mathrm{E}+05$ kg $3.41 \mathrm{E}+02 \quad 3.41 \mathrm{E}+02$ $\mathrm{kg} \quad 3.41 \mathrm{E}+04 \quad 3.41 \mathrm{E}+04$ kg 1.83E+05 kg $\quad 5.84 \mathrm{E}+02$

$\mathrm{kg} \quad 3.61 \mathrm{E}+01$

$\mathrm{kg} \quad 1.81 \mathrm{E}+03$ kg $\quad 8.27 \mathrm{E}+02$ $\mathrm{kg} \quad 1.48 \mathrm{E}+03$ kg 2.48E+03 kg $3.90 \mathrm{E}+02$ $1.02 \mathrm{E}+03$

$\mathrm{kg} \quad 9.68 \mathrm{E}+03$ kg $1.19 \mathrm{E}+02$ $6.21 \mathrm{E}-03$ $3.67 \mathrm{E}+02$ $1.58 \mathrm{E}-02$ $6.80 \mathrm{E}+04$ 6.74E-00 2.77E+01 $7.50 \mathrm{E}-01$ 7.54E-02 $2.28 \mathrm{E}-00$ $6.21 \mathrm{E}-00$ $1.50 \mathrm{E}-00$ $7.20 \mathrm{E}+04$ $2.04 E+01$ $1.49 \mathrm{E}+02$ $1.04 \mathrm{E}+03$ $9.98 \mathrm{E}-02$ $3.29 \mathrm{E}+01$ $1.54 \mathrm{E}+01$ $1.51 \mathrm{E}+03$ 2.25E-01 1.03E-02 $1.20 \mathrm{E}+01$ $2.62 \mathrm{E}+02$ $4.87 \mathrm{E}+01$ $5.83 \mathrm{E}+02$ $3.30 \mathrm{E}-03$ 1.32E-03 $7.93 \mathrm{E}-03$ $1.47 E-03$ $2.24 \mathrm{E}+01$ $8.40 \mathrm{E}-00$ $3.00 \mathrm{E}+04$ $2.67 E+05$ $5.26 \mathrm{E}+01$ 1.92E-04 1.06E-04 $1.16 \mathrm{E}+01$ $1.34 \mathrm{E}+02$ 3.54E-02 1.36E-01 6.65E-01 2.68E-02 2.79E-02 5.80E-01 $2.67 \mathrm{E}+05$ $3.71 \mathrm{E}+01$ $7.89 \mathrm{E}+03$ 3.33E-00 $2.11 \mathrm{E}+01$ $1.87 \mathrm{E}+02$ $3.27 \mathrm{E}+02$ $\mathrm{kg} \quad 1.51 \mathrm{E}+02$

$1.83 \mathrm{E}+05$

NA

$3.61 \mathrm{E}+01$

$1.81 \mathrm{E}+03$

NA
$\mathrm{E}+03$

$1.48 \mathrm{E}+03$

$2.48 \mathrm{E}+03$

$1.02 \mathrm{E}+03$

$9.68 \mathrm{E}+03$

$1.19 \mathrm{E}+02$

$6.21 \mathrm{E}-03$

$3.67 \mathrm{E}+02$

1.58E-02

$6.80 \mathrm{E}+04$

6.74E-00

2.77E+01

7.50E-01

7.54E-02

2.28E-00

6.21E-00

1.50E-00

$7.20 \mathrm{E}+04$

$2.04 \mathrm{E}+01$

$1.49 \mathrm{E}+02$

$1.04 \mathrm{E}+03$

9.98E-02

$3.29 \mathrm{E}+01$

$1.54 \mathrm{E}+01$

$1.51 \mathrm{E}+03$

2.25E-01

1.03E-02

$1.20 \mathrm{E}+01$

2.62E +02

$4.87 \mathrm{E}+01$

$5.83 \mathrm{E}+02$

$3.30 \mathrm{E}-03$

1.32E-03

7.93E-03

$1.47 E-03$

$2.24 \mathrm{E}+01$

8.40E-00

$3.00 \mathrm{E}+04$

$2.67 \mathrm{E}+05$

$5.26 \mathrm{E}+01$

1.92E-04

$1.06 \mathrm{E}-04$

$1.16 \mathrm{E}+01$

$1.34 \mathrm{E}+02$

2.01E-01

$7.69 \mathrm{E}-01$

$3.77 \mathrm{E}-00$

1.52E-01

$1.58 \mathrm{E}-01$

3.23E-00

2.67E+05

$3.71 \mathrm{E}+01$

$7.89 \mathrm{E}+03$

3.33E-00

$2.11 \mathrm{E}+01$

$1.87 \mathrm{E}+02$

$3.26 \mathrm{E}+02$ $1.51 \mathrm{E}+02$
$1 \%-i l e$

$1.00 \mathrm{E}-00$

$10 \%$-ile

$5.36 \mathrm{E}-00$

$6.55 \mathrm{E}+01 \quad 5.49 \mathrm{E}+02$

$3.34 \mathrm{E}+04$

$7.46 \mathrm{E}+04$

$1.48 \mathrm{E}-00$

$2.62 \mathrm{E}+01$

$1.54 \mathrm{E}+03$

$8.74 \mathrm{E}+03$

$6.50 \mathrm{E}+02$

$4.50 \mathrm{E}+04$

3.27E+01

$5.95 \mathrm{E}+02$
$1.24 \mathrm{E}+01$

$3.08 \mathrm{E}+01$

$1.70 \mathrm{E}+02$

$4.10 \mathrm{E}+01$

$4.86 \mathrm{E}+02$

$3.44 \mathrm{E} \div 01$

$4.45 \mathrm{E}+01$

$8.80 \mathrm{E}+01$

$9.22 \mathrm{E}+02$

$4.77 \mathrm{E}+02$

$1.52 \mathrm{E}+02$

$9.08 \mathrm{E}+02$

$0.00 \mathrm{E}+01$

$0.00 \mathrm{E}+01$

$2.58 \mathrm{E}+01$

$0.00 \mathrm{E}+01$

$0.00 \mathrm{E}+01$

$0.00 \mathrm{E}+01$

$0.00 \mathrm{E}+01$

$0.00 \mathrm{E}+01$

$0.00 \mathrm{E}+01$

$0.00 \mathrm{E}+01$

3.73E-00

$0.00 \mathrm{E}+01$

$1.09 \mathrm{E}+04$

$0.00 \mathrm{E}+01$

$0.00 \mathrm{E}+01$

$0.00 \mathrm{E}+01$

$0.00 \mathrm{E}+01$

$0.00 \mathrm{E}+01$

$0.00 \mathrm{E}+01$

$0.00 \mathrm{E}+01$

$0.00 \mathrm{E}+01$

$0.00 \mathrm{E}+01$

$0.00 \mathrm{E}+01$

$0.00 \mathrm{E}+01$

$0.00 \mathrm{E}+01$

$0.00 \mathrm{E}+01$

$0.00 \mathrm{E}+01$

8.71E-05

5.23E-04

$0.00 \mathrm{E}+01$

$0.00 \mathrm{E}+01$

2.40E-03

$0.00 \mathrm{E}+01$

$0.00 \mathrm{E}+01$

$0.00 \mathrm{E}+01$

$0.00 \mathrm{E}+01$

$0.00 \mathrm{E}+01$

$0.00 \mathrm{E}+01$

$4.32 \mathrm{E}+01$

$0.00 \mathrm{E}+01$

$0.00 \mathrm{E}+01$

$0.00 \mathrm{E}+01$

$0.00 \mathrm{E}+01$

$0.00 \mathrm{E}+01$

$0.00 \mathrm{E}+01$

$0.00 \mathrm{E} \div 01$

$0.00 \mathrm{E} \div 01$

3.11E-04

$0.00 \mathrm{E}+01$

6.42E-07

$3.26 \mathrm{E}-06$

2.19E-05

$1.96 \mathrm{E}-06$
$1.01 \mathrm{E}+03$
$3.40 \mathrm{E}+03$

8.55E-00

$1.28 \mathrm{E}-03$

$3.48 \mathrm{E}+01$

3.17E-03

2.13E+04

$1.04 \mathrm{E}-00$

4.10E-00

$1.51 \mathrm{E}-01$

1.51E-02

4.58E-01

5.42E-00

$4.70 \mathrm{E}-01$

$3.87 \mathrm{E}+04$

4.21E-00

$3.07 \mathrm{E}+01$

$2.14 \mathrm{E}+02$

$1.94 \mathrm{E}-02$

$1.65 \mathrm{E}-01$

3.36E-00

$3.29 \mathrm{E}+02$

4.52E-02

$0.00 \mathrm{E}+01$

2.41E-00

$5.26 \mathrm{E}+01$

9.78E-00

$1.17 \mathrm{E}+02$

6.63E-04

4.60E-04

2.76E-03

$1.25 \mathrm{E}-04$

2.45E-00

$7.15 \mathrm{E}-01$

$6.18 \mathrm{E}+03$

$1.65 \mathrm{E}+04$

$1.88 \mathrm{E}-02$

$0.00 \mathrm{E}+01$

$0.00 \mathrm{E}+01$

$1.72 \mathrm{E}-00$

$8.18 \mathrm{E}+01$

$0.00 \mathrm{E}+01$

$0.00 E+01$

$0.00 \mathrm{E}+01$

$0.00 \mathrm{E}+01$

$0.00 E+01$

$0.00 \mathrm{E}+01$

$1.00 \mathrm{E}+04$

$1.86 \mathrm{E}-01$

8.60E-04

6.49E-06

1.32E-05

2.05E-05

1.62E-04

$1.66 \mathrm{E}-05$

50\%-ile

$1.69 \mathrm{E}+01$

$1.79 \mathrm{E}+03$

$1.33 \mathrm{E}+05$

$1.14 \mathrm{E}+02$

$3.06 \mathrm{E}+04$

$1.65 E+05$

$4.94 \mathrm{E}+03$

$7.87 \mathrm{E}+01$

$1.31 \mathrm{E}+04$

$1.91 \mathrm{E}+03$

$1.77 \mathrm{E}+03$

$5.25 E+03$

$4.00 \mathrm{E}+02$

$3.25 \mathrm{E}+03$

$8.95 \mathrm{E}+03$

$2.64 \mathrm{E}+02$

$6.21 \mathrm{E}-03$

$3.78 \mathrm{E}+02$

$1.58 \mathrm{E}-02$ 
Tank Anal. Un

$\begin{array}{ll}\text { SX115 } & \mathrm{F} \\ \text { SX115 } & \mathrm{Fe} \\ \text { SX115 } & \mathrm{Hg} \\ \text { SX115 } & \mathrm{K} \\ \text { SX115 } & \mathrm{La} \\ \text { SX115 } & \mathrm{Mn} \\ \text { SX115 } & \mathrm{Na} \\ \text { SX115 } & \mathrm{Ni} \\ \text { SX115 } & \mathrm{NO2} \\ \text { SX115 } & \text { NO3 } \\ \text { SX115 } & \mathrm{P} \\ \text { SX115 } & \mathrm{Pb} \\ \text { SX115 } & \mathrm{PO4}\end{array}$

SX115 PO4

SX115 S

SX115 Si

SX115 SO4

SX115 Sr

SX115 TOC

SX115 U

SX115 Zr

SX115 Ac227

SX115 Am241

SX115 Am243

SX115 Ba137

SX115 C14

SX115 Cd113

SX115 Cm242

SX115 Cm243

SX115 Cm244

SX115 Co60

SX115 Cs134

SX115 Cs137

SX115 Eu152

SX115 Eu154

SX115 Eu155

SX115 1129

SX115 Nb93

SX115 Ni59

SX115 Ni63

SX115 Np237

SX115 Pa231

SX115 Pu238

SX115 Pu239

SX115 Pu240

SX115 Pu241

SX115 Pu242

SX115 Ra226

SX115 Ra228

SX115 Ru106

SX115 Sb125

SX115 Se79

SX115 Sm151

SX115 Sr89/90

SX115 Tc99

SX115 Th229

SX115 Th232

SX115 Tin126

Sx115 Tritium

SX115 U232

SX115 U233

SX115 U234

SX115 U235

SX115 U236

SX115 U238

SX115 Y90

SX115 Zr93

SY101 Al

SY101 Bi
$B B$ May $\overline{98}$

kg $\quad \overline{9.47 \mathrm{E}-00}$

kg $\quad 1.27 \mathrm{E}+02$

kg 9.90E-03

kg 3.40E+01

kg 8.50E-01

kg 1.05E+02

kg 7.72E+03

kg 9.30E-00

kg $2.40 \mathrm{E}+03$

$\mathrm{kg} \quad 9.62 \mathrm{E}+03$

kg 4.39E+01

gg 2.62E-00

kg $\quad 1.36 \mathrm{E}+02$

kg 3.90E+01

kg $\quad 9.85 \mathrm{E}+01$

kg 1.17E+02

kg 3.30E+01

kg $\quad 1.36 \mathrm{E}+01$

kg $\quad 6.07 \mathrm{E}+02$

kg 5.28E-00

1.71E-04

2.83E-00

8.63E-05

$5.80 \mathrm{E}+03$

3.11E-01

1.21E-00

3.69E-03

$8.45 \mathrm{E}-06$

6.57E-05

2.54E-01

5.29E-02

$6.13 \mathrm{E}+03$

$3.60 \mathrm{E}-01$

6.08E-00

$1.77 \mathrm{E}+01$

4.52E- 03

6.48E-01

4.92E-01

$4.65 \mathrm{E}+01$

1.11E-02

2.51E-04

2.04E-01

2.00E-02

1.82E-00

$1.18 \mathrm{E}+01$

5.59E-05

3.52E-05

3.58E-04

$5.41 \mathrm{E}-05$

8.65E-01

1.69E-01

6.02E+02

$2.27 \mathrm{E}+04$

2.38E-00

8.62E-06

4.79E-06

2.59E-01

5.73E-00

1.60E-03

6.12E-03

3.16E-02

$1.28 \mathrm{E}-03$

$1.24 \mathrm{E}-03$

2.06E-01

$2.27 \mathrm{E}+04$

7.98E-01

$2.19 E+05$

$1.58 \mathrm{E}+03$
$B B$ Sept98

9.46E-00

$1.27 \mathrm{E}+02$

$0.00 \mathrm{E}+01$

$3.40 \mathrm{E}+01$

$0.00 \mathrm{E}+01$

$1.05 \mathrm{E}+02$

$7.71 \mathrm{E}+03$

9.30E-00

$2.40 \mathrm{E}+03$

$9.62 \mathrm{E}+03$

NA

2.62E-00

$1.36 \mathrm{E}+02$

NA

$9.85 E+01$

$1.17 E+02$

$3.30 \mathrm{E}+01$

$1.36 \mathrm{E}+02$

$6.06 \mathrm{E}+02$

5.27E- 00

1.71E-04

2.83E-00

8.63E-05

$5.78 \mathrm{E}+03$

3.11E-01

1.21E-00

3.69E-03

8.45E-05

$6.57 \mathrm{E}-05$

2.54E-01

5.29E-02

$6.11 E+03$

3.60E-01

6.08E-00

$1.77 \mathrm{E}+01$

4.52E-03

$6.48 \mathrm{E}-01$

4.92E-01

$4.65 \mathrm{E}+01$

1.11E-02

2.51E-04

2.04E-01

$1.24 \mathrm{E}+01$

$1.82 \mathrm{E}-00$

$1.18 \mathrm{E}+01$

5.59E-05

3.52E-05

3.58E-04

5.41E-05

8.65E-01

1.69E-01

$6.02 \mathrm{E}+02$

$2.27 \mathrm{E}+04$

2.38E-00

8.62E-06

4.79E-06

2.59E-01

5.73E-00

1.17E-02

4.48E-02

2.31E-01

9.39E-03

9.08E-03

2.02E-01

$2.27 \mathrm{E}+04$

7.98E-01

$2.19 E+05$

$0.00 E+01$

1\%-ile

$10 \%$-ile

50\%-ile

$0.00 \mathrm{E}+01 \quad 3.43 \mathrm{E}-05$

2.65E-06 5.07E-05

$0.00 \mathrm{E}+01 \quad 0.00 \mathrm{E} \div 01$

$1.18 \mathrm{E}-06 \quad 3.01 \mathrm{E}-05$

$3.78 \mathrm{E}-08 \quad 1.54 \mathrm{E}-07$

8.81E-07

$3.79 \mathrm{E}-04$

$4.29 \mathrm{E}-08$

$3.21 \mathrm{E}-05$

$0.00 \mathrm{E}+01$

$1.24 \mathrm{E}-06$

4.06E-08

$1.21 \mathrm{E}-06$

1.21E-06

$2.28 \mathrm{E}-06$

$1.96 \mathrm{E}-06$

$2.79 \mathrm{E}-07$

$4.09 \mathrm{E}-06$

$1.05 \mathrm{E}-05$

$0.00 \mathrm{E}+01$

$0.00 \mathrm{E}+01$

$1.74 \mathrm{E}-07$

$0.00 \mathrm{E}+01$

$0.00 \mathrm{E}+01$

$0.00 \mathrm{E}+01$

$0.00 \mathrm{E}+01$

$0.00 \mathrm{E}+01$

$0.00 \mathrm{E}+01$

$0.00 \mathrm{E}+01$

3.24E-08

$0.00 \mathrm{E}+01$

$1.94 \mathrm{E}-04$

$0.00 \mathrm{E}+01$

$0.00 \mathrm{E}+01$

$0.00 \mathrm{E}+01$

$0.00 E+01$

$0.00 \mathrm{E}+01$

$0.00 \mathrm{E}+01$

$0.00 \mathrm{E}+01$

$0.00 \mathrm{E}+01$

$0.00 \mathrm{E}+01$

$0.00 \mathrm{E}+01$

$0.00 E+01$

$0.00 \mathrm{E}+01$

$0.00 \mathrm{E}+01$

$0.00 \mathrm{E}+01$

$0.00 \mathrm{E}+01$

$0.00 \mathrm{E}+01$

$0.00 \mathrm{E}+01$

$0.00 E+01$

$0.00 \mathrm{E}+01$

$0.00 E+01$

$0.00 \mathrm{E}+01$

$0.00 \mathrm{E}+01$

$0.00 \mathrm{E}+01$

$0.00 \mathrm{E}+01$

$0.00 \mathrm{E}+01$

$0.00 E+01$

$0.00 \mathrm{E}+01$

$0.00 \mathrm{E}+01$

$0.00 \mathrm{E}+01$

$0.00 \mathrm{E}+01$

$0.00 \mathrm{E}+01$

$0.00 \mathrm{E}+01$

$0.00 \mathrm{E}+01$

$0.00 \mathrm{E}+01$

$1.32 \mathrm{E}+05$

$1.46 \mathrm{E}-05$

$8.79 \mathrm{E}-04$

9.66E-07

2.68E-04

$1.18 \mathrm{E}-03$

9.65E-05

1.64E-06

$1.90 \mathrm{E}-04$

2.06E-05

1.89E-05

4.30E-05

$3.08 \mathrm{E}-06$

4.11E-05

7.33E-05

$5.26 \mathrm{E}-07$

$0.00 \mathrm{E}+01$

4.94E-06

$0.00 \mathrm{E}+01$

$0.00 \mathrm{E}+01$

$0.00 \mathrm{E}+01$

$0.00 \mathrm{E}+01$

$0.00 \mathrm{E}+01$

$0.00 \mathrm{E}+01$ 
Tank Anal. Un

$\begin{array}{ll}\text { SY101 } & \text { Ca } \\ \text { SY101 } & \text { C } \\ \text { SY101 } & \text { CO3 } \\ \text { SY101 } & \text { Cr } \\ \text { SY101 } & \text { F } \\ \text { SY101 } & \text { Fe } \\ \text { SY101 } & \text { Hg } \\ \text { SY101 } & \text { K } \\ \text { SY101 } & \text { La } \\ \text { SY101 } & \text { Mn } \\ \text { SY101 } & \text { Na } \\ \text { SY101 } & \text { Ni } \\ \text { SY101 } & \text { NO2 } \\ \text { SY101 } & \text { NO3 } \\ \text { SY101 } & \text { P } \\ \text { SY101 } & \text { Pb } \\ \text { SY101 } & \text { PO4 } \\ \text { SY101 } & \text { S } \\ \text { SY101 } & \text { Si } \\ \text { SY101 } & \text { SO4 } \\ \text { SY101 } & \text { Sr } \\ \text { SY101 } & \text { TOC } \\ \text { SY101 } & \text { U } \\ \text { SY101 } & \text { Zr }\end{array}$

SY101

SY101 Am241

SY101 Am243

SY101 Ba137

SY101 C14

SY101 Cd113

SY101 Cm242

SY101 Cm243

SY101 Cm244

SY101 Co60

SY101 Cs134

SY101 Cs137

SY101 Eu152

SY101 Eu154

SY101 Eu155

SY101 1129

SY101 Nb93

SY101 Ni59

SY101 Ni63

SY101 Np237

SY101 Pa231

SY101 Pu238

SY101 Pu239

SY101 Pu240

SY101 Pu241

SY101 Pu242

SY101 Ra226

SY101 Ra228

SY101 Ru106

SY101 Sb125

SY101 Se79

SY101 Sm15!

SY101 Sr89/90

SY101 TC99

SY101 Th229

SY101 Th232

SY101 Tin126

SY101 Tritium

SY101 U232

SY101 U233

SY101 U234

SY101 U235

SY101 U236

SY101 U238
May $\frac{B B}{98}$

$2 . \overline{41 E+03}$

(c.63E+04

kg 2.52E+05

kg $2.46 \mathrm{E}+04$

kg $7.08 \mathrm{E}+03$

kg 2.08E+03

$\mathrm{kg} \quad 1.03 \mathrm{E}+01$

kg 2.28E+04

$\mathrm{kg} \quad 3.56 \mathrm{E}+01$

kg 1.19E+03

$\mathrm{kg} \quad 1.39 \mathrm{E}+06$

kg 1.03E+03

$\mathrm{kg} \quad 7.44 \mathrm{E}+05$

kg $\quad 8.50 \mathrm{E}+05$

$\mathrm{kg} \quad 1.44 \mathrm{E}+04$

kg $\quad 1.16 \mathrm{E}+03$

kg $\quad 4.46 E+04$

8.87E +03

kg 1.23E+04

$2.66 \mathrm{E}+04$

k $0.00 \mathrm{E}+01$

1.04E+05

$2.65 E+02$

$1.28 \mathrm{E}+02$

5.42E-03

$6.33 \mathrm{E}+02$

$1.23 \mathrm{E}-02$

$2.09 \mathrm{E}+06$

$2.04 \mathrm{E}+02$

$5.26 \mathrm{E}+02$

9.38E-01

8.70E-02

8.41E-01

$2.28 \mathrm{E}+02$

$1.68 \mathrm{E}+01$

$2.21 E+06$

$2.46 \mathrm{E}+01$

$3.73 E+03$

$1.46 \mathrm{E}+03$

2.79E-00

$7.21 \mathrm{E}+01$

$1.30 \mathrm{E}+01$

$1.28 \mathrm{E}+03$

5.24E-00

2.50E-02

8.60E-00

$4.66 \mathrm{E}+01$

Ci Incl. in 239

$5.84 \mathrm{E}+02$

$3.21 \mathrm{E}-03$

8.54E-04

8.65E-01

4.16E-02

$9.89 \mathrm{E}+02$

Ci $2.03 E+01$

Ci $7.14 \mathrm{E}+04$

$1.25 \mathrm{E}+05$

$1.46 \mathrm{E}+04$

Ci 2.03E-02

Ci 5.84E-02

$3.06 \mathrm{E}+01$

Ci $1.38 \mathrm{E}+03$

Ci 4.40E-00

Ci $1.69 \mathrm{E}+01$

$4.75 \mathrm{E}-00$

1.92E-01

$1.49 \mathrm{E}-01$

Ci 5.56E-00
Sept98 $2.41 \mathrm{E}+03$

$5.63 E+04$

$2.52 E+05$

$2.46 \mathrm{E}+04$

$7.08 \mathrm{E}+03$

$2.08 \mathrm{E}+03$

$0.00 \mathrm{E}+01$

$2.28 \mathrm{E}+04$

$3.56 \mathrm{E}+01$

$1.19 \mathrm{E}+03$

$1.39 \mathrm{E}+06$

$1.03 \mathrm{E}+03$

$7.44 \mathrm{E}+05$

$8.50 \mathrm{E}+05$

$$
\text { NA }
$$

$1.16 \mathrm{E}+03$

$4.46 \mathrm{E}+04$$$
\text { NA }
$$

$1.23 \mathrm{E}+04$

$2.66 \mathrm{E}+04$

$0.00 \mathrm{E}+01$

$1.04 \mathrm{E}+05$

$2.65 \mathrm{E}+02$

$1.28 \mathrm{E}+02$

5.42E-03

$6.33 \mathrm{E}+02$

$2.26 \mathrm{E}-02$

$2.09 \mathrm{E}+06$

$2.04 \mathrm{E}+02$

$5.26 \mathrm{E}+02$

$1.72 \mathrm{E}-00$

$1.60 \mathrm{E}-01$

$1.54 \mathrm{E}-00$

$2.28 \mathrm{E}+02$

$1.68 \mathrm{E}+01$

$2.21 \mathrm{E}+06$

$2.46 \mathrm{E}+01$

$3.73 \mathrm{E}+03$

$1.46 \mathrm{E}+03$

2.79E-00

$7.21 \mathrm{E}+01$

$1.30 \mathrm{E}+01$

$1.28 \mathrm{E}+03$

$5.24 \mathrm{E}-00$

2.50E-02

1.17E-00

$3.98 \mathrm{E}+01$

$6.78 \mathrm{E}-00$

$7.96 \mathrm{E}+01$

4.38E-04

8.54E-04

$8.65 E-01$

4.16E-02

$9.89 \mathrm{E}+02$

2.03E+01

$7.14 \mathrm{E}+04$

$1.25 \mathrm{E}+05$

$1.46 \mathrm{E}+03$

2.03E-02

5.84E-02

$3.06 \mathrm{E}+01$

$1.38 \mathrm{E}+03$

9.00E-02

$3.46 \mathrm{E}-01$

9.74E-02

$3.95 \mathrm{E}-03$

3.07E-03

8.84E-02
$1 \%$ ile

1.79E +02

$3.87 \mathrm{E}+04$

1.57E+05

$1.57 \mathrm{E}+04$

$0.00 \mathrm{E}+01$

$0.00 \mathrm{E}+01$

$0.00 \mathrm{E}+01$

$0.00 \mathrm{E}+01$

2.84E-00

$0.00 \mathrm{E}+01$

$8.90 \mathrm{E}+05$

$0.00 \mathrm{E}+01$

$4.58 \mathrm{E}+05$

$3.79 \mathrm{E}+05$

$3.29 \mathrm{E}+03$

6.95E-00

$1.40 \mathrm{E}+03$

$2.76 \mathrm{E}+03$

$0.00 \mathrm{E}+01$

$3.11 \mathrm{E}+03$

$0.00 \mathrm{E}+01$

$6.28 \mathrm{E}+04$

$0.00 \mathrm{E}+01$

$5.44 \mathrm{E}-00$

$0.00 \mathrm{E}+01$

2.72E+02

$3.67 \mathrm{E}-03$

$3.51 \mathrm{E}+05$

$0.00 \mathrm{E}+01$

$0.00 \mathrm{E}+01$

$2.80 \mathrm{E}-01$

2.60E-02

2.51E-01

$3.03 \mathrm{E}+02$

$2.82 \mathrm{E}-00$

$1.88 \mathrm{E}+06$

$0.00 \mathrm{E}+01$

$0.00 \mathrm{E}+01$

$0.00 \mathrm{E}+01$

8.06E-03

$0.00 \mathrm{E}+01$

$0.00 \mathrm{E}+01$

$0.00 \mathrm{E}+01$

$1.56 \mathrm{E}-00$

$0.00 \mathrm{E}+01$

2.57E-00

$1.39 \mathrm{E}+01$

$1.39 \mathrm{E}+01$

$1.74 \mathrm{E}+02$

$9.58 \mathrm{E}-04$

$0.00 \mathrm{E}+01$

$0.00 \mathrm{E}+01$

$0.00 \mathrm{E}+01$

$0.00 \mathrm{E}+01$

$0.00 \mathrm{E}+01$

$0.00 \mathrm{E}+01$

$1.63 \mathrm{E}+05$

$0.00 \mathrm{E}+01$

$0.00 \mathrm{E}+01$

$0.00 \mathrm{E}+01$

$0.00 \mathrm{E}+01$

$1.13 E+03$

$0.00 \mathrm{E}+01$

$0.00 \mathrm{E}+01$

$0.00 \mathrm{E}+01$

$0.00 \mathrm{E}+01$

$0.00 \mathrm{E}+01$

$0.00 \mathrm{E}+01$

$10 \%$-ile

$6.08 E+02$

4.33E+04

$2.09 \mathrm{E}+05$

$1.70 \mathrm{E}+04$

$3.73 \mathrm{E}+02$

$1.80 \mathrm{E}+02$

$0.00 \mathrm{E}+01$

$6.16 \mathrm{E}+03$

$3.65 \mathrm{E}+01$

$0.00 \mathrm{E}+01$

$9.17 \mathrm{E}+05$

$3.97 \mathrm{E}+02$

4.84E+05

$4.58 \mathrm{E}+05$

$7.59 \mathrm{E}+03$

$3.05 E+02$

$1.15 \mathrm{E}+04$

$6.15 \mathrm{E}+03$

$2.01 \mathrm{E}+02$

$1.43 \mathrm{E}+04$

4.06E-00

$6.54 \mathrm{E}+04$

$3.19 \mathrm{E}+01$

8.05E-00

$0.00 \mathrm{E}+01$

$3.01 \mathrm{E}+02$

$7.64 \mathrm{E}-03$

$1.08 \mathrm{E}+06$

$3.17 \mathrm{E}+01$

$0.00 \mathrm{E}+01$

$5.82 \mathrm{E}-01$

5.40E-02

$5.22 \mathrm{E}-01$

$3.41 \mathrm{E}+02$ 
Tank Anal. Un

$\begin{array}{ll}\text { SY101 } & \text { Y90 } \\ \text { SY101 } & \text { Zr93 } \\ \text { SY102 } & \text { Al } \\ \text { SY102 } & \text { Bi } \\ \text { SY102 } & \text { Ca } \\ \text { SY102 } & \text { Cl } \\ \text { SY102 } & \text { CO3 } \\ \text { SY102 } & \text { Cr } \\ \text { SY102 } & \text { F } \\ \text { SY102 } & \text { Fe } \\ \text { SY102 } & \text { Hg } \\ \text { SY102 } & \text { K } \\ \text { SY102 } & \text { La } \\ \text { SY102 } & \text { Mn } \\ \text { SY102 } & \text { Na } \\ \text { SY102 } & \text { Ni } \\ \text { SY102 } & \text { NO2 } \\ \text { SY102 } & \text { NO3 } \\ \text { SY102 } & \text { P } \\ \text { SY102 } & \text { Pb } \\ \text { SY102 } & \text { PO4 } \\ \text { SY102 } & \text { S } \\ \text { SY102 } & \text { Si } \\ \text { SY102 } & \text { SO4 }\end{array}$

SY102 SO4

SY102 $\mathrm{Sr}$

SY102 TOC

SY102 U

SY102 $\mathrm{Zr}$

SY102 Ac227

SY102 Am241

SY102 Am243

SY102 Ba137

SY102 C14

SY102 Cd113

SY102 Cm242

SY102 Cm243

SY102 Cm244

SY102 Co60

SY102 Cs134

SY102 Cs137

SY102 Eu152

SY102 Eul54

SY102 Eu155

SY102 I129

SY102 Nb93

SY102 Ni59

SY102 Ni63

SY102 Np237

SY102 Pa231

SY102 Pu238

SY102 Pu239

SY102 Pu240

SY102 Pu241

SY102 Pu242

SY102 Ra226

SY102 Ra228

SY102 Ru106

SY102 Sb125

SY102 Se79

SY102 Sm151

SY102 Sr89/90

SY102 Tc99

SY102 Th229.

SY102 Th232

SY102 Tin126

SY102 Tritium

SY102 U232

SY102 U233
BB Ci $\frac{1.25 \mathrm{E}+05}{1.92}$ $\begin{array}{ll}\mathrm{Ci} & 9.95 \mathrm{E}+01 \\ \mathrm{~kg} & 1.64 \mathrm{E}+04\end{array}$ kg 1.99E+02

kg 4.10E+03

kg 1.37E+03

kg $1.04 \mathrm{E}+04$

kg $\quad 6.67 \mathrm{E}+03$

kg $1.89 \mathrm{E}+03$

$\mathrm{kg} \quad 1.29 \mathrm{E}+04$

kg 8.07E-01

$\mathrm{kg} \quad 1.72 \mathrm{E}+03$

kg $\quad 3.80 \mathrm{E}+01$

kg 3.43E+03

$\mathrm{kg} \cdot 9.91 \mathrm{E}+04$

kg $\quad 4.67 \mathrm{E}+02$

kg $\quad 1.80 \mathrm{E}+05$

kg $\quad 9.03 \mathrm{E}+04$

kg $\quad 4.07 \mathrm{E}+03$

kg $\quad 9.72 \mathrm{E}+02$

kg $\quad 1.26 \mathrm{E}+04$

kg 1.25E+03

kg $4.32 \mathrm{E}+03$

$\mathrm{kg} \quad 3.76 \mathrm{E}+03$

kg $\quad 6.96 \mathrm{E}+01$

kg $\quad 2.77 \mathrm{E}+03$

kg $\quad 6.20 \mathrm{E}+02$

kg $2.34 \mathrm{E}+02$

Ci 1.76E-04

Ci $2.48 \mathrm{E}+04$

$7.09 \mathrm{E}-04$

$4.83 \mathrm{E}+04$

$2.86 \mathrm{E}-00$

$1.63 \mathrm{E}+01$

$1.72 \mathrm{E}-04$

$1.19 \mathrm{E}-05$

$1.46 \mathrm{E}-04$

$5.87 \mathrm{E}+01$

9.44E-01

$5.10 \mathrm{E}+04$

$7.82 \mathrm{E}-01$

$5.66 \mathrm{E}+02$

$4.93 E+02$

$1.73 \mathrm{E}-01$

2.31E-00

1.74E-01

$1.70 \mathrm{E}+01$

3.72E-01

$1.00 \mathrm{E}-03$

$3.48 \mathrm{E}+02$

$3.17 \mathrm{E}+03$

$\mathrm{Ci}$ Incl. in 239 I

$1.08 \mathrm{E}+01$

5.84E-05

2.78E-05

2.06E-02

$1.22 \mathrm{E}-03$

$3.18 \mathrm{E}+01$

6.41E-01

$2.27 \mathrm{E}+03$

$3.89 \mathrm{E}+04$

$2.04 \mathrm{E}+01$

4.95E-04

2.27E-03

9.75E-01

4.26E-01

4.97E-02

1.91E-01
Sept98

$1.25 \mathrm{E}+05$

$9.95 \mathrm{E}+01$

$1.99 \mathrm{E}+02$

$4.10 \mathrm{E}+03$

$1.37 \mathrm{E}+03$

$1.04 \mathrm{E}+04$

$6.67 \mathrm{E}+03$

$1.89 \mathrm{E}+03$

$1.29 \mathrm{E}+04$

$0.00 \mathrm{E}+01$

$1.72 \mathrm{E}+03$

$3.80 \mathrm{E}+01$

$3.43 \mathrm{E}+03$

$9.91 \mathrm{E}+04$

$4.67 \mathrm{E}+02$

$1.80 \mathrm{E}+05$

9.03E+04

NA

$9.72 \mathrm{E}+02$

$1.26 \mathrm{E}+04$

NA

$4.32 \mathrm{E}+03$

$3.76 \mathrm{E}+03$

$6.96 \mathrm{E}+01$

$2.77 \mathrm{E}+03$

$6.20 \mathrm{E}+02$

$2.34 \mathrm{E}+02$

$1.76 \mathrm{E}-04$

$2.48 \mathrm{E}+04$

$7.09 \mathrm{E}-04$

$4.83 \mathrm{E}+04$

$2.86 \mathrm{E}-00$

$1.63 \mathrm{E}+01$

1.72E-04

1.19E-05

$1.46 \mathrm{E}-04$

$5.87 \mathrm{E}+01$

9.44E-01

$5.10 \mathrm{E}+04$

$7.82 \mathrm{E}-01$

$5.66 \mathrm{E}+02$

$4.93 \mathrm{E}+02$

$1.73 \mathrm{E}-01$

2.31E-00

1.74E-01

$1.70 \mathrm{E}+01$

3.72E-01

1.00E-03

$3.48 \mathrm{E}+02$

$3.17 E+03$

Incl. in 239

$1.08 \mathrm{E}+01$

5.84E-05

2.78E-05

2.06E-02

1.22E-03

$3.18 \mathrm{E}+01$

6.41E-01

$2.27 \mathrm{E}+03$

$3.89 \mathrm{E}+04$

$2.04 \mathrm{E}+01$

4.95E-04

2.27E-03

9.75E-01

$4.26 \mathrm{E}-01$

4.97E-02

I.91E-01
$1.64 \mathrm{E}+04$

1\%-ile

$10 \%$-ile

5

$0.00 \mathrm{E}+01 \quad 0.00 \mathrm{E}+01$

$1.51 \mathrm{E}+02$

6.72E-03

$9.08 \mathrm{E}+02$

2.67E-01

.47E+01

$1.65 \mathrm{E}+02$

$3.67 \mathrm{E}+01$

9.55E-02

$5.04 \mathrm{E}-00$

$0.00 \mathrm{E}+01$

$1.07 \mathrm{E}-00$

$1.09 \mathrm{E}-01$

4.09E-03

$3.73 \mathrm{E}+03$

$7.13 \mathrm{E}-00$

$1.89 \mathrm{E}-00$

$7.89 \mathrm{E}+02$

3.11E-02

$1.07 \mathrm{E}+02$

$2.83 \mathrm{E}+01$

$8.64 \mathrm{E}+02$

$1.03 \mathrm{E}+02$

$6.13 \mathrm{E}+01$

$1.69 \mathrm{E}+02$

$6.95 \mathrm{E}-02$

$3.60 \mathrm{E} \div 01$

8.01E-01

4.87E-02

$8.40 \mathrm{E}+03$

$4.62 \mathrm{E}+01$

$7.46 \mathrm{E}+01$

$4.00 \mathrm{E}+03$

$1.53 \mathrm{E}-01$

$3.71 \mathrm{E}-03 \quad 1.87 \mathrm{E}-01$

$4.89 \mathrm{E}+02$

$1.72 \mathrm{E}+02$

$6.48 \mathrm{E}+02$

$2.11 \mathrm{E}+02$

$1.11 \mathrm{E}-02$

$2.71 E+02$

2.84E-00

$8.55 \mathrm{E}-02$

$4.94 \mathrm{E}+02$

2.37E-01

$5.35 \mathrm{E}+01$

3.07E-03

$1.11 \mathrm{E}-03$

$0.00 \mathrm{E}+01$

$0.00 \mathrm{E}+01$

$0.00 \mathrm{E}+01$

$0.00 \mathrm{E}+01$

$0.00 \mathrm{E}+01$

$0.00 \mathrm{E}+01$

$0.00 \mathrm{E}+01$

$0.00 \mathrm{E}+01$

$0.00 \mathrm{E}+01$

5.04E-05

$0.00 \mathrm{E}+01$

9.36E-02

$0.00 \mathrm{E}+01$

$0.00 \mathrm{E}+01$

$0.00 \mathrm{E}+01$

$0.00 \mathrm{E}+01$

$0.00 E+01$

5.32E-03

5.19E-01

$0.00 \mathrm{E}+01$

$0.00 \mathrm{E}+01$

$0.00 \mathrm{E}+01$

$0.00 \mathrm{E}+01$

$0.00 \mathrm{E}+01$

$0.00 \mathrm{E}+01$

$0.00 \mathrm{E}+01$

3.57E-06

2.64E-03

$0.00 \mathrm{E}+01$

$0.00 \mathrm{E}+01$

$1.53 \mathrm{E}-01$

$0.00 \mathrm{E}+01$

$5.93 E+01$

$0.00 E+01$

$0.00 \mathrm{E}+01$

$0.00 E+01$

$0.00 \mathrm{E}+01$

$1.08 \mathrm{E}-01$

$0.00 \mathrm{E}+01$

$1.11 \mathrm{E}-02$

$0.00 \mathrm{E}+01$

8.70E-03

$1.22 \mathrm{E}-06$

$0.00 \mathrm{E}+01$

$0.00 \mathrm{E}+01$

$1.40 \mathrm{E}-00$

$2.97 \mathrm{E}-07$

2.06E-08

2.52E-07

$1.33 \mathrm{E}-03$

$0.00 \mathrm{E}+01$

$5.36 \mathrm{E}-01$

$0.00 \mathrm{E}+01$ 
Tank Anal. Un

$\begin{array}{ll}\text { SY102 } & \text { U234 } \\ \text { SY102 } & \text { U235 } \\ \text { SY102 } & \text { U236 } \\ \text { SY102 } & \text { U238 } \\ \text { SY102 } & \text { Y90 } \\ \text { SY102 } & \text { Zr93 } \\ \text { SY103 } & \text { Al } \\ \text { SY103 } & \text { Bi } \\ \text { SY103 } & \text { Ca } \\ \text { SY103 } & \text { Cl } \\ \text { SY103 } & \text { CO3 } \\ \text { SY103 } & \text { Cr } \\ \text { SY103 } & \text { F } \\ \text { SY103 } & \text { Fe } \\ \text { SY103 } & \text { Hg } \\ \text { SY103 } & \text { K } \\ \text { SY103 } & \text { La } \\ \text { SY103 } & \text { Mn } \\ \text { SY103 } & \text { Na } \\ \text { SY103 } & \text { Ni } \\ \text { SY103 } & \text { NO2 } \\ \text { SY103 } & \text { NO3 } \\ \text { SY103 } & \text { P } \\ \text { SY103 } & \text { Pb } \\ \text { SY103 } & \text { PO4 } \\ \text { SY103 } & \text { S } \\ \text { SY103 } & \text { Si }\end{array}$

SY103 Si

SY103 SO4

SY103 Sr

SY103 TOC

SY103 U

SY103 Zr

SY103 Ac227

SY103 Am241

SY103 Am243

SY103 Ba137

SY103 C14

SY103 Cd113

SY103 Cm242

SY103 Cm243

SY103 Cm244

SY103 Co60

SY103 Cs134

SY103 Cs137

SY103 Eu152

SY103 Eu154

SY103 Eu155

SY103 I129

SY103 Nb93

SY103 Ni59

SY103 Ni63

SY103 Np237

SY103 Pa231

SY103 Pu238

SY103 Pu239

SY103 Pu240

SY103 Pu241

SY103 Pu242

SY103 Ra226

SY103 Ra228

SY103 Ru106

SY103 Sb125

SY103 Se79

SY103 Sm151

SY103 Sr89/90

SY103 Te99

SY103 Th229

SY103 Th232

\section{BB}

May $\frac{\text { Ma8 }}{1.06 \mathrm{E}-01}$

Ci $4.45 E-03$

Ci 2.59E-03

Ci 1.31E-01

$3.89 \mathrm{E}+04$

$3.16 \mathrm{E}-00$

$1.47 \mathrm{E}+05$

$7.89 \mathrm{E}+02$

$9.39 \mathrm{E}+02$

$3.15 E+04$

$9.46 \mathrm{E}+04$

$2.19 \mathrm{E}+04$

g $3.35 \mathrm{E}+03$

kg $\quad 5.81 \mathrm{E}+03$

kg 5.31E-00

$\mathrm{kg} \quad 1.28 \mathrm{E}+04$

$\mathrm{kg} \quad 1.47 \mathrm{E}+01$

kg $\quad 6.29 \mathrm{E}+02$

kg $7.19 \mathrm{E}+05$

kg 2.90E+02

kg $3.77 \mathrm{E}+05$

kg $5.04 E+05$

kg 4.01E+04

kg $\quad 6.30 \mathrm{E}+02$

kg $\quad 1.24 \mathrm{E}+05$

5.67E +03

$\mathrm{kg} \quad \mathrm{1.07E+02}$

kg 1.70E+04

kg $0.00 \mathrm{E}+01$

3.62E+04

$1.67 \mathrm{E}+03$

$1.23 E+02$

2.75E-03

$1.44 \mathrm{E}+03$

6.32E-03

1.07E+06

$1.05 E+02$

$2.73 E+02$

4.70E-01

4.36E-02

4.21E-01

Ci $1.05 E+02$

Ci $\quad 9.00 \mathrm{E}-00$

Ci $1.13 E+06$

Ci $1.27 \mathrm{E}+01$

Ci 1.62E+03

Ci $1.41 \mathrm{E}+03$

$\mathrm{Ci}$ 2.58E-01

Ci $3.73 E+01$

Ci 6.51E-00

Ci $6.39 \mathrm{E}+02$

Ci $2.74 E-00$

Ci 1.28E-02

Ci 3.54E+01

Ci $1.34 E+02$

Ci Incl. in 239

Ci 2.95E+02

Ci 1.62E-03

Ci $\quad 4.36 E-04$

$\mathrm{Ci} \quad$ 5.56E-01

Ci 2.16E-02

Ci $5.23 E+02$

$\mathrm{Ci} \quad 1.05 \mathrm{E}+01$

Ci $3.69 \mathrm{E}+04$

Ci $7.90 \mathrm{E}+04$

Ci $7.59 E+02$

Ci 1.30E-02

Ci 4.58E-02.
$\frac{B B}{t 98}$

Sept98

4.45E- 03

2.59E-03

1.31E-01

$3.89 \mathrm{E}+04$

$3.16 \mathrm{E}-00$

$1.47 E+05$.

$0.00 \mathrm{E}+01$

$9.39 \mathrm{E}+02$

$3.15 \mathrm{E}+04$

$4.73 E+05$

$2.19 E+04$

$3.35 E+03$

$5.81 \mathrm{E}+03$

$0.00 \mathrm{E}+01$

$1.28 \mathrm{E}+04$

$1.00 \mathrm{E}+02$

$1.15 \mathrm{E}+03$

$7.19 \mathrm{E}+05$

$2.90 \mathrm{E}+02$

$3.77 \mathrm{E}+05$

5.04E+05

NA

$630 \mathrm{E}+02$

$4.05 \mathrm{E}+04$

NA

$1.07 \mathrm{E}+02$

$1.70 \mathrm{E}+04$

8.60E-00

$3.79 E+04$

1.67E+03

$1.23 E+02$

2.75E-03

$1.44 \mathrm{E}+03$

$5.14 \mathrm{E}-02$

$1.07 \mathrm{E}+06$

$1.05 E+02$

$2.73 E+02$

3.83E-00

3.55E-01

3.35E-00

$1.05 \mathrm{E}+02$

$9.00 \mathrm{E}-00$

$1.13 E+06$

$1.27 \mathrm{E}+01$

$1.62 \mathrm{E}+03$

$1.41 \mathrm{E}+03$

2.58E-01

$3.73 E+01$

$6.51 \mathrm{E}-00$

$6.39 \mathrm{E}+02$

$2.74 \mathrm{E}-00$

1.28E-02

$3.54 \mathrm{E}+01$

$1.14 \mathrm{E}+02$

$1.95 \mathrm{E}+01$

$2.29 \mathrm{E}+02$

$1.26 \mathrm{E}-03$

4.36E-04

5.56E-01

2.16E-02

$5.23 \mathrm{E}+02$

$1.05 E+01$

$3.69 E+04$

$7.90 \mathrm{E}+04$

$7.59 \mathrm{E}+02$

1.30E-02

4.58E-02 1\%-ile

$0.00 \mathrm{E}+01$

$0.00 \mathrm{E}+01$

$0.00 \mathrm{E}+01$

$0.00 \mathrm{E}+01$

$1.29 \mathrm{E}+04$

$0.00 \mathrm{E}+01$

$6.88 \mathrm{E}+04$

$0.00 \mathrm{E}+01$

$2.02 \mathrm{E}+02$

$1.81 E+04$

4.12E-01

2.73E-02

$0.00 E+01$

$0.00 \mathrm{E}+01$

$0.00 \mathrm{E}+01$

$2.16 \mathrm{E}+02$

1.73E-04

$0.00 \mathrm{E}+01$

$3.58 \mathrm{E}+05$

$5.11 \mathrm{E}+01$

$2.22 \mathrm{E}+05$

$2.79 \mathrm{E}+05$

3.31E-02

$1.46 \mathrm{E}-03$

4.29E +03

2.90E-02

$1.53 \mathrm{E}-02$

$2.92 \mathrm{E}+03$

$0.00 \mathrm{E}+01$

$1.81 \mathrm{E}+04$

$1.17 \mathrm{E}+02$

$0.00 \mathrm{E}+01$

$0.00 \mathrm{E}+01$

$0.00 \mathrm{E}+01$

$0.00 \mathrm{E}+01$

$0.00 \mathrm{E}+01$

$0.00 \mathrm{E}+01$

$0.00 \mathrm{E}+01$

$0.00 \mathrm{E}+01$

$0.00 \mathrm{E}+01$

$0.00 \mathrm{E}+01$

$3.54 \mathrm{E}+01$

$0.00 \mathrm{E}+01$

$5.77 \mathrm{E}+05$

$0.00 \mathrm{E}+01$

$0.00 \mathrm{E}+01$

$0.00 \mathrm{E}+01$

$0.00 \mathrm{E}+01$

$0.00 \mathrm{E}+01$

$0.00 \mathrm{E}+01$

$0.00 \mathrm{E}+01$

$0.00 \mathrm{E}+01$

$0.00 \mathrm{E}+01$

$0.00 \mathrm{E}+01$

$0.00 \mathrm{E}+01$

$0.00 \mathrm{E}+01$

$0.00 \mathrm{E}+01$

$0.00 \mathrm{E}+01$

$0.00 \mathrm{E}+01$

$0.00 \mathrm{E}+01$

$0.00 \mathrm{E}+01$

$0.00 \mathrm{E}+01$

$0.00 \mathrm{E}+01$

$0.00 \mathrm{E}+01$

$5.79 \mathrm{E}+03$

$0.00 E+01$

$0.00 E+01$

$0.00 \mathrm{E}+01$

10\%-ile

$0.00 \mathrm{E}+01$

$0.00 \mathrm{E}+01$

$0.00 \mathrm{E}+01$

$0.00 \mathrm{E}+01$

$2.21 \mathrm{E}+04$

$0.00 \mathrm{E}+01$

$7.66 \mathrm{E}+04$

$1.53 \mathrm{E}+01$

$2.42 \mathrm{E}+02$

$2.32 \mathrm{E}+04$

$1.38 \mathrm{E}+04$

$1.06 \mathrm{E}+02$

$1.26 \mathrm{E}-02$

$6.94 \mathrm{E}-03$

$0.00 \mathrm{E}+01$

$4.01 \mathrm{E}+03$

8.26E-00

$0.00 \mathrm{E}+01$

$4.08 \mathrm{E}+05$

$7.80 \mathrm{E}+01$

$2.86 \mathrm{E}+05$

$3.80 \mathrm{E}+05$

7.28E- 02

$1.89 \mathrm{E}+01$

$6.78 \mathrm{E}+03$

$1.24 \mathrm{E}+03$

$1.35 \mathrm{E}+02$

$5.84 \mathrm{E}+03$

$1.93 \mathrm{E}-00$

$2.25 E+04$

$2.11 \mathrm{E}+02$

$2.40 \mathrm{E}-00$

$0.00 \mathrm{E}+01$

$3.44 \mathrm{E}-01$

$0.00 \mathrm{E}+01$

$4.98 \mathrm{E}+05$ 
Tank Anal. Un

\begin{tabular}{|c|c|}
\hline Y103 & Tin126 \\
\hline SY103 & Tritium \\
\hline SY103 & U232 \\
\hline SY103 & U233 \\
\hline SY103 & U234 \\
\hline SY103 & U235 \\
\hline SY103 & U236 \\
\hline SY103 & U238 \\
\hline SY103 & Y90 \\
\hline SY103 & Zr93 \\
\hline T101 & Al \\
\hline T101 & $\mathbf{B i}$ \\
\hline T101 & $\mathrm{Ca}$ \\
\hline T101 & Cl \\
\hline T101 & $\mathrm{CO} 3$ \\
\hline T101 & $\mathrm{Cr}$ \\
\hline T101 & $\mathbf{F}$ \\
\hline T101 & $\begin{array}{l}\mathrm{Fe} \\
\mathrm{Ho}\end{array}$ \\
\hline T101 & $\begin{array}{l}\mathbf{H} \mathbf{g} \\
\mathbf{K}\end{array}$ \\
\hline T101 & $\mathbf{L a}$ \\
\hline T101 & Mn \\
\hline T101 & $\mathbf{N a}$ \\
\hline T101 & $\mathrm{Ni}$ \\
\hline T101 & $\mathrm{NO} 2$ \\
\hline T101 & NO3 \\
\hline T101 & $\mathbf{P}$ \\
\hline T101 & $\mathbf{P b}$ \\
\hline T101 & PO4 \\
\hline T101 & $\mathbf{S}$ \\
\hline $\begin{array}{l}\text { T101 } \\
\text { T101 }\end{array}$ & $\begin{array}{l}\text { Si } \\
\text { SO4 }\end{array}$ \\
\hline T101 & $\mathbf{S r}$ \\
\hline T101 & TOC \\
\hline T101 & $\mathbf{U}$ \\
\hline T101 & $\mathbf{Z r}$ \\
\hline T101 & Ac227 \\
\hline T101 & Am241 \\
\hline T101 & Am243 \\
\hline T101 & Ba137 \\
\hline T101 & C14 \\
\hline T101 & Cd113 \\
\hline T101 & $\mathrm{Cm} 242$ \\
\hline T101 & Cm243 \\
\hline T101 & Cm244 \\
\hline T101 & Co60 \\
\hline T101 & Cs134 \\
\hline T101 & Cs137 \\
\hline T101 & Eul52 \\
\hline T101 & Eu154 \\
\hline T101 & Eu155 \\
\hline T101 & 1129 \\
\hline T101 & Nb93 \\
\hline T101 & Nis9 \\
\hline T101 & Ni63 \\
\hline T101 & Np237 \\
\hline T101 & Pa231 \\
\hline T101 & Pu238 \\
\hline T101 & Pu239 \\
\hline T101 & Pu240 \\
\hline T101 & Pu241 \\
\hline T101 & Pu242 \\
\hline T101 & Ra226 \\
\hline T101 & Ra228 \\
\hline T101 & Ru106 \\
\hline T101 & Sb125 \\
\hline $\begin{array}{l}\text { T101 } \\
\text { T101 }\end{array}$ & $\begin{array}{l}\text { Se79 } \\
\text { Sm151 }\end{array}$ \\
\hline
\end{tabular}

\section{$B B$} May $\frac{B 8}{58 \mathrm{E}+01}$ 3.16E-00

2.41E-00

9.22E-00

2.39E-00

9.65E-02

7.52E-02

2.86E-00

$7.90 \mathrm{E}+04$

$5.15 E+01$

$9.10 \mathrm{E}+04$

$1.44 \mathrm{E}+01$

$1.76 \mathrm{E}+03$

$2.16 E+02$

$1.37 \mathrm{E}+04$

$1.38 \mathrm{E}+02$

$6.70 \mathrm{E}+01$

$3.45 \mathrm{E}+03$

$2.93 \mathrm{E}+02$

$1.48 \mathrm{E}+02$

5.30E-00

$1.02 \mathrm{E}+01$

$7.01 \mathrm{E}+04$

$1.62 \mathrm{E}+01$

$1.56 \mathrm{E}+04$

$1.22 \mathrm{E}+05$

$4.10 \mathrm{E}+03$

$8.94 \mathrm{E}+03$

$1.27 \mathrm{E}+04$

$7.25 E+02$

$1.10 \mathrm{E}+03$

2.18E+03

$0.00 \mathrm{E}+01$

$2.35 E+02$

$1.86 \mathrm{E}+04$

$2.00 \mathrm{E}-01$

2.25E-05

1.53E-00

$5.90 \mathrm{E}-05$

$8.80 \mathrm{E}+04$

$1.18 \mathrm{E}-00$

2.49E-00

$5.98 \mathrm{E}-03$

4.50E-04

4.66E-03

$1.34 \mathrm{E}-00$

2.01 E-02

$9.30 \mathrm{E}+04$

2.75E-01

$1.98 \mathrm{E}+01$

$1.64 \mathrm{E}+01$

$1.59 \mathrm{E}-02$

3.24E-01

6.33E-02

6.26E- -00

2.94E-62

9.55E-05

$2.49 \mathrm{E}+01$

$6.92 E+02$

$1.14 \mathrm{E}+02$

$1.02 \mathrm{E}+03$

Ci 2.70E-03

4.48E-06

Ci $\quad 6.48 \mathrm{E}-05$

2.26E-04

Ci $\quad 5.85 \mathrm{E}-00$

$\mathrm{Ci}$ 9.18E-02

$3.24 \mathrm{E}+02$
$\frac{B B}{\text { Sept98 }}$

1\%-ile

$0.00 \mathrm{E}+01$

$10 \%$-ile

50\%-jle

90\%-ile

99\%-ile

6.19E-01

2.37E-00

6.15E-01

2.48E-02

$1.93 \mathrm{E}-02$

5.57E-01

$7.90 \mathrm{E}+04$

$5.15 E+01$

$9.10 \mathrm{E}+04$

$1.44 \mathrm{E}+01$

$1.76 \mathrm{E}+03$

$2.16 \mathrm{E}+02$

$1.37 \mathrm{E}+04$

$1.38 \mathrm{E}+02$

$6.70 \mathrm{E}+01$

$3.45 \mathrm{E}+03$

$2.54 \mathrm{E}+01$

$1.48 \mathrm{E}+02$

$5.28 \mathrm{E}-00$

$1.02 \mathrm{E}+01$

$7.01 \mathrm{E}+04$

$1.62 \mathrm{E}+01$

$1.56 \mathrm{E}+04$

$1.22 \mathrm{E}+05$

NA

2.33E +02

4.14E +03

NA

$1.10 \mathrm{E}+03$

$7.25 \mathrm{E}+02$

$0.00 \mathrm{E}+01$

$2.35 \mathrm{E}+02$

$1.86 \mathrm{E}+04$

$0.00 \mathrm{E}+01$

$2.25 \mathrm{E}-05$

$1.53 \mathrm{E}-00$

$5.90 \mathrm{E}-05$

$8.80 \mathrm{E}+04$

$1.18 \mathrm{E}-00$

2.49E-00

5.98E-03

$4.50 \mathrm{E}-04$

4.66E-03

$1.34 \mathrm{E}-00$

2.01 E-02

$9.30 \mathrm{E}+04$

2.75E-01

$1.98 \mathrm{E}+01$

$1.64 \mathrm{E}+01$

$1.59 \mathrm{E}-02$

3.24E-01

6.33E-02

6.26E-00

2.94E-02

9.55E-05

$2.49 \mathrm{E}+01$

$6.92 \mathrm{E}+02$

$1.14 \mathrm{E}+02$

$1.02 \mathrm{E}+03$

2.70E-03

4.48E-06

6.48E-05

2.26E-04

5.85E-00

9.18E-02

$3.24 \mathrm{E}+02$
$0.00 \mathrm{E}+01$

$0.00 \mathrm{E}+01$

$0.00 \mathrm{E}+01$

$0.00 \mathrm{E}+01$

$0.00 E+01$

$0.00 E+01$

$2.93 \mathrm{E}+04$

$0.00 \mathrm{E}+01$

$1.28 \mathrm{E}+04$

2.15E-00

6.17E+01

$4.95 \mathrm{E}+01$

$1.28 \mathrm{E}+03$

$4.41 \mathrm{E}+01$

$5.39 \mathrm{E}+03$

$1.88 \mathrm{E}+02$

$0.00 \mathrm{E}+01$

$1.94 \mathrm{E}+01$

1.19E-00

$4.96 \mathrm{E}+01$

$3.98 \mathrm{E}+04$

7.55E-00

$5.93 \mathrm{E}+02$

$9.79 \mathrm{E}+02$

$1.58 \mathrm{E}+02$

3.52E-00

$2.59 \mathrm{E}+02$

$5.60 \mathrm{E}+01$

$4.87 \mathrm{E}+01$

$1.52 \mathrm{E}+02$

$6.79 \mathrm{E}-00$

$1.26 \mathrm{E}+02$

$7.15 \mathrm{E}+02$

$3.80 \mathrm{E}-01$

$0.00 \mathrm{E}+01$

7.71E-00

8.04E-07

$0.00 \mathrm{E}+01$

$0.00 \mathrm{E}+01$

$0.00 \mathrm{E}+01$

8.15E-05

6.13E-06

$6.35 \mathrm{E}-05$

9.76E-01

$0.00 \mathrm{E}+01$

$5.32 \mathrm{E}+03$

$0.00 \mathrm{E}+01$

$0.00 E+01$

$0.00 \mathrm{E}+01$

$0.00 \mathrm{E}+01$

$0.00 \mathrm{E}+01$

4.75E-04

4.70E-02

4.01E-04

$0.00 \mathrm{E}+01$

$3.39 \mathrm{E}-01$

$9.43 \mathrm{E}-00$

$1.55 \mathrm{E}-00$

$1.39 \mathrm{E}+01$

3.68E-05

7.31E-08

$1.06 \mathrm{E}-06$

$0.00 \mathrm{E}+01$

$0.00 \mathrm{E}+01$

$0.00 \mathrm{E}+01$

$0.00 \mathrm{E}+0 \mathrm{I}$
2.19E-01

$0.00 \mathrm{E}+01$

$0.00 \mathrm{E}+01$

$0.00 \mathrm{E}+01$

$0.00 \mathrm{E}+01$

$0.00 \mathrm{E}+01$

$0.00 \mathrm{E}+01$

$4.76 \mathrm{E}+04$

$8.37 \mathrm{E}-00$

2.03E+04

$5.45 \mathrm{E}+01$

$1.36 \mathrm{E}+02$

$4.60 \mathrm{E}+02$

$3.84 \mathrm{E}+03$

$2.92 \mathrm{E}+02$

$9.62 \mathrm{E}+03$

$7.93 \mathrm{E}+02$

$0.00 \mathrm{E}+01$

$8.76 \mathrm{E}+01$

2.71E-00

$1.88 \mathrm{E}+02$

$6.61 \mathrm{E}+04$

$2.42 \mathrm{E}+01$

$3.53 \mathrm{E}+03$

$2.06 \mathrm{E}+04$

$5.64 \mathrm{E}+02$

$1.35 \mathrm{E}+01$

$1.10 \mathrm{E} \div 03$

$1.52 \mathrm{E}+02$

$2.14 \mathrm{E}+02$

$3.97 \mathrm{E}+02$

$4.00 \mathrm{E}+01$

$6.44 \mathrm{E}+02$

$1.73 \mathrm{E}+03$

$4.96 \mathrm{E}-00$

$0.00 \mathrm{E}+01$

$1.14 \mathrm{E}+01$

2.07E-05

1.53E+04

$0.00 \mathrm{E}+01$

$0.00 \mathrm{E}+01$

$2.09 \mathrm{E}-03$

$1.58 \mathrm{E}-04$

$1.63 E-03$

1.62E-00

$3.50 \mathrm{E}-03$

$1.69 \mathrm{E}+04$

$0.00 \mathrm{E}+01$

$0.00 \mathrm{E}+01$

$0.00 \mathrm{E}+01$

3.77E-03

$0.00 \mathrm{E}+01$

$1.16 \mathrm{E}-02$

$1.15 \mathrm{E}-00$

$1.03 \mathrm{E}-02$

1.66E-06

8.72E-00

$2.42 \mathrm{E}+02$

$3.99 \mathrm{E}+01$

$3.57 \mathrm{E}+02$

9.45E-04

1.43E-06

2.07E-05

$0.00 E+0$

$5.82 \mathrm{E}-01$

$0.00 \mathrm{E}+01$

$0.00 \mathrm{E}+01$
$0.00 \mathrm{E}+01$

$1.58 \mathrm{E}+01$

$3.16 \mathrm{E}-00$

2.41E-00

9.22E-00

2.39E-00

9.65E-02

7.52E-02

2.86E-00 
Tank Anal. Un

\section{T101 Sr89/90 Ci}

T101 Tc99 Ci 1.18E+05

T101 Th229 Ci 3.05E-06

T101 Th232 Ci 2.15E-05

T101 Tin126 Ci 1.38E-01.

$\begin{array}{llll}\text { T101 } & \text { Tritium } & \mathrm{Ci} & 6.18 \mathrm{E}-00 \\ \text { T101 } & \text { U232 } & \mathrm{Ci} & 2.98 \mathrm{E}-03\end{array}$

$\begin{array}{llll}T 101 & U 233 & C i & 9.60 E-03\end{array}$

$\begin{array}{llll}\text { T101 U234 Ci } & 8.47 E-00\end{array}$

T101 U235 Ci 3.28E-01

T101 U236 Ci 4.93E-01

$\begin{array}{llll}T 101 & \mathrm{U} 238 & \mathrm{Ci} & 6.21 \mathrm{E}-00\end{array}$

$\begin{array}{llll}\text { T101 } & \text { Y90 } & \mathrm{Ci} & 1.18 \mathrm{E}+05\end{array}$

$\begin{array}{llll}\text { T101 } & \text { Zr93 } & \mathrm{Ci} & 4.52 \mathrm{E}-01 \\ \mathrm{T102} & \mathrm{Al} & \mathrm{kg} & 3.85 \mathrm{E}+04\end{array}$

$\mathrm{T102} \mathrm{Bi} \quad \mathrm{kg} \quad 7.16 \mathrm{E}+02$

$\mathrm{T} 102 \mathrm{Ca} \quad \mathrm{kg} \quad 9.51 \mathrm{E}+01$

$\begin{array}{llll}\mathrm{T} 102 & \mathrm{Cl} & \mathrm{kg} & 7.03 \mathrm{E}+01 \\ \mathrm{~T} 102 & \mathrm{CO} 3 & \mathrm{~kg} & 3.69 \mathrm{E}+03\end{array}$

$\begin{array}{llll}\mathrm{T} 102 & \mathrm{Cr} & \mathrm{kg} & 1.88 \mathrm{E}+02\end{array}$

$\begin{array}{lllr}\text { T102 } & \text { F } & \text { kg } & 4.20 E+01 \\ \text { T102 } & \text { Fe } & \text { kg } & 2.33 E+03 \\ \text { T102 } & \text { Hg } & \text { kg } & 8.00 E-01\end{array}$

$\begin{array}{llll}\text { T102 } & \text { Hg } & \text { kg } & 8.00 E-01 \\ \text { T102 } & \mathrm{K} & \mathrm{kg} & 5.30 \mathrm{E}+02\end{array}$

$\begin{array}{llll}\mathrm{T} 102 & \mathrm{La} & \mathrm{kg} & 7.16 \mathrm{E}+01\end{array}$

$\begin{array}{llll}\mathrm{T} 102 & \mathrm{Mn} & \mathrm{kg} & 1.23 \mathrm{E}+02 \\ \mathrm{~T} 102 & \mathrm{Na} & \mathrm{kg} & \mathbf{7 . 2 2 E}+03\end{array}$

T102 Ni

T102

T102 NO3

T102 $P$

$\begin{array}{llll}\mathrm{T} 102 & \mathrm{PO4} & \mathrm{kg} & 2.47 \mathrm{E}+03 \\ \mathrm{T102} & \mathrm{S} & \mathrm{kg} & 4.43 \mathrm{E}+02\end{array}$

$\begin{array}{llll}\mathrm{T} 102 & \mathrm{Si} & \mathrm{kg} & 4.17 \mathrm{E}+02\end{array}$

$\begin{array}{llll}\mathrm{T102} & \mathrm{SO} 4 & \mathrm{~kg} & 1.33 \mathrm{E}+03\end{array}$

$\mathrm{T102} \mathrm{Sr} \quad \mathrm{kg} \quad 7.20 \mathrm{E}-00$

T102 TOC $\mathrm{kg} 1.06 \mathrm{E}+02$

$\begin{array}{llll}\mathrm{T} 102 \mathrm{U} & \mathrm{kg} & 2.86 \mathrm{E}+03\end{array}$

$\begin{array}{llll}\mathrm{T} 102 & \mathrm{Zr} & \mathrm{kg} & 1.43 \mathrm{E}+01\end{array}$

T102 Ac227 Ci 1.06E-02

T102 Am241 Ci 3.29E+01

T102 Am243 Ci 3.32E-07

$\begin{array}{llll}\text { T102 } & \text { Ba137 } & \text { Ci } & 6.90 \mathrm{E}+03\end{array}$

$\begin{array}{llll}\mathrm{T} 102 & \mathrm{C} 14 & \mathrm{Ci} & 5.90 \mathrm{E}-00\end{array}$

$\begin{array}{llll}\text { T102 Cd113 Ci } & \text { 3.06E-02 }\end{array}$

$\begin{array}{llll}\text { T102 Cm242 } & \text { Ci } & 6.11 E-04\end{array}$

$\mathrm{T102} \mathrm{Cm} 243 \mathrm{Ci}$ 1.60E-01

$\begin{array}{llll}\mathrm{T} 102 & \mathrm{Cm} 244 & \mathrm{Ci} & 1.26 \mathrm{E}-05\end{array}$

T102

T102

T102

T102

T102

T102

T102

T102

$\mathrm{T102}$

T102

T10

T10

T102

T102

T102 Pu240

T102 Pu241

T102 Pu242

T102 Ra226

T102 Ra228
$B B$ Sept $\overline{98}$

$\frac{1.18 \mathrm{E}+05}{8.24 \mathrm{E}-00}$

3.05E-06

2.15E-05

1.38E-01

6.18E-00

2.98E-03

$9.60 \mathrm{E}-03$

8.47E-00

3.28E-01

4.93E- 01

6.21E-00

$1.18 \mathrm{E}+05$

4.52E-01

$3.85 \mathrm{E}+04$

$1.26 \mathrm{E}+02$

$9.51 \mathrm{E}+01$

$7.03 \mathrm{E}+01$

$3.69 \mathrm{E}+03$

$1.88 \mathrm{E}+02$

$4.20 \mathrm{E}+01$

$2.33 \mathrm{E}+03$

8.00E-01

4.91E-00

$0.00 \mathrm{E}+01$

$1.23 \mathrm{E}+02$

$7.22 \mathrm{E}+03$

9.00E-00

$2.16 \mathrm{E}+03$

$9.87 \mathrm{E}+03$

$2.47 \mathrm{E}+02$

$8.05 E+02$

4.17E

$4.43 E+02$

2.25E-00

$1.06 \mathrm{E}+02$

$5.80 \mathrm{E}+03$

5.53E-00

1.06E-02

$3.29 \mathrm{E}+01$

$3.40 \mathrm{E}-04$

$6.90 \mathrm{E}+03$

5.90E- 00

3.06E-02

6.27E-01

$6.40 E-03$

1.54E-01

7.63E- 03

2.63E-03

$7.30 E+03$

4.26E-02

$6.30 \mathrm{E}+01$

$7.00 \mathrm{E}+01$

1.04E-04

$6.03 E-03$

2.14E-03

2.06E-01

7.00E-02

1.57E-02

1.51E-01

6.28E-00

1.12E-00

$1.21 \mathrm{E}+01$

3.40E-05

1.11E-06

3.46E-03 1\%-ile

\section{$1.29 \mathrm{E}+02$}

$0.00 \mathrm{E}+01$

$0.00 \mathrm{E}+01$

$0.00 \mathrm{E}+01$

$0.00 \mathrm{E}+01$

3.24E-00

$0.00 \mathrm{E}+01$

$0.00 \mathrm{E}+01$

$0.00 \mathrm{E}+01$

$0.00 \mathrm{E}+01$

$0.00 \mathrm{E}+01$

$0.00 \mathrm{E}+01$

$0.00 \mathrm{E}+01$

$0.00 \mathrm{E}+01$

$5.89 \mathrm{E}-04$

$1.89 \mathrm{E}-05$

7.06E-06

$1.76 \mathrm{E}-05$

$1.73 \mathrm{E}-04$

2.34E-05

3.57E-05

4.69E-05

$0.00 \mathrm{E}+01$

2.24E-05

1.53E-07

1.13E-05

7.12E-04

8.35E-07

2.56E-04

8.28E-04

$1.38 \mathrm{E}-04$

2.11E-06

2.76E-04

1.04E-05

8.91E-06

3.15E-05

2.75E-06

4.30E-05

9.53E-05

$2.02 \mathrm{E}-07$

$0.00 \mathrm{E}+01$

3.64E-06

$0.00 \mathrm{E}+01$

$0.00 \mathrm{E}+01$

$0.00 \mathrm{E}+01$

$0.00 \mathrm{E}+01$

$0.00 \mathrm{E}+01$

$0.00 \mathrm{E}+01$

$0.00 \mathrm{E}+01$

$1.08 \mathrm{E}-07$

$0.00 \mathrm{E}+01$

$1.40 \mathrm{E}-03$

$0.00 E+01$

$0.00 \mathrm{E}+01$

$0.00 \mathrm{E}+01$

$0.00 \mathrm{E}+01$

$0.00 \mathrm{E}+01$

$0.00 E+01$

$0.00 \mathrm{E}+01$

$0.00 E+01$

$0.00 \mathrm{E}+01$

$0.00 E+01$

$0.00 \mathrm{E}+01$

$0.00 \mathrm{E}+01$

$0.00 E+01$

$0.00 E+01$

$0.00 \mathrm{E}+01$

$0.00 \mathrm{E}+01$
$10 \%$-ile

$3.79 E+03$

7.91E-01

5.32E-08

3.75E-07

$0.00 \mathrm{E}+01$

$4.29 \mathrm{E}-00$

5.19E-05

1.67E-04

$1.48 \mathrm{E}-01$

$5.72 \mathrm{E}-03$

8.59E-03

$1.08 \mathrm{E}-01$

$1.13 \mathrm{E}+03$

$0.00 \mathrm{E}+01$

$1.78 \mathrm{E}+03$

4.31E-00

$1.56 \mathrm{E}+01$

$3.01 \mathrm{E}+01$

4.49E+02

$2.48 \mathrm{E}+01$

8.13E-00

$6.50 \mathrm{E}+01$

$6.36 \mathrm{E}-02$

$1.45 \mathrm{E}+01$

2.52E-01

$1.22 \mathrm{E}+01$

$2.69 \mathrm{E}+03$

$1.65 \mathrm{E}-00$

$4.45 \mathrm{E}+02$

$1.43 \mathrm{E}+03$

$1.07 \mathrm{E}+02$

$9.78 \mathrm{E}-01$

$2.08 \mathrm{E}+02$

$2.35 \mathrm{E}+01$

$1.68 \mathrm{E}+01$

$6.26 \mathrm{E}+01$

2.26E-00

$3.77 \mathrm{E}+01$

$2.54 \mathrm{E}+02$

$4.49 \mathrm{E}-01$

$0.00 \mathrm{E}+01$

1.61E-00

$1.96 \mathrm{E}-08$

$7.35 E+02$

7.44E-01

2.43E-03

3.60E-05

9.43E-03

7.43E-07

$2.56 \mathrm{E}-01$

$1.60 \mathrm{E}-01$

$1.94 \mathrm{E}+03$

$0.00 \mathrm{E}+01$

$0.00 \mathrm{E}+01$

$0.00 \mathrm{E}+01$

7.97E-06

4.14E-04

$2.52 \mathrm{E}-04$

2.42E-02

4.13E-03

$7.45 \mathrm{E}-04$

$2.36 \mathrm{E}-01$

$4.36 \mathrm{E}-01$

$1.75 \mathrm{E}-00$

$1.89 \mathrm{E}+01$

$5.31 \mathrm{E}-05$

9.11E-08

$2.84 \mathrm{E}-04$

50\%-ile

$2.70 \mathrm{E}+05$

8.24E-00

3.05E-06

2.15E-05

1.38E-01

6.18E-00

2.98E-03

$9.60 \mathrm{E}-03$

8.47E-00

$3.28 \mathrm{E}-01$

4.93E-01

6.21E-00

1.18E+05

4.52E-01

$1.04 \mathrm{E}+04$

$6.87 \mathrm{E}+01$

$1.13 E+02$

$2.80 \mathrm{E}+02$

$2.97 \mathrm{E}+03$

$2.06 \mathrm{E}+02$ 


\begin{tabular}{|c|c|c|c|c|c|c|c|c|c|c|c|}
\hline ank & Anal. & Un & May98 & Sept98 & $1 \%$-ile & $10 \%$-ile & $50 \%$-ile & $90 \%$-jle & 99\%-ile & Mean & Std Dev \\
\hline 102 & 106 & $\mathrm{Ci}$ & $\overline{1.20 \mathrm{E}-05}$ & $1.20 \mathrm{E}-05$ & $0.00 \mathrm{E}+01$ & $1.27 \mathrm{E}-06$ & $1.20 \mathrm{E}-05$ & $E-05$ & $9 \mathrm{E}-04$ & $2.100-03$ & $3.42 \mathrm{E}-05$ \\
\hline T102 & 125 & $\mathrm{Ci}$ & $3.98 E-02$ & $3.98 \mathrm{E}-02$ & $0.00 \mathrm{E}+01$ & $\mathrm{E}-03$ & 8E-02 & & $5 E-00$ & $1 \mathrm{E}-01$ & $96 \mathrm{E}-01$ \\
\hline r102 & e79 & $\mathrm{Ci}$ & $1.65 \mathrm{E}-03$ & $1.65 \mathrm{E}-03$ & $0.00 \mathrm{E}+01$ & $2.14 \mathrm{E}-04$ & $1.65 \mathrm{E}-03$ & $6.94 E-03$ & $65 \mathrm{E}-02$ & $3.49 \mathrm{E}-03$ & $8.18 E-03$ \\
\hline 102 & $\mathrm{~m} 151$ & $\mathrm{Ci}$ & $6.04 \mathrm{E}-00$ & $6.04 \mathrm{E}-00$ & $0.00 \mathrm{E}+01$ & $0.00 \mathrm{E}+01$ & $6.04 \mathrm{E}-00$ & $2.35 E+01$ & $7.18 \mathrm{E}+01$ & $1.01 \mathrm{E}+01$ & $1.48 \mathrm{E}+01$ \\
\hline T102 & Sr89/90 & $\mathrm{Ci}$ & $.07 E+04$ & $3.07 \mathrm{E}+04$ & $0.00 \mathrm{E}+01$ & $7.96 \mathrm{E}+01$ & $.29 E+04$ & $4.56 \mathrm{E}+05$ & $4.78 E+06$ & $3.83 E+05$ & $2.76 \mathrm{E}+06$ \\
\hline T102 & Te99 & $\mathrm{Ci}$ & 30E-00 & 2.30E-00 & $0.00 \mathrm{E}+01$ & $E-02$ & $2.30 \mathrm{E}-00$ & $9.70 \mathrm{E}-00$ & $36 E+01$ & $E-00$ & \\
\hline T102 & Th229 & $\mathrm{Ci}$ & $1.57 \mathrm{E}-0$ & $1.57 \mathrm{E}-03$ & $0.00 \mathrm{E}+01$ & $E-05$ & $1.57 \mathrm{E}-03$ & & & & \\
\hline 102 & Th232 & $\mathrm{Ci}$ & $1.61 E-04$ & $1.61 \mathrm{E}-04$ & $0.00 \mathrm{E}+01$ & $7.64 \mathrm{E}-06$ & $1.61 \mathrm{E}-04$ & $6.24 \mathrm{E}-04$ & $1.74 \mathrm{E}-03$ & 2.77E-04 & $9 \mathrm{E}-04$ \\
\hline 2 & Tin126 & $\mathrm{Ci}$ & $2.50 \mathrm{E}-03$ & $2.50 \mathrm{E}-03$ & & & $2.50 \mathrm{E}-03$ & & $5.22 \mathrm{E}-02$ & & -02 \\
\hline T102 & Tri & $\mathrm{Ci}$ & $9.00 \mathrm{E}-01$ & $9.00 \mathrm{E}-01$ & $0.00 \mathrm{E}+01$ & $1.46 \mathrm{E}-01$ & $9.00 \mathrm{E}-01$ & & $4.69 \mathrm{E}-00$ & $E-00$ & $E-00$ \\
\hline T102 & U232 & $\mathrm{Ci}$ & $1.79 \mathrm{E}-01$ & 1.79E-01 & $0.00 \mathrm{E}+01$ & $8.50 \mathrm{E}-03$ & 1.79E-01 & -01 & $1.93 E-00$ & $E-01$ & $4.33 \mathrm{E}-01$ \\
\hline T102 & U233 & $\mathrm{Ci}$ & $6.94 \mathrm{E}-01$ & $6.94 \mathrm{E}-01$ & $0.00 \mathrm{E}+01$ & $3.29 \mathrm{E}-02$ & $6.94 \mathrm{E}-01$ & $2.69 \mathrm{E}-00$ & $7.48 \mathrm{E}-00$ & 1.19E-00 & $1.68 \mathrm{E}-00$ \\
\hline $\mathrm{T} 102$ & U234 & $\mathrm{Ci}$ & $1.97 \mathrm{E}-00$ & $1.97 \mathrm{E}-00$ & $0.00 \mathrm{E}+01$ & $9.35 \mathrm{E}-02$ & $1.97 \mathrm{E}-00$ & $E-00$ & $2.12 E+01$ & E- -00 & $6 \mathrm{E}-00$ \\
\hline T102 & U235 & $\mathrm{Ci}$ & $8.51 E-02$ & $8.51 E-02$ & $0.00 \mathrm{E}+01$ & 4.04E-03 & 8.51E-02 & $\varepsilon-01$ & $9.17 \mathrm{E}-01$ & $E-01$ & $E-01$ \\
\hline T102 & U236 & $\mathrm{Ci}$ & 3.47E-02 & $3.47 E-02$ & $0.00 \mathrm{E}+01$ & $1.65 \mathrm{E}-03$ & $3.47 \mathrm{E}-02$ & & $E-01$ & $E-02$ & -02 \\
\hline T102 & U238 & $\mathrm{Ci}$ & $1.93 E-00$ & $1.93 E-00$ & +01 & $\varepsilon-02$ & 1.93 & & +01 & $=00$ & \\
\hline T102 & Y90 & $\mathbf{C i}$ & $3.07 E+04$ & $3.07 E+04$ & +01 & +02 & $3.07 E+04$ & +05 & $6.42 \mathrm{E}+06$ & +05 & +06 \\
\hline T102 & Zr93 & $\mathbf{C i}$ & $7.79 \mathrm{E}-03$ & $7.79 \mathrm{E}-03$ & +01 & $E-04$ & $.79 E-03$ & -02 & $8 E-01$ & $E-02$ & $E-01$ \\
\hline T103 & Al & kg & $3.30 \mathrm{E}+04$ & $3.30 E+04$ & E-04 & $9.78 \mathrm{E}+02$ & $.90 \mathrm{E}+03$ & $\div 04$ & $4.69 \mathrm{E}+04$ & $E+04$ & +04 \\
\hline T103 & $\mathbf{B i}$ & kg & $2.70 \mathrm{E}+01$ & $2.70 \mathrm{E}+01$ & & -00 & -01 & 02 & $3 E+03$ & +02 & +03 \\
\hline 103 & $\mathrm{Ca}$ & kg & $8.46 E+02$ & $8.46 E+02$ & $\varepsilon-06$ & $E-00$ & $8.85 E+01$ & +02 & $E+03$ & +02 & +02 \\
\hline T103 & CI & $\mathrm{kg}$ & $5.78 \mathrm{E}+01$ & $5.78 E+01$ & -06 & +01 & $1.96 E+02$ & +02 & & +02 & \\
\hline T103 & $\mathrm{CO3}$ & kg & $1.83 E+03$ & 03 & & +02 & & & & +03 & +03 \\
\hline T103 & $\mathrm{Cr}$ & $\mathrm{kg}$ & $4.09 E+01$ & $4.09 E+01$ & 06 & +01 & +02 & +02 & $\div 03$ & +02 & +02 \\
\hline T103 & $\mathbf{F}$ & $\mathrm{kg}$ & $9.00 \mathrm{E}-01$ & $8.89 \mathrm{E}-01$ & -05 & +01 & +03 & +03 & +03 & +03 & +03 \\
\hline T103 & $\mathrm{Fe}$ & $\mathrm{kg}$ & $1.67 \mathrm{E}+03$ & $1.67 \mathrm{E}+03$ & $E-05$ & +01 & $7.37 \mathrm{E}+02$ & 103 & $9.84 E+03$ & $E+03$ & +03 \\
\hline T103 & Hg & kg & $7.63 \mathrm{E}+01$ & $1.24 E \div 01$ & +01 & -00 & $7.63 E+01$ & & $\div 03$ & $E+02$ & +02 \\
\hline T103 & $\mathbf{K}$ & kg & $1.59 \mathrm{E}+01$ & $1.59 \mathrm{E}+01$ & & -00 & +02 & & & & +02 \\
\hline T103 & $\mathbf{L a}$ & kg & $0.00 \mathrm{E}+01$ & $0.00 \mathrm{E}+01$ & & & & & & & \\
\hline T103 & Mn & kg & $8.00 \mathrm{E}+01$ & +01 & & -00 & 02 & & -03 & +02 & +02 \\
\hline T103 & $\mathrm{Na}$ & $\mathrm{kg}$ & $3.75 E+03$ & +03 & -04 & +03 & 1.66 & 04 & 04 & +04 & +04 \\
\hline T103 & $\mathbf{N i}$ & kg & $7.30 \mathrm{E}-00$ & $7.30 \mathrm{E}-00$ & -07 & -01 & +01 & 01 & $\div+02$ & +01 & +02 \\
\hline T103 & NO2 & kg & $1.25 E+03$ & +03 & & +02 & & & & +03 & \\
\hline T103 & NO3 & kg & $3.21 E+03$ & $3.21 E+03$ & & & 104 & & & & \\
\hline T103 & $\mathbf{P}$ & kg & $1.55 E+02$ & NA & & +01 & +02 & & +03 & +03 & +03 \\
\hline T103 & $\mathbf{P b}$ & kg & $4.50 \mathrm{E}+01$ & $4.50 \mathrm{E}+01$ & -07 & $E-01$ & $7.17 \mathrm{E}-00$ & 01 & +02 & +01 & +01 \\
\hline T103 & PO4 & $\mathrm{kg}$ & $4.81 E+02$ & $1.57 E+02$ & -05 & +01 & $1.46 \mathrm{E}+03$ & +04 & $E+04$ & $E+03$ & +03 \\
\hline T103 & $\mathbf{S}$ & kg & $2.16 E+02$ & NA & -06 & +01 & $3.46 \mathrm{E}+02$ & 03 & $E+04$ & $E+03$ & +03 \\
\hline T103 & $\mathbf{S i}$ & kg & $3.89 \mathrm{E}+02$ & $3.89 E+02$ & & +01 & -02 & & & & +02 \\
\hline T103 & SO4 & $\mathrm{kg}$ & 6 & 2.1 & & 01 & 02 & & & & \\
\hline T103 & Sr & kg & & $i+01$ & & $-\infty 0$ & 2.3 & & & -01 & +02 \\
\hline T103 & TOC & $\mathrm{kg}$ & $5.70 E+01$ & $i+01$ & -06 & +01 & 102 & 03 & 03 & +02 & +02 \\
\hline T103 & $\mathbf{U}$ & kg & $4.23 E+03$ & $4.23 E+03$ & 4.34E-05 & +02 & $1.77 \mathrm{E}+03$ & .03 & +04 & $E+03$ & +03 \\
\hline T103 & $\mathbf{Z r}$ & $\mathrm{kg}$ & $1.30 \mathrm{E}-02$ & $0.00 \mathrm{E}+01$ & -07 & $E-01$ & -00 & & +02 & $E+01$ & +02 \\
\hline T103 & Ac227 & $\mathrm{Ci}$ & -02 & 02 & & & & & & & \\
\hline $\mathbf{T} 1$ & A & $\mathrm{Ci}$ & & & & & & & & & 103 \\
\hline 3 & Am243 & $\mathrm{Ci}$ & -05 & -05 & & $E-07$ & & & & & \\
\hline T103 & Ba137 & $\mathrm{Ci}$ & $2.69 E+04$ & $2.69 E+04$ & +01 & +03 & 2.69 & 7. & 1.6 & +04 & +04 \\
\hline T103 & C14 & $\mathrm{Ci}$ & $3.04 \mathrm{E}-01$ & $3.04 E-01$ & ;+01 & $\mathrm{E}-02$ & -01 & & -00 & $E-01$ & \\
\hline T103 & Cd113 & $\mathrm{Ci}$ & $6.51 \mathrm{E}-0$ & $\varepsilon-01$ & 01 & $=0$ & 01 & & 01 & 00 & -00 \\
\hline T103 & Cm242 & $\mathrm{Ci}$ & 1.67E-03 & 1.6 & & & -03 & & 02 & -03 & -03 \\
\hline T1 & $\mathrm{Cm} 243$ & $\mathrm{Ci}$ & & & & & & & & & \\
\hline & C & $\mathrm{Ci}$ & & & & 05 & & & & & -03 \\
\hline T103 & Co60 & $\mathrm{Ci}$ & -01 & 01 & & -01 & & & -01 & $-\infty 0$ & -00 \\
\hline T103 & Cs134 & $\mathrm{Ci}$ & $8.26 \mathrm{E}-03$ & $8.26 \mathrm{E}-03$ & +01 & $5.09 \mathrm{E}-04$ & 8.26 & -02 & $\mathrm{E}-02$ & $\mathrm{E}-02$ & -02 \\
\hline T103 & Cs137 & $\mathrm{Ci}$ & $2.84 \mathrm{E} \div 04$ & $2.84 E+04$ & 7E-04 & $1.09 E+03$ & $1.07 E+04$ & 3. & $E+04$ & $E+04$ & $E+04$ \\
\hline T103 & Eu152 & $\mathrm{Ci}$ & $6.81 E-02$ & 6.8 & 01 & +01 & 6.8 & 01 & -00 & $E-01$ & $E-01$ \\
\hline T103 & Eu15 & $\mathrm{Ci}$ & 00 & 5. & & & & & & & +01 \\
\hline T103 & & $\mathrm{Ci}$ & & & & & & & & $:+01$ & +01 \\
\hline T103 & I129 & $\mathrm{Ci}$ & & & & $\sum-04$ & & & & & -03 \\
\hline T103 & $\mathrm{Nb93}$ & $\mathrm{Ci}$ & $8.36 E-02$ & 8.3 & & $E-03$ & & & & $E-01$ & $E-00$ \\
\hline T103 & Nis & $\mathrm{Ci}$ & $1.64 \mathrm{E}-02$ & $1.64 E-02$ & & $1.23 \mathrm{E}-03$ & $1.64 \mathrm{E}-02$ & & -00 & $E-02$ & E-01 \\
\hline T103 & Ni63 & $\mathrm{Ci}$ & $1.62 E-00$ & $1.62 \mathrm{E}-00$ & $0.00 \mathrm{E}+01$ & $1.21 \mathrm{E}-01$ & $1.62 \mathrm{E}-00$ & $E-00$ & $1.03 E+02$ & $0 E-00$ & $1.65 \mathrm{E}+01$ \\
\hline T103 & Np23 & $\mathrm{Ci}$ & $7.38 \mathrm{E}-03$ & $7.38 \mathrm{E}-03$ & $0.00 \mathrm{E}+01$ & $231 E-04$ & $7.38 \mathrm{E}-03$ & $F=0 ?$ & $5.58 \mathrm{E}-02$ & $1.10 \mathrm{E}-02$ & E-02 \\
\hline T103 & Pa231 & $\mathrm{Ci}$ & $1.57 \mathrm{E}-02$ & $1.57 \mathrm{E}-02$ & $0.00 \mathrm{E}+01$ & $0.00 \mathrm{E}+01$ & $1.57 \mathrm{E}-02$ & E-02 & $1.57 \mathrm{E}-01$ & $2.77 \mathrm{E}-02$ & $3.46 \mathrm{E}-02$ \\
\hline $\mathbf{r 1 0 3}$ & Pu238 & $\mathrm{Ci}$ & 4.01E-00 & $4.01 E-00$ & & $E-01$ & & $1.42 \mathrm{E}+01$ & $3.03 E+01$ & $E-00$ & $7 \mathrm{E}-00$ \\
\hline r103 & Pu239 & $\mathrm{Ci}$ & $1.67 E+02$ & $1.67 E+02$ & $0.00 \mathrm{E}+01$ & $5.22 \mathrm{E}-00$ & $1.67 \mathrm{E}+02$ & 5. & 1.2 & $2.49 E+02$ & $2.74 \mathrm{E}+02$ \\
\hline (n) & $u 240$ & $\mathrm{Ci}$ & $.97 E+01$ & $97 E+01$ & $0.00 \mathrm{E}+01$ & $9.29 \mathrm{E}-01$ & $2.97 E+01$ & $1.05 E+02$ & $2.25 E+02$ & $4.42 E+01$ & $4.87 E+01$ \\
\hline
\end{tabular}


Tank Anal. Un

\begin{tabular}{|c|c|c|c|}
\hline 1103 & Рu241 & $\mathrm{Ci}$ & $\frac{M a \overline{98}}{3.21 E+02}$ \\
\hline T103 & Pu242 & $\mathbf{C i}$ & $9.05 E-04$ \\
\hline T103 & Ra226 & $\mathbf{C i}$ & $1.36 \mathrm{E}-06$ \\
\hline T103 & Ra228 & $\mathbf{C i}$ & $3.48 \mathrm{E}-03$ \\
\hline T103 & Ru106 & $\mathbf{C i}$ & $6.78 E-05$ \\
\hline T103 & Sb125 & Ci & $1.49 \mathrm{E}-00$ \\
\hline T103 & Se79 & $\mathbf{C i}$ & 2.38E-02 \\
\hline T103 & Sm151 & $\mathbf{C i}$ & $8.38 E+01$ \\
\hline T103 & Sr89/90 & $\mathbf{C i}$ & $2.71 E+04$ \\
\hline T103 & Te99 & $\mathbf{C i}$ & 2.09E-00 \\
\hline T103 & Th229 & $\mathrm{Ci}$ & $1.57 \mathrm{E}-03$ \\
\hline T103 & Th232 & $\mathbf{C i}$ & $1.68 \mathrm{E}-04$ \\
\hline T103 & Tin126 & $\mathrm{Ci}$ & 3.59E-02 \\
\hline T103 & Tritium & $\mathrm{Ci}$ & 1.70E-00 \\
\hline T103 & U232 & $\mathrm{Ci}$ & 1.79E-01 \\
\hline T103 & U233 & $\mathbf{C i}$ & $6.97 \mathrm{E}-01$ \\
\hline T103 & U234 & $\mathrm{Ci}$ & $1.46 \mathrm{E}-00$ \\
\hline T103 & U235 & $\mathrm{Ci}$ & $6.18 E-02$ \\
\hline T103 & U236 & $\mathrm{Ci}$ & 3.15E-02 \\
\hline T103 & U238 & $\mathrm{Ci}$ & 1.41E-00 \\
\hline T103. & Y90 & $\mathrm{Ci}$ & $2.71 E+04$ \\
\hline T103 & Zr93 & $\mathrm{Ci}$ & 1.17E-01 \\
\hline T104 & Al & kg & $3.50 \mathrm{E}+04$ \\
\hline T104 & $\mathbf{B i}$ & kg & $4.08 E+04$ \\
\hline T104 & $\mathrm{Ca}$ & kg & $3.12 E+03$ \\
\hline T104 & Cl & kg & $1.45 E+03$ \\
\hline T104 & $\mathrm{CO3}$ & kg & $5.97 \mathrm{E}+03$ \\
\hline T104 & $\mathrm{Cr}$ & $\mathrm{kg}$ & $1.94 \mathrm{E}+03$ \\
\hline T104 & $\mathbf{F}$ & kg & $1.85 \mathrm{E}+04$ \\
\hline T104 & $\mathbf{F e}$ & $\mathrm{kg}$ & $1.95 E+04$ \\
\hline T104 & Hg & $\mathrm{kg}$ & $2.62 E+01$ \\
\hline T104 & $\mathbf{K}$ & kg & $1.92 \mathrm{E}+02$ \\
\hline T104 & La & kg & $6.60 \mathrm{E}-03$ \\
\hline T104 & Mn & kg & $1.33 E+02$ \\
\hline T104 & $\mathbf{N a}$ & kg & $1.39 E+05$ \\
\hline T104 & $\mathbf{N i}$ & kg & $2.44 E+01$ \\
\hline T104 & NO2 & kg & $8.81 E+03$ \\
\hline T104 & NO3 & kg & $1.25 E+05$ \\
\hline T104 & $\mathbf{P}$ & $\mathrm{kg}$ & $1.60 \mathrm{E}+05$ \\
\hline T104 & $\mathbf{P b}$ & kg & $8.70 \mathrm{E}-01$ \\
\hline T104 & P04 & kg & $4.96 E+05$ \\
\hline T104 & $\mathbf{S}$ & kg & $8.42 E+03$ \\
\hline T104 & $\mathbf{S i}$ & $\mathrm{kg}$ & $1.41 E+04$ \\
\hline T104 & SO4 & kg & $2.53 \mathrm{E}+04$ \\
\hline T104 & $\mathbf{S r}$ & kg & $2.13 E+02$ \\
\hline T104 & TOC & kg & $1.15 \mathrm{E}+02$ \\
\hline T104 & $\mathbf{U}$ & kg & $1.94 E+03$ \\
\hline T104 & $\mathbf{Z r}$ & kg & $1.46 \mathrm{E}+02$ \\
\hline T104 & Ac227 & $\mathrm{Ci}$ & $6.45 \mathrm{E}-05$ \\
\hline T104 & Am241 & $\mathrm{Ci}$ & $3.72 \mathrm{E}+01$ \\
\hline T104 & Am243 & $\mathrm{Ci}$ & $1.98 \mathrm{E}-05$ \\
\hline T104 & Ba137 & $\mathrm{Ci}$ & $4.28 E+02$ \\
\hline T104 & C14 & $\mathrm{Ci}$ & $9.68 \mathrm{E}-02$ \\
\hline T104 & Cd113 & $\mathrm{Ci}$ & 1.17E-90 \\
\hline T104 & $\operatorname{Cm} 242$ & $\mathrm{Ci}$ & $2.34 \mathrm{E}-03$ \\
\hline T104 & $\mathrm{Cm} 243$ & $\mathrm{Ci}$ & $1.41 E-04$ \\
\hline T104 & Cm244 & $\mathrm{Ci}$ & $1.21 \mathrm{E}-03$ \\
\hline T104 & Co60 & $\mathrm{Ci}$ & $3.42 \mathrm{E}-01$ \\
\hline T104 & Cs134 & $\mathrm{Ci}$ & $2.40 \mathrm{E}-02$ \\
\hline T104 & Cs137 & $\mathrm{Ci}$ & $4.28 \mathrm{E}+02$ \\
\hline T104 & Eu152 & $\mathrm{Ci}$ & $9.11 E-02$ \\
\hline T104 & Eu154 & $\mathbf{C i}$ & 7.35E-00 \\
\hline T104 & Eu155 & $\mathrm{Ci}$ & $6.36 \mathrm{E}-00$ \\
\hline T104 & 1129 & Ci & $9.98 \mathrm{E}+01$ \\
\hline T104 & Nb93 & $\mathrm{Ci}$ & $2.60 \mathrm{E}-01$ \\
\hline T104 & Ni59 & $\mathrm{Ci}$ & $7.22 \mathrm{E}-02$ \\
\hline T104 & Ni63 & $\mathrm{Ci}$ & $6.64 E-00$ \\
\hline T10M & $\mathrm{Np2}$ & Ci & $2.95 E+02$ \\
\hline
\end{tabular}

\section{Sept98}

\section{$3.21 \mathrm{E}+02$}

9.05E-04

1.36E-06

$3.48 \mathrm{E}-03$

$6.78 \mathrm{E}-05$

1.49E-60

2.38E-02

8.38E +01

$2.71 \mathrm{E}+04$

2.09E-00

1.57E-03

1.68E-04

3.59E-02

1.70E-00

1.79E-01

6.97E-01

$1.46 \mathrm{E}-00$

6.18E-02

3.15E-02

1.41E-00

$2.71 \mathrm{E}+04$

1.17E-01

$3.50 \mathrm{E}+04$

$4.08 \mathrm{E}+04$

$3.12 \mathrm{E}+03$

$1.45 \mathrm{E}+03$

$7.69 \mathrm{E}+03$

$1.94 \mathrm{E}+03$

$1.85 \mathrm{E}+04$

$1.95 E+04$

$2.74 E+02$

$1.92 \mathrm{E}+02$

$0.00 \mathrm{E}+01$

$1.33 E+02$

$1.39 \mathrm{E}+05$

$2.44 E+01$

$8.81 \mathrm{E}+03$

$1.25 E+05$

$$
\text { NA }
$$

1.35E-00

$1.62 \mathrm{E}+05$

NA

$1.41 E+04$

$8.42 \mathrm{E}+03$

$2.13 E+02$

$1.15 \mathrm{E}+02$

$1.94 \mathrm{E}+03$

$1.46 \mathrm{E}+02$

6.45E-05

$3.72 \mathrm{E}+01$

8.83E-04

$4.05 E+02$

9.68E-02

1.17E-00

1.04E-01

$6.28 \mathrm{E}-03$

5.40E-02

$3.42 \mathrm{E}-01$

2.40E-02

$4.28 \mathrm{E}+02$

9.11E-02

7.35E-00

6.36E-00

6.17E-03

2.60E-01

$7.22 \mathrm{E}-02$

6.64E-00

1.52E-02 1\%-ile

$0.00 E+01$

$0.00 \mathrm{E}+01$

$0.00 \mathrm{E}+01$

$0.00 \mathrm{E}+01$

$0.00 \mathrm{E}+01$

$0.00 \mathrm{E}+01$

$0.00 \mathrm{E}+01$

$0.00 \mathrm{E}+01$

$0.00 \mathrm{E}+01$

$0.00 \mathrm{E}+01$

$0.00 \mathrm{E}+01$

$0.00 \mathrm{E}+01$

$0.00 \mathrm{E}+01$

$0.00 \mathrm{E}+01$

$0.00 \mathrm{E}+01$

$0.00 \mathrm{E}+01$

$0.00 \mathrm{E}+01$

$0.00 \mathrm{E}+01$

$0.00 \mathrm{E}+01$

$0.00 \mathrm{E}+01$

$0.00 \mathrm{E}+01$

$0.00 \mathrm{E}+01$

$1.87 \mathrm{E}+02$

$1.80 \mathrm{E}+04$

$1.03 \mathrm{E}+02$

4.77E+02

4.74E +01

3.24E-00

$2.62 \mathrm{E}+02$

$8.42 \mathrm{E}+03$

$0.00 \mathrm{E}+01$

$7.62 \mathrm{E}+01$

$7.48 \mathrm{E}-02$

$3.98 \mathrm{E}-00$

$1.09 \mathrm{E}+05$

2.04E-00

$1.29 \mathrm{E}+03$

$2.46 \mathrm{E}+04$

$7.43 \mathrm{E}+03$

9.03E-00

$3.16 \mathrm{E}+03$

$3.77 \mathrm{E}+02$

$1.22 \mathrm{E}+03$

$2.30 \mathrm{E}+03$

1. $67 \mathrm{E}+01$

$5.30 \mathrm{E}-02$

$7.21 \mathrm{E}+01$

$1.46 \mathrm{E}-00$

$0.00 E+01$

1.36E-00

8.85E-07

$2.83 \mathrm{E}+01$

$0.00 E+01$

$0.00 \mathrm{E}+01$

1.05E-04

6.30E-06

5.41E-05

$0.00 \mathrm{E}+01$

$1.59 \mathrm{E}-03$

$2.90 \mathrm{E}+03$

$0.00 E+01$

$0.00 E+01$

$0.00 E+01$

$0.00 E+01$

$0.00 \mathrm{E}+01$

$0.00 \mathrm{E}+0 \mathrm{I}$

$0.00 E+01$

1.32E+01 10\%-ile

$1.00 \mathrm{E}+01$

2.83E-05

6.91E-08

1.77E-04

2.24E-06

3.05E-02

1.13E-03

$0.00 \mathrm{E}+01$

$1.04 \mathrm{E}+01$

$0.00 E+01$

$0.00 \mathrm{E}+01$

$0.00 E+01$

2.05E-03

$1.62 \mathrm{E}-01$

$0.00 \mathrm{E}+01$

$0.00 \mathrm{E}+01$

$0.00 \mathrm{E}+01$

$0.00 \mathrm{E}+01$

$0.00 \mathrm{E}+01$

$0.00 \mathrm{E}+01$

$8.24 \mathrm{E}-00$

$2.98 \mathrm{E}-03$

$2.41 \mathrm{E}+03$

$2.52 \mathrm{E}+04$

$5.97 \mathrm{E}+02$

$1.33 \mathrm{E}+03$

8.20E +03

$7.89 \mathrm{E}+02$

$2.31 \mathrm{E}+03$

$1.63 \mathrm{E}+04$

2.93E-00

$3.13 \mathrm{E}+02$

$1.73 \mathrm{E}-00$

$6.20 E+01$

$1.48 \mathrm{E}+05$

$1.59 \mathrm{E}+01$

$7.09 \mathrm{E}+03$

1.01E+05

$2.29 \mathrm{E}+04$

$1.38 \mathrm{E}+02$

$1.80 \mathrm{E}+04$

$2.65 \mathrm{E}+03$

$6.32 \mathrm{E}+03$

$9.70 \mathrm{E}+03$

$1.32 \mathrm{E}+02$

$4.40 \mathrm{E}+02$

$9.08 \mathrm{E}+02$

1.30E +01

$1.00 \mathrm{E}-05$

$2.85 \mathrm{E}+01$

6.05E-06

$1.49 \mathrm{E}+02$

$0.00 \mathrm{E}+01$

1.31E-01

7.15E-04

4.31E-05

3.70E-04

2.19E-01

$8.35 \mathrm{E}-03$

$1.35 \mathrm{E}+04$

1.41E-02

$1.14 \mathrm{E}-00$

9.88E-01

$2.90 \mathrm{E}+01$

$0.00 \mathrm{E}+01$

2.08E-02

$1.91 \mathrm{E}-00$

$9.02 \mathrm{E}+01$

\section{$50 \%-i l e$}

$3.21 \mathrm{E}+02$

9.05E-04

$1.36 \mathrm{E}-06$

3.48E-03

6.78E-05

1.49E-00

2.38E-02

8.38E+01

$2.89 \mathrm{E}+04$

2.09E-00

1.57E-03

$1.68 \mathrm{E}-04$

3.59E-02

1.70E-00

1.79E-01

6.97E-01

$1.46 \mathrm{E}-00$

6.18E-02

3.15E-02

1.41E-00

$2.71 E+04$

1.17E-01

$2.92 \mathrm{E}+04$

$3.94 \mathrm{E}+04$

$1.53 \mathrm{E}+03$

$2.38 \mathrm{E}+03$

$2.24 \mathrm{E}+04$

$1.89 \mathrm{E}+03$

$1.47 \mathrm{E}+04$

3.12E+04

2.62E+01

$6.97 \mathrm{E}+02$

$8.22 \mathrm{E}-00$

$1.63 \mathrm{E}+02$

$2.03 E+05$

$4.99 \mathrm{E}+01$

$2.40 \mathrm{E}+04$

$2.89 \mathrm{E}+05$

4.35E +04

$6.78 \mathrm{E}+02$

$4.29 \mathrm{E}+04$

$8.60 \mathrm{E}+03$

$1.57 \mathrm{E}+04$

2.31E+04

4.11E +02

$1.38 \mathrm{E}+03$

$3.78 \mathrm{E}+03$

$1.20 \mathrm{E}+02$

6.45E-05

$1.20 \mathrm{E}+02$

1.98E-05

$4.28 \mathrm{E}+02$

$9.68 \mathrm{E}-02$

1.17E-00

2.34E-03

1.41E-04

1.21E-03

$7.97 \mathrm{E}-00$

2.40E-02

$3.82 \mathrm{E}+04$

9.11E-02

$7.35 \mathrm{E}-00$

6.36E-00

$9.98 \mathrm{E}+01$

2.60E-01

7.22E-02

6.64E-00

$2.95 \mathrm{E}+02$

90\%-ile

$1.14 E+03$

$3.21 \mathrm{E}-03$

5.99E-06

1.53E-02

3.64E-04 


\begin{tabular}{|c|c|c|c|c|c|c|c|c|c|c|c|}
\hline nk & Anal. & Un & May $\frac{B 8}{88}$ & Sept98 & $1 \%$-ile & $10 \%$-ile & $50 \%$-ile & $90 \%$-ile & 99\%-ile & Mean & Std Dev \\
\hline 104 & 231 & $\mathrm{Ci}$ & $\overline{1.57 \mathrm{E}-04}$ & $\overline{1.57 \mathrm{E}-04}$ & $0.00 \mathrm{E}+01$ & $2.73 \mathrm{E}-05$ & E-04 & -04 & UIC-0 & OE-04 & $08 \mathrm{E}-04$ \\
\hline T104 & Pu238 & $\mathrm{Ci}$ & 4.83E-01 & $2.02 \mathrm{E}-00$ & $16 \mathrm{E}-02$ & $1.48 \mathrm{E}-01$ & $4.83 \mathrm{E}-01$ & $82 \mathrm{E}-01$ & $1.92 \mathrm{E}-00$ & $47 \mathrm{E}-01$ & $.79 \mathrm{E}-01$ \\
\hline$\Gamma 104$ & u239 & $\mathbf{C i}$ & $3.67 E+02$ & $2.76 E+02$ & $95 \mathrm{E}-00$ & $2.02 \mathrm{E}+01$ & $6.61 \mathrm{E}+01$ & $4 \mathrm{E}+02$ & $2.62 \mathrm{E}+02$ & $7.48 \mathrm{E}+01$ & $5.18 \mathrm{E}+01$ \\
\hline 104 & $u 240$ & $\mathbf{C i}$ & $6.02 \mathrm{E}-00$ & $2.51 E+01$ & $69 \mathrm{E}-01$ & $1.84 \mathrm{E}-00$ & $3.02 \mathrm{E}-00$ & $22 E+01$ & $2.39 \mathrm{E}+01$ & $6.82 \mathrm{E}-00$ & 4.72E-00 \\
\hline 104 & 1241 & $\mathrm{Ci}$ & $03 \mathrm{E}+01$ & $46 \mathrm{E}+01$ & $7 E-01$ & $6.21 \mathrm{E}-00$ & $.03 E+01$ & $E+01$ & $.06 \mathrm{E}+01$ & $2.30 \mathrm{E}+01$ & $1.59 \mathrm{E}+01$ \\
\hline 104 & 1242 & $\mathrm{Ci}$ & 9.27E-05 & $3.87 \mathrm{E}-04$ & $14 \mathrm{E}-06$ & $2.83 \mathrm{E}-05$ & $9.27 \mathrm{E}-05$ & & $3.68 \mathrm{E}-04$ & $1.05 \mathrm{E}-04$ & $7.27 \mathrm{E}-05$ \\
\hline 104 & 26 & & $1.24 \mathrm{E}-0$ & $1.24 \mathrm{E}-05$ & $E+01$ & 39E-06 & & & $8.97 E-05$ & 05 & \\
\hline Г104 & 8 & C & $9.12 \mathrm{E}-\mathrm{C}$ & $9.12 \mathrm{E}-04$ & & $E-04$ & 4 & & 3 & & \\
\hline 104 & 36 & $\mathrm{Ci}$ & 5.67E-05 & $5.67 \mathrm{E}-05$ & 01 & 05 & - & & 2.0 & .05 & \\
\hline 104 & 125 & $\mathrm{Ci}$ & $1.34 \mathrm{E}-00$ & $1.34 E-00$ & $0.00 \mathrm{E}+01$ & $0.00 \mathrm{E}+01$ & $1.34 E-00$ & -00 & $5.76 \mathrm{E}-00$ & E-00 & $1.28 \mathrm{E}-00$ \\
\hline T104 & 79 & $\mathrm{Ci}$ & $6.80 \mathrm{E}-02$ & $6.80 \mathrm{E}-02$ & $E-04$ & 2.47E-02 & $6.80 \mathrm{E}-02$ & -01 & 4.47E-01 & $7.80 \mathrm{E}-02$ & $6.72 \mathrm{E}-02$ \\
\hline T104 & n151 & $\mathbf{C i}$ & $2.48 \mathrm{E}+02$ & $2.48 \mathrm{E}+02$ & $0.00 E+01$ & $3.85 \mathrm{E}+01$ & $2.48 \mathrm{E}+02$ & $2.10 \mathrm{E}+03$ & $5.78 E+03$ & $7.35 \mathrm{E}+02$ & $1.25 E+03$ \\
\hline T104 & $89 / 90$ & $\mathbf{C i}$ & $.66 \mathrm{E}+03$ & $5.66 \mathrm{E}+03$ & $4.24 \mathrm{E}-00$ & +03 & $4.03 E+04$ & & $7.83 E+05$ & +05 & $E+05$ \\
\hline 104 & c99 & $\mathrm{Ci}$ & 1.24E-00 & $1.24 \mathrm{E}-00$ & $E-02$ & -01 & -00 & & $6.28 \mathrm{E}+01$ & -00 & -00 \\
\hline 104 & 3229 & $\mathbf{C i}$ & $2.16 \mathrm{E}-05$ & 2.16E-05 & +01 & & 05 & & 2.77E-04 & -05 & .05 \\
\hline 104 & 232 & $\mathrm{Ci}$ & $6.00 \mathrm{E}-\mathrm{C}$ & $6.00 \mathrm{E}-05$ & 11 & & $6.0-2>0$ & & & & \\
\hline 104 & 26 & C & $1.02 \mathrm{E}-$ & 1.02 & & & & & & & \\
\hline T104 & um & $\mathbf{C i}$ & 2.73E-00 & $2.73 E-00$ & 0 & 10 & 2.7 & & 00 & .00 & -01 \\
\hline T104 & 2 & $\mathrm{Ci}$ & 5.33E-03 & $1.34 \mathrm{E}-04$ & 01 & 4 & 3 & & 6. & 03 & -02 \\
\hline T104 & U233 & $\mathrm{Ci}$ & $1.87 E-02$ & 4.69E-04 & +01 & $3.25 \mathrm{E}-03$ & $1.87 E-02$ & -02 & $2.40 \mathrm{E}-01$ & $E-02$ & $E-02$ \\
\hline T104 & U234 & $\mathbf{C i}$ & $2.54 \mathrm{E}+01$ & 6.38E-01 & +01 & -00 & 2.54] & & $3.26 \mathrm{E}+02$ & $E+01$ & +01 \\
\hline 104 & U235 & $\mathrm{Ci}$ & $1.13 E-00$ & $2.84 \mathrm{E}-02$ & & & & & +01 & & \\
\hline 104 & U236 & $\mathrm{Ci}$ & 2.17E-01 & $5.45 \mathrm{E}-03$ & -01 & & & & & & \\
\hline T10 & 8 & C & $2.58 \mathrm{E}+01$ & 6.48E-01 & & & 2. & & 2 & & \\
\hline T104 & Y90 & C & $5.66 \mathrm{E}+03$ & $5.66 E+03$ & 01 & 02 & 5.6 & & 05 & -04 & 104 \\
\hline T104 & Zr93 & $\mathrm{C}$ & 3.27E-01 & 3.27E-01 & +01 & 01 & -01 & & -00 & -01 & -01 \\
\hline 105 & A & kg & $1.70 \mathrm{E}+04$ & $2.61 E+04$ & +02 & +03 & +03 & 04 & +04 & -04 & +03 \\
\hline T105 & B1 & kg & $7.50 E+03$ & $3.35 E+03$ & & & 03 & & +04 & & -03 \\
\hline 105 & $\mathrm{Ca}$ & kg & $2.20 \mathrm{E}+03$ & +02 & & 02 & & & & & \\
\hline 105 & C & kg & $2.40 \mathrm{E}+02$ & 12 & & & & & & & \\
\hline 05 & C & $\mathbf{k g}$ & $1.72 \mathrm{E}$ & $9.43 E+03$ & 02 & 03 & 03 & & +04 & & -03 \\
\hline T105 & $\mathrm{Cr}$ & kg & $3.60 \mathrm{E}+02$ & $3.74 E+02$ & +01 & +02 & -02 & 03 & +03 & +02 & +02 \\
\hline T105 & $\mathbf{F}$ & kg & $1.20 \mathrm{E}+03$ & $2.50 E+02$ & +02 & 02 & 2.78 & 03 & $\div+03$ & -03 & +03 \\
\hline T105 & $\mathbf{F e}$ & kg & $8.60 \mathrm{E}+03$ & $9.43 E+03$ & & & & & -04 & 03 & 03 \\
\hline T105 & Hg & kg & $1.00 \mathrm{E}-00$ & -00 & & & & & & & \\
\hline T105 & $\mathbf{K}$ & kg & 1.90 & 2.5 & & & & & & & \\
\hline 105 & L. & kg & $0.00 \mathrm{E}+01$ & $2.02 \mathrm{E}+01$ & -01 & 01 & 00 & & +01 & .00 & -00 \\
\hline T105 & $\mathbf{M n}$ & $\mathbf{k g}$ & $7.00 \mathrm{E}+03$ & $4.04 E+03$ & $E-00$ & +01 & $1.59 E+02$ & -02 & +03 & +02 & +02 \\
\hline T105 & Na & kg & $3.80 \mathrm{E}+04$ & $3.45 E+04$ & -04 & 04 & 4.7 & & +04 & -04 & -04 \\
\hline T105 & $\mathbf{N i}$ & kg & $2.80 \mathrm{E}+01$ & +01 & & & & & 02 & 02 & 02 \\
\hline T105 & $\mathbf{N}$ & kg & +03 & +04 & & & & & & & \\
\hline 105 & & kg & 3. & & & & & & & .04 & -04 \\
\hline T105 & $\mathbf{P}$ & kg & $1.98 E+04$ & NA & +03 & 03 & 8.8 & -04 & +04 & +03 & +03 \\
\hline T105 & $\mathbf{P b}$ & kg & $2.80 \mathrm{E}+02$ & $2.37 \mathrm{E}+02$ & $E-00$ & 01 & +02 & -02 & $i+02$ & 02 & +02 \\
\hline T105 & $\mathbf{P O}$ & $\mathbf{k g}$ & $6.13 E+04$ & $2.29 E+03$ & -03 & 4. & 04 & & -04 & -04 & r03 \\
\hline T105 & $\mathbf{S}$ & kg & 03 & NA & & & & & & & 03 \\
\hline $\mathbf{T 1 0}$ & S & kg & +03 & $1.92 E+03$ & & & & & & & \\
\hline T105 & & kg & & & & & & & & & -03 \\
\hline $\mathbf{T 1}$ & s & $\mathrm{kg}$ & -01 & +01 & +01 & 01 & & 02 & 02 & -02 & +01 \\
\hline T105 & TOC & kg & $0.00 \mathrm{E}+01$ & $1.89 E+03$ & $: 01$ & +02 & -02 & +02 & +02 & $E+02$ & $E+02$ \\
\hline T105 & $\mathbf{U}$ & kg & $1.00 \mathrm{E}+03$ & $3.01 E+03$ & +01 & 2.7 & 9.9 & -03 & +03 & +03 & +03 \\
\hline T105 & $\mathbf{Z r}$ & kg & $2.10 \mathrm{E}+\theta 1$ & $2.34 E+01$ & 01 & 3. & 6.4 & 2 & 02 & & +01 \\
\hline $\mathbf{T}$ & & $i$ & & & & & & & & & \\
\hline $\mathrm{T105}$ & A & $\mathrm{Ci}$ & 5. & & & & & & & & \\
\hline & & $\mathrm{Ci}$ & & & & & & & & 07 & -07 \\
\hline T105 & Ba137 & $\mathrm{Ci}$ & $2.84 E+04$ & $1.26 E+04$ & 03 & 7.1 & 2.8 & & -05 & $E+04$ & +04 \\
\hline T105 & C14 & $\mathrm{Ci}$ & $6.10 \mathrm{E}-01$ & $4.10 \mathrm{E}-01$ & $=01$ & $E-01$ & 01 & 00 & $E-00$ & -01 & -01 \\
\hline T105 & Cd113 & $\mathrm{Ci}$ & $4.26 \mathrm{E}-02$ & $4.26 \mathrm{E}-02$ & $=01$ & 8.2 & 4.26 & 1 & 01 & -02 & -02 \\
\hline T10 & Cm242 & $\mathrm{Ci}$ & $1.07 \mathrm{E}-04$ & $2.87 E-01$ & 06 & 3. & 04 & 4 & 04 & -04 & -04 \\
\hline T105 & C & $\mathrm{Ci}$ & $2.19 \mathrm{E}-06$ & $5.88 \mathrm{E}-03$ & & & 6 & & E-06 & -06 & -06 \\
\hline T10 & C & $\mathrm{Ci}$ & 6.2 & 1.0 & & & & & & & \\
\hline & & $\mathrm{Ci}$ & & & & & & & 02 & $1.22 E+01$ & $E+01$ \\
\hline T105 & Cs134 & $\mathrm{Ci}$ & $1.71 E-04$ & $2.27 \mathrm{E}+01$ & -06 & 05 & 04 & & -04 & E-04 & $1.94 \mathrm{E}-04$ \\
\hline T105 & $\mathrm{Cs}$ & $\mathrm{Ci}$ & $3.00 \mathrm{E}+04$ & $1.33 E+04$ & $1.88 \mathrm{E}+03$ & $5.67 \mathrm{E}+03$ & $1.93 E+04$ & $5.85 \mathrm{E}+04$ & $9.34 \mathrm{E}+04$ & $2.53 \mathrm{E}+04$ & $2.07 E+04$ \\
\hline T105 & Eu152 & $\mathbf{C i}$ & $5.86 \mathrm{E}-03$ & $5.86 \mathrm{E}-03$ & $0.00 E+01$ & $0.00 \mathrm{E}+01$ & $5.86 \mathrm{E}-03$ & $2.80 \mathrm{E}-02$ & E-02 & $E-02$ & 1.34E-02 \\
\hline T105 & Eu154 & $\mathrm{Ci}$ & $1.00 \mathrm{E}+03$ & $7.37 E+02$ & $0.00 \mathrm{E}+01$ & $0.00 \mathrm{E}+01$ & $1.00 \mathrm{E}+03$ & & $9.76 E+03$ & $1.82 E+03$ & $2.28 E+03$ \\
\hline T105 & Eu155 & $\mathrm{Ci}$ & $1.10 \mathrm{E}+03$ & $8.69 E+02$ & $0.00 \mathrm{E}+01$ & $0.00 \mathrm{E}+01$ & $1.10 E+03$ & $5.26 \mathrm{E}+03$ & $1.07 \mathrm{E}+04$ & $2.00 E+03$ & $2.51 E+03$ \\
\hline & & 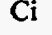 & & $2.22 E-04$ & $.00 E+01$ & $.36 \mathrm{E}-05$ & $2.22 \mathrm{E}-04$ & -04 & $5.12 \mathrm{E}-04$ & -04 & \\
\hline
\end{tabular}


Tank Anal. Un

\begin{tabular}{|c|c|c|c|}
\hline T105 & Nb93 & $\mathrm{Ci}$ & $\frac{\text { Maу } \overline{98}}{1.42 \mathrm{E}-02}$ \\
\hline T105 & Ni59 & $\mathrm{Ci}$ & $4.80 E-03$ \\
\hline T105 & Ni63 & $\mathrm{Ci}$ & 4.35E-01 \\
\hline T105 & Np237 & $\mathrm{Ci}$ & $7.28 \mathrm{E}-04$ \\
\hline T105 & Pa231 & $\mathrm{Ci}$ & $9.88 \mathrm{E}-06$ \\
\hline T105 & Pu238 & $\mathrm{Ci}$ & $1.06 \mathrm{E}-01$ \\
\hline T105 & Pu239 & $\mathrm{Ci}$ & $1.09 \mathrm{E}+02$ \\
\hline T105 & Pu240 & $\mathrm{Ci}$ & $1.68 \mathrm{E}-00$ \\
\hline T105 & Pu241 & $\mathrm{Ci}$ & $2.66 \mathrm{E}-00$ \\
\hline T105 & Pu242 & $\mathrm{Ci}$ & 1.11E-05 \\
\hline T105 & $\operatorname{Ra226}$ & $\mathrm{Ci}$ & $8.76 \mathrm{E}-07$ \\
\hline T105 & $\mathbf{R a 2 2 8}$ & $\mathrm{Ci}$ & $2.25 \mathrm{E}-11$ \\
\hline T105 & Rul06 & $\mathrm{Ci}$ & 1.88E-09 \\
\hline T105 & Sb125 & $\mathrm{Ci}$ & $4.00 \mathrm{E}+02$ \\
\hline T105 & Se79 & $\mathrm{Ci}$ & $3.57 \mathrm{E}-03$ \\
\hline T105 & Sm151 & $\mathrm{Ci}$ & $1.32 \mathrm{E}+01$ \\
\hline T105 & Sr89/90 & $\mathrm{Ci}$ & $1.70 E+05$ \\
\hline T105 & Te99 & $\mathrm{Ci}$ & $2.30 \mathrm{E}+02$ \\
\hline T105 & Th229 & $\mathrm{Ci}$ & 4.37E-09 \\
\hline T105 & Th232 & $\mathrm{Ci}$ & 4.74E-12 \\
\hline T105 & Tin126 & $\mathrm{Ci}$ & 5.38E-03 \\
\hline T105 & Tritium & $\mathrm{Ci}$ & $7.60 \mathrm{E}-00$ \\
\hline T105 & U232 & $\mathrm{Ci}$ & $3.60 \mathrm{E}-05$ \\
\hline T105 & U233 & $\mathrm{Ci}$ & 1.67E-06 \\
\hline T105 & U234 & $\mathrm{Ci}$ & $1.56 \mathrm{E}-00$ \\
\hline T105 & U235 & $\mathbf{C i}$ & $6.88 \mathrm{E}-02$ \\
\hline T105 & U236 & $\mathbf{C i}$ & 1.55E-02 \\
\hline T105 & U238 & $\mathbf{C i}$ & 1.58E-00 \\
\hline T105 & Y90 & $\mathrm{Ci}$ & $1.70 \mathrm{E}+05$ \\
\hline T105 & Zr93 & Ci & $1.69 \mathrm{E}-02$ \\
\hline T106 & Al & kg & $1.38 E+04$ \\
\hline T106 & $\mathbf{B i}$ & kg & $1.00 E+03$ \\
\hline T106 & Ca & $\mathrm{kg}$ & $1.73 E+02$ \\
\hline T106 & Cl & $\mathrm{kg}$ & $1.12 \mathrm{E}+02$ \\
\hline T106 & $\mathrm{CO3}$ & kg & $8.35 E+01$ \\
\hline T106 & $\mathrm{Cr}$ & kg & $6.22 \mathrm{E}+01$ \\
\hline T106 & $\mathbf{F}$ & kg & $5.63 E+02$ \\
\hline T106 & $\mathrm{Fe}$ & kg & $1.30 \mathrm{E}+03$ \\
\hline T106 & $\mathbf{H g}$ & kg & $3.91 \mathrm{E}+01$ \\
\hline T106 & $\mathbf{K}$ & kg & $9.10 \mathrm{E}-00$ \\
\hline T106 & $\mathbf{L a}$ & kg & $6.80 \mathrm{E}+01$ \\
\hline T106 & Mn & kg & $1.73 E+01$ \\
\hline T106 & $\mathbf{N a}$ & kg & $1.02 E+04$ \\
\hline T106 & $\mathrm{Ni}$ & kg & $7.80 \mathrm{E}-00$ \\
\hline T106 & NO2 & kg & $1.28 E+03$ \\
\hline T106 & NO3 & $\mathbf{k g}$ & $3.56 E+03$ \\
\hline T106 & $\mathbf{P}$ & kg & $1.29 E+03$ \\
\hline T106 & $\mathbf{P b}$ & kg & $3.55 \mathrm{E}+02$ \\
\hline T106 & PO4 & kg & $3.99 E+03$ \\
\hline T106 & $\mathbf{S}$ & kg & $2.35 E+02$ \\
\hline T106 & $\mathbf{S i}$ & kg & $4.93 E+02$ \\
\hline T106 & SO4 & kg & $7.04 E+02$ \\
\hline T106 & Sr & kg & $4.12 \mathrm{E}+01$ \\
\hline T106 & TOC & $\mathrm{kg}$ & $4.71 \mathrm{E}+01$ \\
\hline T106 & $\mathbf{U}$ & kg & $8.61 E+02$ \\
\hline T106 & $\mathbf{Z r}$ & kg & $1.19 \mathrm{E}+01$ \\
\hline T106 & Ac227 & $\mathrm{Ci}$ & $1.66 \mathrm{E}-06$ \\
\hline T106 & Am241 & $\mathrm{Ci}$ & $8.85 E-00$ \\
\hline T106 & Am243 & $\mathrm{Ci}$ & $2.46 \mathrm{E}-07$ \\
\hline T106 & Ba137 & $\mathrm{Ci}$ & $1.87 E+03$ \\
\hline T106 & C14 & $\mathrm{Ci}$ & $9.25 E-03$ \\
\hline T106 & Cd113 & $\mathrm{Ci}$ & $2.86 \mathrm{E}-02$ \\
\hline T106 & $\mathrm{Cm} 242$ & $\mathrm{Ci}$ & 2.37E-04 \\
\hline T & Cm243 & Ci & 5.34E-06 \\
\hline T106 & Cm244 & $\mathrm{Ci}$ & $7.30 \mathrm{E}-06$ \\
\hline T106 & Co60 & $\mathrm{Ci}$ & $3.94 \mathrm{E}-03$ \\
\hline T106 & Cs134 & Ci & $3.85 \mathrm{E}-04$ \\
\hline T106 & Cs137 & Ci & $1.98 E+03$ \\
\hline
\end{tabular}

\section{$B B$}

\section{Sept98}

1.42E-02

4.80E-03

4.35E-01

7.28E-04

9.88E-06

4.22E-01

$9.91 \mathrm{E}+01$

6.68E-00

$1.06 \mathrm{E}+01$

4.41E-05

8.76E-07

2.25E-11

$1.88 \mathrm{E}-09$

2.74E+02

3.57E-03

$1.32 \mathrm{E}+01$

$1.15 \mathrm{E}+05$

$1.53 \mathrm{E}+02$

4.37E-09

4.74E-12

5.38E-03

4.94E-00

2.29E-05

1.06E-06

9.89E-01

4.37E-02

9.83E-03

1.01E-00

$1.15 E+05$

$1.69 \mathrm{E}-02$

$1.38 \mathrm{E}+04$

$1.00 \mathrm{E}+03$

$1.73 \mathrm{E}+02$

$1.12 \mathrm{E}+02$

$8.35 E+01$

$6.22 \mathrm{E}+01$

$5.63 \mathrm{E}+02$

$1.30 \mathrm{E}+03$

9.72E-00

9.10E- 00

$0.00 \mathrm{E}+01$

$1.33 E+01$

$1.02 \mathrm{E}+04$

$7.80 \mathrm{E}-00$

$1.28 \mathrm{E}+03$

$3.56 \mathrm{E}+03$

NA

$3.55 \mathrm{E}+02$

$3.99 \mathrm{E}+03$

NA

4.93E+02

$7.04 \mathrm{E}+02$

$3.44 E+01$

$4.71 \mathrm{E}+01$

$8.61 \mathrm{E}+02$

5.77E-01

$1.66 \mathrm{E}-06$

8.85E-00

7.13E-05

$1.87 \mathrm{E}+03$

$9.25 \mathrm{E}-03$

$2.86 \mathrm{E}-02$

$6.88 \mathrm{E}-02$

1.55E-03

2.12E-03

$3.94 \mathrm{E}-03$

3.85E-04

$1.98 \mathrm{E}+03$
$1 \%$-ile

$0.00 \mathrm{E}+01$

$0.00 \mathrm{E}+01$

$0.00 \mathrm{E}+01$

4.03E-05

$0.00 \mathrm{E} \div 01$

$5.87 \mathrm{E}-03$

$1.38 \mathrm{E}-00$

9.31E-02

$1.47 \mathrm{E}-01$

$6.15 \mathrm{E}-07$

$0.00 \mathrm{E}+01$

$0.00 \mathrm{E}+01$

$0.00 \mathrm{E}+01$

$0.00 \mathrm{E}+01$

3.53E-04

$0.00 \mathrm{E}+01$

$8.74 E+02$

$0.00 \mathrm{E}+01$

$0.00 \mathrm{E}+01$

$0.00 \mathrm{E}+01$

$0.00 \mathrm{E}+0 \mathrm{I}$

$4.05 \mathrm{E}-00$

$0.00 \mathrm{E}+01$

$0.00 \mathrm{E}+01$

$0.00 \mathrm{E}+01$

$0.00 \mathrm{E}+01$

$0.00 \mathrm{E}+01$

$0.00 \mathrm{E}+01$

$0.00 \mathrm{E}+01$

$0.00 \mathrm{E}+01$

$2.90 \mathrm{E}+03$

$3.05 \mathrm{E}+02$

$2.03 E+01$

$3.76 \mathrm{E}+01$

$2.40 \mathrm{E}+02$

$3.18 E+01$

$3.55 \mathrm{E}+01$

$2.78 \mathrm{E}+02$

$0.00 \mathrm{E}+01$

$1.56 \mathrm{E}+01$

1.50E-0I

6.68E-00

$5.48 \mathrm{E}+03$

$7.97 \mathrm{E}-01$

$4.20 E+02$

$2.01 E+03$

$3.42 E+02$

$2.46 \mathrm{E}-00$

$3.31 \mathrm{E}+02$

$3.90 E+01$

$9.09 \mathrm{E}+01$

$1.28 \mathrm{E}+02$

$5.52 \mathrm{E}-00$

2.61E+01

$1.01 \mathrm{E}+02$

2.2IE-0I

$0.00 \mathrm{E}+0 \mathrm{I}$

$2.80 \mathrm{E}-00$

$1.72 \mathrm{E}-08$

$4.50 \mathrm{E}-00$

$0.00 \mathrm{E}+01$

$0.00 \mathrm{E}+0 \mathrm{I}$

$1.66 \mathrm{E}-05$

3.74E-07

5.11E-07

$3.73 \mathrm{E}-01$

9.26E-07

$1.43 \mathrm{E}+03$ 10\%-ile

\section{$0.00 \mathrm{E}+01$}

6.56E-04

2.05E-04

1.44E-06

$2.98 \mathrm{E}-02$

$7.00 \mathrm{E}-00$

4.73E-01

$7.48 \mathrm{E}-01$

3.12E-06

9.02E-08

2.32E-12

5.32E-10

$0.00 \mathrm{E}+01$

$1.55 \mathrm{E}-03$

$0.00 \mathrm{E}+01$

$7.01 \mathrm{E}+03$

$2.00 \mathrm{E}+01$

$6.38 \mathrm{E}-10$

$6.92 \mathrm{E}-13$

$1.05 \mathrm{E}-03$

5.29E-00

$5.25 \mathrm{E}-06$

2.44E-07

$2.28 \mathrm{E}-01$

$1.00 \mathrm{E}-02$

$2.26 \mathrm{E}-03$

2.31E-01

$0.00 \mathrm{E}+01$

$0.00 \mathrm{E}+01$

$4.83 \mathrm{E}+03$

$5.44 E+02$

$3.58 \mathrm{E}+01$

$7.96 \mathrm{E}+01$

$6.51 \mathrm{E}+02$

$7.25 \mathrm{E}+01$

$1.17 \mathrm{E}+02$

$5.35 \mathrm{E}+02$

7.17E-00

3.30E+01

$5.71 \mathrm{E}-01$

$2.33 \mathrm{E}+01$

$8.30 \mathrm{E}+03$

2.96E-00

9.71E $\div 02$

$6.65 \mathrm{E}+03$

$6.94 E+02$

$7.51 \mathrm{E}-00$

$7.76 \mathrm{E}+02$

$9.78 \mathrm{E}+01$

$2.25 \mathrm{E}+02$

$3.09 \mathrm{E}+02$

$1.28 \mathrm{E}+01$

$7.33 \mathrm{E}+01$

$2.62 \mathrm{E}+02$

$1.51 \mathrm{E}-00$

1.54E-07.

4.43E-00

8.29E-08

$6.88 \mathrm{E}+02$

$1.09 \mathrm{E}-03$

5.25E-03

7.99E-05

$1.80 \mathrm{E}-06$

2.46E-06

$5.33 \mathrm{E}-01$

$1.42 \mathrm{E}-04$

$3.59 \mathrm{E}+03$
$50 \%$-ile

\subsection{E-02}

$4.80 \mathrm{E}-03$

4.35E-01

7.28E-04

9.88E-06

1.06E-01

$2.49 \mathrm{E}+01$

$1.68 \mathrm{E}-00$

2.66E-00

1.11E-05

8.76E-07

2.25E-11

1.88E-09

$4.00 \mathrm{E}+02$

3.57E-03

1.32E+01

$9.27 \mathrm{E}+04$

$2.30 \mathrm{E}+02$

4.37E-09

4.74E-1?

5.38E-03

7.60E-00

$3.60 \mathrm{E}-05$

1.67E-06

$1.56 \mathrm{E}-00$

6.88E-02

$1.55 \mathrm{E}-02$

$1.58 \mathrm{E}-00$

$1.70 \mathrm{E}+05$

1.69E-02

$7.46 \mathrm{E}+03$

$1.02 \mathrm{E}+03$

$8.61 E+01$

$1.71 \mathrm{E}+02$

$1.32 \mathrm{E}+03$

$1.77 \mathrm{E}+02$

$5.50 \mathrm{E}+02$

$1.04 \mathrm{E}+03$

$3.91 \mathrm{E}+01$

$6.55 \mathrm{E}+01$

$1.13 \mathrm{E}-00$

$1.18 \mathrm{E}+02$

$1.22 \mathrm{E}+04$

8.29E-00

$2.32 \mathrm{E}+03$

$1.47 \mathrm{E}+04$

$1.52 \mathrm{E}+03$

$2.45 \mathrm{E}+01$

$1.90 \mathrm{E}+03$

$2.61 \mathrm{E}+02$

$4.75 \mathrm{E}+02$

$6.88 \mathrm{E}+02$

$3.27 \mathrm{E}+01$

$2.03 E+02$

$6.14 E+02$

8.34E-00

1.66E-06

8.09E-00

$2.46 \mathrm{E}-07$

$1.87 \mathrm{E}+03$

$9.25 \mathrm{E}-03$

2.86E-02

2.37E-04

5.34E-06

$7.30 \mathrm{E}-06$

8.99E-01

3.85E-04

$7.32 \mathrm{E}+03$

\section{$90 \%$-ile}

\subsection{E-02}

$1.60 \mathrm{E}-02$

$1.45 \mathrm{E}-00$ 
Tank Anal. Un

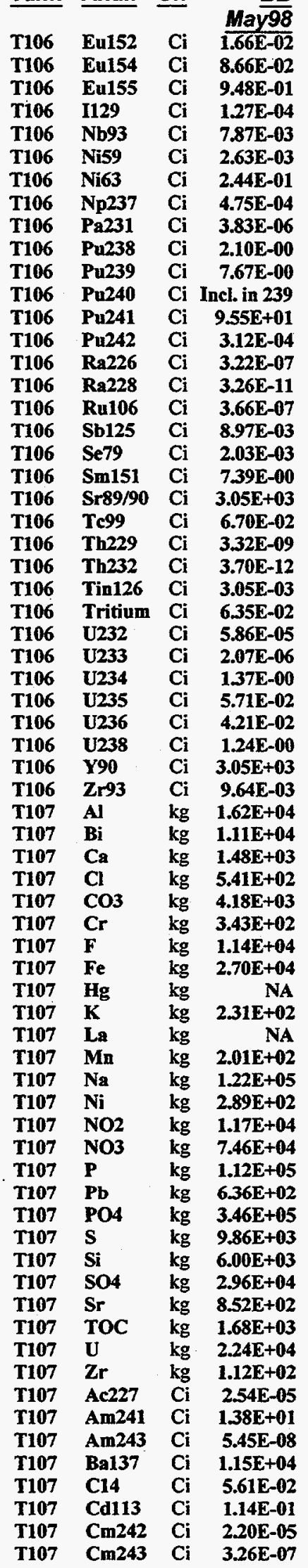

\section{$B B$} Sept98

$\overline{1.66 \mathrm{E}-02}$ 8.66E-02 $9.48 \mathrm{E}-01$

$1.27 \mathrm{E}-04$

$7.87 \mathrm{E}-03$

2.63E-03

2.44E-01

4.75E-04

$3.83 \mathrm{E}-06$

$1.66 \mathrm{E}-01$

$6.66 \mathrm{E}-00$

1.01E-00

7.56E- 00

2.47E-05

3.22E-07

3.26E-11

3.66E-07

8.97E-03

2.03E-03

7.39E-00

$3.05 \mathrm{E}+03$

6.70E-02

3.32E-09

3.70E-12

3.05E-03

$6.35 \mathrm{E}-02$

$1.36 \mathrm{E}-05$

4.82E-07

3.18E-01

1.33E-02

$9.77 \mathrm{E}-03$

2.87E-01

$3.05 E+03$

$9.64 \mathrm{E}-03$

$1.62 \mathrm{E}+04$

$1.11 \mathrm{E}+04$

$1.48 \mathrm{E}+03$

$5.41 \mathrm{E}+02$

4.18E +03

$3.43 \mathrm{E}+02$

$1.14 \mathrm{E}+04$

$2.70 \mathrm{E}+0.4$

$1.42 \mathrm{E}+02$

2.31E +02

$0.00 \mathrm{E}+01$

$2.01 \mathrm{E}+02$

$1.22 \mathrm{E}+05$

$2.89 \mathrm{E}+02$

1.17E+04

$7.46 \mathrm{E}+04$

NA

$6.36 \mathrm{E}+02$

1.13E+05

NA

$6.00 E+03$

$9.86 \mathrm{E}+03$

$8.52 \mathrm{E}+02$

$1.68 \mathrm{E}+03$

$2.24 E+04$

$1.12 \mathrm{E}+02$

2.54E-05

$1.38 \mathrm{E}+01$

3.86E-05

$1.15 \mathrm{E}+04$

5.61E-02

$1.14 \mathrm{E}-01$

$1.56 \mathrm{E}-02$ 2.31E-04

\section{1\%-ile}

$0.00 \mathrm{E}+01$

$0.00 \mathrm{E}+01$

$0.00 \mathrm{E}+01$

$5.05 \mathrm{E}-09$

$0.00 \mathrm{E}+01$

2.27E-04

2.11E-02

3.33E-05

$0.00 \mathrm{E}+01$

$1.47 \mathrm{E}-01$

5.37E-01

5.37E-0?

6.69E-00

2.18E-05

4.10E-08

4.15E-12

$0.00 \mathrm{E}+01$

$0.00 \mathrm{E}+01$

$3.18 \mathrm{E}-04$

$0.00 \mathrm{E}+01$

$7.26 \mathrm{E}+01$

$0.00 \mathrm{E}+01$

$0.00 \mathrm{E}+01$

$0.00 \mathrm{E}+01$

$0.00 \mathrm{E}+01$

$3.09 \mathrm{E}-02$

$0.00 \mathrm{E}+01$

$0.00 \mathrm{E}+01$

$0.00 \mathrm{E}+01$

$0.00 \mathrm{E}+01$

$0.00 \mathrm{E}+0 \mathrm{I}$

$0.00 \mathrm{E}+01$

$6.91 \mathrm{E}-00$

$0.00 \mathrm{E}+01$

$2.59 \mathrm{E}+02$

$5.38 \mathrm{E}+03$

$4.27 \mathrm{E}+0 \mathrm{I}$

$2.08 \mathrm{E}+02$

$8.54 \mathrm{E}+01$

$6.10 \mathrm{E}-00$

$1.61 \mathrm{E}+02$

$2.97 \mathrm{E}+03$

NA

4. $12 \mathrm{E}+01$

1.07E-01

$3.20 \mathrm{E}-00$

$3.57 \mathrm{E}+04$

$1.87 \mathrm{E}-00$

$7.58 \mathrm{E}+02$

$1.14 \mathrm{E}+04$

$2.80 \mathrm{E}+03$

$6.72 \mathrm{E}-00$

$1.39 \mathrm{E}+03$

$9.69 \mathrm{E}+01$

$5.00 \mathrm{E}+02$

$8.49 \mathrm{E}+02$

$7.65 \mathrm{E}-00$

3.10E-03

$1.67 \mathrm{E}+01$

$7.83 \mathrm{E}-01$

$0.00 \mathrm{E}+01$

8.73E-01

3.76E- 09

$7.44 \mathrm{E}+02$

$0.00 \mathrm{E}+01$

$0.00 \mathrm{E}+01$

$1.52 \mathrm{E}-06$

2.25E-08

\section{$10 \%$-ile}

\section{$1.54 \mathrm{E}-03$}

8.01E-03

8.77E-02

4.00E-05

$1.24 \mathrm{E}-03$

9.19E-04

8.53E-02

1.60E-04

6.97E-07

$7.08 \mathrm{E}-01$

2.59E-00

2.59E-00

$3.22 \mathrm{E}+01$

$1.05 \mathrm{E}-04$

$1.16 \mathrm{E}-07$

$1.18 \mathrm{E}-11$

8.86E-08

1.71E-03

8.72E-04

6.83E-01

$9.53 \mathrm{E}+02$

$0.00 \mathrm{E}+01$

$6.04 \mathrm{E}-10$

$6.73 \mathrm{E}-13$

$5.59 \mathrm{E}-04$

4.49E-02

1.07E-05

3.77E-07

2.49E-01

1.04E-02

7.66E-03

2.26E-01

$1.09 \mathrm{E} \div 02$

$1.52 \mathrm{E}-03$

1. $35 \mathrm{E}+03$

$8.94 \mathrm{E}+03$

$2.05 \mathrm{E}+02$

$4.84 \mathrm{E}+02$

$2.83 \mathrm{E}+03$

$2.86 \mathrm{E}+02$

$8.31 \mathrm{E}+02$

$5.97 \mathrm{E}+03$

NA

$1.43 \mathrm{E}+02$

$7.71 \mathrm{E}-01$

$2.23 \mathrm{E}+01$

$5.13 \mathrm{E}+04$

$7.24 \mathrm{E}-00$

$3.01 \mathrm{E}+03$

$3.81 E+04$

$8.40 \mathrm{E}+03$

$5.73 E+01$

$6.30 \mathrm{E}+03$

$9.31 \mathrm{E}+02$

$2.17 \mathrm{E}+03$

$3.50 \mathrm{E}+03$

$4.95 \mathrm{E}+01$

$1.69 \mathrm{E}+02$

$3.20 \mathrm{E}+02$

$5.21 \mathrm{E}-00$

5.09E-06

$1.14 \mathrm{E}+01$

1.73E-08

$3.74 \mathrm{E}+03$

$0.00 \mathrm{E}+01$

1.62E-02

6.98E-06

1.03E-07

\section{0\%-ile}

\section{$1.66 \mathrm{E}-02$}

8.66E-02

9.48E-01

1.27E-04

$7.87 \mathrm{E}-03$

2.63E-03

2.44E-01

4.75E-04

3.83E-06

2.10E-00

7.67E-00

7.67E-00

$9.55 \mathrm{E}+01$

3.12E-04

3.22E-07

$3.26 \mathrm{E}-11$

3.66E-07

8.97E-03

2.03E-03

7.39E-00

$2.63 \mathrm{E}+04$

6.70E-02

3.32E-09

3.70E-12

3.05E-03

$6.35 \mathrm{E}-02$

5.86E-05

2.07E-06

1.37E-00

5.71E-02

4.21E-02

$1.24 \mathrm{E}-00$

$3.05 \mathrm{E}+03$

9.64E- 03

$1.09 \mathrm{E}+04$

$1.45 \mathrm{E}+04$

$5.65 \mathrm{E}+02$

$8.96 \mathrm{E}+02$

8.17E+03

$6.85 \mathrm{E}+02$

$5.40 \mathrm{E}+03$

$1.14 E+04$

$$
\text { NA }
$$

$3.15 \mathrm{E}+02$

3.07E-00

$6.07 \mathrm{E}+01$

$7.69 \mathrm{E}+04$

$2.13 \mathrm{E}+01$

$9.29 \mathrm{E}+03$

$1.06 \mathrm{E}+05$

$1.60 \mathrm{E}+04$

$2.51 \mathrm{E}+02$

$1.58 \mathrm{E}+04$

$3.12 \mathrm{E}+03$

$5.70 \mathrm{E}+03$

$8.56 \mathrm{E}+03$

$1.51 \mathrm{E}+02$

$5.29 \mathrm{E}+02$

$1.40 \mathrm{E}+03$

$3.23 \mathrm{E}+01$

2.54E-05

$5.37 \mathrm{E}+01$

5.45E-08

$1.15 \mathrm{E}+04$

5.61E-02

1.14E-01

2.20E-05

3.26E-07

$90 \%$-ile

$8.08 \mathrm{E}-02$ 
Tank Anal. Un

\begin{tabular}{|c|c|c|c|}
\hline T107 & Cm244 & $\mathrm{Ci}$ & $\frac{\text { Мау } 98}{8.34 \mathrm{E}-07}$ \\
\hline T107 & Co60 & $\mathrm{Ci}$ & $7.82 \mathrm{E}-03$ \\
\hline T107 & Cs134 & $\mathrm{Ci}$ & $6.69 \mathrm{E}-05$ \\
\hline T107 & Cs137 & $\mathrm{Ci}$ & $1.22 \mathrm{E}+04$ \\
\hline T107 & En152 & $\mathrm{Ci}$ & $5.47 \mathrm{E}-03$ \\
\hline T107 & Eu154 & $\mathrm{Ci}$ & $1.05 E-01$ \\
\hline T107 & Eu155 & $\mathrm{Ci}$ & $8.28 E-01$ \\
\hline T107 & I129 & $\mathrm{Ci}$ & $7.22 \mathrm{E}-04$ \\
\hline T107 & Nb93 & $\mathrm{Ci}$ & $4.85 E-02$ \\
\hline T107 & Ni59 & $\mathrm{Ci}$ & $1.60 \mathrm{E}-02$ \\
\hline T107 & Ni63 & $\mathrm{Ci}$ & $1.40 \mathrm{E}-00$ \\
\hline T107 & Np237 & $\mathrm{Ci}$ & 2.33E-03 \\
\hline T107 & Pa231 & $\mathrm{Ci}$ & 5.28E-05 \\
\hline T107 & Pu238 & $\mathrm{Ci}$ & $3.47 \mathrm{E}-02$ \\
\hline T107 & Pu239 & $\mathrm{Ci}$ & $1.48 \mathrm{E}+02$ \\
\hline T107 & Pu240 & $\mathrm{Ci}$ & Incl. in 239 \\
\hline T107 & Pu241 & $\mathrm{Ci}$ & 4.10E-01 \\
\hline T107 & Pu242 & $\mathrm{Ci}$ & $1.25 \mathrm{E}-06$ \\
\hline T107 & Ra226 & $\mathrm{Ci}$ & $5.03 E-06$ \\
\hline T107 & $\mathbf{R a 2 2 8}$ & $\mathrm{Ci}$ & 7.45E-11 \\
\hline T107 & Ru106 & $\mathrm{Ci}$ & 1.84E-10 \\
\hline T107 & Sb125 & $\mathrm{Ci}$ & $4.22 \mathrm{E}-03$ \\
\hline T107 & Se79 & $\mathbf{C i}$ & $1.18 \mathrm{E}-02$ \\
\hline T107 & Sm151 & $\mathrm{Ci}$ & $4.46 \mathrm{E}+01$ \\
\hline T107 & Sr89/90 & $\mathrm{Ci}$ & $1.07 E+05$ \\
\hline T107 & Tc99 & $\mathrm{Ci}$ & 3.88E-01 \\
\hline T107 & Th229 & $\mathrm{Ci}$ & $1.44 \mathrm{E}-08$ \\
\hline T107 & Th232 & $\mathrm{Ci}$ & $1.74 \mathrm{E}-11$ \\
\hline T107 & Tin126 & $\mathbf{C i}$ & $1.75 \mathrm{E}-02$ \\
\hline T107 & Tritium & $\mathbf{C i}$ & 2.19E-01 \\
\hline T107 & $\mathrm{U} 232$ & $\mathrm{Ci}$ & $1.22 \mathrm{E}-0.4$ \\
\hline T107 & $\mathbf{U} 233$ & $\mathrm{Ci}$ & $7.33 \mathrm{E}-06$ \\
\hline T107 & U234 & $\mathbf{C i}$ & $1.03 E+01$ \\
\hline T107 & U235 & $\mathbf{C i}$ & 4.64E-01 \\
\hline T107 & U236 & $\mathbf{C i}$ & $6.59 \mathrm{E}-02$ \\
\hline T107 & U238 & $\mathbf{C i}$ & $1.05 E+01$ \\
\hline T107 & Y90 & $\mathrm{Ci}$ & $1.07 E+05$ \\
\hline T107 & Zr93 & $\mathrm{Ci}$ & 5.62E-02 \\
\hline T108 & AI & kg & $2.16 E+03$ \\
\hline T108 & $\mathbf{B i}$ & kg & $1.77 \mathrm{E}+03$ \\
\hline T108 & $\mathrm{Ca}$ & $\mathrm{kg}$ & $1.92 \mathrm{E}+02$ \\
\hline T108 & Cl & kg & 2.05E +02 \\
\hline T108 & $\mathrm{CO3}$ & kg & $1.40 \mathrm{E}+03$ \\
\hline T108 & $\mathrm{Cr}$ & kg & $7.28 \mathrm{E}+01$ \\
\hline T108 & $\mathbf{F}$ & $\mathrm{kg}$ & $2.73 E+03$ \\
\hline T108 & $\mathbf{F e}$ & $\mathrm{kg}$ & $3.19 \mathrm{E}+03$ \\
\hline T108 & $\mathbf{H g}$ & $\mathrm{kg}$ & $1.78 \mathrm{E}-00$ \\
\hline T108 & $\mathbf{K}$ & kg & $6.19 \mathrm{E}+01$ \\
\hline T108 & $\mathbf{L a}$ & kg & $7.10 \mathrm{E}-00$ \\
\hline T108 & $\mathbf{M n}$ & kg & $4.33 E+01$ \\
\hline T108 & $\mathbf{N a}$ & kg & $4.45 E+04$ \\
\hline T108 & $\mathbf{N i}$ & kg & $1.93 E+01$ \\
\hline T108 & NO2 & kg & $1.82 E+03$ \\
\hline T108 & No3 & $\mathrm{kg}$ & $6.67 E+04$ \\
\hline T108 & $\mathbf{P}$ & kg & $2.92 E+04$ \\
\hline T108 & $\mathbf{P b}$ & $\mathrm{kg}$ & $1.28 \mathrm{E}+02$ \\
\hline T108 & PO4 & kg & $9.04 E+04$ \\
\hline T108 & $\mathbf{S}$ & $\mathrm{kg}$ & $1.92 E+03$ \\
\hline T108 & $\mathbf{S i}$ & kg & $9.28 E+02$ \\
\hline T108 & SO4 & $\mathbf{k g}$ & $5.76 E+03$ \\
\hline T108 & Sr & kg & $6.24 \mathrm{E}+01$ \\
\hline T108 & TOC & $\mathrm{kg}$ & $2.03 E+01$ \\
\hline T108 & $\mathbf{U}$ & kg & $1.47 E+03$ \\
\hline T108 & $\mathbf{Z r}$ & $\mathrm{kg}$ & $1.17 \mathrm{E}+01$ \\
\hline T108 & Ac227 & $\mathrm{Ci}$ & $1.52 \mathrm{E}-05$ \\
\hline T108 & Am241 & $\mathrm{Ci}$ & $6.98 E-00$ \\
\hline T108 & Am243 & $\mathbf{C i}$ & 4.97E-07 \\
\hline 10 & Ba137 & $\mathrm{Ci}$ & $9.44 E+02$ \\
\hline
\end{tabular}

BB Sept98 5.91E-04

7.82E-03

6.69E-05

$1.22 \mathrm{E}+04$

5.47E-03

$1.05 \mathrm{E}-01$

8.28E-01

7.22E-04

$4.85 \mathrm{E}-02$

1.60E-02

$1.40 \mathrm{E}-00$

$233 \mathrm{E}-03$

5.28E-05

4.46E-01

$1.40 \mathrm{E}+02$

8.24E-00

5.27E-00

$1.61 \mathrm{E}-05$

5.03E-06

7.45E-11

1.84E-10

4.22E-03

1.18E-02

$4.46 \mathrm{E}+01$

1.07E+05

3.88E-01

1.44E-08

1.74E-11

1.75E-02

2.19E-01

8.75E-05

5.24E-06

7.38E-00

3.32E-01

4.72E-02

7.48E-00

$1.07 \mathrm{E}+05$

5.62E-02

$2.16 E+03$

$1.77 \mathrm{E}+03$

$1.92 \mathrm{E}+02$

$1.19 \mathrm{E}+02$

$1.40 \mathrm{E}+03$

$7.28 \mathrm{E}+01$

$2.73 E+03$

$3.19 \mathrm{E}+03$

6.69E-00

$6.19 \mathrm{E}+01$

$0.00 E+01$

$4.33 E+01$

$4.45 \mathrm{E}+04$

$1.93 E+01$

$1.82 \mathrm{E}+03$

$6.67 E+04$

NA

$1.28 \mathrm{E}+02$

$2.95 E+04$

NA

$9.28 \mathrm{E}+02$

$1.92 \mathrm{E}+03$

$6.24 \mathrm{E}+01$

2.03E-01

$1.47 E+03$

$1.17 \mathrm{E}+01$

1.52E-05

2.71E-01

1.84E-06

$9.44 \mathrm{E}+02$
$1 \%$ ile

\subsection{E-08}

$10 \%-j l e$

$0.00 \mathrm{E}+01$

4.33E-06

$1.44 \mathrm{E}+03$

$0.00 \mathrm{E}+01$

$0.00 \mathrm{E}+01$

$0.00 \mathrm{E}+01$

$0.00 \mathrm{E}+0$

$0.00 \mathrm{E}+01$

$5.16 \mathrm{E}-04$

4.52E-02

1.61E-04

$0.00 \mathrm{E}+01$

2.39E-03

$1.02 \mathrm{E}+01$

$1.02 \mathrm{E}+01$

$2.83 \mathrm{E}-02$

8.62E-08

$0.00 \mathrm{E}+01$

$0.00 \mathrm{E}+01$

$0.00 \mathrm{E}+01$

$0.00 \mathrm{E}+01$

$1.19 \mathrm{E}-04$

$0.00 \mathrm{E}+0 \mathrm{l}$

$2.98 \mathrm{E}+02$

$1.78 \mathrm{E}-02$

$0.00 \mathrm{E}+01$

$0.00 \mathrm{E}+01$

$0.00 \mathrm{E}+01$

$9.13 \mathrm{E}-02$

$0.00 \mathrm{E}+01$

$0.00 \mathrm{E}+01$

$0.00 E+01$

$0.00 E+01$

$0.00 \mathrm{E}+01$

$0.00 \mathrm{E}+01$

$0.00 E+01$

$0.00 \mathrm{E}+01$

4.22E- 00

$6.80 \mathrm{E}+01$

2.75E-00

4.57E-00

3.27E-01

8.94E-01

7.44E-00

4.14E+01

$0.00 E+01$

$1.32 \mathrm{E}-00$

$1.41 \mathrm{E}-03$

$1.43 \mathrm{E}-01$

$3.96 \mathrm{E}+02$

2.93E-06

$4.19 \mathrm{E}+01$

$3.86 \mathrm{E}+02$

$6.30 \mathrm{E}+01$

2.76E-01

6.17E+01

$1.12 \mathrm{E}+01$

$1.17 \mathrm{E}+01$

$3.97 \mathrm{E}+01$

$2.50 \mathrm{E}-01$

$1.49 \mathrm{E}-03$

3.01E-00

2.61E-02

$0.00 E+01$

$1.22 \mathrm{E}-02$

5.53E-09

5.19E-00

\subsection{E-07}

$2.70 \mathrm{E}-01$

$2.18 \mathrm{E}-05$

$6.09 \mathrm{E}+03$

1. $10 E-03$

$2.10 E-02$

$1.66 \mathrm{E}-01$

$2.30 E-04$

$9.88 \mathrm{E}-04$

$4.80 \mathrm{E}-03$

$4.20 \mathrm{E}-01$

7.39E-04

8.71E-06

$1.10 \mathrm{E}-02$

4.70E+01

$4.70 \mathrm{E}+01$

$1.30 \mathrm{E}-01$

3.97E-07

9.30E-07

$1.38 \mathrm{E}-11$

5.47E-11

$0.00 \mathrm{E}+01$

4.13E-03

8.94E-00

$3.05 E+03$

$1.41 \mathrm{E}-01$

2.37E-09

$2.87 \mathrm{E}-12$

2.49E-03

$1.40 \mathrm{E}-01$

2.01E-05

1.21E-06

1.70E-00

$7.65 \mathrm{E}-02$

$1.09 \mathrm{E}-02$

$1.73 \mathrm{E}-00$

$1.76 \mathrm{E}+04$

$1.15 \mathrm{E}-03$

$2.11 \mathrm{E}+02$

$8.86 \mathrm{E}+02$

$2.78 \mathrm{E}+01$

$6.19 \mathrm{E}+01$

$4.03 E+02$

$3.37 \mathrm{E}+01$

$1.79 \mathrm{E}+02$

$6.70 \mathrm{E}+02$

$1.38 \mathrm{E}-01$

$1.66 \mathrm{E}+01$

$1.29 \mathrm{E}-01$

3.05E-00

8.46E-0I

$3.95 \mathrm{E}+02$

$5.19 E+03$

$1.12 \mathrm{E} \div 03$

$1.01 \mathrm{E}+01$

$1.10 E+03$

$1.75 E+02$

$2.61 \mathrm{E}+02$

$5.98 \mathrm{E}+02$

$6.38 \mathrm{E}-0.0$

$2.48 \mathrm{E}+0 \mathrm{I}$

$5.90 \mathrm{E}+01$

6.99E-01

$7.54 \mathrm{E}-07$

1.79E-00

$1.02 \mathrm{E}-07$

$1.63 E+02$
$6.54 E+03$ 50\%-ile

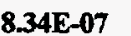

3.25E-00

6.69E-05

$1.81 \mathrm{E}+04$

5.47E-03

1.05E-01

8.28E-01

7.22E-04

4.85E-02

1.60E-02

1.40E-00

2.33E-03

5.28E-05

3.47E-02

$1.48 \mathrm{E}+02$

$1.48 \mathrm{E}+02$

4.10E-01

1.25E-06

5.03E-06

7.45E-11

$1.84 \mathrm{E}-10$

4.22E-03

$1.18 \mathrm{E}-02$

$4.46 \mathrm{E}+01$

$1.62 \mathrm{E}+04$

3.88E-01

$1.44 \mathrm{E}-08$

$1.74 \mathrm{E}-11$

1.75E-02

2.19E-01

1.22E-04

7.33E-06

$1.03 \mathrm{E}+01$

4.64E-01

6.59E-02

$1.05 \mathrm{E}+01$

$1.07 \mathrm{E}+05$

5.62E-02

$2.54 \mathrm{E}+03$

$2.91 \mathrm{E}+03$

$1.40 \mathrm{E}+02$

$2.14 \mathrm{E}+02$

$1.76 \mathrm{E}+03$

$1.53 \mathrm{E}+02$

$1.44 \mathrm{E}+03$

$2.41 \mathrm{E}+03$

$1.78 \mathrm{E}-\mathrm{-00}$

$8.82 E+01$

$1.16 \mathrm{E}-00$

$1.47 \mathrm{E}+01$

$2.10 \mathrm{E}+04$

5.30E-00

$2.24 \mathrm{E}+03$

$2.40 \mathrm{E}+04$

$4.08 \mathrm{E}+03$

$6.30 \mathrm{E}+01$

$4.45 E+03$

$1.01 \mathrm{E}+03$

$1.12 E+03$

$2.94 E+03$

$3.41 \mathrm{E}+01$

$1.43 E+02$

$2.98 E+02$

$1.04 \mathrm{E}+01$

1.52E-05

$1.20 \mathrm{E}+01$

4.97E-07

$9.44 \mathrm{E}+02$

$90 \%$-ile

$99 \%$-ile

$1.76 \mathrm{E}-06$

3.26E-06

4.42E+02

6.23E-04

$1.66 \mathrm{E}+05$ 


\begin{tabular}{|c|c|c|c|c|c|c|c|c|c|c|c|}
\hline ank & nal. & $n$ & $\frac{B B}{\text { May } 98}$ & $\operatorname{Sep} \frac{B B}{\operatorname{Bg}}$ & $1 \%$-ile & $10 \%$-ile & $50 \%$-ile & 90\%-ile & $99 \%$-ile & Mean & td Dev \\
\hline T108 & $\mathrm{Cl4}$ & $\mathbf{C i}$ & $4 \overline{457 \mathrm{E}-02}$ & $\overline{4.57 \mathrm{E}-02}$ & $0.00 E+01$ & $3.30 \mathrm{E}-03$ & 4.57E-02 & $1 E-01$ & 24E-01 & $5.95 \mathrm{E}-02$ & $24 \mathrm{E}-02$ \\
\hline T108 & Cd113 & $\mathrm{Ci}$ & $1.09 \mathrm{E}-01$ & $1.09 \mathrm{E}-01$ & $0.00 \mathrm{E}+01$ & $E-03$ & & & $E-00$ & & \\
\hline T108 & Cm242 & $\mathbf{C i}$ & $1.65 E-04$ & $6.08 \mathrm{E}-04$ & $1.83 \mathrm{E}-06$ & $3.38 \mathrm{E}-05$ & $1.65 E-04$ & $1 \mathrm{E}-04$ & $9.71 \mathrm{E}-04$ & 3E-04 & $1.92 \mathrm{E}-04$ \\
\hline T108 & Cm243 & $\mathbf{C i}$ & 3.35E-06 & $1.24 \mathrm{E}-05$ & $3.73 \mathrm{E}-08$ & $6.87 \mathrm{E}-07$ & 3.35E-06 & $8.96 \mathrm{E}-06$ & $1.97 \mathrm{E}-05$ & 4.33E-06 & $3.90 \mathrm{E}-06$ \\
\hline T108 & $\mathrm{Cm} 244$ & $\mathrm{Ci}$ & $1.09 \mathrm{E}-05$ & $4.04 \mathrm{E}-05$ & $1.21 \mathrm{E}-07$ & $2.24 \mathrm{E}-06$ & $1.09 \mathrm{E}-05$ & $2.92 \mathrm{E}-05$ & $642 \mathrm{~F}-05$ & $1.41 E-05$ & $1.27 \mathrm{E}-05$ \\
\hline T108 & Co60 & $\mathrm{Ci}$ & $3.34 E-00$ & $9.71 \mathrm{E}-03$ & $0.00 \mathrm{E}+01$ & $6.56 \mathrm{E}-02$ & $1.04 \mathrm{E}-00$ & $6 \mathrm{E}-00$ & $8 \mathrm{E}+02$ & $5.53 \mathrm{E}-00$ & $2.06 \mathrm{E}+01$ \\
\hline 8 & Cs134 & $\mathrm{Ci}$ & $3.76 \mathrm{E}-04$ & $3.76 \mathrm{E}-04$ & 2.07E-06 & $6.47 \mathrm{E}-05$ & -04 & E-03 & & E-04 & $E-03$ \\
\hline & Cs137 & $\mathrm{Ci}$ & $98 \mathrm{E}+02$ & $98 \mathrm{E}+02$ & $E+01$ & $95 E+02$ & $5.06 \mathrm{E}+03$ & $2.80 \mathrm{E}+04$ & & $E+04$ & $1.75 \mathrm{E}+04$ \\
\hline & Eu152 & $\mathrm{Ci}$ & $1.05 \mathrm{E}-02$ & $1.05 \mathrm{E}-02$ & $E \div 01$ & $1 E-04$ & $1.05 E-02$ & $.77 \mathrm{E}-02$ & -01 & $3.29 \mathrm{E}-02$ & 5.31E-02 \\
\hline 08 & Eu154 & $\mathbf{C i}$ & $1.10 E+01$ & $1.69 \mathrm{E}-01$ & $E+01$ & $46 \mathrm{E}-01$ & $1.10 E+01$ & $E+02$ & +02 & $E+01$ & $5.56 \mathrm{E}+01$ \\
\hline T108 & Eu155 & Ci & $.15 \mathrm{E}+01$ & $8.54 \mathrm{E}-01$ & $0.00 \mathrm{E}+01$ & & $1.15 E+01$ & $E+02$ & & & $1 E+01$ \\
\hline 108 & 1129 & $\mathrm{Ci}$ & $6.05 E-04$ & $6.05 E-04$ & $0.00 \mathrm{E}+01$ & $1.09 \mathrm{E}-04$ & $6.05 \mathrm{E}-04$ & E-03 & & $E-04$ & $.06 \mathrm{E}-04$ \\
\hline T108 & Nb93 & $\mathbf{C i}$ & 3.94E-02 & $3.94 \mathrm{E}-02$ & $0.00 \mathrm{E}+01$ & $0.00 \mathrm{E}+01$ & $E-02$ & & & E-02 & $5 \mathrm{E}-01$ \\
\hline T108 & Ni59 & $\mathbf{C i}$ & 8.07E-02 & $8.07 \mathrm{E}-02$ & $0.00 \mathrm{E}+01$ & $E-02$ & $E-02$ & E-01 & & $E-01$ & E-01 \\
\hline T108 & Ni63 & $\mathrm{Ci}$ & $7.26 \mathrm{E}-00$ & $7.26 \mathrm{E}-00$ & $0.00 \mathrm{E}+01$ & 00 & & $E+01$ & & +01 & +01 \\
\hline 108 & 237 & $\mathbf{C i}$ & $1.97 \mathrm{E}-03$ & $1.97 \mathrm{E}-03$ & $E-05$ & & & & & -03 & -03 \\
\hline 108 & Pa231 & $\mathbf{C i}$ & 3.27E-05 & $3.27 \mathrm{E}-05$ & $0.00 \mathrm{E}+01$ & & & & & & \\
\hline T108 & Pu238 & $\mathrm{Ci}$ & $3.64 E-02$ & $1.35 E-01$ & $E-04$ & .03 & & .02 & -01 & -02 & \\
\hline T108 & Pu239 & $\mathbf{C i}$ & $6.68 \mathrm{E}-00$ & $2.47 \mathrm{E}+01$ & $E-02$ & & & & & & \\
\hline T108 & Pu240 & $\mathrm{Ci}$ & $5.14 \mathrm{E}-01$ & $1.90 \mathrm{E}-00$ & $5.72 \mathrm{E}-03$ & & & & & & -01 \\
\hline T108 & Pu241 & $\mathbf{C i}$ & $1.23 \mathrm{E}-00$ & $4.56 \mathrm{E}-00$ & $1.37 \mathrm{E}-02$ & & & & & & $E-00$ \\
\hline T108 & Pu242 & $\mathrm{Ci}$ & $5.43 E-06$ & $2.01 E-05$ & -08 & & & & & & \\
\hline T108 & $\operatorname{Ra226}$ & $\mathrm{Ci}$ & $2.96 \mathrm{E}-06$ & $2.96 \mathrm{E}-06$ & +01 & & & & & & -06 \\
\hline T108 & Ra228 & $\mathbf{C i}$ & $4.83 \mathrm{E}-11$ & $4.83 \mathrm{E}-11$ & +01 & & & & & & \\
\hline T108 & Ru106 & $\mathbf{C i}$ & $3.49 \mathrm{E}-09$ & $3.49 \mathrm{E}-09$ & $0.00 \mathrm{E}+01$ & 4.2 & -09 & & & -09 & -09 \\
\hline T108 & Sb125 & $\mathrm{Ci}$ & $8.45 E-03$ & $8.45 \mathrm{E}-03$ & $8+01$ & & & & & & \\
\hline T108 & Se79 & $\mathrm{Ci}$ & $9.78 \mathrm{E}-03$ & $9.78 \mathrm{E}-03$ & +01 & & & & & & $\varepsilon-02$ \\
\hline T108 & Sm151 & $\mathrm{Ci}$ & $3.65 \mathrm{E}+01$ & $3.65 \mathrm{E}+01$ & $0.00 \mathrm{E}+01$ & & 01 & & & & +02 \\
\hline T108 & $9 / 90$ & $\mathrm{Ci}$ & $1.58 E+03$ & $1.58 \mathrm{E}+03$ & 01 & & & & & 104 & \\
\hline T108 & Tc99 & $\mathrm{Ci}$ & $3.22 \mathrm{E}-01$ & $3.22 \mathrm{E}-01$ & -04 & & & & & -01 & -00 \\
\hline T108 & Th229 & $\mathrm{Ci}$ & 9.35E-09 & 9.35E-09 & $0.00 \mathrm{E}+01$ & & 09 & & & & -08 \\
\hline T108 & Th232 & $\mathrm{Ci}$ & 1.35E-11 & 1.35E-11 & $: 01$ & & & & & & -11 \\
\hline T108 & Tin126 & $\mathrm{Ci}$ & $1.47 \mathrm{E}-02$ & 1.47E-02 & $E+01$ & & & & & -02 & \\
\hline T108 & Tritium & $\mathrm{Ci}$ & 2.87E-01 & -01 & & & & & & -01 & \\
\hline T108 & U232 & $\mathrm{Ci}$ & $2.53 \mathrm{E}-05$ & $7.00 \mathrm{E}-06$ & 01 & & & & & 5 & \\
\hline T108 & U233 & $\mathrm{Ci}$ & $1.39 \mathrm{E}-06$ & $3.84 \mathrm{E}-07$ & $E+01$ & -07 & -06 & & & -00 & -06 \\
\hline T108 & U234 & $\mathbf{C i}$ & $1.75 E-00$ & $4.84 \mathrm{E}-01$ & $E+01$ & & & & & -00 & $E-00$ \\
\hline T108 & U235 & $\mathrm{Ci}$ & $7.85 \mathrm{E}-02$ & 2.17E-02 & $E+01$ & & & & & & $E-01$ \\
\hline & U236 & $\mathrm{Ci}$ & $1.26 \mathrm{E}-02$ & $3.49 \mathrm{E}-03$ & +01 & & & & & & \\
\hline & & $\mathbf{C i}$ & 00 & 4.5 & & & & & & & \\
\hline T108 & Y90 & $\mathrm{Ci}$ & $1.58 E+03$ & $1.58 E+03$ & +01 & +01 & 03 & & & 3 & +03 \\
\hline T108 & Zr93 & $\mathrm{Ci}$ & $4.65 \mathrm{E}-02$ & 4.65E-02 & $E+01$ & +01 & -02 & & & & -01 \\
\hline T109 & Al & $\mathrm{kg}$ & $4.77 \mathrm{E}+02$ & $4.77 E+02$ & $E+01$ & -01 & & +04 & & +04 & +04 \\
\hline T109 & $\mathbf{B i}$ & $\mathrm{kg}$ & $6.49 \mathrm{E}+01$ & $6.49 E+01$ & $E+01$ & & & & & +03 & +02 \\
\hline T109 & $\mathrm{Ca}$ & $\mathrm{kg}$ & $1.24 \mathrm{E}+02$ & $1.24 \mathrm{E}+02$ & $:+01$ & & & & & & \\
\hline & Cl & $\mathrm{kg}$ & 1.3 & 02 & & 02 & -02 & 02 & & & \\
\hline T109 & $\mathrm{CO3}$ & $\mathrm{kg}$ & $3.97 \mathrm{E}+03$ & $3.97 \mathrm{E}+03$ & +01 & 01 & +03 & +03 & & +03 & +03 \\
\hline T109 & $\mathrm{Cr}$ & $\mathrm{kg}$ & $1.53 E+01$ & $1.53 \mathrm{E}+01$ & $E+01$ & +01 & -02 & & & +02 & +02 \\
\hline T109 & F & $\mathrm{kg}$ & $4.97 \mathrm{E}+03$ & $4.97 \mathrm{E}+03$ & $E+01$ & 01 & & & & +03 & +03 \\
\hline r109 & $\mathbf{F e}$ & $\mathrm{kg}$ & $2.10 \mathrm{E}+03$ & $2.10 \mathrm{E}+03$ & $E+01$ & & & & & & +03 \\
\hline & Hg & $\mathrm{kg}$ & & $0.00 \mathrm{E}+01$ & $E+01$ & & & & & & \\
\hline T109 & $\mathbf{K}$ & kg & +02 & & & & & & & & \\
\hline & 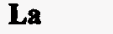 & kg & & & & & & & & & \\
\hline T109 & $\mathbf{M n}$ & $\mathbf{k g}$ & $3.94 \mathrm{E}+02$ & +02 & 01 & 01 & & 02 & 02 & -01 & 01 \\
\hline T109 & $\mathbf{N a}$ & $\mathbf{k g}$ & $7.62 E+04$ & $7.62 \mathrm{E}+04$ & & & & & & & +04 \\
\hline T109 & $\mathbf{N i}$ & kg & $7.64 \mathrm{E}-00$ & $0.00 E+01$ & +01 & & 101 & +02 & & +01 & +01 \\
\hline T109 & NO2 & kg & $1.88 \mathrm{E}+02$ & $1.88 E+02$ & & & & & & & +03 \\
\hline T109 & NO3 & kg & $7.95 E+03$ & $7.95 E+03$ & & & & & & & +04 \\
\hline & $\mathbf{p}$ & $\mathbf{k g}$ & & & & & & & & & \\
\hline T109 & $\mathbf{P b}$ & kg & $1.16 \mathrm{E}+02$ & +02 & & & & & & & +01 \\
\hline T109 & PO4 & kg & $2.88 E+05$ & $9.40 \mathrm{E}+04$ & & & & & & & \\
\hline T109 & $\mathbf{S}$ & kg & $1.97 \mathrm{E}+02$ & 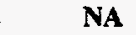 & & & & & & 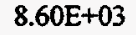 & \\
\hline T109 & $\mathbf{S i}$ & kg & $3.40 \mathrm{E}+02$ & $3.40 \mathrm{E}+02$ & $0.00 E+01$ & $E+01$ & $E+02$ & $E+03$ & $E+03$ & $E+02$ & $E+02$ \\
\hline T109 & SO4 & $\mathbf{k g}$ & $5.91 E+02$ & $1.97 E+02$ & $0.00 \mathrm{E}+0 \mathrm{l}$ & $7.69 \mathrm{E}+01$ & $2.40 \mathrm{E}+04$ & $.6 .61 \mathrm{E}+04$ & $E+05$ & $2.96 \mathrm{E}+04$ & $2.70 \mathrm{E}+04$ \\
\hline T109 & Sr & kg & 3.82E-00 & $0.00 E+01$ & $0.00 \mathrm{E}+01$ & $0.00 \mathrm{E}+01$ & $E+01$ & $E+02$ & & $7.06 \mathrm{E}+01$ & $4 \mathrm{E}+0 \mathrm{l}$ \\
\hline$m e n$ & TO & kg & $5.12 E-01$ & 5.12E-01 & $E+01$ & +01 & +02 & +03 & +03 & $4.44 E+02$ & $E+02$ \\
\hline & $\mathbf{U}$ & kg & $1.91 E+02$ & $1.91 E+02$ & & & & & & +02 & \\
\hline & $\mathbf{Z r}$ & kg & $4.66 \mathrm{E}-00$ & $4.66 \mathrm{E}-00$ & $0.00 \mathrm{E}+01$ & $0.00 E+01$ & $7.14 E-00$ & $E+02$ & $4.94 \mathrm{E}+02$ & $7.54 \mathrm{E}+01$ & $1.16 \mathrm{E}+02$ \\
\hline
\end{tabular}


Tank Anal. Un

\begin{tabular}{|c|c|c|c|}
\hline & & & May 98 \\
\hline & 2241 & $\begin{array}{l}\mathrm{Ci} \\
\mathrm{Ci}\end{array}$ & 05 \\
\hline U & $\begin{array}{l}n 241 \\
0243\end{array}$ & C & 1.79] \\
\hline & 1137 & $c$ & 106 \\
\hline & & & $07 E+03$ \\
\hline & 113 & & \\
\hline & n242 & & \\
\hline 109 & n243 & & 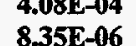 \\
\hline 109 & $\mathrm{n} 244$ & & $73 \mathrm{E}-05$ \\
\hline 109 & Co60 & & 2.21E-02 \\
\hline 109 & Cs134 & & $9.28 \mathrm{E}-04$ \\
\hline 109 & Cs137 & & $1.13 E+03$ \\
\hline 109 & & & $2.47 \mathrm{E}-02$ \\
\hline T109 & & & $3.92 \mathrm{E}-01$ \\
\hline 109 & Eu155 & & 1.90E-00 \\
\hline 109 & 1129 & & $1.30 \mathrm{E}-03$ \\
\hline 109 & Nb93 & & $8.44 \mathrm{E}-02$ \\
\hline 109 & Ni59 & & $1.99 \mathrm{E}-01$ \\
\hline 109 & Ni63 & & $1.79 \mathrm{E}+01$ \\
\hline 109 & 237 & & $4.26 \mathrm{E}-03$ \\
\hline 109 & 31 & & $6.61 \mathrm{E}-0$ \\
\hline 109 & Pu238 & & E-02 \\
\hline T109 & Pu239 & & $1.35 \mathrm{E}+01$ \\
\hline T109 & 10240 & & 1.10E-00 \\
\hline 109 & 1241 & & $2.98 \mathrm{E}-00$ \\
\hline 109 & 10242 & & 1.33E-05 \\
\hline 10 & 26 & & 5.91E-06 \\
\hline 109 & $\mathbf{R a 2 2 8}$ & & 9.87E-11 \\
\hline 109 & Ru106 & $\mathrm{Ci}$ & 8.74E-09 \\
\hline 109 & Sb125 & $\mathrm{Ci}$ & $2.00 \mathrm{E}-02$ \\
\hline 109 & Se79 & $\mathrm{i}$ & $2.10 \mathrm{E}-02$ \\
\hline 109 & Sm151 & i & $7.83 \mathrm{E}+01$ \\
\hline 109 & 0 & C & +03 \\
\hline T109 & Tc99 & C & $6.92 \mathrm{E}-01$ \\
\hline T109 & Th229 & C & 1.91E-08 \\
\hline 109 & Th232 & & $2.86 \mathrm{E}-11$ \\
\hline 109 & Tin126 & Ci & $3.16 \mathrm{E}-02$ \\
\hline 09 & Tritiu & & 01 \\
\hline 109 & $\mathbf{U} 232$ & C & $2.60 \mathrm{E}-05$ \\
\hline ri09 & U233 & $\mathrm{Ci}$ & $1.24 \mathrm{E}-06$ \\
\hline T109 & U234 & C & $1.22 \mathrm{E}-00$ \\
\hline 109 & U235 & c & 5.42E-02 \\
\hline 109 & U236 & & E-02 \\
\hline 19 & U & & \\
\hline T109 & Y90 & c & $2.45 E+03$ \\
\hline 109 & Zr93 & c & 9.99E-02 \\
\hline T110 & A) & k & $5.03 E+03$ \\
\hline 110 & $\mathbf{B i}$ & $\mathbf{K}$ & $4.77 \mathrm{E}+0$ \\
\hline 110 & Ca & & $3.59 \mathrm{E}+03$ \\
\hline 110 & C & & 02 \\
\hline 110 & $\mathrm{CO} 3$ & & +04 \\
\hline T110 & $\mathrm{Cr}$ & $k_{z}$ & $3.56 \mathrm{E}+03$ \\
\hline T110 & $\mathbf{F}$ & kg & $2.35 E+03$ \\
\hline T110 & $\mathrm{Fe}$ & $\mathrm{k}$ & $3.08 \mathrm{E}+04$ \\
\hline T110 & Hg & $\mathbf{k}$ & $2.54 \mathrm{E}-0$ \\
\hline T110 & $\mathbf{K}$ & k & $2.03 E+0$ \\
\hline T110 & $\mathbf{L}$ & & \\
\hline & $\mathbf{M}$ & & $1.09 E+04$ \\
\hline T110 & $\mathrm{N}$ : & k & $9.27 E+04$ \\
\hline T110 & $\mathbf{N i}$ & k & $2.35 E+02$ \\
\hline T110 & NO2 & $\mathrm{kg}$ & $2.56 \mathrm{E}+04$ \\
\hline T11 & NO3 & k & $5.19 E+04$ \\
\hline$[11$ & $\mathbf{P}$ & k & $3.87 E+04$ \\
\hline$[11$ & & & $6.33 E+02$ \\
\hline 11 & & & $1.20 \mathrm{E}+05$ \\
\hline & $\mathbf{S}$ & $\mathbf{k g}$ & $1.13 E+04$ \\
\hline 11 & Si & kg & $1.29 \mathrm{E}+04$ \\
\hline & & & \\
\hline
\end{tabular}

Sept98

3.04E-05

1.79E-01

$1.24 \mathrm{E}-06$

$1.07 \mathrm{E}+03$

9.78E-02

$2.41 \mathrm{E}-01$

4.08E-04

8.35E-06

2.73E-05

2.21E-02

9.28E-04

$1.13 \mathrm{E}+03$

2.47E-02

3.92E-01

$1.90 \mathrm{E}-00$

$1.30 \mathrm{E}-03$

8.44E-02

$1.99 \mathrm{E}-01$

$1.79 \mathrm{E}+01$

$4.26 \mathrm{E}-03$

6.61E-05

8.11E-02

$1.35 \mathrm{E}+01$

$1.10 \mathrm{E}-00$

2.98E-00

1.33E-05

5.91E-06

9.87E-11

8.74E-09

2.00E-02

2.10E-02

$7.83 \mathrm{E}+01$

$2.45 E+03$

6.92E-01

1.91E-08

2.86E-11

$3.16 \mathrm{E}-02$

6.56E-01

1.34E-06

6.36E-08

6.28E-02

2.78E-03

5.88E-04

6.38E-02

$2.45 E+03$

9.99E-02

$5.03 \mathrm{E}+03$

4.77E+04

$3.59 \mathrm{E}+03$

$7.81 \mathrm{E}+02$

$1.68 \mathrm{E}+04$

$3.56 \mathrm{E}+03$

$2.35 E+03$

$3.08 \mathrm{E}+04$

$0.00 \mathrm{E}+01$

$2.03 E+03$

$9.12 \mathrm{E}+03$

$1.09 \mathrm{E}+04$

$9.27 \mathrm{E}+04$

$2.35 \mathrm{E}+02$

$2.56 \mathrm{E}+04$

$5.19 E+04$

NA

$6.33 E+02$

$3.91 E+04$

NA

$1.29 \mathrm{E}+04$

$1.13 E+04$
$1 \%$-ile

$0.00 \mathrm{E}+01$

$0.00 \mathrm{E}+01$

$0.00 \mathrm{E}+01$

$0.00 \mathrm{E}+01$

$0.00 \mathrm{E}+01$

$0.00 \mathrm{E}+01$

$0.00 \mathrm{E}+01$

$0.00 \mathrm{E}+01$

$0.00 \mathrm{E}+01$

$0.00 \mathrm{E}+01$

$0.00 \mathrm{E}+01$

$0.00 \mathrm{E}+01$

$0.00 \mathrm{E}+01$

$0.00 \mathrm{E}+01$

$0.00 \mathrm{E}+01$

$0.00 \mathrm{E}+01$

$0.00 \mathrm{E}+01$

$0.00 \mathrm{E}+01$

$0.00 \mathrm{E}+01$

$0.00 \mathrm{E}+01$

$0.00 \mathrm{E}+01$

$0.00 \mathrm{E}+01$

$0.00 \mathrm{E}+01$

$0.00 \mathrm{E}+01$

$0.00 \mathrm{E}+01$

$0.00 \mathrm{E}+01$

$0.00 \mathrm{E}+01$

$0.00 \mathrm{E}+01$

$0.00 \mathrm{E}+01$

$0.00 \mathrm{E}+01$

$0.00 \mathrm{E}+01$

$0.00 \mathrm{E}+01$

$0.00 E+01$

$0.00 \mathrm{E}+01$

$0.00 \mathrm{E}+01$

$0.00 \mathrm{E}+01$

$0.00 \mathrm{E}+01$

2.03E-02

$0.00 \mathrm{E}+01$

$0.00 \mathrm{E}+01$

$0.00 \mathrm{E}+01$

$0.00 \mathrm{E}+01$

$0.00 \mathrm{E}+01$

$0.00 \mathrm{E}+01$

$0.00 \mathrm{E}+01$

$0.00 \mathrm{E}+01$

$2.60 \mathrm{E}+02$

$1.66 \mathrm{E}+04$

$9.73 \mathrm{E}+01$

$4.07 \mathrm{E}+02$

$4.94 \mathrm{E}+02$

$1.57 \mathrm{E}+02$

5. $63 \mathrm{E}+02$

$7.37 \mathrm{E}+03$

$0.00 \mathrm{E}+01$

$1.89 \mathrm{E}+02$

$4.88 \mathrm{E}+01$

$1.40 \mathrm{E}+02$

$9.19 E+04$

7.07E-00

$9.33 \mathrm{E}+02$

$2.13 E+04$

$6.31 \mathrm{E}+03$

$1.73 \mathrm{E}+01$

$2.22 \mathrm{E}+03$

$4.88 \mathrm{E}+02$

$9.58 \mathrm{E}+02$

$2.08 \mathrm{E}+03$
$10 \%$-ile

$0.00 \mathrm{E}+01$

$0.00 \mathrm{E}+01$

$0.00 \mathrm{E}+01$

$1.09 \mathrm{E}+02$

$0.00 \mathrm{E}+01$

$0.00 \mathrm{E}+01$

$0.00 \mathrm{E}+01$

$0.00 \mathrm{E}+01$

$0.00 \mathrm{E}+01$

$0.00 \mathrm{E}+01$

9.49E-05

$1.02 \mathrm{E}+03$

$0.00 \mathrm{E}+01$

$0.00 \mathrm{E}+01$

$0.00 \mathrm{E}+01$

$3.16 \mathrm{E}-04$

$0.00 \mathrm{E}+01$

$0.00 \mathrm{E}+01$

9.00E+01

$0.00 \mathrm{E}+01$

$0.00 \mathrm{E}+01$

$0.00 \mathrm{E}+01$

$0.00 \mathrm{E}+01$

$0.00 \mathrm{E}+01$

$0.00 E+01$

$0.00 E+01$

$0.00 \mathrm{E}+0 \mathrm{I}$

$0.00 \mathrm{E}+01$

$0.00 \mathrm{E}+01$

$0.00 \mathrm{E} \div 0$ ?

$0.00 \mathrm{E}+01$

$0.00 \mathrm{E}+01$

$0.00 \mathrm{E}+01$

$0.00 \mathrm{E}+01$

$0.00 \mathrm{E}+01$

$0.00 \mathrm{E}+01$

$0.00 \mathrm{E}+01$

2.23E-0l

$0.00 \mathrm{E}+01$

$0.00 \mathrm{E}+01$

$0.00 \mathrm{E}+01$

$0.00 \mathrm{E}+01$

$0.00 \mathrm{E}+0$

$0.00 \mathrm{E}+01$

$0.00 \mathrm{E}+01$

$0.00 \mathrm{E}+01$

$1.95 \mathrm{E}+03$

$2.28 \mathrm{E}+04$

$5.26 \mathrm{E}+02$

$1.13 \mathrm{E}+03$

$6.81 \mathrm{E}+03$

$7.68 \mathrm{E}+02$

$2.33 \mathrm{E}+03$

$1.38 \mathrm{E}+04$

$3.26 \mathrm{E}-01$

$4.67 \mathrm{E}+02$

$1.82 \mathrm{E} \div 02$

$3.35 \mathrm{E}+02$

$1.23 \mathrm{E}+05$

$2.10 \mathrm{E}+01$

$5.90 \mathrm{E}+03$

$8.41 E+04$

$1.96 \mathrm{E}+04$

$1.28 \mathrm{E}+02$

$1.45 \mathrm{E}+04$

$2.36 \mathrm{E}+03$

$5.19 \mathrm{E}+03$

$8.67 \mathrm{E}+03$ 50\%-ile

3.04E-05

6.53E-00

$1.24 \mathrm{E}-06$

$1.07 \mathrm{E}+03$

9.78E-02

2.41E-01

4.08E-04

8.35E-06

2.73E-05

1.43E-00

9.28E-04

$7.02 \mathrm{E}+03$

2.47E-02

$3.92 \mathrm{E}-01$

$1.90 \mathrm{E}-00$

1.30E-03

8.44E-02

$1.99 \mathrm{E}-01$

$1.79 \mathrm{E}+01$

4.26E-03

6.61E-05

8.11E-02

$1.35 \mathrm{E}+01$

1.10E-00

2.98E-00

1.33E-05

5.91E-06

9.87E-11

8.74E-09

$2.00 \mathrm{E}-02$

2.10E-02

$7.83 \mathrm{E}+01$

$1.44 \mathrm{E}+04$

6.92E-01

1.91E-08

2.86E-11

3.16E-02

6.56E-01

2.60E-05

1.24E-06

$1.22 \mathrm{E}-00$

5.42E-02

1.14E-02

$1.24 \mathrm{E}-00$

$2.45 E+03$

9.99E-02

$2.38 \mathrm{E}+04$

$3.46 E+04$

$1.31 E+03$

$2.00 \mathrm{E}+03$

$1.90 \mathrm{E}+04$

$1.68 \mathrm{E}+03$

$1.23 \mathrm{E}+04$

$2.58 \mathrm{E}+04$

2.54E-00

$8.34 \mathrm{E}+02$

$4.64 \mathrm{E}+02$

$6.65 \mathrm{E}+02$

$1.69 \mathrm{E}+05$

$5.06 \mathrm{E}+01$

$1.96 \mathrm{E}+04$

2.42E+05

$3.61 \mathrm{E}+04$

$5.80 \mathrm{E}+02$

$3.59 \mathrm{E}+04$

$7.25 \mathrm{E}+03$

$1.30 \mathrm{E}+04$

$1.92 E+04$

90\%-ile

99\%-ile

$1.62 \mathrm{E}-04$

$6.64 \mathrm{E}+03$

$7.26 \mathrm{E}-06$

$2.88 \mathrm{E}+03$

7.35E-01

$6.18 \mathrm{E}-01$

$2.39 \mathrm{E}-03$

4.89E-05 


\begin{tabular}{|c|c|c|c|c|c|c|c|c|c|c|c|}
\hline$n k$ & nal. & Un & $\frac{B B}{\operatorname{May} 98}$ & $\operatorname{Sept98}$ & $1 \%$-ile & $10 \%$-ile & $50 \%$-ile & 90\%-ile & 99\%-ile & Mean & Std Dev \\
\hline 110 & Sr & kg & $5.44 E+02$ & $5.44 \mathrm{E}+02$ & $3.33 \mathrm{E}+01$ & $1.31 E+02$ & $.62 E+02$ & $8.47 \mathrm{E}+02$ & $2.05 E+03$ & $4.58 \mathrm{E}+02$ & $4.01 E+02$ \\
\hline r110 & TOC & $\mathrm{kg}$ & $5.55 E+03$ & $5.55 \mathrm{E}+03$ & $2 \mathrm{E}+01$ & $E+02$ & $1.27 \mathrm{E}+03$ & $E+03$ & $E+03$ & $30 \mathrm{E}+03$ & $49 E+02$ \\
\hline T110 & $\mathbf{U}$ & $\mathrm{kg}$ & $4.96 \mathrm{E}+03$ & $4.96 E+03$ & $2.82 \mathrm{E}+01$ & $7.28 \mathrm{E}+02$ & $11 \mathrm{E}+03$ & $3 E+04$ & $71 E+04$ & $52 E+03$ & $44 E+03$ \\
\hline T110 & $\mathbf{Z r}$ & kg & $3.08 \mathrm{E}+02$ & $0.00 \mathrm{E}+01$ & $9.45 \mathrm{E}-01$ & $1.03 E+01$ & $98 E+01$ & $3.92 E+02$ & $5.86 \mathrm{E}+02$ & $1.50 \mathrm{E}+02$ & $1.63 E+02$ \\
\hline 110 & Ac227 & $\mathrm{Ci}$ & $6.05 E-06$ & $6.05 \mathrm{E}-06$ & $0.00 E+01$ & $0.00 \mathrm{E}+01$ & $6.05 E-06$ & $2.09 \mathrm{E}-05$ & $4.05 E-05$ & $8.46 \mathrm{E}-06$ & $9.58 \mathrm{E}-06$ \\
\hline 110 & Am241 & $\mathbf{C i}$ & $7.58 \mathrm{E}+01$ & $7.58 \mathrm{E}+01$ & $3.45 \mathrm{E}-00$ & $2.94 E+01$ & $1.22 E+02$ & $4.61 E+02$ & $1.18 \mathrm{E}+03$ & $2.04 E+02$ & $2.60 E+02$ \\
\hline 110 & Am243 & $\mathrm{Ci}$ & $1.40 \mathrm{E}-07$ & $4.95 \mathrm{E}-04$ & $1.09 \mathrm{E}-08$ & $4.56 \mathrm{E}-08$ & $1.40 \mathrm{E}-07$ & $2.81 \mathrm{E}-07$ & $5.83 \mathrm{E}-07$ & $1.58 \mathrm{E}-07$ & $.07 \mathrm{E}-07$ \\
\hline 110 & Ba137 & $\mathbf{C i}$ & $2.66 \mathrm{E}+02$ & $2.66 E+02$ & $1.58 \mathrm{E}+01$ & $9.00 E+01$ & $2.66 E+02$ & $1.83 E+03$ & $2.81 E+03$ & $5.50 \mathrm{E}+02$ & $96 E+02$ \\
\hline 110 & $\mathrm{C14}$ & $\mathrm{Ci}$ & $1.69 \mathrm{E}-02$ & $1.69 \mathrm{E}-02$ & $0.00 E+01$ & $0.00 \mathrm{E}+01$ & $1.69 \mathrm{E}-02$ & $3.51 \mathrm{E}-02$ & $5.39 \mathrm{E}-02$ & $1.78 \mathrm{E}-02$ & 28E-02 \\
\hline 1110 & Cd113 & $\mathrm{Ci}$ & $3.85 \mathrm{E}-02$ & $3.85 \mathrm{E}-02$ & $0.00 \mathrm{E}+01$ & $4.95 \mathrm{E}-03$ & $3.85 \mathrm{E}-02$ & $1.48 \mathrm{E}-01$ & $3.07 \mathrm{E}-01$ & $6.20 \mathrm{E}-02$ & $97 \mathrm{E}-02$ \\
\hline 1110 & $\mathrm{Cm} 242$ & $\mathrm{Ci}$ & $1.74 \mathrm{E}-04$ & $6.05 \mathrm{E}-01$ & $1.36 \mathrm{E}-05$ & $5.66 \mathrm{E}-05$ & $1.74 \mathrm{E}-04$ & $3.49 \mathrm{E}-04$ & $7.25 \mathrm{E}-04$ & $1.97 \mathrm{E}-04$ & $33 E-04$ \\
\hline T110 & $\mathrm{Cm} 243$ & $\mathrm{Ci}$ & $3.54 E-06$ & $1.23 \mathrm{E}-02$ & 2.77E-07 & $1.15 \mathrm{E}-06$ & $3.54 \mathrm{E}-06$ & $7.10 \mathrm{E}-06$ & $1.48 \mathrm{E}-05$ & & 2.70E-06 \\
\hline T110 & Cm244 & $\mathrm{Ci}$ & $3.27 \mathrm{E}-06$ & $1.15 E-02$ & 2.55E-07 & & $3.27 \mathrm{E}-06$ & $6.56 \mathrm{E}-06$ & $1.36 \mathrm{E}-05$ & $3.69 \mathrm{E}-06$ & $2.49 \mathrm{E}-06$ \\
\hline T110 & Co60 & $\mathrm{Ci}$ & $3.24 E-03$ & $3.24 \mathrm{E}-03$ & $5.69 \mathrm{E}-03$ & $4.57 \mathrm{E}-01$ & $6.74 \mathrm{E}-00$ & $1.02 E+02$ & $9.94 E+02$ & $5.13 E+01$ & $1.79 \mathrm{E}+02$ \\
\hline T110 & Cs134 & $\mathrm{Ci}$ & 9.87E-05 & $9.87 \mathrm{E}-05$ & $5.86 \mathrm{E}-06$ & -05 & $9.87 \mathrm{E}-05$ & $E-04$ & $1.04 \mathrm{E}-03$ & E-04 & $E-04$ \\
\hline T110 & Cs137 & $\mathbf{C i}$ & $2.81 E+02$ & $2.81 E+02$ & $2.17 E+03$ & +04 & $3.13 E+04$ & \pm 05 & $3.28 E+05$ & $E+04$ & $E+04$ \\
\hline T110 & 152 & $\mathrm{Ci}$ & $1.02 \mathrm{E}-02$ & $1.02 E-02$ & $0.00 \mathrm{E}+01$ & & $Q-02$ & -02 & $2 \mathrm{E}-02$ & $E-02$ & $E-02$ \\
\hline $\mathbf{T 1 1 0}$ & & $\mathrm{Ci}$ & $5.40 \mathrm{E}-02$ & $5.40 \mathrm{E}-02$ & $0.00 \mathrm{E}+01$ & +01 & $5.40 \mathrm{E}-02$ & $1.86 \mathrm{E}-01$ & $3.61 \mathrm{E}-01$ & $\mathrm{E}-02$ & $E-02$ \\
\hline T110 & Eu155 & $\mathbf{C i}$ & $8.36 \mathrm{E}-01$ & $8.36 \mathrm{E}-01$ & $0.00 E+01$ & $0.00 E+01$ & $8.36 \mathrm{E}-01$ & $E-00$ & $5.59 \mathrm{E}-00$ & $E-00$ & $E-00$ \\
\hline T110 & I129 & $\mathrm{Ci}$ & 2.19E-C & 2.19E-04 & $0.00 E+01$ & $E-05$ & $2.19 \mathrm{E}-04$ & & $5.01 \mathrm{E}-04$ & $E-04$ & \\
\hline T110 & Nb93 & $\mathbf{C i}$ & $1.44 \mathrm{E}-02$ & $1.44 \mathrm{E}-02$ & $0.00 E+01$ & & & & E-01 & $\mathrm{E}-02$ & $E-02$ \\
\hline $\mathrm{T} 110$ & Ni59 & $\mathrm{Ci}$ & $4.80 \mathrm{E}-03$ & $4.80 E-03$ & $4.01 \mathrm{E}-04$ & & & -02 & $E-02$ & E-03 & $\mathrm{E}-02$ \\
\hline T110 & Ni63 & $\mathrm{Ci}$ & 4.28E-01 & $4.28 \mathrm{E}-01$ & E-02 & & & $-\infty 0$ & -00 & $E-01$ & -00 \\
\hline T110 & Np237 & $\mathbf{C i}$ & 7.13E-04 & $7.13 \mathrm{E}-04$ & 05 & & .04 & .03 & & $E-04$ & -04 \\
\hline T110 & Pa231 & $\mathrm{Ci}$ & $1.29 \mathrm{E}-05$ & $1.29 \mathrm{E}-05$ & $0.00 \mathrm{E}+01$ & $E-06$ & -05 & $5.88 \mathrm{E}-05$ & $1.66 \mathrm{E}-0.04$ & $E-05$ & $E-05$ \\
\hline T110 & Pu238 & $\mathbf{C i}$ & $5.41 \mathrm{E}-01$ & $1.20 \mathrm{E}-00$ & $4.23 \mathrm{E}-02$ & $=01$ & & $E-00$ & -00 & & -01 \\
\hline T110 & Pu239 & $\mathbf{C i}$ & $2.47 E+02$ & $2.30 E+02$ & $1.93 E+01$ & +01 & 2.4 & $E+02$ & +03 & & +02 \\
\hline $\mathrm{T} 110$ & Pu240 & $\mathrm{Ci}$ & Incl. in 239 & $1.73 E+01$ & $1.93 E+01$ & -01 & -02 & $E+02$ & $E+03$ & +02 & $E+02$ \\
\hline T110 & Pu241 & $\mathbf{C i}$ & $1.76 E+01$ & $3.92 E+01$ & $E-00$ & & & -01 & +01 & +01 & +01 \\
\hline T110 & Pu242 & $\mathrm{Ci}$ & -05 & $1.72 \mathrm{E}-04$ & 06 & & & & .04 & -05 & -05 \\
\hline T110 & $\operatorname{Ra226}$ & $\mathrm{Ci}$ & 1.19E-06 & 1.19E-06 & $E+01$ & -07 & -06 & -06 & -06 & -06 & \\
\hline T110 & $\operatorname{Ra} 228$ & $\mathrm{Ci}$ & $4.76 \mathrm{E}-11$ & $4.76 \mathrm{E}-11$ & $0.00 E+01$ & -12 & & & 0 & & $:-11$ \\
\hline T110 & Ru106 & $\mathbf{C i}$ & 9.78 & $9.78 \mathrm{E}-10$ & $0.00 E+01$ & & & $E-09$ & E-09 & $E-09$ & -10 \\
\hline T110 & 25 & $\mathrm{Ci}$ & $2.64 \mathrm{E}-03$ & $2.64 \mathrm{E}-03$ & $E+01$ & & & & & -03 & \\
\hline T110 & 79 & $\mathbf{C i}$ & 3.5 & 03 & & & & & -02 & $E-03$ & -03 \\
\hline T110 & Sm151 & $\mathbf{C i}$ & $1.33 E+01$ & $i+01$ & +01 & & 01 & 01 & +01 & +01 & +01 \\
\hline $\mathrm{r} 110$ & Sr89/90 & $\mathbf{C i}$ & $9.18 \mathrm{E}+03$ & $9.18 \mathrm{E}+03$ & $3.02 E+02$ & +03 & +04 & & 6. & -05 & +05 \\
\hline T110 & Tc99 & $\mathrm{Ci}$ & $1.40 \mathrm{E}+01$ & $1.40 E+01$ & $E-00$ & & & & $\div 02$ & +01 & +01 \\
\hline T110 & Th229 & $\mathbf{C i}$ & $9.22 \mathrm{E}-09$ & 9.22E-09 & $E+01$ & & & $E-08$ & $9 \mathrm{E}-07$ & -08 & \\
\hline T110 & Th232 & $\mathbf{C i}$ & 4.9 & 12 & -01 & & & & & & \\
\hline T110 & Tin126 & Ci & 5.32 & 5.32E-03 & +01 & & & -02 & 02 & -03 & -03 \\
\hline T110 & Tritium & $\mathrm{Ci}$ & 4.94E-02 & $4.94 \mathrm{E}-02$ & $E-02$ & -02 & -02 & .02 & -02 & & -02 \\
\hline T110 & U232 & $\mathbf{C i}$ & $6.54 \mathrm{E}-06$ & $2.33 E-05$ & +01 & & & & -05 & & $E-05$ \\
\hline T110 & U233 & $\mathbf{C i}$ & $3.61 \mathrm{E}-07$ & $1.28 \mathrm{E}-06$ & $E+01$ & -08 & & & $5-06$ & -07 & $E-07$ \\
\hline T110 & & $\mathrm{Ci}$ & $4.60 E-01$ & $1.63 E-00$ & $E+01$ & & & & & & -00 \\
\hline T110 & & $\mathrm{Ci}$ & 12 & 02 & & & & & & & \\
\hline $\mathrm{T} 1$ & & $\mathrm{Ci}$ & 3.2 & & 01 & & & & -02 & -03 & -03 \\
\hline T110 & U238 & $\mathrm{Ci}$ & $4.66 \mathrm{E}-01$ & $8-00$ & +01 & 02 & -01 & -00 & $6.00 \mathrm{E}-00$ & $E-01$ & $E-00$ \\
\hline T110 & Y90 & $\mathrm{Ci}$ & $9.18 E+03$ & $9.18 E+03$ & $0.00 \mathrm{E}+01$ & +03 & +03 & & +05 & & +04 \\
\hline T110 & Zr93 & $\mathbf{C i}$ & $1.69 \mathrm{E}-02$ & $1.69 \mathrm{E}-02$ & $E+01$ & & -02 & 2E-01 & 4E-01 & $E-02$ & $\mathrm{E}-02$ \\
\hline T111 & Al & kg & $1.23 E+03$ & $1.23 E+03$ & $2.08 \mathrm{E}+02$ & +03 & +04 & +04 & $3 E \div 04$ & $E+04$ & $E+04$ \\
\hline T111 & $\mathbf{B i}$ & $\mathbf{k g}$ & $5.60 \mathrm{E}+04$ & $5.60 \mathrm{E}+04$ & $2.07 E+04$ & +04 & & & & $E+04$ & +04 \\
\hline TIII & $\mathrm{Ca}$ & kg & & & & & & & & & \\
\hline T111 & Cl & $\mathrm{kg}$ & 9.7 & 9.7 & & & & & & +03 & +02 \\
\hline T111 & $\mathrm{CO} 3$ & $\mathbf{k g}$ & 1.80 & 1.8 & 03 & & & & & & +04 \\
\hline T111 & $\mathrm{Cr}$ & kg & $4.23 E+03$ & $4.23 \mathrm{E}+03$ & $E+02$ & & & $E+03$ & $4.62 \mathrm{E}+03$ & $2.25 \mathrm{E}+03$ & $E+02$ \\
\hline T111 & $F$ & kg & $4.97 E+03$ & $4.97 E+03$ & $E+03$ & +03 & & $E+04$ & $E+04$ & $E \div 04$ & $E+04$ \\
\hline T111 & $\mathbf{F e}$ & kg & $4.00 E+04$ & $4.00 E+04$ & $E+03$ & +04 & +04 & $\div+04$ & & $E \div 04$ & $E+04$ \\
\hline T111 & Hg & $\mathrm{kg}$ & $3.00 \mathrm{E}-00$ & & $0.00 \mathrm{E} \div 01$ & & & $E+01$ & & & $E-00$ \\
\hline T111 & $\mathbf{K}$ & $\mathrm{kg}$ & & & & & & $E+03$ & & +03 & +02 \\
\hline T111 & $\mathbf{L a}$ & kg & $9.12 E+03$ & $1.39 E+03$ & $E+02$ & & +03 & & & $E+03$ & +02 \\
\hline 10 & $\mathbf{M}$ & $\mathbf{k g}$ & $1.37 \mathrm{E}$ & $1.37 E+04$ & & & & & & $E+03$ & \\
\hline T11 & $\mathrm{N}$ & $\mathrm{kg}$ & $8.00 E+04$ & $8.00 E+04$ & $E+05$ & & & $E+05$ & & $E+05$ & $E+04$ \\
\hline T1 & Ni & $\mathbf{k g}$ & $2.85 E+02$ & $2.85 E+02$ & $1.92 E+01$ & 3.9 & & $E+02$ & +02 & $\mathrm{E}+02$ & +02 \\
\hline T111 & $\mathrm{NO} 2$ & $\mathrm{~kg}$ & $1.71 E+03$ & $1.71 E+03$ & $1.02 \mathrm{E}+03$ & $6.82 \mathrm{E}+03$ & $2.25 \mathrm{E}+04$ & $6.44 E+04$ & $1.03 E+05$ & $3.05 \mathrm{E}+04$ & $E+04$ \\
\hline T111 & NO3 & kg & $8.90 E+04$ & $8.90 E+04$ & $2.61 E \div 04$ & $1.03 E+05$ & $2.76 E+05$ & $4.61 E+05$ & $5.89 \mathrm{E}+05$ & $2.78 \mathrm{E}+05$ & 1.37E+05 \\
\hline T111 & $\mathbf{P}$ & kg & $6.80 \mathrm{E}+04$ & NA & $E+03$ & $2.18 \mathrm{E}+04$ & +04 & $6.44 E+04$ & +04 & $4.23 \mathrm{E}+04$ & $1.74 E+04$ \\
\hline & $\mathbf{P b}$ & kg & $7.89 \mathrm{E}+02$ & +02 & $3.06 \mathrm{E}+01$ & $1.61 E+02$ & $6.83 E+02$ & $2.41 E+03$ & $4.81 E+03$ & $1.04 E+03$ & $1.06 E+03$ \\
\hline
\end{tabular}


Tank Anal. Un

\section{T111 PO4 ks}

T111

T111 Si

T111 SO4

T111 Sr

Ti11 TOC

T111 U

T111 Zr

T111 Ac227

T111 Am241

T111 Am243

T111 Ba137

T111 C14

T111 Cd113

T11 Cm242 Ci

T111 Cm243 Ci

T111 Cm244 $\mathrm{Ci}$

T111 C060

T111 Cs134

T111 Cs137

T111 Eu152

T111 Eu154

T111 Eu155

T111 1129

T111 Nb93

T111 Ni59

T111 Ni63

T111 Np237

T111 Pa231

T111 Pu238

T111 Pu239

T111 Pu240

T111 Pu241

T111 Pu242

T111 Ra226

T111 Ra228

T111 Ru106

T111 Sb125

Ti11 Se79

T111 Sm151

T111 Sr89/90

T111 Te99

T111 Th229

Ti11 Th232

T11 Tin126 C

T111 Tritium C

T111 U232

T11 U233

T11 $\mathbf{2 3 4}$

T11 U235

T11 $\mathbf{2 3 6}$

T111 U238

T111 Y90

T111 2r93

T112 Al

$\mathrm{T112} \mathrm{Bi}$

$\mathrm{T} 112 \mathrm{Ca}$

$\mathrm{T112} \mathrm{Cl}$

$\mathrm{T112} \mathrm{CO}$

T112 $\mathrm{Cr}$

I112 F

T112 $\mathrm{Fe}$

$\mathrm{T112} \mathrm{Hg}$

T112 K

T112 La

TI12 Mn

T112 N2

T112 Ni
BB

May 98 2.11E+05

g $7.97 \mathrm{E}+03$

$1.22 \mathrm{E}+04$

$2.39 \mathrm{E}+04$

kg $6.48 \mathrm{E}+02$

$6.80 \mathrm{E}+03$

$6.74 \mathrm{E}+03$

$0.00 \mathrm{E}+01$

5.83E-06

$9.20 \mathrm{E}+01$

2.64E-07

$3.17 \mathrm{E}+02$

NA

4.84E- 02

$3.32 \mathrm{E}-04$

6.81E-06

$6.24 \mathrm{E}-06$

8.00E-01

$1.79 \mathrm{E}-04$

$3.34 \mathrm{E}+02$

$1.76 \mathrm{E}-02$

8.13E-02

1.28E-00

1.67E-02

1.10E-01

Ci 1.20E+01

Ci 8.47E-04

Ci 1.27E-05

Ci 8.03E-01

Ci $3.00 E+02$

Ci Incl. in 239

Ci $3.14 \mathrm{E}+01$

Ci 1.41E-04

Ci. 1.14E-06

Ci 6.08E-11

1.90E-09

Ci 4.20E-03

Ci NA

Ci 1.55E+01

Ci $1.09 \mathrm{E}+04$

$1.70 \mathrm{E}+01$

1.18E-08

$5.64 \mathrm{E}-12$

$6.27 \mathrm{E}-03$

NA

6.98E-06

3.58E-07

Ci 4.08E-01

Ci 1.82E-02

Ci 3.28E-03

Ci 4.14E-01

Ci $1.09 \mathrm{E}+04$

Ci 1.98E-02

kg 1.49E+03

kg 8.37E+03

kg $\quad 5.50 \mathrm{E}+02$

kg 1.38E+02

kg $1.44 \mathrm{E}+03$

kg $6.30 \mathrm{E}+02$

kg 1.10E+02

kg $\quad 4.77 \mathrm{E}+03$

kg 4.57E-01

kg $3.80 \mathrm{E}+02$

kg $1.40 \mathrm{E}+03$

kg 1.74E +03

kg 1.35E+04

kg $4.50 \mathrm{E}+01$
$B B$

Sept98

$6.87 \mathrm{E}+04$

NA
$\mathbf{5}+04$

$1.22 \mathrm{E}+04$
$7.97 \mathrm{E}+03$

$7.97 \mathrm{E}+03$
$6.48 \mathrm{E}+02$

$6.80 \mathrm{E}+03$

$6.74 E+03$

$0.00 \mathrm{E}+01$

5.83E-06

$9.20 \mathrm{E}+01$

6.43E-04

$3.17 \mathrm{E}+02$

1.98E-02

4.84E-02

8.08E-01

$1.66 \mathrm{E}-02$

1.52E-02

8.00E-01

1.79E-04

$3.34 \mathrm{E}+02$

1.76E-02

8.13E-02

1.28E-00

2.59E-04

1.67E-02

1.10E-01

$1.20 \mathrm{E}+01$

8.47E-04

1.27E-05

1.81E-00

$2.76 \mathrm{E}+02$

$2.37 \mathrm{E}+01$

$7.10 \mathrm{E}+01$

3.19E-04

1.14E-06

6.08E-11

1.90E-09

4.20E-03

4.17E-03

$1.55 \mathrm{E}+01$

$1.09 E+04$

$1.70 \mathrm{E}+01$

1.18E-08

$5.64 \mathrm{E}-12$

$6.27 \mathrm{E}-03$

5.45E-02

3.79E-05

1.95E-06

2.22E-00

$9.90 \mathrm{E}-02$

1.78E-02

2.25E-00

$1.09 \mathrm{E}+04$

$1.98 \mathrm{E}-02$

$1.48 \mathrm{E}+03$

8.37E+03

$5.50 \mathrm{E}+02$

$1.38 \mathrm{E}+02$

$1.44 \mathrm{E}+03$

$6.32 \mathrm{E}+02$

$5.82 \mathrm{E}+02$

$4.77 \mathrm{E}+03$

4.62E-01

$3.85 \mathrm{E}+02$

$3.47 \mathrm{E}+03$

$1.73 \mathrm{E}+03$

$2.11 \mathrm{E}+04$

$4.34 E+01$
$1 \%$ ile

$3.52 \mathrm{E}+03$

$4.11 \mathrm{E}+02$

$1.43 \mathrm{E}+03$

$2.43 E+03$

$7.19 \mathrm{E}+01$

$1.70 \mathrm{E}+02$

4.69E+01

$1.31 \mathrm{E}-00$

$0.00 \mathrm{E}+01$

$5.96 \mathrm{E}-00$

4.04E-08

$1.39 \mathrm{E}+0$

$0.00 \mathrm{E}+01$

5.09E-05

1.04E-06

9.56E-07

1.47E-02

7.82E-06

$1.90 \mathrm{E}+03$

$0.00 \mathrm{E}+01$

$0.00 \mathrm{E}+01$

$0.00 \mathrm{E}+01$

$0.00 E+01$

$1.63 \mathrm{E}-02$

$1.78 \mathrm{E}-00$

$1.30 \mathrm{E}-04$

$0.00 \mathrm{E}+01$

$1.23 \mathrm{E}-01$

$4.60 \mathrm{E}+01$

$4.60 \mathrm{E}+01$

4.81E-00

$2.16 \mathrm{E}-05$

$0.00 \mathrm{E}+01$

$0.00 \mathrm{E}+01$

$0.00 \mathrm{E}+01$

$0.00 \mathrm{E}+0$

$0.00 \mathrm{E}+01$

$3.58 \mathrm{E}+02$

$0.00 \mathrm{E}+01$

$0.00 \mathrm{E}+01$

$0.00 \mathrm{E}+01$

$0.00 \mathrm{E}+01$

NA

$0.00 E+01$

$0.00 \mathrm{E}+01$

$0.00 \mathrm{E}+01$

$0.00 \mathrm{E}+01$

$0.00 \mathrm{E}+01$

$0.00 E+01$

$0.00 \mathrm{E}+01$

$0.00 \mathrm{E}+01$

$6.99 \mathrm{E}+01$

$6.86 \mathrm{E}+02$

$3.57 \mathrm{E}+01$

$4.31 \mathrm{E}+01$

$1.83 \mathrm{E}+02$

$4.58 \mathrm{E}+01$

$4.25 \mathrm{E}+01$

$5.61 \mathrm{E}+02$

$0.00 \mathrm{E}+01$

8.10E-00

3.56E-02

$1.42 \mathrm{E}-00$

$4.32 \mathrm{E}+03$

2.77E-00

10\%-ile

$1.63 \mathrm{E}+04$

$2.67 \mathrm{E}+03$

$6.15 \mathrm{E}+03$

$9.72 \mathrm{E}+03$

$1.97 \mathrm{E}+02$

$7.28 \mathrm{E}+02$

$8.31 \mathrm{E}+02$

$1.15 \mathrm{E}+01$

$0.00 \mathrm{E}+01$

$3.42 \mathrm{E}+01$

1.01E-07

$1.07 \mathrm{E}+02$
$\mathrm{NA}$

6.69E-03

1.27E-04

2.61E-06 


\begin{tabular}{|c|c|c|c|c|c|c|c|c|c|c|c|}
\hline Tank & Anal. & $U_{n}$ & May $\frac{B B}{98}$ & Sept98 & $1 \%$-ile & $10 \%$-ile & 50\%-ile & 90\%-ile & 99\%-ile & Mean & Std Dev \\
\hline & $\mathrm{O} 2$ & $\mathbf{k g}$ & $20 \mathrm{E}+03$ & $21 \mathrm{E}+03$ & $E+02$ & $E+03$ & $E+03$ & $+E+04$ & $\mathrm{E}+04$ & $E+03$ & 00E+ \\
\hline 12 & NO3 & $\mathrm{kg}$ & $3.65 E+03$ & $5.66 \mathrm{E}+03$ & & $7 E+04$ & $66 E+04$ & +05 & $E+05$ & 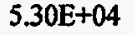 & \\
\hline 1112 & $\mathbf{F}$ & $\mathrm{kg}$ & $1.18 \mathrm{E}+03$ & NA & $E+02$ & $E+03$ & $57 E+03$ & $5 E+04$ & $50 E+04$ & $E+03$ & \\
\hline$\Gamma 112$ & $\mathbf{P b}$ & $\mathrm{kg}$ & $1.15 E+02$ & $1.16 E+02$ & $3.60 \mathrm{E}-00$ & $08 \mathrm{E}+01$ & $11 \mathrm{E}+01$ & $30 \mathrm{E}+02$ & $09 \mathrm{E}+02$ & $47 \mathrm{E}+02$ & $6 \mathrm{E}+02$ \\
\hline T112 & PO4 & $\mathrm{kg}$ & $3.64 \mathrm{E}+03$ & $3.66 \mathrm{E}+03$ & $4.53 E+02$ & $.01 E+03$ & $.17 E+03$ & $1.40 \mathrm{E}+04$ & $.42 \mathrm{E}+04$ & $.97 \mathrm{E}+03$ & $61 \mathrm{E}+03$ \\
\hline 112 & $\mathbf{S}$ & $\mathrm{k}$ & $60 \mathrm{E}+02$ & NA & $.21 E+01$ & $64 \mathrm{E}+02$ & $.26 E+03$ & $90 \mathrm{E}+03$ & $04 E+03$ & $1.58 \mathrm{E}+03$ & $13 E+03$ \\
\hline 112 & $\mathbf{S i}$ & $\mathrm{kg}$ & $.56 \mathrm{E}+03$ & $E+03$ & $1.07 \mathrm{E}+02$ & $38 E+02$ & $84 E+03$ & $3.76 E+03$ & $84 E+03$ & 2. & $E+03$ \\
\hline 112 & SO4 & $\mathrm{kg}$ & $2.88 \mathrm{E}+03$ & $.87 \mathrm{E}+03$ & $3.72 E+02$ & $9 \mathrm{E}+03$ & $0 E+03$ & $\mathrm{E} \div 03$ & $1.78 \mathrm{E} \div 04$ & $3.97 \mathrm{E}+03$ & $3.22 \mathrm{E}+03$ \\
\hline 112 & $\mathbf{s}$ & kg & $9.20 \mathrm{~F}$ & $9.10 \mathrm{E}+01$ & -00 & $3 \mathrm{E}+01$ & +01 & $E+02$ & $3.73 E \div 02$ & 01 & $7.52 \mathrm{E}+01$ \\
\hline $\mathbf{T 1 1 2}$ & C & kg & 1.0 & $1.01 \mathrm{E}+03$ & & $6 \mathrm{E}+01$ & +02 & & -02 & & $E+02$ \\
\hline $\mathrm{r112}$ & $\mathbf{U}$ & kg & $9.25 \mathrm{E}-$ & $9.25 E+02$ & & $3 E+02$ & +02 & $E+03$ & $5 E+03$ & $E+02$ & $E+03$ \\
\hline 112 & $\mathbf{Z r}$ & $\mathrm{kg}$ & $5.00 \mathrm{E}+01$ & $0.00 \mathrm{E}+01$ & $2.27 \mathrm{E}-01$ & $1.86 \mathrm{E}-00$ & $2.53 E+01$ & $0 \mathrm{E} \div 02$ & $2.67 \mathrm{E}+02$ & $4.64 \mathrm{E}+01$ & $5.90 \mathrm{E}+01$ \\
\hline T112 & Ac227 & $\mathrm{Ci}$ & $6.27 E-07$ & $6.27 \mathrm{E}-07$ & $0.00 \mathrm{E}+01$ & $00 \mathrm{E}+01$ & 27E-07 & $63 \mathrm{E}-06$ & 27E-06 & $1.37 \mathrm{E}-06$ & $1.94 E-06$ \\
\hline T112 & Am241 & $\mathrm{Ci}$ & $2.80 \mathrm{E}-02$ & $2.76 E-02$ & $2.37 \mathrm{E}-01$ & $49 \mathrm{E}-00$ & $2.75 E+01$ & $1.14 \mathrm{E}+03$ & $3.66 \mathrm{E}+03$ & $3.44 \mathrm{E}+02$ & $7.55 \mathrm{E}+02$ \\
\hline 112 & Am243 & $\mathrm{Ci}$ & $5.40 \mathrm{E}-08$ & $2.00 \mathrm{E}-07$ & $E-09$ & 4E-08 & $E-08$ & E- 07 & $64 \mathrm{E}-07$ & -08 & E-08 \\
\hline $112>->$ & Ba137 & $\mathrm{Ci}$ & $.64 E+01$ & $4.62 \mathrm{E}+01$ & $E+01$ & E-00 & +01 & $E+02$ & $2 E+02$ & & $E+01$ \\
\hline 112 & C & $\mathrm{Ci}$ & 2.8 & 2.83E-03 & -01 & E-04 & & & $9 \mathrm{E}_{2} \longrightarrow$ & & .03 \\
\hline $\mathbf{T}$ & & $\mathrm{Ci}$ & 7.4 & $7.45 \mathrm{E}-03$ & & & & & 02. & & \\
\hline T112 & 242 & $\mathrm{Ci}$ & $6.88 \mathrm{E}-05$ & 2.55E-04 & 3 & E-05 & 10 & 04 & -04 & 9. & -05 \\
\hline T112 & Cm243 & $\mathrm{Ci}$ & 1.42E-06 & 5.25E-06 & $E-08$ & E-07 & 06 & $E-06$ & $9.586-00$ & $1.99 \mathrm{E}-06$ & $1.94 \mathrm{E}-06$ \\
\hline T112 & $\mathrm{Cm} 244$ & $\mathrm{Ci}$ & $1.29 \mathrm{E}-06$ & $4.76 \mathrm{E}-06$ & $5.89 \mathrm{E}-08$ & $3.19 \mathrm{E}-07$ & 1.29E-06 & $3.97 \mathrm{E}-06$ & 8.70E-06 & $1.81 \mathrm{E}-06$ & $1.76 \mathrm{E}-06$ \\
\hline T112 & Co60 & $\mathrm{Ci}$ & $7.62 \mathrm{E}-04$ & $7.62 E-04$ & $E-05$ & E-01 & -00 & +01 & $1.65 \mathrm{E}+02$ & $E-00$ & $E+01$ \\
\hline 11 & Cs134 & $\mathrm{Ci}$ & $3.56 \mathrm{E}-05$ & $3.56 \mathrm{E}-05$ & +01 & E-06 & 05 & $\mathrm{E}-0$ & $E_{-} 0$ & & \\
\hline T112 & $c$ & $\mathrm{Ci}$ & $4.91 \mathrm{E}+01$ & $4.88 \mathrm{E}+01$ & 3. & $E+03$ & 14 & $E+04$ & $E+04$ & & 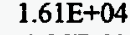 \\
\hline T112 & & $\mathrm{Ci}$ & E-03 & $3.47 \mathrm{E}-03$ & & 01 & & & & & \\
\hline $\mathbf{T}$ & & $\mathrm{Ci}$ & 1. & 1.4 & & 1 & 02 & 2 & 11 & .02 & \\
\hline T112 & Eu155 & $\mathrm{Ci}$ & 2.33E-01 & 2.33E-01 & +01 & $0.00 \mathrm{E}+01$ & -01 & $E-00$ & $.44 \mathrm{E}-00$ & $E-01$ & E-01 \\
\hline T112 & 1129 & $\mathrm{Ci}$ & 3.72E-05 & 3.72E-05 & $E+01$ & E-05 & E-05 & E-05 & $1.14 \mathrm{E}-04$ & $E-05$ & E-05 \\
\hline T112 & $\mathrm{Nb93}$ & $\mathrm{Ci}$ & -03 & $2.37 \mathrm{E}-03$ & 01 & $E+01$ & & & & & \\
\hline T112 & & c & 8.03E-04 & 8.03E-04 & & $E-04$ & & & & & \\
\hline 2 & & 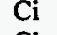 & 2 & 7.3 & & 12 & & & & & \\
\hline T112 & Np237 & $\mathrm{Ci}$ & $1.23 \mathrm{E}-04$ & $1.23 \mathrm{E}-04$ & 06 & $E-05$ & 04 & 04 & 4 & 04 & -04 \\
\hline T112 & P2231 & $\mathrm{Ci}$ & 1.41E-06 & 1.41E-06 & $E+01$ & E-07 & -06 & $E-06$ & $2.00 \mathrm{E}-05$ & -06 & E-06 \\
\hline T112 & Pu238 & $\mathrm{Ci}$ & 5.40E-01 & $5.39 \mathrm{E}-01$ & -02 & $E-01$ & 01 & $E-00$ & $E-00$ & 01 & $E-01$ \\
\hline T112 & 239 & $\mathrm{i}$ & $6.78 \mathrm{E}+01$ & $6.70 \mathrm{E}+01$ & & +01 & & & & & +01 \\
\hline T112 & 240 & c & 00 & & & & & & & & \\
\hline $\mathbf{T}$ & & & 0 & 2 & & 00 & & 01 & -01 & & -00 \\
\hline 112 & Puz42 & $\mathrm{Ci}$ & 2.92E-05 & $1.08 \mathrm{E}-04$ & 06 & E-06 & .05 & $E-05$ & -04 & -05 & -05 \\
\hline T112 & Ra226 & $\mathrm{Ci}$ & 1.21E-07 & 1.21E-07 & $: 01$ & $9.67 \mathrm{E}-09$ & 07 & -07 & $-0 /$ & -07 & $E-07$ \\
\hline T112 & $\operatorname{Ra228}$ & $\mathrm{Ci}$ & $9.57 \mathrm{E}-12$ & 9.57E-12 & +01 & 7. & & & & & -11 \\
\hline T112 & & $\mathrm{Ci}$ & 10 & 10 & & & & & & & \\
\hline $\mathbf{T}$ & & $\mathrm{Ci}$ & & 7.7 & & & & & & 04 & -04 \\
\hline T11 & & $\mathrm{Ci}$ & -04 & 5.97 & 05 & $E-04$ & 04 & $E-03$ & 3 & $7.30 \mathrm{E}-04$ & $E-04$ \\
\hline T112 & Sml51 & $\mathrm{Ci}$ & $2.21 \mathrm{E}-00$ & $2.21 \mathrm{E}-00$ & $E+01$ & $E+01$ & -00 & $E+01$ & $E+01$ & $E-00$ & -00 \\
\hline T112 & Sr $89 / 90$ & $\mathrm{Ci}$ & $1.59 E+03$ & $1.58 \mathrm{E}+03$ & +01 & $E+03$ & -04 & $i+05$ & E & $i+04$ & $E+04$ \\
\hline T112 & Te99 & $\mathrm{Ci}$ & $2.60 \mathrm{E}-00$ & 2.56E-00 & 02 & & & & & & $E+01$ \\
\hline T1 & & $\mathrm{Ci}$ & & & & & & & & & \\
\hline $\mathbf{T}$ & & c & & & & & & & & 12 & -12 \\
\hline & & $\mathrm{Ci}$ & 04 & 9.0 & & E-04 & & -03 & & & -03 \\
\hline T112 & ium & $\mathrm{Ci}$ & $7.20 \mathrm{E}-03$ & $7.20 \mathrm{E}-03$ & $E-03$ & $2 \mathrm{E}-03$ & 03 & $E-02$ & $1.65 \mathrm{E}-02$ & -03 & $E-03$ \\
\hline T112 & U232 & $\mathrm{Ci}$ & 8.84E-07 & 7.31E-06 & +01 & |E-07 & 07 & $E-06$ & EE-05 & -06 & E-06 \\
\hline T112 & U233 & $\mathrm{Ci}$ & 4.05E-08 & 3.35E-07 & 01 & $E-0$ & 98 & -07 & & 7.42E-08 & $8-07$ \\
\hline T11 & $\mathbf{U}$ & $\mathrm{Ci}$ & -02 & 3.0 & & $\mathrm{E}-03$ & & & & & -02 \\
\hline T1 & & C & & & & & & & & & \\
\hline$T$ & & & & & & & & & & & \\
\hline & $\mathbf{U}$ & $\mathrm{Ci}$ & 3.74E-02 & 3.09E-01 & & 03 & & -01 & & -02 & -02 \\
\hline T112 & Y90 & $\mathrm{Ci}$ & $1.59 \mathrm{E}+03$ & $1.58 \mathrm{E}+03$ & $E+01$ & $0.00 \mathrm{E}+01$ & 1.5 & $E+03$ & $E+04$ & $3.06 \mathrm{E}+03$ & $E+03$ \\
\hline T112 & Zr93 & $\mathrm{Ci}$ & 2.83E-03 & $2.83 \mathrm{E}-03$ & $0.00 \mathrm{E}+01$ & $0.00 \mathrm{E}+01$ & $2.83 \mathrm{E}-03$ & $E-02$ & $3.43 E-02$ & 5 & E-03 \\
\hline T20 & Al & $\mathrm{kg}$ & $4.73 E+02$ & $1.40 \mathrm{E}+01$ & $E+01$ & $4 \mathrm{E}-00$ & 01 & $E+03$ & +03 & 2 & +02 \\
\hline T201 & $\mathbf{B i}$ & $\mathbf{k g}$ & $1.30 E+04$ & $1.66 \mathrm{E}+04$ & +02 & -03 & 03 & +03 & +04 & -03 & $=03$ \\
\hline T201 & Ca & kg & $1.68 E+03$ & $1.73 E+02$ & & -01 & & & $i+03$ & & +02 \\
\hline & C & $k$ & & & & & & & +02 & & $\$+01$ \\
\hline T201 & $\mathrm{CO3}$ & kg & $1.55 E+03$ & $5.64 \mathrm{E}+02$ & $E+01$ & $E+02$ & & $E+03$ & $5.89 \mathrm{E}+03$ & $1.75 \mathrm{E}+03$ & $5 E+03$ \\
\hline $\mathrm{T} 20$ & $\mathrm{Cr}$ & kg & $4.59 \mathrm{E}+02$ & $7.46 E+02$ & $1.29 E+02$ & $2.46 \mathrm{E}+02$ & & $4 E+02$ & $7.32 \mathrm{E}+02$ & $4.11 E+02$ & $1.32 \mathrm{E}+02$ \\
\hline $\mathrm{T} 2$ & $\mathbf{F}$ & kg & $8.02 E+02$ & $7.08 \mathrm{E}+02$ & $4.35 E+01$ & $5.60 \mathrm{E}+02$ & & $E+02$ & $283 \mathrm{~F}$ & $1 \mathrm{E}+02$ & $6.83 E+02$ \\
\hline T20 & $\mathbf{F e}$ & $\mathrm{kg}$ & $1.84 E+03$ & $1.38 E+03$ & $1.42 E+02$ & $2.73 E+02$ & $5.14 E+02$ & $1.92 E+03$ & $3.86 \mathrm{E} \div 03$ & $8.07 \mathrm{E}+02$ & $7.68 E+02$ \\
\hline T20 & Hg & kg & $0.00 E+01$ & $0.00 \mathrm{E}+01$ & $0.00 \mathrm{E}+01$ & $0.00 \mathrm{E}+01$ & $0.00 \mathrm{E}+01$ & $0.00 \mathrm{E}+01$ & $0.00 \mathrm{E}+01$ & $0.00 \mathrm{E}+01$ & $0.00 E+01$ \\
\hline & & ng & & & $5.72 E+01$ & $1.11 \mathrm{E}+02$ & $6.72 E+02$ & $39 E+02$ & $1.07 \mathrm{E}+03$ & $6.54 \mathrm{E}+02$ & \\
\hline
\end{tabular}


Tank Anal. Un

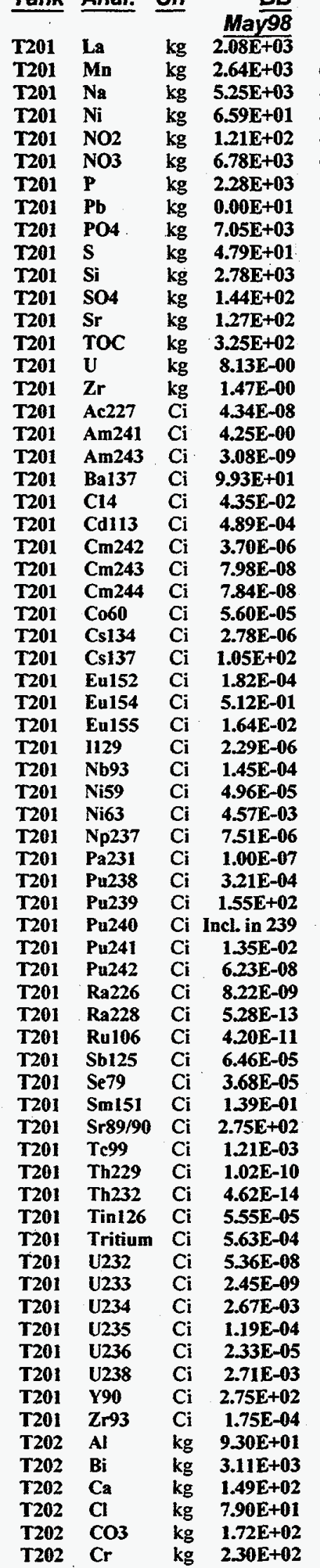

\section{Sept98}

$1.24 \mathrm{E}+03$
$6.18 \mathrm{E}+03$

$4.50 \mathrm{E}+03$

$8.78 \mathrm{E}+01$

$4.38 \mathrm{E}+01$

$6.73 E+03$

NA

$2.96 \mathrm{E}+01$

$1.94 \mathrm{E}+03$

NA

$2.59 \mathrm{E}+02$

$3.86 \mathrm{E}+01$

$1.56 \mathrm{E}+02$

$4.24 \mathrm{E}+01$

8.13E-00

$0.00 \mathrm{E}+01$

4.34E-08

4.32E-00

3.51E-05

6.60E-00

4.41E-02

4.89E-04

4.22E- 02

9.08E-04

8.92E-04

2.73E-01

3.32E-01

6.98E-00

$1.82 \mathrm{E}-04$

6.11E-01

4.57E-01

2.29E-06

1.45E-04

9.56E-04

2.62E-02

7.51E-06

1.00E-07

4.85E-01

$9.28 \mathrm{E}+01$

8.14E-00

$2.69 \mathrm{E}+01$

1.24E-04

8.22E-09

$5.28 \mathrm{E}-13$

$4.20 \mathrm{E}-11$

$6.46 \mathrm{E}-05$

$3.68 \mathrm{E}-05$

1.39E-01

$2.17 \mathrm{E}+01$

1.21E-03

1.02E-10

$4.62 \mathrm{E}-14$

$5.55 \mathrm{E}-05$

2.86E-00

5.36E-08

2.45E-09

2.67E-03

$1.19 \mathrm{E}-04$

2.33E-05

2.71E-03

$2.17 \mathrm{E}+01$

1.75E-04

7.12E-00

$4.04 \mathrm{E}+03$

$3.06 \mathrm{E}+01$

$6.83 \mathrm{E}+01$

$1.03 \mathrm{E}+03$ $3.71 E+02$
$1 \%$ ile

\section{$2.40 \mathrm{E}+02$}

$2.49 \mathrm{E}+02$

$2.45 \mathrm{E}+03$

$3.26 \mathrm{E}-00$

$2.26 \mathrm{E}-01$

$2.13 \mathrm{E}+03$

$1.87 \mathrm{E}+02$

1.51E-04

4.17E+01

$5.36 \mathrm{E}-01$

2.01E-00

$1.65 \mathrm{E}+01$

$5.65 \mathrm{E}-00$

$1.16 \mathrm{E}+01$

$0.00 \mathrm{E}+01$

$2.39 \mathrm{E}-02$

$0.00 \mathrm{E}+01$

3.39E-01

$5.46 \mathrm{E}-10$

$0.00 \mathrm{E}+01$

$0.00 \mathrm{E}+01$

$0.00 \mathrm{E}+01$

6.56E-07

1.42E-08

$1.39 \mathrm{E}-08$

$0.00 \mathrm{E}+01$

$0.00 \mathrm{E}+01$

4.97E-01

$0.00 E+01$

$0.00 \mathrm{E}+01$

$0.00 \mathrm{E}+01$

1.15E-06

2.77E-05

$0.00 \mathrm{E}+01$

$0.00 \mathrm{E}+01$

$1.33 \mathrm{E}-06$

$0.00 \mathrm{E}+01$

$5.69 \mathrm{E}-05$

$2.75 \mathrm{E}+01$

$2.75 \mathrm{E}+01$

2.39E-03

1.10E-08

$0.00 \mathrm{E}+01$

$0.00 \mathrm{E}+01$

9.16E-12

$0.00 \mathrm{E}+01$

6.02E-06

$0.00 \mathrm{E}+01$

$0.00 \mathrm{E}+01$

$0.00 \mathrm{E}+01$

$0.00 \mathrm{E}+01$

$0.00 \mathrm{E}+01$

$0.00 \mathrm{E}+01$

$3.78 \mathrm{E}-04$

$0.00 \mathrm{E}+01$

$0.00 \mathrm{E}+01$

$0.00 \mathrm{E}+01$

$0.00 \mathrm{E}+01$

$0.00 \mathrm{E}+01$

$0.00 \mathrm{E}+01$

$0.00 \mathrm{E}+01$

3.35E-05

$0.00 \mathrm{E}+01$

$5.35 \mathrm{E}+02$

$1.58 \mathrm{E}-03$

$3.48 \mathrm{E}+01$

$6.80 \mathrm{E}+01$

$1.01 \mathrm{E}+02$

\section{$10 \%$-ile}

7.42E+02

$8.46 \mathrm{E}+02$

$3.14 \mathrm{E}+03$

$1.13 \mathrm{E}+01$

$3.46 \mathrm{E}+01$

$5.00 \mathrm{E}+03$

$2.33 \mathrm{E}+02$

$1.40 \mathrm{E}-00$

$1.99 \mathrm{E}+02$

$6.32 \mathrm{E}-00$

$4.56 \mathrm{E}+01$

$4.08 \mathrm{E}+01$

$3.18 \mathrm{E}+01$

$6.86 \mathrm{E}+01$

7.12E-01

$6.35 \mathrm{E}-02$

$0.00 \mathrm{E}+01$

$1.43 \mathrm{E}-00$

$1.49 \mathrm{E}-09$

$4.59 \mathrm{E}-00$

$8.00 \mathrm{E}-03$

$6.07 \mathrm{E}-05$

1.80E-06

$3.87 \mathrm{E}-08$

$3.80 \mathrm{E}-08$

$2.30 \mathrm{E}-02$

1.28E-07

$1.44 \mathrm{E}+01$

$0.00 \mathrm{E} \div 01$

$0.00 \mathrm{E}+01$

$0.00 \mathrm{E}+01$

$1.68 \mathrm{E}-06$

7.38E-05

3.34E-06

3.07E-04

3.64E-06

$1.72 \mathrm{E}-08$

$1.56 \mathrm{E}-04$

$7.52 \mathrm{E}+01$

$7.52 \mathrm{E}+01$

$6.55 \mathrm{E}-03$

3.02E-08

$0.00 \mathrm{E}+01$

$0.00 \mathrm{E}+01$

2.07E-11

$0.00 \mathrm{E}+01$

$1.56 \mathrm{E}-05$

$0.00 \mathrm{E}+01$

$1.74 \mathrm{E}+01$

4.32E-05

1.75E-11

$7.94 \mathrm{E}-15$

$6.89 \mathrm{E}-06$

4.63E-04

$9.22 \mathrm{E}-09$

4.21E-10

4.59E-04

2.05E-05

4.01E-06

4.66E-04

8.23E-00

8.90E-05

$1.39 \mathrm{E}-00$

$1.92 \mathrm{E}+03$

$7.56 \mathrm{E}-00$

4.72E+01

$2.87 \mathrm{E}+02$

$1.74 \mathrm{E}+02$ 50\%-ile

$1.28 \mathrm{E}+03$

$1.63 \mathrm{E}+03$

$4.27 \mathrm{E}+03$

$2.44 \mathrm{E}+01$

$1.57 \mathrm{E}+02$

$7.12 \mathrm{E}+03$

$3.19 \mathrm{E}+02$

$1.11 \mathrm{E}+01$

4.10E +02

$2.13 \mathrm{E}+01$

$1.38 \mathrm{E}+02$

$9.48 \mathrm{E}+01$

$6.34 \mathrm{E}+01$

$3.33 \mathrm{E}+02$

4.09E-00

1.25E-01

4.34E-08

4.14E-00

3.08E-09

$9.93 \mathrm{E}+01$

4.35E-02

4.89E-104

3.70E-06

$7.98 \mathrm{E}-08$

7.84E-08

1.52E-01

2.78E-06

$2.92 \mathrm{E}+02$

1.82E-04

5.12E-01

1.64E-02

2.29E-06

1.45E-04

4.96E-05

4.57E- 03

7.51E-06

1.00E-07

3.21E-04

$1.55 \mathrm{E}+02$

$1.55 \mathrm{E}+02$

1.35E-02

6.23E-08

8.22E-09

5.28E-13

4. $20 \mathrm{E}-11$

6.46E-05

3.68E-05

$1.39 \mathrm{E}-01$

5.47E+02

$1.21 \mathrm{E}-03$

1.02E-10

4.62E-14

5.55E-05

5.63E-04

5.36E-08

2.45E-09

2.67E-03

1.19E-04

2.33E-05

2.71E-03

$2.75 \mathrm{E}+02$

$1.75 \mathrm{E}-04$

$7.72 \mathrm{E}+01$

$3.72 \mathrm{E}+03$

$4.27 \mathrm{E}+01$

$6.36 \mathrm{E}+01$

$9.93 \mathrm{E}+02$

$2.94 \mathrm{E}+02$

\section{$90 \%$-ile}

$1.86 \mathrm{E}+03$

$2.56 \mathrm{E}+03$

$6.47 \mathrm{E}+03$

4.60E+01

$6.50 E+02$

$1.43 \mathrm{E}+04$ 
Tank Anal. Un

\begin{tabular}{|c|c|}
\hline T202 & $\mathbf{F}$ \\
\hline $\mathrm{T} 202$ & Fe \\
\hline T202 & $\mathrm{Hg}$ \\
\hline T202 & K \\
\hline $\mathrm{T} 202$ & $\mathbf{L a}$ \\
\hline T202 & Mn \\
\hline $\mathrm{T} 202$ & $\mathrm{Na}$ \\
\hline T202 & $\mathrm{Ni}$ \\
\hline T202 & NO2 \\
\hline T202 & $\mathrm{NO3}$ \\
\hline T202 & $\mathbf{P}$ \\
\hline T202 & $\mathbf{P b}$ \\
\hline T202 & PO4 \\
\hline T202 & $\mathbf{S}$ \\
\hline T202 & $\mathbf{S i}$ \\
\hline T202 & SO4 \\
\hline T202 & $\mathbf{S r}$ \\
\hline T202 & TOC \\
\hline T202 & $\mathbf{U}$ \\
\hline T202 & $\mathbf{Z r}$ \\
\hline T202 & Ac227 \\
\hline T202 & Am241 \\
\hline T202 & $\operatorname{Am} 243$ \\
\hline T202 & Ba137 \\
\hline T202 & C14 \\
\hline T202 & Cd113 \\
\hline T202 & $\mathrm{Cm} 242$ \\
\hline T202 & $\mathrm{Cm} 243$ \\
\hline T202 & $\mathrm{Cm} 244$ \\
\hline T202 & $\mathrm{C} 060$ \\
\hline T202 & Cs134 \\
\hline T202 & Cs137 \\
\hline T202 & Eu152 \\
\hline T202 & Eu154 \\
\hline T202 & Eu155 \\
\hline T202 & 1129 \\
\hline T202 & Nb93 \\
\hline T202 & Ni59 \\
\hline T202 & Ni63 \\
\hline T202 & Np237 \\
\hline $\mathrm{T202}$ & Pa231 \\
\hline T202 & Pu238 \\
\hline T202 & Pu239 \\
\hline T202 & Pu240 \\
\hline T202 & Pu241 \\
\hline T202 & Pu242 \\
\hline T202 & $\operatorname{Ra} 226$ \\
\hline T202 & $\operatorname{Ra} 228$ \\
\hline T202 & Ru106 \\
\hline $\mathrm{T} 202$ & Sb125 \\
\hline T202 & Se79 \\
\hline $\mathrm{T} 202$ & Sm151 \\
\hline T202 & Sr89/90 \\
\hline T202 & Tc99 \\
\hline T202 & Th229 \\
\hline T202 & Th232 \\
\hline T202 & Tin126 \\
\hline T202 & Tritium \\
\hline T202 & U232 \\
\hline T202 & U233 \\
\hline T202 & U234 \\
\hline T202 & U235 \\
\hline $\mathrm{T} 202$ & U236 \\
\hline T202 & U238 \\
\hline T202 & Y90 \\
\hline T202 & Zr93 \\
\hline T20 & A) \\
\hline
\end{tabular}

$\mathbf{T 2 0 3} \mathrm{Bi} \quad \mathrm{k}$

\section{May $\frac{B 8}{88}$} $5 . \overline{93 \mathrm{E}+02}$ $6.22 \mathrm{E}+02$ 3.00E-02 $6.31 \mathrm{E}+02$

$1.25 E+03$

- $1.25 \mathrm{E}+03$

$3.53 \mathrm{E}+03$

$1.91 \mathrm{E}+01$

$5.20 E+01$

$6.02 E+03$

$8.43 E+02$

$6.00 \mathrm{E}+01$

$2.61 \mathrm{E}+03$

$1.34 E+02$

$3.11 E+02$

4.02E+02

$5.50 \mathrm{E}+01$

$2.69 \mathrm{E}+02$

$2.05 \mathrm{E}+01$

5.40E-01

3.25E-08

6.14E-00

2.31E-09

2.30E-00

1.31E-04

3.67E-04

2.78E-06

5.99E-08

5.88E-08

4.20E-05

2.09E-06

2.40E-00

1.37E-04

6.74E-04

$1.23 \mathrm{E}-02$

1.72E-06

1.08E-04

3.72E-05

3.43E-03

5.63E-06

7.51E-08

2.40E-04

$1.90 \mathrm{E}+01$

3.06E-03

1.01E-02

4.67E-08

$6.16 \mathrm{E}-09$

3.96E-13

3.15E-11

4.84E-05

2.76E-05

1.04E-01

$3.49 \mathrm{E}+02$

9.09E-04

7.67E-11

3.46E-14

4.16E-05

4.22E-04

4.02E-08

1.83E-09

2.00E-03

8.92E-05

$1.75 \mathrm{E}-05$

2.03E-03

$3.49 \mathrm{E}+02$

$1.31 \mathrm{E}-04$

8.03E-00

$6.43 E+03$
Sept98

$6.47 \mathrm{E}+02$

$7.51 \mathrm{E}+02$

$0.00 \mathrm{E}+01$

$7.04 \mathrm{E}+02$

$1.91 E+03$

$1.46 \mathrm{E}+03$

$3.54 \mathrm{E}+03$

1.30E+01

5.17E+01

6.47E+03

NA

5.72E-00

$7.21 \mathrm{E}+02$

NA

$1.84 \mathrm{E}+02$

$1.09 \mathrm{E}+02$

$5.00 \mathrm{E}+01$

$3.42 \mathrm{E}+01$

$1.01 \mathrm{E}+01$

2.00E-01

3.25E-08

3.06E-00

2.49E-05

2.88E-00

3.11E-02

3.67E-04

2.99E-02

6.43E-04

6.32E-04

1.93E-01

2.35E-01

3.04E-00

1.37E-04

4.32E-01

3.23E-01

1.72E-06

1.08E-04

6.76E-04

1.85E-02

5.63E-06

$7.51 \mathrm{E}-08$

3.43E-01

$1.69 \mathrm{E}+01$

1.48E-00

4.91E-00

2.27E-05

6.16E-09

3.96E-13

3.15E-11

4.84E-05

2.76E-05

$1.04 \mathrm{E}-01$

2.70E-01

9.09E-04

7.67E-11

3.46E-14

4.16E-05

2.02E-00

$6.66 \mathrm{E}-08$

3.04E-09

3.32E-03

1.48E-04

2.90E-05

3.37E-03

2.70E-01

1.31E-04

9.17E-00

$7.94 \mathrm{E}+03$ 1\%-ile

$10 \%$-ile

$2.11 \mathrm{E}+01 \quad 3.92 \mathrm{E}+02 \quad 5.51 \mathrm{E}+02$

$9.96 \mathrm{E}+01 \quad 1.99 \mathrm{E}+02 \quad 3.68 \mathrm{E}+02$

$0.00 \mathrm{E}+01 \quad 3.46 \mathrm{E}-03$

$4.81 \mathrm{E}+01 \quad 2.78 \mathrm{E}+02$

$1.70 \mathrm{E}+02 \quad 5.28 \mathrm{E}+02$

$1.58 \mathrm{E}+02$

$1.71 \mathrm{E}+03$

2.47E-00

$5.89 \mathrm{E}+02$

$2.23 \mathrm{E}+03$

8.33E-00

$1.08 \mathrm{E}-00 \quad 2.67 \mathrm{E}+01$

$1.45 \mathrm{E}+03$

$3.55 \mathrm{E}+03$

$1.29 \mathrm{E}+02$

$1.67 \mathrm{E}+02$

$9.99 \mathrm{E}-01$

$4.02 \mathrm{E}+01$

$1.50 \mathrm{E}+02$

$3.76 \mathrm{E}-01$

$4.58 \mathrm{E}-00$

$3.19 \mathrm{E}-01$

1.13E+01

$3.19 \mathrm{E}+01$

6.62E-00

$2.21 \mathrm{E}+01$

$2.61 \mathrm{E}-03$

$1.88 \mathrm{E}-02$

$4.89 \mathrm{E}+01$

$6.00 \mathrm{E}-01$

4.41E-02

$0.00 E+01$

2.77E-01

$1.10 \mathrm{E}-00$

$1.13 \mathrm{E}-09$

$0.00 \mathrm{E}+01$

$0.00 \mathrm{E}+01$

$5.76 \mathrm{E}-02$

$1.97 \mathrm{E}-05$

$0.00 \mathrm{E}+01$

$5.14 E-07$

$1.11 E-08$

1.09E-08

4.23E-05

$1.36 \mathrm{E}-06$

2.93E-08

2.87E-08

6.10E-05

$0.00 \mathrm{E}+01$

$7.40 \mathrm{E}-02$

$0.00 \mathrm{E}+01$

$1.85 \mathrm{E}-02$

5.23E-08

$9.69 \mathrm{E}-00$

$0.00 \mathrm{E}+0$

$0.00 \mathrm{E}+01$

8.35E-07

2.17E-05

$0.00 \mathrm{E}+01$

$0.00 E+01$

$1.22 \mathrm{E}-06$

5.10E-05

4.21E-06

3.89E-04

2.75E-06

$1.04 \mathrm{E}-06$

$0.00 \mathrm{E}+01$

4.43E-05

1.41E-08

$1.17 \mathrm{E}-04$

6.43E-03 1.70E-02

$5.65 \mathrm{E}-04$

$1.50 \mathrm{E}-03$

$1.87 \mathrm{E}-03 \quad 4.93 \mathrm{E}-03$

8.63E-09 2.28E-08

$0.00 \mathrm{E}+01$

$0.00 \mathrm{E}+01$

$0.00 \mathrm{E}+01$

$0.00 \mathrm{E}+01$

6.72E-12 1.59E-11

$0.00 \mathrm{E}+01 \quad 0.00 \mathrm{E}+01$

4.13E-06 1.13E-05

$0.00 \mathrm{E}+01$

$0.00 \mathrm{E}+01$

$0.00 \mathrm{E}+01$

$2.29 \mathrm{E}+01$

$0.00 \mathrm{E}+01$

$0.00 \mathrm{E}+01$

3.11E-05

1.44E-11

$0.00 \mathrm{E}+01 \quad 6.51 \mathrm{E}-15$

$0.00 \mathrm{E}+01 \quad 4.80 \mathrm{E}-06$

2.63E-04 3.41E-04

$0.00 \mathrm{E}+01 \quad 7.57 \mathrm{E}-09$

$0.00 \mathrm{E}+01 \quad 3.44 \mathrm{E}-10$

$0.00 \mathrm{E}+01 \quad 3.76 \mathrm{E}-04$

$0.00 \mathrm{E}+01 \quad 1.68 \mathrm{E}-05$

$0.00 \mathrm{E}+01 \quad 3.29 \mathrm{E}-06$

$0.00 \mathrm{E}+01 \quad 3.82 \mathrm{E}-04$

$0.00 \mathrm{E}+01$

$3.82 \mathrm{E}-04$
$1.92 \mathrm{E}+01$

$6.18 \mathrm{E}-05$

2.63E-05

$1.72 \mathrm{E}-02$

2.06E-00

$9.47 \mathrm{E}+02 \quad 3.34 \mathrm{E}+03$

$3.00 \mathrm{E}-02$

$4.78 \mathrm{E}+02$

$9.08 \mathrm{E}+02$

$1.15 E+03$

3.13E+03

$1.76 \mathrm{E}+01$

$1.31 \mathrm{E}+02$

$5.27 \mathrm{E}+03$

$2.36 \mathrm{E}+02$

8.51E-00

$2.97 \mathrm{E}+02$

$1.69 \mathrm{E}+01$

$1.00 \mathrm{E}+02$

$7.09 \mathrm{E}+01$

$4.54 \mathrm{E}+01$

$2.33 \mathrm{E}+02$

3.15E-00

9.34E-02

3.25E-08

3.06E-00

2.31E-09

2.30E-00

1.31E-04

3.67E-04

2.78E-06

$5.99 \mathrm{E}-08$

$5.88 \mathrm{E}-08$

$1.20 \mathrm{E}-01$

2.09E-06

$3.25 \mathrm{E}+02$

1.37E-04

6.74E-04

1.23E-02

1.72E-06 


\begin{tabular}{|c|c|c|c|c|c|c|}
\hline & & & May98 & Sept98 & 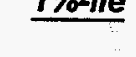 & 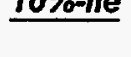 \\
\hline 203 & $\mathrm{Ca}$ & $\mathrm{kg}$ & $3.43 \mathrm{E}+01$ & $5.64 \mathrm{E}+01$ & $1.96 \mathrm{E}-02$ & $1.25 \mathrm{E}+01$ \\
\hline 203 & c & $\mathrm{kg}$ & $1.35 E+02$ & $1.07 \mathrm{E}+02$ & $5.76 \mathrm{E}+01$ & $8.07 E+01$ \\
\hline 203 & $\cos$ & kg & $5.28 \mathrm{E}+02$ & $1.29 E+03$ & $3 E+01$ & $5.49 \mathrm{E}+02$ \\
\hline 203 & $\mathrm{Cr}$ & $\mathbf{k g}$ & $4.76 E+02$ & $6.18 E+02$ & $1.58 \mathrm{E}+02$ & $3.07 \mathrm{E}+02$ \\
\hline 203 & $\mathbf{F}$ & $\mathbf{k g}$ & $1.21 E+03$ & $1.03 E+03$ & $4.35 \mathrm{E}+01$ & $6.69 \mathrm{E}+02$ \\
\hline 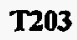 & $\mathrm{Fe}$ & $\mathrm{kg}$ & $6.81 \mathrm{E}+02$ & $1.11 E+03$ & $1.73 E+02$ & \\
\hline 20 & $\mathbf{H g}$ & k & $0.00 \mathrm{E}+01$ & $0.00 \mathrm{E}+01$ & $0.00 \mathrm{E}+01$ & OEE+01 \\
\hline 203 & $\mathbf{K}$ & & $8.03 E+02$ & $1.12 E+03$ & $6.86 \mathrm{E}+01$ & $2 \mathrm{E}+02$ \\
\hline 20 & $\mathbf{L a}$ & 1 & $1.61 \mathrm{E}+03$ & $2.00 \mathrm{E}+03$ & $2.93 \mathrm{E}+02$ & $9.03 E+02$ \\
\hline 203 & $\mathbf{M n}$ & & $2.18 E+03$ & $2.62 E+03$ & $2.66 \mathrm{E}+02$ & $1.04 E+03$ \\
\hline 20 & $\mathrm{Na}$ & $\mathrm{kg}$ & $4.57 \mathrm{E}+03$ & $5.67 E+03$ & $2.91 E+03$ & $3.76 \mathrm{E}+03$ \\
\hline 203 & $\mathrm{Ni}$ & $k$ & $2.82 \mathrm{E}+01$ & $2.41 E+01$ & 4.40E- 00 & $1.37 \mathrm{E}+01$ \\
\hline 203 & $\mathrm{NO} 2$ & kg & $1.17 E+02$ & $4.81 \mathrm{E}+01$ & $2.75 E-01$ & $E+01$ \\
\hline 203 & NO3 & $\mathrm{kg}$ & 1.001 & $1.05 E+04$ & & $: 03$ \\
\hline 203 & $\mathbf{P}$ & $\mathrm{kg}$ & 1.0 & NA & +02 & +02 \\
\hline 20 & $\mathbf{P b}$ & n & 0.0 & 3.39E-00 & +01 & -00 \\
\hline 80 & PO4 & Ki & $3.19 \mathrm{I}$ & $1.19 E+03$ & $5.00 \mathrm{E}+01$ & $2.38 \mathrm{E}+02$ \\
\hline 20 & $\mathbf{S}$ & $\mathrm{kg}$ & 1.1 & NA & $E-01$ & $2 E-00$ \\
\hline 203 & $\mathbf{S i}$ & $\mathrm{kg}$ & $1.42 E+02$ & $2.61 E+02$ & $E-00$ & $E+01$ \\
\hline 203 & SO4 & $\mathrm{kg}$ & $3.30 \mathrm{E}+02$ & $7.14 E+01$ & & $6 E+01$ \\
\hline 20 & Sr & $\mathrm{kg}$ & $7.61 E+01$ & $9.08 E+01$ & & +01 \\
\hline 20 & TO & $\mathrm{kg}$ & $3.38 \mathrm{E}+03$ & $6.84 E+01$ & -00 & +01 \\
\hline 20 & $\mathbf{U}$ & k & $1.02 \mathrm{E}+01$ & $1.02 \mathrm{E}+01$ & $E+01$ & $E-01$ \\
\hline 20 & $\mathbf{Z r}$ & k & $0.00 \mathrm{E}+01$ & $0.00 \mathrm{E}+01$ & $E-02$ & $7.68 \mathrm{E}-02$ \\
\hline 205 & Ac227 & 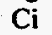 & $5.42 \mathrm{E}-08$ & $5.42 \mathrm{E}-08$ & $E+01$ & $0.00 \mathrm{E}+01$ \\
\hline 20. & Am241 & $\mathrm{Ci}$ & $4.74 E-04$ & $5.85 E-00$ & & $E-00$ \\
\hline 203 & Am & $c$ & 3.86E-09 & $4.75 \mathrm{E}-0 \mathrm{~S}$ & & \\
\hline 203 & & $c$ & +01 & E-00 & -01 & -00 \\
\hline 203 & C14 & $c$ & 2.18E-04 & $5.15 E-02$ & -01 & -05 \\
\hline 20: & CdII3 & c & $6.12 \mathrm{E}-04$ & 6.12E-04 & $E+01$ & $E-05$ \\
\hline 02 & $\mathrm{Cm} 242$ & $c$ & 4.63E-06 & $5.71 \mathrm{~K}$ & $E-07$ & E-06 \\
\hline 203 & $\mathrm{Cm} 243$ & $c$ & 9.98E-08 & $1.23 E-03$ & .08 & $E-08$ \\
\hline & $\mathrm{Cr}$ & $c$ & -08 & 1.2 & & \\
\hline 203 & $\mathrm{Co}$ & $c$ & $7.00 \mathrm{E}-05$ & -01 & & -02 \\
\hline 203 & CsI & $c$ & $3.48 \mathrm{E}-06$ & 3.88E-01 & $E+01$ & $E-07$ \\
\hline 203 & Cs13 & 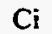 & $2.58 \mathrm{E}+01$ & $3.22 \mathrm{E}-00$ & $E-01$ & $E+01$ \\
\hline 30. & Eul52 & $\mathrm{C}$ & $2.28 \mathrm{E}-04$ & $2.28 \mathrm{E}-04$ & +01 & $E+01$ \\
\hline 203 & Eul54 & $\mathrm{Ci}$ & 1.12E-03 & $7.14 \mathrm{E}-01$ & +01 & $E+01$ \\
\hline 03 & E & $c$ & 02 & & & +01 \\
\hline 203 & 1129 & $c$ & $2.86 \mathrm{E}-06$ & $2.86 \mathrm{E}-06$ & -06 & -06 \\
\hline 203 & $\mathrm{Nb93}$ & $c$ & $1.81 \mathrm{E}-04$ & $1.81 E-04$ & -05 & -05 \\
\hline 203 & Ni59 & $c$ & $6.20 E-05$ & $1.12 \mathrm{E}-03$ & +01 & $E-06$ \\
\hline 203 & $\mathbf{N}$ & $\mathrm{C}$ & $5.72 E-03$ & $3.06 \mathrm{E}-02$ & +01 & E-04 \\
\hline 03 & $\mathbf{N}$ & $\mathrm{Ci}$ & .06 & 9.39E-06 & & -06 \\
\hline 203 & $\mathbf{P}$ & $\mathrm{Ci}$ & $1.25 E-07$ & $1.25 E-07$ & & -08 \\
\hline 203 & Pu238 & $\mathrm{Ci}$ & 04 & $5.67 \mathrm{E}-01$ & & \\
\hline 203 & Pu239 & $\mathrm{Ci}$ & $5.80 \mathrm{E}-02$ & $2.66 \mathrm{E}+01$ & -02 & $E-02$ \\
\hline 203 & Puz40 & $\mathrm{Ci}$ & $5.10 E-03$ & 2.33E-00 & $E-03$ & $E-03$ \\
\hline 203 & Pu241 & $\mathrm{Ci}$ & $1.68 \mathrm{E}-02$ & $7.71 E-00$ & -03 & $E-03$ \\
\hline 203 & Puz & C & & & -08 & $E-08$ \\
\hline 003 & & $\mathrm{Ci}$ & & & & $E+01$ \\
\hline 203 & & $\mathrm{Ci}$ & & -13 & & +01 \\
\hline 203 & Ru106 & $\mathrm{Ci}$ & 5.25 & $5.25 \mathrm{E}-11$ & -11 & \\
\hline 203 & Sb125 & $\mathrm{Ci}$ & $8.07 E-05$ & $8.07 E-05$ & +01 & $E+01$ \\
\hline 203 & Se79 & $\mathrm{Ci}$ & $4.60 \mathrm{E}-05$ & $4.60 \mathrm{E}-05$ & -06 & E-05 \\
\hline 203 & Sm15 & C & $1.74 \mathrm{E}-01$ & $1.74 \mathrm{E}-01$ & +01 & +01 \\
\hline $\mathbf{T 2 0}$ & Sr89/9 & $\mathrm{Ci}$ & 2.28 & 4.61 & & +01 \\
\hline 2 & Tc99 & $\mathrm{Ci}$ & & & & $E-05$ \\
\hline 2 & Th229 & $\mathrm{Ci}$ & $1.28 \mathrm{E}$ & $1.28 \mathrm{E}-10$ & +01 & E-11 \\
\hline 203 & Th232 & $\mathrm{Ci}$ & $5.77 E-14$ & $5.77 \mathrm{E}-14$ & $\div 01$ & $2 \mathrm{E}-15$ \\
\hline & Ti & $\mathrm{Ci}$ & $6.94 E-05$ & $6.94 E-05$ & & \\
\hline & & $\mathrm{C}$ & $7.04 \mathrm{E}-0$ & $3.34 \mathrm{E}-00$ & & \\
\hline & U232 & $\mathrm{Ci}$ & $6.70 E-08$ & $6.70 \mathrm{E}-08$ & $0.00 \mathrm{E}+01$ & $1.06 \mathrm{E}-08$ \\
\hline & U233 & C & $3.06 \mathrm{E}-0$ & $3.06 \mathrm{E}-09$ & $E+01$ & $E-10$ \\
\hline 120 & U234 & $\mathrm{Ci}$ & $3.34 E-03$ & $3.34 \mathrm{E}-03$ & $0.00 \mathrm{E}+01$ & $5.28 \mathrm{E}-04$ \\
\hline & $\mathbf{U} 235$ & $\mathrm{Ci}$ & $1.49 E-04$ & $1.49 \mathrm{E}-04$ & $0.00 \mathrm{E}+01$ & $2.35 \mathrm{E}-0.5$ \\
\hline & U236 & $\mathrm{C}$ & $2.91 \mathrm{E}-05$ & $2.91 \mathrm{E}-05$ & $0.00 E \div 01$ & $4.60 \mathrm{E}-06$ \\
\hline & U238 & $\mathrm{Ci}$ & $3.39 \mathrm{E}-03$ & $3.39 E-03$ & $0.00 E+01$ & $5.36 \mathrm{E}-04$ \\
\hline
\end{tabular}

$50 \%$-ile

\section{$6.61 \mathrm{E}+01$}

$1.04 \mathrm{E}+02$

$1.68 \mathrm{E}+03$

$4.89 \mathrm{E}+02$

$9.41 \mathrm{E}+02$

$6.07 \mathrm{E}+02$

$0.00 \mathrm{E}+01$

$8.20 \mathrm{E}+02$

$1.57 \mathrm{E}+03$

$1.97 \mathrm{E}+03$

5.13E +03

$2.90 \mathrm{E}+01$

$1.85 E+02$

$8.71 \mathrm{E}+03$

$3.82 \mathrm{E}+02$

$1.30 \mathrm{E}+01$

$4.87 \mathrm{E}+02$

$2.66 \mathrm{E}+01$

$1.67 \mathrm{E}+02$

$1.13 E+02$

$7.62 \mathrm{E}+01$

$4.06 \mathrm{E}+02$

4.94E-00

1.49E-01

5.42E-08

5.09E-00

3.86E-09

$2.44 \mathrm{E}+01$

2.18E-04

6.12E-04

4.63E-06

9.98E-08

9.80E-08

1.77E-01

3.48E-06

$2.80 \mathrm{E}+02$

$2.28 \mathrm{E}-04$

1.12E-03

2.00E-02

2.86E-06

1.81E-04

6.20E-05

5.72E-03

9.39E-06

1.25E-07

4.01E-04

5.80E-02

5.10E-03

1.68E-02

7.79E-08

1.03E-08

6.61E-13

5.25E-11

8.07E-05

4.60E-05

1.74E-01

$6.97 E+02$

$1.51 E-03$

1.28E-10

5.77E-14

6.94E-05

7.04E-04

6.70E-08

3.06E-09

3.34E-03

1.49E-04

2.91E-05

$3.39 \mathrm{E}-03$ 90\%-ile

\section{$4.06 \mathrm{E}+02$}

$1.56 \mathrm{E}+02$

$4.72 \mathrm{E}+03$

$6.98 \mathrm{E}+02$

$1.12 \mathrm{E}+03$

$2.33 \mathrm{E}+03$

$0.00 \mathrm{E}+01$

$1.08 \mathrm{E}+03$

$2.26 \mathrm{E}+03$

$3.15 \mathrm{E}+03$

$7.64 \mathrm{E}+03$

$5.51 \mathrm{E}+01$

$6.61 \mathrm{E}+02$

$1.45 \mathrm{E}+04$

$7.68 \mathrm{E}+02$

$1.28 \mathrm{E}+02$

$1.40 \mathrm{E}+03$

$1.56 \mathrm{E}+02$

$4.12 \mathrm{E}+02$

$4.36 \mathrm{E}+02$

$1.20 \mathrm{E}+02$

$9.94 \mathrm{E}+02$

$8.58 \mathrm{E}+01$

$1.32 \mathrm{E}+01$

1.77E-07

$1.51 \mathrm{E}+02$

8.02E-09

$2.99 \mathrm{E}+02$

6.93E-04

6.01E-03

9.62E-06

2.07E-07

2.04E-07

$2.18 \mathrm{E}-00$

4.27E-05

$3.43 \mathrm{E}+03$

7.43E-04

3.65E-03

6.52E-02

4.48E-06

$1.60 \mathrm{E}-02$

$1.56 \mathrm{E}-04$

1.44E-02

$1.95 \mathrm{E}-05$

2.18E-06

8.33E-04

$1.21 \mathrm{E}-01$

$1.06 \mathrm{E}-02$

$3.49 \mathrm{E}-02$

1.62E-07

2.38E-08

1.53E- 12

2.13E-10

$1.86 \mathrm{E}-04$

1.79E-04

$5.67 \mathrm{E}-01$

$1.52 \mathrm{E}+04$

3.31E-03

2.23E-09

$1.01 \mathrm{E}-12$

6.81E-04

8.03E-04

1.17E-06

$5.34 \mathrm{E}-08$

$5.82 \mathrm{E}-02$

2.60E-03

5.07E-04

5.91E-02

\section{9\%-ile}

\section{$2.44 E+03$}

$3.18 \mathrm{E}+02$

$7.12 \mathrm{E}+03$

$8.79 \mathrm{E}+02$

$3.21 \mathrm{E}+03$

$4.61 \mathrm{E}+03$

$0.00 E+01$

$1.31 E+03$

$3.20 \mathrm{E}+03$

$4.77 \mathrm{E}+03$

$1.16 \mathrm{E}+04$

$9.99 \mathrm{E}+01$

$5.42 \mathrm{E}+03$

$3.00 \mathrm{E} \div 04$

$2.38 \mathrm{E}+03$

1.00E +03

$4.27 \mathrm{E}+03$

$5.16 E+02$

$2.21 \mathrm{E}+03$

$1.37 \mathrm{E}+03$

$1.58 \mathrm{E}+02$

$2.61 \mathrm{E}+03$

$2.51 \mathrm{E}+02$

$4.20 \mathrm{E}+01$

$3.46 \mathrm{E}-07$

$6.13 \mathrm{E}+02$

$2.08 \mathrm{E}-08$

$5.15 \mathrm{E}+02$

1.07E-03

4.71E-02

2.49E-05

5.37E-07

5.27E-07

$6.88 \mathrm{E}-00$

7.35E-05

$5.91 \mathrm{E}+03$

$1.46 \mathrm{E}-03$

7.15E-03

$1.28 \mathrm{E}-01$

9.49E-06

5.12E-02

3.17E-04

2.93E-02

5.05E-05

6.38E-06

$2.16 \mathrm{E}-03$

3.12E-01

2.74E-02

$9.04 \mathrm{E}-02$

4.19E-07

3.59E-08

2.30E-12

4. $25 \mathrm{E}-10$

3.90E-04

$5.64 \mathrm{E}-04$

$1.11 \mathrm{E}-00$

4.44E+04

$5.80 \mathrm{E}-03$

6.53E-09

2.94E-12

$5.34 \mathrm{E}-03$

$9.00 \mathrm{E}-04$

3.42E-06

$1.56 \mathrm{E}-07$

$1.70 \mathrm{E}-01$

7.60E-03

$1.49 \mathrm{E}-03$

$1.73 \mathrm{E}-01$
Mean StdDev

$2.09 \mathrm{E}+02 \quad 4.53 \mathrm{E}+02$

$1.20 \mathrm{E}+02 \quad 8.39 \mathrm{E}+01$

$2.10 \mathrm{E}+03 \quad 1.62 \mathrm{E}+03$

$4.99 \mathrm{E}+02 \quad 1.57 \mathrm{E}+02$

$9.88 \mathrm{E}+02 \quad 8.31 \mathrm{E}+02$

$9.69 \mathrm{E}+02 \quad 9.25 \mathrm{E}+02$

$0.00 \mathrm{E}+01 \quad 0.00 \mathrm{E}+01$

$7.97 \mathrm{E}+02 \quad 2.54 \mathrm{E}+02$

$1.58 \mathrm{E}+03 \quad 5.55 \mathrm{E}+02$

$2.06 \mathrm{E}+03 \quad 8.96 \mathrm{E}+02$

$5.48 \mathrm{E}+03 \quad 1.74 \mathrm{E}+03$

$3.28 \mathrm{E}+01 \quad 1.88 \mathrm{E}+01$

$4.56 \mathrm{E}+02 \quad 1.02 \mathrm{E}+03$

$1.02 \mathrm{E}+04 \quad 7.51 \mathrm{E}+03$

$5.02 \mathrm{E}+02 \quad 3.66 \mathrm{E}+02$

$6.32 \mathrm{E}+01 \quad 1.65 \mathrm{E}+02$

$7.14 \mathrm{E}+02 \quad 7.91 \mathrm{E}+02$

6.37E+01 1.01E+02

$2.91 \mathrm{E}+02 \quad 5.98 \mathrm{E}+02$

$2.13 \mathrm{E}+02 \quad 2.83 \mathrm{E}+02$

$7.77 \mathrm{E}+01 \quad 3.19 \mathrm{E}+01$

$5.57 \mathrm{E}+02 \quad 5.87 \mathrm{E}+02$

$2.87 \mathrm{E}+01 \quad 5.61 \mathrm{E}+01$

3.59E-00 8.96E-00

$7.25 \mathrm{E}-08 \quad 7.79 \mathrm{E}-08$

$4.97 \mathrm{E}+01 \quad 1.30 \mathrm{E}+02$

4.77E-09 3.54E-09

$8.75 \mathrm{E}+01 \quad 1.29 \mathrm{E}+02$

$2.86 \mathrm{E}-04 \quad 2.52 \mathrm{E}-04$

$2.97 \mathrm{E}-03 \quad 7.75 \mathrm{E}-03$

$5.72 \mathrm{E}-06 \quad 4.25 \mathrm{E}-06$

$1.23 \mathrm{E}-07 \quad 9.16 \mathrm{E}-08$

$1.21 \mathrm{E}-07 \quad 9.00 \mathrm{E}-08$

7.34E-01 1.50E-00

$125 \mathrm{E}-05 \quad 1.84 \mathrm{E}-05$

$1.00 \mathrm{E}+03 \quad 1.48 \mathrm{E}+03$

$3.05 \mathrm{E}-04 \quad 3.28 \mathrm{E}-04$

$1.50 \mathrm{E}-03 \quad 1.61 \mathrm{E}-03$

$2.67 \mathrm{E}-02 \quad 2.87 \mathrm{E}-02$

3.35E-06 2.60E-06

4.38E-03 $1.09 \mathrm{E}-02$

$7.66 \mathrm{E}-05 \quad 6.65 \mathrm{E}-05$

7.07E-03 6.14E-03

$1.16 \mathrm{E}-05 \quad 8.62 \mathrm{E}-06$

7.30E-07 1.42E-06

$4.95 \mathrm{E}-04 \quad 3.68 \mathrm{E}-04$

$7.16 \mathrm{E}-02 \quad 5.33 \mathrm{E}-02$

$6.30 \mathrm{E}-03 \quad 4.68 \mathrm{E}-03$

$2.07 \mathrm{E}-02 \quad 1.54 \mathrm{E}-02$

9.62E-08 7.15E-08

$1.14 \mathrm{E}-08 \quad 8.96 \mathrm{E}-09$

$7.32 \mathrm{E}-13 \quad 5.75 \mathrm{E}-13$

$8.62 \mathrm{E}-11 \quad 8.60 \mathrm{E}-11$

9.38E-05 8.19E-05

8.71E-05 1.17E-04

2.33E-01 2.50E-01

$4.77 E+03 \quad 9.25 E+03$

$1.70 \mathrm{E}-03 \quad 1.30 \mathrm{E}-03$

$7.47 \mathrm{E}-10 \quad 1.46 \mathrm{E}-09$

3.37E-13 6.57E-13

3.37E-04 8.79E-04

$6.96 \mathrm{E}-04 \quad 9.06 \mathrm{E}-05$

$391 \mathrm{E}-07 \quad 7.63 \mathrm{E}-07$

$1.79 \mathrm{E}-08 \quad 3.49 \mathrm{E}-08$

$1.95 \mathrm{E}-02 \quad 3.81 \mathrm{E}-02$

8.70E-04 1.70E-03

$1.70 \mathrm{E}-04 \quad 3.32 \mathrm{E}-04$

$1.98 \mathrm{E}-02 \quad 3.86 \mathrm{E}-02$ 
Tank Anal. Un

\begin{tabular}{|c|c|c|c|}
\hline & & & \\
\hline T203 & Y90 & $\mathrm{Ci}$ & $\frac{\text { Mav98 }}{2.28 \mathrm{E}+01}$ \\
\hline $\mathrm{T} 203$ & Zr93 & $\mathrm{Ci}$ & $2.18 \mathrm{E}-04$ \\
\hline T204 & Al & $\mathrm{kg}$ & $1.09 \mathrm{E}+01$ \\
\hline T204 & $\mathbf{B i}$ & $\mathrm{kg}$ & $8.13 E+03$ \\
\hline T204 & $\mathrm{Ca}$ & kg & $5.11 E+01$ \\
\hline T204 & Cl & kg & $1.18 E+02$ \\
\hline T204 & $\mathrm{CO3}$ & kg & $2.10 \mathrm{E}+03$ \\
\hline T204 & $\mathrm{Cr}$ & kg & $5.43 E+02$ \\
\hline T204 & $\mathbf{F}$ & kg & $1.20 \mathrm{E}+03$ \\
\hline T204 & $\mathbf{F e}$ & $\mathrm{kg}$ & $6.38 \mathrm{E}+02$ \\
\hline T204 & Hg & $\mathrm{kg}$ & $0.00 \mathrm{E}+01$ \\
\hline T204 & $\mathbf{K}$ & kg & \\
\hline T204 & La & kg & \\
\hline T204 & $\mathbf{M n}$ & $\mathrm{kg}$ & $2.48 E+03$ \\
\hline T204 & $\mathbf{N a}$ & kg & $4.47 E+03$ \\
\hline T204 & $\mathbf{N i}$ & $\mathrm{kg}$ & $3.91 \mathrm{E}+01$ \\
\hline T204 & NO2 & kg & $1.22 E+02$ \\
\hline T204 & NO3 & kg & $8.97 E+03$ \\
\hline T204 & $\mathbf{P}$ & kg & $1.19 E+03$ \\
\hline T204 & Pb & $\mathrm{kg}$ & 0. \\
\hline T204 & PO4 & kg & $3.68 \mathrm{E}+03$ \\
\hline T204 & $\mathbf{S}$ & kg & $1.11 \mathrm{E}+02$ \\
\hline T204 & $\mathbf{S i}$ & $\mathrm{kg}$ & $1.79 E+02$ \\
\hline $\mathbf{2 0 4}$ & SO4 & $\mathrm{kg}$ & $3.33 E+02$ \\
\hline T204 & $\mathbf{S r}$ & kg & $6.54 \mathrm{E}+01$ \\
\hline T204 & TOC & kg & 3.67 \\
\hline T204 & $\mathbf{U}$ & kg & 1.10 \\
\hline T204 & $\mathbf{Z r}$ & $\mathrm{kg}$ & $0.00 E+01$ \\
\hline T204 & Ac227 & $\mathrm{Ci}$ & $5.89 E-08$ \\
\hline T204 & Am241 & $\mathrm{Ci}$ & 5.15 \\
\hline T204 & Am243 & $\mathrm{Ci}$ & $4.19 \mathrm{E}-09$ \\
\hline T204 & Ba137 & $\mathrm{Ci}$ & $2.65 \mathrm{E}+01$ \\
\hline T204 & C14 & $\mathrm{Ci}$ & 2.3 \\
\hline T204 & Cd113 & $\mathrm{Ci}$ & $6.64 \mathrm{E}-04$ \\
\hline T204 & $\mathrm{Cm} 242$ & $\mathrm{Ci}$ & 5.03E-06 \\
\hline T204 & $\mathrm{Cm} 243$ & $\mathrm{Ci}$ & $1.08 E-07$ \\
\hline T204 & $\mathrm{Cm} 244$ & $\mathrm{Ci}$ & $1.06 \mathrm{E}-07$ \\
\hline T204 & Co60 & $\mathrm{Ci}$ & $7.60 \mathrm{E}-05$ \\
\hline T204 & Cs134 & $\mathrm{Ci}$ & 3.78E-06 \\
\hline T204 & Cs137 & $\mathrm{Ci}$ & $2.81 \mathrm{E}+01$ \\
\hline T204 & Eu152 & $\mathrm{Ci}$ & $2.47 \mathrm{E}-04$ \\
\hline T204 & Eu154 & $\mathrm{Ci}$ & $1.22 \mathrm{E}-03$ \\
\hline T204 & Eu155 & $\mathrm{Ci}$ & 2.2 \\
\hline T204 & 1129 & $\mathrm{Ci}$ & 3.10E-06 \\
\hline T204 & $\mathrm{Nb93}$ & $\mathrm{Ci}$ & $1.96 \mathrm{E}-04$ \\
\hline T204 & Ni59 & $\mathrm{Ci}$ & $6.73 E-05$ \\
\hline T204 & Ni63 & C & $6.21 \mathrm{E}-03$ \\
\hline 2204 & Np237 & $\mathrm{Ci}$ & -05 \\
\hline T204 & Pa231 & $C i$ & $1.36 \mathrm{E}-07$ \\
\hline T204 & Pu238 & c & 4.3 \\
\hline T204 & Pu239 & $\mathrm{Ci}$ & $6.30 \mathrm{E}-02$ \\
\hline T204 & Pu240 & $\mathrm{Ci}$ & 5.53E-03 \\
\hline T204 & Pu241 & $\mathrm{Ci}$ & $1.83 \mathrm{E}-02$ \\
\hline T204 & Pu242 & $\mathbf{C i}$ & $8.45 E-08$ \\
\hline T204 & Ra226 & C & 1.12E-08 \\
\hline T204 & Ra228 & C & 7.17 \\
\hline T204 & Ru106 & $\mathrm{Ci}$ & $5.70 \mathrm{E}-11$ \\
\hline T204 & Sb125 & $\mathrm{Ci}$ & $8.77 E-05$ \\
\hline T204 & Se79 & $\mathrm{Ci}$ & $5.00 \mathrm{E}-05$ \\
\hline T204 & Sm151 & $\mathbf{C i}$ & $1.89 \mathrm{E}-01$ \\
\hline T204 & Sr89/90 & $\mathrm{Ci}$ & $2.47 \mathrm{E}+01$ \\
\hline T204 & Tc99 & $\mathrm{Ci}$ & $1.64 E-03$ \\
\hline T204 & Th229 & $\mathrm{Ci}$ & 1.39E-10 \\
\hline T204 & Th232 & $\mathrm{Ci}$ & $6.27 \mathrm{E}$ \\
\hline T204 & Tin126 & $\mathrm{Ci}$ & $7.53 E-05$ \\
\hline T204 & Tritium & $\mathrm{Ci}$ & $7.64 E-04$ \\
\hline T204 & U232 & $\mathrm{Ci}$ & $7.27 \mathrm{E}-08$ \\
\hline$m n$ & U23 & $\mathrm{Ci}$ & 3.3 \\
\hline
\end{tabular}

$B B$ Sept98

\subsection{E-01}

2.18E-04

9.33E-00

$8.96 \mathrm{E}+03$

$3.59 \mathrm{E}+01$

$1.17 \mathrm{E}+02$

$1.22 \mathrm{E}+03$

$7.81 \mathrm{E}+02$

$1.03 E+03$

$7.03 \mathrm{E}+02$

$0.00 \mathrm{E}+01$

$1.07 E+03$

$1.27 \mathrm{E}-01$

$2.45 \mathrm{E}+03$

$5.53 \mathrm{E}+03$

$4.20 \mathrm{E}+01$

$4.94 \mathrm{E}+01$

$9.61 \mathrm{E}+03$

NA

$5.40 \mathrm{E}+01$

1.31E+03

NA

$2.61 \mathrm{E}+02$

$6.32 \mathrm{E}+01$

$8.70 \mathrm{E}+01$

$5.43 E+01$

$1.10 \mathrm{E}+01$

$0.00 \mathrm{E}+01$

$5.89 \mathrm{E}-08$

$4.25 \mathrm{E}-00$

$3.45 \mathrm{E}-05$

$1.40 \mathrm{E}-00$

5.50E-02

6.64E-04

4.15E-02

8.94E-04

8.78E-04

3.41E-01

4.14E-01

$1.48 \mathrm{E}-00$

2.47E-04

7.62E-01

5.71E-01

3.10E-06

$1.96 \mathrm{E}-04$

1.19E-03

3.27E-02

1.02E-05

$1.36 \mathrm{E}-07$

6.06E-01

$1.98 \mathrm{E}+01$

$1.74 \mathrm{E}-00$

$5.75 \mathrm{E}-00$

2.66E-05

1.12E-08

7.17E-13

5.70E-11

8.77E-05

$5.00 \mathrm{E}-05$

$1.89 \mathrm{E}-01$

8.82E-01

$1.64 \mathrm{E}-03$

1.39E-10

6.27E-14

7.53E-05

3.57E-00

7.27E-08

3.32E-09 1\%-ile

$0.00 \mathrm{E}+01$

4.94E-05

$0.00 \mathrm{E}+01$

$1.04 \mathrm{E}+03$

$0.00 \mathrm{E}+01$

$6.32 \mathrm{E}+01$

$6.84 \mathrm{E}+01$

$1.68 \mathrm{E}+02$

$4.31 \mathrm{E}+01$

$2.02 \mathrm{E}+02$

$0.00 \mathrm{E}+01$

$7.16 \mathrm{E}+01$

$3.00 \mathrm{E}+02$

$2.83 \mathrm{E}+02$

$3.29 \mathrm{E}+03$

$5.53 \mathrm{E}-00$

$3.00 \mathrm{E}-01$

$3.14 \mathrm{E}+03$

$2.44 \mathrm{E}+02$

1.07E-04

$5.75 \mathrm{E}+01$

8.39E-01

$1.79 \mathrm{E}-01$

$2.25 \mathrm{E}+01$

$6.54 \mathrm{E}-00$

$0.00 \mathrm{E}+01$

$4.40 \mathrm{E}-02$

$0.00 \mathrm{E}+01$

3.51E-01

8.85E-10

$0.00 \mathrm{E}+01$

$0.00 \mathrm{E}+01$

$0.00 \mathrm{E}+01$

1.06E-06

2.28E-08

$2.24 \mathrm{E}-08$

$0.00 \mathrm{E}+01$

$0.00 \mathrm{E}+01$

2.14E-01

$0.00 \mathrm{E}+01$

$0.00 \mathrm{E}+01$

$0.00 \mathrm{E}+01$

1.59E-06

5.51E-05

$0.00 \mathrm{E}+01$

$0.00 \mathrm{E}+01$

2.15E-06

$0.00 \mathrm{E}+01$

9.19E-0S

1.33E-02

$1.17 \mathrm{E}-03$

$3.87 \mathrm{E}-03$

$1.79 \mathrm{E}-08$

$0.00 \mathrm{E}+01$

$0.00 \mathrm{E}+01$

$1.48 \mathrm{E}-11$

$0.00 \mathrm{E}+01$

8.92E-06

$0.00 \mathrm{E}+01$

$0.00 \mathrm{E}+01$

$0.00 \mathrm{E}+01$

$0.00 \mathrm{E}+01$

$0.00 \mathrm{E}+01$

$0.00 \mathrm{E}+01$

$4.95 \mathrm{E}-04$

$0.00 \mathrm{E}+01$

$0.00 \mathrm{E}+01$

\section{$1.06 \mathrm{E}-00$}

1.13E-04

2.19E-00

$3.71 E+03$

$1.40 \mathrm{E}+01$

$8.63 \mathrm{E}+01$

$5.89 \mathrm{E}+02$

$3.17 \mathrm{E}+02$

$7.49 \mathrm{E}+02$

$3.58 \mathrm{E}+02$

$0.00 \mathrm{E}+01$

$5.42 \mathrm{E}+02$

$9.91 \mathrm{E}+02$

$1.11 \mathrm{E}+03$

4.12E+03

$1.47 \mathrm{E}+01$

$4.75 \mathrm{E}+01$

$6.82 \mathrm{E}+03$

$3.07 \mathrm{E}+02$

$1.53 \mathrm{E}-00$

$2.57 \mathrm{E}+02$

$7.97 \mathrm{E}-00$

$6.12 \mathrm{E}+01$

$5.20 \mathrm{E}+01$

$4.04 \mathrm{E}+01$

$8.67 \mathrm{E}+01$

$9.50 \mathrm{E}-01$

$7.98 \mathrm{E}-02$

$0.00 \mathrm{E}+01$

1.83E-00

2.21E-09

2.08E-00

$4.55 \mathrm{E}-05$

$6.92 \mathrm{E}-05$

2.65E-06

$5.69 \mathrm{E}-08$

$5.59 \mathrm{E}-08$

2.94E-02

$2.97 \mathrm{E}-07$

$2.18 \mathrm{E}+01$

$0.00 \mathrm{E}+01$

$0.00 \mathrm{E}+01$

$0.00 \mathrm{E}+01$

2.31E-06

1.00E-04

7.35E-06

6.79E-04

5.38E-06

$2.28 \mathrm{E}-08$

2.29E-04

3.32E-02

$2.92 \mathrm{E}-03$

9.65E-03

4.45E-08

$0.00 E+01$

$0.00 \mathrm{E}+01$

2.94E-11

$0.00 \mathrm{E}+01$

2.14E-05

$0.00 \mathrm{E}+01$

$3.03 E+01$

3.75E-05

2.33E-11

$1.05 \mathrm{E}-14$

7.85E-06

6.23E-04

1.22E-08

$5.57 \mathrm{E}-10$ 50\%-ile

90\%-ile

99\%-ile

Mean StdDev

$2.28 \mathrm{E}+01 \quad 4.98 \mathrm{E}+02$

2.18E-04 1.93E-02

$6.67 \mathrm{E}+01$

$6.99 \mathrm{E}+03$

$7.13 \mathrm{E}+01$

$1.18 \mathrm{E}+03$

$1.14 \mathrm{E}+04$

$11.66 \mathrm{E}+02$

$1.81 \mathrm{E}+03 \quad 5.02 \mathrm{E}+03$

$5.28 \mathrm{E}+02$

$1.02 \mathrm{E}+03$

$6.51 \mathrm{E}+02$

$0.00 \mathrm{E}+01$

$8.92 \mathrm{E}+02$

$1.71 E+03$

$2.17 \mathrm{E}+03$

$5.53 \mathrm{E}+03$

$3.12 \mathrm{E}+01$

$1.90 \mathrm{E}+02$

$9.48 E+03$

4.12E+02

$1.45 \mathrm{E}+01$

$5.38 \mathrm{E}+02$

$2.76 \mathrm{E}+01$

$1.81 \mathrm{E}+02$

$1.19 \mathrm{E}+02$

$8.36 \mathrm{E}+01$

$4.41 \mathrm{E}+02$

5.53E-00

1.56E-01

5.89E-08

5.55E-00

4.19E-09

$2.65 \mathrm{E}+01$

2.37E-04

$6.64 \mathrm{E}-04$

5.03E-06

1.08E-07

$1.06 \mathrm{E}-07$

$1.85 \mathrm{E}-01$

3.78E-06

$2.70 \mathrm{E}+02$

2.47E-04

$1.22 \mathrm{E}-03$

2.23E-02

3.10E-06

1.96E-04

6.73E-05

$6.21 \mathrm{E}-03$

1.02E-05

1.36E-07

4.35E-04

$6.30 \mathrm{E}-02$

5.53E-03

1.83E-02

8.45E-08

1.12E-08

7.17E-13

5.70E-11

8.77E-05

5.00E-05

1.89E-01

$7.08 \mathrm{E}+02$

1.64E-03

1.39E-10

6.27E-14

7.53E-05

7.64E-04

7.27E-08

3.32E-09

$7.62 \mathrm{E}+02$

$1.22 \mathrm{E}+03$

$2.52 \mathrm{E}+03$ 
Tank Anal. Un

\begin{tabular}{ll} 
T204 & U234 \\
T204 & U235 \\
T204 & U236 \\
T204 & U238 \\
T204 & Y90 \\
T204 & Zr93 \\
\hline
\end{tabular}

TX101 Al

TX101 Bi

TX101 Ca

TX101 CI

TX101 CO3

TX101 Cr

TX101 F

TX101 Fe

TX101 Hg

TX101 K

TX101 La

TX101 Mn

TX101 Na

TX101 Ni

TX101 NO2

TX101 NO3

TX101 P

TX101 Pb

TX101 PO4

TX101 S

TX101 Si

TX101 SO4

TX101 Sr

TX101 TOC

TX101 U

TX101 Zr

TX101 Ac227

TX101 Am241

TX101 Am243

TX101 Ba137

TX101 C14

TX101 Cd113

TX101 Cm242

TX101 Cm243

TX101 Cm244

TX101 Co60

TX101 Cs134

TX101 Cs137

TX101 Eu152

TX101 Eu154

TX101 Eu155

TX101 I129

TX101 Nb93

TX101 Ni59

TX101 Ni63

TX101 Np237

TX101 Pa231

TX101 Pu238

TX101 Pu239

TX101 Pu240

TX101 Pu241

TX101 Pu242

TX101 Ra226

TX101 Ra228

TX101 Ru106

TX101 Sb125

TX101 Se79

TX101 Sm151

TX101 Sr89/90

TX101 Te99

TX101 Th229

TX101 Th232
$B B$

May $\overline{98}$

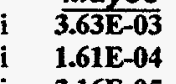

3.16E-05

3.68E-03

$2.47 \mathrm{E}+01$

$2.37 \mathrm{E}-04$

$5.22 \mathrm{E}+04$

$8.29 \mathrm{E}-00$

$1.59 \mathrm{E}+02$

$1.62 E+03$

$4.53 \mathrm{E}+03$

$1.07 \mathrm{E}+03$

$2.21 \mathrm{E}+02$

$8.66 \mathrm{E}+02$

5.59E-02

$3.57 \mathrm{E}+02$

1.27E-01

$6.83 E+02$

$6.64 E+04$

$6.70 E+01$

$1.78 \mathrm{E}+04$

$8.68 \mathrm{E}+04$

$4.84 E+02$

$2.90 \mathrm{E}+01$

$1.50 \mathrm{E}+03$

$7.00 \mathrm{E}+02$

$6.37 \mathrm{E}+02$

$2.10 \mathrm{E}+03$

$2.10 \mathrm{E}+02$

$1.63 \mathrm{E}+03$

$3.86 \mathrm{E}+03$

$6.95 \mathrm{E}-01$

1.30E-03

$1.15 \mathrm{E}+02$

8.68E-05

$6.81 \mathrm{E}+04$

$1.80 \mathrm{E}-00$

$4.86 \mathrm{E}-00$

$4.75 \mathrm{E}-02$

$1.43 \mathrm{E}-03$

$5.20 \mathrm{E}-03$

$1.51 \mathrm{E}-00$

9.16E-02

$7.21 \mathrm{E}+04$

1.98E-00

i $2.65 \mathrm{E}+01$

$9.53 \mathrm{E}+01$

Ci 2.41E-02

9.14E-01

i $\quad 4.01 \mathrm{E}-00$

Ci $3.75 \mathrm{E}+02$

$\mathrm{Ci} \quad 5.82 \mathrm{E}-02$

$\mathrm{Ci}$ 3.39E-04

Ci 1.80E-00

Ci $2.32 \mathrm{E}+02$

$4.45 \mathrm{E}+01$

Ci $1.08 \mathrm{E}+02$

Ci 5.00E-04

$\mathrm{Ci}$ 2.93E-04

$\mathrm{Ci} \quad 4.20 \mathrm{E}-03$

Ci $\quad 2.24 \mathrm{E}-04$

Ci 5.78E-00

$\mathrm{Ci} \quad 2.45 \mathrm{E}-01$

i $8.71 \mathrm{E}+02$

i $1.43 E+05$

Ci 1.25E+01

Ci 9.90E-05

Ci $2.78 \mathrm{E}-04$
$B B$

\section{Sept98}

$\overline{3.63 \mathrm{E}-03}$
$1.61 \mathrm{E}-04$

3.16E-05

3.68E-03

8.82E-01

2.37E-04

$5.22 \mathrm{E}+04$

8.29E-00

$1.59 \mathrm{E}+02$

$1.62 \mathrm{E}+03$

$4.53 \mathrm{E}+03$

$1.07 \mathrm{E}+03$

$2.21 E+02$

$8.66 \mathrm{E}+02$

$0.00 \mathrm{E}+01$

$3.57 \mathrm{E}+02$

$0.00 \mathrm{E}+01$

$6.83 \mathrm{E}+02$

$6.64 \mathrm{E}+04$

$6.70 \mathrm{E}+01$

$1.78 \mathrm{E}+04$

$8.68 \mathrm{E}+04$

NA

$2.30 \mathrm{E}+01$

$1.38 \mathrm{E}+03$

NA

$6.37 \mathrm{E}+02$

2.10E+03

$2.10 \mathrm{E}+02$

$1.63 \mathrm{E}+03$

$3.86 \mathrm{E}+03$

6.95E-01

1.30E-03

$1.15 E+02$

8.68E-05

$6.81 \mathrm{E}+04$

$1.80 \mathrm{E}-00$

4.86E-00

4.75E-02

1.43E-03

$5.20 \mathrm{E}-03$

1.51E-00

9.16E-02

$7.21 E+04$

$1.98 \mathrm{E}-00$

2.65E+01

$9.53 \mathrm{E}+01$

2.41E-02

9.14E-01

4.01E-00

$3.75 E+02$

5.82E- 02

$3.39 \mathrm{E}-04$

$1.80 \mathrm{E}-00$

$2.32 \mathrm{E}+02$

$4.45 E+01$

$1.08 \mathrm{E}+02$

5.00E-04

2.93E-04

4.20E-03

2.24E-04

$5.78 \mathrm{E}-00$

2.45E-01

$8.71 E+02$

$2.50 \mathrm{E}+05$

$1.25 \mathrm{E}+01$

9.90E-05

2.78E-04 1\%-ile

$0.00 \mathrm{E}+01$

$0.00 \mathrm{E}+01$

$0.00 \mathrm{E}+01$

$0.00 \mathrm{E}+01$

$0.00 \mathrm{E}+01$

$6.66 \mathrm{E}-05$

$1.53 \mathrm{E}+04$

7.04E-02

$9.17 \mathrm{E}+01$

$8.09 \mathrm{E}+01$

$1.60 \mathrm{E}+03$

$6.92 \mathrm{E}+01$

$4.23 \mathrm{E}-00$

$2.60 \mathrm{E}+02$

$1.56 \mathrm{E}-03$

$5.78 \mathrm{E}+01$

$10.00 \mathrm{E}-01$

9.87E-00

$1.96 \mathrm{E}+04$

$1.03 E+01$

$1.08 \mathrm{E}+03$

7.33E +02

$1.34 \mathrm{E}+02$

$1.73 \mathrm{E}-00$

$4.07 \mathrm{E}+02$

$5.84 \mathrm{E}+01$

$1.84 \mathrm{E}+01$

$1.21 \mathrm{E}+02$

$1.25 \mathrm{E}-00$

$1.59 \mathrm{E}+02$

8.51E+02

9.30E-03

$0.00 \mathrm{E}+01$

$1.27 \mathrm{E}+01$

1.17E-06

$0.00 \mathrm{E}+01$

$0.00 \mathrm{E}+01$

$1.36 \mathrm{E}-01$

6.42E-04

$1.93 \mathrm{E}-05$

$7.03 \mathrm{E}-05$

$1.68 \mathrm{E}-00$

$0.00 \mathrm{E}+01$

$5.36 \mathrm{E}+03$

$0.00 \mathrm{E}+01$

$0.00 \mathrm{E}+01$

$0.00 \mathrm{E}+01$

$0.00 \mathrm{E}+01$

$0.00 \mathrm{E}+01$

3.79E-01

$3.55 \mathrm{E}+01$

7.86E-04

$0.00 \mathrm{E}+01$

2.43E- 02

$3.13 \mathrm{E}-00$

6.01E-01

$1.46 \mathrm{E}-00$

6.76E-06

$0.00 \mathrm{E}+01$

$0.00 \mathrm{E}+01$

$1.08 \mathrm{E}-05$

$0.00 \mathrm{E}+01$

$1.10 \mathrm{E}-02$

$0.00 \mathrm{E}+01$

6.01E-01

$0.00 \mathrm{E}+01$

$0.00 \mathrm{E}+01$

$0.00 \mathrm{E}+01$

10\%-ile

$6.09 \mathrm{E}-04$

2.70E-05

$5.30 \mathrm{E}-06$

6.17E-04

9.86E-01

1.21E-04

$2.70 \mathrm{E}+04$

$3.70 \mathrm{E}+01$

$1.72 \mathrm{E}+02$

$2.83 \mathrm{E}+02$

$4.55 \mathrm{E}+03$

$3.04 \mathrm{E}+02$

$1.12 \mathrm{E}+02$

$6.63 \mathrm{E}+02$

$1.39 \mathrm{E}-02$

$1.91 \mathrm{E}+02$

3.42E-00 
Tank Anal. Un

$\begin{array}{ll}\text { TX101 } & \text { Tin126 } \\ \text { TX101 } & \text { Tritium } \\ \text { TX101 } & \text { U232 } \\ \text { TX101 } & \text { U233 } \\ \text { TX101 } & \text { U234 } \\ \text { TX101 } & \text { U235 } \\ \text { TX101 } & \text { U236 } \\ \text { TX101 } & \text { U238 } \\ \text { TX101 } & \text { Y90 } \\ \text { TX101 } & \text { Zr93 } \\ \text { TX102 } & \text { Al } \\ \text { TX102 } & \text { Bi } \\ \text { TX102 } & \text { Ca }\end{array}$

TX102 Cl

$\mathrm{rX102} \mathrm{CO} 3$

TX102 Cr

TX102 F

TX102 Fe

TX102 Hg

TX102 K

TX102 La

TX102 Mn

TX102 $\mathrm{Na}$

TX102 Ni

TX102 NO2

TX102 NO3

TX102 P

TX102 Pb

TX102 PO4

TX102 S

TX102 Si

TX102 $\mathrm{SO4}$

TX102 Sr

TX102 TOC

TX102 U

TX102 $\mathrm{Zr}$

TX102 Ac227

TX102 Am241

TX102 Am243

TX102 Ba137

TX102 C14

TX102 Cd113

TX102 Cm242

TX102 Cm243

TX102 Cm244

TX102 Co60

TX102 Cs134

TX102 Cs137

TX102 Eu152

TX102 Eu154

TX102 Eu155

TX102 1129

TX102 Nb93

TX102 Ni59

TX102 Ni63

TX102 Np237

TX102 Pa231

TX102 Pu238

TX102 Pu239

TX102 Pu240

TX102 Pu241

TX102 Pu242

TX102 Ra226

TX102 Ra228

TX102 Ru106

TX102 Sb125

TX102 Se79

TX102 Sm151
May $\frac{B B}{98}$

3.74E-01

$1.61 \mathrm{E}+01$

2.21E-02

8.47E-02

$1.62 \mathrm{E}-00$

7:27E-02

$1.13 E-02$

$1.64 \mathrm{E}-00$

$1.43 \mathrm{E}+05$

1.18E-00

$3.84 \mathrm{E}+04$

$2.98 \mathrm{E}+02$

$4.16 \mathrm{E}+02$

$7.30 \mathrm{E}+03$

$7.56 \mathrm{E}+04$

$1.83 \mathrm{E}+03$

$2.68 \mathrm{E}+03$

$1.03 E+03$

kg 1.56E-00

kg $2.37 \mathrm{E}+03$

kg 1.50E-04

kg 3.31E+02

kg $\quad 2.94 \mathrm{E}+05$

kg $1.27 \mathrm{E}+02$

kg 4.29E+04

kg $\quad 4.28 \mathrm{E}+05$

kg $3.46 \mathrm{E}+03$

kg $1.45 \mathrm{E}+02$

kg 1.07E+04

kg $7.57 \mathrm{E}+03$

2.33E+02

$\mathrm{kg} \quad 2.27 \mathrm{E}+04$

3.18E-04

kg 1.29E+04

g $\quad 5.40 \mathrm{E}+03$

$\mathrm{kg} \quad 9.63 \mathrm{E}+01$

5.83E-04

$3.51 \mathrm{E}+01$

$1.23 \mathrm{E}-03$

$1.47 \mathrm{E}+05$

$1.79 \mathrm{E}+01$

$5.07 \mathrm{E}+01$

$1.04 \mathrm{E}-01$

9.58E-03

8.18E-02

$2.04 \mathrm{E}+01$

2.49E-00

$1.55 \mathrm{E}+05$

2.72E-00

$3.52 \mathrm{E}+02$

$1.63 \mathrm{E}+02$

2.46E-01

$6.96 \mathrm{E}-00$

1.32E-00

$1.29 \mathrm{E}+02$

4.56E-01

2.62E-03

8.51E-01

$2.97 E+01$

5.00E-00

$5.69 \mathrm{E}+01$

3.13E-04

9.04E-05

1.38E-01

3.88E-03

8.91E +01

$1.96 \mathrm{E}-00$

$6.89 \mathrm{E}+03$
Sept98

3.74E-01

$1.61 \mathrm{E}+01$

1.74E-02

$6.66 \mathrm{E}-02$

$1.27 \mathrm{E}-00$

5.72E-02

8.91E-03

1.29E-00

$2.50 \mathrm{E}+05$

$1.18 \mathrm{E}-00$

$3.84 \mathrm{E}+04$

$2.90 \mathrm{E}+02$

4.16E+02

$7.30 \mathrm{E}+03$

$7.56 \mathrm{E}+04$

$1.83 \mathrm{E}+03$

$2.68 \mathrm{E}+03$

$1.03 \mathrm{E}+03$

$0.00 \mathrm{E}+01$

$2.37 \mathrm{E}+03$

2.81E-00

$3.31 \mathrm{E}+02$

$2.94 \mathrm{E}+05$

$1.27 \mathrm{E}+02$

$4.29 \mathrm{E}+04$

$4.28 \mathrm{E}+05$

NA

$1.52 \mathrm{E}+02$

1.07E+04 NA

$2.33 \mathrm{E}+02$

$2.27 E+04$

$1.67 \mathrm{E}-00$

$1.29 \mathrm{E}+04$

$4.67 \mathrm{E}+03$

$2.71 \mathrm{E}+01$

5.83E-04

$3.51 \mathrm{E}+01$

1.23E-03

$1.47 \mathrm{E}+05$

$1.79 \mathrm{E}+01$

$5.07 \mathrm{E}+01$

1.04E-01

9.58E-03

8.18E-02

$2.04 \mathrm{E}+01$

2.49E-00

$1.55 \mathrm{E}+05$

2.72E-00

$3.52 \mathrm{E}+02$

$1.63 \mathrm{E}+02$

2.46E-01

$6.96 \mathrm{E}-00$

$1.32 \mathrm{E}-00$

$1.29 \mathrm{E}+02$

4.56E-01

2.62E-03

8.51E-01

$2.97 \mathrm{E}+01$

5.00E-00

$5.69 \mathrm{E}+01$

3.13E-04

9.04E-05

1.38E-01

$3.88 \mathrm{E}-03$

$8.91 \mathrm{E}+01$

$1.96 \mathrm{E}-00$ $6.89 E+03$

1\%-ile

$10 \%$-ille

50\%-ile

90\%-ile

$99 \%$-ile

1.05E-02

$5.14 \mathrm{E}-00$

9.28E- 02

$9.25 \mathrm{E}-00$

$.00 \mathrm{E}+01$

$2.74 \mathrm{E}-03$

$1.05 \mathrm{E}-02$

$0.00 \mathrm{E}+01$

$0.00 \mathrm{E}+01$

2.01E-01

9.01E-03

$.00 \mathrm{E}+01$

$1.40 \mathrm{E}-03$

$.00 \mathrm{E}+01$

$2.03 \mathrm{E}-01$

$0.00 E+01$

$0.00 \mathrm{E}+01$

$9.67 \mathrm{E}+02$

$0.00 \mathrm{E}+01$

3.61E-0.0

$1.66 \mathrm{E}-01$

$2.42 \mathrm{E}+02$

4.96E-02

$7.92 \mathrm{E}-03$

$0.00 \mathrm{E}+01$

$0.00 \mathrm{E}+01$

1.52E-01

$4.38 \mathrm{E}-04$

$0.00 \mathrm{E}+01$

$5.68 \mathrm{E}+04$

2.98E-02

6.31E-01

$1.53 \mathrm{E}+01$

$1.92 \mathrm{E}+03$

$1.67 \mathrm{E}-02$

$1.29 \mathrm{E}+04$

3.86E-02

$8.73 \mathrm{E}+01$

3. $47 \mathrm{E}+01$

$2.93 \mathrm{E}+03$

$9.33 \mathrm{E}-00$

$1.73 \mathrm{E}-01$

$2.92 \mathrm{E}+01$

$0.00 \mathrm{E}+01$

$8.25 \mathrm{E}+02$

$2.03 \mathrm{E}-02$

$1.64 \mathrm{E}-02$

$1.35 \mathrm{E}+05$

3.21E-01

$1.80 \mathrm{E}+03$

$1.78 \mathrm{E}+01$

$8.14 \mathrm{E}+03$

$1.32 \mathrm{E}+02$

$0.00 \mathrm{E}+01$

$1.71 \mathrm{E}-01$

$5.36 \mathrm{E}+02$

$2.68 \mathrm{E}-00 \quad 5.45 \mathrm{E}+01$

$0.00 \mathrm{E}+01$

$2.46 \mathrm{E}-00$

4.61E-01

$1.45 \mathrm{E}+02$

$2.20 \mathrm{E}-02$

$8.21 \mathrm{E}-02 \quad 1.16 \mathrm{E}+03$

$0.00 \mathrm{E}+01 \quad 6.45 \mathrm{E}+02$

$0.00 \mathrm{E}+01$

$0.00 \mathrm{E}+01$

$4.85 \mathrm{E}-03$

$0.00 \mathrm{E}+01$

$0.00 \mathrm{E}+01$

$0.00 \mathrm{E}+01$

$0.00 \mathrm{E}+01$

$0.00 \mathrm{E}+01$

$0.00 \mathrm{E}+01$

$0.00 \mathrm{E}+01$

$0.00 \mathrm{E}+01$

$0.00 \mathrm{E}+01$

$0.00 \mathrm{E}+01$

$0.00 \mathrm{E}+01$

$4.79 \mathrm{E}-00$

$0.00 \mathrm{E}+01$

$0.00 \mathrm{E}+01$

$0.00 \mathrm{E}+01$

$0.00 \mathrm{E}+01$

$0.00 \mathrm{E}+01$

$0.00 \mathrm{E}+01$

$0.00 \mathrm{E}+01$

$0.00 \mathrm{E}+01$

$0.00 \mathrm{E}+01$

$0.00 \mathrm{E}+01$

$0.00 \mathrm{E}+01$

$0.00 \mathrm{E}+01$

$0.00 \mathrm{E}+01$ 
Tank Anal. Un

TX102 Sr89/90 Ci

TX102 Te99

TX102 Th229

TX102 Th232 Ci

TX102 Tin126 Ci

TX102 Tritium

TX102 U232

TX102 U233

TX102 U234

TX102 U235

TX102 U236

TX102 U238

TX102 Y90

TX102 Zr93

TX103 Al

TX103 Bi

TX103 Ca

TX103 Cl

TX103 $\mathrm{CO}$

TX103 Cr

TX103 F

TX103 Fe

TX103 Hg

TX103 K

TX103 La

TX103 Mn

TX103 $\mathrm{Na}$

TX103 Ni

TX103 $\mathrm{NO}_{2}$

TX103 NO3

TX103 P

TX103 Pb

TX103 PO4

TX103 S

TX103 Si

TX103 SO4

TX103 Sr

TX103 TOC

TX103 U

TX103 Zr

TX103 Ac227

TX103 Am241

TX103 Am243

TX103 Ba137

TX103 $\mathrm{Cl} 14$

TX103 Cd113

TX103 Cm242

TX103 Cm243

TX103 Cm244

TX103 Co60

TX103 Cs134

TX103 Cs137

TX103 Eu152

TX103 Eu154

TX103 Eu155

TX103 I129

TX103 Nb93

TX103 Nis9

TX103 Ni63

TX103 Np237

TX103 Pa231

TX103 Pu238

TX103 Pu239

TX103 Pu240

TX103 Pu241

TX103 Pu242

TX103 Ra226

TX103 Ra228
$\underline{B B}$

May98

$1.28 \mathrm{E}+02$

3.19E-03

8.45E-03

2.95E-00

$1.28 \mathrm{E}+02$

$6.89 \mathrm{E}-01$

2.64E-00

1.61E-00

Ci $\quad 6.95 \mathrm{E}-02$

2.52E-02

1.74E-00

$6.95 \mathrm{E}+04$

9.64E-00

$2.73 E+04$

$7.30 \mathrm{E}+01$

$3.00 \mathrm{E}+02$

$5.19 E+03$

$5.41 \mathrm{E}+04$

$1.30 \mathrm{E}+03$

$2.08 \mathrm{E}+03$

$2.01 E+02$

kg $\quad 5.20 \mathrm{E}-01$

$1.69 \mathrm{E}+03$

2.81E-00

$2.47 \mathrm{E}+02$

g $2.13 \mathrm{E}+05$

$9.10 \mathrm{E}+01$

kg $3.05 E+04$

kg $3.11 \mathrm{E}+05$

kg $9.99 \mathrm{E}+03$

kg $4.87 \mathrm{E}+01$

$3.10 \mathrm{E}+04$

kg $1.64 E+04$

kg $\quad 1.89 \mathrm{E}+02$

$\mathrm{kg} \quad 4.92 \mathrm{E}+04$

kg $\quad 6.86 \mathrm{E}-00$

kg $\quad 9.13 \mathrm{E}+03$

3.95E+02

kg 1.73E+01

Ci 1.88E-04

Ci $1.16 \mathrm{E}+01$

Ci 3.92E-04

Ci $5.32 \mathrm{E}+04$

6.12E-00

$1.59 \mathrm{E}+01$

2.81E-02

2.61E-03

2.62E-02

$6.80 \mathrm{E}-00$

5.15E-01

$5.62 E+04$

$7.22 \mathrm{E}-01$

$1.10 E+02$

$4.29 E+01$

8.40E-02

$2.22 \mathrm{E}-00$

Ci 4.55E-01

Ci $4.46 \mathrm{E}+01$

Ci 1.56E-01

Ci 8.33E-04

Ci 2.62E-01

$\mathrm{Ci} \quad 9.91 \mathrm{E}-00$

$\mathrm{Ci}$ 1.60E-00

Ci $1.73 \mathrm{E}+01$

Ci 9.48E-05

Ci 2.90E-05

Ci $4.29 \mathrm{E}-02$
Sept98

\section{$6.95 \bar{E}+04$}

$1.28 \mathrm{E}+02$

3.19E-03

$8.45 \mathrm{E}-03$

2.95E-00

$1.28 \mathrm{E}+02$

$6.89 \mathrm{E}-01$

2.64E-00

1.61E-00

6.95E-02

2.52E-02

$1.56 \mathrm{E}-00$

$6.95 \mathrm{E}+04$

$9.64 \mathrm{E}-00$

$2.73 \mathrm{E}+04$

$4.04 \mathrm{E}+01$

$3.00 \mathrm{E}+02$

$5.19 \mathrm{E}+03$

$5.41 \mathrm{E}+04$

$1.30 \mathrm{E}+03$

$2.08 \mathrm{E}+03$

$2.01 \mathrm{E}+02$

$0.00 \mathrm{E}+01$

$1.69 \mathrm{E}+03$

$0.00 \mathrm{E}+01$

$2.47 \mathrm{E}+02$

$2.13 E+05$

$9.10 \mathrm{E}+01$

$3.05 E+04$

$3.11 \mathrm{E}+05$

NA

$4.87 \mathrm{E}+01$

$1.01 \mathrm{E}+04$

NA

$1.89 \mathrm{E}+02$

$1.64 \mathrm{E}+04$

4.99E-01

$9.13 E+03$

$3.95 E+02$

$1.73 E+01$

$1.88 \mathrm{E}-04$

$1.16 \mathrm{E}+01$

3.92E-04

$5.32 \mathrm{E}+04$

6.12E-00

$1.59 \mathrm{E}+01$

2.81E-02

2.61E-03

2.62E-02

$6.80 \mathrm{E}-00$

5.15E-01

$5.62 \mathrm{E}+04$

$7.22 \mathrm{E}-01$

$1.10 E+02$

$4.29 \mathrm{E}+01$

8.40E-02

2.22E-00

4.55E- 01

$4.46 \mathrm{E}+01$

1.56E-01

8.33E-04

2.62E-01

9.91E-00

1.60E-00

$1.73 E+01$

9.48E-05

2.90E-05

$4.29 \mathrm{E}-02$
$1 \%$-ile

$0.00 \mathrm{E}+01$

$0.00 \mathrm{E}+01$

$0.00 \mathrm{E}+01$

$0.00 \mathrm{E}+01$

$0.00 \mathrm{E}+01$

$2.27 \mathrm{E}+01$

$0.00 \mathrm{E}+01$

$0.00 \mathrm{E}+01$

$0.00 \mathrm{E}+01$

$0.00 \mathrm{E}+01$

$0.00 \mathrm{E}+01$

$0.00 \mathrm{E}+01$

$0.00 \mathrm{E}+01$

$0.00 \mathrm{E}+01$

$0.00 \mathrm{E}+01$

$0.00 \mathrm{E}+01$

$0.00 \mathrm{E}+01$

$0.00 \mathrm{E}+01$

$0.00 \mathrm{E}+01$

$0.00 \mathrm{E}+01$

$0.00 \mathrm{E}+01$

$0.00 \mathrm{E}+01$

$0.00 \mathrm{E}+01$

$0.00 \mathrm{E}+0 \mathrm{I}$

$0.00 \mathrm{E}+01$

$0.00 \mathrm{E}+01$

$2.32 \mathrm{E}+04$

$0.00 \mathrm{E}+01$

$0.00 \mathrm{E}+01$

$0.00 \mathrm{E}+01$

$0.00 \mathrm{E}+01$

$0.00 \mathrm{E}+01$

$0.00 \mathrm{E}+01$

$0.00 \mathrm{E}+01$

$0.00 \mathrm{E}+01$

$0.00 \mathrm{E}+01$

$0.00 \mathrm{E}+01$

$0.00 \mathrm{E}+01$

$0.00 \mathrm{E}+01$

$0.00 \mathrm{E}+01$

$0.00 \mathrm{E}+01$

$0.00 \mathrm{E}+01$

$0.00 \mathrm{E}+01$

$0.00 \mathrm{E}+01$

$0.00 \mathrm{E}+01$

$0.00 \mathrm{E}+01$

$0.00 \mathrm{E}+01$

$0.00 \mathrm{E}+01$

$0.00 \mathrm{E}+01$

$0.00 \mathrm{E}+01$

$0.00 \mathrm{E}+01$

$0.00 \mathrm{E}+01$

$0.00 \mathrm{E}+01$

$0.00 \mathrm{E}+01$

$0.00 \mathrm{E}+01$

$0.00 \mathrm{E}+01$

$0.00 \mathrm{E}+01$

$0.00 \mathrm{E}+01$

$0.00 \mathrm{E}+01$

$0.00 \mathrm{E}+01$

$0.00 \mathrm{E}+01$

$0.00 \mathrm{E}+01$

$0.00 \mathrm{E}+01$
$0.00 \mathrm{E}+01$

$0.00 \mathrm{E}+01$

$0.00 \mathrm{E}+01$

$0.00 \mathrm{E}+01$

$0.00 E+01$

10\%-ile

$6.82 \mathrm{E}+01$

$0.00 \mathrm{E}+01$

$0.00 \mathrm{E}+01$

$0.00 \mathrm{E}+01$

$0.00 \mathrm{E}+01$

$6.42 \mathrm{E}+01$

$0.00 \mathrm{E}+01$

$0.00 \mathrm{E}+01$

$0.00 \mathrm{E}+01$

$0.00 \mathrm{E}+01$

$0.00 \mathrm{E}+01$

$0.00 \mathrm{E}+01$

$0.00 \mathrm{E}+01$ 
Tank Anal. Un

\begin{tabular}{ll} 
TX103 & Ru106 \\
TX103 & Sb125 \\
TX103 & Se79 \\
TX103 & Sm151 \\
TX103 & Sr89/90 \\
TX103 & Tc99 \\
TX103 & Th229 \\
TX103 & Th232 \\
TX103 & Tin126 \\
TX103 & Tritium \\
TX103 & U232 \\
TX103 & U233 \\
TX103 & U234 \\
TX103 & U235 \\
TX103 & U236 \\
TX103 & U238 \\
TX103 & Y90 \\
TX103 & Zr93 \\
TX104 & Al \\
TX104 & Bi \\
\hline
\end{tabular}

TX104 Ca

TX104 Cl

TX104 $\mathrm{CO3}$

TX104 Cr

TX104 F

TX104 Fe

TX104 Hg

TX104 K

TX104 La

TX104 Mn

TX104 Na

TX104 Ni

TX104 NO2

TX104 NO3

TX104 P

TX104 Pb

TX104 PO4

TX104 S

TX104 Si

TX104 SO4

TX104 Sr

TX104 TOC

TX104 U

TX104 Zr

TX104 Ac227

TX104 Am241

TX104 Am243

TX104 Ba137

TX104 C14

TX104 Cd113

TX104 Cm242

TX104 Cm243

TX104 Cm244

TX104 Co60

TX104 Cs134

TX104 Cs137

TX104 Eu152

TX104 Eu154

TX104 Eu155

TX104 I129

TX104 Nb93

TX104 Ni59

TX104 Ni63

TX104 Np237

TX104 Pa231

TX104 Puz38

TX104 Pu239

TX104 Pu240

\section{May $\frac{B B}{98}$}

$1.24 \mathrm{E}-03$

2.92E+01

6.23E-01

2.19E+03

$2.08 \mathrm{E}+04$

$4.36 \mathrm{E}+01$

9.96E-04

2.65E-03

9.41E-01

4.19E+01

2.16E-01

8.29E-01

2.50E-01

1.03E-02

$6.60 \mathrm{E}-03$

2.88E-01

$2.08 \mathrm{E}+04$

3.06E-00

$2.04 \mathrm{E}+04$

$5.99 \mathrm{E}+01$

kg 1.22E+02

kg $\quad 1.87 \mathrm{E}+03$

kg 1.69E+04

kg $\quad 6.29 \mathrm{E}+02$

kg

kg

4.18E+02

kg $5.66 \mathrm{E}+02$

kg 3.04E-05

kg 2.32E+02

7.55E+04

g $4.20 \mathrm{E}+01$

kg 1.30E+04

$\mathrm{kg}$

kg 8.17E+02

$\mathrm{kg} \quad 2.53 \mathrm{E}+03$

kg 1.70E +03

kg 2.02E+02

kg $5.11 \mathrm{E}+03$

kg 5.26E+01

kg 2.99E+03

kg

kg 1.94E+01

Ci 1.50E-04

7.82E-00

2.78E-04

i $4.06 \mathrm{E}+04$

Ci 4.01E-00

$1.09 \mathrm{E}+01$

2.17E-02

i $2.00 \mathrm{E}-03$

1.80E-02

4.33E-00

i $4.93 \mathrm{E}-01$

4.30E+04

5.66E-01

Ci 7.45E+01

$3.43 E+01$

Ci 5.28E-02

Ci 1.53E-00

Ci 2.92E-01

Ci $2.86 \mathrm{E}+01$

Ci 9.88E-02

Ci 5.87E-04

Ci 1.82E-01

Ci 6.55E-00

Ci 1.08E-00

\section{Sept98}

1.24E-03

$2.92 \mathrm{E}+01$

6.23E-01

$2.19 \mathrm{E}+03$

$2.08 \mathrm{E}+04$

$4.36 \mathrm{E}+01$

9.96E-04

2.65E-03

9.41E-01

$4.19 \mathrm{E}+01$

$1.24 \mathrm{E}-01$

4.74E-01

1.43E-01

5.89E-03

3.77E-03

1.32E-01

$2.08 \mathrm{E}+04$

3.06E-00

$2.04 \mathrm{E}+04$

$6.18 E+01$

$1.22 \mathrm{E}+02$

$1.87 \mathrm{E}+03$

$1.69 \mathrm{E}+04$

$6.29 \mathrm{E}+02$

$5.97 \mathrm{E}+02$

$4.18 \mathrm{E}+02$

$0.00 \mathrm{E}+01$

$5.66 \mathrm{E}+02$

$0.00 \mathrm{E}+01$

2.32E+02

$7.55 \mathrm{E}+04$

$4.20 \mathrm{E}+01$

$1.30 \mathrm{E}+04$

$1.08 \mathrm{E}+05$

NA

3.65E+01

$2.53 E+03$

NA

2.02E+02

$5.11 \mathrm{E}+03$

$5.26 \mathrm{E}+01$

$2.99 \mathrm{E}+03$

$1.04 \mathrm{E}+03$

5.78E-00

1.50E-04

7.82E-00

2.78E-04

$4.06 \mathrm{E}+04$

4.01E-00

$1.09 \mathrm{E}+01$

2.17E-02

2.00E-03

$1.80 \mathrm{E}-02$

4.33E- 00

4.93E-01

$430 \mathrm{E}+04$

5.66E-01

$7.45 \mathrm{E}+01$

$3.43 E+01$

5.28E- 02

$1.53 E-00$

2.92E-01

$2.86 \mathrm{E}+01$

9.88E-02

5.87E-04

$1.82 \mathrm{E}-01$

6.55E-00

$1.08 E-00$ 1\%-ile

$0.00 \mathrm{E}+01$
$0.00 \mathrm{E}+01$

$0.00 \mathrm{E}+01$

$0.00 \mathrm{E}+01$

$0.00 \mathrm{E}+01$

$0.00 \mathrm{E}+01$

$0.00 \mathrm{E}+01$

$0.00 \mathrm{E}+01$

$0.00 \mathrm{E}+01$

$0.00 \mathrm{E}+01$

$0.00 \mathrm{E} \div 01$

$0.00 \mathrm{E}+01$

$0.00 \mathrm{E}+01$

$0.00 \mathrm{E}+01$

$0.00 \mathrm{E}+01$

$0.00 \mathrm{E}+01$

$0.00 \mathrm{E}+01$

$0.00 \mathrm{E}+01$

$2.67 \mathrm{E}+02$

$0.00 \mathrm{E}+01$

8.62E-00

$1.41 \mathrm{E}-00$

$1.08 \mathrm{E}+03$

8.25E-02

$7.12 \mathrm{E}-02$

$0.00 \mathrm{E}+01$

$0.00 \mathrm{E}+01$

$1.56 \mathrm{E}-01$

$0.00 \mathrm{E}+01$

$0.00 \mathrm{E}+01$

$1.07 \mathrm{E}+04$

6.07E-03

8.96E-01

$7.21 \mathrm{E}+01$

$6.00 \mathrm{E}+01$

$0.00 \mathrm{E}+01$

$1.39 \mathrm{E}+02$

$3.08 \mathrm{E}-00$

$0.00 \mathrm{E}+01$

$1.46 \mathrm{E}+01$

$0.00 \mathrm{E}+01$

7.81E-01

$0.00 \mathrm{E}+01$

$0.00 \mathrm{E}+01$

$0.00 \mathrm{E}+01$

$0.00 \mathrm{E}+01$

$0.00 \mathrm{E}+01$

$0.00 \mathrm{E}+01$

$0.00 \mathrm{E}+01$

$0.00 \mathrm{E}+01$

$0.00 \mathrm{E}+01$

$0.00 E+01$

$0.00 \mathrm{E}+01$

$0.00 E+01$

$0.00 \mathrm{E}+01$

$2.81 \mathrm{E}+01$

$0.00 \mathrm{E}+01$

$0.00 \mathrm{E}+01$

$0.00 \mathrm{E}+01$

$0.00 \mathrm{E}+01$

$0.00 \mathrm{E}+01$

$0.00 E+01$

$0.00 \mathrm{E}+01$

$0.00 \mathrm{E}+01$

$0.00 E+01$

$0.00 E+01$

$0.00 E+01$

$0.00 \mathrm{E}+01$

$10 \%$-ile

$0.00 \mathrm{E}+01$

$0.00 \mathrm{E}+01$

$0.00 \mathrm{E}+01$

$0.00 \mathrm{E}+01$

$0.00 \mathrm{E}+01$

$0.00 \mathrm{E}+01$

$0.00 \mathrm{E}+01$

$0.00 \mathrm{E}+01$

$0.00 \mathrm{E}+01$

2.00E-00

$0.00 \mathrm{E}+01$

$0.00 \mathrm{E}+01$

$0.00 \mathrm{E}+01$

$0.00 \mathrm{E}+01$

$0.00 \mathrm{E}+01$

$0.00 \mathrm{E}+01$

$0.00 \mathrm{E}+01$

$0.00 \mathrm{E}+01$

$2.36 \mathrm{E}+03$

1.23E-01

$1.07 \mathrm{E}+02$

$2.77 \mathrm{E}+01$

$6.05 \mathrm{E}+03$

$1.42 \mathrm{E}+01$

$9.50 \mathrm{E}-01$

$1.08 \mathrm{E}+02$

$0.00 \mathrm{E}+01$

$1.54 \mathrm{E}+02$

$6.05 \mathrm{E}-02$

$8.98 \mathrm{E}-02$ 


\begin{tabular}{|c|c|c|c|c|c|c|c|c|c|c|c|}
\hline nk & Anal. & $U_{n}$ & $\frac{B B}{\operatorname{Bay} 98}$ & $\frac{B B}{p t 98}$ & 1\%-ile & $10 \%$-ile & $0 \%-j l e$ & $90 \%$-ile & $9 \%$-jle & Mean & td Dev \\
\hline & & $\mathrm{Ci}$ & & $1 E+01$ & -01 & -02 & +01 & $E+01$ & +02 & +01 & $5 E+$ \\
\hline s & $\mathbf{P u} \mathbf{z}$ & $\mathrm{Ci}$ & $77 \mathrm{E}-05$ & $6.67 \mathrm{E}-05$ & $E+01$ & -08 & -05 & -04 & 34 & 1.2 & $7 \mathrm{E}-$ \\
\hline & Ra226 & $\mathrm{Ci}$ & DE-05 & $.90 \mathrm{E}-05$ & $i+01$ & & $0 \mathrm{E}-05$ & 04 & & 4.33E-05 & $E-05$ \\
\hline & Ra228 & $\mathrm{Ci}$ & & & & & -02 & & 01 & & \\
\hline TX104 & Ru106 & $\mathrm{Ci}$ & 52E-04 & $8.52 \mathrm{E}-04$ & $0 \mathrm{E}+01$ & $2 E-05$ & 2E-04 & $99 \mathrm{E}-03$ & $71 \mathrm{E}-03$ & $1.43 \mathrm{E}-03$ & $3.38 \mathrm{E}-03$ \\
\hline & Sb125 & $\mathrm{Ci}$ & $39 E+01$ & $89 E+01$ & & & $89 \mathrm{E}+01$ & 02 & 02 & $5.83 \mathrm{E}+01$ & $8.09 E+01$ \\
\hline TX104. & Se79 & $\mathbf{C i}$ & $9 E-01$ & $.29 \mathrm{E}-01$ & $E+01$ & $1 \mathrm{E}-03$ & $29 \mathrm{E}-01$ & $8 \mathrm{E}-00$ & 00 & il & $4.85 \mathrm{E}-01$ \\
\hline TX104 & Sm151 & $\mathbf{C i}$ & $1.51 E+03$ & $1.51 E+03$ & $0.00 \mathrm{E}+01$ & $0 \mathrm{E}+01$ & $1.51 E+03$ & $16 \mathrm{E}+03$ & $.52 \mathrm{E}+04$ & $3.05 \mathrm{E}+03$ & $3.88 \mathrm{E}+03$ \\
\hline TX104 & & $\mathrm{Ci}$ & & $47 \mathrm{E}+04$ & $E+01$ & & $\mathbf{E}+\mathbf{0 4}$ & & $\mathrm{E}+04$ & & \\
\hline $\mathbf{K 1 0 4}$ & Te99 & $\mathbf{C i}$ & $4 \mathrm{E}+01$ & $74 \mathrm{E}+01$ & $E+01$ & $E+01$ & $4 \mathrm{E}+01$ & +02 & $2 \mathrm{E}+02$ & $E+01$ & $1 E+01$ \\
\hline TX104 & Th229 & $\mathbf{C i}$ & $6.79 \mathrm{E}-04$ & $6.79 \mathrm{E}-04$ & $0 \mathrm{E}+01$ & & & & & & $.13 \mathrm{E}-03$ \\
\hline & Th232 & $\mathrm{Ci}$ & & & & & & & & & \\
\hline TX104 & Tin126 & $\mathbf{C i}$ & $6.48 \mathrm{E}-01$ & $6.48 \mathrm{E}-01$ & $E+01$ & $E+01$ & & & & 00 & $E-00$ \\
\hline TX104 & Tritium & $\mathbf{C i}$ & $2.75 E+01$ & $75 E+01$ & & $33 E+01$ & & & & & $.44 E+01$ \\
\hline $\mathbf{T X}$ & U232 & $\mathbf{C i}$ & & & & & & & & & \\
\hline TX104 & U233 & $\mathrm{Ci}$ & $5.63 \mathrm{E}-01$ & 34E-02 & 01 & & & & & & \\
\hline 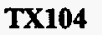 & nat & & $00 \mathrm{ng}$ & & & & & & & +01 & +01 \\
\hline $\mathbf{T X}$ & $\mathbf{U} 2$ & $\mathbf{C i}$ & 4 & & & & & & & & \\
\hline TX104 & U236 & $\mathrm{Ci}$ & $6.37 \mathrm{E}-02$ & 2.311 & & & & & & & \\
\hline K104 & U238 & $\mathbf{C i}$ & $9.63 \mathrm{E}-00$ & $47 \mathrm{E}-01$ & +01 & -01 & & & $9 E+01$ & 01 & +01 \\
\hline TX104 & Y90 & $\mathbf{C i}$ & $47 E+04$ & Tr & & & & & & & \\
\hline TX104 & Zr93 & $\mathbf{C i}$ & 2.1 & & & & & & & & \\
\hline TX105 & Al & $\mathbf{k g}$ & $1.08 \mathrm{E}+05$ & $08 \mathrm{E}+05$ & -03 & +04 & -04 & & -05 & +04 & \\
\hline & $\mathbf{B i}$ & & $2.60 \mathrm{E}+02$ & 1.30 & & & & & & & \\
\hline & $\mathrm{Ca}$ & & $1.17 \mathrm{E}$ & 3 & & & & & & & \\
\hline TX105 & Cl & $\mathrm{kg}$ & $2.05 E+04$ & $2.05 E+04$ & & & & & & & \\
\hline TX105 & 03 & 10 & $2.13 E+05$ & & & & & & & & \\
\hline TX1 & $\mathrm{Cr}$ & $\mathrm{kg}$ & $5.15 E+03$ & & & & & & & & \\
\hline TX1 & & 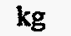 & 7.55 & $.55 E+03$ & & & & & & & \\
\hline TX1 & Fe & & $2.90 \mathrm{E}+03$ & $.90 E+03$ & & & & & & & \\
\hline & $\mathrm{dg}$ & $\mathrm{kg}$ & & & & & & & & & \\
\hline & $\mathbf{K}$ & $\mathrm{k}$ & 6.67 & 3 & & & & & & & \\
\hline TX105 & $\mathbf{L a}$ & $\mathrm{kg}$ & $1.34 E+02$ & +01 & & & & & & & \\
\hline TX105 & Mn & kg & $9.32 E+02$ & & & & & & & & \\
\hline & $\mathbf{N a}$ & $\mathrm{kg}$ & & & & & & & & & \\
\hline $\mathbf{T X}$ & $\mathbf{N i}$ & $\mathrm{kg}$ & 3.5 & & & & & & & & \\
\hline $\mathbf{T X}$ & No: & $\mathrm{k}$ & -05 & & & & & & & 105 & \\
\hline & $\mathbf{N}$ & & & & & & & & & & \\
\hline & & $\mathrm{kg}$ & & & & & & & & & \\
\hline 5 & $\mathbf{P b}$ & & 02 & $36 E+02$ & & & & & & & \\
\hline & PO & & & +04 & & & & & & & \\
\hline & & & & & & & & & & & \\
\hline & & & 6.5 & $6.56 \mathrm{E}+02$ & & & & & & & \\
\hline & 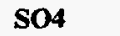 & & & & & & & & & & \\
\hline & & & & & & & & & & & \\
\hline & TOC & & 3.62 & & & & & & & & \\
\hline & & & & & & & & & & & \\
\hline & $=$ & & & & & & & & & & \\
\hline & & & & & & & & & & & \\
\hline & & & & & & & & & & & \\
\hline & & & & & & & & & & & \\
\hline & & & & & & & & & & & \\
\hline & & & & & & & & & & & \\
\hline & & & & & & & & & & & \\
\hline & & & & & & & & & & & \\
\hline & & & & & & & & & & & \\
\hline & & & & & & & & & & & \\
\hline & & & & & & & & & & & \\
\hline & & & & & & & & & & & \\
\hline & & & & & & & & & & & \\
\hline & & & & & & & & & & & \\
\hline & & & & & & & & & & & \\
\hline & & & & & & & & & & & \\
\hline & & C & & & & & & & & & \\
\hline & & c & & & & & & & & & \\
\hline & & c & & & & & & & & & \\
\hline & 10 & c & & & & & & & & & \\
\hline & & c & $0-v 0$ & $30 \mathrm{r}$ & & $00 \mathrm{E}+01$ & & & & & $c=r$ \\
\hline
\end{tabular}


Tank Anal. Un

$\begin{array}{ll}\text { TX105 } & \text { Pa231 } \\ \text { TX105 } & \text { Pu238 } \\ \text { TX105 } & \text { Pu239 } \\ \text { TX105 } & \text { Pu240 } \\ \text { TX105 } & \text { Pu241 } \\ \text { TX105 } & \text { Pu242 } \\ \text { TX105 } & \text { Ra226 } \\ \text { TX105 } & \text { Ra228 } \\ \text { TX105 } & \text { Ru106 } \\ \text { TX105 } & \text { Sb125 } \\ \text { TX105 } & \text { Se79 } \\ \text { TX105 } & \text { Sm151 } \\ \text { TX105 } & \text { Sr89/90 } \\ \text { TX105 } & \text { Tc99 }\end{array}$

TX105 Th229

TX105 Th232

TX105 Tin126

TX105 Tritium

TX105 U232

TX105 U233

TX105 U234

TX105 U235

TX105 U236

TX105 U238

TX105 Y90

TX105 Zr93

TX106 Al

TX106 Bi

TX106 Ca

TX106 Cl

TX106 CO3

TX106 Cr

TX106 F

TX106 Fe

TX106 $\mathrm{Hg}$

TX106 K

TX106 La

TX106 Mn

TX106 Na

TX106 Ni

TX106 NO2

TX106 NO3

TX106 P

TX106 Pb

TX106 PO4

TX106 S

TX106 Si

TX106 SO4

TX106 Sr

TX106 TOC

TX106 U

TX106 $\mathrm{Zr}$

TX106 Ac227

TX106 Am241

TX106 Am243

TX106 Ba137

TX106 C14

TX106 Cd113

TX106 Cm242

TX106 Cm243

TX106 Cm244

TX106 Co60

TX106 Cs134

TX106 Cs137

TX106 Eu152

TX106 Eu154

TX106 Eu155

TX106 I129

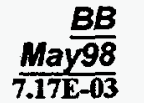

2.095-00

$7.54 \mathrm{E}+01$

$1.25 \mathrm{E}+01$

$1.37 \mathrm{E}+02$

$7.49 \mathrm{E}-04$

2.51E-04

4.09E-01

9.88E-03

$2.35 \mathrm{E}+02$

5.20E-00

$1.83 \mathrm{E}+04$

$1.68 \mathrm{E}+05$

$3.60 \mathrm{E}+02$

9.48E-03

2.51E-02

7.84E-00

$3.54 \mathrm{E}+02$

2.05E-00

$7.84 \mathrm{E}-00$

5.83E-00

2.54E-01

8.08E-02

$6.25 \mathrm{E}-00$

$1.68 \mathrm{E}+05$

$2.55 \mathrm{E}+01$

$6.05 \mathrm{E}+04$

$4.63 \mathrm{E}+02$

$6.53 \mathrm{E}+02$

$1.15 \mathrm{E}+04$

$1.19 \mathrm{E}+05$

$2.88 \mathrm{E}+03$

$4.22 \mathrm{E}+03$

$1.62 \mathrm{E}+03$

2.44E-00

$3.73 E+03$

$7.50 \mathrm{E}+01$

$5.21 \mathrm{E}+02$

$4.63 \mathrm{E}+05$

$2.00 \mathrm{E}+02$

$6.75 \mathrm{E}+104$

$6.74 \mathrm{E}+05$

$5.43 E+03$

$3.00 \mathrm{E}+02$

$1.68 \mathrm{E}+04$

$1.19 \mathrm{E}+04$

$3.67 \mathrm{E}+02$

$3.58 \mathrm{E}+04$

$1.50 \mathrm{E}+01$

$2.02 \mathrm{E}+04$

$4.69 \mathrm{E}+03$

$1.50 \mathrm{E}+02$

9.72E-04

$5.40 \mathrm{E}+01$

$1.85 \mathrm{E}-03$

$2.31 \mathrm{E}+05$

$2.77 \mathrm{E}+01$

$7.46 \mathrm{E}+01$

1.37E-01

1.25E-02

$1.21 \mathrm{E}-01$

3.70E-00

2.75E-00

$2.44 \mathrm{E}+05$

3.59E-00

$5.08 \mathrm{E}+02$

$2.12 \mathrm{E}+02$

3.80E-01

\section{$B B$}

Sept98

2.09E-00

$7.54 \mathrm{E}+01$

$1.25 \mathrm{E}+01$

$1.37 \mathrm{E}+02$

$7.49 \mathrm{E}-04$

2.51E-04

4.09E-01

9.88E-03

$2.35 E+02$

5.20E-00

$1.83 \mathrm{E}+04$

$1.68 \mathrm{E}+05$

$3.60 \mathrm{E}+02$

9.48E-03

2.51E-02

7.84E-00

$3.54 E+02$

$1.82 \mathrm{E}-01$

$6.98 \mathrm{E}-01$

5.19E-01

2.26E-02

7.19E-03

5.07E-01

$1.68 \mathrm{E}+05$

$2.55 \mathrm{E}+01$

$6.05 \mathrm{E}+04$

$4.62 \mathrm{E}+02$

$6.53 \mathrm{E}+02$

$1.15 \mathrm{E}+04$

$1.19 \mathrm{E}+05$

$2.88 E+03$

$4.22 \mathrm{E}+03$

$1.62 \mathrm{E}+03$

$0.00 \mathrm{E}+01$

3.73E +03

$0.00 \mathrm{E}+01$

$5.21 \mathrm{E}+02$

4.63E +05

$2.00 \mathrm{E}+02$

$6.75 \mathrm{E}+04$

$6.74 \mathrm{E}+05$

$$
\text { NA }
$$

$2.42 \mathrm{E}+02$

$1.68 \mathrm{E}+04$

NA

$3.67 \mathrm{E}+02$

$3.58 \mathrm{E}+04$

2.69E-00

2.02E+04

$3.99 \mathrm{E}+03$

$4.32 \mathrm{E}+01$

9.72E-04

$5.40 \mathrm{E}+01$

$1.85 \mathrm{E}-03$

$2.31 E+05$

$2.77 \mathrm{E}+01$

$7.46 \mathrm{E}+01$

1.37E-01

1.25E-02

$1.21 \mathrm{E}-01$

$3.07 \mathrm{E}+01$

2.75E-00

$2.44 \mathrm{E}+05$

$3.59 \mathrm{E}-00$

$5.08 \mathrm{E}+02$

2.12E +02

$3.80 \mathrm{E}-01$
$1 \%$-ile

$0.00 \mathrm{E}+01$

$0.00 \mathrm{E}+01$

$0.00 \mathrm{E}+01$

$0.00 \mathrm{E}+01$

$0.00 \mathrm{E}+01$

$0.00 \mathrm{E}+01$

$0.00 \mathrm{E}+01$

$0.00 \mathrm{E}+01$

$0.00 \mathrm{E}+01$

$0.00 \mathrm{E}+01$

$0.00 \mathrm{E}+01$

$0.00 \mathrm{E}+01$

7.48E-01

$0.00 \mathrm{E}+01$

$0.00 \mathrm{E}+01$

$0.00 \mathrm{E}+01$

$0.00 \mathrm{E}+01$

$9.25 \mathrm{E}+01$

$0.00 \mathrm{E}+01$

$0.00 \mathrm{E}+01$

$0.00 \mathrm{E}+01$

$0.00 \mathrm{E}+01$

$0.00 \mathrm{E}+01$

$0.00 \mathrm{E}+01$

$0.00 \mathrm{E}+01$

$0.00 \mathrm{E}+01$

$4.64 \mathrm{E}+03$

4.28E-02

$1.31 \mathrm{E}+01$

$1.06 \mathrm{E}+01$

$4.35 E+02$

$1.06 \mathrm{E}+01$

$7.82 \mathrm{E}-01$

$1.93 \mathrm{E}+01$

$0.00 \mathrm{E}+01$

$1.18 \mathrm{E}+01$

9.13E-02

2.24E-00

$1.27 \mathrm{E}+05$

4.59E-01

$1.81 \mathrm{E}+02$

$4.45 \mathrm{E}+02$

$5.45 \mathrm{E}+01$

$254 \mathrm{E}-01$

$7.35 E+01$

$1.01 \mathrm{E}+01$

4.21E-00

$2.48 E+01$

1.95E-01

$2.36 \mathrm{E}+01$

$1.27 \mathrm{E}+02$

4.29E-03

$0.00 \mathrm{E}+01$

$3.29 \mathrm{E}-01$

$0.00 \mathrm{E}+01$

$0.00 \mathrm{E}+01$

$0.00 \mathrm{E}+01$

$0.00 \mathrm{E}+01$

$0.00 \mathrm{E}+01$

$0.00 \mathrm{E}+01$

$0.00 \mathrm{E}+01$

$7.16 \mathrm{E}-02$

$0.00 E+01$

$9.25 E+02$

$0.00 E+0$ !

$0.00 \mathrm{E}+01$

$0.00 E+01$

$0.00 \mathrm{E}+01$

$10 \%$ ile

$0.00 \mathrm{E}+01$

$0.00 \mathrm{E}+01$

$0.00 \mathrm{E}+01$

$0.00 \mathrm{E}+01$

$0.00 \mathrm{E}+01$

$0.00 \mathrm{E}+01$

$0.00 \mathrm{E}+01$

$0.00 \mathrm{E}+01$

$0.00 \mathrm{E}+01$

$0.00 \mathrm{E}+01$

$0.00 \mathrm{E}+01$

$0.00 \mathrm{E}+01$

$2.72 \mathrm{E}+02$

$0.00 \mathrm{E}+01$

$0.00 \mathrm{E}+01$

$0.00 \mathrm{E}+01$

$0.00 \mathrm{E}+01$

$2.06 \mathrm{E}+02$

$0.00 \mathrm{E}+01$

$0.00 \mathrm{E}+01$

$0.00 \mathrm{E}+01$

$0.00 \mathrm{E}+01$

$0.00 \mathrm{E} \div 01$

$0.00 \mathrm{E}+01$

$0.00 \mathrm{E}+01$ 
Tank Anal. Un

$\begin{array}{ll}\text { TX106 } & \text { Nb93 } \\ \text { TX106 } & \text { Ni59 } \\ \text { TX106 } & \text { Ni63 } \\ \text { TX106 } & \text { Np237 } \\ \text { TX106 } & \text { Pa231 } \\ \text { TX106 } & \text { Pu238 } \\ \text { TX106 } & \text { Pu239 } \\ \text { TX106 } & \text { Pu240 } \\ \text { TX106 } & \text { Pu241 } \\ \text { TX106 } & \text { Pu242 } \\ \text { TX106 } & \text { Ra226 } \\ \text { TX106 } & \text { Ra228 } \\ \text { TX106 } & \text { Ru106 } \\ \text { TX106 } & \text { Sb125 } \\ \text { TX106 } & \text { Se79 } \\ \text { TX106 } & \text { Sm151 } \\ \text { TX106 } & \text { Sr89/90 } \\ \text { TX106 } & \text { Tc99 }\end{array}$

TX106 Teg9

TX106 Th229

TX106 Th232

TX106 Tin126

TX106 Tritium

TX106 U232

TX106 U233

TX106 U234

TX106 U235

TX106 U236

TX106 U238

TX106 Y90

TX106 Zr93

TX107 A]

TX107 Bi

TX107 Ca

TX107 Cl

TX107 CO3

TX107 $\mathrm{Cr}$

TX107 F

TX107 Fe

TX107 $\mathrm{Hg}$

TX107 K

TX107 La

TX107 Mn

TX107 Na

TX107 Ni

TX107 NO2

TX107 NO3

TX107 P

TX107 Pb

TX107 $\mathrm{PO4}$

TX107 S

TX107 Si

TX107 SO4

TX107 Sr

TX107 TOC

TX107 U

TX107 $\mathrm{Zr}$

TX107 Ac227

TX107 Am241

TX107 Am243

TX107 Ba137

TX107 C14

TX107 Cd113

TX107 Cm242

TX107 Cm243

TX107 Cm244

TX107 Co60

TX107 Cs134

TX107 Cs137
BB

Ci $\begin{aligned} & \text { May98 } \\ & \text { Ci } \\ & \text { 2.32E-05 }\end{aligned}$

Ci $2.26 \mathrm{E}+02$

Ci 7.11E-01

Ci 4.00E-03

Ci 1.31E-00

$5.02 \mathrm{E}+01$

Ci $\quad 8.22 \mathrm{E}-00$

$8.60 \mathrm{E}+01$

$4.66 \mathrm{E}-04$

1.55E-04

2.19E-01

5.71E-03

$1.32 \mathrm{E}+02$

2.94E-00

$1.03 E+04$

$1.12 \mathrm{E}+05$

$1.97 \mathrm{E}+02$

5.08E-03

1.35E-02

$4.44 \mathrm{E}-00$

$1.96 \mathrm{E}+02$

$1.10 \mathrm{E}-00$

4.20E-00

Ci 1.42E-00

$\mathrm{Ci}$ 5.96E-02

Ci 3.26E-02

Ci 1.62E-00

Ci 1.12E+05

Ci 1.44E+01

kg 1.03E+04

kg $\quad 6.80 \mathrm{E}-00$

kg $\quad 6.80 \mathrm{E}+01$

kg 1.07E+03

k $\quad 9.98 \mathrm{E}+03$

kg $3.40 \mathrm{E}+02$

kg $\quad 3.53 \mathrm{E}+02$

kg 2.19E+02

kg 3.56E-02

kg 3.29E+02

kg 3.43E-06

kg 1.14E+02

kg 4.32E+04

kg 2.30E+01

kg 7.17E+03

kg $6.19 \mathrm{E}+04$

kg 4.75E+02

kg 3.32E-00

$\mathrm{kg} \quad 1.47 \mathrm{E}+03$

kg 1.01E+03

$\mathrm{kg} \quad 9.60 \mathrm{E}+01$

kg 3.02E+03

$\mathrm{kg}$ 7.22E-07

kg $\quad 1.75 E+03$

$\mathrm{kg} \quad 4.82 \mathrm{E}+02$

kg 2.18E-00

Ci 2.48E-05

8.83E-01

Ci 3.10E-05

Ci $2.29 \mathrm{E}+04$

Ci 5.16E-01

Ci 124E-00

Ci 2.39E-03

Ci 2.20E-04

Ci 2.01E-03

Ci 4.91E-01

Ci 5.32E-02

Ci 2.42E+04
$B B$ Sept98 $1.05 E+01$
$2.32 \mathrm{E}-00$ $2.26 \mathrm{E}+02$

7.11E-01

4.00E-03

1.31E-00

$5.02 \mathrm{E}+01$

8.22E-00

$8.60 \mathrm{E}+01$

4.66E-04

1.55E-04

2.19E-01

5.71E-03

$1.32 \mathrm{E}+02$

2.94E-00

$1.03 \mathrm{E}+04$

$1.12 \mathrm{E}+05$

$1.97 \mathrm{E}+02$

5.08E-03

1.35E-02

4.44E-00

$1.96 E+02$

1.10E-00

$4.20 \mathrm{E}-00$

$1.42 \mathrm{E}-00$

5.96E-02

3.26E-02

1.33E-00

$1.12 \mathrm{E}+05$

$1.44 \mathrm{E}+01$

$1.03 E+04$

7.06E-00

$6.80 \mathrm{E}+01$

$1.07 \mathrm{E}+03$

$9.98 \mathrm{E}+03$

$3.40 \mathrm{E}+02$

$3.53 \mathrm{E}+02$

$2.19 \mathrm{E}+02$

$0.00 \mathrm{E}+01$

$3.29 \mathrm{E}+02$

$0.00 \mathrm{E}+01$

$1.14 E+02$

$4.32 \mathrm{E}+04$

2.30E +01

7.17E +03

$6.19 \mathrm{E}+04$

NA

3.71E-00

$1.47 \mathrm{E}+03$

NA

$9.60 \mathrm{E}+01$

$3.02 \mathrm{E}+03$

4.10E-02

$1.75 \mathrm{E}+03$

$4.82 \mathrm{E}+02$

6.60E-01

2.48E-05

8.83E-01

3.10E-05

$2.29 \mathrm{E}+04$

$5.16 \mathrm{E}-01$

$1.24 \mathrm{E}-00$

2.39E-03

2.20E-04

2.01E-03

4.91E-01

5.32E-02

$2.42 E+04$

\section{1\%-ile}

$0.00 \mathrm{E}+01$

$0.00 \mathrm{E}+01$

$0.00 \mathrm{E}+01$

$0.00 \mathrm{E}+01$

$0.00 \mathrm{E}+01$

$0.00 \mathrm{E}+01$

$0.00 \mathrm{E}+01$

$0.00 \mathrm{E}+01$

$0.00 \mathrm{E}+01$

$0.00 \mathrm{E}+01$

$0.00 \mathrm{E}+01$

$0.00 \mathrm{E}+01$

$0.00 \mathrm{E}+01$

$0.00 \mathrm{E}+01$

$0.00 \mathrm{E}+01$

$0.00 \mathrm{E}+01$

4.41E-01

$0.00 \mathrm{E}+01$

$0.00 \mathrm{E}+01$

$0.00 \mathrm{E}+0$

$0.00 \mathrm{E}+01$

$4.62 E+01$

$0.00 \mathrm{E}+01$

$0.00 \mathrm{E}+01$

$0.00 \mathrm{E}+01$

$0.00 \mathrm{E}+0 \mathrm{I}$

$0.00 \mathrm{E}+01$

$0.00 \mathrm{E}+01$

$0.00 \mathrm{E}+01$

$0.00 \mathrm{E}+01$

$7.75 \mathrm{E}+01$

$0.00 \mathrm{E}+01$

1.52E-01

3.51E-01

$1.15 \mathrm{E}+02$

$5.75 \mathrm{E}-03$

$1.42 \mathrm{E}+02$

$0.00 \mathrm{E}+01$

$0.00 \mathrm{E}+01$

$1.08 \mathrm{E}-02$

$5.71 \mathrm{E}-04$

$1.30 \mathrm{E}-02$

$1.32 \mathrm{E}+03$

$0.00 \mathrm{E}+01$

$1.13 \mathrm{E}-0 \mathrm{l}$

$1.93 E+01$

$3.39 \mathrm{E}-00$

$0.00 E+01$

$2.60 \mathrm{E}-00$

$9.09 \mathrm{E}-01$

$0.00 E+01$

$3.86 \mathrm{E}-01$

$0.00 \mathrm{E}+01$

3.96E-02

$0.00 E+01$

$0.00 \mathrm{E}+01$

$0.00 E+0.1$

$0.00 \mathrm{E}+0.1$

$0.00 E+01$

$0.00 E+01$

$0.00 \mathrm{E}+0.1$

$0.00 E+01$

$0.00 \mathrm{E}+01$

$0.00 E+01$

$0.00 \mathrm{E}+01$

$0.00 \mathrm{E}+01$

$0.00 \mathrm{E}+01$

2.10E-00

$10 \%$-ile

$0.00 \mathrm{E}+01 \quad 1.05 \mathrm{E}+01$

$0.00 \mathrm{E}+01 \quad 2.32 \mathrm{E}-00$

$0.00 \mathrm{E}+01$

$2.23 \mathrm{E}-03$

$0.00 \mathrm{E}+01$

4.12E-03

$1.58 \mathrm{E}-01$

2.58E-02

2.70E-01

$1.46 \mathrm{E}-06$

$0.00 \mathrm{E}+01$

$0.00 \mathrm{E}+01$

$0.00 \mathrm{E}+01$

$0.00 \mathrm{E}+01$

$0.00 \mathrm{E}+01$

$0.00 \mathrm{E}+01$

$1.50 \mathrm{E}+04$

$0.00 \mathrm{E}+01$

$0.00 \mathrm{E}+01$

$0.00 \mathrm{E}+01$

$0.00 \mathrm{E}+01$

$1.10 \mathrm{E}+02$

$0.00 \mathrm{E}+01$

$0.00 \mathrm{E}+01$

$0.00 \mathrm{E}+01$

$0.00 \mathrm{E}+0 \mathrm{l}$

$0.00 \mathrm{E}+01$

$0.00 \mathrm{E}+01$

$0.00 \mathrm{E}+01$ 
Tank Anal. Un

$\begin{array}{ll}\text { TX107 } & \text { Eu152 } \\ \text { TX107 } & \text { Eu154 } \\ \text { TX107 } & \text { Eu155 } \\ \text { TX107 } & \text { I129 } \\ \text { TX107 } & \text { Nb93 } \\ \text { TX107 } & \text { Ni59 } \\ \text { TX107 } & \text { Ni63 } \\ \text { TX107 } & \text { Np237 } \\ \text { TX107 } & \text { Pa231 } \\ \text { TX107 } & \text { Pu238 } \\ \text { TX107 } & \text { Pu239 } \\ \text { TX107 } & \text { Pu240 } \\ \text { TX107 } & \text { Pu241 } \\ \text { TX107 } & \text { Pu242 } \\ \text { TX107 } & \text { Ra226 } \\ \text { TX107 } & \text { Ra228 } \\ \text { TX107 } & \text { Ru106 } \\ \text { TX107 } & \text { Sb125 } \\ \text { TX107 } & \text { Se79 }\end{array}$

TX107 Sm151

TX107 Sr89/90

TX107 Te99

TX107 Th229

TX107 Th232

TX107 Tin126

TX107 Tritium

TX107 U232

TX107 U233

TX107 U234

TX107 U235

TX107 U236

TX107 U238

TX107 Y90

TX107 Zr93

TX108 Al

TX108 Bi

TX108 Ca

TX108 Cl

TX108 $\mathrm{CO} 3$

TX108 Cr

TX108 F

TX108 Fe

TX108 Hg

TX108 K

TX108 La

TX108 Mn

TX108 Na

TX108 $\mathrm{Ni}$

TX108 NO2

TX108 NO3

TX108 P

TX108 Pb

TX108 PO4

TX108 S

TX108 Si

TX108 $\mathrm{SO4}$

TX108 Sr

TX108 TOC

TX108 U

TX108 Zr

TX108 Ac227

TX108 Am241

TX108 Am243

TX108 Ba137

TX108 C14

TX108 Cd113

TX108 Cm242

TX108 Cm243

\section{May $\frac{B 8}{98}$ \\ 6.37E-102}

8.36E-00

3.98E-00

$6.09 \mathrm{E}-03$

1.79E-01

3.54E-02

3.44E-00

1.15E-02

7.34E-05

2.05E-02

8.10E-01

1.25E-01

$1.35 \mathrm{E}-00$

$7.43 \mathrm{E}-06$

6.37E-06

3.35E-03

9.51E-05

2.13E-00

4.99E-02

$1.76 \mathrm{E}+02$

$1.71 E+03$

3.16E-00

7.76E-05

2.06E-04

7.53E-02

3.13E-00

$1.68 \mathrm{E}-02$

6.43E-02

4.18E-00

1.88E-01

2.70E-02

4.23E-00

$1.71 \mathrm{E}+03$

2.44E-01

$2.28 \mathrm{E}+04$

$6.95 E+01$

$3.83 E+02$

$4.35 \mathrm{E}+03$

$4.46 \mathrm{E}+04$

$1.09 \mathrm{E}+03$

$1.60 \mathrm{E}+03$

$1.54 \mathrm{E}+03$

7.72E-01

$1.40 \mathrm{E}+03$

$3.66 \mathrm{E}+01$

$2.01 \mathrm{E}+02$

$1.78 \mathrm{E}+05$

$7.79 \mathrm{E}+01$

$2.60 \mathrm{E}+04$

$2.60 \mathrm{E}+05$

$9.40 \mathrm{E}+03$

$1.31 \mathrm{E}+02$

$2.91 E+04$

$1.41 \mathrm{E}+04$

$1.73 \mathrm{E}+02$

$4.23 \mathrm{E}+04$

$2.56 \mathrm{E}+01$

$7.61 \mathrm{E}+03$

$7.20 \mathrm{E}+02$

$1.51 \mathrm{E}+01$

$2.66 \mathrm{E}-04$

$1.51 \mathrm{E}+01$

5.09E-04

$8.70 \mathrm{E}+04$

8.21E-00

2.13E+01

3.52E-02

$3.26 \mathrm{E}-03$
Sept98

6.37E-02

$8.36 \mathrm{E}-00$

3.98E-00

$6.09 \mathrm{E}-03$

1.79E-01

3.54E-02

3.44E-00

1.15E-02

7.34E-05

2.05E-02

8.10E-01

$1.25 \mathrm{E}-01$

1.35E-00

7.43E-66

6.37E-06

3.35E-03

9.51E-05

2.13E-00

4.99E-02

$1.76 \mathrm{E}+02$

$1.71 \mathrm{E}+03$

$3.16 \mathrm{E}-00$

7.76E-05

2.06E-04

7.53E-02

3.13E-00

6.40E-04

2.45E-03

1.59E-01

7.15E-03

1.03E-03

1.61E-01

$1.71 \mathrm{E}+03$

2.44E-01

$2.28 \mathrm{E}+04$

$4.23 \mathrm{E}+01$

$3.83 \mathrm{E}+02$

$4.35 \mathrm{E}+03$

$4.46 \mathrm{E}+04$

$1.09 \mathrm{E}+03$

$1.60 \mathrm{E}+03$

$1.54 \mathrm{E}+03$

$0.00 E+01$

$1.40 \mathrm{E}+03$

$0.00 \mathrm{E}+01$

$2.01 E+02$

$1.78 \mathrm{E}+05$

$7.79 \mathrm{E}+01$

$2.60 \mathrm{E}+04$

$2.60 \mathrm{E}+05$

NA

9.23E+01

$9.50 \mathrm{E}+03$

NA

$1.73 E+02$

$1.41 \mathrm{E}+04$

2.08E+01

$7.61 \mathrm{E}+03$

$7.20 \mathrm{E}+02$

$1.51 \mathrm{E}+01$

2.66E-04

$1.51 \mathrm{E}+01$

5.09E-04

$8.70 \mathrm{E}+04$

$8.21 \mathrm{E}-00$

2.13E+01

3.52E-02

$3.26 \mathrm{E}-03$

1\%-ile

$10 \%$-ile

8.3

$0.00 \mathrm{E}+01 \quad 0.00 \mathrm{E}+01$

$0.00 \mathrm{E}+01 \quad 0.00 \mathrm{E}+01$

$0.00 \mathrm{E}+01 \quad 0.00 \mathrm{E}+01$

$0.00 \mathrm{E}+01 \quad 0.00 \mathrm{E}+01$

$0.00 \mathrm{E}+01 \quad 2.80 \mathrm{E}-04$

$0.00 \mathrm{E}+01 \quad 2.72 \mathrm{E}-02$

$0.00 \mathrm{E}+01$

9.99E-05

5.49E-06

$1.78 \mathrm{E}-04$

$0.00 \mathrm{E}+01$

$0.00 \mathrm{E} \div 01$

$7.04 \mathrm{E}-03$

$1.09 \mathrm{E}-03$

$0.00 \mathrm{E}+01$

$1.17 \mathrm{E}-02$

$6.46 \mathrm{E}-08$

$0.00 \mathrm{E}+0$

$0.00 \mathrm{E}+01$

$0.00 \mathrm{E}+01$

$0.00 \mathrm{E}+01$

$0.00 \mathrm{E}+01$

$0.00 \mathrm{E}+01 \quad 0.00 \mathrm{E}+01$

$0.00 \mathrm{E}+01$

$0.00 \mathrm{E}+01$

$0.00 \mathrm{E}+01 \quad 0.00 \mathrm{E}+01$

$0.00 \mathrm{E}+01 \quad 0.00 \mathrm{E}+01$

$0.00 \mathrm{E}+01$

$1.20 \mathrm{E}-01$

$0.00 \mathrm{E}+01 \quad 0.00 \mathrm{E}+01$

$0.00 \mathrm{E}+01 \quad 5.81 \mathrm{E}-06$

$0.00 \mathrm{E}+0 \mathrm{I} \quad 1.54 \mathrm{E}-05$

$0.00 \mathrm{E}+01 \quad 0.00 \mathrm{E}+01$

$1.05 \mathrm{E}-01 \quad 1.07 \mathrm{E}-00$

$0.00 \mathrm{E}+01 \quad 1.26 \mathrm{E}-03$

$0.00 \mathrm{E}+01 \quad 4.81 \mathrm{E}-03$

$0.00 \mathrm{E}+01 \quad 3.13 \mathrm{E}-01$

$0.00 \mathrm{E}+01 \quad 1.41 \mathrm{E}-02$

$0.00 \mathrm{E}+01 \quad 2.02 \mathrm{E}-03$

$0.00 \mathrm{E}+01 \quad 3.17 \mathrm{E}-01$

$0.00 \mathrm{E}+01 \quad 0.00 \mathrm{E}+01$

$0.00 \mathrm{E}+01 \quad 0.00 \mathrm{E}+01$

$1.39 \mathrm{E}+03 \quad 8.66 \mathrm{E}+03$

$5.41 \mathrm{E}+01 \quad 1.81 \mathrm{E}+02$

$1.08 \mathrm{E}+01 \quad 9.77 \mathrm{E}+01$

8.56E-00 4.91E+01

$3.46 \mathrm{E}+02 \quad 3.63 \mathrm{E}+03$

$5.44 \mathrm{E}-00 \quad 2.51 \mathrm{E}+01$

$1.21 \mathrm{E}+01 \quad 8.44 \mathrm{E}+01$

$8.78 \mathrm{E}+01 \quad 3.00 \mathrm{E}+02$

$0.00 \mathrm{E}+01 \quad 0.00 \mathrm{E}+01$

6.43E-00 4.89E+02

$1.15 \mathrm{E}-02 \quad 1.32 \mathrm{E}-0 \mathrm{I}$

2.36E-01 1.00E-00

$4.61 \mathrm{E}+04 \quad 9.04 \mathrm{E}+04$

$2.20 \mathrm{E}-01 \quad 8.35 \mathrm{E}-01$

$8.18 \mathrm{E}+01 \quad 1.44 \mathrm{E}+03$

$1.28 \mathrm{E}+03 \quad 9.37 \mathrm{E}+03$

$1.80 \mathrm{E}+02 \quad 5.81 \mathrm{E}+02$

4.00E-01 3.63E-00

$2.41 \mathrm{E}+02 \quad 8.69 \mathrm{E}+02$

$3.98 \mathrm{E}+01 \quad 1.58 \mathrm{E}+02$

$2.65 \mathrm{E}+01 \quad 1.10 \mathrm{E}+02$

$1.22 \mathrm{E}+02 \quad 3.80 \mathrm{E}+02$

5.32E-01 2.59E-00

$6.59 E-00 \quad 6.73 E+02$

$1.96 \mathrm{E}+01 \quad 6.42 \mathrm{E}+02$

2.39E-02

$0.00 E+01$

$1.24 \mathrm{E}-02$

$1.55 \mathrm{E}-07$

$0.00 E+01$

$0.00 E+01$

$0.00 \mathrm{E}+01$

1.07E-05

2.19E-01

$0.00 \mathrm{E}+01$

3.11E-01

$1.82 \mathrm{E}-06$

$0.00 \mathrm{E}+01$

$0.00 \mathrm{E}+01$

$0.00 \mathrm{E}+01$

$1.26 \mathrm{E}-04$

9.90E-07 1.17E-05

6.37E-02

8.36E-00

$3.98 \mathrm{E}-00$

$6.09 \mathrm{E}-03$

1.79E-01

3.54E-02

3.44E-00

$1.15 \mathrm{E}-02$

7.34E-05

2.05E-02

8.10E-01 


\section{Tank Anal. Un}

$\begin{array}{ll}\text { TX108 } & \text { Cm244 } \\ \text { TX108 } & \text { Co60 } \\ \text { TX108 } & \text { Cs134 } \\ \text { TX108 } & \text { Cs137 } \\ \text { TX108 } & \text { Eu152 } \\ \text { TX108 } & \text { Eu154 } \\ \text { TX108 } & \text { Eu155 } \\ \text { TX108 } & \text { 1129 } \\ \text { TX108 } & \text { Nb93 }\end{array}$

TX108 Ni63

TX108 Np237

TX108 Pa231

TX108 Pu238

TX108 Pu239

TX108 Pu240

TX108 Pu241

TX108 Pu242

TX108 Ra226

TX108 Ra228

TX108 Ru106

TX108 Sb125

TX108 Se79

TX108 Sm151

TX108 Sr89/90

TX108 Tc99

TX108 Th229

TX108 Th232

TX108 Tin126

TX108 Tritium

TX108 U232

TX108 U233

TX108 U234

TX108 U235

TX108 U236

TX108 U238

TX108 Y90

TX108 Zr93

TX109 Al

TX109 Bi

TX109 Ca

TX109 Cl

TX109 CO3

TX109 Cr

TX109 F

TX109 Fe

TX109. Hg

TX109 K

TX109 La

TX109 Mn

TX109 Na

TX109 Ni

TX109 NO2

TX109 NO3

TX109 P

TX109 Pb

TX109 PO4

TX109 S

TX109 Si

TX109 SO4

TX109 Sr

TX109 TOC

TX109 U

TX109 Zr

TX109 Ac227

TX109 Am241

TX109 Am243

TX109 Bal37
May $\frac{B B}{88}$

3.38E-02

8.96E-00

6.47E-01

$9.20 \mathrm{E}+04$

$9.20 \mathrm{E}-01$

$1.44 \mathrm{E}+02$

$5.47 \mathrm{E}+01$

1.13E-01

2.99E-00

$6.28 \mathrm{E}-01$

$6.15 E+01$

2.11E-01

1.17E-03

3.36E-01

$1.22 \mathrm{E}+01$

2.01E-00

$2.21 \mathrm{E}+01$

1.20E-04

4.12E-05

6.77E-02

$1.59 \mathrm{E}-03$

$3.80 \mathrm{E}+01$

$8.40 \mathrm{E}-01$

$2.71 E+04$

$5.85 \mathrm{E}+01$

$1.57 \mathrm{E}-03$

4.15E-03

Ci 1.27E-00

$5.74 \mathrm{E}+01$

Ci 3.38E-01

Ci 1.30E-00

Ci 1.31E-00

Ci 5.78E-02

$\mathrm{Ci} \quad 1.55 \mathrm{E}-02$

Ci 1.39E-00

Ci $2.71 \mathrm{E}+04$

Ci 4.12E-00

kg 3.31E+04

kg 3.14E+04

kg 2.25E+03

kg 1.23E+03

kg $\quad 1.56 \mathrm{E}+04$

kg $1.28 \mathrm{E}+03$

kg 2.03E+04

kg $\quad 3.89 \mathrm{E}+04$

kg 2.70E-01

kg $3.29 \mathrm{E}+02$

kg $0.00 \mathrm{E}+01$

kg $2.80 \mathrm{E}+02$

kg 1.98E+05

kg 2.83E+02

kg 1.61E+04

kg 1.35E+05

$\mathrm{kg} \quad 1.75 \mathrm{E}+05$

kg 1.32E+03

kg 5.42E+05

kg $\quad 1.39 \mathrm{E}+04$

kg 1.28E+04

kg 4.17E+04

kg $\quad 9.90 \mathrm{E}+02$

kg $\quad 1.70 \mathrm{E}+03$

kg 2.78E+04

kg $1.63 \mathrm{E}+02$

4.98E-05

3.19E+01

2.29E-06

$1.14 E+04$
Sept98

3.38E-02

$8.96 \mathrm{E}-00$

6.47E-01

$9.20 \mathrm{E}+04$

9.20E-01

$1.44 \mathrm{E}+02$

$5.47 \mathrm{E}+01$

$1.13 \mathrm{E}-01$

2.99E-00

6.28E-01

$6.15 E+01$

2.11E-01

1.17E-03

$3.36 \mathrm{E}-01$

$1.22 \mathrm{E}+01$

2.01E-00

$2.21 \mathrm{E}+01$

1.20E-04

4.12E-05

6.77E-02

1.59E-03

$3.80 \mathrm{E}+01$

$8.40 \mathrm{E}-01$

$2.96 \mathrm{E}+03$

2.71E+04

$5.85 \mathrm{E}+01$

1.57E-03

4.15E-03

1.27E-00

$5.74 \mathrm{E}+01$

6.27E-02

2.40E-01

2.44E-01

1.07E-02

2.88E-03

2.40E-01

$2.71 E+04$

4.12E-00

$3.31 E+04$

$3.14 E+04$

$2.25 E+03$

$1.23 \mathrm{E}+03$

$1.56 \mathrm{E}+04$

$1.28 \mathrm{E}+03$

2.03E+04

$3.89 \mathrm{E}+04$

$1.22 \mathrm{E}+02$

$3.29 \mathrm{E}+02$

$0.00 \mathrm{E}+01$

$2.80 \mathrm{E}+02$

$1.98 \mathrm{E}+05$

$2.83 \mathrm{E}+02$

$1.61 \mathrm{E}+04$

$1.35 E+05$

$$
\text { NA }
$$

$1.32 \mathrm{E}+03$

$1.77 \mathrm{E}+05$

NA

$1.28 \mathrm{E}+04$

$1.39 \mathrm{E}+04$

$9.90 \mathrm{E}+02$

$1.70 \mathrm{E}+03$

$2.78 \mathrm{E}+04$

$1.63 \mathrm{E}+02$

4.98E-05

$3.19 \mathrm{E}+01$

2.23E-04

$1.14 E+04$
$1 \%$-ile

1.03E-05

$1.09 \mathrm{E}-03$

$0.00 \mathrm{E}+01$

$1.02 \mathrm{E}+02$

$0.00 \mathrm{E}+01$

$0.00 \mathrm{E}+01$

$0.00 \mathrm{E}+01$

$0.00 \mathrm{E}+0 \mathrm{I}$

$0.00 \mathrm{E}+01$

$0.00 \mathrm{E}+01$

$0.00 \mathrm{E}+01$

6.41E-05

$0.00 \mathrm{E}+01$

1.02E-04

$3.70 \mathrm{E}-03$

$6.10 \mathrm{E}-04$

$6.71 \mathrm{E}-03$

$3.64 \mathrm{E}-08$

$0.00 \mathrm{E}+01$

$0.00 \mathrm{E}+01$

$0.00 \mathrm{E}+01$

$0.00 \mathrm{E}+01$

$0.00 \mathrm{E}+01$

$0.00 E+01$

$3.55 \mathrm{E}+01$

$0.00 \mathrm{E}+01$

$0.00 \mathrm{E}+01$

$0.00 \mathrm{E}+01$

$0.00 E+01$

$1.67 E+01$

$0.00 \mathrm{E}+01$

$0.00 E+01$

$0.00 E+01$

$0.00 \mathrm{E}+01$

$0.00 \mathrm{E}+01$

$0.00 \mathrm{E}+01$

$0.00 \mathrm{E}+01$

$0.00 \mathrm{E}+01$

$2.42 \mathrm{E}+02$

$1.33 \mathrm{E}+04$

$1.18 \mathrm{E}+02$

$4.12 \mathrm{E}+02$

$2.65 \mathrm{E}-01$

$1.40 \mathrm{E}+01$

$1.98 \mathrm{E}+02$

$7.44 \mathrm{E}+03$

$0.00 \mathrm{E}+01$

$6.22 \mathrm{E}+01$

9.44E- 02

4.80E-00

$8.44 \mathrm{E}+04$

$2.65 \mathrm{E}-00$

$7.39 \mathrm{E}+02$

$2.58 \mathrm{E}+04$

$6.18 \mathrm{E}+03$

9.79E-00

$2.62 E+03$

$2.35 E+02$

$8.45 \mathrm{E}+02$

$1.77 \mathrm{E}+03$

$1.41 E \div 01$

3.02E-03

$1.67 \mathrm{E}+01$

$1.85 \mathrm{E}-00$

$0.00 \mathrm{E}+01$

$1.45 \mathrm{E}-00$

$9.58 \mathrm{E}-08$

$7.41 \mathrm{E}+02$

$10 \%$-ile

1.21E-04

$5.54 \mathrm{E}-02$

$0.00 \mathrm{E}+01$

$3.92 \mathrm{E}+03$

$0.00 \mathrm{E}+01$

$0.00 \mathrm{E}+01$

$0.00 E+01$

$0.00 \mathrm{E}+01$

$0.00 \mathrm{E}+01$

$0.00 \mathrm{E}+01$

$0.00 \mathrm{E}+01$

$7.56 \mathrm{E}-04$

$0.00 \mathrm{E}+01$

$1.20 \mathrm{E}-03$

4.37E-02

$7.20 \mathrm{E}-03$

7.92E-02

4.30E-07

$0.00 \mathrm{E}+01$

$0.00 \mathrm{E}+01$ 
Tank Anal. Un

$\begin{array}{ll}\text { TX109 } & \text { C14 } \\ \text { TX109 } & \text { Cd113 } \\ \text { TX109 } & \text { Cm242 } \\ \text { TX109 } & \text { Cm243 } \\ \text { TX109 } & \text { Cm244 } \\ \text { TX109 } & \text { Co60 } \\ \text { TX109 } & \text { Cs134 } \\ \text { TX109 } & \text { Cs137 } \\ \text { TX109 } & \text { Eu152 } \\ \text { TX109 } & \text { Eu154 } \\ \text { TX109 } & \text { Eu155 } \\ \text { TX109 } & \text { I129 }\end{array}$

TX109 Nb93

TX109 Ni59

TX109 Ni63

TX109 Np237

TX109 Pa231

TX109 Pu238

TX109 Pu239

TX109 Pu240

TX109 Pu241

TX109 Pu242

TX109 Ra226

TX109 Ra228

TX109 Ru106

TX109 Sb125

TX109 Se79

TX109 Sm151

TX109 Sr89/90

TX109 Tc99

TX109 Th229

TX109 Th232

TX109 Tin126

TX109 Tritium

TX109 U232

TX109 U233

TX109 U234

TX109 U235

TX109 U236

TX109 U238

TX109 Y90

TX109 Zr93

TX110 Al

TX110 Bi

TX110 Ca

TX110 Cl

TX110 CO3

TX110 Cr

TX110 F

TX110 Fe

TX110 Hg

TX110 K

TX110 La

TX110 Mn

TX110 Na

TX110 Ni

TX110 NO2

TX110 NO3

TX110 P

TX110 Pb

TX110 $\mathrm{PO4}$

TX110 S

TX110 Si

TX110 SO4

TX110 Sr

TX110 TOC

$\operatorname{rx110} U$

TX110 Zr
$B B$

MaY98

2.30E-01

4.16E-01

$9.20 \mathrm{E}-04$

$1.88 \mathrm{E}-05$

5.41E-05

2.00E-00

2.40E-00

$1.21 \mathrm{E}+04$

5.04E-02

$7.70 \mathrm{E}-00$

6.05E-00

$9.40 \mathrm{E}+01$

1.44E-01

4.83E-02

4.36E-00

$2.79 E+02$

$1.08 \mathrm{E}-04$

$1.45 E+02$

$2.76 \mathrm{E}+02$

Incl. in 239

$1.70 \mathrm{E}+01$

7.70E-05

9.76E-06

1.95E-10

$7.75 \mathrm{E}+01$

3.61E-02

$3.60 \mathrm{E}-01$

$1.33 \mathrm{E}+02$

$1.10 \mathrm{E}+05$

1.18E-00

3.79E-08

$4.84 \mathrm{E}-11$

5.39E-02

1.20E-00

4.12E-04

2.05E-05

$2.20 \mathrm{E}+01$

9.81E-01

$1.88 \mathrm{E}-01$

2.24E+01

$1.10 \mathrm{E}+05$

$1.70 \mathrm{E}-01$

$7.85 \mathrm{E}+04$

$3.21 \mathrm{E}+03$

$1.03 \mathrm{E}+03$

$1.44 \mathrm{E}+04$

$1.50 \mathrm{E}+05$

$3.71 \mathrm{E}+03$

$7.22 \mathrm{E}+03$

$2.24 E+03$

5.10E-00

$4.68 \mathrm{E}+03$

$9.40 \mathrm{E}+01$

$6.80 \mathrm{E}+02$

$5.96 \mathrm{E}+05$

$2.80 \mathrm{E}+02$

$8.56 \mathrm{E}+04$

$8.52 \mathrm{E}+05$

$3.76 \mathrm{E}+04$

$3.00 \mathrm{E}+02$

$1.16 \mathrm{E}+05$

$4.59 \mathrm{E}+04$

$1.70 \mathrm{E}+03$

$1.38 \mathrm{E}+05$

$1.14 \mathrm{E}+02$

$2.54 \mathrm{E}+04$

$3.65 \mathrm{E}+03$

$6.30 \mathrm{E}+01$
$\frac{B B}{498}$

2.30E-01

4.16E-01

8.96E-02

$1.83 \mathrm{E}-03$

5.27E-03

3.89E-02

$1.50 \mathrm{E}-03$

$1.21 \mathrm{E}+04$

5.04E-02

$7.70 \mathrm{E}-00$

6.05E-00

2.22E-03

$1.44 \mathrm{E}-01$

4.83E-02

4.36E-00

7.28E-03

1.08E-04

1.82E-00

2.53E+02

$2.30 \mathrm{E}+01$

$7.51 \mathrm{E}+01$

3.41E-04

9.76E-06

$1.95 \mathrm{E}-10$

1.62E-08

3.61E-02

$3.58 \mathrm{E}-02$

$1.33 \mathrm{E}+02$

$1.10 \mathrm{E}+05$

1.18E-00

3.79E-08

4.84E-11

5.39E-02

1.20E-00

1.71E-04

8.49E-06

9.15E-00

4.07E-01

$7.81 \mathrm{E}-02$

9.28E-00

$1.10 \mathrm{E}+05$

$1.70 \mathrm{E}-01$

$7.85 \mathrm{E}+04$

$3.21 \mathrm{E}+03$

$1.03 \mathrm{E}+03$

$1.44 \mathrm{E}+04$

$1.50 \mathrm{E}+05$

$3.71 \mathrm{E}+03$

$7.22 \mathrm{E}+03$

$3.33 \mathrm{E}+03$

$1.18 \mathrm{E}+01$

$4.68 \mathrm{E}+03$

$0.00 \mathrm{E}+01$

$6.80 \mathrm{E}+02$

$5.96 \mathrm{E}+05$

$2.80 \mathrm{E}+02$

$8.56 \mathrm{E}+04$

$8.52 \mathrm{E}+05$

NA

$3.00 \mathrm{E}+02$

$3.80 \mathrm{E}+04$

NA

$1.70 \mathrm{E}+03$

$4.59 \mathrm{E}+04$

$9.86 \mathrm{E}+01$

$2.54 \mathrm{E}+04$

$3.65 \mathrm{E}+03$

$6.30 \mathrm{E}+01$ 1\%-ile

$10 \%$-ile

$50 \%$ ille

$0.00 \mathrm{E}+01$

$0.00 \mathrm{E}+01$

3.85E-05

$7.86 \mathrm{E}-07$

2.26E-06

$0.00 \mathrm{E}+01$

$1.56 \mathrm{E}-01$

$2.62 \mathrm{E}+03$

$0.00 \mathrm{E}+01$

$0.00 \mathrm{E}+01$

$0.00 \mathrm{E}+01$

$0.00 \mathrm{E}+01$

$0.00 \mathrm{E}+01$

$7.96 \mathrm{E}-04$

7.19E-02

$1.17 \mathrm{E}+01$

$0.00 \mathrm{E}+01$

6.06E-00

$1.15 \mathrm{E}+01$

$1.15 \mathrm{E}+01$

7.11E-01

3.22E-06

$0.00 \mathrm{E}+01$

$0.00 \mathrm{E}+01$

$0.00 \mathrm{E}+01$

$0.00 \mathrm{E}+01$

$2.68 \mathrm{E}-03$

$0.00 \mathrm{E}+01$

$3.93 \mathrm{E}+01$

$3.01 \mathrm{E}-02$

$0.00 \mathrm{E}+01$

$0.00 E+01$

$0.00 \mathrm{E}+01$

$5.84 \mathrm{E}-01$

$0.00 \mathrm{E}+01$

$0.00 \mathrm{E}+01$

$0.00 \mathrm{E}+01$

$0.00 \mathrm{E}+01$

$0.00 \mathrm{E}+01$

$0.00 \mathrm{E}+01$

$0.00 \mathrm{E}+01$

$0.00 \mathrm{E}+01$

$6.94 \mathrm{E}+03$

$1.13 \mathrm{E}+03$

4.14E+01

8.21E+01

$5.54 \mathrm{E}+02$

$4.58 \mathrm{E}+01$

$8.21 E+01$

$8.06 \mathrm{E}+02$

$0.00 \mathrm{E}+01$

$4.68 \mathrm{E}+01$

$6.16 \mathrm{E}-02$

$1.57 \mathrm{E}-00$

$1.68 \mathrm{E}+05$

4.36E-01

$8.13 \mathrm{E}+02$

$8.79 \mathrm{E}+03$

$1.34 \mathrm{E}+03$

$2.80 \mathrm{E}-00$

$6.76 \mathrm{E}+02$

$1.23 \mathrm{E}+02$

$2.42 \mathrm{E}+02$

$4.12 \mathrm{E}+02$

4.88E-00

$5.70 \mathrm{E}+01$

$2.25 \mathrm{E}+01$

1.95E-01

1.17E-02

$5.20 \mathrm{E}-02$

2.82E-04

$5.76 \mathrm{E}-06$

$1.66 \mathrm{E}-05$

2.64E-01

7.73E-01

$1.14 \mathrm{E}+04$

9.60E-03

$1.47 \mathrm{E}-00$

$1.15 \mathrm{E}-00$

$3.08 \mathrm{E}+0.1$

7.34E-03

$1.45 \mathrm{E}-02$

$1.31 \mathrm{E}-00$

$8.56 \mathrm{E}+01$

$1.99 \mathrm{E}-05$

$4.45 \mathrm{E}+01$

$8.46 \mathrm{E}+01$

$8.46 \mathrm{E}+01$

$5.21 \mathrm{E}-00$ 
Tank Anal. Un

$\begin{array}{ll}\text { TX110 } & \text { Ac227 } \\ \text { TX110 } & \text { Am241 } \\ \text { TX110 } & \text { Am243 } \\ \text { TX110 } & \text { Ba137 } \\ \text { TX110 } & \text { C14 } \\ \text { TX110 } & \text { Cd113 } \\ \text { TX110 } & \text { Cm242 } \\ \text { TX110 } & \text { Cm243 } \\ \text { TX110 } & \text { Cm244 } \\ \text { TX110 } & \text { Co60 } \\ \text { TX110 } & \text { Cs134 } \\ \text { TX110 } & \text { Cs137 } \\ \text { TX110 } & \text { Eu152 } \\ \text { TX110 } & \text { Eu154 } \\ \text { TX110 } & \text { Eu155 } \\ \text { TX110 } & 1129 \\ \text { TX110 } & \text { Nb93 } \\ \text { TX110 } & \text { Ni59 } \\ \text { TX110 } & \text { Ni63 }\end{array}$

TX110 Ni63

TX110 Np237

TX110 Pa231

TX110 Pu238

TX110 Pu239

TX110 Pu240

TX110 Pu241

TX110 Pu242

TX110 Ra226

TX110 Ra228

TX110 Ru106

TX110 Sb125

TX110 Se79

TX110 Sm151

TX110 Sr89/90

TX110 Te99

TX110 Th229

TX110 Th232

TX110 Tin126

TX110 Tritium

TX110 U232

TX110 U233

TX110 U234

TX110 U235

TX110 U236

TX110 U238

TX110 Y90

TX110 Zr93

TX111 Al

TXIII Bi

TX111 Ca

TX111 Cl

TX111 $\mathrm{CO} 3$

TX111 $\mathrm{Cr}$

TX111 F

TX111 Fe

TX111 Hg

TX111 K

TX111 La

TX111 Mn

TX111 Na

TX111 Ni

TX111 NO2

TX111 NO3

TX111 $P$

TX111 Pb

TX111 PO4

TX111 S

TX111 Si

TX111 SO4
May $\frac{B B}{98}$

$1.02 \mathrm{E}-03$

$6.25 \mathrm{E}+01$

2.22E-03

$2.89 \mathrm{E}+05$

3.07E+01

$8.67 \mathrm{E}+01$

1.73E-01

1.60E-02

$1.44 \mathrm{E}-01$

$3.48 \mathrm{E}+01$

2.60E-00

$3.05 \mathrm{E}+05$

$4.49 \mathrm{E}-00$

$5.96 \mathrm{E}+02$

$2.68 \mathrm{E}+02$

4.22E-01

$1.21 \mathrm{E}+01$

2.30E-00

$2.25 E+02$

$7.86 \mathrm{E}-01$

4.54E-03

$1.50 \mathrm{E}-00$

$5.71 \mathrm{E}+01$

9.14E-00

$9.88 \mathrm{E}+01$

5.43E-04

1.58E-04

2.36E-01

6.81E-04

$1.52 \mathrm{E}+02$

3.40E-00

$1.20 \mathrm{E}+04$

$1.25 \mathrm{E}+05$

$2.19 E+02$

5.47E-03

1.45E-02

5.14E-00

$2.20 \mathrm{E}+02$

1.18E-00

4.53E-00

3.07E-00

1.32E-01

5.12E-02

$3.31 \mathrm{E}-00$

$1.25 \mathrm{E}+05$

$1.67 \mathrm{E}+01$

$6.17 \mathrm{E}+04$

$3.66 \mathrm{E}+03$

$8.80 \mathrm{E}+02$

1.11E+04

$1.16 \mathrm{E}+05$

$2.90 \mathrm{E}+03$

$6.32 \mathrm{E}+03$

$2.06 \mathrm{E}+03$

4.30E-00

$3.61 E+03$

$7.43 E+01$

$5.30 \mathrm{E}+02$

$4.66 \mathrm{E}+05$

$2.20 \mathrm{E}+02$

kg 6.65E+04

$\mathrm{kg} \quad 6.61 \mathrm{E}+05$

kg $3.56 \mathrm{E}+04$

$\mathrm{kg} \quad 2.20 \mathrm{E}+02$

kg 1.10E +05

$\mathrm{kg} \quad 3.58 \mathrm{E}+04$

kg $\quad 1.80 \mathrm{E}+03$

kg
Sept98

1.02E-03

$6.25 \mathrm{E}+01$

$2.22 \mathrm{E}-03$

$2.89 \mathrm{E}+05$

$3.07 \mathrm{E}+01$

$8.67 \mathrm{E}+01$

$1.73 E-01$

1.60E-02

$1.44 \mathrm{E}-01$

$3.48 \mathrm{E}+01$

2.60E-00

$3.05 \mathrm{E}+05$

4.49E-00

$5.96 \mathrm{E}+02$

$2.68 \mathrm{E}+02$

$4.22 \mathrm{E}-01$

$1.21 \mathrm{E}+01$

2.30E-00

$2.25 \mathrm{E}+02$

$7.86 \mathrm{E}-01$

4.54E-03

1.50E-00

5.71E+01

9.14E-00

$9.88 \mathrm{E}+01$

$5.43 \mathrm{E}-04$

1.58E-04

2.36E-01

6.81E-03

$1.52 \mathrm{E}+02$

$3.40 \mathrm{E}-00$

$1.20 \mathrm{E}+04$

$1.20 \mathrm{E}+05$

$2.19 \mathrm{E}+02$

$5.47 \mathrm{E}-03$

$1.45 \mathrm{E}-02$

5.14E-00

$2.20 \mathrm{E}+02$

$4.82 \mathrm{E}-01$

$1.85 \mathrm{E}-00$

1.25E-00

$537 \mathrm{E}-02$

2.08E-02

$122 \mathrm{E}-00$

$1.20 \mathrm{E}+05$

$1.67 \mathrm{E}+01$

$6.17 \mathrm{E}+04$

$3.66 \mathrm{E}+03$

$8.80 \mathrm{E}+02$

$1.11 E+04$

$1.16 \mathrm{E}+05$

$2.90 \mathrm{E}+03$

$6.32 \mathrm{E}+03$

$3.63 \mathrm{E}+03$

$1.37 \mathrm{E}+01$

$3.61 \mathrm{E}+03$

$0.00 \mathrm{E}+01$

$5.30 \mathrm{E}+02$

$4.66 \mathrm{E}+05$

$2.20 \mathrm{E}+02$

$6.65 \mathrm{E}+04$

$6.61 \mathrm{E}+05$

NA

$2.20 \mathrm{E}+02$

$3.60 \mathrm{E}+04$

NA

$1.80 \mathrm{E}+03$

$3.58 \mathrm{E}+04$

1\%-ile

\section{$0.00 \mathrm{E}+01$}

$10 \%$-ile

50\%-ile

90\%-ile

4.76E-01

$0.00 \mathrm{E}+01 \quad 1.02 \mathrm{E}-03$

$3.36 \mathrm{E}-00$

$1.60 \mathrm{E}+02$

2.22E-03

$0.00 \mathrm{E}+01$

$0.00 \mathrm{E}+01$

$0.00 \mathrm{E}+01$

$0.00 \mathrm{E}+01$

$2.91 \mathrm{E}-04$

$0.00 \mathrm{E}+01$

$0.00 \mathrm{E}+01$

$1.72 \mathrm{E}-03$

$2.89 \mathrm{E}+05$

$3.07 \mathrm{E}+01$

8.67E+01

1.73E-01

$1.59 \mathrm{E}-04$

$1.44 \mathrm{E}-03$

1.60E-02

2.42E-04

$0.00 \mathrm{E}+01$

$0.00 \mathrm{E}+01$

$4.26 \mathrm{E}-01$

$0.00 \mathrm{E}+01$

$1.44 \mathrm{E}-01$

$1.36 \mathrm{E}+03$

$0.00 \mathrm{E}+01$

$1.54 \mathrm{E}+04$

$0.00 \mathrm{E}+01$

$6.74 \mathrm{E}+01$

2.60E-00

$0.00 \mathrm{E}+01$

$0.00 \mathrm{E}+01$

$0.00 \mathrm{E}+01$

$0.00 \mathrm{E}+01$

$0.00 \mathrm{E}+01$

$0.00 \mathrm{E}+01$

$1.32 \mathrm{E}-03$

$0.00 \mathrm{E}+01$

2.52E-03

$9.60 \mathrm{E}-02$

$1.54 \mathrm{E}-02$

$0.00 \mathrm{E}+01$

$0.00 \mathrm{E}+01$

$0.00 \mathrm{E}+01$

$0.00 \mathrm{E}+01$

$0.00 \mathrm{E}+01$

$0.00 \mathrm{E}+01$

$7.83 \mathrm{E}-03$

$0.00 \mathrm{E}+01$

$1.50 \mathrm{E}-02$

$5.69 \mathrm{E}-01$

$9.11 \mathrm{E}-02$

9.85E-01

5.41E-06

9.12E-07

$0.00 \mathrm{E}+01$

$0.00 \mathrm{E}+01$

$0.00 \mathrm{E}+01$

$0.00 \mathrm{E}+01 \quad 5.13 \mathrm{E}-05$

$0.00 \mathrm{E}+01 \quad 0.00 \mathrm{E}+01$

$0.00 \mathrm{E}+01$

$0.00 \mathrm{E}+01$

$4.22 \mathrm{E}+02$

$0.00 \mathrm{E}+01$

$0.00 E+01$

$9.32 E+03$

$0.00 \mathrm{E}+01$

$0.00 \mathrm{E}+01$

$0.00 \mathrm{E}+01$

$0.00 \mathrm{E}+01$

$0.00 \mathrm{E}+01$

$0.00 \mathrm{E}+01$

$0.00 \mathrm{E}+01$

$0.00 \mathrm{E}+01$

$6.34 \mathrm{E}+01$

$1.31 \mathrm{E}+02$

$0.00 E+01$

$0.00 \mathrm{E}+0$

$0.00 E+01$

$0.00 \mathrm{E}+01$

$0.00 E+0$

$0.00 \mathrm{E}+01 \cdot 0.00 \mathrm{E}+01$

$0.00 \mathrm{E}+01$

$0.00 \mathrm{E}+01$

$0.00 \mathrm{E}+01$

$0.00 \mathrm{E}+01$

$4.49 \mathrm{E}+03$

$1.52 \mathrm{E}+03$

$4.68 \mathrm{E}+01$

$1.05 \mathrm{E}+02$

$6.76 \mathrm{E}+02$

$5.42 \mathrm{E}+01$

$1.12 \mathrm{E}+02$ 
Tank Anal. Un

\begin{tabular}{ll|l}
\hline & & \\
TX111 & Sr & \\
TX111 & TOC & \\
TX111 & U & \\
TX111 & Zr & \\
TX111 & Ac227 & \\
TX111 & Am241 \\
TX111 & Am243 \\
TX111 & Ba137 \\
TX111 & C14 \\
TX111 & Cd113 \\
TX111 & Cm242 \\
TX111 & Cm243 \\
TX111 & Cm244 \\
TX111 & Co60 \\
TX111 & Cs134 \\
TX111 & Cs137 \\
TX111 & Eu152 \\
TX111 & Eu154 \\
TX111 & Eu155 \\
TX111 & I129 \\
TX111 & Nb93 \\
TX111 & Ni59 \\
TX111 & Ni63
\end{tabular}

TX111 Np237

TX111 Pa231

TX111 Pu238

TX111 Pu239

TX111 Pu240

TX111 Pu241

TX111 Pu242

TX111 Ra226

TX111 Ra228

TX111 Ru106

TX111 Sb125

TX111 Se79

TX111 Sm151

TX111 Sr89/90

TX111 Te99

TX111 Th229

TX111 Th232

TX111 Tin126

TX111 Tritium

TX111 U232

TX111 U233

TX111 U234

TX111 U235

TX111 U236

TX111 U238

TX111 Y90

TX111 Zr93

TX112 Al

TX112 Bi

TX112 Ca

$\mathrm{TX112} \mathrm{Cl}$

$\mathrm{TX} 112 \mathrm{CO} 3$

TX112 Cr

TX112 F

TX112 Fe

TX112 Hg

TX112 K

TX112 La

TX112 Mn

TX112 Na

TX112 Ni

TX112 NO2

TX112 NO3

TX112 P

TX112 Pb
May $\frac{B B}{98}$

$1 \overline{25 \mathrm{E}+02}$

$1.96 \mathrm{E}+04$

$2.82 \mathrm{E}+03$

$5.40 \mathrm{E}+01$

8.66E-04

$5.31 \mathrm{E}+01$

$1.90 \mathrm{E}-03$

$2.23 \mathrm{E}+05$

$2.58 \mathrm{E}+01$

$7.36 \mathrm{E}+01$

$1.50 \mathrm{E}-01$

$1.38 \mathrm{E}-02$

$1.23 \mathrm{E}-01$

$2.93 \mathrm{E}+01$

$2.00 \mathrm{E}-00$

2.35E+05

3.89E-00

$5.07 \mathrm{E}+02$

$2.33 \mathrm{E}+02$

$3.54 \mathrm{E}-01$

$1.03 \mathrm{E}+01$

$1.93 \mathrm{E}-00$

$1.89 \mathrm{E}+02$

6.61E-01

3.84E-03

1.31E-00

$5.21 \mathrm{E}+01$

8.15E-00

$8.59 \mathrm{E}+01$

4.71E-04

$1.34 \mathrm{E}-04$

$1.97 \mathrm{E}-01$

5.82E-03

$1.28 \mathrm{E}+02$

2.89E-00

$1.02 \mathrm{E}+04$

$1.03 \mathrm{E}+05$

$1.84 \mathrm{E}+02$

4.56E-03

$1.21 \mathrm{E}-02$

4.36E-00

$1.86 \mathrm{E}+02$

9.85E-01

3.78E-00

3.19E-00

1.38E-01

5.09E-02

3.41E-00

$1.03 \mathrm{E}+05$

$1.42 E+01$

$1.11 E+05$

$3.35 \mathrm{E}+02$

$1.24 \mathrm{E}+03$

$2.11 E+04$

$2.22 \mathrm{E}+05$

$5.29 \mathrm{E}+03$

$9.32 \mathrm{E}+03$

$1.69 \mathrm{E}+03$

$5.00 \mathrm{E}-00$

$6.89 \mathrm{E}+03$

$1.45 \mathrm{E}+02$

$1.06 E+03$

$8.86 \mathrm{E}+05$

$3.70 \mathrm{E}+02$

$1.24 \mathrm{E}+05$

$1.29 \mathrm{E}+06$

$5.29 \mathrm{E}+04$

$6.23 E+02$
$B B$ Sept98

$1.13 \mathrm{E}+02$

$1.96 \mathrm{E}+04$

$9.41 \mathrm{E}+03$

$5.40 \mathrm{E}+01$

8.66E-04

$5.31 \mathrm{E}+01$

$1.90 \mathrm{E}-03$

$2.23 E+05$

$2.58 \mathrm{E}+01$

$7.36 \mathrm{E}+01$

1.50E-01

$1.38 \mathrm{E}-02$

$1.23 \mathrm{E}-01$

2.93E+01

2.00E-00

2.35E+05

$3.89 \mathrm{E}-00$

$5.07 \mathrm{E}+02$

2.33E+02

3.54E-01

$1.03 \mathrm{E}+01$

$1.93 \mathrm{E}-00$

$1.89 \mathrm{E}+02$

6.61E-01

3.84E-03

1.31E-00

$5.21 \mathrm{E}+01$

8.15E-00

$8.59 \mathrm{E}+01$

4.71E-04

1.34E-04

1.97E-01

$5.82 \mathrm{E}-03$

$1.28 \mathrm{E}+02$

2.89E-00

$1.02 \mathrm{E}+04$

$1.03 \mathrm{E}+05$

$1.84 \mathrm{E}+02$

4.56E-03

1.21E-02

4.36E-00

$1.86 \mathrm{E}+02$

9.85E-01

3.78E-00

3.19E-00

1.38E-01

5.09E-02

3.14E-00

$1.03 \mathrm{E}+05$

$-1.42 \mathrm{E}+01$

$1.11 E+05$

$2.02 \mathrm{E}+02$

$1.24 \mathrm{E}+03$

2.11E+04

$2.22 \mathrm{E}+05$

$5.29 \mathrm{E}+03$

$9.32 \mathrm{E}+03$

$1.69 \mathrm{E}+03$

$0.00 \mathrm{E}+01$

$6.88 \mathrm{E}+03$

$0.00 \mathrm{E}+01$

$1.06 \mathrm{E}+03$

$8.86 \mathrm{E}+05$

$3.70 \mathrm{E}+02$

$1.24 \mathrm{E}+05$

$1.29 \mathrm{E}+06$

NA

$5.40 \mathrm{E}+02$ 1\%-ile

7.03E-00

$5.19 \mathrm{E}+01$

$1.85 \mathrm{E}+01$

2.49E-01

$0.00 \mathrm{E}+01$

$3.21 \mathrm{E}-01$

4.51E-06

$0.00 \mathrm{E}+01$

$0.00 \mathrm{E}+01$

$0.00 \mathrm{E}+01$

$3.56 \mathrm{E}-04$

3.27E-05

2.92E-04

$0.00 \mathrm{E}+01$

$0.00 \mathrm{E}+01$

$1.64 \mathrm{E}+03$

$0.00 \mathrm{E}+01$

$0.00 \mathrm{E}+01$

$0.00 \mathrm{E}+01$

$0.00 \mathrm{E}+01$

$0.00 \mathrm{E}+01$

$0.00 \mathrm{E}+01$

$0.00 \mathrm{E}+01$

$1.57 \mathrm{E}-03$

$0.00 \mathrm{E}+01$

3.11E-03

$1.24 \mathrm{E}-01$

$1.93 \mathrm{E}-02$

2.04E-01

$1.12 \mathrm{E}-06$

$0.00 \mathrm{E}+01$

$0.00 \mathrm{E}+01$

$0.00 \mathrm{E}+01$

$0.00 \mathrm{E}+01$

$0.00 \mathrm{E}+01$

$0.00 \mathrm{E}+01$

$3.86 \mathrm{E}+02$

$0.00 \mathrm{E}+01$

$0.00 \mathrm{E}+01$

$0.00 \mathrm{E}+01$

$0.00 \mathrm{E}+01$

$6.13 \mathrm{E}+01$

$0.00 \mathrm{E}+01$

$0.00 \mathrm{E}+01$

$0.00 \mathrm{E}+01$

$0.00 \mathrm{E}+01$

$0.00 \mathrm{E}+01$

$0.00 \mathrm{E}+01$

$0.00 \mathrm{E}+01$

$0.00 \mathrm{E}+01$

4.15E+03

$0.00 \mathrm{E}+01$

$0.00 \mathrm{E}+01$

$0.00 \mathrm{E}+01$

$0.00 \mathrm{E}+01$

$0.00 \mathrm{E}+01$

$0.00 \mathrm{E}+01$

$0.00 \mathrm{E}+0$

$0.00 \mathrm{E}+01$

$0.00 \mathrm{E}+01$

$0.00 \mathrm{E}+01$

$0.00 \mathrm{E}+01$

$2.43 \mathrm{E}+05$

$0.00 \mathrm{E}+01$

$0.00 \mathrm{E}+01$

$0.00 \mathrm{E}+01$

$0.00 \mathrm{E}+01$

$0.00 \mathrm{E}+01$

$10 \%$-ile

$2.81 \mathrm{E}+01$

$2.08 \mathrm{E}+03$

$2.17 \mathrm{E}+02$

$2.11 \mathrm{E}-00$

$0.00 \mathrm{E}+01$

4.92E-00

$2.89 \mathrm{E}-05$

$0.00 \mathrm{E}+01$

$0.00 \mathrm{E}+01$

$0.00 \mathrm{E}+01$

2.28E-03

2.10E-04

$1.87 \mathrm{E}-03$

4.59E-01

$0.00 \mathrm{E}+01$

$1.88 \mathrm{E}+04$

$0.00 \mathrm{E}+01$

$0.00 \mathrm{E}+01$

$0.00 \mathrm{E}+01$

$0.00 \mathrm{E}+01$

$0.00 \mathrm{E}+01$

$0.00 \mathrm{E}+01$

$0.00 \mathrm{E}+01$

1.01E-02

$0.00 \mathrm{E}+01$

$1.99 \mathrm{E}-02$

$7.92 \mathrm{E}-01$

1.24E-01

1.31E-00

7.16E-06

$0.00 \mathrm{E}+01$

$0.00 \mathrm{E}+01$

$5.20 \mathrm{E}-04$

2.23E-00 
Tank Anal. Un

TX112 PO4

TX112 S

TX112 Si

TX112 504

TX112 Sr

TX112 TOC

TX112 U

TX112 $\mathrm{Zr}$

TX112 Ac227

TX112 Am241

TX112 Am243

TX112 Ba137

TX112 C14

TX112 Cd113

TX112 Cm242

TX112 Cm243

TX112 Cm244

TX112 Co60

TX112 Cs134

TX112 Cs137

TX112 Eu152

TX112 Eu154

TX112 Eu155

TX112 I129

TX112 Nb93

TX112 Ni59

TX112 Ni63

TX112 Np237

TX112 Pa231

TX112 Pu238

TX112 Pu239

TX112 Pu240

TX112 Pu241

TX112 Pu242

TX112 Ra226

TX112 Ra228

TX112 Ru106

TX112 Sb125

TX112 Se79

TX112 Sm151

TX112 Sr89/90

TX112 Te99

TX112 Th229

TX112 Th232

TX112 Tin126

TX112 Tritium

TX112 U232

TX112 v233

TX112 U234

TX112 U235

TX112 U236

TX112 U238

TX112 Y90

TX112 Zr93

TX113 Al

$\mathrm{TX} 113 \mathrm{Bi}$

TX113 Ca

$\mathrm{TX} 113 \mathrm{CI}$

$\mathrm{TX}_{113} \mathrm{CO} 3$

TX113 Cr

TX113 F

TX113 Fe

TX113 Hg

TX113 K

TX113 La

TX113 Mn

TX113 Na

TXI13 Ni
May $\frac{B 8}{98}$

$1.64 \mathrm{E}+05$
$6.76 \mathrm{E}+04$

$8.84 \mathrm{E}+02$

$2.03 E+05$

$2.90 \mathrm{E}+01$

$3.70 \mathrm{E}+04$

$1.66 \mathrm{E}+03$

$7.10 \mathrm{E}+01$

$1.68 \mathrm{E}-03$

$9.76 \mathrm{E}+01$

$3.29 \mathrm{E}-03$

$4.24 \mathrm{E}+05$

$5.21 \mathrm{E}+01$

$1.36 \mathrm{E}+02$

$2.27 \mathrm{E}-01$

2.11E-02

2.17E-01

$5.71 \mathrm{E}+01$

5.30E-00

$4.48 \mathrm{E}+05$

$5.88 \mathrm{E}-00$

$9.20 \mathrm{E}+02$

$3.49 \mathrm{E}+02$

$7.17 \mathrm{E}-01$

$1.91 \mathrm{E}+01$

4.06E-00

$3.97 \mathrm{E}+02$

$1.34 \mathrm{E}-00$

$7.42 \mathrm{E}-03$

$2.19 \mathrm{E}-00$

$8.34 \mathrm{E}+01$

$1.34 \mathrm{E}+01$

$1.43 E+02$

$7.79 \mathrm{E}-04$

2.58E-04

4.22E-01

1.02E-02

$2.43 \mathrm{E}+02$

5.37E-00

$1.89 \mathrm{E}+04$

$1.74 \mathrm{E}+05$

$3.72 \mathrm{E}+02$

$9.78 \mathrm{E}-03$

2.59E-02

8.11E-00

$3.65 \mathrm{E}+02$

$2.11 \mathrm{E}-00$

8.09E-00

2.23E-00

$9.16 \mathrm{E}-02$

6.06E-02

2.61E-00

$1.74 \mathrm{E}+05$

$2.64 \mathrm{E}+01$

$9.09 \mathrm{E}+04$

$1.52 \mathrm{E}+04$

$1.88 \mathrm{E}+03$

$1.49 \mathrm{E}+04$

$1.55 \mathrm{E}+05$

$4.19 \mathrm{E}+03$

$1.49 \mathrm{E}+04$

$7.53 \mathrm{E}+03$

$1.21 \mathrm{E}+01$

$4.79 \mathrm{E}+03$

$\quad 1.03 E+02$

$7.80 \mathrm{E}+02$

$6.69 \mathrm{E}+05$

$3.80 \mathrm{E}+02$
Sept98

$5.35 \mathrm{E}+04$

NA

$8.84 \mathrm{E}+02$

$6.76 E+04$

4.17E-00

$3.70 \mathrm{E}+04$

$1.66 E+03$

$7.10 \mathrm{E}+01$

1.68E-03

$9.76 \mathrm{E}+01$

3.29E-03

4.24E+05

$5.21 \mathrm{E}+01$

$1.36 \mathrm{E}+02$

2.27E-01

2.11E-02

2.17E-01

5.71E+01

$5.30 \mathrm{E}-00$

$4.48 \mathrm{E}+05$

$5.88 \mathrm{E}-00$

$9.20 \mathrm{E}+02$

$3.49 \mathrm{E}+02$

$7.17 \mathrm{E}-01$

$1.91 \mathrm{E}+01$

4.06E-00

$3.97 E+02$

$1.34 \mathrm{E}-00$

$7.42 \mathrm{E}-03$

2.19E-00

8.34E+01

$1.34 \mathrm{E}+01$

$1.43 E+02$

7.79E-04

2.58E-04

$4.22 \mathrm{E}-01$

1.02E-02

$2.43 E+02$

5.37E- 90

$1.89 \mathrm{E}+04$

$1.74 \mathrm{E}+05$

$3.72 \mathrm{E}+02$

9.78E-03

2.59E-02

8.11E-00

$3.65 E+02$

5.71E-01

2.19E-00

6.03E-01

2.48E-02

1.64E-02

5.54E-01

$1.74 \mathrm{E}+05$

$2.64 \mathrm{E}+01$

$9.09 E+04$

$1.52 \mathrm{E}+04$

$1.88 \mathrm{E}+03$

$1.49 \mathrm{E}+04$

$1.55 \mathrm{E}+05$

$4.19 E+03$

$1.49 \mathrm{E}+04$

$1.38 \mathrm{E}+04$

$5.83 \mathrm{E}+01$

$4.79 \mathrm{E}+03$

$0.00 \mathrm{E}+01$

$7.80 \mathrm{E}+02$

$6.69 \mathrm{E}+05$

$3.80 \mathrm{E}+02$

1\%-ile

$10 \%$-ile

50\%-ile

$0.00 \mathrm{E}+01$

2.38E-00

$6.75 E+04$

$2.60 \mathrm{E}+04$

$8.51 \mathrm{E}+03$

$0.00 \mathrm{E}+01$

$0.00 E+01$

$8.00 \mathrm{E}+04$

$6.09 \mathrm{E}+02$

$0.00 \mathrm{E}+01$

4.13E-00

$0.00 \mathrm{E}+01$

$2.09 \mathrm{E}+04$

$0.00 E+01$

$3.70 \mathrm{E}+03$

$3.03 E+04$

$0.00 \mathrm{E}+01$

$0.00 \mathrm{E}+01$

$1.06 \mathrm{E}+04$

$0.00 \mathrm{E}+01$

$0.00 \mathrm{E}+01$

$1.68 \mathrm{E}-03$

$0.00 \mathrm{E}+01 \quad 2.01 \mathrm{E}+02$

$0.00 \mathrm{E}+01 \quad 3.29 \mathrm{E}-03$

$0.00 \mathrm{E}+01$

$0.00 \mathrm{E}+01$

$0.00 E+01$

$4.24 E+05$

$0.00 \mathrm{E}+01$

$0.00 \mathrm{E}+01$

$5.21 \mathrm{E}+01$

$0.00 \mathrm{E}+01 \quad 0.00 \mathrm{E}+01 \quad 1.36 \mathrm{E}+02$

$0.00 \mathrm{E}+01$

$0.00 \mathrm{E}+01$

$0.00 \mathrm{E}+0$

2.27E-01

$0.00 \mathrm{E}+01 \quad 2.11 \mathrm{E}-02$

$0.00 \mathrm{E}+01$

2.17E-01

$0.00 \mathrm{E}+01$

$0.00 \mathrm{E}+0 \mathrm{I}$

$0.00 \mathrm{E}+01$

$0.00 \mathrm{E}+01$

$0.00 \mathrm{E}+01$

$0.00 \mathrm{E}+0$

$9.88 \mathrm{E}+01$

$3.34 \mathrm{E}+03$

$0.00 \mathrm{E}+01$

$5.30 \mathrm{E}-00$

$5.21 E+05$

$0.00 \mathrm{E}+01$

$0.00 \mathrm{E}+01$

$0.00 \mathrm{E}+01$

$0.00 \mathrm{E}+01$

$0.00 \mathrm{E}+01$

$0.00 \mathrm{E}+01$

$0.00 \mathrm{E}+01$

$0.00 \mathrm{E}+01$

$0.00 \mathrm{E}+01$

$0.00 \mathrm{E}+01$

$0.00 \mathrm{E}+01$

$0.00 \mathrm{E}+01$

$0.00 \mathrm{E}+01$

$0.00 \mathrm{E}+01$

$0.00 \mathrm{E}+01$

$0.00 \mathrm{E}+01$

$0.00 \mathrm{E}+01$

$0.00 \mathrm{E}+01$

$0.00 \mathrm{E}+01$

$0.00 \mathrm{E}+01$

$0.00 \mathrm{E}+01$

$0.00 \mathrm{E}+01$

$0.00 \mathrm{E}+01$

$0.00 \mathrm{E}+01$

$7.83 \mathrm{E}+01$

$0.00 \mathrm{E}+01$

$0.00 \mathrm{E}+01$

$0.00 \mathrm{E}+01$

$0.00 \mathrm{E}+01$

$0.00 \mathrm{E}+01$

$0.00 \mathrm{E}+01$

$0.00 \mathrm{E}+01$

$0.00 \mathrm{E}+01$ 
Tank Anal. Un

\begin{tabular}{|c|c|}
\hline $\begin{array}{l}\text { TX113 } \\
\text { TX113 }\end{array}$ & $\begin{array}{l}\text { NO2 } \\
\text { NO3 }\end{array}$ \\
\hline $\begin{array}{l}\text { TX113 } \\
\text { TX113 }\end{array}$ & $\begin{array}{l}\mathbf{P} \\
\mathbf{P b}\end{array}$ \\
\hline TX113 & PO4 \\
\hline TX113 & 0 \\
\hline TX113 & $\mathbf{S i}$ \\
\hline TX113 & SO4 \\
\hline TX113 & Sr \\
\hline TX113 & TOC \\
\hline TX113 & \\
\hline TX113 & Ac227 \\
\hline TX113 & $\operatorname{Am} 241$ \\
\hline TX113 & Am243 \\
\hline $\begin{array}{l}\text { TX113 } \\
\text { TX113 }\end{array}$ & $\begin{array}{l}\text { Ba137 } \\
\text { C14 }\end{array}$ \\
\hline
\end{tabular}

TX113 C14

TX113 Cd113

TX113 Cm242

TX113 Cm243

TX113 Cm244

TX113 Co60

TX113 Cs134

TX113 Cs137

TX113 Eu152

TX113 Eu154

TX113 Eu155

TX113 1129

TX113 Nb93

TX113 Ni59

TX113 Ni63

TX113 Np237

TX113 Pa231

TX113 Pu238

TX113 Pu239

TX113 Pu240

TX113 Pu241

TX113 Pu242

TX113 Ra226

TX113 Ra228

TX113 Ru106

TX113 Sb125

TX113 Se79

TX113 Sml51

TX113 Sr89/90

TX113 Te99

TX113 Th229

TX113 Th232

TX113 Tin126

TX113 Tritium

TX113 U232

TX113 U233

TX113 U234

TX113 U235

TX113 U236

TX113 U238

TX113 Y90

TX113 Zr93

TX114 Al

TX114 Bi

TX114 Ca

TX114 Cl

TX114 CO3

TX114 Cr

TX114 F

TX114 Fe

TX114 Hg

TX114 K

\section{May $\frac{B B}{98}$ \\ $9 \overline{15 \mathrm{E}+04}$}

$9.02 \mathrm{E}+05$

$1.04 \mathrm{E}+05$

$1.00 \mathrm{E}+03$

$3.22 \mathrm{E}+05$

$5.11 \mathrm{E}+04$

$6.55 \mathrm{E}+03$

$1.53 \mathrm{E}+05$

$4.90 \mathrm{E}+02$

$2.59 \mathrm{E}+04$

$1.93 \mathrm{E}+03$

$1.25 \mathrm{E}+02$

1.14E-03

$6.77 \mathrm{E}+01$

2.33E-03

$2.92 \mathrm{E}+05$

$3.42 \mathrm{E}+01$

$9.32 \mathrm{E}+01$

1.73E-01

1.60E-02

1.52E-01

$3.80 \mathrm{E}+01$

$3.80 \mathrm{E}-00$

$3.09 \mathrm{E}+05$

4.50E-00

$6.35 \mathrm{E}+02$

$2.69 \mathrm{E}+02$

4.69E-01

$1.31 \mathrm{E}+01$

2.60E-00

2.54E+02

8.78E-01

4.99E-03

$1.83 \mathrm{E}-00$

$9.25 \mathrm{E}+01$

$1.28 \mathrm{E}+01$

$1.14 \mathrm{E}+02$

6.16E-04

1.75E-04

2.68E-01

7.17E-03

$1.64 \mathrm{E}+02$

3.68E-00

$1.29 \mathrm{E}+04$

$1.33 \mathrm{E}+05$

$2.43 E+02$

6.21E-03

$1.65 \mathrm{E}-02$

Ci 5.55E-00

$2.42 \mathrm{E}+02$

1.34E-00

5.14E-00

$1.12 \mathrm{E}+01$

4.91E-01

1.39E-01

Ci 1.16E+01

$1.33 E+05$

Ci $1.80 \mathrm{E}+01$

$8.49 \mathrm{E}+04$

kg $\quad 6.95 \mathrm{E}+02$

$1.04 \mathrm{E}+03$

1.61E+04

$1.74 E+05$

4.04E+03

$9.89 \mathrm{E}+03$

kg 2.64E+03

g.00E-00

kg $\quad 5.28 \mathrm{E}+03$
Sept98

$9.15 \mathrm{E}+04$

$9.02 \mathrm{E}+05$

NA

$9.26 \mathrm{E}+02$

$1.05 E+05$

NA

6.55E+03

$5.11 \mathrm{E}+04$

$4.90 \mathrm{E}+02$

$2.59 \mathrm{E}+04$

$1.93 \mathrm{E}+03$

$1.25 \mathrm{E}+02$

1.14E-03

$6.77 \mathrm{E}+01$

2.33E-03

$2.92 \mathrm{E}+05$

$3.42 \mathrm{E}+01$

$9.32 \mathrm{E}+01$

1.73E-01

$1.60 \mathrm{E}-02$

1.52E-01

$3.80 \mathrm{E}+01$

2.63E-00

$3.09 E+05$

4.50E-00

$6.35 \mathrm{E}+02$

$2.69 \mathrm{E}+02$

4.69E-01

$1.31 \mathrm{E}+01$

2.60E-00

$2.54 \mathrm{E}+02$

8.78E-01

4.99E-03

$1.83 \mathrm{E}-00$

$9.25 E+01$

$1.28 \mathrm{E}+01$

$1.14 \mathrm{E}+02$

6.16E-04

1.75E-04

2.68E-01

7.17E-03

$1.64 \mathrm{E}+02$

3.68E-00

$1.29 \mathrm{E}+04$

$1.33 \mathrm{E}+05$

$2.43 \mathrm{E}+02$

6.21E-03

1.65E-02

5.55E-00

2.42E+02

7.68E-02

2.94E-01

$6.42 \mathrm{E}-01$

2.81E-02

7.98E-03

6.44E-01

$1.33 \mathrm{E}+05$

$1.80 \mathrm{E}+01$

$8.49 \mathrm{E}+04$

$5.94 \mathrm{E}+02$

$1.04 \mathrm{E}+03$

$1.61 \mathrm{E}+04$

$1.74 E+05$

$4.04 E+03$

$9.89 \mathrm{E}+03$

$2.64 \mathrm{E}+03$

1.27E-00

$5.28 \mathrm{E}+03$ 1\%-ile

$10 \%$-ile

50\%-ile

$2.97 \mathrm{E}+03$

$3.05 \mathrm{E}+04$

$6.36 \mathrm{E}+03$

$1.63 E+01$

$6.49 \mathrm{E}+03$

$7.03 \mathrm{E}+02$

$1.84 \mathrm{E}+03$

$2.90 \mathrm{E}+03$

$3.61 \mathrm{E}+01$

$2.84 \mathrm{E}+02$

$1.56 \mathrm{E}+02$

$1.18 \mathrm{E}-00$

$0.00 \mathrm{E}+01$

8.25E-01

1.77E-05

$0.00 \mathrm{E}+01$

$0.00 \mathrm{E}+01$

$0.00 \mathrm{E}+01$

$1.32 \mathrm{E}-03$

1.22E-04

$1.16 \mathrm{E}-03$

$0.00 \mathrm{E}+01$

$0.00 \mathrm{E}+01$

$6.06 \mathrm{E}+03$

$0.00 \mathrm{E}+01$

$0.00 \mathrm{E}+01$

$0.00 \mathrm{E}+01$

$0.00 \mathrm{E}+01$

$0.00 \mathrm{E}+01$

$0.00 \mathrm{E}+01$

$0.00 \mathrm{E}+01$

6.68E-03

$0.00 \mathrm{E}+01$

1.39E-02

$7.03 \mathrm{E}-01$

9.73E-02

8.67E-01

4.68E-06

$0.00 \mathrm{E}+01$

$0.00 \mathrm{E}+01$

$0.00 \mathrm{E}+01$

$0.00 \mathrm{E}+01$

$0.00 E+01$

$0.00 \mathrm{E}+01$

$2.35 \mathrm{E}+03$

$0.00 \mathrm{E}+01$

$0.00 \mathrm{E}+01$

$0.00 \mathrm{E}+01$

$0.00 \mathrm{E}+01$

$1.13 \mathrm{E}+02$

$0.00 \mathrm{E}+01$

$0.00 \mathrm{E}+01$

$0.00 \mathrm{E}+01$

$0.00 \mathrm{E}+01$

$0.00 \mathrm{E}+01$

$0.00 \mathrm{E}+01$

$0.00 \mathrm{E}+01$

$0.00 \mathrm{E}+01$

$4.34 \mathrm{E}+03$

$5.79 \mathrm{E}+01$

3.13E-00

5.72E-00

$3.29 \mathrm{E}+01$

$3.00 \mathrm{E}-00$

$5.77 \mathrm{E}-00$

$4.23 \mathrm{E}+01$

$0.00 \mathrm{E}+01$

$3.82 \mathrm{E}-00$

$1.73 \mathrm{E}+04$

$1.66 \mathrm{E}+05$

$1.48 \mathrm{E}+04$

$1.55 \mathrm{E}+02$

$1.77 \mathrm{E}+04$

$3.89 \mathrm{E}+03$

$4.86 \mathrm{E}+03$

$9.31 E+03$

$1.30 \mathrm{E}+02$

$3.03 \mathrm{E}+03$

$1.14 \mathrm{E}+03$

$8.48 \mathrm{E}-00$

$0.00 \mathrm{E}+01$

$2.02 \mathrm{E}+01$

$1.03 \mathrm{E}-04$

$8.14 E+02$

$0.00 \mathrm{E}+01$

$0.00 \mathrm{E}+01$

7.64E-03

$7.06 \mathrm{E}-04$

6.71E-03

$1.81 \mathrm{E}-00$

1.06E-02

$4.62 \mathrm{E}+04$

$0.00 \mathrm{E}+01$ 
Tank Anal. Un

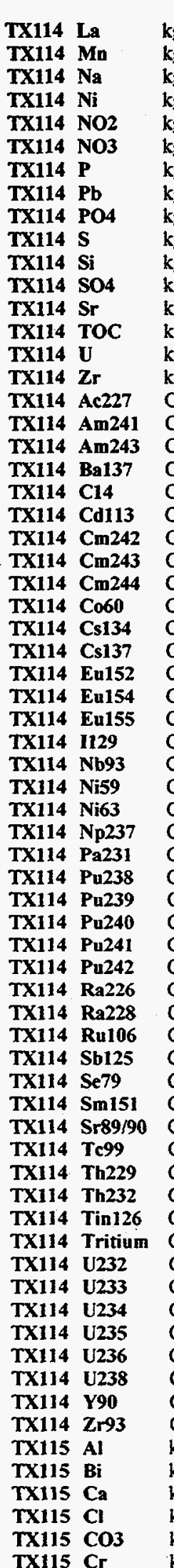

May $\frac{B B}{98}$ $\begin{array}{ll}\mathrm{kg} & \mathbf{2 . 1 0 \mathrm { E } - 0 4} \\ \mathrm{kg} & 9.85 \mathrm{E}+02\end{array}$ kg 7.36E+05 kg 2.88E+02 $\mathrm{kg} \quad 9.47 \mathrm{E}+04$ $\mathrm{kg} \quad 1.08 \mathrm{E}+06$ 7.92E+04 kg 3.35E+02 g 2.45E+05 kg 5.49E+04 kg $\quad 1.15 \mathrm{E}+03$ g $1.65 \mathrm{E}+05$ 3.70E+01 $2.80 \mathrm{E}+04$ $1.70 \mathrm{E}+03$ g.90E+01 $1.29 \mathrm{E}-03$ $7.65 \mathrm{E}+01$ Ci 2.64E-03 i $3.86 \mathrm{E}+05$ Ci $3.86 \mathrm{E}+01$ 1.05E+02 $1.96 \mathrm{E}-01$ 1.81E-02 1.72E-01 $4.30 \mathrm{E}+01$ $6.40 \mathrm{E}-00$ $4.08 \mathrm{E}+05$

Ci 5.10E-00 Ci $7.19 \mathrm{E}+02$

Ci $3.04 \mathrm{E}+02$ Ci 5.30E-01

Ci $1.48 \mathrm{E}+01$

Ci 3.10E-00

Ci $3.02 E+02$

Ci 9.92E-01

5.65E-03

$\mathrm{Ci} \quad 1.81 \mathrm{E}-00$

$\mathrm{Ci} \quad 7.54 \mathrm{E}+01$

Ci $1.14 \mathrm{E}+01$

Ci 1.18E+02

Ci 6.42E-04

$\mathrm{Ci} \quad 2.00 \mathrm{E}-04$

Ci 3.02E-01

Ci 8.12E-03

Ci $1.85 \mathrm{E}+02$

Ci $4.16 E-00$

Ci 1.46E+04 $1.42 \mathrm{E}+05$ $2.75 \mathrm{E}+02$ 7.01E-03 $1.86 \mathrm{E}-02$ $6.28 \mathrm{E}-00$ $2.74 E+02$

\section{Ci 1.51E-00} $5.80 \mathrm{E}-00$

Ci 2.69E-00

$\mathrm{Ci}$ 1.14E-01

Ci 5.42E-02

Ci 2.98E-00

Ci 1.42E+05

Ci $2.04 \mathrm{E}+01$

$\mathrm{kg} \quad 1.01 \mathrm{E}+05$

kg 2.42E+02

kg $\quad 1.09 \mathrm{E}+03$

kg $\quad 1.91 \mathrm{E}+04$

kg $\quad 1.98 \mathrm{E}+05$

$\mathrm{kg} \quad 4.80 \mathrm{E}+03$
Sept98

$0.00 \mathrm{E}+01$

$9.85 \mathrm{E}+02$

$7.36 \mathrm{E}+05$

$2.88 \mathrm{E}+02$

$9.47 \mathrm{E}+04$

$1.08 \mathrm{E}+06$

NA

3.35E+02

$8.00 \mathrm{E}+04$

NA

1.15E+03

$5.49 \mathrm{E}+04$

$1.38 \mathrm{E}+01$

$2.80 \mathrm{E}+04$

$1.70 \mathrm{E}+03$

$5.90 \mathrm{E}+01$

$1.29 \mathrm{E}-03$

$7.65 \mathrm{E}+01$

2.64E-03

$3.86 \mathrm{E}+05$

$3.86 \mathrm{E}+01$

$1.05 E+02$

1.96E-01

1.81E-02

1.72E-01

$4.30 \mathrm{E}+01$

6.40E-00

$4.08 \mathrm{E}+05$

5.10E-00

$7.19 \mathrm{E}+02$

$3.04 \mathrm{E}+02$

$5.30 \mathrm{E}-01$

$1.48 \mathrm{E}+01$

3.10E-00

$3.02 \mathrm{E}+02$

$9.92 \mathrm{E}-01$

5.65E-03

$1.81 \mathrm{E}-00$

$7.54 \mathrm{E}+01$

1.14E +01

$1.18 \mathrm{E}+02$

$6.42 \mathrm{E}-04$

2.00E-04

3.02E-01

8.12E-03

$1.85 \mathrm{E}+02$

4.16E-00

$1.46 \mathrm{E}+04$

$1.42 \mathrm{E}+05$

$2.75 \mathrm{E}+02$

7.01E-03

$1.86 \mathrm{E}-02$

6.28E-00

$2.74 \mathrm{E}+02$

3.34E-01

$1.28 \mathrm{E}-00$

5.93E-01

2.51E-02

1.19E-02

5.68E-01

$1.42 \mathrm{E}+05$

$2.04 \mathrm{E}+01$

$1.01 \mathrm{E}+05$

$1.21 \mathrm{E}+02$

$1.09 \mathrm{E}+03$

$1.91 E+04$

$1.98 \mathrm{E}+05$

$4.80 \mathrm{E}+03$ 1\%-ile

6.49E-03

5.84E-02

$1.95 \mathrm{E}+05$

3.43E-02

$7.13 \mathrm{E}+01$

$6.64 \mathrm{E}+02$

$7.25 \mathrm{E}+01$

$2.36 \mathrm{E}-01$

$6.78 \mathrm{E}+01$

$1.03 \mathrm{E}+01$

$1.81 \mathrm{E}+01$

$4.05 \mathrm{E}+01$

2.42E-01

$4.80 \mathrm{E}-00$

2.22E-00

1.32E-02

$0.00 \mathrm{E}+01$

2.45E-02

$0.00 \mathrm{E}+01$

$0.00 \mathrm{E}+01$

$0.00 \mathrm{E}+01$

$0.00 \mathrm{E}+01$

$0.00 \mathrm{E}+01$

$0.00 \mathrm{E}+01$

$0.00 \mathrm{E}+01$

$0.00 \mathrm{E}+01$

$0.00 \mathrm{E}+01$

$5.96 \mathrm{E}+01$

$0.00 \mathrm{E}+01$

$0.00 \mathrm{E}+01$

$0.00 \mathrm{E}+01$

$0.00 \mathrm{E}+01$

$0.00 \mathrm{E}+01$

$0.00 \mathrm{E}+01$

$0.00 \mathrm{E}+01$

$0.00 \mathrm{E}+01$

$0.00 \mathrm{E}+01$

$0.00 \mathrm{E}+01$

$0.00 \mathrm{E}+01$

$0.00 \mathrm{E}+01$

$0.00 \mathrm{E}+01$

$0.00 \mathrm{E}+01$

$0.00 \mathrm{E}+01$

$0.00 \mathrm{E}+01$

$0.00 \mathrm{E}+01$

$0.00 \mathrm{E}+0$

$0.00 \mathrm{E}+01$

$0.00 \mathrm{E}+0$

2.47E+01

$0.00 \mathrm{E}+01$

$0.00 \mathrm{E}+01$

$0.00 \mathrm{E}+01$

$0.00 \mathrm{E}+01$

$5.82 \mathrm{E}+01$

$0.00 \mathrm{E}+01$

$0.00 \mathrm{E}+01$

$0.00 \mathrm{E}+01$

$0.00 \mathrm{E}+01$

$0.00 \mathrm{E}+01$

$0.00 \mathrm{E}+01$

$0.00 \mathrm{E}+01$

$0.00 \mathrm{E}+01$

$4.10 \mathrm{E}+03$

$1.25 \mathrm{E}+02$

$1.65 \mathrm{E}+01$

$1.87 \mathrm{E}+01$

$1.25 \mathrm{E}+02$

$1.57 \mathrm{E}+01$
$10 \%$-ile

4.41E-02.

$5.46 \mathrm{E}-01$

$4.14 \mathrm{E}+05$

2.31E-01

$7.05 \mathrm{E}+02$

$3.33 \mathrm{E}+04$

$2.71 \mathrm{E}+02$

2.50E-00

$3.15 \mathrm{E}+02$

$6.15 \mathrm{E}+01$

$8.56 \mathrm{E} \div 01$

$1.79 E+02$

2.14E-00

$3.24 \mathrm{E}+03$

$1.57 \mathrm{E}+01$

1.29E-01

$0.00 \mathrm{E}+01$

$3.06 \mathrm{E}-01$

7.67E-07

$0.00 \mathrm{E}+01$

$0.00 \mathrm{E}+01$

$0.00 \mathrm{E}+01$

$5.70 \mathrm{E}-05$

$5.26 \mathrm{E}-06$

5.00E-05

2.83E-02

$0.00 \mathrm{E}+01$

$3.06 \mathrm{E}+03$

$0.00 \mathrm{E}+01$

$0.00 \mathrm{E}+01$

$0.00 \mathrm{E}+01$

$0.00 \mathrm{E}+01$

$0.00 \mathrm{E}+01$

$0.00 E+01$

$0.00 \mathrm{E}+01$

2.88E-04

$0.00 \mathrm{E}+01$

$5.26 \mathrm{E}-04$

2.19E-02

3.31E-03

3.43E-02

$1.87 \mathrm{E}-07$

$0.00 \mathrm{E}+01$

$0.00 \mathrm{E}+01$

$0.00 E+01$

$2.97 \mathrm{E}+01$

$0.00 \mathrm{E}+01$

$0.00 \mathrm{E}+01$

$1.28 \mathrm{E}+03$

$0.00 \mathrm{E}+01$

$0.00 \mathrm{E}+01$

$0.00 \mathrm{E}+01$

$0.00 \mathrm{E}+01$

$1.55 \mathrm{E}+02$

$0.00 \mathrm{E}+01$

$0.00 \mathrm{E}+01$

$0.00 \mathrm{E}+01$

$0.00 \mathrm{E}+01$

$0.00 \mathrm{E}+01$

$0.00 \mathrm{E}+01$

$0.00 \mathrm{E}+01$

$0.00 \mathrm{E}+01$

4.12E+04

$3.97 \mathrm{E}+02$

1. $52 \mathrm{E}+02$

$8.04 \mathrm{E}+01$

$7.47 \mathrm{E}+02$

$7.55 \mathrm{E}+01$

50\%-ile

$2.86 \mathrm{E}+02$

$2.09 \mathrm{E}+03$

$6.98 \mathrm{E}+05$

$1.06 \mathrm{E}+03$

$1.08 \mathrm{E}+05$

$6.85 \mathrm{E}+05$

$2.06 \mathrm{E}+04$

$4.43 \mathrm{E}+02$

5.62E+04 
Tank Anal. Un

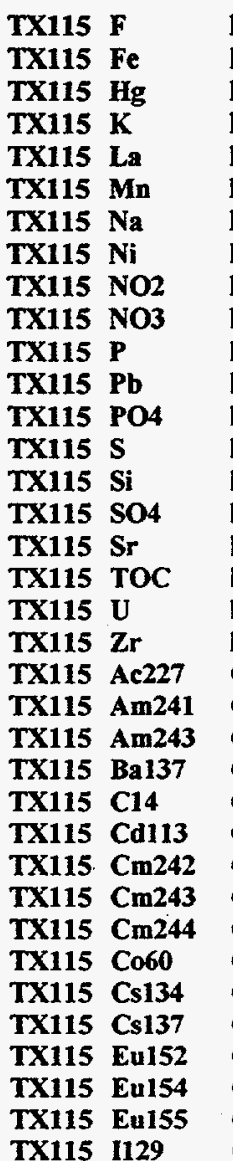

TX115 I129

TX115 Nb93

TX115 Ni59

TX115 Ni63

TX115 Np237

TX115 Pa231

TX115 Pu238

TX115 Pu239

TX115 Pu240

TX115 Pu241

TX115 Pu242

TX115 Ra226

TX115 Ra228

TX115 Ru106

TX115 Sb125

TX115 Se79

TX115 Sm151

TX115 Sr89/90

TX115 Te99

TX115 Th229

TX115 Th232

TX115 Tin126

TX115 Tritium

TX115 U232

TX115 U233

TX115 U234

TX115 U235

TX115 U236

TX115 U238

TX115 Y90

TX115 Zr93

TX116 Al

TX116 Bi

\section{May $\frac{B B}{98}$}

$7.03 \mathrm{E}+03$

$3.48 \mathrm{E}+03$

5.18E-00

$6.21 \mathrm{E}+03$

$1.25 \mathrm{E}+02$

$8.67 \mathrm{E}+02$

$7.70 \mathrm{E}+05$

3.33E +02

$1.12 \mathrm{E}+05$

$1.12 \mathrm{E}+06$

$9.04 \mathrm{E}+03$

$4.99 \mathrm{E}+02$

$2.80 \mathrm{E}+04$

$1.99 \mathrm{E}+04$

$6.11 \mathrm{E}+02$

$5.96 \mathrm{E}+04$

$2.40 \mathrm{E}+01$

3.37E+04

$6.25 \mathrm{E}+03$

$6.30 \mathrm{E}+01$

$1.51 \mathrm{E}-03$

8.82E +01

2.98E-03

$3.88 E+05$

$4.70 \mathrm{E}+01$

$1.23 \mathrm{E}+02$

2.06E-01

1.91E-02

$1.97 \mathrm{E}-01$

$5.15 E+01$

3.84E-00

4.07E+05

5.34E-00

$8.31 \mathrm{E}+02$

$3.17 \mathrm{E}+02$

6.46E-01

$1.73 \mathrm{E}+01$

3.58E-00

$3.51 \mathrm{E}+02$

$1.21 \mathrm{E}-00$

6.68E-03

1.95E-00

$7.04 \mathrm{E}+01$

1.17E +01

$1.29 \mathrm{E}+02$

7.00E-04

2.31E-04

$3.80 \mathrm{E}-01$

$9.24 \mathrm{E}-03$

$2.19 \mathrm{E}+02$

4.85E-00

$1.71 E+04$

$1.57 \mathrm{E}+05$

$3.35 \mathrm{E}+02$

8.81E-03

2.33E-02

7.32E-00

$3.30 E+02$

$1.90 \mathrm{E}-00$

$7.28 \mathrm{E}-00$

$1.56 \mathrm{E}-00$

$\mathrm{Ci} \quad$ 6.25E-02

Ci 5.04E-02

Ci 1.89E-00

Ci $1.57 \mathrm{E}+05$

Ci $2.38 E+01$

kg $\quad 6.94 \mathrm{E}+04$

kg $\quad 9.36 \mathrm{E}+02$
Sept98

$7.03 \mathrm{E}+03$

$3.61 \mathrm{E}+03$

$0.00 \mathrm{E}+01$

$6.21 \mathrm{E}+03$

$0.00 \mathrm{E}+01$

$8.67 \mathrm{E}+02$

$7.70 \mathrm{E}+05$

$3.33 \mathrm{E}+02$

1.12E +05

1.12E+06

$$
\text { NA }
$$

$4.20 \mathrm{E}+02$

$2.80 \mathrm{E}+04$

NA

6.11E+02

$5.96 \mathrm{E}+04$

$3.76 \mathrm{E}-00$

3.37E+04

$4.15 \mathrm{E}+03$

$6.30 \mathrm{E}+01$

1.51E-03

8.82E+01

2.98E-03

$3.88 \mathrm{E}+05$

$4.70 \mathrm{E}+01$

$1.23 \mathrm{E}+02$

2.06E-01

1.91E-02

$1.97 \mathrm{E}-01$

$5.15 \mathrm{E}+01$

3.84E-00

$4.07 \mathrm{E}+05$

5.34E-00

8.31E+02

$3.17 \mathrm{E}+02$

$6.46 \mathrm{E}-01$

$1.73 \mathrm{E}+01$

3.58E-00

$3.51 \mathrm{E}+02$

1.21E-00

6.68E-03

1.95E-00

$7.04 \mathrm{E}+01$

$1.17 E+01$

$1.29 \mathrm{E}+02$

7.00E-04

2.31E-04

$3.80 \mathrm{E}-01$

9.24E-03

$2.19 \mathrm{E}+02$

4.85E-00

$1.71 \mathrm{E}+04$

$1.57 \mathrm{E}+05$

$3.35 \mathrm{E}+02$

$8.81 \mathrm{E}-03$

2.33E-02

7.32E-00

$3.30 \mathrm{E}+02$

$1.90 \mathrm{E}-00$

$7.28 \mathrm{E}-00$

$1.56 \mathrm{E}-00$

$6.25 \mathrm{E}-02$

5.04E-02

1.38E-00

$1.57 \mathrm{E}+05$

2.38E+01

$6.94 E+04$

$8.71 E+02$ 1\%-ile

$10 \%$-ile

50\%-ile

90\%-ile

$1.42 \mathrm{E}+01 \quad 2.03 \mathrm{E}+02$

$1.51 \mathrm{E}+02 \quad 4.44 \mathrm{E}+02$

$0.00 \mathrm{E}+01 \quad 0.00 \mathrm{E}+01$

6.43E-00 2.46E+03

$2.56 \mathrm{E}-02 \quad 1.36 \mathrm{E}-01$

$2.83 \mathrm{E}-01 \quad 1.60 \mathrm{E}-00$

$2.20 \mathrm{E}+05$

7.97E-01

$1.60 \mathrm{E}+02$

$1.87 \mathrm{E}+03$

$1.42 \mathrm{E}+02$

$1.01 \mathrm{E}-00$

$1.39 \mathrm{E}+02$

$3.84 \mathrm{E}+01$

4.12E+01

$1.08 \mathrm{E}+02$

9.06E-01

$1.80 \mathrm{E}+01$

2.73E-00

7.35E-02

$0.00 \mathrm{E}+01$

8.19E-02

$0.00 \mathrm{E}+01$

$0.00 \mathrm{E}+01$

$0.00 \mathrm{E}+01$

$0.00 \mathrm{E} \div 01$

$0.00 \mathrm{E}+01$

$0.00 \mathrm{E} \div 01$

$0.00 \mathrm{E}+01$

2.50E-05

$0.00 \mathrm{E}+01$

$1.84 E+02$

$0.00 \mathrm{E}+01$

$0.00 \mathrm{E}+01$

$0.00 \mathrm{E}+01$

$0.00 \mathrm{E}+01$

$0.00 \mathrm{E}+01$

$0.00 \mathrm{E}+01$

$0.00 \mathrm{E}+01$

$0.00 \mathrm{E}+01$

$0.00 \mathrm{E}+01$

$0.00 \mathrm{E}+01$

$0.00 \mathrm{E}+01$

$0.00 \mathrm{E}+01$

$0.00 \mathrm{E}+01$

$0.00 \mathrm{E}+01$

$0.00 \mathrm{E}+01$

$0.00 \mathrm{E}+01$

$0.00 \mathrm{E}+01$

$0.00 E+01$

$0.00 \mathrm{E}+01$

$0.00 \mathrm{E}+01$

$3.63 \mathrm{E}+01$

$0.00 \mathrm{E}+01$

$0.00 \mathrm{E}+01$

$0.00 \mathrm{E}+01$

$0.00 \mathrm{E}+01$

$7.51 \mathrm{E}+01$

$0.00 \mathrm{E}+01$

$0.00 \mathrm{E}+01$

$0.00 \mathrm{E}+01$

$0.00 \mathrm{E}+01$

$0.00 \mathrm{E}+0 \mathrm{I}$

$0.00 \mathrm{E}+01$

$0.00 \mathrm{E}+01$

$0.00 \mathrm{E}+01$

$1.45 \mathrm{E}+01$

$6.60 \mathrm{E}-02$

$4.39 \mathrm{E}+05$

$7.96 \mathrm{E}-00$

$2.25 \mathrm{E}+03$

$3.03 \mathrm{E}+04$

$7.11 \mathrm{E}+02$

6.61E-00

$8.89 \mathrm{E}+02$

$2.12 \mathrm{E}+02$

$2.32 \mathrm{E}+02$

$4.69 \mathrm{E}+02$

$5.26 \mathrm{E}-00$

$3.34 \mathrm{E}+03$

$3.43 \mathrm{E}+01$

$3.70 \mathrm{E}-01$

$0.00 \mathrm{E}+01$

7.05E-01

3.93E-06

$0.00 \mathrm{E}+01$

$0.00 \mathrm{E}+01$

$0.00 \mathrm{E}+01$

2.71E-04

2.52E-05

2.60E-04

$1.03 \mathrm{E}-01$

$0.00 \mathrm{E}+01$

$7.07 \mathrm{E}+03$

$0.00 \mathrm{E}+01$

$0.00 \mathrm{E}+01$

$0.00 \mathrm{E}+01$

$0.00 \mathrm{E}+01$

$0.00 E+01$

$0.00 \mathrm{E}+01$

$0.00 \mathrm{E}+01$

$1.59 \mathrm{E}-03$

$0.00 \mathrm{E}+01$

2.57E-03

9.27E-02

1.54E-02

1.70E-01

9.22E- 07

$0.00 \mathrm{E}+01$

$0.00 E+01$

$0.00 \mathrm{E}+01$ 
Tank Anal. Un

\begin{tabular}{ll|l} 
TX116 & Ca \\
TX116 & Cl \\
TX116 & CO3 \\
TX116 & Cr \\
TX116 & F \\
TX116 & Fe \\
TX116 & Hg \\
TX116 & K \\
TX116 & La \\
TX116 & Mn \\
TX116 & Na \\
TX116 & Ni \\
TX116 & NO2 \\
TX116 & NO3 \\
TX116 & P \\
TX116 & Pb \\
TX116 & PO4 \\
TX116 & S \\
TX116 & Si \\
TX116 & SO4 \\
TX116 & Sr \\
TX116 & TOC \\
TX116 & U \\
TX116 & Zr \\
TX116 & Ac227 \\
TX116 & Am241 \\
TX116 & Am243 \\
TX116 & Ba137 \\
TX116 & C14 \\
TX116 & Cd13
\end{tabular}

TX116 C14

TX116 Cd113

Tx116 Cm242

TX116 Cm243

TX116 Cm244

TX116 Co60

TX116 Cs134

TX116 Cs137

TX116 Eu152

TX116 Eu154

TX116 Eu155

TX116 I129

TX116 Nb93

TX116 Nis9

TX116 Ni63

TX116 Np237

TX116 Pa231

TX116 Pu238

TX116 Pu239

TX116 Pu240

TX116 Pu241

TX116 Pu242

TX116 Ra226

TX116 Ra228

TX116 Ru106

TX116 Sb125

TX116 Se79

TX116 Sm151

TX116 Sr89/90

TX116 Te99

TX116 Th229

TX116 Th232

TX116 Tin126

TX116 Tritium

TX116 U232

TX116 U233

TX116 U234

TX116 U235

TX116 U236

TX116 U238
May $\frac{B 8}{98}$

\section{kg 1.11E+03 \\ kg $\quad 1.11 \mathrm{E}+04$}

kg $1.60 \mathrm{E}+05$

$\mathrm{kg} \quad 9.66 \mathrm{E}+02$

kg 1.19E+04

kg 1.02E+04

kg 3.00E-00

kg $\quad 3.87 \mathrm{E}+03$

kg 1.00E-04

kg 1.73E+03

$\mathrm{kg} \quad 7.95 \mathrm{E}+05$

$\begin{array}{ll}\mathrm{kg} & 2.18 \mathrm{E}+02 \\ \mathrm{~kg}\end{array}$

$\begin{array}{ll}\mathrm{kg} & 1.40 \mathrm{E}+04 \\ \mathrm{~kg} & 1.72 \mathrm{E}+06\end{array}$

$\begin{array}{ll}\mathrm{kg} & 1.72 \mathrm{E}+06 \\ \mathrm{~kg} & 4.42 \mathrm{E}+04\end{array}$

kg $\quad 1.14 \mathrm{E}+03$

$\mathrm{kg} \quad 1.37 \mathrm{E}+05$

kg $\quad 9.94 \mathrm{E}+04$

$\mathrm{kg} \quad 4.28 \mathrm{E}+04$

kg $2.98 \mathrm{E}+05$

kg 4.60E+01

2.87E+03

kg $2.46 \mathrm{E}+03$

$5.80 \mathrm{E}+01$

7.06E-04

$2.77 E+01$

9.41E-04

$2.33 E+05$

$1.75 E+01$

$4.46 \mathrm{E}+01$

$7.04 \mathrm{E}-02$

6.35E-03

6.43E-02

Ci $1.87 \mathrm{E}+01$

$1.31 \mathrm{E}-00$

$2.47 E+05$

$1.93 \mathrm{E}-00$

$2.94 \mathrm{E}+02$

$1.17 \mathrm{E}+02$

2.41 E-01

6.42E-00

2.43E-00

$2.29 E+02$

4.58E-01

2.81 E-03

1.12E-00

$9.89 \mathrm{E}+01$

9.96E-00

$6.06 \mathrm{E}+01$

3.08E-04

1.11E-04

1.67E-01

3.03E-03

$7.85 E+01$

$1.79 \mathrm{E}-00$

$6.32 E+03$

$6.60 \mathrm{E}+04$

$1.25 E+02$

$\mathrm{Ci} \quad 3.88 \mathrm{E}-03$

Ci 1.03E-02

Ci 2.70E-00

$1.19 E+02$

8.29E-01

3.17E- 00

Ci 7.40E- 00

Ci 3.26E-01

Ci 8.34E-02

Ci 7.69E-00
Sept98

$\frac{5 e p t 98}{1.11 E+03}$

1.11E+04

$1.60 \mathrm{E}+05$

$9.66 \mathrm{E}+02$

$1.19 \mathrm{E}+04$

$1.02 \mathrm{E}+04$

$0.00 \mathrm{E}+01$

$3.87 \mathrm{E}+03$

$0.00 \mathrm{E}+01$

$1.73 \mathrm{E}+03$

$7.95 \mathrm{E}+05$

$2.18 \mathrm{E}+02$

$1.40 \mathrm{E}+04$

$1.72 E+06$

NA

$1.05 \mathrm{E}+03$

4.47E+04

NA

$4.28 \mathrm{E}+04$

$9.94 \mathrm{E}+04$

$1.58 \mathrm{E}-00$

$2.87 \mathrm{E}+03$

$2.46 \mathrm{E}+03$

$5.80 \mathrm{E}+01$

$7.06 \mathrm{E}-04$

$2.77 \mathrm{E}+01$

9.41E-04

$2.33 \mathrm{E}+05$

$1.75 \mathrm{E}+01$

$4.46 \mathrm{E}+01$

7.04E-02

6.35E-03

6.43E-02

$1.87 \mathrm{E}+01$

$1.31 \mathrm{E}-00$

$2.47 \mathrm{E}+05$

$1.93 \mathrm{E}-00$

$2.94 \mathrm{E}+02$

$1.17 \mathrm{E}+02$

2.41E-01

6.42E-00

2.43E-00

$2.29 \mathrm{E}+02$

4.58E-01

$2.81 \mathrm{E}-03$

1.12E-00

$9.89 \mathrm{E}+01$

$9.96 \mathrm{E}-00$

$6.06 \mathrm{E}+01$

3.08E-04

1.11E-04

$1.67 E-01$

$3.03 E-03$

$7.85 \mathrm{E}+01$

$1.79 \mathrm{E}-00$

$6.32 \mathrm{E}+03$

$6.60 \mathrm{E}+04$

$1.25 \mathrm{E}+02$

3.88E-03

1.03E-02

2.70E-00

$1.19 \mathrm{E}+02$

9.14E-02

3.50E-01

8.17E-01

3.59E-02

$.9 .20 \mathrm{E}-03$

8.21E-01
$1 \%$-ile

$7.27 \mathrm{E}+01$

$5.38 \mathrm{E}+02$

$5.18 \mathrm{E}-00$

8.64E-03

8.30E-02

$0.00 \mathrm{E}+01$

$0.00 \mathrm{E}+01$

$4.60 \mathrm{E}-01$

$0.00 \mathrm{E}+01$

$4.65 \mathrm{E}-03$

$7.79 \mathrm{E}+04$

$0.00 \mathrm{E}+01$

$1.46 \mathrm{E}-01$

9.81E-00

$4.05 \mathrm{E}+04$

$1.81 \mathrm{E}+02$

2.31E-00

2.68E-01

$4.35 \mathrm{E}+02$

2.21E-00

$0.00 \mathrm{E}+01$

$1.78 \mathrm{E}-01$

$3.94 \mathrm{E}-02$

$0.00 \mathrm{E}+01$

$0.00 \mathrm{E}+01$

$0.00 \mathrm{E}+01$

$0.00 \mathrm{E}+01$

$0.00 \mathrm{E}+01$

$0.00 \mathrm{E}+01$

$0.00 \mathrm{E}+01$

$0.00 \mathrm{E}+01$

$0.00 \mathrm{E}+01$

$0.00 \mathrm{E}+01$

$0.00 \mathrm{E}+01$

$0.00 \mathrm{E}+01$

$3.15 \mathrm{E}-01$

$0.00 \mathrm{E}+01$

$0.00 \mathrm{E}+01$

$0.00 \mathrm{E}+01$

$0.00 \mathrm{E}+01$

$0.00 \mathrm{E}+01$

$0.00 \mathrm{E}+01$

$0.00 \mathrm{E}+01$

$0.00 \mathrm{E}+0$

$0.00 \mathrm{E}+01$

$0.00 \mathrm{E}+01$

$0.00 E+01$

$0.00 \mathrm{E}+01$

$0.00 \mathrm{E}+01$

$0.00 \mathrm{E}+01$

$0.00 \mathrm{E}+01$

$0.00 \mathrm{E}+01$

$0.00 \mathrm{E}+01$

$0.00 \mathrm{E}+01$

$0.00 \mathrm{E}+01$

$0.00 \mathrm{E}+01$

$0.00 \mathrm{E}+01$

$0.00 \mathrm{E}+01$

$0.00 \mathrm{E}+01$

$0.00 \mathrm{E}+01$

$0.00 \mathrm{E}+01$

$1.06 \mathrm{E}+01$

$0.00 \mathrm{E}+01$

$0.00 \mathrm{E}+01$

$0.00 \mathrm{E}+01$

$0.00 \mathrm{E}+01$

$0.00 \mathrm{E}+01$

$0.00 \mathrm{E}+01$

$10 \%$-ile

$8.92 \mathrm{E}+02$

$2.03 \mathrm{E}+03$

$5.27 \mathrm{E}-01$

$5.64 \mathrm{E}-00$

6.38E+02

$0.00 \mathrm{E}+01$

$2.82 \mathrm{E}-00$

$2.54 \mathrm{E}-02$

$1.84 \mathrm{E}-01$

$3.01 \mathrm{E}+05$

7.50E-02

$1.01 \mathrm{E}+02$

$1.26 \mathrm{E}+05$

6.35E +04

$7.51 \mathrm{E}+02$

$4.56 \mathrm{E}+04$

$1.27 \mathrm{E}+03$

$3.01 \mathrm{E}+03$

$5.25 \mathrm{E}+01$

4.87E-02

$3.65 \mathrm{E}-00$

$1.88 \mathrm{E}+03$

$9.13 \mathrm{E}-03$

$0.00 \mathrm{E}+01$

$0.00 \mathrm{E}+01$

$0.00 \mathrm{E}+01$

$0.00 \mathrm{E}+01$

$0.00 \mathrm{E}+01$

$0.00 \mathrm{E}+01$

$0.00 \mathrm{E}+01$

$0.00 \mathrm{E}+01$

$0.00 \mathrm{E}+01$

$2.92 \mathrm{E}-03$

$0.00 \mathrm{E}+01$

$7.14 \mathrm{E}+03$

$0.00 \mathrm{E}+01$

$0.00 \mathrm{E}+01$

$0.00 \mathrm{E}+01$

$7.46 \mathrm{E}-02$

$0.00 \mathrm{E}+01$ 
Tank Anal. Un

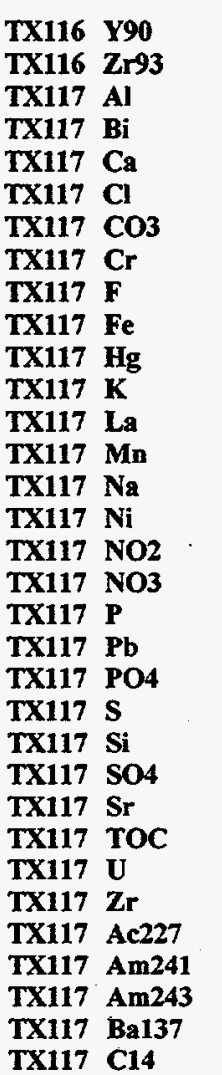

TX117 Cd113

TX117 Cm242

TX117 Cm243

TX117 Cm244

TX117 C060

TX117 Cs134

TX117 Cs137

TX117 Eu152

TX117 Eu154

TX117 Eu155

TX117 I129

TX117 Nb93

TX117 Ni59

TX117 Ni63

TX117 Np237

TX117 Pa231

TX117 Pu238

TX117 Pu239

TX117 Pu240

TX117 Pu241

TX117 Pu242

TX117 Ra226

TX117 Ra228

TX117 Ru106

TX117 Sb125

TX117 Se79

TX117 Sm151

TX117 Sr89/90

TX117 Te99

TX117 Th229

TX117 Th232

TX117 Tin126

TX117 Tritium

TX117 U232

TX117 U233

\section{May $\frac{B 8}{98}$}

$6 . \overline{60 \mathrm{E}+04}$

8.80E-00

$7.34 \mathrm{E}+04$

$6.61 \mathrm{E}+02$

$1.35 \mathrm{E}+03$

$1.39 \mathrm{E}+04$

$1.67 \mathrm{E}+05$

$3.47 \mathrm{E}+03$

$1.63 \mathrm{E}+04$

$6.31 \mathrm{E}+03$

3.75E-00

$4.67 \mathrm{E}+03$

1.39E-04

$1.38 \mathrm{E}+03$

8.17E+05

$2.57 \mathrm{E}+02$

$8.20 \mathrm{E}+04$

$1.21 \mathrm{E}+06$

$1.27 \mathrm{E}+05$

$8.81 \mathrm{E}+02$

$3.92 \mathrm{E}+05$

$5.71 E+04$

$1.96 \mathrm{E}+04$

$1.71 \mathrm{E}+05$

$3.70 \mathrm{E}+01$

$2.37 \mathrm{E}+04$

$1.69 \mathrm{E}+03$

$5.90 \mathrm{E}+01$

9.48E-04

$3.97 \mathrm{E}+01$

$1.35 \mathrm{E}-03$

$2.98 \mathrm{E}+05$

$2.44 E+01$

6.33E+01

9.97E-02

9.09E-03

8.98E-02

$2.62 \mathrm{E}+01$

$1.27 \mathrm{E}+01$

$3.15 \mathrm{E}+05$

2.68E-00

$4.13 E+02$

$1.62 \mathrm{E}+02$

3.36E-01

9.04E-00

2.66E-00

$2.55 \mathrm{E}+02$

6.36E-01

4.02E-03

Ci $\quad 1.25 \mathrm{E}-00$

8.17E+01

9.43E-00

$7.40 \mathrm{E}+01$

3.81 E-04

$1.41 \mathrm{E}-04$

$3.16 \mathrm{E}-01$

4.25E-03

$1.10 \mathrm{E}+02$

2.53E-00

$8.89 E+03$

$8.60 E+04$

$1.74 E+02$

$7.29 \mathrm{E}-03$

i $1.92 \mathrm{E}-02$

$3.81 \mathrm{E}-00$

$1.68 E+02$

$1.54 \mathrm{E}-00$

5.92E-00
Sept98

$6.60 \mathrm{E}+04$

8.80E-00

$7.34 \mathrm{E}+04$

$5.76 \mathrm{E}+02$

$1.35 \mathrm{E}+03$

$1.39 \mathrm{E}+04$

$1.67 \mathrm{E}+05$

$3.47 \mathrm{E}+03$

$1.63 \mathrm{E}+04$

$6.31 \mathrm{E}+03$

$0.00 \mathrm{E}+01$

$4.67 \mathrm{E}+03$

$1.48 \mathrm{E}-00$

$1.38 \mathrm{E}+03$

$8.17 \mathrm{E}+05$

$2.57 \mathrm{E}+02$

$8.20 \mathrm{E}+04$

1.21E+06

NA

8.35E+02

$1.28 \mathrm{E}+05$

NA

$1.96 \mathrm{E}+04$

$5.71 \mathrm{E}+04$

2.06E-00

$2.37 \mathrm{E}+04$

$1.69 \mathrm{E}+03$

$5.90 \mathrm{E}+01$

9.48E-04

$3.97 \mathrm{E}+01$

1.35E-03

$2.98 \mathrm{E}+05$

$2.44 \mathrm{E}+01$

$6.33 E+01$

9.97E- 02

9.09E-03

$8.98 \mathrm{E}-02$

$2.62 \mathrm{E}+01$

$1.27 \mathrm{E}+01$

$3.15 E+05$

2.68E-00

$4.13 E+02$

$1.62 \mathrm{E}+02$

3.36E-01

$9.04 \mathrm{E}-00$

$2.66 \mathrm{E}-00$

$2.55 \mathrm{E}+02$

6.36E-01

4.02E-03

$1.25 E-00$

8.17E+01

9.43E-00

$7.40 \mathrm{E}+01$

3.81E-04

$1.41 \mathrm{E}-04$

3.16E-01

4.25E-03

$1.10 \mathrm{E}+02$

2.53E-00

$8.89 \mathrm{E}+03$

$8.60 \mathrm{E}+04$

$1.74 \mathrm{E}+02$

$7.29 \mathrm{E}-03$

1.92E-02

$3.81 \mathrm{E}-00$

$1.68 \mathrm{E}+02$

1.72E-01

6.58E-01 1\%-ile

$10 \%$-ile

50\%-ile

\section{0\%-ile}

$0.00 \mathrm{E}+01$

$0.00 \mathrm{E}+01$

9.56E-00

2.99E-02

$3.97 \mathrm{E}+01$

$5.42 \mathrm{E}+02$

2.14E-00

4.47E-03

$3.55 \mathrm{E}-02$

$0.00 E+01$

$0.00 \mathrm{E}+01$

2.55E-01

3.25E-05

$0.00 \mathrm{E}+01$

$5.26 \mathrm{E}+04$

$0.00 \mathrm{E}+01$

$1.41 \mathrm{E}-01$

2.71E-00

$4.15 \mathrm{E}+04$

$2.03 \mathrm{E}+02$

$8.98 \mathrm{E}-01$

$7.44 \mathrm{E}-02$

$1.99 \mathrm{E}+02$

$9.20 \mathrm{E}-01$

$0.00 \mathrm{E}+01$

1.92E-02

9.95E-03

$0.00 \mathrm{E}+01$

$0.00 \mathrm{E}+01$

$0.00 \mathrm{E}+01$

$0.00 \mathrm{E}+01$

$0.00 \mathrm{E}+01$

$0.00 \mathrm{E}+01$

$0.00 \mathrm{E}+01$

$0.00 \mathrm{E}+01$

$0.00 \mathrm{E}+01$

$0.00 \mathrm{E}+01$

$0.00 \mathrm{E}+01$

$0.00 E+01$

$9.87 \mathrm{E}-02$

$0.00 \mathrm{E}+01$

$0.00 \mathrm{E}+01$

$0.00 \mathrm{E}+01$

$0.00 \mathrm{E}+01$

$0.00 \mathrm{E}+01$

$0.00 \mathrm{E}+01$

$0.00 \mathrm{E}+01$

$0.00 \mathrm{E}+01$

$0.00 \mathrm{E}+01$

$0.00 E+01$

$0.00 E+01$

$0.00 E+01$

$0.00 E+01$

$0.00 \mathrm{E}+01$

$0.00 \mathrm{E}+01$

$0.00 E+01$

$0.00 \mathrm{E}+01$

$0.00 \mathrm{E}+01$

$0.00 E+01$

$0.00 \mathrm{E}+01$

$0.00 \mathrm{E}+01$

$0.00 \mathrm{E}+01$

$0.00 \mathrm{E}+01$

$0.00 \mathrm{E}+01$

$0.00 \mathrm{E}+01$

$1.11 \mathrm{E}+01$

$0.00 \mathrm{E}+01$

$0.00 E+01$

$0.00 \mathrm{E}+01$

$0.00 \mathrm{E}+01$

$2.32 \mathrm{E}+02$

$1.40 \mathrm{E}+03$

$5.07 \mathrm{E}+02$

$1.92 \mathrm{E}+03$

$2.26 \mathrm{E}+01$

$1.89 \mathrm{E}-01$

4.57E- 00

$3.00 \mathrm{E}+02$

$0.00 \mathrm{E}+01$

1.33E-00

$1.29 \mathrm{E}-02$

2.19E-01

$2.83 \mathrm{E}+05$

3.64E-02

$8.85 \mathrm{E}+01$

$1.18 \mathrm{E}+05$

$6.26 \mathrm{E}+04$

$7.41 \mathrm{E}+02$

$4.55 \mathrm{E}+04$

$1.41 \mathrm{E}+03$

$2.12 \mathrm{E}+03$

$7.89 \mathrm{E}+01$

2.31E-02

$1.27 \mathrm{E}-00$

$1.75 \mathrm{E}+03$

3.91E-03

$0.00 \mathrm{E}+01$

$0.00 \mathrm{E}+01$

$0.00 \mathrm{E}+01$

$3.19 \mathrm{E}+03$

$0.00 \mathrm{E}+01$

$0.00 \mathrm{E}+01$

$0.00 \mathrm{E}+01$

$0.00 \mathrm{E}+01$

$0.00 \mathrm{E}+01$

8.19E-04

$1.36 \mathrm{E}-01$

8.01E+03

$0.00 \mathrm{E}+01$ 
Tank Anal. Un

$\begin{array}{ll}\text { TX117 } & \text { U234 } \\ \text { TX117 } & \text { U235 } \\ \text { TX117 } & \text { U236 } \\ \text { TX117 } & \text { U238 } \\ \text { TX117 } & \text { Y90 } \\ \text { TX117 } & \text { Zr93 } \\ \text { TX118 } & \text { Al } \\ \text { TX118 } & \text { Bi } \\ \text { TX118 } & \text { Ca } \\ \text { TX118 } & \text { Cl } \\ \text { TX118 } & \text { CO3 } \\ \text { TX118 } & \text { Cr } \\ \text { TX118 } & \text { F } \\ \text { TX118 } & \text { Fe } \\ \text { TX118 } & \text { Hg } \\ \text { TX118 } & \text { K } \\ \text { TX118 } & \text { La } \\ \text { TX118 } & \text { Mn } \\ \text { TX118 } & \text { Na } \\ \text { TX118 } & \text { Ni } \\ \text { TX118 } & \text { NO2 } \\ \text { TX118 } & \text { NO3 } \\ \text { TX118 } & \text { P } \\ \text { TX118 } & \text { Pb } \\ \text { TX118 } & \text { PO4 } \\ \text { TX118 } & \text { S } \\ \text { TX118 } & \text { Si } \\ \text { TX118 } & \text { SO4 } \\ \text { TX118 } & \text { Sr } \\ \text { TX118 } & \text { TOC } \\ \text { TX118 } & \text { U } \\ \text { TX118 } & \text { Z } \\ \text { To } \\ \text { To }\end{array}$

TX118 U

TX118 Zr

TX118 Ac227

TX118 Am241

TX118 Am243

TX118 Ba137

TX118 C14

TX118 Cd113

TX118 Cm242

TX118 Cm243

TX118 Cm244

TX118 Co60

TX118 Cs134

TX118 Cs137

TX118 Eu152

TX118 Eu154

TX118 Eu155

TX118 1129

TX118 Nb93

TX118 Ni59

TX118 Ni63

TX118 Np237

TX118 Pa231

TX118 Pu238

TX118 Pu239

TX118 Pu240

TX118 Pu241

TX118. Pu242

TX118 $\mathbf{R a 2 2 6}$

TX118 Ra228

TX118 Ru106

TX118 Sb125

TX118 Se79

TX118 Sm15!

TX118 Sr89/90

TXi18 Te99

TX118 Th229

TX118 Th232
May $\frac{B 8}{98}$

$5.12 \mathrm{E}-00$

2.23E-01

7.04E-02

5.52E-00

$8.61 E+04$

$1.24 \mathrm{E}+01$

$8.49 E+04$

$1.26 \mathrm{E}+02$

$2.67 \mathrm{E}+03$

$8.99 \mathrm{E}+03$

$9.55 E+04$

$2.23 \mathrm{E}+03$

$3.56 \mathrm{E}+03$

$1.43 E+04$

1.00E-00

$2.89 \mathrm{E}+03$

$5.88 \mathrm{E}+01$

$4.20 \mathrm{E}+02$

$3.67 \mathrm{E}+0 \mathrm{~S}$

$9.00 \mathrm{E}+02$

$5.16 \mathrm{E}+04$

5.38E+05

$1.76 \mathrm{E}+04$

$2.44 \mathrm{E}+02$

$5.45 \mathrm{E}+04$

$2.78 \mathrm{E}+04$

$3.26 \mathrm{E}+02$

8.34E+04

$1.17 \mathrm{E}+01$

$1.54 \mathrm{E}+04$

$6.70 \mathrm{E}+02$

$2.92 \mathrm{E}+01$

4.12E-04

$3.81 \mathrm{E}+03$

8.67E-04

$1.76 E+05$

$138 \mathrm{E}+01$

$3.66 \mathrm{E}+01$

6.46E-02

5.96E-03

$5.75 \mathrm{E}-02$

$1.53 E+01$

$1.92 \mathrm{E}-00$

$1.86 \mathrm{E}+05$

$1.70 \mathrm{E}-00$

2.52E-00

$1.01 \mathrm{E}+02$

$1.90 \mathrm{E}-01$

5.11E-00

9.82E-01

$3.59 \mathrm{E}-01$

$3.59 \mathrm{E}-01$

1.85E-03

$5.93 \mathrm{E}-01$

$3.64 \mathrm{E}+03$

$9.10 \mathrm{E}+02$

$3.95 \mathrm{E}+01$

$2.16 \mathrm{E}-04$

6.47E-05

7.71E-02

2.79E- 03

$6.57 \mathrm{E}+01$

1.43E-00

$5.05 E+03$

$4.84 \mathrm{E}+04$

$9.85 \mathrm{E}+0$

$1.80 \mathrm{E}-03$

4.86E- 03
Sept $\overline{98}$ 5.70E-01

2.48E-02

$7.83 E-03$

5.64E-01

$8.61 \mathrm{E}+04$

$1.24 \mathrm{E}+01$

$8.49 \mathrm{E}+04$

$7.04 \mathrm{E}+01$

$2.67 E+03$

$8.99 \mathrm{E}+03$

$9.55 E+04$

$2.23 \mathrm{E}+03$

$3.56 \mathrm{E}+03$

$1.43 E+04$

$0.00 \mathrm{E}+01$

$2.89 \mathrm{E}+03$

$0.00 \mathrm{E}+01$

$4.20 \mathrm{E}+02$

3.67E+05

$9.00 \mathrm{E}+02$

$5.16 \mathrm{E}+04$

$5.38 E+05$

$$
\text { NA }
$$

$1.12 \mathrm{E}+02$

$1.78 \mathrm{E}+04$

NA

$3.26 \mathrm{E}+02$

$2.78 \mathrm{E}+04$

1.16E-00

$1.54 \mathrm{E}+04$

$6.70 \mathrm{E}+02$

$2.92 \mathrm{E}+01$

4.12E-04

$3.81 E+03$

7.62E-04

$1.76 E+05$

$1.38 \mathrm{E}+01$

$3.66 \mathrm{E}+01$

$5.68 \mathrm{E}-02$

5.24E-03

$5.06 \mathrm{E}-02$

$1.53 \mathrm{E}+01$

$1.92 \mathrm{E}-00$

$1.86 \mathrm{E}+05$

1.70E-00

$2.52 \mathrm{E}+02$

$1.01 \mathrm{E}+02$

1.90E-01

5.11E-00

9.82E-01

$9.60 \mathrm{E}+01$

$3.59 \mathrm{E}-01$

$1.85 \mathrm{E}-03$

5.22E-01

$3.64 \mathrm{E}+03$

$9.10 \mathrm{E}+02$

$3.47 E+01$

$1.90 \mathrm{E}-04$

6.47E-05

7.71E- 02

2.79E-03

$6.57 \mathrm{E}+01$

$1.43 E-00$

$5.05 E+03$

$4.84 E+04$

$9.85 \mathrm{E}+01$

$1.80 \mathrm{E}-03$

4.86E-03 1\%-ile

$0.00 \mathrm{E}+01$

$0.00 \mathrm{E}+01$

$0.00 \mathrm{E}+01$

$.00 \mathrm{E}+01$

$0.00 \mathrm{E}+01$

$0.00 \mathrm{E}+01$

$4.69 \mathrm{E}+02$

$3.68 \mathrm{E}-03$

$1.70 \mathrm{E}+01$

$5.26 \mathrm{E}+01$

$4.94 \mathrm{E}+02$

$1.21 \mathrm{E}+01$

$7.97 \mathrm{E}-02$

$1.34 \mathrm{E}-01$

$0.00 \mathrm{E}+01$

2.61E-00

6.41E-04

$9.77 \mathrm{E}-03$

$3.73 E+04$

9.49E-00

$3.22 \mathrm{E}-00$

$3.82 \mathrm{E}+03$

$1.45 \mathrm{E}-01$

5.47E-02

$5.53 \mathrm{E}-01$

2.72E-00

$1.60 \mathrm{E}-03$

$9.32 \mathrm{E}-00$

9.72E-04

$5.13 \mathrm{E}-01$

1.87E-04

3.47E-04

$0.00 \mathrm{E}+01$

$0.00 \mathrm{E}+01$

$0.00 \mathrm{E}+01$

$0.00 \mathrm{E}+01$

$0.00 \mathrm{E}+01$

$0.00 \mathrm{E}+01$

$0.00 \mathrm{E}+01$

$0.00 \mathrm{E}+01$

$0.00 \mathrm{E}+01$

$0.00 \mathrm{E}+01$

$0.00 \mathrm{E}+01$

3.45E-01

$0.00 \mathrm{E}+01$

$0.00 \mathrm{E}+01$

$0.00 \mathrm{E}+01$

$0.00 \mathrm{E}+01$

$0.00 E+01$

$1.73 \mathrm{E}-03$

$6.33 \mathrm{E}-04$

$0.00 \mathrm{E}+01$

$0.00 \mathrm{E}+01$

$0.00 \mathrm{E}+01$

$0.00 \mathrm{E}+01$

$0.00 \mathrm{E}+01$

$0.00 \mathrm{E}+01$

$0.00 \mathrm{E}+01$

$0.00 \mathrm{E}+01$

$0.00 \mathrm{E}+01$

$0.00 \mathrm{E}+01$

$0.00 \mathrm{E}+01$

$0.00 \mathrm{E}+01$

$0.00 \mathrm{E}+01$

$0.00 \mathrm{E}+01$

$0.00 \mathrm{E}+01$

$0.00 \mathrm{E}+01$

$0.00 E+01$

$0.00 \mathrm{E}+01$

$0.00 \mathrm{E}+01$

$0.00 \mathrm{E}+01$

$0.00 \mathrm{E}+01$

$0.00 \mathrm{E}+01$

$.00 \mathrm{E}+01$

$1.12 \mathrm{E}+04$

$2.36 \mathrm{E}-01$

$7.99 \mathrm{E}+02$

$6.64 \mathrm{E}+02$

$2.99 \mathrm{E}+03$

8.48E +01

1.07E-00

$10.00 E+02$

$0.00 \mathrm{E}+01$

$1.34 \mathrm{E}+01$

$5.10 \mathrm{E}-02$

$3.38 \mathrm{E}-01$

$1.51 \mathrm{E}+05$

$2.64 \mathrm{E}+02$

7.44E+01

4.02E+04

4.36E+03

$1.26 \mathrm{E}-00$

$1.20 \mathrm{E}+04$

2.37E+01

$1.71 \mathrm{E}-01$

$6.80 \mathrm{E}+01$

1.41E-01

$8.55 \mathrm{E}-00$

$1.08 \mathrm{E}-02$

5.33E-02

$0.00 \mathrm{E}+01$

4.01E-05

6.62E-08

$0.00 \mathrm{E}+01$

$0.00 \mathrm{E}+01$

$0.00 \mathrm{E}+01$

4.93E-06

$4.55 \mathrm{E}-07$

4.39E-06 
Tank Anal. Un

$\begin{array}{lll}\text { TX118 } & \text { Tin126 } \\ \text { TX118 } & \text { Tritium } \\ \text { TX118 } & \text { U232 } \\ \text { TX118 } & \text { U233 } & \\ \text { TX118 } & \text { U234 } & \text { C } \\ \text { TX118 } & \text { U235 } & \\ \text { TX118 } & \text { U236 } & \\ \text { TX118 } & \text { U238 } & \\ \text { TX118 } & \text { Y90 } & \\ \text { TX118 } & \text { Zr93 } & \\ \text { TY101 } & \text { Al } & \end{array}$

TY101 B

TY101 Ca

TY101 Cl

TY101 CO3

TY101 Cr

TY101 F

TY101 Fe

TY101 Hg

TY101 K

TY101 La

TY101 Mn

TY101 Na

TY101 Ni

TY101 NO2

TY101 NO3

TY101 P

TY101 Pb

TY101 PO4

TY101 S

TY101 Si

TY101 SO4

TY101 Sr

TY101 TOC

TY101 U

TY101 Zr

TY101 Ac227

TY101 Am241

TY101 Am243

TY101 Bal37

TY101 C14

TY101 Cd113

TY101 Cm242

TY101 Cm243

TY101 Cm244

TY101 Co60

TY101 Cs134

TY101 Cs137

TY101 Eu152

TY101 Eu154

TY101 Eu155

TY101 1129

TY101 Nb93

TY101 Ni59

TY101 Ni63

TY101 Np237

TY101 Pa231

TY101 Pu238

TY101 Pu239

TY101 Pu240

TY101 Pu241

TY101 Pu242

TY101 Ra226

TY101 Ra228

TY101 Ru106

TY101 Sb125

TY101 Se79

TY101 Sm151

\section{May $\frac{B B}{98}$}

2.17E-00

$9.83 \mathrm{E}+01$

3.93E-01

$1.51 \mathrm{E}-00$

5.10E-01

2.11E-02

$1.34 \mathrm{E}-02$

5.81E-01

$4.84 \mathrm{E}+04$

7.03E-00

$2.13 E+04$

$1.99 \mathrm{E}+04$

$3.39 \mathrm{E}+03$

$5.50 \mathrm{E}+02$

$6.32 \mathrm{E}+03$

$6.16 \mathrm{E}+03$

$2.47 \mathrm{E}+03$

$2.94 \mathrm{E}+04$

$1.40 \mathrm{E}-00$

$1.39 \mathrm{E}+02$

$0.00 \mathrm{E}+01$

$4.10 \mathrm{E}+02$

$8.88 \mathrm{E}+04$

$3.73 \mathrm{E}+03$

$9.94 \mathrm{E}+03$

$1.06 \mathrm{E}+05$

$1.76 \mathrm{E}+04$

$1.60 \mathrm{E}+02$

$5.44 \mathrm{E}+04$

$1.34 \mathrm{E}+03$

$2.83 E+04$

$4.02 \mathrm{E}+03$

$0.00 \mathrm{E}+01$

$4.90 \mathrm{E}+02$

$1.71 \mathrm{E}+03$

$2.80 \mathrm{E}+02$

3.44E-05

9.37E-00

$1.86 \mathrm{E}-06$

$1.62 \mathrm{E}+02$

3.19E-02

3.13E-01

1.14E-03

2.34E-05

4.27E-05

2.20E-00

7.92E-03

$1.71 \mathrm{E}+02$

6.06E-02

$5.49 \mathrm{E}-01$

4.26E-00

3.44E-02

$1.06 \mathrm{E}-01$

4.00E-01

$3.63 \mathrm{E}+01$

5.39E-03

$7.56 \mathrm{E}-05$

$3.89 \mathrm{E}-01$

$1.40 \mathrm{E}+02$

4.81E-00

$1.66 \mathrm{E}+01$

$7.57 \mathrm{E}-05$

6.67E-06

1.91E-10

1.33E-08

$0.00 \mathrm{E}+01$

2.64E-02

$9.82 \mathrm{E}+01$
$\frac{\frac{B B}{\text { Sept98 }}}{2.17 E-00}$

1.85E-01

7.10E-01

2.41E-01

9.96E-03

6.31E-03

$2.24 \mathrm{E}-01$

$4.84 \mathrm{E}+04$

7.03E-00

$2.13 E+04$

$1.99 \mathrm{E}+04$

$3.24 \mathrm{E}+03$

$5.54 \mathrm{E}+02$

$5.84 E+03$

$6.16 \mathrm{E}+03$

$2.47 \mathrm{E}+03$

$2.94 E+04$

$0.00 \mathrm{E}+01$

$1.24 \mathrm{E}+02$

$0.00 \mathrm{E}+01$

$4.09 \mathrm{E}+02$

$8.88 \mathrm{E}+04$

$3.73 E+03$

8.52E+03

$1.06 \mathrm{E}+05$

NA

$1.64 \mathrm{E}+02$

$5.69 \mathrm{E}+04$

NA

$2.83 E+04$

$4.02 \mathrm{E}+03$

$4.33 \mathrm{E}+01$

$4.86 \mathrm{E}+02$

$1.71 \mathrm{E}+03$

$2.82 \mathrm{E}+02$

$3.44 \mathrm{E}-05$

2.64E-01

$1.86 \mathrm{E}-06$

$1.63 \mathrm{E}+02$

6.38E-01

3.13E-01

1.14E-03

2.34E-05

4.27E-05

2.47E-00

$7.92 \mathrm{E}-03$

$1.72 \mathrm{E}+02$

6.06E-02

5.49E-01

4. $26 \mathrm{E}-00$

3.44E-02

1.06E-01

4.00E-01

$3.63 \mathrm{E}+01$

5.39E-03

7.56E-05

$9.76 \mathrm{E}-01$

$1.28 \mathrm{E}+02$

$1.21 \mathrm{E}+01$

$4.17 E+01$

1.90E-04

6.67E-06

1.91E-10

$1.33 E-08$

$0.00 \mathrm{E}+01$

2.64E-02

$9.82 \mathrm{E}+01$ 1\%-ile

$10 \%-i l e$

$6.90 \mathrm{E}-00$

$0.00 \mathrm{E}+01$

$0.00 \mathrm{E}+01$

$0.00 \mathrm{E}+01$

$0.00 E+01$

$0.00 \mathrm{E}+01$

$0.00 \mathrm{E}+01$

$0.00 \mathrm{E}+01$

$0.00 \mathrm{E}+01$

$9.70 \mathrm{E}+02$

$6.30 \mathrm{E}-01$

2.55E-02

$5.35 \mathrm{E}+01$

$9.24 \mathrm{E}+02$

6.34E-00

$2.84 \mathrm{E}+01$

$5.92 \mathrm{E}+02$

$0.00 \mathrm{E}+01$

8.12E-00

8.14E-01

$1.46 \mathrm{E}-00$

$1.13 E+04$

$0.00 \mathrm{E}+01$

$8.66 \mathrm{E}+03$

$1.28 \mathrm{E}+03$

$3.27 \mathrm{E}-01$

$3.85 \mathrm{E}+01$

$3.56 \mathrm{E}+02$

$1.15 \mathrm{E}-01$

$3.21 \mathrm{E}+01$

4.19E+02

2.01E-03

$1.37 \mathrm{E}-02$

$2.29 \mathrm{E}+02$

$0.00 \mathrm{E}+01$

$0.00 \mathrm{E}+01$

5.31E-00

$0.00 \mathrm{E}+01$

$8.50 \mathrm{E}-00$

$0.00 \mathrm{E}+01$

$0.00 \mathrm{E}+01$

$0.00 \mathrm{E}+01$

$0.00 \mathrm{E}+01$

$0.00 \mathrm{E}+01$

1.02E-00

4.15E-04

$8.03 E+03$

$0.00 \mathrm{E}+01$

$0.00 \mathrm{E}+01$

$0.00 E+01$

$5.40 \mathrm{E}-04$

$0.00 \mathrm{E}+01$

$0.00 \mathrm{E}+01$

$0.00 \mathrm{E}+01$

$0.00 \mathrm{E}+01$

$0.00 E+01$

$0.00 \mathrm{E}+01$

$0.00 \mathrm{E}+01$

$0.00 \mathrm{E}+01$

$0.00 \mathrm{E}+01$

$0.00 \mathrm{E}+01$

$0.00 \mathrm{E}+01$

$0.00 \mathrm{E}+01$

$0.00 \mathrm{E}+01$

$0.00 \mathrm{E}+01$

$0.00 \mathrm{E}+01$

$0.00 \mathrm{E}+01$

\section{$0.00 \mathrm{E}+01$}

$0.00 \mathrm{E}+01$

$0.00 \mathrm{E}+01$

$0.00 \mathrm{E}+01$

$0.00 \mathrm{E}+01$

$0.00 \mathrm{E}+01$

$0.00 \mathrm{E}+01$

$0.00 \mathrm{E}+01$

$1.24 \mathrm{E}-02$

$5.97 \mathrm{E}+03$

$3.58 \mathrm{E}+02$

2.67E+03

3.01E+02

$4.83 \mathrm{E}+03$

7.14E+01

$3.00 \mathrm{E}+02$

$3.99 \mathrm{E}+03$

1.02E-01

$4.00 \mathrm{E}+02$

$5.40 \mathrm{E}-00$

$4.89 \mathrm{E}+01$ 
Tank Anal. Un

$\begin{array}{ll}\text { TY101 } & \text { Sr89/90 } \\ \text { TY101 } & \text { Tc99 } \\ \text { TY101 } & \text { Th229 } \\ \text { TY101 } & \text { Th232 } \\ \text { TY101 } & \text { Tin126 } \\ \text { TY101 } & \text { Tritium } \\ \text { TY101 } & \text { U232 } \\ \text { TY101 } & \text { U233 } \\ \text { TY101 } & \text { U234 } \\ \text { TY101 } & \text { U235 } \\ \text { TY101 } & \text { U236 } \\ \text { TY101 } & \text { U238 } \\ \text { TY101 } & \text { Y90 } \\ \text { TY101 } & \text { Zr93 }\end{array}$

TY102 Al

TY102 Bi

TY102 Ca

TY102 Cl

TY102 CO3

TY102 Cr

TY102 F

TY102 Fe

TY102 $\mathrm{Hg}$

TY102 K

TY102 La

TY102 Mn

TY102 Na

TY102 Ni

TY102 NO2

TY102 NO3

TY102 P

TY102 Pb

TY102 PO4

TY102 S

TY102 Si

TY102 SO4

TY102 Sr

TY102 TOC

TY102 U

TY102 Zr

TY102 Ac227

TY102 Am241

TY102 Am243

TY102 Ba137

TY102 C14

TY102 Cd113

TY102 Cm242

TY102 Cm243

TY102 Cm244

TY102 C060

TY102 Cs134

TY102 Cs137

TY102 Eu152

TY102 Eu15

TY102 Eu155

TY102 I129

TY102 Nb93

TY102 Ni59

TY102 Ni63

TY102 Np237

TY102 Pa231

TY102 Pu238

TY102 Pu239

TY102 Pu240

TY102 Pu241

TY102 Pu242

TY102 Ra226

TY102 Ra228 $\frac{\text { May }}{208}$

5.91E-01

3.72E-08

3.54E-11

3.98E-02

6.67E-01

3.02E-04

$1.38 \mathrm{E}-05$

$1.26 \mathrm{E}+01$

5.59E-01

$1.29 \mathrm{E}-01$

$1.29 \mathrm{E}+01$

$2.01 \mathrm{E}+04$

$1.26 \mathrm{E}-01$

$1.88 \mathrm{E}+02$

$1.81 \mathrm{E}+01$

$1.14 \mathrm{E}+02$

$1.24 \mathrm{E}+03$

$1.62 \mathrm{E}+04$

2.62E +01

$2.10 \mathrm{E}+03$

$3.99 \mathrm{E}+02$

3.62E-01

$4.27 E+02$

1.49E-05

$1.61 \mathrm{E}+01$

$9.38 \mathrm{E}+04$

$7.51 \mathrm{E}-00$

$7.35 E+03$

$1.50 \mathrm{E}+05$

$1.33 E+04$

$2.91 E+01$

4.11E+04

$5.90 \mathrm{E}+03$

$9.88 \mathrm{E}+01$

$1.77 \mathrm{E}+04$

4.44E-00

$1.49 \mathrm{E}+02$

$8.02 E+01$

3.71E-00

1.00E-04

$2.16 \mathrm{E}-00$

1.67E-04

$1.19 \mathrm{E}+03$

3.98E-01

7.01E-00

$1.16 \mathrm{E}-02$

1.07E-03

1.10E-02

3.42E-01

2.11E-01

$1.26 \mathrm{E}+03$

Ci 3.08E-01

$4.68 \mathrm{E}+01$

Ci $1.85 \mathrm{E}+01$

Ci $\quad 8.65 E-03$

Ci 1.01E-00

Ci 3.01E-01

Ci $\quad 2.87 \mathrm{E}+01$

Ci $\quad 7.03 \mathrm{E}-02$

Ci 4.08E-04

Ci $1.50 \mathrm{E}-01$

Ci 6.50E-01

Ci Incl. in 239

Ci 8.69E-00

Ci 4.59E-05

Ci 1.59E-05

Ci 2.15E-02
$B B$

Sept98

$7.47 \mathrm{E}+03$
$5.39 \mathrm{E}-00$

3.72E-08

3.54E-11

3.98E-02

6.67E-01

1.34E-05

6.15E-07

5.62E-01

2.48E-02

5.72E-03

5.71E-01

$7.47 \mathrm{E}+03$

$1.26 \mathrm{E}-01$

$1.88 \mathrm{E}+02$

$1.81 \mathrm{E}+01$

$1.14 \mathrm{E}+02$

$1.24 \mathrm{E}+03$

$1.62 \mathrm{E}+04$

$2.62 \mathrm{E}+01$

$2.10 \mathrm{E}+03$

$3.99 \mathrm{E}+02$

$0.00 \mathrm{E}+01$

4.27E +02

$1.97 \mathrm{E}-05$

$1.61 E+01$

$9.38 \mathrm{E}+04$

7.51E-00

$7.35 \mathrm{E}+03$

$1.50 \mathrm{E}+05$

NA

$2.91 \mathrm{E}+01$

$1.34 \mathrm{E}+04$

NA

$9.88 \mathrm{E}+01$

$5.90 \mathrm{E}+03$

4.44E-00

$1.49 E+02$

8.02E+01

$3.71 \mathrm{E}-00$

$1.00 \mathrm{E}-04$

$2.16 \mathrm{E}-00$

7.19 E- 05

$1.19 \mathrm{E}+03$

$3.98 \mathrm{E}-01$

7.01E-00

5.00 E-03

4.58E-04

$4.74 \mathrm{E}-03$

3.42E-01

2.11E-01

$1.26 \mathrm{E}+03$

3.08E-01

$4.68 \mathrm{E}+01$

$1.85 \mathrm{E}+01$

8.65E-03

$1.01 \mathrm{E}-00$

3.01E-01

$2.87 \mathrm{E}+01$

7.03E-02

4.08E-04

8.19E-03

5.84E-01

6.57E-02

4.75E-01

2.51E-06

1.59E-05

2.15E-02

$1 \%$-ile

$3.99 \mathrm{E}+03$

$10 \%$-ile

\subsection{E+04}

$1.40 \mathrm{E}-01$

$0.00 E+01$

$0.00 \mathrm{E}+01$

$0.00 \mathrm{E}+01$

$0.00 \mathrm{E}+01$

2.61E-01

$0.00 \mathrm{E}+01$

$0.00 \mathrm{E}+01$

$0.00 \mathrm{E}+01$

$0.00 \mathrm{E}+01$

$0.00 \mathrm{E}+01$

$0.00 \mathrm{E}+01$

$0.00 \mathrm{E}+01$

$0.00 \mathrm{E}+01$

3.92E-00

$2.09 \mathrm{E}-00$

4.42E-00

$5.92 \mathrm{E}+01$

$4.84 \mathrm{E}-00$

$5.82 \mathrm{E}-02$

$5.71 \mathrm{E}-01$

4.70E-00

$0.00 \mathrm{E}+01$

$7.25 \mathrm{E}-02$

4.83E- 03

$4.54 \mathrm{E}-02$

$5.19 \mathrm{E}+03$

2.85E-01

$2.47 \mathrm{E}+01$

$1.78 \mathrm{E}+02$

$3.17 \mathrm{E}+03$

$0.00 \mathrm{E}+01$

$2.89 \mathrm{E}-03$

4.27E-01

$0.00 \mathrm{E}+01$

$0.00 \mathrm{E}+01$

$0.00 \mathrm{E}+01$

$0.00 \mathrm{E}+01$

$0.00 \mathrm{E}+01$

$0.00 \mathrm{E}+01$

$1.58 \mathrm{E}+03$

7.77E-03

$1.60 \mathrm{E}+02$

$1.44 \mathrm{E}+02$

$6.37 \mathrm{E}+01$

$2.12 \mathrm{E}+02$

$5.14 \mathrm{E}+01$

$1.95 \mathrm{E}-00$

$3.85 \mathrm{E} \div 01$

$8.50 \mathrm{E}+01$

$6.46 \mathrm{E}-03$

$2.97 \mathrm{E}-00$

$7.56 \mathrm{E}-02$

$1.49 \mathrm{E}-00$

$2.50 \mathrm{E}+04$

$1.05 \mathrm{E}+01$

$3.79 \mathrm{E}+02$

$1.32 \mathrm{E}+04$

$5.88 \mathrm{E}+03$

$2.45 \mathrm{E}+01$

$7.84 \mathrm{E}+01$

$4.46 \mathrm{E}+03$

$1.15 \mathrm{E}-00$

$1.56 \mathrm{E}+02$

$1.39 \mathrm{E}+02$

4.54E-00

$1.86 \mathrm{E}+02$

$4.24 \mathrm{E}-00$

$7.01 \mathrm{E}-01$

$6.08 \mathrm{E}+0 \mathrm{I}$

$3.43 \mathrm{E}+01$

$2.93 \mathrm{E}+02$

$0.00 \mathrm{E}+01 \quad 1.02 \mathrm{E}-01$

$0.00 \mathrm{E}+01$

$0.00 \mathrm{E}+01$

2.85E-01

$\begin{array}{ll}2.22 \mathrm{E}-02 & 2.85 \mathrm{E}-01 \\ 6.11 \mathrm{E}-07 & 1.24 \mathrm{E}-05\end{array}$

$0.00 \mathrm{E}+01 \quad 2.18 \mathrm{E}+02$

$0.00 \mathrm{E}+01 \quad 4.95 \mathrm{E}-03$

$0.00 \mathrm{E}+01 \quad 125 \mathrm{E}-01$

4.24E-05 8.61E-04

$3.91 \mathrm{E}-06 \quad 7.94 \mathrm{E}-05$

4.02E-05

$4.54 \mathrm{E}-03$

$8.17 \mathrm{E}-04$

3.09E-02

$0.00 \mathrm{E}+01 \quad 3.87 \mathrm{E}-02$

$2.48 \mathrm{E}+02$

$0.00 \mathrm{E}+01$

$0.00 \mathrm{E}+01$

$0.00 \mathrm{E}+01$

$0.00 \mathrm{E}+01$ 
Tank Anal. Un

$\begin{array}{ll}\text { TY102 } & \text { Ru106 } \\ \text { TY102 } & \text { Sb125 } \\ \text { TY102 } & \text { Se79 } \\ \text { TY102 } & \text { Sm151 } \\ \text { TY102 } & \text { Sr89/90 } \\ \text { TY102 } & \text { Tc99 } \\ \text { TY102 } & \text { Th229 } \\ \text { TY102 } & \text { Th232 } \\ \text { TY102 } & \text { Tin126 } \\ \text { TY102 } & \text { Tritium } \\ \text { TY102 } & \text { U232 } \\ \text { TY102 } & \text { U233 } \\ \text { TY102 } & \text { U234 } \\ \text { TY102 } & \text { U235 } \\ \text { TY102 } & \text { U236 } \\ \text { TY102 } & \text { U238 } \\ \text { TY102 } & \text { Y90 } \\ \text { TY102 } & \text { Zr93 } \\ \text { TY103 } & \text { Al }\end{array}$

TY103 $\mathrm{Bi}$

TY103 Ca

TY103 Cl

TY103 CO3

TY103 Cr

TY103 F

TY103 Fe

TY103 Hg

TY103 K

TY103 La

TY103 Mn

TY103 Na

TY103 Ni

TY103 NO2

TY103 NO3

TY103 P

TY103 Pb

TY103 PO4

TY103 S

TY103 Si

TY103 SO4

TY103 Sr

TY103 TOC

TY103 U

TY103 Zr

TY103 Ac227

TY103 Am241

TY103 Am243

TY103 Ba137

TY103 C14

TY103 Cd113

TY103 Cm242

TY103 Cm243

TY103 Cm244

TY103 Co60

TY103 Cs134

TY103 Cs137

TY103 Eu152

TY103 Eu154

TY103 Eu155

TY103 I129

TY103 Nb93

TY103 Ni59

TY103 Ni63

TY103 Np237

TY103 Pa231

TY103 Pu238

TY103 Pu239

TY103 Pu240

\section{May $\frac{B B}{98}$}

5.18E-04 $1.23 \mathrm{E}+01$

2.82E-01

$9.96 \mathrm{E}+02$

$1.72 \mathrm{E}+03$

3.59E-01

4.98E-04

1.32E-03

$4.26 \mathrm{E}-01$

$1.89 \mathrm{E}+01$

1.08E-01

4.12E-01

$6.99 \mathrm{E}-01$

$3.06 \mathrm{E}-02$

8.57E-03

$7.28 \mathrm{E}-01$

$1.72 \mathrm{E}+03$

1.38E-00

$2.97 \mathrm{E}+03$

2.72E+04

$5.05 E+03$

$1.16 \mathrm{E}+03$

6.17E+03

$\quad 9.77 \mathrm{E}+02$

$1.40 \mathrm{E}+03$

$2.40 \mathrm{E}+04$

kg 3.35E-01

kg 3.89E+02

kg 1.97E-05

kg $1.68 \mathrm{E}+02$

1.15E+05

kg 2.34E+03

kg $4.22 E+04$

kg 7.93E+04

kg

kg $2.45 \mathrm{E}+05$

kg $\quad 9.59 \mathrm{E}+03$

kg $\quad 5.68 \mathrm{E}+03$

kg 2.88E+04

kg 6.93E-01

kg 1.15E+03

kg. $\quad 1.80 \mathrm{E}+04$

(c.48E+02

Ci 1.36E-04

$3.35 E+01$

2.01E-04

$1.41 \mathrm{E}+04$

$1.04 \mathrm{E}-00$

8.98E-00

1.73E-02

1.35E-03

1.34E-02

$1.53 \mathrm{E}-00$

2.57E-01

$1.49 \mathrm{E}+04$

5.52E-01

$5.91 \mathrm{E}+01$

$3.56 \mathrm{E}+01$

4.82E-02

1.30E-00

4.41E-01

$4.20 \mathrm{E}+01$

$9.23 \mathrm{E}-02$

5.41E-04

$\mathrm{Ci}$ 3.52E-01

$1.86 \mathrm{E}+02$

Ci Incl. in 239
$B B$ Sept98

5.18E-04 $1.23 \mathrm{E}+01$

2.82E-01

$9.96 \mathrm{E}+02$

$1.72 \mathrm{E}+03$

3.59E-01

4.98E-04

1.32E-03

4.26E-01

$1.89 \mathrm{E}+01$

4.12E-03

$1.58 \mathrm{E}-02$

2.68E-02

$1.17 \mathrm{E}-03$

$3.28 \mathrm{E}-04$

2.68E-02

$1.72 \mathrm{E}+03$

$1.38 \mathrm{E}-00$

$2.97 \mathrm{E}+03$

2.72E+04

$5.05 \mathrm{E}+03$

$1.16 \mathrm{E}+03$

6.17E+03

$9.77 \mathrm{E}+02$

$1.40 \mathrm{E}+03$

$2.40 \mathrm{E}+04$

$0.00 \mathrm{E}+01$

$3.89 \mathrm{E}+02$

$0.00 \mathrm{E}+01$

$1.68 \mathrm{E}+02$

$1.15 \mathrm{E}+05$

$2.34 \mathrm{E}+03$

$4.22 \mathrm{E}+04$

$1.49 \mathrm{E}+05$

NA

$5.18 \mathrm{E}+02$

8.01E+04

NA

$5.68 \mathrm{E}+03$

$9.59 \mathrm{E}+03$

$2.04 \mathrm{E}+02$

$1.15 \mathrm{E}+03$

$1.80 \mathrm{E}+04$

$2.48 \mathrm{E} \div 02$

$1.36 \mathrm{E}-04$

$3.35 \mathrm{E}+01$

$1.12 \mathrm{E}-03$

$1.41 \mathrm{E}+04$

$1.04 \mathrm{E}-00$

$8.98 \mathrm{E}-00$

$9.68 \mathrm{E}-02$

$7.53 \mathrm{E}-03$

$7.51 \mathrm{E}-02$

$1.53 \mathrm{E}-00$

2.57E-01

$1.49 E+04$

5.52E-01

$5.91 \mathrm{E}+01$

$3.56 \mathrm{E}+01$

4.82E-02

$1.30 \mathrm{E}-00$

4.41E-01

$4.20 \mathrm{E}+01$

9.23E-02

5.41E-04

$1.86 \mathrm{E}-00$

$1.68 E+02$

$1.80 \mathrm{E}+01$

\section{1\%-ile}

$0.00 \mathrm{E}+01$

$0.00 \mathrm{E}+01 \quad 0.00 \mathrm{E}+01$

$0.00 \mathrm{E}+01$

$0.00 \mathrm{E}+01$

1.35E+02

$0.00 \mathrm{E}+01$

$0.00 \mathrm{E}+01$

$0.00 \mathrm{E}+01$

$0.00 \mathrm{E}+01$

$1.93 \mathrm{E}-00$

$0.00 \mathrm{E}+01$

$0.00 \mathrm{E}+01$

$0.00 \mathrm{E}+01$

$0.00 \mathrm{E}+01$

$0.00 E+01$

$0.00 \mathrm{E}+01$

$0.00 \mathrm{E}+01$

$0.00 \mathrm{E}+01$

$6.36 \mathrm{E}+03$

$2.71 E+03$

$2.91 \mathrm{E}+02$

$3.79 \mathrm{E}+02$

$4.20 \mathrm{E}+03$

$1.71 \mathrm{E}+02$

$3.92 \mathrm{E}+02$

$3.58 \mathrm{E}+03$

$0.00 \mathrm{E}+01$

$2.53 \mathrm{E}+02$

$2.24 \mathrm{E}-00$

$1.70 \mathrm{E}+01$

$6.62 \mathrm{E}+04$

$7.36 \mathrm{E}+01$

$8.88 E+03$

2.62E+04

$4.35 \mathrm{E}+03$

$1.01 \mathrm{E}+02$

$3.53 \mathrm{E}+03$

$6.75 \mathrm{E}+02$

$9.65 \mathrm{E}+02$

$2.68 \mathrm{E}+03$

5.77E+01

4.83E+02

$1.05 \mathrm{E}+03$

$2.98 \mathrm{E}-00$

$0.00 \mathrm{E}+01$

$1.28 \mathrm{E}+01$

$1.71 \mathrm{E}-05$

$1.34 \mathrm{E}+03$

$0.00 \mathrm{E}+01$

$0.00 \mathrm{E}+01$

$1.47 \mathrm{E}-03$

$1.15 \mathrm{E}-04$

1. $14 \mathrm{E}-03$

$1.30 \mathrm{E}-00$

2.45E-02

$1.93 \mathrm{E}+04$

$0.00 \mathrm{E}+01$

$0.00 \mathrm{E}+01$

$0.00 E+01$

4.51E-03

$0.00 \mathrm{E}+01$

$0.00 \mathrm{E}+01$

$0.00 \mathrm{E}+01$

$7.85 \mathrm{E}-03$

$0.00 \mathrm{E}+01$

2.99E-02

$1.58 \mathrm{E}+01$

$1.58 \mathrm{E}+01$

$10 \%$-ile $0.00 \mathrm{E}+01$ $0.00 \mathrm{E}+01$ $0.00 \mathrm{E}+01$ $1.39 \mathrm{E}+03$ $0.00 \mathrm{E}+01$

$0.00 \mathrm{E}+01$

$0.00 \mathrm{E}+01$

7.61E-03

$7.76 \mathrm{E}-00$

$0.00 \mathrm{E}+01$

$0.00 \mathrm{E}+01$

$0.00 \mathrm{E}+01$

$0.00 \mathrm{E}+01$

$0.00 \mathrm{E}+01$

$0.00 E+01$

$0.00 \mathrm{E}+01$

$0.00 \mathrm{E}+01$

$1.16 \mathrm{E}+04$

3. $70 \mathrm{E}+03$

$1.74 \mathrm{E}+03$

$7.38 \mathrm{E}+02$

$1.04 \mathrm{E}+04$

4.70E +02

$1.91 \mathrm{E}+03$

$6.60 \mathrm{E}+03$

3.33E-02

8.01E +02

5.18E-04 
Tank Anal. Un

$\begin{array}{ll}\text { TY103 } & \text { Pu241 } \\ \text { TY103 } & \text { Pu242 } \\ \text { TY103 } & \text { Ra226 } \\ \text { TY103 } & \text { Ra228 } \\ \text { TY103 } & \text { Ru106 } \\ \text { TY103 } & \text { Sb125 } \\ \text { TY103 } & \text { Se79 }\end{array}$

TY103

TY103 Sr89/90

TY103 Te99

TY103 Th229

TY103 Th232

TY103 Tin126

TY103 Tritium

TY103 U232

TY103 U233

TY103 U234

TY103 U235

TY103 U236

TY103 U238

TY103 Y90

TY103 Zr93

TY104 Al

TY104 Bi

TY104 Ca

TY104 CI

TY104 CO3

TY104 Cr

TY104 F

TY104 Fe

TY104 Hg

TY104 K

TY104 La

TY104 Mn

TY104 Na

TY104 Ni

TY104 NO2

TY104 NO3

TY104 P

TY104 Pb

TY104 PO4

TY104 S

TY104 Si

TY104 SO4

TY104 Sr

TY104 TOC

TY104 U

TY104 Zr

TY104 Ac227

TY104 Am241

TY104 Am243

TY104 Ba137

TY104 C14

TY104 Cd113

TY104 Cm242

TY104 Cm243

TY104 Cm244

TY104 Co60

TY104 Cs134

TY104 Cs137

TY104 Eu152

TY104 Eu154

TY104 Eu155

TY104 1129

TY104 Nb93

TY104 Ni59

TY104 Ni63

TY104 Np237
May $\frac{B B}{98}$

\section{$1 \overline{84 \mathrm{E}+01}$}

i 9.13E-05

2.18E-05

Ci 3.05E-02

Ci 6.29E-04

Ci $1.56 \mathrm{E}+01$

Ci 3.64E-01

Ci $1.28 E+03$

$9.43 E+04$

Ci $1.64 \mathrm{E}+01$

Ci 7.04E-04

Ci 8.50E-03

Ci 5.48E-01

Ci $2.45 \mathrm{E}+01$

Ci 1.52E-01

Ci 5.82E-01

Ci 7.75E-00

Ci 3.42E-01

Ci 8.20E-02

Ci 7.90E-00

Ci $\quad 9.43 E+04$

Ci 1.78E-00

kg 2.67E+03

kg $\quad 5.24 \mathrm{E}+03$

kg 1.76E+03

kg 2.05E+02

$\mathrm{kg} \quad 5.50 \mathrm{E}+03$

kg 4.15E+02

kg $\quad 1.57 \mathrm{E}+03$

kg $\quad 9.59 \mathrm{E}+03$

kg $\quad 0.00 \mathrm{E}+01$

kg 3.14E+01

kg $0.00 \mathrm{E}+01$

kg $\quad 5.09 \mathrm{E}+02$

kg $3.32 \mathrm{E}+04$

kg 4.38E+02

$\mathrm{kg} \quad 3.59 \mathrm{E}+03$

kg $\quad 1.37 \mathrm{E}+04$

kg $1.62 \mathrm{E}+03$

kg $1.69 \mathrm{E}+02$

$\mathrm{kg} \quad 5.01 \mathrm{E}+03$

kg 5.13E +02

$\mathrm{kg} \quad 3.74 \mathrm{E}+02$

kg $\quad 1.54 \mathrm{E}+03$

$\mathrm{kg} \quad 0.00 \mathrm{E}+01$

kg $\quad 5.67 \mathrm{E}+02$

kg $5.74 E+03$

kg $5.32 \mathrm{E}+01$

Ci 8.86E-06

$9.71 \mathrm{E}-00$

5.78E-0?

$1.04 \mathrm{E}+04$

9.36E-01

8.90E-02

9.93E-04

2.04E-05

1.37E-05

2.22E-00

3.04E-03

$1.10 \mathrm{E}+04$

$5.28 \mathrm{E}-02$

$1.64 \mathrm{E}-01$

$3.84 \mathrm{E}-00$

$4.58 \mathrm{E}-04$

2.93E- 02

$1.05 \mathrm{E}-01$

9.61E-00

1.51E-03
$\frac{B B}{t 98}$

Sept98

$4.82 \mathrm{E}-04$

2.18E-05

3.05E-02

$6.29 \mathrm{E}-04$

$156 \mathrm{E}+01$

3.64E-01

$1.28 \mathrm{E}+03$

$9.43 \mathrm{E}+04$

$1.64 \mathrm{E}+01$

7.04E-04

1.85E-03

5.48E-01

$2.45 \mathrm{E}+01$

1.16E-01

4.45E-01

5.92E-00

2.61E-01

6.27E-02

6.01E-00

$9.43 E+04$

$1.78 \mathrm{E}-00$

$2.67 E+03$

$5.24 \mathrm{E}+03$

$1.76 \mathrm{E}+03$

2.05E +02

$5.50 \mathrm{E}+03$

$4.15 \mathrm{E}+02$

$1.57 \mathrm{E}+03$

$9.59 \mathrm{E}+03$

$0.00 \mathrm{E}+01$

$3.14 \mathrm{E}+01$

$0.00 \mathrm{E}+01$

$5.09 \mathrm{E}+02$

$3.32 \mathrm{E}+04$

$4.38 \mathrm{E}+02$

$3.59 \mathrm{E}+03$

1.37E+04

NA

$1.69 \mathrm{E}+02$

$5.01 E+03$

NA

$3.74 E+02$

$1.54 \mathrm{E}+03$

$4.91 \mathrm{E}+01$

$5.67 \mathrm{E}+02$

$5.74 \mathrm{E}+03$

5.32E+01

8.86E-06

9.71E-00

6.93E-05

$1.04 \mathrm{E}+04$

9.36E-01

8.90E-02

1.19E-01

2.45E-03

$1.65 E-03$

$2.22 E-00$

3.04E-03

$1.10 \mathrm{E}+04$

5.28E-02

1.64E-01

$3.84 \mathrm{E}-00$

4.58E-04

2.93E-02

1.05E-01

9.61E-00

$1.51 \mathrm{E}-03$ 1\%-ile

$1.57 \mathrm{E}-00$

$10 \%$-ile

$7.77 \mathrm{E}-06$

$0.00 \mathrm{E}+01$

$0.00 \mathrm{E}+01$

$0.00 \mathrm{E}+0$

$0.00 \mathrm{E}+01$

$0.00 \mathrm{E}+01$

$0.00 \mathrm{E} \div 01$

$2.33 E+04$

$0.00 \mathrm{E}+01$

$0.00 \mathrm{E}+01$

$0.00 \mathrm{E}+01$

$0.00 \mathrm{E}+01$

$1.52 \mathrm{E}+01$

$0.00 \mathrm{E}+01$

$0.00 E+01$

$0.00 E+01$

$0.00 \mathrm{E}+01$

$0.00 \mathrm{E}+01$

$0.00 \mathrm{E}+01$

$0.00 \mathrm{E}+01$

$0.00 E+01$

$8.22 \mathrm{E}+01$

$3.44 \mathrm{E}+01$

$1.13 \mathrm{E}+01$

$1.38 \mathrm{E}+01$

8.14E+01

$2.45 E-00$

$1.89 \mathrm{E}+01$

$1.30 \mathrm{E}+02$

$0.00 \mathrm{E}+01$

$3.58 \mathrm{E}-00$

8.01E-02

$1.67 \mathrm{E}-00$

$7.51 E+02$

6.62E-01

$2.71 \mathrm{E}+02$

$2.79 \mathrm{E}+02$

$8.58 \mathrm{E}+01$

4.27E-00

$1.04 \mathrm{E}+02$

$1.21 \mathrm{E}+0 \mathrm{I}$

1. $32 \mathrm{E}+01$

$7.19 E+01$

$1.69 \mathrm{E}-00$

6.25E-00

$4.84 E+01$

$1.25 \mathrm{E}-01$

$0.00 E+01$

$7.32 \mathrm{E}-01$

8.14E-09

$3.65 \mathrm{E}+0 \mathrm{I}$

$0.00 \mathrm{E}+01$

$0.00 E \div 01$

1.40E-05

2.87E-07

1.93E-07

4.61E-02

1.07E-0S

$8.43 \mathrm{E}+02$

$0.00 \mathrm{E}+0 \mathrm{I}$

$0.00 \mathrm{E}+01$

$0.00 \mathrm{E}+01$

$4.59 \mathrm{E}-06$

8.02E-0S

$0.00 \mathrm{E}+01$

$0.00 \mathrm{E}+0 \mathrm{l}$

2.13E-05

4.27E-00

2.12E-05

$0.00 \mathrm{E}+01$

$0.00 \mathrm{E}+01$

$1.44 \mathrm{E}-04$

$5.26 \mathrm{E}-01$

$1.02 \mathrm{E}-01$

$0.00 \mathrm{E}+01$

$7.75 \mathrm{E}+04$

$1.58 \mathrm{E}-00$

$0.00 \mathrm{E}+01$

$0.00 E+01$

$5.45 \mathrm{E}-02$

$1.92 \mathrm{E}+01$

$0.00 \mathrm{E}+01$

$0.00 \mathrm{E}+01$

$0.00 E+01$

$0.00 \mathrm{E}+01$ 
Tank Anal. Un

TY104 Pa231

TY104 Pu238

TY104 Pu239

TY104 Pu240

TY104 Pu241

TY104 Pu242

TY104 Ra226.

TY104 Ra228

TY104 Ru106

TY104 Sb125

TY104 Se79

TY104 Sm151

TY104 Sr89/90

TY104 Te99

TY104 Th229

TY104 Th232

TY104 Tin126

TY104 Tritium

TY104 U232

TY104 U233

TY104 U234

TY104 U235

TY104 U236

TY104 U238

TY104 Y90

TY104 Zr93

TY105 Al

TY105 Bi

TY105 Ca

TY105 Cl

TY105 CO3

TY105 Cr

TY105 F

TY105 Fe

TY105 Hg

TY105 K

TY105 La

TY105 Mn

TY105 Na

TY105 Ni

TY105 NO2

TY105 NO3

TY105 P

TY105 Pb

TY105 PO4

TY105 S

TY105 Si

TY105 SO4

TY105 Sr

TY105 TOC

TY105 U

TY105 Zr

TY105 Ac227

TY105 Am241

TY105 Am243

TY105 Ba137

TY105 C14

TY105 Cd113

TY105 Cm242

TY105 Cm243

TY105 Cm244

TY105 Co60

TY105 Cs134

TY105 Cs137

TY105 Eu152

TY105 Eu154

TY105 Eu155

TY105 1129

\section{BB} May $\frac{B 8}{98}$

$1.96 \mathrm{E}-05$

1.36E-01

$1.69 \mathrm{E}+01$

1.65E-00

$5.97 \mathrm{E}-00$

2.73E-05

1.72E-06

1.84E-10

4.13E-09

8.59E-03

$7.36 \mathrm{E}-03$

2.73E+01

$3.18 \mathrm{E}+04$

9.06E-00

3.57E-08

9.72E-12

1.11E-02

$1.74 \mathrm{E}-01$

1.17E-04

5.36E-06

4.87E-00

2.15E-01

4.99E-02

4.95E-00

$3.18 \mathrm{E}+04$

3.49E-02

2.55E+03

$5.12 \mathrm{E}+02$

$8.37 \mathrm{E}+03$

$2.19 \mathrm{E}+03$

$1.96 \mathrm{E}+04$

$1.77 \mathrm{E}+02$

$0.00 \mathrm{E}+01$

$2.80 \mathrm{E}+04$

$0.00 \mathrm{E}+01$

$4.00 \mathrm{E}+02$

$0.00 \mathrm{E}+01$

$2.00 \mathrm{E}+02$

$1.74 \mathrm{E}+05$

$1.12 E+02$

$7.73 E+03$

$2.38 \mathrm{E}+05$

$5.13 E+04$

$5.12 E+02$

$1.59 \mathrm{E}+05$

$2.94 \mathrm{E}+03$

$4.92 \mathrm{E}+02$

$8.83 E+03$

$0.00 \mathrm{E}+01$

$1.08 \mathrm{E}+03$

$7.22 \mathrm{E}+03$

$0.00 \mathrm{E}+01$

1.32E-04

8.62E-01

6.01E-06

$9.70 \mathrm{E}+04$

9.00E-01

1.10E-00

8.09E-03

$1.66 \mathrm{E}-04$

1.42E-04

7.50E-00

8.90E-04

Ci $1.03 \mathrm{E}+05$

Ci 4.42E-01

Ci 1.85E-00

$3.33 \mathrm{E}+01$

4.60E-02
1.96E-05

3.88E-01

$4.83 \mathrm{E}+01$

4.71E-00

$1.70 \mathrm{E}+01$

7.78E-05

1.72E-06

1.84E-10

4.13E-09

8.59E-03

7.36E-03

2.73E+01

$3.18 \mathrm{E}+04$

9.06E-00

3.57E-08

9.72E-12

1.11E-02

1.74E-01

4.54E-05

2.08E-06

1.89E-00

8.33E-02

1.93E-02

1.92E-00

$3.18 \mathrm{E}+04$

3.49E-02

$2.56 \mathrm{E}+03$

$5.22 \mathrm{E}+02$

$8.37 \mathrm{E}+03$

$2.19 \mathrm{E}+03$

$1.96 \mathrm{E}+04$

$0.00 \mathrm{E}+01$

$4.08 \mathrm{E}+04$

$0.00 \mathrm{E}+01$

4.00E +02

$0.00 \mathrm{E}+01$

$2.18 \mathrm{E}+02$

2.18E +05

$1.14 \mathrm{E}+02$

$7.73 \mathrm{E}+03$

2.38E +05

NA

$1.78 \mathrm{E}+05$

NA

4.92E+02

$8.83 \mathrm{E}+03$

$7.89 \mathrm{E}+02$

$1.08 \mathrm{E}+03$

$3.25 E+04$

$0.00 \mathrm{E}+01$

1.32E-04

8.62E-01

6.01E-06

$1.02 \mathrm{E}+04$

1.05E-00

1.10E-00

8.09E-03

$1.66 \mathrm{E}-04$

1.42E-04

6.65E-00

$8.90 \mathrm{E}-04$

$1.05 E+04$

4.42E-01

$1.85 \mathrm{E}-00$

$3.33 \mathrm{E}+01$

$4.60 \mathrm{E}-02$

\section{Sept98}

$1.78 \mathrm{E}+02$
$1 \%$-ile

$10 \%-j l e$

\section{$0.00 \mathrm{E}+01$}

$1.91 \mathrm{E}-03$

2.38E-01

2.32E-02

8.40E-02

$3.84 \mathrm{E}-07$

$0.00 E+01$

$0.00 \mathrm{E}+01$

$0.00 \mathrm{E}+01$

$0.00 \mathrm{E}+01$

$0.00 \mathrm{E}+01$

$0.00 \mathrm{E}+01$

$8.75 \mathrm{E}+02$

$6.40 \mathrm{E}-02$

$0.00 \mathrm{E}+01$

$0.00 \mathrm{E}+01$

$0.00 \mathrm{E}+01$

4.50E-03

$0.00 \mathrm{E}+01$

$0.00 \mathrm{E}+01$

$0.00 \mathrm{E}+01$

$0.00 \mathrm{E}+01$

$0.00 \mathrm{E}+01$

$0.00 \mathrm{E}+01$

$0.00 \mathrm{E}+01$

9.55E-05

$1.87 \mathrm{E}+02$

$9.11 \mathrm{E}+03$

$6.04 \mathrm{E}+01$

$2.59 \mathrm{E}+02$

$3.25 \mathrm{E}+01$

4. $29 \mathrm{E}-00$

$1.25 \mathrm{E}+02$

$4.36 \mathrm{E}+03$

$0.00 \mathrm{E}+0 \mathrm{I}$

$3.73 \mathrm{E}+01$

6.09E-02

2.09E-00

$5.74 \mathrm{E}+04$

$1.00 \mathrm{E}-00$

$7.36 \mathrm{E}+02$

$1.26 \mathrm{E}+04$

$3.64 \mathrm{E}+03$

$6.77 \mathrm{E}-00$

$2.29 \mathrm{E}+03$

$1.82 E+02$

$6.53 E+02$

$1.37 E+03$

9.93E-00

$1.13 \mathrm{E}-01$

$2.61 \mathrm{E}+01$

$1.37 \mathrm{E}-00$

$0.00 \mathrm{E}+01$

$6.52 \mathrm{E}-01$

2.02E-07

$6.32 E+03$

$0.00 \mathrm{E}+0 \mathrm{I}$

$0.00 E+01$

$2.72 \mathrm{E}-04$

5.58E-06

4.78E-06

$0.00 E+01$

$5.80 \mathrm{E}-05$

$1.52 \mathrm{E}+03$

$0.00 \mathrm{E}+01$

$0.00 \mathrm{E}+01$

$0.00 \mathrm{E}+01$

$0.00 \mathrm{E}+01$

\subsection{E-07}

2.71E-02

$3.36 \mathrm{E}-00$

$3.28 \mathrm{E}-01$

$1.19 \mathrm{E}-00$

5.43E-06

4.84E-08

$5.17 \mathrm{E}-12$

5.07E-10 
Tank Anal. Un

$\begin{array}{ll}\text { TY105 } & \text { Nb93 } \\ \text { TY105 } & \text { Ni59 } \\ \text { TY105 } & \text { Ni63 } \\ \text { TY105 } & \text { Np237 } \\ \text { TY105 } & \text { Pa231 } \\ \text { TY105 } & \text { Pu238 } \\ \text { TY105 } & \text { Pu239 } \\ \text { TY105 } & \text { Pu240 } \\ \text { TY105 } & \text { Pu241 } \\ \text { TY105 } & \text { Pu242 } \\ \text { TY105 } & \text { Ra226 } \\ \text { TY105 } & \text { R2228 } \\ \text { TY105 } & \text { Ru106 } \\ \text { TY105 } & \text { Sb125 } \\ \text { TY105 } & \text { Se79 } \\ \text { TY105 } & \text { Sm151 } \\ \text { TY105 } & \text { Sr89/90 } \\ \text { TY105 } & \text { T99 }\end{array}$

TY105 Tc99

TY105 Th229

TY165 Th232

TY105 Tin126

TY105 Tritium

TY105 U232

TY105 U233

TY105 U234

TY105 U235

TY105 U236

TY105 U238

TY105 Y90

TY105 Zr93

TY106 Al

TY106 Bi

TY106 Ca

TY106 Cl

TY106 CO3

TY106 Cr

TY106 F

TY106 Fe

TY106 Hg

TY106 K

TY106 La

TY106 Mn

TY106 Na

TY106 Ni

TY106 NO2

TY106 NO3

TY106 $P$

TY106 Pb

TY106 PO4

TY106 S

TY106 Si

TY106 SO4

TY106 Sr

TY106 TOC

TY106 U

TY106 Zr

TY106 Ac227

TY106 Am241

TY106 Am243

TY106 Ba137

TY106 C14

TY106 Cd113

TY106 Cm242

TY106 Cm243

TY106 Cm244

TY106 Co60

TY106 Cs134

TY106 Cs137
May $\frac{B B}{98}$

3.79E-01

1.27E-01

$1.15 E+01$

$1.92 \mathrm{E}-02$

2.86E-04

2.54E-02

$3.53 \mathrm{E}+01$

3.31E-01

$9.99 \mathrm{E}-01$

4.50E-06

2.58E-05

1.69E-09

$4.27 \mathrm{E}-08$

9.49E-02

9.45E-02

$3.52 \mathrm{E}+02$

$2.56 \mathrm{E}+05$

4.40E-00

3.27E-07

$1.28 \mathrm{E}-10$

1.42E-01

3.24E-00

1.46E-06

7.24E-08

$7.75 \mathrm{E}-02$

3.45E-03

6.66E-04

$7.86 \mathrm{E}-02$

$2.56 \mathrm{E}+05$

4.49E-01

$6.03 \mathrm{E}+02$

$4.62 \mathrm{E}+01$

$4.60 \mathrm{E}+02$

$1.33 \mathrm{E}+02$

$1.10 \mathrm{E}+02$

$1.25 \mathrm{E}+01$

$0.00 \mathrm{E}+01$

$4.03 E+03$

$0.00 E+01$

$1.06 \mathrm{E}-00$

$0.00 E+01$

$4.88 E+01$

9.25E-00

6.02E-00

$6.19 E+02$

$1.50 \mathrm{E}+04$

$5.15 E+03$

$3.38 \mathrm{E}+01$

$1.59 \mathrm{E}+04$

$5.07 \mathrm{E}+02$

$7.87 \mathrm{E}+03$

$1.52 \mathrm{E}+03$

$5.73 \mathrm{E}+01$

$2.02 \mathrm{E}+02$

$7.27 \mathrm{E}+02$

$5.71 E+01$

3.51E-07

4.22E-00

$1.60 \mathrm{E}-08$

$1.44 \mathrm{E}+03$

1.03E-01

2.92E-03

5.00E-05

1.02E-06

3.79E-07

7.41E-01

3.17E-06

$3.17 \mathrm{E}-06$
$1.53 \mathrm{E}+03$
Sept98

$3.79 \mathrm{E}-01$

$1.27 \mathrm{E}-01$

$1.15 E+01$

$1.92 \mathrm{E}-02$

$2.86 \mathrm{E}-04$

5.02E-01

$7.64 \mathrm{E}+01$

6.56E-00

$1.98 \mathrm{E}+01$

8.90E-05

2.58E-05

1.69E-09

4.27E-08

9.49E-02

$9.45 \mathrm{E}-02$

$3.52 \mathrm{E}+02$

$2.51 \mathrm{E}+05$

$4.40 \mathrm{E}+01$

3.27E-07

$1.28 \mathrm{E}-10$

1.42E-01

$3.24 \mathrm{E}-00$

2.02E-04

9.99E-06

$1.07 \mathrm{E}+01$

4.76E-01

9.19E-02

$1.09 \mathrm{E}+01$

$2.56 \mathrm{E}+05$

4.49E-01

$6.03 \mathrm{E}+02$

$4.62 \mathrm{E}+01$

$2.37 E+02$

$1.33 \mathrm{E}+02$

$1.10 \mathrm{E}+02$

$1.26 \mathrm{E}+01$

$0.00 \mathrm{E}+01$

$4.01 E+03$

$0.00 \mathrm{E}+01$

$1.06 \mathrm{E}-00$

$0.00 \mathrm{E}+01$

$4.88 \mathrm{E}+01$

$9.28 \mathrm{E}+03$

$6.01 \mathrm{E}-00$

$6.19 \mathrm{E}+02$

$1.49 \mathrm{E}+04$

$$
\text { NA }
$$

$3.38 \mathrm{E}+01$

$5.22 E+03$

NA

1.17E+04

$1.52 \mathrm{E}+03$

$1.35 \mathrm{E}+01$

$2.01 \mathrm{E}+02$

$7.27 \mathrm{E}+02$

$5.71 \mathrm{E}+01$

3.51E-07

2.29E-03

1.60E-08

$1.51 \mathrm{E}+03$

$1.03 \mathrm{E}-01$

2.92E-03

5.00E-05

1.02E-06

3.79E-07

2.73E-04

3.17E-06

$1.59 \mathrm{E}+03$
$1 \%$-ile

\section{$0.00 \mathrm{E}+01$}

$10 \%$-ile

$0.00 \mathrm{E}+01$

$0.00 \mathrm{E}+01$

$6.46 \mathrm{E}-04$

$0.00 \mathrm{E}+01$

8.54E-04

$1.30 \mathrm{E}-01$

$1.11 \mathrm{E}-02$

3.36E-02

$1.51 \mathrm{E}-07$

$0.00 \mathrm{E}+01$

$0.00 \mathrm{E}+01$

$0.00 \mathrm{E}+01$

$0.00 \mathrm{E}+01$

2.12E-03

$0.00 \mathrm{E}+01$

4.11E-00

$7.38 \mathrm{E}-02$

$0.00 \mathrm{E}+01$

$0.00 \mathrm{E}+01$

$0.00 \mathrm{E}+01$

$1.68 \mathrm{E}-00$

$0.00 \mathrm{E}+01$

$0.00 \mathrm{E}+01$

$0.00 \mathrm{E}+01$

$0.00 \mathrm{E}+01$

$0.00 \mathrm{E}+01$

$0.00 \mathrm{E}+01$

$0.00 \mathrm{E}+01$

$0.00 \mathrm{E}+01$

7.83E-06

2.63E-06

5.57E-06

2.18E-07

$3.45 \mathrm{E}-06$

1.20E-07

$5.40 \mathrm{E}-07$

8.45E-06

$0.00 \mathrm{E}+01$

$1.26 \mathrm{E}-07$

$1.30 \mathrm{E}-09$

$1.83 \mathrm{E}-08$

2.94E-04

8.07E-09

2.50E-06

2.68E-05

2.59E-06

2.54E-08

3.36E-06

$5.99 \mathrm{E}-07$

6.40E-06

$1.78 \mathrm{E}-06$

2.11E-08

9.66E-08

1.17E-07

3.51E-09

$0.00 \mathrm{E}+01$

6.75E-09

$0.00 \mathrm{E}+01$

$0.00 \mathrm{E}+01$

$0.00 \mathrm{E}+0 \mathrm{l}$

$0.00 \mathrm{E}+0 \mathrm{I}$

$0.00 \mathrm{E}+01$

$0.00 \mathrm{E}+0 \mathrm{l}$

$0.00 \mathrm{E}+01$

2.52E-10

$0.00 \mathrm{E}+01$

$3.46 \mathrm{E}-06$

\section{$0.00 \mathrm{E}+01$}

$3.68 \mathrm{E}-02$

$3.33 \mathrm{E}-00$

$6.43 \mathrm{E}-03$

5.52E-05

8.50E-03

$1.29 \mathrm{E}-00$

1.11E-01

3.34E-01

1.51E-06

4.03E-06

2.64E-10

1.12E-08

$0.00 \mathrm{E}+01$

3.62E-02

$5.25 \mathrm{E}+01$

$3.74 \mathrm{E}+03$

$1.64 \mathrm{E}-00$

6.32E-08

2.47E-11

$1.75 \mathrm{E}-02$

2.22E-00

2.82E-07

$1.40 \mathrm{E}-08$

1.50E-02

6.66E-04 
Tank Anal. Un

$\begin{array}{ll}\text { TY106 } & \text { Eu152 } \\ \text { TY106 } & \text { Eu154 } \\ \text { TY106 } & \text { Eu155 } \\ \text { TY106 } & \text { I129 } \\ \text { TY106 } & \text { Nb93 } \\ \text { TY106 } & \text { Ni59 } \\ \text { TY106 } & \text { Ni63 } \\ \text { TY106 } & \text { Np237 } \\ \text { TY106 } & \text { Pa231 } \\ \text { TY106 } & \text { Pu238 } \\ \text { TY106 } & \text { Pu239 } \\ \text { TY106 } & \text { Pu240 }\end{array}$

TY106 Pu241

TY106 Pu242

TY106 Ra226

TY106 Ra228

TY106 Ru106

TY106 Sb125

TY106 Se79

TY106 Sm151

TY106 Sr89/90

TY106 Te99

TY106 Th229

TY106 Th232

TY106 Tin126

TY106 Tritium

TY106 U232

TY106 U233

TY106 U234

TY106 U235

TY106 U236

TY106 U238

TY106 Y90

TY106 Zr93

U101 Al

U101 Bi
U101

$\mathrm{U} 101$
$\mathrm{Cl}$

$\mathrm{U101} \mathrm{CO} 3$

$\mathrm{U} 101 \mathrm{Cr}$

U101 F

U101 Fe

U101 Hg

U101 K

U101 La

U101 Mn

U101 Na

U101 Ni

U101 NO2

U101 NO3

$\begin{array}{ll}\text { U101 } & P \\ \text { U101 } & \mathrm{Pb}\end{array}$

U101 PO4

U101 $S$

U101 Si

U101 SO4

U101 Sr

U101 TOC

U101 U

U101 $\mathrm{Zr}$

U101 Ac227

U101 Am241

U101 Am243

U101 Ba137

U101, $\mathrm{Cl} 4$

v101 Cal13

v101 Cm242

v101 Cm243

\section{Mav $\frac{B B}{98}$ \\ 2.73E-03}

$4.92 \mathrm{E}-03$

2.06E-01

5.86E-00

1.01E-03

3.06E-02

$3.06 \mathrm{E}-02$

5.11E-05

7.62E-07

6.76E-05

$3.54 \mathrm{E}-00$

i Incl. in 239

$2.66 \mathrm{E}-03$

$1.20 \mathrm{E}-08$

$6.86 \mathrm{E}-08$

1.04E-11

1.14E-10

2.53E-04

2.52E-04

9.37E-01

$9.42 \mathrm{E}+03$

$1.07 \mathrm{E}+01$

2.02E-09

3.40E-13

3.79E-04

8.63E-03

$3.89 \mathrm{E}-09$

1.93E-10

2.06E-04

9.18E-06

$1.77 \mathrm{E}-06$

2.09E-04

$9.42 \mathrm{E}+03$

$1.20 \mathrm{E}-03$

$1.48 \mathrm{E}+04$

$0.00 \mathrm{E}+01$

$3.95 \mathrm{E}+01$

$3.49 \mathrm{E}+02$

$6.10 \mathrm{E}+02$

$2.83 E+02$

$1.77 \mathrm{E}+01$

$2.38 \mathrm{E}+02$

$1.86 \mathrm{E}-02$

6.37E+01

$0.00 \mathrm{E}+01$

$1.96 \mathrm{E}+02$

$1.44 \mathrm{E}+04$

$1.74 \mathrm{E}+01$

$4.49 \mathrm{E}+03$

$1.81 \mathrm{E}+04$

$8.23 E+01$

$4.89 \mathrm{E}-00$

2.55E +02

$7.30 \mathrm{E}+01$

$1.84 \mathrm{E}+02$

$2.19 \mathrm{E}+02$

$6.49 \mathrm{E}+01$

$2.55 \mathrm{E}+02$

$1.13 \mathrm{E}+03$

$9.86 \mathrm{E}-00$

2.86E-05

9.77E-03

2.73E-08

$1.08 \mathrm{E}+04$

2.27E-01

5.73E-02

2.10E-05

3.12E-07
$B B$

Sept98

2.73E-03

4.92E-03

$2.06 \mathrm{E}-01$

$1.56 \mathrm{E}-05$

$1.01 \mathrm{E}-03$

3.39E-04

3.06E-02

5.11E-05

7.62E-07

2.17E-02

3.30E-00

2.83E-01

8.52E-01

3.82E-06

$6.86 \mathrm{E}-08$

$1.04 \mathrm{E}-11$

1.14E-10

2.53E-04

2.52E-04

9.37E-01

$9.76 \mathrm{E}+03$

$1.07 \mathrm{E}+01$

2.02E-09

3.40E-13

3.79E-04

8.63E-03

4.51E-06

2.23E-07

2.39E-01

1.06E-02

$2.06 \mathrm{E}-03$

2.43E-01

$9.76 \mathrm{E}+03$

$1.20 \mathrm{E}-03$

$1.48 \mathrm{E}+04$

$0.00 \mathrm{E}+01$

$3.95 E+01$

$3.49 \mathrm{E}+02$

$6.10 \mathrm{E}+02$

$2.83 \mathrm{E}+02$

$1.77 \mathrm{E}+01$

$2.38 \mathrm{E}+02$

$0.00 \mathrm{E}+01$

6.37E+01

$0.00 \mathrm{E}+01$

$1.96 \mathrm{E}+02$

$1.44 \mathrm{E}+04$

$1.74 \mathrm{E}+01$

$4.49 \mathrm{E}+03$

$1.81 \mathrm{E}+04$

NA

$4.89 \mathrm{E}-00$

2.55E+02

NA

$1.84 \mathrm{E}+02$

$2.19 \mathrm{E}+02$

$6.49 \mathrm{E}+01$

$2.55 E+02$

$1.13 \mathrm{E}+03$

$9.86 \mathrm{E}-00$

$2.86 \mathrm{E}-05$

2.57E-00

7.17E-06

$1.08 \mathrm{E}+04$

2.27E-01

5.73E-02

5.52E-03

8.19E-65
$1 \%$ ile

$0.00 \mathrm{E}+01$

$0.00 \mathrm{E}+01$

$0.00 \mathrm{E}+01$

$0.00 \mathrm{E}+01$

$0.00 \mathrm{E}+01$

$0.00 \mathrm{E}+01$

$0.00 \mathrm{E}+01$

$0.00 \mathrm{E}+01$

$0.00 \mathrm{E}+01$

$0.00 \mathrm{E}+01$

$0.00 \mathrm{E}+01$

$0.00 \mathrm{E}+01$

$0.00 \mathrm{E}+01$

$0.00 \mathrm{E}+01$

$0.00 \mathrm{E}+01$

$0.00 \mathrm{E}+01$

$0.00 \mathrm{E}+01$

$0.00 \mathrm{E}+01$

$0.00 \mathrm{E}+01$

$0.00 \mathrm{E}+01$

2.40E-06

$0.00 \mathrm{E}+01$

$0.00 \mathrm{E}+01$

$0.00 \mathrm{E}+01$

$0.00 \mathrm{E}+01$

$0.00 \mathrm{E}+01$

$0.00 \mathrm{E}+01$

$0.00 E+01$

$0.00 \mathrm{E}+01$

$0.00 E+01$

$0.00 \mathrm{E}+01$

$0.00 \mathrm{E}+01$

$0.00 \mathrm{E}+01$

$0.00 \mathrm{E}+01$

$1.90 \mathrm{E}-0 \mathrm{I}$

$0.00 \mathrm{E}+0$

2.97E-02

$3.88 \mathrm{E}-01$

$1.44 \mathrm{E}+02$

2.01E-02

2.64E-02

2.03E-02

$0.00 \mathrm{E}+01$

$7.20 \mathrm{E}-02$

$1.57 \mathrm{E}-05$

$0.00 E+01$

$9.61 \mathrm{E}+02$

$1.60 \mathrm{E}-03$

$3.93 \mathrm{E}-0 \mathrm{I}$

$3.41 \mathrm{E}+01$

$1.28 \mathrm{E}+01$

$0.00 \mathrm{E}+01$

2.27E-01

3.04E-00

3.07E-03

$1.56 \mathrm{E}-01$

$0.00 \mathrm{E}+01$

$9.79 \mathrm{E}-03$

$0.00 \mathrm{E}+01$

$0.00 \mathrm{E}+0$

$0.00 \mathrm{E}+01$

$0.00 E+01$

$0.00 \mathrm{E}+01$

$0.00 \mathrm{E}+01$

$1.32 \mathrm{E}-03$

$0.00 \mathrm{E}+0 \mathrm{I}$

$0.00 \mathrm{E}+01$

$0.00 \mathrm{E}+01$

$10 \%$-ile

$0.00 \mathrm{E}+01$

$0.00 \mathrm{E}+01$

$0.00 \mathrm{E}+01$

1.65E-01

6.29E-06

4.43E-04

4.43E-04

$1.37 \mathrm{E}-06$

2.62E-09

$1.81 \mathrm{E}-06$

9.48E-02

$9.48 \mathrm{E}-02$

7.13E-05

$3.21 \mathrm{E}-10$

1.37E-09

2.08E-13

3.08E-12

$0.00 \mathrm{E}+01$

5.23E-06

$0.00 E+01$

$3.25 \mathrm{E}-00$

2.24E-01

6.94E-12 
Tank Anal. Un

\begin{tabular}{|c|c|c|c|}
\hline U101 & $\mathrm{Cm} 244$ & $\mathrm{Ci}$ & $\frac{\text { Мау } \overline{98}}{4.18 \mathrm{E}-07}$ \\
\hline U101 & $\mathrm{C} 060$ & $\mathrm{Ci}$ & $3.92 \mathrm{E}-03$ \\
\hline U101 & Cs134 & $\mathrm{Ci}$ & $2.26 \mathrm{E}-06$ \\
\hline U101 & Cs137 & $\mathrm{Ci}$ & $1.14 E+04$ \\
\hline U101 & Eu152 & $\mathrm{Ci}$ & $5.23 E-03$ \\
\hline U101 & Eu154 & $\mathrm{Ci}$ & $5.27 \mathrm{E}-02$ \\
\hline U101 & Eu155 & $\mathrm{Ci}$ & $7.91 \mathrm{E}-01$ \\
\hline U101 & 1129 & $\mathrm{Ci}$ & $3.62 \mathrm{E}-04$ \\
\hline U101 & $\mathrm{Nb93}$ & $\mathrm{Ci}$ & $2.43 \mathrm{E}-02$ \\
\hline U101 & Ni59 & $\mathrm{Ci}$ & $8.01 E-03$ \\
\hline U101 & Ni63 & $\mathrm{Ci}$ & $7.03 \mathrm{E}-01$ \\
\hline U101 & Np237 & $\mathrm{Ci}$ & $1.17 \mathrm{E}-03$ \\
\hline U101 & Pa231 & $\mathrm{Ci}$ & $2.65 \mathrm{E}-05$ \\
\hline U101 & Pu238 & $\mathrm{Ci}$ & 8.81E-04 \\
\hline U101 & Pu239 & $\mathrm{Ci}$ & $2.76 \mathrm{E}-01$ \\
\hline U101 & Pu240 & $\mathrm{Ci}$ & $1.63 E-02$ \\
\hline U101 & Pu241 & $\mathbf{C i}$ & $1.04 \mathrm{E}-02$ \\
\hline U101 & Pu242 & $\mathrm{Ci}$ & $3.18 E-08$ \\
\hline U101 & Ra226 & $\mathrm{Ci}$ & 1.14E-05 \\
\hline U101 & Ra228 & $\mathrm{Ci}$ & $7.13 \mathrm{E}-11$ \\
\hline U101 & Ru106 & $\mathrm{Ci}$ & $9.24 E-11$ \\
\hline U101 & Sb125 & $C \mathrm{i}$ & 2.12E-03 \\
\hline U101 & Se79 & $C \mathrm{i}$ & 5.92E-03 \\
\hline U101 & Sm151 & $\mathbf{C i}$ & $2.24 E+01$ \\
\hline U101 & Sr89/90 & $\mathrm{Ci}$ & $4.25 E+04$ \\
\hline U101 & Te99 & Ci & $1.94 \mathrm{E}-01$ \\
\hline U101 & Th229 & $\mathrm{Ci}$ & 1.37E-08 \\
\hline U101 & Th232 & $C \mathrm{i}$ & 8.74E-12 \\
\hline U101 & Tin126 & $\mathrm{Ci}$ & 8.79E-03 \\
\hline U101 & Tritium & $\mathrm{Ci}$ & $7.91 \mathrm{E}-02$ \\
\hline U101 & U232 & $\mathrm{Ci}$ & $1.36 \mathrm{E}-04$ \\
\hline U101 & $\mathbf{U 2 3 3}$ & $\mathrm{Ci}$ & $8.12 E-06$ \\
\hline U10I & U234 & $\mathrm{Ci}$ & $1.14 E+01$ \\
\hline U101 & U235 & $\mathrm{Ci}$ & $5.15 \mathrm{E}-01$ \\
\hline U101 & U236 & $\mathrm{Ci}$ & $7.31 \mathrm{E}-02$ \\
\hline U101 & U238 & $C i$ & $1.16 E+01$ \\
\hline U101 & Y90 & $\mathrm{Ci}$ & $4.54 E+04$ \\
\hline U101 & Zr93 & $\mathrm{Ci}$ & 2.82E-02 \\
\hline $\mathrm{U} 102$ & Al & kg & $5.91 E+04$ \\
\hline U102 & $\mathbf{B i}$ & kg & $1.00 E+02$ \\
\hline U102 & $\mathrm{Ca}$ & kg & $6.80 \mathrm{E}+02$ \\
\hline U102 & Cl & kg & $9.55 E+03$ \\
\hline U102 & $\mathrm{CO3}$ & $\mathbf{k g}$ & $9.92 E+04$ \\
\hline U102 & $\mathrm{Cr}$ & $\mathrm{kg}$ & $5.98 \mathrm{E}+03$ \\
\hline U102 & $\mathbf{F}$ & $\mathrm{kg}$ & $1.35 \mathrm{E}+03$ \\
\hline U102 & $\mathbf{F e}$ & kg & $1.44 \mathrm{E}+03$ \\
\hline U102 & $\mathbf{H g}$ & $\mathrm{kg}$ & 8.60E-00 \\
\hline U102 & $\mathbf{K}$ & kg & $3.41 \mathrm{E}+03$ \\
\hline U102 & La & $\mathrm{kg}$ & $5.50 \mathrm{E}-01$ \\
\hline U102 & $\mathbf{M n}$ & kg & $6.35 \mathrm{E}+02$ \\
\hline U102 & $\mathbf{N a}$ & $\mathrm{kg}$ & $5.51 \mathrm{E}+05$ \\
\hline U102 & $\mathbf{N i}$ & $\mathrm{kg}$ & $2.06 \mathrm{E}+02$ \\
\hline U102 & $\mathrm{NO} 2$ & $\mathrm{~kg}$ & $1.07 E+05$ \\
\hline U102 & NO3 & kg & $6.69 E+05$ \\
\hline U102 & $\mathbf{P}$ & kg & $8.07 E+03$ \\
\hline U102 & $\mathbf{P b}$ & $\mathrm{kg}$ & $1.48 E+02$ \\
\hline U102 & PO4 & $\mathrm{kg}$ & $2.50 \mathrm{E}+04$ \\
\hline $\mathrm{U102}$ & $\mathbf{S}$ & kg & $1.00 E+04$ \\
\hline $\mathrm{U} 102$ & $\mathrm{Si}$ & kg & $6.94 E+02$ \\
\hline U102 & SO4 & $\mathrm{kg}$ & $3.00 \mathrm{E}+04$ \\
\hline U102 & Sr & kg & $1.17 E+02$ \\
\hline $\mathrm{U} 102$ & TOC & kg & $1.64 E+04$ \\
\hline U102 & $\mathbf{U}$ & kg & $2.14 E+03$ \\
\hline U102 & $\mathbf{Z r}$ & kg & $3.53 E+01$ \\
\hline U102 & $\mathbf{A}<22$ & $\mathrm{Ci}$ & $1.40 \mathrm{E}-03$ \\
\hline $\mathrm{U102}$ & $A m 241$ & $\mathrm{Ci}$ & $8.43 E+01$ \\
\hline U102 & Am243 & $\mathrm{Ci}$ & $3.00 \mathrm{E}-03$ \\
\hline U102 & Ba137 & $c$ & \\
\hline
\end{tabular}

\section{BB \\ Sept98}

3.92E-03

2.26E-06

$1.14 \mathrm{E}+04$

5.23E-03

5.27E-02

7.91E-01

3.62E-04

2.43E-02

8.01E-03

7.03E-01

1.17E-03

2.65E-05

2.31E-01

$7.25 \mathrm{E}+01$

4.27E-00

2.73E-00

8.35E-06

1.14E-05

7.13E-11

9.24E-11

2.12E-03

$5.92 \mathrm{E}-03$

2.24E+01

$4.25 E+04$

1.94E-01

1.37E-08

8.74E-12

8.79E-03

7.91E-02

4.42E-06

2.64E-07

3.73E-01

1.68E-02

2.38E-03

$3.77 \mathrm{E}-01$

$4.25 \mathrm{E}+04$

2.82E-02

$5.91 \mathrm{E}+04$

$4.43 E+02$

$6.79 E+02$

$9.54 \mathrm{E}+03$

$9.91 \mathrm{E}+04$

$5.97 E+03$

$3.34 \mathrm{E}+01$

$1.44 \mathrm{E}+03$

0.00E +01

$3.44 \mathrm{E}+03$

$7.63 \mathrm{E}+01$

$6.35 \mathrm{E}+02$

$5.59 \mathrm{E}+05$

2.05E+02

$1.07 \mathrm{E}+05$

$6.68 \mathrm{E}+05$

NA

$1.48 \mathrm{E}+02$

$2.48 \mathrm{E}+04$

NA

$6.94 \mathrm{E}+02$

$3.00 \mathrm{E}+04$

$1.17 \mathrm{E}+02$

$1.64 \mathrm{E}+04$

$2.43 E+03$

$3.53 E+01$

1.40E-03

$2.70 \mathrm{E}+02$

9.59E-03

$3.50 \mathrm{E}+05$
$1 \%$-ile

$0.00 \mathrm{E}+01$

$0.00 \mathrm{E}+01$

$0.00 \mathrm{E}+01$

$5.50 \mathrm{E}-00$

$0.00 \mathrm{E}+01$

$0.00 \mathrm{E}+01$

$0.00 \mathrm{E}+01$

3.67E-06

$0.00 \mathrm{E}+01$

$0.00 \mathrm{E}+01$

$0.00 E+01$

$0.00 \mathrm{E}+01$

$0.00 \mathrm{E}+01$

$0.00 \mathrm{E}+01$

$0.00 \mathrm{E}+01$

$0.00 \mathrm{E}+01$

$0.00 \mathrm{E}+01$

$0.00 \mathrm{E}+01$

$0.00 \mathrm{E}+01$

$0.00 \mathrm{E}+01$

$0.00 \mathrm{E}+01$

$0.00 \mathrm{E}+01$

$0.00 \mathrm{E}+01$

$0.00 \mathrm{E}+01$

$0.00 \mathrm{E}+01$

$0.00 \mathrm{E}+01$

$0.00 \mathrm{E}+01$

$0.00 \mathrm{E}+01$

$0.00 \mathrm{E}+01$

5.27E-03

$0.00 \mathrm{E}+01$

$0.00 \mathrm{E}+01$

$0.00 \mathrm{E}+01$

$0.00 \mathrm{E}+01$

$0.00 \mathrm{E}+01$

$0.00 \mathrm{E}+01$

$0.00 \mathrm{E}+01$

$0.00 \mathrm{E}+01$

$1.72 \mathrm{E}+04$

2.58E-01

$2.51 \mathrm{E}+02$

$7.60 \mathrm{E}+03$

$6.02 \mathrm{E}+04$

$3.36 \mathrm{E}+03$

$4.40 \mathrm{E}-01$

$1.67 \mathrm{E}+01$

$0.00 \mathrm{E}+01$

$1.40 \mathrm{E}+02$

$6.12 \mathrm{E}-01$

1.12E-01

$4.95 E+05$

8.78E-00

$7.29 E+04$

$5.06 \mathrm{E}+05$

$5.04 \mathrm{E}+03$

$5.79 \mathrm{E}+01$

$1.73 \mathrm{E}+04$

$5.55 \mathrm{E}+03$

8.10E-00

$1.42 E+04$

1.05E-01

$1.32 \mathrm{E}+04$

$1.19 \mathrm{E}+02$

4.67E-02

$0.00 \mathrm{E}+01$

$1.02 \mathrm{E}-00$

2.61E-04

$2.88 E+04$
$10 \%-i l e$

3.58E-09

$1.23 \mathrm{E}-03$

8.81E-08

$1.02 \mathrm{E}+02$

$0.00 \mathrm{E}+01$

$0.00 \mathrm{E}+01$

$0.00 \mathrm{E}+01$

3.25E-05

2.12E-04

$1.22 \mathrm{E}-03$

1.07E-01

1.00E-05

$0.00 E+01$

7.54E-06

2.36E-03

1.39E-04

8.90E-05

2.72E-10

$7.26 \mathrm{E}-07$

4.54E- 12

$7.06 \mathrm{E}-12$

2.61E-05

8.86E-04

$0.00 \mathrm{E}+01$

$1.37 \mathrm{E}+01$

$3.44 \mathrm{E}-03$

$0.00 \mathrm{E}+01$

$0.00 \mathrm{E}+01$

6.52E-04

$2.66 \mathrm{E}-02$

$0.00 \mathrm{E}+01$

$0.00 \mathrm{E}+01$

$0.00 \mathrm{E}+01$

$0.00 \mathrm{E}+01$

$0.00 \mathrm{E}+01$

$0.00 \mathrm{E}+01$

$5.45 \mathrm{E}+02$

2.47E-04

$2.36 \mathrm{E}+04$

1.71E-00

$6.07 \mathrm{E}+02$

$8.10 \mathrm{E}+03$

$8.26 E+04$

$4.28 \mathrm{E}+03$

$1.68 \mathrm{E}+01$

$7.85 \mathrm{E}+02$

$0.00 \mathrm{E}+01$

$6.01 \mathrm{E}+02$

4.54E-00

$1.16 \mathrm{E}+01$

$5.13 E+05$

$1.94 \mathrm{E}+01$

8.35E+04

$5.58 \mathrm{E}+05$

$7.53 \mathrm{E}+03$

$1.14 \mathrm{E}+02$

$2.36 \mathrm{E}+04$

$7.94 \mathrm{E}+03$

$3.73 E+02$

$2.21 E+04$

2.03E-01

$1.44 \mathrm{E}+04$

$2.37 \mathrm{E}+03$

$1.67 \mathrm{E}-01$

$0.00 \mathrm{E}+01$

1.17E-00

$1.47 \mathrm{E}-03$

$1.54 \mathrm{E}+05$ 50\%-ile

4.18E-07

1.47E-01

2.26E-06

2.35E+03

5.23E-03

5.27E-02

7.91E-01

3.62E-04

2.43E- 02

8.01E-03

7.03E-01

1.17E-03

2.65E-05

8.81E-04

2.76E-01

1.63E-02

1.04E-02

3.18E-08

1.14E-05

7.13E-11

9.24E-11

2.12E-03

5.92E-03

2.24E+01

$9.27 E+02$

$1.94 \mathrm{E}-01$

1.37E-08

8.74E-12

8.79E-03

$7.91 \mathrm{E}-02$

1.36E-04

8.12E-06

$1.14 \mathrm{E}+01$

5.15E-01

7.31E-02

$1.16 \mathrm{E}+01$

$4.54 \mathrm{E}+04$

2.82E-02

$3.13 E+04$

$5.75 E+01$

$1.27 \mathrm{E}+03$

$8.73 E+03$

$1.18 \mathrm{E}+05$

$5.35 \mathrm{E}+03$

$1.50 \mathrm{E}+03$

$2.49 \mathrm{E}+03$

8.60E-00

$2.81 \mathrm{E}+03$

$3.26 \mathrm{E}+01$

$3.23 \mathrm{E}+02$

$5.39 \mathrm{E}+05$

$2.08 \mathrm{E}+02$

$9.65 \mathrm{E}+04$

$6.23 E+05$

$1.11 E+04$

$1.80 \mathrm{E}+02$

$3.48 \mathrm{E}+04$

$1.10 E+04$

$1.59 \mathrm{E}+03$

3.12E+04

$1.15 E+01$

$1.60 \mathrm{E}+04$

$1.95 E+04$

$3.03 E+01$

1.40E-03

$3.99 \mathrm{E}+03$

3.00E-03

$2.90 \mathrm{E}+05$

90\%-ile

$6.70 \mathrm{E}-06$

$1.55 \mathrm{E}-00$

$1.76 \mathrm{E}-05$

$1.83 \mathrm{E}+04$

$4.16 \mathrm{E}-02$

$4.19 \mathrm{E}-01$

$6.28 \mathrm{E}-00$

2.78E-03 
Tank Anal. Un

\begin{tabular}{|c|c|}
\hline U102 & C14 \\
\hline $\mathrm{U102}$ & Cd113 \\
\hline U102 & Cm242 \\
\hline U102 & $\mathrm{Cm} 243$ \\
\hline U102 & Cm244 \\
\hline U102 & Co60 \\
\hline U102 & Cs134 \\
\hline U102 & Cs137 \\
\hline $\mathbf{U 1 0 2}$ & Eu152 \\
\hline U102 & Eu154 \\
\hline U102 & Eu155 \\
\hline U102 & I129 \\
\hline $\mathrm{U} 102$ & Nb93 \\
\hline U102 & Ni59 \\
\hline U102 & Ni63 \\
\hline $\mathbf{U 1 0 2}$ & Np237 \\
\hline U102 & Pa231 \\
\hline U102 & Pu238 \\
\hline U102 & Pu239 \\
\hline U102 & Pu240 \\
\hline U102 & Pu241 \\
\hline U102 & Pu242 \\
\hline U102 & Ra226 \\
\hline U102 & $\operatorname{Ra} 228$ \\
\hline U102 & Ru106 \\
\hline U102 & Sb125 \\
\hline U102 & Se79 \\
\hline U102 & Sm151 \\
\hline U102 & Sr89/90 \\
\hline U102 & Tc99 \\
\hline U102 & Th229 \\
\hline U102 & Th232 \\
\hline U102 & $\operatorname{Tin} 126$ \\
\hline U102 & Tritium \\
\hline U102 & U232 \\
\hline U102 & U233 \\
\hline U102 & U234 \\
\hline U102 & U235 \\
\hline U102 & U236 \\
\hline U102 & U238 \\
\hline U102 & Y90 \\
\hline U102 & Zr93 \\
\hline U103 & Al \\
\hline U103 & $\mathbf{B i}$ \\
\hline U103 & $\mathrm{Ca}$ \\
\hline U103 & Cl \\
\hline vio3 & $\mathrm{CO3}$ \\
\hline U103 & $\mathrm{Cr}$ \\
\hline U103 & $\mathbf{F}$ \\
\hline U103 & $\mathrm{Fe}$ \\
\hline U103 & Hg \\
\hline U103 & $\mathbf{K}$ \\
\hline U103 & $\mathbf{L a}$ \\
\hline U103 & Mn \\
\hline U103 & $\mathbf{N a}$ \\
\hline U103 & $\mathbf{N i}$ \\
\hline U103 & $\mathrm{NO} 2$ \\
\hline U103 & NO3 \\
\hline U103 & $\mathbf{P}$ \\
\hline U103 & $\mathbf{P b}$ \\
\hline U103 & PO4 \\
\hline U103 & $\mathbf{S}$ \\
\hline $\mathrm{U} 103$ & Si \\
\hline U103 & SO4 \\
\hline U103 & Sr \\
\hline U103 & TO \\
\hline U103 & $\mathbf{U}$ \\
\hline U103 & $\mathbf{Z r}$ \\
\hline
\end{tabular}

$\frac{B B}{98}$ $4 . \overline{95 \mathrm{E}+01}$ $1.27 \mathrm{E}+02$ 2.25E-01 2.09E-02 2.03E-01 $5.49 \mathrm{E}+01$

4.09E-00 $3.07 \mathrm{E}+05$

5.91E-00 $8.96 \mathrm{E}+02$ $3.52 \mathrm{E}+02$ $6.74 \mathrm{E}-01$ $1.75 E+01$ $3.24 \mathrm{E}-00$

$3.18 \mathrm{E}+02$

1.26E-00

$6.21 \mathrm{E}-03$

2.06E-00

$3.51 \mathrm{E}+02$

$1.20 \mathrm{E}+01$

$1.39 \mathrm{E}+02$

7.65E-04

2.33E-04

2.26E-01

$9.97 \mathrm{E}-03$

$2.37 E+02$

$4.92 \mathrm{E}-00$

$1.73 E+04$

$8.10 \mathrm{E}+04$

$3.50 \mathrm{E}+02$

$5.30 \mathrm{E}-03$

1.50E-02

$7.43 \mathrm{E}-00$

$3.35 E+02$

$1.16 \mathrm{E}-00$

4.44E-00

$2.36 \mathrm{E}+01$

$1.06 \mathrm{E}-00$

$1.81 \mathrm{E}-01$

$2.41 E+01$

8.10E +04

$2.41 \mathrm{E}+01$

$5.82 \mathrm{E}+04$

$2.73 E+02$

$8.18 \mathrm{E}+02$

$1.05 E+04$

$6.14 \mathrm{E}+04$

$1.33 E+04$

$1.21 \mathrm{E}+04$

$3.90 \mathrm{E}+03$

3.52E-00

$2.99 \mathrm{E}+03$

$7.63 \mathrm{E}+01$

$1.76 \mathrm{E}+03$

$5.15 \mathrm{E}+05$

$3.67 \mathrm{E}+02$

$1.45 \mathrm{E}+05$

$6.29 \mathrm{E}+05$

$2.38 \mathrm{E}+04$

4.33E+02

$7.38 \mathrm{E}+04$

$1.17 \mathrm{E}+04$

$4.32 \mathrm{E}+03$

$3.51 \mathrm{E}+04$

$1.25 \mathrm{E}+02$

$1.97 \mathrm{E}+04$

4.12E+03

$1.12 \mathrm{E}+02$
$\underline{B B}$ Sept98 $4.95 \mathrm{E}+01$

$1.27 \mathrm{E}+02$

$7.20 \mathrm{E}-01$

6.67E-02

6.50E-01

$5.49 \mathrm{E}+01$

4.09E-00

$3.70 \mathrm{E}+05$

5.91E-00

$8.96 \mathrm{E}+02$

$3.52 \mathrm{E}+02$

$6.74 \mathrm{E}-01$

$1.75 \mathrm{E}+01$

3.24E-00

$3.18 \mathrm{E}+02$

$1.26 \mathrm{E}-00$

6.21E-03

6.59E-00

$2.26 \mathrm{E}+02$

$3.82 \mathrm{E}+01$

$4.45 E+02$

2.45E-03

2.33E-04

2.26E-01

9.97E-03

$2.37 \mathrm{E}+02$

4.92E-00

$1.73 \mathrm{E}+04$

$1.66 \mathrm{E}+05$

$3.50 \mathrm{E}+02$

$5.30 \mathrm{E}-03$

$1.50 \mathrm{E}-02$

7.43E-00

$3.35 \mathrm{E}+02$

$3.96 \mathrm{E}-02$

1.52E-01

8.06E-01

$3.60 \mathrm{E}-02$

$6.20 \mathrm{E}-03$

8.11E-01

$1.66 \mathrm{E}+05$

$2.41 \mathrm{E}+01$

$5.82 \mathrm{E}+04$

$2.73 \mathrm{E}+02$

$8.18 \mathrm{E}+02$

$1.05 \mathrm{E}+04$

$6.14 \mathrm{E}+04$

1.33E+04

$1.21 E+04$

$3.90 \mathrm{E}+03$

$0.00 \mathrm{E}+01$

$2.99 \mathrm{E}+03$

$0.00 \mathrm{E}+01$

$1.76 E+03$

$5.15 \mathrm{E}+05$

$3.67 \mathrm{E}+02$

$1.45 \mathrm{E}+05$

$6.29 \mathrm{E}+05$

NA

4.33E +02

$7.38 \mathrm{E}+0$

NA

$4.32 E+03$

$3.51 E+04$

$1.25 E+02$

$1.97 \mathrm{E}+04$

4.12E +03

1.12E+02

1\%-ile

$10 \%$-ile

50\%-ile

90\%-ile $9.56 \mathrm{E}-00 \quad 2.51 \mathrm{E}+01 \quad 4.95 \mathrm{E}+01 \quad 1.14 \mathrm{E}+02$ $0.00 \mathrm{E}+01$ $1.96 \mathrm{E}-02$ $1.82 \mathrm{E}-03$

$1.76 \mathrm{E}-02$

$1.52 \mathrm{E}-00$ $0.00 \mathrm{E}+01$

$1.10 \mathrm{E}-01$

$1.02 \mathrm{E}-02$

$1.27 \mathrm{E}+02$

$2.66 \mathrm{E}+02$

2.25E-01 3.40E-01

2.09E-02 3.16E-02

$9.95 \mathrm{E}-02$
$1.95 \mathrm{E}-00$

2.03E-01

3.07E-01

$3.81 \mathrm{E}+01$

$4.09 \mathrm{E}-00$

$6.20 \mathrm{E}-00$

$2.82 \mathrm{E}+05$

$0.00 \mathrm{E}+01$

$3.06 \mathrm{E}+05$

$0.00 \mathrm{E}+01$

$3.32 \mathrm{E}+05$

$3.61 \mathrm{E}+05$

$0.00 \mathrm{E}+01$

$0.00 \mathrm{E}+01$

3.05E-01

5.91E-00

$2.21 \mathrm{E}+01$

$8.96 \mathrm{E}+02 \quad 3.35 \mathrm{E}+03$

$3.52 \mathrm{E}+02$

$6.74 \mathrm{E}-01$

$1.31 \mathrm{E}+03$

$1.04 \mathrm{E}-00$

.25E-02

$0.00 \mathrm{E}+01$

$0.00 \mathrm{E}+01$

$1.75 E+01$

3.24E-00

$.00 \mathrm{E}+01$

$0.00 \mathrm{E}+01$

$1.09 \mathrm{E}-01$

$0.00 \mathrm{E}+01$

$6.18 \mathrm{E}-01$

$3.18 \mathrm{E}+02$

$1.26 \mathrm{E}-00$

$3.80 \mathrm{E}+02$

8.53E-00

8.37E+02

$1.90 \mathrm{E}-00$

1.00E+01

$0.00 \mathrm{E}+01$

$1.01 \mathrm{E}-00$

6.21E-03

$3.05 \mathrm{E}+01$

$1.72 \mathrm{E}+02$

2.06E-00

2.22E-02

$5.30 \mathrm{E}+02$

$1.04 \mathrm{E}-00$

$5.88 \mathrm{E}-00$

$6.81 \mathrm{E}+01$

$6.65 \mathrm{E}-05$

$0.00 \mathrm{E}+01$

3.75E-04

$0.00 \mathrm{E} \div 01$

$1.20 \mathrm{E}+01$

$1.39 \mathrm{E}+02$

7.65E-04

$1.81 \mathrm{E}+01$

$2.10 \mathrm{E}+02$

$1.16 \mathrm{E}-03$

2.33E-04

$0.00 \mathrm{E}+01$

$0.00 \mathrm{E}+01$

$0.00 \mathrm{E}+01$

2.59E-01

$0.00 \mathrm{E}+01$

$5.57 \mathrm{E}+02$

$0.00 \mathrm{E}+01$

$0.00 \mathrm{E}+01$

$0.00 \mathrm{E}+01$

$0.00 \mathrm{E}+01$

$2.67 \mathrm{E}+02$

$0.00 \mathrm{E}+01$

$0.00 \mathrm{E}+01$

$0.00 \mathrm{E}+01$

$0.00 \mathrm{E}+01$

$0.00 \mathrm{E}+01$

$0.00 \mathrm{E}+01$

$0.00 \mathrm{E}+01$

$0.00 \mathrm{E}+01$

$3.75 \mathrm{E}+04$

$1.89 \mathrm{E}-04$

$6.04 \mathrm{E} \div 02$

$9.92 \mathrm{E}+03$

$6.26 \mathrm{E}+04$

$6.50 \mathrm{E}+03$

$1.50 \mathrm{E}-01$

$8.09 \mathrm{E}+02$

$0.00 \mathrm{E}+01$

4.26E-01

$3.66 \mathrm{E}+01$

$1.96 \mathrm{E}-01$

$5.13 E+05$

2.27E-02

$1.11 \mathrm{E}+05$

$3.06 \mathrm{E}-03$

2.26E-01

$2.30 \mathrm{E}-03$

2.23E- 00

4.03E-02

$0.00 \mathrm{E}+01$

2.42E-00

$0.00 \mathrm{E}+01$

$6.34 \mathrm{E}+02$

$0.00 \mathrm{E}+01$

$0.00 \mathrm{E}+01$

$0.00 \mathrm{E}+01$

$0.00 \mathrm{E}+01$

$2.93 \mathrm{E}+02$

$0.00 \mathrm{E}+01$

$0.00 \mathrm{E}+01$

$0.00 \mathrm{E}+01$

$0.00 \mathrm{E}+01$

$0.00 \mathrm{E}+01$

$0.00 \mathrm{E}+01$

$0.00 \mathrm{E}+01$

$0.00 \mathrm{E}+01$

$4.53 \mathrm{E}+04$

$1.85 E-01$

$1.08 \mathrm{E}+03$

$1.06 \mathrm{E}+04$

$9.31 \mathrm{E}+04$

$7.60 \mathrm{E}+03$

$1.16 \mathrm{E}-00$

$2.21 \mathrm{E}+03$

$0.00 \mathrm{E}+01$

$3.08 E+02$

$6.92 \mathrm{E}+01$

$2.13 \mathrm{E}+02$

$5.33 \mathrm{E}+05$

4.94E-00

$1.25 \mathrm{E}+05$

$5.61 \mathrm{E}+05$ 


\begin{tabular}{|c|c|}
\hline U103 & Ac227 \\
\hline U103 & Am241 \\
\hline U103 & Am243 \\
\hline U103 & Ba137 \\
\hline U103 & C14 \\
\hline U103 & Cd113 \\
\hline U103 & $\mathrm{Cm}_{242}$ \\
\hline U103 & Cm243 \\
\hline U103 & Cm244 \\
\hline U103 & Co60 \\
\hline U103 & Cs134 \\
\hline U103 & Cs137 \\
\hline U103 & Eu152 \\
\hline U103 & Eu154 \\
\hline U103 & Eu155 \\
\hline U103 & 1129 \\
\hline U103 & Nb93 \\
\hline U103 & Ni59 \\
\hline U103 & Ni63 \\
\hline U103 & Np237 \\
\hline U103 & Pa231 \\
\hline U103 & Pu238 \\
\hline v103 & Pu239 \\
\hline U103 & Pu240 \\
\hline U103 & Pu241 \\
\hline U103 & Pu242 \\
\hline U103 & Ra226 \\
\hline U103 & Ra228 \\
\hline U103 & Ru106 \\
\hline U103 & Sb125 \\
\hline U103 & Se79 \\
\hline U103 & Sm151 \\
\hline U103 & Sr89/90 \\
\hline U103 & Te99 \\
\hline U103 & Th229 \\
\hline U103 & Th232 \\
\hline U103 & Tin126 \\
\hline U103 & Tritium \\
\hline U103 & U232 \\
\hline U103 & U233 \\
\hline U103 & U234 \\
\hline U103 & U235 \\
\hline U103 & U236 \\
\hline U103 & U238 \\
\hline U103 & Y90 \\
\hline U103 & Zr93 \\
\hline U104 & Al \\
\hline U104 & $\mathbf{B i}$ \\
\hline U104 & $\mathrm{Ca}$ \\
\hline U104 & $\mathrm{Cl}$ \\
\hline U104 & $\mathrm{CO} 3$ \\
\hline U104 & $\mathrm{Cr}$ \\
\hline v104 & $\mathbf{F}$ \\
\hline v104 & $\mathrm{Fe}$ \\
\hline v104 & $\mathbf{H g}$ \\
\hline U104 & $\mathbf{K}$ \\
\hline v104 & La \\
\hline U104 & Mn \\
\hline U104 & $\mathbf{N a}$ \\
\hline U104 & $\mathbf{N i}$ \\
\hline U104 & $\mathrm{NO} 2$ \\
\hline U104 & NO3 \\
\hline U104 & $\mathbf{P}$ \\
\hline U104 & $\mathbf{P b}$ \\
\hline $\mathrm{U} 104$ & PO4 \\
\hline 0104 & $S$ \\
\hline $\begin{array}{l}U 104 \\
\text { U104 }\end{array}$ & \\
\hline
\end{tabular}

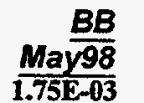

\subsection{E-03}

$1.91 \mathrm{E}+03$

3.79E-03

$3.89 \mathrm{E}+05$

$9.27 \mathrm{E}+01$

$1.61 \mathrm{E}+02$

2.85E-01

2.64E-02

2.57E-01

$6.96 \mathrm{E}+01$

5.09E-00

$4.11 \mathrm{E}+05$

7.46E-00

$1.13 \mathrm{E}+03$

$4.43 E+02$

8.56E-01

$2.22 \mathrm{E}+01$

$4.09 \mathrm{E}-00$

$4.01 \mathrm{E}+02$

1.61E-00

7.84E-03

2.60E-00

$6.10 \mathrm{E}+01$

$1.51 \mathrm{E}+01$

$1.76 \mathrm{E}+02$

9.67E-04

2.84E-04

2.92E-01

1.26E-02

$3.01 \mathrm{E}+02$

6.23E-00

$2.19 \mathrm{E}+04$

$3.11 E+05$

$4.44 \mathrm{E}+02$

6.83E-03

$1.92 \mathrm{E}-02$

9.42E-00

$4.27 \mathrm{E}+02$

$1.49 \mathrm{E}-00$

5.71E-00

$1.82 \mathrm{E}+01$

8.11E-01

1.55E-01

$1.87 \mathrm{E}+01$

$3.11 E+05$

$3.06 \mathrm{E}+01$

$3.47 \mathrm{E}+04$

$2.96 \mathrm{E}+01$

$5.02 \mathrm{E}+02$

$2.08 E+03$

$1.61 \mathrm{E}+04$

8.77E +02

$5.63 \mathrm{E}+02$

$4.59 \mathrm{E}+03$

9.60E-01

$5.86 \mathrm{E}+02$

$1.24 \mathrm{E}+01$

$4.22 \mathrm{E}+02$

$8.45 \mathrm{E}+04$

$5.68 \mathrm{E}+01$

$1.66 \mathrm{E}+04$

1.18E+05

$8.33 E+02$

$4.65 \mathrm{E}+01$

$2.58 \mathrm{E}+03$

$1.64 \mathrm{E}+03$

$2.38 E+04$ $4.91 \mathrm{E}+03$
Sept98

$1.75 \mathrm{E}-03$

$1.07 \mathrm{E}+02$

$3.79 \mathrm{E}-03$

$3.89 \mathrm{E}+05$

$6.27 \mathrm{E}+01$

$1.61 \mathrm{E}+02$

2.85E-01

2.64E- 02

2.57E-01

$6.96 \mathrm{E}+01$

$5.09 \mathrm{E}-00$

$4.11 \mathrm{E}+05$

$7.46 \mathrm{E}-00$

$1.13 \mathrm{E}+03$

$4.43 \mathrm{E}+02$

8.56E-01

$2.22 \mathrm{E}+01$

4.09E-00

$4.01 \mathrm{E}+02$

1.61E-00

7.84E-03

$2.60 \mathrm{E}-00$

$8.93 E+01$

$1.51 \mathrm{E}+01$

$1.76 \mathrm{E}+02$

9.67E-04

2.84E-04

2.92E-01

1.26E-02

$3.01 E+02$

6.23E-00

$2.19 \mathrm{E}+04$

$3.46 \mathrm{E}+05$

$4.44 \mathrm{E}+02$

$6.83 \mathrm{E}-03$

1.92E-02

9.42E-00

$4.27 \mathrm{E}+02$

1.12E-01

4.30E-01

1.37E-00

6.11E-02

1.17E-02

$1.38 \mathrm{E}-00$

$3.46 \mathrm{E}+05$

$3.06 \mathrm{E}+01$

$3.47 \mathrm{E}+04$

$0.00 \mathrm{E}+01$

$5.02 \mathrm{E}+02$

$2.08 E+03$

$1.61 \mathrm{E}+04$

$8.77 E+02$

$5.63 \mathrm{E}+02$

$4.59 \mathrm{E}+03$

$0.00 \mathrm{E}+0 \mathrm{I}$

$5.86 \mathrm{E}+02$

$2.46 \mathrm{E}+01$

$4.22 \mathrm{E}+02$

$8.45 E+04$

$5.68 \mathrm{E}+01$

$1.66 \mathrm{E}+04$

1.18E+05

NA

4.65E +01

$2.58 E+03$

NA

$2.38 \mathrm{E}+04$ $4.91 E+03$
$1 \%$ ile

\section{$0.00 \mathrm{E}+01$}

$0.00 \mathrm{E}+01$

8.00E-04

$0.00 \mathrm{E}+01$

$0.00 \mathrm{E}+01$

$0.00 \mathrm{E}+01$

$6.01 \mathrm{E}-02$

$5.57 \mathrm{E}-03$

5.42E-02

$0.00 \mathrm{E}+01$

$0.00 \mathrm{E}+01$

$3.59 \mathrm{E}+02$

$0.00 \mathrm{E}+01$

$0.00 \mathrm{E}+01$

$0.00 \mathrm{E}+01$

2.33E-02

$0.00 \mathrm{E}+01$

$0.00 \mathrm{E}+01$

$0.00 \mathrm{E}+01$

$3.40 \mathrm{E}-01$

$0.00 \mathrm{E}+01$

$5.49 \mathrm{E}-01$

$1.29 \mathrm{E}+01$

3.19E-00

$3.71 \mathrm{E}+01$

2.04E-04

$0.00 \mathrm{E}+01$

$0.00 \mathrm{E}+01$

$1.83 \mathrm{E}-03$

$0.00 E+01$

$0.00 \mathrm{E}+01$

$0.00 \mathrm{E}+01$

$0.00 \mathrm{E}+01$

$0.00 \mathrm{E}+01$

$0.00 \mathrm{E}+01$

$0.00 \mathrm{E}+01$

$0.00 \mathrm{E}+01$

$3.58 \mathrm{E}+02$

$0.00 \mathrm{E}+01$

$0.00 \mathrm{E}+01$

$0.00 \mathrm{E}+01$

$0.00 \mathrm{E}+01$

$0.00 \mathrm{E}+01$

$0.00 \mathrm{E}+01$

$0.00 \mathrm{E}+01$

$0.00 \mathrm{E}+01$

$2.63 \mathrm{E}+02$

$6.96 \mathrm{E}-02$

$1.68 \mathrm{E}+02$

$3.24 \mathrm{E}-00$

$9.09 \mathrm{E}+03$

7.91E-00

4.77E- 01

$4.58 \mathrm{E}+01$

$0.00 \mathrm{E}+01$

3.55E-00

3.66E-02

$7.89 \mathrm{E}-02$

$5.39 \mathrm{E}+04$

4.07E-01

$4.08 \mathrm{E}+02$

$6.88 \mathrm{E}+02$

$5.52 \mathrm{E}+02$

4.37E-01

$9.53 \mathrm{E}+02$

$2.65 \mathrm{E}+02$

3.89E-00 $4.98 \mathrm{E}+02$
$10 \%$-ile

\section{$0.00 \mathrm{E}+01$}

2.02E-03

2.13E-03

8.38E+04

$3.55 \mathrm{E}+01$

$0.00 \mathrm{E}+01$

1.60E-01

$1.49 \mathrm{E}-02$

$1.45 \mathrm{E}-01$

8.65E-03

$1.10 \mathrm{E}-00$

$1.65 \mathrm{E}+05$

$0.00 \mathrm{E}+01$

$0.00 \mathrm{E}+01$

$0.00 \mathrm{E}+01$

$4.08 \mathrm{E}-01$

$0.00 \mathrm{E}+01$

$0.00 \mathrm{E}+01$

$0.00 \mathrm{E}+01$

$9.06 \mathrm{E}-01$

$0.00 \mathrm{E}+01$

$1.46 \mathrm{E}-00$

$3.43 \mathrm{E}+01$

$8.50 \mathrm{E}-00$

$9.90 \mathrm{E}+01$

5.44E-04

$0.00 \mathrm{E}+01$

$0.00 \mathrm{E}+01$

$6.27 \mathrm{E}-03$

$0.00 \mathrm{E}+01$

2.59E-00

$0.00 \mathrm{E}+01$

$6.70 \mathrm{E}-00$

$0.00 \mathrm{E}+01$

$0.00 \mathrm{E}+01$

$0.00 \mathrm{E}+01$

$0.00 \mathrm{E}+01$

$3.89 \mathrm{E}+02$

$0.00 \mathrm{E}+0 \mathrm{I}$

$0.00 \mathrm{E}+01$

$0.00 E+01$

$0.00 \mathrm{E}+01$

$0.00 \mathrm{E}+01$

$0.00 \mathrm{E}+01$

$0.00 \mathrm{E}+01$

$0.00 E+01$

$2.12 E+03$

$5.59 \mathrm{E}-01$

$4.35 \mathrm{E}+02$

$7.61 \mathrm{E}+01$

$1.41 \mathrm{E} \div 04$

$2.95 \mathrm{E}+02$

$2.26 \mathrm{E}-00$

$5.30 \mathrm{E}+02$

$0.00 \mathrm{E}+01$

$6.20 \mathrm{E}+01$

$3.71 \mathrm{E}-01$

$5.89 \mathrm{E}-01$

$6.90 \mathrm{E}+04$

$5.53 \mathrm{E}-00$

$7.01 \mathrm{E}+03$

$1.27 \mathrm{E}+04$

$1.11 \mathrm{E}+03$

$5.57 \mathrm{E}-00$

$2.64 \mathrm{E}+03$

$5.39 \mathrm{E}+02$

$2.08 \mathrm{E}+02$

$1.45 \mathrm{E}+03$

$50 \%$-ile

\subsection{E-03}

1.36E+04

3.79E-03

$3.89 \mathrm{E}+05$

$9.27 \mathrm{E}+01$ 
Tank Anal. Un

\begin{tabular}{|c|c|c|c|}
\hline & & & - \\
\hline 104 & $\mathbf{S r}$ & & $1.15 E+0$ \\
\hline 104 & TOC & & $3.01 \mathrm{E}+0$ \\
\hline 104 & & & $17 \mathbf{E}+0$ \\
\hline 04 & $\mathbf{Z r}$ & & $27 E+0$ \\
\hline 04 & 247 & & $.63 \mathrm{E}$ \\
\hline 04 & 1241 & & $.60 \mathrm{E}-$ \\
\hline 04 & n243 & & $2.92 \mathrm{E}-0$ \\
\hline & 137 & $\pi^{\circ}$ & $4.87 \mathrm{E}+0$ \\
\hline 04 & C14 & & 4.88E- \\
\hline 04 & Cd113 & 0 & $1.48 \mathrm{E}+0$ \\
\hline 04 & $n 242$ & $\mathrm{Ci}$ & 1.80 \\
\hline 34 & $n 243$ & C & 1.37 \\
\hline & 244 & & \\
\hline & & & \\
\hline 4 & & & 8.24 \\
\hline 44 & 137 & C & $5.15 \mathrm{~K}$ \\
\hline & 152 & $\mathrm{Ci}$ & 6.07E-0 \\
\hline & & C & $81 E+0$ \\
\hline & 155 & 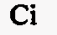 & $3.82 E+0$ \\
\hline & & & 6.2 \\
\hline & & $\mathrm{Ci}$ & .17 \\
\hline & & - & $5.65 \mathrm{E}-0]$ \\
\hline & Ni63 & 0 & $5.50 \mathrm{E}+0$ \\
\hline & 237 & & \\
\hline & & & 1.01 \\
\hline & & & \\
\hline & & & 7. \\
\hline 14 & Pu240 & & 1.19 \\
\hline 04 & Pu241 & & 1.201 \\
\hline & Pu242 & & \\
\hline & 226 & & \\
\hline & & & \\
\hline & & & \\
\hline 14 & Sb125 & & $1.59 \mathrm{E}+0$ \\
\hline 04 & Se79 & & 6.05 \\
\hline & Sm151 & & \\
\hline 4 & 190 & c & \\
\hline & & & 3.27 \\
\hline & 229 & & 04 \\
\hline 94 & Th232 & & \\
\hline & 126 & $\mathbf{C i}$ & 9.1 \\
\hline & 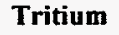 & $\mathbf{C i}$ & \\
\hline & & $\Omega^{\circ}$ & \\
\hline & & 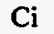 & $3.97 \mathrm{E}-01$ \\
\hline & 34 & 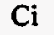 & $2.11 E+01$ \\
\hline & & 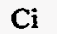 & $9.47 E-01$ \\
\hline & & $\mathrm{Ci}$ & -01 \\
\hline & & & +01 \\
\hline & & & \\
\hline & & & \\
\hline & A & & 4.4 \\
\hline & $\mathbf{b}$ & k & $2.31 E+02$ \\
\hline & c & 5 & $6.88 \mathrm{E}+02$ \\
\hline & 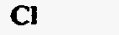 & & \\
\hline & & & \\
\hline & & & \\
\hline & $\mathbf{F}$ & & $3.01 \mathrm{E}+03$ \\
\hline & $\mathbf{F}$ & & $7.10 \mathrm{E}+03$ \\
\hline & $\mathbf{H}$ & & $3.19 \mathrm{E}-0$ \\
\hline & $\mathbf{K}$ & kg & $3.68 \mathrm{E}+03$ \\
\hline & La & kg & $9.82 \mathrm{E}+01$ \\
\hline & $\mathbf{M n}$ & & $2.50 \mathrm{E}+0$ \\
\hline & $\mathbf{N}$ & & $5.02 \mathrm{E}+0$ \\
\hline & $N$ & & $3.65 \mathrm{E}+0$ \\
\hline & & & $1.80 \mathrm{E}+0$ \\
\hline & & & \\
\hline & $P$ & Ki & \\
\hline & & & \\
\hline
\end{tabular}

\section{BB} Sept98 $1.15 E+02$ $3.01 \mathrm{E}+03$ $2.17 E+03$ $2.27 \mathrm{E}+01$

2.63E-04

$9.60 \mathrm{E}-00$

2.92E-04

$4.87 \mathrm{E}+04$

$4.88 \mathrm{E}-00$

$1.48 \mathrm{E}+01$

1.80E-02

$1.37 \mathrm{E}-03$

1.69E-02

4.30E-00

8.24E-01

$5.15 \mathrm{E}+04$

6.07E-01

$7.81 \mathrm{E}+01$

$3.82 \mathrm{E}+01$

6.29E-02

2.17E-00

5.65E-01

$5.50 \mathrm{E}+01$

1.23E-01

1.01E-03

2.01E-01

7.99E-00

1.19E-00

$1.20 \mathrm{E}+01$

5.95E-05

5.01E-05

2.61E-03

7.02E-04

$1.59 \mathrm{E}+01$

6.05E-01

$2.13 E+03$

$7.72 E+04$

$3.27 \mathrm{E}+01$

1.52E-04

6.97E-04

9.10E-01

$3.80 \mathrm{E}+01$

3.52E-03

1.35E-02

7.17E-01

3.22E-02

$4.89 \mathrm{E}-03$

$7.24 \mathrm{E}-01$

$7.72 \mathrm{E}+04$

2.99E-00

$4.48 \mathrm{E}+04$

$5.41 E+01$

$6.88 \mathrm{E}+02$

$1.05 \mathrm{E}+04$

$1.26 \mathrm{E}+05$

$6.29 \mathrm{E}+03$

$3.01 E+03$

$7.10 \mathrm{E}+03$

$0.00 \mathrm{E}+01$

$3.68 \mathrm{E}+03$

$5.16 \mathrm{E}+01$

$2.50 \mathrm{E}+03$

$5.02 E+05$

$3.65 \mathrm{E}+02$

$1.80 \mathrm{E}+05$

$7.52 E+05$

NA

$4.92 \mathrm{E}+02$ 1\%-ile

\section{$1.59 \mathrm{E}-02$}

$2.59 \mathrm{E}-00$

4.65E-03

$9.22 \mathrm{E}-03$

$0.00 E+01$

$0.00 \mathrm{E}+01$

$0.00 \mathrm{E}+01$

$0.00 \mathrm{E}+01$

$1.44 \mathrm{E}-00$

$0.00 \mathrm{E}+01$

$0.00 \mathrm{E}+01$

$0.00 \mathrm{E}+01$

$0.00 \mathrm{E}+01$

8.34E-04

$0.00 \mathrm{E}+01$

$4.24 \mathrm{E}+02$

$0.00 \mathrm{E}+01$

$0.00 \mathrm{E}+01$

$0.00 \mathrm{E}+01$

$0.00 \mathrm{E}+01$

$0.00 \mathrm{E}+01$

$0.00 \mathrm{E}+01$

$0.00 \mathrm{E}+01$
$0.00 \mathrm{E}+01$

$0.00 \mathrm{E}+01$

$0.00 \mathrm{E}+01$

$0.00 \mathrm{E}+01$

$0.00 \mathrm{E}+01$

$0.00 \mathrm{E}+01$

$0.00 \mathrm{E}+01$

$0.00 \mathrm{E}+01$

$0.00 \mathrm{E}+01$

5.20E-06

$0.00 \mathrm{E}+01$

7.04E-02

$0.00 \mathrm{E}+01$

$0.00 \mathrm{E}+01$

$0.00 \mathrm{E}+01$

$0.00 \mathrm{E}+01$

$0.00 \mathrm{E}+01$

$0.00 \mathrm{E}+01$

$1.50 \mathrm{E}+01$

$0.00 \mathrm{E}+01$

$0.00 \mathrm{E}+01$

$0.00 \mathrm{E}+01$

$0.00 \mathrm{E}+01$

$0.00 \mathrm{E}+01$

$0.00 \mathrm{E}+01$

$0.00 \mathrm{E}+01$

$0.00 \mathrm{E}+01$

$1.75 \mathrm{E}+04$

3.94E-01

$2.06 \mathrm{E}+02$

$7.40 \mathrm{E}+03$

$6.27 \mathrm{E}+04$

$3.48 \mathrm{E}+03$

2.88E-01

$3.39 \mathrm{E}+03$

$0.00 \mathrm{E}+01$

$1.45 \mathrm{E}+02$

3.32E-01

$1.43 \mathrm{E}+03$

$3.77 \mathrm{E}+05$

2.21E-00

$6.79 \mathrm{E}+04$

$5.22 \mathrm{E}+05$

$5.05 \mathrm{E}+03$

$2.49 \mathrm{E}+02$
$10 \%$-ile

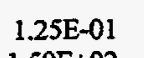

$1.59 \mathrm{E}+02$

$1.52 \mathrm{E}+03$

6.17E-02

$0.00 \mathrm{E}+01$

2.14E-03

$1.94 \mathrm{E}-06$

$6.18 \mathrm{E}+03$

2.40E-00

$0.00 \mathrm{E}+01$

1.19E-04

$9.08 \mathrm{E}-06$

1.12E-04

$1.22 \mathrm{E}-01$

$1.05 \mathrm{E}-01$

$1.41 \mathrm{E}+04$

$0.00 \mathrm{E}+01$

$0.00 \mathrm{E}+01$

$0.00 \mathrm{E}+01$

$0.00 \mathrm{E}+01$

$0.00 \mathrm{E}+01$

$0.00 \mathrm{E}+01$

$0.00 \mathrm{E}+01$

8.15E-04

$0.00 \mathrm{E}+01$

1.33E-03

5.30E-02

$7.89 \mathrm{E}-03$

$7.96 \mathrm{E}-02$

$3.94 \mathrm{E}-07$

$1.41 E-06$

7.33E-05

$1.45 \mathrm{E}-04$

$0.00 \mathrm{E}+01$

2.45E-01

$0.00 \mathrm{E}+01$

$2.28 \mathrm{E}+02$

$0.00 \mathrm{E}+01$

$0.00 \mathrm{E}+01$

$0.00 \mathrm{E}+01$

$0.00 E+01$

2.37E+01

$0.00 E+01$

$0.00 \mathrm{E}+01$

$0.00 E+01$

$0.00 \mathrm{E}+01$

$0.00 \mathrm{E}+01$

$0.00 \mathrm{E}+01$

$0.00 \mathrm{E}+01$

$0.00 \mathrm{E}+01$

$2.28 \mathrm{E}+04$

$3.06 \mathrm{E}-00$

$5.51 \mathrm{E}+02$

$8.03 \mathrm{E}+03$

8.37E+04

$4.23 \mathrm{E}+03$

$3.93 \mathrm{E}+01$

$4.33 \mathrm{E}+03$

$0.00 \mathrm{E}+01$

7.37E+02

$1.33 \mathrm{E}+01$

$1.68 \mathrm{E}+03$

$4.01 \mathrm{E}+05$

$3.02 \mathrm{E}+01$

$7.86 \mathrm{E}+04$

$5.67 \mathrm{E}+05$

$7.18 \mathrm{E}+03$

$2.99 \mathrm{E}+02$ 50\%-ile

2.58E-00

$2.30 \mathrm{E}+01$
$5.80 \mathrm{E}+03$
$5.35 \mathrm{E}+04$
$1.41 \mathrm{E}+02$

$5.80 \mathrm{E}+03$

$1.70 \mathrm{E}+04 \quad 5.35 \mathrm{E}+04$

$1.56 \mathrm{E}+01$

2.63E-04

$2.84 \mathrm{E}+01$

2.92E-04

$4.87 \mathrm{E}+04$

4.88E-00

$1.48 \mathrm{E}+01$

$1.80 \mathrm{E}-02$

$1.37 \mathrm{E}-03$

$1.69 \mathrm{E}-02$

$1.58 \mathrm{E}+01$

8.24E-01

$5.42 \mathrm{E}+04$

6.07E-01

$7.81 \mathrm{E}+01$

$3.82 \mathrm{E}+01$

6.29E-02

2.17E-00

$5.65 \mathrm{E}-01$

$5.50 \mathrm{E}+01$

$1.23 \mathrm{E}-01$

1.01E-03

2.01E-01

$7.99 \mathrm{E}-00$

1.19E-00

$1.20 \mathrm{E}+01$

5.95E-05

5.01E-05

2.61E-03

$7.02 \mathrm{E}-04$

$1.59 \mathrm{E}+01$

6.05E-01

$2.13 \mathrm{E}+03$

$8.58 \mathrm{E}+03$

$3.27 \mathrm{E}+01$

$1.52 \mathrm{E}-04$

6.97E-04

9.10E-01

$3.80 \mathrm{E}+0$

1.04E-01

3.97E-01

$2.11 \mathrm{E}+01$

9.47E-01

$1.44 \mathrm{E}-01$

$2.13 E+01$

$7.72 \mathrm{E}+04$

2.99E-00

$2.96 \mathrm{E}+04$

$5.95 \mathrm{E}+01$

$1.09 \mathrm{E}+03$

$8.76 \mathrm{E}+03$

$1.13 \mathrm{E}+05$

$5.29 \mathrm{E}+03$

$1.64 \mathrm{E}+03$

$5.79 \mathrm{E}+03$

3.19E-00

$2.95 \mathrm{E}+03$

$4.25 E+01$

$2.01 E+03$

$4.31 E+05$

$2.23 \mathrm{E}+02$

$9.08 \mathrm{E}+04$

$6.32 \mathrm{E}+05$

$1.07 \mathrm{E}+04$

$3.65 \mathrm{E}+02$

$1.06 \mathrm{E}-03$

$2.12 \mathrm{E}+03$

8.33E-04

$9.72 \mathrm{E}+04$

$2.06 \mathrm{E}+01$ 
Tank Anal. Un

\begin{tabular}{|c|c|}
\hline U105 & PO4 \\
\hline U105 & $\mathbf{S}$ \\
\hline U105 & $\mathbf{S i}$ \\
\hline U105 & SO4 \\
\hline U105 & $\mathrm{Sr}$ \\
\hline U105 & TOC \\
\hline U105 & $\mathbf{U}$ \\
\hline U105 & $\mathbf{Z r}$ \\
\hline U105 & Ac227 \\
\hline U105 & Am241 \\
\hline U105 & Am243 \\
\hline U105 & Ba137 \\
\hline U105 & C14 \\
\hline U105 & Cd113 \\
\hline U105 & $\mathrm{Cm} 242$ \\
\hline U105 & Cm243 \\
\hline U105 & $\mathrm{Cm} 244$ \\
\hline U105 & Co60 \\
\hline U105 & Cs134 \\
\hline U105 & Cs137 \\
\hline U105 & Eu152 \\
\hline U105 & Eu154 \\
\hline U105 & Eu155 \\
\hline U105 & 1129 \\
\hline U105 & Nb93 \\
\hline U105 & Ni59 \\
\hline U105 & Ni63 \\
\hline U105 & Np237 \\
\hline U105 & Pa231 \\
\hline U105 & Pu238 \\
\hline U105 & Pu239 \\
\hline U105 & Puz240 \\
\hline U105 & Pu241 \\
\hline U105 & Pu242 \\
\hline U105 & Ra226 \\
\hline U105 & Ra228 \\
\hline U105 & Rul06 \\
\hline U105 & Sb125 \\
\hline U105. & Se79 \\
\hline U105 & Sm15I \\
\hline U105 & Sr89/90 \\
\hline U105 & Te99 \\
\hline v105 & Th229 \\
\hline U105 & Th232 \\
\hline U105 & Tin126 \\
\hline U105 & Tritium \\
\hline U105 & U232 \\
\hline U105 & U233 \\
\hline U105 & U234 \\
\hline U105 & U235 \\
\hline U105 & U236 \\
\hline U105 & U238 \\
\hline U105 & Y90 \\
\hline U105 & Zr93 \\
\hline U106 & Al \\
\hline U106 & $\mathbf{B i}$ \\
\hline U106 & $\mathrm{Ca}$ \\
\hline U106 & $\mathrm{Cl}$ \\
\hline $\begin{array}{l}\text { U106 } \\
\text { U106 }\end{array}$ & $\mathrm{Cr}$ \\
\hline U106 & $\mathbf{F}$ \\
\hline U106 & Fe \\
\hline U106 & $\mathbf{H g}$ \\
\hline U106 & $\mathbf{K}$ \\
\hline U106 & $\mathbf{L a}$ \\
\hline U106 & Mn \\
\hline U106 & $\mathrm{Na}$ \\
\hline 106 & \\
\hline
\end{tabular}

May $\frac{B B}{98}$ $9.22 \mathrm{E}+04$ $3.13 E+04$ $7.93 \mathrm{E}+02$ $9.39 \mathrm{E}+04$ $1.89 \mathrm{E}+01$ $3.00 \mathrm{E}+04$ $5.56 \mathrm{E}+03$ $8.49 \mathrm{E}+01$ $1.74 \mathrm{E}-03$ $1.07 \mathrm{E}+02$ 3.82E-03 $3.83 \mathrm{E}+05$ $6.29 \mathrm{E}+01$ $1.61 \mathrm{E}+02$ 2.87E-01 2.66E-02 2.59E-01 $7.01 \mathrm{E}+01$ 5.09E-00 $4.05 E+05$ $7.50 \mathrm{E}-00$

$1.14 \mathrm{E}+03$ $4.45 \mathrm{E}+02$ 8.60E-01 $2.22 \mathrm{E}+01$ 4.08E-00 $4.00 \mathrm{E}+02$

$1.61 \mathrm{E}-00$

7.83E-03

2.61E-00

$8.95 \mathrm{E}+01$

$1.52 \mathrm{E}+01$

$1.77 \mathrm{E}+02$

9.74E- 04

2.84E-04

2.90E-01

$1.27 \mathrm{E}-02$

$3.03 E+02$

$6.25 \mathrm{E}-00$

$2.20 \mathrm{E}+04$

$1.54 \mathrm{E}+05$

$4.46 \mathrm{E}+02$

6.79E-03

1.92E-02

9.44E-00

$4.27 \mathrm{E}+02$

1.47E-00

5.65E-00

$1.82 \mathrm{E}+01$

8.10E-01

1.54E-01

$1.87 \mathrm{E}+01$

$1.54 \mathrm{E}+05$

3.07E+01

$1.57 \mathrm{E}+04$

$5.68 \mathrm{E}+01$

$5.10 \mathrm{E}+02$

$3.81 \mathrm{E}+03$

$5.44 \mathrm{E}+04$

$3.52 \mathrm{E}+03$

$4.18 \mathrm{E}+03$

$4.05 E+03$

$1.54 \mathrm{E}-00$

$1.86 \mathrm{E}+03$

$5.16 \mathrm{E}+01$

$1.53 E+03$

$2.58 \mathrm{E}+05$

$3.89 \mathrm{E}+02$ $\frac{\frac{B B}{\text { Sept98 }}}{3.01 \mathrm{E}+04}$

NA

$7.93 E+02$

3.13E+04

2.09E-00

$3.00 \mathrm{E}+04$

$5.56 \mathrm{E}+03$

$8.49 \mathrm{E}+01$

1.74E-03

$8.95 \mathrm{E}+02$

3.19E-02

$3.83 \mathrm{E}+05$

$6.29 \mathrm{E}+01$

$1.61 \mathrm{E}+02$

2.39E-00

2.22E-01

2.16E-00

$7.01 \mathrm{E}+01$

5.09E-00

$4.04 \mathrm{E}+05$

7.50E-00

$1.14 \mathrm{E}+03$

$4.45 E+02$

8.60E-01

$2.22 \mathrm{E}+01$

4.08E-00

4.00E +02

1.61E-00

7.83E-03

$2.18 \mathrm{E}+01$

$7.47 \mathrm{E}+02$

$1.27 \mathrm{E}+02$

$1.48 \mathrm{E}+03$

8.13E-03

2.84E-04

2.90E-01

1.27E-02

$3.03 E+02$

$6.25 \mathrm{E}-00$

$2.20 \mathrm{E}+04$

$1.54 \mathrm{E}+05$

$4.46 \mathrm{E}+02$

$6.79 \mathrm{E}-03$

$1.92 \mathrm{E}-02$

9.44E-00

$4.27 \mathrm{E}+02$

1.50E-01

5.75E-01

$1.85 \mathrm{E}-00$

8.25E-02

1.57E-02

$1.86 \mathrm{E}-00$

$1.54 \mathrm{E}+0.5$

$3.07 \mathrm{E}+01$

$1.57 \mathrm{E}+04$

$0.00 \mathrm{E}+01$

$5.10 \mathrm{E}+02$

$3.81 \mathrm{E}+03$

$5.44 E+04$

$3.52 \mathrm{E}+03$

$4.18 \mathrm{E}+03$

$4.05 E+03$

$0.00 \mathrm{E}+01$

$1.86 \mathrm{E}+03$

$0.00 \mathrm{E}+01$

$1.53 \mathrm{E}+03$

$2.58 \mathrm{E}+05$ $3.89 \mathrm{E}+02$ 1\%-ile

$1.74 \mathrm{E}+04$

2.50E+03

7.61E-00

$1.30 \mathrm{E}+04$

$0.00 \mathrm{E}+01$

$2.09 \mathrm{E}+04$

$7.06 \mathrm{E}+01$

$8.29 \mathrm{E}-03$

$0.00 \mathrm{E}+01$

$5.80 \mathrm{E}+03$

2.94E-03

$7.38 \mathrm{E}+04$

8.47E-00

$0.00 \mathrm{E}+01$

2.21E-01

2.04E-02

$1.99 \mathrm{E}-01$

$3.57 \mathrm{E}+01$

9.80E-01

3.07E+05

$0.00 E+01$

$0.00 \mathrm{E}+01$

$0.00 \mathrm{E}+01$

$6.08 \mathrm{E}-03$

$0.00 \mathrm{E}+01$

$0.00 \mathrm{E}+01$

$0.00 \mathrm{E}+01$

$1.24 \mathrm{E}-00$

$0.00 E+01$

2.01E-00

$6.88 \mathrm{E}+01$

$1.17 E+01$

$1.36 \mathrm{E}+02$

$7.49 \mathrm{E}-04$

$0.00 \mathrm{E}+01$

$0.00 \mathrm{E}+01$

$6.83 \mathrm{E}-03$

$0.00 \mathrm{E}+01$

$2.49 \mathrm{E}-01$

$0.00 \mathrm{E}+01$

$0.00 \mathrm{E}+01$

$0.00 \mathrm{E}+01$

$0.00 \mathrm{E}+01$

$0.00 \mathrm{E}+01$

$0.00 \mathrm{E}+01$

$3.29 \mathrm{E}+02$

$0.00 \mathrm{E}+01$

$0.00 \mathrm{E}+01$

$0.00 \mathrm{E}+01$

$0.00 \mathrm{E}+01$

$0.00 \mathrm{E}+01$

$0.00 \mathrm{E}+01$

$0.00 \mathrm{E}+01$

$0.00 \mathrm{E}+01$

$7.28 \mathrm{E}+03$

4.19E-01

$1.38 \mathrm{E}+02$

$2.41 \mathrm{E}+03$

$3.97 \mathrm{E}+04$

$1.76 \mathrm{E}+03$

2.31E-01

$2.03 \mathrm{E}+03$

$0.00 \mathrm{E}+01$

$2.09 \mathrm{E}+01$

$1.38 \mathrm{E}-00$

$7.14 \mathrm{E}+02$

$2.25 \mathrm{E}+05$

$2.64 \mathrm{E}+01$
$10 \%$-ile

2.47E+04

$4.91 \mathrm{E}+03$

$2.54 \mathrm{E}+02$

$1.96 \mathrm{E}+04$

$1.19 \mathrm{E}-01$

$2.26 \mathrm{E}+04$

$1.71 \mathrm{E}+03$

2.06E-01

$0.00 \mathrm{E}+01$

$1.56 \mathrm{E}+04$

3.32E-03

$1.96 \mathrm{E}+05$

$3.10 \mathrm{E}+01$

$0.00 \mathrm{E}+01$

2.49E-01

2.31E-02

2.25E-01

$5.00 \mathrm{E}+01$

2.60E-00

$3.28 \mathrm{E}+05$

$0.00 \mathrm{E}+01$

$0.00 \mathrm{E}+01$

$0.00 \mathrm{E}+01$

4.04E-01

$0.00 \mathrm{E}+01$

$0.00 \mathrm{E}+01$

$0.00 \mathrm{E}+01$

$1.40 \mathrm{E}-00$

$0.00 \mathrm{E}+01$

2.27E- 00

$7.78 \mathrm{E}+01$

$1.32 \mathrm{E}+01$

$1.54 \mathrm{E}+02$

8.46E-04

$0.00 \mathrm{E}+01$

$0.00 \mathrm{E}+01$

$9.13 \mathrm{E}-03$

$0.00 \mathrm{E}+01$

2.97E-00

$0.00 \mathrm{E}+01$

$6.13 \mathrm{E}+02$

$0.00 \mathrm{E}+01$

$0.00 \mathrm{E}+01$

$0.00 \mathrm{E}+01$

$0.00 \mathrm{E}+01$

$3.69 \mathrm{E}+02$

$0.00 \mathrm{E}+01$

$0.00 \mathrm{E}+01$

$0.00 \mathrm{E}+01$

$0.00 \mathrm{E}+01$

$0.00 \mathrm{E}+01$

$0.00 \mathrm{E}+01$

$0.00 \mathrm{E}+01$

$0.00 \mathrm{E}+01$

$1.13 \mathrm{E}+04$

$1.80 \mathrm{E}-00$

$3.68 \mathrm{E}+02$

$2.75 \mathrm{E}+03$

$5.16 \mathrm{E}+04$

$2.36 \mathrm{E}+03$

$1.66 \mathrm{E}+01$

$2.59 \mathrm{E}+03$

$0.00 \mathrm{E}+01$

4.12E+02

$1.44 \mathrm{E}+01$

$9.05 \mathrm{E}+02$

$2.34 E+05$

$1.28 \mathrm{E}+02$

\section{$50 \%$-ile}

$3.44 \mathrm{E}+04$

$7.63 \mathrm{E}+03$

$1.41 \mathrm{E}+03$

$2.75 E+04$

9.89E-00

$2.46 \mathrm{E}+04$

$1.49 \mathrm{E}+04$

$3.09 E+01$ 
Tank Anal. Un

\begin{tabular}{|c|c|}
\hline U106 & NO2 \\
\hline U106 & NO3 \\
\hline U106 & $\mathbf{P}$ \\
\hline U106 & $\mathbf{P b}$ \\
\hline U106 & PO4 \\
\hline U106 & S \\
\hline U106 & $\mathbf{S i}$ \\
\hline U106 & SO4 \\
\hline U106 & Sr \\
\hline U106 & TOC \\
\hline U106 & $\mathbf{U}$ \\
\hline U106 & $\mathbf{Z r}$ \\
\hline U106 & Ac227 \\
\hline U106 & Am241 \\
\hline U106 & $\operatorname{Am} 243$ \\
\hline U106 & Ba137 \\
\hline U106 & C14 \\
\hline U106 & Cd113 \\
\hline U106 & $\mathrm{Cm} 242$ \\
\hline U106 & $\mathrm{Cm} 243$ \\
\hline U106 & $\mathrm{Cm} 244$ \\
\hline U106 & Co60 \\
\hline U106 & Cs134 \\
\hline U106 & Cs137 \\
\hline U106 & Eu152 \\
\hline U106 & Eu154 \\
\hline U106 & Eu155 \\
\hline U106 & 1129 \\
\hline U106 & Nb93 \\
\hline U106 & Ni59 \\
\hline U106 & Ni63 \\
\hline U106 & Np237 \\
\hline U106 & Pa231 \\
\hline U106 & Pu238 \\
\hline U106 & Pu239 \\
\hline U106 & Pu240 \\
\hline U106 & Pu241 \\
\hline U106 & Pu242 \\
\hline U106 & Ra226 \\
\hline U106 & $\operatorname{Ra} 228$ \\
\hline U106 & Ru106 \\
\hline U106 & Sb125 \\
\hline U106 & Se79 \\
\hline U106 & Sm15I \\
\hline U106 & Sr89/90 \\
\hline U106 & Tc99 \\
\hline U106 & Th229 \\
\hline U106 & Th232 \\
\hline U106 & Tin126 \\
\hline U106 & Tritium \\
\hline U106 & U232 \\
\hline U106 & U233 \\
\hline U106 & U234 \\
\hline U106 & U235 \\
\hline U106 & U236 \\
\hline U106 & U238 \\
\hline U106 & Y90 \\
\hline U106 & Zr93 \\
\hline U107 & Al \\
\hline U107 & $\mathbf{B i}$ \\
\hline U107 & $\mathrm{Ca}$ \\
\hline U107 & $\mathrm{Cl}$ \\
\hline U107 & $\mathrm{CO} 3$ \\
\hline U107 & $\mathrm{Cr}$ \\
\hline U107 & $\mathbf{F}$ \\
\hline U107 & $\mathbf{F e}$ \\
\hline U107 & $\mathbf{H g}$ \\
\hline U107 & $\mathbf{K}$ \\
\hline
\end{tabular}

1\%-ile

\section{May $\overline{98}$}

kg $\quad \overline{67 \mathrm{87E}+04}$

kg $2.86 \mathrm{E}+05$

kg 3.97E+03

kg 4.22E+02

kg 1.23E+04

kg $\quad 4.27 \mathrm{E}+03$

kg 2.28E+02

kg $1.28 \mathrm{E}+04$

kg 6.70E-00

g $2.96 \mathrm{E}+04$

k 1.01E+03

kg $1.33 E+02$

$1.10 \mathrm{E}-03$

$2.28 \mathrm{E}+03$

2.56E-03

$2.00 \mathrm{E}+05$

$4.20 \mathrm{E}+01$

$1.05 \mathrm{E}+02$

$1.86 \mathrm{E}-01$

$1.74 \mathrm{E}-02$

1.72E-01

$1.82 \mathrm{E}+02$

3.00E-00

2.15E +05

4.86E-00

$7.59 \mathrm{E}+02$

$2.88 \mathrm{E}+02$

5.74E-01

$1.45 \mathrm{E}+01$

2.57E-00

$2.52 \mathrm{E}+02$

1.07E-00

4.97E-03

1.71E-00

$5.70 \mathrm{E}+01$

$9.74 \mathrm{E}-00$

1.17E-00

6.44E-04

$1.82 \mathrm{E}-04$

$1.66 \mathrm{E}-01$

8.63E-00

$2.05 \mathrm{E}+02$

4.07E-00

$1.43 E+04$

$1.06 \mathrm{E}+05$

2.97E+02

$3.91 \mathrm{E}-03$

1.18E-02

6.15E-00

$2.79 \mathrm{E}+02$

8.30E-01

3.18E-00

$1.44 \mathrm{E}+01$

6.45E-01

$1.15 E-01$

$1.48 \mathrm{E}+01$

$1.06 \mathrm{E}+05$

$1.99 \mathrm{E}+01$

$3.76 \mathrm{E}+04$

$2.47 \mathrm{E}+02$

$7.38 \mathrm{E}+02$

$5.74 \mathrm{E}+03$

$3.17 \mathrm{E}+04$

$5.08 \mathrm{E}+03$

$9.16 \mathrm{E}+02$

$1.90 \mathrm{E}+03$

$2.38 \mathrm{E}+02$ $2.07 E+03$
Sept98

$6.87 \mathrm{E}+04$

$2.86 \mathrm{E}+05$

NA

$22 \mathrm{E}+02$

$1.23 \mathrm{E}+04$

NA

$2.28 \mathrm{E}+02$

$1.28 \mathrm{E}+04$

6.70E-00

$2.96 \mathrm{E}+04$

$1.01 \mathrm{E}+03$

$1.33 \mathrm{E}+02$

1.10E-03

$7.85 \mathrm{E}+02$

2.91E-02

2.03E+05

$4.20 \mathrm{E}+01$

$1.05 E+02$

2.12E-00

$1.98 \mathrm{E}-01$

$1.97 \mathrm{E}-00$

$1.82 \mathrm{E}+02$

$3.00 \mathrm{E}-00$

2.15E+05

4.86E- 00

$1.99 \mathrm{E}+03$

$2.88 \mathrm{E}+02$

5.74E-01

$1.45 \mathrm{E}+01$

2.57E-00

2.52E+02

1.07E-00

4.97E-03

$1.95 \mathrm{E}+01$

$6.50 \mathrm{E}+02$

$1.11 E+02$

$1.33 E+03$

7.34E-63

1.82E-04

1.66E-01

8.63E-03

$2.05 E+02$

4.07E-00

$1.43 E+04$

$1.06 \mathrm{E}+05$

$2.97 \mathrm{E}+02$

3.91E-03

1.18E-02

6.15E-00

$2.79 \mathrm{E}+02$

$1.93 \mathrm{E}-02$

$7.38 \mathrm{E}-02$

3.35E-01

1.50E-02

2.66E-03

3.37E-01

$1.06 \mathrm{E}+05$

$1.99 \mathrm{E}+01$

$3.76 \mathrm{E}+04$

$1.04 \mathrm{E}+02$

$7.38 \mathrm{E}+02$

$5.74 E+03$

$3.17 \mathrm{E}+04$

$5.08 \mathrm{E}+03$

$9.16 \mathrm{E}+02$

$1.90 \mathrm{E}+03$

$5.52 \mathrm{E}+01$

$2.07 E+03$
$4.22 \mathrm{E}+04$

$1.75 \mathrm{E}+05$

$2.12 \mathrm{E}+03$

$2.80 \mathrm{E}+02$

$2.21 \mathrm{E}+03$

$9.66 \mathrm{E}+02$

$5.53 \mathrm{E}-01$

$1.61 \mathrm{E}+03$

2.17E-01

$2.37 \mathrm{E}+04$

$5.85 \mathrm{E}-01$

1.43E-01

$0.00 \mathrm{E}+01$

$5.73 \mathrm{E}-00$

$2.00 \mathrm{E}-03$

2.32E+04

9.06E-00

$0.00 \mathrm{E}+01$

$1.45 \mathrm{E}-01$

$1.36 \mathrm{E}-02$

$1.34 \mathrm{E}-01$

$9.64 \mathrm{E}+0 \mathrm{I}$

$3.48 \mathrm{E}-01$

$1.58 \mathrm{E}+05$

$0.00 \mathrm{E}+01$

$0.00 \mathrm{E}+01$

$0.00 \mathrm{E}+01$

$1.76 \mathrm{E}-02$

$0.00 \mathrm{E}+01$

$0.00 \mathrm{E}+01$

$0.00 \mathrm{E}+01$

8.34E-01

$0.00 \mathrm{E}+01$

$1.33 \mathrm{E}-00$

$4.44 \mathrm{E}+0 \mathrm{I}$

$7.59 \mathrm{E}-00$

9.12E-01

5.02E-04

$5.40 \mathrm{E}-06$

$4.92 \mathrm{E}-03$

4.42E-00

$0.00 \mathrm{E}+01$

$0.00 \mathrm{E}+01$

$0.00 \mathrm{E}+01$

$3.40 \mathrm{E}+03$

$0.00 \mathrm{E}+01$

$0.00 \mathrm{E}+01$

$0.00 \mathrm{E}+01$

$0.00 \mathrm{E}+01$

$2.24 \mathrm{E}+02$

$0.00 \mathrm{E}+01$

$0.00 \mathrm{E}+01$

$0.00 \mathrm{E}+01$

$0.00 E+01$

$0.00 E+01$

$0.00 \mathrm{E}+01$

$0.00 \mathrm{E}+01$

$0.00 \mathrm{E}+01$

$3.53 \mathrm{E}+04$

2.69E-00

$3.12 \mathrm{E}+02$

$5.17 \mathrm{E}+03$

$1.66 \mathrm{E}+04$

$4.25 \mathrm{E}+03$

$5.15 \mathrm{E}+01$

$7.60 E+02$

$0.00 \mathrm{E}+01$

$3.12 \mathrm{E} \div 02$
$10 \%$-ile

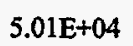

$2.11 \mathrm{E}+05$

$3.60 \mathrm{E}+03$

$3.18 \mathrm{E}+02$

$7.37 \mathrm{E}+03$

$2.22 \mathrm{E}+03$

$5.09 \mathrm{E}-00$

$5.78 \mathrm{E}+03$

2.79E-01

$2.47 \mathrm{E}+04$

$1.47 \mathrm{E}+03$

$2.16 \mathrm{E}-01$

$0.00 \mathrm{E}+01$

$6.38 \mathrm{E}-00$

2.24E-03

1.03E+05

$2.16 \mathrm{E}+01$

$0.00 \mathrm{E}+01$

1.63E-01

1.53E-02

$1.51 \mathrm{E}-01$

$1.09 \mathrm{E}+02$

$1.55 \mathrm{E}-00$

$1.74 \mathrm{E}+05$

$0.00 \mathrm{E}+01$

$0.00 \mathrm{E}+01$

$0.00 \mathrm{E}+01$

2.74E- 01

$0.00 \mathrm{E}+01$

$0.00 E+01$

$0.00 \mathrm{E}+01$

9.38E-01

$0.00 \mathrm{E}+01$

$1.50 \mathrm{E}-00$

$5.00 \mathrm{E}+01$

8.54E-00

$1.03 \mathrm{E}-00$

$5.65 \mathrm{E}-04$

7.28E-06

6.64E-03

5.82E-00

6.15E-00

1.43E-00

$0.00 \mathrm{E}+01$

$3.84 \mathrm{E}+03$

$0.00 \mathrm{E}+01$

$0.00 \mathrm{E}+01$

$0.00 \mathrm{E}+01$

$0.00 \mathrm{E}+01$

$2.45 \mathrm{E}+02$

$0.00 \mathrm{E}+01$

$0.00 \mathrm{E}+01$

$0.00 \mathrm{E}+01$

$0.00 \mathrm{E}+01$

$0.00 \mathrm{E}+01$

$0.00 \mathrm{E}+01$

$0.00 \mathrm{E}+01$

$0.00 \mathrm{E}+01$

$5.12 \mathrm{E}+04$

$7.14 \mathrm{E}+01$

$6.14 \mathrm{E}+02$

$5.87 \mathrm{E}+03$

$3.67 \mathrm{E}+04$

$5.11 \mathrm{E}+03$

$2.48 \mathrm{E}+02$

$1.82 \mathrm{E}+03$

$6.92 \mathrm{E}+01$

$8.85 \mathrm{E}+02$

50\%-ile

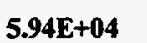

2.51E+05

$5.97 \mathrm{E}+03$

$3.68 \mathrm{E}+02$

$1.41 \mathrm{E}+04$

$3.85 \mathrm{E}+03$

$4.25 \mathrm{E}+02$

$1.11 E+04$ 
Tank Anal. Un

\begin{tabular}{|c|c|}
\hline U107 & La \\
\hline U107 & Mn \\
\hline U107 & $\mathbf{N a}$ \\
\hline U107 & $\mathbf{N i}$ \\
\hline U107 & NO2 \\
\hline U107 & NO3 \\
\hline U107 & $\mathbf{P}$ \\
\hline U107 & $\mathbf{P b}$ \\
\hline U107 & PO4 \\
\hline U107 & $\mathbf{s}$ \\
\hline U107 & Si \\
\hline U107 & SO4 \\
\hline U107 & Sr \\
\hline U107 & TOC \\
\hline U107 & $\mathbf{U}$ \\
\hline U107 & $\mathbf{Z r}$ \\
\hline U107 & Ac227 \\
\hline U107 & Am241 \\
\hline U107 & Am243 \\
\hline $\mathbf{U 1 0 7}$ & Ba137 \\
\hline $\mathrm{U} 107$ & C14 \\
\hline U107 & Cd113 \\
\hline $\mathbf{U 1 0 7}$ & $\mathrm{Cm} 242$ \\
\hline U107 & $\mathrm{Cm} 243$ \\
\hline U107 & Cm244 \\
\hline U107 & Co60 \\
\hline U107 & Cs134 \\
\hline U107 & Cs137 \\
\hline U107 & Eu152 \\
\hline U107 & Eu154 \\
\hline U107 & Eul55 \\
\hline U107 & I129 \\
\hline U107 & $\mathrm{Nb} 93$ \\
\hline U107 & Ni59 \\
\hline U107 & Ni63 \\
\hline U107 & Np237 \\
\hline U107 & Pa231 \\
\hline U107 & Pu238 \\
\hline U107 & Pu239 \\
\hline U107 & Pu240 \\
\hline U107 & Pu241 \\
\hline U107 & Pu242 \\
\hline U107 & $\operatorname{Ra226}$ \\
\hline U107 & $\operatorname{Ra} 228$ \\
\hline U107 & Ru106 \\
\hline U107 & Sb125 \\
\hline U107 & Se79 \\
\hline U107 & Sml5i \\
\hline U107 & Sr89/90 \\
\hline U107 & Te99 \\
\hline U107 & Th229 \\
\hline U107 & Th232 \\
\hline U107 & Tin126 \\
\hline U107 & Tritium \\
\hline U107 & U232 \\
\hline U107 & U233 \\
\hline U107 & U234 \\
\hline v107 & U235 \\
\hline $\mathbf{U 1 0 7}$ & U236 \\
\hline U107 & U238 \\
\hline V107 & Y90 \\
\hline U107 & Zr93 \\
\hline U108 & Al \\
\hline U108 & $\mathbf{B i}$ \\
\hline $\mathrm{U108}$ & $\mathrm{Ca}$ \\
\hline v108 & Cl \\
\hline U108 & $\mathrm{CO3}$ \\
\hline Jino & $\mathrm{Cr}$ \\
\hline
\end{tabular}

May 98 $6.90 \mathrm{E}+01$ $6.77 \mathrm{E}+02$ $4.44 E+05$ $5.75 E+01$ $6.36 \mathrm{E}+04$ $1.03 \mathrm{E}+06$ $2.59 \mathrm{E}+04$ $8.41 \mathrm{E}+02$ $8.03 E+04$ $1.01 \mathrm{E}+04$ $6.37 \mathrm{E}+02$ $3.03 \mathrm{E}+04$ $1.60 \mathrm{E}+01$ $4.82 \mathrm{E}+03$ $7.47 \mathrm{E}+02$ $1.94 \mathrm{E}+01$ $1.30 \mathrm{E}-03$ $1.36 \mathrm{E}+03$ $2.83 E-03$

$2.06 \mathrm{E}+05$

$4.83 \mathrm{E}+01$

$1.24 \mathrm{E}+02$

2.11E-01

1.95E-02

$1.92 \mathrm{E}-01$

$4.16 \mathrm{E}+01$

3.65E-00

$2.18 \mathrm{E}+05$

$5.60 \mathrm{E}-00$

$1.90 \mathrm{E}+02$

$8.15 E+02$

6.64E-01

$1.71 \mathrm{E}+01$

3.13E-00

$3.07 E+02$

$1.26 \mathrm{E}-00$

$5.96 \mathrm{E}-03$

9.18E-00

$5.90 \mathrm{E}-03$

Ci Incl. in 239

$5.31 \mathrm{E}+02$

2.42E-03

2.06E-04

2.01E-01

9.52E-03

$2.30 \mathrm{E}+02$

$4.80 \mathrm{E}-00$

$1.69 \mathrm{E}+04$

$5.72 \mathrm{E}+01$

$3.44 E+02$

4.72E- 03

1.34E-02

$7.25 \mathrm{E}-00$

$3.35 \mathrm{E}+02$

1.03E-00

3.96E-00

5.41E-00

2.28E-01

1.30E-01

5.49E-00

$5.72 \mathrm{E}+01$

$2.36 \mathrm{E}+01$

$5.12 \mathrm{E}+04$

$4.37 \mathrm{E}+02$

4.47E+02

$1.09 \mathrm{E}+04$

$1.19 \mathrm{E}+05$

$1.21 E+04$
BB

Sept98

$0.00 \mathrm{E}+01$
$6.77 \mathrm{E}+02$

$4.44 \mathrm{E}+05$

5.75E+01

$6.36 \mathrm{E}+04$

$1.03 E+06$

NA

$8.41 \mathrm{E}+02$

$2.62 \mathrm{E}+04$

$$
\text { NA }
$$

$6.37 \mathrm{E}+02$

$1.01 E+04$

3.84E-00

$4.82 \mathrm{E}+03$

$7.86 \mathrm{E}+02$

2.30E-00

$1.30 \mathrm{E}-03$

$2.58 \mathrm{E}+01$

8.94E-04

$1.99 \mathrm{E}+05$

$4.83 \mathrm{E}+01$

$1.24 \mathrm{E}+02$

6.67E-02

6.15E-03

6.07E-02

$5.35 \mathrm{E}+01$

3.65E-00

$2.10 \mathrm{E}+05$

$5.60 \mathrm{E}-00$

$8.69 \mathrm{E}+02$

$3.30 \mathrm{E}+02$

6.64E-01

$1.71 \mathrm{E}+01$

$3.13 \mathrm{E}-00$

$3.07 \mathrm{E}+02$

$1.26 \mathrm{E}-00$

$5.96 \mathrm{E}-03$

$2.90 \mathrm{E}-00$

$1.67 \mathrm{E}+02$

$2.42 \mathrm{E}+01$

$1.68 \mathrm{E}+02$

7.63E-04

2.06E-04

2.01E-01

9.52E-03

$2.30 \mathrm{E}+02$

$4.80 \mathrm{E}-00$

$1.69 \mathrm{E}+04$

$1.60 \mathrm{E}+05$

$3.44 \mathrm{E}+02$

$4.72 \mathrm{E}-03$

1.34E-02

$7.25 \mathrm{E}-00$

$3.35 E+02$

5.22E-02

2.00E-01

2.73E-01

1.15E-02

$6.56 \mathrm{E}-03$

2.62E-01

$1.60 \mathrm{E}+05$

$2.36 \mathrm{E}+01$

$5.12 \mathrm{E}+04$

$2.94 \mathrm{E}+02$

$4.47 \mathrm{E}+02$

$1.09 \mathrm{E}+04$

$1.19 \mathrm{E}+05$

$1.21 E+04$ 1\%-ile

$4.54 \mathrm{E}-00$

$3.48 \mathrm{E}+02$

$3.42 \mathrm{E}+05$

$3.18 \mathrm{E}+01$

$5.45 \mathrm{E}+04$

$6.44 \mathrm{E}+05$

$4.00 \mathrm{E}+03$

$5.09 \mathrm{E}+01$

$1.53 \mathrm{E}+04$

$4.04 \mathrm{E}+02$

$1.17 \mathrm{E}+02$

$1.67 \mathrm{E}+03$

$5.00 \mathrm{E}-01$

$2.83 \mathrm{E}+03$

$9.41 \mathrm{E}+02$

2.32E-01

$0.00 \mathrm{E}+01$

$1.87 \mathrm{E}+01$

4.21E-04

$0.00 \mathrm{E}+01$

$0.00 \mathrm{E}+01$

$0.00 \mathrm{E}+01$

3.14E-02

2.90E-03

2.85E-02

3.51E-00

$0.00 \mathrm{E}+01$

$7.56 \mathrm{E}+04$

$0.00 \mathrm{E}+01$

$0.00 \mathrm{E}+01$

$0.00 \mathrm{E}+01$

4.11E-02

$0.00 \mathrm{E}+01$

1.19E-01

$1.17 \mathrm{E}+01$

1.87E-01

$0.00 \mathrm{E}+01$

1.36E-00

8.77E-04

8.77E-04

$7.89 \mathrm{E}+01$

$3.60 \mathrm{E}-04$

$0.00 \mathrm{E}+01$

$0.00 \mathrm{E}+01$

$0.00 \mathrm{E}+01$

$5.28 \mathrm{E}-01$

$0.00 \mathrm{E}+01$

$0.00 \mathrm{E}+01$

$2.98 \mathrm{E}+01$

$0.00 \mathrm{E}+01$

$0.00 \mathrm{E}+01$

$0.00 \mathrm{E}+01$

$0.00 \mathrm{E}+01$

$2.49 \mathrm{E}+02$

$0.00 \mathrm{E}+01$

$0.00 \mathrm{E}+01$

$0.00 \mathrm{E}+01$

$0.00 \mathrm{E}+01$

$0.00 \mathrm{E}+01$

$0.00 \mathrm{E}+01$

$0.00 \mathrm{E}+01$

$0.00 \mathrm{E}+01$

$4.81 \mathrm{E}+04$

$1.58 \mathrm{E}-00$

$2.01 \mathrm{E}+02$

$9.22 \mathrm{E}+03$

$7.02 \mathrm{E}+04$

$9.57 \mathrm{E}+03$ 10\%-ile

7.14E-00

$7.16 \mathrm{E}+02$

$3.75 \mathrm{E}+05$

$9.77 \mathrm{E}+01$

$6.60 \mathrm{E}+04$

$7.10 \mathrm{E}+05$

$6.50 \mathrm{E}+03$

$1.10 \mathrm{E}+02$

$2.16 \mathrm{E}+04$

$1.03 \mathrm{E}+03$

$6.26 \mathrm{E}+02$

$8.08 \mathrm{E}+03$

$3.96 \mathrm{E}+01$

$4.62 \mathrm{E}+03$

$2.09 \mathrm{E}+03$

$5.40 \mathrm{E}-00$

$0.00 \mathrm{E}+01$

$2.39 \mathrm{E}+01$

1.35E-03

$7.87 \mathrm{E}+04$

4.47E-00

$3.61 \mathrm{E}+01$

1.00E-01

9.27E-03

9.13E-02

$5.22 \mathrm{E}-00$

1.39E-00

$1.45 \mathrm{E}+05$

$0.00 \mathrm{E}+01$

$0.00 \mathrm{E}+01$

$0.00 \mathrm{E}+01$

2.89E-01

$0.00 \mathrm{E}+01$

7.42E-01

$7.28 \mathrm{E}+01$

5.99E-01

7.53E-04

4.36E-00

$2.80 \mathrm{E}-03$

$2.80 \mathrm{E}-03$

$2.52 \mathrm{E}+02$

$1.15 \mathrm{E}-03$

4.64E-05

$4.52 \mathrm{E}-02$

3.34E-03

$4.22 \mathrm{E}+01$

$1.42 \mathrm{E}-00$

$0.00 \mathrm{E}+01$

$3.38 \mathrm{E}+03$

$4.51 \mathrm{E}+01$

$5.96 \mathrm{E}-04$

$1.69 \mathrm{E}-03$

$2.11 \mathrm{E}-00$

$2.83 \mathrm{E}+02$

$1.30 \mathrm{E}-01$

$5.00 \mathrm{E}-01$

$6.83 \mathrm{E}-01$

$2.88 \mathrm{E}-02$

1.64E-02

$6.93 \mathrm{E}-01$

1.35E-00

$0.00 \mathrm{E}+01$

$5.47 \mathrm{E}+04$

$4.13 \mathrm{E}+01$

$5.45 \mathrm{E}+02$

$9.75 \mathrm{E}+03$

$9.27 \mathrm{E} \div 04$

$1.05 \mathrm{E}+04$

50\%-ile

$3.57 \mathrm{E}+01$

$1.43 E+03$

$4.16 \mathrm{E}+05$

$3.51 \mathrm{E}+02$

$8.28 \mathrm{E}+04$

$8.04 \mathrm{E}+05$

$9.68 \mathrm{E}+03$

$2.00 \mathrm{E}+02$ 
Tank Anal. Un

\begin{tabular}{|c|c|}
\hline U108 & $\mathbf{F}$ \\
\hline U108 & $\mathbf{F e}$ \\
\hline U108 & $\mathbf{H g}$ \\
\hline U108 & $\mathrm{K}$ \\
\hline U108 & $\mathbf{L a}$ \\
\hline U108 & $\mathbf{M n}$ \\
\hline U108 & $\mathbf{N a}$ \\
\hline U108 & $\mathbf{N i}$ \\
\hline U108 & NO2 \\
\hline U108 & NO3 \\
\hline U108 & $\mathbf{P}$ \\
\hline U108 & $\mathbf{P b}$ \\
\hline U108 & PO4 \\
\hline U108 & $\mathbf{S}$ \\
\hline U108 & $\mathbf{S i}$ \\
\hline U108 & SO4 \\
\hline U108 & Sr \\
\hline U108 & TOC \\
\hline U108 & $\mathbf{U}$ \\
\hline U108 & $\mathbf{Z r}$ \\
\hline U108 & Ac227 \\
\hline U108 & Am241 \\
\hline U108 & Am243 \\
\hline U108 & Ba137 \\
\hline U108 & C14 \\
\hline U108 & Cd113 \\
\hline U108 & $\mathrm{Cm} 242$ \\
\hline U108 & Cm243 \\
\hline U108 & Cm244 \\
\hline U108 & Co60 \\
\hline U108 & Cs134 \\
\hline U108 & Cs137 \\
\hline U108 & Eu152 \\
\hline U108 & Eu154 \\
\hline U108 & Eu155 \\
\hline U108 & 1129 \\
\hline U108 & Nb93 \\
\hline U108 & Ni59 \\
\hline U108 & Ni63 \\
\hline U108 & Np237 \\
\hline U108 & Pa231 \\
\hline U108 & Pu238 \\
\hline U108 & Pu239 \\
\hline U108 & Pu240 \\
\hline U108 & Pu241 \\
\hline U108 & Pu242 \\
\hline U108 & $\operatorname{Ra226}$ \\
\hline U108 & $\operatorname{Ra228}$ \\
\hline U108 & Ru106 \\
\hline U108 & Sb125 \\
\hline $\mathrm{U108}$ & Se79 \\
\hline U108 & Sm151 \\
\hline U108 & Sr89/90 \\
\hline U108 & Te99 \\
\hline U108 & Th229 \\
\hline U108 & Th232 \\
\hline U108 & Tin126 \\
\hline U108 & Tritium \\
\hline U108 & U232 \\
\hline U108 & U233 \\
\hline U108 & U234 \\
\hline U108 & U235 \\
\hline U108 & U236 \\
\hline U108 & U238 \\
\hline 4108 & Y90 \\
\hline U108 & Zr93 \\
\hline
\end{tabular}

U109 Bi

\section{May $\frac{B B}{98}$}

$2 \overline{84 \mathrm{E}+03}$

$5.07 \mathrm{E}+02$

$2.21 E+02$

$4.80 \mathrm{E}+03$

$1.17 \mathrm{E}+02$

$1.85 E+02$

$7.26 \mathrm{E}+05$

$1.50 \mathrm{E}+02$

$1.58 \mathrm{E}+05$

$8.80 E+05$

$1.63 \mathrm{E}+04$

$2.34 \mathrm{E}+02$

$5.05 \mathrm{E}+04$

$1.48 \mathrm{E}+04$

$8.30 \mathrm{E}+02$

$4.44 \mathrm{E}+04$

$2.34 \mathrm{E}+01$

$1.29 \mathrm{E}+04$

$5.38 \mathrm{E}+02$

$2.34 E+01$

$1.92 \mathrm{E}-03$

$1.93 \mathrm{E}+03$

4.38E-03

$3.90 E+05$

$7.63 E+01$

$1.90 \mathrm{E}+02$

$3.28 \mathrm{E}-01$

3.05E-02

$3.03 E-01$

$6.15 \mathrm{E}+01$

5.17E-00

$4.11 \mathrm{E}+05$

8.68E-00

$2.41 \mathrm{E}+02$

$8.84 \mathrm{E}+02$

1.05E-00

$2.60 E+01$

$4.70 \mathrm{E}-00$

$4.62 \mathrm{E}+02$

$1.96 \mathrm{E}-00$

8.90E-03

$2.15 E+01$

$6.15 \mathrm{E}+02$

$1.02 \mathrm{E}+02$

$9.62 \mathrm{E}+02$

3.14E-03

3.04E-04

2.69E-01

$1.51 \mathrm{E}-02$

$3.68 \mathrm{E}+02$

$7.31 \mathrm{E}-00$

$2.57 \mathrm{E}+04$

$2.94 \mathrm{E}+04$

$5.42 E+02$

$6.34 \mathrm{E}-03$

1.83E-02

$1.11 E+01$

$5.13 E+02$

$1.40 \mathrm{E}-00$

5.39E-00

8.72E-00

3.46E-01

4.23E-01

7.33E-00

$2.94 \mathrm{E}+04$

Ci 3.59E+0

kg $\quad 5.77 \mathrm{E}+04$

kg 3.00E+02
$B B$ Sept98

$2.84 \mathrm{E}+03$

$5.07 E+02$

$1.89 \mathrm{E}+01$

$4.80 \mathrm{E}+03$

$0.00 \mathrm{E}+01$

$1.85 \mathrm{E}+02$

$7.26 \mathrm{E}+05$

$1.50 \mathrm{E}+02$

$1.58 \mathrm{E}+05$

$8.80 E+05$

NA

$2.34 \mathrm{E}+02$

$5.05 E+04$

NA

8.30E+02

$4.44 \mathrm{E}+04$

$7.46 \mathrm{E}-01$

$1.29 \mathrm{E}+04$

$5.38 \mathrm{E}+02$

$2.34 \mathrm{E}+01$

$1.92 \mathrm{E}-03$

$2.18 \mathrm{E}+01$

$7.79 \mathrm{E}-04$

$4.49 \mathrm{E}+05$

$7.63 \mathrm{E}+01$

$1.90 \mathrm{E}+02$

$5.84 \mathrm{E}-02$

5.42E-03

5.39E-02

$6.15 \mathrm{E}+01$

5.17E-00

4.75E+05

8.68E-00

$2.41 \mathrm{E}+02$

$9.02 \mathrm{E}+01$

$1.05 \mathrm{E}-00$

$2.60 \mathrm{E}+01$

4.70E-00

$4.62 E+02$

$1.96 \mathrm{E}-00$

8.90E-03

3.83E-00

$1.09 \mathrm{E}+02$

$1.82 \mathrm{E}+01$

$1.71 \mathrm{E}+02$

5.58E-04

3.04E-04

2.69E-01

1.51E-02

$3.68 \mathrm{E}+02$

$7.31 \mathrm{E}-00$

$2.57 E+04$

$3.11 E+04$

$5.42 E+02$

$6.34 \mathrm{E}-03$

$1.83 \mathrm{E}-02$

$1.11 E+01$

$5.13 E+02$

$3.65 \mathrm{E}-02$

$1.40 \mathrm{E}-01$

$2.26 E-01$

8.98E-03

$1.10 \mathrm{E}-02$

1.79E-01

$3.11 \mathrm{E}+04$

$3.59 \mathrm{E}+01$

$5.77 \mathrm{E}+04$

$3.00 \mathrm{E}+02$ 1\%-ile

$2.02 \mathrm{E}+01$
$2.20 \mathrm{E}+02$

$0.00 \mathrm{E}+01$

2.73E +02

$1.98 \mathrm{E}-00$

$1.66 \mathrm{E}+01$

$6.66 \mathrm{E}+05$

$5.17 \mathrm{E}-00$

$1.24 \mathrm{E}+05$

$6.97 \mathrm{E}+05$

$7.09 \mathrm{E}+03$

4.47E-00

2.62E+04

$1.80 \mathrm{E}+02$

$7.87 \mathrm{E}+01$

$2.52 \mathrm{E}+04$

2.67E-01

$9.79 \mathrm{E}+03$

$7.27 \mathrm{E}+02$

$1.36 \mathrm{E}-01$

$0.00 \mathrm{E}+01$

$7.49 \mathrm{E}-00$

$1.22 \mathrm{E}-04$

$2.87 \mathrm{E}+04$

$0.00 \mathrm{E}+01$

$0.00 \mathrm{E}+01$

9.12E-03

8.48E-04

8.43E-03

$1.89 \mathrm{E}-00$

$3.81 \mathrm{E}-01$

$3.79 \mathrm{E}+05$

$0.00 \mathrm{E}+01$

$0.00 \mathrm{E}+01$

$0.00 \mathrm{E}+01$

3.50E-02

$0.00 \mathrm{E}+01$

$0.00 \mathrm{E}+01$

$0.00 \mathrm{E}+01$

5.45E-02

$0.00 E+01$

$5.98 \mathrm{E}-01$

1.71E +01

$2.84 \mathrm{E}-00$

$2.68 E+01$

8.73E-05

$0.00 \mathrm{E}+01$

$0.00 \mathrm{E}+01$

$0.00 \mathrm{E}+01$

$0.00 \mathrm{E}+01$

$0.00 \mathrm{E}+01$

$0.00 E+01$

$2.14 \mathrm{E}+01$

$0.00 \mathrm{E}+01$

$0.00 \mathrm{E}+01$

$0.00 \mathrm{E}+01$

$0.00 \mathrm{E}+01$

4.35E+02

$0.00 \mathrm{E}+01$

$0.00 \mathrm{E}+01$

$0.00 \mathrm{E}+01$

$0.00 \mathrm{E}+01$

$0.00 \mathrm{E}+01$

$0.00 \mathrm{E}+01$

$0.00 \mathrm{E}+01$

$0.00 \mathrm{E}+01$

$3.47 \mathrm{E}+04$

$2.44 \mathrm{E}-00$
$10 \%$-ile

$1.72 \mathrm{E}+02$

$2.90 \mathrm{E}+03$

$0.00 \mathrm{E}+01$

$7.23 \mathrm{E}+02$

$3.09 \mathrm{E}-00$

$1.85 \mathrm{E}+02$

$6.80 \mathrm{E}+05$

$1.92 \mathrm{E}+01$

1.34E+05

$7.43 E+05$

$9.56 \mathrm{E}+03$

$5.02 \mathrm{E}+01$

3.33E+04

$2.35 \mathrm{E}+03$

$5.60 \mathrm{E}+02$

$3.24 \mathrm{E}+04$

$1.94 \mathrm{E}+01$

1.12E+04

$1.72 \mathrm{E}+03$

$2.36 \mathrm{E}-00$

$0.00 \mathrm{E}+01$

9.51E-00

$1.46 \mathrm{E}-03$

$2.02 \mathrm{E}+05$

$1.09 \mathrm{E}+01$

$0.00 \mathrm{E}+01$

1.09E-01

1.02E-02

$1.01 E-01$

3.74E-00

$2.68 \mathrm{E}-00$

$4.06 \mathrm{E}+05$

$0.00 \mathrm{E}+01$

$0.00 \mathrm{E}+01$

$0.00 \mathrm{E}+01$

4.90E-01

$0.00 \mathrm{E}+01$

$0.00 \mathrm{E}+01$

$0.00 \mathrm{E}+01$

6.53E-01

1.15E-03

7.16E-00

$2.05 \mathrm{E}+02$

$3.40 \mathrm{E}+01$

$3.20 \mathrm{E}+02$

1.05E-03

5.34E-05

$4.72 \mathrm{E}-02$

$1.87 \mathrm{E}-03$

4.93E+01

$3.09 \mathrm{E}-00$

$0.00 \mathrm{E}+01$

8.22E+02

$0.00 \mathrm{E}+01$

8.21E-04

2.37E-03

$0.00 \mathrm{E}+01$

$4.69 \mathrm{E}+02$

$1.81 \mathrm{E}-01$

6.98E-01

$1.13 E-00$

4.48E-02

5.48E-02

9.50E-01

$2.06 \mathrm{E}+02$

$0.00 \mathrm{E}+01$

4.19E+04

$3.27 \mathrm{E}+01$

50\%-ile

$2.21 \mathrm{E}+02$

$3.91 \mathrm{E}+03$

4.04E+03 


\begin{tabular}{|c|c|c|c|}
\hline & & & $\operatorname{1a} \overline{98}$ \\
\hline 109 & $\mathrm{Ca}$ & $\mathrm{kg}$ & $\overline{7.72 \mathrm{E}+02}$ \\
\hline & & 1 & $1.02 \mathrm{E}+04$ \\
\hline 09 & $\mathrm{CO3}$ & & $10 \mathrm{E}+05$ \\
\hline 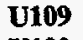 & $\mathrm{Cr}$ & & $E+04$ \\
\hline & $\mathbf{F}$ & & $2.13 E+03$ \\
\hline 09 & $\mathrm{Fe}$ & & $4.72 \mathrm{E}+03$ \\
\hline 09 & Ig & & $7.79 \mathrm{E}+01$ \\
\hline 09 & $Y$ & & $2.90 \mathrm{E}+03$ \\
\hline 109 & $\mathbf{L a}$ & & $1.26 \mathrm{E}+01$ \\
\hline 109 & & & $1.33 E+03$ \\
\hline 109 & & & $6.47 \mathrm{E}+05$ \\
\hline 109 & $\mathbf{N i}$ & & $3.28 \mathrm{E}+02$ \\
\hline 109 & & & \\
\hline 9 & & & \\
\hline 109 & $\mathbf{P}$ & & \\
\hline 109 & $\mathbf{P b}$ & & \\
\hline 109 & PO4 & & \\
\hline 109 & & & \\
\hline 109 & $\mathbf{S i}$ & $g$ & 4.9 \\
\hline 109 & & $g$ & \\
\hline 9 & & & \\
\hline 109 & & & 9.8 \\
\hline 109 & 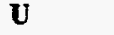 & & 1.2 \\
\hline 109 & $\mathbf{Z r}$ & & 9.6 \\
\hline 1109 & $A c ?$ & & \\
\hline 109 & & & 1.3 \\
\hline 9 & & & \\
\hline 109 & Bal & & 3.2 \\
\hline 109 & & & $8.26 E+01$ \\
\hline 109 & Cd11 & & 2.0 \\
\hline 109 & $\mathrm{Cm} 2$ & & \\
\hline 9 & 8 & & \\
\hline 9 & 0 & & \\
\hline 109 & Co60 & & 5.0 \\
\hline 109 & C & & \\
\hline & C & & \\
\hline 109 & 5 & $i$ & \\
\hline 9 & $\mathbf{E}$ & & \\
\hline 09 & & & \\
\hline 109 & 1129 & & \\
\hline 109 & Nb9 & $\mathrm{Ci}$ & \\
\hline & & Ci & \\
\hline U109 & Ni63 & & \\
\hline U109 & & & \\
\hline 109 & & & \\
\hline U109 & Pu2: & $\mathrm{Ci}$ & \\
\hline U109 & Pu2: & & \\
\hline U109 & Puz & & \\
\hline U109 & Pu241 & 0 & \\
\hline & & & \\
\hline 9 & $\mathbf{F}$ & & \\
\hline 19 & $\mathbf{R}$ & & \\
\hline U109 & $\mathbf{R}$ & 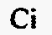 & \\
\hline U109 & Sb125 & $\mathrm{Ci}$ & +02 \\
\hline U109 & Se79 & c & \\
\hline & & S: & \\
\hline & & 0 & \\
\hline 09 & & c & \\
\hline 0 & $\mathbf{T}$ & & \\
\hline$r$ & $\mathbf{T}$ & $c$ & \\
\hline $\mathbf{U}$ & Tin! & $\mathrm{Ci}$ & $E+01$ \\
\hline U109 & Tritiun & $\mathrm{Ci}$ & +02 \\
\hline U109 & U232 & $\mathrm{Ci}$ & E-00 \\
\hline & & $\mathrm{Ci}$ & \\
\hline & & 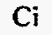 & \\
\hline & & & \\
\hline & & c & \\
\hline & U238 & $\mathrm{Ci}$ & \\
\hline
\end{tabular}

\section{Septo8}

$\frac{\text { Sept } 98}{7.72 \mathrm{E}+02}$

$1.02 \mathrm{E}+04$

$1.10 \mathrm{E}+05$

$1.08 \mathrm{E}+04$

$2.13 E+03$

$4.72 \mathrm{E}+03$

$1.74 \mathrm{E}+01$

$2.90 \mathrm{E}+03$

$0.00 \mathrm{E}+01$

$1.33 \mathrm{E}+03$

6.47E+05

$3.28 \mathrm{E}+02$

$1.16 \mathrm{E}+05$

$9.28 \mathrm{E}+05$

$$
\text { NA }
$$

$3.83 E+02$

$2.41 E+04$

NA

$4.91 \mathrm{E}+03$

$3.42 E+04$

$1.29 \mathrm{E}+02$

$9.86 \mathrm{E}+03$

$1.25 \mathrm{E}+03$

$9.65 \mathrm{E}+01$

2.14E-03

$3.32 \mathrm{E}+01$

$1.21 \mathrm{E}-03$

$3.21 \mathrm{E}+05$

$8.26 \mathrm{E}+01$

$2.09 \mathrm{E}+02$

9.02E-02

8.39E-03

8.30E-02

$9.22 \mathrm{E}+01$

$6.04 \mathrm{E}-00$

$3.39 \mathrm{E}+05$

9.60E-00

$1.49 E+03$

$5.69 \mathrm{E}+02$

1.13E-00

$2.87 \mathrm{E}+01$

5.14E-00

$5.04 E+02$

2.12E-00

9.79E-03

1.39E-00

$6.34 \mathrm{E}+01$

9.76E-00

8.71E+01

4.41E-04

$3.46 \mathrm{E}-04$

$2.80 \mathrm{E}-01$

$1.67 \mathrm{E}-02$

$3.99 \mathrm{E}+02$

8.06E-00

$2.84 \mathrm{E}+04$

$8.22 \mathrm{E}+04$

$5.86 \mathrm{E}+02$

6.62E-03

$1.88 \mathrm{E}-02$

$1.22 \mathrm{E}+01$

$5.59 \mathrm{E}+02$

$3.99 \mathrm{E}-02$

$1.53 \mathrm{E}-01$

4.18E-01

1.85E-02

4.44E-03

4.17E-01 1\%-ile

\section{$3.03 \mathrm{E}+02$}

$4.43 \mathrm{E}+02$

$7.38 \mathrm{E}+04$

$7.11 \mathrm{E}+03$

$1.36 \mathrm{E}+01$

$5.96 \mathrm{E}+02$

$0.00 \mathrm{E}+01$

$9.01 \mathrm{E}+01$

$1.86 \mathrm{E}-00$

$1.23 \mathrm{E}+01$

$5.03 \mathrm{E}+05$

9.60E-00

$1.26 \mathrm{E}+04$

$6.20 \mathrm{E}+05$

$6.51 \mathrm{E}+03$

$5.39 \mathrm{E}-00$

$5.51 \mathrm{E}+03$

$1.15 \mathrm{E}+03$

$2.96 \mathrm{E}+01$

$1.05 \mathrm{E}+03$

9.78E-01

$7.39 \mathrm{E}+03$

$7.28 \mathrm{E}+02$

2.91E-01

$0.00 \mathrm{E}+01$

$1.50 \mathrm{E}+01$

$2.86 \mathrm{E}-04$

$4.80 \mathrm{E}+04$

$0.00 \mathrm{E}+01$

$0.00 \mathrm{E}+01$

2.13E-02

$1.98 \mathrm{E}-03$

$1.95 \mathrm{E}-02$

$1.03 \mathrm{E}+01$

9.02E-01

$2.64 \mathrm{E}+05$

$0.00 \mathrm{E}+01$

$0.00 \mathrm{E}+01$

$0.00 \mathrm{E}+01$

$0.00 \mathrm{E}+01$

$0.00 \mathrm{E}+01$

$0.00 \mathrm{E}+01$

$0.00 \mathrm{E}+01$

$1.22 \mathrm{E}-01$

$0.00 E+01$

$3.26 \mathrm{E}-01$

$1.49 \mathrm{E}+01$

2.30E-00

$2.05 \mathrm{E}+01$

$1.04 \mathrm{E}-04$

$0.00 \mathrm{E}+01$

$0.00 \mathrm{E}+01$

2.01E-03

$0.00 \mathrm{E}+01$

$0.00 \mathrm{E}+01$

$0.00 \mathrm{E}+01$

$5.07 \mathrm{E}+03$

$0.00 \mathrm{E}+01$

$0.00 \mathrm{E}+01$

$0.00 \mathrm{E}+01$

$0.00 \mathrm{E}+01$

$4.50 \mathrm{E}+02$

$0.00 \mathrm{E}+01$

$0.00 \mathrm{E}+01$

$0.00 \mathrm{E}+01$

$0.00 \mathrm{E}+01$

$0.00 \mathrm{E}+01$

$0.00 \mathrm{E}+01$
$10 \%$-ile

$6.68 \mathrm{E}+02$

$1.48 \mathrm{E}+03$

$9.60 \mathrm{E}+04$

$8.04 \mathrm{E}+03$

$1.18 \mathrm{E}+02$

$1.59 \mathrm{E}+03$

$0.00 \mathrm{E}+01$

8.27E+02

$3.68 \mathrm{E}-00$

$1.12 \mathrm{E}+02$

$5.23 \mathrm{E}+05$

$3.68 \mathrm{E}+01$

$7.88 \mathrm{E}+04$

$6.65 \mathrm{E}+05$

$8.92 \mathrm{E}+03$

$2.79 \mathrm{E}+01$

$1.45 \mathrm{E}+04$

$4.05 \mathrm{E}+03$

$2.53 \mathrm{E}+02$

$8.36 \mathrm{E}+03$

$1.67 \mathrm{E}+01$

$9.04 \mathrm{E}+03$

$3.32 \mathrm{E}+03$

2.44E-00

$0.00 \mathrm{E}+01$

$1.70 \mathrm{E}+01$

1.55E-03

$1.60 \mathrm{E}+05$

$3.65 \mathrm{E}+01$

$0.00 \mathrm{E}+01$

1.15E-01

1.07E-02

$1.06 \mathrm{E}-01$

$1.18 E+01$

$3.01 \mathrm{E}-00$

$2.84 \mathrm{E}+05$

$0.00 \mathrm{E}+01$

$0.00 \mathrm{E}+01$

$0.00 \mathrm{E}+01$

$0.00 \mathrm{E}+01$

$0.00 \mathrm{E}+01$

$0.00 \mathrm{E}+01$

$0.00 \mathrm{E}+01$

6.64E-01

$1.72 \mathrm{E}-04$

1.77E-00

$8.08 \mathrm{E}+01$

$1.25 \mathrm{E}+01$

$1.11 \mathrm{E}+02$

5.64E-04

$5.06 \mathrm{E}-05$

4.09E-02

7.32E- 03

$4.29 \mathrm{E}+01$

$1.76 \mathrm{E}-00$

$0.00 \mathrm{E}+01$

$1.21 \mathrm{E}+04$

$0.00 \mathrm{E}+01$

$1.16 \mathrm{E}-04$

3.30E-04

$0.00 \mathrm{E}+01$

$4.99 \mathrm{E}+02$

2.62E-02

$1.00 \mathrm{E}-01$

2.74E-01

1.21E-02

2.92E-03

$2.81 \mathrm{E}-01$
$50 \%$-ile

\section{$1.24 \mathrm{E}+03$}

$1.41 \mathrm{E}+04$

$1.24 \mathrm{E}+05$

$9.04 \mathrm{E}+03$

$3.99 \mathrm{E}+03$

3.34E +03

$7.79 \mathrm{E}+01$

$5.80 \mathrm{E}+03$

$5.62 \mathrm{E}+01$

$4.27 \mathrm{E}+02$

$5.44 \mathrm{E}+05$

$4.63 \mathrm{E}+02$

$1.72 E+05$

$7.25 \mathrm{E}+05$

1.30E+04

$4.32 \mathrm{E}+02$

$2.83 \mathrm{E}+04$

$7.28 \mathrm{E}+03$

$1.25 \mathrm{E}+03$

$2.07 \mathrm{E}+04$

$8.81 \mathrm{E}+01$

$1.12 \mathrm{E}+04$

$1.29 \mathrm{E}+04$

$2.03 \mathrm{E}+02$

2.14E-03

$2.95 \mathrm{E}+02$

4.95E-03

$3.21 \mathrm{E}+05$

$8.26 \mathrm{E}+01$

$2.09 \mathrm{E}+02$

$3.68 \mathrm{E}-01$

3.42E-02

3.38E-01

$1.63 \mathrm{E}+02$

$6.04 \mathrm{E}-00$

$3.13 \mathrm{E}+05$

9.60E-00

$1.49 \mathrm{E}+03$

$5.69 \mathrm{E}+02$

$1.13 \mathrm{E}-00$

$2.87 \mathrm{E}+01$

5.14E-00

$9.22 \mathrm{E}+01$

2.12E-00

9.79E-03

$5.65 \mathrm{E}-00$

$2.58 \mathrm{E}+02$

$3.98 \mathrm{E}+01$

$3.55 \mathrm{E}+02$

$1.80 \mathrm{E}-03$

$3.46 \mathrm{E}-04$

2.80E-01

$1.67 \mathrm{E}-02$

$3.99 \mathrm{E}+02$

8.06E-00

$2.84 \mathrm{E}+04$

$1.47 \mathrm{E}+05$

$5.86 \mathrm{E}+02$

6.62E-03

$1.88 \mathrm{E}-02$

$1.22 \mathrm{E}+01$

$5.59 \mathrm{E}+02$

1.49E-00

5.71E-00

$1.56 \mathrm{E}+01$

6.91E-01

1.66E-01

$1.60 \mathrm{E}+01$

90\%-ile

$4.12 \mathrm{E}+03$

$2.72 \mathrm{E}+04$

$1.76 \mathrm{E}+05$

$1.02 \mathrm{E}+04$

$8.83 \mathrm{E}+03$ 
Tank Anal. Un

\begin{tabular}{|c|c|}
\hline U109 & Y90 \\
\hline U109 & Zr93 \\
\hline U110 & Al \\
\hline U110 & $\mathbf{B i}$ \\
\hline U110 & $\mathrm{Ca}$ \\
\hline U110 & Cl \\
\hline U110 & $\mathrm{CO} 3$ \\
\hline U110 & $\mathrm{Cr}$ \\
\hline U110 & F \\
\hline U110 & Fe \\
\hline U110 & Hg \\
\hline U110 & $\mathbf{K}$ \\
\hline U110 & La \\
\hline U110 & Mn \\
\hline U110 & $\mathbf{N a}$ \\
\hline U110 & $\mathrm{Ni}$ \\
\hline U110 & NO2 \\
\hline U110 & NO3 \\
\hline U110 & $\mathbf{P}$ \\
\hline U110 & $\mathbf{P b}$ \\
\hline U110 & PO4 \\
\hline U110 & $\mathbf{S}$ \\
\hline U110 & $\mathbf{S i}$ \\
\hline U110 & SO4 \\
\hline U110 & $\mathrm{Sr}$ \\
\hline U110 & TOC \\
\hline U110 & $\mathbf{U}$ \\
\hline U110 & $\mathbf{Z r}_{\mathbf{r}}$ \\
\hline U110 & Ac227 \\
\hline U110 & Am241 \\
\hline U110 & Am243 \\
\hline U110 & Ba137 \\
\hline U110 & C14 \\
\hline U110 & Cd113 \\
\hline U110 & $\mathrm{Cm} 242$ \\
\hline U110 & Cm243 \\
\hline U110 & $\mathrm{Cm} 244$ \\
\hline U110 & Co60 \\
\hline U110 & Cs134 \\
\hline U110 & Cs137 \\
\hline U110 & Eu152 \\
\hline U110 & Eul54 \\
\hline U110 & Eul55 \\
\hline U110 & 1129 \\
\hline U110 & Nb93 \\
\hline U110 & Ni59 \\
\hline U110 & Ni63 \\
\hline U110 & Np237 \\
\hline U110 & Pa231 \\
\hline U110 & Pu238 \\
\hline U110 & Pu239 \\
\hline U110 & Pu240 \\
\hline U110 & Pu241 \\
\hline U110 & Pu242 \\
\hline U110 & $\operatorname{Ra} 226$ \\
\hline U110 & $\operatorname{Ra228}$ \\
\hline U110 & Ru106 \\
\hline U110 & Sb125 \\
\hline U110 & Se79 \\
\hline U110 & Sm151 \\
\hline U110 & Sr89/90 \\
\hline U110 & Te99 \\
\hline U110 & Th229 \\
\hline U110 & Th232 \\
\hline U110 & Tin126 \\
\hline U110 & Tritium \\
\hline U110 & U232 \\
\hline U110 & U233 \\
\hline
\end{tabular}

BB $8 \overline{24 \mathrm{E}+04}$ $3.95 \mathrm{E}+01$ $1.50 \mathrm{E}+05$ $2.10 \mathrm{E}+04$ kg $3.30 \mathrm{E}+03$ $1.00 \mathrm{E}+03$

$$
4.50 \mathrm{E}+03
$$$$
6.30 \mathrm{E}+02
$$

$7.20 \mathrm{E}+03$

1.30E+04

$$
\text { 3.00E-00 }
$$$$
7.80 \mathrm{E}+01
$$

$0.00 \mathrm{E}+01$

$4.20 \mathrm{E}+03$

$1.30 \mathrm{E}+02$

$9.40 \mathrm{E}+03$

$4.60 \mathrm{E}+04$

$4.65 \mathrm{E}+04$

$1.10 \mathrm{E}+03$

$1.44 \mathrm{E}+05$

$2.50 \mathrm{E}+03$

$2.30 \mathrm{E}+04$

$7.50 \mathrm{E}+03$

$5.20 \mathrm{E}+02$

$9.80 \mathrm{E}+02$

$1.10 \mathrm{E}+04$

$3.80 E+02$

5.52E-05

$9.00 \mathrm{E}+01$

$8.14 \mathrm{E}-08$

$2.50 \mathrm{E}+04$

3.50E-01

1.71E-01

4.37E-05

6.49E-07

1.25E-06

1.17E-02

6.59E-05

$2.60 \mathrm{E}+04$

$1.09 \mathrm{E}-02$

1.57E-01

1.65E-00

$1.08 \mathrm{E}-03$

$7.24 \mathrm{E}-02$

2.39E-02

2.10E-00

3.48E-03

$7.89 \mathrm{E}-05$

3.38E-02

$2.60 \mathrm{E}+02$

Ci Incl. in 239

4.00E-01

1.22E-06

1.72E-05

$1.48 \mathrm{E}-10$

2.75E-10

6.30E-03

$1.76 \mathrm{E}-02$

$6.66 E+01$

$3.40 E+05$

$7.30 \mathrm{E}-00$

2.86E-08

2.60E-11

2.62E-02

2.94E-01

2.64E-04

1.58E-05
BB Sept98 $8.22 \mathrm{E}+04$ $3.95 \mathrm{E}+01$ $1.54 \mathrm{E}+05$ 2.12E+04 $3.29 \mathrm{E}+03$ $1.05 \mathrm{E}+03$ $4.47 \mathrm{E}+03$ $6.29 \mathrm{E}+02$ $7.25 \mathrm{E}+03$ $1.27 \mathrm{E}+04$ 3.04E-00 $1.62 \mathrm{E}+02$ 2.70E+01 4.19E+03

$1.14 \mathrm{E}+05$

$1.27 \mathrm{E}+02$

$9.40 \mathrm{E}+03$

$4.64 \mathrm{E}+04$ NA

$1.12 \mathrm{E}+03$ $4.83 E+04$ NA

$2.28 \mathrm{E}+04$

$2.60 \mathrm{E}+03$

$5.19 \mathrm{E}+02$

9.82E +02

$1.13 E+04$

$3.82 \mathrm{E}+02$

5.52E-05

$8.89 \mathrm{E}+01$

2.49E-04

$2.51 \mathrm{E}+04$

3.52E-01

1.71E-01

1.34E-01

1.98E-03

$3.80 \mathrm{E}-03$

$1.17 \mathrm{E}-02$

6.59E-05

$2.65 \mathrm{E}+04$

1.09E-02

$1.57 \mathrm{E}-01$

$1.65 \mathrm{E}-00$

$1.08 \mathrm{E}-03$

$7.24 \mathrm{E}-02$

2.39E-02

2.10E-60

$3.48 \mathrm{E}-03$

$7.89 \mathrm{E}-05$

7.75E-01

$2.43 E+02$

$1.43 E+01$

9.16E-00

2.80E-05

1.72E-05

$1.48 \mathrm{E}-10$

2.75E-10

$6.30 \mathrm{E}-03$

$1.76 \mathrm{E}-02$

$6.66 \mathrm{E}+01$

$3.42 \mathrm{E}+05$

$7.35 \mathrm{E}-00$

2.86E-08

2.60E-11

2.62E-02

2.94E-01

4.42E-05

2.64E-06 1\%-ile

$2.57 \mathrm{E}+03$

$0.00 \mathrm{E}+01$

$1.95 \mathrm{E}+03$

$5.68 \mathrm{E}+03$

$2.15 \mathrm{E}+02$

$2.57 \mathrm{E}+02$

$3.61 \mathrm{E}+03$

$1.04 \mathrm{E}+02$

$1.30 \mathrm{E}+02$

$3.30 \mathrm{E}+03$

$0.00 \mathrm{E}+01$

$5.53 \mathrm{E}+01$

$5.00 \mathrm{E}-01$

$2.92 \mathrm{E}+01$

$4.71 \mathrm{E}+04$

6.53E-00

$1.16 \mathrm{E}+03$

$1.41 \mathrm{E}+04$

$5.09 \mathrm{E}+03$

$1.45 \mathrm{E}+01$

4.17E+03

$3.21 \mathrm{E}+02$

$5.35 \mathrm{E}+02$

$1.86 \mathrm{E}+03$

$1.94 \mathrm{E}+01$

$6.23 \mathrm{E}+01$

$8.36 \mathrm{E}+02$

$1.83 \mathrm{E}-00$

$0.00 \mathrm{E}+01$

4.17E-00

9.96E-09

$5.14 \mathrm{E}+03$

$4.78 \mathrm{E}-02$

$0.00 \mathrm{E}+01$

$5.35 \mathrm{E}-06$

7.94E-08

$1.53 \mathrm{E}-07$

2.34E-01

$1.36 \mathrm{E}-05$

4.57E+03

$0.00 \mathrm{E}+01$

$0.00 \mathrm{E}+01$

$0.00 \mathrm{E}+01$

$0.00 \mathrm{E}+01$

$0.00 \mathrm{E}+01$

3.63E-03

$3.19 \mathrm{E}-01$

$4.26 \mathrm{E}-04$

$0.00 \mathrm{E}+01$

4.14E- 03

$3.18 \mathrm{E}+01$

$3.18 \mathrm{E}+01$

$4.89 \mathrm{E}-02$

$1.49 \mathrm{E}-07$

$0.00 \mathrm{E}+01$

$0.00 \mathrm{E}+01$

$0.00 \mathrm{E}+01$

$0.00 \mathrm{E}+01$

$1.96 \mathrm{E}-03$

$0.00 \mathrm{E}+01$

$5.40 \mathrm{E}+02$

$1.00 \mathrm{E}-00$

$0.00 \mathrm{E}+01$

$0.00 \mathrm{E}+01$

$0.00 \mathrm{E}+01$

$1.66 \mathrm{E}-01$

$0.00 \mathrm{E}+01$

$0.00 \mathrm{E}+01$
$10 \%$-ile

$6.52 \mathrm{E}+03$

$0.00 \mathrm{E}+01$

$5.58 \mathrm{E}+03$

$8.60 \mathrm{E}+03$

$4.63 \mathrm{E}+02$

$5.68 \mathrm{E}+02$

$8.27 \mathrm{E}+03$

$3.78 \mathrm{E}+02$

$9.26 \mathrm{E}+02$

6.61E+03

4.60E-01

$1.50 \mathrm{E}+02$

$1.56 \mathrm{E}-00$

$6.07 \mathrm{E}+01$

$6.31 \mathrm{E}+04$

$1.39 \mathrm{E}+01$

$4.02 E+03$

$4.35 \mathrm{E}+04$

$9.91 \mathrm{E}+03$

$5.82 \mathrm{E}+01$

$1.02 \mathrm{E}+04$

$1.26 \mathrm{E}+03$

$2.24 \mathrm{E}+03$

$4.44 \mathrm{E}+03$

$6.41 \mathrm{E}+01$

$2.86 \mathrm{E}+02$

$3.26 \mathrm{E}+03$

$6.58 \mathrm{E}-00$

1.31E-05

$1.53 \mathrm{E}+01$

3.47E-08

$1.16 \mathrm{E}+04$

$1.45 \mathrm{E}-01$

2.62E-02

$1.86 \mathrm{E}-05$

2.77E-07

5.33E-07

$7.87 \mathrm{E}-01$

3.05E-05

$9.66 \mathrm{E}+03$

2.59E-03

3.74E-02

3.93E-01

4.19E-04

$1.88 \mathrm{E}-03$

9.21E-03

8.09E-01

$1.48 \mathrm{E}-03$

$0.00 \mathrm{E}+01$

$1.44 \mathrm{E}-02$

$1.11 \mathrm{E}+02$

$1.11 \mathrm{E}+02$

1.71E-01

$5.20 \mathrm{E}-07$

4.22E-06

$3.63 \mathrm{E}-11$

9.82E-11

$0.00 \mathrm{E}+01$

$7.45 \mathrm{E}-03$

$1.58 \mathrm{E}+01$

$8.42 \mathrm{E}+03$

$2.49 \mathrm{E}-00$

$0.00 \mathrm{E}+01$

$0.00 \mathrm{E}+01$

4.02E-03

$2.09 \mathrm{E}-01$

$0.00 \mathrm{E}+01$

$0.00 \mathrm{E}+01$

50\%-ile

$8.24 \mathrm{E}+04$

$3.95 \mathrm{E}+01$

$1.58 \mathrm{E}+04$

$1.34 \mathrm{E}+04$

$9.83 \mathrm{E}+02$

$9.22 \mathrm{E}+02$ 
Tank Anal. Un

\begin{tabular}{|c|c|c|c|c|}
\hline U110 & U234 & $\mathrm{Ci}$ & $2 \frac{\text { Мау }}{2.23 \mathrm{E}+01}$ & $\frac{\text { Sept98 }}{3.73 E-60}$ \\
\hline U110 & U235 & $\mathrm{Ci}$ & $1.00 \mathrm{E}-00$ & $1.68 \mathrm{E}-01$ \\
\hline U110 & U236 & $\mathrm{Ci}$ & $1.42 \mathrm{E}-01$ & $2.38 E-02$ \\
\hline U110 & U238 & $\mathrm{Ci}$ & $2.25 \mathrm{E}+01$ & $3.77 \mathrm{E}-00$ \\
\hline U110 & Y90 & $\mathrm{Ci}$ & $3.40 E+05$ & $3.42 E+05$ \\
\hline U110 & Zr93 & $\mathrm{Ci}$ & $8.41 E-02$ & $8.41 \mathrm{E}-02$ \\
\hline U111 & Al & kg & $3.58 \mathrm{E}+04$ & $3.58 E+04$ \\
\hline U111 & Bi & kg & $3.04 \mathrm{E}+03$ & $3.04 \mathrm{E}+03$ \\
\hline U111 & $\mathrm{Ca}$ & kg & $5.73 E+02$ & $5.80 \mathrm{E}+02$ \\
\hline U111 & Cl & kg & $6.76 E+03$ & $6.76 E+03$ \\
\hline U111 & $\mathrm{CO} 3$ & kg & $2.37 \mathrm{E}+04$ & $2.37 E+04$ \\
\hline U111 & $\mathbf{C r}$ & $\mathrm{kg}$ & $7.72 E+03$ & $7.72 E+03$ \\
\hline U111 & F & kg & $4.84 E+03$ & $4.84 \mathrm{E}+03$ \\
\hline U111 & Fe & kg & $3.70 \mathrm{E}+03$ & $3.70 \mathrm{E}+03$ \\
\hline U111 & $\mathbf{H g}$ & kg & $2.66 \mathrm{E}-00$ & $4.14 \mathrm{E}-00$ \\
\hline U111 & $\mathbf{K}$ & kg & $1.96 \mathrm{E}+03$ & $1.96 E+03$ \\
\hline U111 & $\mathbf{L a}$ & kg & $2.70 \mathrm{E}+01$ & $0.00 \mathrm{E}+01$ \\
\hline U111 & Mn & kg & $1.34 E+03$ & $1.34 E+03$ \\
\hline U111 & $\mathbf{N a}$ & $\mathbf{k g}$ & $3.60 E+05$ & $3.60 \mathrm{E}+05$ \\
\hline U111 & $\mathbf{N i}$ & kg & $4.87 E+02$ & $4.87 E+02$ \\
\hline U111 & NO2 & kg & $8.45 E+04$ & $8.45 E+04$ \\
\hline U111 & NO3 & kg & $5.47 E+05$ & $5.47 E+05$ \\
\hline U111 & $\mathbf{P}$ & kg & $4.19 E+04$ & $\mathbf{N A}$ \\
\hline U111 & $\mathbf{P b}$ & kg & $3.52 \mathrm{E}+02$ & $3.52 E+02$ \\
\hline U111 & PO4 & kg & $1.30 \mathrm{E}+05$ & $4.23 E+04$ \\
\hline U111 & $\mathbf{S}$ & kg & $2.25 E+04$ & NA \\
\hline U111 & Si & kg & $2.40 E+03$ & $2.40 E+03$ \\
\hline U111 & SO4 & kg & $6.75 E+04$ & $2.25 E+04$ \\
\hline U111 & Sr & kg & $1.07 E+02$ & $1.07 E+02$ \\
\hline U111 & TOC & kg & $9.93 E+03$ & $9.93 E+03$ \\
\hline U111 & $\mathbf{U}$ & kg & $3.29 E+03$ & $3.29 E+03$ \\
\hline U111 & $\mathbf{Z r}$ & kg & $4.70 \mathrm{E}+01$ & $4.70 \mathrm{E}+01$ \\
\hline U111 & Ac227 & $\mathrm{Ci}$ & $1.10 \mathrm{E}-03$ & $1.10 \mathrm{E}-03$ \\
\hline U111 & Am241 & $\mathrm{Ci}$ & $5.49 \mathrm{E}+01$ & $5.49 \mathrm{E}+01$ \\
\hline U111 & Am243 & $\mathrm{Ci}$ & $1.89 \mathrm{E}-03$ & $1.89 \mathrm{E}-03$ \\
\hline U111 & Ba137 & $\mathrm{Ci}$ & $2.34 E+05$ & $2.34 E+05$ \\
\hline v111 & C14 & $\mathrm{Ci}$ & $3.23 \mathrm{E}+01$ & $3.23 \mathrm{E}+01$ \\
\hline U111 & Cd113 & $\mathrm{Ci}$ & $8.30 E+01$ & $8.30 \mathrm{E}+01$ \\
\hline U111 & Cm242 & $\mathrm{Ci}$ & $1.48 \mathrm{E}-01$ & $1.48 \mathrm{E}-01$ \\
\hline U111 & Cm243 & $\mathbf{C i}$ & $1.31 \mathrm{E}-02$ & $1.31 \mathrm{E}-02$ \\
\hline U111 & $\mathrm{Cm} 244$ & $\mathrm{Ci}$ & $1.28 \mathrm{E}-01$ & $1.28 \mathrm{E}-01$ \\
\hline U111 & Co60 & $\mathrm{Ci}$ & $3.56 \mathrm{E}+01$ & $3.56 \mathrm{E}+0.1$ \\
\hline U111 & Cs134 & $C \mathrm{i}$ & $2.46 \mathrm{E}-00$ & $2.46 E-00$ \\
\hline U111 & Cs137 & $\mathrm{Ci}$ & $2.48 E+05$ & $2.48 E+05$ \\
\hline U111 & Eu152 & $\mathrm{Ci}$ & $4.05 E-00$ & $4.05 E-00$ \\
\hline U111 & Eu154 & $\mathrm{Ci}$ & $5.80 E+02$ & $5.80 E+02$ \\
\hline U111 & Eu155 & $\mathrm{Ci}$ & $2.35 \mathrm{E}+02$ & $2.35 E+02$ \\
\hline U111 & 1129 & $\mathrm{Ci}$ & 4.44E-01 & 4.44E-01 \\
\hline U111 & Nb93 & $\mathrm{Ci}$ & $1.16 E+01$ & $1.16 E+01$ \\
\hline U111 & Ni59 & $\mathrm{Ci}$ & 2.82E-00 & 2.82E-00 \\
\hline U111 & Ni63 & $\mathrm{Ci}$ & $2.73 E+02$ & $2.73 E+02$ \\
\hline U111 & Np237 & $\mathrm{Ci}$ & 8.45E-01 & $8.45 E-01$ \\
\hline U111 & Pa231 & $\mathrm{Ci}$ & $4.02 \mathrm{E}-03$ & $4.02 \mathrm{E}-03$ \\
\hline U111 & Pu238 & $\mathrm{Ci}$ & $1.64 \mathrm{E}-00$ & $1.64 E-00$ \\
\hline U111 & Pu239 & $\mathrm{Ci}$ & $6.90 \mathrm{E}+01$ & $6.90 E+01$ \\
\hline U111 & Pu240 & $\mathrm{Ci}$ & $1.10 E+01$ & $1.10 \mathrm{E}+01$ \\
\hline U111 & Pu241 & $\mathrm{Ci}$ & $1.08 E+02$ & $1.08 \mathrm{E}+02$ \\
\hline U111 & Pu242 & $\mathrm{Ci}$ & 5.75E-04 & 5.75E-04 \\
\hline U111 & Ra226 & $\mathrm{Ci}$ & $1.91 \mathrm{E}-04$ & $1.91 \mathrm{E}-04$ \\
\hline U111 & Ra228 & $\mathrm{Ci}$ & $1.28 \mathrm{E}-01$ & $1.28 \mathrm{E}-01$ \\
\hline U111 & Ru106 & $\mathrm{Ci}$ & $6.33 E-03$ & $6.33 E-03$ \\
\hline U111 & Sb125 & $\mathrm{Ci}$ & $1.53 E+02$ & $1.53 \mathrm{E}+02$ \\
\hline U111 & Se79 & $\mathrm{Ci}$ & $3.24 E-00$ & $3.24 \mathrm{E}-00$ \\
\hline U111 & Sm151 & $\mathrm{Ci}$ & $1.14 E+04$ & $1.14 E+04$ \\
\hline U111 & Sr89/90 & $\mathrm{Ci}$ & $2.21 E+05$ & $2.66 \mathrm{E}+05$ \\
\hline U111 & Tc99 & $\mathrm{Ci}$ & $2.30 \mathrm{E}+02$ & $2.30 \mathrm{E}+02$ \\
\hline U111 & Th229 & $\mathrm{Ci}$ & $3.01 \mathrm{E}-03$ & $3.01 E-03$ \\
\hline U111 & Th232 & $\mathrm{Ci}$ & 8.52E-03 & $8.52 E-03$ \\
\hline
\end{tabular}

$1 \%$-ile

$0.00 \mathrm{E}+01$

$0.00 \mathrm{E}+01$

$0.00 \mathrm{E}+01$

$0.00 \mathrm{E}+01$

$8.05 \mathrm{E}+02$

$0.00 \mathrm{E}+01$

$1.22 \mathrm{E}+04$

$2.84 \mathrm{E}+02$

$3.10 \mathrm{E}+01$

$7.75 \mathrm{E}+01$

$7.34 \mathrm{E}+02$

$6.80 \mathrm{E}+01$

8.45E+01

$3.33 \mathrm{E}+02$

$0.00 \mathrm{E}+01$

$6.01 \mathrm{E}+01$

$3.96 \mathrm{E}-01$

$7.36 \mathrm{E}-00$

$1.26 \mathrm{E}+05$

$1.56 \mathrm{E}-00$

$1.05 E+03$

$5.87 \mathrm{E}+03$

$5.98 \mathrm{E}+02$

$3.17 \mathrm{E}-00$

$6.48 \mathrm{E}+02$

$8.42 \mathrm{E}+01$

$1.90 \mathrm{E}+02$

$2.39 \mathrm{E}+02$

$7.59 \mathrm{E}-00$

$1.19 \mathrm{E}+02$

$1.84 \mathrm{E}+02$

7.14E-01

$0.00 \mathrm{E}+01$

2.63E-00

$9.47 \mathrm{E}-06$

$0.00 \mathrm{E}+01$

$0.00 \mathrm{E}+01$

$0.00 \mathrm{E}+01$

7.42E-04

$6.57 \mathrm{E}-05$

6.42E-04

$3.88 \mathrm{E}-01$

$0.00 \mathrm{E}+01$

$5.18 \mathrm{E}+03$

$0.00 \mathrm{E}+01$

$0.00 \mathrm{E}+01$

$0.00 \mathrm{E}+01$

$0.00 \mathrm{E}+01$

$0.00 \mathrm{E}+01$

$0.00 \mathrm{E}+01$

$0.00 \mathrm{E}+01$

4.24E-03

$0.00 \mathrm{E}+01$

$8.22 \mathrm{E}-03$

$3.46 \mathrm{E}-01$

5.51E-02

5.41E-01

$2.88 \mathrm{E}-06$

$0.00 \mathrm{E}+01$

$0.00 \mathrm{E}+01$

$0.00 \mathrm{E}+01$

$0.00 \mathrm{E}+01$

$0.00 \mathrm{E}+01$

$0.00 \mathrm{E}+01$

$3.67 \mathrm{E}+02$

$0.00 \mathrm{E}+01$

$0.00 \mathrm{E}+01$

$0.00 \mathrm{E}+01$
$10 \%$-ile

$0.00 \mathrm{E}+01$

$0.00 \mathrm{E}+01$

$0.00 \mathrm{E}+01$

$0.00 \mathrm{E}+01$

$4.06 \mathrm{E}+04$

2.18E-03

$3.24 \mathrm{E}+04$

$7.54 \mathrm{E}+02$

$1.57 \mathrm{E}+02$

$2.92 \mathrm{E}+02$

$2.62 \mathrm{E}+03$

$2.73 E+02$

$7.00 \mathrm{E}+02$

$9.14 \mathrm{E}+02$

$0.00 \mathrm{E}+01$

$1.33 \mathrm{E}+03$

$1.01 \mathrm{E}-00$

$5.92 \mathrm{E}+01$

$2.49 \mathrm{E}+05$

6.32E-00

$4.95 \mathrm{E}+03$

$3.68 \mathrm{E}+04$

$1.68 \mathrm{E}+03$

$1.49 \mathrm{E}+01$

$2.30 \mathrm{E}+03$

$3.79 \mathrm{E}+02$

$5.37 \mathrm{E}+02$

$9.09 \mathrm{E}+02$

$2.97 \mathrm{E}+01$

$2.03 E+03$

$5.15 E+02$

3.13E-00

$0.00 \mathrm{E}+01$

$5.77 \mathrm{E}-00$

3.32E-05

$0.00 \mathrm{E}+01$

$0.00 E+01$

$0.00 \mathrm{E}+01$

$2.60 \mathrm{E}-03$

2.30E-04

2.25E-03

9.10E-01

$0.00 \mathrm{E}+01$

$1.71 \mathrm{E}+04$

$0.00 \mathrm{E}+01$

$0.00 \mathrm{E}+01$

$0.00 \mathrm{E}+01$

$0.00 E+01$

$0.00 \mathrm{E}+01$

$0.00 \mathrm{E}+01$

$0.00 \mathrm{E}+01$

$1.48 \mathrm{E}-02$

$0.00 \mathrm{E}+01$

2.88E-02

$1.21 \mathrm{E}-00$

1.93E-01

$1.90 \mathrm{E}-00$

1.01E-05

$0.00 \mathrm{E}+01$

$0.00 \mathrm{E}+01$

$0.00 \mathrm{E}+01$

7.89E- 00

$0.00 \mathrm{E}+01$

$0.00 \mathrm{E}+01$

3.32E+04

$0.00 \mathrm{E}+01$

$0.00 E+01$

$0.00 \mathrm{E}+01$ 50\%-ile

$2.23 \mathrm{E}+01$

1.00E-00

1.42E-01

2.25E+01

$3.40 \mathrm{E}+05$

8.41E-02

$5.71 \mathrm{E}+04$

$1.71 \mathrm{E}+03$

$1.24 \mathrm{E}+03$

$4.18 \mathrm{E}+03$

$1.40 \mathrm{E}+05$

$6.69 \mathrm{E}+03$

$1.58 \mathrm{E}+04$

$6.80 \mathrm{E}+03$

2.66E-00

$4.86 \mathrm{E}+03$

$1.63 \mathrm{E}+02$

$1.42 \mathrm{E}+03$

4.07E+05

$6.15 \mathrm{E}+02$

6.57E+04

4.00E+05

$1.33 \mathrm{E}+04$

$2.87 \mathrm{E}+02$

$3.41 \mathrm{E}+04$

$1.23 \mathrm{E}+04$

$4.87 \mathrm{E}+03$

$3.81 \mathrm{E}+04$

$3.34 \mathrm{E}+02$

$9.94 \mathrm{E}+03$

$1.55 \mathrm{E}+04$

$4.99 \mathrm{E}+03$

$1.10 \mathrm{E}-03$

$1.29 \mathrm{E}+02$

$1.89 \mathrm{E}-03$

$2.34 \mathrm{E}+05$

$3.23 \mathrm{E}+01$

$8.30 \mathrm{E}+01$

$1.48 \mathrm{E}-01$

$1.31 \mathrm{E}-02$

1.28E-01

4.64E+01

2.46E-00

2.52E+05

4.05E-00

$5.80 \mathrm{E}+02$

$2.35 \mathrm{E}+02$

4.44E-01

$1.16 \mathrm{E}+01$

2.82E-00

$2.73 E+02$

8.45E-01

4.02E-03

1.64E-00

$6.90 \mathrm{E}+01$

$1.10 \mathrm{E}+01$

$1.08 E+02$

5.75E-04

1.91E-04

$1.28 \mathrm{E}-01$

6.33E-03

$1.53 \mathrm{E}+02$

$3.24 \mathrm{E}-00$

$1.14 \mathrm{E}+04$

$2.25 E+05$

$2.30 \mathrm{E}+02$

3.01E-03

8.52E-03

\section{$90 \%$-ile}

$6.92 \mathrm{E}+01$

$3.10 \mathrm{E}-00$

4.41E-01

$6.98 \mathrm{E}+01$

$1.75 \mathrm{E}+06$

4.68E-01

$8.40 \mathrm{E}+04$

$3.42 \mathrm{E}+03$

$2.44 \mathrm{E}+03$

$8.96 \mathrm{E}+03$

$2.93 \mathrm{E}+05$

$1.46 \mathrm{E}+04$

$3.89 \mathrm{E}+04$

$1.80 \mathrm{E}+04$

$1.05 \mathrm{E}+01$

$8.71 E+03$

$5.01 \mathrm{E}+02$

$5.18 \mathrm{E}+03$

$5.92 \mathrm{E}+05$

$1.76 \mathrm{E}+03$

$1.30 \mathrm{E}+05$

8.17E+05

3.17E+04

$1.03 \mathrm{E}+03$

$7.79 \mathrm{E}+04$

$2.81 \mathrm{E}+04$

$1.17 \mathrm{E}+04$

8.75E+04

$9.24 \mathrm{E}+02$

$1.85 \mathrm{E}+04$

$4.91 \mathrm{E}+04$

$2.84 \mathrm{E}+04$

5.02E-03

$5.90 \mathrm{E}+03$

7.37E-03

$5.06 \mathrm{E}+05$

$7.82 \mathrm{E}+01$

$3.26 \mathrm{E}+02$

$5.77 \mathrm{E}-01$

5.11E-02

$4.99 \mathrm{E}-01$

1.52E+02

5.32E-00

$5.12 E+05$

$1.85 \mathrm{E}+01$

$2.65 E+03$

$1.07 \mathrm{E}+03$

$1.02 \mathrm{E}-00$

$7.38 \mathrm{E}+01$

9.45E-00

$9.15 \mathrm{E}+02$

3.30E-00

1.51E-02

6.40E-00

$2.69 \mathrm{E}+02$

$4.29 \mathrm{E}+01$

$4.21 \mathrm{E}+02$

$2.24 \mathrm{E}-03$

$6.24 \mathrm{E}-04$

4.18E-01

1.84E-02

$4.12 \mathrm{E}+02$

$7.89 \mathrm{E}-00$

$5.20 \mathrm{E}+04$

$6.04 E+05$

$9.16 \mathrm{E}+02$

1.13E-02

$3.20 \mathrm{E}-02$

99\%-ile

$1.63 \mathrm{E}+02$

$7.30 \mathrm{E}-00$

$1.04 \mathrm{E}-00$

$1.64 \mathrm{E}+02$

$1.18 \mathrm{E}+07$

8.71E-01

$1.07 \mathrm{E}+05$

$6.15 \mathrm{E}+03$ 


\begin{tabular}{|c|c|c|c|c|c|c|c|c|c|c|c|}
\hline ank & nal. & $\underline{n}$ & May $\frac{B B}{98}$ & Sept98 & $\%$-ile & $10 \%$-ile & $50 \%$-ile & $90 \%$-ile & 99\%-ile & Mean & Std Dev \\
\hline U111 & in126 & $\mathrm{Ci}$ & $4.90 \mathrm{E}-00$ & $4.90 \mathrm{E}-00$ & $0.00 \mathrm{E}+01$ & $0.00 \mathrm{E}+01$ & $4.90 \mathrm{E}-00$ & $1.93 \mathrm{E}+01$ & $3.24 \mathrm{E}+01$ & $4 E-00$ & $8.20 \mathrm{E}-00$ \\
\hline U111 & Tritium & $\mathrm{Ci}$ & $2.27 \mathrm{E}+02$ & $2.27 \mathrm{E}+02$ & $7.03 E+01$ & $1.35 \mathrm{E}+02$ & $2.27 \mathrm{E}+02$ & $\mathrm{E}+02$ & $17 \mathrm{E}+02$ & $E+02$ & $7.37 \mathrm{E}+01$ \\
\hline U111 & U232 & $\mathrm{Ci}$ & $6.64 \mathrm{E}-01$ & $4.86 \mathrm{E}-01$ & $0.00 \mathrm{E}+01$ & $0.00 \mathrm{E}+01$ & $6.64 \mathrm{E}-01$ & $2.50 \mathrm{E}-00$ & $E-00$ & 9.53E-01 & $1.05 \mathrm{E}-00$ \\
\hline U111 & U233 & $\mathrm{Ci}$ & 2.55E-00 & $1.86 \mathrm{E}-00$ & $0.00 \mathrm{E}+01$ & $0.00 \mathrm{E}+01$ & 2.55E-00 & $9.58 \mathrm{E}-00$ & $1.53 E+01$ & $3.66 \mathrm{E}-00$ & $4.05 \mathrm{E}-00$ \\
\hline U111 & U234 & $\mathrm{Ci}$ & $1.56 \mathrm{E}-00$ & 1.14E-00 & $0.00 \mathrm{E}+01$ & $0.00 \mathrm{E}+01$ & $1.56 \mathrm{E}-00$ & $5.86 \mathrm{E}-00$ & $9.36 \mathrm{E}-00$ & $2.24 \mathrm{E}-00$ & $2.48 \mathrm{E}-00$ \\
\hline U111 & U235 & $\mathrm{Ci}$ & $6.68 \mathrm{E}-02$ & $4.89 E-02$ & $0.00 \mathrm{E}+01$ & $0.00 \mathrm{E}+01$ & $6.68 \mathrm{E}-02$ & $2.51 \mathrm{E}-01$ & & $9.58 \mathrm{E}-02$ & \\
\hline U111 & U236 & $\mathbf{C i}$ & 2.91E-02 & 2.13E-02 & $E+01$ & & & & & -02 & \\
\hline U111 & U238 & $\mathrm{Ci}$ & $1.69 \mathrm{E}-00$ & $1.10 \mathrm{E}-00$ & $0.00 \mathrm{E}+01$ & $0.00 \mathrm{E}+01$ & $1.69 \mathrm{E}-00$ & 6.35 & +01 & -00 & -00 \\
\hline U111 & Y90 & $\mathrm{Ci}$ & $E+05$ & $2.66 \mathrm{E}+05$ & $0.00 \mathrm{E}+01$ & 104 & +05 & +05 & +06 & 4.17E+05 & +06 \\
\hline U111 & Zr93 & $\mathbf{C i}$ & $1.59 E+01$ & $1.59 \mathrm{E}+01$ & $0.00 \mathrm{E}+01$ & $0.00 E+01$ & $1.59 E+01$ & $1.01 \mathrm{E}+02$ & $1.71 \mathrm{E}+02$ & $E+01$ & $\div 01$ \\
\hline U112 & Al & kg & $1.12 E+04$ & $4.06 E+04$ & $1.46 \mathrm{E}+03$ & $4.43 E+03$ & $9.97 E+03$ & $1.84 \mathrm{E}+04$ & $2.66 \mathrm{E}+04$ & $1.07 E+04$ & $5.57 \mathrm{E}+03$ \\
\hline U112 & $\mathbf{B i}$ & kg & $2.94 E+03$ & $1.11 E+03$ & $3.81 E+02$ & $E+03$ & $2.59 E+03$ & $3 E+03$ & $E+03$ & $2.97 \mathrm{E}+03$ & $E+03$ \\
\hline U112 & $\mathrm{Ca}$ & kg & $2.25 \mathrm{E}+02$ & $5.24 \mathrm{E}+02$ & $2.19 E+01$ & $6.16 \mathrm{E}+01$ & $1.61 E+02$ & $4.14 \mathrm{E}+02$ & $E+02$ & $2.09 E+02$ & $E+02$ \\
\hline U112 & Cl & kg & $3.92 \mathrm{E}+02$ & $1.72 E+02$ & $4.96 \mathrm{E}+01$ & $E+02$ & $2.94 E+02$ & $5.58 \mathrm{E}+02$ & $9.00 E+02$ & $3.24 \mathrm{E}+02$ & $E+02$ \\
\hline U112 & $\mathrm{CO3}$ & kg & $1.07 E+03$ & $1.51 E+03$ & $2.38 \mathrm{E}+02$ & +02 & +03 & $E+03$ & $E+03$ & +03 & $E+03$ \\
\hline U112 & $\mathrm{Cr}$ & kg & $2.50 \mathrm{E}+02$ & $1.23 \mathrm{E}+02$ & +01 & +02 & -02 & $8+02$ & +03 & -02 & +02 \\
\hline U112 & $\mathbf{F}$ & kg & $1.35 \mathrm{E}+03$ & $2.34 E+03$ & +01 & +02 & +03 & +03 & +03 & +03 & +03 \\
\hline U112 & $\mathbf{F e}$ & kg & $2.07 E+03$ & $1.39 E+03$ & $3.13 E+02$ & +02 & +03 & +03 & $E+03$ & +03 & +03 \\
\hline U112 & $\mathrm{Hg}$ & kg & $2.12 \mathrm{E}+01$ & $1.46 \mathrm{E}+01$ & $0.00 \mathrm{E}+01$ & -00 & +01 & +01 & +02 & +01 & +01 \\
\hline U112 & $\mathbf{K}$ & kg & $1.36 E+02$ & $1.17 E+02$ & $E+01$ & +01 & +02 & +02 & +02 & +02 & +02 \\
\hline U112 & La & kg & 3.50E-01 & $0.00 E+01$ & $1.72 \mathrm{E}-01$ & $E-01$ & $E-00$ & & -01 & & $8-00$ \\
\hline U112 & Mn & kg & $1.26 \mathrm{E}+02$ & $5.34 E+01$ & $E-00$ & & +02 & & 02 & & +02 \\
\hline U112 & $\mathbf{N a}$ & kg & $2.33 E+04$ & $2.21 E+04$ & +03 & +04 & +04 & -04 & 04 & -04 & +03 \\
\hline U112 & $\mathrm{Ni}$ & kg & $1.45 \mathrm{E}+01$ & $3.15 E+01$ & $=-00$ & -00 & +01 & +01 & +02 & +01 & +01 \\
\hline U112 & NO2 & $\mathrm{kg}$ & $3.84 \mathrm{E}+03$ & $1.17 E+03$ & $3.44 \mathrm{E}+02$ & +03 & +03 & +03 & $E+04$ & $:+03$ & $E+03$ \\
\hline Uns & NO3 & kg & $3.02 E+04$ & $E+04$ & +03 & & & & & & +04 \\
\hline U112 & $\mathbf{P}$ & kg & $5.03 E+03$ & NA & $:+02$ & & 03 & & +04 & & \\
\hline U112 & $\mathbf{P b}$ & kg & $4.80 \mathrm{E}+01$ & $4.62 E+02$ & $E-00$ & +01 & +01 & 102 & +02 & +01 & H01 \\
\hline 2 & PO4 & kg & $1.56 E+04$ & $2.05 E+04$ & 02 & & & & & & \\
\hline U112 & $\mathbf{S}$ & kg & $2.73 E+03$ & NA & +01 & 02 & 02 & & -03 & 02 & -02 \\
\hline U112 & $\mathbf{S i}$ & kg & $1.37 E+03$ & $7.21 E+02$ & $E+02$ & +02 & +03 & +03 & +03 & +03 & +02 \\
\hline 0112 & SO4 & kg & $8.19 E+03$ & $8.36 \mathrm{E}+02$ & +02 & +02 & +03 & & 104 & & +03 \\
\hline U112 & $\mathbf{S r}$ & kg & $5.93 E+01$ & $1.97 E+01$ & $E-00$ & +01 & & & & & \\
\hline U112 & TOC & kg & $2.92 \mathrm{E}+02$ & $4.58 \mathrm{E}+02$ & +01 & & & & & & \\
\hline U112 & $\mathbf{U}$ & $\mathbf{k g}$ & 9.30 & $2.62 E+03$ & 01 & & & & & -03 & \\
\hline U112 & $\mathbf{Z r}$ & kg & $3.60 \mathrm{E}+01$ & $5.16 \mathrm{E}+01$ & .01 & 00 & 01 & 01 & 02 & -01 & +01 \\
\hline U112 & Ac227 & $\mathbf{C i}$ & $1.31 \mathrm{E}-04$ & $1.31 E-04$ & $0.00 E+01$ & -05 & -04 & .04 & -03 & -04 & -04 \\
\hline U112 & $\operatorname{Am} 241$ & $\mathrm{Ci}$ & 2.27E-01 & $4.24 E-02$ & & & 01 & & 02 & -01 & +02 \\
\hline U112 & Am243 & $\mathbf{C i}$ & 2.10E-06 & $3.92 E-07$ & & & & & & & \\
\hline U112 & Ba137 & $\mathbf{C i}$ & $1.50 E+04$ & $1.81 E+04$ & & & & & & & \\
\hline 2 & C14 & $\mathbf{C i}$ & $7.29 \mathrm{E}-02$ & $E-02$ & & & & & & .02 & -02 \\
\hline U112 & Cd113 & $\mathrm{Ci}$ & 2.15E-01 & 2.15E-01 & -01 & 02 & 01 & 01 & -00 & -01 & -01 \\
\hline U112 & Cm242 & $\mathrm{Ci}$ & 4.35E- 03 & $8.13 E-04$ & -04 & 03 & .03 & & .02 & -03 & -03 \\
\hline U112 & Cm243 & $\mathrm{C}$ & -05 & $1.86 \mathrm{E}-05$ & & & & & & -04 & -05 \\
\hline U112 & $\mathrm{Cm} 2$ & $\mathrm{Ci}$ & 7.4 & 1.40 & & & & & & & \\
\hline & Co60 & $\mathrm{Ci}$ & 12 & 2.62 & & & & & & & +01 \\
\hline U112 & 134 & $\mathrm{Ci}$ & 04 & $7.70 \mathrm{E}-04$ & & & & & & -04 & -04 \\
\hline U112 & Cs137 & $\mathbf{C i}$ & $1.59 E+04$ & $1.91 E+04$ & +02 & -03 & 104 & 04 & -04 & 104 & +03 \\
\hline U112 & Eu152 & $\mathbf{C i}$ & $1.90 \mathrm{E}-01$ & $1.90 \mathrm{E}-01$ & +01 & & & & & & \\
\hline U112 & Eu154 & $\mathbf{C} \mathbf{j}$ & $6.18 \mathrm{E}-01$ & $6.18 \mathrm{E}-01$ & $8+01$ & & & & & -00 & $E-00$ \\
\hline $\mathbf{U 1 1 2}$ & Eu155 & $\mathrm{Ci}$ & -00 & & +01 & & & -01 & +02 & +01 & +01 \\
\hline U1] & & $\mathrm{Ci}$ & & & & & & & & & \\
\hline $\mathbf{v}$ & & 0 & 6.0 & & & & & & & -01 & -01 \\
\hline U112 & & $\mathrm{Ci}$ & 3.97E-01 & $3.97 \mathrm{E}-01$ & $E-02$ & -01 & -01 & .00 & +01 & -01 & $E-00$ \\
\hline U112 & Ni63 & $\mathrm{Ci}$ & $3.71 E+01$ & $3.71 \mathrm{E}+01$ & $=00$ & +01 & $i+01$ & 1. & +03 & +01 & +02 \\
\hline U112 & Np237 & $\mathrm{Ci}$ & $3.28 \mathrm{E}-03$ & $3.28 \mathrm{E}-03$ & .04 & -03 & & & -02 & & -03 \\
\hline U112 & Pa231 & $\mathrm{Ci}$ & $2.97 E-05$ & $2.97 \mathrm{E}-05$ & +01 & & & -04 & & -05 & -05 \\
\hline U112 & Pu238 & $\mathrm{Ci}$ & $7.52 \mathrm{E}-01$ & $1.40 \mathrm{E}-01$ & & & & -00 & & -01 & -01 \\
\hline U112 & Pu239 & $\mathrm{Ci}$ & $5.1 !$ & & & & & & & & +01 \\
\hline U112 & Pu240 & $\mathrm{Ci}$ & & & & & & & +01 & $=00$ & $\varepsilon-00$ \\
\hline & Pu241 & $\mathrm{Ci}$ & $4.21 E+01$ & $7.86 \mathrm{E}-00$ & & & & 01 & +02 & +01 & +01 \\
\hline & Pu242 & $\mathrm{Ci}$ & $1.82 \mathrm{E}-04$ & $3.40 \mathrm{E}-05$ & E-05 & & & .04 & $\mathrm{E}-04$ & -44 & -04 \\
\hline & Ra226 & $\mathrm{Ci}$ & $2.96 \mathrm{E}-05$ & $2.96 \mathrm{E}-05$ & & & & & & & \\
\hline U112 & Ra228 & $\mathrm{Ci}$ & $2.66 \mathrm{E}-10$ & $2.66 \mathrm{E}-10$ & & & & -10 & -09 & & $E-10$ \\
\hline U112 & Ru106 & $\mathrm{Ci}$ & $7.24 E-08$ & $7.24 \mathrm{E}-08$ & $0.00 \mathrm{E}+01$ & $1.60 \mathrm{E}-08$ & $7.24 E-08$ & 1.72E-07 & $2.93 \mathrm{E}-07$ & $8.60 \mathrm{E}-08$ & $6.49 \mathrm{E}-08$ \\
\hline U112 & Sb125 & $\mathrm{Ci}$ & $3.73 E-02$ & $3.73 E-02$ & $0.00 \mathrm{E}+01$ & $3.40 \mathrm{E}-03$ & -02 & $1.02 \mathrm{E}-01$ & $1.97 \mathrm{E}-01$ & 4.75E-02 & $\mathrm{E}-02$ \\
\hline U112 & Se79 & $\mathrm{Ci}$ & $1.55 \mathrm{E}-02$ & $1.55 E-02$ & $E-04$ & $E-03$ & $E-02$ & $3.61 E-02$ & $E-01$ & $2.05 \mathrm{E}-02$ & $2.06 \mathrm{E}-02$ \\
\hline 1112 & Sm151 & $\mathrm{Ci}$ & $5.57 \mathrm{E}+01$ & $5.57 \mathrm{E}+01$ & $0.00 \mathrm{E}+01$ & 9.32E-00 & $5.57 E+01$ & $2.72 E+02$ & $7.10 \mathrm{E}+02$ & $1.07 \mathrm{E}+02$ & $1.47 \mathrm{E}+02$ \\
\hline
\end{tabular}


Tank Anal. Un

\begin{tabular}{|c|c|c|c|}
\hline & Sr89/90 & $C i$ & May98 \\
\hline $\begin{array}{l}0112 \\
\text { U112 }\end{array}$ & Te99 & $\mathrm{Ci}$ & $5.10 \mathrm{E}-01$ \\
\hline U112 & Th2299 & $\mathrm{Ci}$ & $5.05 \mathrm{E}-08$ \\
\hline U112 & Th232 & $\mathrm{Ci}$ & $1.98 \mathrm{E}-11$ \\
\hline U112 & Tin126 & $\mathrm{Ci}$ & $2.37 \mathrm{E}-02$ \\
\hline U112 & Tritium & $C \mathrm{i}$ & $8.39 \mathrm{E}-01$ \\
\hline U112 & $\mathbf{U} 232$ & $C i$ & $3.77 \mathrm{E}-05$ \\
\hline U112 & U233 & $\mathrm{Ci}$ & $1.92 \mathrm{E}-06$ \\
\hline U112 & U234 & $C i$ & $2.27 \mathrm{E}-00$ \\
\hline U112 & U235 & $\mathrm{Ci}$ & 1.01E-01 \\
\hline U112 & U236 & $\mathrm{Ci}$ & $1.98 \mathrm{E}-02$ \\
\hline U112 & U238 & $\mathrm{Ci}$ & $2.29 \mathrm{E}-00$ \\
\hline U112 & Y90 & $\mathrm{Ci}$ & $1.71 E+04$ \\
\hline U112 & Zr93 & $\mathrm{Ci}$ & 7.32E-02 \\
\hline U201 & Al & kg & $3.24 E+03$ \\
\hline U201 & $\mathbf{B i}$ & $\mathrm{kg}$ & $1.53 E+01$ \\
\hline U201 & $\mathbf{C a}$ & $\mathrm{kg}$ & $1.42 \mathrm{E}+01$ \\
\hline U201 & Cl & $\mathrm{kg}$ & $4.22 \mathrm{E}+01$ \\
\hline U201 & $\mathrm{CO} 3$ & $\mathrm{~kg}$ & $1.10 E+02$ \\
\hline U201 & $\mathrm{Cr}$ & kg & $3.21 \mathrm{E}+01$ \\
\hline U201 & $\mathbf{F}$ & $\mathrm{kg}$ & $3.51 E+01$ \\
\hline U201 & Fe & kg & $4.59 \mathrm{E}+01$ \\
\hline U201 & Hg & $\mathrm{kg}$ & $1.24 E+01$ \\
\hline U201 & $\mathbf{K}$ & kg & $8.00 \mathrm{E}-00$ \\
\hline U201 & La & $\mathrm{kg}$ & $0.00 \mathrm{E}+01$ \\
\hline U201 & Mn & kg & $1.07 \mathrm{E}+01$ \\
\hline U201 & $\mathbf{N a}$ & $\mathrm{kg}$ & $1.63 E+03$ \\
\hline U201 & $\mathbf{N i}$ & kg & $3.43 E+01$ \\
\hline U201 & NO2 & kg & $5.18 E+02$ \\
\hline U201 & NO3 & kg & $2.13 E+03$ \\
\hline U201 & $\mathbf{P}$ & kg & $1.58 \mathrm{E}+01$ \\
\hline U201 & $\mathbf{P b}$ & kg & $6.00 E+01$ \\
\hline U201 & PO4 & kg & $4.88 \mathrm{E}+01$ \\
\hline U201 & $\mathbf{S}$ & kg & $1.11 \mathrm{E}+01$ \\
\hline U201 & $\mathbf{S i}$ & kg & $4.00 \mathrm{E}+01$ \\
\hline U201 & SO4 & kg & $3.33 E+01$ \\
\hline U201 & $\mathbf{S r}$ & kg & $6.84 \mathrm{E}-00$ \\
\hline U201 & TOC & kg & $2.70 E+01$ \\
\hline $\mathbf{U} 201$ & $\mathbf{U}$ & kg & $1.37 \mathrm{E}+02$ \\
\hline U201 & $\mathbf{Z r}$ & kg & $1.56 \mathrm{E}-00$ \\
\hline U201 & Ac227 & $\mathrm{Ci}$ & 2.21E-07 \\
\hline U201 & $\operatorname{Am} 241$ & $\mathrm{Ci}$ & $6.23 \mathrm{E}-03$ \\
\hline U201 & Am243 & $\mathrm{Ci}$ & $5.68 \mathrm{E}-08$ \\
\hline U201 & Ba137 & $\mathbf{C i}$ & $2.62 E+03$ \\
\hline U201 & C14 & $\mathrm{Ci}$ & $1.66 \mathrm{E}-03$ \\
\hline U201 & Cd113 & $\mathrm{Ci}$ & $5.34 \mathrm{E}-03$ \\
\hline U201 & Cm242 & $\mathrm{Ci}$ & $5.53 \mathrm{E}-05$ \\
\hline U201 & $\mathrm{Cm} 243$ & $\mathrm{Ci}$ & $1.26 \mathrm{E}-06$ \\
\hline U201 & $\mathrm{Cm} 244$ & $\mathbf{C i}$ & $1.98 \mathrm{E}-06$ \\
\hline U201 & Co60 & $\mathrm{Ci}$ & $7.18 E-04$ \\
\hline U201 & Cs134 & $\mathrm{Ci}$ & 2.52E-05 \\
\hline U201 & Cs137 & $\mathbf{C i}$ & $2.77 E+03$ \\
\hline U201 & Eu152 & $\mathrm{Ci}$ & $2.65 \mathrm{E}-03$ \\
\hline U201 & Eu154 & Ci & $1.75 E-02$ \\
\hline U201 & Eu155 & $\mathbf{C i}$ & $1.26 \mathrm{E}-01$ \\
\hline U201 & 1129 & $\mathbf{C i}$ & $2.29 \mathrm{E}-05$ \\
\hline U201 & Nb93 & $\mathrm{Ci}$ & $1.39 E-03$ \\
\hline U201 & Ni59 & $\mathbf{C i}$ & $4.70 \mathrm{E}-04$ \\
\hline U201 & Ni63 & $\mathbf{C i}$ & $4.40 \mathrm{E}-02$ \\
\hline U201 & Np237 & $\mathrm{Ci}$ & $8.35 E-05$ \\
\hline U201 & Pa231 & $\mathrm{Ci}$ & $5.24 \mathrm{E}-07$ \\
\hline U201 & Pu238 & $\mathrm{Ci}$ & $3.80 \mathrm{E}-01$ \\
\hline U201 & Pu239 & $\mathbf{C i}$ & $2.43 E+01$ \\
\hline U201 & Pu240 & $\mathrm{Ci}$ & $3.43 E-00$ \\
\hline U201 & Pu241 & $\mathrm{Ci}$ & $2.10 \mathrm{E}+0 \mathrm{I}$ \\
\hline U201 & Pu242 & $\mathrm{Ci}$ & $8.90 \mathrm{E}-05$ \\
\hline U201 & Ra226 & $\mathrm{Ci}$ & 4.30E-08 \\
\hline U201 & $\mathbf{R a 2 2 8}$ & $\mathrm{Ci}$ & $4.42 \mathrm{E}-12$ \\
\hline
\end{tabular}

\section{Sept98}

$6.76 \mathrm{E}+04$

5.10E-01

5.05E-08

$1.98 \mathrm{E}-11$

2.37E-02

8.39E-01

1.44E-05

7.35E-07

8.69E-01

3.88E-02

$7.57 \mathrm{E}-03$

8.75E-01

$6.76 \mathrm{E}+04$

7.32E-02

$3.24 \mathrm{E}+03$

$1.53 \mathrm{E}+01$

$1.42 \mathrm{E}+01$

$4.22 \mathrm{E}+01$

$1.10 \mathrm{E}+02$

$3.21 \mathrm{E}+01$

$3.51 \mathrm{E}+01$

$4.59 \mathrm{E}+01$

2.91E-00

8.00E-00

$0.00 \mathrm{E}+01$

$1.07 \mathrm{E}+01$

$1.63 \mathrm{E}+03$

$3.43 \mathrm{E}+01$

$5.18 \mathrm{E}+02$

$2.13 E+03$

NA

$6.00 \mathrm{E}+01$

$4.88 \mathrm{E}+0 \mathrm{I}$

NA

$4.00 \mathrm{E}+01$
$3.33 \mathrm{E}+01$

$6.84 \mathrm{E}-00$

$2.70 \mathrm{E}+01$

$1.37 \mathrm{E}+02$

$1.56 \mathrm{E}-00$

2.21E-07

2.96E-00

$2.70 \mathrm{E}-05$

$1.06 \mathrm{E}+03$

$1.66 \mathrm{E}-03$

5.34E-03

2.65E-02

6.00E-04

9.41 E-04

7.18E-04

2.52E-05

$1.12 \mathrm{E}+03$

2.65E-03

1.75E-02

1.26E-01

2.29E-05

1.39E-03

4.70E-04

4.40E-02

8.35E-05

5.24E-07

9.46E-02

$6.06 \mathrm{E}-00$

8.55E-01

5.22E-00

2.22E-05

4.30E-08

4.42E-12
$1 \%$-ile

\section{$1.37 \mathrm{E}+02$}

$0.00 \mathrm{E}+01$

$0.00 \mathrm{E}+01$

$0.00 \mathrm{E}+01$

$0.00 \mathrm{E}+01$

$1.44 \mathrm{E}-01$

$0.00 \mathrm{E}+01$

$0.00 \mathrm{E}+01$

$0.00 \mathrm{E}+01$

$0.00 \mathrm{E}+01$

$0.00 \mathrm{E}+01$

$0.00 \mathrm{E}+01$

$5.68 \mathrm{E}+01$

$0.00 E+01$

$1.36 \mathrm{E}+02$

$0.00 \mathrm{E}+01$

2.40E-01

$5.52 \mathrm{E}-01$

4.26E- 00

7.27E-01

4.31E-01

5.95E-01

$0.00 \mathrm{E}+01$

$5.66 \mathrm{E}-01$

1.07E-02

$1.06 \mathrm{E}-01$

$9.30 \mathrm{E}+01$

4.94E-04

1. $23 \mathrm{E}+01$

3.16E-01

3.21E-01

$5.43 \mathrm{E}-03$

$5.22 \mathrm{E}-01$

7.16E-01

$3.70 \mathrm{E}-01$

1.73E-00

7.04E-04

1. 13E-00

2.03E-00

4.43E-04

$0.00 \mathrm{E}+01$

$8.50 \mathrm{E}-02$

$0.00 \mathrm{E}+0 \mathrm{I}$

$0.00 \mathrm{E}+0.1$

$0.00 \mathrm{E}+01$

$0.00 E+01$

$0.00 \mathrm{E}+01$

$0.00 \mathrm{E}+0 \mathrm{i}$

$0.00 E+01$

1.63E-02

$0.00 E+01$

$5.43 \mathrm{E}+01$

$0.00 \mathrm{E}+0 \mathrm{i}$

$0.00 \mathrm{E}+01$

$0.00 \mathrm{E}+01$

$0.00 \mathrm{E}+01$

$0.00 \mathrm{E}+01$

$0.00 E+01$

$0.00 \mathrm{E}+01$

$0.00 E+01$

$0.00 \mathrm{E}+01$

$0.00 \mathrm{E}+01$

$0.00 E+01$

$0.00 E+01$

$0.00 E+01$

$0.00 \mathrm{E}+01$

$0.00 \mathrm{E}+0 \mathrm{l}$

$0.00 E+01$
$10 \%$-ile

\section{$2.12 \mathrm{E}+03$}

4.91E-02

6.26E-09

2.45E- 12

3.30E-03

$4.13 \mathrm{E}-01$

4.67E-06

2.38E-07

2.81E-01

$1.25 \mathrm{E}-02$

2.45E-03

$2.84 \mathrm{E}-01$

$1.03 \mathrm{E}+03$

9.02E-03

$8.27 \mathrm{E}+02$

$1.05 \mathrm{E}-00$

2.74E-00

$7.68 \mathrm{E}-00$

$9.96 \mathrm{E}+01$

7.72E-00

$5.92 \mathrm{E}-00$

$1.37 \mathrm{E}+01$

2.32E-00

$6.14 \mathrm{E}-00$

1.14E-01

4.24E-00

$1.01 \mathrm{E}+03$

$3.49 \mathrm{E}-01$

$1.21 \mathrm{E}+02$

$2.52 \mathrm{E}+02$

$1.08 \mathrm{E}+01$

2.32E-01

$1.85 \mathrm{E}+01$

4.71E-00

$7.25 \mathrm{E}-00$

$1.12 \mathrm{E}+01$

8.12E-01

$1.52 \mathrm{E}+01$

$3.74 \mathrm{E}+01$

2.05E-01

4.87E- 08

$5.96 \mathrm{E}-01$

6.40E-09

$5.87 \mathrm{E}+02$

2.77E-04

1.00E-03

6.23E-06

$1.42 \mathrm{E}-07$

2.23E-07

8.77E-02

5.64E-06

$6.18 \mathrm{E}+02$

5.84E-04

$3.86 \mathrm{E}-03$

2.78E-02

2.83E-06

2.07E-04

9.33E-05

8.73E-03

9.41E-06

4.93E-08

4.28E-02

2.74E-00

$3.87 \mathrm{E}-01$

2.37E-00

$1.00 \mathrm{E}-05$

$6.27 \mathrm{E}-09$

$6.45 \mathrm{E}-13$ 50\%-ile

$3.51 \mathrm{E}+04$

5.10E-01

5.05E-08

1.98E-11

2.37E-02

8.39E-01

3.77E-05

1.92E-06

2.27E-00

1.01E-01

1.98E-02

2.29E-00

$1.71 \mathrm{E}+04$

7.32E-02

$2.10 \mathrm{E}+03$

$1.41 \mathrm{E}+01$

7.80E-00

$3.88 \mathrm{E}+01$

$3.61 \mathrm{E}+02$

$3.88 \mathrm{E}+01$

$1.50 E+02$

$9.83 \mathrm{E}+01$

$1.24 E+01$

$2.60 \mathrm{E}+01$

3.46E-01

$2.91 \mathrm{E}+01$

$2.65 E+03$

1.73E-00

$5.36 \mathrm{E}+02$

$2.08 \mathrm{E}+03$

$6.91 \mathrm{E}+01$

1.18E-00

1.31E +02

2.25E+01

$3.20 \mathrm{E}+01$

$6.58 \mathrm{E}+01$

5.25E-00

$6.88 \mathrm{E}+01$

$1.44 \mathrm{E}+02$

1.32E-00

2.21E-07

$1.66 \mathrm{E}-00$

5.68E-08

$2.62 \mathrm{E}+03$

$1.66 \mathrm{E}-03$

5.34E-03

5.53E-05

1.26E-06

1.98E-06

2.29E-01

2.52E-05

$2.01 \mathrm{E}+03$

2.65E-03

$1.75 \mathrm{E}-02$

1.26E-01

2.29E-05

1.39E-03

4.70E-04

4.40E-02

8.35E-05

5.24E-07

$3.80 \mathrm{E}-01$

$2.43 \mathrm{E}+01$

$3.43 \mathrm{E}-00$

$2.10 \mathrm{E}+01$

8.90E-05

4.30E-08

4.42E-12

90\%-ile

99\%-ile

Mean

\section{$3.23 E+05$}

$2.76 \mathrm{E}+06$

4.04E-00

$2.16 \mathrm{E}+05$ 
Tank Anal. Un

\begin{tabular}{|c|c|}
\hline U201 & Ru106 \\
\hline U201 & Sb125 \\
\hline U201 & Se79 \\
\hline U201 & Sm151 \\
\hline U201 & Sr89/90 \\
\hline U201 & Tc99 \\
\hline U201 & Th229 \\
\hline U201 & Th232 \\
\hline U201 & Tin126 \\
\hline U201 & Tritium \\
\hline U201 & U232 \\
\hline U201 & U233 \\
\hline U201 & U234 \\
\hline U201 & U235 \\
\hline U201 & U236 \\
\hline U201 & U238 \\
\hline & Y90 \\
\hline U201 & Zr93 \\
\hline U202 & Al \\
\hline & Bi \\
\hline U202 & $\mathrm{Ca}$ \\
\hline U202 & CI \\
\hline U202 & $\mathrm{CO} 3$ \\
\hline U202 & $\mathrm{Cr}$ \\
\hline U202 & $\mathbf{F}$ \\
\hline U202 & $\mathrm{Fe}$ \\
\hline U202 & $\mathbf{H g}$ \\
\hline U202 & $\mathbf{K}$ \\
\hline U202 & $\mathbf{L a}$ \\
\hline U202 & Mn \\
\hline U202 & $\mathrm{Na}$ \\
\hline U202 & $\mathbf{N i}$ \\
\hline U202 & $\mathrm{NO} 2$ \\
\hline U202 & NO3 \\
\hline U202 & $\mathbf{P}$ \\
\hline U202 & $\mathbf{P b}$ \\
\hline U202 & P04 \\
\hline U202 & $\mathbf{s}$ \\
\hline U202 & $\mathbf{S i}$ \\
\hline U202 & SO4 \\
\hline U202 & $\mathbf{S r}$ \\
\hline U202 & TOC \\
\hline U202 & $\mathbf{U}$ \\
\hline U202 & $\mathbf{Z r}$ \\
\hline U202 & Ac227 \\
\hline U202 & Am241 \\
\hline $\mathbf{U} 202$ & Am 243 \\
\hline U202 & Ba137 \\
\hline U202 & C14 \\
\hline U202 & Cd113 \\
\hline U202 & $\mathrm{Cm} 242$ \\
\hline U202 & $\mathrm{Cm} 243$ \\
\hline U202 & $\operatorname{Cm} 244$ \\
\hline U202 & Co60 \\
\hline U202 & Cs134 \\
\hline U202 & Cs137 \\
\hline U202 & Eu152 \\
\hline U202 & Eu154 \\
\hline U202 & Eu155 \\
\hline U202 & 1129 \\
\hline U202 & Nb93 \\
\hline U202 & Ni59 \\
\hline $\mathrm{U} 202$ & Ni63 \\
\hline U202 & Np237 \\
\hline U202 & Pa231 \\
\hline J20 & Pu238 \\
\hline & \\
\hline $\mathrm{J} 202$ & Pu240 \\
\hline
\end{tabular}

- $\frac{B B}{88}$ 4.19E-09 1.20E-03 3.62E-04 $1.29 \mathrm{E}-00$ $3.07 \mathrm{E}+03$ 1.19E-02 6.25E-10 6.14E-13 5.51E-04 9.79E-06 3.62E-07 2.24E-01 9.52E-03 4.93E-03 2.18E-01

3.07E+03 Ci 1.71E-03 g $3.24 \mathrm{E}+03$ 1.53E+01 i.42E+01 4.22E+01 1.10E+02 g 3.21E+01 3.51E+01 g $4.59 \mathrm{E}+01$ g $1.24 E+01$ g 8.00E-00 $0.00 \mathrm{E}+01$ $1.07 E+01$ $1.63 \mathrm{E}+03$ $3.43 \mathrm{E}+01$ $5.18 \mathrm{E}+02$ 2.13E+03 $1.58 \mathrm{E}+01$ $6.00 \mathrm{E}+01$ $4.88 \mathrm{E}+01$ 1.11E+01 $4.00 \mathrm{E}+01$ $3.33 E+01$ $6.84 \mathrm{E}-00$ $2.70 \mathrm{E}+01$ $1.37 \mathrm{E}+02$ $1.56 \mathrm{E}-00$

2.20E-07 6.19E-03 5.64E-08 $2.62 E+03$

$1.65 \mathrm{E}-03$ 5.31E-03 5.53E- 05 $1.26 \mathrm{E}-06$ 1.98E-06 7.14E-04 2.50E-05 $2.77 \mathrm{E}+03$ 2.65E-03 $1.74 \mathrm{E}-02$ $1.26 \mathrm{E}-01$ 2.27E-05 1.38E-03 4.68E-04 4.39E-02 8.30E-05 5.21E-07 3.80E-01 $\mathrm{Ci} \quad 2.44 \mathrm{E}+01$ 3.43E-00
BB $\frac{\text { Sept98 }}{4.19 E-09}$ $1.20 \mathrm{E}-03$ $3.62 \mathrm{E}-04$ $1.29 \mathrm{E}-00$ $4.71 \mathrm{E}+03$ 1.19E-02 $6.25 \mathrm{E}-10$ $6.14 \mathrm{E}-13$ 5.51E-04 1.55E-02 2.05E-06 7.59E-08 $4.70 \mathrm{E}-02$ 2.00E-03 1.03E-03 4.57E-02 $4.71 \mathrm{E}+03$ $1.71 E-03$ $3.24 E+03$ $1.53 \mathrm{E}+01$ $1.42 \mathrm{E}+01$ $4.22 \mathrm{E}+01$ $1.10 \mathrm{E}+02$ $3.21 \mathrm{E}+01$ $3.51 \mathrm{E}+01$ $4.59 \mathrm{E}+01$ 2.91E-00 8.00E-00 $0.00 \mathrm{E}+01$ $1.07 \mathrm{E}+01$ $1.63 \mathrm{E}+03$ $3.43 \mathrm{E}+01$ $5.18 \mathrm{E}+02$ $2.13 E+03$ NA

6.00E +01 $4.88 \mathrm{E}+01$ NA $4.00 E+01$ $3.33 E+01$ $6.84 \mathrm{E}-00$ $2.70 \mathrm{E}+01$ 1.37E +02 $1.56 \mathrm{E}-00$ 2.20E-07 2.96E-00 2.70E-05 $1.06 \mathrm{E}+03$ 1.65E-03 5.31E-03 $2.65 E-02$ 6.04E-04 9.41E-04 7.14E-04 2.50E-05 $1.12 \mathrm{E}+03$ 2.65E-03 $1.74 \mathrm{E}-02$ $1.26 \mathrm{E}-01$ 2.27E-05 $1.38 \mathrm{E}-03$ 4.68E-04 4.39E-02 8.30E-05 5.21E-07 9.46E-02 6.06E-00 8.55E-01 1\%-ile $0.00 \mathrm{E}+01$ $0.00 \mathrm{E}+01$ 9.35E-06 $0.00 \mathrm{E}+01$ $0.00 \mathrm{E}+01$ $0.00 \mathrm{E}+01$ $0.00 \mathrm{E}+01$ $0.00 \mathrm{E}+01$ $0.00 \mathrm{E}+01$ 8.49E-04 $0.00 \mathrm{E}+01$ $0.00 \mathrm{E}+01$ $0.00 \mathrm{E}+01$ $0.00 \mathrm{E}+01$ $0.00 \mathrm{E}+01$ $0.00 \mathrm{E}+01$

$0.00 E+01$

$0.00 \mathrm{E}+01$

$1.85 \mathrm{E}+02$

$7.11 \mathrm{E}-03$

8.45E-01

$1.56 \mathrm{E}-00$

2.34E-01

$1.05 \mathrm{E}-00$ $0.00 \mathrm{E}+01$ $9.60 \mathrm{E}-01$ $0.00 \mathrm{E}+01$ $1.06 \mathrm{E}-00$ $1.87 \mathrm{E}-02$ 4.47E-01 $2.29 \mathrm{E}+02$ $1.68 \mathrm{E}-02$ $1.10 \mathrm{E}+01$ 2.00E-00 $2.76 \mathrm{E}-01$ $2.86 \mathrm{E}-02$ $1.17 \mathrm{E}-00$ $7.68 \mathrm{E}-01$ $6.42 \mathrm{E}-01$ $1.87 \mathrm{E}-00$ 2.17E-02 2.80E-00 $4.18 \mathrm{E}-00$ $0.00 E+01$ $0.00 E+01$ 2.17E-01 $0.00 \mathrm{E}+01$ $0.00 \mathrm{E}+01$ $0.00 \mathrm{E}+01$ $0.00 \mathrm{E}+01$ $0.00 \mathrm{E}+01$ $0.00 \mathrm{E}+01$ $0.00 \mathrm{E}+01$ 2.61E-02 $0.00 \mathrm{E}+01$ $1.02 \mathrm{E}+02$ $0.00 \mathrm{E}+01$ $0.00 \mathrm{E}+01$ $0.00 \mathrm{E}+0$ i $0.00 \mathrm{E}+01$ $0.00 \mathrm{E}+01$ $0.00 \mathrm{E}+01$ $0.00 \mathrm{E}+01$ $0.00 \mathrm{E}+01$ $0.00 \mathrm{E}+01$ $0.00 \mathrm{E}+01$ $0.00 \mathrm{E}+01$ $0.00 \mathrm{E}+01$
$10 \%$-ile $4.42 \mathrm{E}-10$
$8.74 \mathrm{E}-05$
$6.13 \mathrm{E}-05$
$2.84 \mathrm{E}-01$
$5.59 \mathrm{E}-01$
$2.98 \mathrm{E}-04$
$5.88 \mathrm{E}-11$

$5.78 \mathrm{E}-14$

1.03E-04

$5.71 \mathrm{E}-03$

9.22E-07

3.41E-08

2.11E-02

8.96E-04

4.64E-04

$2.05 \mathrm{E}-02$

$0.00 \mathrm{E}+01$

$2.55 \mathrm{E}-04$

$9.70 \mathrm{E}+02$

$1.83 \mathrm{E}-00$

$3.58 \mathrm{E}-00$

$1.27 \mathrm{E}+01$

$8.66 \mathrm{E}+01$

9.94E-00

3.02E-00

$1.97 \mathrm{E}+01$

2.02E-00

6.33E-00

1.12E-01

$6.08 \mathrm{E}-00$

$1.11 \mathrm{E}+03$

6.36E-01

$1.85 \mathrm{E}+02$

$6.36 \mathrm{E}+02$

$1.90 \mathrm{E}+01$

4.03E-01

$2.94 \mathrm{E}+01$

$4.94 \mathrm{E}-00$

$7.54 \mathrm{E}-00$

$1.22 \mathrm{E}+01$

$1.39 \mathrm{E}-00$

$1.45 \mathrm{E}+01$

4.08E +01

1.82E-01

7.53E-09

6.77E-01

9.15E-09

$6.76 \mathrm{E}+02$

1.71E-04

8.67E-04

8.97E-06

2.04E-07

3.21E-07

1.12E-0I

6.45E-06

$8.75 \mathrm{E}+02$

9.07E-05

$5.95 \mathrm{E}-04$

4.31E-03

3.81E-06

$1.93 \mathrm{E}-04$

1.03E-04

9.66E-03

$1.35 \mathrm{E}-05$

4.83E-08

$6.16 \mathrm{E}-02$

3.96E-00

$5.56 \mathrm{E}-01$

50\%-ile

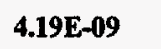

$1.20 \mathrm{E}-03$

3.62E-04

$1.29 \mathrm{E}-00$

$6.37 \mathrm{E}+03$

1.19E-02

6.25E-10

6.14E-13

5.51E-04

1.55E-02

9.79E-06

3.62E-07

2.24E-01

9.52E-03 
Tank Anal. Un

U202 Pu241 Ci $\frac{\text { May } 2.10 \mathrm{E}+01}{8.90}$

U202 Pu242 Ci 8.90E-05

$\begin{array}{llll}\mathrm{U} 202 & \mathrm{R} 2226 & \mathrm{Ci} & 4.28 \mathrm{E}-08\end{array}$

$\begin{array}{llll}\text { U202 } & \text { Ra228 } & \text { Ci } & 4.42 E-12\end{array}$

$\begin{array}{llll}\text { U202 Sb125 Ci 1.19E-03 } & \text { C }\end{array}$

$\begin{array}{llll}\text { U202 Se79 } & \text { Ci } & \text { 3.59E-04 }\end{array}$

$\begin{array}{llll}\text { U202 } & \text { Sm151 } & \mathrm{Ci} & 1.28 \mathrm{E}-00 \\ \text { U202 } & \text { Sr89/90 } & \mathrm{Ci} & 3.07 \mathrm{E}+03\end{array}$

$\begin{array}{llll}\text { U202 } & \text { Tc99 } & \text { Ci } & 1.19 \mathrm{E}-02 \\ \text { U202 } & \text { Th229 } & \text { Ci } & 625 \mathrm{E}-10\end{array}$

$\begin{array}{llll}\text { U202 } & \text { Th229 } & \text { Ci } & 6.25 E-10 \\ \text { U202 } & \text { Th232 } & \text { Ci } & 6.11 E-13\end{array}$

$\begin{array}{llll}\text { U202 } & \text { Tin126 } & \mathrm{Ci}_{\mathrm{i}} & 5.47 \mathrm{E}-04 \\ \text { U202 } & \text { Tritium } & \mathrm{Ci} & 1.53 \mathrm{E}-02\end{array}$

$\begin{array}{llll}\text { U202 } & \text { Tritium } & \mathrm{Ci} & 1.53 \mathrm{E}-02 \\ \mathrm{U} 202 & \mathrm{U} 232 & \mathrm{Ci} & 9.79 \mathrm{E}-06\end{array}$

$\begin{array}{llll}\text { U202 } & \text { U233 } & \mathrm{Ci}_{i} & 3.62 \mathrm{E}-07 \\ \mathrm{U} 202 & \mathrm{U} 234 & \mathrm{Ci} & 2.24 \mathrm{E}-01\end{array}$

$\begin{array}{llll}\mathrm{U} 202 & \mathrm{U} 235 & \mathrm{Ci} & 9.52 \mathrm{E}-03 \\ \mathrm{U} 202 & \mathrm{U} 236 & \mathrm{Ci} & 4.93 \mathrm{E}-03\end{array}$

$\begin{array}{llll}\mathrm{U} 202 & \mathrm{U} 236 & \mathrm{Ci} & 4.93 \mathrm{E}-03 \\ \mathrm{U} 202 & \mathrm{U} 238 & \mathrm{Ci} & 2.18 \mathrm{E}-01\end{array}$

$\begin{array}{llll}\text { U202 } & \text { U238 } & C i & 2.18 E-01 \\ \text { U202 } & \text { Y90 } & C i & 3.07 E+03\end{array}$

$\begin{array}{llll}\mathrm{U} 202 & \mathrm{Zr} 93 & \mathrm{Ci} & 1.70 \mathrm{E}-03 \\ \mathrm{U} 203 & \mathrm{Al} & \mathrm{kg} & 1.62 \mathrm{E}+03\end{array}$

U203 Bi $\mathrm{kg}$ 7.64E-00

$\begin{array}{llll}\mathrm{U} 203 & \mathrm{Ca} & \mathrm{kg} & \mathbf{7 . 1 1 E}-00\end{array}$

$\begin{array}{llll}203 & \mathrm{Cl} & \mathrm{kg} & 2.11 \mathrm{E}+01\end{array}$

$\mathrm{U203} \mathrm{CO} 3 \mathrm{~kg} \quad 6.28 \mathrm{E}+01$

$\begin{array}{llll}\mathrm{U} 203 & \mathrm{Cr} & \mathrm{kg} & 1.39 \mathrm{E}+01\end{array}$

$\begin{array}{llll}U 203 & F & \mathrm{~kg} & 1.75 \mathrm{E}+01\end{array}$

$\mathrm{U203} F \mathrm{~kg} 2.29 \mathrm{E}+01$

$\begin{array}{llll}\mathrm{U} 203 & \mathrm{Hg} & \mathrm{kg} & 6.20 \mathrm{E}-00\end{array}$

U203 $\mathrm{K} \quad \mathrm{kg} \quad 5.60 \mathrm{E}-00$

$\mathrm{U} 203 \mathrm{La}$ kg $0.00 \mathrm{E}+01$

U203 Mn kg 5.37E-00

$\begin{array}{llll}\mathrm{U} 203 & \mathrm{Na} & \mathrm{kg} & 8.16 \mathrm{E}+02\end{array}$

$\begin{array}{llll}\mathrm{U} 203 & \mathrm{Ni} & \mathrm{kg} & 1.72 \mathrm{E}+01\end{array}$

$\mathrm{U} 203 \mathrm{NO2} \mathrm{kg} \quad 2.58 \mathrm{E}+02$

$\mathrm{U} 203 \mathrm{NO3} \mathrm{kg} \quad 1.07 \mathrm{E}+03$

$\begin{array}{llll}\mathrm{U} 203 & P & \mathrm{~kg} & 7.88 \mathrm{E}-00\end{array}$

U203 Pb

$\mathrm{U} 203 \mathrm{PO}$

U203 S

U203 Si

U203

U203

$\mathrm{U} 203$

U203

$\mathrm{U} 203$

U203

U203

U203

U203

U203

U203

U203

U203

U203

U203

U203

U203

U203

U203

U203

U203

U203

U203

1203

U203 Np237

$2.44 E+01$

$\mathrm{kg} \quad 5.57 \mathrm{E}-00$

SO4

Tr

U

Ac227

Am241

Am243

Ba137

C14

CdII3

Cm242

Cm243

Cm244

Co60

Cs134

Eu152

Eu154

Eu155

I129

Nb93

Ni59
$B B$

\section{Sept98}

2.22E-05

4.28E-08

4.42E-12

4.17E-09

1.19E-03

3.59E-04

$1.28 \mathrm{E}-00$

$4.71 \mathrm{E}+03$

1.19E-02

6.25E-10

6.11E-13

5.47E-04

$.53 \mathrm{E}-02$

2.05E-06

7.59E-08

4.70E-02

2.00E-03

1.03E-03

4.57E-02

$4.71 \mathrm{E}+03$

1.70E-03

$1.62 \mathrm{E}+03$

7.64E-00

7.11E-00

$2.11 \mathrm{E}+01$

$6.28 \mathrm{E}+01$

$1.39 \mathrm{E}+01$

$1.75 \mathrm{E}+01$

$2.29 \mathrm{E}+01$

1.45E-00

5.60E-00

$0.00 \mathrm{E}+01$

5.37E-00

$8.16 \mathrm{E}+02$

1.72E+01

$2.58 \mathrm{E}+02$

$1.07 \mathrm{E}+03$

NA

$3.00 E+01$

$2.44 \mathrm{E}+01$

$1 \%$ ile

$0.00 \mathrm{E}+01$

$0.00 \mathrm{E}+01 \quad 7.44 \mathrm{E}-05$

$0.00 \mathrm{E}+01 \quad 7.66 \mathrm{E}-13$

$0.00 \mathrm{E}+01 \quad 5.72 \mathrm{E}-10$

$0.00 \mathrm{E}+01 \quad 1.17 \mathrm{E}-04$

8.86E-06 6.83E-05

$0.00 \mathrm{E}+01 \quad 4.38 \mathrm{E}-02$

$0.00 \mathrm{E}+01 \quad 1.35 \mathrm{E}+01$

$0.00 \mathrm{E}+01 \quad 2.30 \mathrm{E}-04$

$0.00 \mathrm{E}+01 \quad 5.79 \mathrm{E}-11$

$0.00 \mathrm{E}+01 \quad 5.66 \mathrm{E}-14$

$0.00 E+01$

$1.26 \mathrm{E}-03$

$0.00 \mathrm{E}+01$

$0.00 \mathrm{E}+01$

$0.00 \mathrm{E}+01$

$0.00 \mathrm{E}+01$

$0.00 \mathrm{E}+01$
$0.00 \mathrm{E}+01$

$0.00 \mathrm{E}+01$

$0.00 \mathrm{E}+01$

$3.80 \mathrm{E}-04$

$0.00 \mathrm{E}+01$

$1.01 E-06$

1.07E-05

$5.36 \mathrm{E}-05$

7.75E-06

1.55E-06

$5.98 \mathrm{E}-06$

$0.00 \mathrm{E}+01$

2.01E-06

4.93E-08

$2.50 \mathrm{E}-06$

6.44E-04

$1.88 \mathrm{E}-07$

$1.44 \mathrm{E}-04$

4.07E-04

9.01E-06

$1.68 \mathrm{E}-07$

$1.96 \mathrm{E}-05$

4.74E-06

$1.95 E+01$

$1.67 \mathrm{E}+01$

3.42E-00

$3.16 \mathrm{E}-06$

1.47E-05

2.60E-07

8.13E-06

$1.97 \mathrm{E}-05$

$6.87 \mathrm{E}+01$

$7.80 \mathrm{E}-01$

2.30E-06

$1.50 \mathrm{E}-00$

1.40E-05

$5.30 \mathrm{E}+02$

2.55E-02

$7.90 \mathrm{E}-02$

1.15E-03

2.63E-05

5.01E-04

1.01E-02

2.61E-04

$5.60 E+02$

3.16E-03

2.44E-01

1.50E-01

3.44E-04

2.10E-02

$3.45 \mathrm{E}-03$

$0.00 \mathrm{E}+01$

$0.00 \mathrm{E}+01$

2.03E-07

$0.00 \mathrm{E}+01$

$0.00 \mathrm{E}+01$

$0.00 \mathrm{E}+01$

$0.00 \mathrm{E}+01$

$0.00 \mathrm{E}+01$

$0.00 \mathrm{E}+01$

$0.00 E+01$

4.72E-08

$0.00 \mathrm{E}+01$

$3.66 \mathrm{E}-04$

$0.00 \mathrm{E}+01$

$0.00 \mathrm{E}+01$

$0.00 \mathrm{E}+01$

$0.00 \mathrm{E}+01$

$0.00 \mathrm{E}+01$

$0.00 \mathrm{E}+01$

$0.00 \mathrm{E}+01$

$0.00 \mathrm{E}+01$

$8.93 \mathrm{E}-05$

5.76E-03

9.07E-07

3.35E-08

2.07E-02

8.82E-04

$4.57 \mathrm{E}-04$

2.02E-02

$3.70 \mathrm{E}-00$

2.37E-04

$1.30 \mathrm{E}+02$

9.05E-02

3.81E-01

3.3IE-00

$1.68 \mathrm{E}+01$

$1.55 \mathrm{E}-00$

4.62E-01

2.10E-00

3.55E-01

9.92E-01

$1.36 \mathrm{E}-02$

$6.39 \mathrm{E}-01$

$1.99 \mathrm{E}+02$

6.81E-02

$4.81 \mathrm{E}+01$

$1.29 \mathrm{E}+02$

$2.64 \mathrm{E}-00$

4.85E-02

7.32E-00

1.10E-00

$1.21 \mathrm{E}-00$

2.95E-00

9.86E-02

2.04E-00

$5.16 \mathrm{E}-00$

1.69E-02

$0.00 \mathrm{E}+01$

$1.02 \mathrm{E}-01$

2.35E-08

$1.38 \mathrm{E}+02$

$0.00 \mathrm{E}+01$

4.52E-03

$1.92 \mathrm{E}-06$

4.39E-08

8.39E-07

2.11E-02

2.76E-05

$1.80 \mathrm{E}+02$

$0.00 E+01$

$0.00 \mathrm{E}+01$

$0.00 \mathrm{E}+01$

1.14E-05

7.94E-04

2.79E-04

2.60E-02

1.17E-03

$50 \%$-ile

$2.10 \mathrm{E}+01$

428E-08 $\quad 2.40 \mathrm{E}-04$

4.42E-12 1.25E-11

4.17E-09 1.50E-08

$1.19 \mathrm{E}-03 \quad 1.87 \mathrm{E}-02$

3.59E-04 1.33E-03

$1.28 \mathrm{E}-00$

$9.09 \mathrm{E}+03$

1.19E-02

6.25E-10

6.11E-13

5.47E-04

1.53E-02 
Tank Anal. Un

\begin{tabular}{|c|c|}
\hline U203 & Pa231 \\
\hline U203 & Pu238 \\
\hline U203 & Pu239 \\
\hline U203 & Pu240 \\
\hline U203 & Pu241 \\
\hline U203 & Pu242 \\
\hline U203 & Ra226 \\
\hline U203 & $\operatorname{Ra228}$ \\
\hline U203 & Ru106 \\
\hline U203 & Sb125 \\
\hline U203 & Se79 \\
\hline U203 & Sm151 \\
\hline U203 & Sr89/90 \\
\hline U203 & Tc99 \\
\hline U203 & Th229 \\
\hline U203 & Th232 \\
\hline U203 & Tin126 \\
\hline U203 & Tritium \\
\hline U203 & U232 \\
\hline $\mathbf{U} 203$ & $\mathbf{U} 233$ \\
\hline U203 & U234 \\
\hline U203 & U235 \\
\hline U203 & U236 \\
\hline U203 & U238 \\
\hline U203 & Y90 \\
\hline U203 & Zr93 \\
\hline U204 & Al \\
\hline U204 & $\mathbf{B i}$ \\
\hline U204 & Ca \\
\hline U204 & Cl \\
\hline U204 & $\mathrm{CO3}$ \\
\hline U204 & $\mathrm{Cr}$ \\
\hline U204 & F \\
\hline U204 & $\mathbf{F e}$ \\
\hline U204 & $\mathbf{H g}$ \\
\hline U204 & $\mathbf{K}$ \\
\hline U204 & $\mathbf{L a}$ \\
\hline U204 & $\mathbf{M n}$ \\
\hline U204 & $\mathbf{N a}$ \\
\hline U204 & $\mathbf{N i}$ \\
\hline U204 & NO2 \\
\hline U204 & NO3 \\
\hline U204 & $\mathbf{P}$ \\
\hline U204 & $\mathbf{P b}$ \\
\hline U204 & PO4 \\
\hline U204 & $\mathbf{S}$ \\
\hline U204 & $\mathbf{S i}$ \\
\hline U204 & SO4 \\
\hline U204 & Sr \\
\hline U204 & TOC \\
\hline U204 & $\mathbf{U}$ \\
\hline U204 & $\mathbf{Z r}$ \\
\hline U204 & Ac227 \\
\hline U204 & Am241 \\
\hline U204 & $\operatorname{Am} 243$ \\
\hline U204 & Ba137: \\
\hline U204 & C14 \\
\hline U204 & Cd113 \\
\hline U204 & $\mathrm{Cm} 242$ \\
\hline U204 & Cm243 \\
\hline U204 & Cm244 \\
\hline U204 & Co60 \\
\hline U204 & Cs134 \\
\hline U204 & Cs137 \\
\hline U204 & Eu152 \\
\hline U204 & Eu154 \\
\hline U204 & Eu155 \\
\hline U204 & 1129 \\
\hline
\end{tabular}

\section{May $\frac{B 8}{98}$}

8.19E-06

1.91E-01

$1.38 \mathrm{E}+01$

1.73E-00

$1.06 \mathrm{E}+01$

4.48E-05

4.01E-07

4.67E-12

3.01E-08

1.50E-02

5.43E-03

$1.94 \mathrm{E}+01$

$1.53 \mathrm{E}+03$

1.79E-01

7.78E-10

$6.84 \mathrm{E}-12$

8.35E-03

3.23E-01

5.00E-06

1.85E-07

1.15E-01

$4.87 \mathrm{E}-03$

2.51E-03

1.12E-01

$1.53 \mathrm{E}+03$

2.57E-02

$2.19 \mathrm{E}+03$

$1.19 \mathrm{E}+01$

$1.25 \mathrm{E}+01$

1.00E-00

$5.49 \mathrm{E}+01$

$3.88 \mathrm{E}-00$

$3.97 \mathrm{E}+01$

$2.70 \mathrm{E}+01$

$6.20 \mathrm{E}-00$

2.18E-00

$0.00 \mathrm{E}+01$

$8.10 \mathrm{E}-01$

$1.80 \mathrm{E}+02$

$3.90 \mathrm{E}+01$

$3.00 \mathrm{E}+01$

$1.19 \mathrm{E}+02$

$6.88 \mathrm{E}-00$

$7.24 \mathrm{E}+01$

$2.13 \mathrm{E}+01$

1.70E-00

2.37E+01

5.09E-00

3.40E-01

4.67E-00

$1.40 \mathrm{E}+01$

2.60E-01

1.10E-07

$3.09 E-03$

2.82E-08

$3.72 E+01$

8.26E-04

2.60E-03

2.77E-05

6.31E-07

9.83E-07

3.57E-04

1.25E-05

$3.93 \mathrm{E}+01$

$1.30 \mathrm{E}-03$

8.70E-03

6.30E-02

1.14E-05
Sept98

$8.19 \mathrm{E}-06$

4.73E- 02

3.03E-00

$4.28 \mathrm{E}-01$

2.61E-00

1.11E-05

4.01E-07

4.67E-12

3.01E-08

1.50E-02

5.43E-03

$1.94 \mathrm{E}+01$

$2.35 \mathrm{E}+03$

1.79E-01

7.78E-10

6.84E-12

8.35E-03

3.23E-01

1.03E-06

$3.80 \mathrm{E}-08$

2.35E-02

$1.00 \mathrm{E}-03$

5.16E-04

2.29E-02

$2.35 \mathrm{E}+03$

2.57E-02

$2.19 \mathrm{E}+03$

$1.19 E+01$

$1.25 \mathrm{E}+01$

$1.00 \mathrm{E}-00$

$5.49 \mathrm{E}+01$

3.88E-00

$3.97 \mathrm{E}+01$

$2.70 \mathrm{E}+01$

$1.45 \mathrm{E}-00$

2.18E-00

$0.00 \mathrm{E}+01$

8.10E-01

$1.80 \mathrm{E}+02$

$3.90 \mathrm{E}+01$

$3.00 \mathrm{E}+01$

1.19E+02

$$
\text { NA }
$$

$7.24 \mathrm{E}+01$

$2.13 E+01$

NA

$2.37 \mathrm{E}+01$

$5.09 \mathrm{E}-00$

3.40E-01

4.67E-00

$1.40 \mathrm{E}+01$

2.60E-01

1.10E-07

2.09E-04

1.90E-09

3.72E +01

8.26E-04

2.65E-03

1.87E-06

4.26E-08

6.63E-08

3.57E-04

1.25E-05

$3.93 \mathrm{E}+01$

1.33E-03

8.70E-03

6.30E-02

1.14E-05
$1 \%$-ile $10 \%$-ile

50\%-ile

90\% ile

$99 \%$-ile

$0.00 \mathrm{E}+01 \quad 0.00 \mathrm{E}+01$

$0.00 \mathrm{E}+01$

$5.27 \mathrm{E}-03$

$3.81 \mathrm{E}-01$

4.77E-02

$0.00 \mathrm{E}+01$

$0.00 \mathrm{E} \div 01$

$0.00 \mathrm{E}+01$

$2.92 \mathrm{E}-01$

$1.24 \mathrm{E}-06$

$1.80 \mathrm{E}-08$

$2.09 \mathrm{E}-13$

$0.00 \mathrm{E}+01$

$2.19 \mathrm{E}-09$

$0.00 \mathrm{E}+01$

$0.00 \mathrm{E}+01$

2.60E-04

$0.00 \mathrm{E}+01$

$0.00 E+01$

$3.75 \mathrm{E}-04$

$0.00 \mathrm{E}+01$

$0.00 \mathrm{E}+01$

$0.00 \mathrm{E}+01$

$0.00 \mathrm{E}+01$

$0.00 \mathrm{E}+01$

$0.00 \mathrm{E}+0 \mathrm{I}$

$0.00 \mathrm{E}+01$

$0.00 \mathrm{E}+01$

$0.00 \mathrm{E}+01$

$0.00 \mathrm{E}+01$

$0.00 \mathrm{E}+01$

$0.00 \mathrm{E}+01$

$0.00 \mathrm{E}+01$

$0.00 \mathrm{E}+01$

$0.00 \mathrm{E}+01$

3.61E-04

$0.00 \mathrm{E}+01$

$1.83 \mathrm{E}-06$

8.65E-06

3.95E-05

$7.29 \mathrm{E}-06$

$0.00 \mathrm{E}+01$

1.31E-05

$0.00 \mathrm{E} \div 01$

2.72E-06

4.63E-08

4. $25 \mathrm{E}-06$

5.37E-04

$1.46 \mathrm{E}-07$

1.18E-04

8.77E-05

$6.99 \mathrm{E}-06$

$1.45 \mathrm{E}-07$

2.37E-05

$1.86 \mathrm{E}-06$

3.27E-06

5.83E-06

$5.46 \mathrm{E}-07$

5.82E-06

1.82E-05

$0.00 \mathrm{E}+01$

$0.00 \mathrm{E}+01$

2.54E-07

$0.00 E+01$

$0.00 \mathrm{E}+01$

$0.00 \mathrm{E}+01$

$0.00 E+01$

$0.00 E+01$

$0.00 E+01$

$0.00 E+01$

5.19E-08

$0.00 \mathrm{E}+01$

5.76E-04

$0.00 E+01$

$0.00 \mathrm{E}+01$

$0.00 \mathrm{E}+01$

4.17E-03

1.22E-05

$0.00 \mathrm{E}+01$

$0.00 \mathrm{E}+01$

4.78E-04

4.30E-02

$0.00 \mathrm{E}+01$

$0.00 \mathrm{E}+01$

$0.00 \mathrm{E}+01$

$0.00 \mathrm{E}+01$

$0.00 \mathrm{E}+01$

$0.00 \mathrm{E}+01$

$0.00 \mathrm{E}+01$

9.72E-04

$1.21 \mathrm{E}+02$

$2.20 \mathrm{E}-01$

$6.59 \mathrm{E}-01$

2.07E-00

$1.22 \mathrm{E}+01$

$1.93 \mathrm{E}-00$

$3.81 \mathrm{E}-01$

2.95E-00

2.95E-01

1.19E-00

1.59E-02

9.02E-01

$1.91 \mathrm{E}+02$

$1.09 \mathrm{E}-01$

$3.18 \mathrm{E}+01$

$9.94 \mathrm{E}+01$

$3.27 \mathrm{E}-00$

8.51E-02

5.88E-00

$1.02 \mathrm{E}-00$

$1.05 E-00$

$2.88 \mathrm{E}-00$

$2.26 \mathrm{E}-01$

$1.76 \mathrm{E}-00$

5.24E-00

1.68E-02

$0.00 E+01$

$1.10 \mathrm{E}-01$

$1.09 \mathrm{E}-09$

3. $33 \mathrm{E}-00$

$1.95 \mathrm{E}-05$

$1.24 \mathrm{E}-04$

1.07E-06

2.44E-08

$3.80 \mathrm{E}-08$

2.01E-02

1.12E-06

$1.78 \mathrm{E}+02$

$0.00 \mathrm{E}+01$

$0.00 \mathrm{E}+01$

$0.00 E+01$

$0.00 \mathrm{E}+01 \quad 5.39 \mathrm{E}-07$

\subsection{E-06}

1.91E-01

$1.38 \mathrm{E}+01$

$1.73 E-00$

$1.06 \mathrm{E}+01$

4.48E-05

4.01E-07

4.67E-12

3.01E-08

1.50E-02

5.43E-03 


\begin{tabular}{|c|c|c|c|c|c|c|c|c|c|c|c|}
\hline Tank & Anal. & $U_{n}$ & May $\frac{B B}{98}$ & Sept98 & 1\%-jle & $10 \%$-ile & $50 \%$-ile & $90 \%$-ile & 99\%-ile & Mean & Std Dev \\
\hline 04 & 993 & $\mathrm{Ci}$ & $5.91 \mathrm{E}-04$ & $6.91 \mathrm{E}-04$ & $0.00 \mathrm{E}+01$ & $1.57 \mathrm{E}-05$ & -04 & $E-03$ & $E-02$ & 3 & $1.12 E-02$ \\
\hline 04 & Ni59 & $\mathrm{Ci}$ & $34 E-04$ & $2.34 E-04$ & $E+01$ & $E-05$ & & $\mathrm{E}-03$ & & & $1.62 \mathrm{E}-03$ \\
\hline 204 & Ni63 & $\mathrm{Ci}$ & $2.20 \mathrm{E}-02$ & $2.20 \mathrm{E}-02$ & $0.00 \mathrm{E}+01$ & $1.59 \mathrm{E}-03$ & $\mathrm{E}-02$ & E-01 & $9.43 E-01$ & & $1.52 \mathrm{E}-01$ \\
\hline 204 & Np237 & $\mathrm{Ci}$ & 4.15E-05 & $4.15 E-05$ & $0.00 \mathrm{E}+01$ & $1.60 \mathrm{E}-06$ & 4.15E-05 & $1.67 \mathrm{E}-04$ & 4.12E-04 & $E-05$ & $8.77 \mathrm{E}-05$ \\
\hline 204 & Pa231 & $\mathrm{Ci}$ & 2.60E-07 & $2.60 \mathrm{E}-07$ & $0.00 \mathrm{E}+01$ & $0.00 \mathrm{E}+01$ & $2.60 \mathrm{E}-07$ & E-06 & $2.67 \mathrm{E}-06$ & $E-07$ & $6.02 \mathrm{E}-07$ \\
\hline 204 & Pu238 & Ci & $1.90 \mathrm{E}-01$ & $1.28 \mathrm{E}-02$ & $0.00 \mathrm{E}+01$ & $34 \mathrm{E}-03$ & $1.90 \mathrm{E}-01$ & 7.63E-01 & $1.89 \mathrm{E}-00$ & $3.20 \mathrm{E}-01$ & 4.01E-01 \\
\hline 204 & Pu239 & $\mathrm{Ci}$ & $1.18 \mathrm{E}-00$ & $8.21 \mathrm{E}-01$ & $0.00 \mathrm{E}+01$ & $\mathrm{E}-02$ & 1.18E-00 & 4.74E-00 & $1.17 \mathrm{E}+01$ & $8-00$ & 2.49E-00 \\
\hline 204 & Pu240 & $\mathbf{C i}$ & acl. in 239 & $1.16 \mathrm{E}-01$ & $0.00 \mathrm{E}+01$ & 02 & 1.18E-00 & $4.74 E-00$ & $1.17 \mathrm{E}+01$ & -00 & $E-00$ \\
\hline 204 & 1 & $\mathbf{C i}$ & 01 & 7. & 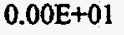 & & 1 & 01 & & & +01 \\
\hline 204 & 242 & $\mathrm{Ci}$ & 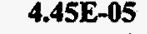 & 3.0 & & & & 04 & 4 & & E-05 \\
\hline 204 & 226 & $\mathrm{Ci}$ & Q-08 & $2.14 \mathrm{E}$ & 0.0 & & 2.1 & 08 & 5. & & -07 \\
\hline 204 & 228 & $\mathrm{Ci}$ & $2.21 \mathrm{E}-12$ & $2.21 \mathrm{E}-12$ & 0.0 & 1.2 & 2.2 & -12 & 6.1 & 4. & $1.20 \mathrm{E}-11$ \\
\hline 204 & 106 & $\mathbf{C i}$ & $2.09 \mathrm{E}-09$ & 2.09E-09 & $0.00 E+01$ & $4.01 \mathrm{E}-11$ & 2.09E-09 & $E-09$ & 2.1 & 09 & $4.49 \mathrm{E}-09$ \\
\hline 204 & 25 & $\mathrm{Ci}$ & $5.97 \mathrm{E}-04$ & $5.97 \mathrm{E}-04$ & +01 & .05 & 5.9 & -03 & $5.30 \mathrm{E}-02$ & 03 & $9.30 \mathrm{E}-03$ \\
\hline 204 & 9 & $\mathbf{C i}$ & -04 & $1.80 \mathrm{E}-04$ & 0.0 & 1.5 & 1.8 & -04 & 3 & & $1 E-03$ \\
\hline 204 & 51 & Ci & 6. & 6.4 & 0. & 0. & & -00 & 00 & 00 & $5 E-00$ \\
\hline 204 & 90 & $\mathbf{C i}$ & & 1. & & & & 04 & & & 04 \\
\hline & & Ci & & & & & & & & & 20 \\
\hline 204 & Th229 & $\mathbf{C i}$ & $3.12 \mathrm{E}-10$ & 3.12E-10 & & & 3.1 & -09 & 09 & 10 & $7.22 \mathrm{E}-10$ \\
\hline 3204 & Th232 & $\mathbf{C i}$ & 3.05E-13 & $3.05 \mathrm{E}-13$ & $0.00 \mathrm{E}+01$ & +01 & $3.05 \mathrm{I}$ & $1.24 \mathrm{E}-12$ & 3.13E-12 & -13 & $7.06 \mathrm{E}-13$ \\
\hline 3204 & Tin126 & $\mathbf{C} \mathbf{i}$ & $2.73 E-04$ & 2.73E-04 & $0.00 \mathrm{E}+01$ & $1.30 \mathrm{E}-05$ & 2.73 & $1.90 \mathrm{E}-03$ & $4.81 \mathrm{E}-03$ & 6.8 & $8 \mathrm{E}-04$ \\
\hline J204 & Tritium & $\mathrm{Ci}$ & $7.70 \mathrm{E}-03$ & $7.70 \mathrm{E}-03$ & $0.00 \mathrm{E}+0 \mathrm{I}$ & $E-03$ & 7.701 & $2.20 \mathrm{E}-02$ & 02 & 02 & $9.18 \mathrm{E}-03$ \\
\hline J204 & $\mathbf{U} 232$ & Ci & 4.89E-06 & 2.10E-07 & 0.06 & +01 & 4.8 & & & & E-05 \\
\hline J204 & 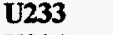 & $\mathrm{Ci}$ & & & & & & & & & 07 \\
\hline J204 & 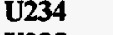 & 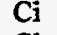 & & & & & & & & & \\
\hline $\mathrm{J} 204$ & 235 & Ci & & & $E+01$ & +01 & & 02 & 4.93E-02 & & E-02 \\
\hline J204 & U236 & $\mathbf{C i}$ & $2.50 \mathrm{E}-03$ & $1.06 \mathrm{E}-04$ & $0.00 \mathrm{E}+01$ & & $2.50 \mathrm{E}$ & $\mathrm{E}-02$ & $2.57 \mathrm{E}-02$ & & $5.79 \mathrm{E}-03$ \\
\hline $\mathrm{U20}$ & U238 & $\mathrm{Ci}$ & $1.09 \mathrm{E}-01$ & $4.67 \mathrm{E}-03$ & $0.00 E+01$ & $0.00 E+01$ & $1.09 \mathrm{E}-01$ & $4.43 \mathrm{E}-01$ & $1.12 \mathrm{E}-00$ & 1.8 & $2.52 \mathrm{E}-01$ \\
\hline ? & Y90 & $\mathbf{C i}$ & 1.10E-01 & $1.10 \mathrm{E}-01$ & $0.00 \mathrm{E}+01$ & $2.39 E-05$ & & $1.34 \mathrm{E}-00$ & $1.03 E+01$ & $7.24 \mathrm{E}-01$ & $2.60 \mathrm{E}-00$ \\
\hline & 03 & & & & II & 05 & -04 & -02 & -02 & & \\
\hline
\end{tabular}




\section{Distribution}

No. of Copies

\section{OFFSITE}

2 Office of Scientific and Technical Information

\section{ONSITE}

Lockheed Martin Hanford Company

J.W. Cammann

R2-11

B. C. Simpson

R2-12
No. of Copies

\section{ONSITE}

Pacific Northwest National Laboratory

B. G. Amidan

K5-12

G. Chen

K5-12

T. J. DeForest

K7-97

T. A. Ferryman

K5-12 (15)

S. A. Hartley

K5-12

J. G. Hill

K7-94

C. A. Lo Presti

K5-12

Technical Report Files (5) 